
H. M.

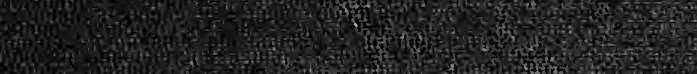
O. W

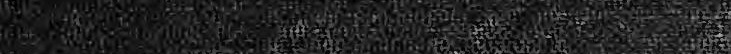
OPing

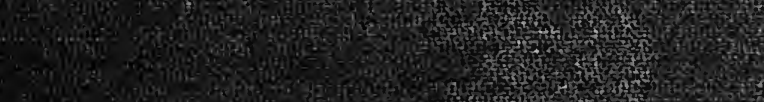
P. N. T2.

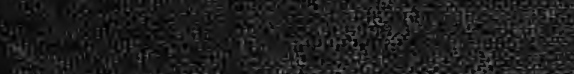

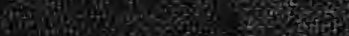
3. Whe oritives A (5) (4) (9.5) 过

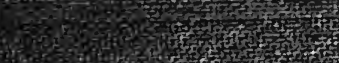

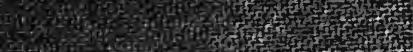
Fin 7.9.5.

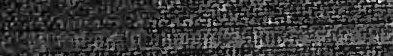
4.

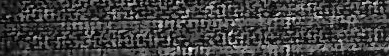
SOA (0) 


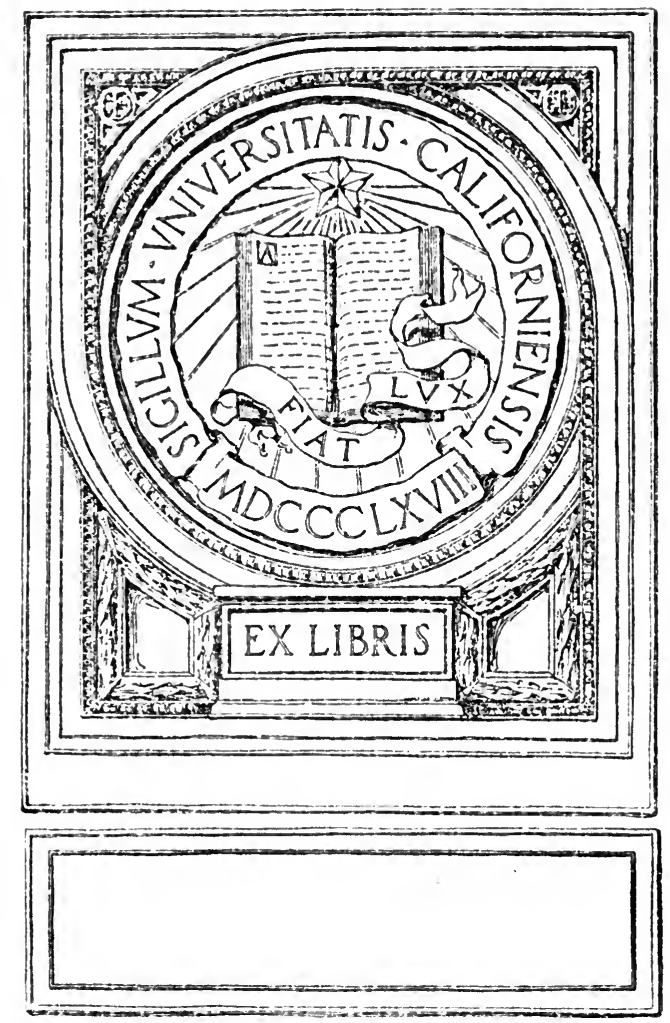




Digitized by the Internet Archive in 2007 with funding from Microsoft Corporation 



\section{THE TREATMENT OF ACUTE INFECTIOUS DISEASES}




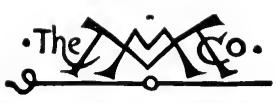

THE MACMILLAN COMPANY

NEW YORK - BOSTON - CHICAGO - DALLAS

ATLANTA - SAN FRANCISCO

MACMILLAN \& CO., Limited

LONDON - BOMBAY - CALCUTTA MELBOURNE

THE MACMILLAN CO. OF CANADA, Ltd. rORONTO 


\title{
THE TREATMENT OF ACUTE INFECTIOUS DISEASES
}

\author{
BY \\ FRANK SHERMAN MEARA, M. D., Рн.D.
}

Professor of Clinical Medicine and formerly Professor of Therapeutics in the Cornell University Medical College in New York City. Consulting Physician to Bellevue Hospital, New York; to the Mountainside Hospital, Montclair, N. J.; to the Morristown Memorial Hospital, Morristown, N. J.; to Overlook

Hospital, Summit, N. J.; to Dover Gencral Hospital, Dover,

N. J.; Mount Vernon Hospital, Mount Vernon, N. Y.; Lawrence Hospital, Bronxville, N. Y.; Associate Attending

Physician to St. Luke's Hospital, New York City

SECOND EDITION, REVISED

\section{ANem \\ THE MACMILLAN COMPANY \\ 1921}




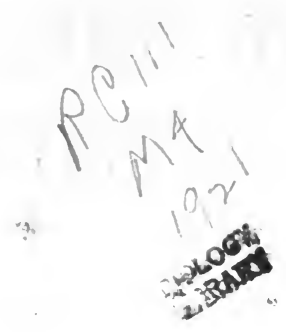

Copyright, 1916 and 1921

Br THE MACMILLAN COMPANY

Set up and printed. Published January, 1916.

Reprinted with corrections, August. 1916;

September, 1917.

Second Edition completely revised and reset Published, June, 1921. 
To the memory of my father SHERMAN T. MEARA

whose encouragement was my inspiration this book is dedicated 



\section{PREFACE TO THE SECOND EDITION}

IN the revision of a book on Acute Infectious Diseases a review of the literature on the recent epidemic of influenza, as well as the camp epidemics of measles, streptococcus pneumonia, meningitis and other infectious processes treated in this book, entails a task of no inconsiderable magnitude.

It is hoped that these subjects will be found brought quite up to date. Moreover, it has seemed wise to include the more common acute infections of the respiratory tract, coryza, tonsillitis, laryngitis, and tracheo-bronchitis, and to add a chapter on acute pleurisy; furthermore, two clinical complexes recently brought to our attention, the one probably incident upon the influenza epidemic, the other consequent upon military operations, encephalitis lethargica and trench fever have been added and for the sake of completeness two less commonly encountered infections that have been the object of much careful research work, ratbite fever and Rocky Mountain spotted fever. It has been deemed advisable to retain the chapter on Grip as written. It covers a picture seen each winter as the result of an acute infection from one of several organisms but does not adequately present epidemic influenza. For this reason a new chapter has been devoted to the latter.

As a result of the camp epidemics of measles, streptococcus pneumonia has been studied as never before and it has been deemed proper to consider this apart from pneumococcus pneumonia.

It gives me great pleasure to acknowledge here my indebtedness to Prof. Oscar W. Bethea of Tulane University, whose great courtesy, kindly criticisms, and helpful suggestions have contributed much of value to this revision.

My thanks are further due to my assistant, Dr. Connie M. Guion, without whose intelligent coöperation, the completion of my task would have been still longer delayed and to the fidelity and tireless devotion of my secretary, Mrs. Mary Nielsen. Finally I have to express my appreciation to the helpfulness of Dr. Minor B. Hill for his article on Intubation, to Dr. Alfred S. Taylor for his treatise on empyema and to Dr. William Parks, Dr. Malcolm Goodridge and Dr. Arthur W. Bingham for many helpful suggestions.

The kindly letters of many friends have been a compensation for the efforts already expended on this work and a stimulus for the added labor of revision. 



\section{PREFACE TO FIRST EDITION}

To the layman the word "physician" connotes a therapeutist; one whose professional end and aim it is to cure the sick. If he thinks of the physician at all as a student of the science of medicine or as a diagnostician it is only in the sense that he is busied with matters that shall lead to the prevention, cure or alleviation of disease; and yet every practitioner of medicine, seeking assistance in the exercise of his art, feels the disproportion of the much that is offered him of the cause, the course and the consequences of disease and the little that is afforded of the practical application of this knowledge to its legitimate uses, getting the sick man well. As a teacher of therapeutics I have always felt that this branch of medicine has been unduly neglected both in the college curriculum and in the text-book. With the text-book my experience has been that it either speaks in generalities assuming knowledge on the part of the student or physician, when such an assumption should annul its very reason for being, or it catalogues and compiles endless measures and procedures without effort to exercise a judgment of their relative values. The few exceptions to this statement make themselves conspicuous.

Successfully or not, it has been the attempt of the author to avoid these extremes of error.

Each chapter has been made to deal with an individual disease in a thoroughly practical manner; each little detail of procedure being explained so that the reader may actually apply it. Moreover, the reason for the procedure, as based on our latest information, both with respect to physical therapy and drugs, the author has sought to give.

Constant and confusing reference to the literature has been avoided, but an effort to give due credit has been made.

The book, finally, must be looked on as an expression of the author's individual opinion and will be didactic rather than critical.

A summary at the end of each chapter is designed to add to its usefulness as a ready reference for the busy practitioner and as a review to the student.

F. S. M. 



\section{CONTENTS}

CHAPTER

I Febrile Conditions.

II Diet in Acute Infectious

III Acute Rheumatic Fever. . . . . . . . . . . . . . . . . . . . . 30

IV Acute Rhinitis.......................... 71

V Tonsillitis and Vincent's Angina . . . . . . . . . . . . 80

VI AcUte LaRYNGitis.......................... 94

VII Acute Bronchitis and Tracheitis................. 101

VIII Pleurisy (Pleuritis) $\ldots \ldots \ldots \ldots \ldots \ldots \ldots \ldots \ldots \ldots \ldots \ldots \ldots$

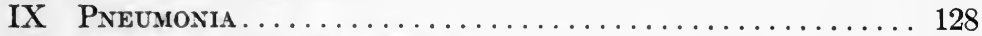

X Streptococcus Pneumonia.................... 208

XI The Treatment of Grip or Sporadic Influenza .......216

XII EPIDEMIC INFLUENZA.......................... 235

XIII Encephalitis Lethargica..................... 271

XIV Typhoid and Paratyphoid Fevers............... 277

XV Malaria............................ 322

XVI Dysentery - Bacillary and Ayebic .............. 353

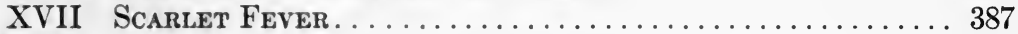

XVIII DiphTHERIA............................. 443

XIX Measles............................. 487

XX Rubella (German Measles)................. 512

XXI Varicella (Chicken PoX)................... 514

XXII Pertussis (Whooping Cough)................ 520

XXIII MUMPS (PAROTITIS)........................ 545

XXIV Glandular Fever......................... 555

XXV Cerebro-Spinal Meningitis.................... 560

XXVI Poliomyelitis (Infantile Paralysis) .............. 590

XXVII Small Pox (Variola)....................... 608

XXVIII TYPHUS FEVER........................... 625

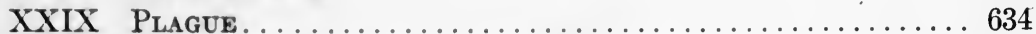

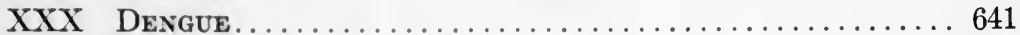

XXXi Asiatic Cholera...........................6 646

XXXII Malta Fever............................. 656

XXXIII Ŕocky Mountain Spotted Fever................ 664

XXXIV LEPROSY.............................. 670

XXXV ANThrax..............................678

XXXVI GLaNdERS or FARCY ....................... 687

XXXVII Foot and Mouth Disease. . . . . . . . . . . . . . . . . 693

XXXVIII PsitTacosis ..................................698

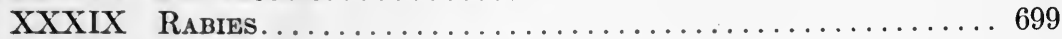

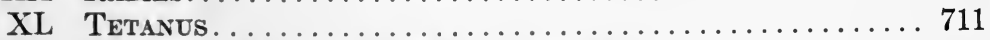

XLI INfECTIOUS JAUndice (WeIL's Disease)............. 728

XLII Yellow FeVER............................... 735

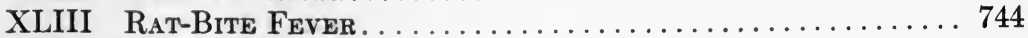

XLIV Trench Fever.................................. 751

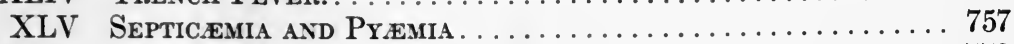

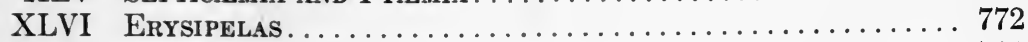

INDEX................................. 781 



\section{THE TREATMENT OF ACUTE INFECTIOUS DISEASES}





\section{TREATMENT OF ACUTE INFECTIOUS DISEASES}

\section{CHAPTER I}

\section{FEBRILE CONDITIONS}

\section{GENERAL PRINCIPLES}

IT is one of the most hopeful features of modern therapy that it is taking cognizance of the fact that the organism has been perfecting means of defense since first it became the seat of disease and that these means of defense are to be read not merely in the subtile elaboration of antitoxic bodies in the tissues and the marshalling of counter-forces in the blood elements, but also in the alteration of the functions of organs. Emesis as a symptom is primarily an effort to unburden the stomach of deleterious substances; diarrhea is protective in the same sense; cough removes secretions which are both irritants and mechanical impediments to the respiration; pain ensures rest to an affected part and so on. Interference with these symptoms must be undertaken with discretion lest it become pernicious. It is the knowledge of when to let alone and when to interfere that constitutes the art of therapy. Emesis, diarrhea and cough may become a source of exhaustion; pain which has pointed the way to the trouble and hinted the need of rest be illy borne when continued and fever rise to degrees that are incompatible with its purpose and constitute hyperpyrexia. At this point, treatment steps in to modify and assist, not to disregard Nature's signals.

Fever, as the physician knows it, is almost invariably the result of bacterial action, so cannot be dissociated from toxemia; but pyrexia and toxemia show but little parallelism; indeed, the worst forms of toxemia may be accompanied by no pyrexia at all, while a relatively high temperature may be seen with a minimal toxemia. Briefly, then, unless the degree of temperature is inordinately high, that is, constitutes a hyperpyrexia, the treatment of fever is not an antipyretic treatment but an antitoxemic treatment, and such fall of temperature as accompanies our efforts is incidental to them and not the object at which we aimed.

The treatment of fever involves the appreciation of certain broad principles that in the main are physiological. They are not numer- 
ous, but they are of funciamental importance. We shall take them up seriatim. (1) rest; (2) diet, including the ingestion of water; (3) fresh air; (4) water locally applied, that is hydrotherapy; (5) drugs; (6) hyperpyrexia. I think it little matters what name we give the febrile process or the organism inducing it. The procedures are much the same.

Rest. It is a valuable form of mental exercise for a physician to pause occasionally and review some of the fundamental facts of physiology that are relevant to the problem in hand. First one should recall the fact that the sum total of the body's activities are but the expression of the conversion of potential energy of the foodstuffs into these different forms of energies; that the body observes an accurate balance between the intake and output; that the law of conservation of energy obtains in the human body as well as elsewhere, and, therefore, when the intake is lessened, the expenditure should be diminished and useless forms of work should be avoided that useful purposes may be subserved.

All this means that in fever rest is imperative and that, as the body cells are expending energy in the elaboration of protective substances and the processes of repair are active in inflamed tissues, muscular unrest and muscular work should be avoided. Physiologists tell us that carbon dioxide, an end product of the combustion of foodstuffs in the production of energy, may be taken as a measure of the energy of work and heat arising in the body and that the same man who during sleep eliminates 22 grams of carbon dioxide an hour will, when awake and exercising the greatest amount of muscular relaxation possible, eliminate 31 grams, and under conditions that we ordinarily term rest, such as most patients observe, eliminates 38 grams. These figures are cited to call attention to what a relative term "rest" is.

Again, in febrile conditions, the vascular and other vital centres are all too often sought out and when we recall the lessened number of heart beats in recumbency and slight fall of pressure under the same circumstances we appreciate the economy exerted in behalf of these organs by rest.

Rest means something more than going to bed, though that is imperative. It means that the bed must be comfortable, so that energy shall not be wasted in the effort to maintain strained positions or avoid uncomfortable ones; it means competent nursing; it means measures directed at sleeplessness and at delirium. Mental rest is equally important, for though the loss of energy in the mental processes per se is minute, strained and restless muscles are the results of anxiety and concern, as, indeed, are perverted functions in many or- 
gans. Leave the sick room to the sick; exclude solicitous friends, Job's comforters, mourners before the fact. Avoid the introduction of business matters or domestic concerns, and let quiet and order reign. The contrast between a well-ordered, neat, cool, sick-room, and a hot stuffy room with six to ten visitors, gas-jets in full action and Babel and Chaos regnant is one of the most striking that can be witnessed.

Diet." See Chap. II, Diet in Acute Infectious Disease. Again let us appeal to physiology for facts. They are (1) That the amount of work done and the amount of heat given off by the human body each day is derived from the food taken in that day or stored in the body from previous meals and that that store is not inexhaustible. (2) That the amount of energy demanded by a man at rest each day amounts on the average to 33 calories ${ }^{1}$ per kilo of body weight which in a man of average weight amounts to 2,300 calories. (3) That a sick man at rest requires as many calories of energy as a well man. (4) That fever makes certain demands in excess of those obtaining in health, because the increased temperature per se, that is, pyrexia above $102^{\circ} \mathrm{F}$. increases the output some 25 per cent. on an average, thus raising our caloric requirements to 40 per kilo or about 2,800 for the man of average weight. (5) That the toxins of disease are destructive of body tissue in varying degrees, a destruction which may be overcome or minimized by a sufficient intake of food. (6) That the amount of protein needed each day in health is about 100 grams. (7) That in fever the requirement is about the same or may be a trifle less, 75 to 85 grams, which is enough to prevent protein loss when enough carbohydrates are added. The appreciation of these facts is increasingly important in the prolonged fevers, such as typhoid, certain bronchopneumonias or septic conditions.

One other fact is of special importance in orientating us correctly with reference to this subject; namely, that such studies as have been undertaken to determine the efficiency of the processes of digestion and assimilation of the foodstuffs in fever show that, except at the onset, these processes are scarcely impaired.

Briefly, in the beginning of fever, do not push the food; respect the meaning of anorexia; but later increase daily to the approximation of theoretical needs. Remember that there are other foods than milk that are easily digested, including cereals, bread, butter, eggs.

Water is an important item in the treatment of fever; all the water that the patient wants and his wants should be determined by offering him water at frequent intervals, as his cerebral condition may be such as to prevent him asking for the amount he needs.

${ }^{1}$ This calorie is the large calorie and is that amount of heat that will raise one kilo of water through $1^{\circ} \mathrm{C}$. 
Studies of the water intake in certain infections such as pneumonia show that the amounts are sometimes extraordinary, 3,000 to 5,000 c.c. a day, and that the patients who are given a sufficiency seem to do best.

Fresh Air. That the sick require fresh air would seem a thesis too simple to require emphasis, and yet many physicians make no protest against the vitiation of the sick room by a swarm of visitors and burning gas-jets and make only a half-hearted appeal for an open window.

The bad effects of ill ventilation are due in part to the accumulation of carbon dioxide gas. In the country pure air contains about 3 volumes of the gas to 10,000 volumes of air. In rooms and work-shops it may rise to over 30 volumes, and in the night with the gas burning to nearly 50 .

We consider it niggardly in the hospital if we do not allow 1,000 cubic feet to each sick infant, but how many of our patients get 1,000 cubic feet of fresh air to themselves in the sick room?

The increase of carbon dioxide is not the only evil in ill ventilation, for contamination of the air with bacteria and varied forms of dirt must ensue; while heat, moisture, or stagnation and odors add their depressing effects.

But there is another quality to fresh air when taken in the open that adds materially to its value; it is the element of cold given either by the temperature of the air or by the effects of its movement. Pembrey, in a masterly article on Respiratory Exchange in Hill's Recent Advances in Physiology and Biochemistry, says, "The success of the open-air treatment depends it would seem, not so much upon any greater purity of the air as upon free exposure; the open air increases the combustion and respiratory exchange improves the appetite and augments the metabolism."

More than that, it is an every-day demonstration to the students in our ward that the exposure of the febrile patient to the open, cold, clear air will induce a rise of blood pressure over that obtained in a well-ventilated ward equal to or better than that obtained from our usual vasomotor stimulants, with the added advantage of being continuously sustained which does not obtain with the drugs.

I am convinced of the very great value of the open air treatment of fever. The pulse and respiration improve, the cerebral intoxication diminishes and the patients are almost universal in their commendation of it.

To reap success, however, the technique must be correct. The patient's bed must be so made as to keep the body always warm, by enclosing the bed in impermeable material like rubber sheeting or paper, the use of the hot water bottle, and by sheltering from winds. The good effects, I believe, come from the action of cold on the nerves of the 
mucous membrane of the nose or of the face, producing reflexly an improved vascular condition.

Water Locally Applied, Hydrotherapy. I am firmly convinced of the efficacy of remedial measures, operating on the various functions through the surface of the body, that branch of the healing art included under the head of physical therapy. This field has been grossly neglected for various reasons; in part because of the dominant position occupied by drug administration in the minds of most practitioners and very largely because of the time and effort entailed in the administration of these measures and the acquisition of a proper technique.

Water is a very valuable instrument in combating the evil results of fever. Baruch, whose persistent advocacy of hydrotherapy constitutes a real service to American Medicine and has not received half the recognition it deserves, points out that the value of water lies not so much in its physical constitution as in the fact that it is an admirable material for the conveyance of heat and cold and that strictly speaking, thermotherapy is a better term than hydrotherapy in the use of water. A few facts should be borne in mind in considering hydrotherapy. (1) That we are aiming rather at the toxemia than at the pyrexia. (2) That the reaction to the use of cold water is the sine qua non of success. (3) That friction is as important as the cold in many of the procedures.

The good effects of the cold water are obtained largely through the nervous system. The impulses pouring in through the countless nerves upon the centres result in a slower, stronger heart beat, and an improved vasomotor tone, which can be measured in terms of an increased blood pressure; respiration is deepened, cerebral processes improved, and metabolism enhanced.

Without detailing the various procedures, one may mention as the best known, the Brand bath in typhoid fever and the chest-compress in pneumonia.

Through the cold air and cold water we effect much the same results; the time of year, the facilities of the environment and the reaction of the patients determine an indication for one or the other.

Drugs. Drug administration, like the other measures, is aimed not at the pyrexia but at the toxemia, and that the fever is influenced is due to a relief of the toxemia or the conditions determined by it.

Cathartics occupy an important position among the drugs, because they prevent a stagnation within the bowel and the consequent absorption of putrefactive products.

The toxins of disease impinge upon the vital nervous centres and upon none more certainly than upon the vasomotor centre. Our great dread in 
the severe intoxication, such as typhoid fever, pneumonia, diphtheria, scarlet fever, and sepsis is a circulatory failure.

Circulatory Failure. The term "circulatory" is used advisedly, because upon this we can all agree, but when we try to fix this failure in the heart or in the vasomotor apparatus an abundant opportunity for difference of opinion arises.

It is the belief of some clinicians that in the large majority "of cases it is the vasomotor mechanism that is at fault. For this reason drugs that act either on the vasomotor centre or on the vessels seem rational. Such drugs include caffeine and I think it should be given in sufficient dosage and so administered as to guarantee its arrival at the goal desired. I give it in the form of one of the soluble double salts, that of caffeine and sodium benzoate or caffeine and sodium salicylate, in doses of five grains every four hours, and such studies as I have made upon its effects on blood pressure show that its effects do not last even throughout this period. Next to caffeine, I use a 10 per cent. or 20 per cent. solution of camphor in olive oil or sesame oil and use more than the usual dose, giving at least five grains every four hours, hypodermically, often alternating with the caffeine, thus giving a dose every two hours. Personally I have less faith in strychnine.

That we can exclude the heart in all cases I do not believe and my experience leads me to believe that in all cases of circulatory failure the digitalis group is more reliable than the vaso-motors. I use digitalis in doses of one-half an ounce of the infusion (15 c.c.) three or four times a day or $\mathrm{m}$. $\mathrm{xxx}(2$ c.c.) of the tincture at the same intervals, and appreciating that it will not become operative in less than twentyfour hours, use in urgent cases as an initial dose one-half milligram (gr. 1/120) of strophanthin intramuscularly. This whole question of digitalis medication will be found fully elaborated under Pneumonia, Chap. IX.

No mention has been made so far of the antipyretics.

In the early stage with bounding pulse, aconite may afford some relief by slowing the heart through the vagus, the coal-tar preparations may be used with relief of headache and other pains, but here again the fall of temperature is incidental. These drugs are depressants and should never be used where the circulation is impaired.

Hyperpyrexia. As has been said, fever may be looked upon as of purposeful intent, as a conservative effort on the part of the organism to accomplish something useful to itself. If this be so, measures directed at the fever as such are misdirected if not pernicious, and it will be noted that the treatment of fever outlined in this chapter has been directed at the toxemia accompanying the febrile movement rather than at the 
latter; but as was said in the beginning, any symptoms primarily useful may in the end become harmful; one may say that Nature has overshot the mark. Excessive temperatures fall into this category as well as prolonged sustained temperatures.

Hyperpyrexia threatens cell function and cell integrity as increased protein destruction shows. We see its effects best in insolation and in certain rheumatic fevers. In these cases reduction of heat is life saving.

We have no better method than the direct application of cold in the shape of cold baths or ice rubbed upon the surface of the body. The patient may be put into a bath of $90^{\circ} \mathrm{F}$. and the water cooled down to $70^{\circ} \mathrm{F}$. to $75^{\circ} \mathrm{F}$. The body is kept immersed until the body temperature falls below the danger point, but it is wise to remove the patient when it falls to $102^{\circ} \mathrm{F}$., as collapse may ensue on efforts to reduce it to normal. If such occurs, stimulants and heat are indicated, as in collapse from any other cause.

Antipyretics of the coal-tar group are not comparable in efficiency or safety to cold water, but may be used where it is impossible to use the latter.

No attempt has been made in this chapter to go into details; only generalizations have been laid down with special emphasis given to those branches of therapy commonly neglected, and for this reason serum therapy or vaccine therapy, which are specific for individual disease, have been omitted, but will be considered in their appropriate places.

\section{SUMMARY}

Symptoms of disease are primarily the expression of purposeful intent on the part of organs or tissues working under abnormal conditions; are conservative efforts and should be respected by the therapist.

Interference with symptoms is demanded only when, having subserved their purpose, by continuing they themselves become the source of exhaustion.

Fever is a term ordinarily used to cover both pyrexia and toxemia.

The treatment of fever, then, is the treatment of pyrexia and toxemia, and, except in hyperpyrexia, more especially to toxemia.

The fundamental principles of the treatment of fever are:

1. Rest.

2. Diet.

3. Fresh Air.

4. Hydrotherapy.

5. Drugs.

6. Treatment of hyperpyrexia. 


\section{Rest.}

Lessens demand on muscular and mental energy and conserves energy for purposes of repair and production of immune bodies.

Rest in practice means:

Rest in bed.

Comfortable bed.

Well ventilated room.

Good nursing.

Quiet.

Relief from anxiety and concern.

Sleep.

Diet.

Determined by the realization that the body heat and the performance of the functions of the muscles, organs and tissues demand a source of energy and that the only source of energy is food or the tissues themselves.

Energy demands at rest are the same for a man in health or in illness: about 33 calories per kilo or 2,300 calories for a man of 154 pounds.

Pyrexia increases demands by some 25 per cent. on an average, i. e., about 40 calories per kilo or 2,800 to 3,000 for an adult.

Toxemia by destruction of tissue makes even greater demands, especially in prolonged fevers, and amounts of food equal to 50-60-70 or even more calories per kilo may be given; the amount depending on the patient's ability to handle it and on the gain or loss of weight.

Protein needs are from 65 to 85 grams.

The more prolonged the fever the more urgent the observance of food requirements.

Gastro-intestinal functions, secretion, motility and assimilation are affected but slightly in early days of fever or in profound intoxication.

\section{Rules for Feeding.}

Do not urg $€$ food in early hours or days of fever against the patient's anorexia, but when it disappears appreciate and provide for the demand for food.

Water should be given freely; offered to the patient, not awaiting his request.

\section{Fresh Air.}

Proper ventilation of room-exclusion of visitors. Open air-on verandah or porch-observing the proper technique in bed-making. (See Pneumonia.)

The value of fresh air consists mostly in its stimulating effects on vital centres, reflexly, through its effect on nerve supply to the skin of face and mucous membranes of air passages.

Essential factors.

Live air, moving air. 


\section{Hydrotherapy.}

Effect same as that of air. Water is a conveyor of temperature. Cold stimulates vital centres reflexly through nerves from the skin. Essentials to success: water must be cold; friction must be applied (it is the alternating cold of water and heat of hand that affords stimulation).

Patient must react.

\section{Drugs.}

Cathartics to prevent or relieve stagnation and absorption of products of putrefaction.

Circulatory stimulants.

Vaso-motor stimulants.

Caffeine in form of soluble salt (double salt of sodium salicylate or benzoate and caffeine). Dose gr. iij-v (0.20-0.30 Gm.) every 4,3 or 2 hours.

Camphor in 10 per cent. to 20 per cent. solution in oil (olive or sesame oil) in doses equal to gr. v, $(0.33 \mathrm{Gm}$.) of the camphor every 4,3 or 2 hours.

Heart stimulants. For immediate action strophanthin into muscle or vein. Dose, 1/2 milligram (gr. 1/120). Follow up with or in less urgent cases begin with digitalis infusion 5 ss (15 c.c.) or Tincture m. $x x x$ ( 2 c.c.) three or four times a day until desired effects ensue or signs of toxicity of any kind appear.

Antipyretics-for pain and discomfort at the beginning, in the sthenic stage and even then with caution.

\section{Hyperpyrexia.}

Sudden rise of temperature to $106^{\circ} \mathrm{F}$., or above, or prolonged temperatures of $104^{\circ} \mathrm{F} .-105^{\circ} \mathrm{F}$.

\section{Cold.}

Best as baths. Put patient in at $90^{\circ} \mathrm{F}$., and lower temperature to $75^{\circ} \mathrm{F}$. or $70^{\circ} \mathrm{F}$. Take out when temperature of body falls to $102.5^{\circ} \mathrm{F}$. Slush baths or cold sponges.

Antipyretics indicated only when cold water is inaccessible.

Specific treatment. (See individual diseases.) 


\section{- CHAPTER II}

\section{DIET IN ACUTE INFECTIOUS DISEASES}

FEeding the sick is both a science and an art; a science in so far as it takes cognizance of the great laws of supply and demand in a physiologic sense; an art in so far as it affects the application of these laws to the individual.

Energy. All matter is endowed with a certain amount of energy, which manifests itself differently, under different conditions. Certain chemical substances, by virtue of the nature of the elements that compose them and their relationship to each other, that is, their structure, have the power to produce one or more manifestations of energy. They are said, then, to have potential energy.

To give rise to the manifestations of energy, these substances must have a certain degree of instability or lability; that is, must be capable under definite conditions of undergoing changes in structure, disruptions, etc., all of which give rise to motion.

The substances of which our bodies are composed and food on which they continue to exist are chemical substances of a highly labile character, possessing potential energy, which in the process of catabolism or breaking down, give rise to motion, which conveyed to other bodies is manifested as work or is expressed as heat, to neglect the lesser forms of energy in the human body, such as the electrical.

Energy is as indestructible as matter itself. It can be transformed from one kind to another, but it cannot be destroyed nor yet created anew. Different manifestations of energy have definite quantitative relationships to each other, as Joule's great experiments on the mechanical equivalent of heat showed. He demonstrated that the amount of work necessary to raise $1 \mathrm{Gm}$. of water through $1 \mathrm{~cm}$. of distance was that required to raise $42.55 \mathrm{Gm}$. of water through $1^{\circ} \mathrm{C}$.

I have said that the conversion of the potential energy of the body or of the food into one or the other manifest forms of energy occurs under definite conditions. The definite condition in the body which gives character to its manifest energy is the "vitality" of the cell. Moore ${ }^{1}$ calls the living cell an " energy transformer." He says:

"The living cell is not a peculiarly constructed membrane obeying,

1 Recent Advances in Physiology and Biochemistry, edited by Leonard Hill, London, 1908. 
even where it seems most directly not to obey, the physical laws of diffusion and osmosis; but is an energy machine or transformer by virtue of the operation of which a form of energy appears, peculiar in its manifestations and phenomena to living matter."

The kind of energy that this transformer, the living cell, produces out of the potential energy of the body and its foodstuffs is almost entirely thermokinetic, that of work and heat, and as we can express work in terms of heat, we may reduce the total activities of the body to terms of heat and express them as equal to so many "calories" or heat units.

The "calorie" is a term used to express that amount of heat which is required to raise 1 c.c. or $\mathrm{Gm}$. of water through $1^{\circ} \mathrm{C}$. More properly, this is termed a "small calorie," while the term "large calorie" is used for the amount required to raise 1 kilogram or 1 liter of water through $1^{\circ}$ C., i. e., 1 large calorie equals 1,000 small calories. Calories referred to in this work are large calories.

The Caloric Balance of the Human Body. As has just been said, the human body (the cells collectively) is an energy transformer; or to put it in other terms, the human body is like a steam-engine, consuming fuel for the purpose of doing work and producing heat; but the human body differs from a steam-engine in this, that, lacking fuel, it will consume its own structure up to the point of a complete collapse of the mechanism; so that, to maintain the integrity of the transformer or engine, it is necessary that the output by it of work and heat shall be exactly met by the fuel afforded it. To know the amount of food the human body requires, then, we must know the amount of work it does and the amount of heat it elaborates in a given time.

Now Nature has given to the individual a most amazing regulator to adapt the supply to the demand on which no device of man can ever improve; that is, appetite. When one thinks that day after day and year after year, the body retains a fixed or approximate weight, under the most varying demands of work, and consequently of food, under the guidance of this monitor, one is lost in admiration; but as there are times, in the stress of disease, when this regulator fails or by the dictates of false theories, is disregarded, we need to know what the demands actually are, in order that we may guarantee that they may be met.

The determination of the caloric output in man has been made possible by the use of the calorimeter chamber of Pettenkofer and Voit, elaborated and perfected by Atwater and Benedict, whose work in this field has been the most extensive and accurate ever done, and whose studies of food-values have been accepted by the government as stand- 
ards; and by the study of the respiratory quotient determined by the simpler apparatus of Zuntz.

Work. Still another way to determine the caloric needs of man is to determine the heat values of the foods habitually taken by normal individuals in different callings. These analyses of the average intake and output agree wonderfully well. For men doing light or sedentary work, clerks, professional men, literary men, the requirements are between 2,400 and 2,600 calories; for those whose work is a little more arduous, as machinists, shoemakers and the like, 2,900 to 3,100 calories; for those doing hard muscular work, smiths, masons, etc., 3,300 to 3,600 calories, while those doing very hard work run up much higher, the Maine woodsmen demanding some 7,000 to 8,000 calories. Football players require nearly as much.

What concerns us most is the requirement of a man at rest, for this is the condition in which we find our patients. Rest, too, is a relative term, and as used here means that degree of activity which obtains. when a patient is put to bed, which is a very different state from complete muscular relaxation or that prevailing in sleep. At rest the demands are about 2,300 calories or 33 calories per kilo of body weight, the average man weighing 70 kilos or about 150 pounds.

A fact of prime importance to us as practitioners is that educed from the work of Magnus-Levy, ${ }^{1}$ namely, that the total metabolism is the same in the sick man as in health, so far as the demands of muscular effort and heat production go; but, as we shall see, fever and toxemia increase these demands.

Weight. The caloric requirement of an individual depends not only on the amount of work he does, but also on the size or bulk of his body; and while we have spoken of the requirements of a man at rest as 2,300 calories, it is meant for a man of average weight, while such wide variations as a man of 200 pounds and a woman of 100 pounds are common in our every-day experience. The requirements are best thought of in units of weight, as so many calories per kilo-for a man at rest 33 calories per kilo.

Here, too, certain modifications have to be made, as in the excessively obese, whose fat is hardly to be considered in the same light as the more actively metabolizing tissues.

Age. The figures "33 calories per kilo" obtain only for adults. In the young the growth has to be taken into consideration, which in certain periods is more intensive than in others; e. g., in the first three months of life the demands are about 110 calories per kilo; in the second three months, 100; in the last half of the first year, 90; at the end of the second

1 Quoted from Otto Cohnheim: Die Physiologie der Verdauung und Ernährung. 
year, 75 to 80 ; at 10 years, 60 , and at 14 years, 52 . Indeed, as Cohnheim observes, the boy of 10 requires as much food as the adult who does not do muscular work, while the youth of 14 to 16 must eat 3,000 calories, about one-fourth more than his father who leads an intellectual life.

Body-surface. In consideration of the fact that in all warm-blooded animals the production of heat is the most imperative function of metabolism, and further that the dissipation of it is largely a function of the skin, there stands a relationship between these two, closer than that between metabolism and weight. In a small sphere the surface is greater, proportionally to the contents, than in a large one, for the contents increase as the cube of the radii, while the surfaces increase as the squares, so that in the smaller bodies the radiation is relatively greater and the heat production must be relatively more intense.

So it is that we find in the infant a requirement of 100 calories against 33 in the adult, but when the surfaces are compared, it is found that reckoned in calories per square decimeter, it is about the same from 10 weeks to adult life, 13 to 14 calories per square decimeter (Pfaundler and Schlossmann). The unit of weight is, however, so much more readily obtained that we use it in preference.

Metabolism experiments carried on during rest have shown that whether reckoned on the one unit or the other they are always the same for all individuals, men or women, sick or well. It must be remembered, however, that the conditions of the experiment produce more nearly real rast than obtains in the sick-room. Differences of temperament determine very different degrees of muscular activity, while certain phases of an acute infection, like delirium, become a matter of serious import in the consideration of rest.

As has been said, all this energy manifested as work and heat by the body, resides potentially in and is derived from the food. The food, then, must furnish periodically just what the body has expended in order to keep the latter intact, for if the food is deficient, "the deficit will be made up out of the body itself, as long as it can stand the drain. Our patient at rest, then, must have 2,300 calories of food a day.

Caloric Value of Foodstuffs. The foodstuffs are divided into three classes, fats, carbohydrates, and proteins. Strictly speaking, salts and water are foodstuffs, but they have no caloric value.

Rubner's figures for the caloric value of foodstuffs still stand to-day, such slight modifications as have been made having but little practical significance. They are as follows:

$1 \mathrm{Gm}$. of fat furnishes 9.3 calories.

1. $\mathrm{Gm}$. of carbohydrate furnishes 4.1 calories.

$1 \mathrm{Gm}$. of protein furnishes 4.1 calories. 
With these figures and a table of the average composition of the common foods, like those of Atwater, published by the U. S. Department of Agriculture, it is no difficult task to arrange a dietary. ${ }^{1}$

The first step, then, in arranging a diet in acute infectious diseases, is to provide, as a minimum, as many calories as are demanded by the individual when at rest in health, 33 calories per kilo, or 2,300 in the average man.

A diet must not only contain sufficient heat units, it must also be properly balanced with reference to the three foodstuffs, and of these three, the most important is protein.

Protein Needs. Two facts of great importance are to be kept in mind, which differentiate protein from the other foodstuffs; first, in the adult, under normal circumstances, the daily demand for protein is fixed and does not vary with the amount of work done, as is the case with the other two foodstuffs; and second, there is no provision for a storage of an excess of protein, as is the case with fats and carbohydrates. Therefore, any excess must be catabolized and eliminated.

This daily amount of protein amounts to about $100 \mathrm{Gm}$., or $16 \mathrm{Gm}$. of nitrogen. Voit's figures are $118 \mathrm{Gm}$. of protein as ingested, which, allowing for the amount found in the stools, gives about $100 \mathrm{Gm}$. net. The analysis of dietaries the world over has given a remarkable unanimity of results, which have confirmed Voit's figures.

That a man may perform hard work for months and remain in excellent condition on an amount of protein far less than this, one-half and even less, has been demonstrated by Chittenden in the most elaborate and extensive set of experiments ever caried out on this subject. Physiologists, however, though they must accept the possibility of this low protein need as a fact, are not yet inclined to argue from this demonstration that such an intake would subserve the interests of the organism in the long run to the best advantage.

Functions of Protein. Protein subserves three important functions. First, it furnishes substance, building material, to the body. This is spoken of as the "storage" of nitrogen. Second, it repairs the daily waste of the body, the wear and tear of the machine. Third, it furnishes heat in its combustion, that is, is a fuel.

It is only in early life that the first of these functions, storage, is of prime importance. At that time it is one of the most imperative of physiologic functions. The continuance of growth, when weight may

${ }^{1}$ An excellent table of food values expressed in units of 100 calories may be obtained from the J. A. M. A., Chicago, for 10 cents, entitled Extracts from Practical Dietetics, Irving Fisher.

Food Values: Edwin Locke-is an excellent hand book. 
be stationary or regressive, is a familiar phenomenon to the pediatrist, expressive of the fact that the protein is seized on with avidity even when the fuel-value of the food is insufficient for body needs. It is a curious fact that the actual amount of protein ingested in infancy is about the same month after month, but the amount devoted to storage decreases, while that used for wear and tear increases. In the first two weeks over 75 per cent. is stored; at two months 40 per cent.; at five months 23 per cent. When an individual has attained his or her growth, storage ceases, except on special occasions; these are during pregnancy and lactation, in the establishment of hypertrophy of groups of muscles, as in the training of an athlete, and during convalescence from wasting disease or after a long fast.

The second function, repair of wear and tear, is the important one in adult life. This has to do with maintaining the integrity of the machine as a whole and has nothing to do with the amount of muscular work, so it is a daily constant, regardless of the amount of exertion the body has been put to. It is for this purpose that the food should contain $100 \mathrm{Gm}$. of protein a day in the adult diet.

The third function is incidental. Protein does furnish heat, but it is not an economical source of supply. In the infant the proper amount of protein furnishes about 7 per cent. of the calories; in the adult about 14 per cent.

The reason that protein is not an economical fuel lies in that peculiar property known as its "specific dynamic action." When food is ingested, the physiologic processes sequential to it produce heat. The amount of such heat induced by fat and sugar is slight and negligible, but not so protein. The ingestion of this substance gives rise to a production of heat equal to a trifle more than 30 per cent of its caloric value.

The source of this heat is a matter of dispute, but it would seem to be due to the work of the glands of secretion and to cell activities with which we are less acquainted. Lusk has intimated that in the case of protein it may be in part due to the denitrogenization of the amidobodies. This heat is lost to the body, is waste heat, under the conditions of temperature and physical regulation of heat in which man lives. He cannot utilize this heat for purposes of cell-life.

This loss of heat by the specific dynamic action of protein may be expressed in this way: that to get the caloric value of $100 \mathrm{Gm}$. of protein one must ingest $140 \mathrm{Gm}$. of the food. It is this increased heat that must be dissipated that has led naturally to a limitation of meat diet in hot weather and to its general elimination from the diet of fever.

The second step, then, in arranging a diet in the acute infectious diseases, is to provide enough protein to replace the wear and tear, 
an amount which hardly differs from the general demand in health. It may be placed at 65 to $80 \mathrm{Gm}$.

Fat and carbohydrates differ from protein in two very striking ways as articles of diet; first, when utilized by the body the oxidation is complete and they are eliminated as carbon dioxide or water, while the nitrogenous moiety of the protein is not completely oxidized and entails work on the part of the kidney to eliminate it, which the other foodstuffs do not require; second, an excess of fat and sugar beyond immediate needs is stored in the body as fat and does not demand prompt elimination like the excess of protein.

The proportions of the three foodstuffs in an average dietary is protein $100 \mathrm{Gm}$., fat $50 \mathrm{Gm}$., and carbohydrates 400 to $500 \mathrm{Gm}$., giving in the neighborhood of 3,000 calories.

Milk. No food in infectious diseases has been used so much as milk, and frequently to the exclusion of all others.

An excellent milk will show on analysis about 4 per cent. of fat, 4.5 per cent. of sugar, and 3.5 per cent. of protein, or, to the liter, $40 \mathrm{Gm}$. of fat, $45 \mathrm{Gm}$. of sugar, and $35 \mathrm{Gm}$. of protein. Using Rubner's caloric values as given above, we see that the caloric value of a liter of milk is 700 ; reduced to quarts, there are about 640 calories to the quart, or 20 to the ounce-a convenient figure as a mnemonic.

A liter contains $35 \mathrm{Gm}$. of protein or $1 \mathrm{Gm}$. to the ounce.

It will be seen presently that a low estimate of the caloric needs in fever of an average individual is 3,000. To meet this one would have to administer over a gallon of milk, which would contain over $140 \mathrm{Gm}$. of protein.

These figures sufficiently demonstrate how illy adapted an exclusively milk diet is to meet the needs of an individual suffering from an acute infection.

Standard Portions. When one comes to study the tables of composition of foodstuffs, it is plain that the establishment of a dietary to respect the caloric requirements and the protein needs of a patient is a task outside the time and patience of an active practitioner. It was with an appreciation of this fact that Professor Irving Fisher, of Yale, published his tables of standard portions.

It will be seen from the table representing a few of these articles selected how remarkably the ordinary servings of common articles of food, or at least multiples or simple fractions of them, amount to just 100 calories.

In some of our hospitals the nurses are now instructed to bring food to the patient in these portions, so that the estimate of the daily intake can be easily made with a degree of accuracy that, though not adapted to 
research work, answers all practical purposes, and because the daily exhibition of these portions is an education to both the medical and nursing staffs. It soon becomes an easy matter to think of the ordinary articles of diet in terms of units of 100 calories.

\section{TABLE OF FOOD-VALUES IN UNITS OF 100 CALORIES ${ }^{1}$}

Milk, 5 oz.

Protein in $\mathrm{Gm}$.

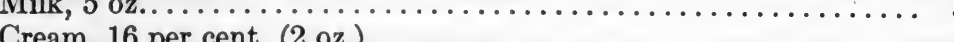

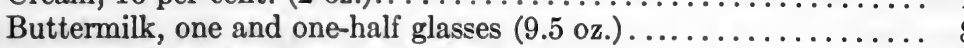

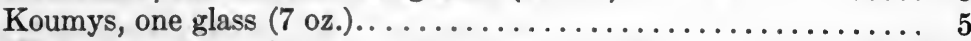

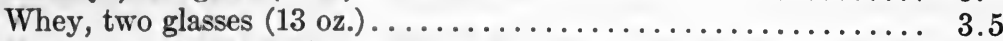

Eggs, one and one-half ............................ 10.

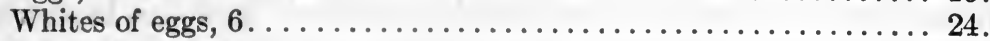

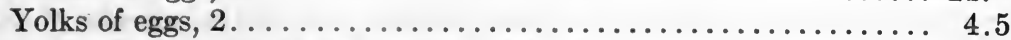

Oatmeal, one and one-half serving $(5.5 \mathrm{oz}.) \ldots \ldots \ldots \ldots \ldots \ldots \ldots, 4.25$

Boiled rice, ordinary cereal dish $(3 \mathrm{oz}.) \ldots \ldots \ldots \ldots \ldots \ldots \ldots \ldots, 2.5$

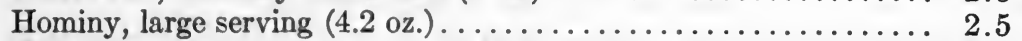

White bread, home made, one thick slice $(1.25$ oz.)........... 3.2

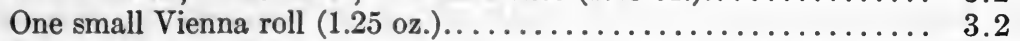

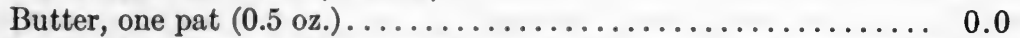

Sugar, three teaspoonfuls, one and one-half lumps $\left(\begin{array}{ll}0.8 \text { oz. }) . . \ldots . . . & 0.0\end{array}\right.$

Macaroni, cooked, average serving.................... 3.0

Spaghetti " " "

Tapioca " " "

Prunes, edible portion, three large.................. 0.9

Lemon juice, 9 oz.............................. 0.0

Oll, one-third ounce............................ 0.0

Codfish, two servings $(5 \mathrm{oz}.) \ldots \ldots \ldots \ldots \ldots \ldots \ldots \ldots \ldots \ldots \ldots \ldots \ldots \ldots . . .23$.

Halibut steaks, one serving $(2.8 \mathrm{oz}.) \ldots \ldots \ldots \ldots \ldots \ldots \ldots \ldots \ldots \ldots . . .15$.

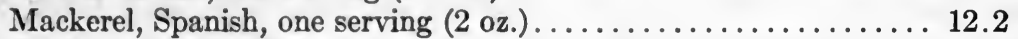

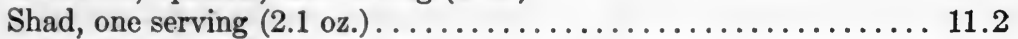

Salmon, small serving $(1.5$ oz.)........................ 7.3

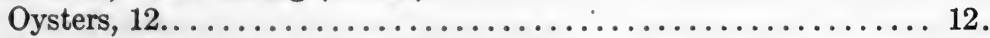

Roast beef, ordinary serving $(1.8 \mathrm{oz}.) \ldots \ldots \ldots \ldots \ldots \ldots \ldots \ldots \ldots \ldots \ldots \ldots .$.

Small sirloin steak $(1.4$ oz.)...................... 7.5

Leg of lamb or mutton, ordinary serving $(1.8 \mathrm{oz}.) \ldots \ldots \ldots \ldots \ldots 10$.

Lamb chop, one, small $(1 \mathrm{oz}.) \ldots \ldots \ldots \ldots \ldots \ldots \ldots \ldots \ldots \ldots \ldots .6 .6$.

Bacon, small serving, medium fat $(0.5$ oz. $) \ldots \ldots \ldots \ldots \ldots \ldots \ldots$. 1.5

Chicken, broiler, edible portion, large serving (3.2 oz.)......... 19 .

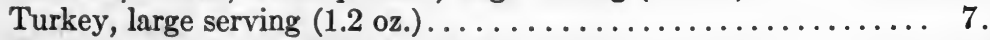

Potato, baked, one, good size $(3$ oz.)................. 3.75

Potato, sweet, baked, one-half average potato $(1.7 \mathrm{oz}.) \ldots \ldots \ldots \ldots . . .61 .5$

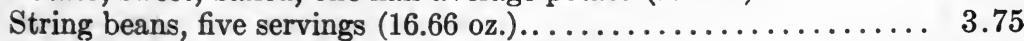

Spinach, two ordinary servings 6.1 oz.) $\ldots \ldots \ldots \ldots \ldots \ldots \ldots \ldots . .7$

${ }^{1}$ Adapted from Prof. Fisher's Tables. Loc. cit. 
Peas, green, one serving $(3 \mathrm{oz.}) \ldots \ldots \ldots \ldots \ldots \ldots \ldots \ldots \ldots \ldots .7$

American or Swiss cheese, 1.5 cubic inches $(0.75$ oz.). . . . . . . 6 .

One baked apple, 3.3 ounces..................... 0.5

The object of our consideration is a patient suffering from an acute infectious disease." So far I have discussed only the food requirements of a man in health at rest. Such a discussion is relevant to this subject, however, because the requirements of the patient are the same plus a certain additional need determined by the infection itself.

There are four factors that enter into the problem of feeding in acute infectious diseases that render it distinct. They are (1) gastro-intestinal disorders; (2) pyrexia; (3) toxemia; (4) starvation.

Starvation in fever has been a tradition in medicine that has lost but little authority with the passage of time and finds wide acceptance today. The legitimacy of such practice I wish to examine.

Disturbance of Gastro-Intestinal Function. It cannot be denied that the gastro-intestinal tract is not indifferent to the effects of the infection. We are all familiar with the dry mouth, the coated tongue, the anorexia and the tympanites of many of the patients.

One feels little inclined to urge food on so unwilling a receptacle and this feeling has plead potently with many practitioners to establish insufficient, often starvation dietaries.

Investigation has shown that the salivary secretion is lessened, that the output of hydrochloric acid is diminished and the motility of the distal portion of the intestine is impaired. It has been claimed that the motility of the stomach, too, is prejudiced (von Leyden and Klemperer), but von Noorden maintains that that has not been the case in the patients he has studied.

More important still, assimilation seems to be good, and von Leyden and Klemperer ${ }^{1}$ found that in different patients with high fever 89 per cent. to 94 per cent. of easily digestible fat was absorbed and 91 per cent of protein. Moreover, they found no carbohydrate in the stools unless excessive amounts had been ingested or diarrhea prevailed.

Taking these data and what I have seen of liberal feeding in febrile conditions, I am convinced that the ability of the alimentary tract to perform its normal functions in fevers has not been duly appreciated and the withholding of food on the supposition that this organ is crippled is unjustifiable.

I do believe that in the incipiency of an acute infection these functions are more in abeyance than later, but that they quickly become competent. I also believe that the state of the mouth and digestion is due in no small measure to an insufficiency of water and is greatly aggravated,

${ }_{1}^{1}$ Von Leyden and Klemperer: Handbuch d. Ernäkrungs-Therap, 1904, ii, 332. 
not by the giving of food, but by the withholding of food, which entails a destruction of body tissue and sets on foot abnormal metabolic processes.

Fever. The term "fever" as ordinarily used, connotes the sum of two distinct factors: (1) pyrexia, and (2) toxemia. These will be discussed separately, and when, under pyrexia the word "fever" is used, it is to be taken in the sense of an elevated temperature alone, i. e., pyrexia.

Pyrexia. There has been an endless controversy from remote times as to the significance of fever, it being contended on the one hand that it was a deleterious factor in the disease, on the other, that it was beneficial and had a meaning that was to be respected; purposeful, the teleologist would say.

This is not the place to discuss at length fever and its relation to disease, but I am in agreement with the conclusions of careful students of the subject that "fever is a specific reaction against injurious materials which affect the tissues"; that it "is in its essentials a protective reaction," to quote from the excellent article on fever by Dr. W. G. MacCallum. ${ }^{1}$

There has been a wealth of work done to prove this statement, which one will find set forth in detail in the article just referred to and in a critical review of the subject by Hermann Ludke. ${ }^{2}$

Pyrexia below $104^{\circ} \mathrm{F}$. is to be let alone; it is useful; above that figure it adds an element of harm and then antipyretic measures are indicated, but not till then. It must be remembered that many so-called antipyretic measures, such as the cold baths, are directed not toward the temperature, but to the circulatory and nervous system, and are of the highest value when these systems are in distress. The phenomena of fever lead rapidly to the assumption that there is a great increase in heat production and a marked retardation of heat dissipation, in other words, a great accumulation of heat in the body. This is only partially so. The actual increase in the oxidative processes is relatively small, while the relation between heat production and heat dissipation is but little interfered with. What seems actually to take place is the regulation of these processes on a higher level, so to speak; what level, that is, at what temperature, depending on the effects of the toxins on the heat-regulating mechanism. This mechanism, however, under such conditions, does not display the same degree of stability as in the normal individual, but a lability that characterizes the temperature curve in infections, and, indeed, gives their individual stamp to them.

${ }^{1}$ MacCallum, W. G.: Arch. Int. Med., 1909, ii, 569, and the Harvey Lectures, 1908-1909.

' Ludke, H.: Ergebn. d. Inn. Med.u. Kinderh; iv, 493. 
I have said that the increase of heat production in fever is relatively small. The figures vary; sometimes there is no increase, but on the average it amounts to about 25 per cent. This might seem considerable did we not pause to consider that in strenuous muscular exercise the increase of heat amounts to several hundred per cent.; for example, the 8,000 calories of the Maine woodsman to the 2,300 at rest.

The fact that such great increase in heat in the normal man causes no rise of temperature shows that the elevated temperature of fever requires an additional factor to that of heat production; that $l t$ is the result of the toxins as well as heat. However, as our patient is at rest, this 25 per cent. increase has to be added to the 2,300 calories allowed him, in order to make good his daily losses, so that it may be said that the requirement of the febrile patient is 40 calories per kilo or 2,800 calories, near enough to 3,000 to make that our guide.

One other effect of pyrexia I wish to mention; that is, its destructive action on protein. As Lusk says, "Infectious fevers are characterized by a toxic destruction of body protein"; but there are three factors concerned in the loss of nitrogen during an acute infectious process: (1) pyrexia, (2) toxins, (3) starvation.

The study of non-toxic fevers, such as are induced by exposure to heat or by puncture of certain parts of the brain, show that the pyrexia can induce protein catabolism, but it seems to demand a temperature of $102^{\circ} \mathrm{F}$. or over to bring about this protein loss, and experimentation has shown that a sufficient feeding can control this loss.

Toxemia. It was recognized that apart from the effect of pyrexia and apart from starvation, or at least in the presence of food sufficient to meet all the theoretical considerations we have so far set forth, there still continued to be a considerable loss of protein in the acute infectious diseases. This loss came to be accepted as inevitable by the ablest investigators in the field and was attributed to the effects of the toxins and was called the "toxic destruction" of protein. Just how the toxins brought this protein loss was and is a matter of conjecture. It has been attributed by Krehl to actual cell destruction; by others to an exciting effect on metabolism by the toxins; by others to an expression of the production of antibodies and other protective substances. Certain it is that it is dissociated from the pyrexia, as such; for it is often intense when there is no rise of temperature. Taken with this destruction it has been suggested that a weakened power of regeneration exaggerates the difference between catabolism and anabolism.

Starvation. This does not belong to the acute infections as a rule, except as it is inflicted on the patient by an insufficient dietary. Energy must be supplied to the body each day and when the food does not do it, 
the body substance does and the protein is called on to do its share at the sacrifice of cell integrity.

At the present moment one of the most intensely interesting fields of medical research is that devoted to metabolism, the building up and breaking down, anabolism and catabolism, the storage of energy and its dispersion; what one writer has called the "most fundamental characteristic of life, energy-traffic." The intimate processes of protoplasm yield their secrets stubbornly, but we know what they have to work on and we know what they yield back; we know the fuel and the ash, the intake and the output, and from the knowledge of these two, we deduce much that has occurred in the process of the change.

Nitrogen is the basic substance on which the body structure is erected. It is ingested with the food and it is expelled in the urine, the stools and in lesser measure by the skin. In health the intake and output balance each other, and the nitrogen eliminated is divided among a number of combinations which maintain a pretty constant relationship to each other. These nitrogen combinations are spoken of as the " nitrogen partition."

In disease the balance of intake and output is disturbed and the relationship of the combination undergoes a change, so that the study of the nitrogen partition in disease is a tree that bears much fruit.

I have said that the nitrogen elimination is divided among three organs, the kidneys, the intestine, and the skin. As for the skin, its output is negligible except when made to functionate in an unusual manner, as in very profuse perspiration. Under such circumstances it has been known to yield as much as 0.75 to $1 \mathrm{Gm}$. of nitrogen.

The stools, however, contain an appreciable amount of nitrogen. Some very curious ideas prevail even among physicians about the feces; e. g., that they represent the residue of the food; that the amount depends entirely on the amount of food ingested; that if there be no food taken there should be no feces. The stools do not represent the residue of food so long as the food is free from indigestible matter. Stools occur in fasting people and a comparison of these stools with those ordinarily passed shows that there is no difference in their composition or relation of constituents, no matter whether there be much or little food taken, and no matter whether that food be protein, carbohydrate or fat. The amount increases but little with the food and in no sense in proportion to the amount ingested. If excesses of food are eaten, a minimum residue may appear and, of course, cellulose or other indigestible substance is found.

The stools are made up of the residues of the digestive juices, mucus, desquamated epithelium, bacteria, the products of bacterial action, salts 
and water. Of the salts, it must be remembered that the intestine is the excretory organ for iron and calcium and for the most part of phosphorus and magnesium.

The nitrogen content varies from 1 to $2 \mathrm{Gm}$., but on the whole is so constant that it has been considered as furnishing a definite per cent. of the nitrogen ingested (15 per cent.) without prejudice to the accuracy of the metabolism study.

It is to the urine that we turn, however, to derive important information; for 80 per cent. to 90 per cent. of the nitrogen is excreted by the kidney as urea, uric acid, ammonia, xanthin bases, creatinin, and amidobodies.

The study of the total excretion and of its individual constituents is of importance.

Total Nitrogen. It has been invariably found that the amount of nitrogen excreted in fever far exceeds the amount ingested, and so much the more, the more severe the process.

It is common enough to find losses of $8 \mathrm{Gm} ., 12 \mathrm{Gm} ., 16 \mathrm{Gm}$., and more of nitrogen a day; that is, in excess of the intake; and, that, too, when on a diet usually considered satisfactory. This latter figure is the amount of nitrogen demanded in Voit's standard dietary, and if it were interpreted in terms of muscle tissue would mean a loss of a pound of such tissue.

Such figures will be found repeatedly in the tables of Shaffer and Coleman's studies on metabolism in typhoid fever, ${ }^{1}$ and those of Wolf and Lambert ${ }^{2}$ on pneumonia.

The study of the total nitrogen and the determination of the "negative nitrogen balance," as it is called, tells us that there has been loss of substance to the body, but it does not tell us what tissues are suffering this loss, nor yet whether the metabolic processes are carried out in a qualitatively normal manner. Such scant information as we have along these lines is derived from the study of the parts that go to make up the total; that is, the nitrogen partition.

It is the small portions that in the past excited but little interest that are now yielding us the most information. One of these portions is the substance creatinin. When Folin ${ }^{3}$ published his classical analyses of thirty normal urines, he called attention to the fact that this substance creatinin remained practically constant, no matter what the amount of

1 Shaffer and Coleman: Arch. Int. Med., 1909, iv, 538.

${ }^{2}$ Wolf and Lambert: Arch. Int. Med., 1910, v, 406.

${ }^{3}$ Folin: Approximately Complete Analysis of Thirty Normal Urines; Laws Governing the Chemical Composition of Urine; a Theory of Protein Metabolism, Am. Jour. Physiol., 1905, xiii, 45, 66, 117. 
protein ingested. On this fact he elaborated the theory that this substance was an indicator of "tissue" or "endogenous" metabolism in contradistinction to "intermediate" or "exogenous" metabolism, of which urea is the chief representative. Another representative of this "constant" or "endogenous" metabolism is neutral sulphur, ${ }^{1}$ and to a lesser extent, uric acid and ethereal sulphates.

Folin showed that this substance was constant in the individual, but varied in different individuals according to body weight. He spoke of it as an "index to the amount of a certain kind of protein metabolism occurring daily in any given individual," ${ }^{2}$ but defined that kind of "protein metabolism" no further.

Shaffer, ${ }^{3}$ however, added to our information a little further, by showing that not only was the output of creatinin in an individual constant from day to day, but also from hour to hour, amounting from 7 to $11 \mathrm{mg}$. of creatinin nitrogen for every kilogram of body weight per twenty-four hours; this he called the "creatinin coefficient." He, moreover, gave reason to believe that the substance was an index, not of total tissue metabolism, as Folin thought, but of one tissue metabolism, that of the muscle, as its output seemed to show a parallelism to the muscular efficiency or strength of the individual. It has nothing to do with the muscular work, but rather with the machine, the muscle itself. $\mathrm{He}$ suggests that it is an index of muscle tone. If one may use an analogy without too close adherence to details, one might consider the creatinin to represent the friction, the daily wear of the muscle machine, not at all the product of its work.

Here was an important clue to follow up. Any substance that showed the ravages of disease in so important a tissue as muscle was worth looking after. Already studies on the substance in pathologic conditions had assisted to suggest the above view of its significance and further work confirmed it.

It was found that creatinin was diminished in conditions of muscular inefficiency, such as amyotonia congenita, myasthenia gravis, muscular dystrophy, ${ }^{4}$ exophthalmic goitre, and in the as yet undeveloped muscles of the infant. ${ }^{5}$

How is it affected by acute infectious fevers?

${ }^{1}$ The importance attributed by Folin to neutral sulphur as an expression of "endogenous" metabolism has more lately been called into question.

See note 3 on page 22 .

${ }^{3}$ Am. Jour. Physiol., 1908, xxiii, 1.

4 Spriggs: The Excretion of Creatinin and Uric Acid in Some Diseases Involving the Muscles, Quart. Jour. Med., 1907-1908, i, 63.

- Amberg and Rowntree: The Excretion of Creatinin in the Infant, Bull. Johns Hopkins Hosp., February, 1910. 
There is a pretty constant agreement among the investigators that it is increased during the height of the fever and is most marked when the nitrogen loss is the greatest, but there is no numerical relationship between the two. During convalescence and even before, there is a diminution in the excretion which may be attributed to muscular weakness. It must be interpreted in the sense that the febrile condition increases the wear and tear of the muscle tissue. This increase in creatinin was determined for typhoid fever, ${ }^{1}$ for pneumonias, and for erysipelas.

So much for increased wear and tear of the protein constituents of the body during the acute infectious process; but is there anything that bespeaks by its presence actual destruction of a definite tissue? There is a substance in the tissue closely allied to creatinin, which differs from it only by the presence of one molecule of water in its structure (creatinin is a dehydration product of creatin), which does not appear normally in the urine, or appears only in traces. This is called creatin.

Creatin is to be found in the urine only in abnormal conditions. It is found in wasting diseases, such as the cachexia of carcinoma, in starvation, and in fevers. It is derived from the muscle and its presence in the urine means destruction of the substance of that structure. It is very striking during the involution of the uterus after childbirth. It was found in all the studies on fevers referred to, and sometimes ran over into convalescence. Its disappearance from the urine is looked on as a good omen, and what interests us as dietitians is that when made to appear in starving animals it has disappeared under a pure carbohydrate diet.

Besides the creatinin, neutral sulphur and uric acid are both increased and may be looked on as further expressions of increased endogenous metabolism.

So far we have considered quantitative changes rather than qualitative in the nitrogen metabolism. Qualitative changes might be taken as evidence of metabolic inefficiency.

Evidence of this is to be looked for in the so-called "rest nitrogen." This "rest nitrogen" or "undetermined nitrogen" is that nitrogen which is left of the total nitrogen when the nitrogen of urea, ammonia, uric acid and creatinin have been subtracted. If the "rest nitrogen" increases it may be considered as being at the expense of the urea, the most important nitrogenous substance, and so indicate a defective deamidation. The amido-bodies are, indeed, the most important of the substances included in the "undetermined nitrogen." There are, besides

${ }^{1}$ Klercher: "Ueber Ausscheidung von Kreatin and Kreatinin in fieberhaften Krankheiten," Ztschr. f. klin. Med., 1909, lxviii, 22. 
leucin and tyrosin, indol, skatol, glycocoll, proteic acids and albumoses, as well as xanthin bases.

Shaffer and Coleman found but little increase in the "rest nitrogen" in typhoid fever and the same was true of pneumonia investigated by Wolf and Lambert (there was absolute increase, but not in disproportion to total nitrogen). It may be said, then, that as a rule the ureaforming function is but little impaired, an important conclusion in considering the ability of the liver to handle the products of protein digestion.

In grave cases, such as were observed in the study of typhoid metabolism by Ewing and Wolf, the "rest nitrogen" did become high and the urea low.

These authors looked on the phenomenon as indicative of inefficiency of the liver urea formation, recalling the high "rest nitrogen" found in acute yellow atrophy.

Such patients probably suffer from an auto-intoxication in addition to the toxemia of the disease.

All this shows that in acute infectious diseases there is a loss to the intrinsic structure of the body and that while such loss is entirely compatible with a perfect urea-forming function-and, indeed, the latter is but rarely impaired even in severe cases-there are yet a certain number of grave cases in which the liver has apparently through grave changes in its structure found itself incapable of dedamiation and abnormal metabolic processes are in evidence.

With this knowledge it should be the effort of the therapeutist to prevent this loss and perversion of function, or at least to mitigate them. Can the administration of food accomplish this, and, if so, how shall it be ordered?

It would seem natural to conjecture that if the body was losing $10 \mathrm{Gm}$., $15 \mathrm{Gm}$., or $20 \mathrm{Gm}$., of nitrogen a day over the intake, such a loss could be made good or prevented by increasing the intake of nitrogen to that amount. The truth of the matter is that neither the amount of the loss nor any other amount, without the help of the other foodstuffs, can bring about a nitrogenous equilibrium in man.

The work of Folin and Chittenden, showing the small amount of nitrogen which is really needed to establish an equilibrium, would lead us rather to use the smallest amount of protein necessary and avoid the breaking down and elimination of the superfluous nitrogen radicles, by organs burdened with disease, and increasing the heat to the fevered organism by the "specific dynamic action" of the protein.

Shaffer and Coleman were able to establish nitrogen equilibrium 
in typhoid fever on from $10 \mathrm{Gm}$. to $15 \mathrm{Gm}$. of nitrogen, when sufficient carbohydrate was used. This amount will be seen to be less than the Voit standard demands, by a considerable margin.

Fat and carbohydrate both spare protein; not, of course, replacing it, for they are both nitrogen-free. Fat spares the combustion of protein to furnish heat; and weight for weight furnishes, as has been shown, over twice as many calories as carbohydrates. The fat of the body is utilized in this manner as a protein-sparer. Emaciation, which bespeaks its disappearance, makes the sufficiency of food intake all the more imperative to prevent destruction of the body protein.

Carbohydrate is a very much more efficient sparer of body protein than fat. Probably much of the demand on protein in the underfed body is for the carbohydrate moiety of its structure. Carbohydrate is imperative to life and must be had at any cost. This the ingested carbohydrate furnishes, so that it both furnishes heat as does the fat, and also subserves other functions which the fat cannot do.

Nitrogen equilibrium has been struck and the subjects kept at from $5 \mathrm{Gm}$. to $8 \mathrm{Gm}$. days and weeks with comfort and apparent health, when enough carbohydrate and fat are taken.

The above statements hold for the protein-sparers in health. In acute infections their influence is not so marked quantitatively. Von Noorden says that in experimentally induced pyrexia the protein metabolism is not limited to the same amount in the presence of carbohydrates as when the temperature is normal.

It would seem possible, then, by covering the caloric needs of a resting patient and adding the 25 per cent. increase induced by pyrexia, to keep a patient in nitrogenous equilibrium. In an average man this would mean, as before stated, about 3,000 calories. In practice, however, it is not true; and it was for this very reason that protein loss still continued in acute infections, when the theoretical food-needs had been liberally met, that investigators in general were convinced that this loss was due to destruction of the cell substance by the poisons of the disease-- "toxic destruction" - and that no diet could prevent it.

That this loss can be prevented and that such a fact impairs the theory of "toxic destruction," the elaborate observations of Shaffer and Coleman show. They prevented nitrogenous loss and the elimination of creatin and increase of neutral sulphur, which are taken as the expression of cell destruction. They found that from sixty to ninety calories per kilo were needed to effect such a result in typhoid fever. Of this amount forty to fifty or even more calories per kilo must be furnished by carbohydrates. They point out that a loss of $10 \mathrm{Gm}$., of nitrogen or $62.50 \mathrm{Gm}$., of protein can furnish but 250 calories, and have no explana- 
tion to offer for the fact that 2,000 calories of carbohydrate are needed to prevent this loss.

It has been proved, then, that in some men suffering from severe acute infectious disease, the usual protein loss can be stayed by the administration of a large quantity of foods.

Vitamines. One of the most recent contributions to the subject of dietetics is the discovery of certain food elements absolutely necessary to nutrition, which are present in minute quantities and have been termed vitamines. There are two groups of vitamines the chemical nature of which has not been identified, but which take their name from the mode of extraction. They are called "fat soluble A" and "water soluble B." Their absence from foodstuffs produces nutritional disorders of which the best known and most carefully studied is Beri Beri and perhaps the most common marasmus. Bread made of bolted flour and cow's milk which constitute so considerable a portion of the dietary of invalidism, are deficient in vitamines. Whole wheat flour, unpolished rice, barley, yellow meal, white potatoes, peas, beans and other legumes, fruit, eggs, all contain vitamines and should enter in some proportion into the dietary. It is understood that vegetables and fruits must be fresh as the canned products are found deficient in vitamines.

In this chapter I have dealt only with those laws that constitute the science of dietetics. The application of these laws to the individual; the appreciation of those factors that make every individual deviate from the type; the adjustment of theory to conditions, constitute the art of dietetics.

\section{SUMMARY}

The body is a mechanism for the conversion of one kind of energy (potential) into other forms of energy, for the most part heat and work (thermo-kinetic).

Total energy may be expressed in terms of heat.

The heat unit is called the calorie.

Small calorie expresses that amount of heat required to raise 1 c.c. or gram of water through $1^{\circ} \mathrm{C}$.

Large calorie equals 1,000 small calories.

The calories referred to in the text are large calories.

The calorie requirements of a man depend on his size, his age and his activities; hence his calling or occupation.

The requirements at rest (in ordinary acceptance of the term) in bed, are 33 calories per kilo of body weight or for man of 70 kilos (154 pounds) 2,300 calories.

The requirements are the same for man at rest, whether he is sick or well. 
Calorie requirements per kilo are much higher in the young.

Calorie requirements per unit of body surface are much the same at all ages.

\section{Arrangement of Diet in Acute Infectious Diseases}

1. Provide as a minimum the calories demanded by the individual at rest; i. e., for adult man 2,300 calories.

2. Provide enough protein to replace wear and tear; i. e., for an adult 65 to 80 grams.

3. Add to calories demanded at rest the amount required by extra heat production of fever; on an average 25 per cent. rest requirement; i. e., for adult, total of 40 calories per kilo or 2,800 to 3,000 calories.

4. Add calories, especially in form of carbohydrates, to shelter proteid loss. May require 60 or more calories per kilo. Amount determined by loss or gain in weight, i. e., 4,000 or more in total.

\section{Protein Needs.}

Voit's standard 118 grams.

Actual need shown by Chittenden to be less by nearly one-half.

\section{Functions of Protein.}

1. Storage of nitrogen, in growing child, in pregnancy, in lactation, in hypertrophy of muscles from exercise, and in convalescence from disease.

2. Repair-wear and tear of day.

3. Fuel-protein is not an economic fuel because of high "specific dynamic action."

Vitamines necessary for an accurately balanced dietary.

\section{Milk.}

Analysis. Fat, 4 per cent.; sugar, 4.5 per cent.; protein, 3.5 per cent. Caloric value, 700 to 1 liter.

$$
\begin{array}{r}
620 \text { to } 1 \text { quart. } \\
20 \text { to } 1 \text { ounce. }
\end{array}
$$

Protein content, 35 grams to 1 liter.

32 grams to 1 quart.

1 gram to 1 ounce.

Not well balanced for sole article of diet.

Disturbance of gastro-intestinal function slight at beginning of fever.

\section{Fever.}

Ordinary use of term connotes effects of 1. pyrexia, 2. Toxemia.

\section{Pyrexia.}

Increases caloric demand even up to 50 per cent. Average, 25 per cent.

Toxemia accelerates destruction of body protein; demands high calorie intake to prevent it. 


\section{Starvation.}

Inflicted on patient by insufficient diet.

Feeding. Early in fever anorexia is a conservative symptom and to be respected.

After first few days gastro-intestinal functions are competent and food should be administered to meet needs.

Water needs are high in fever. It should be given freely. 


\section{CHAPTER III}

\section{ACUTE RHEUMATIC FEVER}

Theories of Rheumatism. 'It is not my purpose to discuss the numerous theories advanced to account for the phenomena of acute rheumatic fever. I will merely state that it is the consensus of opinion at the present time that the disease is an acute infection. Numerous observers have claimed to have isolated the specific organism, among the most insistent of whom are Poynton and Payne.

Etiology. The classification of this "specific" organism has not as yet been fairly settled; indeed, the identity of the "specific" organisms is much in question. Some believe, moreover, that different organisms can produce the same clinical complex called Rheumatic Fever and one observer, Rosenow, maintains the transmutation of members of the streptococcus group into several forms, each inducing some differences in the clinical picture. This will be touched upon again when discussing vaccine therapy. To Poynton's view has been lent the weight of the authority of Osler's new Modern Medicine, to the pages of which he contributes the article on rheumatism, and in which the various views of the etiology of this disorder are set forth in some detail.

Age. About one-half of all cases of rheumatism occur between the ages of fifteen and twenty-five years; about one-quarter in the next decade, that is, between twenty-five and thirty-five years. My own impression, based on considerable contact with children, is that the figures set for childhood are too low, as the disease is peculiarly insidious at this age, and deviates strikingly from the type as established in the adult. The serious complications are quite as common, even more common than in the adult. Of the cases occurring in childhood, 70 per cent. fall between the ages of ten and fifteen years.

I cannot refrain from intercalating a bit of pediatric wisdom at this juncture: (1) Beware of a diagnosis of rheumatism in infancy. It is so rare that, when authentic, it warrants rushing into print, which is saying a good deal. The so-called rheumatic joints of infancy are almost certainly pyogenic, scorbutic, or syphilitic. (2) Scan every child's heart with care, and seek constantly in the histories for tonsillitis, stiff neck, and especially "growing pains." How many children's lives have been sacrificed to that unfortunate term no man can estimate.

How readily rheumatism in children may be overlooked is shown 
by Langmead who, examining 2,556 English school children, found that 5.2 per cent. of them showed evidences of rheumatism and 4.49 per cent. of them in terms of heart disease.

While this percentage is larger than one would, in all probability, find among our children, still among 1,000 children coming to my clinic at Bellevue Hospital just 1 per cent. showed valvular heart disease, for the most part unrecognized.

Symptomatology. That the disease is rather abrupt in its onset, accompanied by fever, that sore throat is not uncommon, that drenching acid sweats may occur, and that the inflamed joints are the pathognomonic sign, is all well known, and is reiterated here merely to emphasize the points of attacks in the application of therapy. The complications are the important events in the course of rheumatism, and will be considered after rules have been laid down for the simple, uncomplicated case.

Therapy. A sick man invites medical attention for two reasons: First, he wants to be cured of his disease; and second, he wants to bemade more comfortable during his illness. To treat a patient intelligently, it goes without saying that a diagnosis is imperative, but the intellectual satisfaction derived from establishing a diagnosis must not lead to a satiety that eschews further effort directed toward relief of the condition. Such a comment is justified by fact. Still another function to be subserved by the physician is the instruction of the patient how to avoid a repetition of the attack. There are certain measures that may be directed toward any acute illness, others that are aimed at the particular disease in question. This order will be preserved.

Rest. In the rheumatism of adults the painful condition of the joints impels rest, willy-nilly, but this by no means obtains in children. Pain, which he who suffers it looks upon as an unmixed evil, is more often a boon than a bane. If one will get in the habit of analyzing the symptoms of disease in terms of efforts on the part of Nature to accomplish a useful purpose, or as expressions of compensations, he will be amazed to see how many hints these symptoms give that they are to be utilized as allies, not combated as enemies.

In rheumatism there are three emphatic reasons why rest should be insisted on: (1) Because the body cells are busied in combating an intoxication, for which their energy should be conserved as much as possible; (2) because certain tissues are undergoing the alteration incident upon inflammation, and are struggling to accomplish repair; and (3) because the spectre of cardiac involvement is never absent from the disease, and we fear that every increment of activity on the part of that organ may heighten the possibility of the dreaded disaster. 
To illustrate the significance of rest, I will cite the following figures: One knows, as a fundamental physiological fact, that the energy and heat of the body are derived from the combustion of the foodstuffs, and that the carbon of these foodstuffs is in large measure eliminated from the body through the lungs as carbon dioxide; so we can collect and estimate the amount of carbon dioxide eliminated in a unit of time, and look upon the results as expressions of the activity of the body cells during that period. This has been done repeatedly, and the same individual who during sleep eliminates 22 grams of carbon dioxide per hour will, when awake and exercising the greatest amount of muscular relaxation possible, eliminate 31 grams, and under conditions ordinarily considered those of rest, 38 grams. In this light, rest assumes a meaning, and the importance of restlessness and loss of sleep in disease is enhanced in dignity.

The two important factors to be considered in estimating the amount of work done by the heart are the amount of blood expelled and the pressure, that is, resistance, to be overcome. The lessened heart rate in recumbency and the diminution, even though moderate, of blood pressure in this attitude will suffice to emphasize the importance of rest to this organ.

Rest should be in bed. One might suppose this injunction to be superfluous, and yet it is every one's experience not infrequently to find himself in the presence of contentious individuals who demand many reasons, when it might be supposed common sense would dictate; and one might as well write in golden letters on the tablets of his memory that he is to treat individuals, whose very individuality depends on differences, not machines nor yet diseases.

Bed. The bed should be of a height and width most convenient for handling the patient, who is in many instances helpless, and to whom the most gentle handling may constitute torture. A half, or at the most a three-quarter bed, with a woven wire spring, sufficiently stiff to prevent sagging, should be chosen. The standard hospital bed is an admirable example. The mattress should be soft, but resilient and firm. A good hair mattress is preferable. If the patient perspires freely, the bed should be made with thin blankets instead of sheets. The patient should wear a thin flannel nightgown, opened all the way down the front and slit along the sleeves, so that the joints may be exposed for inspection or treatment with the least disturbance, and it is well to throw a thin flannel cape about the shoulders. The nurse should be instructed to put the clothes on this bed with a view to the comfort of the patient, rather than to preserve the symmetrical and esthetic effect so often insisted on in the hospital ward, regardless of the comfort of the 
patient. Often the lightest touch of the clothes is agonizing to the patient, and hoops, cradles, or other contrivances to take the weight of the clothes off the patient must be utilized.

An excellent bed is one of which the so-called Gatch-bed is a type. It consists of an extra frame on which the mattress lies, broken at two places so that it can be raised and maintained by a ratchet. The break at the upper end of the bed affords an excellent bed rest that can be readily maintained at any angle, while a second break at about the bend of the knee allows the raising of the bed at this point which gives the support and prevents the body from sliding down and is a grateful change in the position of the extremities. Such a bed is particularly valuable in cardiac cases with orthopnea.

Room. The best available room should be chosen, with a view to an abundance of light and air, with a southern exposure in the winter, and away from the prevailing winds at all times. The bed should be so placed that the draughts may be avoided but the air not shunned. The therapeutics of light is not duly appreciated. The minds and bodies of many of us are as responsive to its influence as a photographic plate. Air should be admitted to the room freely. There can be no superfluity of fresh air. In summer the windows should be kept wide open. In the winter the room should be frequently aired and kept at $65^{\circ}$ to $70^{\circ} \mathrm{F}$. Personally, I do not hesitate to admit the cold clear air of a winter's day to the sick room, observing proper precautions with reference to the patient's coverings.

Diet. If there is any one field within the province of medicine that promises richer yield than another for the labor expended on it, it is that of dietetics. As yet it is almost virgin, and still such results as have been obtained are of the highest significance. It has been well suggested that if the physician would give the same amount of time, work, and care to the prescription of foods that he does to drugs, enormous benefit would accrue to bis patients.

In the first place, a sick man needs food, and he needs more food than is ordinarily given him. Of course, one grants that there are certain conditions that make the administration of sufficient food difficult or impossible, but that does not obtain in the majority of instances. ${ }^{1}$ As I have said, the energy and heat of the body are derived from the combustion of its foodstuffs, and as energy can be converted into heat, the value of the foodstuffs can be expressed in heat units. Moreover, the amount of energy and heat the body gives off in a day can be measured in terms of heat units; so that we can determine just how much food an individual of a given weight, under varying conditions of activity, ${ }^{1}$ See Chapter II, Diet in Acute Infectious Diseases. 
needs. The term adopted to express a heat unit is the "calorie." The amount of heat that 1 calorie represents is that required to raise 1 kilogram of water from $0^{\circ}$ to $1^{\circ} \mathrm{C}$. This calorie is sometimes spoken of as the "large calorie." The "small calorie" is the amount of heat needed to raise 1 gram of water through $1^{\circ} \mathrm{C}$. of heat; therefore, 1 "large calorie" equals 1,000 "small calories." Unless qualified, the term "calorie" means a "large calorie."

Now, under what is ordinarily known as rest, a man gives off heat in twenty-four hours equivalent to about 33 calories per kilo of body weight; that is, a man weighing 70 kilos, or 154 pounds, will give off about 2,300 calories. This amount of heat must be replaced by his foodstuffs to keep him in equilibrium.

The patient with rheumatism, however, is suffering from fever, and in fever he gives off not only what he does in health at rest, but some 20 to 30 ' per cent. more. If we add 25 per cent. more to our estimated calories at rest, we find the patient's needs are 2,800 to 2,900 calories. We are all aware that in febrile conditions it is customary to put a patient on a milk diet, because the different food constituents-fat, carbohydrates, and proteins-are so well represented in it, because its protein furnishes all the elements, amidobodies, "building stones," necessary to the construction of body protein (which is not true of all food proteins), because it is bland, and because it is easily administered. The physician's instructions frequently are a glass of milk every two hours. A glass is supposed to hold 8 ounces; more commonly, as given, it holds 6 ounces. On this schedule, ten feedings would be exceptional, and eight nearer the actual number; so the patient would get $1 \frac{1}{2}$ to 2 quarts of milk a day. In a quart of milk there are 620 calories of food; in the patient's dietary, 930 to 1,240 calories, or one-third to two-fifths of his needs as calculated. But the case is even worse than this, for, as I have shown, there are reasons why, in fever, a patient's dietetic needs are greater than those set out above.

It is an easy mathematical problem to determine chat to meet the patient's caloric needs with milk would require some 5 quarts of milk, and the administration of over a gallon of milk day after day does not appeal to our common sense.

Moreover, this amount of milk would contain 128 to 160 grams of protein, which is excessive.

To keep within quantitative limits, qualitative changes must be made in the food. The readiest way to do this is to add to milk, cream to furnish more fat, or starch, or sugar, to furnish more carbohydrates. For example, enough milk sugar can be added to the milk to make 10 per cent. without making it disagreeably sweet; or cereals, to make 
gruels or milk soups. Six per cent. of sugar added to milk will give a milk equal to about 860 calories to the quart.

In addition to milk, eggs, cereals, bread and butter, rice, and cereal or milk soups are permissible. An ordinary thick slice of white bread ( $11 / 3$ ounces) furnishes 100 calories; an average pat or ball of butter (a little less than $1 / 2$ ounce) the same; an ordinary helping of boiled rice (4 ounces) as much more, and $11 / 2$ to 2 ounces of cream the same. Three even teaspoonfuls of granulated sugar affords 100 calories. There are 100 calories in a large serving of oatmeal or hominy, and one egg adds 80 calories more.

This necessity for a sufficient diet obtains especially in long-continued fevers, while in the brief fevers of intense character, in which the functions of the digestive organs are impaired, only small amounts of food are to be urged, as the body has a surplus to meet its needs for a short time.

I have introduced these figures to concentrate attention on a branch of our art that has been left too little cultivated and exercised without reason. Milk, milk soups, cereals, bread, and rice form the staple diet in rheumatism. To meat soups, which have scarcely any nutritive value, there are certain theoretical objections, but if their well-known influence in spurring a jaded appetite and stimulating what the Germans call the "appetit-saft," is taken into consideration, I think their administration in small amounts is warranted. With the decrease in the fever, eggs may be used; and in convalescence, fish, meats, and vegetables.

Fluids. Water should be allowed ad libitum unless the heart is decompensated in which case certain restrictions should be made. As more grateful drinks, dilute fruit juices are permissible, orangeade, lemonade or diluted grape-juice. See diet in Summary.

Bowels. When called to attend a patient suffering from an acute infectious process, it is a good rule to assure satisfactory evacuation of the bowels. Just how much additional disturbance a neglect of this measure may induce we do not know, but we are aware of the fact that at times in an individual otherwise well, constipation may incite symptoms akin to acute intoxication, or more commonly, depression, malaise, anorexia, and headache, and we have evidence that products of decomposition in the bowel, normally absorbed and paired, like the indol group, with sulphuric acid, can, when this function of pairing is interfered with in disease, give rise to toxic manifestations.

An active saline, like magnesium sulphate or Epsom salt, sodium sulphate or Glauber's salt, or sodium potassium tartrate or Rochelle salt, in doses of 1 ounce, or, in patients susceptible to saline purgatives, $1 / 2$ 
ounce, may be given, assisted by a soapsuds enema four to six hours later if the salts have not been sufficiently effectual.

"Technique of a Soapsuds Enema. The enema is prepared by agitating white castile or ivory soap in warm water until a good suds is formed. The froth must then be removed because it contains air and, if introduced into the bowel, may cause pain. From one to two quarts of soapsuds are used. This is then poured into a douche bag or fountain syringe to the tubing of which has been attached, by means of a glass connecting tube, a large catheter or rectal tube. The bed should be protected by a rubber sheet and the patient be placed in the dorsal recumbent position, the pan or douche bag be raised to a distance of not over three feet above the patient and in order to avoid getting air into the intestines the fluid should be allowed to run through the tube when it is unclamped before introducing it into the bowel.

"The rectal tube should be lubricated with vaseline or soap emulsion and introduced slowly into the bowel to a distance of about eight or ten inches.

"One to two pints for a child or 2-4 pints for an adult should be allowed to run in slowly, the flow being controlled by a clamp or stopcock attached to the tubing.

When the patient begins to complain about discomfort the flow should be stopped and rectal tube should be removed slowly and gently. The patient being instructed to retain the enema for 10-15 minutes." (Pope and Maxwell.)

It should be remembered that the feces represent an excretion from the bowel of mucus and other substances which represent nitrogenous metabolism, as well as a large content of bacteria, and afford a pretty constant percentage of the total nitrogenous output; that the feces are not mere food residues, and, in fact, normal feces should contain but very little food residue other than indigestible fibers of cellulose, seeds, etc. Hence it is surprising to the patient, and often to the physician, too, to discover so large results from catharsis when the patient has been on a milk diet, or even when on no diet at all. With this knowledge, then, of the formation of feces with a low or easily digestible diet, the necessity of attending to periodical evacuation is emphasized. This may best be done by enemas in most febrile diseases, but with the discomfort incident upon handling the body in rheumatism, further doses of salines may be preferred. I believe that too frequent catharsis by drugs entails an irritation that in itself may become mischievous.

Sleep. Rest cannot be guaranteed unless sleep can be obtained. Of first importance is the environment. If the room is cool, well ventilated, the patient's toilet prepared for the night, the bed arranged, lights turned 
low and noise excluded much will have been done to secure sleep. If this is not effectual, mild hypnotics may be tried such as bromides in doses of $\mathrm{gr} . \mathrm{xv}-\mathrm{xxx}\left(1-2 \mathrm{Gm}\right.$.) or trional ${ }^{1} \mathrm{gr} . \mathrm{v}$ to $\mathrm{gr}$. $\mathrm{xv}$ (0.33-1 Gm.), or chloralamid gr. $\mathrm{xx}-\mathrm{xxx}(1.33-2 \mathrm{Gm}$.) early in the evening, often the lesser dose will be found to be effectual. Adalin in gr. v (0.33 Gm.) doses is a mild hypnotic. Barbital (Veronal) and sodium barbital (Medinal) gr. v-vii ss. $(0.33-0.50 \mathrm{Gm}$.) may be used, but are not favorites of mine.

All too often sleeplessness is due to pain, and sleep can be obtained only by an anodyne. One may use codeine phosphate gr. $1 / 8$ to $\overline{\mathrm{ss}}(0.008-$ $0.030 \mathrm{Gm}$.) by mouth or hypodermically, or in severe cases morphine sulphate gr. 1/8-1/4 (0.008-0.015 Gm.) hypodermically. The doses given are for adults and must be modified for children according to Young's rule or adapted to weight. Young's rule is to divide the age of the child by the age +12 ; e. g., for a child of 2 years $\frac{2}{2+12}=1 / 7$ therefore the child's dose would be $1 / 7$ of the adult dose.

Weight Rule. Dr. Clark's rule is to divide the child's weight by 150 ; i. e., if the child weighs 50 pounds, $\frac{50}{150}=1 / 3$, therefore the dose would be $1 / 3$ of the adult dose.

Cowling divides the figure of the next birthday by 24 ; therefore if the child is $2, \frac{3}{24}=\frac{1}{8}$, therefore the dose of the child is $1 / 8$ of the adult dose.

Specific Treatment. It was once hoped that every disease might be met by a specific drug, and it was once believed that many diseases were cured by specific drugs; but as medicine entered on an era of more searching observation, and had to rest its judgment on scientific criteria, the number of specifics dwindled, until the term "specific treatment" has come to connote the treatment of one disease alone, syphilis.

The Salicylates. When Dr. Maclagan, of Edinburgh, in 1874, began to use salicin, a glucoside of salicylic acid, obtained from the young bark of the willow, in rheumatism, which was quickly followed by the introduction of other forms of salicylic acid, the change that came over the clinical picture of this disease, that turned a bed of racking pain into a couch of relative comfort in a few hours, and a patient to whom the least. touch was agonizing into one who could be handled with relative impunity, warranted the belief that a specific had, indeed, been discovered. It is said that we no longer see rheumatism as it was presented to the

${ }^{1}$ Trional is the official sulphonethyl methane. For the sake of brevity trional is used throughout the book. 
older practitioners, and yet the drug cannot be called specific, if we mean by that one that can eradicate the disease. That its discovery was a boon, no one who has witnessed its effects can for a moment doubt.

Salicylic acid has this structure:<smiles>[C+]1C=CCCC1</smiles>

(4)

is the benzene ring. If we will replace the $\mathrm{H}$ at (2) by an- $\mathrm{OH}$ group, that is, make an alcohol of it, we convert it into a well-known poison, carbolic acid.<smiles>Oc1ccccc1</smiles>

Note what slight changes in a complex group and the introduction of what simple radicles induce potent changes in character. We have but to make another slight change, by introducing an acid radicle at (I), to convert the toxic carbolic acid (which is no acid at all, but an alcohol) into the substance in question, salicylic acid. ${ }^{1}$<smiles>O=C(O)c1ccccc1O</smiles>

I might add that the introduction of an acid radicle into the structure of a toxic alcohol detoxicates it. This is a general law.

This substance is classed among the antipyretics and antiseptics; it is also an anodyne. Its therapeutic value is exercised in all three directions in rheumatism. Salicylic acid should not be administered as such, but in the form of a salt or ester. The effect is the same in kind in all these forms, but certain by-effects determine the use of one

${ }^{1}$ Salicylic acid is not derived in this manner, but from ortho-oxy-benzyl alcohol, $\mathrm{HO} . \mathrm{C}_{6} \mathrm{H}_{4} \cdot \mathrm{CH}_{2} \mathrm{OH}$. The illustration is used to draw attention to the chemical kinship of well-known drugs which are so different in their action. 
or the other. It should be administered in full dose. Its failure may often be attributed to insufficient dosage. Its toxicity is slight. In an adult of average weight I give as much as 20 grains $(1.30 \mathrm{Gm}$.) of one or the other form of the drug every two hours for the first twenty-four hours during the waking period, or even for forty-eight hours. In severe cases even 30 grains $(2 \mathrm{Gm}$.) may be given for the first two or three doses. As the pain subsides the dose may be cut down gradually to 15 , to $10(1 .-0.60 \mathrm{Gm}$.) grains at a dose, how much and how rapidly depending on the progress of events or on signs of toxity. The dose should be well maintained at amounts of 10 grains $(0.60 \mathrm{Gm}$.) every two hours until the active phases, as evidenced by fever, pain, and joint swelling, have passed. Just how this drug acts in rheumatism we do not know, but its effects are so much more prompt and satisfactory in this condition than in any other clinically akin to it, that we are tempted to believe that it has some effect on the materies morbi directly. It is my custom to keep the patients on considerable doses- 5 to 10 grains $(0.30-$ $0.60 \mathrm{Gm}$.) every two or three hours for a week or ten days after the subsidence of acute symptoms, and for four to six weeks on lesser doses of 5 or 10 grains $(0.30-0.60 \mathrm{Gm}$.) three or four times a day, administering the drug much as we do quinine in malaria.

If satisfactory results do not follow these doses they may be pushed to the production of toxic manifestations.

Hanzlik's studies of the toxicity of the salicylates carried on at the request and under a grant from the Committee on Therapeutic Research, Council on Pharmacy and Chemistry, American Medical Association, showed that the toxic dose of Sodium Salicylate in the majority of individuals of both sexes lies between 100 and 200 grains per diem (the mean toxic dose for males nearer 200, for females nearer 150).

These figures are close to my customary dosage.

The dosage for children should be relatively large and may be pushed to toxicity, even in cardiac disease.

The tendency is to give to children too small a dose rather than too large. When men of large experience, like Lees in England, give 200400 and more grains a day to children under sixteen years, it will be appreciated how far short of the danger-mark, if not of efficiency, our usual dosage is.

With this drug, one may administer an alkali. I prefer bicarbonate of soda of which something more than grain for grain should be given; my rule at the beginning is 2 grains of bicarbonate to one of the salicylate. It seems to lessen the irritating effect of the salicylate on the gastrointestinal tract.

The toxic symptoms, except such as constitute an idiosyncrasy are 
disagreeable rather than dangerous or disagreeable long before they are dangerous, giving ample warning to watch or to stop or modify the dosage.

They are: (1) buzzing, roaring in the ears, with varying degrees of deafness, headäche; (2) gastric disturbances, more rarely; (3) cardiac disturbances; (4) respiratory disturbances; (5) cerebral symptoms; (6) renal complications; (7) hemorrhages; and (8) skin involvement. This looks like a formidable array of disasters, and so do tidal waves, cataclysms, and the fall of meteors in the catalogue of everyday possibilities; but, like most apparitions, this list takes less substantial proportion when submitted to light. I will consider them in the reverse order. Skin eruptions after the use of the salicylates are rare; still a diffuse erythema, an urticaria, a hemorrhagic outbreak, and other forms may follow. It will be observed that the three forms specified have all been associated with rheumatism, and it would be difficult to determine in all cases the association of the drug with the rash. They are not dangerous in themselves, and the drug should not be intermitted on their appearance unless the rash is distinctly aggravated by the continuance of the drug.

Retinal hemorrhages are still more uncommon, while epistaxis and other hemorrhagic manifestations have been more frequently reported, and, if severe, might enforce cessation of the drug: Albuminuria and hematuria, which have been attributed to the irritating effects of salicylates, may be and probably are caused by the disease itself, but with their appearance it might be wise to intermit the treatment until it is demonstrated that they do or do not play a part in the disturbance. Scott and Hanzlik have recently called attention to the appearance of albumin, leucocytes and small granular bodies resembling casts in the urine of patients taking salicylates; they note, however, that this promptly clears up after salicylates have been stopped.

I do not feel that these statements should lead to the modification of our salicylate treatment of rheumatism and in a rather large experience in these cases I have never seen renal complications from this cause that were clinically recognizable or eventuated in renal disease. There can be no doubt that now and then the salicylates have induced an active delirium, sometimes like an acute mania. I recall a report of two such cases occurring at Bellevue Hospital, but it is very unusual. It must not be forgotten that delirium intervenes in the course of rheumatism, especially, it is said, with the onset of a pericardial involvement, and associated with hyperpyrexia. Dyspnœa, characterized by slow and labored breathing (see below), has occurred, and suggests the possibility of impurities in the drug, as, indeed, does the slow heart and threatened 
collapse occasionally noted. Here, too, one must keep in mind the involvement of the myocardium, the lung and the pleura, in the disease. However, in either instance, so threatening a condition should indicate a withdrawal of the drug.

I have recently seen four cases of bradycardia and arhythmia in rheumatism; in some of these have obtained the graphic evidence of sinoauricular block. In all cases the block disappeared with the intermission of the salicylate and in one a return was provoked by its resumption. No evidence of circulatory embarrassment was seen in these cases. Sino-auricular block has been reported in other acute infections such as influenza in which no salicylate had been administered. Auriculoventricular block has been repeatedly noted in rheumatism in which no salicylates had been administered and has been attributed to interference of conduction impulses by the Aschoff bodies, the characteristic lesion in rheumatism, whose site of election is in and about the conducting bundle of His.

To sum up, I should say that the above-mentioned conditions are rare; that they may be attributable in most instances to the disease rather than to the drug, or to idiosyncrasies-those peculiar reactions of the individual to drugs, food, and environment that takes him out of his class and constitutes in him an anomaly, and defies foreknowledge. I firmly believe that it is only a minority of the above-mentioned conditions that can be attributed to the drug itself.

It stands otherwise with the first two disturbances enumerated. They are to be attributed to the drug, and their occurrence modifies our action. The ringing in the ears and a mild grade of deafness may be looked upon as a limit of tolerance with comfort rather than a menace. There is no reason to intermit the drug on this account, but if the discomfort is considerable, the dosage should be cut down or stopped. The gastric irritation resulting upon the administration of salicylates is the bete noir of the practitioner. It is for this reason rather than for any other that so many forms of salicylates are in use. There are certain forms of the drug from which one may anticipate more irritation than from others, but, again, the susceptibility of a particular stomach to a particular preparation cannos be predicted with any degree of assurance.

I advise, as a rule, the use of the preparation that has stood best the test of time and experience. In this case it is the sodium salt of the acid, sodium salicylate. I may say at once that the acid itself is too irritating to administer internally. Order the drug alone, in simple solution. Order the dose to be taken well diluted. 
For example, write thus:

R

Sodii Salicylatis..........................

Aquæ destillatæ q. s. ad.....................6. $\boldsymbol{\xi}_{\mathrm{ij}}$

M. et S.-One teaspoonful in water every two hoûrs.

One will note that this calls for a 2-ounce mixture, but, if written in the metric system, that there are just as many grams in this 2-ounce, or 60 c.c., mixture as we want to give grains in one dose. Even the water has as many cubic centimeters as we want to give drops in a dose- 60 , that is, 1 teaspoonful.

Sometimes the salt is better borne and less disagreeable to the taste if a little glycerin is used, as:

R

Sodii Salicylatis.......................

Glycerini...............................

Aquæ destillatæ q. s. ad...................60.

M. et S.-One teaspoonful in water every two hours.

$\bar{a} \bar{a} \bar{\jmath}$ ss.

予

If one has doubts about his patient's ability to get a good salt, he should order the salt made fresh from salicylic acid by adding sodium bicarbonate. This is a very excellent way of writing the prescription:

R

Acidi Salicylici 15.

亏ss.

Sodii Bicarbonatis............................

Aquæ destillatæ q. s. ad.....................60.

q.s.

M. et S.-One teaspoonful in water every two hours.

与 $\mathrm{ij}$

The druggist is to use of the soda what is needed; he adds definite proportions of the two drugs, if he follows the Pharmacopœia, or he simply adds soda to the solution of the acid until effervescence ceases; that is, until no acid is left to liberate the carbon dioxide from the soda.

In the early days of the synthesis of sodium salicylate a good many impurities existed which made such a prescription as was just cited a wise precaution, but Hilprit's study of the different synthetic sodium salicylates at the instigation of the Council of Therapeutic Research of the A. M. A. show that such impurities no longer exist and that their. "investigation would seem to warrant the conclusion that the cheapest commercial synthetic sodium salicylate is the equal of the higher priced brands of the synthetic kind or costly natural product."

If, for any reason, sodium salicylate is not well borne, one may have recourse to another form of the drug. Moreover, it has been occasionally noted that a patient's symptoms which did not yield to one form of salicylate, may to another, e. g., to aspirin after the failure of sodium salicylate. My own preference is for acetylsalicylic acid [aspirin], that 
is, salicylic acid in which the $\mathrm{H}$ of the $\mathrm{OH}$ group has been replaced by an acetic acid radicle, $\mathrm{CH}_{3} \mathrm{CO}$; thus:<smiles>COc1ccccc1C(=O)O</smiles>

This substance is a white powder, formed of small crystalline needles, practically insoluble in water (100 parts) and in acids, so that it passes through the stomach for the most part unchanged, and is broken up in the intestine. It is less irritating to the stomach, but that it should be devoid of all the disadvantages of the sodium salt its chemical structure forbids us to believe. I have in one instance seen a massive angioneurotic œdema of the face follow a single small dose, and have seen such cases reported in the literature since. However, I believe it to be a very valuable form of salicylic acid. It is best prescribed in capsules. The dose is practically the same as the sodium salt, or about 15 grains $(1.0 \mathrm{Gm}$.) for a beginning dose.

The Committee determined the mean toxic dose of aspirin to be a little smaller only than that of sodium salicylate, i. e., 165 grains a day for adult males, 120 for females.

It is said that alkaline salts must not be given at the time of administration because they decompose the drug in the stomach and, therefore, should be given between the doses. As Bastedo has pointed out, this is more or less a theoretical consideration and in the test-tube bicarbonate of soda cannot be shown to decompose the drug. He finds, indeed, that the administration of a little bicarbonate of soda in many instances lessens the irritating effects of the drug in the stomach.

Another excellent preparation of the salicylic acid series is the ester, methyl salicylate, that is, salicylic acid in which the $\mathrm{H}$ of the acid group is replaced by methyl $\mathrm{CH}_{3}$; thus:<smiles>COC(=O)c1ccccc1O</smiles>

Methyl salicylate is a volatile oil that constitutes well over 90 per cent. of the oil of wintergreen, the well-known gaultheria procumbens of our woods, and of the oil of birch, oleum betulæ, obtained from the bark of the sweet birch, betula lenta, or is produced synthetically, and when 
carefully prepared should answer the purposes of the natural oils. Of the three, the oil of wintergreen is, as a rule, preferred. It may be given in capsules, in emulsion, or in milk. I very much prefer the capsules, because in emulsion the decided taste of the drug, which may be agreeable at first, soon palls on the patient.

As for its administration in milk, the same objection obtains, and what is much more important, it violates a rule that I believe one should invariably observe-never give medicine in food, for, if the medicine does disagree, its association in the mind of the patient with the food may produce a disgust for food which may be the mainstay of the case.

The Committee's research placed the mean toxic dose as 120 minims of the oil of gaultheria.

The drug is usually very well borne, but its decided taste, even when given in capsules (for the slight eructations it often induces is a constant reminder) is the chief drawback.

Diplosal is a form of salicylate more lately come into use and as its name suggests contains two salicylic acid radicles; that is, it is a salicylosalicylic acid or salicylic ester of salicylic acid, formed by the condensation of two molecules of salicylic acid, the $\mathrm{H}$ of the acid radical of one molecule being replaced by the second molecule entering by its phenol group $(\mathrm{OH})$ at (1); thus:

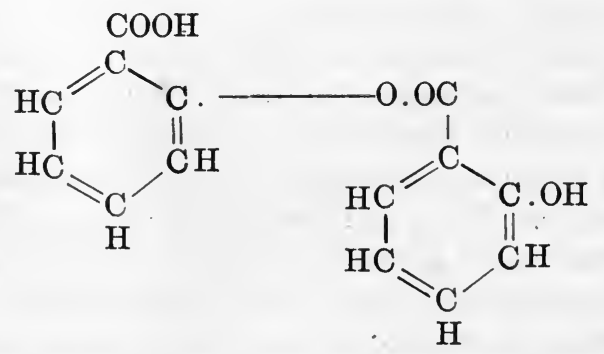

It is an insoluble powder and like aspirin is little affected by acids, so is supposed to pass the stomach unchanged and like the aspirin is broken up by alkalis.

It is best given in capsules and alkali should not be given with the dose but between the doses.

It has the same toxic effects as other salicylates and is given in about half the dose of sodium salicylate.

Hanzlik's studies showed the mean toxic dose per day was 100 grains for adult males and 83 grains for adult females.

I have had but little experience with this particular form of salicylate.

One will rarely have to choose outside of one of these four forms of salicylic acid in the treatment of rheumatism. If he does, the great 
probability is that be has not administered these forms properly, or that the patient cannot stand salicylic acid in any form, or that the series does not meet the needs of this particular case.

I will mention two other well-known preparations: First, the original drug, salicin. This is a glucoside, which can be split up by acids into grape sugar and saligenin, the active principle, which is the alcohol from which salicylic acid is formed, and this formation of the acid goes on in the body after its administration. It is a white powder, bitter to the taste, rather insoluble in water (28 parts), so best administered in capsules. It is well borne, and by many preferred for children. The dose is the same as for the others. Second, another ester, phenylsalicylate, salol that is, salicylic acid in which the $\mathrm{H}$ of the acid group is replaced by phenyl $\mathrm{C}_{6} \mathrm{H}_{5}$; thus:<smiles>O=C(O)c1ccccc1</smiles>

$\mathrm{H}$<smiles>c1ccccc1</smiles>

$\mathrm{H}$

It is a white powder, almost tasteless, and quite insoluble in water. It passes through the stomach for the most part unchanged, and is broken up in the intestine, two-thirds of it appearing as salicylic acid and one-third as carbolic acid. It is administered best in capsules or powders, or can be suspended in mucilage of acacia. It has no advantage over the other forms in rheumatism, and has the disadvantage of affording only two-thirds of its weight as the desired substance, while one-third is the toxic carbolic acid, which can produce its characteristic poisonous symptoms when given in large doses. The dose is about the same as for the other preparations.

As I have already intimated, some patients cannot take salicylic acid in any form. We cannot, for that reason, neglect their need for relief of pain. The three drugs in most common use for such a purpose are acetanilid (antifebrin), antipyrin, and acetphenetidin (phenacetin) (officially acetphenetidinum). Of these three, the first is the most potent, also the most irritating and toxic. These drugs are not given over a long period, as the salicylates are, but as needed, to control pain. Often small doses, frequently repeated, are as efficacious as fewer large doses. Acetanilid may be given in doses of $1 \frac{1}{2}$ grains $(0.1 \mathrm{Gm}$.) every half hour for four doses, or 2 to 3 grains $(0.15-0.2 \mathrm{Gm}$.) every two hours. If there are heart complications, it should not be used. Phenacetin may be used in twice the dose. The dose of antipyrin lies between the two. These drugs should be promptly stopped if cyanosis appears, which is 
well before cardiac or respiratory failure threaten, and, of course, as soon as pain is relieved.

If the pain is severe, rather than push these coal tars to large doses, one should use morphine, in small doses, hypodermically, $1 / 16$ to $1 / 8$ grain $(0.004-0.008 \mathrm{Gm}$.), of the sulphate. Morphine in any illness of length should be used reluctantly and in minimum dose, lest a habit be established. Two other drugs have been much used to control painpotassium iodide and colchicum. Their bad effects on the stomach are too certain, and their beneficial effects on the condition too dubious, to encourage their use.

Another line of treatment, originated in England by Fuller to combat an acidity that at that time was looked upon as an etiological factor in the disease, is the "alkaline treatment." This treatment met with little favor elsewhere in Europe, but was adopted in this country to a considerable extent, and still has some vogue. By many men it is used when the salicylates are not well borne; by others when cardiac complications threaten or exist; and by a very great many in conjunction with the salicylate treatment. Its use rests on empiricism solely.

One should choose the milder alkalis-sodium bicarbonate, potassium citrate, or potassium acetate; for example, 2 grains to 1 of salicylate until the urine reacts alkaline, and then in a little less dose, or enough to continue the urine alkaline.

Acidosis. Miller of London has laid a great stress upon the dangers of acidoses that may obtain with the administration of salicylates which, if it were true would be a matter of serious consideration. Recent work of Hanzlik, however, does not corroborate these findings as he was unable to determine on the administration of toxic doses of salicylates that the reserve alkalinity of the blood was in any way perceptibly altered. One should be warned that the usual ferric chloride test for diacetic acid in the urine of patients taking salicylates elicits a beautiful purple color in the urine that without control may be mistaken for the rich port-wine red of the diacetic acid reaction.

Vomiting. Miller studying vomiting in children taking salicylates found it severe in only 10 per cent. of the cases and what is of great importance that two-thirds of these had severe cardiac dilatation, and drew the conclusion that vomiting depended less on the dose than on involvement of the heart and that the more severe the heart affection the less the salicylate required to induce vomiting. Small doses of bromides combined with the salicylates may lessen the gastric irritation.

It must be remembered that vomiting may be the symptom of cardiac involvement when no salicylates are taken and that cardiac involvement does not contra-indicate the use of salicylates, but it is well to divide the 
total day's dose into smaller and more frequent dosage, or use the rectal or intravenous methods.

Rectal Administration of the Salicylates. Heyn of Cincinnati called attention to this mode of administration in 1912. I have used it with trivial modifications with most gratifying results over a long period of time.

The essence of the procedure is to give the drug in suspension in starch paste with enough opium to lessen rectal irritation and assist in retention. Give a cleansing enema.

Technique. Make a thin starch paste-use it at body temperature. Take 4 to 6 ounces (not an amount too large to be retained readily) and add to it $1 / 2$ to total daily dose, say $3 \mathrm{i}-\mathrm{ij}(4-8 \mathrm{Gm}$.) of the sodium salicylate in powder and one or two minims ( $0.06-0.13$ c.c.) of the tincture of opium. Inject gently into the bowel and hold the buttocks together for a few moments. Repeat at 12 -hour intervals.

Instead of dividing the dose one may give the whole dose in one daily injection.

The amount of salicylates used depends on the same consideration as when given by mouth, i. e., to the point of efficiency or toxicity. The amount of starch-enough to carry the drug and not provoke a movement; the amount of opium - the least to effect retention.

Alkalis (bicarbonate of soda) should be given in same amounts by mouth as if the salicylates were so administered.

Intravenous Administration. Still another method of administering salicylates, the intravenous, I recommend on the advocacy of my colleague, Conner, of New York, although I am entirely lacking in personal experience with it. Conner does not urge it in preference to the more time-honored mode of administration, but in those cases where success has not followed the latter or the stomach rejects it. I simply quote in substance and largely in words his technique as published in The Medical Record, February 21, 1914.

The drug chosen is a chemically pure crystalline sodium salicylate dissolved in distilled water (preferably recently distilled) which has been freshly sterilized by boiling, to a 20 per cent. solution. Such a solution will keep well several days if protected from the light and will remain colorless.

A rubber ligature is placed around the arm tight enough to obstruct the venous flow, making the veins at the bend of the elbow stand out prominently. The desired result is more effectually attained if the arm is allowed to hang down and the fist is opened and closed.

The skin over the vein is then sterilized by painting with Tincture of Iodine. The best syringe to use is one made entirely of glass, holding 
10 c.c. and supplied with a fine (small, bright, sharp) hypodermic needle. These, of course, are sterilized.

The arm is extended fully and fixing the vein below by pressure with the left thumb, the operator thrusts the needle into the vein in the direction of the venous flow, and makes certain of entrance into the vein by drawing a drop of blood into the syringe; and then injects. Pressure is made for a moment over the site of the injection to prevent leakage of blood into the subcutaneous tissue and the iodine washed off with alcohol.

Fifteen, twenty, or even thirty grains are given at 12 or 8 hour intervals. - No unpleasant effects were met with in Dr. Conner's experience, even after 120 grains a day.

The relief from pain is striking and prompt and the stomach is not upset.

The same vein can be used again and again, provided a small clean needle is used.

Symptomatic Treatment. The symptoms that give character to this disease are those referable to the joints. The improvement in the local manifestations of the disorder under salicylates constitutes one of the most satisfactory evidences of their potency, and yet the resolution of these parts often lingers well behind the disappearance of the fever and the pain; and, moreover, much can be done during the height of the disturbance to ameliorate the discomfort, hasten the resolution, and prevent bad sequels.

Rest. For an inflamed joint, just as for a broken bone, rest is imperative. Pain, which I have said is Nature's agent, impels rest, but when pain is banished or mitigated under our ministrations, the patient uses the joint too early, and often to his great detriment. The position of the limb in semiflexion is one involuntarily chosen as the most comfortable, and may be preserved during the acuteness of the attack. Various devices are used to maintain a single position. We can bolster the limb by putting pillows under the knee or on either side, or accomplish the same by the use of the Gatch bed, and find similar arrangements for the other joints. This, of course, cannot assure a high degree of immobility, and we can secure better results with splints, well padded and carefully applied. The success of these devices depends on the care and skill with which they are applied, and if simply suggested by you and left to the devices of unskilled hands in the application will be far worse than useless. Still another way to attain the desired end is by applying stiff bandages of plaster-of-Paris or starch. Again, much care must be taken in the application, as the parts cannot be daily inspected, and rough folds in the bandage, bits of dried plaster next the skin, which is moist with 
the excessive perspiration, can induce sores of serious import. These casts must be reapplied as the effusion in the joint disappears.

In a considerable experience with rheumatism, I have very rarely had to have recourse to splints.

The joints should be protected by some material that prevents chilling or rapid changes of temperature, such as flannel bandages. At the Presbyterian Hospital in this city (New York) a layer of cotton 1-1 1/2 inch thick, covered with gauze is wrapped around the joint like a binder and secured with safety pins. This affords, as well, easy access to the parts. (Swift.)

Heat and Cold. The patient's testimony is sufficient evidence of the comfort these measures afford, whatever opinion may be entertained with reference to their curative qualities and the rationale of their action. From the standpoint of comfort the reaction of different patients to heat or to cold differs widely. To one patient, with a painful joint, eold gives almost instant relief, while in another the pain is intensified, and finds relief from heat, and vice versa. Continuous cold exercises considerable anesthetic effect, and may be secured by the application of the ice coil, or the more readily obtained and manipulated ice bag. Ice bags of various shapes may be obtained, but the circular ice bag does well for most purposes. The ice bag must be properly filled in order to make its application efficient. The ice should be cracked in pieces not larger than the end of one's thumb, and enough to cover the bottom of the bag. Enough cold water is poured on this to enable one to force all the air out of the bag and screw the cap down to the level of the water. This procedure leaves the bag supple, so that it may be wrapped around the part, which the presence of air makes impossible. Protect the part with a thin layer of vaseline or oil and a thin layer of cloth.' A long-continued direct application of ice to the skin may do damage to that structure. Heat is best applied by fomentations. A couple of layers of flannel are wrung out of boiling water in a wringer made of a crash towel, and applied snugly to the joint. This is repeated three or four times, at intervals of ten to fifteen minutes. The parts are then sponged with water at about $75^{\circ} \mathrm{F}$., and wrapped in flannel or non-absorbent cotton. A soothing application is the cold compress. This is done by wringing two or more layers of linen or old cotton eloth or cheesecloth out out of water at about $60^{\circ} \mathrm{F}$. and applying snugly to the part. This in turn is covered by dry flannel. These applications are renewed about once an hour. The effect of cold is momentary; the vessels soon dilating and conveying heat to the surface, warm the compress to the temperature of the part drying it. The reaction induces a hyperemia, the value of which will be touched on at some future time. The joints should at all 
times be well protected from changes in temperature. This is best done by wrapping them in layers of non-conducting material, like flannel or non-obsorbent cotton or cotton batten. The number of drugs that have been used locally are legion. I will purposely refrain from mentioning more than one or two that I have found helpful." Perhaps the most common application is methyl salicylate. That it does any more than any other volatile oil, by inducing a hypermia, I doubt. That the salicylates may be absorbed by the skin I have proved to my own satisfaction, but not in such amounts as to make that the object of the application. The methyl salicylate may be applied pure, or in the form of an ointment. The following is one in much use:

R

Methylis Salicylatis......................f $\mathbf{Z i}$

Mentholis..............................

M. et.adde

Petrolati q.s. ad.......................... $\mathrm{z}$

Tere bene simul.

Sig.-Apply as directed

An ointment containing ichthyol has been much praised. For example:

R

Ichthyolis.................................

Petrolati q. s. ad.............................

M.

Sig.-Apply as directed.

Counterirritation. Counterirritation is a very old remedial measure which has survived the rise and fall of countless therapeutic efforts, and the very persistency of which, in this Nihilistic age, speaks for its reality. It is indicated rather in the subacute stages of joint inflammation than in the acute. Of the many means of inducing it, I will mention two only as worthy consideration-the cautery and the fly blister. Of the two, the former is much the better, as being easier of application, easier of control, less likely to be followed by bad results locally, and entailing no danger from absorption. The cautery is flicked lightly over the part, care being taken to avoid severe blistering or deep burns. The part is then smeared with vaseline, oil, or ointment.

In applying the blister (ceratum cantharadis), it is cut about one inch square. In making the application to the knee, four such might be used, one above and one below on either side. Shave and cleanse the part. Oil the edges of the blister and apply a little vaseline to the skin adjacent to the blister, to avoid its spreading. Leave the blister in position for: four to six hours, and if a blister has not formed in the skin by this time apply a warm poultice to the part, which will hasten its formation. 
Puncture the blister on its dependent edge, evacuating the serum but not destroying the protecting epithelium. Dress with oil. Some of the disadvantages of the blister have just been set forth, and in the presence of a damaged kidney, as may occur in the course of rheumatism, the dangerous irritating effect of this drug on the parenchyma of the kidney, which it causes in the course of its excretion, must be kept in mind.

Pressure. When an effusion is slow to absorb, one may hasten the result at times by applying a snug bandage of flannel or rubber, which will exert a continuous but moderate pressure.

In persistent effusions, and effusions will sometimes persist after both temperature and pain have ceased, and in the acute stage with excessive and painful effusion a paracentesis is indicated.

\section{PARACENTESIS OF THE KNEE-JOINT}

"In synovitis the joint is considerably distended and there are four prominences about the patella, two on each side, above and below. The upper inner swelling is usually the most prominent as there is more space for the collection of fluid in this situation. In aspirating fluid from the knee-joint the object is to insert the needle so as to be sure to get the fluid and at the same time to give the least opportunity for damage to the joint cartilage. These objects are best attained in either one of the upper swellings. My personal preference is for the upper inner one. The aspirating needle should be from 8 to $10 \mathrm{~cm}$. long. It should be of moderate calibre and should have a point not too long and sharp. It should have an obturator so that one may assure himself of the patency of the needle when in the joint cavity. The joint is painted with tincture of iodine over a space $5 \mathrm{~cm}$. in diameter with its centre at the site of puncture. It goes without saying that the operator's hands, the needle and everything used must be scrupulously sterilized. The needle is held firmly in the operator's hand with the index finger on the shaft of the needle about $4 \mathrm{~cm}$. from its point. The base of the needle rests in the palm of the hand so that it is used somewhat after the manner of a shoemaker's awl. With the thumb and index finger of the other hand the operator steadies the skin at the site of puncture. The skin at the site of puncture may be previously anæsthetized by injecting a few drops of novocaine 1 per cent. If the skin appears to be tough, as it is in a certain proportion of instances, a sharp pointed scalpel may be used to puncture the skin after the novocaine has been injected. This renders the insertion of the needle very much easier, as it is always the skin which gives most resistance. The needle is inserted in a direction upward, inward and backward through a point at the summit of the swelling. By 
following this course upward, backward and inward, one gets into the joint obliquely and there is less likelihood of damaging the articular cartilage of the inner condyle of the femur. There is always plenty of effusion in this neighborhood for aspiration and when the needle is later withdrawn the oblique entrance into the joint causes a valve effect so that there is no leakage of the infectious material into the periarticular structures. After the needle has been inserted into the joint sufficient fluid for the purpose of examination, culture, etc., is obtained. While the needle is in the joint it is well worth while to entirely evacuate the fluid contents as this will give great relief to the patient's symptoms for a time. At the end of the procedure the needle is withdrawn in the line of its insertion, a small sterile pad is placed over the puncture hole, and the entire joint is wrapped in a bandage which causes moderate compression." (Personal communication from Dr. Alfred S. Taylor.)

Often the effusion will not recur in the subacute cases, while the relief to the patient in the acute cases is highly gratifying. The procedure, simple as it is, should be hedged about by all the precautions of a major operation, for an infection of the joint by a pyogenic organism is a serious matter.

If paracentesis be done and the opportunity affords, cultures of the aspirated fluid should be made for the identification of the organism and its use as a vaccine. Personally, I have been very rarely rewarded by culture of the joint fluid in the acute cases.

Later Measures. Chronic rheumatism is spoken of by both the layman and the physician as of common occurrence. In truth, chronic rheumatism as a sequence of true acute rheumatism is a rarity. Some men believe it never occurs and for a time I was of this opinion; but recent studies in my wards have convinced me that it is an occasional happening. If it does occur care must be exercised to prevent ankylosis. Splints must be occasionally removed and plasters and bandages taken off. Gentle manipulation of the joint must be carried out; or intelligent massage, hot fomentations, hot air baths, and baking had recourse to to facilitate absorption and resolution. In these later stages it is still believed that the iodide of potassium may do good.

Better in my estimation is the use of vaccines (see below).

\section{COMPLICATIONS}

Hyperpyrexia. The sudden onset of excessively high temperature, with extreme restlessness, headache, vomiting, delirium, and later coma, suggestive of meningitis, while occurring in the course of other febrile processes, is relatively common in rheumatism. It 
must be treated promptly and on the same principle as a sunstroke, that is, by a rapid withdrawal of heat. This is effectually done only by the use of cold baths or packs. The patient is put into a tub at $65^{\circ} \mathrm{F}$., or, if the shock is too great, the water may be warmed to $80^{\circ} \mathrm{F}$., and as the water warms from the patient's body heat, the temperature is kept down by adding ice to it. The patient should be kept in the bath until the temperature falls several degrees. If a fall to about $102^{\circ} \mathrm{F}$. can be attained, the patient should be removed from the bath, as the temperature will often continue to fall. During the bath, ice or cold water should be applied to the head. The bath should be repeated as often as the temperature rises to between $104^{\circ} \mathrm{F}$. and $105^{\circ} \mathrm{F}$. If the patient becomes chilled, is cyanosed, the temperature falls well below normal, or collapse threatens, he should be removed from the bath, put in warm blankets, heat applied, and stimulants freely used. A cold pack, the wet sheet in which the patient is wrapped being continuously rubbed with pieces of ice until the desired drop is obtained, is sometimes quite as efficacious as the bath.

The delirium that accompanies hyperpyrexia may be ameliorated or controlled by the measures just advised for that condition. If, however, the delirium still continues one may try hyoscine hydrobromate in doses of gr. $1 / 200$ to $1 / 150(0.0003-0.00045 \mathrm{Gm}$.) provided that the heart is not involved; or better yet morphine in doses of gr. $1 / 8$ to gr. 1/3 $(0.008$ to $0.02 \mathrm{Gm})$. These are adult doses. A measure which one frequently has recourse to in the treatment of delirium in acute infectious diseases with very excellent result is Lumbar Puncture; for the technique of which see Cerebro-spinal meningitis, Chap. XXII.

There may be in these cases a serious meningitis with increased intracranial pressure or one may assume that the toxins of the disease are irritating the meninges which find relief on withdrawal of the cerebrospinal fluid containing toxins.

Cardiac Complications. The frequency of these complications, and their gravity, threatening not merely the patient's life in the present attack, but, worse yet, his future, dooming him to a life of invalidism and dependence, make them by far the most important features of the attack, and haunt the physician from the incipiency of the disease. $A$ visit should never be completed without a careful examination of the heart. Changes in rate, rhythm, quality of sounds, or the appearance of adventitious sounds, should immediately put the physician on his guard. It cannot be too emphatically insisted that the mildest attack, as judged by fever, pain, joint implications, and general discomfort, may still be accompanied by grievous heart complications.

Statistics vary, but from reliable sources it is gathered that peri- 
carditis occurs in 15 per cent. of the cases, and endocarditis in over 50 per cent.; in children under ten years, in as high as 75 to 80 per cent.

Lees insists that in every case of rheumatism dilatation of the left ventricle can be made out. It is, he says "an inevitable manifestation of the disease and one of its earliest sỳmptoms" (British Med. Journ., October 12,1912), and, certainly, the more one gives attention to alteration of cardiac sounds and outline the more convinced he is of the very high per cent. of cardiac involvement.

It must be remembered that a more proper term for what actually exists in the heart is pancarditis, for the whole structure is likely to be implicated. The treatment of these conditions is the same as when occurring under other circumstances, and I will merely add that when there are signs of cardiac decompensation I am a firm believer in the use of the digitalis series in the acute cardiac disease in the same large doses as in the chronic form. The only question to be discussed at this juncture is the use of salicylates. Some authors fear the depressing effects of the drug. Others still consider that the appearance of the cardiac complications indicates no change in the treatment. Personally, I have always continued the salicylates; and in the same liberal doses as in uncomplicated cases.

I have never been convinced of any bad results by so doing unless the rare occurrence of the bradycardia mentioned be called such. If salicylates have any effect on the disease organisms themselves, it would seem as if their use in the serious complications of the disease was the more urgent.

Any case of rheumatism in whom a cardiac complication is evident should be kept in bed two months, even though joint manifestations and temperature promptly disappear, and as much longer as the cardiac condition and symptoms of the infection continue.

While cardiac murmurs have great significance, they need not necessarily be present when the heart is affected; hence, the great importance of familiarity with the first and second sounds of the heart and their variation when the myocardium is involved and an appreciation of changes in cardiac outline.

While the presence of murmurs should indicate heart involvement (with the exception perhaps of certain murmurs in the 2nd left intercostal space) they need not necessarily mean endocarditis or valvular change; for a goodly per cent. of them disappear and are probably attributable to hyperæmia of the valves or such an affection of the myocardium as entails a relative insufficiency of the valves. The statistics of some authors (Kemp) show that nearly half of the cases with cardiac complications clear up their signs before leaving the hospital and that no mean 
proportion of those not cleared up at the time of discharge have no permanent damage to the valves.

Aortic valve lesions show more myocardial involvement than the mitral (Brooks).

If the murmur appears early in the disease, it is more likely to be the expression of valvular endocarditis than if it occurs later, at which time relative insufficiency from loss of tone may well obtain.

Aortic insufficiency, double valve lesions, and pericarditis increase the gravity, but the repetitions of the cardiac involvement is what constitutes the greatest danger.

When all active manifestations of the rheumatism have ceased and the patient has been kept in bed six weeks to two months beyond this, he may be allowed up in a chair and then on his feet, but always guided by the cardiac response; for a pulse rate out of all proportion to the effort made betokens a cardiac irritability that gives warning of the prematurity of the effort.

In case the heart decompensates, it is to be treated as a decompensated heart under other circumstances.

The gravity of these cardiac complications has been accentuated by the studies of Dunn ${ }^{1}$ on over 300 cases of cardiac disease of rheumatic origin. He states that the immediate mortality of rheumatic cardiac disease is about 20 per cent. and that the final mortality of the affection followed at least ten years is 60 per cent. The mortality is seen chiefly during childhood. After young adult life is reached it falls to only 7 per cent. Most patients who weather the greater dangers of childhood and arrive at adult life get on remarkably well and this is attributed to an adaptation that takes place between the patient and his heart during the period of growth. Moreover, this author declares the earlier the cardiac lesion is acquired the more likely is the patient, if adult life is achieved, to lead an active and normal existence.

After-Treatment of cardiac disease in children has been well discussed by Dunn. ${ }^{1}$ It is largely a question of prophylaxis. With our present conviction that the tonsil is the usual portal of entry, it seems logical to remove the tonsil, if the patient has been the subject of tonsillitis or if the tonsil shows evidence of disease.

One of the most important items of the after treatment of the heart in rheumatism is the regulation of his activities. One feels that he is constantly steering between the Scylla of over-strain and the Charybdis of under-exercise.

I believe Dunn is correct in concluding that the danger of the former is less than the latter. I heartily favor his plan of regulated exercise

${ }^{1}$ American Journal of Diseases of Children, August, 1913. 
beginning soon after the disappearance of symptoms. At first with passive movements against resistance, and later active exercise always within the limits of the heart's demonstrated capacity to cope with it. Overstepping the limits is shown by rapidity of heart action and dyspnea. If a child can resume the normal activities of childhood without provoking these symptoms, he should be allowed to pursue them. Coddling merely because heart murmurs are heard really does the child great harm. Exposures to damp cold likely to provoke recurrence of rheumatism must be avoided or a removal to a climate where rheumatism is less prevalent is advisable when it is feasible to carry out this plan.

Pulmonary Complications. Pneumonia and pleurisy occur in a considerable number of cases, some authors giving the figures as high as 10 per cent. - I, myself, have seen it frequently in the severe rheumatism, and always with cardiac involvement. The figure 10 per cent. represents merely the incidence in the rheuumatic fever in general, but in cardiac complications and especially severe cases, they are much more frequent accompaniments. Pleurisy and pneumonia occur rarely if the heart is not affected. When the heart is the seat of endocardial changes only, the incidence is probably less than 10 per cent. but with pericarditis, which always means a severe infection, pleurisy or pneumonia or both probably occur in half of the cases while if both pericardium and endocardium are involved the figures rise to a still higher point.

Pericarditis, pleurisies, pneumonias, like subcutaneous nodules, are expressions of a virulent infection. The pleurisy may be an extension from a pericarditis, but this is by no means the necessary origin and probably in the majority of instances pleurisy is an expression of direct infection. Pneumonia as a complication is more frequent than pleurisy, though they often occur together as one might assume. The pleurisy is usually dry, but there may a serous exudate sufficient to tap. Both the pleurisy and the pneumonia are more likely to be on the left side. The pneumonia is very often a gradual development and the signs are often atypical; for example, a fall of temperature by crisis is not the rule, the physical signs may clear up as rapidly as they appear; the cough is not characteristic and the sputum is not rusty. My own experience has been that the physical signs are those of a lobar pneumonia with a massive pleural exudate and it has seemed to me that it does not so characteristically involve the whole lobe as a primary lobar pneumonia.

Sometimes the type may be that of a broncho-pneumonia. .Pulmonary edema may occur and at times infarctions. Bronchitis occasionally is a complication.

The treatment of these conditions, the pleurisies, pneumonias, pulmonary edemas, infarctions and bronchitis is such as would be instituted 
under other circumstances, and does not call for an intermission of the salicylates (see Prieumonia, Chap. IX; Bronchitis, Chap. VII).

Other Complications. Sore throat, if severe is to be treated as described under Scarlet Fever (see Chap. XVII and Tonsillitis, Chap. V).

The mouth throughout the illness should be kept scrupulously clean (see Pneumonia, Chap. IX).

Other complications are numerous but rare.

The skin eruptions, urticaria, erythema multiforme or nodosum, and purpuras require no special treatment.

Hemorrhages from the nose, stomach, bowel, kidney may occur but rarely demand interference.

Venous thrombosis is a rare complication and is treated as under other circumstances. Once in my experience an embolism of the popliteal artery occurred necessitating amputation.

Anæmia is a striking feature of the disease, and indicates in convalescence the use of iron and iron-containing foods.

Sweats of a severe type have long been associated in the minds of physicians with rheumatism, and may require especial consideration. They are very acid, and may cause considerable irritation. Sponging with a mild alkaline solution, as 1 per cent. sodium bicarbonate, gives relief. The skin should be kept dry with one of the numerous powders that contain talcum, or one made of equal parts of zinc oxide and starch. If the sweating is very severe, atropine may be used in doses of $1 / 100$ to $1 / 50$ grain. $(0.0006-0.0015 \mathrm{Gm}$.)

Vaccine Therapy. All the phenomena of acute rheumatic fever seen to me to demonstrate that it is an infectious process and yet, in spite of the careful work that has been done to determine the organism concerned, the positive findings are not very convincing. Nevertheless it seems wise to me to continue to make blood cultures during the febrile period and to take cultures from the crypts of the tonsils either in situ or after enucleation; from teeth; from excised subcutaneous nodules; from sinuses, ears or other obvious foci of infection. Joint fluids have been repeatedly cultured but are almost invariably negative. If these cultures are positive, most particularly those from the blood or deep crypts of excised tonsils and obvious contamination can be excluded, I am accustomed to have vaccines made from them and by their administration give the patient at least the benefit of the doubt in this debatable question of the specificity of the organism. It must be remembered, more particularly in adults, but by no means in them exclusively, that a toxic arthritis derived from any of the foci mentioned may closely simulate rheumatic arthritis, though the heart is very rarely involved. 
And in these cases vaccine therapy after the removal of the focus of infection, is of real value. I am accustomed to administer these vaccines when the case has gone on to a long continued infection or is subacute in its manifestations or between the exacerbations of the rheumatism. $\mathrm{My}$ best results have been obtained with vaccines cultured from the deep crypts of the excised tonsils, but here of course, the enucleation of the tonsil itself is probably the more potent factor in the improvement.

The Dosage. The first dose should be tentative. I begin with $5,000,000-10,000,000$, though some authors prefer so low a dose as 1,000 ,000 . It is my custom to give the dose twice a week. The increase in dose depends upon the reaction. If there is no reaction one may increase the dose to $10,000,000,20,000,000,40,000,000,75,000,000,125,000,000$, $200,000,000$, and then add $100,000,000$, or more, to each dose.

If there is a slight local reaction one advances more cautiously; if a slight general reaction, do not increase the next dose; if a marked general reaction, wait for two or three days after it has subsided and begin again with a lesser dose.

I have myself provoked an unfortunate exacerbation of symptoms with too large a beginning dose (in this case $50,000,000$ ).

The reaction may be a reddened and tender area at the site of injection or a recrudescence of local lesions, e. g., in joints or a general reaction of fever,' accelerated pulse and the other manifestations of slight infection. More serious consequences can follow careless dosing.

The number of injections is a matter of judgment and depends on results. I prefer eight to twelve doses and sometimes more.

The technique is that of any hypodermic injection, a clean syringe and needle and a clean site of injection.

The syringe should be graduated like a tuberculin syringe to hundredths of a c.c.

When the heart is involved one should be even more careful in the dosage.

Intravenous Injections of Foreign Protein (Foreign Protein Therapy), Shock Terapy. The use of non-specific vaccine or foreign protein has been recently agitated in the treatment of acute rheumatic arthritis as well as in other acute infectious processes, especially. typhoid fever. Various proteins have been utilized, both from certain foodstuffs and bacterial bodies. All of these, regardless of their origin, when given intravenously produce a striking reaction. Indeed, favorable results from the use of such foreign proteins seem to depend on these reactions. Shortly after the injection there is a chill, accompanied by a leucopenia; in an hour or two a febrile reaction which lasts about 2-3 
hours and accompanying this a sharp rise in the leucocyte count, which gradually returns to normal in 24 to 48 hours. In my ward at Bellevue, typhoid bacilli or sometimes, and especially when a gonococcus arthritis is a possibility in the case, the bodies of killed gonococci are used as the foreign protein. After the reaction, in favorable cases, there is a fall of temperature and a striking amelioration of symptoms; that is, a disappearance of pain and a diminution of the swelling in the joint. Cecil, reporting on 30 cases in our ward at Bellevue Hospital, found 40 per cent. were relieved of their symptoms without recourse to salicylates. In the rest it was necessary to supplement the vaccine treatment with the salicylates. Unhappily, nearly all these cases that had improved, relapsed in some measure. We can only theorize about how these beneficial results ensue, but it seems probable that these injections in some way distribute antibodies, sometimes spoken of as "a mobilization." Moreover, both the febrile reaction excited and the leucocytosis that follows are themselves useful instruments in combating the infection. Our dosage has been 25,000,000 typhoid bacilli, with increasing doses to $50,000,000,75,000,000$, etc., at intervals of a day or two after the subsidence of the preceding reaction. While the results are not all that might be desired and in a case with cardiac complications should be undertaken with great care, nevertheless it is a measure that may afford prompt and lasting relief in a sufficient number to warrant its continuance.

\section{CHOREA}

Chorea is often looked upon and treated as a separate and distinct disease, but chorea minor or Sydenham's chorea, long appreciated as mysteriously connected with rheumatism and as commonly complicated by the same kind of cardiac disease as acute rheumatism is now pretty generally looked upon as but one manifestation of rheumatism.

Treatment then of chorea minor is the treatment of rheumatism except that rest is even more insisted on and more difficult to attain when both fever and pain are absent.

Isolation is a very important part of the treatment. This is a guarantee of freedom from excitement. All visitors and relations are excluded and the child sees only the mother or nurse. How absolute. this shall be, how long continued depends on the progress of the case and the temperament of the patient.

As avoidance of excitement as well as physical rest is the desideratum, much depends on the temperament of the mother or nurse. This does not mean that the patient is imprisoned and such isolation can be carried 
on in the open air or the patient sent away to the country with the nurse and isolation effected there. In acute cases, however, the darkened quiet room is preferable.

The diet should be sufficient and that of the healthy child in the milder cases and in the acuter cases sufficient too, but chosen as in the febrile cases.

These measures alone are often sufficient. They should be persisted in until effectual or for six weeks to two months have passed. Of course a time comes when the need of light and air makes us abandon the closer confinement, or the child's depression may forbid its continuance.

Local Measures. Warm baths, especially before sleeping are often sedative in their effects and sometimes hot packs have a similar result.

In febrile cases cool sponges may be of value.

Drugs. As a rheumatic manifestation salicylates are indicated as in acute rheumatism, but there is quite a widespread belief that of the salicylates acetyl salicylic acid (aspirin) has a decided superiority over the others. The dosiage should be that specified for rheumatism.

Time honored, too, is the use of arsenic. It may be given in any form, but the preparation perhaps to be preferred is the liquor potassii arsenitis or Fowler's solution. The dose begins with $\mathrm{m}$. ij to iij (0.130.20 c.c.) increasing up to the point of tolerance. The urine is carefully watched meanwhile and with any evidence of albuminuria the drug is promptly stopped. If the stomach or bowels are affected or there is puffiness under the eyes we stop until the symptoms disappear and begin again on half the dose.

Vaccines. I have had but a limited experience with the use of vaccines in chorea, but believe there is a field for them in chorea, when administered with the above precautions.

I deplore the use of mixtures of organisms of unknown value and strength so widely advertised by commercial interests.

Treatment of Symptoms. Restlessness may sometimes be controlled by the use of luminal in half grain doses $(0.03 \mathrm{Gm}$.) three or four times a day. In adults slightly larger doses may be used. Three cases in my practice have shown diploplia even on these doses which quickly cleared up when the drug was omitted.

When the patient is very restless and the movements severe, one may use choral, best by rectum, in doses of gr. $\mathrm{v}-\mathrm{xxx}(0.30-2 \mathrm{Gm}$.) according to age, remembering that children bear relatively large doses. Codeine is sometimes very effective in controlling the movements. Dosage should be appropriate to age and it is to be remembered that it is very much more effective when given hypodermically. In an adult the dose ranges from $1 / 8$ to 1 grain $(0.008-0.06 \mathrm{Gm}$.) of the phosphate. 
For the worst forms and the maniacal type morphine sulphate in doses of gr. 1/16-1/4 (0.004-0.016 Gm.) or hyoscine hydrobromide gr. 1/200-gr. 1/100 (0.0003-0.0006 Gm.) may be necessary.

At times the patients movements are so violent that measures have to be taken to restrict them lest the patient throw himself from the bed and suffer injury. In the hospital side boards have to be attached to the bed and lined with pads or pillows to prevent trauma following the violent motion. In children the same precaution should be taken with the crib. I have seen the skin on the bony parts, over the heels, malleoli, elbows, actually cornified from the constant attrition. One is likely to get sores difficult to manage. The part liable to damage should be padded.

Auto-Sero-Therapy: This method has been recently advocated. Goodman describes his method as follows: Withdraw 50 c.c. of blood, centrifugalize, pipette off serum and place on ice. Do a lumbar puncture and withdraw 15-20 c.c. of fluid. Heat the serum to body temperature and slowly inject (into the subarachnoid space) taking 10-15 minutes, 15-18 c.c. of fluid. In a series of 30 cases so treated, he reported 20 cases cured and 7 improved. By cure he defines the cessation of all twitching in a week. It is assumed that some ferment or antibody present in the serum is introduced directly to the site of irritation.

Convalescence from Rheumatism. The patient should be kept in bed for some time after the symptoms have subsided, one or two weeks; if the heart has been involved, longer, as detailed above.

The diet should be increased to include green vegetables, later eggs, fish, and meat if they have not already been allowed. It should be simple in its character and in the manner of its preparation. It should be sufficient, but not excessive, nor should the patient be teased to stuff by palatable dishes. A change of environment often helps to establish convalescence, but the patient should not be hurried away too soon or exposed to discomforts in bis new surroundings for the mere sake of the change.

Prophylaxis. The avoidance of rheumatism, so lamentable in its consequences, must rest upon our education of the public in hygiene, through the schools and other agencies. The gospel of fresh air must be preached, clean bodies, proper clothing, avoidance of neglect, such as remaining in wet and damp clothing. More than this, parents and teachers must be made to understand the meaning of chorea, sore throats, stiff necks, and "growing pains" in children, and the results of neglect.

The physician should be the teacher, as the term "doctor" implies, 
both in his daily walks and in the more public capacity of lecturer, health officer, school inspector, etc. The school inspectorship is rife with the possibilities of infinite good.

The upper air passages afford the portal of entry of the vast majority of rheumatic infections.

Tonsils and adenoids hypertrophied to the point of obstruction, even though they have never been the seat of acute infection should be removed.

Tonsils and adenoids that have been the seat of infection whether accompanied or followed by rheumatism or not should be removed.

Tonsils and adenoids in all cases of rheumatism should be removed between the attacks.

There is a difference of opinion expressed by excellent observers as to the advisability of removing tonsils during an acute attack of rheumatic fever. That such a procedure may entail additional infection cannot be denied, nor does it seem advisable to submit a patient to the other exigencies of an operative procedure during the height of an infection. Finally foreign body pneumonia is an all too frequent sequel of tonsillar enucleation even in health. But when the infection is persistent or the heart involved and other measures fail to control the infectious process it becomes a question as to whether the danger of leaving a focus of infection is not greater than the dangers entailed in its removal. Hence the difference of opinion. The most brilliant result I have ever seen in promptly controlling an acute rheumatic fever with serious cardiac involvement, lasting for 10 to 12 weeks, followed a tonsillar enucleation, which was done at a time when an acute appendical attack necessitated an operation for removal of that organ.

I favor complete enucleation of the tonsil. The tissue should be carefully preserved in a sterile container to be submitted to bacteriological investigation for the purpose of identification and preparation of vaccines.

Sinuses should be examined and any abnormality of the nose attended to.

The teeth, especially that condition known as pyorrhea alveolaris contribute to infection, though in my experience the so-called toxic arthritis has more commonly been associated with this infection than the clinically true rheumatism.

These should have dental consideration and it is a condition that puts to the test the best dental skill.

Some investigators believe that bronchial infection and intestinal infection point the road to rheumatic infection and certainly these conditions demand attention. 
Rheumatic children, especially, when showing nervousness should not be urged at school.

Moon has sensibly remarked that they should do no evening work and should rest in the middle of the day.

When in these cases headache and poor sleep intervene or there is a frank exacerbation of nervousness the little patient should be taken out of school until matters improve.

Damp cold has long been known to provoke rheumatism and recurrences of rheumatism and Rosenow declares that exposure to cold after injection of rabbits with the rheumatic organism increases the percentage and degree of joint involvement.

This of course accentuates the necessity of avoidance of exposure to cold and wet; the importance of promptly changing wet stockings and clothes, removal from cold damp houses and localities and the importance of warm clothing, and especially underclothing containing wool.

\section{Rest.}

\section{SUMMARY}

Quiet.

Exclusion of visitors.

Mental rest.

Freedom from annoyance, conversation, business cares in the adult, efforts at amusement in children.

Good nursing.

\section{Bed.}

Hospital type preferred. Gatch bed, see text.

Woven wire spring.

Firm mattress.

Flannel blankets.

Flannel nightgown - open down front or side.

\section{Room.}

Light and air.

Avoid draughts.

Temperature $65^{\circ} \mathrm{F}$. to $70^{\circ} \mathrm{F}$.

Open air.

\section{Diet.}

Calories-approximate 3,000 in adult and more if well taken.

Protein 65 to $75 \mathrm{Gm}$.

During early hours with high fever and anorexia don't push food.

Milk-640 calories to 1 quart. Protein $33 \mathrm{Gm}$.

Sugar-120 calories to 1 ounce.

Cereals-about 100 calories to large serving. Average: protein $4 \mathrm{Gm}$. Rice-about 100 calories to large serving. 
Oatmeal cooked-100 calories to 5.5 ounces. Protein, $4.25 \mathrm{Gm}$.

Oatmeal dry-1 oz. - 120 calories.

Cream-16\%, calories, 50 per ounce. Protein, $1 \mathrm{Gm}$.

Cream-40\%, calories, 120 per ounce. Protein, $1 \mathrm{Gm}$.

Eggs-70-89 calories, each. Protein, $7 \mathrm{Gm}$.

Eggs-Yolk, 50-65 calories.

Eggs, Whites-16-25 calories.

Flours in general approximate 100 calories to the ounce.. Protein, $3 \mathrm{Gm}$.

Bread-100 calories to thick slice $(1 \& 1 / 3$ ounce). Protein, $4 \mathrm{Gm}$.

Butter-100 calories to pat (scant $1 / 2$ ounce). 230 calories to the ounce.

Milk, soups, mutton broths, chicken broths thickened with rice or cereals.

Vegetables
Fish as temperature approaches normal.

Meats after temperature has been normal a few days.

\section{Bowels.}

Salts-Epsom, Rochelle, Glauber's 5 ss.-j (15-30 Gm.) followed by enema of plain water or soapsuds if needed.

LATER.

Enemata.

Salines.

Hunyadi water-other mild equivalents on the market, liquor magnesii citratis, 5 viii-xii (240-360 c.c.), Seidlitz powders or, if obstinate, salts mentioned above and enemata to follow.

\section{"Specific" Treatment.}

Sodium salicylate gr. $\mathrm{xx}(1.33 \mathrm{Gm}$.) every two hours. As pain subsides cut gradually to $\mathrm{gr}$. $\mathrm{xv}$, then gr. $\mathrm{x}(1.00-0.66 \mathrm{Gm}$.) every two hours.

Keep at gr. x $(0.66 \mathrm{Gm}$.) until all active phases have past.

After symptoms have disappeared gr. $\mathrm{x}$ or $\mathrm{v}(0.66-0.33 \mathrm{Gm}$.) every two or three hours for a week or ten days.

Then for four to six weeks give gr. $\mathrm{x}$ or $\mathrm{v}(0.66$ or $0.33 \mathrm{Gm}$.) three or four times a day. Continue with alkali (see below).

If satisfactory results do not ensue increase dose to production of toxic manifestations. This may be anticipated in the adult in the neighborhood of 150 to 200 grains a day, depending on the sex, weight, etc.

Administer alkali; citrate of potash, acetate of potash, bicarbonate of soda, enough to render urine alkaline and keep it alkaline or neutral.

Rule - two grains of the alkali, preferably bicarbonate, for one of the salicylate.

Toxic manifestations. (See text.)

R

Sodii Salicylatis....................... そ ss.

Aquæ, q. s. ad........................ f $\mathrm{g}_{\mathrm{j}}$ ii

M.

S. Teaspoonful in water every two hours. 
R

Acidi Salicylici...................... 3 iv

Sodii Bicarbonatis..................... 3 iii

Aquæ, q. s. ad....................... f 3 ii

M.

R

S. Teaspoonful in water every two hours.

Acidi Acetylsalicylici.....................gr. cl

Ft cap. no. $\mathrm{xxx}$.

S. Three (3) or four (4) every two hours.

Give alkaline as above, but between the doses, to avoid incompatibility, or push dose to toxic manifestations. This may be anticipated in the adult near 120 or 160 grains a day depending on sex (weight). Toxic manifestations (see text).

Methyl salicylate or oil of gaultheria in $\mathrm{m} . \mathrm{xv}-\mathrm{xx}$ (1.00-1.30 c.c.) every two hours.

P

Olei Gaultheriæ................... $3 \mathrm{v}$

Ft. cap. no. $x x x$.

S. Two (2) every two hours.

R

Olei Gaultheriæ................... f 3 iv

Acaciæ, q. s.

Aquæ, q. s. ad....................... f 5 ii

M. ft. emul.

$\mathrm{P}$

S. Teaspoonful every two hours.

Olei Gaultheriæ.................... f 3 iv

Acaciæ................................

Potassii Citratis..................... 节

Aquæ, q. s. ad........................ f $\mathrm{z}^{\mathrm{j}}$ iv

M. ft. emul.

S. Teaspoonful every two hours.

Give bicarbonate or other alkali gr. ii to m. i of the oil; may push to production of toxic manifestations.

These may be looked for at about 120 minims ( 8 c.c.) a day.

For toxic manifestations. (See text.)

Diplosal-(salicylsalicylic acid) gr. x (0.66 Gm.) every two hours.

R

Diplosal...........................20.00

$3 \mathrm{v}$

Divide in capsules no. $\mathrm{xxx}$.

S. One every two hours.

Or push to toxic manifestations, which may be anticipated as dose approximates 80 to 100 grains a day depending on sex (weight).

Administer alkali between doses. Dose about gr. iv $(0.25 \mathrm{Gm}$.) per grain diplosal.

Toxic manifestations. (See text.) 


\section{Rectal administration of salicylates.}

Use a thin starch paste at body temperature, 5 iv-vj (120-180 c.c.).

Add 1/2 daily dose of salicylate, e. g., $3 \mathrm{i}(4 \mathrm{Gm}$.).

Add $\mathrm{m}$. $\mathrm{i}$ to ii of tincture of opium if needed to retain.

Inject slowly; hold buttocks together for a few minutes.

Dose every twelve hours.

or

Give whole dose, e. g., 3 ii $(8.00 \mathrm{Gm}$.) in the same amount of starch paste once a day.

Alkalis, e. g., bicarbonate of soda, is given at two-hour intervals by the mouth, the total day's dose to amount to two grains to one of the salicylates.

\section{Intravenous administration.}

Technique. (See text.)

Use when salicylates cannot be borne for any reason as in the case of idiosyncrasies.

\section{Drugs to relieve pain.}

Acetanilid, gr. iss. $(0.10 \mathrm{Gm}$.) every $1 / 2$ hour for 4 doses; repeat at six or eight hour intervals, or gr. ii-iii (0.15-0.20 Gm.) every two hours. Give in capsules or powders.

Acetphenetidin (Phenacetin), gr. iii to $\mathrm{v}(0.20-0.30 \mathrm{Gm}$.) every two hours. Give in capsules or powders.

Antipyrin - gr. iii-iv $(0.20 \mathrm{Gm}$.) every two hours. Give in capsules or solution.

For severe pain.

Morphine sulphate best hypodermically, gr. 1/16-gr. to $1 / 8$ (0.0040.008 ) adult dose.

\section{Symptomatic treatment.}

Joints.

Rest.

Semi-flexion.

Pillows, cushions, sand bags.

Carefully padded splints.

Cold.

Ice bag or ice coil.

Cold compresses.

Use of Gatch bed.

Fomentations.

Protection with non-absorbent cotton and flannel bandages.

Drugs.

Methyl Salicylate or oil of wintergreen.

R

Methyl Salicylatis.

Menthol........................... $\bar{a} \bar{a} 15$ per cent.

Petrolati q. s. ad......................... 30.00 ₹i.

M. et fiat unguentum.

S. Local use. 
Ichthyol.

R

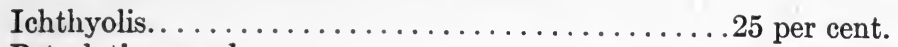

Petrolati q. s. ad........................ 30.00 รi

M. et fiat unguentum.

S. Local use.

\section{Counterirritation.}

Cautery.

Fly-blister.

Pressure.

Flannel bandage.

Rubber bandage.

\section{Persistent effusions.}

Paracentesis-For Technique, see text.

Later Measures.

Gentle manipulation.

Massage.

Fomentations.

Baking.

Vaccines.

\section{Complications.}

Hyperpyrexia.

Cold baths.

Put in tub at $65^{\circ} \mathrm{F}$., or to save shock at $80^{\circ} \mathrm{F}$., cooling it to $65^{\circ} \mathrm{F} .$, gradually.

Keep temperature of water down by adding ice.

Take patient out when temperature is $102^{\circ} \mathrm{F}$.

Repeat when patient's temperature rises to $104^{\circ} \mathrm{F}$. or $105^{\circ} \mathrm{F}$. Cold packs.

Sheet wrung out of cold water and wrapped about patient's body and then rubbed with pieces of ice.

\section{Cardiac complications.}

Continue salicylates in full dose as described above.

Continue in bed two months at least, after all other signs of rheumatism have disappeared, and as much longer as the condition of the heart may seem to warrant.

Allow first to sit up, then to get on feet, but always guided by evidences of heart's stability.

Increased rate and tumultuousness of beat out of proportion to effort made, indicates continuance of rest.

If heart decompensates it is to be treated like a decompensated heart under other circumstances.

The acute febrile process does not contra-indicate the use of digitalis in full doses.

Digitalis, full dose the equivalent of gr. ix-xii (0.60-0.80 Gm.) daily 
for three or four days or until signs of accumulation or desired results ensue, then stop two or three days and resume with smaller dose gr. iii (0.20 Gm.) a day (adult dose).

\section{After treatment.}

Tonsilectomy.

Avoidance of damp cold.

Change of climate.

Regulated exercise beginning soon after disappearance of symptoms.

Passive first, then active within limit of demonstrated cardiac capacity.

\section{Pneumonia and pleurisy.}

Treat as under other circumstances.

Continue salicylates in full doses.

\section{Sore throat.}

Careful oral toilet. (See Pneumonia, Chap. IX.) Tonsillitis, Chap. V. When severe treat as in Scarlet Fever. (See Chap. XVI.)

\section{Insomnia.}

Trional, gr. $\mathrm{x}-\mathrm{xx}(0.66-1.33 \mathrm{Gm}$.) in early evening and repeat if needed two or three hours later (adult dose).

Give in capsule or powder.

Chloralamid, gr. $\mathrm{xx}-\mathrm{xxx}(1.33-2 . \mathrm{Gm}$.) in early evening; repeat, if needed, in two to three hours (adult dose).

When pain is present use morphine hypodermically gr. 1/16 to gr. $1 / 4$ (0.004-0.015 Gm.) (adult dose).

\section{Anæmia.}

Iron. Blaud's pill (Pil. ferri carbonatis), or 'Vallet's Mass (Massa ferri carbonatis) gr. $\mathrm{v}-\mathrm{x}(0.33-0.66 \mathrm{Gm}$.) three times a day.

In children lesser doses of same, or bitter wine of iron (Vinum ferri armarum) 3i-iii (4-12 c.c.), three times a day.

\section{Sweats.}

Sponging with 1 per cent. bicarbonate of soda.

Talcum powder.

Zinc oxide and starch equal parts. Apply locally.

Atropine sulphate gr. $1 / 100$ to $1 / 50$ (0.0006-0.0013 Gm.).

\section{Vaccine therapy.}

Use only autogenous vaccines.

Seek organisms by cultures.

1. from blood.

2. from joint fluid.

3. from subcutaneous nodules.

4. from depths of tonsilar crypts.

5. from sites of obvious infection as teeth, ears, sinuses.

Time to use vaccines.

During subacute stage or between exacerbations. 
Dosage.

1st dose tentative, $5,000,000-10,000,000$.

In cardiac case $1,000,000-5,000,000$.

Frequency.

Twice a week.

Succeeding doses.

In the absence of reactions, double the doses up to $150,000,000$ $200,000,000$ then increase by $100,000,000$ at a dose.

Reaction. (See text.)

If reaction is slight and local do not increase next dose.

If reaction is severe and general wait until all signs of reaction have subsided for two or three days and begin with lesser dose.

Number of doses.

Depends on results and is a matter of judgment, 10 to 12 or perhaps more.

Technique - that of any hypodermic medication; cleanliness of instruments, operator and site of operation.

Syringe graduated to fractions of a c.c., e. g., tuberculin syringe.

In cardiac cases.

Beginning dosage lower.

Advance in dosage more cautiously.

Foreign Protein Therapy. (See Text.)

Tonsillectomy. (See Text.)

\section{Chorea.}

Rest in bed.

Isolation.

Diet. Liberal if afebrile; as in rheumatism if febrile (see above). Local measures.

Warm baths especially before sleeping, or two or three times a Drugs. day. Hot packs once a day. Cool sponges in febrile cases.

Salicylates in full doses as in rheumatism. Acetyl salicylic acid (Aspirin) the form preferred.

Arsenic; any form.

Fowler's Solution (Liq. potassium arsenitis), m. ii-iii (0.12-0.20 c.c.), as a beginning dose, increase a minim a day or a minim a dose a day up to the point of tolerance; that is, disturbance of stomach or bowels or puffiness under the eyes.

Watch urine for albumin.

Then stop until symptoms disappear; begin with $1 / 2$ largest dose and increase again.

Vaccines.

As in rheumatism, but with less dose and cautious increase.

\section{Autoserotherapy.}

(See text.)

Marked restlessness and violent movements.

Chloral, gr. v-gr. $\mathrm{xxx}(0.33-2.00 \mathrm{Gm}$.) in $\mathrm{J}$-iiii $(60-90$ c.c.) of warm milk by rectum at night. (Children take large doses relative to their age.) 
Most severe and maniacal cases.

Morphine sulphate, gr. $1 / 16$ to gr. $1 / 4$ (0.004-0.015).

Hyoscine hydrobromide, gr. 1/200 to gr. 1/100 (0.0003-0.0006 Gm.).

Convalescence in rheumatism.

In bed one or two weeks after symptoms subside.

In cardiac cases longer, as above.

Diet. Made more liberal. Change of environment to be considered.

\section{Prophylaxis.}

Education of public.

Fresh air.

Cleanliness.

Warm clothing.

Avoidance of chilling.

Inspection of upper air passages.

Elimination of tonsils and adenoids.

Teeth to be attended to.

Avoidance of excitement and urging in school of the nervous children already infected.

After treatment of the heart.

See text. 


\section{CHAPTER IV}

\section{ACUTE RHINITIS-CORYZA}

ThIs affection, known to the laity as a "cold in the head," is one of the most infectious diseases to which we are exposed and, as immunity seems but short-lived, these two facts contribute to make it the most common of our afflictions.

What the etiological agent is, is not known. Pathogenic organisms in abundance have been recovered from the secretions of the nasal passages during an acute rhinitis, but no one of these has been proven to be the etiological agent. A strong plea has been made for a filterable virus, and such it may well prove to be; but it remains for the future to definitely settle that question. Moreover, it is possible that the rhinitis that accompanies or ushers in certain other acute infections, such as measles or influenza, may be actuated by other organisms than those responsible for a primary attack.

The common cause for an infection is direct exposure to an individual suffering an attack. Some men are inclined to think that that is the only mode of infection. Others believe that many are in a carrier state, harboring the infecting organism as most of us do Type IV pneumococcus and afford opportunity for infection by lowering resistance as is the case with Type IV pneumococcus. Such a lowering of resistance follows exposure and chilling, such as wet feet and wet clothes, remaining in draughts when the skin is warm and moist and it has seemed to me that conditions causing intense congestion of the tissues of the nasal mucous membrane, like uncontrolled sneezing, open portals to infection. Nasal obstruction, adenoids or possibly old sinus infections, may predispose to attacks.

Symptomatology-The symptoms are local and in the severe forms, general. They are so familiar to every reader that an attempt to picture them is an act of supererogation. The stuffed nose, especially following nasal obstruction, the thin watery secretion that wets handkerchief after handkerchief, and excoriates the nasal openings, the dull frontal headache, and in severe cases, the conjunctival congestion, the miserable malaise, or even general aches and pains, with perhaps a slight fever, constitute the catalogue of events.

The seriousness of an attack lies in the fact that it weakens the bar- 
riers of resistance to other infections that invade the rest of the air passages and contiguous structures.

Treatment-Early or abortive. The object of this is to promote hyperemia and a leucocytosis, two reactions of protective significance. To be effectual the treatment must be prompt and in the early hours of the attack.

A hot bath or a hot mustard foot-bath (for technique, see index) is taken; after which the patient should go to bed. The bed must be warm and the patient should get between blankets or put on a flannel night dress or pajamas. A hot water bottle should be placed at the feet and hot drinks should be taken - hot lemonade with or without a dash of whisky, ( 5 ss. -15 c.c.) or hot weak tea or hot Imperial drink (a tablespoonful of cream of tartar in two pints of boiling water-it is poorly soluble in cold water-to which is added lemon juice or lemon peel and sugar to the taste). All this induces sweating, diaphoresis, and for the same purpose Dover's powder (Pulv. ipecac et opii) has long been a favorite drug of many. It may be given to an adult in the full dose of gr. $x(0.66 \mathrm{Gm}$.) or in any fractions of this dose at frequent intervals until the whole amount is taken.

Great care must be taken when perspiring freely to avoid exposure; so the treatment should not be undertaken except in warm rooms and a warm and properly prepared bed.

Aches and pain are best treated with acetylsalicylic acid (aspirin) in doses of gr. v-gr. $\mathrm{x}(0.33-0.66 \mathrm{Gm}$.) at 2 or 3 hour intervals or small doses of a coal-tar preparation at frequent intervals; e. g., acetanilid gr. i ss. (0.10 Gm.), antipyrin gr. ii $(0.120 \mathrm{Gm}$.), acetphenetidin gr. iii $(0.20 \mathrm{Gm}$.) at hourly intervals for 4 doses, then every 2 hours in such a prescription as follows:

$\mathbf{R}$

Acetanilidi....................... 50

Sodii Bicarbonatis.................. 1.00

Caffeinæ Citratæ.................... 0.50

(gr. $\mathrm{xx}$ iiss.)

(gr. xv)

M. et div. in cap. no. xv.

(gr. viiss.)

S. One every hour for 4 doses, then every 2 hours.

or:

R

Codeinæ Sulphatis...................... 0.125

Acetphenetidini (phenacetin) ..............00

Acidi acetylsalicylici (aspirin) ............5.00

(gr. ii)

(gr. xlv)

M. et div. in cap. no. xv.

(gr. lxxv)

S. One every 2 or 3 hours. 
When the secretion is excessive, if the physician does not prescribe, the patient is pretty sure to take one of the multitudinous rhinitis or coryza tablets on the market, the bases of which are belladonna, camphor, aconite, and often quinine and small doses of opium.

Local Treatment. The object of this is to promote drainage and ventilation and so afford comfort. It is effected by applications made directly to the sites involved, by sprays or by inhalations.

Direct Applications. It is well to precede all applications by a cleansing spray of normal salt solution, Seiler's tablets, or one of the many alkaline solutions on the market which contain solutions of both alkaline salts and certain volatile oils. Argyrol 15 per cent. to 25 per cent. applied on a cotton swab on an applicator, or used as a spray seems at present to be the choice for the purpose. In addition to this it is by some clinicians dropped in the conjunctival sacs, thus gaining access to the nasal mucous membrane by way of the lachrymal ducts.

Drops of adrenalin (epinephrin) 1-1000 into either nostril, or used as a spray or applied on a pledget of cotton on an applicator and left in contact with the mucous membrane for a few seconds, astringe these structures and allow of ventilation. Cocaine has been used for the same purpose; but there are too many objections to it to permit of recommendation; 1 per cent. solution of the hydrochloride is that commonly used. Antipyrin, 3 per cent. solution, is used for the same purpose and is less objectionable than the cocaine; at times the two are combined in the strengths specified.

The constringency induced by the above applications is maintained by ointments or sprays the essential ingredient of which is menthol.

Ointments.-As useful will be found the following:

R

Aristol. ............................1.00

Mentholis...........................0.10

Petrolati q. s. ad...................... 15.00

(gr. xv)

(gr. iss.)

M. S. Local use.

(ॐss.)

or:

R
Mentholis
.0 .20
(gr. iii)
Olei Pini Pumilionis...........................
Olei Rosæ............................0.060
Petrolati........................... 30.00

M. S. Local use.

Sprays. One may use 1 per cent. each of menthol, camphor and oil of eucalyptus in Benzoinol or liquid petrolatum. 
or:

$\mathbf{R}$

"Mentholis........................gr. $\mathbf{x x x}$

(2.0 Gm.)

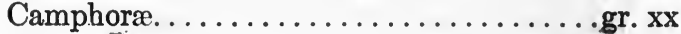

(1.30 Gm.)

Eucalyptolis.................. $\mathbf{x x}$

(1.30 c.c.)

Olei Rosæ.......................... ii

Benzoinol q. s. ad..................

$(0.20$ c.c.)

(60.00 c.c.)

M. S. Use in oil atomizer."

(Coakley.)

If this is found too strong the menthol may be reduced to $\mathrm{gr} . \mathrm{x}-\mathrm{xv}$ $(0.66-1$. Gm.) or the whole diluted with more benzoinol to an agreeable strength.

Special oil atomizers must be used for all these.

Inhalations. Simple steam inhalations may be found grateful, or compound tincture of benzoin, or the oil of pine, one or two teaspoonfuls on the hot water of the inhaler, or a few drops of the alcoholic saturated solution of menthol in the same manner, or such prescription as follows:

$R_{*}$

Olei Pini Sylvestris................

Olei Eucalypt....................

Mentholis......................... $x$

Creosoti.

m. $x$

Tr. Benzoin Comp. q. s. ad............... ii

( 2.00 c.c.)

(15.00 c.c.)

(0.66 Gm.)

(0.66 c.c.) lations one may use pitchers, carafes, kettles, with stiff paper rolled for a funnel, or a croup kettle, or one of the simple and inexpensive inhalers on the market.

Later, when the discharge becomes thickened, warm alkaline sprays may be used such as follows:

$R_{4}$

"Sodii Bicarbonatis

Sodii Biboratis .................a gr. xxxii $\quad(2 \mathrm{Gm}$.

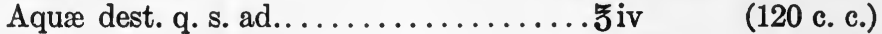

M. S. Use as spray."

(Coakley.)

or one of the mild alkaline "antiseptics" of the trade. If there is still much obstruction to the nasal passages, adrenalin (epinephrin) or antipyrin as directed above. Such a spray as follows may prove of value:

R
Iodine
0.06
Menthol
(gr.i)
Liquid petrolatum
(gr. i ss.)
M. S. Spray from oil atomizer with hard rubber parts.

Nasal irrigations and douches are deplored as being likely to induce otitis. 
Sore Throat. If a sore throat accompanies the coryza, the specified inhalations may be used. If a tonsillitis, see Chap. V. If a cough without definite signs in chest, one may assume a tracheitis and give such a mixture as follows:

R

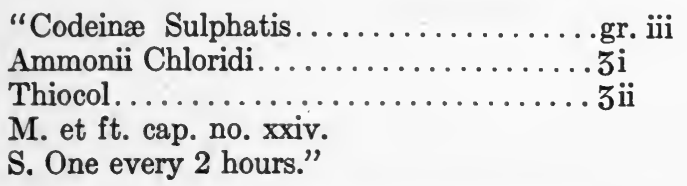

S. One every 2 hours."

(Bingham.)

For detailed treatment, see Tracheitis, Chap. VII.

Complications. As has been said the respiratory tree at large may be involved or the contiguous structures. See Bronchitis, Pneumonia, Laryngitis, Tonsillitis (Chap. VII, IX, VI, V).

Sinusitis. This is a not uncommon complication. The frontals, ethmoids, or sphenoid sinuses, or antra of Highmore may become involved. The methods detailed above of local applications to facilitate ventilation and drainage are of considerable prophylactic value; but clear evidences of sinus involvement requires expert attention. To those who must rely on their own management, special treatises on this subject are recommended.

Antral Involvement. Efforts at drainage should first be made before having recourse to radical procedure. See details under Grip, Chap. XI. For lavage and surgical procedure consult special treatises.

Otitis. This is always to be feared, especially in young children. Irrigations and douches should not be used in the nose. Instructions should be given to blow nose gently, one side at a time, to avoid forcing infection into Eustachian tubes and antra or sinuses. When antra are involved try to suck discharges back into pharynx, to expel by mouth rather than blow nose.

Prophylaxis. Rooms should be kept at $68^{\circ}$ to $70^{\circ}$ and as in most steam heated apartments the air is deprived of its moisture an effort should be made to replace this by suitable devices.

Removal of obstructions - such as tonsils, adenoids, and the rest of infected tissue of Waldeyer's ring; attention to hypertrophied turbinates, deviated septa, and chronically infected sinuses and antra.

Personal Hygiene. Training the vaso-motor supply of superficial vessels, so that the vessels will quickly respond to changes in environment. This is done by daily cold baths, sleeping in well-ventilated rooms, windows open at all times of year, exercise in the open air, avoidance of overclothing, especially about the neck. 
Dress lightly in house, and on going out add clothing suitable to the weather.

Underclothing should be of silk or cotton mesh or if it contains wool should be very light.

\section{Treatment.}

\section{SUMMARY}

Early or abortive.

Must be begun early.

Hot bath.

Hot mustard foot bath.

For technique see Pneumonia, Chap. IX.

Patient must go to bed promptly.

Bed to be made up with blankets rather than sheets or patient to wear flannel night clothes.

Hot water bottle at feet.

Hot drinks to be taken.

Lemonade with whisky, 5 ss. (15 c.c.).

Imperial drink (one tablespoon of cream of tartar in one quart of boiling water, and sugar to taste).

Dover's powder gr. $x(0.66 \mathrm{Gm}$.) or in small fractions at frequent intervals until this amount is taken.

Avoid exposure if this treatment is taken.

\section{Aches and pains.}

Acetylsalicylic acid (aspirin) gr. v-gr. x (0.33-0.66 Gm.) every two or three hours.

Or coal-tar preparations, acetanilid gr. iss. (0.10 Gm.), antipyrin, gr. ii $(0.120 \mathrm{Gm}$.), acetphenetidin (phenacetin) gr. iii $(0.20 \mathrm{Gm}$.).

Give any one of these at hourly intervals for 4 doses and then every 2 hours.

or:

R

Acetanilidi.................... 1.50

Sodii Bicarbonatis................1.00

Caffeinæ Citratæ................. 0.50

(gr. xxiiss.)

(gr. $\mathrm{xv}$ )

(gr. viiss.)

M. et div. in cap. no. $\mathrm{xv}$

S. One every hour for four doses and then one every two hours.

or:

R

Codeinæ Sulphatis.................... 0.125

Acetphenetidini (Phenacetin)............2.50

Acidi Acetylsalicylici (Aspirin) ...........5.0

(gr. ii)

(gr. xl)

(gr. lxxv)

M. et div. in cap. no. xv.

S. One every two or three hours.

For excessive secretions combinations of belladona, aconite, quinine, 
opium, in one of the multitudinous formulas offered by pharmaceutical houses may be tried.

\section{Local applications.}

Precede by cleansing sprays of normal saline or one of the alkaline solutions on the market.

Argyrol $15 \%-25 \%$ on swab of applicator or as spray. Same may be dropped in conjunctival sacs.

To relieve obstruction.

Adrenalin (epinephrin).

1:1000 dropped in either nostril or sprayed or applied by applicator and left in contact with mucous membrane for a few seconds.

Or antipyrin, $3 \%$ solution.

The effects of the above may be continued by sprays or ointments with menthol as the chief ingredient.

R
Aristol
1.0
(gr. $\mathrm{xv})$
Mentholis.
$0.10 \quad$ (gr. iss.)
Petrolati q. s. ad
15.00
M.
(负ss.)
S. Local use.

or:

R
Mentholis
0.20
Olei Pini Pumilionis...................... 0.125
(gr. iii)
(m. ii)
Olei Rosæ................................
(m. i)
Petrolati........................... 30.00
M.
S. Loral use.

As sprays one may use $1 \%$ each of menthol, camphor, and oil of eucalyptus in benzoinol or liquid petrolatum.

or:

R
"Mentholis
.gr. $x x x$
(2 Gm.)
Camphoræ............................ $\mathrm{xx}$
(1.3 Gm.)
Eucalytolis
m. $x x$
Olei Rosæ
m. iii
Benzoinol q. s. ad
予 $\mathrm{ii}$
(1.3 c.c.)
( 0.2 c.c.)
(60 c.c.)

M.

S. Use in oil atomizer."

(Coakley.)

If this is too strong reduce the menthol to gr. $\mathrm{x}-\mathrm{xv}(0.66-1 \mathrm{Gm}$.$) or$ dilute with benzoinol.

\section{Inhalations.}

Simple steam.

Compound tincture of benzoin. 
Oil of pine.

One or two teaspoonfuls of either on the hot water of inhaler or one or two drops of saturated alcoholic solution of menthol in the same manner.

or:

R

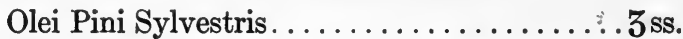

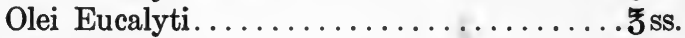

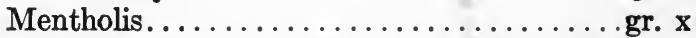

Tr of Benzoin Co. q. s. ad................

M. S. 20 drops in boiling water for inhalation.

(2.00 c.c.)

(15.00 c.c.)

(0.66 Gm.)

(60.00 c.c.)

For inhalers use pitchers, kettles with stiff paper rolled, or one of the simple inhalers on the market.

\section{Later treatment.}

Warm alkaline sprays, e. g.

R

Sodii Bicarbonatis

Sodii Biboratis................. āā gr. xxxii

Aquæ q. s. ad.................... 节iv

(2.0 Gm.)

M.

(120 c.s.)

S. Use as spray.

(Coakley.)

May use mild alkaline antiseptics of the trade.

For obstruction of the nasal passages use adrenalin or antipyrin as above.

or:

R

Iodine ................................

Menthol...........................

Liquid Petrolatum..................60.00 (ร ii)

M.

S. Spray from oil atomizer with hard rubber parts.

Do not use nasal irrigations and douches lest they induce otitis. Sore throat.

Inhalations as above. See Tonsillitis (Chap. V).

Cough.

With no signs in the chest, probably tracheitis.

R

Codeinæ Sulphatis..................... iii

Ammonii Chloridi...................

Thiocol.........................

(0.20 Gm.)

$(4.00 \mathrm{Gm}$.

M. et ft. cap. no. xxiv.

(8.00 Gm.)

Or see Tracheitis, Chap. VII.

\section{Complications.}

Laryngitis. (See Chap. VI.)

Tonsillitis. (See Chap. V.) 
Bronchitis. (See Chap. VII.)

Pneumonia. (See Chap. IX.)

Sinusitis.

Local applications.

Persistent cases or suspected empyema, see special treatises.

Antrum Involvement.

Effect drainage. See Influenza, Chap. XII.

Otitis.

Always suspect in young children.

Routine examination of ear.

Avoid douches.

Blow nose gently and one side at a time.

Suck discharge back into the pharynx rather than blow nose.

Prophylaxis.

Temperature of rooms $68^{\circ} \mathrm{F}$. to $70^{\circ} \mathrm{F}$.

Replace moisture in air by one of the suitable devices on the market. Removal of obstructions - such as tonsils, adenoids, and hypertrophied turbinates.

Treat chronically infected sinuses and antra.

\section{Personal hygiene.}

Train the vasomotor supply of the superficial vessels by cold baths, sleeping in well ventilated rooms, exercise in open air, avoid overdressing in the house and on going out add clothing suitable to the weather.

Underclothing should be of silk or cotton mesh or if it contains wool it should be very light. 


\section{CHAPTER V : \\ TONSILLITIS AND VINंCENT'S ANGINA \\ TONSILLITIS}

BETweEn the mouth and the larynx stands the wall of adenoid tissue, designed as a barrier against infection. This is distributed in a circle about the tube and is known as Waldeyer's ring. It accumulates in masses on either side known as the faucial tonsils; above, where it is commonly spoken of as the adenoid or adenoids, its structure usually showing a central and lateral lobes; and below, where it is known as the lingual tonsil. Lymphatics from the mouth drain into these structures whose function it is to check infection coming from these sites. Infections which force these barriels pass to the lymphatic channels and glands beyond. Their importance to the welfare of the body is readily understood; but when from repeated infections they become the sites of chronic infection, harboring organisms ready to take advantage of any lowering of the individual's resistance they become a menace to the integrity of many remote organs and all too frequently to life. It is generally appreciated that the tonsils harbor such infection, but it is too commonly forgotten, especially in the adult, that the adenoid, and still more the lingual tonsil may be chronically infected to continue the infection, after complete enucleation of the faucial tonsils.

However, because the faucial tonsils are most commonly and most obviously affected we speak of this "sore throat" as Tonsillitis.

The infecting organisms are in the majority of instances streptococcus hemolyticus, but the invaders may be streptococcus non-hemolyticus, (viridans) Staphylococcus aureus or micrococcus catarrhalis.

The structure of the tonsil with its deep crypts, which one may think of as the prints of fingers thrust deeply into dough, favors the reception and retention of pathogenic bacteria.

The tonsils become swollen and reddened and bulge out from between the pillars of the fauces, the peritonsillar tissue is also infiltrated and reddened. The sites of the plugged crypts appear as white spots, and afford the designation of follicular tonsillitis. Often the exudation from adjacent follicles coalesce. The tissue between these white areas is red and swollen.

The differential diagnosis from a diphtheritic throat is often very 
difficult and many times can be made only by cultures and microscopical examination of smears.

The characteristics of a typical tonsillitis are an exudate of a yellowish color separated by a deep red mucous membrane giving a patchy appearance and confined to the tonsils. It may be removed without causing any bleeding.

The sore throat of diphtheria, on the other hand, has a membrane of an ashen gray color. It usually starts at one point and spreads out over the tonsil and may extend onto the pillars of the fauces, the edge of the soft palate and uvula. It is firmly adherent and when removed leaves a bleeding surface.

The worst form of streptococcus hemolyticus infections are called septic sore throat and are commonly seen in milk-borne epidemic mastitis in cows.

The cervical glands, receiving the lymphatics draining the tonsils, become enlarged and tender and in severe infections may break down into abscesses, which may point and discharge on the surface.

Bullowa insists that the gland which receives the drainage from the tonsil is situated high up in the triangle made by the sterno-cleido mastoid and the posterior belly of the digastric muscle and not the one at the angle of the lower jaw commonly called the tonsillar gland.

It must be remembered that a tonsillitis may be the expression of rheumatism, a diphtheria, an early symptom of scarlet fever, a Vincent's angina, or the precursor of an infection of the respiratory tree and that syphilitic sore throat, either the primary lesion or secondary, and acute lymphatic leukæmia may lead to an error of diagnosis.

Symptomatology. The onset is often abrupt and the evidences of infection severe. Chilly sensations or a chill followed by fever, headache, general muscular pains like a grip infection and sore throat constitute the symptomatology. The tonsils are swollen and red and the crypts may be plugged or a membrane be present.

To be sure, many attacks are much lighter and patients may insist on keeping about. But acute tonsillitis is never a trivial affair and the involvement of remote structures, such as the kidney or the heart or joints may occur in what seems like a mild attack.

Treatment. The patient should be confined to bed even in a light attack. Children and all the members of the family who are susceptible to sore tbroats, or infection of the air passages of any kind should be kept away from the patient. In fact, there is little common sense in allowing any person to come into contact with a patient suffering from an infectious process unless it is to act in the capacity of a nurse.

Cultures and Precautionary Measures. Text-book descriptions 
of the differential diagnosis between follicular tonsillitis and diphtheria lead you to wonder how a mistake could ever be made or to marvel that a differentiation can ever be achieved.

Experientia docet and the man who has seen much of these two conditions rarely"errs; but for the general run of us the knowledge that a net is always laid for the unwary and that a text-book follicular tonsillitis may be diphtheritic, plead for the culture tube in the physician's bag and the use of it as a routine in dealing with sore throats. On the other hand the throat highly suspicious of diphtheria may turn out on culture to be streptococcal in origin; for this reason, if cultures cannot be taken, or reports are likely to be delayed, or in children or adults showing prostration, let the patient have benefit of the doubt and administer antitoxin at once. (See Diphtheria, Chap. XVIII.)

Diet should be light. The condition of the throat causes so much pain on swallowing that all articles of food must necessarily be fluid, or at the most semi-solid; milk, broths, cereals, custards; jellies, ice-cream, milk toast are the basis of such a dietary and one may be referred to the diet lists given in the chapters on Typhoid Fever and Pneumonia for the several items. It is amazing how much flesh is lost during the short attack of tonsillitis of three to six days and theoretically a dietary should take these needs into consideration; but when swallowing is torture, knowing it to be an affection of relatively short course, one must be satisfied with what the patient can take without great discomfort. Sometimes the food is preferred hot, sometimes cold, sometimes as fluid, again with a little consistency and the frequency must be dictated by individual considerations.

Drink. Water, alkaline water and such fruit juices as the inflamed structures accept without discomfort are indicated in such quantities as the patient can readily take.

Bowels: At the beginning of the illness it is well to move the bowels with a mild saline, such as Hunyadi water or one of its many equivalents on the market. This may be preceded, if one so chooses, by a small dose of calomel gr. 1/10-1/4 (0.006-0.015 Gm.) at 10 or 15 minute intervals until one grain is taken. Liquor magnesii citratis 5 viii (240 c.c.), a Seidlitz powder, or one of the more drastic salts, Epsom, Rochelle, Glauber's or Sodium Phosphate in $\varsigma_{\mathrm{ss}}$, $(15 \mathrm{Gm}$.) doses may be preferred. Throughout the illness the bowels may be regulated with enemata, liquid petrolatum, cascara, aloin, phenolphthalein or a mild saline water.

Drugs. The salicylates have always been favorites in the treatment of tonsillitis; in fact perhaps, because as a local manifestation of rheumatism it seems to yield to this so-called specific and in part be- 
cause of the general anodyne effects of the group. It may be given as sodium salicylate, acetylsalicylic acid (aspirin), salicin, diplosal or other form, my choice being one of the first two. The usual dose is gr. v-x (0.33-0.66 Gm.) at two-hour intervals or it may be used as in rheumatism. (For details and prescriptions see Acute Rheumatic Fever, Chap. III). A favorite prescription of mine combines small doses of tincture of aconite with salicylate

R

Tincturæ Aconiti......................

Sodii Salicylatis.....................5.00

Aquæ destillatæ, q. s. ad.............60.00

(m. xvi)

(gr. $\mathrm{lxxx}$ )

M.

(כ)

S. One teaspoonful every two hours. (Delafield.)

In the early hours of the infection, the so-called sthenic period, coaltar preparations may be used for the general aches and pains. Acetanilid, antipyrin, acetphenetidin (phenacetin) alone or combined with bicarbonate of soda to lessen their irritating effects on the stomach and for its antidotal action or with citrated caffeine which increases the anodyne action and is assumed to counteract the depressing action of the coal-tar drugs on the circulation, an assumption which is more than dubious.

I prefer to give doses smaller than the usual text-book doses, but at frequent intervals, e. g.

R

Acetanilidi .......................... 1.50

Sodii Bicarbonatis................... 1.00

Caffeinæ Citratæ......................50

(gr. xxiiss)

(gr. xv)

(gr. viiss.)

M. et div. in cap. no. xv.

S. As directed.

I order one of these given every $1 / 2$ hour for four doses, then every hour for four doses, then every two hours. (Adult dose.)

Fever. This is usually not so high as to need interference. The drugs above mentioned ameliorate it, though not given for that particular purpose. If the fever gives the patient discomfort, it is to be met by cool or cold sponges of water; following which an alcohol rub is found gratifying.

Care of the Body. A daily sponge with castile soap and warm water should be given. The nose should be freed from secretions by the use of cotton swabs on wooden tooth-picks as applicators, moistened with a saturated solution of boric acid (4 per cent.) or $1 / 2$ to $1 / 4$ strength Dobell's solution. The mouth should be cleansed by rinsing and gargling with the same solutions and food removed from the interstices of the 
teeth and from between the gums and the cheeks and lips by the use of cotton swabs on wooden applicators.

Insomnia. It cannot be too often insisted that sleep is rest and rest is repair. As a rule the milder hypnotics are sufficient; bromides gr. $\mathrm{xv}(1 \mathrm{Gm}$.$) , trional gr. \mathrm{x}-\mathrm{xv}\left(0.33-1^{\circ} \mathrm{Gm}.\right)$, adalin gr. $\mathrm{v}(0.33$ Gm.), chloralamid gr. $\mathrm{xx}(1.33 \mathrm{Gm}$.), or somewhat more potent preparations; barbital (veronal) gr. v-vii ss. $(0.33-0.5 \mathrm{Gm}$.) or barbital sodium (medinal) in the same doses. If the pain in the throat is keeping the patient awake codeine phosphate gr. $1 / 4(0.015 \mathrm{Gm}$.) or morphine sulphate gr. $1 / 8(0.008 \mathrm{Gm}$.) hypodermically is indicated.

Local Treatment. It not infrequently happens that an application of a strong solution of silver nitrate to the tonsils, if done in the first few hours will abort or decidedly ameliorate the process. This solution should be 25 per cent. to 50 per cent. and applied liberally with a swab. Others use a weaker solution of silver nitrate 40 to 60 grains (2.33 to $4 \mathrm{Gm}$.) to $5 \mathrm{i}(30$ c.c.) and make the application three times a day.

I advise the stronger solution in the first 12 hours and after that rely on hot irrigations of normal saline or boric acid 2 per cent. to 4 per cent. at $100^{\circ} \mathrm{F}$. to $115^{\circ} \mathrm{F}$. and if the throat is very red $115^{\circ} \mathrm{F}$. to $118^{\circ} \mathrm{F}$., every hour or two while awake. For efficiency much depends on technique. I refer the reader to the section on angina under Scarlet Fever, Chap. XVII where the treatment by irrigations, inhalations, sprays and gargles as well as external applications is taken up in detail.

I would add as an excellent gargle, though my faith in gargles alone is small, one made up as follows:-

R.

Sodii Salicylatis

Sodii Bicarbonatis

Sodii Biboratis.................... àa 30

One teasponful in $3 / 4$ glass of hot water to gargle. (Goodridge.)

Phenol 1-100 solution used as a gargle is said to relieve pain. (Hare.)

If properly applied the throat compress is of practical value where there is much pain. The technique is given by Baruch as follows:

"Although this compress is probably more frequently applied than the other, it is remarkable how little its rationale is understood and how imperfectly it is applied. The usual method is to fold a handkerchief or napkin into a narrow bandage, dip it in cold water, wring it out, and wind it around the neck, securing it by pin. In a very short time the movements of the patient displace the bandage, which has been applied loosely to prevent choking, so that it loses its shape, allowing air to enter freely from above; more or less chilling is then produced and the 
compress dries rapidly. As will be seen in the description of the therapeutic indication of the throat compress, the object is defeated by this imperfect application, unless it is intended to treat some tracheal or laryngeal trouble. When intended for the treatment of tonsillitis, diphtheria and other pharyngeal affections, the throat compress should be applied as follows: A piece of old thin linen, of sufficient length to reach from below the ear on one side to the same point on the left, is folded into a bandage of four layers. A piece of flannel, eight by twenty-four inches, provided with a slit for each ear, is also made ready. These bandages are fitted by actual measurement to the patient's head, so that they may pass under the chin from ear to ear. The linen compress bandage is now wrung out of water at $\left(60^{\circ} \mathrm{F}\right)$ and laid upon the middle of the dry flannel bandage. While the wet bandage is placed under the chin, the flannel bandage is unrolled from the top of the head and passed over the right side of the head (the right ear being made to protrude through the slit) and then passed under the chin to the left side, where the left ear is also allowed to protrude (the slit being made longer than actually needed, to insure perfect apposition of the bandage and prevent pressure on the ear). The entire bandage is now drawn firmly over the head and secured by pins. Two sets of bandages are required - one being allowed to dry while the other is in use.

"In children and restless patients, additional security is afforded by a circular turn around the head forming a bandage to which the throat compress may be pinned." Baruch. "Hydrotherapy."

Adenitis. (See Scarlet Fever, Chap. XVII.)

Nephritis. (See Scarlet Fever.)

Convalescence. (See Scarlet Fever.)

Tonsillectomy. (See Scarlet Fever.)

Septic Sore Throat. The virulent forms of streptococcus hemolyticus infection that have received this designation are associated with such degrees of toxemia that they require special consideration. With these, too, the more serious complications are likely to ensue.

Circulation. Precisely the same problem is presented here as in Scarlet Fever (unless endocarditis has intervened, for which see below). The reader is referred to the treatment of failing circulation in that disease. (Chap. XVII.)

Furthermore a daily examination of the heart even well into convalescence should be made and frequent examination of the urine and especially in convalescence. This is the time, too, when the productive nephritis, secondary to a scarlet fever, the rash of which may have been overlooked is likely to develop.

Toxemia. Beside the supportive treatment one has to consider 
the value of the specific measures we have at hand. This question, too, is discussed under Scarlet Fever. (Chap. XVII.)

We are dealing with a streptococcus hemolyticus and may have recourse to three efforts of doubtful value, but with certain theoretical justifications behind them, e. g., the administration of polyvalent streptococcus serum, the use of autogenous or polyvalent vaccines, and transfusions. Details of these procedures will be found under the chapters specified.

Complications and Sequellæ. As has been said a given case of tonsilitis may usher in a rheumatic infection and be followed by a rheumatic arthritis, endocarditis, chorea or other manifestations. On the other hand an infectious arthritis may occur and an acute malignant endocarditis or if the infecting organism is streptococcus non-hemolyticus (viridans) a subacute bacterial endocarditis.

Nephritis. An acute exudative or an acute productive nephritis may occur, which latter may pass over to a chronic form. This is to be treated as under other circumstances. (See Scarlet Fever, Chap. XVII.)

Septicæmia is not a rare result of a septic sore throat. This subject is considered in Chap. XLV.

Complications due to the involvement of more contiguous structures are:

Otitis media. In young children in whom this process may occur without pain, a routine examination of the ear should be made. Early incision of the drum is indicated. The treatment is fully considered under Scarlet Fever. (Chap. XVII.)

Adenitis. This, too, will be found fully discussed under Scarlet Fever.

Peritonsillar Abscess. The local signs and symptoms are aggravated. The pain is intense and may extend upward to the ear if the pus burrows in that direction. The mouth is opened with difficulty, swallowing is painful and feeding almost impossible so that with the toxemia and loss of food, weakness and loss of flesh are very marked. The anterior pillars of the fauces are red or purple and swollen and the soft palate often involved. There is a bulging of the tissues concerned frequently to the midline. The uvula may be swollen and reddened.

The incision is made in the most prominent portion of the swelling. The soft palate and other tissues may be treated by application of 10 per cent. cocaine hydrochloride solution, but this unhappily does not do much to lessen the pain of incision. A sharp-pointed curved bistoury may be used, the blade being protected with adhesive plaster to within a half inch of the point. The incision is made from above downward and should be nearly vertical to avoid injuring important blood vessels. It 
begins at a point above the soft palate where it joins the uvula and $3 / 8$ of an inch outside of the inner margin of the anterior pillar of the fauces.

The incision should be deep to the extent of the exposed portion of the blade.

Pus should follow the incision but it is possible that the failure to evacuate pus is due to an unsuccessful incision or to the fact that a pus pocket has not yet formed. A failure to make a successful incision is often due to the patient's drawing back to avoid the pain and therefore the head should be firmly supported to prevent this.

If there is much offensive pus Coakley advises syringing or sponging the cavity with 1:5000 phenol (carbolic acid) and inserts a strip of iodoform gauze to prevent too rapid closure. If no pus follows the incision it may appear a day or two later. Relief will be afforded by a lessened congestion following incision.

If pus burrows downward in the posterior pillar, an incision is made over the point of greatest bulging.

Convalescence. Both physician and patient err in hastening the period of convalescence. Even after short attacks the patient plainly shows the effects for a long time. In the World War, Army surgeons studying return to fitness of patients after tonsillitis, found the period prolonged to an extent that amazed the civilian practitioner and made him realize the injustice he has regularly done his patients. Fresh air, good food a simple tonic of strychnine sulphate gr. 1/60-1/30 (0.001$0.002 \mathrm{Gm}$.) three times a day or such a prescription as follows may be ordered:

$\mathrm{R}$

Tr. Nucis Vomicæ.......................20

Sodii Glycerophosphatis.................20

Aq., dest. q. s. ad....................120

$\mathrm{M}$.

S. One teaspoonful three times a day.

A red cell count and hemoglobin determination should be made, and if there is anemia, iron in some form is indicated. Arsenic may be combined with it. My favorite is Vallet's mass and arsenious acid, e. g.:

R

Massæ Ferri Carbonatis................. 10.00

Acidi Trioxidi........................0.045

M. Massa fiat. div. in cap. no. $\mathrm{xxx}$.

3iiss.

gr. $3 / 4$

S. One or two, three times a day, after meals.

Chronic Tonsillitis. This is not a treatise on chronic conditions, but one cannot discuss tonsillitis in any form without voicing the danger that lurks in a chronically infected tonsil. The mere cataloging of such 
sequences as chronic arthritis, chronic nephritis, rheumatism or chorea, with their endocarditis, subacute bacterial endocarditis, constant infection of the upper air passages with repeated colds and bronchitis as well as otitis and âdenitis and the suceptibility of diseased tonsil to tuberculous infection, is plea enough for the removal of such a danger spot.

It is but a reversal of the above statement to say that the indications for removal of the tonsils are:

1. When they are causing obstruction.

2. When they are the seat of chronic infection.

3. Repeated attacks of acute tonsillitis.

4. Rheumatic fever with tonsils infected. (For discussion of time for removal see Acute Rheumatic Fever, Chap. III.)

6. Repeated infection of the upper air passages.

7. Chronic rheumatic endocarditis, as a prophylactic measure.

8. Chronic nephritis or acute nephritis if secondary to tonsilar infection.

9. Chronic cervical adenitis, tuberculous or simple.

9. The operation is a tonsillectomy; enucleation; and all diseased tissue of Waldeyer's ring, adenoid and lingual tonsil should be removed at the same time.

\section{SUMMARY}

\section{Treatment.}

Confine patient to bed in even a light attack.

Isolate from all of the family who are not acting in the capacity of nurse.

Make cultures and smears from all sore throats.

Administer diphtheria antitoxin to any patient who has a suspicious throat that cannot be cultured for any reason.

\section{Diet.}

Fluid or semi-solid.

Broths, cereals, custards, jellies, ice cream, and toast form the basis of such a dietary. (See Typhoid, Chap. XIV.)

\section{Fluids.}

Water, alkaline waters, fruit juices in such quantities as may be taken with comfort.

\section{Bowels.}

At the beginning of the illness give a mild saline such as Hunyadi water.

May precede the above by a small dose of calomel gr. 1/10-1/4 (0.006$0.015 \mathrm{Gm}$.) at 10-15 minute intervals until a grain is taken.

Liquor Magnesii Citratis, 5 viii (240 c.c.) or Epsom, Rochelle or Glauber's Salt, 5 ss. $(15 \mathrm{Gm}$.) may be preferred. 
Throughout the illness regulate bowels with enemata, liquid petrolatum, cascara, aloin, phenolphthalein or a mild saline water.

\section{Drugs.}

Salicylates as sodium salt, acetyl salicylic acid (aspirin), salicin, diplosal. Dosage gr. $\mathbf{v}-\mathbf{x}(0.33-0.66 \mathrm{Gm}$.) at two-hour intervals as in rheumatism (see Chap. III) or:

R

Tincturæ Aconiti....................... (m. xvi)

Sodii Salicylatis....................5.00 (m. lxxx)

Aquæ Destillatæ q. s. ad.................60.00 (ङ ii)

M. et S. One teaspoonful every two hours. (Delafield.)

Or the coal-tar preparations in the sthenic period for aches and pains. Acetanilid, antipyrin, acetphenetidin (phenacetin) alone or comAs

bined with sodium bicarbonate and citrated caffeine.

R

Acetanilidi...................... 1.50

Sodii Bicarbonatis.................... 1.00

Caffeinæ Citratæ.................... 0.50

M. et div. in chart. no. xv.

S. One every half hour for four doses, then every hour for four doses, then every two hours for an adult.

Phenacetin (acetphenetidin) double the dose of acetanilid. Antipyrin, $11 / 2$ the dose of acetanilid, may be used in solution

\section{Fever.}

Cool or cold sponges followed by alcohol rub if fever gives discomfort.

\section{Care of the body.}

Daily sponge with castile soap and warm water.

Nose freed from secretions by cotton swabs with boric acid 4 per cent. or $1 / 2$ to $1 / 4$ strength of Dobell's solution.

Mouth cleansed with same solutions on cotton applicator and sordes and food particles removed from interstices, etc., with tooth picks.

\section{Insomnia.}

Rest imperative.

Milder hypnotics usually sufficient:

Bromides gr. xv (1 Gm.).

Trional gr. $\mathrm{x}-\mathrm{xv}(0.66-1 \mathrm{Gm}$.).

Chloralamid gr. xx (1.33 Gm.).

Or more potent preparations as barbital (veronal) gr. v-vii ss. (0.33$0.5 \mathrm{Gm}$.) or barbital sodium (medinal) in same doses. Codeine phosphate gr. $1 / 4(0.015 \mathrm{Gm}$.) or morphine sulphate gr. 1/8 (0.008 $\mathrm{Gm}$.) hypodermically if the pain in the throat disturbs sleep.

\section{Local treatment.}

$25-50 \%$ solution of silver nitrate applied on a swab to the tonsils may abort or ameliorate the process if used in the first few hours. 
Guiacol and glycerin equal parts may be used for the same purpose and in the same way.

Silver nitrate solution containing 40 to 60 grains (2.33 to $4 \mathrm{Gm}$.) in one ounce $(30$ c.c.) of water may be used in place of the stronger soution and applied three times a day. I prefer the stronger solution in the first 12 hours followed by hot $\left(110^{\circ} \mathrm{F} .-115^{\circ} \mathrm{F}\right.$.) normal saline every hour or two while awake. For details, see Scarlet Fever, Chap. XVII, text and summary.

\section{Throat compress.}

(See text.)

\section{Gargles.}

$\mathbf{R}$

Sodii Salicylatis......................

Sodii Bicarbonatis. . . . . . . . . . . . . . . . . .

Sodii Biboratis................... $\bar{a} .30 .00$

M. et S. Dissolve one teaspoonful in three-quarters glass of hot water. Use as a gargle. (Goodridge.)

Phenol solution 1:100 used as a gargle, Hare says, will relieve pain. Adenitis; nephritis, convalescence, tonsillectomy. (See Scarlet Fever, Chap. XVII.)

Pus may be removed from crypts by suction.

\section{Heart.}

Should be examined daily well into convalescence. (See text.)

\section{Urine.}

Examine frequently during the attack and in convalescence. (See text.)

Septic sore throat. (See text.)

Circulation. (See Scarlet Fever, Chap. XVII.)

Toxemia. (See text and Scarlet Fever, Chap. XVII.)

Complications and sequelæ. (See text.)

Peritonsillar abscess. Incision. (See text.)

Convalescence.

Do not hasten even after a light attack. Fresh air, good food, a simple tonic such as strychnine sulphate gr. 1/60-1/30 (0.001-.002 Gm.) three times a day, or:

$-\mathbf{R}$

Tr. Nucis Vomicæ......................20

Sodii.Glycerophosphatis . . . . . . . . . . . . . . . . 20

Aq. dest. q. s. ad. ...................120 (丂 iv)

M. et S. One teaspoonful three times a day.

If anemic, give iron or iron and arsenic as Vallet's mass and arsenious acid. 
$\mathbf{R}$

Massæ Ferri Crabonatis............ 10.00

Acidi Trioxidi.................... 0.0045

Massa fiat, div. in cap. no. xxx.

(3iiss.)

(gr. $3 / 4$ )

S. One or two three times a day.

Chronic tonsillitis.

Enucleation of the tonsils and the complete removal of the adenoid tissue in Waldeyer's ring.

\section{VINCENT'S ANGINA}

This condition, which is not infrequently encountered and diagnosed as either acute follicular tonsillitis or diphtheria, is on the tonsil but a local expression of what may be a more widespread oral infection and in some instances an uncontrollable and fatal one, called noma or cancrum oris. It will be briefly described here. The affection is characterized by the formation of a necrotic membrane that most commonly is found upon the tonsils, though by no means confined to these structures as it may invade the fauces, gums, cheeks and indeed any part of the mouth, pharynx and in rarer instances larynx, trachea or bronchi.

It simulates closely one of two conditions and to one unfamiliar with Vincent's angina the error in diagnosis is almost invariable; when superficial it will be called diphtheria and when ulcerative, and it is often a deep, punched out ulcer, it will be called syphilitic.

The membrane simulating diphtheria is ashy gray or has a yellowish or even greenish tinge, is easily removable and then discloses the bleeding surface of the superficial ulceration. When the ulcers are deep they are found filled with a gray pultaceous material and both forms untreated are curiously persistent.

In the severe types, occurring especially after measles or in the course of certain blood diseases, notably leukæmia, the extent and swiftness of the process is appalling; soft tissue and bones, cheeks, gums, alveolar processes, melting before its advance.

In the mild cases there are not many general symptoms, only a very trifling rise of temperature; the one symptom being the persistent sore throat; but in the severe type intoxication ensues with fatal results. The organisms concerned in this necrosis are easily detectable and so characteristic that they cannot be mistaken. There is a fusiform bacillus (bacillus fusiformis), it is double pointed, thicker in the middle and often of a beaded or barred appearance from deep staining of granules.

These fusiform bacilli are always accompanied by spirilla that look like the spirochetæ pallidæ but have fewer twists and wider spirals.

It has not yet been definitely determined whether the occurrence of these two forms constitute a symbiosis or whether they are different 
stages in the development of a single organism. They are found in normal mouths where they seem to be saprophytic, but under certain conditions become pathogenic.

The conditions that determine pathogenicity in these organisms, are an unhygienic mouth, neglected teeth, the lowered resistance accompanying or following certain diseases, as leukæmia, measles, scarlet fever, diphtheria.

Treatment. Milder cases only require local treatment and the prompt manner in which a long standing and annoying condition yields to simple procedures is most satisfactory.

Remove the membrane with a swab dipped in 1 per cent. cocaine hydrochloride as the ulcerations are sensitive, often exquisitely so, and apply directly to the ulcer one of the following substances:

Chromic Acid, 5 per cent. solution. This is one of the best applications. Repeat daily until the ulcer heals.

Silver Nitrate, 10 per cent. to 25 per cent. solution applied in the same manner.

Zinc Sulphate, 5 per cent. solution. I have had no experience with this application.

Tincture of Iodine. This is applicable in the milder cases and on small areas.

With all this, proper care of the mouth must be instituted. The dentist should be consulted and all affected teeth receive proper treatment. A neglect of these precautions invites relapse.

As malnutrition and the lowered state induced by infectious diseases are predisposing factors, a sufficiency of a properly selected diet is of first importance in these conditions, together with fresh air and sunlight.

Severe Cases. When the necrosis is deep and rapid the condition is appalling and as unyielding as the milder cases are yielding.

Salvarsan is indicated in these cases. The technique and dosage is the same as in syphilis. The success that meets the use of arsenical compounds speaks for the spirochetal nature of the infecting organism.

Cancrum oris is discussed under the heading of Measles, Chap. XIX, where the radical measures to stay the process are detailed.

\section{SUMMARY}

Mild cases.

Do not require confinement to bed, nor, in the absence of constitutional manifestations, to the house.

\section{Local treatment.}

Remove membrane with swab dipped in 1 per cent. cocaine to relieve pain of the procedure. 
Applications.

Chromic acid, 5 per cent. solution to the bared ulcer daily until healing is established.

Silver nitrate, 10 per cent. to 25 per cent. solutions, in the same manner.

Zinc sulphate, 5 per cent. solution in the same manner.

Tincture of iodine: on small areas.

Oral hygiene.

Dental supervision.

Abundant diet in cases of malnutrition and convalescence from infectious diseases.

Fresh air and sunlight.

\section{Severe cases.}

Confine to bed.

For choice of bed, room, care of body and maintenance of the circulation see Pneumonia, Chap. IX.

\section{Salvarsan.}

Technique and dosage same as in syphilis.

\section{Cancrum oris.}

Radical surgical procedure. (See same under Measles, Chap. XIX.)

Bismuth subnitrate paste. (See Measles, Chap. XIX.) 


\section{- CHAPTER VI}

\section{ACUTE LARYNGITIS}

THE larynx may be the seat of an acute inflammation, either as a primary process or secondary to or a part of an infection elsewhere in or general throughout the respiratory tract. It may then be found with a tracheitis or bronchitis or an item in a "cold" or "sore throat."

If patients are prone to laryngitis we suspect foci of infection in tonsils, adenoids, especially in children, and in sinuses or nasal passages. Irritating gases can produce a very acute inflammation.

Predisposing causes are to be found in exposure to cold, damp and high winds and excessive use of the voice. Not infrequently the attacks seem to have some connection with indigestion and are prone to occur in the gouty.

Symptomatology. Sore throat, more marked on swallowing, with hoarseness and cough, and in severe cases pain, are the chief symptoms. In young children from 1 to 5 years of age there is commonly in addition to the catarrhal inflammation a laryngeal spasm, due to reflex irritability, a spasmodic croup with a harsh, hollow or metallic quality to the cough, dyspnœa and inspiratory stridor. During the attack the child may look desperately ill and always sounds a note of alarm to the parent who sees it for the first time. It is always more severe at night, a remission occurring during the day, beginning with the early morning hours.

There may be a slight temperature, especially in children, of $100^{\circ} \mathrm{F}$. to $101^{\circ} \mathrm{F}$.

The laryngoscope discloses a redness of the mucosa and swelling and redness of the vocal cords.

This is not the place to go into detail of differential diagnosis, but one must keep in mind tuberculosis of the larynx with its ulceration of cords and arytenoids; syphilis with its gummatous infiltration; edema of the glottis and, in children, diphtheria. Croup carrying on into the day should be labeled diphtheria and the practitioner is wise to take a culture in all cases of croup. In infants, with manifestations of rickets, laryngismus stridulus is to be thought of. The general practitioner must be as familiar with the laryngoscope as he is with the stethoscope. These instruments, with the otoscope and ophthalmoscope, are as much a part of the equipment of the general practitioner as of the specialist. The 
former can read headlines with them, if not fine print and from headlines we gain the essential news.

Treatment. Rest; rest of the body at large and rest of the organ concerned. The patient should be ordered to bed; ordered because, perchance deeming it a matter of no great importance, he will not listen to language that is hortatory, but only to that expressed in the imperative.

To rest the organ involved the patient should be instructed not to talk, and what is of equal importance the people about him should be ordered not to make him talk more than is absolutely necessary and then in a whisper.

Smoking during the attack should be forbidden, an injunction illy received by the confirmed cigarette smoker. Irritating smoke or gases must be avoided, even if it necessitates removal of the patient from the environment.

Bowels. A free catharsis should be administered at the beginning of the attack, either one of the well-known salts, Epsom (magnesium sulphate), Rochelle (sodium and potassium-tartrate), Glauber's (sodium sulphate) or sodium phosphate in doses of 5 ss. (15 Gm.) in $3 / 4$ glass water or full doses of one of the many saline cathartics on the market of the type of Hunyadi water; later, milder doses or enemata may be relied upon.

Abortive Treatment. A hot full bath or a hot foot-bath or a hot mustard foot-bath is often taken to abort the process and in my estimation is seldom or never successful, but the patient may be rendered more comfortable by the process.

If a full bath is taken the patient must get at once into a warm bed preferably between blankets or into a flannel night dress or pajamas, have a hot water bottle put at the feet and take hot drinks; weak tea, hot lemonade to which a dash of whisky may or may not do more than break the drought. The same precautions should be taken with the foot-baths, as the object is to induce a gentle perspiration, which in a cold bed and a cold room and cotton night clothes might well aggravate the condition.

The mustard foot-bath is prepared as follows:-

"Mustard Baths and Foot-Baths. To prepare a mustard bath or footbath: Dissolve mustard in hot water in the proportion of two tablespoons of the former to a gallon of the latter. Stir well just before giving the bath.

"To give a foot-bath: Fold the bed-clothes up from the foot of the bed to above the patient's knees, replacing them with a double blanket. (Be careful not to expose the patient while doing this.) Turn part of the 
doubled blanket over the feet and back under the legs. Flex the knees and place the foot tub, half filled with water $115^{\circ} \mathrm{F}$., lengthwise on the bed, between the folds of the blanket. Lift the feet with one hand and, with the other, draw the tub under them. Put them into the water slowly, that they may become gradually: accustomed to the high temperature. Fold the blanket around the tub and knees, and bring down the bed-clothes. In about ten minutes add hot water, being careful not to pour it in near the feet. The bath lasts about twenty minutes. Take out the feet in the same manner as you put them in, drying them well, and place a hot-water bag against them." Maxwell and Pope, Practical Nursing.

In the early dry stage the cough is irritating and painful and sedative measures are indicated:

Inhalations. The inhalation of plain steam is gratifying or one may add compound tincture of benzoin, oil of pine or oil of eucalyptus, $3 \mathrm{i}-3$ ii $(4-8$ c.c.) on the surface of the water. One may use one of the many inhalers on the market, some of which are very inexpensive, or devise one from a kettle, pitcher or carafe to which a cone is affixed, made of a roll of stiff paper by which the steam is led to the face of the patient, lying on his side.

In children a croup kettle, one of the best of which is that devised by Holt, is the safest and most convenient. The child may be put into a tent made by a sheet thrown over the head of the bed, to the post of which laths may be attached to give elevation or over a clothes horse placed about the head of the bed or even over an open umbrella. Children must not be kept for long periods in these confined spaces, the damp, warm air of which becomes depressing.

In the early stages the drug which seems to afford the most relief is ipecac. A good prescription is as follows:

\section{(Adult dose)}

$\mathrm{R}$-Vini Ipecac..................... 10.00

Potassii Acetatis................... 10.00

Aquæ q. s. ad ....................... 120.00

M. et S. 3ii (2 teaspoonfuls) every three hours.

(3iiss.)

(3iiss.)

(马iv.)

A favorite prescription of the late Dr. Francis Delafield was tartar emetic and ipecac each gr. 1/100 (0.0006 Gm.) every half hour.

Cough. If the cough is excessive one may have recourse to codeine in doses of $\mathrm{gr} .1 / 8-1 / 4(0.008-0.015 \mathrm{Gm}$.) of the phosphate, or heroine in doses of gr. 1/16-gr. 1/12 (0.004-0.005 Gm.) of the hydrochloride. It is only the exceptional case, that not yielding to the above measures and losing sleep, requires morphine sulphate in gr. 1/8 (0.008 
Gm.) doses hypodermically. A local application of equal parts of spirits of turpentine, spirits of camphor and olive oil, well mixed and sprinkled liberally on a square of flannel, laid upon the chest and pinned to the night clothes may be found to ameliorate the cough.

Local Treatment. Cold compresses to the larynx are often effectual. They should be applied as described by Baruch in his $H y$ drotherapy.

"The Wet Compress, Technique. Two or more folds of old linen, thin or thick as may be required, and of the necessary size and shape to conform to the part which is to be treated, are formed into a compress. Cotton cloth is objectionable because it does not receive or hold moisture so well. If, however, linen is not available, the oldest and most worn cotton cloth should be selected. The compress is wrung out of water of the required temperature $\left(60^{\circ}-75^{\circ} \mathrm{F}\right.$.) and is covered with flannel or with a dry piece of linen of the same shape, but an inch or two wider and of sufficient length to secure the wet cloth snugly when pinned. It is a sine ua qnon of the cold wet compress that air be excluded from it, because the vaporization of water contained in it by warmth of the skin renders the latter extremely sensitive to chilling, which is the usual result of the imperfectly applied compress. The compress is renewed every hour as a rule, but this depends on each individual case. Before removal a fresh compress should be laid in readiness. A cardinal rule to guide in the renewal of the compress, applicable in all cases, is that the latter should be warm before removal. If it has not been warmed by an hour's apposition with the skin, it must either remain or be removed without renewal."

Ice Collar. A special ice bag made to apply as a collar may be used.

For technique in filling the bag and its application, see Pneumonia, Chapter IX.

Fomentations. Application of fomentation. Cut two or three thicknesses of flannel, sufficiently large to surround the neck. This flannel is placed in a crash towel, boiling water poured upon it, the ends of the towel twisted in opposite directions to squeeze the water from the flannel. The skin is smeared with vaseline or sweet oil and the flannels applied with dry flannels outside, all of which are kept in place by a binder. These are replaced as fast as they become cool. Care must be taken that not enough hot water is left in them to drip down upon the skin and burn.

Sprays. Perhaps the best spray is one containing menthol gr. ii in $\xi_{\mathrm{i}} \mathrm{i}$ of liquid petrolatum (albolene). 
$\mathrm{R}$
Ac. Carbolici (Phenol) ................... gr. iii
Menthol............................ vr. v
0.33
Benzoinol ........................ $\mathrm{z}$.
30.00
M. et S. Spray the throat while taking a deep breath.

In children steam or medicated inhalations as above are indicated. Hot fomentations, using the technique given above, and small doses of ipecac and tartar emetic āa gr. 1/100 (0.0006 Gm.) at 4 to 6 hour intervals and, if there is spasm, antipyrin $\mathrm{gr} . \mathrm{i}(0.060 \mathrm{Gm}$.) and sodium bromide gr. iii $(0.20 \mathrm{Gm}$.) to a dram of water 4 c.c. $)$ may be given at four-hour intervals.

The spasmodic croup is interrupted by the use of emetics, the safest and best is syrup of ipicac in teaspoonful doses at 15 minute intervals until vomiting occurs. There is rarely occasion for alarm, unless the croup is diphtheritic.

Complications. The one of alarming significance is edema of the larynx, more commonly occurring after the inhalation of irritating gases.

The measures for relief must be prompt. They are:

Scarification. "When the dyspnea is severe no time should be lost in scarifying the edematous tissue. This should be done by first spraying the back of the throat freely with a 20 per cent. solution of cocaine, and instructing the patient to swallow any excess of the fluid, so that some of the solution may bathe the edematous tissue in the aryepiglottic folds. A cotton-wound laryngeal applicator should be dipped in a 20 per cent. solution of cocaine and the swollen region of the larynx brushed with the solution, the tongue being protruded and the laryngeal mirror employed in order to paint accurately the swollen areas. Sometimes the edema will be markedly diminished owing to the constringent action that this drug has upon the mucous membrane. It is not safe to rely upon this entirely, for in an hour or so, when the effects of the cocaine have passed off, the edema usually reappears in a more aggravated form. Five minutes after the cocainization the parts should be well illuminated with a laryngeal mirror and a curved, concealed laryngeal knife should be introduced, the knife being concealed during the introduction, and only pushed forward to scarify the tissues when it has reached the areas of greatest swelling. Hemorrhage and transudation of serum follow, the swelling diminishes, and respiration becomes easier. If this procedure does not diminish the edema and cyanosis deepens, then tracheotomy should be promptly performed." Coakley, Diseases of the Nose and Throat. 
Intubation. (For technique see section under Diphtheria (Chap. XVIII.)

Tracheotomy. If this operation is to be performed, a textbook on surgery should be consulted.

\section{SUMMARY}

\section{Treatment.}

Rest of the body at large and of the organ concerned is imperative. If speaking is necessary, do so in a whisper.

Smoking is forbidden.

Irritating smoke or gases must be avoided.

\section{Bowels.}

An initial catharsis with a saline, e. g., Epsom, Rochelle or Glauber's salt in doses 5 ss. (15 Gm.) in $3 / 4$ glass water.

Later, enemata or milder salines.

\section{Abortive treatment.}

Hot full bath or hot foot-bath or hot mustard foot-bath may abort the process. Technique-see text.

Follow by going to bed at once between hot blankets or in warm flannel night clothes with hot water bag at feet.

Take hot drinks such as lemonade containing whisky.

\section{Inhalations.}

Plain steam or add compound tincture of benzoin, oil of pine or oil of eucalytus $3 \mathrm{i}-\mathrm{ii}(4-8$ c.c.) to the water in an inhaler or in a kettle, pitcher, carafe, etc., to which is attached a cone of stiff paper by which steam is led to the patient's face. For children the Holt Croup Kettle is best, or use a tent. (See text.)

Ipecac, best drug in early stages.

$R$
Vini Ipecac
10.00
(3iiss.)
Potassii Acetatis.................... 10.00
(3iiss.)

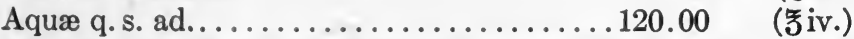
M. et S. Two teaspoonfuls every three hours for an adult. Or Del- afield's favorite prescription of tartar emetic and ipecac, each gr. 1/100 (0.0006 Gm.) given every half hour.

\section{Cough.}

If excessive, give codeine phosphate gr. 1/8-1/4 (0.008-0.015 Gm.) or heroine hydrochloride gr. 1/16-1/12 (0.004-0.005 Gm.).

For loss of sleep due to cough not yielding to above, morphine sulphate gr. $1 / 8(0.008 \mathrm{Gm}$.) hypodermically.

Local application of oil of turpentine, spirits of camphor and olive oil equal parts on a warm flannel laid on the chest and pinned to the night clothes. 


\section{Local treatment.}

Cold compresses to the larynx. (See text.)

Ice collar. (See text.)

Hot fomentations. (See text.)

\section{Sprays.}

Menthol gr. ii $(0.120 \mathrm{Gm}$.) in $5 \mathrm{i}(30$ c.c.) of liquid petrolatum (albolene) or

R

Ac. Carbolici.............................. gr. iii

Menthol.................................. v

Benzoinol...............................

M. et S. Spray the throat while taking a deep breath." (Coakley.)

For children.

Inhalations as given above.

Ipecac and tartar emetic of each gr.1/100(0.0006 Gm.) every 4-6 hours.

If there is spasm give antipyrin gr. $\mathrm{i}(0.060 \mathrm{Gm}$.) and sodium bromide gr. iii $(0.20 \mathrm{Gm}$.) to a dram (4 c.c.) of water every 4-6 hours.

\section{Complications.}

Edema of the larynx.

Scarification. (See text.)

Intubation. (See Diptheria, Chap. XVIII.)

Tracheotomy. Consult special surgery text-book. 


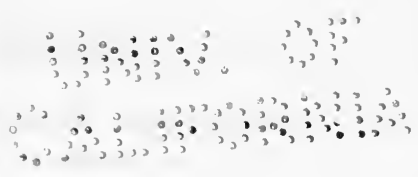

\section{CHAPTER VII}

\section{ACUTE BRONCHITIS AND TRACHEITIS}

These conditions will be considered together as the processes are identical and the terms are merely referable to regional distribution. The pathology consists of a catarrhal inflammation of the whole bronchial tree with a thick mucoid exudation, becoming thinner as the process advances toward resolution or becoming muco-purulent. It is usually confined to the larger and medium sized tubes; but in children and the elderly is prone to involve the smaller bronchi, when it is spoken of as a capillary bronchitis, and is, indeed, a broncho-pneumonia, though the areas of consolidation may be too small to give it the physical signs. Not infrequently the process is limited to the trachea (tracheitis) and the larger tubes; commonly the infection begins as a coryza and spreads downward, involving the larynx in its progress.

The bronchial involvement is always bilateral though in exceptional instances physical signs may be confined to one side and to a limited region. When these signs are so limited it is not justifiable to make a diagnosis of bronchitis until broncho-pneumonia and tuberculosis are excluded. If localized bronchitis occurs at the bases, the diagnosis should be broncho-pneumonia, if in the upper lobe, tuberculosis and more particularly at the apices; but broncho-pneumonia and a so-called grip pneumonia, a partial consolidation of the lobar type, may affect one upper lobe to simulate closely a tuberculosis. The infecting organisms may be the influenza bacillus, pneumococcus, streptococcus, micrococcus catarrhalis and more rarely others.

The inhalation of irritating gases may be the direct cause, as was witnessed on so large a scale amid the barbarities of the late war.

Among the predisposing causes are exposures to wet and cold, causing chilling, to which fatigue contributes, hence its frequency in Spring and Autumn with their sudden changes, overheating of rooms, overdressing, close confinement; causing sluggish vaso-motor response when exposed to cold. In children, adenoids and hypertrophied tonsils predispose to infection. (Holt.)

The immediate cause in the vast majority of instances is direct exposure to infection from others suffering from coryza and bronchitis; especially in crowded conveyances and places of amusement.

Bronchitis, too, is a common and early accompaniment of several 
- Infeut oas diseases, typhoid fever, malaria, measles, whooping cough. Tuberculosis, we are reminded, may begin as an acute bronchitis. (Norris and Landis.)

Symptoms vary with the severity and extent of the process. The one cardinal symptom is cough. This cough is at first dry; if the trachea is much inflamed it is racking and persistent and accompanied by a sense of soreness under the sternum and some oppression in that region. A coryza may or may not have preceded the cough. There are general symptoms of malaise and aches and pains, as after a mild grip, but they are not a feature. Fever may be trivial or rise to $102^{\circ} \mathrm{F}$. or $103^{\circ} \mathrm{F}$. or higher if the small bronchi are involved. As the process progresses the cough becomes looser and a mucoid or muco-purulent expectoration follows.

Physical Signs are such as are determined almost exclusively by auscultation. They are prolongation of expiration and rales, at first sibilant, later moist.

Treatment. A bronchitis, unless very widespread or a capillary bronchitis (broncho-pneumonia), is too often looked upon as a trivial affair. That in children it may be the precursor of measles or whooping cough or in adults an early manifestation of typhoid or tuberculosis should make us cautious; but if a primary process, it may, through neglect, lead to pneumonia or go into a chronic form, with the disagreeable sequences of bronchiectasis, emphysema and asthma.

The patient should certainly be confined to the house and, if there is any febrile reaction, to the bed until it has subsided for at least three days.

If the process is intense and the small bronchi and bronchioles are involved the case should be treated as a pneumonia. (See Chap. IX.)

Room. The room should be chosen with reference to a sufficient ventilation and access to sunlight. No matter how mild the case, it should not be shared with another person for sleeping purposes. Dry sweeping should never be allowed in a sick room; damp cloths should be used. Smoking should not be allowed. It seems ludicrous that the warning should have to be made; but to anyone who has had to treat the confirmed cigarette smoker it will seem less ludicrous than difficult. The habit of inhalation, practically universal among cigarette smokers, gives rise to further irritation of the inflamed air passages and also antagonizes the physician's best efforts.

Talking should be discouraged, as it aggravates the cough and encourages spread to the smaller bronchi.

In milder cases a specially selected bed, so desirable in long and serious infections, is not imperative. 
Diet. Except in severe cases no peculiar modification in the usual diet is necessary. In severe cases the diet is arranged as in pneumonia.

Precautions. It must be remembered that bronchitis is an infectious disease and that the infection is conveyed by the secretions of the patient. For the safety of the family, isolation is highly desirable. Certainly during the early days of the acute process it is well to keep all members of the family except those who act in the capacity of nurse, and certainly all visitors, out of the sick room. Members of the family and the patient should be told that coughing, sneezing and even conversation at close range convey the infection as a fine spray through several feet distance; that kissing, petting, hand-shaking, contact with the patient's soiled linen, towels and handkerchiefs, with table utensils, convey the infection directly. This information warns others from unnecessary contact and instructs the patient to cover the mouth and nose when coughing and sneezing.

All the secretions and expectorations should be caught on pieces of gauze or old cloths, placed in a paper bag and burned. Handkerchiefs and linen should be immersed in water until such time as they can be boiled to sterilize them; table utensils should be boiled and kept separate and a separate thermometer kept for the patient and when not in use should be immersed in an antiseptic solution, e. g. carbolic acid, 1 to 20 .

Early Measures. It has long been a habit of the layman, encouraged by the physician, to try to abort a "cold" by taking a hot bath, getting to bed between warm blankets, putting a hot water bag or bottle at the feet and taking a hot drink to encourage perspiration. I have never convinced myself that an infection could be so aborted, but it may relieve the discomforts incident upon a beginning infection. Unless, however, a warm bed and its accessories are at hand, a hot bath followed by chilling may do great harm.

Bowels. It is well to move the bowels with a mild saline; one of the many laxatives on the market of which Hunyadi water is a type, or with liquor magnesii citratis 3 viii (240 c.c.) or moderate doses 3 ii-iv (8 to $15 \mathrm{Gm}$.) of Epsom salt, sodium sulphate (Glauber's salt), Rochelle salt or sodium phosphate.

Headaches and General Aches and Pains. These are not constant and as a rule not severe. My custom is to use small but frequent doses of acetanilid, which I usually prescribe in this form.

$\mathrm{R}$

Acetanilidi'......................... 1.50

Sodii Bicarbonatis.................... 1.00

Caffeinæ Citratæ......................50

gr. xxiiss.

gr. $\mathrm{xv}$

gr. viiss.

M. et div. in capsulas no. xv.

S. As directed. 
These capsules are given at half hour intervals until four are given and then every two hours. They are continued only during the first 24 hours. For the same purpose acetphenetidin (phenacetin) in three grain $(0.20 \mathrm{Gm}$.) doses, combined with bicarbonate of soda and citrated caffeine in the doses given above may be used. If it is desired to give medicine in solution, antipyrin in 2 grain doses $(0.120 \mathrm{Gm}$.) may be substituted. If the headache is severe single large doses may be administered, e. g., acetphenetidin (phenacetin) gr. x (0.66 Gm.) with citrated caffeine gr. iii $(0.20 \mathrm{Gm}$.). This should not be repeated more than once at an interval of two hours.

Aspirin (acetylsalicylic acid), as being less depressing than the coal-tar preparations, is preferred by many men. It is an excellent drug for the purpose. The dose is gr. $\mathrm{v}$ to gr. $\mathrm{x}(0.33$ to $0.66 \mathrm{Gm}$.) at 2,3 or 4-hour intervals. Quinine has had a similar usage and is not infrequently combined with the coal-tar preparations and with the salicylates, all in small doses. Personally I have not been much impressed with the supposed synergistic effects of such combinations and have preferred to learn the possibilities of a single drug to becoming wise in the labyrinth of polypharmacy.

At the beginning of the process Dover's powder (Pulv. ipecac et opii) has long been employed; sometimes in a full dose of gr. x $(0.66 \mathrm{Gm}$.) or in divided doses of gr. i-gr. iiss. (0.060-0.150 Gm.). It induces a mild perspiration, is an anodyne and an hypnotic.

An ice-bag applied to the head may relieve the headache. The circular ice-bag is the best. For technique of filling see Pneumonia Chap. IX.

Local Treatment. Except in the case of children I have not been much impressed with the efficacy of local measures.

Cupping. The best plea for cupping is the faith of the layman, yes, and of many physicians, the world over, in the value of the cups. In my Bellevue experience it was the exception to find a patient brought. in from the East side of New York, the great immigrant quarter, who, suffering from bronchitis or pneumonia, had not been liberally and well cupped. One must at least be tolerant to long continued customs. Moreover, that the application of the cup does influence the circulatory states in the chest would seem attested by the sudden relief of pulmonary edema one sees occasionally follow. For these reasons I do not say nay, if my fellow practitioners say yea.

Counterirritation. The form I am accustomed to employ is the mustard paste and this almost exclusively in children. However, if the adult, who has had bronchitis and tracheitis has found comfort or relief in mustard paste applied to the chest I am quite willing to 
indulge his preference. The strength should be one part of mustard to 3,4 or 5 parts of flour, mixed with cold or luke-warm water, the strength depending on the sensitiveness of the skin. (For technique see Chap. XIV.) The mustard leaf is a more convenient form, but its strength cannot be varied. (For technique of application see Chap. IX.) These applications should not burn, but be left on until the skin is well reddened, and reapplied at 4 to 6 hour intervals. Vaseline (liquid petrolatum) or olive oil is applied to the skin in the intervals.

Liniments. Those that are counterirritants will do no more, if as much, as mustard, and are decidedly mussy. Those that are volatile have another usage, locally applied, of which I will speak presently.

Poultices. I mention but to condemn. I doubt their therapeutic value in the condition under consideration, while they burden the chest with an unnecessary weight, leave the superficial vessels atonic and dilated and often are left on to become a cold, clammy, disagreeable annoyance.

Cold. The only way I should care to apply cold to the chest in bronchitis is as a hydrotherapeutic measure, in the shape of a cold compress. (For technique see Pneumonia, Chap. IX.)

Inhalations. These are especially indicated for the laryngitis and tracheitis and the hard dry cough and substernal soreness that accompany these conditions.

I have long been wedded to an "old wives" "prescription for tracheitis, so common an accompaniment of a "cold" without further implication of the bronchial tree. The ingredients are often found in the household or easily obtained. Take a tablespoonful each of oil of turpentine, spirits of camphor and olive oil; mix them well and sprinkle liberally upon a piece of flannel, put this on the skin of the sternal region direct, pin the flannel to the night dress and leave on overnight. Of course a prescription of equal parts of these ingredients may be ordered. The volatile substances are inhaled during sleep and their soothing effects with loosening of the expectorations are very manifest the next morning. It does not irritate the skin unless the patient has some idiosyncracy towards its ingredients.

One of the blandest of inhalations, and to many an agreeable one, is the compound tincture of benzoin $3 \mathrm{i}$ or 3 ii ( 4 c.c. to 8 c.c.) of hot water in one of the numerous devices that may be extemporaneously devised (see Whooping Cough, Chap. XXII), or one of the numerous croup kettles or inhalers on the market.

Such volatile substances as eucalyptol, oil of pine, a teaspoonful or two in hot water or a few drops of a saturated alcoholic solution of menthol or creosote on a dampened sponge of an inhaler are of value. These 
drugs may be combined as menthol 1 pt., eucalyptol 2 pts., and benzoinol $3 \mathrm{pts}$., or menthol, eucalyptol, terebene and chloroform equal parts.

A very excellent inhalation is one of equal parts of alcohol, chloroform and creosote (Delafield). A few drops on a moistened sponge of a Robinson's inhaler, or kindred device, a small mask of perforated zinc over mouth and nose worn ad libitum.

Cough. Nothing controls cough better than the morphine derivatives of which codeine is the best, because the safest while at the same time effectual. It is usually prescribed as the phosphate either alone or in combination with expectorants; if alone it may be given in tablet form or in solution, the dose ranging from gr. 1/12 to gr. $1 / 4(0.005$ to $0.015 \mathrm{Gm}$.) according to the severity of the process, which again determines the frequency of 2,3 or 4 hour intervals. Heroine is more depresant to the respiratory centre than codeine, but in some instances decidedly more potent to control the cough. It is given as the hydrochloride in the dose of gr. $1 / 16$ to gr. $1 / 8(0.004$ to $0.008 \mathrm{Gm}$.) and the interval the same as in the case of codeine. In very stubborn cases, exhausting the patient and sacrificing sleep, morphine sulphate may be needed in doses of gr. 1/24 to g. 1/12 (0.0025 to $0.005 \mathrm{Gm}$.) at four-hour intervals, or exceptionally it may require an occasional hypodermic injection of gr. $1 / 8(0.008 \mathrm{Gm}$.). I have seen an intense cough in bronchitis accompanying influenza yield to 2 per cent. cocaine hydrochloride as a spray that had bidden defiance to morphine and its derivatives.

It must be remembered that cough is a symptom of purposeful significance, as most symptoms are, and that its purpose is to expel secretions laden with infecting organisms. For this reason cough that accomplishes this end, should be left alone; it is the dry, racking, exhausting cough that requires interference.

Expectorants. I suspect that my usage of expectorants has been based rather on unreasoning reverence for hoary tradition than on conviction of their therapeutic value. Depressant, sedative or nauseating expectorants, such as ipecac, tartar emetic, apomorphine and potassium salts were supposed to be indicated in the early stages and stimulating expectorants, the ammonium salts in the later stages. An example of the former is the Brown mixture (Mistura Glycyrrhizæ Comp.) which contains antimony and is ordered in doses of $\bar{\jmath}$ ss. in water every four hours. In spite of indications signified above it is often used as a vehicle for simulating expectorants (see below); another example is:

$\mathrm{R}$

Syrupi Ipecacuanhæ................ 3 vi

Potassii Citratis..................... 3 iii

Aquæ q. s. ad......................... 
M.

S. A dessertspoonful (3ii or 8 c.c.) every 4 hours.

As examples of the stimulating expectorants:

$\mathrm{R}$
Ammonii Chloridi............. 3 iss. -3 iiss.
Codeinæ Phosphatis...............gr. iv

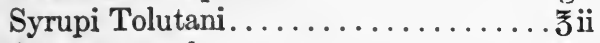
Aquæ q. s. ad.................. 兮iv
M. et S. $3 \mathrm{i}$ every $3-4$ hours in water.

If the cough is not annoying the codeine may be left out. Another such prescription is as follows:

$\mathrm{R}$

Ammonii Chloridi............... gr. xxxvi-3i

Misturæ Glycyrrhizæ Comp........ ₹vi

$(2.40-4$ c.c.)

M. et S. 5 ss. (1 tablespoonful) every 4 hours.

In no type of prescription has more ingenuity of combinations been exercised than in expectorant mixtures with the result that the patient suffers from the physician's transference of affections from one alluring prescription to another.

Children should be put to bed in a room with the temperature at $70^{\circ} \mathrm{F}$. Counter-irritation in the shape of mustard paste, one part of mustard to six parts of flour in infants, one to five or four in older children, I believe to be of more value than in adults. It should be applied all over the chest, left on until the skin is slightly reddened and reapplied once in four to six hours. Liquid petrolatum or olive oil being applied in the interval.

Do not use poultices and pneumonia jackets.

Inhalations. Compound tincture of benzoin is the best. Creosote too may be tried. (See Whooping Cough, Chap. XXII, for details.)

Expectorants should be used sparingly in children and in infants and young children not at all. In older children in the early stages ipecac and antimony may be used and that especially when there is spasmodic tendency in the larynx or bronchi. One may use tablets of ipecac and tartar emetic each gr. 1/100 (0.0006 Gm.) at 2 to 4 hour intervals. In later stages with a view to stimulating expectoration ammonium chloride gr. $\mathrm{i}$ to gr. iii $(0.060-0.20 \mathrm{Gm}$.) in Brown mixture $3 \mathrm{i}$ to $3 \mathrm{ii}$ ( $4-8$ c.c.) may be used at 3 to 4 hour intervals. Children are much more prone to bronchial spasm as they are more prone to croup than adults when they suffer from a catarrhal inflammation. Sedatives, such as the tartar emetic and ipecac or small doses of antipyrin and 
sodium bromide, say antipyrin gr. i $(0.060 \mathrm{Gm}$.) to sodium bromide gr. iii $(0.20 \mathrm{Gm}$.) at 4 hour intervals lessen this tendency, but if an asthmatic attack does occur adrenalin (epinephrin) intramuscularly in doses of $\mathrm{m}$. ii-m. v (0.130-3 c.c.) is most effectual to afford relief.

A preparation often used is the Elixir Terpini Hydratis cum Heroina of the national formulary. It contains in each dram (4 c.c.) 1 grain $(0.06 \mathrm{Gm}$.) of terpin hydrate and $1 / 24$ grain of heroine.

Young children prone to have colds should have diseased tonsillar and adenoid tissues removed. Their sleeping room should not be too cold, not below $60^{\circ} \mathrm{F}$., the skin should be hardened by gradually accustoming it to the cold bath. Underclothing should contain woolen, but be light of weight. Overclothing in the house is pernicious. Sufficient extra clothing is added when exposed to cold. Damp or wet stockings or clothing should be promptly changed.

These same prophylactic measures obtain for adults, attention to abnormalities in the upper air passages, cold baths, open air exercise, regulation of clothing, avoiding exposure to damp cold. In some instances the recovery of a suspicious organism from the sputum during the acute attack may be utilized for the production of a vaccine to be used prophylactically between the attacks.

\section{SUMMARY}

\section{Treatment.}

Confine to house and, if any fever, to bed for three days after temperature is normal.

\section{Room.}

Chosen with regard to light and sun and nearness to bath room.

No smoking allowed.

Talking to be discouraged.

\section{Diet.}

Unless severe no special directions required.

If severe, treat as in pneumonia. (See Chap. IX.)

\section{Precautions.}

Isolate patient from all but those acting as nurse.

The secretions from mouth, nose and eyes convey the infection and the patient should be instructed to cover the mouth and nose when coughing or sneezing with gauze or old cloths, which should be placed in paper bags and burned.

All linen should be immersed in water until it can be boiled.

Boil all table utensils and keep separate.

Separate thermometer used and kept immersed in an antiseptic solution (phenol 1:20). 


\section{Early measures.}

Hot bath, hot drinks, etc., as summarized under Acute Rhinitis, Chap. IV.

Bowels.

Mild saline such as Hunyadi or liquor magnesii citratis 5 viii (240 c.c.) or Epsom, Rochelle or Glauber's salt, 3ii-iv (8-15 Gm.).

\section{Aches and pains.}

R

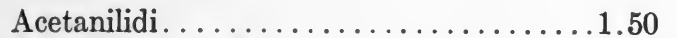

Sodii Bicarbonatis................. 1.00

(gr. xxiiss.)

Caffeinæ Citratæ ................. $0.50 \quad$ (gr. viiss.)

M. et div. in cap. no. xv.

S. One every half hour for 4 doses, then every hour for 4 doses and then every two hours for first 24 hours.

Or acetphenetidin (phenacetin) in three grain doses $(0.20 \mathrm{Gm}$.) or combine with sodium bicarbonate and citrated caffeine in doses given above.

Or antipyrin grains two $(0.120 \mathrm{Gm}$.) may be given in solution, or if headache is severe single large doses of acetphenetidin, gr. $\mathrm{x}(0.66$ Gm.) with citrated caffeine gr. iii $(0.20 \mathrm{Gm}$.) and repeat once after two hours if necessary.

Aspirin (acetylsalicylic acid) gr. v-x (0.33-0.66 Gm.) at 2, 3, or 4 hour intervals.

Dover's powder (Pulv. ipecac et opii) gr. x (0.66 Gm.) or in divided doses of gr. i-ii ss. (0.60-0.150 Gm.).

Ice bag to head.

(See Typhoid Fever, Chap. XIV.)

\section{Local treatment.}

Cupping. (See Pneumonia, Chap. IX.)

Counterirritation.

Mustard paste (1 part mustard to 3,4 or 5 parts flour mixed with luke warm water). (For technique see Pneumonia, Chap. IX.)

Cold Compress.

(See Pneumonia, Chap. IX.)

Ice collar.

(See text.)

Hot fomentations.

(See text.)

Inhalations.

(See Acute Rhinitis, Chap. IV.)

Flannel wet with equal parts of oil of turpentine, spirits of camphor and olive oil. Lay on chest and pin in the night dress and inbale the vapor.

Compound tincture of benzoin or eucalyptol or oil of pine $3 \mathbf{i}$ (4 c.c.) in boiling water, or

A few drops of a saturated alcoholic solution of menthol or,

Creosote on a dampened sponge of an inhaler or, 
Combine menthol, 1 part, eucalyptol, 2 parts, and benzoinol, 3 parts or,

Menthol, eucalyptol and chloroform equal parts or,

Equal parts of alcohol, chloroform, and creosote. (Delafield.)

Put a few drops of any of these on a moistened sponge of a Robinson inhaler or kindred device and wear over nose and mouth ad libitum.

\section{Cough.}

Codeine phosphate gr. $1 / 12$ to gr. $1 / 4(0.005-0.015 \mathrm{Gm}$.) every 2,3 or 4 hours according to the severity.

Heroine hydrochloride gr. 1/16-1/8 (0.004-0.008 Gm.) every 2, 3 or 4 hours.

Morphine sulphate gr. 1/24-1/12 (0.0025-0.005 Gm.) at four-hour intervals if the above is not efficacious. Give gr. $1 / 8$ hypodermically if necessary to effect sleep.

Cocaine hydrochloride solution, 2 per cent., used as a spray may relieve a stubborn cough. Use two or three jets every two to four hours. Have patient expectorate excess after spraying.

\section{Expectorants.}

Brown mixture $₹$ ss. in water every 4 hours or,

R

Syrupi Tolutani.....................

Aquæ q. s. ad.........................

M. et S. 3i every $3-4$ hours in water.

$\mathrm{R}$

Syrupi Ipecacuanhæ.................

Potassii Citratis......................

Aquæ q. s. ad........................

M. et S. a dessertspoonful (3ii-8 c.c.) every four hours.

R

Ammonii Chloridi.............gr. xxxvi-3i

Misturæ Glycyrrhizæ Comp...... ₹ vi

M. et S. 3 ss. (1 teaspoonful) every 4 hours.

\section{Children.}

To bed in room at temperature of $70^{\circ} \mathrm{F}$.

Mustard paste one part mustard to one to six parts of flour.

For technique see Pneumonia. (See text.)

\section{Inhalations.}

Compound tincture of benzoin on water or creosote. (See above and Whooping Cough, Chap. XXII.)

\section{Expectorants.}

Do not use in infants and young children.

For older children with spasmodic tendency use tablets of ipecac and tartar emetic gr. 1/100 (0.0006 Gm.) of each every 2 to 4 hours. Later stages use ammonium chloride gr. i to iii $(0.06-0.20 \mathrm{Gm}$.) in Brown mixture $3 \mathrm{i}-3$ ii ( $4-8$ c.c.) every 3 to 4 hours. 
Or, antipyrin gr. i (0.060 Gm.) and sodium bromide gr. iii (0.20 Gm.) every 4 hours to lessen tendency to bronchial spasm.

For asthmatic attack give adrenalin (epinephrin) 1:1000 intramuscularly in doses of $\mathrm{m}$. $\mathrm{ii}-\mathrm{v}(0.130-3$ c.c.).

\section{Prophylaxis.}

Removal of diseased tonsilar and adenoid tissue.

Current deformities in upper air passages.

Sleeping room not too cold, not below $60^{\circ} \mathrm{F}$.

Cold baths or sponges.

Underclothes to contain wool and be of light weight.

Add sufficient extra clothing on going out of doors.

Change damp or wet clothing promptly.

If pathogenic organism can be isolated make a vaccine to be used between attacks. 


\section{CHAPTER VIII}

\section{PLEURISY (PLEURITIS)}

Pleurisy or pleuritis as the latter name implies is an inflammation of the pleura, characterized by the usual phenomena of congestion and the exudation of fibrin, serums and the cellular constituents of the blood, diapedesis and emigration.

When the exudate is largely fibrinous the process is called a fibrinous or plastic pleurisy or in less technical parlance a "dry pleurisy." When the exudation of serum predominates the process is called a serous or serofibrinous pleurisy, as the amount of fibrin in the exudate is small or more abundant. It is commonly termed a "pleurisy with effusion." When the emigration of white blood cells is the dominant feature it is called a purulent pleurisy or an empyema.

Pleurisies may be primary or secondary. Nearly all pleurisies are secondary, originating from processes starting in the lung, pericardium, mediastinum, liver, or brought by the blood stream. So-called primary pleurisies, especially those characterized by an exudation of serum, are in the vast majority of cases tubercular in origin. The other organisms responsible for pleurisy are in the main pneumococcus and streptococcus. Other bacteria than these play a very minor rôle. The end results of pleurisies are resolution to a normal condition or commonly proliferation with adhesions or more complete organization with partial or complete obliteration of the pleural sac.

Fibrinous Pleurisy is the least serious of the types, both as to its course, inconveniencies and sequences. It must not be forgotten in any case, even of dry pleurisy, that the cause in the vast majority of cases of "primary pleurisy" is tuberculosis, and every effort should be made to determine its presence or absence. Whether exposure to cold alone in the absence of an infecting organism can cause fibrinous pleurisy is open to serious doubt; and yet this is often offered as an adequate explanation.

As has been said, the secondary cases arise from infections in the lung, pericardium, and mediastinum, and also from the peritoneum, wounds in the chest walls and in the course of cardio-renal disease. The symptoms are superadded to those of the disease to which the pleurisy is secondary, but in primary cases the dry pleurisies are not as severe as the forms with effusion. 
Pain is the most characteristic symptom, most commonly felt in the axilla or in the mammary regions; more rarely in the back. Especially is it to be remembered that diaphragmatic pleurisy is full of pitfalls and gins for the unwary; for an involvement of the central part of that structure gives rise to pain in the shoulder and trapezius muscle with tenderness of the trapezius ridge, brought about by reflexes through the phrenic and fourth cervical, while the peripheral portion causes pain in the lower costal region, the lumbar region and abdomen. The muscles of the abdomen may be rigid and painful to palpation. As no friction rub may be heard, diagnoses referable to the kidney, gall-bladder, appendix and other structures are frequent enough.

Constitutional symptoms as a rule are not marked, the temperature, at the most, trivial. Some malaise may be experienced.

Cough occurs in most cases, whether due to the affected pleura or to an infected lung is a question. Severe pain on breathing may cause shallow, rapid breathing, with some dyspnœa. The classical physical sign is the friction sound - commonly a crepitant or subcrepitant ralemore rarely a coarse rub or a sound like a mucous rale. Change in percussion with dulness occurs even when the fibrin is very abundant. The leucocytic count is usually slightly increased $-12,000$ to 20,000 .

Treatment. In the milder forms the patient may be allowed out of bed, but should be confined to the house. If there is any elevation of temperature he should be put to bed. In severe attacks the patient will require no urging to remain in bed. Here he will commonly be found lying on the affected side in the unconscious effort to splint that side, as by so limiting the respiratory excursion he lessens the pain incident upon the movement of the pleural surfaces upon each other. This very fact gives us a hint as to proper procedure, as will be detailed below.

For the choice of a room with reference to light and air the reader is referred to the article on pneumonia; but emphasis must be laid on the open air treatment because the case, if a primary one; is in all probability tuberculous. The same rules as are laid down in standard treatises on tuberculosis are applicable in these cases; in brief, they are open air day and night with proper attention to shelter against high winds, dust clouds and inclement weather. If proper care cannot be given at home or if the patient so prefers, sanitarium treatment should be sought.

Diet. In febrile cases the diet is like that of other acute infectious diseases-see Chap. II; Pneumonia, Chap. IX; Typhoid Fever, Chap. XIV. If there is no fever the diet should be liberal; such as we would offer to a tuberculous patient, milk, eggs, cereals, breadstuffs, meat and vegetable soups and some meat, fish, fowl, and vegetables, fruits and light desserts. 
Drinks. Water, and fruit juices may be given ad libitum. Cocoa may be allowed, very weak tea, and milk may be flavored with coffee.

Bowels. The bowels should be moved when the patient is first seen. One may give castor oil or calomel followed by saline or salines alone. The calomel may be given in a single dose of gr. ii-iii $(0.120-0.2$ $\mathrm{Gm}$.) or as I prefer in $1 / 4$ grain $(0.015 \mathrm{Gm}$.) doses at quarter-hour intervals for six doses. I fancy this lesser total so given is as effectual as the larger doses given at once. One follows the calomel in 4 to 6 hours, or if the calomel is given at night, the next morning, by salts. One may use Rochelle, Epsom, or Glauber's salt in 3/4 to one glass of water. Or milder salines may be used such as the solution of magnesium citrate 8-12 ounces or a Seidlitz powder or one of the many saline laxatives on the market, of which Hunyadi water is a type. If a satisfactory movement does not follow each day or the stools are foul or otherwise abnormal, an enema may be given every other day, or one of the milder salines. If the condition of the stools seems to demand it colonic irrigations may be given. The physician should have some accurate knowledge of the state of the patient's bowels in every acute disease; but I am not sure that routine catharsis, even as described above, may not be too drastic. The object really should be to secure good movements with the least and gentlest assistance possible.

Local Measures. These have been applied for a double purpose; to exert a modifying influence on the inflammatory process in the pleura and to afford relief from pain; one may argue about the possibility or probability of attaining the first end arrived at; but certainly some of these local measures do relieve pain. One of the most effectual of these is strapping the chest.

Strapping the Chest. This manuœvre accomplishes what nature is trying to do, namely, to splint the chest as explained above. The technique is as follows. One uses zinc oxide adhesive plaster, the wellknown surgeons' adhesive. The strip must be wide enough to extend from the axilla to the edge of the ribs, about 5 inches in the adult and long enough to encircle the affected side, overlapping the opposite chest some 3 inches both in front and behind. The patient, if he is able to be out of bed, stands before the operator, who holds the sheet of adhesive stretched and smooth as he faces the patient's affected side; one end of the strip is applied some 3 inches to the unaffected side of the patient's spinous processes and firmly secured; the patient is then asked to breathe out as completely as possible and during his continued expiratory phase to turn his body slowly to face the operator who smoothly applies the taut adhesive strip as he turns, overlapping the opposite side in front. 
Thus applied during expiration the motion of the affected chest is greatly restricted and the relief immediate.

If it is not wise to have the patient out of bed, he may sit up in bed and the technique described is imitated as best possible under the circumstances.

When the patient is very stout, and in women with large breasts, the application of the adhesive in a single sheet cannot be smoothly made. One uses in these cases three or four strips which overlap each other and conform more readily to the contour of the chest. If the chest is hairy, it should be shaven before the plaster is applied. This facilitates its removal when the time comes. It may be pulled off by a sudden strong jerk or if there is much hair or the patient fearful, it may be softened before removal by benzine or ether. If strapping cannot be obtained, a snug binder or towel may be tried as a substitute. If the strapping is not comfortable or if for any reason it does not seem feasible to apply it, the application of cold is usually found gratifying.

The Ice Bag. Perhaps the best method of applying cold is by the ice bag. The circular ice bag with a metallic cup is the one I prefer. The ice should be cracked to the size of the end of one's thumb and enough put into the bag to cover the bottom. Only enough water is added to cover the ice; the air is then expressed and the metallic cap screwed on. This affords a pliable bag, which one filled with air does not, and clings closely to the underlying surface. A layer or two of linen is put over the bag to intervene between it and the skin and the whole secured to the chest by a towel or binder. The bag should be taken off at intervals of two or three hours, lest too long continuous application damage the tissues. When the bag is taken off, one should apply oil or vaseline to the skin. The use of salt in the bag is to be deprecated. As our chief aim in making use of these local measures is to afford comfort, if cold fails to afford it, one may try heat.

Heat. Hot water bag. This is an article rarely wanting in a household and, of course, readily applied. One or two directions, however, will not be out of place. Only partially fill the bag with hot water and by squeezing it, expel the air and screw in the plug to render the bag flaccid and pliable; place flannel between bag and skin to prevent burning it; keep in place by a binder.

Electric Pad. This clever device, that may be utilized in any modern dwelling house of city or town, furnished with current, is most easy of application-light, dry and easily regulated to the desired temperature.

Fomentations. For the technique, see Pneumonia, Chap. IX. Smear the skin with oil or vaseline before applying and cover with a dry 
flannel to retain the heat. These are removed as fast as they become cool. A series of these are applied at intervals, dry flannel being put in their place in the meantime. The disadvantage of fomentations lies in the necessity of continual renewal. One may get a more continuous effect by the hot poultice.

Poultice. For technique, see Pneumonia, Chap. IX. A poultice will keep its heat, when properly made, about an hour. "When it is removed a dry flannel is put over the site previously smeared with olive oil or vaseline. Huge, heavy poultices, and such, allowed to remain on long after they have become cold are not only useless but harmful. Another time-honored local measure is counterirritation.

Counterirritants. Perhaps the simplest, most easily applied and one almost always to be found in any household is mustard. When mustard is at hand the paste is the best form in which to apply it. Its strength can be readily regulated by the proportion of flour and mustard used, and its preparation is of the easiest. When a paste cannot be conveniently prepared the mustard leaf (charta sinapis), is readily obtainable and simple of application. For the technique of preparation and application of the mustard leaf and mustard paste, see Pneumonia, Chap. IX.

The mustard is to be left on until the skin is well reddened and when removed, the skin is to be washed with soap and water to free it from any little particles of mustard adhering and then smeared with a thin coat of vaseline or sweet oil. The applications should be repeated at four-hour intervals or even three-hour intervals, if the physician hopes to influence the pathological processes; if relief of pain alone is aimed at, the number and frequency of the application depends on results.

Iodine. The tincture has been used as a counterirritant. It offers no advantages over mustard; it is less easily regulated and more likely to set up a disagreeable dermatitis.

The Cautery. Either the acutal cautery or the electro-cautery offers certain advantages as a counterirritant. The cautery makes a more decided impression on the sensory nerves than the mustard and may relieve pain when mustard fails. It is flicked lightly over the area of pain, just reddening the skin or at the most inducing a very slight blister. The part is then treated to a coat of vaseline, or sweet oil, and covered with a layer of gauze. The procedure is easy, it is neat, and contrary to the patient's anticipations of an ordeal by fire, being provocative of but little pain in the application.

Blisters. Blisters at one time in much vogue have fallen largely into disuse. I do not think that they effect any more than the measure just described; on the other hand the site of their application is often 
sore and may leave a sore surface, necessitating protective dressings and adding discomfort rather than comfort to the patient.

For the technique of applying blisters see Rheumatism, Chap. III. Another local measure still much in use among the laity, especially among our recent European immigrants, is cupping. In my service at Bellevue Hospital it is the exception to find a patient drawn from the great East side, who is admitted for pneumonia or pleurisy, who has not been thoroughly and well cupped. This cupping may have been done by a physician or by a lay hand. The rationale of the procedure rests only on theory and but little definite knowledge. It may modify the distribution of blood in the inflamed pleura as is claimed for it, but it is at best a dubious assumption or it may operate through the nerve reflexes. The best results seem to follow the early application of cups in early and sudden attacks.

For technique of cupping see Pneumonia, Chap. IX.

Wet cups and leeches have been used to effect the same purpose, but are not to be recommended.

Drugs. For relief of pain. If the local measures are not successful, one must have recourse to drugs.

When the pleurisy is a part of a rheumatic attack, salicylates are indicated, or as salicylate therapy will have been instituted already, they are to be continued. A pleurisy may occur as an early rheumatic manifestation or accompany some other particular manifestation of rheumatism; for example, tonsillitis, or in a rheumatic subject without other expressions of the infection, and in such cases salicylates should be administered as in rheumatism. For details see Acute Rheumatic Fever, Chap. III. However, the practitioner must keep in mind the far more common cause, tuberculosis, which may well occur in a patient who has had rheumatism. As I write a young man with a marked mitral stenosis from a previous attack of rheumatism just developed a fibrinous pleurisy without other rheumatic symptoms; but an X-ray picture of the chest shows two old healed tubercular lesions of the lung and emphasizes the necessity for caution in these cases. From whatever origin, the pain of pleurisy may be relieved by the use of the salicylates. My preference is the acetylsalicylic acid (aspirin) in doses of gr. $\mathrm{x}$ (0.66 Gm.) every two or three hours until its value is demonstrated or its failure calls for its interruption. Sodium salicylate in doses of gr. $\mathrm{x}-\mathrm{xv}(0.66-1 \mathrm{Gm}$.) at the same intervals may be used. This may be given with bicarbonate of soda, two grains of the latter to one of the salicylate, or given alone.

For the use of the various forms of the salicylates and prescriptions of the same see Acute Rheumatic Fever, Chap. III. 
The salicylates have the advantage of being the least harmful of the anodynes.

Coal-Tar Preparations. When the circulation is in any degree imperilled, these should not be used; but in a primary pleurisy circulatory embarrassment is very rare. The coal-tar preparations may be used then with safety in reasonable doses. I should prefer to use them only when the salicylates fail or when for some reason, such as an idiosyncracy or gastric distress, the salicylates may not be used.

If the pain is severe it may be necessary to use a single large dose of phenacetin (acetphenetidin) gr. $\mathrm{xv}(1 \mathrm{Gm}$.) or of antipyrin gr. viiss. $(0.5 \mathrm{Gm}$.) or of acetanilid gr. $\mathrm{v}(0.33 \mathrm{Gm}$.); but as a rule I believe better results follow smaller doses frequently repeated, e. g., phenacetin gr. iii $(0.2 \mathrm{Gm}$.) or acetanilid gr. iss. $(0.10 \mathrm{Gm}$.) with which one may combine, if he so chooses, a grain $(0.06 \mathrm{Gm}$.) of sodium bicarbonate and a half grain $(0.030 \mathrm{Gm}$.) of citrated caffeine. Antipyrin, which has the advantage of being soluble in water, can be given in gr. ii $(0.120 \mathrm{Gm}$.) doses. These doses may be repeated at first at $1 / 4$ to $1 / 2$ hour intervals, depending on the severity of the pain, for four doses, then at hourly intervals for the same number (4), and then every two hours.

If the pain does not yield to these drugs one should have recourse to codeine phosphate in doses of gr. 1/8 to gr. 1/2 (0.008-0.03 Gm.) hypodermically or better intramuscularly.

If, as may well happen, codeine fails to afford relief, one must have recourse to morphine given as the sulphate intramuscularly, in doses of gr. $1 / 8$ to gr. $1 / 4(0.008-0.015 \mathrm{Gm}$.).

Cough. Dyspnœa. Both these symptoms in an uncomplicated pleurisy are due to the painful act of breathing and are to be treated in the same manner as, or rather, are being treated simultaneously with the pain.

With the disappearance of symptoms which in some instances may be fairly prompt, our obligations to the patient does not come to an end.

The patient in all primary cases as has been said, is a tuberculosis suspect. If no pulmonary signs have at any time been detected, then the patient should be treated as tuberculous, in a sanatorium if possible, or if not, at home, until we can be sure that quiescence of the lesion has been obtained.

\section{PLEURISY WITH EFFUSION}

A primary pleurisy with effusion is almost certainly tuberculous, though, occurring in a rheumatic subject, it may. be the first expression of a reinfection. The constitutional symptoms are as a rule more marked and the course more prolonged. 
A patient with an effusion is likely of his own volition to seek the bed; but it is not a rare happening to find a patient up and about and endeavoring to attend to his duties with one pleural sac nearly filled with fluid.

The general care of the patient, the diet, the local measures, the use of drugs and the relief of distressing symptoms are the same in the serofibrinous cases as in the fibrinous.

Throughout the course of the disease the patient should be treated as a case of pulmonary tuberculosis. Fresh air night and day, and in the open as much of the day as possible, and an abundance of good food, of which milk and eggs form an essential part, are indicated.

Dr. Francis Delafield was accustomed to take issue with most authorities of his time on at least three points. First, he gave all subacute cases solid food; second, he kept his patients with effusion out of bed a good deal, even if the afternoon temperature was $102^{\circ} \mathrm{F}$. He thought that the fluid was better absorbed and that the patients ran down less when they were not closely confined to the bed; but all his patients had been relieved of considerable accumulations, for he believed in early aspiration, his third point.

All these points are worthy of consideration.

The key-note of the treatment is thoracentesis. It is generally agreed that withdrawal of the fluid is indicated when so much has accumulated as to embarrass the respiration or circulation as shown by cyanosis, dyspnœa or cardiac weakness, or when the heart is frankly displaced, even in the absence of dyspnœa, or when the fluid has persisted for two or three weeks in spite of all efforts to expedite its absorption. But I would follow the teaching of Dr. Francis Delafield that thoracentesis should be done early; that nothing is to be gained by delay but much sacrificed by waiting.

I quote his own words which succinctly define his position and voice his advice: "I believe that aspiration is to be used not simply to remove fluid from the chest, but to cure pleurisy is a morbid process" and further "this means that as aspiration is the treatment of pleurisy, it is to be performed as soon as the presence of fluid in the chest is to be made out." (Am. Journal of Med. Science, 1902, Vol. CXXIV.)

Such an early removal of fluid not only shortens the disease, but prevents in no small measure the formation of pleuritic adhesions, which are commonly very extensive after expectant treatment, with the development of bronchiectatic cavities; prevents the chronic interstitial pneumonia and secondary tuberculosis of the lung which a long compression of that structure facilitates.

Dr. Delafield's teaching was to aspirate as soon as the chest was half 
full, either in the acute or subacute case. He would not wait for embarrassment of respiration or circulation, but even on the second or third day of the attack, if the chest was half full, evacuate.

In such a case one should withdraw all that the aspirator will recover, but if the chest is full take off only 8 to 16 ounces (250-500 c.c.) at the first aspiration to avoid serious disturbance of the heart action. . The rest of the fluid may be absorbed; if it reaccumulates, further tappings are indicated.

Exploratory Puncture. This should always be done before the aspiration of the fluid is undertaken. No man is so skilled in physical signs that he can score 100 per cent. in his diagnoses; so far from it, indeed, that to the best of diagnosticians the fruit of their experience is humbleness of spirit. Confirm the diagnosis of an effusion by an exploratory puncture and if fluid is withdrawn submit it to microscopical and bacteriological examination.

If the fluid is clear an aspiration is indicated; if purulent, sooner or later an operation.

If the fluid is turbid, some judgment must be exercised. If the fluid cultures out streptococci it is almost certain to become an empyema and is to be treated as described under streptococcus Empyema. (See Chap. X.)

If the fluid cultures out pneumococci, which grow readily and the leucocytes are abundant, and some necrotic, it should be treated as a pneumococcus empyema. (See Chap. IX.)

If, however, the leucocytes are few and the pneumococci do not grow well on culture, a thoracentesis should be done. It might be mentioned that sometimes the little fluid withdrawn in an exploratory puncture seems to initiate absorption. It is too rare a happening, however, to warrant delay of aspiration.

For the Technique of Aspiration, I quote from my own contribution on Pleurisy to Hare's Modern Treatment, Vol. I, p. 734.

"For aspiration some modification of the Potain apparatus is commonly utilized. The essentials of a successful aspirator are a needle and a trocar and cannula, to which a rubber tube is attached, passing to a bottle or other container, which in turn can be exhausted by a pump or other device.

It is well to have a piece of glass tubing introduced into the rubber tube to enable one to see how the fluid is running.

The needle is the easier of introduction, but has the disadvantage of wounding the lung if it comes in contact with it, and of easily getting plugged with fibrin. The trocar and cannula are more difficult to intro- 
duce and of more intricate mechanism, but free from the above objections.

The needle should be about three inches long, and longer for a thick chest, and one-sixteenth to one-twelf th inch in diameter.

The tube should be of thick firm rubber, and about a yard long. There should be a clamp attached with which to regulate the flow-an artery clamp will do.

The bottle should be 1500-2000 cubic centimeters' capacity and can be readily graduated by applying strips of adhesive plaster at the levels of given quantities previously determined.

The bottle may be exhausted by a pump, or, better yet, by a clever device of Dr. Karl Connell, namely, by burning alcohol which has been run in small quantity about the inside of the bottle and stoppering as the flame dies out. The stopper is of rubber or of cork and is perforated by a glass tube, to which the rubber tube is attached; the rubber tube is clamped, of course, to prevent the advent of air into the bottle.

All this apparatus must be carefully sterilized. The operator's hands and the field of operation should receive the same precautions with reference to cleanliness as in surgical procedures of greater magnitude. Boiling for the instruments and soap and water and alcohol for the skin, are the best, and any antiseptics that may interfere with the cultures desired are, of course, to be avoided.

The hypodermic needle, with such stimulants as may be needed, should be at hand.

The best position for the patient is a sitting posture, with the hands on the opposite shoulders, to widen the intercostal spaces. The site of election is in the fifth or sixth intercostal spaces, in the midaxillary line or in the seventh space, between the posterior axillary line and the scapular line. This site avoids the dome of the diagram, which rises as high as the fourth in the nipple line, the sixth in the midaxillary, and the eighth in the scapular.

Another site advised is an inch in front of the posterior axillary line, in the space that lies just below the angle of the scapula. It is easy to reach, the space is wide and is remote from important organs.

The average chest-wall will vary from four-fifths inch to twice that thickness. Musser has given the distance of vessels at the root of the lung at a level of the fourth costochondral junction and the seventh dorsal vertebræ as 4 inches on the right side in the midaxillary line and $23 / 4$ inches on the left. In the posterior axillary line the distance on the right is $51 / 4$ inches, on the left 5 inches. As our sites are below this level the danger of puncture is less.

A local anesthetic is rarely necessary, but, if the patient is insistent 
or nervous, an ethyl chloride spray may be used to freeze the part, or a dilute cocaine solution used to infiltrate the underlying tissue.

The space to be entered should be clearly palpated, the thumb of one hand determining the edge of the rib below, while the nail serves as a guide for the point of the needle, so that it shall be kept away from the intercostal artery that hugs the rib. The needle or trocar, with the blunt end held against the palm of the hand, and the forefinger marking off the probable thickness of the chest wall, is thrust quickly and firmly in until non-resistance to its advance tells of the entrance into the cavity.

In some cases the skin may be so thick that a slight incision may be needed to introduce the point of the needle.

The coupling should now be made, or, if already made, the clamp should be loosened gradually, that the flow may be slow.

The amount of fluid to be withdrawn depends on the individual case. It depends on how early it is done, how much fluid is in the chest, and how the patient reacts to the operation.

Most of the accidents may be attributed to the sudden return of the blood to the vessels of the compressed lung. Hence, if the fluid is withdrawn very soon after its appearance, the probability of much compression is lessened, and, if no untoward symptoms set in during the procedures, all may be allowed to drain out that will. If the amount of fluid is large, the compression will be greater, and still more if it has been left for a long time.

Much, too, depends on the rapidity of the aspiration, for if the fluid is drained off slowly, the expanding lung and its filling vessels have time to readjust themselves to the new condition in some measure.

If there is much fluid and everything goes on well, it is rarely necessary to take more than 1,500 cubic centimeters, and the better part of half an hour should be consumed in doing so. The balance of the fluid will often disappear promptly; if not, another tapping can be done.

If, however, either coughing or severe pain or dyspnœa or hemoptysis occurs, or heart weakness ensues, the procedure should be stopped at once and the patient put in a recumbent position.

A slight feeling of faintness may arise from fear of operation. This is to be relieved by a little whisky or a glass of cold water.

Pneumothorax may arise from puncture of the lung, entrance being made into a bronchus or cavity, or air enter by the needle or trocar.

While fatal cases are known to the literature, they are very rare and the accident need not cause undue alarm. Sometimes subcutaneous emphysema may be caused by air following the needle from the punctured lung on its withdrawal. I have seen these results on more than one 
occasion. Sometimes the absorption of air was rapid; again it lingered for some days, but in no case have I seen alarming symptoms.

More rare and more formidable is the appearance of pulmonary edema with abundant albuminous expectoration. This may occur during the tapping or just after, and, in rare instances, later. The most plausible explanation. is transudation from the congested capillaries, whose walls have undergone impairment of their integrity during compression.

It is more likely after withdrawal of large amounts of fluid, and especially when rapidly withdrawn. There may be cyanosis, dyspn œa, rapid weak heart and fall of blood pressure.

Another accident, fortunately rare, but frequent enough to make its mention of importance, is the sudden onset without warning, of cyanosis and dyspnœea with weak heart, fall of pressure, unconsciousness and death. Numerous theories have been advanced to account for it, but none have been so suggestive as those of Joseph Lewis and Dean Capps, as the result of experiments carried on in the Laboratory of Experimental Therapeutics of Chicago University.

They attributed these accidents to the result of irritation of the inflamed pleura by the instrument used.

The lessons they draw are that great care should be taken not to wound the pleura by scratching with the needle and for that reason the cannula is preferable to the needle.

This condition is to be met like that of shock or collapse from other causes, the most valuable drug being adrenalin." (See also Pneumonia, Chap. IX, and Empyema, Chap. X.)

After the aspiration we anticipate a rapid improvement in the patient's symptoms, drop in the temperature and absorption of the remaining fluid. Unfortunately, the fluid may re-collect in some cases and necessitate further aspirations.

After the fluid was removed, or in small accumulations, it was Dr. Delafield's routine to administer diuretics, acetate of potash, caffeine, digitalis and others, at the same time restricting the fluid. I must confess that this measure has not appealed to me strongly, but I can see no objections to the use of diuretics. The acetate of potash or the citrate of potash could be used in $\mathrm{gr} . \mathbf{x v}-\mathbf{x x x}(1-2 \mathrm{Gm}$.) doses at two or three hour intervals until the urine becomes alkaline.

Caffeine, citrated, gr. ii ss.-iii (0.150-0.2 Gm.) or the double salt of caffeine and sodium benzoate or salicylate gr. v (0.33 Gm.) may be given three times a day, or diuretin (the double salt of sodium salicylate and theobromin) or agurin (the double salt of sodium acetate and theobromin) in doses of gr. viuss. $-x(0.5-0.66 \mathrm{Gm}$.) three times a day or theocine (Theophylline) gr. v (0.33 Gm.), three times a day, but these should 
not be continued over more than two or three days at a time and theocin but one or two days.

Digitalis may be administered as the infusion 5 ss. (15 cc.) three times a day for two or three days or the tincture in doses of $\mathrm{m}$. $\mathrm{xxx}$ ( 2 c.c.) for the same time at the same intervals.

At times the patient seems unable to control the process until a change of climate or, at least, of environment is effected.

Autoserotherapy, the injection under the skin of the patient of 1 or 2 c.c. of the fluid aspirated from his chest, repeated every second day for four, five or six times, a species of vaccination, I do not feel able to recommend. I have had no experience with the measure and have not been impressed with the literature on the subject.

Convalescence. This is not established as long as there is pain, fever, persistent effusion or pulmonary complications.

If the patient has been confined to bed, he should not be allowed up until these symptoms have disappeared, unless persistent fluid alone remains, when getting up does seem at times to hasten absorption. (See Dr. Delafield's teaching above.)

Expanding the Lung. Compression of the lung and pleural adhesions remain after the absorption of the fluid to an extent dependent on the promptness of or delay in aspiration.

Inflation of the lung and a freer play between pleural surfaces is effected by breathing exercises. They must be undertaken gradually and if pain is elicited the patient should desist for the time.

For the Technique I quote from Dr. Fred Lord's excellent article on pleurisy in Osler's Modern Medicine, Vol. III, p. 832: "Forced inspiration from six to twelve times every two hours may serve as a beginning. Later, with each inspiration, the outstretched arm on the diseased side may be elevated to a horizontal, then to a vertical position, with coincident compression of the sound side by the other arm. Lateral deviation of the spine toward the sound side during inspiration more forcibly puts the diseased side on the stretch. It may be combined with a similar movement, holding both arms outstretched at right angles with the body. This, as well as torsion of the trunk with the arms similarly placed, should, however, be preceded by simpler exercises and practiced only late in the course."

Another method, and a simple one, to effect the same purpose, is to have the patient sit on an armchair with the sound side fixed against the arm of the chair by grasping the rung of the chair on that side with the hand, while deep inspirations are taken. With the sound side thus splinted, the force of the inspiration is expended on expanding the compressed lung and stretching the adhesions. 
A time-honored method for expanding the lung is blowing through a rubber tube attached to a bottle containing water and transferring the water by this means to another bottle attached to the first by a rubber connection. These bottles should contain about a gallon each.

After Treatment. The primary case is tuberculous. For this reason treatment of the patient at a sanatarium or at home, just as a case of pulmonary tuberculosis is treated, until the patient is in excellent physical condition and pulmonary complications have been eliminated or stayed, is the proper procedure.

If this cannot be done fresh air, good food, as much rest as is compatible with the patient's necessary activities, sleep in a freely ventilated room, instructions as to proper hygienic measures, clothing, and attention to colds, and finally careful reëxamination of the lung from time to time, must be insisted on.

Acute Purulent Pleurisy. Empyema. The key-note of the treatment of these cases is evacuation of pus by operative procedure. (See Pneumococcus Empyema under Pneumonia, Chap. IX and Streptococcus Empyema, Chap. X.)

\section{SUMMARY}

\section{Dry Pleurisy}

\section{Confine to the house.}

Milder cases need not remain in bed unless a temperature occurs. Severe cases confined to bed.

Room, bed, care of the body.

(See Pneumonia, Chap. IX.)

\section{Diet.}

(See Chaps. II, IX and XIV.)

If no fever the diet should be liberal and such as is given a tuberculous patient. (See text.)

\section{Drinks.}

Water, fruit juices ad libitum.

Very weak tea.

Milk flavored with coffee.

\section{Bowels.}

Initial cathartic of castor oil $\xi_{\text {ss. }}$ or calomel gr. ii -iii (0.120-02 Gm.) as one dose or gr. $1 / 4(0.015 \mathrm{Gm}$.) at quarter-hour intervals for six doses.

Follow calomel in 4 to 6 hours by salts, Epsom, Glauber or Rochelle salt, 5 ss. (15 Gm.). or magnesium citrate $气$ viii-xii (240-300 Gm.). 
If satisfactory daily stool does not follow, an enema may be given every other day or mild salines be used.

Colonic irrigation if nature of stool demands it.

\section{Local measures.}

Strapping the chest. (See text.)

Ice bag. (See text.)

Heat. (See text.)

Hot water bag.

Electric pad.

Fomentations.

Poultices.

Counterirritants. (See text.)

Mustard plaster.

Iodine.

Actual cautery.

Blisters.

\section{Drugs.}

Salicylates. (See Acute Rheumatic Fever, Chap. III.)

Acetyl salicylic acid (aspirin) gr. x (0.66 Gm.) every two or three hours.

Sodium salicylate gr. $\mathrm{x}-\mathrm{xv}(0.66-1.0 \mathrm{Gm}$.) at same intervals.

\section{Coal Tar Preparations. (See text.)}

Do not use if circulation is impaired.

If pain is severe, phenacetin (acetphenetidin) $\mathrm{gr} . \mathrm{xv}(1 \mathrm{Gm}$.$) or acetani-$ lid gr. v $(0.33 \mathrm{Gm}$.) or antipyrin gr. viiss. $(0.5 \mathrm{Gm}$.).

Better to use repeated small doses as phenacetin gr. iii $(0.2 \mathrm{Gm}$.) or acetanilid gr. iss. $(0.10 \mathrm{Gm}$.) combined with $1 \mathrm{gr}$. $(0.06 \mathrm{Gm}$.) of sodium bicarbonate and citrated caffeine, gr. ss. $(0.03 \mathrm{Gm}$.) or antipyrin gr. ii $(0.120 \mathrm{Gm}$.) (which is soluble).

These small doses may be repeated every $1 / 4$ to $1 / 2$ hour for four doses, depending on the severity of the pain, then at hourly intervals for four doses and then every two hours.

If pain is not relieved by the above, give codeine phosphate gr. 1/8-1/2 $(0.008-0.03 \mathrm{Gm}$.) hypodermically or intra-muscularly, or morphine sulphate gr. $1 / 8-1 / 4(0.008-0.015 \mathrm{Gm}$.) intramuscularly

\section{Cough and Dyspnca.}

Due to pain of the pleurisy.

Relieved by above measures.

\section{Pleurisy with Effusion}

General care, diet and local measures as outlined above.

Treat as case of tuberculosis.

Fresh air, sunlight, abundance of good food.

Dr. Delafield differed from his contemporaries in three points: e. g.

1. Performing early aspiration. 
2. Giving solid food to all subacute cases. 3. Keeping patients out of bed a good deal even when temperature
was $102^{\circ} \mathrm{F}$.

\section{Thoracentesis.}

(See text.)

(See Pneumonia, Chap. IX.)

(See Streptococcus Pneumonia, Chap. X.)

(See Influenzal Pneumonia, Chap. XII.)

\section{Exploratory Puncture.}

Should always precede aspiration.

Culture fluid.

(See text.)

Dr. Delafield gave diuretics after aspiration. (See text.)

Autoserotherapy.

Not recommended.

\section{Convalescence.}

Do not allow up until fever, pain, the effusion or pulmonary complications have disappeared. (See text.)

Expanding the lung.

See text.

After treatment.

If the etiology is tuberculosis, treat patient as under other circumstances.

Sanitarium treatment best.

\section{Acute Purulent Pleurisy-Empyema}

Operation for evacuation is essential. (See Pneumonia, Chap. IX.) 


\section{CHAPTER IX}

\section{PNEUMONIA}

The term Pneumonia as commonly used means an acute infection of the lung. Such an infection may be primary or may be secondary to another infection. From the pathological standpoint two great groups of pneumonias are recognized: Lobar Pneumonia and Bronchopneumonia.

Lobar pneumonia is a form of pneumonia commonly met with in adult life and forms a considerable percentage of the pneumonias of childhood.

Broncho-pneumonia is particularly common in infancy, early childhood and in old age. It is, too, the common form of secondary pneumonia.

Lobar pneumonia is characterized pathologically, by an intra-alveolar exudate containing a great deal of fibrin and no interstitial infiltration.

Broncho-pneumonia is characterized by a lesser intra-alveolar exudate with far less fibrin and much interstitial infiltration of the bronchial tissue and alveoli.

Pleurisy is common in lobar pneumonia; in broncho-pneumonia the incidence depends on the nature of the infecting organism, virulency and the nature of the primary disease to which the broncho-pneumonia is secondary.

Etiologically, lobar pneumonia is due to a group of organisms, having, morphologically and culturally, features in common, but biologically divided into a number of groups each inducing a specific reaction on the part of the tissues. These are called in general pneumococci and include the streptococcus mucosus capsulatus or as it is sometimes called, pneumococcus mucosus capsulatus. A small group of cases is caused by the bacillus of Friedlander.

Broncho-pneumonia is caused by a variety of organisms, including the pneumococcus, various types of streptococci, as well as other organisms, such as the staphylococcus the micrococcus catarrhalis, the influenza bacillus, colon bacillus and other rarer types.

From the standpoint of prognosis, immediate and remote, it is desirable to determine the pathological process, but the most urgent problem that faces the practitioner is to establish the infecting organism as upon 
the success of this depends the application of such specific treatment as we have at our command.

\section{THERAPY}

\section{Specific Treatment}

Few diseases have been the object of more intensive study in the past five years than pneumonia. The war with its epidemics of primary and secondary pneumonia and the recent epidemic of influenzal pneumonia, both among the troops and in civil life, have added much to our information of this disease. The common form of epidemic pneumonia as met in civil practice, is due, with rare exception, to the pneumococcus. As has been said, these pneumococci form groups closely allied to each other, both in their appearance and in the character of the pathological change they provoke, but they have subtler differences of great significance both as regards the virulency of their toxins and the nature of these toxins and of substances elaborated by the tissues as mechanisms of defense, i. e., the immune bodies. We speak of these differences as being specific, meaning that each group is a distinct entity; one might say we are dealing with so many different infections.

Among British investigators, as many as eleven different types have been described. But, in this country at present, for practical purposes, we utilize the classification of Cole and his co-workers. They have determined four types of organisms, termed Type I., Type II, Type III, Type IV. Types I, II and III may be considered as homogeneous though some sub-groups in Type II have been described. Type III is the streptococcus mucosus capsulatus or pneumococcus mucosus capsulatus previously mentioned. Type IV is heterogeneous, made up of a large and undetermined number of types. Type IV includes those forms of pneumococci which have long been known to be resident in normal mouths. In the absence of epidemics they give rise to the pneumonias following upon undue exposure which constitute the majority of the cases as seen in rural practice.

Types I, II and III are contagious and follow only when an individual has been exposed to those who are suffering from a pneumonia due to that given type or have recovered from such a pneumonia, but still carry in the secretions of their upper air passages virulent organisms. Such persons are technically called carriers. Of the various types mentioned Type $I$ is the most common, constituting roughly one-third of the cases with a mortality of 25-30 per cent. Type III is the most virulent, affording the highest mortality, 56 per cent., but fortunately is respon- 
sible for only 10 per cent. or thereabouts, of the cases. The type giving the lowest mortality (14 per cent.) is Type IV. Up to the present time we have been able to elaborate a serum efficient as a therapeutic agent only from Type I. While statistics of mortality reduction vary greatly, one would scarcely be guilty of exaggeration if he claimed a reduction from 25 per cent. to one-half or one-third that number.

The efficiency of serum treatment depends largely upon the promptness of its administration. For this reason the first step taken by the practitioner should be the determination of the nature of the organism he is dealing with in a given case of pneumonia and if a pneumococcus, its type. His ability to do this will depend largely upon the laboratory facilities at his command. Where these are excellent he should have immediate recourse to blood and sputum cultures. Taken early, blood cultures are positive in the vast majority of cases. After early days of infection positive blood cultures indicate a severe infection. Moreover, the severity of the infection may be gauged by the number of colonies on the plate. As soon as a pure culture is obtained, the organisms should be typed. Sputum sufficient for typing and cultures may be difficult to get in the early hours of the illness. This makes the blood cultures doubly valuable, but as soon as the sputum is collected it should be submitted to typing and culture. Numerous techniques have been devised to expedite the typing of the organisms, for the saving of a few hours may mean the saving of a life, and the delay necessitated by growing out the organisms may sacrifice the patient. Typing depends on three varieties of reactions, namely, agglutination and precipitation, using sputum or urine, and the color reaction in blood.

The agglutination method depends on the clumping of the organisms when exposed to immune serum previously prepared against this organism.

The precipitation method is based on the discovery that pneumococci produce soluble precipitin antigens when growing on broth, in the peritoneal cavity or in sputum. These antigens are also in the urine in a certain per cent. of cases. This precipitinogen is specific and will cause a cloudy precipitate to form in the anti-pneumococcus serum of the type to which belong the pneumococci elaborating it.

Rosenow, and later Longcope, pointed out that pneumococci produce a coagulum when grown in the serum of patients sick with pneumonia. The chemical reaction involved has not yet been explained fully. It may be due to acids or to a precipitinogen. The authors of the blood method of typing pneumococci have used this as a working principle and found that it is specific for different types of pneumococci. The change consists in the formation of hemoglobin derivatives which give a dark 
brownish red gelatinous clot. The reaction may be evident in two hours, but the average time is from 6-8 hours. The technique ${ }^{1}$ of this test is still in the experimental stage, but it promises to be of great value as it is often very difficult to get sufficient sputum early in the disease when the serum would be most beneficial.

The simplest test for typing sputum is the Krumwiede test and the technique may be adopted by the general practitioner. ${ }^{2}$ Where it is

${ }^{1}$ Loewe, Hirschfeld, \& Wallach; J. A. M. A., Vo. 13, No. 3. July 19, 1919, p. 170.

2 Technique of Krumwiede-Valentine method of typing pneumococci in sputum.

1. Sputum-free from saliva as possible, by coughing repeatedly and spitting at once into sterile Petri dish.

2. Send to laboratory immediately. If delay is unavoidable, keep on ice.

\section{Procedure :}

1. Make direct smears from the sputum at once. Stain one by Gram's method and a second with Hiss' capsule stain. This gives an idea of the bacteriology and is a guide in procedure. Group III may sometimes be recognized by the large distinct capsule seen on both smears.

\section{"Technic:}

"From 3 to 10 c.c. of the sputum depending on the amount available, is poured from the sputum container into a test tube. This is placed in boiling water for several minutes or longer until a more or less firm coagulum results, which will occur, if the specimen is a suitable one. The coagulum is then broken up with a heavy platinum wire or glass rod, and saline is added. Just enough saline should be added so that, on subsequent centrifuging, there will be sufficient fluid to carry out the test. If too much is added, the resulting antigen may be too dilute. In some instances little or no saline is necessary, as sufficient fluid separates from the coagulum.

"After the addition of the saline, the tube is again placed in boiling water for a few minutes to extract the soluble antigen from the coagulum, the tube being shaken several times during the heating. The broken clot is then thrown down by centrifuge, and the clear supernatant fluid used for the test. To hasten the appearance of the reaction and to obtain a reaction even should the antigen be dilute, we layer the antigen over the 'type' serums, using the latter undiluted. Two-tenths c.c. of the three 'type' serums, are placed in narrow test tubes, and the antigen added from a capillary tube with a rubber teat. If the tubes containing the serum are tilted and the antigen dropped slowly on the side of the tube just above the serum, no difficulty will be encountered in obtaining sharp layers, as the undiluted serum is sufficiently higher in its specific gravity. The tubes are then placed in the water bath at from $50^{\circ}$ to $55^{\circ} \mathrm{C}$., and observed after several minutes.

"If a fixed type was present in the sputum, and should the sputum have been rich in antigen, a definite contact ring is seen in the tube containing the homologous serum. With sputums less rich in antigen, the ring may develop more slowly, and it will be less marked. Some experience is necessary in detecting 
possible it is wise to confirm this test by the mouse method which is a more dependable one although it requires more time before a report can be made and necessitates using white mice. ${ }^{1}$ If this is not feasible one may use the artificial mouse method. For the details of the technique one should consult text-books on laboratory methods.

Urine may also be used where sputum is not available and in view of the fact that the technique ${ }^{2}$ is simple and rapid it would seem an advantage to test the urine for precipitins as soon as a case is suspected of being pneumonia, for Quigley says that they may be present as early as the second day of the disease.

If the organism is found to be Type I, immune serum ${ }^{3}$ should be

the less marked contact rings and in differentiating them from an apparent ring which may be confusing, if one of the sera is darker in color, giving thus a sharper contrast with the supernatant antigen. The true ring is more or less opaque, and this quality can be seen by tilting the tubes and looking at the area of contact against a dark background, for example; the lower edge of a dark shade raised to just above the level of the eyes. The advantage of the ring test is that a ring may be evident, whereas definite clouding or a visible precipitate may appear only after longer incubation or may be so slight even after an hour's incubation as to leave one in doubt. It is well to shake the tubes after twenty minutes as many of the specimens will show definite clouding or precipitate either at once or on further incubation, thus checking the ring reading." Krumwiede and Valentine, J. A. M. A., Vol. 70, No. 8, p. 573, February 23, 1918.

${ }^{1}$ Blake: Jour. Exper. Med., Vol. 26, No. 1, July, 1917.

${ }^{2}$ Small quantities of urine are clarified by centrifugation and stratified with equal amounts of immune serum of the different types in small agglutination tubes, incubated in the water bath at $37^{\circ} \mathrm{C}$ for one hour and observed at intervals. The precipitate usually appears in 15 minutes, but may need to wait one-half to one hour. (It is essential that all the reagents used should be clean.) Quigley got 81 per cent. of positive results in the urines of 82 cases of Types I, II, III. The strength of the reaction gradually increased during three or four days and persisted two to ten days and gradually disappeared. The day of its appearance is not regular, the earliest was on the second day of the illness. Quigley: J.Infect. Dis., Sept. 18.

${ }^{3}$ The Production of Antipneumococcus Serum. The choice of animal to be used in the production of an antipneumococcus serum has been determined by the facts; that we must have a healthy animal, free from a tendency toward joint infections, and a large animal, capable of furnishing a comparatively large volume of blood at rather frequent intervals. To meet these requirements a draft horse has been found most suitable.

The nature of the antigen to be used has been the subject of much experimentation. At present, injections of both killed and living pneumococci are considered necessaly to produce a serum containing the required strength of agglutinating bodies and the proper protecting power; that is, a serum, that, when diluted 1:200, will cause an agglutination of pneumococci Type I. and 0.2 c.c. of which will protect a mouse against 0.1 c.c. of a virulent culture of pneumococci. 
administered at once. The importance of these procedures pleads for community laboratories in more sparsely settled districts. If it is not possible for the practitioner to determine the type of the organism in a case in which the clinical evidence is positive for lobar pneumonia, he has to consider the advisability of administering Type I on the percentual chance of the case concerned being due to Type I. Under no circumstance is the practitioner justified in neglecting the typing where it is possible, but if Type I furnishes 25-33 per cent. of the cases of epidemic lobar pneumonia it does not seem to me that the patient should be deprived of the chance that such a choice affords. It must be remembered, however, as has been mentioned, that in country districts where it can be proven that the patient has not been subjected very recently to infection in town or city, that Type I cases are relatively rare and Type IV the rule. In any case all precautions should be taken in the administration of the serum to avoid the accidents that can occur to those that are anaphylactic. Patients should be instructed to tell their physicians that they have had serum should the occasion ever arise for a second administration of serum of any kind derived from a horse. This is done in anticipation of anaphylactic manifestations.

Administration of Serum. If it has been determined in any

The bacteria used for the preparation of the antigen must be virulent for man: they must not have been long under cultivation; must have gone through but few passages in animals; the broth culture must be only 12-15 hours old; the virulency is tested on mice or rabbits.

The method of procedure is then: to select a sound draft horse and test him for glanders by complement fixation.

Under sterile precautions to draw 20 c.c. of blood from the jugular vein and to keep this for a control. To inject intravenously daily for six days 20-30 c.c. of an emulsion of killed pneumoccocci from 50 c.c. of a 12-15 hour old culture of virulent pneumococci; to let the animal rest for seven days and then repeat the injections. On the sixth day of the second series, to draw a specimen of blood and test for agglutination and protective power. If it measures up to the standard, bleed the horse. This has rarely occurred and it is usually necessary to give 1-3 or more series of live organisms in this manner: 8-10 days after the second series of injections, give a series of three intravenous injections of a live broth culture, beginning with 20 c.c. and doubling the dose each day (that is, 40,80 c.c.) unless the temperature rises above $104.9^{\circ} \mathrm{F}$. If the temperature is: above this level, decrease the dosage accordingly.

Wait six days the after first injection, take a specimen of blood as before; if it is up to standard, bleed the horse, if not, repeat the injection of live bacteria, using respectively $100,150,200$ c.c. of the broth, being guided by temperature as before; wait six days and test the blood as before; if it is weak give a third series of live cultures and of the same volume as the second series and test the blood as before. After bleeding the horse, allow it to be quiet for 3-4 days and then give series of three injections of living bacteria and after seven days test the serum. If it is of the desired strength, bleed the horse again. 
given case to administer serum, the first step to be taken is to discover whether the patient is or is not sensitized to horse serum. This is a step too often neglected, but the neglect always entails a risk that not infrequently results in discomforts and alarm on the part of the patient, and may in rare instances be followed by sudden death. For this reason it is important to take a careful history of the patient. One should never fail to make inquiry as to the previous administration of serum and the history of asthmatic attacks should be particularly elicited. The previous administration of serum entails severe urticaria in many cases; anaphylactic shock must be the rarest of occurrences. In asthmatics sensitized to horse emanations, however, much more violent reactions, severe asthma and death may ensue, unless the patients are properly desensitized. As the technique of the desensitization is not difficult and entails only a little more time at the bedside every conscientious practitioner will have recourse to this precaution before going further.

This sensitization or as it is sometimes technically termed anaphylactic state constitutes one of the most interesting chapters in modern medical research. It may well be of the nature of, or a step towards, immunity as we witness it in infectious diseases. The rationale of the condition would then depend upon the body's effort to dispose of foreign substances whenever introduced into the blood stream and tissues by any avenue of entrance. Protein substances, whether derived from bacterial bodies as in disease or from other sources, are destroyed within the living tissues by substances akin to ferments, which are elaborated by the body cells on the occasion of the demand. These substances are termed immune bodies, while the protein that has given rise to their production is called the antigen. The action of the immune body upon the antigen results directly or indirectly in the formation of toxic bodies, giving rise to certain manifestations. These considerations are at present largely theoretical. The immune substances are assumed to attach themselves to the body cells until they are produced in such numbers as to more than satisfy the affinities of the body cells when the excess circulates free in the blood stream. At this stage immunity is established and the immune substances, combining with the foreign protein, dispose of it without injury to the body cells, but if the excess of immune bodies circulating free in the blood has not yet been attained, then the foreign protein is acted upon by the immune bodies attached to the body cells which are thus brought into intimate contact with the toxic substances elaborated, to their detriment. This halfway stage is called anaphylaxis and begins in 10-14 days after the administration of serum and continues indefinitely. (In case one is in doubt about the time for a second intravenous injection of serum one should inject subcutaneously 
every third day, $1 / 2$ to 1 c.c. of horse serum to perpetuate the immunity established.) The manifestations of anaphylaxis vary in degree and kind. They may be violent, constituting the condition known as anaphylactic shock, characterized by, in some instances, sudden collapse and death, occurring with lightning-like rapidity; or it may be less violent and cause suffusion of the face, marked restlessness, increased heart rate and not infrequently a most distressing dyspnœa, due to the sharp contraction of the bronchial muscles which results in extreme distension of the alveoli from which the air in expiration cannot escape as a result of the valve-like actions of the folds of soft mucous membrane. In such a case I have witnessed the escape of air into the mediastinum and fascial tissues of the neck. These symptoms may or may not be followed by a fatal issue from a circulatory collapse. Massive urticaria is a common manifestation.

Determination of Sensitization. The technique followed in determining sensitization is here outlined: use a syringe, graduated in hundredths, - the sub Q tuberculin syringe is a good example,-with a very fine needle, a No. 28 is to be preferred. Cleanse the skin over the forearm with alcohol, not wiping vigorously, and carefully avoiding making the skin red. Inject intradermally 0.02 c.c. of a solution of horse serum (or if this cannot be obtained the antipneumococcus serum may be utilized for this purpose) which has been diluted $1: 10$ with sterile normal saline solution ( 0.9 per cent.), i. e., 0.002 c.c. of undiluted horse serum. It must be remembered not to inject under the skin, but between its layers. As a control, some two inches distant from the site and at the same level, inject an equal volume of sterile saline in the same manner. If the injection is correctly done there will appear a small, blanched wheal, showing depressions of the hair follicles. This wheal disappears in a few minutes as the fluid diffuses into the tissues.

If the patient is sensitive there will develop in a half an hour to an hour an urticarial wheal at the site of the injection; this will be surrounded by an area of erythema and the degree of sensitiveness is roughly proportional to the size of the reaction. If there is no reaction we proceed to the administration of the serum.

Desensitization. If a positive reaction has been obtained we endeavor to desensitize the patient. If the reaction is very slight or doubtful, the very slow introduction of serum noted below is sufficient or, and this procedure is advisable in any case, one should give $1 / 2$ to 1 c.c. of the horse serum (or antipneumococcic serum) subcutaneously at least an hour before the intravenous introduction of the antipneumococcus serum. If the patient has a striking reaction or if there is a distinct history of asthma and more especially horse asthma, even greater 
precautions should be taken by desensitizing the patient with repeated small injections. Besredka prefers to attain this end by introducing the serum intravenously, because immunity can be established by it in 10 to 15 minutes and followed at once by a large dose of serum without removing the needle from the vein. His directions $₫$ are:-

"We begin by testing this sensitiveness by introducing intravenously as small a dose as is desired-0.1 c.c. of serum, for example. (The serum is diluted to ten times its volume with physiological saline solution, and 1 c.c. of this solution is injected.) If the patient does not react at the end of three to five minutes, another injection of 0.3 c.c. of serum is given (3 c.c. of the dilution) without withdrawing the cannula. We wait again two minutes, and if nothing happens we inject 1 c.c. of serum (10 c.c. of dilution). At this moment anti-anaphylactic immunity is acquired, but for further security after a further interval of two minutes, we make a last injection of 2.5 c.c. of serum ( 25 c.c. of dilution). Whatever may have been the degree of the patient's hypersensitiveness before this vaccination, we can be certain that he will now tolerate, without the least trouble $20-40$ c.c. of undiluted serum at any stage of the illness." Besredka states that vaccination to obtain immunity may be given subcutaneously by injecting 1 to 5 c.c. of serum, but that one should wait four hours before injecting the full dosage. Some individuals are so extremely sensitive to subcutaneous injections that the only safe method is the intravenous one.

The method of desensitization used by Cole and his co-workers and described in Monograph No. 7 of the Rockefeller Institute for Medical Research, is as follows:

"This should consist in giving extremely small amounts of serum subcutaneously at $1 / 2$ hour intervals, doubling the size of the dose at each injection. One can safely begin with the injection of 0.025 c.c. of serum. If no reaction follows the injection of 1 c.c., subsequent doses may be given intravenously, also at $1 / 2$ hour intervals, beginning with 0.1 c.c. and doubling the dose at each injection. If a general reaction recurs, or cyanosis, dyspncea or increased rapidity of the heart rate supervenes, the injections should be suspended for 2 to 4 hours, depending upon the severity of the reaction, and then be resumed, starting with the same dose as that producing the reaction. After 25 c.c. of serum have been given in these small doses, after a lapse of 4 hours, 50 c.c. may be given, followed by the regular dose 6 to 8 hours later."

Serum Administration. Precautions. This is a surgical procedure and surgical precaution should be used. Sterile gloves, towels and gauze are very desirable though not absolutely essential. They all may be obtained by methods detailed below. 
Serum very frequently contains a sediment and one should avoid stirring this up; if sterile pipettes are at hand it is well to pipette the serum from the bottle rather than pouring it out.

As the serum is quite viscous, it flows better through the needle if it is diluted. As a diluent we use normal saline. It is absolutely essential that this solution be prepared fresh from freshly distilled and if possible doubly distilled sterile water in order to prevent undesirable reactions as explained hereafter. If proper saline is not obtainable, give the serum undiluted.

Further, to avoid unnecessary reactions the serum should be given at or a little above body temperature, and very slowly.

After sterilization in undistilled water all apparatus should be thoroughly rinsed in the special saline to remove the plain water which tends to cause unfavorable reactions.

Inspect the patient's arm before starting the work to ascertain how accessible the vein is and hence the apparatus which will be needed.

\section{Apparatus Needed:}

1. Two number 16 or 18 needles, preferably Luer, and two adapters to fit.

2. Three feet of new rubber tubing $5 \mathrm{~mm}$. inside diameter interrupted by three inches of glass tubing which allows one to inspect the flow.

3. One graduate of 100 c.c. capacity.

4. A container: a Kelley flask is best. The barrel of a 60 to 120 c.c. syringe or a three-inch funnel will answer.

5. Two clamps: a Moore and a screw type are best. Two artery clamps will suffice.

6. A tourniquet of rubber or gauze.

7. A 20 c.c. Luer syringe.

8. A hypodermic syringe and needle.

9. Pitcher, beaker or other vessels of 250 c.c. capacity.

10. Rubber gloves.

11. Towels.

12. Gauze.

13. Adrenalin (epinephrin) solution 1:1000.

14. Mercuric bichloride solution 1:1000.

15. 50 per cent. alcohol.

16. 95 per cent. alcohol.

17. Tincture of iodine.

18. Adhesive plaster.

19. Rubber sheet or newspapers. 


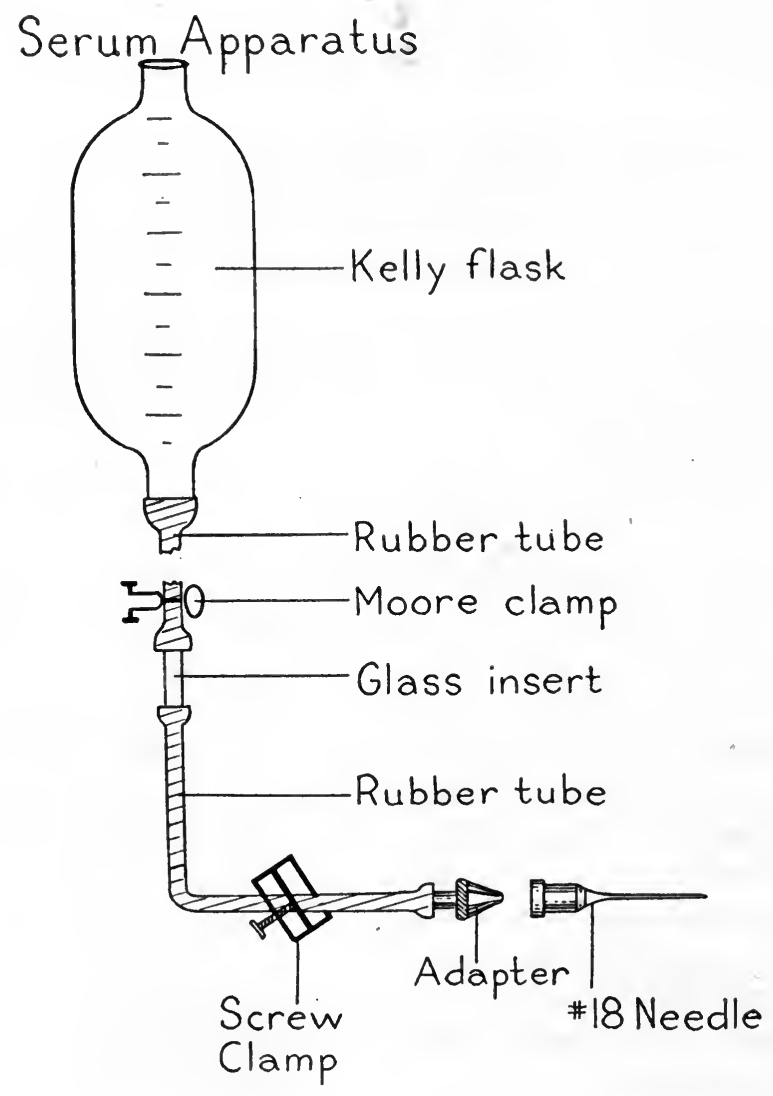




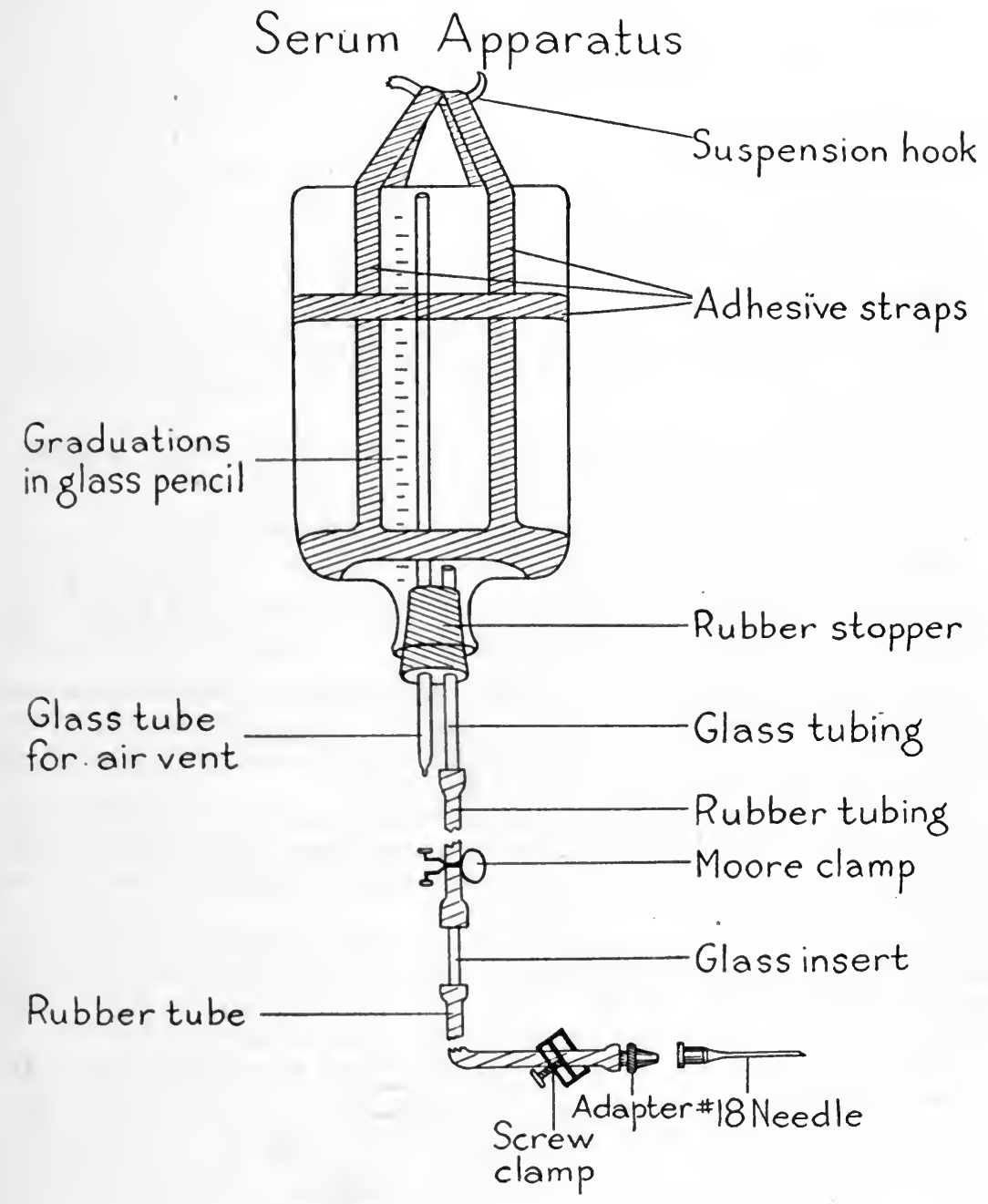


If vein is inaccessible:

1. Two artery clamps.

2. Two pairs of four-inch forceps.

3. One pair of straight scissors.

4. One cannula-Webster style preferred.

5. Sterile catgut, No. I.

6. Sterile bandage.

7. Straight needle.

\section{Assembling of Apparatus.}

(This should not be done in the patient's room.)

(See diagrams I and II.)

Connect the container ${ }^{1}$ with the rubber tubing which is interrupted by the glass tubing inserted about four or five inches from the end distal to the container.

Apply loosely the Moore clamp to the long rubber tubing and the screw clamp to the shorter piece as shown in the diagram.

Connect the adapter to the short length of the rubber tube.

Sterilizing Apparatus. Place the apparatus thus set up, the graduate and pitcher, etc., in a vessel that can be closed and pour over them warm water sufficient to just cover them. Close tightly and boil for 20

1 A much more convenient device, if opportunity affords, utilizes as a container a flat-bottom bottle of 500 c.c. capacity. The advantages of this are that it can be set flat, that it can be used as a mixing vessel and so does away with an extra vessel and an unnecessary transference of the serum.

The setting up of the apparatus is simple: through a two-hole rubber stopper insert two glass tubes, one of which extends to the bottom of the bottle and acts as an air vent and the other goes only just through the stopper and carries the serum.

The procedure is the same as given above, i. e., the rubber tubing is connected with the short glass tube; the system is rinsed with saline and the serum and saline are measured into the bottle and mixed. The bottle and tubing are put in water at $110^{\circ} \mathrm{F}$., taking care to preserve the sterility of the adapter.

When ready to give the serum the bottle is inverted and suspended at about 24 inches above the patient's arm. The needle is put on the adapter and the rubber tube raised and lowered until all the air bubbles are removed from the tubing as shown by the cessation of bubbling of the serum when the tubing is raised. Then adjust the screw clamp so that 15 drops of the serum flow per minute from the needle. Leave the screw clamp thus adjusted and apply the Moore clamp. Proceed as given above.

It is a matter of convenience in judging the rate of flow, if graduations are made on the bottle with a glass pencil. (See Diagram II.)

The Lederle Antitoxin Laboratories now furnish a most convenient intravenous outfit for administering antipneumococcus serum. It is accompanied by specific directions and one may have recourse to it in any emergency where the above apparatus is not available. 
minutes. If one has no sterile goods, immerse towel, gauze, etc., in 1:1000 solution of mercuric bichloride for at least 10 minutes. In the meantime prepare the serum and patient. A few minutes before needles are needed, drop them in boiling water, cover and allow them to boil for five minutes, pour off the water and let them stand in the covered sterilizer.

I. Preparation of the Serum. Immerse the sealed bottle of serum for 10 minutes in bichloride of mercury solution 1:1000, at a temperature of $110^{\circ} \mathrm{F}$. (Higher temperature will coagulate the serum.)

Remove the bottle from the bichloride, dry on sterile gauze and pour sterile normal salt solution over the stopper to remove the bichloride and again dry the stopper with sterile gauze. Remove the stopper, pour the first few cubic centimeters of the serum into a waste pan to avoid contamination from the mouth of the bottle. (Do not shake the bottle and disturb the sediment in the serum.)

Measure 90 to 100 c.c. of the serum in the sterile graduate, pour into the sterile pitcher and add an equal volume of freshly distilled and freshly sterilized normal ( 0.9 per cent.) saline solution at a temperature of $110^{\circ} \mathrm{F}$. and mix thoroughly. (If proper saline is not obtainable, give serum undiluted.) Cover pitcher with sterile cloth and stand it in a pan of water at $110^{\circ} \mathrm{F}$. until it is to be used.

When the apparatus is sterile drain off all the plain water that it contains and rinse it with freshly distilled sterile normal saline solution. Then replace all the air in the tubes by saline, connect the adapter with the needle and adjust the screw clamp so that 15 drops of saline run from the needle in one minute when the container is held two feet above the needle. Leave the clamp thus adjusted. When the saline stands just at the top of the neck of the container apply the Moore clamp. Remove the needle and place in 50 per cent. alcohol.

At the right of the patient's bed place all materials to be used on a table, covered with a sterile towel and have several small flat sterile pans, in one of which is 50 per cent. alcohol.

\section{Preparation of Patient and Operator:}

1. Place the patient as nearly flat as possible and in bed so that his arm is near the edge of the bed. Place the rubber sheet or newspaper, covered by a towel, under the arm.

2. Apply a tourniquet to the arm so that the pulse is not decreased in size.

3. By inspection and more especially by palpation determine the largest and most accessible vein. The vein may be made prominent by the patient's opening and closing his fist or by massaging the arm from the fingers proximally. Having selected the vein, outline the centre of it by a thin line of tincture of iodine. Remove the tourniquet. Carefully 
cleanse the skin over the cubital fossa with 50 per cent. alcohol. Let it dry and then paint with tincture of iodine an area of two inches square over the vein selected. Cover this sterile area with sterile gauze and have someone hold the patient's arm still.

4. The operator now washes his hands thoroughly with soap and water, followed by an immersion in 50 per cent. alcohol and allows them to dry naturally. Put on sterile gloves, if available.

5. The completion of the preparation of the patient:

1. Have an assistant raise the patient's arm, protecting the sterile zone.

2. Lay one of the sterile towels under the arm; lay arm on it.

3. Have assistant apply tourniquet as before and remove sterile gauze from arm.

4. Lay one sterile towel over the forearm and a second over the arm, thus leaving the site of injection exposed.

5. Have assistant hold the patient's hand to prevent his moving the arm.

III. Pour the Serum Into the Container and if a Kelly Flask is used, it may be suspended from a peg of a clothes tree or by some other device. Surround the flask with a hot water bag or electric pad and lay the rubber tubing in water at $110^{\circ} \mathrm{F}$, taking precaution to retain the sterility of the adapter.

If a funnel is used as a reservoir pour the serum into it only so fast as to keep an inch or two in it and have the pitcher with the bulk of the serum sitting in the hot water. Be careful to never allow the serum to get below the neck of the funnel and thus admit air into the tubing.

Fill the hypodermic syringe with the adrenalin solution ready for an emergency if the patient should develop a dyspnœa, cyanosis, etc.

IV. Injection. With right thumb and forefinger grasp the butt of the needle (avoiding at all times touching the body of the needle), and with the forefinger and thumb of the left hand, pinch up the skin over the vein, with a quick motion insert the needle through the skin only. Now steady the vein by placing the forefinger and thumb of the left hand on each side of the vein just above point of puncture. With right hand hold the needle at an angle of about $60^{\circ}$ to the vein. Should you fail to enter the vein in the first trial, withdraw the needle a short distance (but never outside of the skin) and repeat the attempt, until the blood flows freely from the needle. Then quickly connect the adapter to the needle, release tourniquet, remove Moore and artery clamp and note the time the fluid starts to flow. Observe the patient carefully for the development of cyanosis, dyspnœa, pallor, urticaria or rapid pulse. If any of these symptoms develop interrupt the flow of serum for 15 minutes. If 
they disappear continue the injection. If they become worse, give a hypodermic injection of 15 minims of adrenalin and desensitize the patient by Besredka's method as given above.

If no untoward symptoms develop, after 15 minutes the screw clamp is removed and the remainder of the serum given in 15 or 20 minutes.

Care must be taken to hold the needle steady and the patient watched so that he will not break the technique.

When the fluid is about gone, apply the Moore clamp and remove the needle from the vein. Immediately apply gencle pressure with gauze just below the point of entrance to avoid blood oozing through the puncture in the vein into the subcutaneous tissues. After a few minutes remove the pressure and wash off the iodine with 95 per cent. alcohol and apply a small sterile pad over the puncture and secure with a few turns of bandage or adhesive straps.

In case a vein cannot be pierced with a needle as described, cut down on the vein. If this is necessary one follows this procedure:-apply tourniquet, make a one-inch incision parallel to the long axis of the arm, starting at about the middle of the cubital fossa and extending directly downward; this will expose the median basilic vein, running obliquely inward. Attempt to pierce the exposed vein with the needle; if impossible, with a small artery clamp separate the posterior surface of this vein from the subcutaneous tissues and insert beneath it a small pair of forceps. With No. 1 catgut ligate the vein distal to the forceps. Pinch up a small piece of the centre of the proximal part of the vein and with scissors make a minute snip in it. Insert immediately the cannula and secure it firmly with a ligature. Proceed as with the needle method above. When the fluid is all in, apply the Moore clamp to the rubber tubing; remove the cannula, ligate the vein centrally and extirpate the portion between the ligatures.

Remove any blood in the wound with normal saline, close the skin with two catgut sutures, paint with iodine and apply adhesive plaster.

Reaction after Serum Administration. While it is our desire to avoid disagreeable reactions after serum administrations and our technique is designed for that end, nevertheless, certain reactions more or less beyond our control may ensue. There are three groups of such reactions recognized. The first, a reaction that occurs during or shortly after the introduction of the serum, which is sometimes termed a nonspecific serum intoxication or thermal reaction. This reaction is accompanied by much the same phenomena as one witnesses after salvarsan administration. In this latter instance it is believed that certain organic substances in the distilled water used are responsible for the reaction 
and for this reason the saline used to dilute the serum should be freshly prepared from freshly distilled water, if it can be obtained. Certain specimens of sera, perhaps derived from the same horse on different occasions for unknown reasons may provoke the reaction. Again it is said that fresh serum is more likely to do so, than serum properly ripened.

Furthermore, body temperature and slow administration seem to lessen the incidence of these reactions.

The symptoms may occur during the administration or one or two hours later; they are initiated by chill, followed by a high temperature which in turn is followed by an abrupt drop, sometimes $6^{\circ}-8^{\circ} \mathrm{F}$., followed by sweating and usually a marked improvement. It simulates a malarial paroxysm.

There may be restlessness, dyspnœa and suffusion of the face and occasionally vomiting.

Should any of these symptoms appear during the administration of the serum, it should be discontinued an hour or two and then resumed and given more slowly.

To ameliorate the symptoms one gives one-half c.c. (m. vii of adrenalin (epinephrin) intramuscularly or atropin sulphate gr. 1/100 (0.0006 $\mathrm{Gm}$.) by the same method. This reaction has no serious significance nor does it militate against the efficiency of the treatment. It does, however, alarm the practitioner who may fear that he is facing a true anaphylaxis.

The second group of the reactions is the so-called serum sickness or serum disease, which is a later manifestation. This ordinarily occurs 7-14 days after the serum injection; sometimes as late as a month. In some instances the reactions occur early in the administration of the serum.

In the early cases the reaction is technically called an accelerated reaction. This reaction is in all probability not an anaphylactic manifestation, but in the period of its development, the long incubation period and in its manifestations, simulates the phenomena of certain acute infectious diseases, for example, measles and scarlet fever. It happens to some degree in about 50 per cent. of the cases; it is rarely severe and never leaves any sequelæ. The symptoms are a mild elevation of temperature, at times to $103^{\circ} \mathrm{F}$., skin rashes, of which the urticaria is the most characteristic and annoying, sometimes angioneuretic edema, although the eruption may be macular or erythematous; joint involvement is frequent enough. It simulates a mild or moderately severe attack of articular rheumatism, in which, it will be recalled, similar skin rashes occur. Albumin may appear in the urine, but one need not look upon this as a serious kidney involvement. The discomforts of the urticaria may be relieved by the usual methods; bathing in soda solu- 
tions in varying strengths, application of aqueous solutions of phenol (carbolic acid) 1-2 per cent. or phenol in olive oil, or vaseline 5-10 per cent., while in some instances adrenalin given intramuscularly in doses of one-half to one c.c. (m. vii-xv) seems efficacious.

The third group of reactions are those which are attributed to the condition of sensitization described above and most commonly termed anaphylaxis.

As it was explained that this condition was due to the introduction of foreign protein into the tissues, it is understood that previous administration may in some instances induce a greater or less degree of this sensitization. Fortunately, however, in the human being this rarely induces the most severe reaction called anaphylactic shock. Shock is more likely to occur in individuals sensitized to horse emanations, especially in those in whom this sensitization expresses itself as asthma, hence the very great importance of determining the presence or absence of such attacks in every patient to whom serum is to be administered.

It has already been described how such patients can be desensitized. Anaphylactic shock occurs during the administration or immediately after and is recognized by the suffusion of the face, the profound restlessness, shortness of breath and rapid heart or by pallor with symptoms of collapse. Sometimes the symptoms of shock occur after the administration, but usually fairly promptly. Adrenalin (epinephin), 1-1000 intramuscularly, in doses of 15 minims (1 c.c.) should be given at once. If the pulse is soft and the blood pressure low, strophanthin, $1 / 2$ milligram (gr. 1/120) intravenously, or digitalis in some of its forms equivalent to 3 to 5 grains $(0.2-0.33 \mathrm{Gm}$.) of the drug, e. g., tincture diluted with saline two or three times, digitoxin gr. $1 / 100$ to $1 / 60(0.0006-0.001$ $\mathrm{Gm}$.) in $\mathrm{m} . \mathrm{xv}$ (1.0 c.c.) of alcohol diluted four times with saline, or solutions of properly assayed commercial preparations, should be given intravenously. Heat should be applied to the extremities, hot towels laid across the abdomen, in other words treated as shock.

If, as commonly occurs, intense asthma is the dominant feature of the reaction, adrenalin in doses of 15-30 minims (1-2 c.c.) should be given intramuscularly, atropin gr. $1 / 50(0.0012 \mathrm{Gm}$.) intramuscularly, morphine sulphate gr. $1 / 8$ to $1 / 4(0.008-0.015 \mathrm{Gm}$.) subcutaneously or intramuscularly. If, in spite of these measures, cyanosis deepens and the dyspnœa increases, it must be recalled that the air vesicles are over distended and unable to empty themselves of air. It would be logical to express the air by force. This may be done by encircling the chest with one's arms and expressing the air rhythmically. This I have seen done in one instance with the most satisfactory results.

If everything goes well, within an hour or two after the administration 
of the serum there is a slight rise of temperature, followed by a marked fall, the patient's mental condition is improved, the heart rate is slower and the respiration is slower and less labored. Furthermore the blood stream is rendered free from organisms, unless there was a marked septicemia; andd the process in the lung may be arrested. To what these changes are due, we have no adequate explanation. We know that the power of agglutination is markedly increased and that the pneumococci are more susceptible to phagocytosis, due to the increased production of opsonins.

Frequency. We repeat the serum, if after an interval of 8-10 hours there is a secondary rise of temperature with an increase in the symptoms of the disease, or we repeat the injections after this interval if there has been no improvement in the condition.

Dosage. The usual dosage is 60-100 c.c. As the serum, unlike drugs, has no toxic effects, the dosage is determined by what in practice has been found sufficient and by the limitation of expense. Larger doses therefore would be indicated in the more toxic stages. In the severe cases the interval between doses is usually 8 hours or less. In the cases showing improvement, the physician's judgment must determine the interval. As the disease advances it has been found, that larger and larger doses are required to get equivalent results.

The general principles of therapy applicable to the one form are also applicable to the other. Certain details of one or the other form of pneumonia may demand modifications or differences in treatment. These will be considered.

Rest. First of these in importance is rest. Rest means rest in bed in a quiet environment. When the patient is very sick it means avoidance of every muscular effort, even turning in bed being done by the nurse or attendant; use of the bed-pan and urinal; absolute interdiction of the effort to arise to attend to these matters. Twice I have witnessed edema of the lungs precipitated by the act of turning the patient for purposes of examination. In very sick patients as infrequent examination of the chest as is compatible with an appreciation of the progress of the disease is desirable. Rest means competent nursing; it means freedom from worry, anxiety, introduction of business cares and concerns; it means the exclusion of all visitors and of all individuals who are not concerned in the care and comfort of the patient.

Bed. Rest in bed is rest only when the bed is comfortable. The type of bed is best illustrated by the hospital bed. It is a half or at most three-quarters bed with a firm woven wire spring, and a firm but resilient mattress. The bed-clothes must be applied smoothly and without wrinkles; the clothes upon the patient chosen to avoid excess of weight 
and should be no more than afford maximum comfort to the patient; only one pillow should be used. For purposes of cleanliness as well as comfort a rubber sheet should be placed across the bed. The width of this sheet should be such as to reach from the pillow to the bend of the patient's knees and sufficiently long to tuck under the mattress nicely. Over this sheet should be placed a draw-sheet, a long sheet folded lengthwise with width enough to cover the rubber sheet and so long that it may be drawn from the one side to the other from time to time to bring a cool surface to the patient's body or to remove a soiled spot.

During the period of convalescence when the patient is allowed a bed rest, the inevitable tendency to slide in the bed, costs the patient much energy.

One may have recourse to the device of placing a rolled blanket under the knees or between the feet and the foot of the bed as a support. Numerous foot rests have been devised for this purpose, but none are satisfactory. A bed whose frame is so devised as to be raised at its upper end to afford a back rest and at a point corresponding to the patient's knees to give support to the flexed limbs, is in my estimation, by far the most satisfactory device. This type of bed is illustrated by the Gatch bed.

Room. The room should be chosen with reference to sufficiency of light and air.

It should be stripped of all unnecessary furniture; if possible, it should be so near the bathroom as to facilitate the many demands of nursing.

Even better is a room from which a verandah or porch may be approached, on to which the patient may be wheeled, if necessary.

Temperature of the Room. This should be about $65^{\circ} \mathrm{F}$. unless the patient is submitted to cold air treatment. At whatever temperature the room is kept, the patient should be so placed as to avoid drafts.

Care of the Body. The care of the body is of prime importance as it means comfort to the patient and a more efficient performance of important functions. This is the particular province of the nurse, but when a nurse cannot be in attendance it is the business of the physician to explain the details to whomsoever is to act as nurse, of all that constitutes the proper care of the body.

Bath. A sponge bath should be given for purposes of cleanliness, as well as comfort every day. The only excuse for omission of the bath is such a weakened condition on the part of the patient that even the little manipulation concerned in it becomes to him a source of exhaustion and danger.

The temperature of the room when the bath is given should be from $65^{\circ} \mathrm{F}$. to $70^{\circ} \mathrm{F}$. If this can not be effected the bath may be given be- 
tween blankets. Tepid water and castile soap are used and the skin gently wiped or dabbed dry with a towel.

Special attention should be given to the eyes, the ears, the parts of the body most pressed upon and likely to become the seat of bed-sores; the genitals and the anus.

Mouth. The care of the mouth is of great importance, especially in early toxic cases, when the patient is stuporous or comatose, the mouth dry, the tongue coated, the lips and teeth covered with sordes and in these cases secondary infection affecting the parotid gland and the middle ear and possibly complicating bronchi and lungs with secondary invasion, as well as imperilling the digestive tract, are to be constantly kept in mind.

In all cases the mouth should be rinsed after each feeding with some mild antiseptic solution, such as a saturated solution of boric acid, 4 per cent. or half-saturated solution, 2 per cent, or one may use Dobell's solution, $1 / 2$ or $1 / 4$ strength.

The teeth, too, should receive attention. The interstices freed from particles of food that may undergo decomposition. The best way to do this is to use closely rolled swabs of absorbent cotton on wooden toothpicks; these being wet with the solutions named. A soft tooth brush may be used, but care has to be exercised to avoid trauma of the gums or lips.

Tongue. When the tongue is much coated and when there is sordes upon the teeth or lips, half-strength solution of peroxide of hydrogen should be applied to soften the deposit and the excess scraped away with the edge of a whalebone or similar instrument and then the tongue and lips should be cleansed with the above solution. For a very dry mouth a mixture of equal parts of liquid petrolatum (albolene) and 2 per cent. boric acid solution flavored with lemon juice is an excellent application. When the breath is fetid or there is much stomatitis an application of an antiseptic solution as follows is excellent:

Phenol (Carbolic Acid) watery solution 1-20.........

Glycerin ..........................

Boric Acid, saturated watery solution............ viii

M.

S. Use as directed.

Collections of mucus in the naso-pharynx and the back of the mouth are best removed with cotton swabs on long applicators, which have been saturated with some of the mild antiseptic solutions.

Gargles do not reach back to the naso-pharynx and rarely reach the tonsil. The back of the mouth, then, should be cleansed by the use of a spray, the tongue being depressed with an applicator, while the spray is 
used. A boric acid solution or a Dobell's solution may be used for this purpose.

Nose. Attention also, should be given to the nose; for if this is plugged with secretions, the patient is obliged to breathe through the mouth and the dryness of the mouth and coated tongue is aggravated. Dried secretions should be softened by the application of vaseline or sweet oil and then the nose is cleansed with a cotton swab on a toothpick or other applicator, which has been saturated in one of the solutions mentioned.

Sprays, too, may be used. Irrigations should be avoided, lest, especially if the patient is somewhat stuporous, the solutions are forced into the Eustachian tube, carrying with them infection.

Fissures and herpetic eruptions should be treated with the solutions and then anointed with the oil or vaseline (petrolatum).

Eyes are to be kept clean by the use of half strength or saturated boric acid solution.

Care of the genitals requires a careful cleansing after every defecation and in the female after micturition. The same solutions may be used for this purpose. If there are excoriations a carbolic acid solution $1 / 2$ per cent. to 1 per cent. may be used, then the parts are to be kept dry and dusting powder applied to the skin about the parts.

Diet. The dietetic demands are quite different in the acute lobar pneumonias, of short course, marked by much intoxication, and the longer continued broncho-pneumonias or the unresolved pneumonias or pneumonias with complications that prolong the course, for a considerable period of time.

The impairment of digestion and assimilation is much less than is usually assumed. Such impairment as there is lasts only during the early hours, the first day or two, of an acute process or when the intoxication is very intense. During this time there is often gastric distress and usually anorexia. It is better at this time not to force the food, but after this period has passed certain theoretical considerations have to be kept in mind. These are that a man at rest, suffering from an infectious process expends as much energy as a well man at rest, but in addition to this the febrile process, when the temperature is above $102^{\circ} \mathrm{F}$. or $102.5^{\circ} \mathrm{F}$., increases the need of food intake some 25 per cent.

These two requirements amount to about forty calories per kilo of body weight or some 2,800 to 3,000 calories for the adult male of average weight; but in addition to this the acute infection induces a protein loss that can be prevented only by increasing the food intake even above the amounts already mentioned. No amount of protein intake will make good for this protein loss unless a sufficiency of carbohydrate 
should be administered with the protein. Under these circumstances protein intake of from seventy to ninety grams are sufficient.

We rely particularly upon milk. One quart of milk gives about 640 calories or about twenty calories to the ounce. It is obvious, however, that if we were to use milk alone the quantity necessary to supply the number of calories required would be inordinate. It becomes necessary, then, to add other articles of food, which have a high calorie content and a low protein content. One of the simplest means of obtaining such a result is to add cream.

Gravity cream, 16 per cent. cream, affords about fifty calories to the ounce. Milk sugar is another substance that can be added to the milk, each ounce giving 120 calories.

A half ounce of milk sugar and one ounce of gravity cream added to each glass of milk increase the calorie content a little over one hundred. Other substances that may be added are cereals, such as thoroughly cooked oatmeal, or hominy or rice; an ordinary serving of these articles of food, varying from three to five ounces, according to the nature of the cereal adds another one hundred calories. When cream and sugar are added to such a serving, the calorie content is greatly increased; as two ounces of cream and three teaspoonfuls of granulated sugar offer each an additional one hundred calories.

One may give eggs, raw or soft cooked. A coddled egg, that is, an egg which is put into boiling water and then set aside from the stove and allowed to cook as the water cools for five minutes thus acquiring a creamy consistency makes an excellent addition to the dietary; eggs and milk in the shape of cup custard, broths made of cereals and milk; bread given as toast or as milk toast or plain bread and butter, all add materially.

Various milk preparations may be used in place of milk, such as matzoon, koumys, zoolak, buttermilk.

If the patient's appetite craves them, meat soups, mutton broth or chicken broth may be given, but it must be remembered that they have very little food value hence rice or other cereal-flour may be cooked into them to give them some caloric value.

If tympanites, often an early symptom of the disease, is increased out of proportion to the other evidences of the intoxication, one should suspect the milk sugar as being responsible.

If diarrhea ensues one cuts down the fat; while both fat and sugar may be responsible for gastric distress and vomiting. Under these circumstances these e'ements must be cut out until the symptoms disappear and then gradually replaced.

In a lobar pneumonia of short course, then, we give an abundance 
of water in the early days, with small amounts of milk. If the stomach be irritable, this milk had better be diluted either with plain water, an alkaline water or with cereal water. The food is gradually increased, but no effort is made to reach a high calorie diet; but in prolonged pneumonias, broncho-pneumonias, unresolved pneumonias and complicated pneumonias, one treats them much as he would a typhoid fever case.

Drinks. Water is rarely given in sufficient quantities. It ought to be offered to the patient every hour or two and this particularly as the patient is often so stuporous as not to ask for water that he sorely needs. It is surprising to see how much these patients will take when the water is offered them. It unquestionably affords a stimulus to diuresis, and diaphoresis and keeps the bowels in better condition, lessens tympanites, keeps the tongue and mouth moister and is in every way beneficial.

Fruit juices, lemonade, orangeade, Imperial drink can be given as freely as water and these latter afford an excellent means of conveying sugar which increases the food intake.

Two to three quarts of fluid a day should be given and, if the patient will take more, three, four or five willingly, he should be allowed it.

Care of the Bowels. Attention to the bowels in pneumonia is almost as imperative as in typhoid fever; for tympanites is almost as common in the one as in the other. The toxins of pneumonia affect the large bowel and its motility characteristically; a moderate grade of paresis of the colon obtains in a very large per cent. of the cases, occurring early in the course of the pneumonia.

The danger that accrues to this condition is entailed by the displacement of the diaphragm upward with consequent encroachment on the thoracic space, favoring congestion, hypostatic pneumonia and lessening the respiratory excursion of the already impaired lung.

The heart, too, undergoes a certain degree of displacement by the crowding upward of the diaphragm and its operation is, consequently, made more difficult.

Stasis, too, in the large intestine favors fermentation which in turn aggravates the tympanites and permits of absorption of products of decomposition and putrefaction which add their own increment of insult to the already toxic organism.

When the case is first seen, then, a catharsis of calomel or salts should be given. Three-quarters of an ounce to an ounce (20-30 Gm.) of Rochelle salt or Epsom salt or (and particularly if there be any nausea) small doses of calomel; for example, grain $1 / 4(0.015 \mathrm{Gm}$.) every $1 / 4$ hour until six or eight are taken or in children gr. $1 / 10(0.006 \mathrm{Gm}$. every ten minutes until one grain is taken and this followed in three or four hours by a half ounce $(15 \mathrm{Gm}$.) of one of the salts mentioned in a 
half to three-quarters of a glass of water. Later it is better to rely on the enema to keep the bowel in condition; or one may give liquid petrolatum in tablespoonful doses or more if needed to effect the result although there is no objection to the use of salts for the same purpose occasionally.

Tympanites. This symptom, because of the danger "it entails, must receive attention. It is less marked in those cases that have a sufficiency of water given them.

The diet, too, should be inspected with reference to the quantity of fats or milk sugar it contains, either of which may be responsible in some measure for the collection of gases, and should be diminished or eliminated from the dietary.

The enemata themselves offer much relief and their effects are enhanced by the addition of small quantities of the oil of turpentine. I use 5 ss. $-5 \mathrm{i}$ (15-30 c.c.) of turpentine in a pint of soapsuds. One makes a thick lather with castile soap and a small amount of water, stirs in slowly the amount of turpentine to be used and then adds water up to a pint or more if desired. In this way the turpentine is kept in emulsion. Other enemas for the same purpose that may be found effectual are, 2 teaspoonfuls of the essence of peppermint to 2 quarts of warm saline or 3 ss. ( 2 c.c.) of tincture of asafœtida in 2 quarts of normal saline or tap water. A very excellent enema to relieve tympanites, to which the surgeon often has recourse, is made of milk and molasses, using a cupful, (200-250 c.c.) of each. One warms the molasses until its consistency is thinned and adds warm milk, the temperature being above body temperature. It is best given with a funnel and tube.

Rectal Tubes may also be inserted, just as in typhoid fever.

Shattuck speaks enthusiastically of the efficiency of pure glycerin in doses of one to two ounces (30-60 c.c.) by rectum for the relief of tympanites.

Stupes, too, are to be used for this purpose; for the technique see Typhoid Fever, Chap. XIV.

Turpentine may, also, be given by way of the mouth in capsules, of 5 to 10 minims $(0.30-0.60$ c.c.) each or in an emulsion. The disagreeable taste may be covered by adding a suitable amount of cinnamon or some other aromatic substance.

Strychnine for its tonic effect on the musculature of the bowel may be used in doses of gr. $1 / 30(0.002 \mathrm{Gm}$.) three or four times a day.

Pituitrin or pituitary extract which contracts the smooth muscle substance of the intestine often acts happily in this condition, but the results are not always certain. As the drug is credited with a stimulating effect upon blood pressure, no harm will come from its use, except in the 
Murphy Drip Apparatus

Glass tube for

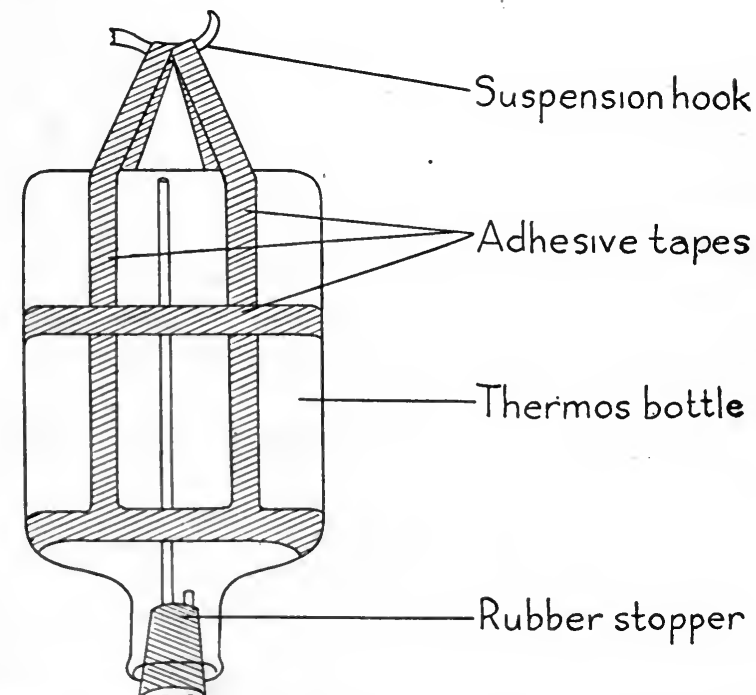
air vent

Murphy drip or Calcium Chloride bulb Rubber tubing

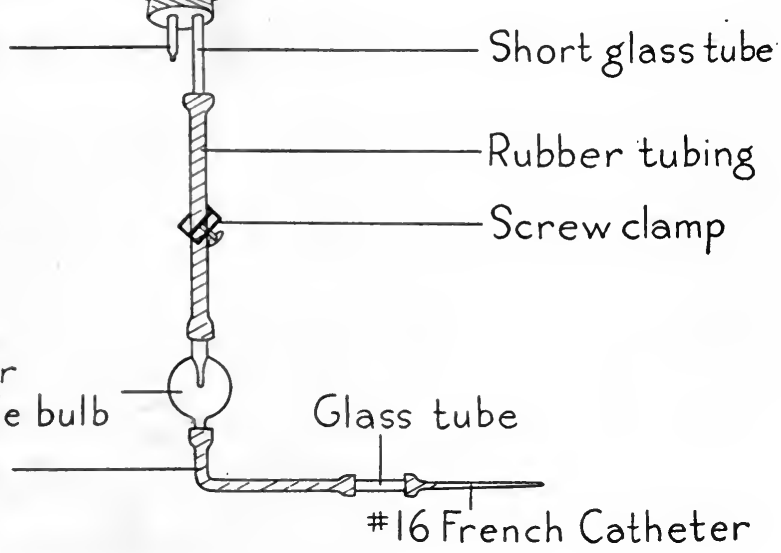


case of pregnant women. The usual dose is 1 c.c. of the extract hypodermically, which may be obtained in the various pharmaceutical houses. A failure to accomplish the desired result may be due to the poor quality of the preparation used and should not discourage one from further trial of another preparation.

A Murphy drip, with the bag held about a foot above the bed may be used for the same purpose. The temperature of the saline used should be about that of the body or slightly above, and the solution may be kept warm in the bag by applying hot water bottle about it or inserting an electric bulb in it. Even a better method for maintaining temperature of the drip is a thermos bottle. One should remember that the radiation of heat as the solution passes through a long tube is excessive. It is the temperature of the solution as it enters the bowels that we are concerned with; short tubes of large calibre or protection of the tube by allowing it to rest in hot water are devices to subserve this end.

The apparatus needed for the administration of Murphy drip is simple; it consists of:

1. A container for the fluid.

2. Two feet rubber tubing $5 \mathrm{~mm}$. in diameter.

3. Number 16 French cathether or plain rubber tip of an ordinary douche bag.

4. Screw clamp or artery clamp.

5. Glass coupling like one of the illustrations, or a Murphy drip bulb or calcium chloride bulb.

The simplest container to use is a thermos bottle, as it maintains the water at the desired temperature for a long time. However, one can use any ordinary bottle that will hold a pint to a quart. The neck of the bottle is filled with the two-hole stopper, carrying two pieces of glass tubing, one being so short that it is just flush with the inner end of the stopper and the other extending to the bottom of the bottle and pulled out to a capillary. To the outer end of the short tube is attached the rubber tubing, which is connected with the glass coupling which in turn is joined to a No. 16 French catheter. Now insert the French catheter 6 " into the rectum and start the water flowing at the rate of 30 drops a minute into the glass coupling, controlling the flow by a screw clamp or artery clamp. The water should be about $105^{\circ} \mathrm{F}-108^{\circ} \mathrm{F}$. as it enters the rectum. If the thermos is not used one may use an electric pad or hot water bottle around the container. The flow should be at such a rate that the desired amount of fluid will be given in 24 hours, 30 drops per minute running continuously will deliver about 5.5 pints in 24 hours. (See diagram of apparatus.) 
The container should hang not more than 18 inches above the rectum of the patient.

The patient should be in a recumbent or semi-recumbent position.

The solution used may be a 0.5 per cent. salt solution, or a 3 per cent. sodium bicarbonate with 5 per cent. glucose in either solution.

The rate depends on the amount.

Symptomatic Treatment. Before touching upon the individual symptoms I will mention those measures which enhance the functions of the body as a whole, that increase its powers of resistance.

The Open-Air Treatment. I am convinced that there is no measure comparable in its effects on the vital centres to fresh, live air in the open.

The open air to have its stimulating effect or to have its maximum stimulating effect must be cold and dry and in motion. The constant play of the currents of cold dry air, impinging on the nerve endings of the face and the upper air passages send their impulses to the vaso-motor, respiratory and other vital centres acting upon them as stimulants.

Fresh air, alone, desirable as it may be, such as one gets by having the windows of a room or ward open does not give the same results as the air out-of-doors.

As the temperature rises and as the humidity increases the effects of the open air diminish.

Sufficient reports have been made, and my own observations have confirmed them, to assure us that as measured by the improvement in blood pressure there is no drug classed as a circulatory stimulant, the effects of which are so decided, so sustained, as the effects of fresh air.

Not only does the pulse show a better quality and become slower, but the respiratory act is deepened, the nervous manifestations lessened, cyanosis is diminished, and the patient in every way shows improvement.

However, to effect the desired results, a technique is to be carefully observed. The preparation of the bed and the patient in the bed is all important.

When, as is usual, a patient in this treatment is exposed to the winter air, our effor ts are directed against any possible chilling of the surface of the body and extremities; for if this occurs our efforts are defeated and our patients may be truly endangered.

The Bed. A half bed, preferably of the hospital type, is stripped to the woven wire spring. Upon these springs is spread a large blanket, extending well beyond the sides of the bed and well beyond the foot. Upon this blanket is laid rubber sheeting or thick papers, that extend beyond the sides and foot as far as the blanket does. Upon these papers. 
is laid the mattress and then the bed is made in the usual way with every care for the smoothness of the sheet and the draw sheet.

The patient, prepared as presently to be mentioned, is placed in this bed and then the blanket and the rubber sheet within it are drawn up around the mattress, bed and patient in such a way as to form a complete envelope and, in fact, folded much as one would an envelope.

The patient has been in the meantime clad in an under suit of flannel, with stockings, and a hot water bottle is placed at the feet. Upon his head is a hood; only his face is left exposed. One low pillow is afforded him. If there be high winds, an effort should be made at affording a shelter by the use of screens or the position of the bed; while some shelter to the head can be procured by the use of pillows on either side of the head, making an inverted letter $\mathrm{V}$. The object of all this is to prevent air getting at the patient's skin to chill him.

The patient must be kept under constant observation, and more particularly if he is in any degree delirious, lest he disarrange his clothes and become chilled or lest in his delirium he leave his bed and injure himself.

Nurses and attendants must be clad for out-of-doors.

For purposes of examination the patient should be drawn into the room and the temperature of the room raised to $65^{\circ} \mathrm{F}$. or $70^{\circ} \mathrm{F}$. before he is exposed; and the same precaution should be taken when the bed-pan or the urinal is to be used or the toilet of the body performed.

When the conveniences are not at hand for such a treatment, on the verandah or the porch or fire-escape or roof, one substitutes for this treatment a room, the windows of which are wide open or the sash taken out, the window space being filled with cheese-cloth screens.

It is common to see a rise of blood-pressure of $10-20 \mathrm{~mm}$. $\mathrm{Hg}$., or even more, shortly after the patient leaves the ward or room. This will persist until he is brought back into the ward and it is certainly curious, as well as interesting that, although the ward or room, into which he is brought is thoroughly ventilated, the blood pressure will gradually go down several $\mathrm{mm}$. after he has been taken from the open air. It has been objected that patients with a severe cough find the cough exaggerated by the exposure to cold air. In my experience this has certainly not been the rule, though in some instances it has seemed to be true. If the cough is aggravated or the patient finds the exposure to the open air decidedly distasteful, it is proper to give them the shelter of the room.

In the very old or very feeble great care should be exercised to see that the technique is perfect, for chilling of the surface of the skin may grievously imperil these patients. In this measure, as in any other, 
common sense must be exercised and the reaction of the patient is the true criterion of success, or failure.

Hydrotherapy. Another therapeutic measure having similar results, effected in a similar manner, is hydrotherapy.

I quote from my article contributed to the New York Medical Journal for Jan. 8, 1910:

"Water is used locally (1) to serve the purposes of cleanliness and hygiene in the daily bath; (2) to afford momentary comfort, cooling the skin and refreshing the patient, and (3) to influence the disease."

This last is the most important and can be done by means of the sheet bath, tub-baths, cold sponging, compresses and other devices.

In this country we owe largely to Baruch's persistency what benefit is accruing to us from this mode of treatment. He maintains, and logically, that good results can come only from observance of the technique.

His own preference in pneumonia is the chest compress. I cannot do better than quote his precise directions and urge that they be followed exactly rather than attempt to duplicate measures only vaguely referred to.

"The chest compress is prepared by cutting three folds of old linen of a sufficient size to fit the entire chest from the clavicle down to the umbilicus, with arm holes in the region of the axillæ, made by exact measure from one axilla to the other, sufficiently deep to allow the upper edge of the compress to reach above the clavicles and admit of the junction of the flap thus formed on each side to cover the shoulders. Two such jackets and two pieces of closely woven, thin flannel of the same shape, but an inch wider and longer should be provided and fitted to the patient. One of the linen compresses is rolled up and soaked in a basin of water at $60^{\circ} \mathrm{F}$. and wrung out so that it remains quite damp without dripping. The flannel is now stretched out upon an even surface and the wet compress put upon it, so that there remains an edge of flannel about an inch wide all around. Both are rolled together halfway, while the patient is gently turned upon his left side with the precaution of not allowing any exertion on his part, the compress is so placed upon the bed that the rolled part lies in close proximity to the left side of the patient and the lower edge of the left slit is under the left axilla. Now the patient is quietly turned upon his back, so as to release the rolled up portion. The latter is now unrolled and both edges of the compress are brought upward upon the front of the chest and are thus made to envelope the latter snugly. The flannel cover which has been allowed to lie upon the bed during the application of the wet compress is now brought forward, so as to cover the latter. It is secured by two safety-pins in front 
and one pin upon each shoulder." He goes on to say that it should fit snugly, but not so tightly as to embarrass breathing. As long as the rectal temperature is above $99.5^{\circ} \mathrm{F}$. it is to be renewed about every hour. A fresh compress should always be ready for an immediate application, so that the chest shall not be exposed. Before taking it off, insert the finger beneath the compress to see if it is thoroughly warm as it is not to be renewed if it is still cool. Dr. Baruch further adds: "In the average case a temperature of $60^{\circ} \mathrm{F}$. will be appropriate. Should the patient evince stupor or muttering delirium, a lower temperature should be adopted and the chest should receive one or more dashes of colder water before the renewal of each compress. The same procedure is indicated in broncho-pneumonia, when the bronchi are blocked by secretions or cyanosis exists. A higher temperature than $60^{\circ} \mathrm{F}$. may be used if there is much jactitation, insomnia or excitability. In the latter event great benefit will accrue from allowing the compress to remain two hours and moistening it more thoroughly before application, thus converting the compress into a soothing fomentation that is not relaxing like a poultice." If the patient remains cold and blue and the compress does not become warm, then the flannel alone is applied until the skin becomes warm and a mustard foot-bath may be utilized.

The rationale of the procedure is put as follows:

It is asserted that "when the cold compress is applied, there is a rapid contraction of the cutaneous vessels, which raises the tension at once, which eventuates in a tonic dilation of these vessels, which is evidenced by a ruddy hue of the skin. This dilation differs decidedly from that relaxed condition of the cutaneous vessels produced by warm poultices. The latter relax the vaso-constrictors, producing a paretic condition of the vessels or a stasis, while cold applications stimulate the vaso-dilators, giving rise to an active dilatation, with maintenance of tone of the vessels, an active hyperæmia, by reason of which the blood is propelled more vigorously through them." The heart is relieved by the increased tone in the vessels. It has been shown that the pulmonary vessels are supplied by nerves rising from the second to the seventh dorsals; the somatic supply is distributed to the skin of the chest and abdomen as far as the umbilicus. Through the effect on this area of the cold compress undoubtedly influences are exerted reflexly on the pulmonary vessels. The improvement is seen in a diminution of the nervous manifestations, deepening of respiration, with relief of dyspnœa; better tension in the arteries, better sleep, improved appetite and freer functioning of skin and kidneys.

The sheet bath, which is applied to the body at large rather than to merely the chest as in the chest compress, is another excellent measure, 
not only to control excessive temperature, but also to afford stimulation to circulation, respiration and depressed or toxic cerebral centres. No better description can be given of the technique than in Dr. Baruch's own words taken from his admirable book Hydrotherapy as follows: "Technique:-One side of the patient's bed or adjoining cot is protected by a rubber sheet. A blanket is spread upon the latter. Several linen sheets, . . . a basin, a bucket of water of the required temperature, a cup and a sponge are placed upon a chair. . The sheet, being partly wrung out of water of from $50^{\circ}$ to $80^{\circ} \mathrm{F}$., according to the effect aimed at, is spread upon the bed as rapidly as possible to prevent a change of its temperature. The head and face of the patient being bathed with ice water and a wet turban wrapped around his head, he is laid upon the wet sheet and is wrapped with it in the following manner: the patient is directed to hold both arms above his head. The upper left border of the sheet is now brought close under the left axilla and laid across the front of his chest, reaching beyond the axillary line of the right side, the lower portion is placed over the pelvis and the edge tucked in between the lower extremities. The arms are brought down and placed alongside the body, from which they are separated by the intervening sheet. Now the right portion of the sheet is carried across the body above and below, enveloping the arms and shoulders as well as the lower extremities. The right upper border of the sheet is firmly drawn over the left shoulder and securely tucked under the latter; the lower end is tucked underneath the heels. In this manner the patient is snugly enveloped in the wet sheet and no uncovered parts of the body lie in apposition. The arms may, in feeble patients, be left out altogether and simply bathed during the process. This will also facilitate the application of the sheet. The first impression will be a shock to the peripheral nerves, caused by the sudden contact of the cold, wet sheet. A deep gasping inspiration, and a little shivering follow. These are readily overcome by the patient's own high temperature, and their removal is now aided by the manipulations of the bath nurse, who, with outstretched hands, gently, but firmly and gradually sweeps over the wet sheet, passing over the entire body successively. Small portions of the body should then be rubbed in this manner until they warm up. So soon as any part of the body becomes thoroughly warmed, water from $50^{\circ}$ to $60^{\circ} \mathrm{F}$., according to the condition of the patient, is poured from a cup or squeezed from a sponge over it and rubbing is resumed. When the treated part ceases to warm under the friction, the attendant proceeds to another part. These gentle, but firm passes or friction over successive parts of the body are alternated with the pouring on of quantities of cold water, until the entire body feels cooled and the patient shivers. Rigor and chattering of teeth must 
always be avoided, because they are an evidence of muscular contraction, and of a too decided temperature difference between the central and peripheral portions of the body, which causes the former to rise and thus counterbalance the effect. Friction prevents this objectionable feature of all cold baths and enables us by the renewed application of cold water upon the warm part of the sheet to maintain the cooling effeet. After its termination, the patient is rapidly dried, the sheets and blankets and rubber sheets are withdrawn by rolling him upon his side and without disturbance. It is not necessary to apply friction in drying, except to the extremities. We have in the sheet bath an admirable antifebrile procedure, the effect of which may be greatly enhanced by allowing the patient to remain in it, leaving him, without drying at all, packed snugly in the blanket and wet sheet for half an hour. Its mildness as compared with the full cold baths, renders it more acceptable to the patient and his friends and it may thus be utilized as a valuable initiatory measure, intermediate between the ablution and the half-bath."

The sheet bath may be repeated at four-hour intervals or at greater or lesser intervals, according to temperature. The rationale of the treatment is the same as that described under the chest compress above.

The cold baths may be utilized for the same purpose, but the movable tub is difficult to secure in private practice while it necessitates more handling of the patient which is not always desirable in the highly toxic states.

Personally, I am not willing to forego the benefit of the open-air treatment for the hydrotherapeutic; for one can see that hourly and half-hourly changes of compresses or frequently administered sheet baths are scarcely compatible with what I have described as the open-air treatment. As I have said before I believe that the success of the openair treatment is due in no small measure to the cold; so, in the warmer seasons the cold-water treatment could be better applied. Again, where it was evident that the open-air treatment was not meeting our expectations, nervous symptoms, excitability, and so forth, increasing, or stupor deepening, we can have recourse to hydrotherapy.

Moreover, the applications of compresses or the giving of sheet baths day and night, when a patient needs sleep, does not accord with my definition of rest. I believe that real results can be obtained from the less nagging procedure of the open-air treatment.

Symptomatic Treatment. As has been said, symptoms may be looked upon as purposeful expressions of the body's efforts to accomplish something useful for itself; but that it is possible for Nature in this effort to overshoot the mark and for the symptoms to become, per se, a source of danger. 
Fever. There is abundant reason to believe that the febrile reaction favors the efforts of the body to kill the invading organism and to protect itself against its ravages. In a frank pneumonia, a certain amount of temperature may be looked upon as a favorable reaction; certain it is that a low temperature, in the presence of a severe intoxication, offers an ominous outlook. A patient, then, with lobar pneumonia, running a temperature from $104^{\circ} \mathrm{F}$. to $105^{\circ} \mathrm{F}$. requires no interference to affect the temperature itself.

If, however, the temperature rises much above these points or the disease is prolonged and the temperature sustained, pyrexia, per se, becomes a source of danger and must be combated. There is but one antipyretic that is warrantable under these conditions and that is cold water. One may have recourse to the cold bath, given after the manner of the Brand bath or its modification, the Zeimssen's bath, or the slush, given in bed, just as described under Typhoid Fever. (See Chap. XIV.) It must be remembered, however, that it is our wish only to bring the temperature down below the danger point and that if the patient is too long exposed to the cold, his temperature may become subnormal and he may go into collapse. It is a good rule, then, to remove the patient from the tub, when his temperature falls to $102^{\circ} \mathrm{F}$. to $102.5^{\circ} \mathrm{F}$. When the cold bath or slush cannot be used, the cold pack or sheet bath may be tried; a sheet wrung out of cold water, wrapped about the patient and then the surface of the sheet rubbed down with pieces of ice, as detailed above.

Less effectual are simple cold sponges.

Rectal injections of cold water have some value in lowering temperature.

The only justification for the use of drugs for reducing temperature is an environment that does not permit of the use of cold water.

The drugs used are those commonly classified as antipyretics; antipyrin, acetanilid and phenacetin (acetphenetidin), and these must be used cautiously because of their depressing effect upon the circulation.

Cough. The cough of pneumonia may be due to bronchitis or to an accompanying pleurisy. In lobar pneumonia there is an abundant pleural exudate as a rule and cough due to it is common. In bronchopneumonia there is usually a considerable element of bronchitis and this is, of course, a common source of the cough. (See Bronchitis, Chap.VII.)

Treatment of Pleurisy. For the cough of pleurisy the treatment is aimed at a relief of this condition.

Rest to the pleural surfaces is of prime importance and this can be attained by strapping the chest; thus lessening the movement of one pleural surface upon the other. Next, we have recourse to local applications, which are supposed to act reflexly upon the nervous and 
vascular supply of the pleura to lessen pain and possibly modify the processes of inflammation. They are counterirritation with the actual cautery, lightly flicked along the course of the intercostal nerves; the application of mustard paste or plaster; in children in the proportion of one part of mustard to four or five of flour; in adults, one part in three, to equal parts, left on for ten or fifteen minutes or until the surface is reddened and reapplied at two, three, or four hour intervals.

Heat in the shape of poultices, fomentations and the hot water bottle or cold by application of the ice bag.

Strapping the Chest. If the patient is not too ill he should be in the sitting position in bed. If this position is not feasible, he should lie on the non-affected side.

The plaster to be used is the "so-called" zinc oxide plaster, because the least irritating. A strip about five inches wide that will reach from the axillary fold to the edge of the rib is cut long enough to enclose the affected side, overlapping in front and behind for two or three inches. One end of this strip is applied two or three inches beyond the spine on the unaffected side, the patient is instructed to breathe out and hold the breath, while the operator holding the strip smooth to avoid any wrinkles applies it snugly to the affected side, carrying it two or three inches beyond the mid-line in front. It is sometimes better to use strips two or three inches wide, overlapping them like clapboards on a house; this particularly where the conformity of the chest, much adipose tissue, or the large breasts of a female, make the application without wrinkles difficult or impossible.

If there is much hair upon the chest, this should be shaven before the strip is applied, to facilitate its removal when necessary.

The Application of Mustard. One may use the mustard leaf (charta sinapis), although I think the paste is preferable.

The mustard leaf is dipped into luke-warm water to initiate the elaboration of the oil of mustard, a single layer of gauze applied to its surface and two or three to its back, and applied, the mustard side towards the chest.

The paste is made by mixing one tablespoonful of mustard to two, three, or four of flour with cold water to the consistency of a paste and spreading it nicely on gauze or cheesecloth. This is covered with gauze and applied to the chest. Care must be taken to use cold or luke-warm water, as heat kills the ferment concerned in the elaboration of the oil. The paste is kept on ten or fifteen minutes, then removed and to the skin is applied a layer of sweet oil or vaseline.

If the skin is very sensitive, the white of an egg or a little oil or vaseline added to the paste will make it less irritating. 
Application of the Poultice. Take three cups of water and two and one-half cups of flax-seed meal. Bring the water to a boil and add the meal slowly, stirring it all the time with a spoon. When the mixture is so thick that, poured from a spoon it falls drop by drop, it is beaten quickly to make it light and then is spread evenly over a muslin or gauze, being made about one-quarter inch in thickness. A margin of gauze of some two inches should be left all around to turn back over the edge of the poultice. The poultice is then covered with gauze, this being cut to have a margin of three inches to fold in and turn under the muslin, between it and the protector. The protector is usually a piece of oil-silk or old flannel, which serves to keep in the heat. A binder is placed under the patient and a poultice is brought to him on a hot plate to keep it warm, the heat is tested against the face of the nurse, to determine that it is not so hot as to burn the patient; it is then applied slowly, so that the heat can be better borne and the binder brought up around the poultice.

The poultice should not be kept on longer than an hour, because it grows cold by that time, unless some device like a hot water bottle partially filled to avoid weight or a Japanese hand-stove is applied to it to keep the heat up. When the poultice is removed, the site of its application is smeared with oil or vaseline and covered with a dry flannel.

Application of a Fomentation. Cut two or three thicknesses of flannel, sufficiently large to cover the chest. This flannel is placed in a crash towel, boiling water poured upon it, the ends of the towel twisted in opposite directions to squeeze the water from the flannel; the skin is smeared with vaseline or sweet oil and the flannels applied with dry flannel outside, all of which are kept in place by a binder. These are renewed as fast as they become cool. Care must be taken that not enough hot water is left in them to drip down upon the skin and burn.

Application of the Ice-Bag. Perhaps the best bag to use is the circular ice bag with a circular metallic cap; the nine-inch English ice bag is the best.form. The bottom of the bag is covered with ice cracked to the size of the end of one's thumb; over this is poured just enough water to cover the ice, then the bag is compressed so that the water comes to the surface of the opening, thus excluding all the air. The cap is then screwed on. This exclusion of the air leaves the bag supple and makes it easily applicable to any curved surface. The bag is then covered with a layer of cheesecloth or linen, to intervene between the bag and the skin and the bag is held on by a binder or by a towel. This should be taken off from time to time, at intervals of at least 
two or three hours, as continuous cold can produce damage to the skin and the underlying tissues. When the bag is taken off, oil may be applied. (For details of these and similar procedures, one should have recourse tô such an excellent little book as that of Practical Nursing of Maxwell and Pope.)

When these local measures are not efficacious and the pain and the cough is severe, it is necessary to have recourse to some anodyne. Perhaps one of the mildest and most effectual is codeine, which may be given in doses of gr. 1/8 to gr. $1 / 4(0.008-0.015 \mathrm{Gm}$.) at two-hour intervals.

If these are not effectual when given by the mouth they may be given hypodermically.

In the most severe cases, however, it is necessary to use morphine. It is well to begin with as small a dose as will be effectual, gr. 1/16 to gr. $1 / 12(0.004$ to $0.008 \mathrm{Gm}$.). This can be repeated at four-hour intervals; and if necessary the dose increased to gr. $1 / 8$, gr. $1 / 6$ or gr. $1 / 4$ $(0.008,0.010,0.015 \mathrm{Gm}$.).

The Treatment of Bronchitis. While the cough due to pleurisy is exhausting and has no purpose in view, the cough due to bronchitis is of very real use to the patient, in that it empties the bronchi of secretions. A certain amount of coughing, then, that effects this end is desirable; but when the irritation continues and induces exhaustion an effort must be made to remove it. This cough is not infrequently relieved by the application of a mustard paste just as described above. Another measure of great value, where the bronchitis is intense and the congestion of the bronchi very marked, in those cases where the narrowing of the bronchi simulate a spasmodic asthma or not infrequently leads one to feel the possibility of edema, is a vigorous cupping, all over the chest. Wet cupping was once much in vogue in these conditions, but to-day is rarely made use of. The dry cupping is the measure of which I speak.

Technique of Cupping. There are special cups made for this purpose, but it is scarcely necessary to obtain these, for any small glass, such as a medicine glass, wine glass, egg-glass will answer. It is desirable that they should be of rather thin glass, lest their own weight should be so great as to displace them. The site of the cup should be cleaned before the application. One produces a partial vacuum in the cup and one of the simplest means is to drop a shred of absorbent cotton into the cup, light it and, while it is burning, invert the cup and clap it upon the part to which it is to be applied.

Another method is to smear the inside of the cup with a thin film of alcohol. This is best done with absorbent cotton on an applicator, 
dipped into alcohol and swabbed on the inside of the glass, then light it and apply while it is aflame. One should be very careful, however, to have no excess of alcohol, as a drop of it running on to the skin may cause quite a severe burn.

Another excellent modification is to light over an alcohol flame the cotton swab saturated with alcohol and quickly rub this burning swab around the interior surface of the glass, avoiding the rim of the cup, lest it become hot. This cup is then rapidly applied while the vacuum still obtains.

Following the application of the cup, the skin is drawn up into the cup and becomes deeply congested; the negative pressure may be so great as to form an ooze of serum on the surface, standing in little drops. This, however, is not desirable, although it does no harm.

The cups remain until the skin looks well congested. In order to take off the cup one depresses the skin at the edge of the cup to let in the air. If the cup is to be reapplied, it must be thoroughly wiped free of the moisture produced by combustion of the alcohol.

The improvement following this measure is sometimes very striking.

Wet-Cups. If the patient is plethoric and cyanotic, the wet-cups may be used with benefit. A special apparatus is supplied for this purpose which consists of an instrument with a small number of lancet blades which, when the instrument is. applied to the skin and a spring touched, spring forth and incise the skin.

Over this bleeding surface a dry cup is applied in the manner detailed above, sucking the blood out of the wound, acting as an artificial leech. It is rarely necessary to repeat this procedure.

Inhalations. Inhalations of steam or medicated steam are often found grateful and relieve the cough materially.

For this purpose one may use the common croup-kettle or some simpler substitute on the market, or manufacture a device by using a kettle or pitcher with a paper cone to conduct the steam. In children the steam may be led into a tent which is made by throwing a sheet over the four posts or over laths attached to the four bed-posts to give it elevation or by throwing a sheet over an open umbrella under which the child lies.

Medication used in the steam may be either the compound tincture of benzoin, oil of pine or oil of eucalyptus. One places one or two drams (4-8 c.c.) of such upon the surface of the water. In specially devised croup-kettles, a sponge is placed in the nozzle, to which when moistened the medication is applied; steam circulating through this takes up the medicament and is inhaled by the patient.

Another excellent prescription is: 
R

Mentholis.

Camphoræ..........................āa $3 \mathbf{i}$

Tinct. Benzoini Co.q. s. ad....................

S. Use a teaspoonful to a pitcher of hot water as an inhalation.

One of the most effectual inhalations with which I am acquainted is one used by Dr. Francis Delafield and consists of the following prescription:

$\mathrm{R}$

Alcohol .

Chloroform .

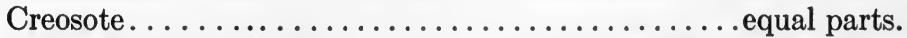

S. Put 10 drops of this upon the moistened sponge of a Robinson's inhaler or some kindred device.

The inhaler is a perforated zinc sheet, bent to fit over the mouth and nose and this may be worn by the patient as long as it gives relief.

If these local procedures and inhalations do not afford relief one has to have recourse to sedatives.

Drugs. Codeine and morphine may be used in the same doses as for the cough of pleurisy. Sometimes relief is afforded by heroine when neither morphine nor its derivative codeine is effectual. It should only be used when the others fail and it should be remembered that it is a very decided respiratory depressant. The dose usually is gr. 1/20 to $1 / 12,(0.003$ to $0.005 \mathrm{Gm}$.) of the hydrochloride. Terpin hydrate is sometimes combined with heroine with happy results. It is best administered as the Elixir Terpini Hydratis cum Heroina of the national formulary, each dram (4 c.c.) of which contains 1 grain $(0.06 \mathrm{Gm}$.) of terpin hydrate and grains $1 / 24(0.004 \mathrm{Gm}$.) of heroine, the dose being 1 to 2 drams ( 4 to 8 c.c.) in water at three-hour intervals to meet the need.

Personally I have very little faith in the expectorants and in pneumonia never order them.

Those, however, who do believe in expectorants, will find in ammonium chloride or carbonate the favorites for this purpose and these may be given in 3 to 5 grain $(0.20-0.30 \mathrm{Gm}$.) doses every two or three hours. It is often well combined with the syrup of Tolu, e. g.:

$\mathrm{R}$

Ammonii Chloridi.......................gr. xlv

Syrupi Tolutani....................

Aquæ q. s. ad......................... 万ui

M.

S. Teaspoonful in water every three hours.

Pain. Pain in pneumonia is a manifestation of pleurisy. (For treat- 
ment of pleurisy see Chap. VIII and Summary.) It is apt to occur early. Sometimes it is trivial and sometimes may not occur at all. Again the pain is most intense and lasts for several days.

An inflammation of the parietal pleura gives rise to pain through the intercostal nerves over the site supplied. There is some pain on deep pressure much more marked in a purulent inflammation. The hyperasthesia is moderate.

It must not be forgotten that a pleurisy occurring on the diaphragmatic surface by reflex through the lower dorsals, especially the last dorsal by its lateral cutaneous branch gives rise to pain in the epigastrium and lower parts of the abdomen; simulating lesions here, such as gastric ulcer, gall bladder disease and appendicitis. The hyperasthesia, with increased pain on light pressure, is decidedly less than when the abdominal parietal peritoneum is involved. However, the diagnosis of appendicitis is commonly made and before the signs of consolidation are obvious often offers difficulties in differentiation.

Pain, too, through the dorsi lumbar nerve, felt in the lumbar region, may suggest renal lesions. Furthermore, pain and tenderness in the supraclavicular region may be attributed to involvement of the diaphragmatic surface, the pain being a reflex derived through the phrenic supply.

This pain in the supraclavicular region, trapezius crest, occurring at the same time and on the same side as the abdominal or lumbar pain suggests the right diagnosis. The involvement of the precordium may also give rise to pain which will be discussed when pericarditis as a complication is considered.

Local Measures. These are identical with those detailed to relieve the cough of pleurisy; namely, application of heat, the application of cold, counterirritation and cupping. In the severe cases the use of codeine and morphine as detailed above.

Toxemia. The degree of toxemia depends in each instance upon the virulence of the toxins on the one hand and the degree of resistance on the other. As we have no means at present of modifying the virulence, except so far as the use of antitoxin in Type I pneumonia cases is efficacious, we must devote our attention to increasing the powers of resistance. This is effected by carrying out all these measures that have been touched upon; giving the body its maximum rest, giving the body a sufficiency of fuel in terms of food, giving the tissues a maximum of oxygen as afforded by fresh air and keeping in good order the eliminating organs; that is, attention to the bowels, attention to the kidneys, and attention to the skin; and it must not be forgotten that a sufficiency of water intake subserves all these purposes to no small degree. 
The use of drugs to enhance these functions, except the use of cathartics as mentioned, are indicated only when the organs show a faltering in their functions or when it is known that these organs are impaired in the individual when infected by the pneumococcus.

The effect of toxins on the nervous system is striking and not the least upon the cerebrum; and while it is true that a considerable area of lung tissue may be involved with practically no delirium or stupor, as a rule one or the other obtains to some degree and in a large per cent. of cases to a very marked degree.

Delirium, restlessness and loss of sleep, headache and stupor have all to be taken into consideration. When delirium obtains the patient must be very carefully guarded as he may leave the bed; throw himself from a window or otherwise injure himself. It is in the milder degrees of delirium, in which the patient, sick as he is, may show no little craftiness, that the greatest danger exists. This emphasizes the necessity for watchfulness when the patient is submitted to the open-air treatment.

The open-air treatment, however, or the hydrotherapeutic measures described, lessen the degree of cerebral disturbances, while sufficiency of food, water and attention to the bowels add still more to the amelioration of this condition.

The application of the ice bag or ice coil to the head has at times a sedative effect. When all the symptoms are marked one has to keep in mind the possible involvement of the meninges; a differential diagnosis between a true meningitis and a meningismus is not easy. The latter term is used often to cover both the irritating effects of the toxin on the brain without evidence of inflammatory reaction in the cerebrospinal fluid and also for a serous meningitis. Many of the signs of true meningitis are commonly manifested in a meningism and one can only be certain of the true state of affairs by a spinal puncture; and a lumbar tap has great value as a therapeutic measure, even if the condition is that of meningismus. While I do not advocate spinal puncture without sufficiently urgent reasons, I think the procedure is distinctly indicated in the condition under discussion. The relief of headaches, restlessness and the amelioration of the delirium is often very striking.

(For the procedure see Meningitis, Chap. XXV.)

Drugs. In the milder forms of delirium, codeine phosphate is sufficient and best administered hypodermically in doses of gr. $1 / 8$ to $1 / 4$ $(0.008$ to $0.015 \mathrm{Gm}$.). In the more severe forms, one must have recourse to morphine sulphate. It should be used as sparingly and in as small doses as will accomplish the object. One may begin, then, with a dose of gr. $1 / 16$ to gr. $1 / 12$ (0.004 to $0.005 \mathrm{Gm}$.).

It is possible that in some individual cases the morphine may not 
be effectual or may aggravate the restlessness. In such a case one may have recourse to hyoscine hydrobromide gr. $1 / 200$ to gr. 1/100 (0.0003 to $0.0006 \mathrm{Gm}$ ). This drug is not always reliable.

Sleeplessness and Restlessness. For milder grades one may use bromides, either dividing the dose throughout the day or giving a larger dose at night. In the latter case one gives in water gr. $\mathrm{xxx}$ to $3 \mathrm{i}(2$ to $4 \mathrm{Gm}$.) of potassium bromide or a mixture of bromides, potassium, sodium and ammonium, each gr. $\mathrm{x}(0.60 \mathrm{Gm}$.).

If the bromides are not effectual, one should try the milder hypnotics, such as trional, sulphonal, ${ }^{1}$ chloralamid, veronal, or in alcoholic cases paraldehyde.

Trional, more soluble, more prompt and more active than sulphonal, is given in doses of gr. $\mathrm{x}-\mathrm{xv}(0.60-1 \mathrm{Gm}$.) in a little wine or whisky or suspended in a warm drink in the early evening and if not effectual the dose may be repeated in two hours. Sometimes the same dose begun earlier in the day and distributed in 2 grain doses at 2 hour intervals is equally or more effectual. If the patient shows no disposition for sleep when the hour for sleep arrives, he may be given the larger dose of 10 $(0.60 \mathrm{Gm}$.) grains, but this latter reënforcement frequently is not necessary.

Again 5 grain $(0.33 \mathrm{Gm}$.) doses taken after the last meal of the day is sufficient to initiate sleep in the milder cases.

Chloralamid is another excellent hypnotic, given in doses of $\mathrm{gr} . \mathrm{xx}$ to gr. $\operatorname{xxx}(1.30$ to $2 \mathrm{Gm}$.), in a cold menstruum, as heat breaks it up. This is given like trional and repeated in the same way if needed.

Veronal, or the American made product barbital, is a fairly potent hypnotic which has now come into extensive use. The dose is $5-71 / 2$ grains, which may be administered in capsule or eachet or put on the tongue and washed down with water. This drug is rather slowly excreted and a drowsiness may continue well into the next day. This hypnotic has never been a favorite of mine because of the prolonged, and to the patient often disagreeable effects, among which are ataxia, hallucinations and tremor. I have always considered it more toxic than the other hypnotics mentioned.

Medinal or barbital sodium, the latter name to be preferred, is a modification of veronal and far more soluble in water (1:5). This increased solubility permits of its use by rectum or hypodermatically. The dose is the same as that of barbital even when given hypodermatically. My experience with this drug has been limited and I have never given it hypodermatically.

${ }^{1}$ The term sulphonal will be used throughout this book in place of the offcial name sulfonmethane. 
Another mild hypnotic which I have come to look upon with some favor is adalin (brom-di-ethyl-acetyl-carbamide). The bromine radial affords the sedative effects of bromides and the ethyl groups act as hypnotics. "It is rather mild in its action, rather freely soluble in alcohol, but not in water. The dose is $5-15$ grains $(0.33-1 \mathrm{Gm}$.), given in capsules or tablets or in powder form, washed down with a little water. It is said when taken in large doses for a considerable time to cause the usual skin lesions attributable to bromides.

In alcoholics paraldehyde seems to have an especially happy effect. It may be given in doses of 3 ij to $3 \mathrm{iv}$. It is soluble 1 in 8 in water and in whisky or brandy. For ordinary usage the dose is somewhat smaller, but the bad taste and odor on the breath makes it objectionable.

Of chloral, on account of its depressing effect on the medullary centres, I am wary, except in the sthenic period of the fever.

One should not allow the patient's strength to be sacrificed by loss of sleep night after night, while the results of one and another of the milder hypnotics is awaited, but should use the most effectual of all hypnotics, morphine.

Just as in delirium, the smallest dose to effect the desired result is given. Begin with a dose of gr. $1 / 12$ to gr. $1 / 8$ (0.005 to $0.008 \mathrm{Gm}$.).

Not too much stress can be laid on the importance of securing sleep. The waste of energy from restlessness is a matter of moment and the evil results of loss of sleep so well known to all of us is greatly exaggerated in sickness.

Headache. The ice cap or coil may be effectual or bromide in doses of gr. $\mathrm{xv}$ to gr. $\mathrm{xx}$ (1 to $1.30 \mathrm{Gm}$.).

Coal tars, such as phenacetin, acetanilid and antipyrin on account of their depressant action on the circulation should be used only in the sthenic periods.

As safer than coal-tar preparations, small doses, 5-10 grains (0.33$0.66 \mathrm{Gm}$.) of acetyl salicylic acid (aspirin) in capsules may be administered.

For the very severe headaches, codeine phosphate or sulphate or morphine sulphate in small doses are the best drugs to use. Severe headaches should always arouse the suspicion of an implication of the meninges. This may be only the so-called serous meningitis, sometimes termed meningismus, or a true pneumococcus meningitis. If there are other evidences of meningitis, one should tap the cord. In the serous meningitis cases this procedure often affords prompt relief to headache, restlessness, sleeplessness and delirium.

Circulation. It is the belief of the present day that in circulatory failure the vaso-motor apparatus is in the vast majority of 
instances the part affected and that only in a small percentage of the cases is the circulatory failure due to myocardial inefficiency. This, however, does occur and, moreover, the heart may be the seat of myocardial change before the patient is the victim of pneumonia. It is not easy, even by a careful physical examination to determine in a given instance, whether the myocardium or the vaso-motor apparatus is at fault. Theoretically, the differentiation should be of great importance, as on the one hand one would appeal to vaso-motor stimulants, on the other to cardiac stimulants; but practically, it makes very little difference, for the vaso-motor stimulants that we now have recourse to are but feeble assistants at the best, while I am more and more convinced that whether the heart be affected or not, in a given case of circulatory failure, the members of the digitalis series are the most valuable drugs under the circumstances.

It is my custom with the first appreciable evidences of circulatory disturbance to begin digitalis. Since this sentence was written in the first edition, early digitalization of the heart in pneumonia has become more or less a routine in many of our best hospitals and in many of the camps during the late war.

It is imperative that this drug should be obtained from an absolutely reliable source and that it should be freshly prepared. It would be desirable to have all preparations of digitalis physiologically assayed, but this is rarely done at the present time when fresh preparations are dispensed, but stock preparations of digitalis or the active principles of digitalis or strophanthin put on the market by many drug houses under different commercial designations are so standardized that stock preparations may be used. It is the only way by which we can estimate accurately in terms of digitalis our dosage in the use of these latter preparations. It must be remembered that when digitalis is administered by the mouth it is slow of absorption.

Unless the case is very light and the circulation in excellent condition I believe it good practice to put the heart under the influence of digitalis when the diagnosis is established. Unless there is urgent need of circulatory support one may observe the so-called Eggleston rule for digitalization of the heart, which calls for the administration of about 0.145 c.c. or 2 minims of the tincture of digitalis per pound of patient, the total amount being given in the first 24 to 36 hours. This dose reckoned for a man of 150 pounds should amount to nearly 22 c.c. of the tincture (or 300 minims) or 2.2 grams of the drug (33 grains). The initial dose advised is a third or a half of the total dose, followed in from 4 to 6 hours by a quarter or a third of the total dose, the remainder in a few doses of smaller size at intervals of from 4 to 6 hours. Whether the larger or 
smaller doses, shorter or longer intervals are to be used depends on the urgency of the case. This estimate is made on a drug of high assay. It is unnecessary to slavishly follow the rule, but the rule affords an excellent guide. In milder cases one may divide the total dose over two to three days. In most urgent cases the total dose is administered in 12 to 16 hours. Assuming that the drug is given by the mouth one may expect to see clinical results in 12-36 hours. In many cases of long duration the total quantity of digitalis given to attain desired results may amount to 50 or 60 grains over a period of five to ten days and not infrequently I have given much larger total doses with benefit. We must not consider too closely a limitation of dosage expressed in figures, but administer the drug until the desired results are obtained or some evidence of digitalis intoxication is manifested. To illustrate this dosage concretely one would give an adult patient of average weight, disregarding the excess of weight in stout individuals, 30 grains or 300 minims of the tincture in the 24-36 hours. The first dose would contain 10-15 grains or 100-150 minims, followed in 4-6 hours by $5-7 \frac{1}{2}$ grains or $50-75$ minims of tincture, followed at 4-6 hour intervals by 2 grains or 20 minims. In this way it will be seen, using the larger doses at shorter intervals, that the whole dose is administered in 12-16 hours; using the smaller doses and longer intervals, administered in 48 hours. Many of the older writers have maintained that digitalis exerted no influence on the heart in febrile conditions; that the heart was not slowed and that there was no improvement observed clinically. I believe these conclusions were based upon an entirely inadequate dosage. When these larger doses are given the heart may be distinctly slowed, though slowing does not always occur. Clinical improvement, that is, evidence of a more efficient circulation, a better quality of the pulse and better character of heart sounds are among the commonest of observations. Moreover, there is electrocardiographic evidence that the drug is in actual combination with the heart muscles by the delayed conduction and deformity in the " $\mathrm{T}$ " wave. I believe that Cohn is right in expressing his belief that there is no difference in the principle in the way digitalis acts in febrile and non-febrile cases.

If the circulatory condition of the patient when first seen denotes great peril or if the circulation becomes rapidly bad the drug not having been previously administered, one must have recourse to other than mouth administration to obtain results.

If for any reason the drug may not be given by the mouth, it may be given by the rectum, in starch paste or in warm milk, 4 or 5 ounces, an amount that is readily retained.

If digitalis is given for a considerable period by this method it is 
better to give the daily dosage in one injection or at the most two, because more frequent administration may render the rectum intolerant and the rate of absorption is so slow that there is no danger of toxic results from the size of the dose. If, when first seen the circulation is sadly impaired or the crisis is near at hand, at which time we are more concerned with the possibility of circulatory collapse, I prefer to begin the administration with strophanthin given intramuscularly or intravenously. Strophanthus, although in no way allied botanically or chemically to digitalis is pharmacologically almost identical, so that in administering strophanthus or its active principle, we may think entirely in terms of digitalis as regards rate of absorption and elimination; however, these two drugs differ materially. Given by the mouth the official preparation, tincture of strophanthus, gives varying and uncertain results entirely due to the variability in its absorption. Hatcher has estimated that the average dose of strophanthin given by the mouth to be therapeutically efficient is ten times the dose given under the skin or into a vein. Often a very large part of the drug so administered can be recovered unabsorbed while again very rapid absorption with dangerous or fatal issue may occur. This latter accident would seem to be more common in conditions of congestion of the gastro-intestinal canal. For these reasons I never prescribe strophanthus or strophanthin by the mouth and advise strongly against it. There are several varieties of strophanthus in use only one of which, strophanthus Hispidus or Kombé are official. The official strophanthin derived from this drug is an amorphous body, a reliable product, but not as active nor as constant in its composition as the crystalline strophanthin recovered by the process of Thoms from strophanthus gratus, called strophanthin G or crystalline strophanthin of Thoms, which is said to be identical with ouabain.

Strophanthin, the nature of which is not specified, or strophanthin under proprietary names the assay of which is not specified should not be used. In my own experience the amorphous strophanthin of the pharmacopœia administered intramuscularly or subcutaneously has caused much irritation about the site of injection, but the amorphous strophanthin prepared under the name of Strophanthin Boehringer and handled by Merck supplied in individual ampoules, containing 1 c.c. of a solution of 1 milligram of the glucoside has rarely caused irritation in my hands. Of late ouabain or the erystalline strophanthin of Thoms has been prepared by different American drug houses in solution in ampoules, usually containing $1 / 2$ milligram. These preparations, so far as my experience has gone with them have been reliable. As determined by physiological assay by the cat-heart method of Hatcher ouabain given intramuscularly is about twice as strong as the amorphous strophanthin; when both 
are given intravenously about 1.3 times as strong. This difference determined by the mode of administration, is probably due to the difference in rate of absorption. It is very important that we should realize the difference in potencies of these two forms of strophanthin.

Ouabain is three times as active as digitoxin when administered intravenously. These statements are based on personal communication from Dr. Hatcher. It has been estimated by Hatcher's method that $0.35 \mathrm{mg}$. of digitoxin of Merck is equal to $100 \mathrm{mg}$. of digitalis leaf, i. e., digitoxin is about 300 times as strong as digitalis leaf. According to these figures, then, strophanthin is about 900 times as potent as digitalis leaf. One mg., then, of crystalline strophanthin or ouabain would equal some 13-14 grains of digitalis leaf, while the amorphous atrophanthin administered by vein would be equal to between 10-11 grains. It must be remembered that these figures are not mathematically accurate, but nevertheless serve as useful guides.

When given intramuscularly strophanthin effects may be looked for in 2-3 hours; when by the vein, immediately. Our dosage, then, in the case under discussion would be $1 \mathrm{mg}$. of the amorphous strophanthin or $1 / 2 \mathrm{mg}$. of the crystalline strophanthin into the muscle; doses equivalent to about 10 grains of digitalis. In four or six hours $1 / 2$ this dose may be given. In these urgent cases one should begin at once the administration of the digitalis by the mouth according to the Eggleston rule given above. Strophanthin is rapidly excreted for the most part in 36 hours. Digitalis is slowly absorbed by the mouth, it requiring 12-16 hours even in these large doses to take effect so that one may say that the digitalis action catches up with and carries on that of the strophanthin. In the most urgent cases and this particularly where edema of the lung obtains one uses the drug intravenously, the dosage being about $3 / 4$ of that given by the muscle, although I very frequently give the same dose by the vein as by the muscle, providing that no digitalis has been given before and that there are no evidences in the history or cardiac examination of partial heart block. If digitalis has been previously administered, one must try to determine how much has actually been taken, reckoning the tincture as 10 per cent. of digitalis and the infusion as about 7 grains to the ounce and allowing elimination at about the rate of $11 / 2$ grains a day, making due allowance for the amount accumulated and erring on the side of a conservative dosage one may proceed with administration of strophanthin.

If strophanthin is not at hand, one may use digitoxin for intravenous medication. It is too irritating for subcutaneous or intramuscular injection. One dissolves the digitoxin in 1 c.c. of alcohol and dilutes four times with sterile water for the intravenous injection. The dosage 
to get equivalent effects would be three times that of the strophanthin, i. e., $1 \mathrm{mg}$. to $11 / 2 \mathrm{mg}$ or 1/60-1/40 grain for the initial dose; one-half of that for the second dose in 4-6 hours. The tincture of digitalis diluted in the same way with water may be used intravenously, the doses being 40-60 minims. These intravenous doses are followed by oral administration as above. The therapeutic effect is shown by the general improvement in the patient, relief of congestion, better quality of pulse and respiration, improvement in the heart sounds and in spite of many statements to the contrary, very frequently a definite slowing of the pulse. I have seen a patient with a temperature between $105^{\circ}$ and $106^{\circ} \mathrm{F}$. on the day before her crisis, after a careless or reckless dosage of strophanthin, with a pulse of 70 , which on the day after the crisis fell to 40 and was probably in block. I may add that at no time was there anything but benefit seen even from such an unwarranted dosage. The signs of digitalis accumulation or poisoning are gastro-intestinal irritation, especially vomiting, but one must be cautious not to mistake the vomiting which may occur with a severe pneumonia, especially an influenzal-pneumonia for the effects of digitalis. I have seen a very excellent man stop the digitalis in such a case with diagnosis of digitalis intoxication on an accumulation of 7 grains. Undue slowing of the heart, evidences of heart block, premature systoles coming on during administration of the drug may all be looked upon as expressions of digitalis intoxication, but premature systoles may occur, and heart block as well, as the expression of toxins on the heart. A cardiogram in one instance demonstrated that the coupled beats (a rare phenomenon in a regularly beating heart) which was attributed to digitalis were premature systoles from the auricle while digitalis coupling is almost always due to premature systoles from the ventricle. Far more damage is done by the neglect of sufficient dosage or to excessive timidity than is done by overdosage.

If, as rarely occurs, the pulse drops abruptly, becomes irregular, betokening a heart block, atropine sulphate in doses of one hundredth of a grain $(0.0006 \mathrm{Gm}$.) should be given hypodermically or intramuscularly, which will usually promptly relieve the block, although its recurrence as the atropine effect wears off may necessitate repetition of the doses at three or four hour intervals. This is such a rare occurrence (and I have never seen it in an acute febrile process), that it should not lead one to hesitate in the use of the drug.

I will mention other so-called cardiac stimulants, such as convallaria and sparteine, only to condemn them, as neither their pharmacology nor their therapeutic application has been worked out and in the case of sparteine, we have reason to believe that we are handling a drug which is rather a depressant than a stimulant. 
If proper administration of the digitalis series is not provocative of a satisfactory result or if for any reason they cannot be given or, if the demands are urgent before the drug has an opportunity to take effect, one has recourse to the vaso-motor stimulants". Among these may be mentioned caffeine, camphor, strychnine and adrenalin.

I am growing more and more to distrust the value of the drugs mentioned for the purpose specified with exception perhaps of adrenalin. Caffeine has been very much used. One can usually demonstrate in the presence of a low blood pressure a fairly constant response varying from 10 to 20, rarely more, millimeters of mercury. This occurs within a few minutes, lasts but a few minutes and the effects, as a rule, are gone in twenty minutes to a half hour and, in a few instances, after a longer period. It would seem to me like the application of a whip to a tired horse. No such sustained effects can be demonstrated as after exposure to the cold fresh air.

If used the drug should be given in large doses, gr. iii to gr. v (0.20 to $0.30 \mathrm{Gm}$.) of a double salt either of sodium salicylate or sodium benzoate which are readily soluble in water. This may be injected or given hypodermically or, better yet, intramuscularly at four-hour intervals, or in urgent cases, at three-hour intervals or two-hour intervals. It is maintained by some clinicians that larger doses are needed by coffee drinkers than for those who abstain, which seems a reasonable assumption (Bethea). Given in the form of coffee by the rectum, in cases of threatened collapse it is of peculiar value, when its effects are enhanced by those of heat. The action of the drug is upon the vaso-motor centres. How much effect it has upon the heart output has never been satisfactorily determined.

I have seen peculiar results ensue after a single dose of caffeine to patients with digitalis accumulation, rapid pulse and respiration, great mental perturbation and the appearance of collapse. I no longer order it for patients under digitalis medication. Moreover, caffeine renders many patients wakeful, a most undesirable result in pneumonia. Under no circumstance should it be administered in place of the digitalis series.

Camphor, too, I use in a goodly dosage, if I use it at all, giving 3 to 5 grains $(0.20$ to $0.30 \mathrm{Gm}$.) and preferably 5 , hypodermically or intramuscularly. The best mode of administration is in a 10 per cent. or 20 per cent. solution in oil. Sesame oil makes an excellent vehicle. Camphor has recently been put on the market in mineral oil with, in some instances lamentable results. The mineral oil is not absorbed, acts as a foreign body irritant, causing chronic inflammation of the tissues about the site of injection and the oil finding its way into the tissues adjacent may give rise to extensive inflammation or even necrosis. The results are akin to 
the more familiar ones following paraffin injections. The practitioner should not use these preparations so attractively offered to the profession.

Camphor is given at four-hour intervals or in urgent cases at three to two-hour intervals; indeed, one may alternate caffeine and camphor in the doses. specified, so that one or the other drug is given at two-hour intervals.

In strychnine I have but little faith, as a circulatory stimulant. I will not, however, oppose the usage of its earnest advocates so far as to condemn it. Again it should never be used as a substitute for digitalis. If the drugs mentioned fail it may be tried, in doses of gr. 1/30 to gr. 1/40 $(0.002$ to $0.0015 \mathrm{Gm}$.) hypodermically or intramuscularly, at intervals of three or four hours.

Adrenalin chloride or epinephrin. Epinephrin is the most powerful vaso-motor stimulant that we have, but its effects are very shortlived. It is indicated in urgent cases or in cases of collapse, and I have found its use of great value. Hypodermically its effects are uncertain; intramuscularly fairly certain and then may be given in doses of $\mathrm{m} . \mathrm{x}$ to m. $x v$ ( 0.60 to 1 c.c.). Given into the vein its effects are immediate, out of all proportion to its usage elsewhere. Only 3 or 4 minims $(0.20$ or 0.25 c.c.) should be put into the vein. I have seen a pressure of 60 millimeters rise to over 200 faster than the measurement could follow it, throwing a tremendous and sudden burden on the heart. It cannot take the place of the other drugs for continued usage.

Nitroglycerin, too, has been used, but I decry it, for its physiological action is to heighten the very effects we desire to overcome, for it is a vaso-motor depressant, causing dilatation of the vessels. The only justification for its use is in the case for excessive hypertension with edema of the lungs. In cases of marked essential hypertension the results are sometimes magical in their promptness.

Alcohol, too, I condemn, for the reasons specified in Typhoid Fever (Chap. XIV).

To reiterate, then, success depends on an early appreciation of circulatory distress, the use and liberal use of the digitalis series.

Collapse. This accident is more likely to occur at or near the crisis, although it may possibly occur earlier.

The probability of collapse is lessened in those cases where digitalization of the heart has been effected. When it does occur, vigorous stimulation is indicated. Heat should be applied to the surface of the body, hot water bottles at the feet, hot flannels or hot cloths applied to the extremities, sufficient number of blankets put upon the patient, volatile stimulants, such as the stronger water of ammonia ap- 
plied to a towel and given to inhale; a teaspoonful of aromatic spirits of ammonia may be given in water, this in turn followed by the injection of the most rapidly acting vaso-motor stimulants, such as adrenalin, in doses of $\mathrm{m} . \mathrm{xv}$ (1 c.c.) intramuscularly or in lesser doses of $\mathrm{m}$. iii-iv $(0.20-0.25$ c.c.) into a vein. This may be followed by caffeine or camphor in doses of gr. $v(0.30 \mathrm{Gm}$.) or strychnine, gr. $1 / 30(0.002 \mathrm{Gm}$.), doses into the muscles or a vein for a more permanent effect; these in turn may be followed by strophanthin, gr. $1 / 120$ to $1 / 60(0.0005$ to $0.001 \mathrm{Gm}$.) down into the muscle or into the vein, and this in turn by a digitalis medication, as discussed above under a failing circulation.

Heat applied to the bowel is also of importance and may be given in the shape of a saline irrigation at $110^{\circ} \mathrm{F}$. to which strong black coffee may well be added.

Pulmonary Edema. Pulmonary edema means cardiac distress and vigorous measures should be taken at once to relieve it.

If the patient is plethoric, full blooded, cyanotic, much of the burden may be taken off the heart by venesection of 12 to 16 or more ounces of blood.

It has been our plan of late both in hospital and private practice to administer at once intramuscularly adrenalin in doses of 15 minims, at intervals of 15 minutes for six doses if necessary. We begin at the same time with intravenous doses (preferably), or intramuscular, of strophanthin $1 / 120-1 / 60$ gr. $(0.0005-0.001 \mathrm{Gm}$.) if the patient has not already had digitalis; beginning with not more than half the lesser dose above mentioned if he has had digitalis. These doses are those of amorphous strophanthin. If crystalline strophanthin or ouabain be given, the dose should be $1 / 2$ to $3 / 4$ of that specified. This has proved very satisfactory in our hands. We have seen pulmonary edema clear up very promptly under adrenalin alone and under strophanthin alone, but unfortunately many cases resist one or both drugs and all other drugs.

Atropine Sulphate has long been used in pulmonary edema in doses of gr. 1/100 (0.0006 Gm.) hypodermically or intramuscularly. The results in my experience have not been satisfactory. In persons with hypertension nitroglycerin may give magical effects. The rationale rests on the dilation of the systemic vessels and the constriction of the vessels in the pulmonary circuit. In this way the left heart is relieved, and pulmonary edema, so far as it is dependent on circulatory conditions, is believed to be caused by accumulation in the pulmonary circuit from a failing left heart and a relatively strong right heart. But in pneumonia one feels convinced that pulmonary edema is determined in some of the cases by the effect of the toxins on the pulmonary vessels 
and so far as this is true would lead one to expect less from the circulatory stimulants than when it is due to circulatory inadequacy.

A measure that is credited with some degree of efficiency is a dry cupping of the chest in front and behind. (For technique, see above.) The best position for the patient is a semi-recumbent one. However I have always been impressed with the amount of handling necessary to pursue the cupping, as in itself prejudicial.

Dyspnœa. Dyspnœa may be due to embarrassment of the respiratory centre or to shallow respiration induced by the pain of pleurisy. In the latter case our efforts are aimed at a relief of the pain. It is surprising how much lung tissue may be involved without respiratory disturbance, providing the toxemia is not marked. However, very extensive involvement of the lung must induce disturbance of breathing. It has been most gratifying to see how much respiratory distress is relieved by submitting the patient to the open-air treatment.

Inhalations of oxygen are used for the same purpose. Every now and then there is a rapid and convincing improvement, during the inhalation of oxygen. Probably the success of oxygen depends on the concentration, that is, the oxygen tension. We are in need of an apparatus by which oxygen may be administered in a concentrated form without such discomfort to the patient as those at our command now entail. The simplest form would be to administer it through a soft rubber funnel with a towel to prevent the escape of oxygen between it and the face or the use of the bag ordinarily employed in gas anesthesia. But the fact is the patient illy bears these procedures. (For further technique see Influenzal Pneumonia, Chap. XII. See Summary.)

Respiratory embarrassmant may also be due to pulmonary edema, when it is to be treated as above specified.

\section{Broncho-pneumonia. (Pneumococcus origin.)}

Specific treatment is determined by the same technique as for lobar pneumonia. It has been contended that the nature of the lesion determined quite a different line of treatment and that broncho-pneumonia should not be treated like lobar pneumonia. I am not quite in accord with this teaching, for it has been my experience that a large per cent. of the cases of broncho-pneumonia have greatly benefited by the open-air treatment. I am, however, appreciative of the fact that broncho-pneumonia is peculiarly prone to attack the aged and the weak. These cases, certainly, should not be exposed to the open air, unless great watchfulness is observed to keep up the body heat.

If the technique of the open-air treatment cannot be well carried out, these cases had better not be exposed. Finally, there is a certain number of cases in whom the cough, instead of being improved is made worse. 
These cases do better in a warmer air and should be kept in a room with a temperature from $65^{\circ} \mathrm{F}$. to $70^{\circ} \mathrm{F}$., the air of which is renewed from time to time. This class of cases often do well with inhalations of steam or medicated steam, such as has been specified for bronchitis.

The broncho-pneumonias under discussion are those due to pneumococcus or to the streptococcus and influenza bacillus as"ordinarily seen. When the virulency of these latter organisms is so great as to cause such epidemics as one saw the streptococcus induce in our camps during the late war and the influenza bacillus send sweeping through the camps and our civil population as well, they give special features to the broncho-pneumonia, its complications and sequelæ that require special consideration which will be found under the headings of streptococcus and influenzal-pneumonia.

\section{COMPLICATIONS OF PNEUMONIA}

Fluid in the Chest. Basing one's statement on autopsies one may say that fluid in the chest is a very common happening during or following lobar pneumonia.

Lord states that a small and varying amount of fluid was present in $37 \%$ of a series of autopsies quoted by him. These small collections, however, are rarely recognized during life so that the percentage of cases on which a diagnosis may be made during life is relatively small, but actually contribute a considerable number.

Norris in Modern Medicine, collecting 24,011 cases found pleural effusion in 6.2 per cent. His figures in autopsy cases agree fairly well with Lord, he giving 41.8 per cent. in nearly 1,000 autopsies. If the statement that at least 400 c.c. must be present before giving physical signs be true and if one considers the difficulty in interpreting the physical signs of small collections of fluid in pulmonary consolidation, the discrepancy of these figures is readily understandable. In over 13,000 cases collected by Norris, the fluid was purulent, that is, constituted an empyema in the clinical sense, in 2.2 per cent., while in autopsy findings in nearly 1,000 cases was a little over 5 per cent.

If, then, during a pneumonia, there is an increase in pleural pain, cough and dyspnœa, if there is an accession of temperature, a rise in pulse rate and respiration one should think of the possibility of an accumulation of fluid. There may be a change in the physical signs suggestive of fluid, one may get a flatness and absence of breathing over fluid or one may find bronchial voice and breathing and this latter particularly in children. A displacement of the heart, more readily determined by a rightsided effusion or a well-defined paravertebral triangle of dulness (Grocco's 
sign) increases the possibility of an effusion. Furthermore, if the temperature is maintained beyond the period of anticipated crisis an effusion is the most probable explanation.

Under these circumstances an exploratory puncture should be made. (For technique see Index.) When a portable X-ray apparatus is at hand a Roentgenological examination should be made. Large collections of fluid of course lessen the respiratory space and by increased thoracic pressure and displacement of the heart further interfere with the circulation.

The indications, then, are such pressure symptoms, dyspnœa, cyanosis and cardiac weakness. Even without these symptoms marked displacement of the heart or an effusion beyond two to three weeks' duration should indicate the procedure. Moreover, increasing pressure upon the lung favors thrombosis and embolism, while thickening of the pleura and adhesions are more likely to ensue. For this reason paracentesis is indicated. (For technique see below.) (For the treatment of symptoms incident upon the effusion see Chap. VIII.)

Much more serious than a serofibrinous effusion is the pleural effusion constituting an empyema.

Whenever after Defervescence the temperature and pulse begin to rise and so continue or whenever the temperature is maintained beyond the anticipated crisis, one should always consider empyema the most probable explanation. While septic endocarditis, thrombosis, phlebitis, abscess of the lung, otitis and antral involvement and other less common complications may be the cause, the frequency of the empyema is to be suspected even in the absence of physical signs and repeated and assiduous search should be made for sacculations of pus and the frequency of such sacculations in the interlobar fissure would indicate exploration in this region, when any signs of consolidation persist in this neighborhood. Furthermore it must be remembered that these sacculations may be small and that only repeated explorations will reward the search. The Roentgen ray, of course, affords material assistance in locating many of these collections. When pus is determined evacuation is called for. The technique of the procedure of thoracentesis is as follows:

\section{Apparatus :}

1. 95 per cent. alcohol and tincture of iodine.

2. 1 per cent. cocaine, hydrochloride or novocaine solution.

3. Sub Q syringe and No. 20 needle.

4. Luer syringe, $10-20$ c.c.

5. Paracentesis needle or a lumbar puncture needle to fit the Luer syringe and provided with an adapter to fit rubber tubing. 
6. Two pieces of rubber tubing, each 12 inches long, $3 \mathrm{~mm}$. inside diameter with a wall sufficiently stiff to resist suction.

7. Potain aspirating set or other similar device.

8. Thick-wälled bottle of any size- $-32 \mathrm{oz}$ is convenient.

9. Sterile gauze and adhesive plaster and towels.

10. Sterile gloves.

11. Three sterile test tubes.

One should first of all assemble the aspirating set and test it to be sure that the stopper fits the bottle tightly and that all the rubber connections, adapters, needles and pump fit properly and that the pump is so connected as to create suction in the flask. When once set up disconnect only the needle for sterilization.

Procedure. Observe strict asepsis. The apparatus and gloves may all be sterilized by immersing them in a solution of mercuric bichlorid 1:1000 for ten minutes.

By physical examination one outlines the position of the fluid and determines the site of puncture. In an ordinary accummulation the best site is at the angle of the scapula at the 6th to the 8th interspaces, depending on the height of the fluid. In sacculation one must puncture over the point of greatest dullness.

Position of the Patient depends on his condition: if he has no fever and his cardiac condition is good he may sit erect in bed with his hands clasped around the flexed knees. This position will draw the scapula forward and increase the width of the intercostal spaces. When the general condition of the patient is questionable or the patient feels very nervous or dizzy, it is better to have him on the unaffected side, propped on a pillow in order to arch the lower side and thus increase the intercostal spaces of the affected side.

When the patient is in position, cleanse the site of the puncture with 95 per cent. alcohol, paint three or four inches with tincture of iodine. The operator either puts on sterile gloves or paints the fingers with tincture of iodine after a thorough scrubbing with soap and water. With the left forefinger locate the interspace to be pierced and with tincture of iodine on the applicator outline a circle about $1 \mathrm{~cm}$. in diameter with its centre about one-half centimeter from the upper border of the lower rib. Infiltrate this area with sterile 1 per cent. cocaine hydrochloride, or $1 \%$ novocaine taking care to introduce the solution intradermally and not subcutaneously. Then insert the needle at right angles to the patient's back and inject the cocaine into all the tissues down and through the pleura. After waiting five minutes, introduce the paracentesis needle (attached to the Luer syringe) along the anesthetized path-holding it at right angles to the patient's back. Use a constant firm pressure with a 
slight boring motion. If the resistance of the skin is strong, nick the skin with the point of a scalpel. With experience one soon learns the feeling produced by piercing the pleura. One should not drive the needle in suddenly with force nor any further in the cavity than is necessary. As soon as one thinks the cavity is pierced, try to withdraw fluid with the syringe. Remove 15-20 c.c. of fluid, leave the needle in situ, remove the syringe from the needle and distribute the fluid among the three sterile test tubes for microscopical and bacteriological examination. Connect the needle immediately with the adapter and rubber tube of the suction apparatus, being sure it is on the suction side. (An easy test is to dip the adapter in a dish of alcohol and release the valve for a second just before connecting the needle.)

Maintain a slow stream by gentle suction until the patient shows signs of distress, such as cough, faintness or pain, or until 1000 to 1500 c.c. is removed. Then discontinue the process and withdraw the needle a small distance. If the symptoms abate one may continue cautiously until the symptoms again occur or until the flow with gentle suction is slight.

Occasionally flakes of fibrin will clog the needle and it will be necessary to stop the suction and let a small amount of air be sucked into the cavity or introduce a stylet into the needle. To remove the needle, clamp off the rubber tube to disconnect the suction, then quickly withdraw the needle and cover the puncture at once with sterile gauze. Move skin about puncture back and forth to diminish the chance of the entrance of air. Remove excess iodine with 95 per cent. alcohol. Apply sterile gauze with adhesive straps.

In case one gets a dry tap at first, one cautiously pushes the needle directly in with the suction on. At any time fluid runs one should retain this position until the flow ceases, then continue to slowly push the needle inward until the lung is touched. If no flow appears, withdraw the needle part way and tilt it toward the patient's head at an angle of about 60 degrees and gradually insert it as before. Repeat this process cautiously, directing the needle in all directions until fluid is obtained or until one is convinced there is none to obtain or that it is too thick to run through the needle being used.

It may be necessary to make a second puncture at a different level or in the midaxillary line in the 4 th or 5 th interspace; and it may be wise to use a larger needle the second time.

The Amount to be Withdrawn varies with each individual case. One must be guided by the reaction of the patient. If the patient coughs, has pain or feels faint one should shut off the suction at once as described above. The rate of withdrawal is more important than the volume and 
should be slow enough to allow the circulation in the lungs and the expansion of the lungs to readjust themselves.

Forscheimer says that too rapid aspiration results in an acute congestion of the lungs and a resulting stimulation of the vagus nerve that may cause syncope or even sudden death.

Caution should be taken to avoid scraping the visceral pleura or piercing the lung tissue with the point of the needle and thus injure the vagus nerve fibres, causing reflex stimulation that is carried to the heart and may result in sudden death.

If the puncture is made too low the diaphragm may be pierced. If one does not keep close to the upper border of the lower rib he may pierce the intercostal vessels and nerves that run in the groove on the lower surface of the upper rib.

Pericarditis. Quoting Norris in Modern Medicine, pericarditis occurred in 12.2 per cent. of his autopsies, and in 40,000 cases of pneumonia 2.2 per cent. of pericarditis was determined clinically. Pleurisy is nearly always coincident with the pericarditis. Autopsy records show the purulent exudate almost but not quite as frequent as the sero-fibrinous. This, of course, in no way represents the frequency in all cases of pneumonia. The physical signs are those of pericarditis from any other cause. The most important are the diminution or disappearance of cardiac impulse or increase of flatness and area of cardiac dulness. With this may be heard pericardial friction sounds, though these may be absent in a large degree; dyspnœa and cyanosis with increasing circulatory embarrassment. X-ray examination affords assistance. When these evidences of circulatory difficulties are determined, an exploratory puncture is indicated.

The best site for paracentesis may be determined as follows:- outline the left border of cardiac dulness; locate by palpation or auscultation the apex beat, go in in the 5 th space between these two points about $1 / 2$ inch inside the limit of dulness. At this site one is less likely to strike the heart and should he do so comes into contact with the thick wall of the ventricle. Other sites recommended are, first, the right edge of the sternum, about 1 inch out in the 5 th space. This is the site of the cardiohepatic angle often rendered obtuse by the collection of fluid. The objection to this site is that in case of error in diagnosis one strikes the auricle. This, though by no means a fatal accident, is best avoided. (2) The angle between the xiphoid cartilage and the cartilaginous attachment of the last rib on the left, passing the needle to the posterior border of the xiphoid and then turn it upwards and sharply to the left. This enters the dependent part of the pericardial sac. The objections are that it may hit the internal mammary artery or the heart.

(3) The 
left of sternum in the 5th space either close to the sternum or an inch to an inch and a half out, to avoid the internal mammary artery. One can use a weak solution of cocaine or 1 per cent. novocaine or may freeze the part with ethylchloride. If the skin is thick it is well to make a tiny incision for the needle. If the fluid is sero-fibrinous one may proceed to aspiration. This may be done with the aspiration needle attached to a Potain aspirator or other device. The fluid should be drawn slowly and if this precaution is observed, it may be removed as completely as possible. The tap against the heart can be readily appreciated and the needle withdrawn. All this is to be done with strict surgical asepsis. If the fluid is purulent, it becomes a surgical problem. The sac is opened and evacuated. The incision is $1 \frac{1}{2}$ inches from the sternum in the 5th space.

Small collections of fluid do not require aspirations. Local treatment may be instituted to afford comfort, lessen pain and precordial distress. The three traditional measures are the application of cold, heat, and counterirritation. Cold gives the best results as a rule. It is applied by means of an ice bag, the Leiter coil or cold compress.

The Bag. The circular ice bag, commonly made with a metal cap is probably the best for the purpose. Ice is cracked in pieces, not larger than the end of one's thumb and introduced in quantities sufficient to cover the bottom of the ice bag. Just enough water is added to cover the ice. The air is then expelled by flattening the bag until the water appears at the opening. The cap is then screwed on and the bag will be found to be flaccid and readily applied to the chest of any contour. As it is not easy to retain the ice bag in position without some device and the patient is annoyed by its slipping or fatigued by the constant effort to replace it, one may avoid the discomfort by suspending the bag in a long strip of muslin or cheesecloth folded once lengthwise and fastened around the opposite shoulder by a split in the end of the cloth. Safety pins retain this bag in position in the bandage.

Before applying the ice bag the skin is lubricated with a simple oil or vaseline and the bag covered by a cloth. At first it is applied interruptedly for an hour at a time, later for longer periods and finally continuously. The skin should be watched, for too great cold for a long period may damage that structure. The custom of adding salt to the ice is to be deprecated.

The Leiter tube is simply a coil of rubber tube coiled like an old fashioned lamp mat, one end connected with a reservoir of water above the level of the bed and the other with a receptacle. The water is allowed to run through this continuously or interruptedly.

The Cold Compress is made by wringing out layers of old 
muslin in water at $60^{\circ} \mathrm{F}$. and applied simply to the chest and covered by flannel and renewed every hour. These applications of ten afford relief for the pain and a sedative for the patient and often bring about a slowing of the heart.

Heat. The simplest method of applying heat is by the hot water bag. Care should be taken to fill the bag not more than half full and to remove all the air from the bag by pressing the bottom of the bag flat until the water fills the mouth of the bag. Then insert the stopper; thus the bag will not be so heavy and be easily adapted to any contour. The temperature of the bag should be tested and the bag either covered by a towel or a flannel cover to avoid burning the patient, who is often unconscious.

Hot compresses may be utilized.

Cloths, the size of the site to be covered, are folded so that they may be easily unrolled and are dropped into very hot water $\left(125^{\circ}-\right.$ $150^{\circ} \mathrm{F}$.), removed with a stick and dropped into a bath towel and wrung out by twisting the ends of the towel in different directions. Test the temperature on your own cheek before applying to the chest. Lay them gently on the precordium and cover them with a towel or small blanket and leave until they cool. Repeat until you are sure that you are either obtaining what is desired, or are accomplishing nothing. The Electric Pad is suitable for the purpose and convenient.

Poultices. Application of the poultice. Take three cups of water and two and one-half cups of flaxseed meal. Bring the water to a boil and add the meal slowly, stirring it all the time with a spoon. When the mixture is so thick that, poured from a spoon it falls drop by drop, it is beaten quickly to make it light and then is spread evenly over a muslin or gauze, being made about one-quarter inch in thickness. A margin of gauze of some two inches should be left all around to turn back over the edge of the poultice. The poultice is then covered with gauze, this being cut to have a margin of three inches to fold in and turn under the muslin, between it and the protector. The protector is usually a piece of oil-silk or old flannel which serves to keep in the heat. A binder is placed under the patient and a poultice is brought to him on a hot plate to keep it warm; the heat is tested against the face of the nurse, to determine that it is not so hot as to burn the patient; it is then applied slowly, so that the heat can be better borne and the binder brought up around the poultice.

The poultice should not be kept on longer than an hour, because it grows cold by that time, unless some device like a hot water bottle, partially filled to avoid weight, or a Japanese hand stove is applied to it to keep the heat up. When the poultice is removed the site of its 
application is smeared with oil or vaseline and covered with a dry flannel.

Fomentations. Application of fomentation. Cut two or three thicknesses of flannel, sufficiently large to cover the chest. This flannel is placed in a crash towel, boiling water poured upon it, the ends of the towel twisted in opposite directions to squeeze the water from the flannel. The skin is smeared with vaseline or sweet oil and the flannels applied with dry flannels outside, all of which are kept in place by a binder. These are replaced as fast as they become cool. Care must be taken that not enough hot water is left in them to drip down upon the skin and burn.

Counterirritation. The application of mustard. One may use the mustard leaf (charta sinapis), although I think the paste is preferable. The mustard leaf is dipped into lukewarm water to initiate the elaboration of the oil of mustard, a single layer of gauze applied to its surface, and two or three to its back, and applied, the mustard side toward the chest.

The paste is made by mixing one tablespoonful of mustard to two, three or four of flour with cold water to the consistency of a paste and spreading it nicely on gauze or cheesecloth. This is covered with gauze and applied to the chest. Care must be taken to use cold or lukewarm water, as heat kills the ferment concerned in the elaboration of the oil. The paste is kept on ten or fifteen minutes, then removed and to the skin is applied a layer of sweet oil or vaseline.

If the skin is very sensitive, the white of an egg or a little oil or vaseline added to the paste will make it less irritating.

The electric cautery or the actual cautery often affords relief. The needle of the cautery is held at right angles to the skin and lightly flicked across it so that the resulting mark looks like a pin scratch. If carried out in this way it will not be painful and may be repeated every other day.

\section{EMPYEMA}

\section{(Pneumococcus Type)}

\section{(Dr. Alfred S. TAylor)}

In this type of empyema the fluid almost at the start is purulent and contains much clotted fibrin. Also the lung is adherent to parietal pleura all about the periphery of the empyema cavity. Therefore evacuation of the pus by any one of various procedures is not likely to cause sudden shock or circulatory or respiratory embarrassment because there is no flapping of the mediastinum as a result of allowing air to enter the 
chest. Therefore the problem is one of evacuating the pus and healing the cavity as rapidly as possible. There is a considerable range of choice of procedure according to the opinions of various men, but the one which is straightforward and reliable and the best to use under all circumstances consists of resection of a portion of rib about $7 \mathrm{~cm}$. long. The best rib to resect as a rule is the 8th at the posterior axillary border. This operation can practically always be done by means of local anesthesia. The skin of the chest is prepared as for any operation. The patient is placed upon the sound side with the arm of the diseased side hanging forward over the edge of the table or bed so as to present the rib to be resected in a convenient position for the operator. One may use $1 / 2$ per cent. novacaine with 1 to 100,000 of adrenaline mixed with it. This should be injected primarily into the skin proper along the line of incision and then into the subcutaneous tissues down to the rib. It is also helpful to remember that the nerve supplying cutaneous sensation lies in a groove along the under border of the rib. With a little practice one can readily insert a needle beneath the edge of the rib between the spine and the incision and inject some of the novocaine solution into the nerve or its immediate neighborhood. The same procedure carried out on the nerve beneath the 7th rib will cause a nerve blocking of sensation in the field of operation. One might be able to resect the rib after just blocking the two nerves alone, but the injection of the skin and subcutaneous tissues makes the anesthesia a little more rapid and somewhat more sure. In all operations under local anesthesia it is quite important to allow from 5 to 10 minutes to elapse after injecting the solution before doing any cutting in order to allow the local anesthetic to become diffused and exert its influence. The knife should be sharp and all the instruments in condition to do accurate, quick work. When anesthesia has taken place the incision is made through the skin over the central line of the 8th rib for a distance of about $7 \mathrm{~cm}$., the middle being at the posterior axillary border. This incision is carried straight through all the tissues, including the periosteum. After a certain amount of experience one can make this incision with a single stroke of a sharp scalpel. It is better, however, for one doing this operation only occasionally, to make this incision layer by layer. Great care must be exercised, however, to have the incision follow a vertical plane from the skin to the rib. It must also be remembered that the axis of the rib runs very sharply from below upward and that therefore the incision will have to slant accordingly. When the soft tissues have been divided they are retracted by sharp retractors and the rib is exposed with its periosteum cut along its mid-line. The sharp periosteal elevator is now used to push the periosteum from the middle of the rib upward to the upper border and the lower half downward to the 
lower border of the rib. Care should be exercised to separate the periosteum as a continuous membrane instead of bruising and shredding it as is so often done. When the upper border of the rib has been cleared one should start at the spinal end of the exposure and strip the intercostal muscle from the upper border of the rib. By starting posteriorly and working forward the insertion of the external intercostal muscles is on a slant that will hold the periosteal elevator against the bone. In clearing the lower border of the rib one should begin anteriorly and work backward because the external intercostal muscles will again hold the instrument against the bone. (See Anatomy.) When both the upper and lower borders of the rib have been thus freed of periosteum and intercostal muscles the periosteal elevator is wormed between the periosteum and rib, starting at the lower border, being careful to follow the curve of the groove beneath the lower edge of the rib and then upward until its edge appears above the upper margin of rib. This elevator is then pushed backward toward the spine and forward as far as the rib is exposed. This separates the deeper layer of periosteum from the rib and leaves the portion to be resected entirely bare. The rib is now divided at each end by a bone forcep. Care should be exercised not to crush the bone any more than is necessary, as damaged fragments, which are bound to become bathed in pus, may keep suppuration going for some time or may even be the source of osteomyelitis of the rib. If the deeper layer of periosteum is sensitive to a prick of the knife, it may be injected with some of the anesthetic solution. If, as is usually the case, it is anesthetic from the nerve blocking, a small incision, $1 \mathrm{~cm}$. long, is made in its centre. This incision is made purposely small to permit the escape of pus but to prevent it from coming out too rapidly. Too rapid evacuation is apt to change the pressure conditions within the thorax so quickly as to cause some disturbance of cardiac and respiratory action. After the pus has been permitted to escape somewhat slowly the incision is continued to both ends of the wound. The flaps of parietal pleura are then retracted sufficiently to remove all of the fibrin clots and pus either with the suction apparatus or with the finger or with an ordinary sponge holder. All the manipulations within the abscess cavity are made with the greatest gentleness. Light should be reflected into the cavity so that the fibrin masses can be detached gently and with certainty. The clean removal of all these clots and of the liquid pus will shorten the convalescence materially. The simplest form of drainage consists of two pieces of flexible rubber tubing about $1 \mathrm{~cm}$. in diameter externally. These tubes should be long enough to project slightly into the pleural cavity and allow for their other ends to be joined by a transfixing safety pin. A narrow cotton tape is fastened to the pin and tied around the chest to 
prevent the tubes from falling into the cavity. Most of the wound is sutured until there is just room for two tubes to emerge. Sterile dressings are applied and held on usually by a wide bandage which is pinned not too tightly about the chest. The after-treatment consists of changing the dressings as often as necessary to keep the patient from getting too wet. Every day or every second day the tubes are removed, washed clean and sterilized. The skin about the wound is kept clean and the tubes are replaced. Meanwhile as soon as the patient's general condition permits he is started on blowing exercises, i. e., Wolff's bottles. As soon as his general condition permits he is allowed to sit up and get about. When the discharge has almost disappeared the tubes can be removed. This usually occurs in 10 days or two weeks. This is the simplest procedure and in most ways answers the purpose everywhere. In well-conducted hospitals, one may use plain thoracotomy between the ribs, then force a large rubber drain tube into the cavity so that it fits air tight into the incision, or one may use any one of the various methods for getting vacuum drainage of the empyema cavity. These methods certainly shorten the time of convalescence and keep the patient much drier between dressings, but they are somewhat more fussy and require more constant skilled attention. In the same way the use of Dakin's solution is an advantage in well-conducted hospitals where the technique can be properly carried out, but as a general rule these cases will do very well when treated in the way described.

Pneumococcus Endocarditis commonly occurs as a late complication and whenever after an apparent crisis the temperature rises again it should be thought of together with empyema as the most probable explanation; or crisis failing and the temperature being prolonged and more particularly if it is of an irregular type the same condition should be suspected. There may or may not be chills and sweat. Such an endocarditis constitutes an acute malignant endocarditis with the usual sequence of events-septicemia, septic infarcts and death.

The physical signs are those of valvular inefficiency and stenosis.

The treatment is that of a septicemia. (See Chapter XLV.)

Thrombosis is a rare complication occurring usually in the femoral veins. The treatment is that of rest by fixation of the limb and application of thick layers of non-absorbent cotton or wet dressings such as aluminum acetate.

Embolism with thrombosis of the arteries is another rare accident. A dry gangrene will ensue and if occurring in a limb may necessitate amputation. One such case occurring on our service at Bellevue in an old man was followed by an amputation of the thigh followed by good results. 
Meningitis. Fortunately this is not a common occurrence. On the other hand, it may not be considered as a rarity. The issue is always a fatal one. The treatment aims at relieving the symptoms; repeated lumbar puncture is a valuable contributary to other procedures. (For details see Cerebro-Spinal Meningitis, Chap. XXV.)

Parotitis is another of the relatively uncommon complications and is frequently associated with endocarditis. There is no doubt that lack of attention to oral hygiene is largely the cause. (For treatment see Mumps. Chap. XXIII.)

Arthritis may be an early manifestation or occur at any time during the course or follow upon the pneumonia. It may be of the nature of a toxic arthritis and yield to such measures as are detailed under Acute Rheumatic Fever (Chap. III) or it may be suppurative and demand surgical interference.

Peritonitis is a rare complication and occurs usually in the severer cases. It is sometimes in the upper peritoneum and a direct extension through the diaphragm.

Jaundice is not an uncommon occurrence in pneumonia. It is usually taken to signify an intense infection and may be due to the parenchymatrous degeneration of the liver with obstruction of the biliary duct. The prognosis is correspondingly serious. When, as not infrequently happens, the earliest signs of pneumonia refer to the involvement of the diaphragmatic pleura or the lower portion of the costal pleura, pain is referred to the region of the gall bladder and sensitiveness of the skin and muscles over that structure, leads to the diagnosis of a primary cholecystitis. It is the knowledge of this fact with twenty-four to thirty-six hours of watchful waiting that saves one from an embarrassing diagnosis, especially if surgical procedure is immediately instituted. The jaundice per se requires no treatment.

Abdominal Pain.-The errors of diagnosis incident upon abdominal pain referred from diaphragmatic pleurisy have been discussed under that subject and in the preceding paragraph. One should merely keep in mind in the pneumonia season that acute onsets, with high fever and with pain thought to be due to involvement of gall bladder, appendix, pancreas or to a gastric ulcer may be due to the pneumonic process.

Relapses may occur on the ninth to eleventh day with return of fever and physical signs. It must be remembered that a return of fever shortly after defervescence is probably due to an empyema or possibly an endocarditis.

The treatment is the same as for the original attack. 


\section{CONVALESCENCE}

The period of convalescence appeals to the good judgment of the physician quite as much as any other period of the illness; for the failure to appreciate the possibilities of convalescence may result in immediate disaster or more remote consequences unfortunate for the patient. It must be remembered that after the defervescence resolution must occur, and that the patient should not be allowed to sit up or raise himself up in bed until the resolution is complete, since it is during this period that pulmonary embolism may occur, the result of which is often immediate death, or, if the toxemia has been profound and the myocardium much weakened, cardiac dilatation may ensue.

If the course of the disease has not been peculiarly severe one may say in a general way that a week after resolution has occurred the patient may be allowed out of bed; but if the toxemia has been severe and the condition of the circulation during the disease has been a source of anxiety, possible weakening of the myocardium must be kept in mind and the patient be kept in bed for a longer period. He should be allowed to be bolstered up in bed at first, then allowed to get out of bed, then allowed to get about; but each increment of freedom from restraint should be granted only when the heart shows no irritability on the occasion of increased effort.

Complications such as empyema, abscess of the lung, gangrene of the lung, and so forth, of course, prolong the convalescence.

Care must be given to the dietary, sufficiency of food given and the value of fresh air to the convalescent be fully appreciated.

It is often most difficult to restrain the patient from returning to his work, particularly where much is dependent upon his effort. How soon the patient shall be allowed so to do, rests entirely upon the good judgment of the physician and no rules can be laid down for him in this matter, except that a restoration of strength and weight and well-being in the patient are the criteria.

If there has been much pleurisy the convalescence is likely to be prolonged and pulmonary exercises, deep breathing, or blowing into bottles (see Pleurisy, Chap. VIII) are necessary for the proper expansion of the lungs.

Prophylaxis. It must be remembered that the infecting agent finds its entrance into the body through the air passages.

An examination of the throat and nasal passages of healthy individuals has shown that a very goodly per cent. of such are carriers of the pneumococcus. It is obvious, then, that any abnormalities of the air passages favor a lodgment of the organism and the exercise of their pathological 
activities. Oral hygiene then becomes of importance, proper dental care of the teeth, the use of a tooth-brush, attention to or removal of hypertrophied tonsils, adenoids or other diseased lymphoid tissue, proper treatment of deviated septum, hypertrophied turbinates and other abnormalities of the nasal passages.

It must be remembered, too, that any lowering of the resistance of the body at large favors infection so that attention to diet, exercise, cleanliness of the body on the one hand and avoidance of undue fatigue, mental worry, dissipation, alcoholism, exposures to cold, failure to change cold wet clothing, are all to be avoided.

It must be remembered, too, that the lessened resistance entailed by another illness favors pneumonia as a complication.

There is no question that a large percentage of individuals carry pneumococcus in the upper air passages. The organism increases in virulency and in infectivity in those people who are suffering from any expression of infection, such as common colds, sore throats, bronchitis and pneumonia. Hence, people in health should avoid unnecessary and immediate contact with the sick, avoid kissing, exposure to coughs, use great care in handling the sputum of patients ill with pneumonia or with bronchitis or common colds, avoid crowded places during periods of epidemics of colds. It must be remembered, too, that those who have suffered from a pneumonia once are more prone to repeated attacks.

Prophylaxis, too, is a function of the State and pertains to Boards of Health, Street Cleaning Departments, Building Departments and all such bodies as have to do directly or indirectly with the health of the public at large, while a dissemination of proper knowledge regarding disease is the duty of Boards of Education, Health Officers, and Medical Organizations.

Prophylactic Use of Pneumococcus Vaccines. Many observations on the value of prophylactic vaccination with pneumococcus were made in the Army, but the measure has not won its way into civil practice as yet to anything like the extent of prophylactic typhoid vaccination; and, indeed, the efficiency of the one as contrasted with the other has not up to the present time warranted it. However, such figures as have been published from the various camps where anti-pneumococcus vaccine was used encourages one to the belief that it will be extensively used in civil practice. A personal communication from Cecil, whose experience in this practice has been very large, describes the procedure as follows: The vaccine is made from Types I, II, and III in equal parts, suspended in saline solution. The first dose contains a billion of each, second dose two billion of each, third and fourth doses two and three 
billion of each of Types I and II and one and a half of Type III. The intervals are 5-7 days.

The reactions are much the same as after administrations of typhoid vaccine. (See Chap. XIV.) But the formation of sterile abscesses at the site of injection was not an uncommon happening and of course not a desirable one. This disagreeable sequel was quite avoided by the use of lipovaccines although there is reason to believe that the formation of antibodies is not so active from the latter as from saline vaccines. The lipovaccines consist of an emulsion of centrifugated and dried bacterial bodies in cotton seed oil. With lipovaccines not only are the possibilities of sterile abscesses quite eliminated, but general reactions are much diminished and so much larger doses may be given as to make a single vaccination sufficient. In this case it is customary to give 10 billion of each of the bacteria. Dr. Cecil's figures show-

Incidence of Pnedmonia among the Vaccinated Troops, February 4 to ${ }^{1}$ APRIL 15, 1918 at CAMP Upton

Average strength of command, Feb. 4 to April 15, 1918... . 32,000

No. of troops vaccinated against pneumonia . . . . . . . . . 12.519 (40\%)

" " unvaccinated men (average)................. 19.481 (60\%)

Total No. of Type I-Pneumonias among the unvaccinated troops Feb. 26

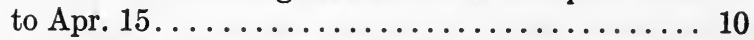

" " " " II and III pneumonias among the unvaccinated troops........................... 16

" " " " I, II, III and IV pneumonias among the unvaccinated troops............................ 59

" " " Streptococcus pneumonias among the unvaccinated troops...............................106

" " " Pneumonias among the unvaccinated troops................173

Incidence of pneumonia among the vaccinated troops, Feb. 4 to Apr. 15

Pneumococcus Type I (developed $24 \mathrm{hrs.} \mathrm{after} \mathrm{first} \mathrm{injection).........} 1$

Pneumococcus Type IV (3 cases receiving only one injection) ....... 9

Streptococcus hemoliticus........................... 6

" viridans............................. 1

Total number of pneumonias among the vaccinated troops......... 17

As pneumonia is responsible for more deaths than any other infectious disease with which we are acquainted, ranking even tuberculosis, one can easily understand the wonderful benefit that would ensue if these figures were sustained by further observation.

Rest.

\section{SUMMARY}

Rest in bed.

Do not allow patient to turn himself if very ill.

${ }^{1}$ Journal of Experimental Medicine, July 1, 1918. 
Exclude visitors.

Permit no subjects of mental concern to be brought to the patient.

Bed.

Half or three-quarter bed.

Hospital type preferred.

Woven wire springs, firm mattress.

Bed clothing, sheets and light weight blankets.

(For technique of bed-making see text.)

In convalescence bed rests are used. The constant tendency to slide indicates some support, rolled blankets under the knees or foot rests; all unsatisfactory.

A bed of the type of the Gatch bed is the best. (See text.)

\section{Room.}

Chosen for light and air.

Stripped of all unnecessary furniture.

Access to porch or verandah desirable.

Temperature about $65^{\circ} \mathrm{F}$.

\section{Specific treatment.}

Determine the organism.

Culture blood; likely to get results in early hours.

Culture sputum.

When the organism is a pneumococcus, proceed to type the pneumococcus.

The determination of the organism and typing should be done at the earliest possible moment.

Technique of typing. (See text.)

Typing may be done from the urine. (For technique, see text.)

\section{Administration of serum.}

At the earliest possible moment after typing determines Type I pneumococcus, give serum.

Shall serum be administered when typing is not possible? (For discussion, see text.)

Determine presence or absence of Sensitization.

Always enquire about asthmatic attacks, or previous serum administration of any kind. These patients are likely to be sensitized.

Sensitization test. (See text.)

If the patient is proven to be sensitized, before serum is given in full dosage he must be desensitized.

Desensitization. (For details, see text.)

Note Besredka's method, intravenous from the start and the Rockefeller Institute method, beginning with subcutaneous doses.

Giving of Serum.

Assembling of apparatus. (See text.)

Sterilizing apparatus. (See text.)

Preparation of serum. (See text.) 
Preparation of patient and operator. (See text.)

Method of injection. (See text.)

\section{Reactions.}

1. Non-specific serum or thermal reaction-much like salvarsan reaction. (For symptoms, see text.)

If it occurs during the serum administration, discontinue for an hour or two, then resume more slowly.

To ameliorate symptoms:

Adrenalin (epinephrin) $1-1000, \mathrm{~m}$. vii ( 0.5 c.c.) intramuscularly.

Atropine sulphate gr. 1/100 (0.0006 Gm.) intramuscularly.

2. Serum sickness or serum disease 7-14 days after administration. (For symptoms, see text.)

Relief of urticaria.

Bathing in solutions of bicarbonate of soda of varying strengths. Apply 1\%-2\% aqueous solutions of phenol (carbolic acid) or $5 \%-10 \%$ solutions of phenol in olive oil or vaseline (liquid petrolatum).

Adrenalin (epinephrin) $1-1000,1 / 2$ to 1 c.c. (m. vii-xv) intramuscularly.

3. Anaphylactic reaction.

Occurs during or immediately after administration.

(For symptoms, see text.)

Adrenalin (epinephrin) 1-1000, 1 c.c. (m. xv) intramuscularly at once.

If pulse is soft and blood pressure low, strophanthin, $1 / 2 \mathrm{mg}$. (gr. $1 / 120)$ intravenously or

Digitalis intravenously in some form in doses equal to 3 to 5 grains of the leaf, e. g., tincture (m. $\mathrm{xxx}-\mathrm{l}$ (2.-3.33 c.c.) diluted with saline 2 or 3 times or digitoxin gr. 1/100-1/60 (0.0006$0.001 \mathrm{mg}$.) in $\mathrm{m} . \mathrm{x}(0.66$ c.c.) alcohol diluted twice with normal saline solution or,

Properly assayed commercial solutions in equivalent doses.

Apply hot cloths to the abdomen; i. e., treat as a shock.

Asthma of an intense type often accompanies it.

Adrenalin (epinephrin) m. $\mathrm{xv}-\mathrm{xxx}(1-2$ c.c.) intramuscularly.

Atropine sulphate gr. $1 / 50(0.0012 \mathrm{Gm}$.) intramuscularly.

Morphine sulphate gr. 1/8-1/4 (0.008-0.015 Gm.).

In unyielding cases air may be expressed rhythmically from chest by force, i. e., compressing the chest with the operator's encircling arms.

Dosage of serum. $60-100$ c.c. ; the larger in toxic cases. There is no toxic dose, the amount is limited only by the supply at hand and demand in the individual case. Late cases demand larger doses. Larger and larger doses are needed as the disease advances, to get equivalent results.

\section{Frequency of dosage.}

Eight hours or less in severe cases. In cases showing improvement the physician's judgment must determine intervals. 


\section{Care of body.}

Sponge baths for cleanliness.

Mouth.

Use mild antiseptic solution 2 to 4 per cent. of boric acid.

$1 / 2$ to $1 / 4$ strength of Dobell's solution.

Teeth.

Keep interstices free from food, use swabs of absorbent cotton or Tongue. wooden toothpicks in above solution.

For coated tongue and sordes on lips and teeth use $1 / 2$ strength of peroxide of hydrogen to soften. Scrape with edge of whalebone and then use above solution.

For very dry mouth, equal parts of albolene and 2 per cent. boric acid solution, flavored with lemon juice.

Fetid breath and marked stomatitis.

Phenol, watery solution 1-20.

Glycerin... ............... āa $\bar{\jmath} \mathrm{i}$

Boric acid saturated watery solution. . ₹ viii (30 c.c.). (240 c.c.).

Naso-pharynx.

Remove collections with cotton swab on long applicators saturated with above solution.

Use sprays of boric acid or Dobell's solution.

Nose.

Soften dried secretions with sweet oil.

Cleanse with cotton swab on toothpicks, using above solution.

Spray, using above solutions.

Avoid irrigations.

Fissures and herpetic eruptions.

Use above solutions and anoint with oil or vaseline.

Eyes.

Cleanse with 2 per cent. boric acid solutions.

Genitals.

Cleanse with boric acid and Dobell's solutions.

To excoriations.

Apply $1 \frac{1}{2}$ per cent. to 2 per cent. phenol solution.

Apply and keep dry with dusting powders.

\section{Diet.}

Do not force food during the early hours.

Do not force food during the high temperature of a lobar pneumonia.

The longer course of a broncho-pneumonia indicates food of a highercaloric content.

Milk.

Each ounce equals 20 calories.

Gravity cream 16 per cent. each ounce equals 50 calories.

Milk sugar each ounce equals 120 calories.

One may add 1 ounce of cream and $1 / 2$ ounce of milk sugar to each glass of milk, thus increasing the calories about 100 . 
Cereals.

Oatmeal.

Hominy.

Farina. *

Rice.

An ordinary serving of which, of 3 to 5 ounces, equals 100 calories.

In addition, eggs, cup custard, broths made of cereals ànd milk, bread and butter.

Milk preparations such as,

Matzoon.

Koumys.

Zoolak.

Buttermilk.

Curds and whey.

If the patient much desires it meat soups such as,

Mutton broth.

Chicken broth to which may be added cereals or rice to add to the caloric value.

Or cream soups.

Desserts.

Tapioca pudding.

Gelatin.

Ice cream.

Prune whip.

Meats.

Brains.

Scraped beef.

Chicken livers.

Fish roe.

Potatoes.

Sweet and Irish.

Summary of diet.

Early days, small amounts of milk, large amounts of water, and milk better diluted with plain water, alkaline water or cereal water.

Increase food gradually.

In prolonged pneumonias, broncho-pneumonias, unresolved pneumonias, complicated pneumonias, increase to a high calorie diet.

Food may be given at two or three hour intervals.

Drinks.

Give water abundantly.

To stuporous or delirious patients offer water every hour or two. Fruit juices.

Lemonade, orangeade, grape juice, imperial drink may be given freely.

Care of bowels.

When first seen.

Calomel, gr. $1 / 4(0.015 \mathrm{Gm}$.) every $1 / 4$ hour for 6 or 8 doses.

In children gr. $1 / 10(0.006 \mathrm{Gm}$.) every 10 minutes for 10 doses. 
In the adult followed in three or four hours by salts, Rochelle or Epsom, $1 / 2$ ounce $(15 \mathrm{Gm}$.) in $1 / 2$ to $3 / 4$ of a glass of water.

In children follow in two to three hours by 4 to 8 ounces (120240 c.c.) of Liq. Magnesii Citratis, or $1 / 2$ to 1 ounce (15-30 c.c.) of milk of magnesia.

Or salts alone may be used, Rochelle, Epsom or Glauber's $3 / 4$ to 1 ounce in adults $(20-30$ c.c.).

Or milk of magnesia $1 / 2$ to 1 ounce (15-30 c.c.) in children.

Later enemata of plain water or soap-suds.

Liquid petrolatum (mineral oil) in doses of one or more tablespoonfuls.

Tympanites.

Suspect the fat of milk, or the milk sugar.

Cut them down or eliminate.

Plain water or soap-suds enemata, or add oil of turpentine $1 / 2$ oz. to 1 oz. (15-30 c.c.) to soap-suds enema.

(For technique see text.)

Peppermint enema-2 teaspoonfuls of essence of peppermint (spiritus menthæ piperitæ) to 2 quarts of warm saline solutions.

Asafœetida enema-3ss. (2 c.c.) of tincture of asafœetida to 2 quarts (2 litres) of normal saline or tap water.

Milk and molasses enema. A cupful of each (200-250 c.c.). Warm the molasses and add warm milk. Have temperature a little above body temperature. Give with funnel and tube.

Rectal tubes.

Glycerin rectal injections $5 \mathrm{i}$ to 5 ii (30-60 c.c.).

Strychnine for tonic effect gr. $1 / 30(0.002 \mathrm{Gm}$.) three times a day or four times a day.

Pituitrin or pituitary extract.

1 c.c. $(\mathrm{m} . \mathrm{xv})$ hypodermically is the usual dose.

Murphy drip. (See text.)

Open air treatment. (See text.)

Bed.

Woven wire spring; over this

1. Large blanket; over this

2. Rubber sheeting or paper; over this

3. Mattress, sheets and blankets.

Fold 1 and 2 over 3 like an envelope.

\section{Patient.}

Flannel undersuit.

Stockings.

Hood.

Low pillow.

Pillows for shelter like an inverted V.

Hot water bottle to feet. 
Screens for high winds.

Must not be left alone.

Nurses and attendants dressed for out of doors.

For toilet, use of bed-pan, examination, draw into room at $65^{\circ} \mathrm{F}$. to $70^{\circ} \mathrm{F}$.

For open-air treatment may use verandah, porch, fire-escape, roof, room with windows out and screened with cheese cloth.

Hydrotheraphy. (See text.)

Chest compresses. Technique, see text.

\section{Symptomatic treatment.}

Fever.

- Let alone unless temperature is above $105^{\circ} \mathrm{F}$. or sustained at about $104^{\circ} \mathrm{F}$.

Brand bath. (See Typhoid Fever, Chap. XIV.)

Take patient out at $102^{\circ} \mathrm{F}$. to $102.5^{\circ} \mathrm{F}$.

Slush.

Pack.

Sheet bath. (For technique, see text.)

Sponges.

Rectal injections of cold water.

\section{Drugs.}

Only when cold water cannot be used.

Phenacetin gr. v or

Antipyrin gr. iv Every 2 hours, but cautiously watch pulse.

or

Acetanilid gr. ii

\section{Cough.}

Due to pleurisy.

Strapping chest. (For technique see text.)

Mustard leaf or paste. (For technique see text.)

Poultice. (For technique see text.)

Fomentations. (For technique see text.)

Ice bag. (For technique see text.)

Codeine phosphate or sulphate, gr. 1/8 to gr. 1/4 (0.008-0.015 Gm.) every two hours.

Morphine sulphate (in very severe) gr. 1/16 to gr. 1/8 (0.004-0.008 $\mathrm{Gm}$.) at 4 hour intervals.

Due to bronchitis.

Its purposefulness. (See text.)

Mustard paste or leaf.

Dry cupping. (For technique see text.)

Wet cupping. (For technique see text.)

(See also Bronchitis, Chap. VIII.) 


\section{Inhalations.}

In croup kettle or similar device. (See text.)

Steam, plain or medicated with, Compound tincture of benzoin, or

Oil of pine, 3 i to 5 ii $(8-15$ c.c.).

or

Oil of eucalyptus

R

Mentholis

Camphoræ ......................... āa $\bar{\jmath}^{i}$

Tr. Benzoini Co. q. s. ad.................... 节ii

S. Use a teaspoonful to a pitcher of hot water as an inhalation.

R

Alcohol.

Chloroform.

Creosote, equal parts.

M. et S. gtt. $\mathrm{x}$ on sponge or inhaler. (See text.)

Drugs.

Codeine phosphate or sulphate

Morphine sulphate

Heroine hydrochloride

Heroine may be combined with terpin hydrate as Elixir Terpini Hydratis cum Heroinal (N. F.). Dose 3i-ii (4-8 c.c.) in water every 3 hours.

Expectorants. (Too much used, rarely indicated.)

$\mathrm{P}$

Ammonii Chloridi.......................gr. xlv

Syrupi Tolutani.....................

Aquæ Destillatæ q. s. ad..................

15.00

60.00

M. et S. One teaspoonful in water every three hours.

\section{Pain.}

Pleurisy.

(See measures for cough of pleurisy above.)

\section{Toxemia.}

Rest.

Diet.

Air.

Care of skin.

Care of bowels.

\section{Delirium.}

Watch patient continuously.

Open-air treatment or

Hydrotherapy. 
Ice bag to head.

Lumbar puncture. (For discussion, see text. For technique, see Cerebro-spinal Meningitis, Chap. XXV.)

\section{Drugs.}

Codeine phosphate gr. $1 / 8$ to gr. $1 / 4(0.008-0.015 \mathrm{Gm}$.) hypodermically. Morphine sulphate (if severe), gr. 1/16 to gr. 1/12 (0.004-0.005 Gm.) hypodermically.

Hyoscine hydrobromide (less reliable and less safe), gr. 1/200 to gr. $1 / 150(0.0003-0.00045 \mathrm{Gm}$.) hypodermically.

\section{Sleeplessness and restlessness.}

Bromides.

Potassium bromides or mixed bromides, gr. xxx $(2 \mathrm{Gm}$.) at night Trional. or gr. xv ( $1 \mathrm{Gm}$.) three times a day.

gr. xv $(1 \mathrm{Gm}$.) in wine, whiskey or warm drink in early evening.

Repeat in two hours if needed.

May begin early in day and give gr. ii $(0.125 \mathrm{Gm}$.) doses at 2 hour intervals and if there is no disposition to sleep in evening reinforce with gr. $\mathrm{x}(0.66 \mathrm{Gm}$.).

Sometimes gr. v $(0.33 \mathrm{Gm}$.) after last meal is effectual.

Chloralamid.

gr. $\mathrm{xx}$ to gr. $\mathrm{xxx}(1.30-2 \mathrm{Gm}$.) in wine, whiskey or cold drink in early evening.

Repeat in two hours if needed.

Barbital (Veronal) gr. v-vii ss. (0.33-0.50 Gm.) in capsule, cachet or dry on tongue, to be washed down with water. Disagreeable after effects are not rare.

Barbital sodium (medinal) a more soluble modification of barbital, gr. v-vii ss. (0.33-0.50 Gm.) by mouth. Has been used by rectum and hypodermically. I have had no experience with the drug by either the rectal or subcutaneous route.

Adalin, gr. V-xv $(0.33-1 \mathrm{Gm}$.) in capsule, tablets or powder-not soluble in water; is in alcohol.

Paraldehyde (in alcoholics) 3 ii to 3 iv (8-15 c.c.) in whiskey, brandy or water ( 1 in 8$)$.

Codeine phosphate.
Morphine sulphate. Same doses as in delirium. (See above.)

\section{Headache.}

Ice cap or cold coil.

Bromides, gr. $\mathrm{xv}$ to gr. $\mathrm{xx}$ ( 1 to $1.30 \mathrm{Gm}$.) in water.

Morphine sulphate (severe) smallest doses effectual.

Codeine phosphate or sulphate gr. $1 / 8(0.008 \mathrm{Gm}$.) by mouth or hypodermically.

Acetylsalicylic acid (aspirin) gr. $\mathrm{v}-\mathrm{x}(0.33-0.66 \mathrm{Gm}$.) in capsules.

May be due to meningismus or serous meningitis and indicate lumbar puncture. (For technique, see Cerebro-spinal Meningitis, Chap. $\mathrm{XXV}$.) 


\section{Circulation.}

It is well to digitalize the heart in any case of pneumonia unless it is obviously a light attack.

$\mathrm{Be}$ sure that the digitalis comes from reliable sources and if a product that has been physiologically assayed is obtainable it is to be preferred.

Preparations should be fresh.

Eggleston's rule for digitalization is about m. ii ( 0.125 c.c.) of reliable tincture per pound of patient. This is roughly 300 minims for an adult (150 pounds).

It should be given in 24 to 36 hours.

First dose 100-150 minims (6-10 c.c.).

Second dose, 75 minims (5 c.c.) 4-6 hours after first.

The remainder of the dose at 4-6 hour intervals, so divided as to get the whole amount in, in 24 to 36 hours.

The urgency of the case determines the larger or smaller doses and the shorter or longer intervals.

Such rules are not to be slavishly followed.

In very urgent cases the whole 300 minims may be administered in 12-16 hours.

Clinical results will follow in 36 hours or 24 or 12 hours if pushed. Many cases need 50 or 60 grains or more to get desired result, especially if the administration is more deliberate.

The drug is continued until results are obtained, or

In non-urgent cases:

Give $\mathrm{m}$. $\mathrm{xxx}(2$ c.c.) of the tincture of $5 \mathrm{ss}$. (15 c.c.) of the infusion every 6 hours for 2 days, every 8 hours for 3 days and then stop or give one dose a day. If desired results follow at any time, stop.

\section{In more urgent cases.}

Make the first dose 3 iss.-ii (6-8 c.c.) or the ticture or 5 iss. -5 ii of infusion and continue as above until results follow.

\section{In most urgent cases.}

\section{Strophanthin.}

There are two varieties-the official amorphous strophanthin and

the crystalline strophanthin of Thoms, said to be identical with ouabain.

An excellent preparation of the amorphous strophanthin is that of Boehringer, in ampoules of 1 c.c. containing $1 \mathrm{mg}$.

Ouabain is put up by several pharmaceutical houses in ampoules containing $1 / 2 \mathrm{mg}$. and $1 \mathrm{mg}$. The ouabain is about one-third more potent than the amorphous strophanthin given by the vein.

Roughly $1 \mathrm{mg}$. of amorphous strophanthin by vein equals 10 grains of digitalis and $1 \mathrm{mg}$. of crystalline strophanthin or ouabain equals 13 to 14 grains of digitalis.

If given into muscle, look for effects in 2-3 hours; if in vein, at once. The following doses assume no digitalis has been previously given. Give $1 \mathrm{mg}$. of amorphous strophanthin or $1 / 2 \mathrm{mg}$. of crystalline stro- 
phanthin or ouabain into the muscle a dose equal to about 10 grains of digitalis. Four to six hours later repeat with $1 / 2$ the first dose. Begin digitalis by mouth according to instructions above at the same time as the first injection.

If immediate effects are desired or edema of the lungs obtains, give by vein about $3 / 4$ of the dose by the muscle and give digitalis by mouth as above.

If digitalis has been given one reckons the amount (tincture is $10 \%-$ infusion about 7 grains to the ounce-digitoxin 300 times as strong as the leaf.) Allow $11 / 2$ to 2 grains a day as the rate of elimination. This is subtracted from the total to estimate the accumulation and so the safe dosage to continue.

Strophanthin is eliminated in 36 hours; hence, does not tend to accumulate like digitalis.

If strophanthin is not at hand use digitoxin intravenously (too irritating for intromuscular or subcutaneous use).

Dose 1 to $11 / 2 \mathrm{mg}$. (gr. $1 / 60$ gr. 1/40) dissolve in 1 c.c. alcohol and dilute 4 times with sterile water.

Tincture digitalis diluted in the same way with water may be used. Dose $\mathrm{m}$. $\mathrm{xl}-\mathrm{lx}$ (2.66-4 c.c.). Follow with digitalis by mouth as above.

If heart block occurs give atropine sulphate gr. $1 / 100$ to gr. 1/60 $(0.00066-0.001 \mathrm{Gm}$.). Read text carefully on digitalis medication. So-called vaso-motor stimulants of relatively little merit.

Give adrenalin (epinephrin) $1-1000, \mathrm{~m} . \mathrm{xv}$ (1 c.c.) into the muscle every 15 minutes for 6 doses if necessary. At the same time if the patient has not had digitalis, give $1 / 2$ to $1 \mathrm{mg}$. (gr. 1/125 gr. 1/60) amorphous strophanthin or $3 / 4$ that amount of crystalline strophanthin or ouabain into the vein. If patient has had digitalis begin with not more than one-half the lesser doses given above.

Atropine sulphate-gr. 1/100 (0.0006 Gm.) intramuscularly.

\section{Caffeine.}

Use soluble double salt of sodium salicylate or sodium benzoate, gr. iii to gr. $\mathrm{v}(0.20-0.30 \mathrm{Gm}$.) into the muscle every four, three or two hours.

\section{Camphor.}

Use a solution (10 per cent. to 20 per cent.) in oil, e. g., sesame oil (never in mineral oil), gr. iii to gr. v $(0.20-0.30 \mathrm{Gm}$.) of the camphor every four, three or two hours, or

Alternate caffeine and camphor as above at two hour intervals.

Strychnine (less valuable), gr.1/40 to gr. 1/30 (0.002-0.0015 Gm.) into muscle every four or three hours.

\section{Sudden collapse.}

Adrenalin $\mathrm{m}$. $\mathrm{x}$ to $\mathrm{m}$. $\mathrm{xv}(0.60-1$ c.c.) into muscle or $\mathrm{m}$. iii to $\mathrm{m}$. iv $(0.20-0.25$ c.c.) into a vein.

Heat to surface of body. 
Hot water bottles or cloths to the feet.

Heat to bowel by injections or irrigations of saline at $110^{\circ} \mathrm{F}$. to which strong black coffee is added.

Ammonia (strong water) on towel flicked before the nose, then

Caffeine or

Camphor or in above doses into muscle.

then

Strychnine

Strophanthin in above dose into muscle.

Pulmonary edema.

If patient is plethoric.

Venesection of 12 to 16 ounces ( $360-500$ c.c.).

Cup chest. Best position semi-recumbent.

See above under circulation.

\section{Dyspnœa.}

Due to pain. (See treatment of pleurisy in summary above.)

Open-ajr treatment.

Inhalations of oxygen.

One may use a funnel around the rim of which a strip of adhesive is attached like a skirt to cover the patient's nose and mouth. Better yet, a small hard rubber tip is placed in one nostril and kept in place by adhesive which at the same time seals the nostril. The other nostril is sealed by the hand of the operator.

Respiratory stimulants.

Caffeine in soluble salt of sodium benzoate or sodium salicylate gr. v (0.33 Gm.) into muscle.

Atropine sulphate, gr. 1/100 (0.0006 Gm.) into muscle.

Strychnine sulph., gr. 1/30 (0.002 Gm.) into muscle.

Due to pulmonary edema. (See above.)

\section{Broncho-pneumonia.}

(For modification of treatment see text.)

Specific treatment.

(See text.)

\section{Complications of lobar pneumonia.}

Fluid in the chest.

Confirm signs by exploration.

(Paracentesis-indications, see text. Technique, see text.)

Treatment of symptoms. (See Empyema.)

Empyema. (See text.)

\section{Pericarditis.}

Indications for paracentesis. (See text.)

Technique. (See text.)

Relief of symptoms. 
Cold.

Ice bag-technique. (See text.)

Leiter coil. (See text.)

Cold compress. (See text.)

Heat.

Hot water bag. (See text.)

Poultices. . (See text.)

Electric pad.

Fomentations.

Counterirritation.

Mustard.

Leaf. (See text.)

Paste. (See text.)

Cautery. (See text.)

\section{Pneumoccocus endocarditis.}

Treatment-that of a septicemia. (See Chap. XLV.)

\section{Thrombosis.}

Rest of part affected by fixation.

Enwrap in thick layers of non-absorbent cotton.

Or:

Wet dressings such as aluminum acetate.

\section{Embolism.}

(See text.)

\section{Meningitis.}

Treatment of symptoms.

(See Cerebro-spinal Meningitis, (Chap. XXV.)

\section{Parotitis.}

Treatment. (See Mumps, Chap. XXIII.)

\section{Arthritis.}

Treat as in Rheumatism. (See Chap. III.)

If purulent, surgery is indicated.

\section{Peritonitis.}

(See text.)

Jaundice. .

(See text.)

Abdominal pain.

(See text.)

\section{Convalescence.}

Patient should not sit up until resolution is complete. Allow to sit up a week after resolution is complete. 
(For management of this period, see text.) Food.

Air.

Return to work. (See text.)

Relapses.

(See'text.)

Prophylactic use of Pneumococcus vaccines. (See text.) 


\section{CHAPTER $\mathrm{X}$ \\ STREPTOCOCCUS PNEUMONIA}

The terrible epidemics of measles that ravished our camps during the late war, afforded an opportunity for the study of the complicating pneumonias never before offered. These were all due to the secondary invasion of the streptococcus hemolyticus although pneumococci may induce the same lesion and an identical pathology was found in many broncho-pneumonias in the influenza epidemic in which pure culture of influenza bacilli was found; nor were these pneumonias confined to measle cases exclusively; on the contrary they constituted in some camps veritable epidemics of primary pneumonia. When I read the careful reports of the morbid anatomy of these cases made by MacCallum, my mind goes back to the insistent teaching of Delafield in my student days as to what constituted the essence of broncho-pneumonia with an admiration that only brightens the halo with which we always saw our great teacher invested. Dr. MacCallum has graciously acknowledged the accuracy and conciseness of Dr. Delafield's description of bronchopneumonia made in 1884. The distinguishing characteristic of this broncho-pneumonia is that it is an interstitial process from the first and is located primarily in the walls of the bronchioles and of the alveoli adjacent. If the walls of the bronchioles be examined, there will be found intense congestion of the vessels, an infiltration with mononuclear cells, a new formation of connective tissue and a new formation of blood vessels. This is a productive lesion, a protective and reparative process. The lumen of the bronchioles contain an exudate of white cells and fibrin in which are enmeshed great numbers of streptococci. In severe cases the lining membrane may become necrotic, forming a pseudo-membrane. The walls of the alveoli adjacent, that is surrounding and radiating from the wall (not those constituting a continuance of the bronchioles, their terminal alveoli), are also infiltrated like the wall of the bronchus with mononuclear cells and new connective tissue; the alveolar cavities contain proliferated and desquamated epithelial cells from its walls together with an exudate containing dense fibrin, red cells and a few mononuclear cells, but streptococci are curiously absent. Organization of this exudate takes place quickly. Atelectasis occurs in those areas that are cut off from air by obstructed bronchi and constitute a prominent feature of the process; an exudate fills the 
air spaces and organizes;--small hemorrhages may occur into infiltrated areas. All this gives rise to the characteristic nodules seen in the gross specimen. When these are crowded together the appearance is that of an extensive and continuous consolidation.

Lymphatics. These channels running from the pleuræ to the nodes at the roots of the lungs, along the interlobular septa, bronchi, and blood vessels, become infected and thrombosed and the pleuræ are infected through them, the bacteria growing by direct extension through the thrombosed lymphatics against the direction of the current. Streptococci are found in numbers in these vessels. Organization occurs in the septa along which they run giving them great definition on section. The blood vessels in the lung are not thrombosed.

Pleuræ. Infection of the pleura is characterized by an effusion of thin turbid fluid, greenish-brown in color, containing many streptococci. It comes on promptly in large quantity, compressing the lung. Organization occurs rapidly here, causing great thickening, dense adhesions and encapsulation of pus.

Necrosis. Portions of the lung may necrose and discharge by the bronchi with excavation and this may break through into the pleura, causing pyopneumothorax. MacCallum describes a second type of streptococcus pneumonia, which he terms lobular and defines as "a patchy consolidation" with an exudate "similar to that which when diffusely distributed over a whole lobe constitutes a lobar consolidation." Streptococci in large numbers are found in the alveoli. There were no interstitial changes but much necrosis of lung tissue. Pleurisy often occurred, but without organization. This lesion may occur uncomplicated or may be mixed up with interstitial broncho-pneumonia.

Empyema. The term has been applied to all the fibrino-purulent exudates of these streptococcal pleurisies. Though thin at first they become distinctly purulent in time. MacCallum states that in acute streptococcus pneumonia cases dying inside of eight days, the pleural cavities are found free from effusion, but if the case lasts from ten days to two weeks or more an exudate was nearly always found. The appearance of fluid in the early stages has been likened to turbid urine, unstrained bouillon, muddy water and thin pea-soup; later it becomes thick and more purulent and when localized often a thick whitish green pus. When the chest is opened and it has become secondarily infected with putrefactive organisms, it becomes foul and often bluish green. Organization is rapid and extensive, causing adhesions and sacculations of pus. When large accumulations of fluid occurred before adhesions formed, the lung became extremely collapsed and flattened against the mediastinal tissues. 
It should be kept in mind that encapsulations of pus between the median surface of the lung and pericardium seems to be common and unless this possibility be kept in mind the signs and symptoms might be overlooked. Similar encapsulation may occur posteriorly between lung and mediastinum and between the inferior surface of the lung and the diaphragm.

Pathology of Other Organs. The larynx may be involved with ulceration of the vocal cords. This was seen in seven cases with measles and caused aphonia.

Tonsils are not commonly involved.

Otitis media is not rare.

Peritonitis seems not to be infrequent; presumably the infection comes through the lymphatics of the diaphragm.

Blood cultures even in severe cases remain sterile, unless taken just before death, thus affording a contrast to the blood findings in pneumococcus lobar pneumonia.

I have dwelt on the pathology of this condition in more detail because it covers many of the pneumonias seen in the influenza epidemic, in the severe form of measles, which seems to induce a peculiar susceptibility to it and lowered resistance toward it, as is seen in its most malignant form. Indeed, death in measles depends largely on pulmonary infection. It is, too, the pneumonia we meet with in whooping cough and diphtheria and occasionally in scarlet fever. It occurs also as a primary infection.

The Symptomatology varies with the virulency of the infection and the extent of the process. In the epidemic cases of primary pneumonia the disease may be almost fulminating in its course; dyspnœa is intense and cyanosis very marked. This is true of the form sometimes designated as capillary bronchitis when the involvement of each bronchiole is like having a ligature tightened about it; so too, when the pleural effusion causes compression and atelectasis.

In the secondary cases the onset is more gradual; but the patient becomes appreciably sicker and the characteristic symptoms of the pulmonary involvement declare themselves. One may expect more dyspnœa, more cough and a more rapid pulse than in lobar pneumonia, a longer course, a more certain empyema and defervescnece by lysis.

\section{TREATMENT}

The case is treated as in lobar pneumonia. There is no specific treatment.

The support of the circulation is urgent and the measures already specified are to be carried out energetically. 
Dyspnœa and cyanosis indicate the use of oxygen; for details of its administration see Influenzal Pneumonia and Summary. Cough is much more troublesome than in lobar pneumonia. For a discussion of its treatment see under Lobar Pneumonia and Influenza Pneumonia. Empyema requires urgent and immediate treatment.

\section{EMPYEMA}

\section{(STREPTOCOCCUS AND INFLUENZA TYPES)}

\section{By Dr. Alfred S. TAYlor}

The conditions in these types of empyema are so different from those in the pneumococcus type that the method of procedure must vary distinctly. The effusion usually occurs to considerable extent before the pneumonic consolidation has resolved. The effusion while virulently infected does not become frankly purulent at so early a stage and therefore adhesions between the lung and pleura do not occur at so early a period as in pneumococcus empyema. Because of this failure of adhesions to form, thoracotomy with resulting entrance of air into the pleural cavity causes not only very marked collapse of the lung on the same side but also causes marked displacement of the mediastinum and heart toward the opposite side with great resulting embarrassment of the respiratory and circulatory functions. The rationale of treatment therefore consists in the following:-

The considerable effusion which occurs early causes marked embarrassment of respiration and circulation and must therefore be relieved. This is best accomplished by aspiration, repeated at such intervals as is indicated by the refilling of the chest with fluid. Preceding aspiration $\mathrm{X}$-Ray pictures of the chest should be taken to determine definitely the situation and amount of fluid in the cavity. As a rule no form of suction apparatus is necessary in evacuating the fluid. A good sized aspirating needle, 8 to $12 \mathrm{~cm}$. long, with a somewhat bluntly beveled point, may be used for the puncture; $1 / 2$ per cent. novocaine solution may be injected into the skin and subcutaneous tissues at the point chosen for puncture, which is usually best placed in the 8th or 9th intercostal space at the posterior axillary border. The aspirating needle is inserted until it is felt to puncture the parietal pleura. As a rule the fluid will begin to flow spontaneously. If it does not a wire may be pushed through the needle to make sure its lumen is patent. If the fluid does not then run one may attach a syringe and cause suction to start the flow. A large syringe of the Janet type may be used with a small segment of rubber tube to connect between the needle and the syringe tip. If necessary 
this syringe may be used repeatedly until the chest is evacuated. This evacuation should take place not too rapidly in order to avoid any sudden change in the pressure conditions within the chest. At the first sign of respiratory or circulatory distress the aspiration should be stopped for the time being. Another simple form of apparatus for aspirating consists in having a bottle which will hold one or two litres with a wide mouth, fitted with a rubber stopper, which can be made air-tight. This stopper is perforated so as to permit the insertion of a glass tube which fits airtight. Connected with the glass tube is a rubber tube, the walls of which are moderately rigid. This tube carries a clamp which shuts it completely. If now a slight amount of alcohol is put in the bottle and the bottle is rolled around until the alcohol covers the inside surface, and then the bottle is placed upon a table, and the operator, holding the rubber stopper, just outside the bottle mouth, holds a lighted match to the mouth of the bottle, the alcohol vapor explodes and the rubber stopper is immediately sucked into the neck of the bottle fitting air-tight so that a distinct vacuum exists within the bottle. When the aspirating needle has been inserted into the pleural effusion the rubber tube may be slipped over the end of the needle and the clamping apparatus on the tube gradually released until the vacuum bottle begins to aspirate the fluid from the chest. This constitutes a simple and usually very effective aspirating outfit. It requires only a moderate amount of practice to obtain a good vacuum. The clamp must be released somewhat gradually because if it were removed at once and if the vacuum within the bottle happened to be very marked, the suction would be too sudden for the comfort of the patient. Aspiration must be repeated with sufficient frequency to relieve the mechanical distress of the patient. When the fluid becomes distinctly purulent, it is usually the case that adhesions have formed between the lung and parietal pleura and thoracotomy may be done with safety. The procedure followed is that described under pneumococcus empyema. In the after-treatment of this streptococcus type of empyema the use of Dakin's solution is of much greater value than in pneumococcus cases inasmuch as it kills off the virulent infection much more promptly and will hasten the result. For a detailed description of the Dakin technique in these cases the reader is referred to the standard works on the subject. Those who are familiar with it can apply it without specific instruction. Those who are not familiar with it should not attempt to gain experience in this type of case. In every respect this type of empyema is apt to be much more troublesome than the usual pneumococcus empyema. There are much more likely to be isolated pockets of pus which frequently do not communicate freely with the main empyema cavity, so that the drainage of the main cavity may 
appear to be progressing very satisfactorily but the patient's general condition does not improve correspondingly. X-Ray pictures will usually indicate the presence of additional pockets. Oftentimes they will also indicate small multiple abscesses in the lung substance. When the trouble is due to pockets of pus in the pleural cavity these must be drained either by opening them by instrumentation through the original thoracotomy wound or by direct approach by means of another operation. The case is thus followed until the wound is healed and the patient's general condition indicates no remaining disturbance. If the Dakin technique is followed it had been found that when the controlling smear shows only from 1 to 4 bacteria to the field the wound may be secondarily sutured even if the cavity is still of some size. In a high percentage of cases so sutured the cavity remains sterile, the lung expands with fair rapidity, and the cavity disappears through absorption of the air content and the healing together of the lung and parietal pleura.

In the small remaining percentage of cases it is necessary to reopen the wound because of some further purulent exudate.

In this streptococcus type of empyema air-tight drainage shows up to the best advantage. It permits free escape of the contents of the empyema cavity and prevents the ingress of any air which might cause collapse of the lung and displacement of the mediastinum to the opposite side inasmuch as the adhesions between the lung and parietal pleura do not appear so early and are not as firm as in the case of pneumococcus empyema. This air-tight drainage is accomplished in many different ways. Many of the methods are complicated, but there are three simple methods which answer the needs very satisfactorily. The simplest method consists in making a stab wound through the 8th intercostal space in the posterior axillary line. This stab wound is made just large enough to permit one to crowd in a rubber drainage tube $1 \mathrm{~cm}$. or $1.5 \mathrm{~cm}$. in diameter, the walls of which are fairly rigid. This tube is pushed in through the stab wound by means of a clamp which folds it to a comparatively small size. It is pushed in so that it protrudes 1 to $2 \mathrm{~cm}$. within the parietal pleura. When the clamp is withdrawn the tube unfolds and fills the wound so that it is air-tight and water-tight. The tube is then supported by adhesive plaster strips attached to it and the skin so as to prevent displacement. The tube is sufficiently long to lead from the wound to a bottle on the floor beneath the bed. In this bottle is placed a sterile, or better, a slightly antiseptic fluid, sufficient to keep the lower end of the tube submerged. There is opportunity for free escape of the empyema fluid but no air can get into the pleural cavity. If one wishes to use Dakin's solution a glass $\mathrm{Y}$ tube may be fitted into any portion of this drainage tube. The arm of the $\mathrm{Y}$ tube through which 
Dakin's solution is instilled, is covered with a rubber tube which is clamped so as to be air-tight and is open only during the instillation of Dakin's solution.

When the patient is able to sit up the bottle of fluid can be placed under the chair and the drainage continued. In some cases, as in children, the patient may be up and about with the drainage tube still in situ and the bottle fastened to him so that he can get about with reasonable comfort. This method while satisfactory in many cases does not serve where there are one or more pockets almost shut off from the main empyema cavity. In these cases which may be detected by proper $\mathrm{X}$-Ray pictures, it is preferable to do rib resection, as described in pneumococcus empyema. The cavity may then be entirely explored and accessory cavities opened so as to give free drainage through the main cavity. The wound is then sutured tight except for sufficient space to carry rubber drainage tube 1 to $1.5 \mathrm{~cm}$. in diameter with fairly rigid walls. In order to insure air-tight closure of the sutured wound a piece of rubber dam $10 \mathrm{~cm}$. square is perforated with a small punch hole at its centre. The drainage tube is pushed through this punch hole which then becomes an air-tight fitting around the tube. For further security the rubber dam may be glued to the tube by using a few drops of chloroform around the joint or more simply by tying a linen thread around the dam and the tube so as to make sure of tight closure. The dam is so placed on the tube that when the tube is inserted through the wound so as to protrude 1 to $2 \mathrm{~cm}$. within the parietal pleura the rubber dam is just flush with the skin surface. This distance varies with the thickness of the tissues of the person operated upon and must be decided at the time of operation. After the tube is inserted and the rubber dam lies spread over the surrounding skin, broad strips of adhesive plaster are used to stick the edges of the rubber dam to the skin. This also gives a water-tight and airtight drainage. The tube is led into a bottle of solution as previously described. Both of these methods keep the patient perfectly dry and uncontaminated by the empyema fluid. A third method, which was modified by the writer from the procedure described by Dr. Lund of Boston gives a perfectly satisfactory air-tight drainage without the use of any apparatus. 'A curved incision is made through the skin and subcutaneous fat over the level of the 9th rib. This flap is dissected up and the skin above it is undermined and retracted up to the level of the 8th rib. The incision is now carried down through the muscles to the rib, 8 to $10 \mathrm{~cm}$. of which is now resected as previously described under pneumococcus empyema. After the rib has been resected the parietal pleura is loosened from the wound upward to beneath the 7th rib and then a rectangular flap of pleura is cut and turned from above downward 
so as to make a facing for the muscular surface denuded by elevating the skin. This pleural flap is held down by a few catgut sutures. It seems to prevent too rapid healing of the skin flap to the underlying muscles. It also creates a good vent hole for the escape of the empyema contents. After the cavity has been explored, and accessory cavities, if present, have been opened into the main cavity, the skin flap is allowed to fall back into place. Its corners are closed by means of silk sutures. The skin flap now acts as a flap valve. Whenever the patient coughs or strains, the contents of the empyema cavity are extruded freely. Whenever inspiration occurs the skin flap immediately falls against the muscle tissues beneath and air-tight closure occurs immediately. This is readily demonstrated on the operating table. A soft ring of cotton is made sufficiently large in diameter to entirely circumscribe the wound and flap valve. This is fastened to the skin by bands of adhesive plaster. Absorbent dressings are now applied over this ring and fastened by a binder. These dressings are changed as often as necessary to keep the patient dry and comfortable. Every second day a pair of blunt flat scissors are slipped beneath the skin flap to make sure that it has not become adherent to the underlying structures to the disadvantage of the drainage. By this method after free vacuation is obtained no air ever gets into the chest and the lung expands and comes down rapidly to its proper position. The patient is free from apparatus and therefore can turn in bed and can sit up with much less discomfort. The only disadvantage lies in the fact that the patient is wet from the discharge a certain amount of time. After the first $48 \mathrm{hrs}$. the discharge is comparatively slight. This has proven an extremely satisfactory method in the hands of a number of men. All of these different procedures may be done with local anesthesia as previously described. General anesthesia may be used according to the judgment of the operating surgeon. In all of these methods also respiratory exercises with the aid of the Wolff bottles may be used, as previously advised. On the whole one of these three methods of air-tight drainage is to be much preferred to the resection of a rib with open drainage.

\section{SUMMARY}

No specific treatment.

For detailed treatment see Lobar Pneumonia, and Influenzal Pneumonia. (Chaps. IX and XII.)

Support of the circulation urgent-measures specified must be undertaken early and energetically.

Dyspnca and cyanosis. Use oxygen. (See Influenzal Pneumonia.)

Empyema.

(See text.) 


\section{CHAPTER XI}

\section{THE TREATMENT OF GRIP OR SPORADIC INFLUENZA ${ }^{1}$}

OF grip or influenza there are two statements to be made which I think no one will attempt to gainsay; first, that of all the acute infectious diseases with which we have to deal, it is the most common; secondly, that no other acute infectious disease is so protean in its manifestations as this ubiquitous malady.

Much confusion has arisen in the use of these two terms, grip and influenza. By most men they are used synonymously, but many insist that influenza is applicable only to that infection caused by the organism of Pfeiffer, the influenza bacillus. If their contention is sustained, then we see this disease relatively infrequently, and what we consider as such should receive another appellation and "grip" might be so used. Now, we do have a common clinical entity, characterized by suddenness of onset, aching pains in the back and limbs, headache, high fever, prostration, some catarrhal symptoms in the respiratory tract and followed by weakness and depression out of proportion to the other symptoms and which may be accompanied or followed by serious involvement of important organs or systems. These attacks are accompanied by the presence of one or the other or of a combination of several organisms, in such numbers and so distributed as to warrant the supposition that they stand in causal relationship to the disease; and among these organisms is the bacillus of Pfeiffer, but in by no means the majority of the cases. Beside this organism may be found the pneumococcus, the streptococcus pyogenes, the streptococcus mucosus capsulatus or the micrococcus catarrhalis. In no way does the attack, with reference to the onset, characteristic pains and aches, prostration, after effects or complications, differ in one case or another sufficiently to guarantee a diagnosis of the organism concerned. Moreover, the influenza bacillus has been found in other conditions that do not present the clinical picture of grip. Whether we are dealing with one or the other bacterium can be determined only by culture. At the present we have no specific treatment for influenza

${ }_{1}^{1}$ This chapter deals with the infections met with each winter, loosely termed grip or influenza and attributed to more than one form of infecting organism. The epidemic influenza as seen in 1889-90 and in 1918-19 is dealt with in the succeeding chapter. 
and find ourselves limited to the effort to afford relief, to prevent complications, or treat them if they do occur.

Therapy. All that follows, then, is applicable to the clinical grip and if I use both terms, it will be understood that they are used synonymously.

Rest. An attack of grip of average severity compels a patient to seek rest and seek it in bed; but in lesser attacks, often termed common colds - and, indeed, the border line between the common cold and grip is by no means clear cut-the patient may insist on keeping about and endeavor to attend to his business. That every patient with a cold in the head should go to bed is absurd, but if the so-called cold is accompanied by signs of intoxication, aches, pains, prostration and temperature out of proportion to the local manifestations, then the diagnosis is warranted, as at least connoting a more serious infection, of which the complications or sequelæ are to be feared, and the patient should be ordered to bed until his temperature is normal, his protests being met by a presentation of the facts and possibilities in the case.

The typical attack of influenza is usually abrupt, sometimes like a bolt out of the blue, ushered in with chilly sensations and decided malaise.

The patient should be put to bed, a hot water bag put at his feet, a drink of hot tea, hot water and a teaspoonful or two of whiskey or a hot lemonade with or without whiskey given, and blankets added to the covering until the febrile reaction begins. With the fever comes the headache, the pains in the back and bones that makes the patient feel as if he had been mauled and bruised, and the discomforts of the elevated temperature. The head is often confused and a mild delirium may occur.

A cool sponge bath or one with tepid water or one containing a little alcohol applied to the whole body or to the face, arms and legs gives some relief. Cold cloths are placed on the forehead to relieve the headache or an ice bag may be used. Bits of cracked ice are sucked or small amounts of cold water taken frequently.

A saline cathartic should be given, in the shape of a Seidlitz powder, a full glass of liquor magnesii citratis, or a half ounce $(15 \mathrm{Gm}$.) of either Rochelle salt or Epsom salt in a half to threefourths of a glass of water.

Diet. At the onset of the attack food should not be offered; and the anorexia should be taken as an indication that the body is not ready to entertain it. After the first day, however, fluids in the shape of milk or gruels may be given and later soups, eggs, and cereals.

When the temperature has subsided the diet should be made liberal, 
for the loss of flesh may have been considerable, and it must be appreciated that the 2,500 or more calories that the body will have, even in the weakened state, must come from somewhere, out of its own tissue, if not provided for in its food.

\section{SYMPTOMATIC TREATMENT}

Sthenic Period of Toxemia.--Drugs. In no other acute infectious disorder do the coal-tar preparations work so happily to the comfort of the patient, as in this.

The sudden fever, the severe headache and the racking pains of body and limbs all indicate these antipyretics and analgesics.

There is no class of drugs more abused or misused than the antipyretics. In a certain number of conditions when properly used they are invaluable, but like most drugs of real worth they are rife with danger when administered carelessly or in ignorance.

The three best known antipyretics are acetanilid, sometimes called antifebrin, antipyrin and phenacetin.

This is also the order of their potency and of their toxicity. From these three have come numerous derivatives, made by introducing a radicle into their structure or substituting one radicle for another, in the effort to enhance their pharmacological value and at the same time diminish their dangers. With them have been combined other drugs of a different pharmacological action in the effort to get the two in one preparation, as for example when antipyrin and chloral are combined to form hypnal to get a substance which will produce sleep in the presence of pain, or salicylic acid and antipyrin are combined to form salipyrin to enhance the value of salicylic acid in the relief of pain in rheumatism.

So quickly and abundantly have these preparations come, so ardently have they been welcomed and praised, so rapidly have they been abandoned that it is difficult to set an appreciation on more than a few of the group.

Personally, I rarely use for any purpose any other than the three first mentioned.

In grip any one may be used; better results are obtained from small doses frequently given than from large doses. Which of these is the better I cannot say, but one will find oneself giving preference to one or the other more and more and that will represent the reliance that comes with familiarity.

In grip my preference is for the most toxic and potent of these threenamely, acetanilid, but I give it in small quantities at frequent intervals. 
The results have been so good, year in and year out, that I have felt no inclination to experiment with the others.

I have no doubt that I should have had just as good reports to offer from one of the other preparations had I accustomed myself to their usage. My favorite prescription runs thus:-

R

Acetanilidi.......................... 1.50

Sodii Bicarbonatis..................... 1.00

Caffeinæ Citratæ....................50

M. et Divide in capsulas no. xv.

gr. xxiiss.

gr. xv.

gr. viiss.

Each of these capsules, then, contains $1 \frac{1}{2}$ grains $(0.10 \mathrm{Gm}$.) of acetanilid, which is a very small dose, one grain of bicarbonate of soda, which possibly lessens the slight irritant effect of acetanilid on the gastric mucous membrane; at any rate it is given on that supposition, and 1/2 grain $(0.030 \mathrm{Gm}$.) of citrated caffeine, for two reasons: First, as a prophylactic, to counteract the slight depressing effect that is induced in some susceptible individuals by acetanilid, and secondly, because it is itself an analgesic.

The fact that caffeine is a circulatory stimulant naturally led to the supposition that its presence in a prescription containing acetanilid or other antipyretic afforded an antidote in some measure to the toxic effects of the latter. Physicians have been influenced by this idea to permit patients to take doses of these antipyretics, when so "sheltered," of a size they would fear to administer, if given alone.

Worth Hale ${ }^{1}$ experimenting on the toxicity of acetanilid when administered alone, when given with caffeine, with sodium bicarbonate or when all three are combined, obtained results that make us pause in the use of the larger doses of these drugs.

In animals he could show that the addition of caffeine to acetanilid caused death by the latter more quickly or in smaller dose, while bicarbonate lessened the toxicity of acetanilid.

In white mice he found acetanilid and sodium bicarbonate the least poisonous, acetanilid alone next, acetanilid, caffeine, and sodium bicarbonate still more, and acetanilid and caffeine most.

I give this capsule, if the attack is severe, every hour for four doses, then every two hours, so that the patient gets only 10 grains $(0.66 \mathrm{Gm}$.) in ten hours, a dose set down in some of the books as a single dose, though I think it rather large.

In the vast majority of cases the effects are very manifest in terms of

${ }^{1}$ Worth Hale: The Effects of Caffeine and Sodium Bicarbonate upon the Toxicity of Acetanilid. The Journal of Pharmacology and Experimental Therapeutics, Vol. I, No. 2. 
relief from discomfort within a very few doses and this is followed soon by a fall in temperature, which is usually marked by, if not before, the next morning.

If such relief occurs, one may give the capsule the next day at three hour intervals and the next at four-hour intervals. In the lighter attacks the drug need not be continued beyond the next day. If the attack is prolonged or complications ensue, the drug should not be too long continued. Such continued usage will do harm. Its benefit is confined to the early, sthenic period of the infection.

This is very important to remember, for one must not entertain the idea for a moment that these drugs cure the disease or have any direct action on the invading organism. They simply make the patient more comfortable and in better condition to meet the infection.

The effects of these drugs on fever is rather interesting, and, indeed, we need once in a while to orientate ourselves with reference to our ideas of fever.

Fever, after all, is a state or condition in which the bodily processes operate at a higher bodily temperature. This state may be variously brought about; but here, as in most of the fevers in which we are interested, it is brought about by the action of the toxins of the infecting organisms on the heat regulating mechanism. We often speak of the patient as "burning up with fever" and entertain the idea that his combustion, his oxidation processes, are greatly increased.

This is not the case. The actual increase in oxidation is slight. While varying in different diseases and individuals, the amount of increase will not average over 25 per cent. of the heat production at rest and as compared with the output of heat during active exercise is a mere bagatelle.

A man at rest with food eliminated 812 grams of carbon dioxide in the day and a man at severe work 3,073 grams of carbon dioxide: over three and a half times as much, and yet all the heat this oxidation represents induced no fever.

Nor, on the other hand, is the output of heat greatly interfered with to account for fever.

What seems actually to occur is that the heat regulation has been set at a higher level, runs on a different plane.

But on this plane the same mechanism for retaining heat when the body temperature is lowered and the same mechanism for getting rid of heat when the body temperature is raised is called into action as when running at a normal level. At this new level, however, the mechanism is not so nicely adjusted and greater variations follow from lesser influences exerted on it; hence, the marked effect of antipyretics in fever that we do not see follow in conditions of health. 
This regulating mechanism is vested in special nerve-tissue somewhere at the base of the cerebrum, in the neighborhood of the corpus striatum.

How antipyretics act on this centre we do not know, but what they do is to knock the regulation down to a lower level, on which the mechanisms of defense against an increase or decrease of heat are called into action, just as on the higher plane before the administration of the drug and as on the lower plane in health.

But as the influence of the drug wears off or the influence of the toxin reasserts itself, the heat regulation is pitched higher and higher to its old level.

We know that the fever is not the disease, only a symptom.

We know that temperature above $102^{\circ} \mathrm{F}$. is accompanied by increased protein destruction, which it may or may not be desirable to interfere with.

We know that the temperature may become so high as to threaten life itself and then certainly should be combated; but we are coming more and more to scrutinize symptoms so as to detect in them expressions of compensatory processes.

That fever was a favorable state in the presence of the toxemia of infection was an idea long entertained, more lately fell into disrepute and, now, most recently, is being advocated by thoughtful men and careful observers.

What our antipyretics do of value in grip is probably less attributable to the lowering of temperature than to the relief of aches, pains, cerebral excitement, all of which mean wear and tear, and so, as I have said, make the patient better able to cope with the infection.

Acetanilid is chemically a very simple body.

It is derived from anilin, $\mathrm{C}_{6} \mathrm{H}_{5} \mathrm{NH}_{2}$. It was discovered that anilin had the property of lowering temperature but that it was at the same time very toxic, inducing collapse.

I have shown how the toxicity of a drug may be altered by the introduction of simple radicles into its constitution without altering its efficiency from a therapeutic standpoint, when discussing the salicylates in the treatment of rheumatism. ${ }^{1}$

Now the introduction of an alkyl radicle to replace an $\mathrm{H}$ of an amido group retards the action of the base, so when the acetic acid radicle $\mathrm{CH}_{3} \mathrm{CO}$ takes the place of an $\mathrm{H}$ in the amido group of anilin, $\mathrm{C}_{6} \mathrm{H}_{5} \mathrm{NH}$ $\left(\mathrm{CH}_{3} \mathrm{CO}\right)$, it retards the action of the active portion of the molecule which is anilin when it is set free, and it does that by offering resistance to the oxidative processes of the organism, by which the setting free of

${ }^{1}$ See Treatment of Rheumatic Fever, Chap. III. 
the anilin occurs. The result is a partial detoxication of the anilin. When the anilin is set free the body further oxidizes it to a paramidophenol, which is anilin with an $\mathrm{OH}$ radicle taking the place of the $\mathrm{H}$ in the ring opposite the $\mathrm{NH}_{2}$ group, the para"position. This oxidation further detoxicates the substance and when finally it becomes paired with sulphuric acid or glycuronic acid the detoxication is complete and the substance is so eliminated in the urine.

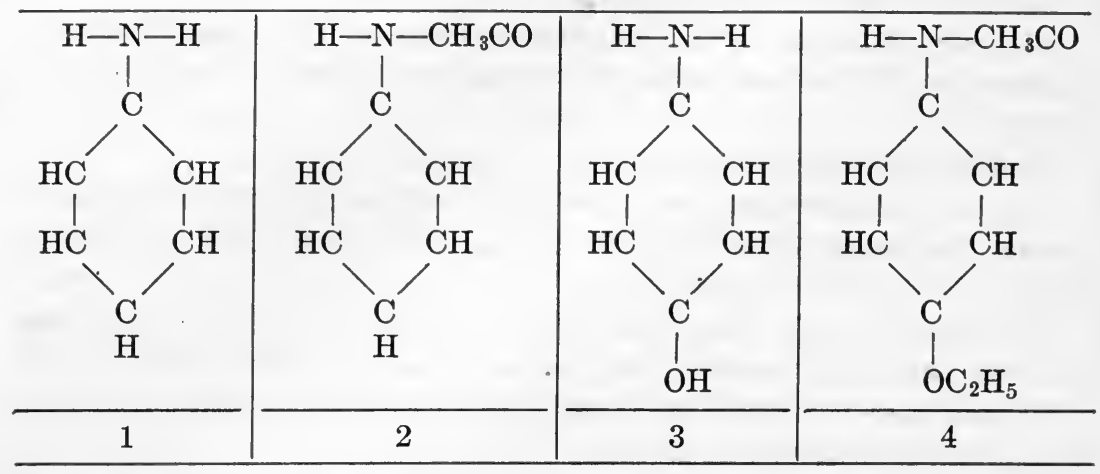

1. Aniline.

2. Acetanilid in which one of the $\mathrm{H}$ atoms in the amido group has been replaced by the alkyl radicle, $\mathrm{CH}_{3} \mathrm{CO}$.

3. Paramidophenol, in which an $\mathrm{H}$ atom in the opposite group (para position) has been replaced by an $\mathrm{OH}$ radicle.

4. Phenacetin, in which the $\mathrm{H}$ atom of the para position of acetanilid has been replaced by an oxyethyl group $\mathrm{OC}_{2} \mathrm{H}_{5}$.

When the chemists appreciated the means by which Nature detoxicated acetanilid, they seized on the paramidophenol, which while less poisonous than anilin was still capable of inducing symptoms in kind and of still changing hemoglobin into methemoglobin like the mother substance and rendered it less toxic by introducing an ethyl radicle into the $\mathrm{OH}$ in the para position and thus produced a body which permitted the paramidophenol to be set free from its molecule still more slowly and therefore was less toxic. The characteristic action, being all the time due to the paramidophenol.

This body is known as acetphenetidin (phenacetin) $\mathrm{C}_{10} \mathrm{H}_{12} \mathrm{NO}_{2}$. One sees then that phenacetin is acetanilid in which the $\mathrm{H}$ of the para position is replaced by an oxyethyl group.

So it is with all this group of drugs. They all owe their activity to the setting free of the paramidophenol, but their efficiency and safety to a nicety of balance between "fast enough" to have action on the tissue and "slow enough" not to poison the tissue. 
So in administering these drugs, the amounts and frequencies with which they are administered make a great difference in the ultimate results.

If one entertains prejudices against acetanilid, one may use the less toxic acetphenetidin (phenacetin) in its place in such a prescription as I have just given in doses of gr. ii to iiss. $(0.125-0.150 \mathrm{Gm}$.); or it may be used alone, as the necessity for the use of the other constituents in this case obtain to a less degree.

Toxic Effects. If these antipyretics be used injudiciously in large amounts or in cases of idiosyncrasy for the drug, certain untoward results may ensue. They are the onset of (1) collapse, (2) cyanosis, (3) a group of lesser disorders.

(1) The collapse may come on abruptly or after certain premonitory symptoms. It is more likely to ensue with acetanilid than with the others and with antipyrin more commonly than with acetphenetidin (phenacetin).

The patient is pale, the skin cold and clammy, the pulse rapid, small and sometimes irregular, the pupils slightly dilated and the patient faint, apathetic or confused. He may remain, however, perfectly conscious. The treatment like that of collapse from any cause is, in brief, application of heat and stimulation. (See Chapter IX.)

(2) Cyanosis. A marked degree of cyanosis is a characteristic symptom of poisoning by these drugs and is most marked after acetanilid, less after acetphenetidin (phenacetin) and least after antipyrin.

It occurs early relative to the more dangerous symptoms and it is not an uncommon experience to see patients who use this class of drugs freely for headache and neuralgias, especially in the shape of some of the proprietary remedies, with blue lips and finger nails, though not conscious of further distress.

The cyanosis is not an indication of respiratory or circulatory embarrassment, but is due to changes in the coloring matter of the blood, to the formation of methemoglobin. Of course, if the dose is sufficiently large, dyspnœa and cardiac failure ensue.

Sodium bicarbonate is supposed to have some antidotal effect. It may be given freely in dram doses at hourly intervals for several doses.

(3) Among the lesser disorders that occasionally appear are eruptions, erythemata, urticaria, or even angioneurotic edema. There may be burning of throat or mouth, nausea or vomiting, excessive perspiration, more rarely apathy or mental confusion or disturbances of hearing.

The eruptions may give rise to a diagnosis of scarlet fever or measles. In the former case the sudden onset and other symptoms may easily 
be diagnosed as grip and the coal-tar preparations given in treatment.

With the appearance of the eruption one might attribute it to the coal-tar produčts. Take no chances in the matter. Treat it as scarlet fever until proven to the contrary. When one has to differentiate between two conditions, one of which is common, and the other unusual, one should always give preference to the former. One should not let the possibility of a shrewd diagnosis run away with sober sense.

By enumerating all the things that may occur after the use of the coal tars, I do not wish it inferred that they are common. They are really safe drugs to use, when used properly. More cannot be said of any drug.

As has been said, there are no end of drugs advised for grip. When there is no specific for a disease, one is impressed with this or that line of treatment in proportion to his knowledge of the man who advocates it and the terms in which he estimates it.

Burney Yeo, whose position as a clinician needs no comment, in his Manual of Medical Treatment, is so thoroughly convinced from his personal experience of the efficacy of quinine after two or three days preliminary treatment with such measures as I have described, his own preference being salicin, that I cannot refrain from mentioning it though I have had no personal experience with it.

He uses it in doses of gr. i to iii (0.060-0.20 Gm.) every three to four hours and prefers to give it in solution in citric acid; lemon juice would answer.

Certainly the doses are neither large nor frequent; the effect of quinine upon temperature and pain and to lessen metabolism and its freedom from bad effects are well known and would lead to no hesitation in its usage.

Tracheitis. Some involvement of the upper air passages or of the trachea is almost constant. In most cases it is slight and relief is afforded by local measures or by inhalations. Tracheitis is not uncommon, causing a nagging cough, and a feeling of rawness or discomfort under the sternum.

I am fond of a simple remedy that works admirably in many instances. A mixture of equal parts of oil of turpentine, spirits of camphor and olive oil is made, a bit of flannel to cover the anterior chest is saturated with it and laid upon the chest, pinned into the night shirt and left on for the night. This may be made in the house. Order a tablespoonful of each, stir with the spoon and saturate the cloth. The patients inhale the fumes all night and marked relief to the feeling of soreness and tightness is afforded, the cough ameliorated and ex- 
pectoration facilitated. Camphorated oil may be used in the same way.

Inhalations of compound tincture of benzoin, or of the oil of pine, a teaspoonful or two on a pitcher of hot water, or a teaspoonful to a pint in an inhaler, or a few drops of the saturated alcoholic solution of menthol on a pitcher of water will prove grateful.

If the cough is more harassing, codeine phosphate may be used in doses of gr. $1 / 8$ to $1 / 4(0.008-0.015 \mathrm{Gm}$.) every two, three or four hours, or heroine hydrochloride (diacetyl morphine hydrochloride) in doses of gr. $1 / 10$ to $1 / 12(0.006-0.015 \mathrm{Gm}$.) at the same intervals.

One of the most striking characteristics of this disease is the weakness and prostration of the patient after even a short course. In many, complications of a mental nature bespeak the profound effect of the poisons on the nerve centres.

Convalescence should not be hurried. Exposure after the attack has subsided, although a matter of two or three days is an invitation to relapse or complication. The danger must be explained to the patient and the demands of his body for further rest, as dictated by his weakness, be listened to, rather than his mental protests and ambitions and business urgencies. If the patient will assume this attitude towards himself, his returning strength will give the word for further indulgence.

In the meantime the diet should be made liberal to make good the ravages of the toxins. Simple well-cooked foods, solid or fluid, are indicated. Light rubs or massage given to the sore and tired muscles, fresh air and plenty of it, in bed or chair at first and later in the walks and, if the convalescence is unduly protracted, a change of air does wonders.

Patients should not get back to work until they feel again the impulse of health. At this juncture tonics are supposed to be indicated. I believe one should feel that the best tonics are to be found in fresh air sunlight, good food and properly proportioned rest and exercise rather than in drugs.

If our patients, however, are not content, after such measures have been honestly adopted, we may try strychnine sulphate in doses of gr. $1 / 40$ to $1 / 30(0.0015-0.002 \mathrm{Gm}$.) three or four times a day or tincture of nux vomica in equivalent dosage may be substituted.

\section{COMPLICATIONS}

Such is the treatment of a moderately severe attack of grip without complications; but it is the complications that makes grip the serious disease it is. 
I have said that it is one of the most protean of diseases we have to deal with.

What organ may be implicated we do not know, but there are a certain number so frequently involved that we should never cease to keep our eyes open for the first indications of such an occurrence.

The respiratory tract in some part of its extent is almost certain to be implicated.

Otitis Media. We have just dwelt on the lesser manifestations of grip. Dependent on infection of the upper air tract is the common occurrence of otitis media. I cannot dwell on all the phases of this important chapter of grip. What I want to emphasize is the vital importance of ever keeping it in mind. In older patients the involvement of the ear is accompanied by pain, so that it is not likely to be overlooked, but in children and especially in younger children this does not hold true. It is the most common experience of a pediatrist to find the first evidence of an otitis in the discharge that has taken place through the ruptured drum.

In the acute infectious diseases of childhood it is good practice to examine the ears periodically and in such diseases as scarlet fever and influenza its neglect is scarcely pardonable. Examination of the child's ear is not the easiest procedure in the world and so is commonly neglected by the general practitioner, but routine use brings its reward in facility acquired.

When treating a young child or infant for influenza, suspect the ear if there is a sudden exacerbation of temperature, often very high in children, or if there is a rise of temperature after the normal has been reached, or the child becomes much sicker without obvious cause for the manifestations. The temperature of otitis in children may be long continued and sometimes with marked excursions.

Let me intercalate here that when I see long continued temperature in children with marked daily excursions, I always think of influenza, otitis media or Bacillus-coli infection and of course, in a malarial district, of malaria.

The incident of otitis seems to vary from epidemic to epidemic and its virulency too.

That mastoiditis is all too frequent a sequel, that sinus thrombosis and meningitis may be, that some of the cases are fulminating should make us appreciate the seriousness of it, and seek special advice. If this is not at hand a paracentesis or incision is simple and should not wait on temporary measures of relief.

Pneumonia. Another result of infection of the respiratory tract, much dreaded and constantly to be watched for is pneumonia. In- 
fluenza pneumonia has a high mortality, which differs in different epidemics.

While lobar pneumonia may occur with grip and run a regular course, the typical pneumonia is a broncho-pneumonia. As a rule this occurs a few days after the onset though it may be earlier.

Grip pneumonia is prone to be insidious. It occurs in scattered foci; it is often incomplete, its physical signs are very irregular, the subjective symptoms are out of all proportion to the objective. It is frequently slow in resolution.

It is because of all these facts that the lungs should be examined daily. In two classes of patients the search should be especially assiduous; in old people and in children. In old people the signs of bronchopneumonia are the least satisfactory. They may be absent entirely and the diagnosis be made on the degree of respiratory embarrassment and intoxication. The cough may or may not be prominent. The temperature may not be elevated.

The signs may be only those of localized bronchitis; that is, râles, or there may be only a little dulness and diminished breathing. One cannot watch the chests of old people confined to bed too closely. They are extremely susceptible to pneumonic processes, made much more so in a grip attack.

In children, too, it must be remembered that the physical signs are often slight and that the diagnosis may have to be made on the rapidity of respiration, prostration or cough.

The irregularity of distribution of the lesion, the incompleteness of the consolidation and the persistency of signs may give rise to the diagnosis of tuberculosis. Such a case in a young man, with consolidation at the left apex, a fairly high temperature with a good deal of excursion, a severe cough and a prolonged course I saw diagnosed as a case of acute miliary tuberculosis to the great distress and alarm of the family, in which tuberculosis had occurred.

Again, the retardation in resolution of these patchy and incomplete consolidations may occasion the same diagnosis.

When these cases are determined, they should be treated as pneumonia arising from other causes. ${ }^{1}$

Tonsilitis, bronchitis and pleurisy are to be treated in the same manner as if arising under other conditions.

Of rhinitis I would add a word.

This is exceedingly common in influenza. It is often very distressing and accompanied by much frontal headache. This usually means an involvement of the sinuses.

${ }^{1}$ See Treatment of Pneumonia, Chap. IX. 
When the frontal sinuses are involved there is pain especially over the inner third of the orbtial ridge and this pain is made worse by blowing the nose; when the ethmoidal sinuses are involved the pain is said to be deeper set or even occipital; when the antrum is involved there is pain in the superior maxillary, especially in the malar region and often a toothache on the affected side. Our efforts should be to endeavor to shrink the mucous membranes enough to open the ducts and passages to these sinuses to allow a drain. This may be done by spraying the mucous membrane with adrenalin 1 to 10,000 and after the drug has exerted its effect spray with an oily solution. I like one advised by Coakley-namely:

R

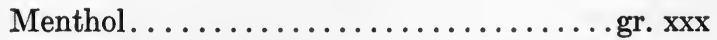

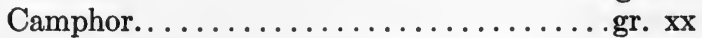

(2. Gm.)

Eucalyptol.................... $x$

(1.30 Gm.)

Olei Rosæ........................ ii

Benzoinol, q. s. ad................. ii

M. et S. Use in an oil atomizer.

(1.30 c.c.)

(0.20 c.c.)

(60. c.c.)

If the antrum is involved, the head should be turned to the sound side, hanging a little over the edge of a pillow to facilitate the discharge. The menthol solution as so given I find at times too strong. It may be reduced $\mathrm{x}$ or $\mathrm{xv}$ grains $(0.60-1 \mathrm{Gm}$.) or the whole may be diluted with more benzoinol.

If the nasal discharge is marked, order extract of belladonna, gr. 1/8 $(0.008 \mathrm{Gm}$.), or atropine sulphate, gr. $1 / 120(0.0005 \mathrm{Gm}$.) every 2 hours, until the discharge stops and then give at intervals of four to six hours to control it.

When giving belladonna so frequently, one should not forget that many people have an idiosyncrasy for it and should recall the early signs of accumulation, pupils widely dilated with blurred vision, erythematous eruption, scarlatiniform in character, excessive dryness of the mouth, and rarely a talkative delirium.

I have seen these coryzas outlast the attack until one despaired of ever giving relief and then clear up in forty-eight hours after changing to a new locality.

Of course one must be assured that there is not a suppurative process in one of the sinuses.

I might add that some of the cases run over into a chronic bronchitis or that small abscess cavities form or bronchiectatic cavities and that a patient may become a veritable bacillus carrier.

The nervous system shows the effect of the toxemia in no inconsiderable number of the cases. 
Neuritis may occur as after any infectious disease and occasionally a true meningitis; but, as strikingly frequent, follow neuralgias. Marked mental depression, which all too often becomes melancholia, or other psychoses make their appearance. These must be dealt with as under other circumstances.

In children one may see symptoms of meningitis which are more commonly due to a meningismus or serous inflammation rather than to the presence of the influenza bacillus or other pyogenic organism.

In such a case a lumbar puncture should be done; first; that by examination of the fluid we may determine the presence or absence of the organism, whether it is purulent or not, that is, whether we have to deal with a purulent or serous meningitis; and second, because such a procedure often brings prompt amelioration of the symptoms. I have seen nothing more striking than the improvement following this procedure. (For technique of lumbar puncture, see Cerebro-Spinal Meningitis, Chap. XXV.)

I would emphasize two facts borne in upon me by my children's service at Bellevue Hospital; first, that this condition of meningismus, under which I include cases of serous meningitis, is extremely common in many acute infections and also in gastro-intestinal disturbances and second, that the differentiation from a suppurative meningitis is in a large percentage of these cases possible only by a lumbar puncture, a procedure followed by more fruitful results than any other measure of clinical diagnosis with which I am familiar.

Prolonged Fever. In some cases the fever drags out to unusual lengths. This is seen at times in children. They should be put in the fresh air as much as possible and their nourishment properly considered.

It is more than doubtful if any drugs have influence on this prolonged intoxication. When all other measures have failed there is one that has results so striking and on the whole so reliable that it needs an emphasis; namely, change of air.

This is more easily attained with children, with whom transportation is a relatively easy problem.

Moreover, a removal to a remote quarter, to an entirely different climate, is not necessary.

Equally good results I have seen by sending cases to places no farther removed from New York than Atlantic City, Lakewood or Greenwich, nor have I been able to determine that the woods or the shore offered any peculiar advantage the one over the other. I have seen a child sent to Lakewood after running a temperature for weeks with prompt subsidence of symptoms, only to relapse on a return home, to as promptly 
subside again after a second trip to Lakewood. There a longer residence effected the cure. These prolonged cases in children associated as they often are with a cough and a slight bronchitis not infrequently arouse the fear of incipient tuberculosis.

\section{PROPHYLAXIS}

Were the mortality of influenza as high as that of tuberculosis or of pneumonia or were the mortality directly traceable to the infection as in the case of typhoid, no doubt we should more fully appreciate the importance of prophylaxis and urge its carrying out, but we do not look upon influenza as a highly dangerous disease and we do not attribute the deaths from pneumonia to the Influenza infection with sufficient emphasis, so little precaution is taken to protect the individual or the community.

There are three ways in which the disease is especially spread, first by those who are a little ill, are suffering from a "common cold" and move about among their fellows, infecting as they go; second, by the very ill who infect other members of the family or attendants because knowledge of the mode of transmission is not known and precautions are not insisted upon by the attending physician; and third, by the carriers of the bacilli, people with a chronic cough, sufferers from chronic bronchitis, bronchiectasis or abscess.

Lord found the organism of Pfeiffer present in 25 to 59 per cent. of unselected cases of chronic cough and expectoration in interepidemic periods.

With the first class of cases, patients should be instructed as to the meaning of "common colds" that occur in epidemics, how easily their cough, with its spray of infecting organisms, can pass on the disease of perhaps fatal severity to those in contact with them; that coughing and sneezing should be done into a handkerchief, that sputum should be destroyed and that intimate contact, kissing, etc., should be seduously avoided.

Perhaps no more certain source of infection exists than the common carriers.

How, wedged into a car or train, in the hours of crowded traffic, with sneezing and coughing all about, with insufficient ventilation or such a one as constitutes a draught to blow over the heated skin, could one escape infection? So far as possible these places should be avoided during an epidemic.

Of the second class of cases, the family should be instructed that the patient is a source of infection and that remaining unnecessarily 
long with the patient increases the danger; the nurse should be instructed to see that the secretions are received into proper receptacles, vessels provided with 5 per cent. carbolic acid, or into cloths that should be burned; that the cloths in contact with the patient should be sterilized by boiling, that his utensils and thermometer be kept separate and that after he has convalesced, the room should be fumigated with formalin.

With reference to the third class of cases, more difficulty exists until the health authorities make more inquiry into the nature of chronic processes and take measures for the public protection.

\section{SUMMARY}

Rest.

Bed. Half bed of iron of hospital type preferred in severely ill.

\section{Room.}

Light and air.

When the attack is trivial the patient should be advised to remain in the house and assured of a shorter course if he goes to bed.

\section{Onset.}

Chill.

Hot drinks, water, tea, lemonade, whiskey or brandy.

Hot water bags to feet, blankets.

\section{Fever.}

Sponge bath with tepid water.

Alcohol rub.

Cold cloths to head.

Cracked ice to suck.

\section{Bowels.}

Saline cathartic.

Seidlitz powders, liquor magnesii citratis 5 viii-xii (240-360 c.c.) Rochelle or Epsom or Glauber's salt $₹$ ss.-i $(15-30 \mathrm{Gm}$.) or begin with Calomel (Hydrargyri chloridum mite), gr. 1/4 (0.015 Gm.) every quarter hour for six doses.

Follow in three to four hours by saline.

\section{Diet.}

No food at onset. Later milk, eggs, cereals, gruels, toast, broths, mutton or chicken, thickened with rice, barley or other flour or cereal.

In prolonged complicated cases consider caloric needs.

(See Chaps. II and XIV.) 


\section{Symptomatic treatment.}

Sthenic period of toxemia.

Drugs.

Antipyretics.

Acetanitid, antipyrin, phenacetin (acetphenetidin). Acetanilid.

In small doses frequently repeated, either alone gr. iss.-ii (0.10$0.15 \mathrm{Gm}$.) or in combination. For example.

R

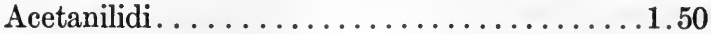

Sodii Bicarbonatis. . . . . . . . . . . . . . 1.00

Caffeinæ Citratæ....................50

gr. xxiiss.

gr. $\mathrm{xv}$

gr. viiss.

M. et divide in capsulas no. $\mathrm{xv}$.

S. One every hour for four doses, then every two hours.

As improvement occurs make the dose every three hours and every four hours.

Phenacetin.

May be given alone in doses of gr. ii-iiss. $(0.125-0.16 \mathrm{Gm}$.) or in combination with sodium bicarbonate and caffeine as above.

Give at same intervals as the acetanilid. Antipyrin.

May use like acetanilid and phenacetin.

Dose when frequently repeated gr. ii-iiss. $(0.125-0.16 \mathrm{Gm}$.). Salicylates.

Especially aspirin in capsules, gr. v $(0.30 \mathrm{Gm}$.) every two hours.

Quinine in capsules, gr. i-iii $(0.060-0.20 \mathrm{Gm}$.) or in solution in citric acid, gr. $\mathrm{x}(0.60 \mathrm{Gm}$.) in lemon juice, every three or four hours after two or three days of preliminary treatment with salicin, e. g., gr. v-viiss. (0.30-0.50 Gm.) (Yeo).

Or this may follow two or three days of acetanilid, phenacetin or antipyrin treatment, outlined above.

\section{Tracheitis.}

Apply to anterior chest, over sternal region flannel saturated with Oil of turpentine,

Spirits of camphor,

Olive oil, equal parts; leave on all night; or camphorated oil applied in the same way.

Inhalations.

Steam from croup kettle, pitcher of hot water with paper cone, or from some simple inhaler. Pour on to this hot water $3 \mathrm{i}-\mathrm{ii}$ (48 c.c.) of compound tincture of benzoin, or oil of pine or oil of eucalyptus.

Inhale as often or as long as it is grateful. Pour on to the hot water a few drops of a saturated alcoholic solution of menthol and inhale. 


\section{Cough.}

Codeine phosphate in solution gr. 1/8-gr. 1/4 (0.008-0.015 Gm.) to $3 i$ (4 c.c.) of water every four, three or two hours according to severity; or codeine sulphate in tablets in same dose.

Or heroine hydrochloride (diacetyl morphine hydrochloride), gr. 1/12$1 / 10 \cdot(0.005-0.006 \mathrm{Gm}$.$) at same intervals.$

\section{Convalescence.}

Care to avoid exposure to inclement weather conditions or to undue exertion or fatigue immediately after an attack, even when mild, lest a recrudescence or complication like severe bronchitis or pneumonia ensue.

\section{Diet.}

Should be made more liberal.

Fresh air.

\section{Massage.}

\section{Tonics.}

Strychnine sulphate, gr. 1/40-gr. 1/30 (0.0015-0.002 Gm.) or tincture of nux vomica $m$. $x-x v(0.60-1$ c.c.) three or four times a day.

\section{Complications.}

\section{Otitis media.}

In children, ears should be examined every day, regardless of symptoms referable to ear.

(The magnifying electric otoscope greatly facilitates these examinations for the general practitioner.)

When the drum is inflamed and bulging, incise the drum (paracentesis or myringotomy).

If in doubt about the indication for the procedure, incise.

\section{Pneumonia.}

Examine the lungs daily and with especial care in the aged and feeble.

Treat as under other circumstances.

(See Pneumonia, Chap. IX.)

Tonsillitis, Bronchitis and Pleurisy. (See Chaps. V, VII and VIII.) Treat as under other circumstances.

\section{Rhinitis.}

Treat as under other circumstances.

\section{Sinusitis.}

Spray the nose with a cleansing solution, 2 per cent. boric acid solution.

Quarter to half strength Dobell's solution, or one of the numerous equivalents on the market. This to clear the mucous surfaces of secretions.

Follow with adrenalin hydrochloride (epinephrin) spray 1-10,000 or stronger $(1-5,000,1-2,000)$ or use any other epinephrin solution. 
(This to shrink the mucous membrane and open the sinuses.) Follow this with sprays to prolong the effect of the adrenalin, e. g.,

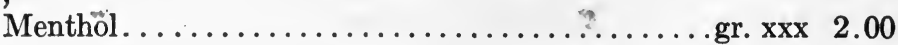

Camphor............................... 1.30

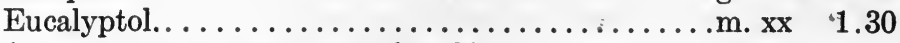

(or one can use 1 per cent. of each)

Olei rosæ (may be omitted) . . . . . . . . . . . . iii $\quad 0.20$

Benzoinol q. s. ad..................... $\quad 60.00$

M. et. S. Use oil atomizer.

If antrum is involved, turn to sound side and hang head off edge of pillow to facilitate discharge.

Extract of belladonna leaves, gr. 1/8 $(0.008 \mathrm{Gm}$.) or

Atropine sulphate, gr. 1/120 (0.0005 Gm.) every six to four hours.

Persistent rhinitis.

Change of locality.

Mental depression.

Requires watchfulness, as true melancholia with its self-destructive impulses may follow.

Meningeal manifestations.

(See text.)

Prolonged Fever.

When not due to complications will often yield to change of locality.

\section{Prophylaxis.}

Instruction in the meaning of "common colds," their infectivity and the condition they may give rise to in another.

Avoidance of too close contact with those suffering from "colds."

Danger of coughing, sneezing and kissing during attacks of "colds."

Danger in crowded cars and crowds during epidemics.

Destruction of secretions from those suffering from Grip.

Avoidance of close contact with Grip patients.

Knowledge that individuals suffering from chronic cough are in a large per cent. of cases "carriers" of the influenza bacillus. 


\section{CHAPTER XII}

\section{EPIDEMIC INFLUENZA}

ONE who has seen the great pandemics of 1889-90 and 1918 are impressed with two facts: First that there are striking differences in the clinical pictures afforded by epidemic cases of influenza and the sporadic cases that we have been accustomed to label influenza. For this reason I have deemed it well to retain in this book the chapter on Grip in which these sporadic cases were included and to write a new chapter on Epidemic Influenza. The second striking fact is that there were many considerable differences in the features, course, complications and sequelæ between these two great epidemics or pandemics and similar great differences between the pandemic of 1918 and the recurrence later.

The clinical and pathological differences naturally gave rise to a question as to the identity of the microörganisms responsible for these different forms. The influenza bacillus of Pfeiffer had not yet been discovered in 1889 and its identity in sporadic cases in 1892 lead to the assumption that it was responsible for the epidemic of 1889-90.

When the epidemic of 1918, having ravished Europe, reached these shores it assumed a malignancy that led many to believe it was due to some unknown virus and that the presence of influenza bacilli were accidental or incidental, but with increasing reports from both Army and civil workers and with improving technique the presence of the bacillus influenza was found to be so constant and the experimental work on monkeys infected with the organism by such workers as Blake and Cecil so exactly reproduced the pulmonary lesions as seen in man, that the Pfeiffer bacillus has come to be pretty generally accepted as the etiological agent of epidemic influenza. However, pneumococci and streptococci and to some extent staphylococci have been so constant invaders that it is difficult to analyze the parts played by each in the character of the attack, the pathologic response and the mortality of the disease.

Pathology. Owing to differences in terminology and the difficulties of word picturing, it is not easy to visualize the lesions in the lung that are characteristic for the influenza organism as given in the literature; moreover, with the usual invasion by pneumococci and streptococci this is further obscured.

The influenza infection attacked peculiarly the upper air passages, the trachea, bronchi and lungs. The trachea and bronchi were intensely 
congested and the cough commonly harassing and of a tracheal type. The nasal mucous membrane, pharynx, tonsils and accessory sinuses might be involved as well, but the escape of these structures from any serious inplieations struck me as rather remärkable.

The walls of the bronchi were found in many cases to be penetrated by the influenza bacillus and the walls weakened to the production of bronchiectasis.

In my earlier 1918 experiences at Bellevue Hospital this epidemic fell upon us with all the horrors of a medieval plague and in spite of all the advantages that modern medicine had brought to assist us in the fight, I again and again felt the sense of overwhelming helplessness and almost despair that our medical forefathers must have experienced. At this time the pathology of the lung explained the high mortality, the fruitless struggle, and has been well described by Symmers. It was a hemorrhagic type of broncho-pneumonia; both lower lobes massive with exudate; their surfaces purple or slate-blue and mottled, little or no fibrin on the pleuræ; cut surfaces smooth and free from fibrin, exuding blood-stained exudate of serum, and red and white cells. This was a confluent type of lobular pneumonia and interspersed with areas of emphysema, often standing out like small bubbles or beads on the surface of the pleuræ. This emphysema was striking on the edge of the lung. The upper lobes were less involved, with consolidation in their lower parts, while the upper portions were very emphysematous. Many of the lungs showed a broncho-pneumonia as defined by Delafield and called by McCallum "Interstitial Broncho-Pneumonia." The process begins in the walls of the bronchioles and spreads to the air vesicles surrounding it. It is an infiltration of mononuclear cells about the walls of the bronchi and walls of the alveoli and about the vessels. The walls become thickened, there is some exudate in the bronchioles and alveoli. Organization begins early. It is distinctly a productive process. Some cases showed a mixed type of lobar and lobular pneumonia. Some showed a pure lobar pneumonia as might be expected from the frequent presence of pneumococci. These were mostly of Type IV. Streptococci were more commonly found in the broncho-pneumonia cases, though the bronchopneumonia may be provoked by pneumococci or influenza bacilli may be found in pure culture.

After the initial epidemic had quieted down and later lighted up again, its characteristics changed in many particulars; this was true of the pathological changes in the lung. The lungs were not so early involved; the pleura was involved in the majority of cases and, in a large percentage of these, effusions were of a semi-purulent or frankly purulent nature. Small pleural and subpleural abscesses were very common, as well as 
abscesses in the substance of the lung and purulent infiltrations in the interlobar and intralobular septa or succulated empyemata; the lungs instead of showing the confluent hemorrhagic and exudative lobular pneumonia of the early epidemic, offered the most extraordinary variety of lesions (Symmers).

Symptomatology. It seems to me as I hark back on that epidemic, as if every case, touched by the hand of Death, sped to its fatal issue; but the truth is all degrees of severity were met with.

The incubation period was short, from one to three or four days; the onset abrupt, the progress uninterrupted throughout the course; or it began like a trivial cold or febrile attack, which subsided after a day or two and then tempted the patient to go out and resume work, whereon he promptly came down with a recrudescence of his infection and a pneumonia. It became my custom to emphatically warn against regarding these lesser attacks as trivial and to urge the patient to remain in the house or even in bed for some time longer; and yet I saw cases who obeyed these instructions implicitly suffer the same sequence of events.

The attack may be very light, then, or on the other hand fulminating, the patients stricken from the start so overwhelmingly that, as Symmers said, some were thought actually to be cases of gas poisoning and tested for evidences of such. These cases were prostrated, deeply cyanotic, became comatose and died and showed at autopsy great dilatation of all the capillaries and a heart immensely dilated on its right side, inducing failure of that organ. One feature that struck me in some of these cases was their parched appearance. It seems as if their tissues were dessicated and they went on to the end often without manifesting a single physical sign referable to any part of the respiratory tract.

In the majority of cases, however, the symptoms were those of a severe grip attack with especial involvement of the trachea and larger bronchi; but with certain features quite peculiar to itself.

With the sudden onset, preceded by nothing more than at times a chilliness, came headache, boring pain in the eye-balls, injected conjunctivæ, backache and pain in the extremities. In the severe types low muttering or active delirium may obtain. Insomnia may be present. At times one sees the picture of meningismus and we saw in the early cases of the epidemic profound mental depression; it being difficult to elicit any response from a perfectly conscious patient; even melancholia and dementia were reported in rare instances. If the respiratory tree was affected, the tracheitis was the most harassing feature, the cough elicited by it being constant, violent and well-nigh uncontrollable. Coryza and sore throats, while reported, were in my experience rare, except in 
the slight attacks mentioned above. Indeed, the escape of the nose and throat seemed to me very striking.

But as more characteristic than the symptoms cited above were the following: first, prostration out of proportion to other symptoms; second, cyanosis, early and out of all proportion to evidences of circulatory failure. It affected the lips, fingers, ears, mucous membranes and the skin of the face and neck and chest, corresponding fairly to the blushing area. The effect was that of duskiness, a red overlaid with a light stroke of blue, sometimes so deep as to suggest coal-tar poisoning from acetanilid or phenacetin. This occurred even in cases with no pulmonary complications. Erythema in this same area was common, mingled to a greater or less degree with the cyanosis. One thing that struck me in the epidemic was the puffy condition of the tissues that seemed almost like air cushions, even if there was no crackling as in the case of the occasional emphysema of the subcutaneous tissues. Epistaxis was not uncommon, in some series amounting to 10 per cent.; and this tendency to hemorrhage was further seen in the occasional vomiting of blood and bloody diarrhea, petechiæ in the mucous membranes, and where the lung was involved, very bloody sputum occurred in many cases. Menstruation seemed to be precipitated by the attack.

The temperature rises abruptly to $102^{\circ} \mathrm{F}$. to $105 \mathrm{~F}$. and may fall as rapidly in 36 to 48 hours, or it rises again after one to three days, usually as an expression of pulmonary involvement. More commonly it falls by lysis in the course of two to seven days, averaging five.

The slow pulse, 100 or under, that accompanied the high fever and the highly toxic state was one of the most striking features of the disease. It gave an erroneous impression of the real state of the circulation and often led to a neglect of circulatory needs. Even when pneumonia set in, the pulse is relatively slow and a convalescence often shows a striking bradycardia. The pulse may accelerate, however, and particularly near a fatal end.

Respiration, like the pulse, was curiously moderate in rate, even with serious pulmonary involvement, though this was not universally the case.

As striking as the slow pulse, was the blood reaction. This was a frank leucopenia or one that remained at normal. It is as striking as in typhoid fever. If a leucocytosis does occur, complications should be suspected.

Retention of urine was not uncommon and had to be kept in mind by the attendant. Profuse sweating might occur and I have seen one patient with a general sudamina, an erythema followed by desqua- 
mation and the separation of casts of the feet as complete as ever seen in scarlet fever, which presented a classical picture of the old English Sweating Sickness that is looked upon by some epidemiologists as an expression of influenza (Crookshank).

When pneumonia develops all symptoms are intensified. So constantly did pneumonia develop that it seemed to me that it was less a complication than an integral part of the infection and that when it did not obtain one might call the case an abortive one. It might come on in the very beginning as in the fulminating cases, or later. It was a common experience to see the initial temperature fall in one to three days, then rise again as evidence of spread to the lungs. If the fever was maintained at the end of six days it was practically certain that pneumonia was present, however scant or deceptive the physical signs. Cyanosis deepened, the patient looked septic, stupor increased, the respiration accelerated, but by no means constantly, the pulse usually remained slow until towards the end, the sputum became bloody, the blood count might increase depending on the character of the secondarily invading organisms or complications.

Death in my experience was more commonly due to the onset of pulmonary edema than from any other cause.

This is not the place to discuss physical signs, which present a great variety of findings; but I will say briefly that I found more constantly than any other a diminished breathing at both bases, earlier and more marked on one side, with a few râles and hyperresonance elsewhere in the lungs. The scattered areas of emphysema in the consolidated lung at first obscured the usual dulness of a consolidation, but this increased later to all degrees. Bronchial voice and breathing were far from constant, but were often met with, indistinct or classically shrill.

Treatment. No stone was left unturned to support and afford relief to these patients and it was just because there was no specific treatment that measures multiplied without limit and it is hard to pass judgment, amid the encomiums and condemnations, as to the value of most of these. Again it is doubtful if we shall ever see a repetition of events as we witnessed it during the pandemic of 1918 , for it differed in many respects from that of 1889-90 and changed very materially in the recrudescences that came in the years following the 1918 pandemic. For that reason one feels himself rather in the attitude of an historian than a guide.

Isolation. Every case should be isolated. During an epidemic an infection that commonly would be designated as a cold, coryza, sore throat should be suspected and isolated. The infection must be promulgated through the secretions of the mouth and nose, by sneezing, 
coughing, kissing, even by conversation at close range, by handkerchief, eating utensils, towels in common use, etc., and all members of a household should be made to understand how each may be a source of danger to the other and how best that danger may be avoided. It is most difficult to impress on the victim of a common cold during an epidemic that if he goes about his usual duties as he has been accustomed to under ordinary conditions, that he is multiplying his chances of a pneumonia, should this "common cold" be of influenza origin; lessening his chances of recovery and imperilling all with whom he comes in contact. How many times during this epidemic has Cassandra and her discredited prophecies come to my mind.

Room. This should be chosen with reference to good ventilation and light and adjacent to a bath room. It should be stripped of all superfluous furnishings, everything being sacrificed to facility in handling the patient and convenience of the nurse and physician.

No members of the family other than those in attendance on the patient should be admitted. The air should be kept fresh and temperature between $60^{\circ} \mathrm{F}$. to $65^{\circ} \mathrm{F}$. Some patients find a colder air more grateful; others suffer from an aggravation of their cough unless it is warmer.

In the hospital a cubical system should be adopted. The patients should be separated by efficient screens or sheets. It seems criminal to permit one patient who may be suffering from a streptococcus pneumonia to cough in the face of another patient across a short space between the beds. Moreover, it prevents the patients from handling the objects within reach that belong to their next-door neighbor that may carry secondary infection. In the wards, too, dry sweeping should be forbidden, but floors had better be scrubbed with water containing compound solution of cresol or some other antiseptic.

Bed. The best bed is the hospital type, a single bed or at most a three-quarter bed with woven wire springs and a firm mattress. The double bed makes the task of the nurse, very great and interferes with proper examinations by the physician. Broken springs, uneven mattresses and feather beds add discomforts for the patient. The woven wire spring is covered with a blanket to make the surface smooth, over this is placed a rubber sheet, extending from the bend of the knees to the pillow, over this a sheet drawn smooth and over this a draw sheet consisting of a long sheet doubled and laid across the bed, the excess of length tucked under one side to be gradually drawn from time to time to the other side, affording a cooling effect to the patient's back or to remove damp or soiled spots. The upper sheet and blankets as light as compatible with warmth complete the bed. The bed should be remade 
each day and refreshed at night. Care must be taken to avoid wrinkles and crumbs of food remaining in the bed. To turn the mattress with the patient in bed, an extra mattress is needed. The patient and mattress are half drawn off the bed, the patient is rolled over on the extra mattress and this pulled into place. All the accessories are now added, by rolling patient on his side, wrinkling the sheet lengthwise, tucking under one side and smoothing half across the bed, then rolling the patient back on the smoothed sheet and drawing the rest of the rolled sheet smooth to the other side. Pillows should be fairly firm, but not hard. A Gatch bed is excellent if it can be secured, as it affords a good bed rest in convalescence. (See index.)

Physician. The physician should protect himself by using a gauze mask while visiting the patient. If he is susceptible, the coughing of the patient in the course of an examination, often directly into his face as the throat is being inspected, in spite of the patient's effort to refrain, make infection almost inevitable without some such device. If he is immune he may still become a carrier and imperil the next patient he sees. The mask may be made of squares of a few thicknesses of gauze large enough to cover mouth and nose with a good margin to spare, to the four corners of which tapes are attached to tie behind the head. $\mathrm{He}$ may take several of these starting on his rounds. Each mask is left after a visit to be destroyed by fire. At the end of his visit he should cleanse his hands thoroughly with soap and hot water which will probably be efficient or he may further disinfect them with alcohol, bichloride, carbolic or lysol. As regards spraying the throat and nose I have my doubts about the value of the measure. The secretions have themselves protective powers and the trauma done the mucous membrane by harsh solutions and the spray nozzles may open the way to infections.

So far as possible he should see other patients than those suffering from influenza at the beginning of the day and on the slightest evidence of a "cold" on his part, he should desist from his visits and make himself a patient; less perhaps for his own sake than for the danger he entails to others, if this cold should turn out to be influenza. I am well aware that such precautions cannot in many instances be regarded; but where they can they should be.

The Nurse. She should so far as possible protect herself. The constant intimate contact with the patient makes precaution on her part even more imperative than on that of the physician. The mask should be used, frequently changed and burned. A gown may well be worn, especially if the exigencies of the household make some contact with other members of the family necessary. Her hands should be frequently cleansed, especially after handling the patient's secretions, feeding the 
patient, handling his dishes and before her own meals, when using a handkerchief and on going off duty. The nails should be cut close and a nailbrush used. One must doubt the efficacy of all these measures, however, when he considers the multitude of opportunities for infection in the long and close contact of nurse and patient. However, it is only just that she should know what may be done to lessen these chances. She should have sufficient time off for rest and exercise in the open air. She should have her meals with some degree of regularity and give proper attention to the condition of her bowels. Two nurses are needed on very sick cases or at least relief by the most competent member of the household. If nurses cannot be gotten the doctor must give explicit instructions to the substitute and warn her in what way she may become a source of peril to the other members of the family.

Warnings to Other Members of the Family. Keep away from patient unless needed to be of assistance, and then do not kiss or expose oneself to the cough. Avoid contact with articles handled by the patient. Always cover the nose and mouth when sneezing or coughing.

Any member showing symptoms that might be interpreted as a beginning infection should be isolated at once. Prophylactic sprays and gargles do not appeal to me, but Sailer's observations of the relatively good result that seemed to follow sprays of 2 per cent. quinine sulphate as compared with others may be worth trying. Enough aromatic sulphuric acid is added to put the sulphate in solution.

Each nostril is sprayed 15 seconds and the throat 30 seconds night and morning.

Care of Patient. The maximum of rest is to be achieved by quiet, a comfortable bed, good nursing, a well-ventilated room and combating insomnia. A cleansing bath of warm water and castile soap should be given daily, the skin gently dabbed dry and a sterile toilet powder applied. Points of pressure in stuporous or comatose patients should receive special care, with gentle rubbing and massage and alcohol rubs.

Mouth. The mouth should be kept clean by gargle and sprays of simple salt solutions, $1 / 2$ strength or full strength (4 per cent.) boric acid solution or Dobell's solution $1 / 2$ to $1 / 4$ strength.

The teeth should be gently brushed, using a simple dentifrice and the interstices of the teeth cleared of food particles.

If the patient is very ill and sordes collect on teeth, lips and tongue one may soften with $1 / 2$ strength of a solution of hydrogen dioxide and scrape the tongue with the edge of a whale-bone. Application of a solution containing 
Phenol 1 in 20 (watery solution)

Glycerin..............................

Boric acid, saturated watery solution............

may be grateful and is antiseptic.

The nasal mucous membrane may be much congested and blocked and should be kept free from secretions. This may be done by softening dried secretions with olive oil on cotton, using a wooden tooth pick as an applicator and then spray with some of the solutions mentioned above and gently cleanse with cotton on an applicator. Sprays containing menthol as the principal ingredient or a mentholated vaseline application may be found desirable. A spray of 1 per cent. menthol and oil of eucalyptus in liquid petrolatum (albolene) may be recommended, or such a prescription as the following:-

\begin{tabular}{|c|c|}
\hline Menthol.......................... & (2 Gm.) \\
\hline ..............gr. xx & $(1.30 \mathrm{Gm})$. \\
\hline 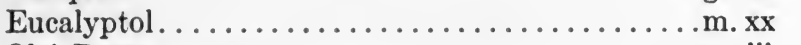 & (1.30 c.c.) \\
\hline Olei Rosæ............................ ii & $(0.20$ c.c. \\
\hline Met. S. Use in an oil atomizer (Coakley). & \\
\hline
\end{tabular}

If there is frontal headache or much turgescence of the nasal mucous membrane use adrenalin chloride (epinephrin) dropped in full strength $(1: 1000)$ or diluted in normal saline or Dobell's solution (1-4 or 1-8) and follow this with the menthol spray.

Eyes should be carefully cleansed with warm water to free from secretions and if the conjunctivæ are much injected one may use boric acid solution diluted one-half or full strength, dropped into the eye or cold compresses saturated with the solution may be laid upon the eyes.

Especial attention should be paid to the extremities in the very ill and the hot water bag placed at the feet if cold. Secretions from the nose and mouth should be received on pieces of gauze, placed in a paper bag and burned. The patient's dishes and table utensils should be boiled after using and bed linen that has been soiled should be put at once in a disinfectant in water and later boiled, at least 10 minutes.

Bowels. The bowels should be opened at the beginning of the illness. In children castor oil may be given or citrate of magnesia. In adults castor oil, 5 ss. -1 (15-30 c.c.) or salines such as Epsom or Rochelle salt in 5 ss. $-\mathrm{i}(15-30 \mathrm{Gm}$.) doses or less drastic salines, such as liquor magnesii citratis $§$ viii $(240$ c.c.) or one of the salines on the market of the Hunyadi water type. Later, enemata or mild salines are indicated if the bowels fail to move as they should daily. The bed 
pan must be insisted on in all very ill patients. The only compromise if the patient cannot or will not use the bed pan is a commode at the bedside with the patient well protected. Some patients who cannot use the pan or those so ill as to make the use of the bed pan onerous (for it is certainly fatiguing to some patients) should be encouraged to let the bowels move into large quilted pads placed under them for that purpose: These are readily folded up without soiling the bed and burned. It should be emphasized that purging is not desirable at any stage, but an assurance of an unloaded bowel is.

Diet. It should consist of milk, milk preparations and milk soups, milk toast, cereals, bread, eggs, cream soups and purées, custards and jellies, orange and pineapple juice, stewed fruits; mutton and chicken broth, cocoa; milk sugar may be used to fortify milk or drinks. (See Typhoid Fever, Chap. XIV.) A suitable diet may be selected from the list given under typhoid fever. Food should not be pushed during the early and highly toxic period, but all allowed that the patient is inclined to take. In the prolonged cases and those with a slowly resolving pneumonia, the caloric needs must be met by the dietary and patients urged to approximate 3000 calories or more.

Fluids. It has been my custom to give fluids freely and in the stuporous patients to offer drinks at frequent intervals. There has been some inveighing against this practice, based on theoretical conditions that do not appeal to me. Water, alkaline waters, fruit juices, such as lemonade and orangeade, grape juice and grape fruit juice and weak tea are permissible.

Aches and pains in the early sthenic stage of the infection may be relieved by drugs classed as antipyretics and coal tars. The object of their administration is not at all to influence the fever, but to relieve the discomforts. All have been tried; the various salicylic acid combinations, quinine, acetanilid or its congeners, separately and in combination. Often the faith in some one such prescription seems to give more comfort to the practitioner than relief to the patient. However, the action of all members of these groups is that of anodynes and their early usage is legitimate. Personally I prefer to use small doses of coal-tar preparations frequently repeated rather than larger doses at rarer intervals; choosing the most potent of the group, actanilid, and giving it in doses of gr. iss. $(0.10 \mathrm{Gm}$.). To lessen its irritating effect on the stomach and for what detoxicating effect it may have, I combine it with small doses of bicarbonate of soda and to enhance its anodyne action add citrated caffeine. For years I have used in the cases of sporadic grip the following prescription for which I am indebted to Dr. Austin Hollis of New York City. 
Acetanilid........................... iss.

Sod. Bicarb.......................... i

Caffeine Citrat....................... ss.

M.

(0.10 Gm.)

$(0.066 \mathrm{Gm}$.

(1.033 Gm.)

Such a dose is given in capsules one every $1 / 2$ hour for four doses, then every hour for four doses, then every two hours.

Small doses of acetphenetidin (phenacetin) in doses of gr. iii $(0.20 \mathrm{Gm}$.) might be substituted or antipyrin gr. ii $(0.125 \mathrm{Gm}$.) which being soluble will allow the prescription to be administered as a fluid. However, it is the coal tar that is the active member and the others may be omitted.

Of the salicylates I prefer acetylsalicylic acid (aspirin) in gr. $\mathrm{v}-\mathrm{x}$ ( 0.33 to $0.66 \mathrm{Gm}$.) doses at two-hour intervals. 'Some men combine the acetanilid and acetylsalicylic acid, giving gr. iss. $(0.10 \mathrm{Gm}$.) of the former and gr. ii $(0.130 \mathrm{Gm}$.) of the latter.

These drugs, all of which are more or less depressant to the circulation, should not be used except in the early period of the infection.

If the pain is considerable and unyielding to the above measures and in any case when associated with any asthenia, codeine and morphine are safer and more effectual, given hypodermatically, codeine phosphate gr.1/8-gr. $1 / 2(0.008$ to $0.030 \mathrm{Gm}$. $)$ and morphine sulphate gr. 1/12 to gr. $1 / 4$ (0.005 to $0.015 \mathrm{Gm}$.).

Headache may be relieved by cold applied to the head, as an ice bag. It may be due to congestion of the nasal passages and be relieved by a few drops of adrenalin (epinephrin) to either nostril.

Bullowa puts three minims of adrenalin (1-1000) into each nostril and 3 minims under the tongue at three-hour intervals. He, like some others, conceives it to be absorbed to produce general effects. If this is so it would seem to me to be an excellent measure; but our own observations on the administration of adrenalin by mouth has rendered me very sceptical of any effect it may have on the circulatory apparatus.

Insomnia.-Loss of sleep soon fatigues and patients with continued insomnia do not do well. It should receive immediate attention and not be allowed to go on night after night without interference. Again, do not be deceived by the report of the nurse or attendant that the patient has really slept, if he himself complains, for quiet does not necessarily mean sleep and sleep may be frequently interrupted.

If the patient is apprehensive of his condition, his "nervousness" may be responsible for his insomnia and bromides may be given, either potassium bromide or the mixed, so-called "triple" bromides, of sodium, potassium and ammonium in equal parts. One may give gr. xv (1 Gm.) in three-quarters of a glass of water early in the evening and repeat two or three hours later, if needed; or gr. $\mathrm{xxx}(2 \mathrm{Gm}$.) may be given at once. 
If this is not effectual, one may give trional, gr. $\mathrm{xv}(1 \mathrm{Gm}$.) suspended in a warm drink or dissolved in a little whiskey or wine or in capsules of gr. $v(0.33 \mathrm{Gm}$.) each or dry as powder on the tongue to be washed down with water. This dose may be given early in the evening and repeated late in the evening, if necessary. If it is effectual, often the patient will sleep the following night without assistance. If used from time to time, small doses gr. v $(0.33 \mathrm{Gm}$.) may prove effectual. Or one may choose chloralamid gr. $\mathrm{xx}-\mathrm{xxx}(1.33-2 \mathrm{Gm}$.) given in the same manner except that a hot menstruum should not be used, as it decomposes the substance. This too, may be repeated. Adalin gr. v (0.33 Gm.), barbital (veronal) gr. v-viiss. $(0.33-0.50 \mathrm{Gm}$.) or sodium barbital (medinal) in the same doses, neither of which latter two are favorites of mine, may be used. If these milder methods are not effectual one should not wait longer, but use morphine sulphate hypodermatically in doses of gr. 1/4 $(0.015 \mathrm{Gm}$.) or divide the dose into gr. $1 / 8(0.008 \mathrm{Gm}$. $)$ to repeat. Codeine phosphate in doses of gr. $1 / 4$ to gr. ss. $(0.015$ to $0.030 \mathrm{Gm}$.) might be substituted. Certain patients who have an idiosyncracy for morphine bear its derivative codeine well.

Delirium or psychoses, developing from insomnia or exhaustion indicate morphine at once. A mild delirium may yield to hyoscine given as the hydrobromide, hypodermically in doses of gr. 1/200 to gr. $1 / 150(0.0003$ to $0.00045 \mathrm{Gm}$.). One should not forget that noise, talking, visitors, heat, light, stuffiness, and an uncomfortable bed may all contribute to insomnia.

Vomiting. In some instances this is an early symptom and may be excessive. Food should be stopped, a mustard paste (one part of mustard and three to four of flour) applied to the pit of the stomach and cracked ice given to relieve thirst. Food should be resumed as milk or buttermilk in teaspoonful doses, at frequent intervals.

Diarrhea is not common and may be controlled by bismuth subnitrate in doses of gr.xv to $3 \mathrm{i}(1-4 \mathrm{Gm}$.) doses at two-hour intervals.

Circulation. In any severe case it is well to digitalize the heart early. This will be discussed under the next section.

Pneumonia. Every case of influenza was a potential pneumonia, which in nearly all severe cases became actual. The pneumonic process may begin at once and was not infrequently fulminating in character; or the progress may be more gradual, delaying commonly to the fifth or sixth day. Again and again a fall of temperature in a day or two after what seemed a slight attack or what was interpreted as a "common cold" was in another day or two followed by a rise ushering in the pneumonic process. Indeed, such a secondary rise nearly always means pneumonia. As I have said, exposure out of doors after the initial attack 
seemed to invite the recurrence; though recurrence happened even without exposure. Any attack in which the temperature remained sustained to the fifth and sixth day practically always meant pneumonia. The absence of convincing physical signs not infrequently lead to a failure of diagnosis.

In fulminating cases one may suspect streptococcus hemolyticus or more rarely staphylococcus aureus; though pneumococcus is not necessarily excluded. In the gradually developing type pneumococci are more commonly found, though streptococci may be the invaders. In every case where the facilities will permit, sputum and blood should be cultured and if pneumococci are recovered, they should be typed. If Type $\mathrm{I}$ is established serum should be given at once.

Pneumococcus serum. For all details for typing and administration of Type I serum see Pneumonia, Chap. IX.

Symptomatology. With the onset of pneumonia the symptoms detailed above are intensified; prostration is increased and cyanosis deepened. The pulse and respiration may be accelerated; but both may remain comparatively slow until near the end. The sputum becomes blood tinged, often the patient spits almost pure blood like an hemoptysis. The blood count may be increased in total white and percentage of polymorphonuclears, but this may be wanting or at least be far less conspicuous than in the ordinary forms.

Circulation. It is my belief that in every acute infectious disease in which the circulation may well be compromised later that a prophylactic administration of digitalis is indicated. In influenza then, unless the attack is obviously light, where even the suspicion of pneumonia occurs and in all severe cases of influenza, even in the absence of anysigns of pneumonia it was my custom to digitalize the heart. One should give some 30 grains of digitalis in the course of two or three days; or, if the case is severe, inside of 36 hours, and when the circulation is actually endangered I begin with intravenous or at the least intramuscular injections of strophanthin $3 / 4$ to $1 \mathrm{mg}$. (gr. 1/80-1/60) and follow at once by digitalis. It may be necessary to give far more than 30 grains in the end. One must judge by the effect. It is to be administered until the pulse is slower or the heart sounds better or the evidences of impairment are lessened or until toxic manifestations, such as nausea and vomiting, bradycardia and cardiac arythmia, premature systoles, are in evidence.

For the details of digitalis medication in this condition see Pneumonia, Chap. IX and the Summary.

If digitalis has been sufficiently administered I believe that other so-called cardiac stimulants, such as camphor, caffeine and strychinine, add little; but I am far from decrying the usage of drugs that good men 
have recommended. Dogmatism is obstructive. What I wish to reiterate again and yet again is that recourse to these drugs is commonly the result of ignorant and inefficient use of the digitalis series. Five drops of a tincture three times a day is not medication: it is nearer murder.

For a discussion for the lesser cardiac stimulants see Pneumonia, Chap. IX. and the Summary.

If the blood pressure is very low as in a certain group of cases, adrenalin (1-1000) into the muscle in doses of $m . x-x v(0.66-1$ c.c.) at two, three or four intervals may help, or it may be given well diluted in saline and slowly into the vein, but the total dose should not be more than m. ii-iii (0.120-0.20 c.c.) at least the first time and the result noted with the sphygmonomanometer. Pituitrin in 1. c.c. doses (m. xv) at 4-6 hour intervals may be tried as well. Sometimes a gentle pressure on the abdomen, for example, with binder and pad and an elevation of the foot of the bed may be helpful. If cyanosis is intense and skin and mucous membranes are congested it would seem logical to take off blood from the veins and the more as we are told by competent pathologists (Symmers) that such cases commonly show at death an enormous dilatation of the right heart. Such cases would find distinct embarrassment from raising the foot of the bed; on the contrary they need a bed-rest. If the patient is conscious the position in which he finds the greatest comfort and least respiratory embarrassment is sure to be the proper one to adopt. It is exceedingly difficult to tell how much good these measures do. The slow pulse gives one the impression of a good circulation and many men believe the heart is rarely involved. However, my own experience has been that edema of the lungs was the common terminal event and while I am unprepared to discuss the rôles of circulatory imbalance and toxemia exerted on pulmonary capillaries in the production of the condition, I feel fortified to a degree against the accident if $I$ have pursued the above measures.

Pulmonary Edema. However difficult it may be to judge of the result of our measures on the general circulation, the rapid change that sometimes follows our efforts in pulmonary edema is a little more convincing. The routine $I$ followed in these cases was as follows: 1. Strophanthin given intravenously. Of the amorphous, official strophanthin $1 \mathrm{mg}$ (gr. 1/60); of the crystalline strophanthin of Thoms or Ouabain ${ }^{1} 1 / 2 \mathrm{mg}$ (gr. 1/120) provided the patient has not had digitalis or but little. If digitalis has been administered, in one-half or threequarters the above dose cautiously. This may be repeated in four hours, if needed. I have seen brilliant results follow. I have seen recurring

${ }_{1}^{1}$ Strophanthin in above doses is marketed in ampoules containing the above dose ready for hypodermic or intravenous use. 
edemas clear up after repeated doses that I should not dare to advise. 2. Adrenalin Chloride (epinephrin). This is given intramuscularly in doses of $\mathrm{m} . \mathrm{xv}$ ( 1 c.c.) every 15 minutes for 6 doses if necessary. Adrenalin is used if the strophanthin has not been successful or if the dosage of digitalis has been such as to make one hesitate to use the strophanthin. I have seen excellent results follow this measure. 3. Atropine. If these measures fail, use atropine sulphate gr. 1/100 (0.0006 Gm.) intramuscularly. This dose may be increased to gr. 1/75-1/50 (0.0009$0.0012 \mathrm{Gm}$.). It may be repeated in four hours. I have not been impressed with atropine. 4. Oxygen inhalation. This I believe to be a useful measure in embarrassment of respiration. It is practically useless as commonly administered with the funnel held in front of the face. It has been shown that the inspired air gains scarcely 2 per eent. by this method. During the epidemic I attached a skirt or broad piece of adhesive plaster to the funnel, thus making a mask. The free surface of the plaster was prevented from sticking by an opposing piece of the adhesive. One may use for the same purpose oil silk attached to the funnel by adhesive. This roughly covered the mouth and nose and must have increased considerably the content of the oxygen in the inspired air; but it had its disadvantages; it was clumsy and hot. Better yet is the method used in treatment of some of the gas cases. A soft rubber tube is inserted in one nostril, attached by a little adhesive. In this way alveolar oxygen could be raised to over 20 per cent. and if the opposite nostril was closed rhythmically with each inspiration by an attendent, the oxygen in the expired air rises to about 60 per cent. (Rudolf). Meltzer has devised an apparatus by which the oxygen in the expired air can be made to rise to 85 per cent. or more. It may be administered for periods of 10 to 15 minutes or as much or as long as affords the patient relief.

Cupping. Excellent observers have been convinced of the value of dry cups in pulmonary edema. (For technique, see Pneumonia, Chap. IX.)

My objection to their use is the disturbance in turning the patient to make the application general. I have twice in my experience noted a pulmonary edema develop under my stethoscope as a result of turning the patient to examine him. I have constantly cautioned my students against frequent and prolonged examinations in pneumonia. Indeed, I make it a rule in the dangerously ill, in whom the diagnosis is obvious, to make no examination of the back, except when the question involves a pleurisy with effusion or empyema.

Cyanosis and Dyspnœa. In general it may be said that cyanosis means insufficient oxygen, anoxemia; we associate it with an inadequate supply of oxygen coming to the blood through some respiratory 
defect or to slowing of the circulation through cardiac inefficiency, change in the form of hemoglobin, such as is produced by the toxic action of coal tars, notably acetanilid, which produces marked cyanosis of a peculiar hue.

In influenza the cyanosis is striking and resembles the cyanosis of acetanilid poisoning in its hue and in its dissociation from respiratory or cardiac embarrassment. It is, however, rather proportionate to the degree of toxemia. It is striking even in cases in which the lung is not involved. When, however, pneumonia obtains, the cyanosis deepens. Under ordinary circumstances this is accompanied by dyspnœa; but less often in influenzal pneumonia than in lobar pneumonia. This may be due to differences in carbon dioxide content of the blood, for the respiratory centre is more susceptible to carbon dioxide increase than oxygen decrease.

Even in normal men neither the red cells nor the plasma is fully saturated. Rudolf says "after all it is the amount of oxygen in the plasma that counts," for it is the intermediary carrier of oxygen between the red cell and tissues. Normally, according to him 100 c.c. of plasma contain 0.35 c.c. of oxygen, but by increasing the oxygen in the inspired air it can be made to take up nearly 3 c.c. Normal undersaturation of the arterial blood amounts to 5 per cent. and in pneumonia may amount to 18 per cent. Finally anoxemia, long continued, is damaging to tissue, especially nerve tissue, as is illustrated by the degenerative processes occurring in the brains of carbon monoxide gas poisoning not immediately fatal. Meakins was able to reduce this undersaturation in pneumonia to just over 3 per cent. by oxygen inhalation.

All this to justify the procedure of oxygen inhalation in the cyanosis and dyspnœa in pneumonia. The mode of administration is discussed above. I have had patients who found so much relief that they begged for the repeated administration of it.

Cough. This is often exceedingly harassing; nothing but severe whooping cough has equalled it in some of the cases. In most instances it may be attributed to the tracheal inflammation. It tires the patient out, interferes with sleep, burdens a weak heart and facilitates the spread of the infection through the bronchial tree. Morphine or its derivatives are indicated. One may try first codeine phosphate in doses of gr. 1/4 to even gr. i (0.015-0.060 Gm.) by mouth or better yet hypodermically, repeating it at 2 to 4 hour intervals, if needed. Heroine has been found to control cough in some cases that would not yield to codeine. The dose of the hydrochloride is gr. 1/12-1/10 (0.005-0.006 Gm.) or with caution more. It must be remembered that this drug is a powerful respiratory repressant. 
Morphine sulphate in the most persistent cases is needed in doses of gr. $1 / 8$ to gr. $1 / 4(0.008-0.015 \mathrm{Gm}$.) hypodermically and as often as it is deemed that the continued cough is causing exhaustion.

Spraying the throat or more properly the trachea, by having the patient breathe deep during a spraying with 2 per cent. cocaine hydrochloride has nicely controlled cases under our observation that resisted all drug medication.

If a generalized bronchitis alone is responsible for the cough it is likely to be less violent and nagging. Cupping the chest (for technique, see Chap. IX) or, and, especially in children, mustard paste (1-8 in children, 1-4 or even stronger in adults) may be tried. (For technique, see Chap. IX.) In pleuritic cases a hard, nagging, unproductive cough may be caused by the exudate. The most effectual treatment is strapping the chest, though counterirritation with mustard may be tried.

Acidosis. We know this not infrequently occurs in pneumonia. If laboratory facilities are at hand the presence or absence of acidosis may be determined, for example by the Van Slyke method of determining carbon dioxide combining power of the blood. When such assistance is not at hand Sellards' method may be applied. This depends on the knowledge that the acidity of a normal urine may be neutralized by the ingestion of 4 to 8 grams of bicarbonate of soda. If one administers 4 grams ( 3 i) of bicarbonate at two-hour intervals, the number of doses required to get a neutral urine gives a rough measure of the degree of acidosis, while at the same time correcting it. I have liked to recommend the administration of biearbonate in the influenzal pneumonias, lest one be caught unawares.

Tympanites. In my experience tympanites was not as constant as in ordinary lobar pneumonia; but it occurred often enough and demanded earnest attention. Its presence added to both the respiratory and circulatory difficulties and contributed to gastro-intestinal disturbances. The methods used to relieve this condition are, first, elimination of easily fermenting foods, such as cereals and sugars, giving of a sufficiency of water; the application of stupes, either plain or turpentine stupes (for technique, see Typhoid Fever, Chap. XIV), and the introduction of the rectal tube some eight or ten inches with an occasional turning of the patient from side to side, a procedure which also lessens hypostatic congestion of the lung and better aëration of the unimpaired air vehicles.

Enemata may be tried, the plain enema, soapsuds enema (for technique, see Index) turpentine enema (for technique, see Index), milk and molasses enema (for Technique, see Index) and peppermint enema (for technique, see Index). Pituitrin in 1 c.c. doses intravenously is often of 
great value. Its stimulating action on the vaso-motor system is an added desideratum. Strychnine sulphate in doses of gr. 1/60-gr. 1/30 (0.001$0.002 \mathrm{Gm}$.) hypodermically is credited with energizing the wall of the gut.

Delayed Resolution was not uncommon, the physical signs and $\mathrm{X}$-ray evidence of persistent consolidation or infiltration lasting weeks after fever has subsided.

Use of Convalescent Serum. Serum obtained from convalescent patients had recently come into use in the treatment of other conditions (see Scarlet Fever, Chap. XVIII); so, in the absence of any serum elaborated from animals except in those cases in which the pneumonia was proven to be caused by Type I pneumococcus, it was natural to think of the whole blood or serum of convalescents as probably containing antibodies utilizable in acute cases.

Transfusion was done in some cases, using the method detailed under Septicemia, Chap. XLV, but more convenient was the use of serum obtained from one individual or, better yet, pooled serum from a large number. It was only in certain hospitals (notably the Naval Hospital in Chelsea, Mass. that this was done, though it was perfectly feasible in any community if health authorities had taken the matter in hand. Pooling has the advantage of higher antibody titre than one might get from a single individual.

Blood was withdrawn ${ }^{1}$ as early as ten days after the temperature was normal and as late as six weeks after in patients in whom a Wassermann had been reported negative and who gave no history or evidences of syphilis or other communicable diseases. The serum was separated and added to a common stock preserved by the addition of 0.3 per cent. strength tricresol and stored in an ice box. It was preferred to obtain serum from patients who had had pneumonia, as the titre was found not so high in simple influenza cases and the content of antibody seemed to be much higher in those who had survived a severe case. The amount used is limited only by the difficulty of obtaining it, but the usual dose was 120 c.c. given one to three or more times at eight-hour intervals, depending on the reaction of the patient. The procedure of administration will be found in detail under Pneumonia, Chap. IX, but the precautions to detect sensitization are not necessary, as here human, not horse serum, is used.

${ }^{1}$ Withdraw serum, put in incubator at body temperature for one hour, at which time 100 c.c. of serum may be separated from 500 c.c. of blood. If there is no haste, place the blood in the refrigerator 5-6-18 hours. Then decant and centrifuge the serum to separate all formed elements; $175-250$ c.c. of serum will separate from 500 c.c. of blood. Add to each 100 c.c. of serum 20 c.c. of a 1.5 per cent. tricresol solution in normal saline. This gives a final tricresol content of 0.3 per cent. The pooled serum will keep in the ice box 5-6 weeks. 
There can be no question that if the serum was used early, its value would be much enhanced; but in private practice the difficulties incident upon getting donors and separating the serum will result in its being chosen only in severe and late cases, unless in case of another epidemic a concerted move on the part of health authorities affords a serum of relatively easy access.

In a paper read before the Massachusetts Medical Society by Redden the results obtained in 100 private cases were cited. In the discussion which ensued issue was taken with the proposition that the statistical evidence was sufficient to prove it superior to other methods including their own experiences; but while taking this ground with reference to the statistics, his critics expressed themselves as impressed by individual instances and frankly said that under similar circumstances they should want the serum administered to themselves. I myself utilized both the transfusion and the convalescent serum in several instances and find it hard to pass judgment on the result, but feel that I, too, if very ill with influenzal pneumonia would be glad to have convalescent serum given me.

Pleurisy and Empyema. In the early epidemic of 1918 pleurisies were relatively rare, but in the later recurrent epidemic were present in 60 per cent. of the cases; in 40 per cent. there were purulent or semipurulent effusions. This extraordinary change in the reaction of the tissues in the first appearance of the epidemic and later was only one of many such differences. In the epidemic of 1918 the physical signs of an effusion were common enough, but the needle went into the enormously congested lung in which fluid within the lung simulated fluid without the lung; but in the recurrence of the epidemic it was not uncommon to overlook fluid in the pleural cavity which occurring over massive consolidations only accentuated the physical signs of consolidation, instead of giving rise to loss of bronchial voice and breathing (Norris and Landis). The displacement of the apex of the heart is a differential point of cardinal significance, as pointing to fluid. The needle settles the question, or if X-ray facilities are at hand, the plate tells the tale.

Not only may the effusion occur early, but it may occur late or after the temperature has fallen. When, then, the temperature is sustained unduly long and takes on a remittent character or when it falls to normal and then shoots up again one should suspect a purulent effusion. It may be diffuse, when its detection by physical signs and needling is comparatively easy or it may be encapsulated, or interlobar when it is not so easy to locate either by one or the other and finds its problem best resolved by the X-ray.

When the needle withdraws sero-purulent or purulent fluid the best procedure seems to be aspiration as a preliminary to later operation. 
This allows the compressed lung to expand again and the displaced heart to come back towards its normal position.

The pleurisies show a tendency to become plastic very quickly, the result is a walling off or localization of the procêss and at the same time makes for dense adhesions and for later crippling of the lung. These empyemata are often, if not usually, due to the rupture of tiny pulmonary abscesses lying very near the surface of the lung; and as these are likely to be multiple and the plastic exudate promptly walls them off, a number of loculated pus collections is the result, which have to be individually emptied and whose walls have to broken down at operation. It is the failure to accomplish this that defeats the operative endeavor and perpetuates the infection. The $\mathrm{X}$-ray affords much assistance in determining sacculated empyemata; but it has decided limitations that the surgeon should appreciate.

When by aspiration the pressure on the circulation has been relieved the immediate danger is over. It seems now to be the judgment of surgeons to wait for further procedure until the acute stages of the infection are passed, unless pressure demands further aspiration. In the meantime the walling off process is more complete and the exudate thickens. When the pus has become of a creamy consistency, operative procedure is much safer.

Lilienthal prefers for aspiration mere evacuation by trochar and cannula to which a rubber tube is later attached, to a Potain apparatus. There is no objection to the small amount of air that enters by the process, in fact it lessens the shock of the procedure and the air. may be expelled by the patient's straining with the glottis closed until bubbles cease to appear from the end of the rubber tube held under water. The instrument is inserted through a small incision between the ribs, in the most dependent part of the chest, the patient lying on the edge of the bed. For continuous drainage, the use of Dakin's solution and thoracotomy, text-books on Surgery should be consulted.

For details of treatment of an influenzal type of empyema the reader is referred to Chap. $\mathrm{X}$ on streptococcus pneumonia.

Abscess of the Lung. The striking difference in the incidence of pulmonary abscess in the beginning of the epidemic and that occurring later is another of the variations difficult to understand.

At first according to Symmers, "intrapulmonary abscesses were virtually unknown accompaniments of the pneumonic process." In the recurrent epidemic he met them in over a third of the cases and when the pleura was involved he met multiple small pleural and subpleural abscesses in nearly half his autopsies.

Of course, many of these resolve, but larger ones account for the con- 
tinuance of fever and evidences of infection in the absence of empyema. The X-ray is of material assistance in determining these. An abscess may rupture into the bronchus and discharge in the expectoration. At times this discharge may be very foul and may run into a chronic process. This, of course, prolongs the disease for an indefinite period and entails loss of flesh and strength and a secondary anemia. The patient should be kept in the open air and sunlight, much as a tuberculosis patient. A sufficiency of diet must be maintained, and it is the physician's duty to know approximately how many calories a day this represents and endeavor to approximate 3,000 calories.

The sputum should be cultured and, if a dominant organism, streptococcus, staphylococcus, Pfeiffer's bacillus or Friedlander's can be secured, a vaccine may be made and administered. (For technique, see Index.)

Drugs are of questionable value and for that reason any that are likely to disturb the stomach should be avoided.

Guiacol Carbonate in gr. ii $(0.120 \mathrm{Gm}$.) doses every three or four hours might be tried and if there is much fetor an inhalation of a mixture composed of equal parts of alcohol, chloroform and creosote, is to be recommended. This is best accomplished by the use of a perforated zinc inhaler, sometimes spoken of as a Robinson's Inhaler, which is worn over the nose and mouth, held on by elastics passed over the ears. One drops 10 drops of the mixture on the moistened sponge of the inhaler to be renewed as needed. The mask may be worn as much of the time as the patient desires.

The abscess may break through into the pleura and be followed by an empyema. This indicates a surgical procedure and presents a little different problem from the usual one. (For technique, see special textbooks.)

Bronchiectasis. This may occur acutely in the course of the disease, prolonging the course and passing into a chronic condition or it may come on gradually, first giving symptoms years after the attack.

Tuberculosis. It seems probable that influenza is responsible for the lighting up of a tuberculous process; but one must keep well in mind that influenza pneumonias in the upper lobe may so simulate the physical signs of tuberculosis, and, indeed, present an X-ray picture so like it, that an error in diagnosis is readily made. This difficulty is enhanced by the fact that such influenza pneumonias are often very slow in resolving. Such patches of pneumonia at the bases should hardly suggest tuberculosis.

In all these upper lobe cases without previous history, one should lean to a favorable diagnosis, but in no way neglect to examine 
the sputum repeatedly and carefully watch the development of the case.

Much more difficult is the problem presented by a persistent infiltration of either upper lobe after influenza and in a patient who is known to have had an old tubercular process. The prognosis here must be most guardedly given. Only the progress of the case and repeated examinations of the sputum can make a decision. Finally, it must be remembered that tuberculous pneumonia may simulate lobar pneumonia. A prolonged course, remittent and intermittent type of fever, anemia, wasting, sweating or an old tubercular history, or hemoptysis or pleurisy in the past give the clue, and examinations of the sputum clinch it. Tuberculosis determined, is to be treated as under other circumstances.

Emphysema. It has been noted how characteristic acute emphysema was in the pulmonary lesions of influenza. Many cases of subcutaneous emphysema were reported. I saw such with extensive infiltration of the tissues of the neck, face and thorax, presenting the peculiar crackle on palpation, characteristic of air in the subcutaneous tissues. The air is derived from ruptured vesicles in the lung, burrowing to the root of the lung, mediastinum and fascial planes of the neck, or may originate from rupture of vesicles beneath the mediastinal pleura or at apices.

The condition needs no interference. The air is later absorbed by the tissue.

Upper Air Passages. It seemed to me that considering the severity of the inflammations affecting bronchi, trachea and lungs that the upper air passages showed a singular freedom from complications. Nasal congestion occurred and probably played its rôle in some of the headaches. Its treatment has been dealt with above. Epistaxis was common enough, but rarely of such proportions as to need interference. However, cases of coryza, tonsillitis, pharyngitis, laryngitis, sinusitis and otitis do occur and are to be treated as under other circumstances. (See these, Chaps. IV, V, VI.)

Heart and Vessels. The heart did not appear so seriously affected as one might have anticipated and yet at autopsy extreme dilatation of the right side of the heart was not uncommon. In the early days of the epidemic the myocardium was deeply congested, but in later cases acute parenchymatous degeneration with flabby and friable muscle was a frequent finding.

The endocardium escaped and the pericardial involvement was not common though it did occur, and in some instances was found to contain a purulent exudate.

The treatment of a failing circulation has been set out above. Peri- 
carditis is treated as under other circumstances. (See Acute Rheumatic Fever, Chap. III.) A relatively slow pulse, maintaining striking regularity, was a feature of the disease during its height; but in convalescence evidences of irritability of its nervous and muscular apparatus was not uncommon. One saw continued bradycardia, tachycardia, premature systoles and sinus arhythm. I saw one case of complete block, not attributable to digitalis, and auricular fibrillation has been reported.

Treatment. Irregularities of the type mentioned above except fibrillation rarely require interference. Premature systoles, if annoying and occurring in convalescence, should indicate the elimination of such excitants as tea, coffee and tobacco; and bromides in moderate doses gr. $\mathrm{x}-\mathrm{xv}(0.066-1 \mathrm{Gm}$.) three times a day may be given. This should be continued but a short time, a week, and a course of strychnine sulphate gr. 1/60-1/30 (0.001-0.002 Gm.) three times a day given after the bromides, may be helpful or bromides gr. $\mathrm{x}(0.66 \mathrm{Gm}$.) and tincture of nux vomica $\mathrm{m}$. $\mathrm{xv}$ (1 c.c.) may be given together three times a day.

Tachycardia may be long persistent. It is important to determine whether this is due to a disordered nerve mechanism or to a weakened myocardium. Dyspnœa on exertion, cardiac dilatation and impairment of sounds and electrocardiographic evidence help us in determining the question. If the myocardium is involved the case should be treated as cardiac inefficiency, rest, regular diet and suitable doses of digitalis. If a simple tachycardia, tonic, fresh air, good food, freedom from worry and regulated rest, exercise and work is all that is needed.

Bradycardia, not due to block, and sinus arhythm requires no interference other than moderation on the part of the patient. Heart block is common to many infectious diseases and disappears of itself as a rule. While it obtains and more particularly if any cardiac decompensation accompanies it, rest should be observed.

Auricular fibrillation may disappear, but is likely to be permanent. I should suspect previous damage to the myocardium. It should be treated as under other circumstances, much depending on evidences of decompensation.

Phlebitis has been reported as a rare happening. It should be treated as detailed in Typhoid Fever. Allbutt tells us that influenza is one of the less common causes of toxic arteriosclerosis, seen more commonly after typhoid; also that it causes an aortitis with involvement of the middle coat as in syphilis and that, as in the latter, aneurisms and angina pectoris may result. This was written before the present epidemic. Whether we shall see such late results of this visitation remains for the future to determine.

Kidneys. Acute parenchymatous degeneration of the kidney 
occurred, more marked later in the epidemic; but I saw no cases of renal inadequacy. Hemmorrhagic nephritis has been reported, however, as occurring early and especially in children; clinical nephritis seems to have been reported more frequently from abroad than in this country. When nephritis occurs it should be treated as if a primary disease. (See Scarlet Fever, Chap. XVII.)

Infections of the Kidney. It has been emphasized, especially by Bugbee, that infections of the kidney were not rare happenings and that they have been all too frequently overlooked. Cases of pyelonephrosis and perinephritic abscesses were observed. Pyelonephritis was recognized by "pain and tenderness in the kidney region; frequent, burning, painful urination, high temperature, chills, malaise" (Bugbee). Cystoscopy confirmed the diagnosis. Colon bacillus was the organism most commonly found, but staphylococcus and possibly influenza bacilli were also noted. Treatment-drainage by catheter, pelvic lavage, urinary antiseptics, colon irrigations and appropriate diet was recommended. Perinephritic abscess gives rise to pain and tenderness in the lumbar region, continued temperature, often chills and often bulging in the flank. Incision and drainage are indicated. Other infections of the genito-urinary tract that have been reported include epididymitis, seminal vesiculitis and prostatic abscesses. For local and surgical treatment one should consult works on genito-urinary diseases.

Nervous System. Meningitis, due to the Pfeiffer organism, is reported from time to time as sporadic cases, but during the recent epidemic, strange to say, no greater number of cases of meningitis due to this organism were noted than on inter-epidemic years. It was noted by Neal in her work under the Department of Health of New York City that a larger number of meningococcus cases were met with during the epidemic than during the same season in other years. This may have been a coincidence or the influenza epidemic may have played a rôle as a contributing factor. Neal noted a considerable number of cases of meningismus (serous meningitis) that occurred, strangely enough, during convalescence rather than at the height of the infection as is usual in the case of meningismus in most acute infections. These cases had all the usual signs, symptoms and cord findings and, as is the usual result, were relieved by simple lumbar puncture.

The treatment of true meningitis cases is symptomatic. (See Cerebrospinal Meningitis, Chap. XXV.) It would be interesting to note the results of intraspinal treatment with convalescent serum in cases of true influenzal meningitis, but no cases have been reported to my knowledge. Meningismus indicates lumbar puncture as a therapeutic procedure.

Encephalitis Lethargica. There seems to be some sort of re- 
lationship between influenza epidemics and encephalitis lethargica. This is discussed under that heading in Chap. XIII.

Neuritis. Affections of a single nerve and polyneuritis has been reported after influenza, and an epidemic of polyneuritis occurred with the present epidemic that seemed to be a peripheral equivalent of encephalitis lethargica.

Mental Disturbance. Aside from the delirium accompanying an attack of influenza, and, indeed, more important are the mental aberrations following it. The disease seems to bring out and precipitate those mental disorders to which a patient may be predisposed.

Delirium may occur early and be mistaken for acute mania. Moderate delirium does not prejudice the outcome, but excessive delirium gives a bad prognosis.

Depression or mental hebetude may characterize the attack and I recall such cases in whom the absolute absence of any response to questions or the absence of effort in patients apparently fully conscious seemed almost wilful.

Psychasthenia accompanied by neurasthenia is the most common sequel. Burr says it is so frequent that it should be considered rather a part and parcel of the disease than a complication. Profound weakness, tachycardia and sweating on effort, disinclination or inability to mental effort, fretfulness or depression, even to melancholia with suicidal tendencies are the usual expressions. Burr says the outlook for complete recovery from psychasthenia and neurasthenia is absolutely good and even from simple melancholia unless delusions of self-accusations occur.

Depressed patients should always be watched lest suicide be attempted.

From a few days to a few weeks after beginning convalescence forms of insanity may appear in the predisposed. It may take the form of confusional insanity in whom after a duration of five to eight weeks the prognosis for recovery is good (Burr).

Influenza seems to bring out in the young the dementia precox to which they have a tendency; in alcoholics, delirium tremens, and in certain old syphilitics, paresis.

Treatment. Food, which especially in the depressed, requires some tact to administer in sufficient quantity. Rest at first, followed by massage and then graduated exercises. Fresh air. Attention to the bowels with mild cathartics especially in the depressed and melancholics who have a tendency to constipation.

In the forms of mental disturbance associated with excitement, the continuous warm bath is of value as a sedative. 
Abdominal Symptoms. Aside from gastro-intestinal disturbances considered above, one meets with occasional cases of abdominal pain that may give rise to confusion. There is a curious hyaline degeneration of the rectus muscle of the abdomen (Zenker's degeneration) into which extravasations of blood sometimes occur and streptococcus infections with abscess formations. This is to be thought of in cases of localized pain in the rectus muscle.

Like any pneumonia in which there is a diaphragmatic pleurisy and involvement of the lowermost area of the costal pleura, one has referred pain that may be mistaken for appendicitis, or one of the several lesions affecting the upper right quadrant. I recall one such in which severe pain in the upper right quadrant with a considerable jaundice simulated cholelithiasis before the pulmonary signs came out.

Otitis Media. While not the common happening that one might expect in a disease affecting the air passages throughout such an extent, otitis media was met with often enough to warn the practitioner to be on the lookout for the condition. In our experience at Bellevue in 500 cases of influenza admitted in October, 1918, 4 per cent. developed otitis media. Of this number fully one-half gave no complaint referable to the ear. These cases were discovered by the routine practice of ear examination established. It must be said that this is a much higher percentage than I met with in private practice. For treatment, the reader is referred to Scarlet Fever, Chap. XVII.

Parotitis. This is a rare happening and although I have no statistics I should conjecture it is no more common than in lobar pneumonia. (For treatment, see Lobar Pneumonia, Chap. IX.)

Abscess of the Muscles. This is an occasional complication. Three such cases occurred in our service at Bellevue. They occurred in the muscles of the calf and in those of the buttocks and as noted above in the recti at the site of the hyaline degeneration of Zenker.

The treatment should consist in immobilization and the application of wet dressings and later incision and drainage.

Convalescence. No period of illness is more commonly neglected than convalescence. In part this is due to the patient, who for reasons of economy wishes to dispense as soon as possible with the doctor's services, and in part to the physician, attracted by more urgent cases and all too often unappreciative of the importance of this period. The careful observations of Army physicians has impressed us with the long periods after infections, which in civil practice we are inclined to treat as trivial, it takes to restore a man to military fitness. I am convinced that we err as a rule in shortening this period to the detriment of the patient. 
Even after the mildest attacks of influenza it is well to keep the patient in bed at least two full days after the temperature is normal and forbid resumption of normal duties for a week. After a severe case or a pneumonia the patient should remain in bed ten days at least and then be allowed to get about gradually. It should require at least two weeks more before any duties are resumed, but in my estimation the period should be prolonged for several weeks with, if possible, a change of environment. The patient should feel and look fit before undertaking his normal activities.

As has been said above the myocardium needs watching and as long as poor heart sounds, arhythms, tachycardias, low blood-pressure or evidences of cardiac incompetency obtain, the patient requires medical care.

Fresh air and sunlight, good food and increasing exercise do away with the necessity for drugs. For many patients a failure to prescribe a tonic constitutes a breach of the contract. For such, I am accustomed to prescribe strychnine sulphate in doses of gr. 1/60-1/30 (0.001-0.002 Gm.) or such a-prescription as follows:

$\mathrm{R}$

Sodii Glycerophosphatis.................

Tr. Nucis Vomicæ................... äa 10

Aq., q. s. ad........................60

(3iiss.)

(3ii)

M.

Sig. One teaspoonful three times a day in water after meals.

Prophylaxis. Theoretically, keeping fit should lessen the incidence of the disease; but when one observed the stalwart specimens in Army and civil life that were affected-and the plague seemed in this epidemic at least to choose the young adult at the very age of physical perfection-one wonders whether physical fitness played any prophylactic rôle whatsoever.

More important was avoiding the conditions that lead to any infection, contact with those already infected and lessening resistance by fatigue, undue exposure, remaining in wet clothes, and exposure to drafts when overheated.

The common carriers, with people herded like the inmates of a slavepen, must be hot-beds of infection; sneezing, coughing, laughing and talking, without effort to prevent spread of secretions of nose and mouth by the use of a handkerchief, constitute ideal conditions. Any place where crowds congregate, theatres and other places of amusement, dances, churches, all endanger at the time of an epidemic.

A man with a "cold" should be mentally labelled "unclean" and shunned accordingly. Delicacy and conventionalities forbid us to say: 
"Sir, you are holding a revolver to my head and are pressing the trigger," but the results unhappily are often the same in either instance.

Kissing, promiscuous handshaking, the use of toilet articles in public places and of half cleaned eating utensils in public eating places are all a menace.

When a case occurs in a family, the measures set out above should be observed.

Vaccines. We could wish that we had an efficient vaccine for prophylactic purposes; but it is hard to convince oneself of the value of those used.

Vaccines of Pfeiffer's organisms and mixed vaccines containing this with pneumococci, streptococci, and staphylococci were used.

The reports are too conflicting to pass judgment on the value of the measure. For example, Rosenow-working under the Mayo Foundation-distributed a polyvalent vaccine of the four types of pneumococci, streptococci and staphylococci, but no influenza bacilli, for prophylactic use by physicians in many states but chiefly in Iowa, Minnesota and Wisconsin who reported back to Rosenow. He reports on a group of 93,476 persons inoculated three times, and over 345,000 who were not vaccinated. The incidence of the disease among the fully inoculated he reports to be one-third of that among the uninoculated, and the mortality among the inoculated to be one-fifth of that among the uninoculated.

Against this optimistic report stands that of McCoy, Director of the Hygienic Laboratories of the U.S.P. H. S. who, using vaccines of the Pfeiffer organism, of the Pfeiffer organism combined with the streptococci and the polyvalent vaccine of Rosenow, reported that he could determine no difference in the incidence of disease and the mortality among the vaccinated and unvaccinated when the tests w ere made under controlled conditions.

Under such circumstances one can but leave the decision to the individual practitioner.

\section{SUMMARY}

\section{Isolation.}

Suspect and isolate every case of cold during an epidemic.

Infection promulgated by secretions.

Guard against conveyance by sneezing, coughing, kissing, handling of handkerchiefs, soiled linen and eating utensils.

\section{Bed room.}

Choose with reference to light, ventilation and adjacent bath room. Strip of all superfluous furnishings. 
Exclude visitors and members of family, except those acting as nurse. Temperature $60^{\circ}-65^{\circ} \mathrm{F}$., colder, if grateful.

In hospital service, separate patient by cubicle system or screens. Forbid dry sweeping.

Scrub floors with water containing compound solution of cresol or . some other antiseptic.

\section{Bed.}

Half or three-quarter width.

Hospital type preferable- Gatch bed a desirable type.

Woven wire spring and even mattress.

For technique of making bed and turning mattress, see text.

\section{Physician.}

Use gauze mask. (For detail, see text.)

Cleanse hands with soap and water and such disinfectants as carbolic, lysol, mercuric bichloride, alcohol.

Visit other patients before influenza cases if possible.

\section{Nurse.}

Wear mask-change frequently.

Wear a gown.

Cleanse hands frequently, especially after handling patient, before feeding patient, going off duty or eating meals.

Cut nails close-use nail brush.

Take rest and exercise in open air.

Eat regularly.

Keep bowels in proper condition.

\section{Rules for the family.}

Keep away from patient unless needed in nursing capacity.

Avoid kissing and exposure to coughs.

Cover nose and mouth when sneezing or coughing as secretions are infectious.

Spray of 2 per cent. quinine sulphate may have prophylactic value.

\section{Care of patient.}

Rest.

Good nursing.

Well ventilated room.

Daily bath of warm water and castile soap.

Keep skin dry with sterile toilet powder.

For points exposed to pressure, massage and gentle alcohol rubs.

\section{Care of the mouth.}

Cleanse with normal saline solution, 4 per cent. boric acid solution, Dobell's solution, one-half to one-quarter strength.

Teeth-brush gently, using simple dentifrice. Free teeth from food particles. 
Cleanse sordes from teeth and tongue by softening with half strength solution of hydrogen dioxide. Scrape tongue with edge of whalebone.

Apply solution of:

R

Phenol ( $1: 20$ watery solution)

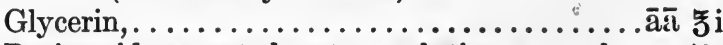

Boric acid saturated watery solution q. s. ad..... J viii

M. et S.

\section{Care of the nose.}

Keep free from secretions.

Soften dried secretions with olive oil on cotton on wooden toothpick as applicator. Spray with solutions mentioned to cleanse mouth. May use spray of 1 per cent. of menthol or eucalyptus in liquid R petrolatum (albolene) or the following: (4)
"Menthol........................gr. xxx
Camphor........................... $\mathrm{xx}$
Eucalyptol.......................gr. $\mathrm{xx}$
Ol. Rosæ.......................... iii
Benzoinol, q. s. ad....................
M. et S. Use in an oil atomizer." (Coakley.)

\section{For frontal headache.}

Drop adrenalin chloride (epinephrin) (1:1000) in nose or dilute with normal saline or Dobell's 1:4 or 1:8. Follow with menthol spray.

\section{Eyes.}

Cleanse with warm water.

Conjunctivitis-boric acid solution 2\%-4\% dropped into the eye or applied as cold compresses.

Secretions from nose, mouth, eyes, should be received on gauze placed in paper bags and burned.

\section{Utensils.}

Should be boiled for ten minutes.

\section{Linen.}

Should be immersed at once in disinfectant and later boiled for ten minutes.

\section{Bowels.}

Should be opened early in the illness.

Children should be given castor oil or magnesium citrate according to age.

Adults, castor oil 5 ss. $-\mathbf{i}$ (15-30 c.c.); Epsom or Rochelle Salt 5 ss.-i (15-30 c.c.).

Liquor Magnesii Citratis, 5 viii (240 c.c.).

Later, enemata if bowels do not move as they should. 
Insist on use of bed pan in all very sick patients.

Bedside commode may be used for those slightly ill.

Large quilted pads may be used for those unable to use the bed pan.

Diet.

Prolonged cases 3,000 or more calories are needed.

Use milk, milk preparations, milk soups, toast, cereals, bread, eggs, cream soups and purées, custards, jellies, pineapple and other fruit juices, mutton and chicken broth, fortify milk with milk sugar.

For further suggestions, see Typhoid Fever, Chap. XIV.

Do not push early and in highly toxic periods.

Prolonged cases require consideration of caloric needs.

Fluids.

Give fluids freely.

Water, alkaline waters, fruit juices, lemonade, orangeade, grapefruit juice, weak tea.

\section{Aches and pains.}

Coal-tar preparations - acetanilid, acetphenetidin (phenacetin), antipyrin.

For example:

R

Acetanilid......................... iss.

Sod. Bicarb........................ i

Caffein. Citrat...................... ss.

M. et $\mathrm{S}$.

May substitute acetphenetidin (phenacetin) in doses of gr. iii $(0.20 \mathrm{Gm}$.) or antipyrin gr. ii $(0.125 \mathrm{Gm}$.).

Combinations with sodium bicarbonate and caffeine may be omitted.

Salicylates.

Perhaps the best is acetylsalicylic acid (aspirin) gr. $\mathrm{v}-\mathrm{x}(0.33-0.66$ Gm.) every two hours.

Combinations of acetanilid gr. iss. $(0.10 \mathrm{Gm}$.) and aspirin gr. ii $(0.125 \mathrm{Gm}$.) at 2 -hour intervals have been recommended.

Discontinue after the early hours (sthenic period).

Later stages, or if pain is severe, codeine phosphate gr. 1/8-1/2 $(0.008-0.030 \mathrm{Gm}$.) or morphine sulphate gr. $1 / 12-1 / 4(0.005-$ $0.015 \mathrm{Gm}$.) hypodermically.

\section{Headache.}

Relieve congestion by adrenalin chloride (epinephrin) 1:1000 solution three minims into each nostril.

\section{Nervousness.}

Bromides. Potassium bromide or triple bromide of potassium, sodium and ammonium gr. $\mathrm{xv}(1 \mathrm{Gm}$.) in $3 / 4$ glass water early in evening. Repeat in 2-3 hours if needed. 
Early in the disease give trional gr. $\mathrm{xv}(1 \mathrm{Gm}$.) in warm drink or in 5 gr. $(0.33 \mathrm{Gm}$.) capsules early in the evening,

or chloralamid gr. $\mathrm{xx}-\mathrm{xxx}(1.33-2 \mathrm{Gm}$.). Repeat in 2 hours if necessary,

or adalin, gr. $\mathrm{v}(0.33 \mathrm{Gm}$.).

In more persistent insomnia, barbital (veronal) gr. v-viiss. $(0.33-0.50$ Gm.).

Sodium barbital (medinal) in the same doses.

In most severe cases, codeine phosphate gr. $1 / 4-1 / 2(0.0015-0.030 \mathrm{Gm}$.). Morphine sulphate gr. 1/4-1/8 (0.015-0.008 Gm.) hypodermically. Repeat if necessary.

\section{Delirium or psychoses.}

Morphine sulphate gr. $1 / 4(0.015 \mathrm{Gm}$.) hypodermically.

Hyoscine hydrobromide, gr. 1/200-1/50 (0.0003-0.00045 Gm.).

Avoid noise, talking, visitors in room. Keep room cool.

Vomiting.

Stop all food.

Mustard paste (one part of mustard to 3-4 parts of flour) apply to pit of stomach.

Cracked ice to relieve thirst.

Food resumed as milk in teaspoonful doses.

Diarrhea.

Bismuth subnitrate gr. $x v-3 i(1-4 \mathrm{Gm}$.) at 3-4 hour intervals.

\section{Circulation.}

Early digitalization in severe cases. (See below.)

\section{Pneumonia.}

Blood culture.

Type sputum and urine. If Type I pneumococcus, give serum. (See Lobar Pneumonia, Chap. IX.)

Early digitalization - 30 gr. digitalis in the course of 2-3 days. In severe cases in the course of 36 hours. When circulation is endangered, intravenous or intramuscular injections of strophanthin $3 / 4$ to $1 \mathrm{mg}$. (gr. $1 / 80-1 / 60$ ). Follow at once by digitalis. (For detailed discussion, see Pneumonia, Chap. IX.)

Other cardiac stimulants-caffeine, strychnine, etc. (See Pneumonia, text and summary, Chap. IX.)

Low blood pressure.

Try adrenalin chloride (epinephrin) $1: 1000 \mathrm{~m} . \mathrm{x}-\mathrm{xv}(0.66-1$ c.c.) at $2,3,4$ hour intervals or $\mathrm{m}$. ii-iii $(0.120-0.20$ c.c.) in saline very slowly into vein.

Or pituitrin in 1 c.c. (m. xv) doses at 4-6 hour intervals.

Gentle pressure on the abdomen as by a binder.

Venesection: with marked cyanosis and deep congestion of superficial vessels of skin and mucous membranes and evidence of dilatation of right heart, venesection is indicated; but moderate amounts of 8-10 ounces should be withdrawn and more later as indicated. 


\section{Pulmonary edema.}

Strophanthin intravenously. Amorphous official preparation $1 \mathrm{mg}$. (gr.1/60), crystalline strophanthin, or oubain, $1 / 2 \mathrm{mg}$. (gr. 1/120). If digitalis has been administered as advised, make the strophanthin one-half the above. If lesser amounts of digitalis have been taken give $3 / 4$ the above dosage. Repeat in four hours if necessary.

Adrenalin chloride (epinephrin) 1:1000 m. xv (1 c.c.) intramuscularly every 15 minutes for 6 doses. May follow the use of strophanthin as given above.

Atropine sulphate, gr. 1/100 (0.0006 Gm.) intramuscularly. May increase the dose to gr. $1 / 75-1 / 50(0.0009-0.0012 \mathrm{Gm}$.). It may be repeated in four hours.

Oxygen inhalations. (See text.)

Cupping. (See text.)

(For technique, see Pneumonia, Chap. IX.)

Cyanosis and Dyspncea.

Oxygen inhalation.

\section{Cough.}

In severe types codeine phosphate, gr. $1 / 4-1(0.015-0.060 \mathrm{Gm}$.) by mouth or hypodermically every 4-6 hours.

Heroine hydrochloride, gr. 1/12-1/10 (0.005-0.006 Gm.) by mouth every 4-6 hours.

Morphine sulphate, gr. 1/8-1/4 (0.008-0.015 Gm.).

Cocaine hydrochloride-spray with 2 per cent. solution. Have patient breathe deeply and use only two or three jets during deep breathing. After an interval have patient expectorate residue.

If from bronchitis, cup chest. (For technique, see Pneumonia, Chap. IX.) In children use a mustard paste $1: 8$ or $1: 4$.

If from pleurisy - strap chest and use counterirritation with mustard 1-4 to 1-8 according to age.

\section{Acidosis.}

Determined by laboratory methods; if not possible, give sodium bicarbonate $3 \mathrm{i}(4 \mathrm{Gm}$.) every 2 hours until urine is alkaline. Two doses should be sufficient for neutralization in normal urine.

\section{Tympanites.}

Cut down on or eliminate sugars.

Administer water abundantly.

Plain or turpentine stupes. (See Typhoid Fever, Chap. XIV.)

Rectal tubes.

Enemata-plain or soap suds, turpentine, milk and molasses, or peppermint. (For technique, see Typhoid Fever, Chap. XIV.)

Pituitrin $\mathrm{m} . \mathrm{v}-\mathrm{xv}$ (0.033-1 c.c.) intramuscularly.

Strychnine sulphate gr. 1/60-1/30 (0.001-0.002 Gm.).

\section{Serum treatment.}

Convalescent serum.

(For mode of obtaining, see text.) 
Dose, 120 c.c.

Intervals of eight hours or more according to reaction.

Administer early.

Mode of administration. (See Pneumonia, Chap. IX.)

Desensitization test not necessary.

\section{Pleurisy and empyema.}

Preliminary aspiration, and operation later after acute stage has passed, unless pressure symptoms demand re-aspiration. Wait until pus has creamy consistency.

Lilienthal method of aspiration.

Trochar and cannula with Potain apparatus attached by rubber tube. Insert through small incision between ribs in most dependent part of chest. Patient lies on edge of bed. Air entering chest is expelled by patient straining with glottis closed until bubbles cease to appear from the end of the rubber tube held under water.

Taylor's method. (See Streptococcus Pneumonia, Chap. X.)

\section{Abscess of lung.}

Treat like a tuberculosis patient-open air and sunlight.

Force the diet to approximate 3,000 calories and more if borne well.

Culture sputum. Use vaccine of the dominant organism.

Beginning dose should be small, not more than 5-10 million.

Dose increased, doubled at first, and administered twice a week, the increase in size depending on reaction and results. (For technique, see Index.)

Guiacol carbonate, gr. ii $(0.120 \mathrm{Gm}$.) every 3 or 4 hours.

Inhalations of equal parts of alcohol chloroform and creosote; use ten drops on a zinc inhaler of the Robinson type. May be used over long period. Especially useful in cases of marked fetor.

Perforation into pleural cavity constitutes an empyema and demands treatment. (See above.)

\section{Emphysema.}

(See text.)

Tuberculosis.

(See text.)

\section{Heart and vessels.}

Failing circulation. (See above and under Pneumonia, Chap. IX.)

Pericarditis. (See Acute Rheumatic Fever, Chap. III.)

Arhythmias - bradycardia, tachycardia, sinus arhythmia, premature systoles require no interference; but premature systoles in convalescence are annoying; hence, eliminate tea, coffee, and tobacco. Bromides gr. $x-x v(0.066-1 \mathrm{Gm}$.) Strychnine sulphate, gr. 1/60-1/30 (0.001-0.002 Gm.) three times a day given after bromides or tincture of nux vomica $\mathrm{m} . \mathrm{xv}$ ( 1 c.c.) may be given with bromides, gr. $x(0.66 \mathrm{Gm}$.$) three times a day.$ 
Tachycardia.

(For discussion, see text.)

Bradycardia.

Requires no interference, even when due to heart block; it usually disappears itself.

Auricular fibrillation may disappear, but usually is permanent.

If the latter, treated as auricular fibrillation under other circumstances.

Phlebitis.

(See Typhoid Fever, Chap. XIV.)

Nephritis.

Rare occurrence. (See Scarlet Fever, Chap. XVII.)

Pyelonephritis.

Drainage by catheter.

Pelvic lavage.

Urinary antiseptics.

Non-irritating diet.

Colon irrigations.

Perinephritic abscess.

Incision and drainage.

Epididymitis, seminal vesiculitis and prostatic abscess.

(See works on genito-urinary diseases.)

Meningitis.

(See text. Treat as in cerebrospinal meningitis. See Chap. XXV.)

Meningism.

Lumbar puncture.

(See Chap. XXV.)

Encephalitis lethargica.

(See Chap. XIII.)

Mental disturbances.

(Consult text.)

Depressed patients should be carefully watched lest suicide be attempted.

Push food.

Continued rest followed by massage. Graduated exercises.

Fresh air.

Attention to bowels.

In cases of excitement, the continuous bath.

Abdominal symptoms.

(See text.)

\section{Convalescence.}

Period of convalescence should not be hastened.

Confine to bed for two days after temperature is normal. Do not allow resumption of duties for one week. 
After severe cases or a pneumonia, keep in bed ten days after temperature is normal and physical signs have disappeared; get about gradually and do not resume duties for several weeks.

Change of environment before this desirable.

Do not discharge until he feels and looks fit,"blood pressure is normal and signs of cardiac incompetency absent.

Fresh air, sunlight, good food.

Graduated exercises.

Tonic, strychnine sulphate, gr. 1/60-1/30 (0.001-0.002 Gm.) or:

R

Sodii Glycerophosphatis

Tr. Nucis Vomicæ ................... āā10 (3iiss.)

Aq., q. s. ad.........................6 60 (䒓ii)

M. et S. One teaspoonful (3i) in water three times a day after meals.

\section{Prophylaxis.}

Avoid those infected.

Avoid fatigue.

Avoid undue exposure, remaining in wet clothes.

Avoid crowded public conveyances and public places.

Talking, laughing, sneezing and coughing of the infected spreads the diseases.

Avoid contact with articles used by infected individual.

Kissing, promiscuous hand-shaking, using public toilet articles, half-cleaned eating utensils and public eating places are a menace.

Vaccines.

(See text.)

Otitis media.

(See text and Scarlet Fever, Chap. XVII.)

\section{Parotitis.}

(See text.)

(See Mumps, Chap. XXIII.)

Abscess of the muscles.

Immobilization.

Wet dressings.

Later incision and drainage. 


\section{CHAPTER XIII}

\section{ENCEPHALITIS LETHARGICA}

Followisg upon the great epidemic of influenza of 1917 and 1918 appeared a lesser epidemic characterized by a widespread focal inflammation of the cerebrospinal nervous system. Similar epidemics have been noted before and their relationship to influenza commented on. An identical causative organism has not been determined, but able epidemiologists, tracing the sequelæ and character of the great plagues of Europe since medieval time, have been so impressed with the relationship noted above as to express their belief that they were but different expressions of the same infection (Crookshank). Others suggest that influenza renders the patient susceptible to invasion by this virus or that the conditions favoring one, favor the other. Others have sought to argue a relationship between this disease and poliomyelitis (HeineMedin Disease), attributing the difference to different strains of the same organism. But all this is mere conjecture. Up to the present time the nature of the virus is unknown. The entrance of the virus is probably through the nose.

The name that has come into very general use was adopted because of the drowsy state shown by many of the early cases that won for the disease the popular appelation of Sleeping Sickness, which, of course, has no relationship to the African disease of that name. The name is inadequate, for many of the cases show no signs of lethargy, but depending on the site of the lesion simulate a variety of diseases of the central nervous system and especially the polio-encephalitic forms of the HeineMedin Disease.

The Pathology. The lesion is a congestion of the meninges and small hemorrhages into both white and gray matter. These are especially common at the base. Microscopically there is cellular infiltration about the vessels. It occurs in any age, even in infancy.

Symptomatology. The onset may be gradual or fairly abrupt and the apparent somnolence, drowsiness or lethargy one of the most characteristic features.

In the first case I saw, this gradually increasing apathy that would cause the patient to interrupt his effort, as if in the act of lacing his shoes, he had fallen asleep, was very striking and the period to the full development when the patient's mask-like face looked like a Parkinson's 
Disease, was very slow. In the second case, deep lethargy came on quickly after striking the head against a heavy piece of machinery and was thought at first to be a fracture of the base of the skull.

The apathy varies in degree from mere drowsiness to what appears to be a complete coma as in the second case listed, and yet the lethargy, while in part real, is largely apparent and our surprise was great in this early case to have the patient address us out of his coma and tell us what had happened during the ten days of what we deemed complete unconsciousness, telling the names of his nurses, the internes and attending physicians. It is not hard to rouse a patient from what seems deep stupor and elicit intelligent responses; but the voice is monotonous and nasal and the speech slow. These cases resembled Parkinson's Disease in their perfectly expressionless faces; the eyelids droop so that the appearance of coma is heightened. The limbs are somewhat rigid and take and retain positions into which they are put, a veritable catatonia. Extended legs and half flexed arms held persistently in these positions was a peculiar feature.

Sometimes mental depression is obvious and delirium may occur. Paradoxical it seems to have a patient suffer from insomnia, who seems to be sleeping continuously; but this is not uncommon and though he may be drowsy and quiet by day, sleeplessness and restlessness harass him by night.

Dizziness is a common symptom and headache is complained of.

Fever is not a striking feature of the disease, but perhaps is always present at some period of the disease though it may be overlooked. It lasts only a few days, but may continue for two to three weeks. It is rarely more than $100^{\circ} \mathrm{F}$. to $102^{\circ} \mathrm{F}$. but on occasion rises to $104^{\circ} \mathrm{F}$. to $105^{\circ} \mathrm{F}$.

The blood count shows as a rule a slight white cell increase though it may be normal, but more marked leucocytosis and polynucleosis have been reported.

The spinal cord fluid is under increased pressure, shows an increase of globulin and a pleocytosis of 10 to 100 of lymphocytic type. Focal symptoms are very varied and lend to a great number of types. It is well, however, to first consider those that are fairly constant in all cases. These are affections of the nuclei and roots of nerves supplying the muscles to the eye (3d, 4th, and 6th) causing external and internal ophthalmoplegia and ptosis. Opthalmoplegia occurs in some 75 per cent. of cases and ptosis is the most common of the occular disturbances. This causes the almost constant symptoms of drooping lids, usually bilateral, diploplia, blurring of vision and strabismus, inequalities in pupils and disturbance of reaction to light. Nystagmus is not unusual. But while 
the oculi motores are so commonly affected as to offer characteristic features to the disease, the other cranial nerve nuclei by no means escape and afford variety to the picture. The involvement of the motor nuclei of the 5th, is shown by weakening of the action of the masseters, so that when the patient is asked to clinch the jaws the muscle masses fail their characteristic hardness; that of the 7 th is shown by varying degrees of facial palsy; of the 8th by deafness and vestibular disturbance-dizziness; of the 9 th by difficulty in swallowing; of the $10 \mathrm{th}$, by resulting tachycardia; of the $12 \mathrm{th}$, by involvement of the tongue.

The ganglia at the base of the brain may be implicated. The masklike expression may be due in part, at least, to the involvement of the globus pallidus (Ramsay Hunt) and I have witnessed in a number of cases twitching of groups of muscle fibres in the face, trunk and limbs corresponding to the picture of para-myoclonus multiplex and occasionally coarser quick rhythmic movements like electrical chorea that may be due to lesions in the thalmic or other basal ganglia.

To add to the complexity and variety of the picture the cortex may be involved not only to produce the general symptoms of drowsiness, depression or delirium, but also by focal lesions, causing varied paralyses, hemiplegias, monoplegias, motor or sensory aphasias, amnesias; or sensory areas are affected with the production of anesthesias or lesions in the cerebellum or its peduncles give rise to ataxias. Meningeal symptoms more rarely occur, but I have seen them fairly pronounced with stiff neck and Kernig's sign and headache. Nor does the spinal cord always escape and there seems to be a distinct polyneuritic type.

To one reading of the multiplicity of neurological manifestations in this disease, it would seem as if a diagnosis was hopeless; but it is surprising how soon a contact with these cases elicits a recognition of the symptom complex as something very definite. Of course, the knowledge of a prevailing epidemic makes a diagnosis fairly sure that would be more than hazardous in a sporadic case.

The course of the disease varies tremendously, from the fulminating, in which death is a matter of hours or days to those that last for many months. The stupor usually lasts two to five weeks. Relapses do not seem to be common, but I have seen one such case.

One should remember in giving a prognosis that recovery is the rule even when the patient seems desperately ill. If the patient survives the first few days, in spite of profound stupor I am inclined to give a good prognosis as to life and a fair one as to complete recovery. The mortality varies in different series, but it averages about 10 per cent.

Sequelæ. Considering the extent of damage done in many 
cases, the residual disturbance is remarkably little. One almost gets in the habit of giving favorable prognoses. However, mental disturbance in children, certain degrees of imbecility, and cranial and spinal paralysis have persisted.

Treatment. There is no specific treatment. Good nursing and the relief of symptoms constitute the sum total of therapy. The disease is long drawn out in many instances, the patient completely helpless and the demands made on watchful and faithful nursing are many and exacting.

Room. A cheerful room, well ventilated, with near access to the bathroom, chosen if possible in the quietest part of the house, is to be selected. In some instances photophobia requires some darkening of the room. Many patients are irritable; indeed, some clinicians recognize an irritable type of the disease. There is often hyperesthesia and in some cases marked headache. Noise causes great discomfort and the patient requires the gentlest handling.

Bed should be of the hospital type or at the most three-quarter, with a good woven wire spring and a firm hair mattress. The mattress should be protected by a rubber sheet and draw sheet. (For details see Chap. IX.) The sheets kept smooth to prevent irritation of the skin or bed sores.

Care of the Body. The patient should have a warm bath with castile soap and water each day. Especial attention should be given to the mouth, teeth and nose and to the genitals and buttocks. (For details, see Pneumonia, Chap. IX.)

The Bowels should be moved by enemata or by readily swallowed laxatives, such as cascara, phenolphthalein, pills containing aloin, or liquid paraffin. Constipation often obtains and must not be neglected.

Diet. Except in cases suffering from dysphagia, food is taken even by those that seem very lethargic. The diet should approximate caloric needs, some 3,000 a day and the articles may be chosen from such a list as is given under Typhoid Fever (Chap. XIV). In dysphagia or deep coma, feeding by nasal tube may be necessary. (See Diphtheria, Chap. XVIII.) Water or fruit drinks or alkaline waters should be given freely and, as the patient's apathy prevents his expressing his needs, it should be given to him up to the amount of two quarts or more a day.

Headache and pains. Headache may be relieved by the use of the ice bag. It and general aches and pains are to be met by the administration of aspirin (acetylsalicylic acid) in gr. v-gr. $\mathrm{x}(0.33-0.66$ $\mathrm{Gm}$.) doses at two-hour intervals, if needed; but coal tars should be used with caution, if at all: Codeine phosphate in doses of Gr. 1/8-gr. 1/4 
(0.008-0.015 Gm.) may be given by mouth or subcutaneously but morphine should be used with great care.

The extent and degree of cerebral and nuclear involvement, depression and lethargy on the one hand or irritability, insomnia or delirium on the other will modify our usage of drugs in accordance with their known pharmacological and toxic properties.

Headaches and pains in chest and extremities may be relieved by lumbar puncture and the measure has come into fairly extensive use.

Lumbar Puncture not only relieves the headache and pains, especially if the spinal fluid is under pressure, but it often lessens the stupor and other symptoms and may be repeated with benefit. The procedure is usually done early for diagnostic purposes and demonstrates its ameliorative effect on the symptoms on that occasion.

Hyperesthesia and paresthesia as well as pain are relieved by warmth, and the discomfort of pressure and irritation by clothes is relieved by raising them by frames.

Irritability and restlessness may be relieved by bromides in doses of $\mathrm{gr} . \mathrm{x}-\mathrm{xxx}(0.66-2 \mathrm{Gm}$.) or by chloral hydrate in tentative doses beginning with gr. $v(0.33 \mathrm{Gm}$.). This would be indicated rather in those cases complicated by sleeplessness.

Insomnia. Quiet, fresh air, properly arranged bed, toilet for the night, bromides and small doses of chloral in the restless may be used. Codeine phosphate gr. 1/8-gr. 1/4 (0.008-0.015 Gm.) may be given by hypodermic method, but morphine had better not be used.

Fever is not a marked feature, but if a source of annoyance, relief may be afforded by cool sponging.

Convalescence is long. Fresh air, good food, massage, hydrotherapy and other measures to meet residual paralyses exercise the ingenuity of the practitioner.

\section{SUMMARY}

Treatment. There is no specific treatment.

Room.

Well ventilated.

Access to bathroom.

In quiet part of house.

Sunlight unless photophobia indicates darkening the room.

Bed.

Hospital type preferred.

Half or three-quarters.

Woven wire springs. 


\section{Care of body.}

Daily warm baths, with castile soap and water.

Mouth, teeth, nose, genitals. (See Pneumonia, Chap. IX.)

\section{Bowels.}

\section{Enemata.}

If patient swallows readily, cascara, phenolthalein, aloin, liquid paraffin in suitable doses.

\section{Diet.}

If effort is made, food is taken by those that seem very lethargic.

Try to approximate the caloric needs of 3,000 calories.

(For items of diet, see Typhoid Fever, Chap. XIV.)

In dysphagia or deep coma nasal feeding may be necessary. (See Diphtheria, Chap. XVIII.)

\section{Drinks.}

Water, alkaline water, fruit juices, orangeade, lemonade, may be given freely.

Try to approximate two quarts or more a day.

\section{Pains and headache.}

Ice bag to head.

Aspirin (acetylsalicylic acid), gr. $\mathbf{v}-\mathrm{x}(0.035-0.066 \mathrm{Gm}$.) if needed. Avoid coal-tar preparations.

Codeine phosphate, gr. 1/8-1/4 (0.008-0.015 Gm.).

Lumbar puncture, besides diagnostic value, relieves headache and pains in extremities and stupor.

\section{Hyperesthesia and paresthesia.}

Warmth.

Raising of bed clothes from the skin by the use of frames.

\section{Irritability and restlessness.}

Bromides, gr. $\mathrm{x}-\mathrm{xxx}(0.66-0.2 \mathrm{Gm}$.).

Chloral hydrate in tentative doses beginning with gr. v (0.33 Gm.).

\section{Insomnia.}

Quiet, fresh air, attention to bed and toilet for the night.

Bromides, gr. xv (1.0 Gm.) early in evening. Repeat if needed.

Chloral hydrate, gr. $\mathrm{v}-\mathrm{x}(0.33-0.66 \mathrm{Gm}$.) early in the evening.

Codeine phosphate, gr. 1/8-1/4 (0.008-0.015) hypodermically.

\section{Fever.}

Rarely needs treatment.

If annoying, cool sponges.

\section{Convalescence.}

Long drawn out.

Fresh air.

Good food.

Massage.

Residual paralysis treated as under other circumstances. 


\section{CHAPTER XIV}

\section{TYPHOID AND PARATYPHOID FEVERS}

\section{TYPHOID FEVER}

Etiology. Typhoid fever, which is one of the commonest diseases in our experience, but less common than in the experience of our medical fathers, thanks to the interest elicited by the work of the research scholar and an awakened sense of civic responsibility, is due to the invasion of the body by a definite bacterium, Bacillus typhosus of Eberth. The gross lesions are referable to the intestinal canal but they are to be looked upon merely as local expressions of a general infection. This is an important therapeutic concept, as it directs our attention to the individual as a whole and spares us useless effort to medicate the intestinal canal, in the hope of curing the disease. The mortality varies in different epidemics and varies in private and hospital practice, but on the whole is in the neighborhood of 15 per cent.

The mortality has been greatly lessened in the Army and Navy where prophylactic vaccination is compulsory.

Pathology. Typhoid fever is a general infection, but its most marked expressions are to be found in the Peyer's patches and solitary follicles of the intestine, which are greatly infiltrated and swollen and may ulcerate with hemorrhage and perforation.

Symptomatology. This must be sought in its details in standard text-books of medicine. Briefly, its onset is usually insidious after an incubation of 10-14 days with a temperature increasing daily throughout the second week, falling in the morning in the third week and falling night and morning throughout the fourth week until normal is reached. Often subjective symptoms are curiously slight compared with the degree of temperature, which runs at $103^{\circ}-104^{\circ} \mathrm{F}$. during its height. Slow pulse, leucopenia, rose spots, enlarged spleen, tympanites, constipation are all characteristics; stupor, delirium and subsultus tendinum in severe cases. Hemorrhages from intestinal ulceration and perforation are dreaded complications. Relapses are not uncommon.

Tympanites may be due to any of the above sources; but milk sugar must be particularly suspected.

Diarrhea is more commonly caused by an excess of fat and cream, but may be due to lactose. 
Certainly, patients sufficiently fed, in my experience, look less toxic, are more content and emerge from their illness less wasted. The nervous manifestations seem to me less pronounced and my impression is that the common complication of hemorrhage and perforation are less frequently met with. Coleman's figures would show hemorrhages to be no less frequent, but decidedly less fatal and perforations more rare. $\mathrm{He}$ claims a drop in mortality from 17.6 per cent. to 8.10 per cent. under high caloric feeding.

Therapy. We are dealing with a self-limited disease, the cure of which depends on the elaboration of specific bodies by the tissue cells of the patient, a result we have not yet been able to duplicate in the laboratory, but which we can influence by the use of vaccines. This does not mean that we cannot further modify the course of the disease. On the contrary, a considerable fall in mortality has come about as the result of skilled care, conscientious watchfulness, competent nursing and the application of definite measures to the relief of symptoms and complications.

Rest. It would seem almost superfluous in a disease of such import as typhoid fever to insist on rest, but it must be remembered that the onset is slow, and in mild cases, or what at the beginning promises to be a mild case, the patient is prone to look upon it as an indisposition, and the physician may be uncertain of his diagnosis, and in the meantime the patient is on his feet, perhaps attempting to attend to his business, or at least protesting against the bed. These cases are the so-called "walking typhoids." Statistics have shown that these cases do not do as well as those receiving attention promptly, and it is probable that what might have been a mild typhoid attack has been converted, by lowering the resistance of the patient, into a severe or fatal case.

Where, then, suspicion is aroused as to the possibility of typhoid fever, the patient should not be permitted to waste his precious strength while one is awaiting the development of diagnostic symptoms or the result of blood examinations. He should be put to bed at once and prepared for what is likely to be a long siege. Real rest can be obtained only by careful and competent nursing, by the exclusion from the sickroom of all business cares and causes for anxiety, and refusal to make the sickroom a reception room for solicitous friends.

The patient is not to leave the bed to go to the toilet, or even to use a commode, but the bed pan is to be insisted on. This trouble comes in the early days of the fever. It will be a source of argumentation and contention, but one's dictum must be firm, backed up by a few words of explanation. The need for rest, the need of sparing the tissues useless oxidation, is emphasized by the length of the disease. 
Bed. While remembering to conserve the strength of the patient, it must not be forgotten that the strength of the attendant ought to be economized, and in no way can this be done to better advantage than in selecting a bed on which the patient can be readily handled. The ideal bed is the half-bed, or single bed, of a convenient height, with strong woven wire springs and a firm springy mattress. A folded blanket will render the surface smooth. Over the blanket, is to be placed a sheet, rubber sheet, and a draw sheet. Sheets should be drawn smooth, for freedom from wrinkles and dryness and cleanliness are great factors in preventing discomfort, and, in very sick patients, the formation of bedsores. Over the patient a sheet and a light blanket is sufficient; and one low pillow, not too soft, makes for comfort. The upper sheet should not be drawn too tightly over the feet of the patient, as is often done to improve the appearance of the bed, to the patient's great discomfort.

Room. The best room is the largest room, with the amount of light most gratifying to the patient; with sunshine in the colder months, and shade in the summer months. It should be well ventilated and free from encumbrances. A hardwood floor is best. If there are carpets, they should be protected in the vicinity of the bed, in consideration of contamination with secretions, which may occur in the ordinary handling of the patient. It is very desirable to have a bathroom near at hand, for the convenience of disinfecting and disposing of secretions, as well as for facilitating hydrotherapeutic measures. The room and bathroom should be screened against flies, whose contamination with the secretions scatter the infection.

If there is a balcony or piazza that may be approached from the room, it meets a great desideratum, for in summer the patient ought to be kept out of doors, and in winter I am convinced of the value of cold air in improving the nervous manifestations and general condition of the patients. I believe we shall use the open-air treatment for all infectious fevers with vast benefit.

The bed should be prepared by placing a blanket on the wire springs, and on this a rubber sheet, each extending well below and on either side of the bed. On these is placed the mattress, on which the bed is made as usual. Over the mattress and its coverings the blanket and rubber sheet are now folded like an envelope, thus preventing the entrance of air under the clothes. A hood is worn by the patient and a hot-water bottle placed at the feet; the nurses are dressed for out of doors.

When there is diarrhea and incontinence of the bowel or bladder, then more constant attention to the bed does not make the open-air treatment feasible. If the patient is very sick or is delirious, a nurse should be in the room day and night, as self-destruction has occurred in this condition. 
Care of the Body. If a properly trained nurse is in attendance, she will exercise all those niceties of her profession with reference to the care of the patient's body and comfort, which will usually improve on one's own suggestions, but it is unjust to the patient to take that for granted, and a quiet survey of the situation and inquiry into the methods used is proper. However, all nurses are not properly trained, and all patients cannot afford the attendanee of a nurse. Under these circumstances, one must know what should be done; one should write out explicitly what is to be done, and then see that the instructions are comprehended and successfully carried out.

The state of the patient's mouth requires constant attention, lest, from a foul condition, it become the source of infection of the mucous membranes and their lymphatic supply, the tonsils, the ears, and the lungs; and, upsetting the stomach, destroys the appetite. The teeth should be brushed two or three times a day. The mouth should be rinsed with water or boric acid solution ( 2 per cent. to 4 per cent.), or one of many mild alkaline and antiseptic washes, after each feeding.

Tongue. If there is a heavy coat on the tongue, such may be removed by careful scraping with the edge of a whalebone. Especial care must be taken to get at the back of the tongue and remove thick mucus from the throat. The cotton swab on a toothpick is a ready means of getting at the dead spaces in the mouth, where food may collect and decompose.

If there is much sordes, and there are fissures, a mild antiseptic containing phenol is of value. Here is one recommended for the purpose:

$R_{k}$

Phenol Solution, 1 to 20,

Glycerin....................... āa

Boric Acid, saturated solution..............

S. To be used as a mouth wash.

Bits of cracked ice are grateful, and the condition is much relieved if the patient gets enough water to drink. The nose is to be kept clear by the use of olive oil on a swab, to soften dried masses, and cleansing with boric acid solution or mild alkaline solutions. Whether hydrotherapeutic measures are pursued or not, the body is to have a cleansing bath with warm water and soap and the sponge.

Bedsores. The formation of bedsores is the bête noire of a good nurse, and this she avoids by constant care of the skin. Moisture about the buttocks is quickly removed, the parts sponged with alcohol or alcohol and water, equal parts, and, if the buttocks have been much soiled, with a mild antiseptic solution-phenol, 1 to 40 , for example. If pressure has been long continued, the skin is rubbed to improve the 
circulation in the part. The parts are then dusted abundantly with a drying powder, like any of the talcum powders used for toilet purposes. The skin of the whole back is treated in this way. Especial attention is paid to the sacrum, the anus, the buttocks, and the heels. The position of the patient should be frequently changed, not only to avoid bedsores, but to lessen the chance of hypostasis in the lungs.

If sores are threatening, rings are used to take the pressure off, but unless used intelligently, they may be more annoying than useful. If bedsores develop, it may be imperative to use a water-bed or air-bed. The excoriated skin may be covered with a drying powder, like aristol, or a bland ointment, like zinc oxide ointment. If more serious sores appear, they are to be treated on surgical principles.

At certain times and in certain places flies are a great annoyance to the patient, and, not only that, but a very real danger to other members of the family or vicinity, by their well-known ability to carry infection from the patient to the food or drink of those about him. Under such circumstances, screens in the room and over the patient are allimportant.

This leads naturally to a consideration of the disposition of the secretions and disinfection of the patient's clothes and utensils.

Disinfection. The stools, the urine, the clothes, the utensils, the bath water, the sputum, and the vomitus have all to be taken into consideration. Stools may be disinfected by 1 to 20 phenol. At least twice the volume of the stool should be used, the stool well broken up and allowed to stand several hours. Chlorinated lime, too, is used. It must be fresh, as it soon deteriorates on exposure to the air. A 1 per cent. solution is required; or, to avoid making exact solutions, a handful is put in the pan with the stool and enough water to cover and mix it with. Bichloride is not reliable for disinfecting stools. Urine is disinfected with 1 to 20 phenol, using one-third to equal amounts. The lesser amount has been shown to be effectual after one and one-half hours' contact. One to 1,000 bichloride, in volumes equal to $1 / 15$ to $1 / 40$ of the urine, standing for an hour and a half, is effectual.

Bath water may be disinfected by adding $1 / 2$ pound of chlorinated lime to a tub (200 litres) of water and allowing it to stand an hour.

Bed linen should be soaked in 1 to 20 phenol for two hours or in formalin, 3 ounces to a gallon, for twelve hours, and then thoroughly boiled. Bed pans, urinals, rectal tubes, and rubber sheets can be disinfected in phenol 1 to 20 and allowed to soak in it.

Knives, forks, spoons, and crockery can be thoroughly boiled. Sputum is best burned; vomitus may be treated with phenol and lime. 
Mattresses can be disinfected after the disease is over with dry heat, and the room should be disinfected.

The nurses should give tub-baths, wearing rubber gloves and a rubber apron. If given with the bare hands, and, indeed, after handling secretions, the hands should be carefully washed and soaked in 1 to 1,000 bichloride.

It must be remembered that the administration of hexamethylenamine (urotropin) by the mouth disinfects an acid urine, but we should not intermit the precautions just named.

Diet. Everyone who has treated typhoid fever has felt that the most urgent problem he has to meet is the dietary. He has consulted authority with the result that he has come away more bewildered than instructed. He has been urged to maintain a low diet, lest too great a burden be thrust on digestive organs and tissues weakened by disease, or damage be done to the inflamed intestinal wall with the production of hemorrhage or perforation; on the other hand, he has been taught to give the patient great freedom in diet, lest strength be sacrificed as the result of starvation. He has read arguments for liquid food, for solid food, for stimulating food, for bland food, until in his despair he chooses some guide with whom he is more or less well acquainted, copies his directions with slavish exactness, and proceeds to feed all patients by this measure.

We are dealing here with a fever and a long fever, during which oxidative changes must be met, either at the expense of food ingested or of the body itself, and during which extensive tissue destruction is going on as the result of the disease; this must be made good by the food or the deficit will increase continuously up to convalescence, or bring about. a bankruptcy, before convalescence can be attained.

The first great fact to be kept in mind in all fevers is that the body demands as much energy and heat in terms of heat units in fever as it does in ordinary rest in health; we will say 33 calories per kilo, or some 2,300 calories for a man of 70 kilos, or 154 pounds; and that as a result of the fever itself, he usually needs some 25 per cent. more, or about 40 calories per kilo, or some 2,800 calories for a 154-pound man. Therefore, he ought to get 2,800 calories to meet this need from his food, unless he takes it out of his own body.

The second great fact with reference to the body needs to be met by the food, is that there must be a certain minimum of protein in the diet. Voit's standard was something over 100 grams per day, but more recent work has shown that even less would do, but that some 70 grams should be afforded. This demands intelligent scrutiny of the food, with the knowledge of its composition, in order that this minimum should be 
observed; or, what is next in importance, that it should not be too much overstepped.

The third great body of facts, in dealing with diet in fever, are those which inform us of the loss of protein to the body in fever and how to shelter it by the food administered. The wasting after fever, even a short fever, and so emphasized after a long one, is one of the most familiar phenomena of disease. The wastage is, of course, due to loss of both fat and protein, but more especially, from the standpoint of importance, of protein.

The destruction in fever may be assigned to three particular causes: (1) Starvation. There can be no doubt that our patients are, in too many instances, underfed. That this cause for protein loss can in a goodly measure be prevented goes without saying. (2) Fever itself. Pyrexia exerts a destructive action on protein of the tissues, as can be determined by studying the nitrogenous output in fever induced artificially in animals, as in hot baths or by puncture of the floor of the fourth ventricle. In disease, however, it is not always easy to separate it from: (3) toxic destruction, meaning the breakdown induced by the toxins of the infecting organisms, in part autolytic, perchance, but the detail of which is still obscure.

The influences of pyrexia can, to a certain degree, of course, be influenced by hydrotherapeutic and other antipyretic measures. The effect of the toxins, until we have specific sera or other specific measures at our command, however, are not under our control ; and yet, it is surprising how near to a nitrogenous equilibrium a patient can be brought in the face of this toxemia, if supplied with the proper food in the proper manner.

I am indebted to the work of Dr. Shaffer and Dr. Coleman for many statements of facts made here, but they are not to be made responsible for any conclusions I may draw.

In the disease in question wasting is marked. A loss of 10 pounds is slight, but losses of $40,50,60$ pounds or more may occur. In this loss, nitrogenous tissue takes a large share. Cases in which the nitrogen loss has been studied, show the equivalent of 7 pounds of pure muscle tissue. in twelve days, of $51 / 2$ pounds of muscle tissue in eight days, and not rarely $11 / 2$ pounds of muscle tissue in a day. This loss may mean not only a deprivation to the body of the functions this tissue subserves, but also disturbances, perhaps toxic in character, induced by the effort. to catabolize this protein. The nitrogen partition offers similarities to those seen in the toxemias of pregnancy, and, to quote Dr. Shaffer's words, "there are severe so-called toxic cases of typhoid fever which terminate with acute yellow atrophy of the liver, a condition which ap- 
pears to be closely associated with a particular type of faulty protein metabolism."

This loss can be avoided, to a greater or less degree, by the food. In considering the foodstuffs, fat, carbohydrates, and protein, one would naturally turn to the proteins to make good a loss of proteins, and, indeed, that is necessary, but to no such degree as would be anticipated. There must be enough protein to meet the daily needs under ordinary circumstances - the 70 grams of which I spoke. There may be a slight storage of excess, which does not obtain in health, but if we give a frank excess, it must be destroyed and eliminated by the organism, and more than that in the process of its catabolism, by virtue of what is known technically as the "specific dynamic action," gives rise to a large amount of heat, which cannot be utiljzed for the purposes of the body, and extra burden is placed to dissipate the heat. The result is that about 140 calories of protein are required to secure 100 calories available for the purposes of the body.

Fat on account of its well-known tendency to induce digestive disturbances and diarrhea, must be used with this fact in mind and yet it is astonishing how much fat some of the patients will take without disturbance of any kind, and Coleman notes that this tolerance is especially enhanced by the third and fourth week as the temperature declines.

According to the best-known investigators in this field, the strongest sparers of body protein are the carbohydrates, and it is upon these that we place our reliance to solve the problem. It has been shown that the absorption of the fats, carbohydrates, and protein in typhoid fever, when severe diarrhea is not present, is very nearly that of normal.

In the feeding experiments carried out along this line of reasoning by Dr. Coleman among the typhoid fever cases in the wards of Bellevue Hospital the carbohydrate selected was milk sugar, because it ferments with difficulty and is less sweet to the taste than cane sugar and more soluble than the starches.

The experiments showed that a nitrogen gain could be attained when the protein intake amounted to 65 to 95 grams, while at the same time the caloric content of the food was high, 4,000 to 5,500 , or 60 to 80 calories per kilo; that is, enough protein was used to meet the daily needs and the loss of nitrogen was stayed by the high carbohydrate content of the food. When the same amount of protein was used with 32 calories per kilo, the loss was considerable. When only 16 grams of protein were used, but with 62 calories per kilo, the loss was greatest.

The basis of the high caloric diet as used in the wards of Bellevue Hospital is milk, cream, eggs, milk sugar, bread, and butter. A quart of milk affords 640 calories. A quart of cream (16 per cent.) about 1,600 
calories; each egg 80 calories; milk sugar about 120 calories to the ounce; white bread, homemade, about 1,225 to the pound-that is 100 calories to a thick slice of $1 \frac{1}{4}$ ounces; butter about 3,600 calories to the pou nd, or 100 calories in a pat a trifle under $1 / 2$ ounce. With such materials various combinations may be made: $1 \frac{1}{4}$ quarts of milk will give 1,000 calories, a pint of cream $800,1 / 2$ pound of sugar about 1,000 more, four eggs 320 more, thus making 3,120 calories. If one wants a few calories more, 250 to 600 can be gotten in 2 to 5 ounces of sugar added to lemonade.

A glass of milk that contains 7 ounces of milk, 1 ounce of cream, and 1 ounce of milk sugar, offers about 310 calories.

Eight such glasses in twenty-four hours will give about 2,500 calories, as high as many care to go. The protein, as well as the calories, in this food must be remembered. The milk contains 35 grams to the quart, and the cream a trifle less, 25 grams; the eggs 8 or 9 grams; the bread, about 9 per cent., or 45 grams to the pound loaf. In the more liberal dietary just suggested, about 100 grams are afforded; in the smaller, 70 . Two eggs added to this would increase the protein to 85 grams.

Dr. Shattuck, of the Massachusetts General Hospital, has long advocated a more liberal dietary in typhoid fever, and can point to quite as good results as the advocates of a restricted or milk diet. I will not cite his dietary in full, but will mention some of its items, which show one how varied we can make our patient's food and relieve a monotony that threatens a loss of appetite and gastro-intestinal disturbances. These are milk and the various milk products, some of which are better borne than others, such as buttermilk, koumys, matzoon, whey or milk with tea, coffee, or cocoa; or the milk can be diluted with lime water, Apollinaris, or Vichy; soups of beef, veal, chicken, tomato, potato, pea, bean, or squash, strained and thickened with powdered rice, arrowroot, wheat-flour, barley, or egg; gruels, ice-cream; eggs, soft boiled or raw, or eggnog; finely minced lean meat or scraped beef, soft crackers with milk or soups, soft puddings, soft toast without crusts, blanc mange, wine jelly, apple sauce and macaroni.

Many of these articles have a low caloric content, and would not serve the purposes of our new teaching, but they show what is safe and what may be used to diversify the dietary.

Each patient must be carefully studied with reference to his dietary. We can make a mathematical problem of his food needs, but not of him. If he is highly toxic and stuporous, if he has gastro-intestinal disturbances, we cannot expect him to ingest or digest the same quantity and quality of food that he would if his disease was of a milder type. We wish to avoid the older policy of frankly starving our patients, while we at the 
same time do not desire to stuff them like a Christmas goose, willy-nilly. I believe if the patient is moderately or severely ill when he comes under our observation, we should begin on milk for the first twenty-four hours, some two quarts; then a little milk sugâr, a dram to a glass, and rapidly more as he shows that he handles it well; and at the same time or shortly after, a little cream - a half ounce to an ounce to a glass, "watching this with especial care; eggs, gruels, or soups, bread or toast or crackers, ice cream, cup-custard, or other milk modifications. If the milk is not well borne, milk preparations, such as koumys, matzoon, buttermilk, or whey, may be used.

If the tongue is coated, if there are eructations, or if diarrhea sets in, the cream should be stopped and the milk skimmed. Of the meat soups, one may say that they have little caloric value, but may improve the appetite. The scraped or minced meats may be allowed, if greatly desired, but they are not an economical form of food in fever, owing to their high "specific dynamic action," and should not be given in the severe toxic cases, as it is believed, on fair grounds for such an assumption, that the liver in some of the cases is impaired and unable to metabolize the protein of the food brought to it, in the normal manner.

At any rate, in such cases the urea nitrogen decreases while the "rest nitrogen" increases, and the giving of meat aggravates the condition and furnishes us in the convalescence of severe cases the so-called "febris carnis," as Ewing and Wolff believe.

I believe a patient should be given as much food as he will take and handle well. If he will take 4,000 calories, he should have it; but with the earliest signs of gastro-intestinal disturbance or disgust for food, one should diminish the food, and do so promptly. The patient's appetite is a valuable guide, and within the range of food mentioned, his likes and dislikes should be considered. Briefly - an estimate should be made of the body's caloric requirements.

Food must be palatable and service attractive. Patients' tastes and food idiosyncrasies must be considered, but the diet must not be subservient to whims. The degree of toxicity of patients modifies the procedure.

If the appetite is large it may be gratified. If the appetite is poor, try to improve it by kind of food and service, but do not force food to the point of producing disgust. If there is frank disgust for the food cut it down for a time.

The desire for food in the first week and during the fastigium is diminished, with the decrease in fever, appetite improves and may be ravishing. The caloric intake should be built up during early days grad- 
ually to theoretical requirements, beginning with 1,000 to 2,000 calories, depending on general condition and increasing 500 calories a day, to 4,000 or 5,000 calories.

With the appearance of gastro-intestinal symptoms modify the diet. These symptoms are anorexia, nausea or vomiting, tympanites and diarrhea.

The disturbance may be due to forcing the feeding; cut down the quantity and lengthen the intervals. The nausea and vomiting may be due to too much sugar; or to too much cream, or eggs are not well borne.

For a veritable mine of information on this subject in both its theoretical considerations and practical applications, Dr. Coleman's article in the American Journal of the Medical Sciences, Jan., 1912, should be consulted.

See Summary for detail of dietary.

Water. Water should be given to the patient ad libitum and more than that, realizing that in his stuporous condition the patient's demands are no measure of his needs, water should be offered to the patient frequently, every hour or two. For this purpose the bent glass tube should be used, but in highly stuporous conditions the water must be administered with a spoon. A patient should be given 2 to 3 quarts of fluids a day and as much more as he will willingly take. Not only is the intake of water necessary for his metabolic needs, but the functions of kidney and skin are sustained by it, while the condition of the mouth is improved by it and constipation much lessened. Other drinks-lemonade, and orangeade and imperial drink may be given and these offer excellent vehicles for sugar, a highly important foodstuff.

Alkaline waters may also be used.

Hydrotherapy. If one had a brief to hold for hydrotherapy, he could not do better than to appeal to its application in typhoid fever. So thoroughly convinced is the American practitioner of its great value in this condition that arguments to maintain this thesis are only of historical value. The mortality of typhoid fever, vary as it may in different epidemics, hospitals, and localities, has, under the old expectant treatment, amounted to 15 per cent. the world over. Since the introduction of the cold-water treatment, where large numbers of cases can be collected, as in great hospital services, the mortality has been cut in half, running about 7.5 per cent. with wonderful uniformity in the hospitals of our larger cities. Statistics have no value unless applied to large numbers. For example, in a certain hospital 100 cases of typhoid were treated by the bath, with a mortality of 1 per cent. One might have attributed this success to some perfection of technique not prevailing elsewhere, but when the same clinician had 
collected 400 cases his published mortality showed 7.8 per cent., or that of the general experience.

The hydrotherapy of typhoid fever, as universally adopted in this country, is based on the Brand bath, or a modification of it, and the greater the modification, the less successful it seems to be. Two emphatic statements with reference to this bath should be made:(1) The essence of the bath lies in the application of cold water and friction, neither one nor the other alone, but both; (2) the purpose of the bath is to improve the condition of the poisoned centres and organs: the heart, lungs, kidneys, and centres in the brain and medulla; incidentally, and to a degree beneficially, to reduce temperature. The last is too often put first, which in this instance does not have biblical justification.

Method. The body should be immersed to the neck; the bath should be given preferably in a tub. In hospital practice, a portable tub on wheels is used. In private practice this is, as a rule, too cumbersome, and a tin bath tub may be used, elevated to nearly the level of the bed on wooden horses, blocks, or other contrivance. Placed alongside of the bed, the floor being protected by a rubber sheet, oilcloth, or carpet, it is filled three-quarters full of water, with buckets, or a hose attached to a tap, if one be conveniently near.

The patient's nightshirt is removed, his genitals covered with a napkin, bound about the body, or the whole body covered by a sheet, under which he is bathed, which latter method is a little more awkward. He is given a half ounce of whiskey, or a cup of hot strong coffee (4 ounces), the face bathed with cold water, a folded bandage a couple of inches wide, bound around the forehead and tied below the occiput, to keep the water applied to the head from running into the eyes and streaming down the face, and he is then ready to be lifted into the bath. To do this, the best way is for one attendant to rest the patient's head on one arm while he raises the upper part of the body by lifting under the shoulders, while another attendant lifts the lower extremities, the patient being requested to stiffen himself out.

Another method, which has advantages if the patient is very stuporous, is to spread a hammock netting under him or a strip of canvas with straps attached, and lift him on this into the tub and by the same means out of it. The head should rest on a water-cushion ring or air-ring.

As soon as the patient is immersed in the bath, the equally important part of the procedure is begun-the friction, gently applied (not kneading) to all parts of the body except the abdomen. The back must not be forgotten. At intervals during the bath, cold water, at $50^{\circ} \mathrm{F}$., should be poured over the head. While in the bath the bed should be prepared for his reception, by placing on it a double blanket, on the 
side he will occupy; a pillow is covered with a towel, the blanket is covered with a linen sheet, and hot-water bottles are placed at the foot.

The patient is then lifted onto the bed, the napkin is removed, and he is surrounded by the sheet, the edges and ends being tucked about the neck and under the arms. He may be allowed to lie in the sheet for five or.ten minutes and then dried with soft towels, or more immediately if the temperature is low and he is shivering. The tub is best emptied by a siphon.

The temperature of the water Brand set at not more than $70^{\circ} \mathrm{F}$., and not less than $65^{\circ} \mathrm{F}$. The frequency he advised was every three hours, if the rectal temperature was above $102.5^{\circ} \mathrm{F}$.; the length of the bath, fifteen minutes. These rules laid down by Brand have met with but slight modifications where satisfactory results are obtained. Slightly lesser temperature of water is used at times, a little higher temperature of the body taken as the indication of the bath, and the initial bath begun at a milder temperature; that is, the tub bath may be given at $80^{\circ} \mathrm{F}$., and in selected cases as low as $70^{\circ} \mathrm{F}$. the bath is continued fifteen minutes, and given every four hours, if the rectal temperature is $103^{\circ} \mathrm{F}$.

Sometimes the tub bath is given at $85^{\circ} \mathrm{F}$. for ten to fifteen minutes, at four-hour intervals, if the body temperature is $102.5^{\circ} \mathrm{F}$.

It is fully appreciated by the advocates of the Brand system that the reaction to a bath at $65^{\circ} \mathrm{F}$. cannot be anticipated in markedly toxemic cases, so that a patient seen in the third week or latter part of the second, who is very toxic, should be subjected to a bath of higher temperature $\left(75^{\circ} \mathrm{F}\right.$. or $80^{\circ} \mathrm{F}$.), and for a shorter time. Our hospitals rarely get patients in the first week, and the higher temperatures adopted are better suited to their class of cases.

If a tub bath cannot be obtained, a substitute for one can be constructed in the bed by running a clothesline around the bed, at a suitable height above its level, attaching a rubber sheet to it by clothes-pins, thus making a tub of the sheet. Other contrivances can be resorted to, to approximate a tub in the bed, as building around the sides and foot and head with rolled blankets, and the use of the rubber sheet as the receptacle for the water. When the patient is put in the water one expects a sudden shock, deep breath, and gasping. These are in themselves beneficial. The patient may shiver, the skin may be shrivelled, and the nails cyanotic, and yet not contraindicate the bath; but if the face is cyanotic, shivering marked, and chattering of the teeth occur, and the patient does not react well on being taken from the bath, then the next bath should be at a higher temperature and less long. Cyanosis of the face or threatened collapse should lead to immediate removal of the patient, warmth to 
the body externally and internally, and stimulants-hot coffee or hot whiskey.

Many untoward symptoms in the bath are due to failure to appreciate the value of, and failure to apply properly the friction during the bath.

The benefit derived from the bath is very obvious and is expressed by improvement in the functions of various organs. No benefit can be expected from any procedure applied to the moribund, and the greatest success in the treatment of typhoid fever by this method is in the early application of it. Many of the severe symptoms seen in cases coming under observation or treatment late, are not observed at all in the cases bathed the first week. The most characteristic effect clinically of typhoid fever is the toxemia impinging on the brain, inducing restlessness, sleeplessness, delirium, stupor, and subsultus tendinum. The stimulating effect on these centres, receiving sensations pouring in from every point of the periphery and nourished by a better blood supply, is shown by an amelioration of every one of these symptoms, better sleep and more quiet, and a clearer mind more alive to its environments.

In typhoid fever the pulse is slow; when rapid, ominous; it is often dichrotic, bespeaking a low vasomotor tone. We stand in dread of heart failure, but the opinion prevails to-day that in acute infectious diseases the heart rarely fails, but that the vasomotor apparatus is the first to falter.

After the tub, the pulse is smaller and harder, losing its dichrotism; the pressure increases by 15 to $20 \mathrm{~mm}$. $\mathrm{Hg}$., and the rate falls. All this is very real, and may be attributed, in all probability, to the effect exerted on the vasomotor centres, though the tonic effect on the vascular walls of the periphery is undoubtedly a factor.

On the respiratory apparatus a beneficial effect is exerted by the fact that the contact with the cold water causes the patient to take deep breaths, which lessen the danger of passive congestion and hypostatic pneumonia that threaten in severe cases. More than that, the respiration of the tissues is enhanced, as increased oxidation can be demonstrated by measuring the oxygen intake and the $\mathrm{CO}_{2}$ output.

The contact of the cold water and the friction improve the circulation in the skin and so improve its functions. The improved condition of the circulation increases the elimination of urine and the toxins of the disease. The temperature is lowered, but this is no longer considered the object of the treatment, and often in the height of the disease the temperature is not influenced, but striking improvement in the general condition is attained, in terms just given.

After all, it is the individual that is being treated, and this means 
that a precise procedure cannot be the optimum for every case. Higher temperature of the water and shorter baths are required for those who stand the shock of the typical bath poorly, for those toxic and advanced in the course of the disease, and for children.

Contraindications for the baths comprise hemorrhages and signs attributable to perforation or peritonitis, all of which conditions demand absolute rest until the proper procedure, surgical or otherwise, can be carried out. Cholecystitis, phlebitis, pleurisy, if accompanied by considerable pain, contraindicate the bath for a like reason. Cyanosis and syncope demand cessation of the bath in which they occur, but if the case is a severe one, the use of water may be continued in a milder form of application. Large bedsores, because of the difficulty of properly treating them, contraindicate the bath. Bronchitis, pneumonia, the milder grades of kidney involvement, and menstruation and pregnancy do not call for a change of procedure.

Ziemssen's Bath. Beside the full bath of Brand, there are certain other methods of applying water in this disease. A slight modification of the Brand bath is the so-called graduated bath of Ziemssen. The details are the same as for the Brand bath, except that the water is warmed to $90^{\circ} \mathrm{F}$., and then, after the patient is in the bath, is cooled down to $70^{\circ} \mathrm{F}$. by adding cold water as the bath proceeds. The bath lasts half an hour with friction. This is a suitable procedure for those who stand the bath poorly, or think they do, and refuse the colder bath, or the very toxic cases of the later stages, or children.

Slush. When, for any reason, it is not feasible to use the tub an approximation to the methods and results of a tub are attained by what is sometimes called a slush. The patient is stripped, a rubber sheet of ample proportions is put under him, the edges of which are elevated by pillows and blankets folded to the formation of a trough. Water is poured into this trough and the patient given a bath with friction, as in a tub, while cold compresses are kept at his head. The water, of course, becomes warm rapidly and is kept cold by adding bits of ice. The temperature cannot be so accurately regulated as in the tub. The head of the bed is elevated, the water drained off, the patient rubbed with a little alcohol, or alcohol and water, after drying and removing the rubber sheet, and then dusted with talcum. These slushes may be very effectual when properly given.

Packs and Sponges. When the baths are refused, or for other reasons cannot be given, packs and sponges may be substituted; but, while these afford comfort and do some good, they fail in the general stimulating effect that comes from the friction of the body immersed in cold water. 
Care of the Alimentary Tract. In the routine treatment of typhoid fever there are no organs whose functions have to be so jealously guarded as those of the alimentary tract, not only because failure of digestion and absorption threaten a fatal issue in so long a disease, but because insufficient elimination and excretion heighten the toxemia, and because diarrhea exhausts and hemorrhage and perforation await upon the advance of the local lesions. Constipation is almost constant in typhoid fever as we see it in New York. Diarrhea, which one might gather from any of the text-books as characteristic, is rather the exception.

Catharsis. If seen early, a catharsis of castor oil, 1 ounce (30 c.c), or Epsom salt, 1 ounce $(30 \mathrm{Gm}$.), may be given. If seen late, a catharsis should be avoided. Enemas of tepid water, of soapsuds, or, in obstinate cases, soapsuds with an ounce of sweet oil, or castor oil, should be given every other day. It is the lower bowel one wishes to empty, not the upper, and this can be done effectually with the enema. The upper bowel, the site of the lesion, like any other inflamed part, should be given as much rest as is compatible with its physiological functioning.

The lower bowel is physiologically differentiated; the lower part forming the reservoir for undigested food, and, what is more important, the excreted matter, the nitrogenous content of which forms an almost constant portion of the total nitrogen output; it is, too, the site of active bacterial action, and is an absorbing surface. We wish to avoid absorption of toxic material of bacterial action, which may add its burden to the already overloaded blood. Nothing is more surprising than the large stools obtained from patients on a milk diet, and still more, the considerable movements obtained when patients are taking little or no food. The patient's plea, to avoid the enema, that he has taken nothing, therefore, can have nothing to evacuate, must be met by explanation of the physiological fact and demonstration of its accuracy.

Tympanites, to some degree, is almost constant, but when marked is usually the expression of the severity of the intoxication. This paretic condition of the bowel, which allows the collection of large quantities of gas, is rife with danger, as it increases the chances of hemorrhage and perforation, and interferes with respiration and cardiac action; pain may be an accompaniment. The free use of water, the attention to periodical evacuation of the bowel, and the general tonic effect of the baths, lessen the severity of this complication.

If milk sugar is being used freely, this should be suspected and cut down or eliminated until its responsibility is determined and the condition relieved. 
When in spite of such precautions, it does obtain, the following measures may be carried out:

1. Introduce for a distance of 12 to 15 inches into the bowel a soft, pliable rubber catheter, taking great care not to use force. The larger bowel is, as a rule, more implicated than the smaller. This will at times permit the escape of considerable amounts of gas. It should be left in ten to twenty minutes, or even a half hour, and may be repeated every two to four hours, as indicated. Turning the patient while the tube is in place may facilitate the escape of gas.

2. The use of stupes. Two or three thicknesses of flannel are wrung out of hot water, as dry as possible, and applied every three or four hours. The technique is as follows: A flannel roller is put under the patient, the abdominal wall smeared with vaseline, the stupes applied, the edges being turned under to prevent dripping; oil silk applied, and the roller brought up over the stupes in the manner of a binder. This should be changed every two or three minutes for ten or fifteen minutes, or every ten or fifteen minutes, for several hours.

3. Turpentine. There are three ways of administering turpentine to relieve this condition: First, in the stupes, by adding a dram of turpentine to the hot water into which the flannels are to be dipped; by sprinkling the stupes with turpentine; or by very lightly passing a bit of absorbent cotton wet with turpentine over the abdomen before applying the stupes. Care must be constantly exercised not to irritate or burn the skin. Careless application of the turpentine will certainly entail such trouble. The second method is by giving turpentine enemas. One-half to one ounce of turpentine is to be used in one pint of soapsuds enema. The method is as follows: Take a little hot water and a piece of castile soap; make a thick soapsuds; add the turpentine slowly, constantly stirring. This makes an emulsion. Add the pint of water, and the emulsion remains stable. Oil enemas are made in the same way. The third method is by the mouth; 10 to 20 drops are used every four hours. It is sometimes dropped on loaf sugar, but the turpentine makes the sugar tough and not easily soluble. It is better given in capsules, 10 minims each $(0.60$ c.c.), or in emulsion, to which a little oil of cinnamon may be added as a flavor, while at the same time it adds to the effect of the turpentine. Thus:

R

Olei Terebinthinæ Rectificati...........10

Olei Cinnamomi, q. s. or............ 0.1

Acaciæ............................. s.

Aq. Destillatæ q. s. ad.............60.0

3 iiss.

gtts. ii or q. s.

q. s.

M. et fiat emulsum

筩

Sig.-One or two teaspoonfuls. 


\section{Milk and Molasses Enema. An expedient often used for tym-} panites after operation is the milk and molasses enema. This is made by adding 8 ounces of milk to 8 ounces of molasses which has been previously warmed, both to make its consistency thinner and to obtain body temperature. This is slowly introduced through a rectal tube inserted not more than 6 or 8 inches.

Peppermint Enema. A fairly effectual enema in tympanites is one in which 1 or 2 teaspoonfuls of spirits of peppermint are added to the quart of warm saline or plain water. (Dr. T. F. Goodwin.)

Pituatrin. Pituatrin, which stimulates the smooth muscle of the intestinal wall, may be used hypodermically in obstinate cases. This substance may be obtained in ampoules ready for hypodermic use. The amount given is usually 1 c.c.

Sometimes a large flat ice bag or the ice coil, brings relief, and if all these measures fail, a hypodermic injection of physostigmine salicylate or sulphate $(1 / 50$ grain) $(0.001 \mathrm{Gm}$.) may be tried. The diet should be materially decreased, milk stopped, and albumin water administered, and water given freely, if the condition is severe.

Diarrhea, fortunately, is not a common complication in typhoid as we see it here. Two or three loose movements a day do not call for interference, except in the matter of regulation of food. The food and neglect of the bowel may be the inciting causes. If the patient is on a milk diet, the fat is more likely to be responsible for the diarrhea than any other constituent of the food, and should be removed. Scalding the milk may help. If the diarrhea is severe, the food should be cut down. Of the astringents, bismuth subnitrate is the best, and should be given in sufficient doses ( 30 grains) $(2 \mathrm{Gm}$.) every two or three hours. This must not be kept up too long, or if administered over a long period, it must be remembered that the bismuth may be retained in the bowel in such quantities as to produce irritation by sheer weight, while the diarrhea still continues; hence, the necessity for irrigations.

Prolonged saline irrigations, by improving diuresis, lessening toxemia, and keeping the bowel clear, may be of distinct advantage. It is tempting to use opium, but it cannot be too emphatically insisted upon that by so doing, pain, the earliest and most important symptom of perforation, may be lacking - to the patient's undoing. It should not be used unless exhaustion from diarrhea and increasing danger of hemorrhage and perforation from peristaltic unrest seem to demand it. Then one may give a few large doses, rather than longcontinued small ones; for example, a pill of opium (1/2 grain) $(0.030$ Gm.) every two hours for three of four doses, or some such marked astringent mixture as one of our well-known clinicians has advised: 
R

Pulv. Opii..................... 0.25

Pulv. Camphoræ.................. 1.00

Plumbi Acetatis.................... 3.00

Bismuth. Subnitrat.................. 30.00

M. et div. in chart no. xv.

Sig.--One every four hours.

(gr. iii $3 / 4$ )

(gr. $\mathrm{xv}$ )

(gr. xlv)

(气 j)

Gastric distress and vomiting are not common. If vomiting does occur, both fats and milk sugar should be suspected and these eliminated from the diet until the symptoms subside and then reintroduce cautiously. Cracked ice, in small pieces, sucked, may be of avail. Then try a mild mustard paste to the epigastrium, one part in four of flour. If these do not succeed, try a bland powder of cerium oxalate (3 grains) $(0.20 \mathrm{Gm}$.), sodium bicarbonate (5 grains) $(0.30 \mathrm{Gm}$.$) , and bismuth subnitrate (10 grains) (0.60 \mathrm{Gm}$. every two hours. If very severe, cocaine hydrochloride (1/5 grain) $(0.01 \mathrm{Gm}$.$) , in tablet form, or one teaspoonful of 0.1$ per cent. solution every two or three hours. Finally stomach washing may succeed when other measures have failed.

Hemorrhage. Few things are more trying than meeting the danger arising from hemorrhage and perforation. Few occurrences demand more judgment and common sense than treating the hemorrhage of typhoid. To judge by the advice given by some authors, the appearance of hemorrhage is to be treated like an invading foe, to be met with every weapon at our command, from stiletto to bludgeon; while more timid counsellors urge letting it severely alone, lest by interference we come off worse than before. It is interesting to note that many competent clinicians both of the past and present have conceived the idea that in non-fatal cases of hemorrhage actual benefit accrued to the bleeding, in terms of improvement in pulse, temperature and general condition, and Rudolph finds authority for the statements that bleeding brings about an increase in the urine, increases the intake of oxygen, hastens coagulation time and enhances the production of anti-bodies in the blood. These facts have suggested venesection in severe cases of typhoid and the measure has actually been carried out, but-with what benefit I am not competent to state. In the first place, in the early stages, a little blood may appear in the stools, resulting from an oozing of the hyperemic patches, and such small amounts at such a period need no treatment. In the latter part of the second week, and during the third, we are anticipating the hemorrhages of real significance from the ulcers and know that they do occur in about 7 per cent. of the cases. If the hemorrhage is mild, that is, small in amount, or well borne 
by the patient, we may have no premonition of it until the stool shows the red blood of the recent bleeding, or the tarry stool of one occurring some hours before.

In these cases, the present bleeding is of less importance than that which may occur later, so that our treatment is largely prophylactic. We cut down the food, we enjoin absolute muscular rest on the part of the patient, and turn him gently when required.

We may apply a light flat ice bag or coil to the abdomen. We may give calcium lactate in doses of 10 grains $(0.60 \mathrm{Gm}$.) three times a day, and in the light of our recent knowledge of the effect of horse serum on coagulation, we may give a dose of 10 to 20 c.c. of it; or, failing that, diphtheria antitoxin in similar amounts. To get the best results from the serum, it should be as fresh as possible. The old serum loses much of its potency in this respect. If fresh horse serum is not available, fresh rabbit's serum may be used. Human serum is the best of all (Welsh).

Antitoxin, because it is likely to be old, is less valuable, and when a concentrated antitoxin, made from the globulins, of no use at all. The usual test for sensitization for horse serum should be made and the history of the past serum administration or asthma investigated. If sensitization is determined, desensitization must be effected. (See Pneumonia, Chap. IX.)

An initial dose of serum after a matter of ten days might possibly establish an anaphylaxis, and this precaution is doubly necessary, when a second dose is given after such an interval. Immunity against anaphylaxis may be established by giving doses of serum-full doses-every day, or second day.

More recently thromboplastin, which is usually prepared from brain tissue (Kephalin), and is a coagulant when locally applied to a bleeding surface, has come into use. It is furnished commonly in vials containing 20 c.c. This should be poured into eight ounces of water and given by mouth several times a day.

If, however, the hemorrhage is severe, as shown by the large quantity of blood passed by the bowel, and even more if general symptoms of hemorrhage prevail, with or without the passage of blood-that is, sudden fall of temperature, pallor, cold sweat, cold extremities, rapid thready pulse, restlessness, and air-hunger, more decided measures must be taken. There is one drug that is of greater value than any other, namely, morphine. The sulphate is to be given in doses of $1 / 4$ grain $(0.015 \mathrm{Gm}$.) under the skin. Morphine is not a styptic, nor has it any effect on the calibre of the vessels or coagulation time, but what it does do is to afford the maximum amount of rest, both to the bleeding bowel 
and to the anxious brain, and to the restless and uneasy body, whose useless movements keep up the blood pressure. This gives the bleeding vessel a chance to do what it is trying to do-form a clot. It is pointed out that perforation occurs in these hemorrhages-one-fifth of the perforations; and that the use of opium blinds the symptoms, and no doubt it does, but, while anticipating such a complication, we cannot sit by and see our patient die from the hemorrhage.

There are many cases that come between these two extremes, and success in those cases is the fruit of experience and judgment. One can only say, when a patient is to be under constant observation for some hours to come, as he ought, hold off the opium as long as possible. If circumstances will not permit remaining by the patient, one should not spare the opium.

The bowels should not be moved for some three days after a hemorrhage, and then with caution. One should get back on the diet slowly and combat meteorism.

When the hemorrhage causes exsanguination, it must be treated like any other hemorrhage-elevation of the foot of the bed, heat to the extremities, saline infusions of 0.9 per cent. sodium chloride solutions, or better yet, Ringer's solutions or transfusion.

The newer methods of transfusion as described by Lindeman (see American Jour. Diseases of Children, Vol. 6, No. 1, July, 1913) and others have so simplified transfusion and so robbed it of the dramatic that the process should be used whenever hemorrhage is repeatedly persistent or dangerously severe.

The essence of the procedure is a rapid transferal by syringe from the vein of the donor to the vein of the patient, or blood is withdrawn into an Erlenmeyer flask or similar receptable in which there is a little potassium or sodium citrate. It may later be transferred to the vein of the patient. (For technique, see Scarlet Fever, Chap. XVII.)

Tests of the blood to determine its congeniality to the recipient must be made first, lest hemolysis ensue. The method is so simple that the transfusion can be repeated as often as needed. Moreover, there are reasons for believing that in a healthy individual a certain amount of anti-bodies are present and certainly complement, which may be diminished in the patient's blood and its replacement thus have an additional beneficial effect on the patient.

Perforation. Even more dangerous than hemorrhage is perforation. It occurs in 2 to 3 per cent. of the cases and causes some 12 per cent. of deaths in typhoid fever. It seems to occur as frequently in cases treated with the bath, but because of the lower mortality of the disease so treated, the percentage of death from perforation rises. If 
one in eight or more of the deaths in typhoid fever are due to perforation, it becomes a moral obligation to know the signs well. One will recall the sudden onset, with acute abdominal pain, like a bolt out of the blue; its paroxysmal character, the rapid rise in the leukocytosis, the two most important early symptoms, I think; then the change in respiration, rapid pulse, distressed expression, local muscle spasm, rigidity, tympanites, with, perhaps, obliteration of the liver dulness.

Every Attack of Abdominal Pain should mean perforation until continuous observation convinces otherwise, for thus its occurrence will not be overlooked. When it occurs, the surgeon should be summoned, an operation performed, and the perforation closed. If satisfied that pain has no such significance, one should consider the possibility of acute cholecystitis. Carter, studying 1,815 cases of typhoid fever, found that in one in 75 abdominal pain had this explanation. It required the same consideration as under other circumstances and may or may not need surgical intervention. It must not be forgotten that perforation can occur in these cases. In three of Carter's cases the pain was due to appendicitis. If the condition is not one requiring surgical intervention then the ice bag or stupes, or a light application of the cautery or small doses of bromides or codeine may be used; or, if more severe, small doses of morphine.

The cerebral disturbances are characteristic of this disease. In the early stages there may be much headache. This is best treated by the ice cap. Sleeplessness is another common occurrence, and, as has been explained, in considering rest, a symptom to be combated.

Hydrotherapy properly applied mitigates this to a great degree. Small doses of bromides may be ased, or trional in 15 to 20 grain doses, or chloralamid in 20 to 30 grain doses; but if it is marked and prolonged, morphine is the best drug to use. Delirium and stupor are best met by the baths. In all decided cerebral symptoms help may be obtained from the tepid bath, with cold water poured over the head; from the ice-bag, from morphine, and, finally, when the delirium is great or the stupor deep, from lumbar puncture, an easy and innocent procedure. Draw off 20 c.c. at least, and more will do no harm, though headache may be aggravated by draining the cord. No delirious patient should be left alone for a moment. Restraint by sheets or other devices are sometimes needed.

Circulation. When the characteristically slow pulse of typhoid fever becomes rapid, going above 100 to 110 to 120 , our concern is elicited. If the circulation is weakening, we shall get a poorer quality of first sound of the heart, perhaps evidences of dilatation and mitral insufficiency, poorer quality of pulse, and a fall in blood pressure. A 
sufficiency of diet, fresh air and hydrotherapy do much to prevent this disaster. If, however, the condition supervenes, stimulants must be given.

As in other infectious diseases the vasomotor apparatus is in the great majority of cases at fault rather than the myocardium and for this reason vasomotor stimulants would seem logically to be indicated. In cold water as applied in the Brand bath we have such a stimulus and in cold, dry, open air another, but all our drugs, classed as vasomotor stimulants are weak assistants, their effects, as a rule, not marked and their action short lived. Personally, I am much more impressed with the digitalis series even when the myocardium is not involved and much more when it is, and I prefer to give them in large quantities when the circulation falters. I give the infusion 3 ss. (15 c.c.) three times a day or the tincture $\mathrm{m}$. $\mathrm{xxx}(2$ c.c.) three times a day for three or four days, or if the patient is under close observation longer, until either improvement in the symptoms occur, better quality of heart sounds, better pulse, improved blood pressure or evidences of accumulation in slower pulse or irregularity of pulse or vomiting or diarrhea ensue.

In urgent circulatory distress I begin with $1 / 2$ to $3 / 4$ milligram (gr. 1/120 to gr. 1/90) of strophanthin into a vein or muscle and begin the digitalis at once.

If one hesitates about these doses $\mathrm{m} . \mathrm{x}(0.66$ c.c.) of the tincture or $3 \mathrm{i}-\mathrm{ii}$ ( $4-8$ c.c.) of the infusion may be used three times a day, day in and day out, but pressing demands are not met by these small doses.

For those who take issue with my use of digitalis in these cases or if satisfactory results do not follow such usage, I recommend as vasomotor stimulants a soluble salt of caffeine, either the double salt of caffeine and sodium salicylate or benzoate in doses of gr. v (0.35 Gm.) into the muscle every four, three or two hours or camphor in solution in oil (sesame or olive) 10 per cent. or 20 per cent. in doses of gr. v (0.66 $\mathrm{Gm}$.) into the muscle at four, three, or two hour intervals; or the caffeine and camphor may be alternated at two hour intervals.

I have less faith in strychnine but it is recommended in doses of the sulphate of gr. 1/40-1/30 (1 $1 / 3-2$ mg.) every three or four hours.

In threatened collapse adrenalin epinephrin 1:1000 may be used in doses of $\mathrm{m} . \mathrm{xv}$ ( 1 c.c.) into a muscle or $\mathrm{m}$. iii-iv $(0.20$ c.c.) into a vein and followed up by caffeine, camphor and strophanthin.

Alcohol requires a special discussion. Briefly, I do not consider it a stimulant in the true sense of the word. Viewed from a chemical, pharmacological, or therapeutic standpoint, I think the weight of evidence is against its stimulating properties. It is hard to conceive that this member of the marsh-gas series, all of whose other members-alkanes, 
alcohols, aldehydes, ketones-are depressant, should furnish this one exception, ethyl alcohol; or two, ethyl alcohol and ethyl ether, which are stimulating. The results of animal experimentation are decided for the depressant êffect of alcohol, while I am convinced that the trend of therapeutic opinion is increasingly in the same direction.

That the stimulating effect of alcohol on the mucous "membrane of the stomach may reflexly stimulate the circulation is left sub judice; that alcohol has a food value is scarcely denied, and, no doubt, has contributed to the patient's strength when the food is insufficient; but neither of these effects are justification for the use of alcohol as it has been administered in typhoid fever. In the so-called typhoid state, characterized by cerebral excitation, sleeplessness, rapid pulse, dry skin, coated tongue, and subsultus tendinum, the picture, to my mind, is one of hyperexcitation of the nervous centres by the toxins of the disease. I believe that the improvement seen in this condition by the judicious use of alcohol is due to the sedative effect of the drug on these centres and not to stimulation. This conception confines the use of alcohol to this stage or state of the infection and decries its use where any of the functions are failing from exhaustion. Personally, I would rather not use it at all in typhoid fever.

The urinary tract is not to be neglected. In the early days of the disease especially, the patient's bladder may be overdistended. Close observation should be directed to this organ, and with the first evidences of retention, efforts should be made, by hot applications over the hypogastrium or by hot enemas, to provoke urination. If such measures fail, catheterization must be done, but should be done by the physician, to avoid by his care the infection that so frequently follows this procedure. Overdistention means stagnation, weakening of motility, and lowered resistance to infection by pathogenic organisms and invites a bacilluria. Bacilluria is a very real menace, more to the community than to the patient himself, as true cystitis or pyelitis of typhoid origin is rare. It occurs in nearly 25 per cent. of the cases, often in such numbers as to render the urine turbid. It is most common as the temperature begins to approach the normal and runs into convalescence. It probably arises by infection through the kidney, the bacilli finding a good culture medium in the urine, especially when the urine is of a low acidity and there is any stagnation, such as might result from neglect of overdistention.

The bacilli tend to disappear spontaneously, probably at such a time as the urine becomes a less favorable medium and the improved tone of the bladder facilitates mechanical removal. However, the condition may remain for weeks, months and, in a few cases, for years. It has been 
shown that a high degree of acidity, and especially an increasing content of organic acid, inhibit their growth. The effort should be made to eliminate them, as a prophylactic measure, for absence of local symptoms make the convalescent an innocent menace to the community. Hexamethylenamine (Urotropin) has been shown to have an astonishing effect on their growth, sometimes clearing up a turbid urine in a day or two. It may be given in doses of 5 to 15 grains for two or three days, a week, or continuously, beginning late in the disease and continuing into convalescence.

A good rule would be, beginning when the temperature approaches normal and continuing for a month after, to give 5 to 10 grains of urotropin (hexamethylenamine) three times a day for three consecutive days each week. One must not forget that some patients show a marked intolerance to this drug in terms of hematuria, marked frequency of urination and pain. It must then, of course, be stopped. When this does not clear up the bacilli, or a true cystitis prevails, bladder irrigations of silver nitrate, 1 to 5,000 daily, or a saturated solution of boric acid, should be instituted.

Vaccines. It is still difficult to estimate the value of vaccine therapy in the treatment of an attack of typhoid fever. That most extraordinary results, one might say dramatic, do occur in some cases, I am myself a witness to; but the results are not constant and the reactions severe, and at times alarming; though I have not witnessed in my own experience any unhappy outcome as a result. Gay has been an advocate of this mode of treatment.

It must be understood that the vaccines in this instance should be used intravenously as the subcutaneous method has not been found equally effectual.

Gay used numerous strains (polyvalent) of B. typhosus which were sensitized, that is, treated with immune serum, washed, killed and precipitated by absolute alcohol, dried to constant weight and ground and endotoxins extracted with carbolated saline and the sediment used. $\mathrm{He}$ conceived vaccine thus prepared to be less toxic and to give less severe reactions than the whole bodies of the organisms.

Others have used the whole bodies of the organisms. My experience has been entirely with these latter. The reaction is the same in kind, however much it may differ in degree.

The reaction consists of a chill coming on a half hour to one hour after the injection, lasting a varying length of time up to a quarter of an hour and accompanied by a rise of temperature of one to three degrees and sometimes to $105^{\circ} \mathrm{F}$., $106^{\circ} \mathrm{F}$. or even higher, which in the more exaggerated cases would indicate too large a dose; the fever is at its 
height in about three hours. 'The pulse accelerates, there may be a little cyanosis and respiratory distress and the blood picture shows a leukopenia to even 2,000 to 3,000 at the height of the fever. In about 12 hours the temperature has fallen to subnormal, accômpanied by sweating, and a general amelioration of symptoms, such as headache and delirium, and the patient may feel perfectly well for the time. The leukopenia is followed by a leucocytosis to as high as 40,000 and a relative polymorphonucleosis.

The beginning dose is $25-50$ million, the repetition depending on the results obtained. If there is no reaction, it may be repeated in two days. If there is a severe reaction, the dose is not increased at the next administration. Otherwise the second dose may be increased 50 per cent. to 100 per cent.

Results. In Gay's series of about 100 treated with his sensitized vaccines just about one third were aborted, one-third benefited and one-third unaffected. The aborted cases averaged a duration of 7 days, those that were benefited 15.8 days and those not benefited 33.1 days.

The mortality in the series was 6.6 per cent., the complications were few, 4 per cent. had hemorrhages and 2 per cent., perforations. Relapses occurred in 10 per cent.

Drugs aimed at the disease itself, intestinal antiseptics, etc., have been left out of consideration, because it is believed that, on the one hand, no good results are to be obtained, while, on the other, harm may be done by the drugs themselves and by the neglect of the more useful measures advised, entailed by reliance on the drugs.

Complications. Bulky monographs have been devoted to the complications of this disease in both medical and surgical fields. In general these complications are to be treated as they would be if primary or under other circumstances.

I will mention among the more common,

Phlebitis. To be treated by rest (fixation of the limb) and application of thick layers of non-absorbent cotton (a dry poultice) or wet dressings such as aluminum acetate or poultices frequently applied.

Conner believes thrombo-phlebitis to be more common than ordinarily accepted, in 10 per cent. to 15 per cent. of all cases and not always recognized. Obscure recurring chills, rises of temperature in convalescence, prolonged and irregular types of fever he believes to be due to such unrecognized phlebitis.

Tender toes is another of its manifestations and, more important, the pleural and pulmonary complications occurring late in the course.

Meningismus is not uncommon in typhoid fever as is the case 
in other severe infections; for example, pneumonia; but though very rare, a true meningitis due to bacillus typhosus may occur as well. The diagnosis is established by the spinal fluid findings. The treatment of both conditions is symptomatic; probably repeated tappings afford some amelioration of symptoms. For further treatment of symptoms see Cerebro-Spinal Meningitis, Chap. XXV.

\section{TREATMENT OF CONVALESCENCE}

After the temperature has been normal for a week or ten days, the patient may be propped up in bed, and in three or four days more be allowed to sit up in the chair, a little longer each day, and if all goes well, in a week he may be allowed to try his feet.

A slight rise in the temperature, day after day, is one of the most nagging features of a convalescence. This may be due to absorption from a neglected bowel, or it may be due to insufficient food, and eminent clinicians long ago pointed out that these patients' temperatures returned to normal when solid food was given them. The older clinician will remember the extreme caution with which each article of food was given the patient in convalescence after a milk or starvation diet and the constant retreats and modifications with every little rise of temperature.

With the patient on a sufficient diet during the fever we have no such problem to deal with. The patient passes into convalescence on a liberal diet, which is maintained to make up losses sustained by his toxemia. Others mend rapidly when kept in the air and light.

Every case must be approached on its merits, and if the pulse shows by its rate and poor quality that the demands on it are too great, the rest must be prolonged and the getting up be more gradual. To get the patient out in the sun and air is certain to facilitate the progress of his convalescence. During this time his bowels must be attended to with the same care as during his illness in bed, at first aiding with enemas, and later with a mild cathartic like cascara. If there is a great deal of anemia, Blaud's pills may be given, 5 grains $(0.30 \mathrm{Gm}$.), three times daily. If the weakness is prolonged, strychnine, $1 / 40$ grain $(0.0015 \mathrm{Gm}$.), three times daily, may be administered.

After the patient has been restored to a considerable degree of strength, he should still be forbidden to return to work too early. A long vacation, if possible of three to six months, should be taken in other surroundings.

Prophylaxis. Typhoid fever is a preventable disease. Its prevention awaits upon the overcoming of an inertia. McCrae divides 
the subject under three heads: (1) General measures, which are for the protection of the community; (2) special measures in connection with the patient; and (3) preventive inoculation. The knowledge that the disease is conveyed through the water, milk, food, especially oysters and green vegetables eaten raw, demand the supervision of the State and the community, through Boards of Health and Sanitary Commissions, supervision of the water supply with reference to policing the water sheds, filtration plants, and all the details dictated by sanitary science. It demands supervision of the milk supply, inspection of the dairies, health of the workers, and a consideration of sewage disposal with reference to oyster beds. It demands notification by physicians to Boards of Health of all cases of typhoid or suspected typhoid fever occurring in a community, and it demands education of the public through schools, lectures, and exhibitions of the part these various factors play in the prevention or spread of the disease.

In connection with the patient arises the question of isolation. Here in New York, as pretty much everywhere else in this country, typhoid fever is treated in the same wards as the general run of cases, and we assume from our familiarity with this condition of affairs that there is no danger in the practice. It is a little startling, then, to be told that the statistics of one of the best conducted hospitals in this country show that from 1.5 per cent. to 2 per cent. of the cases of typhoid fever treated in this hospital are of hospital origin. I have seen this thing occur in my own wards, and so has every attending physician, but still our patients are not isolated. As regards the disinfection of stools, urine, sputum, vomitus, and care of clothing and utensils, all this has been touched upon. If we were faithful in carrying out these measures in every case of typhoid fever, there soon would be no more cases, for, after all, the patient is the true source of typhoid fever.

The attendants about a patient are sources of infection for others unless conscientious in their cleanliness. A great source of danger that might be overlooked are the flies, carrying the infectious material direct on their legs and bodies from the excreta to food and water. Screens are an obvious help in their exclusion.

Carriers. One great menace to the community, the handling of which is no easy problem, is the chronic bacillus carrier. Every effort should be made to discover carriers. That means for one thing tracing to its source every case of typhoid and suspecting all food handlers, those supplying milk, green vegetables, cooks and kitchen help, and recent convalescents. All suspects should have a Widal test and have stools and urine examined.

Should these be found positive their families should receive prophy- 
lactic treatment with vaccines and means must be taken to trace the carrier.

Some patients harbor for months and years virulent bacilli in the urine, and more commonly in the feces, from constant passage into the bowel of bacilli from an infected gall-bladder. Bacilluria and its treatment have been touched upon. Some day, perhaps, Boards of Health will demand to know whether the stools of a typhoid fever patient are free from bacilli before he is allowed the freedom of the community, as they now demand to know whether the Klebs-Lœffler organism is absent from the secretions of diphtheria patients before release from quarantine.

The use of vaccines for the purpose of ridding "carriers" of their infection seems at the present moment to hold out the most promise. Cases of cure have been reported in urinary "carriers," feces "carriers," and old bone lesions.

The urinary cases have succeeded when hexamethylamin (urotropin) failed. The doses ranged from $25,000,000$ (beginning) up to $1,000,000$,000 in six to nine doses at four days to one and two week intervals.

One feces "carrier" was reported cured by the use of bacillus Bulgaricus.

If a gall-bladder carrier is suspected one may confirm the suspicion by the use of an Einhorn tube. The tube is given two hours after a regular breakfast.

The swallowing may be facilitated by having the tube and sinker ice cold and the patient in sitting posture and sipping small amounts of cold water. Usually after four to six hours the duodenal contents may be expressed as an alkaline fluid containing bile. It should be sent at once to a laboratory for examination for the typhoid group. The magnesium sulphate test of Meltzer would seem a valuable aid, in this connection. Crile describes the technique thus:- "The patient is given a duodenal tube to swallow and the stomach contents are aspirated for a routine examination. When this is done, the patient is turned on the right side and a pillow placed under the hips. He is then instructed to massage the epigastric region from the left to the right . . . To relax the pyloric sphincter and thus facilitate entrance to the duodenum, from 20 to $30 \mathrm{~m}$. of benzyl benzoate are given immediately after the tube is swallowed.

When it is ascertained that the duodenum has been reached, usually from three-quarters of an hour to an hour, a solution of 60 c.c. of a 25 per cent. solution of magnesium sulphate is injected through the tube into the duodenum. The tube is then clamped and after three or four minutes preparation is made to collect the specimen of bile. On removing 
the clamp of the tube a flow of fluid is expected, usually with no preparatory aspiration. This back flow consists of a drip of

(1) A return of part of magnesium sulphate injected into the duodenum. Thiš changes to

(2) The "common duct phase"-bile of a consistency of a thin syrup. After 5 or 10 c.c. of bile of this consistency have been cleared a definite change is noted which indicates

(3) The "gall-bladder phase"-bile of a thicker, more ropy consistency, and of a dark color, the amount of which may vary from 25 to 100 c.c. The character again changes to

(4) The "liver phase" - in which the bile is of a lighter, straw color, and much more fluid in consistency.

These changes are quite definite and abrupt. As a routine measure no aspiration is needed, and the outflow of bile occurs spontaneously. When the flow of bile ceases, however, it is always advisable to aspirate gently to see if the flow can again be started. When flowing spontaneously the bile emerges in a series of drops which ebb and flow like the discharge from the ureter." (New York State Journal of Medicine, Oct., 1920, p. 335.) If positive, Nichols recommends giving two grams of sodium bicarbonate three times a day. In two weeks again test the duodenal contents. If the alkaline treatment is not successful one may need to resort to vaccines and, finally, surgery.

One gall-bladder "carrier" was reported cured after repeated exposures of gall-bladder to the X-ray. In some of the gall-bladder "carriers" only drainage of the gall-bladder seemed successful.

It must be remembered as a check upon enthusiasm for any one measure that some of the "carriers" discharge bacilli only intermittently and repeated examinations at not too short intervals must be made before certainty of a case is assured.

Preventive Inoculation. As has been said, serotherapy has not yielded tangible results as yet, but great results have followed the preventive inoculation or vaccination originated and applied to the troops of the British army by Sir A. E. Wright.

His procedure consisted in the culture in bouillon of the organism for four weeks, then killing at $60^{\circ} \mathrm{F}$. Different strains were mixed together, standardized, and two injections made at intervals of two weeks of amounts of 0.5 to 1.5 c.c., the first containing about $1,000,000,000$ bacilli, the second 2,000,000,000.

This was usually injected into the flank, from which might result some redness and pain and involvement of lymph glands draining that territory. There was some constitutional reaction, fever, malaise, nausea and vomiting, but of no significance. 
Statistics seem to show that among some 20,000 inoculated soldiers the incidence of disease and the mortality was about one-half of that of the 150,000 uninoculated.

With certain improvements in technique made by Leishman of the British Army and by our own Army Medical Service under Major Russell, antityphoid inoculation has become the most fruitful and certain preventive treatment since Jenner's introduction of vaccination against small-pox. Compulsory inoculation in our army and navy has fortunately afforded a most convincing body of statistics. Bodies of workers and individuals have been quick to seize the advantage of such a measure, and it is safe to predict that communities will protect themselves by compulsory inoculation.

Statistics are easily available for those who are open-minded.

I will cite the published figures that contrast the conditions in two great Military Camps, one at Jacksonville on the occasion of the SpanishAmerican War in 1898 and the other the recent camp at San Antonio along the Mexican border.

Jacksonville, troops, 10,759. Cases of typhoid, 2,693. Deaths, 248.

San Antonio troops, 12,801. Cases of typhoid, 2. Deaths, 0 .

In Hawaii, in Sept., 1917, an epidemic occurred along a certain water system. Among a population of 4,087 who had been prophylactically vaccinated, only 55 contracted the disease and four died, while among 812 unvaccinated, 45 contracted the disease and seven died. That is, the incidence of the disease was only four times as great in the unvaccinated group and the mortality nearly 9 times as great. (Russell.)

The experience of our army in 1917 and 1918 was most convincing. From Sept. 1, 1917, to May 2, 1919, in an Army whose average strength approximated 2,121,396 there were 213 deaths from typhoid. If the death rate of typhoid that obtained in the Civil War had prevailed the deaths would have been 51,133 while the death rate of the Spanish war would have raised the figures to 68,164 ; one case of typhoid for every 3,756 men in the great war and one for every 7 men in the Spanish war; one death from typhoid among 25,641 men in the great war; one among 71 in the Spanish war. (Russell.)

What a triumph of Modern Medicine these figures bespeak!

Reliable vaccine is now supplied by various Health Boards and by well-known pharmaceutical houses.

It is usual to inoculate in three doses at ten-day intervals (they may be shortened to a week) the first dose being $500,000,000$, the second and third each $1,000,000,000$,

In the army inoculation against paratyphoid A and B is made at the same time as against typhoid. It is well to do this in civil life. In 1917 
in the army there occurred 13 cases of paratyphoid A and $7 \mathrm{~B}$ against 297 cases of typhoid. If this proportion obtains in civil life the precaution is well taken. The bacillus paratyphosus $\mathrm{A}$ and $\mathrm{B}$ are each added $250,000,000$ to the $500,000,000$ of typhoid at the first dose and 500,000,000 each to the $1,000,000,000 \mathrm{~B}$. typhosus in the second and third doses or the proportion may be $750,000,000$ each of $\mathrm{A}$ and $\mathrm{B}$ to $1,000,000,000$ of B. typhosus. One speaks of them as typhoid combined vaccines. The interval of administration may be shortened to seven days or even less in emergency. When given every other day the reaction is likely to be more severe and the second dose the worse. At ten-day intervals the first usually gives the worst reaction.

It might be said that the New York Board of Health supplies paratyphoid vaccine, the bacteria in the proportion of 1,000 million of paratyphoid A and 750 million of para. B.

Reaction may occur in a minority of cases, usuálly mild, rarely severe. These reactions may be local or general.

The local induces a redness and heat about the site of the injection. It rarely needs attention, but if discomforting a simple wet dressing may be applied.

The general reaction is that of any slight infection; a mild "grippe" attack: Major Russell with his vast experience can unqualifiedly pronounce the procedure harmless.

The duration of immunity is placed by Russell at "assuredly" two and a half years and probably longer.

This does not mean that the recognized immune bodies last that long and Russell calls attention to the fact that in cases of recovery from typhoid, though immunity usually lasts for life, "the immune bodies, nevertheless, disappear from the blood of convalescents within a few months." Russell has further called attention to the fact that about one-third of deaths from typhoid occur under twenty years; hence accentuates the necessity for inoculation of children.

Revaccination. In a civil community very young children should be vaccinated. It is well to repeat this vaccination in later childhood, especially if school, travel or work takes the individual into other communities of whose sanitary regulation and their enforcement little is known. It might be repeated in early adult life and especially if a typhoid epidemic prevails. This is following much the procedure advised in revaccinations against small pox. The supposedly more severe reactions in cases of revaccinations are probably exaggerated.

Some observers have called attention to the onset of tuberculosis and other infections after inoculation, but the statistics are not convinc- 
ing to me as being anything more than coincidence or the normal incidence of the disease in question.

An epidemic of typhoid fever at the Trudeau Sanitarium of Tuberculosis in 1917 gave us valuable information on this point. All patients in the sanatarium except those with very active and extensive pulmonary lesions were inoculated prophylactically; 134 patients were so treated. Without going into the details of the reaction I will simply state that no influence of the vaccines on the progress of the tuberculosis could be determined.

Lipovaccines. These are prepared in certain vegetable oils such as olive or sweet almond and it is claimed for them that they provoke less reaction, local or general, that they admit of the administration of a sufficiently large dose to effect immunity by a single injection; that they afford a focus from which immunity proceeds over months; that certain lipoids having detoxicating effects can be incorporated with them and that they do not undergo autolysis and deterioration (Whitman, Fennel and Petersen). As much as 7,500,000,000 can be given at a single dose.

Whether these advantages actually accrue to lipovaccines time must determine.

To individuals going out of their usual environment to an unknown water and food supply, antityphoid inoculation is a wise procedure.

\section{SUMMARY}

Rest.

Bed-how made. (See text.)

Preparation for the open air. (See Pneumonia, Chap. IX.)

Room-Choice. (See text.)

Screens against flies.

\section{Care of the body.}

Mouth.

Washes and sprays of boric acid solution, 2 per cent., 4 per cent., Teeth. or $1 / 2$ strength Dobell's solution.

Brush two or three times a day; cleanse interstices, cotton on wooden tooth pick applicators saturated with boric acid or Dobell's solution.

Tongue.

Soften coat with $1 / 2-1 / 4$ strength peroxide of hydrogen solution, Pharynx. scrape with edge of a whalebone.

Remove mucus with cotton swabs on applicators. 
Sordes and fissures.

Soften sordes with $1 / 2-1 / 4$ strength of peroxide of hydrogen solution; then cleanse with the boric acid or Dobell's solution.

Use mild antiseptic, e. g.,

R

Phenol Solution, 1-20

Glycerin.......................... $\bar{a} \bar{a}{ }^{3} \mathrm{i}$ " 30 c.c.

Boric Acid, saturated solution,............. ₹ viii 240 c.c.

M. et S. To be used as a mouth wash.

Sucking bits of cracked ice.

Nose.

Soften dried secretions with sweet oil on cotton on applicator. Skin.

Cleanse with boric acid or Dobell's solution in sprays or on swabs.

Cleansing bath daily, castile soap and water.

Use drying powder, talcum.

Sponge buttocks with equal parts of alcohol and water.

If buttocks are much soiled, cleanse with phenol solution, 1 to 40 . Bed sores.

Prevention. Clean skin, dry skin, frequent change of position, rubbing of parts most pressed upon to improve the circulation. Smooth bed clothes, mattress and sheets.

Threatening.

Use of rings.

Developed.

Use rings.

Use water bed or air bed.

- Use drying powder on excoriations, e. g., aristol, or

Use ointment such as zinc oxide ointment.

Serious sores.

Surgery.

\section{Disinfection.}

Stools.

One to 20 phenol (carbolic acid). Use twice the bulk of the stool, break up the stool, allow it to stand in the solution for several hours.

Chlorinated lime (must be fresh) 1 per cent. solution or a handful in a bed pan with enough water to cover stool. Let it stand Urine. several hours.

One to 20 phenol, using $1 / 3$ to equal amounts with urine.

Let stand two or more hours.

One to 1,000 bichloride in volume equal to $1 / 15-1 / 40$ of the urine. Let stand two or more hours.

Bath water.

Half pound of chlorinated lime to one tub (200 litres). Stand an hour.

Bed linen.

One to 20 phenol-soak two hours, or formalin 3 ounces to 1 gallon (100 c.c. to 4 litres), soak twelve hours, boil thoroughly. 
Bed-pans, urinals, rectal tubes, rubber sheets, 1 to 20 phenol; allow to stand in it.

Knives, forks, spoons, crockery. Boil.

Sputum. Burn.

Vomitus.

One to 20 phenol.

1 per cent. chlorinated lime.

\section{Precautions for physicians and nurses.}

Wear rubber gloves and aprons in giving baths, and wash hands later with soap and water and with 1 to 1,000 bichloride of mercury.

End of illness.

Mattresses.

Room.

Dry heat-(not practical at home).

Formaldehyde gas.

Diet.

Aim at 3,000 calories (40 calories per kilo) in early weeks and more in latter weeks, as much more as the patient wishes or will take and handle well.

Proteid-70 to 90 grams.

Carbohydrates the mainstay (the protein shelterer).

Fats fairly liberally.

Frequency of feeding.

Every two hours. Some patients will do better at longer intervals. It is better not to disturb the patient's night's rest for feeding.

Milk, the basis of the dietary-1 to 2 quarts a day.

Caloric value -640 to the quart, one glass of 8 ounces equals 160 calories.

Milk enriched by cream and milk sugar.

Cream-16 per cent. gravity, 50 calories per ounce.

Lactose-120 calories per ounce.

One glass containing milk, 7 ounces, 140 calories. cream, 1 ounce, 50 calories. lactose, $1 / 2$ ounce, 60 calories.

Equals 250 calories.

One glass containing milk, 6 ounces, equals 120 calories. cream, 2 ounces, equals 100 calories. lactose, $1 / 2$ ounce, equals 60 calories.

Equals 280 calories. 
One glass containing milk, 6 ounces, equals 120 calories. cream, 2 ounces, equals 100 calories. lactose, 1 ounce, equals 120 calories.

Equals 340 calories.

Eggs may be given up to 4,5 , or 6 a day.

One egg equals 80 calories.

One egg white equals 30 calories.

One egg yolk equals 50 calories.

May be given soft boiled, coddled, or raw, or in soft or baked custard, or eggnog.

Bread, white, home made, equals 1,225 calories to 1 pound.

One slice, $1 \frac{1}{4}$ ounces, equals 100 calories.

Given as toast, milk toast, bread and butter, bread and milk.

Butter equals 3,600 calories to 1 pound.

One pat of butter ( $2 / 5$ ounce) equals 100 calories.

Put on bread and cereals.

Cereals - vary somewhat, but one helping of two heaping tablespoonfuls equal about

Rice-one heaping tablespoonful equals

Potato-baked (medium) equals

Potato-mashed, 11/2 tablespoonfuls, 100 calories. 100 calories. 100 calories. 100 calories.

Table.

Milk-one glass, 8 ounces,

Cream-one ounce,

Sugar-one ounce,

Egg-one,

Bread, one slice, $11 / 4$ ounces,

Butter, one pat, 2/5 ounce,

Cereals, two heaping tablespoonfuls,

Rice, one heaping tablespoonful,

Potato, baked, one,

Potato, mashed, 11/2 tablespoonfuls,

Crackers, Boston, one,

Oysters, ten,

Pilot bread, one,

Soda, Educators, one,

Uneeda biscuits, one,

160 calories.

50 calories.

120 calories.

80 calories.

100 calories.

100 calories.

100 calories.

100 calories.

100 calories.

100 calories.

42 calories.

48 calories.

131 calories.

10 calories.

25 calories.

Ice-cream (average) 2 heaping tablespoonfuls, 200 calories.

Baked custard, 2 heaping tablespoonfuls, 180 calories.

Soft custard, 4 heaping tablespoonfuls, 130 calories.

Bread pudding, 2 heaping tablespoonfuls, 225 calories.

Koumyss, one glass, 7 ounces,

Buttermilk, one glass,

Whey, one glass,

Apple sauce, one ounce,

Macaroni, 2 heaping tablespoonfuls,

100 calories.

80 calories.

50 calories.

50 calories.

100 calories. 
Cocoa, one rounding teaspoon, 5 grains, Cracker, Uneeda, one

Egg white, one egg,

Egg yolk, one egg,

Orange, one large,

Among other articles may be mentioned tea, coffee, cocoa to carry sugar and cream; lemonade, orangeade, wine jelly to carry sugar.

Chicken, veal or mutton broth to stimulate an appetite; thickened with rice, barley, arrowroot to add calories.

Potato, pea and tomato soup, junkets.

Sample diet modelled on Coleman list, avoiding night feeding.

Milk, 7 ounces,

Cream, 1 ounce, $\}$ equals 250 calories, at 5 a. m.

Lactose, $1 / 2$ ounce,

Egg, one,

Toast, two thin slices,

Butter, $11 / 2$ pats,

Coffee with cream, 2 ounces,

Lactose, $2 / 3$ ounce,

Milk as above,

80 calories.

100 calories.

150 calories.

100 calories.

80 calories.

510 calories.

Egg, one,

80 calories.

Mashed potato, $1 / 2$ tablespoonful, 30 calories.

Baked custard, 2 heaping tablespoons,

180 calories.

Toast or bread, two small slices, 100 calories.

Butter, $1 \frac{1}{2}$ pats,

Coffee, cream, 2 ounces,

Lactose, $1 / 4$ ounce,

150 calories.

100 calories.

80 calories.

720 calories.

Milk as above,

Milk as above,

Egg, one,

Cereal, 3 tablespoonfuls,

Cream, 2 ounces,

Sugar (powdered), 1 heaping teaspoonful,

Apple sauce, 1 ounce,

Tea, cream, 2 ounces,

Lactose, $2 / 3$ ounce,

80 calories.

150 calories.

100 calories.

50 calories.

50 calories.

100 calories.

80 calories.

610 calories.

Milk as above,

Milk as above,

at 7 a. m.

at 9 a. m.

510.

250.

250.

at 11 a. $\mathrm{m}$.

720.

at 1 p. m. 250 .

at 3 p. m. 250 .

at $5 \mathrm{p} . \mathrm{m}$.

610.

at 7 p. m. 250 .

at 10 p. m. 250 .

Total 3,340. 


\section{Drinks.}

Water ad libitum; offer hourly when patient does not ask for it, or alkaline waters, lemonade and orangeade and Imperial drink, to which the addition of sugar increases the caloric intake.

Two to three quarts of fluid daily and more if patient will take it. Avoid slavish adherence to exact dietaries.

Vary the food to avoid monotony.

Increase calories in decline of fever.

Add chicken, fish, meat, and vegetables in convalescence; begin with chicken or scraped beef, when temperature is normal or nearly so, and add other articles gradually and tentatively, ${ }^{1}$ such as chops, spinach, asparagus tops, peas, and stringed beans put through a purêe sieve.

\section{Hydrotherapy.}

Brand bath. (For technique, see text.)

Ziemssen's graduated bath. (See text.)

Slush. (See text.)

Packs.

Sponges.

\section{Care of alimentary canal.}

When first seen early.

Castor Oil (Oleum ricini)........... $3 \frac{3 / 4}{4}$ to 1 (20-30 c.c.).

Epsom Salt (Magnesium sulphate)...... $33 / 4$ to $1(20-30 \mathrm{Gm}$.).

Rochelle Salt (Sod. et Pot. tartrate)..... $33 / 4$ to $1(20-30 \mathrm{Gm}$.).

or

Calomel (Hydrargyri chloridum mite), gr. 1/4 (0.015 Gm.) every quarter hour for 6 doses and followed by one of the above.

Later and if seen late.

Enemata of tepid water.

Soapsuds-use castile soap.

Oil enema.

Make thick suds with castile soap and warm water, stir in oil slowly, then add water to 1 or 2 pints.

\section{Tympanites.}

Suspect milk sugar, cut it down or eliminate it.

Rectal tube.

Stupes. (See text.)

Turpentine.

In stupes.

In enemata.

By mouth. (See text.)

In Capsule or Emulsion. m. x.

${ }^{2}$ For caloric values of foodstuffs consult tables of Atwater and his co-workers issued by U. S. Department of Agriculture.

Food Values by Edwin Locke.

Tables of Prof. Irving Fisher in J. A. M. A. April 20, 1907.

Tables of Arnold in J. A. M. A. December 24, 1910.

Coleman in Articles referred to. 
Ice bag.

Ice coil.

Milk and molasses enema.

Each eight ounces, warm.

\section{Peppermint enema.}

$3 \mathrm{i}$ to 3 ii to quart saline.

Pituatrin, minims 5-15.

(0.33-1 c.c.).

\section{Diarrhea.}

Suspect fat.

Cut down or eliminate.

Boiling milk may help.

May have to stop milk.

Saline irrigation, sodium chloride $3 \mathrm{i}(4 \mathrm{Gm}$.) to 1 pint (500 c.c.), prolonged.

Murphy drip.

Astringents.

Bismuth subnitrate, gr. $\mathbf{x x x}(2 \mathrm{Gm}$.) every three or every two hours.

Opium if imperative, pill, gr. 1/2 (0.030) every two hours for 2, 3 or 4 doses.

R
Pulv. Opii.
0.25
(gr. iii $3 / 4$ )
Pulv. Camphoræ................. 1.00
(gr. xv
Plumbi Acetatis
3.00
Bismuth Subnitratis.................30.00
(gr. xlv)

M. et Div. in chartulas no. xv.

S. One every 4 hours.

Gastric distress.

Suspect both fat and lactose. Cut down or eliminate.

\section{Vomiting.}

Cracked ice, sucked.

Mustard paste to epigastrium (1 in 4).

Bismuth subnitrate.......gr. $\mathrm{xv}-\mathrm{xxx}(1-2 \mathrm{Gm}$.).

Sodium bicarbonate........gr. $\mathrm{x}-\mathrm{xx}(0.66-1.33 \mathrm{Gm}$.).

Cerium oxalate...........gr. iii-v (0.20-0.30 Gm.).

or combine..............

R

Bismuthi Subnitratis....................15

Sodii Bicarbonatis.......................10

Cerii Oxalatis........................ 5

M. et Div. in chart no. xv.

S. One every two hours (in the food if given). 
If very severe.

Cocaine hydrochloride - tablet, gr. $1 / 5(0.010 \mathrm{Gm}$.), or $1 / 10$ per cent. solution $3 \mathrm{i}$ ( 4 c.c.) every two hours.

Stomach washing, lavage.

\section{Hemorrhage.}

Early stages, oozing, no treatment.

Serious latter part of second and third week.

Mild.

Cut down food.

Enjoin rest.

Moderately severe.

Ice bag to abdomen.

Calcium lactate, gr. $\mathrm{x}(0.60 \mathrm{Gm}$.) three times a day.

Horse serum, 10-20 c.c. To avoid anaphylaxis in use of serum. (See text.)

Human serum, 10-20 c.c.

Thromboplastin.

20 c.c. in water 3 viii several times a day by mouth.

Severe with symptoms of hemorrhage. (See text.)

Morphine sulphate hypodermically or intramuscularly, gr. 1/4 (0.015 Gm.).

Exsanguination.

Elevate foot of bed.

Heat to extremities.

Physiological salt solution ( $3 \mathrm{i}-\mathrm{Oi})(4 \mathrm{Gm} .-500$ c.c.) hot in the bowel.

Hypodermoclysis of same, or Ringer's solution.

Infusion of same into vein.

Best of all, transfusion. (See text.)

Perforation. (For symptoms, see text.)

Surgical intervention.

\section{Nervous system.}

Headache.

Ice bag.

Sleeplessness.

Less marked with the Brand bath and sufficient diet.

Bromides.

Bromide of potash, gr. $\mathrm{xv}-\mathrm{xxx}(1-2 \mathrm{Gm}$.$) .$

or

R

Potassii Bromidi.

Ammonii Bromidi.

Sodii Bromidi....................

Aquæ destillatæ................... s. ad 60.00

M. et S. $3 \mathrm{i}$ in water. Repeat in two hours if needed. 
Trional-gr. $\mathrm{xv}-\mathrm{xx}(1-1.30 \mathrm{Gm}$.). In a little warm water or in whiskey, brandy or wine or in powder; wash down with water.

Repeat in two hours if needed.

Chlóralamid-gr. $\mathrm{xx}-\mathrm{xxx}(1.30-2 \mathrm{Gm}$.).

In cold water (not hot), in wine, whiskey, or brandy, or in powder and wash down with water.

Repeat if needed in two or three hours.

If marked or prolonged.

Morphine sulphate, gr. 1/16-1/4 (0.005-0.015 Gm.).

Delirium-Restraint.

Cold baths.

Tepid baths with cold water to head.

Ice bag to head.

Morphine hypodermically, gr. 1/8-1/4 (0.008-0.015 Gm.).

\section{Lumbar puncture.}

Draw off 20 c.c. or even more.

(For technique, see Cerebro-Spinal Meningitis, Chap. XXV.)

\section{Stupor.}

Cold baths.

Tepid baths.

- Ice bag to head.

Lumbar puncture.

\section{Circulatory disturbances.}

(For symptoms, see text.)

Prevention.

Sufficient diet.

Cold baths.

Fresh air.

Failing circulation.

Digitalis.

Infusion $₹$ ss. (15 c.c.) three times a day, or tincture $\mathrm{m}$. xxx (2 c.c.) three times a day for three or four days, or longer until improvement in the circulation is seen or accumulation is evidenced. (See text.)

In urgent circulatory distress.

Strophanthin (Boehringer's) (0.0005-0.00075 Gm.) (gr. 1/120-1/90) intramuscularly or intravenously, and then follow with digitalis as above, or crystalline strophanthin (ouabain) $1 / 2$ to $3 / 4$ the above dose.

When either therapeutic or toxic effect is severe, stop using for three or more days, and repeat if needed.

Another method (less satisfactory).

Digitalis infusion $3 \mathrm{i}$-ii or tincture $\mathrm{m} . \mathrm{x}$ three times a day may be given without interruption unless the stomach is irritated.

If satisfactory results do not ensue, or for any reason (idiosyncrasy) digitalis cannot be taken, 
Vasomotor stimulants.

Caffeine sodium salicylate or caffeine sodium benzoate, gr. v $(0.35 \mathrm{Gm}$.) into muscle every four, three or two hours.

Camphor in oil 10 per cent. or 20 per cent., gr. v $(0.35 \mathrm{Gm}$.$) every$ four, three or two hours, or alternate at every two-hour intervals the caffeine and the camphor in gr. v (0.35 Gm.) doses.

Strychnine sulphate (less valuable), gr. 1/60-1/30 (0.001-0.002 Gm.) every three or four hours. Given hypodermically.

Threatened collapse.

Adrenalin (Epinephrin) m. xv (1 c.c.), into a muscle or $\mathrm{m}$. iii-iv $(0.20$ c.c.) into a vein. Follow by caffeine or camphor in gr. $v$ (0.35 Gm.) doses into muscle and by strophanthin $1 / 2 \mathrm{mg}$. (gr. $1 / 120$ ) into muscle or vein.

\section{Urinary tract.}

Bacilluria.

Urotropin as a routine.

Begin as temperature approaches normal and continue for a month after, gr. $\mathrm{v}-\mathrm{x}(0.30-0.60 \mathrm{Gm}$.) three times a day for three consecutive days each week.

If urine is alkaline, add benzoate of sodium or ammonium, gr. $\mathbf{x}$ $(0.60 \mathrm{Gm}$.) to each dose of urotropin.

If this does not clear it up use

Bladder injections of silver nitrate 1-5,000 solution daily, or saturated boric acid solution daily.

\section{Vaccine therapy.}

Intravenous: initial dose 25 to 50 million diluted to 1 c.c. (For intervals and reactions, see text.)

\section{Complications.}

Phlebitis.

Rest-fixation relative or absolute.

Enclose in non-absorbent cotton.

Wet dressings-Aluminium acetate.

Poultices. (See Pneumonia, Chap. IX.)

Meningismus.

Tapping.

Meningitis.

(See Cerebro-Spinal Meningitis, Chap. XXV.)

\section{Convalescence.}

(See diet in Summary.)

After temperature is normal, seven-ten days.

Prop up in bed: three to four days more.

Sit up in chair, a little longer each day, after a week get on feet. If temperature rises, consider bowels; too much meat too early. 
Get out in sun and air.

Watch bowels.

Mild cathartics such as cascara, aloin.

\section{Treat anæmia.}

Blaud's pill (Pil. ferri carb.) gr. v $(0.35 \mathrm{Gm}$.) three times a day.

For weakness.

Strychnine sulphate, gr. 1/60-1/30 (0.001-0.002 Gm.) three times a day,

or tincture of nux vomica $\mathrm{m} . \mathrm{x}-\mathrm{xv}(0.60-1$ c.c.) three times a day. Do not return to work, or admit business matters too soon.

Long vacation of three to six months.

Prophylaxis.

Protection of community.

Supervision of water supply.

Boards Filtration plants. Policing water sheds.

of Supervision of milk supply; inspection of health of Health $\quad$ workers. Inspection of drains.

and

Sanitary

CommisSewage disposal.

sioners.

The patient.

Notification of cases.

Education.

Lectures.

Schools.

Exhibitions.

Isolation.

Disinfection of all discharges and clothes and utensils. (See above.)

Preventing access of flies by screens.

Care on part of attendants.

Treatment of "carriers."

Prophylactic; urotropin as above.

Inspection of urine and stools of convalescents for the organisms.

Vaccines for all "carriers," beginning $25,000,000$, work up to $1,000,000,000$, four to seven day intervals, in six to nine doses.

May try for feces "carriers,"

Bacillus Bulgaricus by mouth; or by rectum in small amount of sugar solution, 2 per cent.

Gall-bladder "carriers."

$\mathrm{X}$-ray cure has been reported.

Drainage of gall-bladder has been necessary and has cured.

Diagnosis established by Einhorn tube. Sodium bicarbonate, 2 grams three times a day. (See text.)

Preventive inoculation, for typhoid.

After 7 to

$$
\text { 1st dose. } \quad 500,000,000 \text {. }
$$

10 days. 2nd dose. $1,000,000,000$.

After 7 to

10 days. 3rd dose. $\quad 1,000,000,000$. 


\section{Preventive inoculation for paratyphoid.}

First dose 500 million of Para A

$$
375 \text { " "6 B }
$$

After 7 to 10 days second dose.

These are often combined in the proportion of 1 billion to typhoid and 750 million each of paratyphoid A and B per c.c.

First dose, one half c.c. and the second and third, 1 c.c. at 7-10 day intervals.

\section{PARATYPHOID FEVER}

Until bacteriological research determined the identity of the paratyphoid organism the clinical entity was submerged in the picture of typhoid fever. In the vast majority of cases this infection of low mortality, not more than 1 per cent., was considered as a light typhoid of short duration and it is doubtful if it ever would have been distinguished on clinical grounds alone, for its symptomatology is almost identical with that of typhoid; a prolonged fever, slow pulse, rose spots, enlarged spleen, hemorrhages, leucopenia, tympanites. No symptom is distinctive. The fever is as a rule less high and less prolonged, the hemorrhages are less profuse, perforations are very rare; but what is all this but the picture of a light typhoid? The complications are the same, such as meningitis, thrombophlebitis, cholecystitis and pyelitis, but the latter and pyelonephritis and cystitis are stubborn in their persistency and the discharge of bacteria long continued.

Pulmonary complications including a considerable percentage of pneumonias, often with pleurisy and sometimes with empyema, are not uncommon. The bacillus paratyphosus may be recovered from the sputum. Such cases are often looked upon as purely pulmonary; the paratyphoid infection being overlooked.

Gastro-intestinal symptoms, too, are likely to be more marked and, indeed, this infection may present the picture of acute gastro-enteritis and even of cholera.

Relapses are rare.

The organisms concerned are two types of bacilli called bacillus paratyphosus A and bacillus paratyphosus B. The former produces acid on suitable media and the latter alkali. Paratyphoid B is a far more common agent than $\mathrm{A}$ in the production of the disease. Paratyphosus B has so many points in common with the bacillus enteritidis of Gärtner that the differentiation depends largely on agglutinating properties.

Infection is conveyed by the feces and urine, through contaminated food and the careless handling of infected excretions. Paratyphoid B at least seems also to be transmitted through infected meat. 
The diagnosis lies in the determination of the bacillus in blood, stool or urine cultures and in agglutination reactions (Widal). It is the only way to distinguish between typhoid fever and paratyphoid fever and the agglutination and cultural characteristics of bacillus A \& B alone distinguish the one infection from the other.

The pathology shows the same implication of the lymphoid tissues in most cases as in typhoid, the same ulcerations, but perhaps more surerficial.

Treatment is in all respects like that in typhoid fever.

Under that heading, too, will be found a discussion of prophylactic vaccination in paratyphoid. 


\section{CHAPTER XV ?}

\section{MALARIA}

From the barks of the various species of cinchona is derived an alkaloid, called quinine, and this quinine is more truly a specific than any other drug in the whole pharmacopeia.

The treatment of malaria resolves itself pretty much into the skillful use of quinine.

The rôle of the mosquito, the anopheles, the varieties of the lowly order of animal life, the sporozoan protozoa (the plasmodium vivax, or tertian parasite, the plasmodium malariæ or quartan parasite and the plasmodium falciparum or estivo-autumnal or malignant parasite), the stereotyped manifestations of chill, fever and sweat and the intermittent or remittent temperature in malarial infection are familiar to us all.

It is only within the last three or four years that, thanks to the labors of Bass, the cultivation of the malarial organisms has become possible and undoubtedly much light of therapeutical value will be shed on the problem by him and those who are using his methods.

While we are interested in the differentiation of the manifestations of the disease, into the tertian, quartan or estivo-autumnal forms, as based on the variety of the invading organism, the degrees of severity, after all, depend not a little on where the infection occurs as well as on what organism is present. In the Northern States we have to treat relatively mild cases; in the South much more severe and some pernicious types; and in the tropics many pernicious cases. The lighter cases, such as predominate in the North, are due to the benign tertian parasite, plasmodium vivax, and much more rarely the quartan plasmodium malariæ, causing intermittent fevers, while the tropical fevers are more commonly due to the malignant tertian, estivo-autumnal organism, p. falciparum, causing a remittent type of fever. However, here in the North one rarely sees the pernicious types of infection, algid, comatose, though estivo-autumnal forms are frequently met with.

Considering first the milder cases as seen here in the North, some of them are so slight that the patient may object to going to bed. The ordinary attack, however, compels the patient to seek rest.

In any case our results are better when the patient does go to bed and is made to observe rest. 
Rest. The difference between the appearance of the shaking, fevered or sweating patient of one day and the apparently well individual of the next, is one of the most striking contrasts of medicine.

During the period of intermission, the patient feels so well that he will often rebel against remaining in bed and greets his physician sitting up and dressed on the occasion of his next visit.

In the milder cases, no strenuous objection to this performance need be voiced; but, if the paroxysm has been severe, the patient should be assured that his remaining at rest facilitates success in aborting the second. In the remittent estivo-autumnal forms it should, of course, be insisted upon. Each individual paroxysm means an intoxication, and as the result of this and the marked pyrexia accompanying it, tissue destruction has been entailed which makes all the more imperative the economy in the body's energies accomplished by rest of the tissues attacked by the organism. The one tissue in which a gross lesion occurs, is the red blood-cell and its destruction induces a degree of anemia. Now, in untreated cases or badly treated cases, emaciation and anemia of a marked grade ensue and the necessity for rest is as urgent as in other infections of long continuance. With prompt treatment this does not obtain, and after an anticipated paroxysm fails to appear the patient may be allowed to get up.

Bed. On account of the brevity of the attack, when well treated one may be less insistent on the nicety of details with reference to the bed than in the infectious diseases of longer continuance, if it entails extra expense in the procuring; but in severe cases, in pernicious types with continued fever, we economize the strength both of the patient and attendant and enhance the success of our treatment if an iron half-bed with woven wire-springs and firm mattress, which is best illustrated by the hospital bed, is used.

Room. If the patient is still in a malarious district his room should be so chosen that the chances of further infection are lessened. By selecting one in the upper part of the house, since the invasion by the mosquito is less likely, and making doubly sure by the use of screens or mosquito netting, this object is accomplished. This, too, affords a certain protection to attendants.

In other respects the room should be chosen in severe cases according to the same criteria that obtain in other acute infections, with a view to size, ventilation, light, access to the open air by veranda and nearness of bath room, simplicity of furnishings and remoteness from disturbing elements of the household.

Diet. The suddenness of the attack, the anorexia, nausea and vomiting contraindicate any effort at nourishment during the paroxysm. 
During the afebrile periods of the intermittent type of fever, semi-solid or solid food of a simple character may be taken, but at any meal occurring within six hours of the anticipated recurrence of the paroxysm the food should-be fluid or semi-solid, as milk, eggs, cereals or toast. This is in consequence of the vomiting that may ensue.

It is interesting to note that Bass and Johns in their studies on the culture of the plasmodium observed that the blood seemed to form a better culture medium immediately after a full meal and this they say corresponds with the clinical fact that the parasites will often disappear from the blood and the paroxysms cease if the patient is put to bed and given a purgative and put on a light diet.

If the fever is of the remittent type, the same precautions should be observed at the periods of paroxysm, but in the intervals the diet, while it should be somewhat restricted in the choice of food; should nevertheless contain sufficient nutritive value in terms of calories and protein.

Care of the Bowels. Calomel should be given either in one dose of gr. iii-v (0.20-0.33 Gm.) or in divided doses of gr. $1 / 4(0.015$ Gm.) every $1 / 2$ hour for six or eight doses, and this may be followed in 3 to 4 hours by a saline. The best time to give the cathartic is between the paroxysms. It should, however, precede the quinine when possible.

\section{SYMPTOMATIC TREATMENT DURING THE PAROXYSM}

The phenomena of the paroxysm, chill, fever, and sweating have been looked upon as the expression of the action of a toxin released by the plasmodia at the time of sporulation, but Wade Brown from his recent studies concludes that they are all in part at least due to the toxic action of the malarial pigment, that is, hematin, derived from the destroyed red blood cells. The three stages of the paroxysm are each provided with their own particular form of discomfort for the patient and demand pretty constant attention and ministration to mitigate their evils.

Chill. Beginning with chilly sensations, followed by shiverings and then shakings, with the skin covered with "goose-flesh," pale, cyanotic, the face pinched and the teeth chattering, the patient can only complain of the cold and beg for warmth.

One does everything possible to afford this, by putting hot-water bottles to the feet, wrapping flannel about the extremities, heaping on blanket after blanket and giving hot drinks, hot water, weak tea, hot weak milk, into which a dash of ginger may be stirred, hot lemonade, a little hot whiskey or brandy. 
The discomforts may be mitigated by the use of codeine phosphate in half grain $(0.033 \mathrm{Gm}$.) doses by mouth or spirits of chloroform in one dram doses.

Vomiting. One other feature of this stage often requires interference-namely, vomiting.

If there is much useless retching, a draught of warm water may help to effect an evacuation of the stomach; then simple measures to stop further vomiting may be pursued, as sipping of effervescent drinks such as ginger ale or Seltzer water or putting a mustard paste on the epigastrium. Bromides in 10 or 15 grains (0.66-1 $\mathrm{Gm}$.) with spirits of chloroform in teaspoonful doses may be of value given by mouth.

If the retching cannot otherwise be overcome, administer morphine hypodermically, in doses of gr. $1 / 12$ or gr. 1/8 (0.005-0.008 $\mathrm{Gm}$.) of the sulphate.

Collapse. In the old and feeble, symptoms of collapse may threaten during the chill. These are to be met by hot drinks of coffee, brandy or whiskey, or a teaspoonful of aromatic spirits of ammonia in water; or a little strong water of ammonia may be inhaled from a towel or handkerchief held a little way from the nose.

This stage lasts from a quarter of an hour in light cases to two hours in very severe ones; and then come occasional flushings of heat and gradually there is established the febrile stage.

Fever. In a few minutes the patient is "burning up with fever," complaining as bitterly of the heat as he was of the cold shortly before. The face is flushed, the whole skin red, the pulse bounding.

The hot-water bottles are removed, blanket after blanket comes off, until only a sheet remains; bits of cracked ice are sucked, cold water or cold lemonade is sipped, cool sponge baths given or sponges of water containing alcohol. A light rub with the hand, using 25 per cent. alcohol in water, affords much comfort.

Headache is the feature of this stage as vomiting is that of the chill, though occurring also in the first stage. This is relieved by cold cloths, wrung out of ice water and applied to the brow. The cold sponging mentioned lessens the intensity of the headache. If, however, it is intense and not lessened by the measures advised and, especially, if delirium accompanies it, one may try codeine phosphate in $1 / 4$ or $1 / 2$ grains doses $(0.015-0.030 \mathrm{Gm}$.) or the morphine sulphate in small doses hypodermically, gr. $1 / 24$ to gr. $1 / 12(0.003-0.005 \mathrm{Gm}$.) may be given. Coal tars should not be used.

This stage lasts usually from four to six hours and then as the fever 
declines, perspiration appears on the face and forehead and the third stage of sweating is ushered in.

Sweat. Soon the whole body is covered with a drenching sweat and, except" for the discomfort of the sweating, the patient grows rapidly better. Relief is afforded the patient in this stage by rubbing the body with dry towels and changing the linen. He may be allowed drinks of water or lemonade freely.

If symptoms of collapse intervene, as but rarely occurs, hot drinks and stimulation as advised during the cold stage should be given. Sleep usually follows this stage.

Blood examination shows a moderate leucocytosis during the attack and leucopenia with large mononuclear increase between the attacks. Parasites may be found at any time during the afebrile period.

Such are the events and their treatment in the milder cases of tertian and quartan fevers of this latitude.

Of the estivo-autumnal fevers of the North, it may be said that they are more severe than the other types, but very rarely pernicious. The nervous symptoms and the aching pains of the limbs and back are more striking and the paroxysm is much longer, usually over twentyfour hours and often permitting but a few bours of intermission or remission.

The chills are not as frank; the rise of temperature less abrupt, delirium or apathy more pronounced and the patient more prostrated. Parasites are found a few hours after the onset of the paroxysm and may be very difficult or impossible to demonstrate later. Such cases oftẹn resemble typhoid fever.

Herpes labialis is common, especially in the benign tertian. The spleen enlarges and is one of the most pathognomonic features of the infection.

Specific Treatment. As I have said quinine is looked upon as more truly a specific than any other drug in use, and yet its specificity is due to effects not confined to the plasmodium malariæ alone, but common to all protoplasm.

In general it is a protoplasmic poison. At first and in slight doses, this action on protoplasm is expressed by an enhancement of function, that is, stimulation; but this in turn, in sufficient dosage, is followed by depression of function, paralysis of same and death. That this action varies in degree in different stages of cell-growth and cell-activity is also true and upon this fact depends its usefulness as a drug.

Now, the protoplasm of the malarial organism seems peculiarly susceptible to it, but by no means equally so at all stages of its development. It is so much more susceptible to quinine than the body-cells, 
that amounts that will kill the plasmodium have no deleterious effects on the latter.

It is during the stage when development and nutrition are most active, that is, in the young stage of the parasite, that quinine is most operative. This fact has a practical bearing on the administration of the drug.

Bass and Johns believe that the quinine does not directly kill the parasites but produces a permeability of the red blood cells to the blood serum which contains an element destructive to the plasmodium.

Time of Administration. Many rules are given by numbers of physicians with reference to the time of administration, and by some of them with an insistence on exactness that would seem to attribute more of the success to this item in technique than to the drug itself. Facts about the absorption and excretion of quinine are as follows: Within thirty minutes evidences of it are found in the urine. In six hours half of it is eliminated, but after that the elimination is more tardy and traces of it can still be found in the urine after seventy-two hours.

Sporulation-the setting free of the young parasites in the bloodstream-occurs at the time of the chill. We should theoretically get the best results by a large dose given three or four hours before the anticipated chill, at a time when the maximum amount of quinine would be in the circulation to act upon the organisms during their egress from the red blood-cells. We may, then, a few hours before the paroxysm give our maximum dose. It goes without saying that this will in no way abort the coming chill, as the organisms responsible for that are protected in the red blood-cells; but it will destroy their offspring and abort the chill for which they would be responsible forty-eight or seventytwo hours later. Bethea is accustomed to give the full day's dose at this time, dividing it into six parts and administering the doses at onehour intervals beginning five hours before the anticipated chill.

Again, as others advise, the quinine may be administered during the decline of the fever. The fever we believe to be coincident with the setting free of the parasite in the blood. The young organisms very quickly attach themselves to the red cells which they seek to penetrate.

If quinine is given at this time, its rapid absorption, as just expressed, brings it into contact with them and effects their destruction.

Still others advise dividing the daily dose, administering a portion, three times a day, every four hours or every six hours. As we have seen, only half the dose ingested is excreted in six hours, so this procedure keeps the blood cinchonized continuously. This method has advantages where the time of the paroxysm is more difficult to determine, as in the remittent or continuous forms, in quotidian infection and in the perni- 
cious types, and particularly if there is a constant migration of plasmodia from cell to cell, as Mary Rowley Lawson contends.

So one may administer the dose (1) a few hours before an expected paroxysm; $;(2)$ at the decline of the fever of a paroxysm; or (3) divide the dose throughout the twenty-four hours.

The object is to have enough quinine in the blood to kill the organism when it is free.

Preparations. There are numerous salts of this alkaloid, representing varying weights of the alkaloid and varying solubility. Most of the salts are sparingly soluble; a few freely. Of these the sulphate which contains about 75 per cent of the alkaloid (74.31 per cent.) is the most commonly used when the drug is administered by the mouth. The more soluble acid hydrochloride or bisulphate, or quinine and urea hydrochloride is used for hypodermic purposes.

The sulphate is practically insoluble in water ( 1 in 800), and is usually administered in capsules or wafers. Pills and tablets are likely to be hard or tough and in this way the drug escapes absorption. The 2 grain $(0.15 \mathrm{Gm}$.) quinine capsule is the favorite unit. There is no question that this drug were better given in solution and, indeed, where the condition of the stomach leads to a suspicion of its impairment and hydrochloric acid may not be secreted, the solution should be used. The drug is freely soluble in any dilute acid, hydrochloric, sulphuric, phosphoric or tartaric. A minim per grain is enough. The objection to this method is the intensely bitter taste. Effort may be made to disguise it. One way is to dissolve the dose in a few grains of citric acid gr. $\mathrm{x}(0.60 \mathrm{Gm}$.) in a little lemon juice, add to it water in which a pinch of bicarbonate of soda has been dissolved and take while effervescing.

The hydrobromide, soluble 1:40 or the bisulphate, soluble in $81 / 2$ parts of water and the dihydrochloride soluble in less than equal parts of water (1 to 0.6 ), may be used instead of the sulphate.

Bethea favors the hydrobromide because of its ready solubility, large quinine content, and possible antidotal effects of the bromide to cinchonism.

Dosage. I am convinced that our dosage for malaria in temperate zones has been too low and accounts for the frequent relapses.

Regardless of the form of infection I advise gr. $\mathrm{xxx}(2 \mathrm{Gm}$.) of quinine a day until the paroxysms cease and at least gr. $\mathrm{xx}(1.30 \mathrm{Gm}$.) a day for ten days to two weeks, and then $\mathrm{gr}$. $\mathrm{xv}(1 \mathrm{Gm}$.) on two successive days of each week for two months after. This amount may be divided into two or three doses a day.

Bass, beginning with the same large doses, 10 grains $(0.66 \mathrm{Gm}$.) three times a day, for three days, then gives 10 grains for each night for eight 
weeks without intermission. This is the fruit of an exceptional clinical experience. Stitt gives the treatment adopted in the Canal Zone where tropical conditions mean severe infections, as follows: 15 grains of quinine three times a day ( 45 grains a day) for a week or until the temperature has been normal five or six days; then 10 grains three times a day for ten or 12 days. Vedder recommends grains xxx daily until symptoms have disappeared and plasmodia are no longer found; then grains $\mathrm{xv}$ daily for two weeks; then grains $\mathrm{x}$ daily for two months at least.

Not all tropical workers, e. g., Manson and Koch, give so large doses as this. Moreover, there seems to be a great variety of opinion about the toxicity of the drug which it is not becoming one whose experience has been confined to the treatment of malaria in the temperate zone to pass upon. Using such doses as I have mentioned in the first paragraph under Dosage, I have never seen toxic results except in rare cases of idiosyncrasy that will be discussed later.

If large doses are not well borne by the stomach, the 24 hour intake may be divided into smaller, but more frequent doses and must be administered by night as well as day to keep the blood properly cinchonized.

Bethea favors more frequent administration-he gives 3 grs. every two hours during the day and every three hours during the sleeping period. After the period of intensive treatment he gives four grains three times a day for about eight weeks.

I believe Wellman is right when he advises a repetition of the treatment thirty or forty days after the initial dose. If a case relapses it means either that the drug was not absorbed or the dose was insufficient. It is better with such a relapsing case to use the drug in solution.

Quinine Immunity or "Fastness." It should be emphatically stated that perhaps as much or more damage can be done by too small doses as by very large ones: for not only is the "cure" as estimated by disappearance of symptoms delayed; but when small doses are given at the beginning of the treatment the organisms gain a resistance or immunity to quinine or as it is sometimes put, become "quinine fast" and thus are able to resist large doses later and so perpetuate the initial attack or prepare the way to relapses.

Quinine in Children. Children require large doses proportionately; Wellman gives one grain for each year of a child's age three times a day. This rule to be followed to ten years.

Bass gives the following dosage: to infants under one year $1 / 2 \mathrm{gr}$. $(0.03 \mathrm{Gm}$.$) ; at one year 1$ grain $(0.06 \mathrm{Gm}) ;$.2 years 2 grains $(0.012 \mathrm{Gm}$.); 3-4 years 3 grains $(0.2 \mathrm{Gm}$.) ; $5-7$ years 4 grains $(0.25 \mathrm{Gm}$.) each night in $3 \mathrm{i}$ (4 c.c.) of aromatic syrup of Yerba Santa. Older children may take it 
in capsules as does the adult. The doses are for 8-10 years 6 grains (0.4 Gm.); $11-14$ years 8 grains (0.5 Gm.). Over this age adult dose. These doses are kept up for eight weeks.

Holt believes in much larger doses. He gives an infant of one year 8 to 12 grains, and says children of five to ten years require nearly as much as an adult.

I should favor the large doses. They can be given to infants and small children suspended in syrup of Yerba Santa, as Tannate, in chocolate lozenges or this prescription for a child of 6 years:

Quininæ Sulphatis..................gr. xxx

Ft. cht. no. xviii

(2 Gm.)

S. Six (6) powders in chocolate syrup each day as directed.

Or it may be given by rectum in solution or in starch paste when one should use two or three times the dose by the mouth. Oral administration is preferable.

Pernicious Infections. As I have said, the pernicious type of the disease is rarely seen in the North; in the South and in the tropics, however, it is much more common. It is caused exclusively by the estivo-autumnal form of the parasite.

One of the best criteria, aside from the severity of the symptoms, for a pernicious infection, is that set by some of the tropical workers; namely, the percentage of infected cells and the number of doubly infected cells.

When 5 per cent. of the red cells are infected the case trespasses upon the dangerous.

The fever is usually irregularly remittent or constant.

The dose recommended is larger than those usually given in temperate climes. While Rogers recommends gr. $\mathrm{x}(0.66 \mathrm{Gm}$.) three times a day, James urges gr. xlv $(3 \mathrm{Gm}$.) a day, in doses of gr. xv $(1 \mathrm{Gm}$.) three times a day for at least ten days and Wellman gives gr. $\mathrm{xx}-\mathrm{xxx}(1.30-2 \mathrm{Gm}$.) for an initial dose, followed by gr. $\mathrm{x}-\mathrm{xx}(0.60-1.30 \mathrm{Gm}$.) every four hours, until the attack clears up (one to four days), then about gr. xx a day.

The profound intoxications are seen more commonly in those who have had repeated attacks or in neglected cases, and in those in whom resistance has been lowered from one cause or another.

Profound depression of all the vital centres is seen, and a particular imposition of the poison on one or the other organ gives a stamp to the picture and affords abundant cause for error in diagnosis.

Thus we have a comatose form in which sudden loss of consciousness resembles apoplexy; apathetic conditions with icterus, like yellow fever; diarrheas that resemble cholera or dysentery; and not infrequently an 
attack characterized by profound prostration, collapse, excessive sweating, sometimes subnormal temperature, the algid form.

In these cases quinine must be used hypodermically or intravenously according to the urgency.

Hypodermic Use. Of the two methods, subcutaneous and intramuscular, the choice should be decidedly for the latter. The rate of absorption is more rapid and the danger of necrosis less. There is now an almost universal condemnation of the subcutaneous route.

The best salt for hypodermic use is the dihydrochloride, which dissolves in less than its own weight in water. Make up a solution as follows:

Quinine dihydrochloride................. $5.0 \quad \mathrm{Gm}$.

Distilled sterile water..................10.0 c.c.

One may take $\mathrm{m} . \mathrm{xv}$ ( 1 c.c.) of the solution given above and dilute to 3iiss. $(10$ c.c.); better 5 ss. $(15$ c.c.) or better yet $5 \mathrm{i}(30$ c.c.) and slowly inject under the skin where the subcutaneous tissue is loose or into the muscle.

The amount needed in the twenty-four hours is some 24 grains, given in divided doses in this way. In worst cases up to gr. $\mathrm{xlv}(3 \mathrm{Gm}$.).

In these severe but less urgent forms, if given by the mouth, the amount should run up to the full doses of Wellman given above.

This method, necessary in this class of cases is not free from disagreeable results and in exceptional circumstances real danger.

As commonly used, the solutions are too concentrated, the result being that a precipitate from the serum is caused by the quinine and the tissues may undergo necrosis.

The more dilute the solution the safer the procedure becomes; it should never be more than 10 per cent. Moreover, too concentrated solutions will not be absorbed.

One should take every precaution to be assured of a sterile needle, syringe and skin, should paint the latter with iodine and inject into the gluteal region or muscles of the back and go down deep into the muscle. There is likely to be some pain and induration but this probability is greatly lessened by the high dilutions.

However, abscesses not infrequently occur in spite of all precautions.

Another preparation of quinine which is excellent for hypodermic use and freely soluble is the quinine and urea hydrochloride to be used in the same dose.

Hypodermoclysis. The same preparation in a gr. $\mathrm{xv}$ (1 Gm.) dose is used in 2 to 3 pints $(1,000-1,500$ c.c.) of salt solution ( $3 \mathrm{i}-0 \mathrm{i}$ 
(4 Gm. -500 c.c.). In diarrheal forms and in collapse this should be an especially valuable method.

Intravenous Use. In severe forms of pernicious malaria, by far the best practice is to put the drug into the vein. This may be done in physiological salt solution. For example:

Quinine Hydrochloride........ $0.50 \mathrm{Gm}$.

Sodium Chloride............. $0.25 \mathrm{Gm}$.

Sterilized, distilled water.......... 30.00 c.c.

(gr. viiss.)

(gr. iii.)

(马i.)

or twice this dilution. In most urgent cases two and four times the dose $\mathrm{gr} . \mathrm{xv}-\mathrm{xxx}(1-2 \mathrm{Gm}$.).

Bass thinks a 10 grain dose is sufficient and much larger doses dangerous. His method of dropping two 5-grain tablets of quinine dihydrochloride into the barrel of a 20 c.c. syringe, drawing up physiological salt solution to dissolve them and then filling to 20 c.c. and injecting, is simple and convenient. Others use a salvarsan apparatus for the purpose.

There seems to be much controversy among authorities as to proper dilutions; some urging the value and safety of 10 per cent. solutions, others pleading for high dilutions 1 to 250 ; critics find danger in one or the other.

Dilutions of 1 to 30 to 1 to 60 seem fairly free from objections. ${ }^{1}$

The dose should be repeated at intervals of a few hours, 2 or 3 up to 6 , until the patient is so far improved as to make oral administrations effectual. This usually occurs in 24 hours. The necessity for frequent intravenous dosage is emphasized by the rapid elimination of quinine so administered, it being only a matter of a few hours.

Inject all at once into the basillic vein made prominent by a ligature as above. (For technique see Rheumatism, Chap. IX.)

Other veins if more accessible may be chosen.

A word more about quinine before we proceed with our subject. This drug is an antipyretic and used as such under certain circumstances. Its antipyretic action is apparently due to the depression of function of protoplasm, of which mention has been made. Some effect, too, upon ferment action can be determined. The result is a lessened output of heat from the tissues, through direct action and not through the intermediation of the heat regulating nervous mechanism. It must be remembered, however, that in malaria the control of temperature is to be attributed entirely to the destruction of the plasmodium and not at all to this pharmacological action.

${ }^{1}$ Some of the pharmaceutical houses now put up in ampoules quinine in solution ready for immediate use. 
Cinchonism. Of more importance to us are those toxic symptoms that may occur when inordinate doses of the drug are given, and so can be avoided; and which occur after very small doses in certain people, whose reaction to the drug constitutes an abnormality which we call an idiosyncrasy, and cannot be avoided. Idiosyncrasy is one of those philological accomplishments, sent as an advance agent to occupy a territory until knowledge shall advance. It is the shadow rather than the substance of a thing, but like many other shadows in affairs temporal it is allowed to usurp and continue to rule as the real thing. It is derived from two Greek words, $i \zeta_{\iota \circ}$, own, and $\sigma v \gamma \rho a \sigma \iota s$, mixture,-having its own mixture, that is a quality peculiar to the individual. What that quality is and how it operates is left for the future to elucidate.

Since we have become more familiar with the phenomena of anaphylaxis, attempts have been made to find analogies in drug idiosyncrasies. They fail so far that they tempt us to apply another term to the phenomena, allergie; but succeed to the extent of furnishing us with a method of detecting sensitization and in some measure of effecting desensitization.

These toxic symptoms are called "cinchonism."

The earliest and most common are ringing or roaring in the ears and a mild degree of deafness. If it amounts to no more than this we give it no consideration, but this may go on to complete deafness.

This has been attributed to congestion and hemorrhages in the tympanum, but Cushny believes, in the light of recent research, that this view is not correct, but that the phenomena are attributable to degenerative changes occurring in the spiral ganglion in the cochlea.

Less frequently than the hearing, the vision is affected. The pupils do not react to light, the field of vision is contracted, the color vision disturbed, and blindness may ensue.

The visible changes in the eye are contraction and even obliteration of the retinal vessels, pallor of the optic disc, sometimes degenerative changes in the retinal nerve-cells, and even atrophy of the optic nerve.

If, as sometimes happens, the disturbance of vision is due to plugging of the retinal vessels by the plasmodia the pupils do react to light and the disc is a greyish red.

Skin eruptions occur in great variety. The most common is an erythema that has been mistaken for scarlatina, an illusion heightened by the fact that it may desquamate; next to that, urticarial wheals. Hemorrhagic rashes also occur, which on occasion may give rise to the diagnosis of a septic process. Less commonly, the drug may cause gastric discomfort of a marked degree and is 
believed at times to cause hemorrhage from the kidneys. In a case of malaria this may give rise to the diagnosis of black water fever and, indeed, Koch maintains that black water fever is due to quinine.

Contraindications to Quinine. When idiosyncrasies do exist to a marked degree they constitute contraindications to the use of the drug.

Further contraindications are any inflammatory process in the middle ear or labyrinth, amblyopia and amaurosis.

Pregnancy is said to contraindicate the use of quinine because of its effect on uterine muscle substance, but the best opinion voices dissent to this statement and the greater danger of abortion and miscarriage from the untreated malaria. If one feels in doubt about the effect of quinine in pregnancy, bromides and opium may be combined with the quinine in treatment.

Other Forms of Quinine. Beside the salts already described mention should be made of cinchona bark; for example, Warburgh's tincture. Many have thought that they got results from Warburgh's tincture that they could not get from quinine. It must be believed that this experience is due to inefficient dosage and usage of the salts of the alkaloid quinine. Other alkaloids of cinchona are not more efficient.

Euquinine or ethylcarbonate of quinine, contains 81 per cent. of quinine, is almost insoluble in water, so almost tasteless. Quinine tannate contains 30 per cent. of quinine. Hence the dose should be $21 / 2$ times as much as the latter. It is insoluble and can be taken by children.

Idiosyncrasies. These are expressed in individuals, after ingestion of small doses of quinine by the symptoms detailed under cinchonism and usually by an intensification of these symptoms. O'Malley and Richey in a case of quinine idiosyncrasy provoked a cutaneous reaction by applying solutions of quinine salts 1-10 to an abrasion just going through the skin, on the flexor surface of the fore-arm, using, of course, a control; this is the common method of determining sensitization to pollens and proteins. The reaction was an area of edema, accompanied by itching and burning, surrounded by a halo of erythema, 3-5 $\mathrm{cm}$. in diameter.

Desensitization was effected by giving $0.005 \mathrm{Gm}$. of a quinine salt with $0.5 \mathrm{Gm}$. of bicarbonate of soda. In one hour and a half $0.1 \mathrm{Gm}$. of the quinine with $0.5 \mathrm{Gm}$. of sodium bicarbonate was given.

Each day the desensitizing doses of $0.005 \mathrm{Gm}$. as given above was administered, but the second dose given one and a half hour later was increased by $0.1 \mathrm{Gm}$. and the same amount $(0.5 \mathrm{Gm}$.) of sodium bicarbonate. Only one dose a day is given. In this way it may be possible to work the dose up to one or two grams. Modifications of this method 
are successful, all being a gradually increasing dose, beginning with a very small one.

\section{SYMPTOMATIC TREATMENT OF PERNICIOUS MALARIA}

The Cerebro-Spinal Types. This includes the delirious and comatose, the hyperpyretic, the irritative, and paralytic suggesting general or focal brain disturbance.

The Comatose Form. This usually follows on delirium and cases in which headache and restlessness have been marked or may come on so abruptly that, like a case of Bass', picked up in the street, may be mistaken for drunkenness. A chill may or may not precede the onset. The pulse is rapid and thready, the heart sounds poor and collapse not uncommonly ensues. The organism found is the estivo-autumnal, but in one of Pepper's cases was the plasmodium vivax. Such attacks are believed to be due to the plugging of the cerebral capillaries by the plasmodia, owing to the size and shape of the Schizontes or the agglutination processes. Intravenous quinine therapy is indicated as described above, beginning with a 10 -grain dose.

Irritative and Paralytic Forms. The irritative and paralytic forms are rife with diagnostic errors, giving rise to general convulsions, epileptiform convulsions, hemiplegia, aphasia, tetanic seizures, cerebellar and bulbar manifestations. The difficulty in these cases lies in establishing a diagnosis. Of course with the previous history of malaria, tropical residence, or the findings of a routine blood examination these difficulties disappear, but one appreciates how often these material, aids are wanting. When the diagnosis is made the treatment is intravenous medication and the treatment of collapse as described.

Collapse. For collapse, stimulation with strophanthin $1 / 2 \mathrm{mg}$. into the vein, strychnine sulphate gr. $1 / 30(0.002 \mathrm{Gm}$. $)$, or caffeine, sodium salicylate, or benzoate gr. v $(0.33 \mathrm{Gm}$.) is required, and in the more urgent cases more rapidly acting agents should be used, such as camphor in 10 per cent. solution in olive or sesame oil or ether gr. iii $(0.2 \mathrm{Gm}$.), epinephrin (adrenalin) 1 to $1,000, \mathrm{~m} . \mathrm{xv}$ (1 c.c.) or ammonia inhalations. Drugs should be given into a vein or into a muscle.

Hyperpyrexia. The symptoms are those of heat stroke and the temperature may rise to $110^{\circ} \mathrm{F}$. These patients pass into coma and the condition is usually a fatal one. It is best controlled by cold baths, at $65^{\circ} \mathrm{F}$. or beginning higher at $85^{\circ} \mathrm{F}$. to $90^{\circ} \mathrm{F}$., and cooling to $65^{\circ} \mathrm{F}$., sponging the head meanwhile. This is continued until the temperature is reduced below the danger point.

For extreme nervousness morphine sulphate is the best, because the least depressing and most effectual of the sedatives. 
Algid Forms are characterized by pallor, cold, clammy skin, small pulse and signs of collapse. Diarrhea of a profuse type with or without vomiting and cramps in the legs simulate cholera; in others the stools contain blood and mucus and are têrmed the dysenteric type; others in which profuse hemorrhages occur are called the hemorrhagic type and if great sweating with collapse is a feature are called the diaphoretic type.

Stitt says cases may even simulate an acute hemorrhagic pancreatitis.

In all these intravenous quinine therapy is indicated as described above and with the treatment of the collapse as detailed under that section.

In the algid form the body heat must be maintained by hot drinks containing coffee, brandy, or whiskey; by blankets and hot bottles or bricks about the body and the hot-water bag at the feet; and by hot rectal injections containing coffee.

Choleraic Form. In the choleraic form the same line of treatment with hypodermoclysis or venous infusion of warm salt solution is indicated.

Morphine is to be injected to control the peristalsis.

Bilious Remittent Fever. This form is characterized by severe vomiting, the vomitus containing bile, the stools and urine charged with bile and jaundice appearing as early as the second day; this form is serious, both because of the fatalities accompanying it and because of the cachexia following upon it.

The treatment is urgent and must be administered intravenously; the vomiting is incompatible with oral administration.

Multiple infections may occur with any of the plasmodia, but seems to be more frequent with the estivo-autumnal. Of course this intensifies the infection, makes for greater destruction of red cells; hence anemia, and blurs the picture.

The treatment is the same as in a simple infection unless its severity counsels intravenous medication.

Kidney Complications occur. Albuminuria is frequent during the attacks, in some 30 per cent. of the tertian and quartan, and 50 per cent. of the estivo-autumnal. This must not be taken too seriously, as real nephritis rarely ensues in the milder forms of the disease. Taking the estivo-autumnal form and the severe infections it induces in the tropics, nephritis, as a sequel, will amount to nearly 3 per cent. and is usually of the chronic parenchymatous variety.

These conditions are to be treated in the same way as if arising from any other cause.

Large liver and large spleen may persist without symptoms of any kind attributable to any interference with their functions. 
Heart. In some cases of pernicious malaria, dilatation of the heart has occurred. This indicates prompt stimulation by the digitalis group, preferably strophanthin by the vein, $1 / 2 \mathrm{mg}$.

Lungs. Bronchitis of a mild type is not uncommon, but more rare and curious is the condition with the signs of a broncho-pneumonia periodically, occurring and yielding to quinine.

Anemia. The marked degree of anemia accompanying malaria has never been satisfactorily explained, as the destruction of cells has been out of all proportion to the number of parasites seen.

Obviously there was another factor operative upon uninfected red cells that made them share in the destruction, some hemorrhagic dissolving substance as Manson put it or a toxin from the plasmodium acting on uninfected cells as Ewing conjectured. Very recently two theories have been advanced, one by Wade Brown who on the basis of experimentation attributes the phenomena or believes them influenced by the hematin set free from the infected cells; and that of Mary Rowley Lawson, who has observed the constant migration of the plasmodium, from cell to cell, resulting in their destruction. This migration is seen in parasites of all ages.

Now and then the anemia is most intense and simulates pernicious anemia. Pepper reports one such case and I recall a very striking example with extensive retinal hemorrhages in my own service at Bellevue Hospital.

After the tertian and quartan infections, repair of the blood is rapid; after the estivo-autumnal form less so.

Good food, air, changes of surroundings, iron and arsenic all help recovery.

Sequelæ. After the severe infections there are certain sequelæ that are possibilities that must be taken into consideration.

The first of these are the mental disturbances, delusional insanity, mania and most common of all melancholia.

Sharp watch must be kept of these exhausted subjects to appreciate any mental aberration, any depression presaging melancholia, with its self-destructive impulses.

\section{RELAPSES}

The relapse is more to be dreaded than the initial attack. It is more fatal, it is more persistent and less amenable to treatment and it finds the patient reduced and less able to resist. Undue exposures to wet and cold, fatigue, and the occurrence of other diseases invite a relapse, but one great factor responsible for relapses is, as James has emphasized, an insufficient quinine treatment of the initial attack. 
He believes that the relapse is due to a renewal of the vitality of the asexual cycle, which is endowed with a large "potential of vitality" and that the asexual cycle can become relatively immune to the protective forces of the body and also to quinine; that is, can become "quininefast."

Others believe that the relapse is simply due to increased numbers of the asexual organisms which have been persistent since the previous attack, but in numbers insufficient to give rise to symptoms.

Relapses may occur (without reinfection) even after years, but are less likely to occur after the malignant tertian; nor do such long intervals intervene between the attacks and relapses in this infection as in the others.

During a relapse the large dose by the vein as above is indicated and a plea is made for larger doses in all forms of the initial infection.

Latent Malaria is a term applied to the continued infection which leads to relapses, often provoked by exposures, change of climate, excesses, surgical operation and parturition.

Masked Malaria is a term applied to certain symptoms, not a part of the usual picture, such as neuralgias, headaches, gastro-intestinal trouble, recurring periodically which seem to disappear on quinine medication.

Malarial Cachexia. There are, however, cases in which repeated attacks have occurred, where the treatment has been neglected or been insufficient, where a chronic poisoning is set up that is termed malarial cachexia. These patients show but few organisms in the blood, and these more commonly during the slight febrile period that interrupt long intervals of normal or subnormal temperature. These are usually the estivo-autumnal form, and are not easy of demonstration. The patients are markedly anemic, with the type of secondary anemia; the face often shows a dirty earth-colored pigmentation. They are emaciated, nervous, depressed, and constantly fatigued physically and mentally inefficient, have a poor appetite and readily get short of breath.

Next to the anemia the enormous size of the spleen is the most notable clinical manifestation. It is easily ruptured or its pedicle may become twisted.

These cases should be removed from the malarious district in which they reside, to one free from infection: to the mountains or to a suitable seashore resort. If this cannot be done, attempts must be made to protect the patient from further infection, that is, bites of mosquitoes, by choosing an upper room, and taking special care to avoid exposure toward nightfall.

Quinine should be begun at once. It has been the custom to give such 
doses as quinine gr. iv three times a day, or in more severe cases up to gr. xvi or gr. $x x$ a day until the slight afternoon rise of temperature that usually accompanies the infection subsides. Then the dose of gr. iv three times a day is cut down to gr. iii and gr. ii three times a day and continued in these doses for weeks.

I think these doses are insufficient, for the cachexia usually means an insufficient quinine administration in the initial attack and the persistence of the malarial organism in a form peculiarly resistant to quinine.

Quinine doses should be as rigorous, then, as in a well-treated initial attack and if this does not answer the use of the drug hypodermically or intravenously in doses of gr. $\mathrm{xx}(1.30 \mathrm{Gm}$.) a day is indicated.

But after all the prophylactic treatment by a sufficiency of quinine in the initial attack is what should be emphasized; together with a repetition of the treatment in 30 or 40 days after the initial attack as advised by Wellman, or 15 grains a day for two successive days each week for two or three months as advised by Deaderick.

Iron and arsenic are of great help in these cases to improve the blood state.

Iron. Iron may be given in the form of a carbonate of iron or other iron preparation. I prefer the carbonate, e. g., pilula ferri carbonatis (Blaud's) gr. v $(0.30 \mathrm{Gm}$.$) one, three times a day.$

Arsenic. Arsenic may be given as Fowler's solution, liquor potassi arsenitis, $\mathrm{m}$. iii ( 0.20 c.c.), increasing $\mathrm{m}$. i ( 0.075 c.c.) each day up to $\mathrm{m}$. viii or $\mathrm{x}(0.50-0.75$ c.c.) three times a day or to the point of some manifestation of intolerance, as suffusion of the eyes or gastric disturbance.

Iron and arsenic may be combined in pill form, e. g. :

$R_{4}$

Arseni Trioxidi....................gr. $3 / 4$

Mass. Ferri Carb.,...................gr. cl

M. ft. cap. no. $\mathrm{xxx}$.

S. One after each meal.

This gives us gr. v $(0.30 \mathrm{Gm}$.) of the mass of carbonate of iron and gr. 1/40 (0.0015 Gm.) of the arsenious acid at a dose.

Another excellent arsenic compound is the cacodylate of soda in doses of gr. ss. to gr. iii a day for 7-14 days which may be repeated after an interval if needed. This is best administered hypodermically. Most pharmaceutical houses furnish the drug in ampoules in solutions ready for hypodermic use.

The nervous manifestations may be helped by strychnine in doses of gr. 1/40 (0.0015 Gm.) to gr. 1/30 (0.0020 Gm.) three times a day, taken with the iron and arsenic; and, indeed, they may all be 
incorporated in one pill or capsule. One should always know that the pills ordered are fresh.

Plenty of fresh air and sunshine are even more important than drugs. Good food and plenty of it to make good the extensive tissue destruction; cool baths, showers, light massage and exercise; at first by walking, later, as the patient's strength returns, by golfing and horse-back riding, and at the shore by swimming complete the treatment.

If the spleen is much enlarged, care must be taken that neither exercise nor occupation shall be of such a violent nature as to threaten its rupture.

Substitutes for Quinine. What is to be done if the patient cannot take quinine?

I hesitate to offer any substitute because there is no other that is even remotely comparable to quinine and the "cannot" is often a prejudice on the part of the patient, or a result of ignorant usage, as colossal dosage, insoluble pills, and nauseous mixture. Again the little disturbances of hearing are interpreted too seriously and further effort to continue the drug is given up. Idiosyncrasies of serious import are very rare, and even these may be dealt with as detailed above.

It is a detriment both to the physician and patient to convey the idea that anything else is nearly as good, or is a fair substitute.

Methylene-blue has been advocated as such. It is a very poor substitute. If, however, we are actually debarred from using quinine, then we may try it in doses of gr. viiss. to gr. $\mathrm{xv}(0.5-1 \mathrm{Gm}$.) a day, divided into three or four portions.

One should remember to warn the patient that the drug will lend color to the urine, a rather startling phenomenon to a patient who is not informed, and that strangury or diarrhea, intervening in the course of treatment, may be due to the drug.

Arsenic, both inorganic, as Fowler's Solution or the oxide and organic as atoxyl and soamin have been used, but with no very encouraging results. Salvarsan is the most efficient arsenical preparation when quinine fails or may not be used. It is given as when used for syphilis at weekly intervals. X-ray or radium treatment of the spleen is said to act as an adjuvant to quinine.

Convalescence. Change of air and especially a removal from a malarious district; open air and increase in the diet, with such drugs for the anemia and such tonics as were named under Cachexia with a repetition of the quinine series as mentioned above are the considerations to be borne in mind during convalescence.

Prophylaxis. We are so well informed now as to the sequence 
of events in malarial infection, as to the rôle of the mosquito, the individual and the malarial organism, that our prophylaxis is clearly defined in measures aimed at the three links in this chain.

Destruction of the Mosquito. The mosquito like Carthage "delenda est" and medical Catos have arisen to reiterate the slogan.

Mosquitoes breed in marshes, ponds, pools or any receptacle of quiet water, even cisterns, buckets or tin-cans.

Where municipalities have become sufficiently interested or in certain territories under control of the Federal government, vast stretches of marshes have been drained for the extermination of the mosquito or, in smaller areas, water-containing depressions have been filled in.

Where communities are ignorant and careless, brigades of mosquito exterminators have been formed, whose business it is to see that cisterns and water receptacles are screened, that small pools are filled, rubbish containing water disposed of or a thin layer of crude petroleum poured on the surface.

Crude petroleum is the most effectual substance used to exterminate the mosquito over extensive surfaces of water. The larvæ of the mosquito have to come to the surface of the water to breathe. The oil prevents their access to the air and fills their air-channels.

One should use about $1 / 2$ pint to every 100 square feet of surface and this should be repeated at two weeks' intervals. Crude carbolic acid has been recommended.

Small fish and tad poles and certain water insects all feed on the pests.

Grass and small bushes harbor mosquitoes, these should be cut about dwellings.

Numerous odorous substances have been used to kill the mosquito in a closed space, the best of these is pyrethrum or pellitory, which contains an acrid resinous-like substance, which when the powder is burned, gives fumes that stupefy them.

Protection of the Individual. Education in schools, public lectures, disseminated literature, explaining to individuals how proper methods may be used in their own environment, is one of the prophylactic measures of the highest import, for through this means both personal and civic responsibility is cultivated.

Swatting with a fly swatter is certainly remunerative exercise in mosquito infested rooms.

Pyrethrum powder in a little alcohol, two pounds per 1,000 cubic feet may be burned in a room closed for four hours; this stupifies the mosquitoes, which are then swept up and burned. (Stitt.)

Sulphur is more certain in its effects when burnt in the proportion 
of two pounds to each 1,000 cubic feet. Damage may be done to metals and delicate fabrics.

If the mosquito cannot be destroyed, the individual may be kept from their attack. This has a double significance. It prevents the man from becoming infected, or being infected, from infecting the mosquito to pass it on to another man.

Isolation may be effected by the use of screens in windows and doors. Copper wire screens are best, 18 meshes to the inch; mosquitoes will pass through a coarser mesh. Also by choosing a room in the upper stories for a sleeping room. If the individual lives in a badly infected district, he should avoid going out after sunset or before sunrise unless his head is protected by a veil worn over the hat, his hands by gloves and his ankles by boots. In the tropical forests such precautions are necessary even by day.

Applications, such as oil of pennyroyal, oil of eucalyptus, camphor, citronella, may be made to the skin, the odor of which is said to keep the insects away, but these are not trustworthy.

$\mathrm{R}$

Oil of Pennyroyal

Oil of Citronella................ āa $\bar{z} \mathrm{i}$

Alcohol or Spirits of Camphor......... viii

M.

S. Apply locally.

Epsom salt one part in two parts of water dabbed on exposed parts and allowed to dry has been recommended.

Quinine Prophylaxis. Going into malarious districts, at the season of the year when infection occurs, or if living in malarious districts, prophylactic doses of quinine should be taken: gr. ii $(0.120$ $\mathrm{Gm}$.) three times a day or slightly larger doses, gr. vii to $\mathrm{x}(0.50-0.60$ $\mathrm{Gm}$.) two or three times a week.

Wellman recommends gr. $\mathrm{xv}$ ( $1 \mathrm{Gm}$.) on two successive days of each week for a period of two months. Various modifications of these interrupted doses have been recommended.

It must be insisted that such prophylaxis is by no means as effectual alone as when combined with protection and the latter should never be neglected if it is possible to carry it out.

Moreover, giving quinine in small doses and interrupted doses induces quinine immunity on the part of the infecting organisms, so that when symptoms of malaria develop it has been repeatedly observed that these cases are harder to cure with quinine, last longer, need more quinine and are more prone to relapse than those who had had no prophylactic treatment. 
Finally, as the infection may die out of itself in certain instances, we know that the body has the power of elaborating protective substances, so that it may repel lesser infections and mitigate severe ones. To do this the body must otherwise be in good health and all measures aimed at excellent physical condition should be considered for those living in exposed districts.

Alcohol does harm and it should not be used in the tropics at all. Arctic explorers tell us it should not be used in the North, so, it would seem that the temperate zone would have a hard battle to justify its designation.

Finally, the malarial organism is attacked in the body of the man, by the use of quinine, before it has had time to multiply and give rise to the characteristics of the disease.

Carriers. It is important in malarial districts, that carriers who may not be manifesting evidences of the disease, should be detected. Blood examinations must determine that.

These cases should be given 15 grains of quinine for two to three successive days each week for three weeks.

\section{BLACKWATER FEVER}

It is not becoming, nay, it is dangerous for me who have had no practical experience with this condition to write dogmatically of its treatment.

The great uncertainty among those who have had an abundant opportunity to study the disease should lead a practitioner taking up tropical work to review the literature with care.

For the student I will merely outline a conservative view of its therapy.

The condition gets its name from the hemoglobinuria, one of its most obvious symptoms.

The whole picture seems to be a tremendous hemolytic crisis, and although some writers have been inclined to look upon it as an entity separate and independent of malaria, this view can scarcely be maintained and malarial infection seems to be the sine qua non, a direct etiological agent. Such an expression of malarial infection, however, needs the intercalation of yet another cause or causes for its eventuation. These have been offered, from vague conjectures (see anemia, above) to conclusions derived from experiment; for example, Bass and Johns, noting the effect on hemolysis of calcium salts added to their cultures, have surmised that an increase of these salts in food and drink might be etiologically concerned in Blackwater Fever. Wade Brown, study- 
ing the effects of alkaline hematin, has considered it an hemolytic element. Others have conjectured a different infection from malaria, such as a piroplasm or a chlamydozoal body. Still others have hypothesized an acidosis affecting the liver, finding support in the observation that the condition is more commonly provoked on the administration of 'acid salts of quinine than when the latter is administered with a base. The views of Koch and others of the part played by quinine in inducing the onset are well known.

Certainly chilling, exposure to sun, fatigue, and alcoholic excesses play a rôle.

It would seem as if individuals, especially Europeans in the Tropics who have had repeated attacks of malaria, had acquired a lessened resistance on the part of their red cells, which renders them more susceptible to whatever toxic influences prevail in malaria or to some such factors as obtain in paroxysmal hemoglobinuria or to an anaphylactic reaction or even to the harmful effects of certain salts of quinine. The form of infection is almost always the malignant tertian, plasmodium falciparum.

Old infection, chilling and quinine administration seem to be the most common factors in the disease.

However, the rôle of quinine must not be misunderstood, as it alone is apparently not a sufficient cause; its inefficient use both prophylactically and therapeutically in malarial infection is largely contributive to the condition.

Symptoms. Its cardinal symptoms are chill, fever, bilious vomiting, with distress in the epigastrium, sweating, jaundice, early and intense, and hemoglobinuria.

Both liver and spleen are somewhat enlarged and tender. Anemia develops rapidly. Most serious symptoms are the development of hiccough and anemia.

Treatment. All rules applicable to the treatment, of malaria (barring quinine) are indicated here, except that the insistency on rest must be greatly emphasized and the patient considered as having sustained a severe hemorrhage (as indeed, he has).

Stimulation is often needed and must be vigorous.

Quinine. As regards the administration of quinine, we are advised to use it vigorously, we are advised to use it cautiously, we are advised to use it only when the parasites are found in the peripheral circulation, and we are advised not to use it at all.

Personally I am much influenced by the attitude of Lovelace (see Archives of Internal Medicine, June, 1913), which seems safe and sane and based on a considerable experience. 
He has been impressed as have others, by the sequence of quinine administration and outbreak of the hemoglobinuria, by the fact that it has never occurred in his experience in the initial attack, but always in cases of relapse and in weakened individuals and in those who took their quinine irregularly as a prophylactic or insufficiently when attacked.

He would, then, under no circumstances give quinine during an attack of Blackwater Fever nor for several days after, not until the urine has cleared up, hemoglobin casts no longer being seen and the icterus quite or nearly cleared up.

Then an effort to stamp out the malarial infection is made by using quinine with great caution.

He begins with 1 grain doses $(0.065 \mathrm{Gm}$.) of quinine tannate (because one of the weakest salts) three times a day, and gradually works up to 20 or 30 grains $(1.30-2 \mathrm{Gm}$.) of the hydrochloride daily.

Those who believe that it is especially the acid salts that provoke the hemoglobinuria confine themselves to the quinine base or the tannate. The treatment thus becomes largely symptomatic. For the chills, fever and sweating, as in the ordinary type, measures to alleviate discomfort are pushed.

Alkaline waters, such as vichy or plain water are given freely, if vomiting does not prevent. Vomiting may be treated as under malaria. If the attack is severe and vomiting continuous, hypodermoclysis, enteroclysis and saline infusion with normal salt is indicated. When food is long rejected glucose into the veins may be needed to maintain nutrition.

If an acidosis is determined, alkalis should be administered. (See Pneumonia, Chap. IX ). In anuria hot fomentations and cups are applied over the kidney. When quinine is deemed unsafe, neo-salvarsan has been advocated and some good results seem to have been obtained.

Prophylactic. The daily use of quinine gr. vi to $\mathrm{x}(0.40-0.60$ $\mathrm{Gm}$.) or gr. $\mathrm{xv}$ once or twice a week, and an efficient treatment of initial attacks.

\section{SUMMARY}

\section{Rest in bed.}

Bed. Preferably single. Hospital type.

Room.

Well ventilated.

Upper story preferred.

Screened.

Freed from encumbrances. 
Diet.

Make no effort to feed during a paroxysm, very light diet, only fluids within six hours of a paroxysm.

In intervals of paroxysms semi-solid food of simple character.

In remittent type of fever (estivo-autumnal) consider caloric needs and protein demands. (See Chap. II.)

\section{Symptomatic treatment during the paroxysm.}

Care of the bowels.

Calomel, gr. iii-v (0.20-0.35 Gm.) or in divided doses, gr. 1/4 (0.015 Gm.) every quarter hour for six or eight doses.

This may be followed in three to four hours by a saline.

Magnesium sulphate (Epsom salt).

Sodium sulphate (Glauber's salt), or

Sodium and potassium tartrate (Rochelle salt) 5 ss. -5 i (15-30 Gm.) in half to three-quarter glass of water.

Best time to give cathartic is between the paroxysms, but before the quinine.

Chill.

\section{Heat.}

Hot water bags or bottles to feet.

Flannels about the extremities.

Blankets.

Hot drinks, water, weak tea, lemonade, whiskey or brandy.

Codeine phosphate, gr. ss. (0.030 Gm.) by mouth. Spirits of chloroform, 3 i (4 c.c.) in water.

Vomiting.

Effervescing drinks, charged water, champagne.

Mustard paste to epigastrium. One part of mustard to three or four of flour; mix with cold water, spread between linen or cheesecloth, leave on ten to fifteen minutes or until well reddened.

Bromides in 10 to 15 grain doses $(0.66-1 \mathrm{Gm}$.) spirits of chloroform, $3 i$ (4 c.c.) in water.

Retching.

Draughts of warm water.

Bicarbonate of soda, $3 \mathrm{j}(4 \mathrm{Gm}$.) in a glass of warm water.

If very severe.

Morphine hypodermically, gr.1/12-1/8 (0.005-0.008 Gm.). Collapse.

Hot drinks, coffee, brandy, whiskey, aromatic spirits of ammonia $3 \mathrm{i}$ (4 c.c.) in 3ii-iv (60-120 c.c.) water.

Fever.

Strong water of ammonia to inhale.

Cold.

Cracked ice to suck.

Cold water, cold lemonade.

Cool sponges of water or water and alcohol.

Light rubs with 25 per cent. alcohol. 
Headache.

Cold cloths to forehead.

Ice bag to head.

Codeine phosphate $1 / 4$ to $1 / 2$ grain $(0.015-0.030 \mathrm{Gm}$.) doses by mouth or hypodermically; or,

If intense, morphine hypodermically, gr. 1/24 to gr. 1/12 (0.003$0.005 \mathrm{Gm}$.)

Sweating.

Change night-dress and sheets.

Rub body with dry towels.

Drinks of water and lemonade.

Collapse. (See under Chill, in Summary.)

\section{Specific treatment.}

Quinine.

May be given a few hours before an expected paroxysm.

May be given at the decline of the fever of the paroxysm, or may be divided into two or more doses during the day.

Oral administration.

Give in capsules, each gr. ii (0.15 Gm.), or

Give in solution; bihydrochlorate is very soluble; bisulphate 1 in 10 ; or dissolve sulphate in any dilute acid, dilute sulphuric, aromatic sulphuric, dilute hydrochloric, phosphoric or tartaric, m. i per gr. i.

Good method.

Dissolve the dose in a few grains, e. g., gr. $x(0.60 \mathrm{Gm}$.) citric acid in lemon juice, add water and a pinch of bicarbonate of soda and drink effervescing.

Dosage.

Gr. $x x x(2 \mathrm{Gm}$.) of quinine a day, until the paroxysms cease, then gr. $\mathrm{xx}(1.30 \mathrm{Gm}$.) a day for ten days to fourteen days, then

gr. xv (1 Gm.) on two successive days (e. g., Saturday and Sunday) each week for two months.

Doses may be divided as gr. $\mathrm{xv}(1 \mathrm{Gm}$.) twice a day, or gr. $\mathrm{x}(0.60$ Gm.) three times a day; or

10 grains $(0.66 \mathrm{Gm}$.) three times a day for three days, then

10 grains $(0.66 \mathrm{Gm}$.) each night for 8 weeks without intermission. (Bass.)

In tropics.

15 grains $(1 \mathrm{Gm}$.) three times a day for a week or until temperature is normal for 5 or 6 days; then 10 grains $(0.66 \mathrm{Gm}$.) three times a day for 10 or 12 days (Stitt in Canal Zone), or:

30 grains $(2 \mathrm{Gm}$.) daily until symptoms have disappeared and plasmodia are no longer found; then 15 grains $(1 \mathrm{Gm}$.) daily for 2 weeks; then 10 grains $(0.66 \mathrm{Gm}$.) daily for 2 months at least. (Vedder.)

Bethea's method. (See text.)

Avoidance of inducing "quinine fastness" in organisms by insufficient early dosage. (See text.) 
Quinine in children.

Gr. i $(0.060 \mathrm{Gm}$.) three times a day for each year of the child's age or, better, larger doses.

(Holt) Infants one year, gr. 8 to $12(0.50-0.75 \mathrm{Gm}$.), five-ten years, almost as much as adult doses.

(Bass) Infants under 1 year-1/2 grain $(0.030 \mathrm{Gm}$.); 1 year, 1 grain $(0.060 \mathrm{Gm}) ;$.2 yrs., 2 grains $(0.012 \mathrm{Gm}$.); $3-4$ yrs., 3 grains $(0.2$ Gm.); 5-7 yrs., 4 grains $(0.25 \mathrm{Gm}$.) each night in aromatic syrup of Yerba Santa. 8-10 yrs., 6 grains $(0.50 \mathrm{Gm}$.) in capsules as adults. Above 14 yrs., adult doses. Doses kept up for 8 weeks. Give quinine in solution by the mouth to infants.

To small children give in syrup of Yerba Santa or by rectum in two to three times the oral dose. Give in solution or starch paste. or

Quinine sulphatis, gr. $\mathrm{xxx}(2 \mathrm{Gm}$.$) .$

Ft. chart. No. xviii.

S. Six (6) powders in chocolate syrup each day as directed. (Bethea).

Treatment of pernicious infections.

Quinine, gr. x $(0.60 \mathrm{Gm}$.) three times a day. (Rogers).

Quinine, gr. xv $(1 \mathrm{Gm}$.) three times a day for at least ten days. (James.) Quinine, gr. xx-xxx (1.30-2 Gm.) for an initial dose, followed by gr. $\mathrm{x}$-xx (0.60-1.30 Gm.) every four hours until attack clears up (one to four days), then gr. $x x$ a day for a series of days. (Wellman.)

The evidence is in favor of the liberal dose.

Very severe forms, comatose, choleraic, algid, etc.

Quinine, subcutaneously, intravenously, or intramuscularly; must be very dilute, 1 in $20-30$ or more.

Subcutaneously or intramuscularly. The latter is always to be preferred.

Use dihydrochloride; e. g., quinine dihydrochloride, $5 \mathrm{Gm}$., distilled sterile water 10 c.c.

Use $\mathrm{m} . \mathrm{xv}$ ( 1 c.c.) of above diluted in water to 3 iiss. (10 c.c.) or better, to $\xi$ ss. ( 15 c.c.) or $5 \mathrm{i}$ (30 c.c.). Never more than 10 per cent: solution. Inject slowly into the muscle or loose subcutaneous tissue.

Quinine and urea hydrochloride - use in same way.

Total daily dose, gr. xxiv-xlv (1.60-3 Gm.).

Hypodermoclysis.

Use gr. xv $(1 \mathrm{Gm}$.) of the hydrochloride of quinine or the bimuriate of quinine and urea in 2 to 3 pints $(1,000-1,500$ c.c.) of salt solution ( $3 \mathrm{i}$ to $\mathrm{Oi}$ ) ( $4 \mathrm{Gm}$. to 500 c.c.) and allow to flow slowly from an irrigator or sterilized fountain syringe into loose tissue under breasts, in abdominal wall, loose tissue of flanks. Temperature at needle should be about $100^{\circ} \mathrm{F}$. A short, large calibred tube from irrigator lessens loss of heat.

This is particularly indicated in choleraic cases and in collapse.

Intravenous use.

High dilution, one in twenty, better thirty or more. (See text.)

Any highly soluble form, as above.

Dose, gr. viiss. $-\mathrm{xxx}(0.50-2 \mathrm{Gm}$.). 
For example.
Hydrochloride of Quinine....... $0.5 \mathrm{Gm}$.
Sodium Chloride............ $0.25 \mathrm{Gm}$.
Distilled Water.................30 c.c.

Inject into basillic vein made prominent by ligature.

Other veins may be chosen.

(For details of technique, see Rheumatism, Chap. III.)

Bass thinks 10 grains (0.66 Gm.) enough-more, dangerous. (For his technique, see text.)

Repeat above doses by vein at 2 or 3 up to 6 hour intervals until improvement makes oral medication feasible. This usually occurs in 24 hours.

(Quinine solutions in ampoules ready for intravenous use are now put up by some of the pharmaceutical houses.)

For toxic manifestations (cinchonism and idiosyncrasies) see text.

For contraindications see text.

Other forms of quinine. (See text.)

Idiosyncrasies-sensitization.

Desensitization. (See text.)

\section{Treatment of collapse.}

Strophanthin, 1/2 mg. (gr. 1/120) into vein.

\section{Symptomatic treatment of pernicious malaria.}

\section{Cerebro-spinal type.}

\section{Comatose type.}

Intravenous quinine therapy.

Collapse.

Stimulants.

Strophanthin, gr. 1/120 (0.0005 Gm.) hypodermically.

Caffeine sodium salicylate or caffeine sodium benzoate, gr. $\mathrm{v}$ $(0.30 \mathrm{Gm}$.$) hypodermically.$

Camphor (10 per cent. -20 per cent. in oil), gr. v (0.30 Gm.) hypodermically.

Strychnine sulphate or nitrate, gr.1/30 (0.002 Gm.) hypodermically.

More urgent cases.

Inhalations of strong water of ammonia on towel.

Adrenalin, m. xv (1 c.c.) intramuscularly or $\mathrm{m}$. iii intravenously. Hyperpyrexia.

Cold baths at $65^{\circ} \mathrm{F}$. or at $85^{\circ} \mathrm{F}$. $-90^{\circ}$. F. and cooling to $65^{\circ} \mathrm{F}$.

Ice in tub to keep temperature down.

Sponge head with cold water during bath.

Take patient out at $102.5^{\circ} \mathrm{F} .-103^{\circ} \mathrm{F}$.

\section{Algid form.}

\section{Heat.}

Hot drinks; water, coffee, lemonade, brandy or whiskey.

Hot water bottles to feet and extremities. 
Blankets.

Hot rectal injections $105^{\circ}$ F. $-110^{\circ}$ F. of water or salt solution containing strong coffee.

Choleraic form.

Same as algid, with hypodermoclysis or venous infusion of warm salt solution (.6 per cent.).

Morphine-gr. 1/8-1/4 (0.008-0.015 Gm.).

Bilious remittent fever. See text.

\section{Complications.}

Kidney. (See text.)

Heart.

Dilatation is a rare occurrence.

Strophanthin by the vein $1 / 2 \mathrm{mg}$. (gr. $1 / 120$ ) or digitalis by the same method. (See Pneumonia, Chap. IX.)

Lungs. (See text.)

Sequelæ.

Extreme nervousness.

Morphine, gr. 1/16-1/8 (0.005-0.008 Gm.).

Keep sharp watch on exhausted patients, who develop psychoses.

Anemia.

Abundant diet.

Fresh air.

Change of surroundings.

- Iron and arsenic. (See below under Cachexia.)

\section{Relapses.}

More resistant to quinine than initial attack.

Treat intravenously.

(See above in Summary.)

\section{Latent malaria.}

(See text.)

\section{Masked malaria.}

(See text.)

\section{Malarial cachexia.}

Remove from a malarial district or protect from further infection. (See Prophylaxis.)

Quinine.

Treat as rigorously as a severe initial attack. (See Summary, above.)

If this does not answer use the drug intravenously in doses of $\mathbf{g r}$. xxiiss-gr. $\mathrm{xxx}(1.50-2 \mathrm{Gm}$.) a day.

When slight temperature has subsided or a course equal to that. given in a severe initial attack has been administered. 


\section{Treat anemia.}

Iron.

Pills of carbonate of iron (Blaud's) or Vallet's mass (Massa ferri carbonatis), gr. $\mathrm{v}$ to $\mathrm{x}(0.30-0.60 \mathrm{Gm}$.) three times a day.

Arsenic.

Fowler's Solution (Liq. potassii arsenitis) m. iii (0.2 c.c.) three times a day, increasing $\mathrm{m}$. i (0.060 c.c.) a day up to $\mathrm{m}$. viii-x (0.50-0.75 Gm.) three times a day or to point of tolerance (suffusion of eyes, puffiness under eyes, gastro-intestinal disturbances).

Combined iron and arsenic.

R

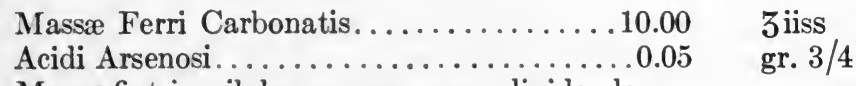

Massa fiat in pilulas numero. $\mathrm{xxx}$, dividenda.

S. One or two three times a day.

Cacodylate of soda, gr. ss. to gir. iii a day for 7 to 14 days. Give hypodermically. This may be repeated after an interval if needed.

Nervous manifestations.

Strychnine sulphate, gr. 1/30 (0.002 Gm.) three times a day.

Fresh air.

Abundant diet.

Cool baths.

Massage.

Graduated exercises.

If the spleen is much enlarged, patient should be warned against possible rupture by violent exercise or effort.

Substitutes for quinine.

None efficient.

Methylene blue, gr. viiss. to gr. xv (0.50-1 Gm.) in divided doses.

Arsenic.

Fowler's solution.

Atoxyl.

Soamin.

Salvarsan. Given as in syphilis at weekly intervals.

$\mathrm{X}$-ray or radium treatment of the spleen as an adjuvant.

\section{Convalescence.}

(See under cachexia, change of air, diet, hygiene, iron, arsenic, etc.)

\section{Prophylaxis.}

Destruction of the mosquito.

Draining marshes.

Filling in depressions.

Screening receptacles of water.

Kerosene in pools.

Small fish and tadpoles in ponds and pools feed on the larvæ. Keep 
grass cut around dwellings and remove small bushes from immediate vicinity.

Pyrethrum and sulphur. (See text.)

Protection of the individual.

Education.

Sleeping in upper rooms.

Screening rooms.

Avoid going out after sunset and before sunrise unless protected.

Head with veil.

Hands with gloves.

Ankles with boots.

Applications to skin (very little value). (See text.)

Prophylactic doses of quinine most important, gr. xv (1 Gm.) quinine on two successive days each week or gr. x (0.60 Gm.) every day.

Keep body in good health.

Avoid alcohol.

Carriers.

Should be given 15 grains ( $1 \mathrm{Gm}$.) of quinine for two or three successive days each week for 3 weeks.

\section{Blackwater Fever}

\section{Treatment.}

All rules for treatment of malaria applicable except quinine.

\section{Use of quinine.}

1. Lovelace's rules. (See text for detail.)

No quinine during the attack and for several days after. Then gr. i $(0.060 \mathrm{Gm}$.) of the tannate of quinine three times a day and gradually work up to $\mathrm{gr} . \mathrm{xx}-\mathrm{xxx}(1.50-2 \mathrm{Gm}$.) of the hydrochloride daily.

Treat chills, fever, and sweating as given under Malaria.

Give alkaline waters or plain water freely.

Vomiting.

See under Malaria. If severe and continuous (Hypodermoclysis,) enteroclysis and infusion with normal saline solution.

If food is long rejected, give glucose into veins. (See Cerebro Spinal Meningitis, Chap. XXV.)

Acidosis.

Administer alkalines.

(See Pneumonia, Chap. IX.)

Anuria.

Hot fomentations and cups over kidneys. (For technique of applying fomentations and cups, see Scarlet Fever and Pneumonia, Chaps. XVII and IX.)

Salvarsan.

Has been used in place of quinine when latter was feared.

Prophylaxis.

Daily use of quinine, gr. $x(0.60 \mathrm{Gm}$.), and an efficient treatment of the initial attack.

Relapses and chronic cases are candidates for Blackwater Fever. 


\section{CHAPTER XVI}

\section{DYSENTERY-BACILLARY AND AMEBIC}

\section{BACILLARY DYSENTERY}

Dysentery is a term used to designate a morbid condition, characterized by inflammation of the colon and, occasionally, of the lower end of the small intestine as well; hence, it is a colitis or enterocolitis.

It is an acute infectious process which, on etiological grounds, can be divided into two diseases: (1) Amebic dysentery, due to the invasion of the mucous membrane and neighboring tissues by a protozoön, the Entamœba histolytica, and (2) bacillary dysentery, due to the pathogenic action on the same structure of a bacillus, or of members of a group of bacilli, spoken of as the Bacillus dysenteriæ.

The pathological changes of either type have certain distinguishing marks of their own; the symptoms vary somewhat and the sequelæ are not identical; and yet the two are so nearly alike that without the finer aids to diagnosis, the use of the microscope and culture, they may readily be confused.

The bacillary form is the one peculiar to this climate and to the United States, though amebic dysentery is every year more frequently met with even in this city and among those who have not been in the tropics; first, because the stools of dysentery are constantly more carefully examined and, secondly, because, since the acquisition of tropical possessions, more patients are invalided home to disseminate the disease.

In 1898 Shiga in Japan announced the isolation from the stools of dysentery patients of the bacillus etiologically concerned. In 1900 Kruse identified the same organism in dysentery cases in Germany; while Flexner, Strong and Muszson in the same year announced the finding of an allied organism, having some cultural and serological differences in the stools of dysentery patients in Manila. Other bacilli with certain differences, the Y. organism of Hiss, have since been reported and during the late war still others. The medical service of our own army issued four different sera for the identification and treatment of these cases.

Culturally the bacillus dysenteriæ has many points of similarity to the typhoid bacillus. It is however a non-motile organism with 
certain other slight morphological variations. The two main types of the bacillus dysenteriæ are the acid strains of Flexner-Strong (producing acid in mannite media) and the non-acid strain of Shiga-Krause (not producing acid in mannite media).

From a practical standpoint the serological differentiation, that is, the power of a serum produced through a given strain to agglutinate bacilli of that strain is more important than the cultural and, of course, identifies the serum to be used therapeutically at the same time. When the serum of the patient's blood is used to agglutinate the stock strains and so identify the invading organism it must be remembered that these agglutinating substances in the serum are usually not marked before the sixth or seventh day of the disease.

Diagnosis is determined then by bacteriological examination of the stools early in the disease and by agglutination tests at the end of the first week and after.

Pathology. The process is an acute catarrhal inflammation of the large intestine and, in the tropics, of the lower parts of the ileum with congestion, sero-purulent exudate, fibrin formation and coagulation necrosis with superficial ulcerations. The ulceration is more intense in the neighborhood of the ileo-cæcal valve, sigmoid flexure and rectum.

The short incubation of two or three days, more rarely delayed as long as seven, the rather sudden onset, with fever, diarrhea, colicky pain and tenesmus, the appearance shortly of mucus in the stools, streaked with blood, later looking like chopped or shredded beef that has been washed out in water, are merely mentioned here to recall what the condition and symptoms are which we have to consider in the treatment.

There is a moderate leucocytosis with slight polymorphonuclear increase; exceptionally the lymphocytes predominate.

Therapy. There are few self-limited infectious diseases that are accompanied by such continuous, nagging discomfort and distress as dysentery. Our ingenuity is exercised to the utmost, not merely in the efforts to shorten the process, but even in attempts to afford some comfort. For success much depends on ability to secure rest.

Rest. One might assume that the train of events in dysentery would compel rest, and so, indeed, in severe cases it does, but in the cases that in their incipiency promise to run a mild course it is often difficult to get the patient to bed; and his failure to accede to such advice may determine a very severe process in what might otherwise have been relatively benign.

Rest in every case of acute infectious diseases is desired both for the body at large, suffering from the effects of the toxins of disease and of pyrexia, to shelter metabolism by avoiding unnecessary work and so 
diminishing the demand for food to meet such metabolic needs and sparing the organs concerned in digestion, assimilation and utilization of these foods, but also for the particular organs or tissues that chance to be the sites of lesions caused by the disease; in this case, the large intestine. Now, the large intestine is a receptacle for certain materials, in part passed on from above, in part excreted from its walls. In this material fermentative and putrefactive processes are going on as the result of bacterial action. From this material, water and certain substances in solution are being absorbed. This structure, the bowel, is in a condition of active muscular exercise, the site of peristalsis, of a swinging pendulum movement, and finally is pressed upon and massaged by contiguous structures as the result of the action of the muscles surrounding the abdominal cavity, from which arises so great benefit from walking and gymnastics directed to the abdominal muscles in constipation. It will be our effort to diminish the activities of the large intestine, that is, to secure rest. By putting the patient to bed we avoid the squeezing and massaging due to muscular action and so take away one stimulus to increased peristalsis; an important factor, considering the hyperactivity of this function, upon which the frequency of the movements and the colicky pain so largely depends.

Even the bed is a bed of unrest, for the griping pain is always forcing the patient to seek some position to relieve it.

Bed. Far more work is to be done about this bed by nurse or attendant than about the bed even of a typhoid fever case. The constant use of the bed pan, the soiling that necessitates changing of the bed linen, the soothing applications to be made, the irrigations to be given, all demand as much economy of effort as can be procured by favoring conditions to work under. The bed, then, should be chosen with care. It must be a narrow or half bed, best of the hospital type, which is about 6 feet 6 inches long, 36 inches wide and 24 to 26 inches high. The spring of woven wire must be stiff and the mattress of hair preferably firm. Over this is placed the sheet, long enough to tuck well under and draw smooth and taut. If the surface of the mattress is not smooth a folded blanket under the sheet will make it so.

Over the sheet is put a rubber-sheeting that should extend from the pillow to the bend of the patient's knees and wide enough to tuck under. Over this comes the important draw-sheet, a long sheet folded lengthwise and laid across the bed covering the rubber-sheeting and tucked far under one side. This sheet can be drawn toward the other side, little by little, to afford a cool fresh surface under the patient from time to time, or, if soiled, can be replaced without remaking the 
whole bed. Over the patient is placed the upper sheet and one or two blankets arranged with a view to comfort.

It is very well to have a second bed of the same kind, to which a patient can be drawn while the mattress and clothes of the first are airing.

Room. Every sick-room should be large, well ventilated, and should get sunshine. As, however, the disease is far more common in the summer than at any other time of the year, it is well to select a room in which there shall be shade the most of the day. Morning sun and afternoon shade are, of course, ideal. If there is a balcony on which the patient can be wheeled it will, if it is properly screened, serve as the best room possible in the warm season.

A bath room contiguous to the sick-room is extremely desirable, saving much labor of fetching and carrying as is obvious.

The odors, which in some cases are very offensive to the patient as well as to attendants, may be mitigated by good ventilation, by the burning of joss-sticks, or by one of the many deodorants on the market, whose claim to disinfect the community when a few drops are shaken here and there must not be taken seriously.

Remembering that this is an infectious disease, that the infecting agent is exclusively in the stool and that the transmitting agent is commonly the fly contaminated with the feces, the room and bath room should be screened and war waged on the fly with every instrument and device of extermination.

Nurse. The nurse or attendant should be thoroughly instructed as to the source of danger to herself and to others. She should wear rubber gloves and a gown whenever handling stools or cleansing the patient after a stool. If she cannot avail herself of this protection, she should carefully clean her hands with especial reference to her nails, after each performance. The urine does not contain the bacilli, but the stools, bed linen, dishes handled by the patient, bed pan, rectal tubes, thermometer, should be sterilized just as in a case of typhoid fever. (See Typhoid Fever, Chap. XIV, for details.) All food left by the patient should be promptly burned.

The Physician. He, too, should protect himself by rubber gloves and gown when examining the patient and inspecting the stools, and carefully disinfect his hands on leaving.

Care of the Body. A cleansing bath should be given each day, if there is a skilled hand to administer it or the manipulation does not cause too much exhaustion.

It is given between blankets, the patient divested of his night-shirt, being rolled on one and covered by another. Soap and warm water 
at $110^{\circ} \mathrm{F}$. is used, one part of the body after the other being exposed and attended to and then dried.

The feet may be placed in a small foot-tub in the bed, the knees being drawn up. After the bath the skin may be rubbed with alcohol and dusted with drying powder-one of the numerous talcum or toilet powders in use.

An approximation to this detail can be attained by an attendant in the family, if care is exercised.

The mouth should be attended to by rinsing after every feeding and, if in bad shape, carefully cleansed by gauze or absorbent cotton around the finger of the nurse or on some applicator after dipping into some cleansing solution, like boric acid 2 per cent. to 4 per cent., a Dobell's solution, or half strength Listerine antiseptic solution N. F. If very bad, peroxide diluted one-half with water or salt solution may be used before the mouth-wash.

When the mouth is very dry, equal parts of liquid petrolatum (albolene) and 2 per cent. boric acid solution with a little lemon juice gives relief. Glycerin is sometimes used, but if the mouth is very dry, it may be aggravated by the glycerin which takes up water so readily.

Constancy of attention and patience are needed to nurse these cases properly, because the restless patient is disarranging the clothes and seeking a comfortable position every moment. The knees are often drawn up to lessen the tension of the abdominal muscles, a hard position to maintain as the feet slip on the smooth sheet. A folded blanket or pillow under the knees gives comfort, or a foot rest placed at the bottom of the bed. These devices, however, are equally unsatisfactory. A gatch bed (see index) raised to support the knees is much more satisfactory, but when the stools are frequent, constant lowering to place the bed pan, makes it an onerous undertaking.

Rings and air-cushions may be needed to take the pressure off the bony parts daily becoming more accentuated.

Nothing is more striking and real than the intangible something we call the "atmosphere" of the room, and it is the ability of the trained hand to secure order, neatness, quiet and calm that gives courage both to the patient and doctor.

Frequent visitors, garrulous friends, Job's comforters, pedlars of similar experiences, id genus omne, are to be excluded as a part of the treatment.

The bed pan in this disease is the most important article in our armamentarium, and its skilful use is imperative. The distressing condition that obtains in some of these cases is hard to appreciate unless 
witnessed. I have seen stools coming every fifteen minutes with tenesmus that deprived the patient of all sense of relief after the stool, and keeping up for hours, until the patient begged that the pan be not removed at all.

The pan should be warmed before using, the patient's knees flexed and a hand placed under the lower part of the back to raise him. The same thing is done on taking the pan away. Either a clean pan can then be slipped under the patient or a towel may be placed under the pan to. begin with, upon one or the other of which the patient lies while the parts are carefully cleansed, dried, rubbed with alcohol and dusted.

It is very essential in this disease that the patient's body and abdomen should be kept warm, as chilling aggravates the diarrhea. Woolen material should be worn over the abdomen. If the feet are cold the hot-water bottle is to be used.

Diet. During the first few days of the attack, when the stomach is often very sensitive to food, and nausea and vomiting are a feature, and while the evidences of intoxication are most marked and peristalsis most active, the quantity of food is of less importance than the quality.

The food must be bland, non-irritating and non-stimulating to the gastro-intestinal tract, must be readily assimilable and leave but little residue, and must be concentrated, especially with reference to its protein content. All these conditions are met by milk better than by any other food.

It has been my lot to have a great many cases of dysentery to treat. Most of them, to be sure, were of the moderate type, but not all.

It has been my routine to put the patient on a milk diet, ordering the milk boiled and given every two hours, the patient is to take what he will of 8 ounces.

Why boiled milk should be any better, if as good, as clean raw milk in this condition, I cannot offer adequate explanation. I can only plead handing a leaf out of the book of my own experience. If the patient prefers it raw, or boiled milk distresses him, I do not persist in my prejudices. At the beginning of the attack, especially if there is much fever, I dilute it $1 / 4$ or $1 / 3$ with plain boiled water, or barley water or rice water. Preferably plain water. If there is a coated tongue or flatulence or the milk does not seem well borne, I suspect that the fat is not well borne and have the milk skimmed.

I believe the experience of our pediatric practice with reference to intolerance for fats can afford us practical information in its usage in adults.

When the temperature disappears and the stools have Tost their 
diarrheal character, one may allow barley jelly, and thoroughly boiled rice; later, toast, then an egg, then a chop and then gradually the resumption of a normal diet.

When, however, a case runs a chronic course, the same needs for sufficient foods to make good the body losses prevail as in typhoid fever. These will be taken up shortly.

All food should be given warm, for if cold or very hot, peristalsis is aggravated.

I am aware that many will take exception to milk as the main article of diet in this disease and especially and perhaps with more justice the pediatrician. I am not prepared to dogmatize. Others prefer during the acute stages only albumin or rice water or barley water sweetened with lactose, thin chicken broth, tea sweetened with lactose, meat juice, expressed from rare steak; while the exceptional case does better on a diet that upsets all our dietetic theories; casein would seem appropriate in infants. If the milk diet I have suggested does not agree, one may fall back on such a diet as has just been quoted.

Milk sugar may be added to the foods given so as to increase the caloric value.

If there is much gastric disturbance, all foods must be stopped, until it is removed.

Water should be given freely, as it must be appreciated that there is a constant and great loss of water to the tissues. It should not be given too cold or in too large amounts at once, for reasons just expressed.

Care of the Bowels. As the treatment of the disease resolves itself pretty much into the treatment of the bowel, the preliminary catharsis only will be touched upon here and the rest taken up under symptomatic treatment.

The object of the preliminary catharsis is to clean out the intestinal canal as far as possible before beginning treatment. That the patient has diarrhea and frequent diarrhea is by no means proof that the intestine is clear. It is undoubtedly Nature's intent that it should be, and the diarrhea is but an expression of the intestine's effort to rid itself of irritation, which effort may be futile or abortive, without help.

There are three cathartics especially recommended for this purpose; calomel, salines, and castor oil.

Personally, I am emphatically in favor of castor oil. I prefer it because I believe it as effectual as the others in evacuating the bowel; because I believe it less irritating than the others and because I am convinced that it has an after-constipating effect. 
A paper read at the American Pediatric Association by Abt of Chicago dealt with the study of the effects of repeated doses of these three cathartics in infants in doses usually used in diarrhea, with reference to irritating effects produced, as expressed by the presence of mucus and occult blood. After the second dose-the doses given at two-hour intervals-calomel was followed by increasing amounts both of blood and mucus, salines by less and castor oil by least and but little unless in large and long continued doses.

I would not convey the impression that this occurs in the adult enough to decry their use, for I think that would be unfair.

If there is nausea and vomiting accompanying the attack, one gives calomel in divided doses, gr. 1/4 or gr. 1/10 every ten or fifteen minutes until 1 or $11 / 4 \mathrm{gr}$. are given, for the anti-emetic effect of the drug as well as its cathartic; then, if the stomach is quiet, give the oil, or failing that, salts.

Castor Oil never was looked upon as a delicacy and the very sight or mention of it so keenly associated in the minds of many with the ordeals of childhood that it is flatly refused or taken under bitter protest. Because of this fact, so-called tasteless preparations are offered or its taste is covered or it is given in capsules.

There are several ways of giving castor oil while at the same time disguising its taste, and this may be so successfully done at times, as to make it desirable to administer it without announcing its character. Many vehicles, however, like hot black coffee, sarsaparilla, soda or beer are unsuited to the conditions at hand, while to give it in milk is to violate a rule that cannot be too emphatically insisted upon"never give disagreeable medicines in a food."

The disguise is not complete and the association may turn the patient against an important food.

An excellent method is to put a teaspoonful of brandy, whiskey, wine, lemon-juice, orange-juice, pineapple juice, or peppermint water in a wine or egg glass, let it run all around the surface to wet it; pour on to that 5 teaspoonfuls of castor oil from a spoon previously wetted in hot water to let it run easily, over the oil another teaspoonful of the same substance, and instruct the patient to take it quickly, wiping the lips and allowing him to suck an orange or lemon.

The dose is, then, $1 / 4$ to 1 ounce.

Specific Treatment. Serum. It is difficult as yet to pass judgment on the value of serum in bacillary dysentery. To be in any degree efficient, the serum must have been prepared from the strain etiologically concerned in the individual case. One would naturally expect better results from serum elaborated against that strain alone than 
from a polyvalent serum, though this may be used for want of better. It is used at present only in the severe cases, and then only after a history of previous serum administration or of asthma has been considered, a test for sensitization made and desensitization precautions observed (see Pneumonia, Chap. IX for details and treatment of anaphylactic phenomena.) The dose is 40 to 60 c.c. of serum diluted with equal amounts of normal saline given intravenously. Doses of $20-40$ c.c. are given each succeeding day if deemed necessary. (For technique, see Pneumonia, Chap. IX.) Polyvalent serum has been used in doses of $80-120$ c.c. into the subcutaneous tissues. Some authorities (NolfJ. A. M. A., Oct. 18, 1919, Vol. 73, No. 16) find vaccine treatment far more efficacious than the serum treatment in acute cases.

Vaccine Therapy. The vaccine used by Nolf in the Belgian army in 1918 was made from dysentery baccilli isolated from the intestine wherever possible. When autogenous vaccines could not be made, a vaccine from the Flexner bacillus type was used. No vaccine was used of the Shiga type. He gave the vaccine in every case which did not yield in one week to dietetic and drug treatment. It was administered intravenously at four-day intervals in very small doses at first, 10,000 , increasing slowly; $30,000,50,000,100,000$ and so on. In cases of moderate severity it was rarely necessary to go beyond 500,000 to effect a cure, but in stubborn cases one may increase to $10,000,000$, before the desired result is attained. Best results are obtained by early treatment. In one series quoted, there was a complete cure in a few weeks' time and only 2 deaths among 500 cases so treated. Fever drops by lysis with some exacerbation on the day of inoculations and the next day following. Nolf emphasizes that in cases thus treated in 1918 they were able to avoid the tendency to chronicity experienced in their cases treated in 1917.

Empirical Treatment. One drug in dysentery has given me more satisfaction than any other, and that is castor oil.

The following prescription advised by Dr. Francis Delafield of this city has been especially satisfactory:

R

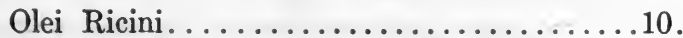

Phenylis Salicylatis................... 2.50

Tr. Opii Deodorati....................

亏 iiss.

gr. xxxvii

M. et div. in capsulas no. xv.

m. $\mathrm{xv}$.

S. One every two hours.

The results are truly most gratifying and I have had to have recourse to no other prescription in a large series of cases of dysentery, but that 
does not include the severest types described, or the tropical variety (amebic).

One capsule is given every two hours and when the stools begin to diminish materially, one every three hours and with still further improvement every four hours.

I believe the improvement is due to the small doses of oil, for the results are very satisfactory without the opium; and one may give the prescription without the opium in the milder cases and when the stools begin materially to decrease in number. The small dose of opium, equal to $1 / 10 \mathrm{gr}$. $(0.006 \mathrm{Gm}$.) does, however, adds its own effect and relieves the pain.

I am not convinced of any particular value on the part of phenyl salicylate (salol). Perhaps it adds its own anodyne and antipyretic effect; any antiseptic virtue, I doubt.

There is one very important adjuvant to this treatment, also insisted upon by its advocate-namely, the administration every second day or at least every third day, of further large doses of castor oil as in the beginning.

The small doses are unquestionably constipating and the diminution of the stools is the result we see, as well as improvement in their character. We have to remember also that in the meantime fecal matter is collecting, mucus, bacterial toxins and bacteria themselves are gathering, and damage from these sources with a recrudescence of the disturbance will follow unless evacuation is attained at suitable periods.

Somewhat similar to this castor oil treatment is one warmly advocated in some quarters, but about which I can speak less authoritatively; namely, the saline treatment.

Sodium sulphate or magnesium sulphate is given in a half ounce or ounce dose, then followed by one-dram doses of the salt every two hours, three hours or four hours as improvement occurs, or dram doses every hour can be given until the initial catharsis is accomplished.

Symptomatic Treatment. Pain. Between the constantly recurring griping, twisting, colicky pains and the incessant nagging of the tenesmus, exhaustion threatens; so that the symptom is one of the most urgent in its demands for relief.

This may be afforded by topical applications or drug administration.

Heat to the abdomen is one of the best measures we have at our command. It can be applied in the shape of hot fomentations, turpentine stupes or thin poultices.

To give a fomentation take two pieces of thick flannel; an old blanket affords just the material we want; cut into squares to cover the abdomen. 
Have ready in addition another piece of flannel for a cover or protector and a towel with sticks sewed across either end to answer as a wringer.

Lay the flannel squares in the towel, pour boiling water over them, saturating them thoroughly and then quickly wring out in the towel by twisting the sticks in opposite directions to each other. The wringing must be complete and no water be left in the flannel. Take this out quickly, shake it once in the air, place it on the abdomen and cover with the protector. Before the fomentations are applied, the skin may well be lubricated with vaseline.

It is helpful to have the patient in a blanket which can be brought up over the fomentations.

These may be applied every ten or fifteen minutes until relief is afforded.

Turpentine stupes are prepared in much the same way, a dram of turpentine having been added to the hot water into which the flannels are to be dipped or the stupes may be sprinkled with a little turpentine after they have been wrung out or a bit of absorbent cotton wet with turpentine may be passed lightly over the abdomen before the stupes are applied. Careless application of the turpentine may result in a burning of the skin.

The frequency of application and the length of time they are to be left on will depend on the reaction of the skin to the turpentine and the efficacy of the measure.

These devices not only lessen the spasm, but by so doing diminish the frequency of the stools.

Comfort may also be afforded by warm rectal irrigations, at $100^{\circ} \mathrm{F}$. to $105^{\circ} \mathrm{F}$. of plain water or, better, physiological salt solution, one teaspoonful ( $1 \mathrm{dram}$ ) of salt to the pint ( $4 \mathrm{Gm}$. to 500 c.c.).

These irrigations are considered by some men as an important item in the treatment of the inflamed colon and are especially in vogue in children's practice. I have had to have recourse to them in adult practice, in the acute stage but rarely, finding the prescription given above together with fomentations sufficient.

While at times the irrigation does good, I prefer to do without it if possible, because it can be overdone and one feels that often when it is done, the bowel gets more excitation than rest by the procedure.

The tenesmus frequently requires drug treatment and we prefer to give it locally at first. This can be done by the suppository containing gr. ss. $(0.030 \mathrm{Gm}$.) of opium or in more severe cases by the morphine suppository which contains gr. $1 / 4(0.015 \mathrm{Gm}$.) or by one combining opium and belladonna, e. g.: 
R

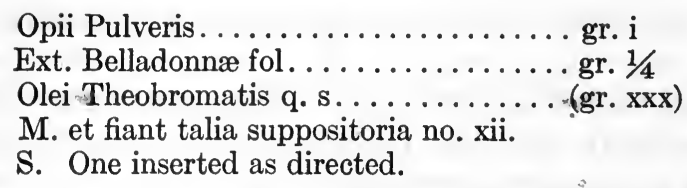


One warning must be offered born of experience.

Be sure that at least every second or third day the bowel is emptied by a dose of castor oil or salts, for these large doses of bismuth collect in the bowel and by sheer weight do harm to the gut. One of my most vivid recollections is seeing a case to which bismuth had been given for weeks without catharsis and with the continuance of the symptoms to a point of dubious prognosis.

A large dose of oil was followed by movements that literally filled the bed-pan with black masses of bismuth that looked like fresh tarconcrete and must have weighed pounds. Recovery started from the time of this relief.

Upon opium we are dependent for our best results.

It diminishes peristalsis and so the cause of pain and tenesmus, it diminishes the stools, it lessens pain and permits rest and sleep; rest for the bowel, rest for the body and rest for the mind.

There are three things that should be emphasized about the use of opium in these cases:

1. Never use it until the bowel has been efficiently emptied by one of the cathartics advised.

2. Use it in the smallest amounts that will get results. I have rarely had to use it in greater amounts than $1 / 10$ of a grain $(0.006 \mathrm{Gm}$.), that is, one drop of the tincture every two hours.

3. Do not advise the patient of the fact that you are using it. The profound effects of opium that will permit him to appreciate that he is getting it will not be manifested in these doses.

Again the necessity of the cathartic at intervals of two or three days should be emphasized.

Severe cases may take on a choleraic character. Beside profuse watery stools causing a veritable dehydration there is vomiting, cyanosis, weak, rapid pulse, cold extremities and muscular cramps. These cases indicate fluid for the tissues.

It may be given as a hypodermoclysis, one to two quarts of normal saline solution or an infusion of normal saline into the vein. If the stomach will retain it, fluids by the mouth are pushed. Heat should be applied to the extremities, morphine in doses of gr. $1 / 4(0.015 \mathrm{Gm}$.) hypodermically to control the flux, which may well be combined with atropine sulphate gr. 1/150 (0.0004 Gm.). Strophanthin gr. 1/120 (1/2 $\mathrm{mg}$.) given into the vein or digitalis administered in the same manner in doses of 3 to 5 grains. Adrenalin intramuscularly in doses of $\mathrm{m} . \mathrm{x}$ to $\mathrm{xv}$ at quarter hour intervals for some half dozen doses to sustain the blood pressure or small doses $\mathrm{m}$. ii-iii cautiously introduced through the wall of the tube of the infusion apparatus when giving the saline intrave- 
nously. Hypertonic glucose solution (25 per cent., 250 c.c. or 5 viii) should theoretically be of value, but I have had no personal experience with the measure.

Heart. In severe cases the myocardium may be seriously damaged by the toxins, which induces circulatory failure; digitalis should be used to digitalizing effects. For details of circulatory stimulants and their administration see Pneumonia, Chap. IX.

Duration. Most cases run a course of a few days to a week or a trifle more, a certain number continue three, four, five or six weeks and then cease or pass over into a chronic form.

Subacute and Chronic Form. When a case is passing over into a chronic form, if seen for the first time, the effects of such medication as has been advised may be tried long enough to test its worth.

Vaccines. The same vaccine is used as in the acute cases. The initial dose and the time intervals should be the same but often the final dosage must be raised to much higher figures -5 and 10 billion. Nolf thus succeeded in effecting complete cures even in the most chronic cases. He first used these vaccines subcutaneously in a dosage starting with a million bacteria and raising it progressively to 5 and 10 billion. The results were very satisfactory as a rule, but in some cases there was a relapse which he found was intractable to the subcutaneous therapy but yielded promptly and permanently to the intravenous injections.

If these efforts are of no avail, make use of topical measures and give as little medicine as possible, especially avoiding opium on account of the danger of the habit.

In the chronic form the use of the irrigation is more valuable than in the acute.

There is one thing about the irrigation and the use of all topical measures to be insisted upon and that is, if used in the acute stage assiduously the chronicity of the process may be due to the irritation these very measures keep up, and a cessation of all local treatment be followed by rapid improvement. Again, when these methods are pursued in a chronic case, improvement may be seen up to a certain point and then cease or the condition grow worse. Here again stop the treatment and study the effect.

The irrigation is to be done with the patient in the dorsal position with the hips elevated and on a douche-pan, or lying across the bed on a rubber sheet so arranged as to form a gutter for the discharge of water.

Sometimes the bowel is better filled by putting the patient on his left side and then turning him to his right, so as to seek the assistance of gravity in following the line of the colon. 
If the patient is fairly strong, the more trying knee-chest or elbow position may be used.

The tube should be long and soft, a rectal tube, a stomach siphon or a large rubber catheter will answer.

The tube is warmed, lubricated and passed carefully into the bowel, as any roughness will cause a contraction and defeat the purpose. If it meets with an obstruction, wait until a spasm ceases and then try again. If difficulty is met with let a little water pass in to distend the gut and try again. Pass it in 4 or 5 inches.

The tube is then or has previously been attached to a $\mathrm{Y}$ tube, to the other two limbs of which are attached the fountain syringe and the drainage tube. The bag contains for a simple irrigation plain water or better a physiological salt solution, a level teaspoonful of salt to the pint, at a temperature of $100^{\circ} \mathrm{F}$. It should be held about three feet above the level of the patient's body. The water is allowed to flow in and out, by alternatingly pinching the outflow tube and the inflow tube. Discomfort on the part of the patient tells when enough has been introduced at any one time.

If irrigation of the lower part of the intestine alone is aimed at, a two-way tube like the Kemp tube is a great convenience.

About two quarts may be used at a time; in some cases, even more.

The irrigation may be followed by an astringent.

The best astringent is silver nitrate. It should be used in increasing strengths, 1 to 2,000 at first and increasing up to 1 in 500 or 1 in 400 or 300 .

Use one or two pints. It may be introduced through the same syringe and tube. The tube should be pinched to keep the solution in contact with the bowel a few minutes or left in for the patient to pass.

If salt solution is used at first, it must all be discharged or the silver will be neutralized by it.

In this case, if such difficulty is constantly met with, use plain water. If the solution of silver gives great pain it is too strong and may be neutralized by salt solution.

The treatment may be tried daily in the weaker solutions and then less frequently. Intermit the treatment occasionally to let the mucous membrane recover from any irritation by the silver that might have been excessive.

If success is not achieved with the nitrate, after an intermission of a few days, some organic salts may be used, e. g., protargol in a 1-500 solution. Some clinicians prefer the organic salts to the nitrate.

The protracted cases are often due to ulcers which will not heal under this milder application; hence, an effort should be made by the 
aid of the proctoscope or sigmoidoscope to detect and treat directly any ulcer within reach. Here strong silver in 25 per cent. solution or the stick may be used.

There are two other important matters to be taken into consideration in the treatment of chronic dysentery-namely, change in environment and the diet.

In chronic dysentery, particularly in the tropics, when the laboratory findings fail to establish a diagnosis between bacillary and amebic dysentery it is worth while to administer a few doses of emetin on the possibilities of establishing a therapeutic diagnosis (see amebic dysentery).

Change in Environment. What those subtle influences are that determine improvement in a new environment, seen in those patients fighting to overcome a lingering infection of one kind or another, defies analysis at present.

While climate, fresh air, sunlight and other physical conditions may be appealed to, they are not always sufficient. The effect upon the mind induced by a change certainly has something to do with the results. I recall a bitter struggle with dysentery, occurring in a patient attacked away from home, dragging out to eight or ten weeks in whom the home-going, though effected on a stretcher, brought health and strength in a manner little short of the miraculous, and such examples every practitioner of medicine can recall.

Diet in the prolonged or chronic cases is an important problem.

During the progress of this disease the patient loses much flesh, to which the low diet adopted to spare the inflamed bowel, has been a factor.

Loss of body substance in infectious diseases is due to (1) pyrexia, (2) toxemia, and (3) starvation. Now, in these prolonged cases the fever has waned and the signs of general toxemia, other than the residual weakness, have subsided, and because the patient has still too many loose stools.with mucus, he is kept on the same restricted diet.

The diet is milk, perhaps two quarts of it with about 1,280 calories and 70 gms. of proteid. Now how can a man, whose daily needs are well over 2,000 calories, make up 1,000 deficit out of his own tissues day after day and still afford energy enough for successful resistance and repair? The proposition is absurd, and I am convinced that in a large percentage of these cases that drag along, starvation is at the bottom of it.

I look through dietary after dietary and see milk, cautiously a little egg and still more cautiously a little scraped meat offered, and no mention made of the use to which the carbohydrates could be put. I do 
not think we should be alarmed at the addition of a little sugar to the milk, and, if we fear fermentation, we can add milk-sugar which is not easily fermentable. Every ounce is worth 120 calories and we could add three ounces to the two quarts of milk without provoking trouble or even distaste.

The cereals should be used-barley, rice, farina, bread and butter, cereal soups; eggs may be given, each worth 60 to 70 calories. A small chop is easily digested and modern physiology emphasizes how easily proteid is digested and assimilated and there are 100 calories more.

Beef, mutton, chicken, and potatoes leave but little residue, and if the patient does well on this more liberal diet, give him some of the green vegetables, puréed at first-peas, or small stringed beans-and let us see if they do not improve rather than aggravate the condition.

What should be emphasized is that the body may suffer more from the absence of food than the intestine from its presence, and that practically all the food above mentioned is absorbed from the small intestine which is not the seat of the lesion and so does not arrive at the point of irritation at all; that when the acuteness of the process has subsided, one should consider the dietetic need of the patient, and see that he gets it, at first in food that leaves but little residue, and then permitting other foods little by little. We are humiliated sometimes to find that these chronic cases improve when first they escape our hands.

Prophylaxis. The bacillus seems to be water-borne, and so when an epidemic occurs the same precautions should be taken as in that other water-borne infection-typhoid fever. Water should be boiled, milk inspected or boiled, green vegetables thoroughly cleaned.

In all epidemics cooks and others handling food should be examined to determine whether or no they are carriers and no one suffering from diarrhea, even of a mild type, should be allowed to come in contact with food supplies.

In an epidemic all convalescents should be suspected of being carriers. All people handling foods should be so suspected. Precautions to be taken by nurses, doctors or others about the patients have been detailed. Toilets, as being possibly contaminated with infected feces, must be kept rigidly clean with antiseptics. Infection, too, may come from contact, and the stools should be destroyed and clothes and utensils, etc. (see Typhoid Fever, Chap. XIV), treated in the same manner as in typhoid.

Especial care should be taken by nurses handling infants lest the infection be carried by handling soiled linen.

Flies are carriers and should be kept from the sick room by screens, from the food supply by screens, and exterminated by every known 
means. All stools should be disinfected and this is doubly imperative when stools are disposed of in outhouses as is frequent in the country or in cesspools, accessible to the fly.

\section{SUMMARY}

\section{Bacillary Dysentery}

Rest.

In bed.

Bed.

Half bed; hospital type best.

Woven wire springs.

Hair mattress.

Rubber sheet.

Draw sheet.

Sheets; light blanket; low pillow.

\section{Room.}

Well ventilated; shaded balcony; adjacent bathroom.

Care of the body.

Bath.

Castile soap and warm water daily.

Alcohol rubs, especially over points of pressure in emaciated cases. Talcum or other drying powders.

Mouth.

Rinse after every feeding; use boric acid solution 2-4 per cent. strength. Dobell's Solution.

Teeth brushed twice a day.

Interstices of teeth freed from food particles. Use small cotton swabs on wooden tooth-picks as applicators.

\section{Tongue.}

Soften coat with one-half strength peroxide of hydrogen.

Cleanse with boric acid solution or Dobell's.

Dry mouth.

Equal parts of 2 per cent. boric acid solution and abolene flavored with lemon juice.

Points of pressure - threatened bed sores. Change of position; rubbing; rubber rings; air cushions.

Exclude visitors.

Use of bed-pan imperative. (Technique, see text.)

Woolen material over abdomen.

Nurse should wear rubber gloves or disinfect hands.

Physician should wear gloves when examining patient or disinfect hands thoroughly. 


\section{Diet}

Boiled milk, diluted $1 / 2$ to $1 / 3$ or $1 / 4$ with plain water, barley water or rice water.

Eight ounces every two hours.

Next add milk sugar $1 / 2$ ounce to 1 ounce to each glass to increase calories.

If there is gastric distress, flatulency, coated tongue or curds in stools, skim the milk or cut out sugar or both. If marked, stop all food for a time.

If milk is not well borne use barley water, rice water, arrowroot water, egg-albumin, thin chicken broth (free from fat) mutton broth (free from fat). Gradually thicken the cereal waters and boil them into the broths. Add milk-sugar to the cereal waters. Add toast.

All food should be given warm, not hot or cold.

When temperature is normal and stools take on substance, add boiled rice, then toast, then chop, then puréed vegetables and then normal diet.

Water should be given freely throughout; administered with the chill taken off.

\section{Care of the bowels.}

Preliminary catharsis.

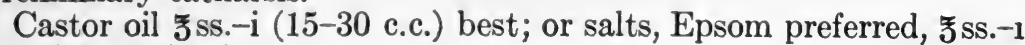
(15-30 Gm.)

If there is nausea or vomiting, calomel, gr. $1 / 4$ or $1 / 10(0.015-0.006$ $\mathrm{Gm}$.) at fifteen or ten minute intervals until gr. $\mathrm{i}$ to gr. iss. (0.060$0.10 \mathrm{Gm}$.) be given (acts as an antiemetic as well as a cathartic).

Follow in two or three hours by castor oil or salts, as above.

How to disguise castor oil. (See text.)

\section{Specific treatment.}

Serum best prepared from strain concerned. Polyvalent used for want of better. Dose 40-60 c.c. with equal amounts of normal saline. Method intravenous. Frequency-repeated if necessary each day, 40-60 c.c. Technique (see Pneumonia, Chap. IX). Polyvalent 80-100 c.c. has been used subcutaneously.

Vaccine therapy should be an autogenous vaccine when possible. Method-intravenous. Dosage small at first, 10,000 increasing 30,000; 50,$000 ; 100,000$ up to 10 million.

Frequency, 4-day intervals. Early treatment most efficacious.

\section{Empirical treatment.}

Castor oil in small doses frequently and in large doses at intervals.

R

Olei Ricini........................10.00

Phenylis Salicylatis................... 2.50

Tr. Opii Deodorati...................... 1.00

M. et. div. in capsulas no. xv.
亏 iiss.

gr. xxxvii

m. $x v$ 
One every two hours; with improvement every three hours; then every four hours.

If of mild grade leave out opium.

Every second day castor oil 5 ss. $-\mathrm{i}$ (15-30 c.c.).

\section{Saline treatment.}

Magnesium sulphate (Epsom salt) or sodium sulphate.. (Glauber's salt) 3 ss.-i (15-30 Gm.); follow by $3 \mathrm{i}^{\prime}(4 \mathrm{Gm}$.) doses every two hours; then as improvement occurs three and four hours.

\section{Symptomatic treatment.}

Pain-colic.

\section{Topical applications.}

Heat.

Fomentations. (Technique, see text.)

Turpentine stupes. (Technique, see text.)

Thin poultices. (Technique, see Pneumonia, Chap. IX.)

Rectal irrigations of plain water in salt solutions (3i-0i) (4 Gm. to 500 c.c.) at $100^{\circ} \mathrm{F}$. to $105^{\circ} \mathrm{F}$.

Drugs, when severe.

Morphine sulphate hypodermically, least quantity effectual, gr. $1 / 16$ to gr. $1 / 4(0.004-0.015 \mathrm{Gm}$.).

\section{Tenesmus.}

Warm rectal irrigations as above for pain.

Suppositories of opium, gr. ss. (0.030).

Or

P

Opii Pulveris..................... i

Ext. Belladonnæ fol . . . . . . . . . ........ gr. $1 / 4$

Olii Theobromatis, q. s............... (gr. $\mathrm{xxx})$

M. et fiant talia suppositoria no. xii.

S. Insert one as directed.

Rectal injections of opium in starch paste.

Tr. opii $\mathrm{m}$. $\mathrm{xv}(1$ c.c.) in $\bar{\jmath} \mathrm{j}$-ii (30-60 c.c.) starch mucilage and inject slowly.

Morphine sulphate, gr. 1/16 (0.004 Gm.) to gr. 1/4 (0.015 Gm.) hypodermically.

Benzyl benzoate 20 per cent. alcoholic solution 10-30 drops three times a day.

\section{Diarrhea.}

Usually satisfactorily controlled by the small doses of castor oil and salol as described above under empirical treatment.

Bismuth subnitrate.

Bismuth subnitrate, gr. xxx $(2 \mathrm{Gm}$.) every two hours. Administered in a little water; a shake diffuses the powder throughout the liquid evenly. 
Every second or third day give castor oil (preferably) or salt, 5 ss.-i Opium. (15-30 Gm.).

As given with castor oil and salol, as above, under empirical treatment, it is usually sufficient. If not, increase the opium or give it with the bismuth.

1. Never use opium until the bowel is emptied by oil or salts.

2. Use it in smallest quantities that are effectual.

3. Do not advise the patient of its use.

Severe cases with desiccation of tissues, hypodermoclysis of normal saline; intravenous infusion of saline; push fluids by mouth if possible. Heat to extremities. Morphine sulphate gr. 1/4 (0.015 Gm.). Atropine sulphate, gr. 1/150 (0.0004 Gm.).

\section{Collapse.}

Strophanthin, gr. 1/120 (1/2 mg.) intravenously or digitalis, gr. 3-5 (0.20-0.33 Gm.) intravenously. Adrenalin hydrochloride intramuscularly $\mathrm{m} . \mathrm{x}-\mathrm{m} . \mathrm{xv}(0.66-1.0$ c.c.) every 15 minutes for 6 doses intravenously $\mathrm{m}$. ii to iii into infusion tube. Hypertonic glucose solution 25 per cent. ( 250 c.c. or 5 viii).

Heart.

Circulatory failure, digitalis. (See Pneumonia, Chap. IX.)

\section{Subacute and chronic stages.}

Irrigations. Technique. (See text.)

Plain water or salt solution first; then silver nitrate, at first 1-2,000, gradually increasing up to 1-500 or, stronger, 1-300.

Use 1 or 2 pints at $100^{\circ} \mathrm{F}$.

If it causes pain neutralize the silver in the bowel by an enema of salt solution ( $3 \mathrm{i}-0 \mathrm{i})$ ( $4 \mathrm{Gm} .500$ c.c.).

Frequency; daily in weaker solution, less frequently with stronger.

Intermit occasionally, as irritation of astringents may be cause of continued diarrhea.

Protargol 1:500 instead of the silver nitrate.

\section{Severe cases.}

Vaccine therapy as in the acute cases. Raise dosage gradually if necessary to 5-10 billion intravenously. (See text.)

\section{Ulcers.}

Often the cause of protracted cases. Use protoscope or sigmoidoscope to detect ulcer and apply medication. Apply 25 per cent. solution of silver nitrate or the silver stick. In cases of doubtful etiology emetine may be used to establish therapeutic diagnosis. (See Amebic Dysentery.)

Change of environment. 
Diet.

Increased to meet caloric demands. (See Diet in Acute Infectious Diseases, Chap. II.)

If on milk add milk sugar 5 ss.-i $(15-30 \mathrm{Gm}$.) to each glass ( (240 c.c.), and then cereals, barley, farina, then rice, bread and butter, cereal soups, then mutton and chicken broth with rice, barley, arrowroot, or flour to thicken; then eggs, chop, chicken, potatoes, peas and beans (at first puréed) and even a more liberal diet.

\section{Prophylaxis.}

Bacillus seems to be water-borne.

Take same precautions in an epidemic as in a typhoid epidemic.

Boil water.

Drink only "inspected" or boiled milk.

Thoroughly cleanse all green vegetables or exclude them from diet.

Carriers should be detected and not allowed to handle food supplies. Suspect convalescents as carriers.

Disinfect stools. (See Typhoid Fever, Chap. XIV.)

Disinfect articles. (See Typhoid Fever, Chap. XIV.)

Disinfect hands after handling patients and excretions.

Screen room from flies.

Screen latrines, outhouses and stools from flies.

Screen food supply from flies.

\section{AMEBIC DYSENTERY}

The clinical course of the two types of dysentery, bacillary and amebic, show much in common; but the former is usually more abrupt in onset, runs a more acute course and is accompanied by a higher fever and is of briefer duration, while in the latter often the acute attack is preceded by relapsing diarrhea and the locality in which it was contracted (tropical and sub-tropical countries) and its prolonged course makes the diagnosis of amebic dysentery more than probable and the hepatitis makes it certain; still, and, especially, in temperate zones, only the recovery of the specific organisms from the stool can make the diagnosis certain in the overwhelming majority of cases.

The specific organism is the Entamœba histolytica.

There are other entamœbæ, for a long time undifferentiated from histolytica and so credited with causation of the disease.

They are the entamœba coli, a parasitic organism and a non-parsitic group called the limax, and as these occur in the stools of a goodly percentage of normal men and so in the stools of many cases of bacillary dysentery, it is a matter of importance to differentiate them; the more so as the treatment most efficacious in amebic dysentery has no effect on the progress of bacillary dysentery, and, vice versa, the treatment 
described for bacillary dysentery does not affect the Entamoba histolytica at all.

When, however, this differentiation by finding an entamœba and identifying it is impossible one must rely on the criteria mentioned and when in doubt give the patient the benefit of the doubt and direct the treatment as in amebic dysentery; as the emetin will do no harm if no good.

Moreover, I do not know any reason why the empiric treatment given under bacillary dysentery should not be combined with the emetin treatment in a doubtful case.

The pathology of amebic dysentery is an invasion of the mucous membrane of the large intestine, rarely to be found above the ileocæcal valve, in mild cases confined to the neighborhood of the cæcum, but in severe cases involving the whole large intestine. The organisms burrow through the mucosa, in the submucosa cause a necrosis undermining the mucosa, and the lesion is accompanied by proliferative changes. Sometimes the muscular coat is invaded and rarely the intestine is perforated. Thrombi form in the portal vessels, cutting off the blood supply to the mucosa, thus giving rise to ulcers and furnishing emboli to be carried to the liver, setting up abscesses in that organ. The appendix is involved in a small per cent. of the cases.

Symptomatology. Most cases begin insidiously with a history of recurring abdominal pains and diarrhea. The stools are pasty and of moderate number, there is no fever, but a loss of strength and secondary anemia.

The blood shows a moderate leucocytosis with an increase in large mononuclears. Decrease of mononuclears looks toward a cure (Stitt). In the lesser number of cases the onset is abrupt, the stools are thinner, brown and greenish and may contain blood and mucus and be accompanied by severe griping. There is a febrile reaction in these cases, but not as high as in the bacillary form and without the striking evidences of the toxemia of the latter.

All the rules laid down for the conservation of the patient's forces and for his comfort obtain in the one form of dysentery as in the other.

For the accomplishment of rest, the choice and preparation of the bed and the room, the care of the body in all its details and the diet the reader is referred to the first section of this chapter, Bacillary Dysentery.

As regards the diet, however, the severity and the prolonged course of amebic dysentery make the plea for sufficiency more emphatic, so that with boiling of milk or elimination of it when not well borne, the rules for diet laid down under Typhoid Fever (see Chap. XIV) should 
be a good guide. During the acute stage, however, a fluid diet is indicated and such as is laid down under bacillary dysentery.

The care of the bowels and the importance of the preliminary catharsis obtain in the one as in the other with equal force.

As amœbæ are often situated high in the bowel, they may not be found in the early stools. It becomes desirable to dislodge them with a saline purge and this is to be preferred to castor oil; for the oil as an initial purge makes the examination difficult. Of course, it will be remembered that flakes of blood and mucus are the best materials to afford the amœbæ and that it must be examined on a warm stage within a few minutes to determine the important diagnostic point of active motility. The character of the cysts are important, too; for the entamœba coli contains eight nuclei while the histolytica has not more than four.

Treatment. Very recently a treatment has come into vogue that promises to be as nearly specific in this disease as quinine is in malaria and yet it is the off spring of an older, I might say "traditional" treatment in some parts of the world. This older treatment rested on the use of ipecac and the more recent on one of its active principles, emetine.

Ipecac had impressed many men practicing in India and elsewhere in the tropics as without a peer in its curative effects in Tropical Dysentery while others found it uncertain or disappointing. We know now that this discrepancy was due to the varying amounts of emetin in the ipecac used, as well, perhaps, as to the difficulties in assuring a sufficient absorption of this drug so actively emetic.

Within a very few years there has been a revival in the ipecac treatment of dysentery and the results reported were encouraging. The drug was given in pill form coated with phenyl salicylate (salol) to make it insoluble in the stomach. The instructions of Brown and Zeiler for its use are as follows: Use a salol coating $1 / 16$ inch for the pills (thicker coating may make it too resistant and the pill passes in the stool intact; thinner coating disintegrates in the stomach).

Ipecac derived from different sources contains varying amounts of emetine. The Brazilian variety is said to have a very high content.

They begin with $60-80$ grains $(4-5.30 \mathrm{Gm}$.) taken at bed-time and decrease the dose 5 grains $(0.30 \mathrm{Gm}$. $)$ a day until a dose of 10 grains $(0.60 \mathrm{Gm}$.) is reached.

The patient should be at rest in bed and no solid food or milk be given for at least six hours previous. No opiate, they say, is necessary.

The action of emetine is so much more certain and satisfactory that the only justification for the use of ipecac is the inability to get emetine.

Emetine Treatment. Of the active principles of ipecac emetine 
is found to be the one, and only one, that is amebicidal; while that action that gave to it its name, i. e., the emetic, does not belong to it at all, but to another active principle, cephaëlin.

The credit for the use of emetine in amebic dysentery is due to Rogers, an English physician.

While one could wish a larger body of statistics to justify such unqualified praise as is bestowed on this treatment, still such results as are accruing to its usage urge us to unhesitatingly recommend it.

Amid the gropings after the best dosage and intervals of administrations, I shall choose Vedder's as at present a most satisfactory guide.

One may use either of the two salts of emetine, the hydrobromide or the hydrochloride, but the latter is the more soluble and so preferable for hypodermic use.

The dose is gr. $1 / 3(0.020 \mathrm{Gm}$.), intramuscularly, three times a day for ten days.

If at the end of this time the case seems clinically cured, the treatment is discontinued. If not increase the dose.

The cure is very rapid, often within the week, affording a striking contrast to the prolonged course of the disease under the old therapeusis. The stools in a day or two change in consistency and the amœbæ quickly disappear in the favorable cases.

There is no vomiting or discomfort with this dose of the drug. Among the American Expeditionary forces larger doses were used, the rule being to give $1 / 2$ grain $(0.030 \mathrm{Gm}$.) of emetine hydrochloride twice a day for 6 or 12 days, the length of administration being determined more or less by the gravity of the disease. If vegetative forms persisted, the treatment was repeated after an interval. If cysts were found in convalescence and in the case of chronic carriers emetine bismuth iodide was given in doses of 3 to 4 grains $(0.2-0.26 \mathrm{Gm}$.) up to 36 to 40 grains (2.30 to $2.60 \mathrm{Gm}$.) total.

Much the same rule was observed in the treatment of the English troops in the Mediterranean during the war. They were given hypodermically 1 grain of emetine daily for 10 days. Sometimes the dose was divided into $1 / 2$ grain morning and night.

Sellards, discussing the subject in Medical Clinics of North America, Jan. '18, advises even more liberal administration, namely, 1 grain of hydrochloride of emetine $(0.060 \mathrm{Gm}$.) morning and night for one or two days and then one grain $(0.06 \mathrm{Gm}$.) at night for 12 to 14 days, then after an interval of two to four weeks as a matter of precaution give a second course of 1 grain $(0.06 \mathrm{Gm}$.) daily for two weeks. This is given into the muscle.

In view of the late development, months or years, of hepatic abscess, 
Stitt advises giving a grain of emetine on two or three successive days each month following the acute attack as a prophylactic measure.

Bethea's Method. Bethea calls attention to the four factors to be considered in treatment; namely (1) the ampebæ in the intestinal wall; (2) the amœbæ in the bowel content, crevices, etc.; (3) mixed infection; (4) ulceration. The first are reached by the blood stream and the most suitable agent for destruction is emetine hydrochloride, which he gives in half grain doses for an adult deep into a muscle, preferably the gluteal. This dose is repeated daily for 24 days and then two or three a week for several weeks.

The amœbæ in the bowel content he thinks are best reached by the use of ipecac in pill form and is begun only after the acute symptoms have subsided, usually in one to three days. His prescription calls for pills and is as follows:

R

Ipecacuanhæ Pulv................gr. ccl

Phenylis Salicylatis.................. s.

$(16 \mathrm{Gm}$.

Ft. pil. ent. no. L.

M. et S. Ten (10) at night as directed.

Ten such pills are given on the first, second, third, fifth, eighth, twelfth, etc., nights.

Vomiting may occur in this treatment.

If the coating of the salol is too thick the pills may pass through the intestine unchanged. It may be necessary to use bromide before taking the pills. In some instances the ipecac seems to provoke a rapid peristalsis. To obviate nausea and quiet peristalsis he used the following prescription:

$\mathrm{R}$

Sodii Bromidi..................... $3 \mathbf{i}$

Tr. Opii Deod...................... f $3 \mathrm{i}$

Aquæ Menth. Pip. q. s. ad................. f f iv

M. et S. One (1) tablespoonful 2 hours before taking pills.

If nausea does occur the ice bag and cold water applied to the abdomen, Seltzer, ginger ale, and other effervescent drinks are used.

Colon irrigations of quinine hydrochloride 1:1000 to 1:500 about a half a gallon an hour or two before taking the pills.

Ulcers and mixed infections are treated as in bacillary dysentery.

The most convincing proof of the specific effect of the drug upon the entamoba histolytica is the results in the cases complicated by Hepatitis, hepatic abscess. Abscesses occur in the liver in about 20 per cent. of the cases. In a certain small percentage of the cases 
10 per cent. to 40 per cent., no history of dysentery is obtainable, but as the abscess may occur years after an attack which may have been mild and as autopsies show the old lesions of dysentery in cases when no history was obtainable one can understand this hiatus in the history.

Some $35-40$ per cent. are double or multiple and they vary in size from very small foci of pus to such as contain a pint or a quart.

Untreated abscesses show a tendency to rupture, most commonly into the lung and pleural cavity, next into the peritoneal cavity, into stomach and intestines, rarely into vena cava, pericardium, kidney; that is into any structures adjacent to the liver surfaces.

The symptoms are a low grade irregular fever, and when this follows on a dysentery the diagnosis is fairly certain; often with sweating, tenderness or pain over the liver, an irregular enlargement of the liver, usually of the right lobe, without splenic enlargement; pain referred to the right shoulder, if the abscess is near the upper surface of the liver; to the appendix, if near the under surface; or to the gastric region, if the left lobe (Stitt). When implicating the pleura the cough, sweat, fever, anemia and wasting certainly suggests tuberculosis.

Jaundice is rare in contrast to suppurative cholangitis for which it may be mistaken. Aspiration of an abscess withdraws a dark chocolate colored pus. This pus rarely contains the amœbæ, which are to be found in the necrotic walls and appear after two or three days of drainage.

Of sixteen cases reported 100 per cent. were cured. Remembering the mortality of these cases set by different observers at figures varying from 30 per cent. to 80 per cent. and that the best surgical results cannot boast of better figures than 25 per cent. mortality, one does not wonder at the enthusiasm that led Vedder to say that "any amebic patient who is not moribund can be cured by emetine." Such results sound almost too good to be true and such successful treatment assumes an appreciation of the presuppurative stage of the hepatitis and its prompt treatment.

The dose of emetine in liver abscess and mode of administration and intervals is the same as in the uncomplicated dysentery. If pus has already been formed it must be evacuated, and the cavity drained; but as the cause for suppuration is removed in the elimination of the amœbæ under emetine the discharge ceases, the wound heals and the cure is effected.

One of two methods are in vogue in the surgical treatment of hepatic abscesses: (1) Drainage by trochar and cannula. (2) Open incision. Quoting Stitt briefly (Diagnostics and Treatment of Tropical Diseases). The procedure in (i) is as follows: "Under a local or general anæsthetic aspiration is made over obvious site of abscess, or this not in evidence, 
in the 8th or 9th intercostal spaces in right anterior axillary line, passing backward, inward and slightly upward. The needle should have a bore of $1 / 8$ inch and should be $31 / 2$ inches long. A larger needle endangers the vena cava. If necessary, mutiple punctures may be made. If pus is obtained the needle is left in situ and through a small skin incision along the course of the needle is passed a 4 to 5 inch trochar and cannula with a bore of $3 / 8$ inch. The trochar is withdrawn, a fenestrated rubber drain passed through the cannula, which is in turn withdrawn, the tube transfixed with a safety pin to anchor the tube and dressing applied."

Some men irrigate the cavity with 1-1000 emetine hydrochloride solution or 1-1000 quinine solution. For the open incision one should consult authorities on surgical technique, which is out of the province of this book.

In cases clinically cured the amœbæ do not always disappear from the stools; that is, the patients become "carriers" and as such are a menace to the community.

In some of these cases there occur, and some say, as a rule, even after months or years, relapses.

Relapses. A relapse is to be treated as the original attack by a repetition of the series of emetine. Such treatment, unfortunately is not always successful. Indeed, emetine has its decided limitations. Sellards says that it is almost without action in the extremely acute, fulminating cases, even when given into the vein in 2 grain doses and also in the extremely chronic.

Explanation for this is not yet forthcoming, but he believes death in the acute cases is due to a secondary bateriemia, the amœbiasis per se being rarely fatal.

There is a limit to the dosage too. The toxic dose is set by Rogers as 15 grains in an adult man and as it has a cumulative action the intermissions after 10-14 days dosage usually observed is logical. Sometimes disagreeable results follow the first dose of the drug in people presumably susceptible. There are usually hyperasthesiæ and paræsthesiæ.

Emetine is irritating and must be given into the muscle and every aseptic precaution must be observed to prevent infection. In obstinate cases one seeks adjuvants to the emetine in some other amebacide.

Ipecac given by the bowel at the same time as emetine intramuscularly. The object is to bring the emetine into contact with amœbæ in necrotic areas in the colon that cannot be reached by the blood stream.

Lawson advises its use in the following manner: 60-120 grains of 
powdered ipecac is put into 24 ounces (720 c.c.) water; this is kept hot, but not boiled, for an hour. This whole amount, while warm is introduced into the bowel and retained as long as possible. It should be introduced slowly with the patient lying on the left side. This is repeated daily.

Ipecac may be taken by the mouth while emetine is given intramuscularly, hoping thereby to get a local effect as well as by the blood stream. It may be given as salol (phenyl salicylate) coated pills or as emetine bismuth iodide. The doses of both ipecac and emetine should be somewhat lowered when given thus together.

Quinine may be given as high enemata, using 1-5000 up to 1-1000 solution of a soluble salt, such as dihydrochloride and urea or bisulphate in amounts of one to two quarts (liters.)

Edsall has called attention to the rarity of relapses in cases of hepatic abscesses.

\section{OTHER DRUGS THAN EMETINE}

Quinine Treatment. Brook of the Army reported on most gratifying results following the use of quinine given by mouth.

He called attention to the known amebicidal effects of a solution of quinine and attributed its failure when given by the rectum to its inability to reach the organism in the tissues.

His dosage is gr. $\mathrm{xxii}-\mathrm{xxx}(1.5-2 \mathrm{Gm}$.) a day for six days.

He stops a week and then repeats the series; especially in chronic cases.

He believes that the special field of quinine is in chronic cases and those that relapse after emetine. He, too, believes that emetine is a specific in acute cases, but not as certain in the chronic, while in all cases he believes quinine in $2 \mathrm{Gm}$. doses (gr. $\mathrm{xxx}$ ) per day to be as effcient as ipecac.

Benzyl Benzoate. Haughwout and his co-workers in the Philippines advise the use of benzyl benzoate in this form of dysentery, either alone or in combination with emetine.

They used a 20 per cent. alcoholic solution of the drug in doses of 10 to 30 drops, three times a day after eating. Their experience was entirely limited to acute cases, in which they got satisfactory results with no ill effects.

Subjective symptoms rapidly improved as the sedative effect of the drug on smooth muscle might lead us to anticipate; and in so far replaces opium; but more than that the amœbæ disappeared from the stool as promptly. Why this should occur is open to speculation. 
They look upon it as valuable adjuvant to ipecac or emetine, which they use much as above described. They further advise benzyl benzoate as a sedative in the bacillary form. In very acute dysenteries before diagnosis is established they give serum to secure what advantage is possible, if the case should prove to be bacillary.

Bitter Bush (Chaparro Amargosa) a shrub from Mexico and Texas used as an infusion of the dried leaves and stems to about the color of weak tea, given liberally by mouth and rectum has been used with rather astonishing results according to some observers even in cases resistant to emetine. Infusion is made from the roots, branches, foliage and fruit of the chaparro amargosa by boiling for 30-60 minutes. The weight used varies with the season and dryness of the plant. The color of the infusion should be that of moderately weak tea.

Routine of the treatment is as follows: The patient is put to bed, if possible; given a liquid or semi-solid diet the day before and during the treatment. Three or four hours before treatment is begun magnesium sulphate $5 \mathrm{i}(30 \mathrm{Gm}$.) is given and it is repeated every two or three days.

A half hour before each meal and at bed time six to eight ounces of the infusion of bitter bush is given by mouth. An enema of the infusion of bitter bush (500 to 2000 c.c.) is given in the knee chest position twice a day. This position should be held for five to ten minutes and the enema retained as long as possible.

If there is any fecal matter in the rectum the enema should be preceded by an irrigation.

Bismuth subnitrate in $3 \mathrm{i}$ doses 3 to 4 times a day has been advised.

Protargol 1-500 has been used by the bowel. Saline irrigation to keep the bowels as free as possible from necrotic material is advised by some workers.

Complications and Sequelæ except the hepatic abscesses (discussed above), are rare. Primary abscesses have been known to occur elsewhere than the liver; for example, in the spleen and the brain, skin and muscles. In the brain the symptoms are those of brain tumor. Meningitis does not occur.

A rare sequel to amebic dysentery is a polyarthritis. Considering how often secondary bacterial invasion occurs in this form of dysentery, it seems fair to conclude that these are the cause of the arthritis rather than amœbæ. (For treatment see Rheumatism, Chap. IV.)

Carriers. If emetine is so decided in its toxic effect on the entamœbæ it would seem logical to bring it into direct contact with the organisms in the stools in the case of carriers, but emetine in any dilution has proven too irritating to the intestine to make its use feasible. 
High irrigations of silver nitrate and of quinine have been found efficient.

Silver nitrate may be used as in bacillary dysentery in strength of 1 to 2,000 up to 1 to 500 or even 300 .

Quinine solutions, on which the hopes of the therapeutists were once placed, while unable to reach the amœbæ in the tissues, can reach them and kill them in the stools and afford a means of attacking the carrier cases.

The solution should be from 1 to 2,000 , up to 1 to 500 .

Oil of chenopodium in doses of 16 minims ( 1 c.c.) at threehour intervals has been recommended. It should be preceded by a purge of magnesium sulphate, and followed by castor oil.

Surgery. Since the introduction of emetine the surgical procedure on the liver is a matter of far less magnitude, while the operations on the bowel, such as appendicostomy for colonic irrigations are less commonly indicated.

In the most intractible cases the appendicostomy is the operation of choice. This is followed with irrigations of various kinds, normal saline, 1 per cent. bicarbonate of soda or boracic acid for cleansing purposes and then by 1 to 10,000 permanganate of potash, protargol 1-500, silver nitrate $1-5000$ to $1-2000$, quinine solutions as above.

Prophylaxis. With the knowledge that infection comes through the ingesta, boiling of all water and cooking of all foods, together with disinfection of the excreta and scrupulous cleanliness in the handling of the same constitutes prophylaxis. Persistent effort must be made by screening to keep flies from contact with stools on the one hand and food on the other. War of extermination should be made on the fly. Detection and treatment of the carrier (see above) constitutes no small part of prophylaxis. It is the carrier and convalescent that scatter encysted amœbæ broadcast. The number of these organisms in a stool is extraordinary. In the study of cases among the troops it was estimated that 330,000 to $45,000,000$ were discharged daily by carriers.

\section{SUMMARY}

Amebic Dysentery

Rest.

Bed.

Room.

Care of the body.

Diet.

(See Bacillary Dysentery and in prolonged cases see Typhoid Fever.) 


\section{Care of the bowels.}

Preliminary catharsis.

Use salines to dislodge amœbæ high in bowel, thus facilitating examination of stools for amœbæ. Castor oil makes examination difficult. Epsom salt is the best, 5 ss. to $5 \mathrm{i}(15$ to $30 \mathrm{Gm}$.) in a glass of water.

\section{Specific treatment.}

Emetine hydrochloride (or hydrobromide).

Dose, gr. 1/3 $(0.20 \mathrm{Gm}$.) three times a day for ten days.

Mode of administration, intramuscularly.

If at the end of this time the case seems clinically cured stop treatment.

If at the end of this time the case does not seem clinically cured, increase the dose to gr. $1 / 2(0.030 \mathrm{Gm}$.). (Vedder.)

When emetine is not procurable use the ipecac treatment.

Dose 60 to 80 grains (4-5.30 Gm.) a day, taken in single dose at bed-time and decrease the dose gr. $\mathrm{v}(0.33 \mathrm{Gm}$.) each day until a dose of $\mathrm{gr} . \mathrm{x}(0.66 \mathrm{Gm}$.) is reached.

Mode of administration, by mouth in gr. $\mathrm{v}(0.33 \mathrm{Gm}$.), pills coated with phenyl salicylate (salol), 1/16 inch thick.

Patient at rest in bed and no solid food or milk given for at least six Or, hours previously (no opiate necessary).

Emetine hydrochloride, $1 / 2$ grain $(0.030 \mathrm{Gm}$.) intramuscularly twice a day for 6 to 12 days.

If cysts persist and in chronic carriers, use:

Emetine bismuth iodide-dose, 3 to 4 grains $(0.2-0.26 \mathrm{Gm}$.) up to 36 to Or, 40 grains $(2.30-2.60 \mathrm{Gm}$.) total. (American Expeditionary Forces.)

Emetine hydrochloride intramuscularly, gr. i $(0.060 \mathrm{Gm}$.) daily for 10 days or $1 / 2$ doses morning and night. (English troops in Mediterranean.)

Or,

Emetine hydrochloride, gr. i $(0.060 \mathrm{Gm}$.) intramuscularly morning and night for two days, then gr. $\mathrm{i}(0.060 \mathrm{Gm}$.) at night for 12 to 14 days then stop for 2 to 4 weeks and resume gr. i $(0.060 \mathrm{Gm}$.) daily for two weeks. (Sellards.)

To prevent late hepatic abscess give emetine hydrochloride, gr. i Or, intramuscularly for 2 or 3 days in succession each month. (Stitt.)

To get amœbæ in intestinal wall-Emetine Hydrochloride, gr. ss. $(0.030 \mathrm{Gm}$.) intramuscularly daily for 24 days, then 2 or 3 injections And, a week for several weeks.

To get amœbæ in bowel content-Ipecac.

$\mathrm{R}_{4}$

Ipecacuanhæ Pulv...................gr. cel

Phenylis Salicylatis..................... q.

Ft. pil. ent. no. L.

M. et S. Ten (10) at night as directed. 
Give 10 pills the first, second, third, fifth, eighth, twelfth, etc., nights. If nausea or diarrhea occur with any dose, to obviate recurrence:

R

Sodii Bromidi......................

Tr. Opii Deod.........................

Aquæ Menth. Pip. q. s................... 万ै

M. et S.' One (1) tablespoonful two hours before taking pills.

Also, for nausea:

Ice bag or cold water to abdomen.

Seltzer, ginger ale and other effervescing drinks.

Quinine.

Colon irrigations of quinine hydrochloride 1-1000 to 1-500 about $1 / 2$ gallon an hour or two before taking the ipecac pills.

Ulcers and mixed infections treated as in Bacillary Dysentery (Bethea).

\section{Hepatitis-Liver abscess.}

For prophylactic treatment, see Stitt's recommendations above.

Emetine.

Dosage and administration same as in uncomplicated dysentery.

(See above.)

Abscess aspirated or incised and drained.

(For technique of operation, see text.)

\section{Relapses.}

Treated by emetine in the same way as the initial attack.

Ipecac by bowel.

60 to 120 grains (4-8 Gm.) of the powdered ipecac in 24 ounces (720 c.c.) of water kept hot (not boiled) for an hour is introduced into the bowel and retained as long as possible. (Lawson.)

Ipecac by mouth. (See Bethea's method above.)

Quinine in high enemata, 1-5000 up to 1-1000 of a soluble salt, the dihydrochloride and urea or the bisulphate take 1 to 2 quarts (liters).

Brook claims quinine (as above) to be more efficacious than emetine in relapses.

Other drugs than emetine.

Quinine treatment of Brook.

Dose, gr. xxii-xxx (1.50-2 Gm.) a day for six days.

Stop a week, then repeat the series.

\section{Benzyl benzoate.}

20 per cent. solution in alcohol. Use $10-30$ drops three times a day after meals.

Bitter bush.

(See text.) 


\section{Bismuth subnitrate.}

$3 i$ three or four times a day.

Protargol.

1:500 solution used by the bowel.

Symptomatic treatment.

Pain and colic.

Tenesmus.

Diarrhea.

(See Bacillary Dysentery above.)

Sub-acute and chronic stages with ulceration.

Silver nitrate. (See Bacillary Dysentery above.)

Solutions of quinine by the bowel, 1-2000 up to 1-1000.

Diet in chronic cases.

(See Bacillary Dysentery above and Typhoid Fever, Chap. XIV.)

\section{Carriers.}

Colon irrigations of silver nitrate, 1-2000 up to 1-300, increasing rapidly if the bowel shows toleration.

Irrigations with quinine solution, 1-1000 up to 1-500.

Oil of Chenopodium.

Dose - m. xvi (1 c.c.) every three hours. Precede by magnesium sulphate and follow by castor oil.

Surgery.

In most intractible cases appendicostomy followed by irrigations of normal saline solution or 1 per cent. bicarbonate of soda or boracic acid solutions to cleanse bowel and then followed by 110000 permanganate of potash solution, or protargol, 1-500 or silver nitrate $1-5000$ to $1-2000$, quinine solutions aos above.

\section{Prophylaxis.}

Boiling of drinking water.

Cooking of all foods.

Disinfection of excreta.

Cleanliness of hands.

Keeping flies from stools by screening.

War of extermination on the fly.

Detection and treatment of the carriers. (See text.) 


\section{CHAPTER XVII}

\section{SCARLET FEVER}

THE causative agent in scarlet fever remains unknown up to the present time, but the close association of the streptococcus pyogenes with this disease and its certain etiological relationship to many of the most serious manifestations, concomitant or sequential, of the disease, makes it of great importance from the standpoint of therapy.

The infection probably enters the body through the nose and mouth and is conveyed by the secretions from these organs in the act of coughing, sneezing, talking, spitting or by objects contaminated with these secretions, such as eating utensils, handkerchiefs and towels. The period of greatest infection is probably at the time of the early rash. There are in all probability chronic carriers and especially convalescents with chronic ear discharge, discharging gland or persistent catarrhal symptoms of nose, throat and bronchi.

The period of incubation is an important one, because it measures the time during which a child exposed to scarlet fever may become a source of infection to other children and the time during which contact with other children should be avoided.

Unfortunately, there is no precise agreement as to this period or, perhaps, the period itself varies considerably, resulting in statements by various observers that range from one day to three weeks. Excellent authority may be quoted for two to four days and ten to fourteen days. $\mathrm{My}$ inclination is to anticipate the shorter period; but, considering it from the standpoint of possible danger to others, to set the limit of isolation of the exposed at three weeks.

This may be an excessive caution as some of our best State Departments of Health set the period at eight days.

The onset is usually abrupt, beginning with vomiting in the vast majority of the cases, a sore throat, a considerable rise of temperature, and in twelve to twenty-four hours an erythematous eruption.

Unfortunately for the clinician, the diagnosis is often made difficult by deviations from the type. The eruption may be delayed for three, four or five days or be atypical or evanescent. The sudden onset with vomiting, sore throat and a rise of temperature, should always make one suspect scarlet fever. The bright injection of the throat, 
and an increasing leucocytosis and polynucleosis enhance the probability. McCollom lays great stress on the hypertrophied papillæ at the tip and edge of the tongue, like small grains of cayenne pepper scattered upon it. If the eruption has been evanescent, overlooked or doubtful, the papillæ growing daily more distinct, the increasing eosinophilia as the temperature disappears and the leucocytosis decreases, the evidences of desquamation or the late onset of nephritis finally fixes the diagnosis and determines certain precautions for the patient and those who might be infected by him.

Distribution of the Family. The diagnosis once made or with good reason suspected, immediate measures must be taken to protect other members of the family, who have not had scarlet fever.

The ages of the individual members of a family determine different precautions.

With reference to the adults, two facts are to be kept in mind; first, that recurrence is so rare that those who have had it have little to fear for themselves, but may convey the disease to others; hence, adult members of the family should come in contact with the patient as little as possible and those who may come in contact with children outside should keep remote from the patient, or, if the contact with other children is intimate, should remove from the house during the course of the disease. Teachers should remove from contact with the patient and notify the Public Health authorities. They should not return to their work until they receive the consent of these authorities. No person, who has come into contact with scarlet fever, should handle food supplies for eight days.

Second, that susceptibility decreases with years; McCollom's figures show that of 5,000 cases less than 5 per cent. occurred after twenty-four. The adult, then, who has not had the disease, runs a risk which should be avoided if possible, but a risk that is so slight that it yields to pressing exigencies.

The other children in the family should be removed to another house and there kept from contact with other children until the longest period of incubation, which we may take as long as three weeks, has passed, although very excellent authority is content with eight days. (Osborne, Epidemiologist of Mass. State Board of Health.) Their return home will depend on the patient's length of illness and upon the complications, which, themselves, may lengthen the period of infectivity, e. g., discharging ears.

They should not be allowed to go to school until this incubation period has passed or, if there is communication between them and the patient through other members of the household, they should not return to 
school until the patient is recovered and the danger of their own infection is passed.

When it is not possible to remove the other children, every contact with the patient, direct or indirect, must be avoided to the best of the ability of those concerned. It has been claimed that isolation can be effected more certainly in scarlet fever than in measles, but it must be remembered that the susceptibility to the former infection is not so great as is the case in the latter; hence, dependence on such isolation cannot be offered as a reason for keeping other children at home, if it be possible to remove them.

It must be remembered, too, that a light case in one patient in no way assures that the infection will prove light in the others; an assumption to which parents are peculiarly prone.

All contacts, adults and children, should have their throats and skins inspected and temperatures taken two or three times a day at least during the first week, to determine the early symptoms of the disease. If this inspection is made by the physician in attendance on the case it should be done on his arrival and before he has seen the patient to lessen the possibility of becoming himself the conveyor of the infection.

\section{THERAPY}

Room. To begin right is to win half the battle and an unwillingness to inconvenience the rest of the family must not stand in the way of the patient's needs. That room in the house which will meet the demands of the physician, nurse and patient best must be given up to him. The demands of the patient are space, ventilation, light, cleanliness; those of the nurse are economy of effort in managing the room; access to a bath-room devoted to the patient; nearness of her own room; those of the physician, ease of ingress and egress with minimum danger to the family.

This ideal can be attained in commodious quarters, but only approximated elsewhere.

The room must be large enough with enough windows to avoid being stuffy and afford good ventilation, without exposure to draughts. A top floor room is excellent or one with approach to a balcony or one at the side of the house affording a special entrance. Moreover, sunlight as well as air must have access to the room. Both cleanliness and economy of effort are afforded by stripping the room of furniture, carpet, hangings, pictures, etc. The floor may be bare or covered with carpet lining and over this unbleached muslin. An open fire-place has its advantages for the sick room. 
Isolation is made more complete by sealing doors leading to other rooms or hallways, except that in use. This sealing may be done with strips of paper laid along the cracks and reënforced to several thicknesses. Gum tragacanth makes an excellent paste, is easily removed and does not stain or injure paint.

The admission to all rooms used by the patient, i. e., the sick room, the bath-room, the nurse's room, should be protected by sheets. If the first entrance is to the bath-room and then to the sick room, both entrances may well be protected. An arrangement that has advantages is two sheets to the door entering the sick room; one on the inside and one on the outside of the entrance or portal. The one attached to the top and right side, the other to the top and left side.

The floor and woodwork should be rubbed down with damp cloths or cloths saturated with 1 to 1,000 bichloride of mercury from time to time and the cloths burned. If the floor is covered, then the same strength bichloride may be sprinkled on the covering from time to time. It is customary to saturate the inner sheet with 1 to 20 carbolic or with bichloride solution, but the heavy odor of the carbolic and the amount of toxic material that must be introduced into the room to keep the sheets wet through all the weeks of illness makes it objectionable.

It would seem better to replace the sheets from time to time, destroying the old, if made of cheap material or sterilizing them if of good material.

The Nurse. In a disease necessitating so close confinement and demanding so close attention, there should be both a day and a night nurse. If this be not feasible, a member of the family should lend assistance, but then should be as completely isolated from the rest of the family as the nurse. When nursing devolves on the mother or other member of the family their isolation from the rest of the family is a duty that must be emphasized by the physician and, all the more, because it is so difficult to make the necessity understood and observed.

In the presence of the patient the nurse should wear a gown and a cap that covers the hair completely and in the care of a bad throat and in the handling of secretions, rubber gloves.

An occasional antiseptic spray to the throat is a wise precaution. The nurse should not come in contact with other members of the family or with people outside the sick room unless the occasion be imperative and then she should subject herself to the same kind of disinfection as on leaving the case. The nurse should not sleep or eat in the sick room, but should have a room adjacent to the sick room devoted to herself.

Taking air or exercise, the nurse should choose that time of day and 
those localities that minimize the possibility of contact with susceptible individuals. Clothes should be changed and hair washed.

The Physician. In an outer room the physician should leave his overcoat or, better yet, his coat and vest, and entering the bathroom or some small ante-room where his accouterments for the sick room are left, don a gown reaching the floor, and tight at wrists and neck. In addition he should have a cap to cover the hair, and a pair of rubber gloves if he is to. examine the throat, as he ought to do. Wearing rubber shoes and turning up the trousers are precautions worth observing. All instruments used in routine examination, including the stethoscope, should be left in the sick room or bath-room and disinfected before using again.

While an honest and conscientious examination of the patient should be made, undue loitering and prolonged examinations only add to the peril of some other patient.

On leaving the patient, the physician leaves the gown, cap, shoes and gloves in the bath-room or ante-room, and washes his face and hands thoroughly with soap and water. This is more important than the antiseptic to follow, in the efficacy of which the physician's faith is often of too childlike simplicity.

Following the soap and water, alcohol, preferably $50 \%$, furnishes an excellent antiseptic, or bichloride in the strength of 1 to 1000 or carbolic or lysol in the strength of 1 per cent.; but to both the carbolic and lysol the objection of the clinging and disagreeable odor attaches, while they are less efficacious than those mentioned.

When a gown may not be obtained, a sheet may be arranged about the person in such a way as to effect the same purpose as a gown. In the absence of any such protection or in a visit to a suspected case, the overeoat and street gloves may be worn.

Full duty to the public who trust the physician is done only when he changes his clothes and better yet takes a full bath before seeing other children. Unfortunately, this is a rule rarely followed; hence, only the most rigid precautions on occasions of visits to the scarlet-fever patient can minimize the danger entailed by such a failure of proper observances. He should plan to see the case only early or late or allow some interval between this visit and the next on a child, spent in the open air. He should not take obstetrical cases or do surgery.

Precautions in the Sick Room. Articles in common use about the patient, when of such a nature as to permit it, should be kept in an antiseptic solution. The thermometer must be left with the patient. No effort at disinfection will justify its use among other patients. It may be kept in carbolic, 2 per cent. or stronger up to saturation, 1 in 
20. The tongue depressor should be of wood or glass. If of wood, to be destroyed by burning after each usage; if of glass, kept in carbolic like the thermometer. Syringe nozzles whether of hard rubber or glass (and they should not be of glass when used to irrigate the throats of young children, lest they be bitten and broken), are to be treated in the same manner.

Dishes, knives, fork, spoons used by the patient or nurse should be boiled for at least a half hour. A small gas stove in the bathroom or adjoining room facilitates the procedure. If the utensils are removed from the sick room to be boiled, they should first be soaked in 1-20 phenol (carbolic) for 20 minutes to $1 / 2$ hour.

Urinals, bed-pans and sputum cups should be disinfected with phenol (carbolic) one in twenty and the same strength carbolic should stand in them when not in use.

Bed-linen should be soaked overnight in phenol (carbolic), 1-50 to $1-20$, and then boiled for a half hour before being sent to the wash.

Discharges from the mouth, nose and ears are better caught on pieces of gauze or cloth that can be burned at once.

Cats, dogs and birds should be excluded. Only a few books or toys and such only as may be destroyed later are permissible.

Temperature of the Room. Thorough ventilation with avoidance of draughts upon the patient is the desideratum; $65^{\circ} \mathrm{F}$. to $70^{\circ} \mathrm{F}$. are the figures usually given and are useful to prevent overheating in the colder months, but in the winter, cold itself is not dangerous but helpful, providing the patient's body is properly protected and the temperature brought up to $70^{\circ} \mathrm{F}$. before the body is exposed for any purpose.

Bed. See Pneumonia, Chap. IX. The weight of clotbing is determined by the comfort of the patient and the temperature of the room.

Patient. The patient must be put to bed; an injunction, which while unnecessary in. severe cases, meets with opposition in the mild ones. An explanation of the consequences, in terms of kidney complications, especially, will persuade the parents.

A tepid sponge bath is given each day, which, given on a blanket, exposing one part after another, in a warm room, entails no danger.

Nightgown. This should be of flannel and if this is too irritating to the skin, a thin cotton or silk undervest may be worn under it.

Diet. No person in contact with the patient should prepare the food for the family nor should the nurse come into contact with the general food supply of the family.

The diet in scarlet fever is determined by the same fundamental princi- 
ples as obtains in the arrangement of a dietary in other acute infections, except so far as the frequency of nephritis may modify it: (See Diet in Acute Infectious Disease, Chap. II) i. e., in the first few days of the infection, when anorexia is marked or the angina entails much suffering from the act of deglutition, the feeding should not be pushed beyond the patient's inclination; but after that period has passed, the theoretical needs of the body must be taken into consideration.

The frequency and severity of the nephritis which comes late in the course of scarlet fever determines greater precautions in scarlet fever than in any other of the acute infections.

Many authors write as if any article of food other than milk might precipitate an attack of nephritis. I think we are convinced to-day that the nephritis is due to the toxins of the disease or to other infectious organisms or their toxins accompanying the disease, such as the streptococcus, whose action would not be modified favorably by an insufficiency in quantity or quality of food. That a constant examination of the urine for the first, least evidence of nephritis should be made, ought to be emphasized and when such evidence appears, then the kidney should be given the greatest degree of rest compatible with the imperative needs of the rest of the organism, like any other damaged organ.

The consensus of opinion is that milk has proved itself, empirically, to be the best single article of food in scarlet fever; but that it should be the only article of food in scarlet fever or that it has proven to be specific as a preventative of nephritis is doubtful.

The three great functions of the kidney are the elimination of water, of salts and of nitrogen. It is desirable to heap no excess of the one or the other upon the kidney. They are nicely proportioned in milk; for example, 2 quarts of milk furnishes about the average water intake for twenty-four hours. In these 2 quarts of milk are about 70 grams of proteid, or 11 grams of nitrogen. While this amount of proteid is below the average proteid intake of a man in health, it is enough to maintain him in health, as Chittenden's experiments showed and in disease a "luxus consumption" should be avoided as putting a burden on the kidney. In these 2 quarts of milk are about 3 grams of salt.

This amount is way below the average salt intake, but in health we use salt largely as a condiment and so small amounts as here given more than meet the actual physiological needs. Moreover, sodium chloride is illy excreted when the tubules of the kidney become impaired.

Two quarts of milk, however, furnish but 1,280 calories of food; less than 50 per cent. of the demands of a man of average weight in fever. If sufficient milk were given to meet the caloric needs of 3,000 calories, twice as much proteid and twice as much water as is required 
would be administered. To meet the caloric needs, either the milk must be fortified or other articles added. The milk may be fortified by adding to each glass milk-sugar or cream or both. One ounce of cream, 16 per cent., 50 calories, 7 ounces of milk, 140 calories and $1 / 2$ ounce of milk-sugar, 60 calories, makes 250 calories to each glass of 8 ounces or 2,000 calories to the 2 quarts.

Additional sugar may be introduced in such drinks as lemonade or orangeade.

Other milk preparations, cereal gruels, well-cooked cereals (oatmeal has been objected to an account of a high purin content), bread and butter, milk toast are suitable for the dietary. Among the cereals and flours are to be mentioned arrowroot, rice, barley, cornstarch, flour; farina, imperial granum; all of which can be made into gruels; or they may be used without the milk in the shape of jellies of barley flour; tapioca, sago or thoroughly cooked farina, rice, cornmeal.

Vanilla ice-cream or lemon ice may be allowed from time to time.

The basis of the dietary, then, is milk; modifications of milk, such as koumys, buttermilk, matzoon, zoolak, cereal gruels, cereals, bread and butter and milk toast being added when the milk disagrees or when the appetite lags from the monotony of the diet.

After the period when a nephritis is likely to ensue, more articles may be added to the diet; eggs, custard, potatoes, other vegetables; oysters, fish and finally meat.

Meat soups should be avoided at all stages, because they contain largely, almost exclusively, nitrogenous extractives which have little or no nutritional value, but must be excreted by the kidneys.

\section{CALORIC VALUES}

Milk.

Cream, gravity

Koumys . . . .

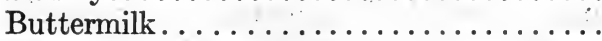

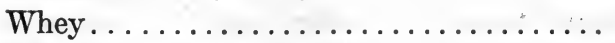

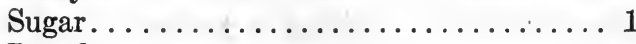

Bread..................... 100 Calories per slice

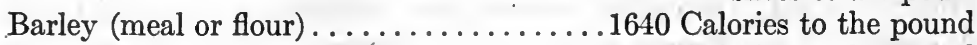

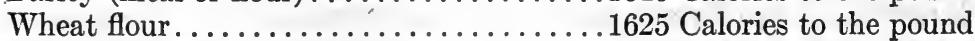

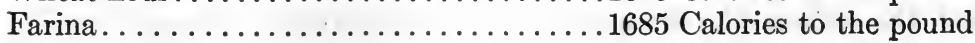
Baked custard............... 183 Calories to 2 heaping tablespoonfuls

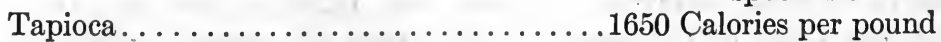

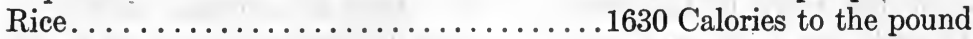

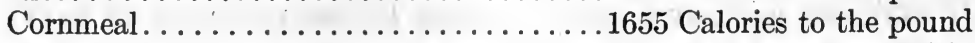
Butter...................... 119 Calories per ball $(1 / 2$ ounce) 
Oysters...................... 88 Calories per dozen

Fish...................... 100-150 Calories per $1 / 4$ pound

Eggs...................... $70-80$ Calories per egg

Potatoes (white)................... About 100 Calories for medium size

(4 to 5 ounces)

The caloric requirements in short acute infections may be disregarded.

The caloric requirements in prolonged infections are some 25 per cent. more than in health and often much more is taken with benefit.

We try to meet the theoretical requirements, while not disregarding the patient's appetite and wishes too violently, but if the patient will take more than these requirements willingly and handles it well we should give it to him.

Water. Water or lemonade or orangeade or the juice of grape fruit or grapes may be given freely. Imperial drink, a tablespoonful of cream of tartar (acid potassium tartrate) to 3 pints of boiling water (to get it in solution) to which sugar and lemon peel is added to suit the flavor, is a grateful drink and a good diuretic.

The amount of fluid to be taken should be well above the 2 quarts taken in health. Drink should be offered every hour and the patient allowed to take such quantities as he will. It must not be left to the patient to ask, as he is often too sick to do so.

Skin. The patient should have a sponge bath once or twice a day both for comfort and cleanliness. It should be done in a warm room, between blankets and as a precaution against chilling, which in a fever seems to me over-exaggerated, one part of the body at a time is exposed for the bath.

Itching and burning of the skin is often an annoying feature of the exanthem. This may be allayed by sponging with a solution of soda bicarbonate, a teaspoonful in 3 pints of water, or a bran. bath, a handful of bran in a muslin or cheesecloth bag immersed in a gallon of water until it becomes slightly milky in appearance, or the skin can be kept well dusted with a rice or talcum powder.

Calamine lotion, $3 \mathrm{i}(4 \mathrm{Gm}$.$) calamine to 1$ pint $(500$ c.c.) of lime water. For extreme itching 1 per cent. to 2 per cent. phenol in olive oil.

When exfoliation begins cocoa-butter, or vaseline may be applied; if 1 per cent. to 2 per cent. phenol be added to the latter it allays the itching and burning. Cold cream (ointment of rose water) affords an excellent application.

Soaking in warm water helps to get off the peeling skin; picking, rubbing and scraping the skin to expedite the desquamation is not justifiable, for it wounds the skin and opens antra of infection. 
The care of the mouth, throat and nose is of supreme importance, because the throat is often the seat of streptococcus or other pyogenic invasion, which, neglected, may permit of the spread of the infection by direct extension, into the ears; air passages, nose and accessory sinuses, while by way of the blood stream and lymphatics, the glands, joints, kidneys and heart may be attacked.

All procedures must be thoroughly and conscientiously carried out, but with extreme gentleness, because of the pain produced by carelessness, but much more because the wounded surface invites the entrance of infection. For the same reason, mild applications are indicated.

Physiological salt solution $3 \mathrm{i}$ to $0 \mathrm{i}(4 \mathrm{Gm}$. to 500 c.c.), boric acid solution, 2 per cent. to 4 per cent., or half strength Dobell's Solution (a weak solution of bicarbonate of soda and borax, $\overline{a a} 3$ ii to $0 \mathrm{i}(8 \mathrm{Gm}$.500 c.c.) with about $1 / 3$ of 1 per cent. phenol (carbolic acid) seem to me to be the safest substances to use whether in the shape of simple mouth wash, spray or irrigation. Any of the mild alkaline solutions, so-called antiseptic solutions, marketed under various names offer an agreeable substitute.

After taking food the mouth should be rinsed with one of these solutions, the teeth cleansed with a cotton swab on a wooden tooth pick wet in the solution and the dead spaces between lips and teeth and teeth and cheeks searched for particles of food or collections of secretions by the same means.

The sicker the patient the more painstaking should be this search.

For sordes on lips or teeth and for the thick coat upon the tongue, half strength solution of hydrogen dioxide (official) should be applied before the milder solutions. Much of the coat may be removed from the tongue by gently scraping the tongue after this treatment with the edge of a whalebone, but there must be no violence.

When the mouth is very dry equal parts of 2 per cent. boric acid solution and albolene (liquid petrolatum) with a little lemon juice added is efficacious and pleasing. If the breath is fetid, the mouth foul and stomatitis in evidence one may make an application of the following solution:

R

Phenol (watery solution 1 in 20)

Glycerin........................ $\overline{a \bar{a}} 3 \mathrm{i}$

Boric Acid (saturated watery sol.)........ 5 viii

M.

(240 c.c.)

This is followed by the milder solution.

The same solutions may be used for the nose; dried secretions being previously moistened and softened by application of sweet oil or vaseline. 
Application should be made with cotton on a tooth pick and very gently. Sprays may also be used.

Irrigations are to be avoided unless special indications arise, as the solution may make its way with infectious material into the Eustachian tube and set up middle ear disease.

For the throat gargles are of little use. The spray is much better and irrigations of hot saline solution $3 \mathrm{i}$ to $0 \mathrm{i}(4 \mathrm{Gm}$. -500 c.c.) are safer here and very grateful. The milder solutions are to be used unless the angina is severe.

The genitals should be carefully inspected and kept clean by the use of the same solutions.

Bowels. When first seen a free catharsis should be induced, one or two watery stools. One may begin with calomel in gr. $1 / 4(0.015$ Gm.) doses every $1 / 4$ hour for four or five doses and follow with salts.

Calomel is easily taken and well borne by children in these doses and if there is nausea the calomel acts as an antiemetic. In the child this dose may be followed by milk of magnesia $₹$ ss. ( 15 c.c.) or liq. magnesii citratis 5 vi to viii (180 c.c. -240 c.c.) or Rochelle salt 3 ii (8 Gm.). In the adult the doses of salt are doubled or Epsom salt or Glauber's salt may be given in doses of $5 \mathrm{ss} .(15 \mathrm{Gm}$.).

If nausea continues, cracked ice, or bicarbonate of soda in doses of gr. $\mathrm{x}-\mathrm{xv}(0.60-1 \mathrm{Gm}$.$) , may be used, or small doses of bismuth gr. \mathrm{xv}$ $(1 \mathrm{Gm}$.) or of cerium oxalate gr. v $(0.30 \mathrm{Gm}$.) are of value. An excellent prescription calls for a combination,

R
Bismuth Subnitratis..................... $\mathrm{xv}$
(1.00 Gm.)
Sodii Bicarbonatis.................... $\mathrm{x}$
(0.60 Gm.)
Cerii Oxalatis
(0.30 Gm.)
M.

This may be given stirred in a little water and repeated at two-hour intervals if needed.

The bowels should be kept open by the use of enemata every other day or by mild cathartics, such as liq. magnesii citratis, Hunyadi water or cascara.

\section{TREATMENT OF SYMPTOMS}

Fever. It is well to bear in mind that the symptoms of disease are often of conservative significance, of purposeful intent, subserving some definite function in combating the agents of disease or the results of their invasion, and that interference is indicated only when this purpose has failed and when pyrexia, too, is adding a burden, not to be endured. 
Fever, so far as we can read its signs, points rather to the establishment of a condition in an acute infection which enhances the efforts and efficacy of the various modes of reaction against the causative agent and its products. If we accept this view there is no reason to attack temperature under all circumstances and, as often, with measures themselves harmful. But we have to learn that this useful temperature may, under conditions, become in itself a danger. These conditions are excessive temperature or long sustained temperature and that the term, hyperpyrexia is a relative one; for a febrile reaction of $105^{\circ} \mathrm{F}$. would scarcely. be considered as hyperpyrexia if of short duration; it becomes a decided hyperpyrexia if sustained for days. Another fact to be borne in mind is that the fever is loaded with the onus of the toxic effects of the materies morbi upon the nervous system and upon the cardio-vascular and respiratory apparatus. The measures that empiricism has directed against fever are successful because they combat the effects of toxemia upon the organs mentioned.

A moderate degree of fever, then, $104^{\circ} \mathrm{F}$. or below is to be let alone; unless accompanied by other evidences of toxemia, but. when above this and sustained, efforts are to be made to reduce it. (For more detailed discussion of fever see Chap. I.)

'The one measure that outranks any other' as an antipyretic both in its efficacy and safety is the application of cold through 'water as the conveying agent.

For infants sponging is best; for children the pack and sponging; for early and middle adult life the pack or bath.

Neither the eruption nor the fear of kidney complications should contraindicate the measure.

We are discussing here hyperpyrexia as such and the object of any procedure is extraction of heat. It is well with children to begin with water relatively warm, $95^{\circ} \mathrm{F}$. to $90^{\circ} \mathrm{F}$. and increase the degree of cold, to $80^{\circ} \mathrm{F}$., $75^{\circ} \mathrm{F}$. and $70^{\circ} \mathrm{F}$. or colder, the degree of cold and the length of the bath depending on the result in terms of reduced temperature and the reaction of the patient.

The patient is to be carefully watched. When the temperature falls to $102^{\circ} \mathrm{F}$. or thereabouts, the bath should be interrupted.

If the patient becomes thoroughly chilled or shows any evidences of collapse, the procedure should stop, the patient be wrapped in a dry blanket, heat placed at the extremities and hot drinks given.

When high temperature is accompanied by collapse and clammy extremities, heat should be applied to the extremities and cold in the shape of compresses or sponges to the trunk.

Angina. Strictly speaking angina is a complication of scarlet 
fever, but a complication so frequent that it may well be considered as characteristic.

We are considering here not the erythematous angina, which has just been touched upon in considering the care of the mouth, but the membranous or gangrenous angina. A membranous angina may occur in every degree of severity, from a small patch here and there to a spreading membrane, covering tonsils, palate, pharyngeal wall and invading the nose and Eustachian tubes. The gangrenous type may be secondary to the membranous or occur as primarily such.

The invasion of the nose and the sinuses, the middle ear, the accompanying adenitis, the possible pneumonia, the ensuing nephritis, the severity of the toxemia and the fact that not only the streptococcus but the Klebs-Löffler bacillus may be concerned in the formation of the membrane make the condition not infrequently a most serious menace to life, demanding the most conscientious and scrupulous consideration.

In the first place let it be emphasized that the presence of membrane should mean diphtheria until excluded and while in the majority of cases the diphtheria bacillus is not present the percentage in which it is, especially in hospital practice which represents patients drawn from the poorer and congested districts, is alarmingly large.

One should never trust to clinical descriptions for differentiation, but take a culture in each instance. If such facilities are not at hand, the patient should be given the benefit of the doubt and a sufficient dose of antitoxin, 10,000 units. If the case is a very severe one and unchecked by cultural data, the antitoxin should be used as in a case of diphtheria.

When the culture is taken, wait upon the report and, if positive, treat as a diphtheria. If there is any laryngeal involvement; it is almost sure to be diphtheritic and the antitoxin should be used at once, not awaiting the return on the culture. Give 10,000 units and repeat in eight hours if no improvement or in four or six if getting worse.

Whether the membrane is due to streptococcus alone or to both it and the diphtheria bacillus, the procedure is the same, in other respects than in the administration of the serum.

Two facts are to be kept in mind. 1. That cleanliness of the parts is the desideratum and that the mildest solutions and the mildest manipulations must be used. 2. That the local condition can be aggravated and its spread and invasion of other structures be facilitated by injury to the parts, either through the irritating or corrosive effects of the applications or the mechanical injury done in the application; and that it is extremely doubtful if any of the so-called antiseptics 
have any effect to kill appreciable amounts of the infecting organisms.

The modes of application are irrigations, gargles, sprays, inhalation of vapor and topical application with a swab.

The most reliable of all these modes of application is the irrigation and the most useful substance hot physiological salt solution, one teaspoonful of common salt, sodium chloride, to the pint of water.

The patient's head is supported by pillow or rest unless too sick, then the head may be turned to one side, a towel tied about the neck, a pus-basin or other receptacle held beneath the chin and the stream of saline directed from a fountain syringe or irrigator held a few inches above the head, but not high enough to exert force, through a hard rubber nozzle, or through a rubber tube, such as a catheter, against the affected parts, the tongue being gently depressed with a wooden or glass tongue depressor. If very young children are so treated, the nozzle may be placed between the molar teeth and the stream directed to the tonsils and pharynx. The excess runs out into the basin.

The amount used should be 1 pint to 2 quarts, according to the demands and the effects on the patient. The temperature should be as hot as can be comfortably borne. One must determine this empirically, beginning with a temperature of $110^{\circ} \mathrm{F}$. to $115^{\circ} \mathrm{F}$. in the adult and $100^{\circ} \mathrm{F}$. in the young child.

The frequency every two to three hours in the day and every four hours at night.

Not only does this clear the throat of secretions and wash away loose membrane, but it relieves the pain in no small measure and induces a moderate degree of hyperæmia, which may itself be a factor in resolution.

Half saturated boric acid solution, 2 per cent., or Dobell's solution diluted from two to four times may be used as the irrigating fluid.

If there is much tenacious mucus in the throat, an abundant spray or preliminary irrigation with a solution of sodium bicarbonate $\mathrm{gr}$. $\mathrm{xx}$ $(1.30 \mathrm{Gm}$.) to the ounce or one containing

Sodii Bicarbonatis

Sodii Biboratis....................... $\bar{a}$ gr. xxx $2 \mathrm{Gm}$.

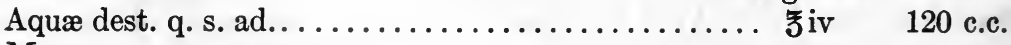

M.

(L. Browne in Coakley on Diseases of the Nose and Throat.)

may be used to rid the throat of this material before the saline irrigation is given.

If there is a considerable deposit of membrane or in any case, applications, preferably by spray or very gently with the swab, of full strength 
of peroxide of hydrogen may be used. It may be better borne in full strength on the swab than in the spray and the latter diluted one to two or three times.

In a bad throat, then, one may get rid of the tenacious mucus by the alkaline spray, then touch the exudate with the peroxide of hydrogen and finally use the hot saline irrigation. If the process is fatiguing, or causes discomfort, it must be simplified as much as is consistent with results.

Sprays of saline, boric acid solution or Dobell's as above may be given between the irrigations as often as they prove grateful to the patient.

Inhalations of hot vapors are sometimes appreciated by the patient. It may be merely steam or steam medicated with compound tincture of benzoin, $3 \mathrm{i}$ to the pint of hot water or oil of eucalpytus.

These may be used in a croup kettle or one of the simple devices on the market or a common kettle with a cone at the nozzle to direct the steam or a pitcher of hot water with a cone over it.

Gargles are in no sense a substitute for irrigation or spray, first, because they do not reach the affected part thoroughly and, second, because the act of gargling is in itself often very painful. If the patient, however, finds relief by such a measure there is no contraindication. One uses the same substances as in the spray or irrigation.

Cracked ice, held in the mouth and sucked, often gives great comfort.

Cold applied to the neck in the shape or compresses or coils or icebags may afford great relief. They must be properly applied and not allowed to annoy.

If heat is the more grateful, it may be applied in the shape of hot cloths.

In gangrenous cases one may use an irrigation of potassium permanganate 1 to 2,000 before using the saline irrigation.

Sprays of adrenalin (epinephrin) of varying strengths have been advised to diminish congestion and afford relief. I have been disappointed in their results.

Alcohol diluted six or eight times used as a gargle is said to be of value.

Touching small gangrenous patches with tincture of iodine has been mentioned as helpful.

Application of Löfler's solution once or twice a day is a method long pursued.

(Löffler's Solution. Menthol 10; solve in toluol ad 36 c.c., alcohol absolut. 60 c.c., Liq. ferri chlor. 4 c.c.)

I purposely refrain from mentioning many escharotics that have been advised, as I believe their use is fraught with danger. 
Nose. Here again cleanliness is the purpose of the measures used and here even more gentleness than in the care of the throat must be exercised. The best solution is, as in the throat, physiological salt solution or 2 per cent. boric acid solution or a Dobell's solution diluted two to four times.

Irrigation, so valuable a measure in the care of the throat, is to be deprecated as a routine in the nose, because of the ease with which the irrigation fluid runs into the Eustachian tube, carrying with it infection to set up otitis media and its sequelæ.

The spray is the best vehicle here and, about the anterior nares, most gentle swabbing.

When membrane is present a solution of hydrogen dioxide diluted three or four times may be applied or sprayed before the cleansing spray.

If the condition of the nasal passages is such as to make imperative other measures than the spray, e. g., in young children with profuse persistent discharge, the irrigation may be adopted with extreme precautions. One uses the same cleansing solutions mentioned, directs the patient to keep the mouth open during the whole procedure, : which lessens the danger of forcing fluid into the tubes, begins, on the side the more obstructed to avoid damming back, holds the irrigator at the least height to just make the fluid pass through, and uses a glass nasal tip or a soft rubber catheter with multiple holes cut in it. Once in four to six hours is often enough.

So good an authority as McCollom advises in membranous rhinitis insufflations of calomel.

A muco-purulent, sero-purulent or serous rhinitis occurs in 10 per cent. to 20 per cent. of the cases. The turbinates are œdematous, the discharges acrid and excoriating. In the majority of the cases it developes in convalescence, when otherwise the patient is considered cured. Its secretions are highly infectious and responsible for many return cases. The measures of cleanliness mentioned above are used, but recent work with vaccine therapy gives encouraging results. The staphylococcus aureus is present in the vast majority of the cases. From cultures of it and other organisms present an autogenous vaccine may be made, or in the absence of these facilities the stock staphylococcus vaccine or bacterin may be used. The initial dose is $50,000,000$ to $100,000,000$, according to age and condition. The doses may be given twice a week or every fifth or sixth day. Usually three injections suffice for a cure. Slight febrile reactions may be anticipated.

No doubt the sinuses are often involved and a persistent temperature in the absence of obvious cause, such as the ear, should make one think of 
the maxillary, ethmoid and even the frontal and sphenoidal sinuses, in spite of the late development of these sinuses in the child.

Cardio-Vascular Apparatus. There has been much discussion about the relative parts played by the heart and vaso-motor mechanism in circulatory failure. I interpret the results of such discussions and experiments as follows: that while the myocardium may be the seat of parenchymatous or interstitial change, such lesions are relatively rare and as the cause of death extremely rare. That circulatory failure may be considered as practically always due to vaso-motor failure and that the result of experimentation points to the vaso-motor center as the particular part of the apparatus at fault.

In one group of cases the heart sounds may be clear and strong to the end, bespeaking competency on the part of that organ, when the vasomotor center has been so impaired as to make the cardiac effort futile. Pallor, cold extremities, a small, empty rapid pulse with a low blood pressure bespeak the condition. In another group, the first sound of the heart may be muffled or almost disappear, or may be split; the pulmonary second sound may be split and accentuated; there may develop a systolic murmur at the apex and the heart may show a tachycardia, bradycardia or arythmia. Yet these signs may not mean and, in the great majority of cases do not mean, any intrinsic change in the heart structure or its functional capacity, but may be explained by that same loss of tone that obtains in the vascular system, and the murmur be one or relative insufficiency of the auriculo-ventricular valves, from relaxation of the rings.

In hearts in wbich loss of tone occurs, the unsupported thin conus arteriosus feels the effect most markedly and its contact with the chest wall immediately overlying at the second left intercastal space gives occasion to the murmur.

Very slight dilatation may sometimes be made out and in the area of the conus denote its dilatation. The apparent impairment of cardiac action is due to loss of normal resistance ahead in the dilated peripheral vessels.

Theoretically, then, our attention should be directed to the vasomotor system in particular.

It is our misfortune that we have no drug that can do for the vasomotor system what digitalis does for the heart.

The drugs credited with effects upon the vaso-motors are caffeine, camphor, strychnine and adrenalin.

None of these are as reliable as we could wish, all are evanescent in their effects, necessitating frequent dosage and to each is denied by many competent men the effect credited to it, if we except adrenalin. 
Digitalis. But let us suppose that there is no improvement in the circulatory condition in response to these measures. Shall we use digitalis? I should say decidedly yes. It may be objected first, that if the heart is not impaired it is questionable whether digitalis can enhance its functions; but even though the chances of its impairment are statistically small, there is still the possibility that it is to some degree and may be helped by digitalis; second, that its effect on blood pressure through its action on the vaso-motor apparatus in man is in doubt, but still that it has such an action in animal experimentation is accepted, and the benefit of the doubt should be given in serious cases; third, that its effect on the heart is lessened in fever, e. g., the inhibitory action of the vagus enhanced by the drug may be set aside in fever, because under the influence of the toxins, the inhibitory action is said to be lost in part or whole.

I question the accuracy of this statement; for I have repeatedly slowed the heart in fever by sufficient doses of digitalis; moreover, we now have electrocardiographic evidence ef the pharmacological effect of the drug on the heart in acute infectious diseases. In the presence of so much doubt our anticipations must be tempered, but it seems to me that any drug which under other circumstances has so decided an effect to improve a faltering circulation, should not be left untried because of theoretical considerations.

Whatever the cause may be of circulatory collapse in acute infectious disease and however digitalis may operate in this condition it is my conviction strengthened by a considerable experience that this drug and its pharmacological equivalent strophanthin are the only reliable agents applicable to the condition.

The action of digitalis is slow, however administered, and if we are accepting it as an agent to be used, it must be begun before the condition has become dire. I feel that it may well be begun when frank evidences of failing circulation set in as an adjuvant to the vaso-motor stimulants mentioned.

The dose in an adult should be 5 ss. (15 c.c.) of the infusion three times a day or four times a day; that is, the equivalent of $101 / 2$ to 14 grains $(0.66$ to $1 \mathrm{Gm}$.) a day. Any official preparation may be used in equivalent doses. It must always be prepared fresh from a fresh green leaf. Such doses may be kept up for 12 to 15 doses.

For very rapid effects, strophanthin may be used in doses of gr. 1/120 $(0.0005 \mathrm{Gm}$.) into muscle or vein, with the anticipation of a digitalis effect beginning within the hour. Such doses should not be repeated except with caution, in less than twelve hours. Digitalis may be begun at once in the above doses, as its effects will be delayed for some thirtysix hours. 
For the child of five years give about one-quarter this dose of digitalis or strophanthin.

A more elaborate discussion of and more detailed instructions for the use of digitalis in any acute infectious disease will be found under pneumonia (Chap. IX).

Caffeine may be used if digitalis fails. I am a little loath to use it when large doses of digitalis have been given, as I think there is an incompatability between the drugs, at least in some individuals, including rapid heart, dyspnœa and alarming evidences of circulatory insufficiency. I advise then that small doses be administered tentatively at first to assure oneself that no such incompatibility exists. Its action is complicated and not constant in effect. I quote the authority of Meyer and Gottlieb's Experimental Pharmacology as an explanation for both these statements. Its action, they say, is a combination of the following factors: " 1 . Stimulation of the vaso-motor centers: Constriction of the arterioles and as a consequence under certain circumstances a rise of blood pressure. 2. Effects upon the heart in a multifold manner: (a) Stimulation of the cardiac inhibitory vagus centers; Slowing of the pulse. (b) Stimulation of the peripheral accelerating cardiac ganglia: Quickening of the pulse rate; according to the circumstances and to individual factors the one or the other action predominates. (c) A change of the heart muscle, whose diastolic capacity decreases and whose systolic energy increases: as a consequence, there is usually a diminution of output per beat and lessening of blood pressure. (d) Dilatation of the coronary vessels.

"If the vascular contraction dominates, the result will be a rise of blood pressure above the norm; but if the vaso-constrictor centers are but little excitable or paralysed through some pharmacological agent, like alcohol, then caffeine will bring a fall in the blood pressure as a rule."

In man, in moderate doses, a slowing of the pulse ensues with a diminished output per unit of time. It is only the effect on the vaso-motor centers that dominate the blood pressure that justifies its use. This effect is brought about through the splanchnic vessels. Many other territories, such as the renal vessels and coronaries are dilated. (Sollman.)

Caffeine is best given in the form of one of the soluble double salts of sodium benzoate and caffeine or sodium salicylate and caffeine. It should be given hypodermically or for more rapid action, intramuscularly, in doses of gr. iii to gr. v (0.2 to $0.33 \mathrm{Gm}$.); this is equal to about half the amount of pure caffeine. In a child of five years gr. ss. $-\mathrm{i}(0.030-$ $0.060 \mathrm{Gm}$.); at ten years gr. i-ii $(0.060-0.120 \mathrm{Gm})$. It should be repeated at two-hour intervals in urgent cases, for the effect rarely lasts 
beyond this period. One may anticipate an effect within five minutes, arriving at a maximum within the half hour and amounting to 10 to $20 \mathrm{~mm} . \mathrm{Hg}$. It may last two hours, but in my observations often a much less time.

Camphor. This is less reliable than caffeine, but is my second choice. It should be given in doses of gr. iii to gr. $\mathrm{v}(0,20-0.30 \mathrm{Gm}$.) in the adult and gr. ss. to i $(0.030-0.060 \mathrm{Gm}$.) in a child of five years, and gr. $\mathrm{i}$ to ii $(0.060-0.120 \mathrm{Gm}$.) at ten years. It is best given in a solution of 10 to 20 per cent. in olive or sesame oil, or in 10 per cent of ether hypodermically or intramuscularly and should be repeated every two hours in urgent cases. One should never use camphor put up in paraffin (mineral oil) as extensive necrosis of tissues have followed upon its administration. The results when they are positive are in my observations very like those of caffeine, a rise of pressure in a few minutes, reaching a maximum within the half hour to 10 to $20 \mathrm{~mm}$. $\mathrm{Hg}$. and lasting from forty-five minutes to over an hour.

One may alternate these drugs, each every four hours, thus one or the other every two hours.

Strychnine is the third choice. It should be given as sulphate in doses of gr. 1/60 to gr. 1/30 (0.001-0.002 Gm.) every three hours in the adult and in doses of gr. $1 / 200$ to gr. $1 / 150(0.0003-0.00045 \mathrm{Gm}$.) at five years and double this dose at ten years. Personally, I place but little reliance on this drug.

Adrenalin (Epinephrin) is not appropriate for continued dose. Its effects are prompt and evanescent. It has value in sudden collapse and acts on the vessels themselves, when the center cannot respond to stimulation. Its effects vary with the mode of administration.

In my studies I have seen no effects when given by mouth or rectum. The effect is uncertain under the skin; it may be decided and prompt or much delayed and slight or fail. It is more certain when given into the muscle and most certain by the vein. The dose by the skin is $\mathrm{m}$. $\mathrm{x}$ (0.65 c.c.) of $1: 1000$ solution; by the muscle slightly less; as an initial dose $\mathrm{m}$. vii or viii $(0.5$ c.c.). By the vein $\mathrm{m}$. ii or iii $(0.120-0.2$ c.c.). This is the dose for an adult. For the child of five years one-quarter the dose, at ten years or twelve years one-half.

There is danger in the careless handling of this drug and more especially in the presence of a weak heart. I have seen in an adult, moribund, a dose of $\mathrm{m}$. vii $(0.5$ c.c.) into the vein shoot a pressure of 60 to $70 \mathrm{~mm}$. to $230 \mathrm{~mm}$. faster than it could be taken. Such strains on the heart may cause a failure on the part of that organ.

Alcohol I believe to be a depressant and condemn.

Cold in the shape of packs or cold air with proper precautions 
of bed making have excellent results on the circulation. See Open Air Treatment. (Pneumonia, Chap. IX.)

Nervous Symptoms. These depend upon the severity of the attack. In moderate grades of the infection there may be some stupor, but in the more severe, delirium of the active type or a low muttering delirium.

There is nothing better for the cerebral manifestations of the toxemia than cold water as described under the treatment of hyperpyrexia, in the shape of packs, sponges or baths.

An ice-cap applied to the head has a sedative effect.

If there is much restlessness bromides may be used, gr. iii $(0.20 \mathrm{Gm}$.) to gr. v (0.30 Gm.) three or four times a day in children, gr. $x v$ to gr. $\mathrm{xx}(1-1.30 \mathrm{Gm}$.$) in adults. Sodium, or potassium salts may be used$ or equal parts of sodium, potassium and ammonium.

Small doses of acetphenetidin (phenacetin), gr. i to gr. ii (0.060-0.120 $\mathrm{Gm}$.) at four-hour intervals may be given to children, but the temptation to use drugs instead of such measures as hydrotherapy must be combated.

Sleeplessness. For milder grades the effects of the baths are often sufficient. Instead of the cold bath, sponging with warm water may prove more sedative.

The small doses of bromides or acetphenetidin (phenacetin) advised may prove effectual. In adults, trional, gr. $x$ to $\mathrm{gr}$. $\mathrm{xv}(0.60-1 \mathrm{Gm}$.) to be repeated in two or three hours, if necessary, or chloralamid, gr. $\mathrm{xx}$ to $\mathrm{gr} . \mathrm{xxx}(1.30-2 \mathrm{Gm}$.) to be repeated in the same time if not effectual.

For wild delirium, sacrificing sleep and rest and imperiling the patient by the exhausion provoked morphine sulphate must be given, in the smallest dose effectual. In the adult gr. 1/8 (0.008 Gm.) hypodermically to be repeated in two or three hours if needed, in the child of six, gr. $1 / 48$ to gr. $1 / 24(0.0015-0.003 \mathrm{Gm}$.)

\section{SERUM AND VACCINE THERAPY}

The achievements of modern medicine in serum and vaccine therapy naturally turn our minds to these fields in the consideration of so dire a malady as scarlet fever. As yet neither of these methods have yielded satisfactory results in this disease. This will be readily understood when we consider that the causative agent of scarlet fever is not yet known and that streptococcic infections during the acute stage are but little amenable to vaccines. With regard to vaccines we may quote the statement of Weaver in Musser and Kelly's Practical Treatment that after a considerable experience with vaccines he concludes that 
their use early in scarlet fever does not stay the usual complications but that they are useful in the treatment of the subacute and chronic stages of some of the streptococcus complications of the disease.

Immune Human Serum, however, gives a more hopeful outlook; it is derived from patients recently convalescent from the disease and is presumed to contain antibodies against the scarlet fever virus and streptococci that have been associated with it.

While statistics so far accumulated are inadequate from which to draw definite conclusions the workers have been competent and careful observers and their convictions are worthy of serious consideration.

The serum is obtained by withdrawing blood from patients in convalescence during the fourth week. Patients who have been septic are excluded and care taken to determine each donor free from tuberculosis. The serum is tested for syphilis, and its sterility determined. Serum from several patients is pooled, as the antibody content of individuals vary and we have no way of determining that content; this is stored in an ice box. The modes of administration are by the vein as practiced by Reiss and Jungman in amounts to 40 to 100 c.c., following the technique given under pneumonia, Chap. IX. Emphasis must be laid on the increased efficacy of early administration. Weaver, more recently, following the same details of preparation, preferred the intramuscular route, giving an average dose of 60 c.c. into the muscles of the outer side of the thigh. He divided the dose between the sides. Usually one dose sufficed, but in a few a second was given.

Personally I should prefer the intravenous route if the vein can be readily entered; but if the results are as satisfactory as they are reported to be by the intramuscular route, one can readily appreciate how this method would come into more general usage.

Zingher made use of the whole blood withdrawn in the second and third weeks of convalescence instead of the serum. He withdrew blood from the veins at the bend of the elbow, citrated at once with 1 c.c. of 10 per cent. sodium citrate to each 30 c.c. of blood and introduced this fresh blood in doses of 70 to 240 c.c. distributed among several large muscle masses. This blood even kept in the ice box deteriorates after one to two months. All of these investigators seem equally enthusiastic. The best results, of course, are in those who are toxic, not septic. There is a prompt fall of temperature. In the toxic cases this fall is likely to be permanent, in the septic cases to be followed by a secondary rise. The fall begins about 2-4 hours after the injection and reaches its maximum in 12 to 24 hours. Accompanying this are amelioration of the general condition, lessening of cyanosis, diminution or disappearance of delirium and change in the appearance of the patient. Even septic cases show 
improvement if the serum is used early and the recurring temperature is usually less high and the course seems to be shortened, but if administered late, in septic cases, no good results ensue.

Normal Human Blood. Zingher, however, says that even in the late septic cases, with extensive throat exudate, higher septic temperature, poor circulation, dusky skin, often with running ears and enlarged cervical glands the use of fresh normal citrated blood is followed by a definite beneficial effect in some desperately ill patients. His doses are $4 \mathrm{oz}$. up to 4 years and 6 to $8 \mathrm{oz}$. in older children and adults, which may be repeated at intervals of 4 to 5 days if needed. This is given into the muscles.

The general practitioner, unless he has ready access to a scarlet fever service will probably still delay the use of serum to the late case of which he despairs. He should keep this method in mind in all cases that appear severe at the onset.

A polyvalent streptococcus serum, in the production of which some thirty types of streptococci obtained from scarlet fever cases have been used has been tried in the Annaskinderspital in Vienna since 1902 with gratifying results. This is known as the Moser serum.

The statistics in this hospital are quoted as 8 per cent. against 13 per cent. in the other hospitals of the city. Such results would seem extremely satisfactory, for the gain must logically be attributed to the successful combating of the streptococcus toxemia. The Moser serum has never been obtainable in this country, which is regrettable considering the long and satisfactory usage in so reliable a service.

In this country a polyvalent serum from different sources has been used, e. g., that of the New York Board of Health.

Personally, I am inclined to use the polyvalent serum from such reliable sources, because, in the absence of any known harm, I feel, in desperate cases, the patient should be given the benefit of the doubt.

The quantity used depends on the make and is specified in each instance.

Otitis. So frequent a complication is otitis in scarlet fever that the possibility of its onset should not be forgotten from the day the case is taken in hand. The frequency varies in different epidemics and has decided relation to the severity of the throat symptoms. In large bodies of statistics the incidence of the complication has been set at figures varying from 10 per cent. to 33 per cent., while in cases that may be termed severe the percentage runs to 50 per cent. and 75 per cent.

It should be laid down as a cardinal rule that the ears should be examined daily with the otoscope. Few simple procedures repay the effort expended on them comparable to otoscopy. The small size of 
the auditory canal in the little patients and the difficulty of determining landmarks discourage many practitioners in making the effort. Of very great assistance are the magnifying otoscopes with electric light attachment, easily portable with a small battery? These instruments bring out the field with remarkable distinctness.

This examination becomes the more imperative when one appreciates that the condition may occur without pain and the first premonition of its existence be determined by a discharge. Before this discharge appears, however, opportunity for a spread into adjacent structures has been given and the rupture of the drum may sacrifice the integrity of that structure beyond repair. Again, patients who are very ill or who are delirious may not voice the pain and discomfort they feel and thus give no clue to the condition.

As a prophylactic measure I would reiterate the warning given in considering the care of the nasal passages, that irrigation may induce the otitis and if the state of the nasal passages seem to demand the use of an irrigation, it must be done with extreme care and with the patient's mouth open.

With appearance of bulging of the drum, congestion and reddening of the drum, a loss of lustre and macerated appearance of the drum, an incision is indicated. To the general practitioner a detailed description of the drum appearances that do or do not indicate paracentesis is often confusing. It seems to me that a decided deviation from the normal in the appearance of the drum and especially if accompanied by pain in the ear and a rise of temperature ought, in a disease in which the sequelæ of otitis are so serious, to demand immediate interference.

Technique. The external auditory canal should be cleansed first with cotton on an applicator with 1 to 1,000 bichloride. A curved bistory or a paracentesis knife is used. The incision should begin posteriorly and below at the end of the malleus, sweep upward along the edge of the drum to Shrapnell's membrane, through it and out along the upper and posterior wall. Do not use wicks and drains or pack the canal tight with cotton.

Irrigations should be instituted every 2 hours by day and every 4 hours by night with 1 to 5,000 bichloride of mercury or with boric acid solution, 2 per cent. to 4 per cent. A pint should be used, from an irrigator or fountain syringe held 2 to $21 / 2$ feet above the level of the ear As the ear improves, the frequency may be decreased to every 4 or 6 or 8 hours.

In irrigating a child's ear the ear should be drawn down and back; in an adult up and back.

With a skilled hand the pain is but momentary though intense; a 
whiff of chloroform or ether may be given or ethyl chloride and the incision done during the primary stages of anæsthesia.

For the pain of earache, dry heat is the best application. This may be afforded in the shape of a hot salt-bag, a hot water bottle or hot plate. Irrigations and instillation are to be deprecated, because the maceration ensuing blinds the landmarks and makes both the examination and incision more difficult. If, however, the pain is not relieved in this manner, one may try a device advised by Yeo. Heat a large sized wine-glass with hot water; pour this out and then into the hot glass place a pledget of cotton, pouring on this 10 to 20 drops of chloroform and then apply the whole closely over the ear, or phenol (carbolic) in glycerin 5 per cent. gtt. ii-iii.

If irrigations must be used, use first simple hot water, then 4 per cent. cocaine hydrochloride.

The natural course of the discharge in older children and adults is one to two weeks, but much longer in young children. In these, however, it usually ceases within twelve weeks.

After the drum is incised, a cessation of discharge with a rise of temperature, pain over the mastoid or swelling there denotes an implication of that structure; but swelling may be absent, pain or tenderness slight and the discharge profuse and yet the mastoid involved. A rise of temperature, not otherwise accounted for, should lead to strong suspicions of the mastoid and surgical advice and interference sought. Involvement of the sinus, of the meninges and the brain are all possibilities that keep us awake to the slightest signs of the same with a view to expert opinion and surgical intervention.

It must be remembered that these discharges are highly infectious and should be treated with 1 to 20 carbolic or 1 to 1,000 bichloride and what can be burned should so be disposed of.

Vaccines. The very fact that these discharges are so infectious; that they detain the patient in the hospital so long; that in the end a small per cent. must be released on the supposition that the discharges are no longer infectious, only to demonstrate that the supposition is not always correct makes any treatment that has theoretical promise welcome.

Good evidence is forthcoming that the recently developed vaccine therapy is fulfilling this theoretical promise to some degree. This is not the place to cite the literature at length on the subject, but an extremely instructive consideration of this subject will be found in an article in the Journal of the A. M. A., April 11, 1911, by Weston and Kolmer, entitled "The Treatment of Suppurative Otitis Media (Scarlatinal) by Bacterial Vaccines (Bacterines)." Their conclusions were "that the best time, all 
things considered, for commencing vaccine treatment in cases of otitis media, is from the eighth to the sixteenth day of the discharge," thus agreeing with nearly all observers, that the subacute stage is that in which the most favorable results ensue. "That continued high fever, nephritis, toxemia and various intercurrent affections are contra-indications to the administration of vaccines." "That under vaccine treatment, three times as many patients are cured within thirty days and permitted to go home as under the usual treatment. This means that the average residence of the patient in the hospital has been considerably reduced."

The organisms most commonly met are staphylococcus aureus and albus, bacillus pseudodiphtheriæ, streptococcus pyogenes and bacillus pyocyaneus.

The vaccine should be autogenous and one finds that recultures do not always show the same organism or show additional organisms. The staphylococcus is often found early, a week later the bacillus pseudodiphtheriæ often intrudes and as a still later comer, as in many suppurative conditions, appears the bacillus pyocyaneus; hence, the value of recultures and fresh vaccines. The initial dose depends on the organism, being small with the streptococcus, $5,000,000$ to $10,000,000$, larger with the staphylococcus, $20,000,000$ to $50,000,000$, and a little more with the others mentioned. The doses should be administered twice a week or every 5 or 6 days. The increase depends on the reaction. The reaction is (1) general, a slight fever. (2) Local; an increase in the discharge. (3) With some organisms, such as the streptococcus, a local redness at the site of injection. It is desired to increase the dose so that little or no reaction occurs.

The ear should not be syringed or medicated during the treatment, as, in the lighting up of the process, as a part of the reaction, a spread into adjacent structures is facilitated by the syringing.

The most cases are cured within five or six doses; a few may require a dozen and a very few more.

Adenitis. A certain amount of involvement of the lymphatic glands always occurs and especially of the cervical glands.

When the angina is severe the adenitis may be very marked. Usually, however, this inflammation of the gland that occurs at the height of the fever subsides without suppurating. Curiously enough the serious involvement of the glands is a late manifestation, occurring during desquamation, most commonly in the third or fourth week or even later, and, indeed, may occur in the same glands which, enlarged during the period of maximum intoxication, had resolved. Those at the angle of the lower jaw are particularly affected. It is this adenitis that so fre- 
quently goes on to suppuration. Whenever a temperature occurs during convalescence this is the one territory the involvement of which must be kept in mind.

Cold. The best application to be made at once is cold in the shape of ice in the ice-bag or bladder-skins. There should be a layer of flannel between the ice and the skin. Direct contact or too long contact may damage the skin.

While remaining skeptical about the value of drugs locally applied, my choice is for ichthyol, painted on pure or in the shape of a 25 per cent. ointment.

There is a difference of opinion about the value of hot applications, such as poultices. My own feeling is that it is useless and looks away from good surgical practice.

At the very first sign of suppuration, incise.

The glands may not suppurate, but become gangrenous, the tissue overlying breaking down; or there may be a great deal of periadenitis and cellulitis with brawny induration.

There is a difference of opinion about the advisability of incision in these cases. Such brawny masses will often resolve, but if the toxemia is increasing and the process spreading, free incision into the tissue should be made.

Arthritis. An arthritis, a so-called scarlatinal rheumatism, occurs in about 4 per cent. of the cases, coming on as a rule in the second week of the attack. In the majority of cases it may be classed as a toxic arthritis; in rare instances it is a septic process.

It resembles the gonococcal type of arthritis rather than that of true rheumatism, affecting the small joints of the fingers and wrists, as well as the large ones. There is as a rule only slight swelling and redness; rarely an effusion of serum, very rarely pus. It lasts as a rule only three or four days, exceptionally a week.

It may be treated on the same principles as the arthritis of rheumatism (see Rheumatic Fever, Chap. III), namely, rest to the joint, by splints or other devices, pillows, folded blankets, local applications of ice, or a snug bandage if there is effusion; if not, methyl salicylate painted on and the joint wrapped in a thick layer of cotton batten and the internal administration of sodium salicylate or other form of salicylate, such as acetyl salicylic acid (aspirin), salicin, oil of wintergreen, be begun in doses of gr. $v$ to viiss. $(0.33-050 \mathrm{Gm}$.) every two hours to a child of ten years or twelve years, half the dose at five years, and gr. $\mathrm{x}$ to $\mathrm{gr} . \mathrm{xv}(0.66-1.0 \mathrm{Gm}$.) in adults. This may be given with or without alkaline salts. $^{1}$ If with, give sodium bicarbonate in double the doses

${ }^{1}$ Alkaline salts are said to be incompatible with acetyl salicylic acid but prob- 
of the salicylates, until the urine is alkaline and then diminish the dose gradually, just keeping the urine alkaline.

As the symptoms subside lessen the dose.

If the joints suppurate they must be treated on surgical principles. Aspiration under surgical precautions should determine the presence of the suspected pus. The joint may then be aspirated through a canula and thoroughly irrigated with sterile salt solution. It may be well to inject a half ounce of 10 per cent. formalin in glycerin. If the joint does not resolve under these procedures it should be opened.

Myositis may occur instead of the arthritis, most commonly a lumbago. This is to be treated locally and by internal medication like the arthritis.

Nephritis. Scarlatinal nephritis adds much to the dread in which scarlet fever is held, not only because of the immediate danger entailed by it, but also because of the haunting possibility of the insidious development of a chronic process, it may be, long after every indication of kidney damage has passed and because its onset comes at the time when we are beginning to congratulate ourselves on a happy convalescence.

Early in the fever a slight albuminuria, such as one may get in any acute infection, from acute degenerative processes of no lasting or important significance in the parenchyma occurs. This need give little alarm, but the increase of albumin at the height of the disease or at the beginning of the third week or the beginning of the fourth has a different meaning.

Delafield describes the nephritis occurring at the height of the disease as an acute exudative nephritis-(non-productive) and while this may prove fatal, if it does not, the lesion clears up entirely; but the late nephritis, post scarlatinal, he says is an acute productive nephritis, which, if not fatal, is likely to go over into a chronic nephritis and it is this feature that makes this late nephritis so serious.

Every day throughout the continuance of the infection and even more imperatively throughout the convalescence the urine should be examined for albumin. It is well to have the paraphernalia for the simple heat and acetic acid or the cold nitric acid test in the sick room or bath-room and do the test at each daily visit.

We must keep in mind, too, that a mild attack can in no way warrant the assumption that nephritis and severe nephritis will not occur. In fact, there seems to be a dissociation in the degrees of severity of the attack and the onset of the nephritis.

ably small doses are not objectionable. For discussion see Acute Rheumatic Fever, Chap. III. 
We know that the severe form of nephritis is most common in the second lustrum and that the appearance of albumin in children after ten is more likely to be an albuminuria simply; hence, redoubled vigilance in the younger children.

Nephritis is said to occur in 10 per cent of the cases.

Treatment.really begins with the beginning of the attack for we believe that.the efficiency of the nursing, care in the diet, a sufficiency of water ingested, especially care of the mouth, firmness in keeping the patient in bed during the convalescence, avoidance of draughts upon the patient's back, all tend to make the incidence of the nephritis much less.

The type of the late nephritis is, as has been said, an acute productive nephritis. Its onset may be insidious or abrupt. Everything is going nicely in convalescence when suddenly an attack of vomiting, a rise of temperature or in rarer instances a convulsion tell of the onset; or the daily examination is rewarded by the first indication in terms of albumin, casts and blood.

With the more insidious attacks a dropsy develops; in the more fulminating cases uremic manifestations. The urine is diminished. Fortunately the vast majority of those attacked recover, the urine becomes normal in amount in about ten days and becomes free from blood and albumin in two to six weeks.

Studies of the renal function, by the phthalein test, determination of urea nitrogen or non-protein nitrogen and creatinin in the blood and observation of blood pressure will give one some idea of the seriousness of the lesion. Normal phthaleins run from 60-80; urea nitrogen less than $20 \mathrm{mg}$.; non-protein nitrogen less then $35 \mathrm{mg}$. and creatinin $2 \mathrm{mg}$. or less in each 100 c.c. of blood. In uncomplicated cases of scarlet fever Veeder and Johnston found that the systolic pressure in young children averaged about $90 \mathrm{~mm}$., in older, $110 \mathrm{~mm}$.

When the kidney function is impaired, the phthalein figures fall, the nitrogen increases and in very severe cases the creatinin increases and the blood pressure is likely to go up.

In all cases of acute nephritis early or late certain definite lines of action are to be pursued.

1. Rest the kidney, by utilizing other organs to perform its functions vicariously in some measure and add as little as possible to its burden by the proper arrangement of dietary.

2. To treat the symptoms that cause distress or danger.

3. To support the strength of the patient during the attack.

To consider the last first, this object is attained by a more rigid definition of rest, if the rules have been relaxed in beginning convalescence; 
by using blankets on the bed and a flannel night-gown, if one is not already in use and by giving the warm sponge bath between the blankets with a renewal of the precautions taken earlier in the illness.

Diet. If the onset is explosive with ${ }_{\text {nausea, }}$ vomiting and fever and with anorexia, no food should be offered until the gastric symptoms subside. During this time cracked ice may be given to mitigate the nausea and vomiting and furnish some water to the tissues. If there is suppression or marked oliguria, only water with sugar and fruit juices, the sugar affording some food value, should be given and it should be remembered that if the kidney has lost its function for eliminating water and is even shunting it into the tissues to the production of anasarca, the ingestion of large quantities of water will only add to their burden. The amount of water should be increased as the increased water output betokens a restoration of function.

We may then begin to give milk in increasing quantities. Our problem from this stage on is to approximate in the diet the caloric needs of the body by a food that shall offer the least amount of work for the kidneys.

We may give milk, with some cream if well borne, rice, arrowroot, cornmeal or oatmeal gruels, bread or zwieback and butter and sugar.

Van Noorden has suggested as a suitable diet in an adult 1,500 grams of milk (1 $1 / 2$ quarts), 375 grams of cream (12 oz.), 50 grams of rice, 50 grams of butter, 20 grams of sugar, which has a heat value of 2,900 calories, as much as that in over 4 quarts of milk. In a child of twelve the demands are less, some 1,600 calories, and the cream should be used much more sparingly.

The kidney eliminates water, salts and nitrogen. Such a dietary is economical in all these substances, considering its high caloric value.

If there is œdema, all the articles taken should be salt free, salt free bread and butter and no salt added to the gruels. Milk contains one and one-half grams of salt to the liter and enough for the metabolic needs.

If the cedema continues still, cut down on the quantity of fluid. I have seen an odema kept up simply because the intake of water was forced beyond the powers of elimination of a kidney in the process of resolution.

Drinks. Water in large quantities is often advised as a sort of a panacea. When œedema is present it should be much limited. The blood will recover water from the œdematous tissues to meet its needs. When the kidney begins to eliminate freely, a freer intake of water will be followed by a diuresis and in uræmia without œdema this may be a desirable means of effecting it. There is rarely danger in giving water 
enough to meet the demands of the patient's thirst. Plain or aërated water, mineral waters, lemonade, orangeade or imperial drink may be used.

As convalescence is established other cereals, vegetables, and lastly fish and meat may be added.

Rest to the kidney is afforded, as shown by the choice of the dietary and is further accorded by the assistance lent in elimination by the other emunctories.

Purgation. One of these emunctories is the intestine. The movements should be rendered watery and fairly copious by the use of salts, such as Rochelle salt, Epsom salt or sodium phosphate, in doses of $5 \mathrm{ss}$. to i $(15-30 \mathrm{Gm}$.) in adult and half the dose to the child. Compound jalap powder is also of use, especially in the adult in doses of $3 \mathrm{i}(4 \mathrm{Gm}$.). It must be remembered that purgation may be carried to the point of exhaustion and do more harm than good.

Diaphoresis. The second great emunctory is the skin.

To effect copious sweating perhaps the best measure is the hot pack. It can be given once or twice a day, depending on the urgency of the attacks. The technique follows:

"Hot Packs. To give the hot pack: Cover the patient with a blanket, folding down the upper bed-clothes to the foot of the bed. Slip two blankets with a rubber between them under him. These must extend from the head to the feet. Put an ice-cap or an ice-compress on his head, changing the latter every two minutes. Line a foot tub with a large rubber sheet-rubber side upward. Put in the tub hot-water bags -four, if possible. Soak two small blankets-one of which is kept doubled-in water $150^{\circ} \mathrm{F}$, leaving out two ends to hold while twisting. Wring the blankets quite dry, put them in the tub with the hot-water bags, and cover with the ends of the rubber sheet in order that they may be kept hot while being taken to the bedside. Slip the doubled blanket under the patient. Stretch the other blanket over his chest and around his arms and legs, without exposing him, and tuck it snuglyaround him, especially at the neck. Place one of the hot-water bags at his feet, one under his knees and one in each axilla and cover all with the rubber which has been lining the tub. Draw up the ends of the under blankets and rubber tightly around the patient, tuck them in and pull up the bed-clothes. Take the pulse frequently at the temporal artery. Encourage the patient to drink copiously-hot drinks, seltzer or vichy. After 20 or 30 minutes remove the wet blankets and rubbers, and roll the dry blankets tightly around the patient. Let him remain thus for an hour, keeping the ice-bag at his head and hot-water bag at his feet. At the end of the hour give him an alcohol rub and remove the blanket. 
Rubbing the body with alcohol under such circumstances, energises the nerve centres and transforms the passive activity of the skin into active vascular excitability." Practical Nursing, Maxwell-Pope, p. 122.

"Modified Hot Packs. Modified hot packs are sometimes given in connection with diaphoretic drugs, to further their action. To apply such a pack, remove the patient's night gown, roll him in a hot dry blanket place hot-water bags at his feet and along his sides, and cover him with a rubber sheet tucking it firmly under the mattress. Leave him thus for half an hour, an hour or longer if necessary." Ibid., p. 123.

The difficulty in a household of achieving the technique of a hot pack often results in the patient's being rolled in a blanket cold by the time it is accomplished. I have found the following modification excellent:

A blanket folded to a little more than the width of the patient's body is wrung out as described, quickly laid on the rubber sheet and the patient, rolled in a dry blanket, laid upon it. Over the patient are placed more blankets and sometimes an impermeable rubber sheet over these. The whole procedure lasts about an hour. During the pack water is given and ice kept at the head.

Another procedure is the hot-air bath, to which some patients respond better than to the pack.

"To give a hot-air bath in bed, the following articles will be needed: An ice cap.

A hot water-bag and cover.

Three blankets.

Two large rubber blankets.

Bed cradles, the number depending on their size.

A bath-thermometer.

A hot-air pipe and support.

Asbestos to put around the top of the pipe.

A Bunsen burner or alcohol lamp.

Hot drinks.

For a vapor bath a croup kettle will be needed instead of the hot-air pipe, and a gas or large alcohol stove will be better than a Bunsen burner.

In a private house the elbow of a stove pipe, five or six inches in diameter, can be substituted for the hot-air pipe, and an old screen, clothes-horse or wooden chairs for the bed cradle.

Method of Giving Bath. Cover the patient with a blanket. Fold down and remove the top bed clothes. Put a sufficient number of bed cradles over him to extend from his neck to his feet and cover these with rubber. Draw out the blanket covering him and pass it up over the cradle under the rubber. Take off his nightgown, put the ice-cap on 
his head and the hot-water bag, covered, at his feet, wrapping the latter in a portion of the blanket on which he is lying. Hang the atmospheric thermometer on the cradle at the top. Draw the ends of the rubber and blanket, which are under the patient, up over the cradle, under the rubber and blankets covering it. Tuck in the latter under the patient on both sides and around the shoulders and neck. At the bottom, tuck them in under the mattress folding them around the air pipe. Put the top end of the air pipe in under the cradle three or.four inches and cover this part of the pipe and as much more of it as the clothes are likely to touch; with asbestos or cold blanket dampened. Tie the pipe to the cradle at least four inches above the feet. See to it that the feet and lower part of the legs are securely covered and apart. Put the bed clothes over the cradle. Tuck them in only at the foot and treat them there in the same manner as the blanket, taking care that the asbestos protects them from the hot pipe. Put the lamp or burner in the pipe and light it, so regulating it that the temperature inside the cradle will be raised from $150-175^{\circ} \mathrm{F}$. Give the patient hot drinks of Vichy while he is in the bath and watch his pulse carefully. The bath is generally continued twenty minutes after the stated temperature is reached. The after treatment is the same as for the pack." Practical Nursing, Maxwell-Pope, p. 124.

Where there is electricity the most convenient sort of heat is from electric light bulbs suspended from the top of the cradle. Three 100 watt 120 Volt bulbs hung 12 to 14 inches apart will suffice to give the desired temperature. Care must be used to avoid the patient or the bedding coming in contact with these bulbs. The common wire cage used to protect electric light bulbs will serve well.

Another procedure is the hot bath, beginning at $95^{\circ} \mathrm{F}$. and slowly increasing the heat to $100^{\circ} \mathrm{F}$. This is kept up for ten or fifteen minutes, then the patient wrapped in a dry or hot moist blanket or pack and continued for another half hour.

It has been again and again noted by clinicians that the hot pack in the œedematous has seemed to precipitate an attack of uræmia. Close observation must be kept upon the patient for any evidence of the same, headaches, twitchings, nausea and vomiting or hypertension.

Drugs. Pilocarpine has been used and will usually induce a marked diaphoresis. It is, however, fraught with dangers, the most imminent of which is œedema of the lungs, and may well be dispensed with.

Treatment of symptoms that cause distress or danger.

Congestion of the kidneys as evidenced by diminished urine or at times by lumbar pain.

Cupping. Two or more cups may be placed over the kidney 
region on either side and left on for 15 to 20 minutes or until the capillaries are well dilated. (For technique of cupping, see Pneumonia, Chap. IX.)

Wet cupping or the application of leeches is advised by some authorities. I am not convinced that the letting of blood here relieves congestion in the kidneys better than venesection elsewhere. So far as the withdrawal of blood relieves the circulation in the presence of hypertension or uræmia, these measures do good. (For the technique of wet cupping and leeching, see Pneumonia, Chap. IX.)

Such good as these measures do, I believe is to be referred to reflex processes, starting in the skin area operated on and impinging on the deep vessels.

Counterirritation. For this purpose hot poultices, mustard paste or hot fomentations may be used. (For the technique of applying poultices, fomentations, mustard paste, see Pneumonia, Chap. IX.)

Diuretics. When the acutest stages have passed, we may venture to encourage diuresis by the mildest of diuretics, milk and water, and the drinks mentioned. These may be followed by the alkaline salts, potassium citrate, potassium acetate or bicarbonate of potash or sodium in doses of $\mathrm{gr}$. $\mathrm{xx}$ to $\mathrm{xxx}(1.30-2 \mathrm{Gm}$.) every two or three hours, or the dose for children according to age until the urine reacts alkaline, then reduce until an amount is continued just enough to keep the urine alkaline. These alkaline salts are often mixed in equal parts.

The use of diuretics of the purin series, theobromine, caffeine, etc., in acute nephritis I advise against. They have been shown to be distinctly irritating to the epithelium of the kidney. If used at all in the subacute stages it should be with caution; then one may use the double salt of sodium salicylate and theobromine or diuretin in doses of gr. $v$ to gr. $x(0.30-0.60 \mathrm{Gm}$.), according to age, three times a day for 2 days only, or the double salt of sodium acetate and theobromine, in the same doses, or theocine gr. v $(0.33 \mathrm{Gm}$.) three times a day for one day only.

If the heart is weak caffeine in the form of the soluble double salts of sodium benzoate or sodium salicylate gr. iii to $\mathrm{v}(0.20-0.30 \mathrm{Gm}$.) three or four times a day or gr. i to gr. ii $(0.060-0.120 \mathrm{Gm}$.) in a child of five years has been advised. The same objection to its use obtains, however, as in the case of diuretin and theocine. Caffeine has a selective action on the renal vessels causing dilatation, facilitating blood supply and diuresis. If the heart, however, is a factor in the condition, increasing congestion of the kidney, by its own decompensation, digitalis is emphatically indicated and is to be used as described under the cardiovascular apparatus.

CEdema is as a rule not massive and is relieved by the measures 
already mentioned. It is possible, however, for fluid to accumulate in the subcutaneous tissues and in the serous sacks to a degree demanding immediate interference.

If the anasarca is marked or obstinate, the tissues should be drained By far the neatest method is by the Southey tubes. I have seen the most gratifying results follow this procedure in a young child when all other measures failed.

Southey tubes are small silver or silver-plated trocars and cannulas, about 1-1 1/2 inches long of small calibre, usually four in a set. They are introduced one behind each internal and external malleolus, at such an angle as to drain well. The trochar is withdrawn and the cannula secured by a bit of silk tied about a groove near the end for that purpose and secured with adhesive plaster. No blood should be drawn; crystal clear water flows freely, and the quantity in 24 hours is often amazing, amounting to quarts. Tissues so infiltrated scarcely feel the introduction of the tube.

When Southey tubes are not obtainable, multiple punctures, 6-12 on the dependent part of each leg with a bistoury protected $1 / 8^{\prime \prime}$ to $1 / 4^{\prime \prime}$ from the point with adhesive plaster may be made or the parts may be cross-hatched with a scalpel. The methods are cruder and it is less easy to prevent infection. None should draw blood.

If the legs hang down or are bent at the knee when the patient is recumbent drainage is facilitated. Whatever the method used, strict asepsis must be observed in the procedure and the legs dressed with loose gauze handkerchiefs or fluff. With any signs of irritation about the puncture withdraw the tubes.

If the hydrothorax is sufficient to embarrass either the circulation or respiration, it should be removed by a paracentesis.

The same procedure must be undertaken for ascites, if the fluid in the peritoneal cavity embarrasses respiration or cardiac action.

Uræmia. With the first premonition of uræmia one should have recourse to vigorous purgation and diaphoresis. With hypertension, twitching foretelling convulsions, venesection is indicated, the amount. depending on the age and size, 10 to 16 ounces in the adult, 3 to 6 in a child of five years. This may be followed by a saline infusion of twice the amount taken; but is not imperative in the stronger patients.

The muscular twitching demands sedatives, chloral, given by the rectum in a couple of ounces of warm milk, gr. $x x x$ to $3 \mathrm{i}(2-4 \mathrm{Gm}$.) in the adult, gr. $v$ to gr. $x(0.30-0.60 \mathrm{Gm}$.) in the child of five to six years.

This can be repeated in a couple of hours if needed.

The hot pack often affords relief, but the patient's condition, pulse, respiration, color and his general response should be studied. The 
length of the time, $1 / 4$ to one hour, and number; one, two or three a day, depends on the patient's response to the procedure. (Technique, see above.)

Convulsions. The one drug efficacious at the moment is chloroform, continued until the attack ceases. This is followed at once by a hypodermic of morphine sulphate to preyent recurrence, in the adult gr. 1/4 (0.015 Gm.) and repeat in a half hour if needed; in the child gr. $1 / 24(0.0025 \mathrm{Gm}$.$) and in an hour gr. 1 / 48(0.0015 \mathrm{Gm}$.) if needed.

An enteroclysis of normal salt solution, two quarts at a time and several times a day, given at $104^{\circ} \mathrm{F}$. to $108^{\circ} \mathrm{F}$. acts often as a vigorous diuretic and assists in elimination of the toxic agent.

With hypertension, amelioration of the condition may follow upon the administration of nitroglycerin, gr. $1 / 100$ to gr. 1/50 (0.0006-0.0015 Gm.) every two hours in the adult or gr. 1/200 (0.0003 Gm.) every hour for five or six doses at five years.

Headache is best relieved by an ice bag to the head or the nape of the neck. Small doses of the milder coal-tars may be used cautiously, acetphenetidin (phenacetin) gr. i to gr. iss. $(0.060-0.10 \mathrm{Gm}$.) every hour for three or four doses in the child; gr. v $(0.30 \mathrm{Gm}$.) at a dose in the adult. Codeine phosphate gr. $1 / 4(0.015 \mathrm{Gm}$.) hypodermically for an adult or according to age for children is useful.

Lumbar Puncture. Recurring convulsions or excruciating headache may find relief in a lumbar puncture, a comparatively simple procedure in the child. (For technique, see Cerebro-Spinal Meningitis, Chap. XXV.)

Nausea and Vomiting. This is an expression of uræmia and will improve with the elimination of the causative agent. For immediate help, we stop all effort at food administration, give cracked ice, apply a mustard paste to epigastrium of one part of mustard to four, five or six of flour, depending on the sensitiveness of the skin, mixed with cold or tepid water and leave until the part is reddened, or give internally bismuth subnitrate, gr. $x$ to $\mathrm{gr} . \mathrm{xv}(0.60-1 \mathrm{Gm}$.), bicarbonate of soda gr. $v$ to gr. $x(0.30-0.60 \mathrm{Gm}$.), or oxalate of cerium, gr. ii to gr. $\mathrm{v}(0.15-$ $0.30 \mathrm{Gm}$.), or they may be combined, as

\footnotetext{
Bismuth Subnitratis.............................

Sodii Bicarbonatis...........................

: Cerii Oxalatis............................. 5 .

5ss.

gr. cl

M. et div. in chart. no. xv.

S. One every two hours in a little water or food.
}

Rare complications in the course of the nephritis, such as pneumonia, pleurisy, endo- or peri-carditis must be treated as if primary. Edema of lungs occurs as a cause of death in nephritis. 
Anæmia is a striking feature of a nephritis. In convalescence iron is indicated. It may be given as Blaud's pill or Vallet's mass in doses of gr. iii to gr. v $(0.20-0.30 \mathrm{Gm}$.) three times a day or as Basham's mixture, Liquor Ferri et Ammonii Acetatis, which contains 4 per cent. tincture of ferric chloride, converted into acetate. Dose $3 \mathrm{i}$ to iv (4-8 c.c.) three times a day.

Convalescence. After an acute attack great care must be taken to prevent a recurrence by too early exposure. The patient should remain in bed until albuminuria ceases or for four or five weeks. If the albumin persists after that, one must consider the depressing effect of too long confinement to bed and the patient be allowed to sit up tentatively, the albumin, casts, total quantity and specific gravity being carefully noted with each increase of license. If the condition is not aggravated, the patient may be allowed about the house or out of doors in suitable weather; but with the slightest indication of recurrence should be put to bed again. If the evidences of nephritis continue and the patient is able to take advantage of a change of climate he may be removed to such a place as allows much out of doors life.

Woolen underclothing must be worn and outer clothing carefully adapted to the weather. Warnings against remaining in wet clothes and undue exposure to the weather must be reiterated. On the occasion of any acute infection the kidneys should be watched with especial care. From time to time, for years after, the urine should be examined at intervals of 3 to 6 months to appreciate the insidious advance of a chronic process.

The sum total of prophylaxis is after all the observance of the laws of hygiene, clean bodies, fresh air, good food and right living.

The Heart. Mention has been made of the heart in considering the cardio-vascular apparatus and its part in nephritis has been touched upon; but in addition to these there may rarely be an endocarditis (in something less than $1 / 2$ per cent.) and still more rarely a pericarditis; the cardiac complications are most common in septic cases, and with post-scarlatinal nephritis and streptococcus pneumonia.

Myocardial changes occur as well. All this may be found with or without articular involvement. Acute dilatation may be the cause of death. (Holt.) More rarely there may be a toxic arteriosclerosis or even an aortitis.

The treatment of these conditions are the same as under other circumstances, rest, the ice bag, and if the myocardium becomes involved and shows signs of weakening, digitalis. (See Acute Rheumatic Fever, Chap. III.)

Broncho-Pneumonia is likely to follow upon septic cases, but 
may more rarely happen during desquamation. It is a far less serious complication than in measles or whooping cough.

The treatment is as elsewhere. (See Pneumonia; Chap. IX.)

Pleurisy occurs not infrequently and occasionally, early or late, an empyema, always a grave condition, demanding prompt operative interference. (See Pneumonia, Chap. IX.)

Still more rarely one meets noma. It is to be combated by curetting and the application of fuming nitric acid and if this fails to stop its extension, excision must be done. McGuire advises application of thick paste of bismuth subnitrate and water. Chorea, peritonitis, symmetrical gangrene and other very rare happenings are to be met as under other conditions.

Relapses. Relapses may occur, as often, it is said by some authorities, as 1 per cent. It is most usual in the fourth or fifth week, but may be delayed until the sixth or seventh. It is in all particulars like the original attack and is to be treated in the same manner.

Recurrences are possible, usually many years later, but are very rare.

Discharge of Patient. The patient should not be released from isolation until desquamation is completed and all discharges from the mucous membranes or suppurating tissues, organs or structures cease.

Even in the mildest cases, however soon the desquamation may seem complete, the patient should be isolated at least four weeks and six weeks is safer. In fact the average case has to be isolated for this time. But even then, if the desquamation is not complete (and the feet have especially to be attended to) isolation must continue. It is to be remembered, as before mentioned, that harsh measures to effect desquamation may set up a dermatitis and defeat the purpose. If, however, when desquamation is quite at an end, there is a discharging nose, ear or gland, the patient must not be released until this ceases.

When all symptoms have ceased and the patient is released from quarantine, if a child, he should not mingle with other children for a month to come nor sleep with them for three months. (Holt.)

When the child is ready for release he is given a thorough soap and water bath and then one of phenol (carbolic) 1 to 50 or bichloride 1 to 5,000 . The scalp and hair are thoroughly shampooed.

No more excellent guide for routine procedure can be taken than Northrup's description of that in use in some of the New York Hospitals. (Nothnagel's Encyclopedia of Practical Medicine, 1902, English Translation, 611, quoted from Weaver in Musser and Kelly's Practical Treatment.) "During the week preceding discharge patients have their ears 
irrigated with bichloride solution 1 to $8,000^{1}$ and the scalp shampooed on alternate days." When discharged

"(1) The ears are irrigated with bichloride solution 1 to 8,000;

(2) the scalp is shampooed with soap and water;

(3) the scalp is shampooed with bichloride 1 to 2,000 ;

(4) a tub-bath is given of soap and water;

(5) a tub-bath is given of bichloride solution 1 to 8,000 for twenty minutes;

(6) a sponge-bath is given of bichloride solution 1 to 2,000;

(7) the bichloride is sponged off with sterile water;

(8) a nasal spray is given of bichloride solution 1 to 8,000;

(9) the mouth is cleansed with saturated solution of boric acid."

The child is then taken into a clean room and clothed in clean clothes.

Fumigation and Sterilization. Washable clothing should be disinfected by steam or by boiling an hour. Mattresses, heavy blankets, pillows and articles that cannot be washed should be steamed where it is possible to have recourse to a properly constructed plant.

All articles that have little value should be burned and toys certainly. No child should ever be given a toy once in the sick room, no matter how sterilized.

Metal objects may be immersed in strong phenol (carbolic acid). The room should be cleaned by having the floors, woodwork and the smooth walls washed down with cloths wrung out of 1 to 2,000 bichloride solution. Then the room should be fumigated with sulphur or formaldehyde. These gases should be generated in the presence of moisture to be effectual.

Sulphur destroys many fabrics and injures other articles and is less effectual than formaldehyde. Where it is possible skilled assistance should be sought in this important procedure; where this may not be obtained, the room is to be sealed with strips about the doors and windows, the key holes plugged and formaldehyde gas generated by some of the contrivances on the market. Candles are made for this purpose, but too implicit faith in their efficacy must not be granted. They should be burned in the presence of moisture, such as may be obtained from a dish of water over an alcohol lamp, and the room sealed for some twelve hours.

Such fumigation does not excuse one from the duty of repainting, repapering, or recalcimining this room.

Fumigation. At the present moment there is a considerable controversy among the authorities as to the necessity of terminal fumigation; The Board of Health of the City of New York, following the lead of Chapin of Providence, R. I., considers terminal fumigation in all

${ }_{1}^{1}$ This in cases in which there may be no otorrhea. 
infectious diseases except anterior poliomyelitis, typhus fever and small pox as unnecessary, providing proper isolation of the patient during the disease with destruction of infectious discharges has been properly carried out. There can be no question that if these precautions are heeded the necessity for terminal fumigation is materially lessened.

Beginning in 1914 the New York Board of Health ceased to fumigate in the Boroughs of Manhattan and the Bronx after all the minor contagious diseases including scarlet fever, diphtheria and measles while the practice was continued in the Borough of Brooklyn. After a long series of cases had been investigated the Health authorities of New York could determine no particular difference in the number of late cases reported from controls. This was in agreement with the observations of Chapin in Providence. Moreover, it is well known that most of the pathogenic organisms have but slight viability after separation from the host and after exposure for an appreciable time to drying and sunlight.

In place, then, of putting reliance upon fumigation at the end of the clinical course of the disease emphasis is laid on the attention to the discharges during the course of the disease and thorough cleansing with soap and water or boiling of the articles that have been soiled by discharges from the patient.

On the other hand at the date of this writing the Health Department of the District of Columbia still continues to disinfect premises after diphtheria, scarlet fever and the major contagious diseases.

In a treatise of this kind the author feels that he can only put the pros and cons before the reader and would advise that in those environments where the rules and regulations laid down for the isolation of patients and destruction of discharges cannot be carried out or are wilfully neglected terminal fumigation be carried out, but would emphasize the inefficiency of most of these terminal fumigations and the great danger of considering them as excuses for neglect of instructions heretofore cited.

Prophylaxis. This subject has already been covered in part as regards disposition of contacts, discharge of patient and fumigation. It includes, furthermore, school inspection of children daily with prompt attention to early signs of infection, following up absentees from school to determine sickness and its nature, supervision of milk supply, enforcement of law against spitting in public places and public lectures on modes of conveyance of disease. 
Incubation.

\section{SUMMARY}

Two to four days. Ten to twelve days.

Period of isolation of those exposed should be eight days. An excess of precaution would set it at three weeks.

Onset and diagnosis.

Vomiting, fever, sore throat.

Erythematous eruption (twelve to twenty-four hours after onset).

Throat shows bright injection.

Tongue shows hypertrophied papillæ at tip and edges.

Leucocytosis and polynucleosis.

Later.

Desquamation.

Eosinophilia.

Nephritis.

Distribution of the family.

Adults. Those who have not had Scarlet Fever should come in contact with the patient as little as is compatible with their duty. All adults should avoid contact with children outside. If contact with children outside is imperative, adults should remove from the environment of infection during its course.

Teachers in the family should move from contact with patient and - notify health authorities and resume working only with their consent.

No member in contact with case should handle food supply for eight days.

Other children of the family.

Removed to another house, until end of illness.

Kept from contact with other children for eight days. Excess of precaution sets it at three weeks.

Should not go to school for three weeks from beginning of isolation. Should not go to school at all during patient's illness, if there is any communication between them and members of the family in contact with the patient or from the home of the patient.

If it is not possible to remove the children to another house, avoid direct or indirect contact with patient.

All contacts should have throats and skin inspected and temperature taken two to three times a day for a week to determine early signs and symptoms of disease. Physician should attend to them before seeing patient.

Room.

Sufficient air space. Good ventilation. Light. Top floor excellent.

Nearness to a bath-room desirable.

Affording ingress and exit without passing through family apartments desirable. Approach through balcony or verandah for example. 
Open fire place a convenience.

Strip room of furniture, carpet, adornments.

Floor bare or covered with carpet lining and over this unbleached muslin.

Sealing doors, except those in use.

Entrance to all rooms protected by sheets.

One sheet on outside of doorway attached to top and right side.

One sheet inside of doorway attached to top and left side or viceversa.

Floor and woodwork.

Rubbed from time to time with 1-1,000 bichloride of mercury (burn the cloths used).

If the floor is covered sprinkle with same solution.

\section{The nurse.}

One for day and one for night or one with a member of the family. If member of family must be nurse, she must be isolated from the rest of the family. She should wear gown and cap.

Handling secretions she should wear rubber gloves.

She should use an occasional throat spray of a mild antiseptic, 2-4 per cent. boric acid solution, or half strength Dobell's solution. Must not come in contact with other members of the family or susceptible people outside. Should not sleep or eat in the sick room.

Should have own room adjacent.

Should take air and exercise. Should change clothes in going out and if coming in contact with others should wash hair.

\section{The physician.}

Leave overcoat and coat and vest in bath-room.

Don gown, long and tight at wrists rubber gloves. Failing this a sheet.

Failing this wear overcoat and street gloves, cap.

Wear rubbers, and turn up trousers.

Leave all instruments in sick room or bath-room (including stethoscope). Disinfect before using again.

Visit as short as compatible with proper examinations.

On leaving. Wash face and hands with soap and water.

Follow with alcohol or bichloride of mercury $1-1,000$ or phenol or lysol 1 per cent.

A careful physician will change his clothes and take a bath before seeing other children. At least plan to see Scarlet Fever cases either early or late in the day. Spend some time later in the open air, and do not visit a child immediately.

Do not take an obstetric case.

\section{Precautions in the sick room.}

Thermometer. Leave the thermometer in the sick room always.

Keep it in 2 per cent. to 5 per cent. phenol solution.

Tongue depressors. Should be of wood or glass; better wooden ones. Keep glass in phenol solution 2 to 5 per cent. 
Syringe nozzles. Glass (not to be used for young children). Hard rubber.

Keep in phenol 2 to 5 per cent. solution.

Dishes, knives, forks, spoons, etc., boiled for half an hour or more. If sent out of sick room to boil, soak in 5 per cent. phenol half an hour.

Urinals, bed-pans, sputum cups, rectal tubes; disinfect and allow to stand in 1-20 phenol.

Bed-linen, night dresses, towels, handkerchiefs, etc.

Soak over night in 2 to 5 per cent. phenol, then boil for half an hour before sending to the wash.

Discharges from mouth, nose, ears caught in pieces of gauze or cloth and burned.

Domestic pets excluded.

Books, toys, etc., destroyed after illness.

\section{Temperature of room.}

Cold air desirable if the body is well protected.

When body is exposed for any purpose temperature should be at $70^{\circ} \mathrm{F}$.

\section{Bed.}

For technique of bed-making, see Chap. IX.

\section{Patient.}

Keep in bed no matter how mild the case may be, especially on account of the kidneys.

Sponge bath with tepid water daily. In a warm room, under a blanket, exposing one part after another.

Nightgown.

Should be flannel or cotton or silk undervest under the flannel nightgown.

Diet.

Person preparing food for family should not come into contact with patient.

Nurse should not handle general food supply of family.

For fundamental principles see Chap. II.

Do not push feeding in first few days.

Milk.

Milk has proven empirically to be the best article of diet in scarlet fever. Two quarts of milk equal 1,280 calories; adding 1 ounce of cream and $1 / 2$ ounce of milk sugar to each glass of milk increases the caloric value to 2,000 .

Milk modifications, such as koumys, buttermilk, whey, may be substituted in part or in whole.

Cereals and cereal gruels, arrowroot, rice, barley, cornstarch, wheat flour, farina, imperial granum, jellies of barley flour, tapioca, sago. Vanilla ice cream. 
Feed at two-hour intervals. Do not interrupt sleep at night.

For caloric values see text.

After the period for nephritis has passed, add eggs, custard, potatoes, other vegetables, oysters, fish and finally meat.

Give no meat soups.

Drinks.

Water freely.

Lemonade, orangeade, juice of grape-fruit, grape juice.

Imperial drink (cream of tartar, a tablespoonful to 3 pints of boiling water, add sugar and lemon juice to suit taste).

Give 2 quarts or more of fluid a day.

Offer water every hour.

\section{Care of skin.}

Sponge bath with tepid water once or twice a day.

Itching and burning.

Cocoa butter.

Cold cream.

Sponge with sodium bicarbonate 3i-0iii (4 Gm.-1,500 c.c.).

Bran bath. Handful of common bran in a clean cloth or muslin bag, swished about in 1 gallon (4,000 c.c.) water until slightly milky.

Calamine $3 \mathrm{i}(4 \mathrm{Gm}$.) to 1 pint (500 c.c.) of lime water.

Severe itching, 1 . per cent. or 2 per cent. phenol in olive oil; $1 / 2$ per cent. dilution of liquor cresolis compositus (Sturtevant).

\section{Desquamation.}

Cocoa butter.

Vaseline (petrolatum) may add

Phenol 1 per cent. or 2 per cent. if itching is severe.

Soaking hands and feet in warm water.

Do not pick, rub or scrape skin; may cause infection.

Apply to soles of feet over night 6 per cent. salicylic acid ointment (Sturtevant).

Itching of scalp.

Wash with alcohol, rub in white vaseline (Sturtevant).

\section{Care of mouth.}

Cleansing solutions.

Physiological salt solution (3i-0i) (4 Gm.-500 c.c.).

Boric acid solution 2 per cent. to 4 per cent.

Dobell's solution (Sod. bicarb. and borax $\bar{a} \bar{a}$ zii-0i (8 c.c.-500 c.c.) with about $1 / 3$ per cent. phenol) quarter to half strength.

Rinse the mouth with one of the solutions after each feeding.

Dead spaces between cheeks and teeth searched.

Teeth cleansed; interstices freed from food; use cotton swab on wooden tooth picks, soaked with a cleansing solution.

Coated tongue and sordes on lips and teeth, one-half strength peroxide of hydrogen (official) to soften, then cleansing solution.

Scrape tongue with edge of whalebone after coat is softened.

Very dry mouth. 
Two per cent. boric acid solution and liquid petrolatum (albolene) equal parts; add lemon juice to flavor.

Fetid breath, foul mouth, stomatitis.

Apply following solution:

R

Phenol (water solution 1-20).

Glycerin....................... àa $3^{i}$

Boric Acid (saturated watery solution) .........

Follow this with a milder solution as above.

\section{Care of nose.}

Dried secretions softened with olive oil or vaseline.

Follow with cleansing solutions on cotton swabs or as a spray.

Do not use irrigations.

\section{Care of throat.}

Spray with cleansing solutions.

Irrigate with hot physiological salt solutions $\left(110^{\circ} \mathrm{F}\right.$.) or 4 per cent. sod. bicarb. solution. Bag a foot above the bed.

Gargles of little use.

For Angina, see below.

\section{Care of eyes.}

Cleansed with boric acid solution, 2 per cent. to 4 per cent.

\section{Conjunctivitis.}

Cleanse with boric acid solution; drop a drop of epinephrin (adrenalin) chloride in eye and follow with 5 per cent. to 10 per cent. argyrol.

\section{Care of genitals.}

Nurse or physician should inspect them each day.

Use boric acid solution, 2 to 4 per cent.

\section{Care of bowels.}

When first seen.

Calomel, gr. 1/4 (0.015 Gm.) every quarter hour for five or six doses (well borne by children).

Follow with salts in three to four hours; in adults Epsom, Rochelle or Glauber's 5 ss.-i (15-30 Gm.) in one-quarter glass of water.

In children follow in two hours with

Milk of Magnesia 5 ss. (15 c.c.).

Liquor Magnesii Citratis 5 vi-viii (180-240 c.c.).

Rochelle Salt 3 ii $(8 \mathrm{Gm}$.) in one-half glass of water.

Later keep open with enemata or mild cathartic, Liq. magnesii citratis, Hunyadi water or cascara.

\section{For nausea.}

The calomel in divided doses as above.

Sodium Bicarbonate........gr. xv Bismuth Subnitrate.........gr. xv Cerium Oxalate...........gr. v
$(1 \mathrm{Gm}$.$) in 5$ ii $(60$ c.c.) water, or

(1 Gm.), or

$(0.30 \mathrm{Gm}$.$) or in combination, e. \mathrm{g}$. 
R

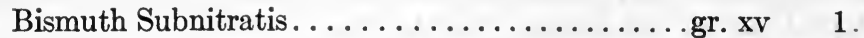

Sodii Bicarbonatis...................... x $\quad 0.60$

Cerii Oxalatis........................ $\quad 0.30$

M. Give one such, stirred in a little water, every two hours.

When severe:

Large dose of Bismuth Subnitrate, e. g.

Subnitrate of bismuth $3 i(4 \mathrm{Gm}$.)

Six drops of 1 per cent. cocaine hydrochloride in a wine-glass of water every fifteen minutes for four doses (Sturtevant).

\section{Treatment of fever.}

Is largely treatment of toxemia.

Treatment of pyrexia per se.

When temperature is excessive, hyperpyrexia.

When long sustained above $104^{\circ} \mathrm{F}$.

\section{Cold.}

Infants, sponge baths.

Children, cold packs and sponge baths.

Early or middle adult life, the bath or cold pack.

With children begin with relatively warm water $95^{\circ} \mathrm{F}$. to $90^{\circ} \mathrm{F}$. and increase the cold to $80^{\circ} \mathrm{F}$. to $75^{\circ} \mathrm{F}$. to $70^{\circ} \mathrm{F}$. or colder.

Discontinue the bath or pack when patient's temperature is $102^{\circ} \mathrm{F}$.

If patient shows evidence of collapse stop the bath, wrap patient in dry blanket; apply heat to extremities. Give hot drinks.

\section{Treatment of angina.}

When membrane is present always take culture for diphtheria bacillus. If culture cannot be taken, give 10,000 units of diphtheria anti-toxin.

If case is very severe and no culture can be taken treat as a case of diphtheria. If laryngeal symptoms are present it is pretty surely diphtheria. Take culture, but do not wait for return but give diphtheria antitoxin 10,000 units and repeat in eight or six or four hours, according to severity. Keep the throat clean but use the mildest solutions. Do not use astringents.

Avoid injury to throat by solutions or procedure.

Solutions.

Sodium Chloride $3 \mathrm{i}-0 \mathrm{i}$ ( $4 \mathrm{Gm}$.- 500 c.c.).

Two per cent. to 4 per cent. boric acid solution; half to quarter strength Dobell's solution.

Mode of application.

Irrigations, gargles, sprays, topical application with swabs.

Inhalation.

Irrigation with hot salt solution the best.

Boric acid and Dobell's solution can be used in the same way.

If there is much tenacious mucus give

Preliminary irrigation or spray with

Sodium bicarbonate solution, gr. $x x(1.30 \mathrm{Gm}$.) to $5 \mathrm{i}(30$ c.c. $)$ 
or

Sodii Bicarbonatis.

Sodii Biboratis................ āà gr. xxxii

Aq. Destillat.q. s. ad............... 万ैiv

If there is much membrane apply gently peroxide of hydrogen with a swab or an applicator.

Use full strength (official) or spray with same diluted one, two or three times.

Then follow with alkaline irrigation as above to clear mucus.

Then follow with hot saline solution as above.

To afford comfort. Apply between the irrigations sprays of any of the cleansing solutions named.

Inhalations of hot steam, medicated with compound tincture of benzoin or oil of eucalyptus or oil of pine. $3 i$ ( 4 c.c.) of any of these on hot water or on sponge of croup kettle amount of water indifferent.

Use croup kettle.

Simple inhalers.

Common kettle with cone of paper attached to spout.

Pitcher of hot water with cone of paper over opening.

Gargles far less efficacious than other methods.

Use same cleansing solutions named.

Cracked ice.

Cold applied to neck as compresses.

Coils.

Ice-bags (throat-bags).

Heat applied to neck.

Fomentations. (See Chap. IX or XIV.) (Modify to suit site of application.)

Gangrenous cases

Irrigate with potassium permanganate solution 1-2,000.

(Other suggestions in gangrenous cases have been,

Sprays of adrenalin.

Gargles of alcohol diluted six to eight times.

Touching with Tr. Iodine.

Application of Löffler's solution once or twice a day.)

\section{Rhinitis.}

Cleansing solutions same as throat.

Sprays best, or swab.

Do not use irrigations unless specially indicated.

If membrane is present, smear and culture for diphtheria.

Spray with peroxide of hydrogen diluted three or four times.

Then use cleansing spray.

When nares are blocked or purulent discharge is profuse, irrigate.

(For procedure, see text.)

Late Rhinitis, muco-purulent, sero-purulent or severe.

Is infectious.

Same measures of cleanliness. 
Staphylococcus aureus usually present.

Vaccines.

$50,000,000$ first dose.

$100,000,000$

$200,000,000$

$300,000,000$

Twice a week.

Usually three or four doses enough.

If temperature continues think of sinuses.

\section{Circulatory failure.}

Digitalis.

If the need is immediate and urgent give

Strophanthin (Boehringer preferred), gr. 1/120-1/60 (0.0005-0.001 $\mathrm{Gm}$.) into muscle.

This dose should not be repeated more than once, at an interval of twelve hours.

Follow the first dose by digitalis, either the infusion, the tincture or the leaf.

Give three or four times a day.

Dose.

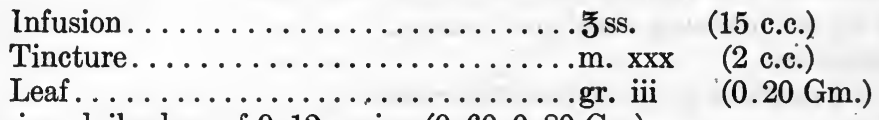

This is a daily dose of $9-12$ grains $(0.60-0.80 \mathrm{Gm})$.

Keep this up for twelve to fifteen doses (three to four days) or until desired results are obtained or some evidences of toxicity obtain.

Child of five years, a quarter dose.

If not urgent omit strophanthin. Begin with digitalis.

I am becoming more and more convinced of the value of digitalis in circulatory failure of acute infections and am giving it preference to the vaso-motor stimulants mentioned.

(See Pneumonia, Chap. IX.)

Usually vaso-motor.

Vaso-motor stimulants.

Caffeine.

Soluble double salt of sodium salicylate or sodium benzoate and caffeine.

Adult dose, gr. v $(0.30 \mathrm{Gm}$.) into muscle.

Child of five years, gr. ss.-i $(0.030-0.060 \mathrm{Gm}$.).

Child of eleven years, gr. i-ii (0.060-0.13 Gm.).

Frequency every four, three or two hours.

Camphor.

In solution in olive oil or sesame oil 10 per cent. or 20 per cent. never in paraffin oil; or in ether, 10 per cent.

Adult dose, gr. iii-v (0.20-0.30 Gm.).

Child of five years, gr. ss.-i (0.030-0.060 Gm.). 
Child of eleven years, gr. i-ii (0.060-0.130 Gm.).

Give hypodermically or intramuscularly.

Frequency every four, three or two hours.

Caffeine and camphor alternately.

Strychnine sulphate or nitrate.

Adult dose, gr. 1/60-1/30 (0.001-0.002 Gm.).

Child five years, gr. $1 / 200-1 / 150(0.0003-0.00045 \mathrm{Gm}$.)

Child eleven years, gr. 1/100-1/80 (0.0006-0.0008 Gm.).

Adrenalin (epinephrin).

In collapse.

Give intramuscularly, 1:1000 solution.

Dose $\mathrm{m} . \mathrm{x}$ (0.60 c.c.).

or

Intravenously $\mathrm{m}$. ii-iii $(0.150-0.20 \mathrm{Gm}$.).

At ten years, a half dose.

At five years, a quarter dose.

\section{Nervous symptoms.}

Stupor and delirium.

Cold air.

Cold water.

Sponges.

Packs.

Baths.

Ice-bag or coil to head.

\section{Restlessness.}

Bromides of potash, sodium, or ammonium or a mixture of equal parts of each.

Dose, gr. $\mathrm{xv}-\mathrm{xx}(1-1.30 \mathrm{Gm}$.) three or four times a day for adults; gr. iii-v (0.20-0.30 Gm.) three or four times a day for children.

Phenacetin, gr. i-ii (0.060-0.130 Gm.) at four-hour intervals may be given to children.

\section{Sleeplessness.}

Cold baths.

Cold sponges.

Warm sponges.

Bromides. (See restlessness.)

Phenacetin. (See restlessness.)

Trional, gr. $\mathrm{x}-\mathrm{xv}(0.60-1 \mathrm{Gm}$.) in capsules or in a little warm water or wine, whisky or brandy.

Repeat in two hours if needed (adult).

Chloralamid, gr. xx-gr. $\mathrm{xxx}(1.30-2 \mathrm{Gm}$.) in powder, cold water or wine, whisky or brandy, and repeat in two hours if needed (adult).

Wild delirium.

Morphine sulphate, gr. $1 / 8(0.008 \mathrm{Gm}$. $)$ hypodermically for adult; gr. 1/48-1/24 (0.0015-0.003 Gm.) for child. 


\section{Specific treatment.}

Convalescent serum.

Convalescent blood. (See text.)

Normal human blood.

Vaccines.

Indicated in some of the subacute and chronic streptococcus complications of the disease. (See below.)

Polyvalent sera.

Moser serum.

New York Board of Health serum. (See text.)

\section{Otitis.}

Examine ears daily.

With involvement of drum. (See text.)

Incise.

For technique and after care, see text.

Earache.

Dry heat, to avoid maceration of drum.

Hot salt bag.

Hot water bag. Applied to the ear.

Hot plate.

Take a wine-glass made hot by dipping into hot water, put in a pledget of cotton; in this 10-20 drops of chloroform; apply inverted glass over ear. (Yeo.)

Drop into ear two or three drops of 5 per cent. phenol in glycerin. If irrigations are used, use hot water first and drop in 4 per cent. cocaine hydrochloride solution after.

Mastoid. (See text for symptoms.)

Surgical procedure.

Remember that the discharges from the ear are highly infectious.

Destroy by burning or 1-20 carbolic or $1-1,000$ bichloride of mercury.

$\left.\begin{array}{l}\text { Sinus involvement. } \\ \text { Cerebral abscess. }\end{array}\right\}$ Surgical procedure.

Vaccine treatment of discharge from ear.

Determine organism by culture.

It may be staphylococcus aureus.

Staphylococcus albus.

Bacillus pseudo-diphtheriæ.

Bacillus pyocyaneus.

Streptococcus pyogenes.

Vaccine should be autogenous when possible and should be fresh.

Time to begin 8th to 16 th day of discharge.

Contraindications.

High fever.

Nephritis. 
Toxemia.

Intercurrent affections.

Dose beginning.

Streptococcus $\quad 5,000,000$.

Staphylococcus $20,000,000$.

Increase depends on reaction; no reaction, double each dose for three or four doses; then add 20,000,000-30,000,000 streptococci at a dose; 50,000,000-100,000,000 staphylococci at a dose.

Frequency, twice a week or every five or six days.

Duration, six to twelve doses.

Reaction. (See text.)

\section{Adenitis.}

The severe suppurating forms are later manifestations, third or fourth week or later.

Cold.

Ice bags or bladders.

\section{Heat.}

Ichthyol, 25 per cent. in vaseline to pure product painted on.

$\left.\begin{array}{l}\text { Fomentations. } \\ \text { Poultices. }\end{array}\right\}$ (See text as to value.)

Wet dressings.

Huge, very wet dressings at the temperature of melting ice.

Use following solution for dressings.

Plumbi Acetatis.................. 60 ร

Aluminis Pulveris................. 60 5

Alcoholis........................250 5viii

Glycerini......................... 120 万

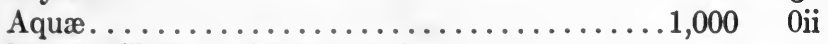

M. et. Shake. (Sturtevant.)

At the first sign of suppuration incise.

\section{Arthritis.}

Usually in second week.

Treat as in rheumatism. (See Chap. III.)

Suppuration is rare.

Treat on surgical principles. (See text.)

Myositis.

Local treatment and medication as in arthritis.

Nephritis.

Examine urine daily. Keep reagents for albumin test at the patient's. Severe attacks occur late in third or early in fourth week.

Prophylactic.

Good nursing.

Bed during convalescence.

Avoidance of draughts.

Diet largely of milk. 
Treatment.

1. Rest to the functions of the kidney.

2. Treat symptoms.

3. Support patient.

Patient is put between blankets.

Flannel nightgown.

Diet.

If explosive with nausea and vomiting give only cracked ice until these symptoms subside.

\section{Suppression or oliguria.}

No more water than the kidney eliminates.

Food, only sugar added to water or fruit juices.

Increase water intake as urine output increases.

Then begin to add milk tentatively, observing effect on total output of urine and albumin.

\section{Edema.}

Make all articles salt-free; salt-free bread, salt-free butter. 'With increasing œdema cut down water intake.

\section{Diet outlined for Scarlet Fever.}

Milk and cereals, bread and butter, are suitable for the nephritis.

Drinks.

Plain water, mineral waters (no sodium chloride) lemonade, orangeade, Imperial drink.

Amount not more than 1 pint to $1 \frac{1}{2}$ pints more than urine output.

Purgation.

Fairly copious watery movements by salts.

Rochelle, Epsom or Glauber's salt, or sodium phosphate.

Dose, 5 ss. $-\mathrm{i}$ (15-30 Gm.) in three-quarter glass of water for adults, half this dose for children,

or

Compound Jalap powder, $3 \mathrm{i}$ in warm water (adult dose).

Purgation must not induce fatigue.

\section{Diaphoresis.}

Hot pack once or twice a day. (Technique, see text.)

Hot air bath. (Technique, see text.)

Hot bath.

Begin at $95^{\circ} \mathrm{F}$. for ten or fifteen minutes. Then wrap in a dry or hot moist blanket or pack for another half-hour.

\section{Drugs.}

Pilocarpine, only when pack is not attainable, has its dangers.

\section{Cupping.} (See text.)

Especially indicated in early stages of congestion and acute suppression; two or more cups over either kidney for fifteen to twenty minutes or until capillaries are well dilated. (For technique, see Chap. IX.) 
Counterirritation.

Hot poultices. (For technique, see Chap. IX.)

Fomentations. (For technique, see Chap. IX.)

Mustard paste. (For technique, see Chap. IX.)

\section{Diuresis.}

After acutest stages have passed.

Mildest diuretics first.

Water and drinks mentioned, milk.

Then alkaline salts.

Potassium citrate.

Potassium acetate.

Potassium bicarbonate.

Sodium bicarbonate.

Any one or combination.

Dose, gr. $\mathrm{xx}-\mathrm{xxx}$ (1.30-2 Gm.) every two to three hours.

Children half dose.

Continue until urine reacts alkaline; then reduce dose in frequency, but keep urine just alkaline.

Little later.

Diuretin, gr. $\mathrm{v}-\mathrm{x}(0.30-0.60 \mathrm{Gm}$.) three times a day.

(The smaller dose in children or even half this.)

Agurin.

Same dose and frequency as diuretin.

Theocin. Same frequency as diuretin for one day only. Dose gr. v $(0.35 \mathrm{Gm}$.) adult.

Caffeine; especially if the heart is weak.

Use double salt of sodium salicylate or sodium benzoate or citrated caffeine.

Dose, ii-v (0.20-0.30 Gm.).

Frequency, three times a day.

Child of five years, dose, gr. i-gr. ii $(0.060-0.120 \mathrm{Gm}$.) three or four times a day.

\section{CEdema.}

Diaphoresis.

Diuresis.

Purgation.

Hydrothorax, paracentesis.

Ascites, paracentesis.

Anascara, scarification.

Southey's tubes. (For technique, see text.)

\section{Uræmia.}

Purgation. $\}$ (See above.)

Hypertension and twitching foretelling convulsions.

Venesection.

Adult, 10 to 20 ounces.

Child (five years), 3 to 6 ounces. 
Sedatives.

Chloral by rectum.

Dose, gr. $x x x-3 i(2-4 \mathrm{Gm}$.).

Child (five years), gr. v-gr. x $(0.30-0.60 \mathrm{Gm}$.).

Give.in 2 ounces of warm milk or starch paste.

Repeat in two hours if needed.

\section{Convulsions.}

During the convulsion.

Chloroform inhalation until the attack ceases.

Follow at once by

Morphine sulphate, gr. 1/4 (0.015 Gm.).

Repeat in half hour if needed.

Child (even infant), gr. 1/48-gr. 1/24 (0.0015-0.0030 Gm.).

Repeat in an hour if needed.

\section{Enteroclysis.}

Salt solution, $3 \mathrm{i}-0 \mathrm{i}\left(4 \mathrm{Gm} .-500\right.$ c.c.) 2 quarts (2 litres) at $104^{\circ} \mathrm{F}$ $108^{\circ} \mathrm{F}$. several times a day.

\section{Lumbar puncture.}

(Technique, see Cerebro-spinal Meningitis, Chap. XXV.)

\section{Hypertension.}

Nitroglycerin, gr. 1/100-gr. 1/50 (0.0006-0.0015 Gm.) every two hours. Child of five years, gr. 1/200 (0.0003 Gm.).

\section{Headache.}

Ice bag to head and nape of neck.

Acetphenetidin (Phenacetin) cautiously, gr. v (0.30 Gm.) every hour for three or four doses.

Child, gr. i-iss. (0.060-0.10 Gm.).

When excruciating, lumbar puncture.

\section{Nausea and vomiting.}

Cracked ice.

Mustard paste to epigastrium 1-4, 5, or 6 of flour.

For technique, see Chap. IX.

Bismuth, gr. $\mathrm{x}-\mathrm{xv}-\mathrm{lx}(0.60-1-4 \mathrm{Gm}$.).

Sodii bicarbonatis, gr. v-lx $(0.30-4 \mathrm{Gm}$.).

Oxalate of cerium, gr. iii-v (0.20-0.30 Gm.).

Or combined as

$\mathrm{R}$

Bismuth Subnitratis................15

Sodii Bicarbonatis..................10

Cerii Oxalatis...................5

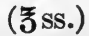

(3iiss.)

M. et div. in chart no. xv.

(gr. lxxv)

One in a little water or milk every two hours. 
Pneumonia.

Pleurisy.

Endocarditis.

Pericarditis.

Rare complications.

Treat as primary.

\section{Anemia.}

In convalescence give iron, Blaud's pill (pil. ferri carbonatis), or Vallet's mass (massæ ferri carbonatis), gr. iii-v (0.20-0.30 Gm.) three times a day. (Adult dose.) Basham's mixture (liq. ferri et ammonii acetatis), $3 \mathrm{i}-\mathrm{iv}$ (4-15 c.c.).

Convalescence from nephritis.

Remain in bed until albuminuria ceases, or for four to five weeks. After that allow up tentatively, then about house, then out of doors. Watch effects on albumin, casts, and total quantity. If aggravated, put back to bed.

Wear woolen underclothing. Adapt clothing to weather. Avoid exposure to weather. Change wet clothing at once.

Watch kidneys on occasion of any illness. Examine urine every three to six months. Other complications.

Endocarditis. Rare.

Treat as under other circumstances.

(See Rheumatic Fever, Chap. III.)

\section{Bronchopneumonia.}

Treatment. (See Pneumonia, Chap. IX and Streptococcus Pneumonia, Chap. X.)

\section{Pleurisy.}

Treatment. (See Pneumonia, Chap. IX.)

\section{Noma.}

Application of bismuth subnitrate and water to form thick paste; apply several times a day.

Curetting.

Fuming nitric acid application, excision.

Relapses.

Treat like initial attack.

Discharge of patient.

Four weeks in the mildest case.

Six weeks is safer. 
If still desquamating at the end of six weeks, isolation must continue until desquamation ceases.

If nose, ears or glands are discharging when desquamation ceases isolation must continue still until the discharges cease.

When patient is released from quarantîne, he must not mingle with other children for a month after.

Should not sleep with other children for three months (Holt).

Preparation of the child for release.

Soap and water bath, then carbolic acid (phenol) solution (1-50) bath,

or

Bichloride of mercury bath (1-5000).

Head shampooed.

Northrop's rules.

1. Ears irrigated with bichloride 1-8000.

2. Scalp shampooed with soap and water.

3. Scalp shampooed with bichloride 1-2000.

4. Tub bath of soap and water.

5. Tub bath of bichloride 1-8000 for twenty minutes.

6. Sponge bath of bichloride 1-2000.

7. Bichloride sponged off with sterile water.

8. Nasal spray of bichloride 1-8000.

9. Mouth cleansed with saturated solution of boric acid.

10. Taken to a clean room and clad in clean clothes.

\section{Fumigation and sterilization.}

(For discussion, see text.)

Washable clothing boiled one hour.

Mattresses, pillow, heavy blankets, etc., sterilized by steam under pressure.

Toys. Always destroy; never attempt to sterilize.

Metal objects. Sterilize in 1-20 phenol.

Room. Smooth walls, woodwork and floors washed with 1-2000 bichloride, then

Fumigated with formaldehyde or sulphur.

Formaldehyde best.

Obtain skilled assistance when possible; otherwise seal room, windows and doors with strips of paper glued together by mucilage of tragacanth over cracks.

Burn formaldehyde candles or use other generator.

Must be burned in presence of moisture (water over alcohol lamp). Sulphur less reliable and more injurious to fabrics.

After fumigation.

Repaper, repaint and rekalsomine. 


\section{CHAPTER XVIII}

\section{DIPHTHERIA}

Since the genius of Jenner made the treatment of Smallpox a rare experience for the general practitioner, no other triumph of medicine is comparable to serum-therapy in Diphtheria. Where four children died before the introduction of diphtheria antitoxin, but one dies to-day and the statistics that show a fall in mortality from 40 per cent. in preantitoxin days to 10 per cent. at the present do but scant justice to the efficacy of this treatment, because so much depends on the promptness and mode of administration of antitoxin, that the figures are vitiated by the neglected cases. More recently the method devised by Schick to determine beforehand what individuals are susceptible to diphtheria and the mode of inducing active immunity lessen still further the terrors of this dread disease.

While the organism responsible for the disease may be found in a certain per cent. of cases distributed to various organs of the body, the disease can scarcely be called a septicæmia, but essentially a toxemia, the toxins in which are elaborated at the site of the membrane formation in the upper air-passages.

Success in treatment depends essentially on an early appreciation of the condition and an early appreciation can be had only by a fidelity to thoroughness in routine procedure. The early symptoms are not distinctive; a malaise, a headache, a chilliness and a sore throat; but the sore throat is often trivial or indeed does not occur and in infants there is little to suggest that the throat is the seat of the trouble.

Malaise, a little temperature and a depression and apathy out of proportion to the temperature may be all the child offers to one's observation. The finding of the local lesion comes, then, often enough as a surprise, awarding the routine of examination of the throat.

There are two examinations too often overlooked or slurred that should be made in every instance of infection in infancy, unless the lesion is more than obvious and even then, in search of complications; namely, the ear and the throat. Humiliation comes to almost every man who wilfully or inadvertently overlooks these procedures.

In the case of the ear not a little skill is needed to see at all or intelligently with the older otoscopes, but with the more modern magnifying otoscopes, changes in the ear can be scarcely overlooked. The examina- 
tion of the throat is more commonly made, but is often a meaningless convention, carelessly done. In the infant a little skill in placing the depressor well back on the tongue gives a momentary, but excellent view; but often this is insufficient and a conscientious examination is put off rather than persist with the crying and struggling child. The tonsils, the pillars of the fauces and the pharynx must be seen.

Such a routine does not allow the condition to be overlooked.

But there is one other fact on which much emphasis must be laid, namely, that diphtheria may be present when the clinician is quite sure that he is dealing with a follicular tonsillitis and, more, diphtheria may be present when no exudate, membranous or follicular is evident and hence, in any inflammation of the throat in a child, even when only catarrhal, a culture should be made. Again and especially in infants, nasal diphtheria may be present when no membrane is anywhere visible and a persistent nasal discharge, particularly if excortiating or bloody should demand a culture. Finally, a laryngitis, more particularly of a croupy character and certainly if persisting in the daytime should make a culture imperative.

The Culture. A tube of fresh culture medium must be obtained; the suspected parts thoroughly rubbed with the swab, the surface of the medium thoroughly smeared with the contaminated swab; great care must be taken to bring the swab into contact with no other object in the procedure; the culture submitted to a competent bacteriologist for incubation and diagnosis.

The Family. When diphtheria is determined in any one individual, all the members of his family at once become suspects. The children are to be kept from school and adults who have intimate relations with children, such as teachers, should temporarily interrupt their occupation.

A Schick reaction should be done on all members of the family and those who react positively should be given an immunizing dose of serum and measures taken to induce active immunization.

Authorities should be informed of the case and inspection of the school or suspected groups of people, who may be the source of infection, should be made.

Children should be isolated until cultures can be taken from their throats and a report made on the cultures. Adults in the family should submit to the same procedure. In this way those who are "carriers" can be determined and can be kept in isolation until their throats are free from infection.

All the children in the family who react to Schick should be immunized with antitoxin, 500 units for the infants and 1,000 units for the older 
children and adults. All adults in intimate contact with the case who react positively should be immunized.

Children, free from infection as proved by negative cultures and with negative Schick, should be removed from the house, if possible, lest they become carriers. It is wise to repeat such cultures at least once. The immunization should be repeated every two weeks in those reacting positively to Schick unless active immunization is practiced. When a Schick reaction cannot be practiced all the children of the household and adults in close contact with the patient should receive immunizing doses, this should be repeated every two weeks in succession. Children found to be infected or to be carriers should be isolated, but never with the sick case, and the isolation of these cases should be separate.

The Schick Reaction. This reaction depends upon the fact that if diphtheria toxin is introduced into the skin it causes an irritation in the tissues of that structure, which is easily recognized, unless there is circulating in the blood and tissue juices of the individual inoculated antitoxin, which, neutralizing the toxin, shelters the tissues from its noxious action and thus prevents a reaction.

Briefly then a (positive) reaction means absence of antitoxin or in other words susceptibility to diphtheria; an absence of (negative) reaction means the presence of antitoxin or immunity.

This important contribution to the warfare upon diphtheria was made by Schick in 1913.

The details to follow are derived largely from the publications of the Department of Health of the City of New York.

Technique. The material used is a fresh solution of diphtheria toxin containing $1 / 50$ the minimum lethal dose for a 250 gram guinea-pig. This should be contained in 0.2 c.c. of the diluent. How to attain such proportion should be found in instructions accompanying the material furnished; e. g., the Health Department of the City of New York furnishes the toxin in capillary tubes, the contents of which when expelled into 10 c.c. of a normal saline affords such a dilution that 0.2 c.c. of this dilution contains the desired 1/50 M. L. D. (minimum lethal dose.) One tube will answer for many tests and the saline solution will keep 12 hours if kept cool in the ice box.

The syringe used should, of course, be graduated to fractions of a c.c. such as a "Record" or a Sub Q. tuberculin syringe, though an ordinary syringe can be used if necessary. A fine steel or platinum iridium needle is selected, a \#26 gauge, $1 / 4$ or $1 / 2$ inch in length is excellent. The flexor surface of the arm chosen and prepared by cleansing with alcohol.

The injection must be intradermal, between the layers of the skin, not subcutaneous. To effect this, one sees, a fine needle is required and 
its insertion should be so superficial that one may see the oval opening of the needle through this superficial layer of the skin and the injection fluid should raise a wheal that brings out pitting of the openings of the hair follicles.

The positive reaction is indicated by a trace of redness at the site of the injection in 12 to 24 hours, distinct in 24 to 48 hours and at its height on the 3rd or 4th day when it presents a circumscribed area of redness with some infiltration one to two centimeters in diameter. This continues for 7 to 14 days. After this it gradually disappears until only a brownish scaling area is left, which persists for 3 to 6 weeks.

The negative reaction is determined by failure to respond to the irritating effects of the toxin and, as has been said, means immunity. Repeated tests over a period of years makes it almost certain that this natural immunity in individuals over three years of age is permanent.

The susceptibility at various ages to diphtheria as determined by the Schick reaction is shown in the following table published by the New York Department of Health:

$$
\text { Age }
$$

Susceptible

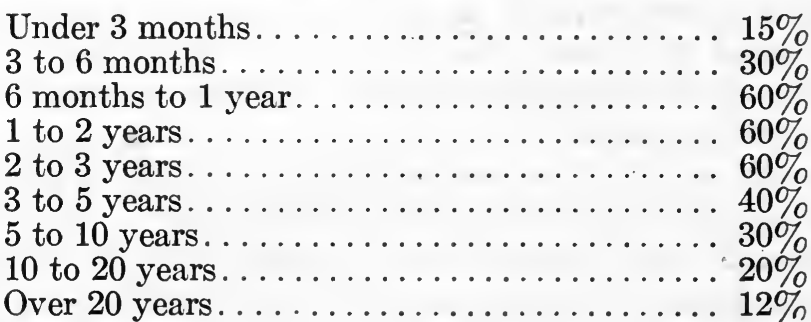

The low susceptibility under 6 is attributable to antitoxin from the mother's blood still persistent in that of the infant.

The pseudo reaction. Unhappily the determination of a positive reaction is embarrassed by another reaction in the skin provoked not by the diphtheria toxin, but by the protein substance of autolyzed diphtheria bacilli to which a certain number of individuals are anaphylactic. It is easy enough to state differences in the true and false reactions; but at first, before the eye becomes practiced, the differentiation may present difficulties.

The pseudo reaction occurs earlier, in 6 to 18 hours; it make its full development in 36 to 48 hours and disappears on the 3rd or 4th day. It leaves a little brownish discoloration but rarely scales. When at its height it shows a dusky red centre with a secondary areola; it somewhat resembles a hive (urticaria). 
Patients who develop only this false reaction are immune. It has the significance of a negative reaction.

Here again we are met with the annoying fact that a patient may react to both the toxin and the protein of the autolyzed bacilli; that is, he may show a combined reaction. This combination of positive and pseudo reaction shows features of both. There is more central redness and more infiltration; the pseudo element fades and leaves the brown, scaling area of the true.

Control. One may obtain and use diphtheria toxin heated at $75^{\circ} \mathrm{C}$. for 5 minutes to control the Schick reaction. This heating destroys the toxin but not the protein products of the autolyzed bacilli. This is diluted in saline and injected, in the same amount and manner as the toxin, in the other arm or far enough away from the site of the other injection to avoid intermingling. A negative reaction to the toxin will also give a negative reaction to the heated toxin. A positive reaction only to the toxin will give a negative reaction to the heated toxin.

A pseudo reaction to the toxin will give also a pseudo reaction to the heated toxin and the comparison of the reactions and their course will determine their nature.

A combined reaction to the toxin will call out a pseudo reaction to the heated toxin. The difference in character and course of the two reactions will determine their nature.

Of course the control may be used after the unheated toxin has given a doubtful reaction. If such controls are not used, any doubtful case should be retested or failing this or being still in doubt, any doubtful reaction should be treated as a true one.

One can see at once the great value of the Schick reaction; how readily in hospitals, institutions and schools the immunes and non-immunes can be separated; what a great saving in antitoxin this means; how it leads to preventive treatment of the non-immunes and what a relief it must afford to the family into whose midst diphtheria has been brought.

Active Immunization. This, of course, is practiced on those only who give a positive reaction to the Schick test.

It is brought about by introducing small amounts of toxin under the skin, which provokes the formation of antitoxin. Diphtheria toxin, however, is so virulent that very small quantities carefully given and cautiously increased, necessitating a considerable period of time in its performance, are alone feasible; but fortunately, it was found that if antitoxin sufficient to neutralize the toxin to the point of not being poisonous was administered with it, this toxin-antitoxin combination 
lost but little of its efficacy in eliciting antitoxin formation by the tissues and as very much more toxin could be used in the combination a great deal more antitoxin was manufactured by the body in a much briefer period. The injection used at any age is about 400 times the fatal dose for a half-grown guinea pig and the amount of antitoxin required to neutralize it is about 4 units. The dose is 1 c.c. of the toxin-antitoxin so prepared, repeated every 7 days for 3 doses. For children under one year, 0.5 c.c. at weekly intervals for 3 doses. The method, a subcutaneous injection into any convenient site, such as the insertion of the deltoid.

Reactions. These are usually less marked than after the more commonly used typhoid vaccine and like the latter consist of more or less redness and swelling at the site of injection with or without a constitutional reaction, such as one attributes to a mild infection. It lasts one to three days.

Infants as a rule have neither local nor constitutional reactions. Older children and adults suffer the reaction in something less than one-third of the cases.

The acquisition and duration of immunity. Antitoxin is slow in its formation. Rarely is the amount protective in less than three weeks and as a rule not until the second month and in some cases dallying along even to the sixth month.

Tested three months after the injection nearly 75 per cent. are immune after one injection, 90 per cent. after two and 95 per cent. after three. The Department of Health found that in young infants harboring their mothers' antitoxin the immunizing powers were not as complete, giving 50 per cent. immune only after a year later.

The best period for active immunization is 6 months to 5 years.

Antitoxin once started by this method seems to keep on forming and 90 per cent. of a small number (100) observed by the Department were immune after 4 years.

Active immunization has certainly won a second great battle in our campaign against this disease; for owing to certain factors, seemingly almost impossible to overcome, the results of antitoxin administration had come to an impasse as shown by the unchanging statistics of mortality in diphtheria for a number of years past; antitoxin cut the mortality more than a half, from about 28 per cent. to a little under 12 per cent., but the neglect of families to call medical assistance until late in the first week, when antitoxin can do but little, or even delay until the patient was moribund or failure to recognize the disease on the part of the physician are all factors contributing to mortality that it seems impossible to correct by mere words of warning. 
It is easy to see how by determining susceptible individuals by the Schick reaction applied in schools, hospitals, institutions, in communities the seat of an epidemic, and in families invaded, the definite determination of susceptibility brings the weapons of fear and moral obligation to enforce active immunization to prevent spread. It will be seen in discussing carriers how impossible it is to eliminate these from a community and how safety can be found only in a community rendered immune to diphtheria by active immunization. The Schick and immunization to diphtheria should be as compulsory as vaccination against small pox and the results would probably be equally efficacious.

If the case cannot be isolated in the home it should be removed to a hospital, if one is accessible. Treated at home one chooses a

Room. The choice, preparation and maintenance of the room in a case of diphtheria is the same as in a case of scarlet fever. (See Scarlet Fever, Chap. XVII.)

Nurse. Identical instructions should be given and precautions taken as in a case of scarlet fever. (See Scarlet Fever, Chap. XVII.)

It is to be remembered, however, that the great danger comes from the secretions of the throat and nose and every precaution must be taken in the treatment of the throat or nose to avoid receiving a cough or sneeze direct in the face. All the secretions should be received on cloths that may be burned.

The nurse may use mild sprays and gargles as a precautionary measure, but no astringents. It is to be remembered that the healthy mucous membrane is resistant to invasion by the bacillus, but when abraded, irritated or inflamed becomes the site of infection.

A wise measure on the part of the nurse is to submit to a Schick reaction and in case it proves positive to accept an immunizing dose of antitoxin, 1,000 units, repeated at an interval of three weeks.

A nurse liable to contact with diphtheria should receive active immunization if the Schick reaction indicates it.

The bacillus is not air borne and the antiseptic bath and shampoo when the nurse is likely to meet others outside the house is not so imperative as in the case of scarlet fever, but she should not see children on these occasions nor come into so close contact with others that she might convey bacilli from her own throat, by coughing, sneezing, close conversation or kissing.

Physician. Such precautions as the physician takes in visiting a scarlet fever case he takes in treating diphtheria. (See Scarlet Fever, Chap. XVII.)

$\mathrm{He}$, too, remembers that his danger lies in examining the throat and in the cough he provokes. He may use mild gargles or sprays, 
one-half strength Dobell's solution or saturated solution of boric acid; but if the patient has coughed in his face he should certainly have recourse to an immunizing dose of antitoxin, 1,000 units, if he has not already done so as a matter of precaution.

The physician who in his practice is likely to treat diphtheria should by a Schick reaction determine his susceptibility and in case of a positive reaction fortify himself against infection by active immunization.

With the knowledge of the organism, its origin and mode of transmission, he finds the antiseptic bath after the case less compelling than in scarlet fever, but is doubly cautious about his mouth and throat and the danger of conveying infection from them, as he may well do in his own family.

Precautions in the Sick Room and the temperature of the room are the same as those observed in scarlet fever. (See Scarlet Fever, Chap. XVII.)

\section{Bed. (See Chap. IX.)}

Patient. The patient must be made to go to bed, no matter how light the attack may appear to be, explanations being made to him, or to the parents; of the insidious effects of the disease on the cardiovascular apparatus and the kidney and the meaning of rest, such only as the bed can afford, to these structures.

Bath. A warm sponge bath of soap and water is given each day for cleansing purposes.

Nightgown. Such an one as can be easily opened for examination of the heart and lungs without effort to the patient.

To the parents or friends the physician must talk frankly of the dangers that any physical effort on the part of the patient entails in terms of cardiac failure. They must understand that this danger is greatest as convalescence approaches. It is the only way in which the insistency of the patient and especially the little patient can be met.

The rest, even in the mild cases, must be absolute; the patient remaining in recumbency, not allowed to turn himself without help, if the case has in any measure been severe and, indeed, in all cases the bed-pan must be insisted on.

During the height of the illness only one low pillow should be allowed and another not until the end of two weeks and not even then if he has been very ill or there are any indications of nerve involvements.

Diet. There are certain fundamental principles that determine the dietary in all acute febrile diseases. An occasional review of these principles is extremely helpful in doing justice to a field of therapy grossly neglected. (See Diet in Acute Febrile Diseases, Chap. II.)

Tissue destruction in acute infections is due to three causes: (1) 
pyrexia; (2) toxemia; (3) starvation. In diphtheria the first factor is feebly operative, for as a rule there is little fever and in many of the worst cases none at all; but the second factor, the operation of toxins is perhaps more potent than in any of the common infections, while the third factor is enhanced by the difficulty of swallowing in angina.

Moreover, it has been shown that tissue destruction continues well beyond the febrile period, as if a late autolysis had occurred in tissue attacked by the toxin earlier in the infection. This tissue destruction both early and late has been lessened or stayed by a sufficiency of diet and considering the important organs attacked by the toxins, heart, kidney, nerve tissue, enough food to meet the daily metabolic demands and furnish material for repair is in this disease especially urgent.

The details of such a dietary are to be found under Diet in scarlet fever (see Scarlet Fever, Chap. XVII), and needs no modification for diphtheria.

In diphtheria, however, both the angina and pharyngeal paralysis may make the feeding exceedingly difficult. If from pain, regurgitation through the nose and disinclination, the diminution of food intake threatens the strength, the food must be administered by gavage or by rectum. The stomach tube is the best means to effect this end. In the adult one may feed by the tube, milk 500 c.c., sugar 50 grams, and one egg three times a day, or one may fortify this food by adding milk sugar 1 to 2 ounces and cream 1 to 2 ounces or even more, at each feeding. In this way one may get in well over 2,000 calories.

In young infants the stomach tube is preferable, but over the age of three years more difficult than the use of a nasal tube.

Rectal feeding is not so satisfactory, but when the feeding by the mouth is difficult or impossible may answer in some measure for a brief period.

When swallowing is painful an insufficiency of water is ingested and a pint of water twice a day by the rectum or a Murphy drip may supply the needy tissues.

A nursing infant affected should be taken from the breast but fed the mother's milk obtained by the use of a pump or by expression and if this is insufficient, the milk of a wet nurse can be used to advantage. Mixed feeding may be cautiously undertaken, but it is a bad time to institute artificial feeding of any kind.

Water or diluted fruit juices should be given as freely as the patient wishes and, indeed, should be offered every hour or two, as apathy induces on the part of the patient neglect.

Mouth, Throat and Nose. The toilet of the mouth, throat and nose is important. It is rather prophylactic than curative. When 
properly carried out, it lessens the probability of mixed infections and extensions.

Whatever is done must be done with care and gentleness; for any trauma suffered by the invaded tissues, enhances the spread and severity of the diphtheritic process and invites the invasion by other organisms.

The purpose is cleanliness and nothing subserves this.purpose better than physiological salt solution, as spray, or irrigation. Dobell's solution in one-half or one-quarter strength may be used for the same purpose.

After taking food the mouth should be rinsed with one or the other of these solutions and the teeth, the spaces between the teeth and the dead spaces in the mouth, cleansed of particles of food by cotton on a tooth-pick as an applicator soaked in these solutions.

When there is sordes, the milder applications may well be preceded by half strength hydrogen peroxide. The softened coat on the tongue may be removed in part by scraping with the edge of a whalebone.

A dry mouth is relieved by equal parts of 2 per cent. boric acid solution and albolene (liquid petrolatum) to which a little lemon juice has been added; if there is fetor or a foul stomatitis the following prescription is useful:

$\mathrm{R}$

Phenol (watery solution 1 in 20)

Glycerin.........................

Boric Acid (saturated watery sol.)........... M.

Follow this with the milder applications.

For the nose use the same mild solutions with the applicator or spray, but avoid the douche. For dried secretions, use a little olive oil on an applicator to soften before using the saline or Dobell's.

The genitals are freed from secretions by the use of the same solutions.

With angina or nasal involvement the above procedures are modified or supplemented.

Bowels. Putrefactive processes in the large intestine, enhanced by constipation and possibly by other conditions attendant upon infection will only add to the burden the patient has to carry; hence, attention to the bowel early in the illness is of importance.

Calomel and salts may be given to the production of one or two loose movements.

Calomel in gr. $1 / 4(0.015 \mathrm{Gm}$.$) doses every 15$ minutes until 1 or $11 / 4$ grains $(0.060-0.075 \mathrm{Gm}$.) are taken work well in the child and may be followed in 2 or 3 hours by a half ounce of milk of magnesia.

In young children or adults, salts, Epsom or Rochelle, in doses of 
5ss. to i $(15-30 \mathrm{Gm}$.) in a half to three-quarter glass of water are efficacious or we may give $11 / 2$ to 2 grains $(0.10-0.120 \mathrm{Gm}$.) of calomel and follow in about 3 or 4 hours by the above dose of salts.

The bowels should be kept open either by the use of an enema or a mild saline water of the type of Hunyadi or Liq. Magnesii Citratis given every other day.

Serum Treatment. The success of modern medicine is the reward of an effort to discover Nature's method of combating disease. Nowhere has that success been more unqualified than in the field of serum therapy in its application to diphtheria.

The bacillus diphtheriæ is one of the relatively few organisms which secretes its poison to operate at a distance from the site of its growth and multiplication. This deleterious material is called a toxin. Many more pathogenic organisms exert their toxic effect upon the host by a material so closely associated with their substance that its liberation depends on the destruction or damage of their own structure. These toxins are called endo-toxins. We do not know the chemical nature or intimate structure of the toxins, but we know something of their effects and none are more remarkable than their effect to excite in the tissues of the animal invaded the production of substances that neutralize their toxic action, that is, antitoxins.

Invading the tissues of the upper air-passages, the diphtheria bacillus finds a suitable soil for multiplication. Here it secretes its toxin, which operating locally causes inflammation with destruction of tissue, which produces the characteristic membrane and passing into the circulation attacks various tissues, for some of which it has an especial affinity and upon which it exerts its toxic effects peculiarly; such are nervous tissue and those of the heart and kidney.

These toxic molecules are believed to consist of a nucleus with certain chemical groups, assumed to be akin to the side chains of a benzol ring. One of these side chains has an affinity for certain side chains in the molecules composing the tissues concerned and effect through these an attachment of the toxin to the cell. This side chain, seizing the cell, as it does, is called a haptophore group. The toxin, too, has another side chain, which thus brought into intimate contact with the cell exerts an injurious effect upon it. This is the toxophore, or poison bearing group. The damage done the cell by this toxophore group, if it does not kill it, stimulates it to reparative processes. The part repaired is the group damaged, that is, the haptophore group of the cell, seized by the haptophore group of the toxin. This process of repair is, however, in excess of actual need and the superfluity of haptophore groups of the cell are set free from it into the blood stream and, combining with the toxins not yet 
attached, divert them from the cells. This substance, that is, these haptophores of the cells, are called antitoxin.

When the antitoxin has been manufactured in sufficient abundance to neutralize successfully the toxin, the animal is said to have acquired an immunity and this process is one of active immunization, active because the tissues have been physiologically active in the production of their own mechanism of defense.

But if the blood of the animal, so cured, or the blood of an animal in whom the same process had been induced by introducing the toxin gradually, that is, giving him the disease by degrees and repeatedly inducing ever increasing degrees of immunity, is drawn off and this antitoxic substance be isolated, it can in turn be injected into another animal and neutralize toxins circulating in his blood or tissue fluids and render him immune; but as this latter animal's tissues have not been actively engaged in the manufacture of this antitoxin injected, he is said to have a passive immunity conferred on him.

Diphtheria antitoxin, then, is manufactured by the horse in the process of acquiring an active immunity to the diphtheria toxins injected into the tissues in increasing doses. This is withdrawn, submitted to certain processes for isolation and preservation and used in the treatment of diphtheria in man, on whom its injection bestows a passive immunity.

Methods of preparation have improved since first this agent was offered to therapy and appreciation of its close relation to certain globulins in the serum has made it possible, by isolating these, to avoid in some measure certain disagreeable results attendant on its usage and has at the same time procured a more concentrated product.

Some measure of its activity had of course to be sought, and the unit that has been established is in terms of its power to neutralize toxic doses in definite animals of definite weights.

The unit agreed upon is the amount of antitoxin which will just neutralize 100 minimal fatal doses of toxin for a 250-gram guinea-pig.

Antitoxin coming from reliable sources is fairly stable, if properly preserved. According to Park if kept cold and not exposed to light and air, it will not deteriorate more than 30 per cent. in a year. It loses some 10 per cent. in two months. Allowance may be made for this in dosage.

Dose. We cannot estimate the amount of toxin in a given case and so cannot use it as a measure of the quantity of antitoxin to be used. Our dose is established empirically largely.

The dose is not determined by age, except in very young children, under two years, when it is slightly less.

Park and Biggs think weight should have some consideration. 
No amount is known to be injurious in any measure. The limit of the dose is set by the needs of the case and by expense.

The dose is modified by two considerations:

1. The severity of the disease.

2. The day of the disease when first seen.

The tendency in this country is to be liberal in the dosage. It is a good rule when in doubt whether to give a larger or smaller dose to choose the larger.

When Seen Early. In mild cases, that is, in cases of simple congestion from which the bacillus has been recovered, or in those with a small patch confined to one tonsil, give 3,000 to 5,000 units.

This should be repeated in twelve hours if there is no sign of improvement. If better, one may wait for twelve hours longer, repeating the dose if improvement does not continue.

A More Severe Case, such as involves both tonsils, requires a larger dose 5,000 to 6,000 units and should be seen again in six hours. If spreading, repeat; if not, wait another six hours. If no signs of improving, give the second dose and repeat at six or twelve hour intervals until the improvement is satisfactory.

If the process has spread from the tonsils onto the pillars or pharynx give 8,000 units and repeat at six to twelve hour intervals as above.

If a pharyngeal case shows (1) decided toxic symptoms or (2), if there are any laryngeal manifestations, such as hoarse cry, stridor or laryngeal cough or (3), if in addition to pharyngeal involvement there is nasal involvement give 10,000 units.

In these cases and especially in the laryngeal, the case should be continuously watched, both to be prompt in repeating the dose and to intervene with the intubating tube, if needed.

The dose should be repeated every six hours until there is improvement and if the spread is increasing one may repeat in four hours.

In malignant cases, cases in which the spread is very rapid and the symptoms toxic 15,000 to 20,000 units should be given.

If the dose can be given intravenously the result will be best. If the vein cannot be entered, as is the case often in small children, go into the muscles of the buttock, as the wide net-work of veins in the muscle facilitates absorption.

These doses are repeated at four, six or twelve hour intervals and are sometimes carried to total amounts of 60,000 to 100,000 units. Apparently hopeless cases sometimes rally under this large dosage. Both McCollom of Boston and Weaver of Chicago are advocates of these large doses. 
In severe or laryngeal cases under two years the dose is 5,000 to 6,000 units, repeated at six, eight or twelve hour intervals.

Cases Seen Late. Every day of delay increases the dangers, both in terms of death and complications, heart, kidney or nerve involvements.

When antitoxin is administered on the first day the results are most brilliant, the mortality amounting to only a little over 1 per cent. and in some considerable series with no mortality.

But on the second day the dangers have increased and the statistics show about 4 per cent. to 5 per cent. and in some series a still higher.

The mortality of cases treated on the third day has doubled and tripled over the second, running from 9 per cent. to 13 per cent. and on the next day still higher, 15 per cent., 17 per cent., even 24 per cent. in some series.

This means larger doses with each day. Holt has said if the case has been three days ill he should have three times the ordinary dose.

The tendency, I think, is toward liberal dosage. Park has pointed out that the "larger the amount injected into the tissues the quicker will a considerable amount be absorbed into the blood and pass into the body fluids. Only a small percentage of what is in the blood passes out of the vessels into the tissue fluids. It is for this reason that, if we would neutralize toxin that has passed from the blood stream, but has not yet united with the tissue cells, very much more must be given than the amount of antitoxin that would be required in a test-tube to neutralize the toxin."

Moreover it has been shown that the toxin is not a simple body, but that a certain portion, called protoxoid has to be neutralized before the toxin is affected and neutralized and that then there is a portion, called toxon, which is believed to be responsible for the toxic changes in nerve tissue which does not combine with antitoxin until all the toxin molecules are satisfied. It is necessary, then, to give such large doses as may reasonably assure us that this portion, the toxon, shall be neutralized. It must be remembered that the body is at the same time manufacturing its own antitoxin which may have increased the richness of antitoxin content of the blood enormously.

The following is the dosage advised by the Board of Health of the City of New York and quoted from its circular sent with its diphtheria antitoxin: 
Amount of Antitoxin Required in the Treatment of a Case

\begin{tabular}{|c|c|c|c|c|}
\hline & Mild Cases & Moderate & * Severe & * Malignant \\
\hline $\begin{array}{l}\text { Infants, } 10 \text { to } 30 \mathrm{lbs} \text {. in weight } \\
\text { (under } 2 \text { years of age)........ }\end{array}$ & $\left\{\begin{array}{c}2,000 \text { units } \\
\text { to } \\
3,000 \text { units }\end{array}\right.$ & $\begin{array}{c}3,000 \text { units } \\
\text { to } \\
5,000 \text { units }\end{array}$ & $\begin{array}{c}5,000 \text { units } \\
\text { to } \\
10,000 \text { units }\end{array}$ & $\begin{array}{c}7,500 \text { units } \\
\text { to } \\
10,000 \text { units }\end{array}$ \\
\hline $\begin{array}{l}\text { Children, } 30 \text { to } 90 \mathrm{lbs} \text {. in weight } \\
\text { (under } 15 \text { years of age) } . . . .\end{array}$ & $\left\{\begin{array}{c}3,000 \text { units } \\
\text { to } \\
4,000 \text { units }\end{array}\right.$ & $\begin{array}{c}4,000 \text { units } \\
\text { to } \\
10,000 \text { units }\end{array}$ & $\begin{array}{c}10,000 \text { units } \\
\text { to } \\
15,000 \text { units }\end{array}$ & $\mid \begin{array}{c}10,000 \text { units } \\
\text { to } \\
20,000 \text { units }\end{array}$ \\
\hline Adults, $90 \mathrm{lbs}$. and over in weight & $\left\{\begin{array}{c}3,000 \text { units } \\
\text { to } \\
5,000 \text { units }\end{array}\right.$ & $\begin{array}{c}5,000 \text { units } \\
\text { to } \\
10,000 \text { units }\end{array}$ & $\begin{array}{c}10,000 \text { units } \\
\text { to } \\
20,000 \text { units }\end{array}$ & $\mid \begin{array}{c}20,000 \text { units } \\
\text { to } \\
40,000 \text { units }\end{array}$ \\
\hline
\end{tabular}

*When given intravenously one-half the amounts stated.

Cases of laryngeal diphtheria, moderate cases seen late at the time of the first injection, and cases of diphtheria occurring as a complication of the exanthemata should be classified and treated as "severe" cases.

It is recommended that the methods of administration be as follows:

Mild Cases-Subcutaneous or intramuscular.

Moderate Cases-Intramuscular or subcutaneous.

Severe Cases-Intramuscular or $1 / 2$ intravenous and $1 / 2$ intramuscular or subcutaneous.

Malignant Cases-Intravenous or intramuscular.

Some point on the surface of the body should be chosen for the injection, as where there is an abundance of subcutaneous cellular tissue. the abdomen or in trascapular region. Before the remedy is administered, the skin should be sterilized at the point of injection with tincture of iodine or other disinfectant. The syringe should be thoroughly sterilized, It is better not to employ massage over the point of injection.

\section{The Early Administration of Antitoxin}

The earlier the remedy is administered the more certain and rapid is the effect. In cases of any severity where diphtheria is suspected, and in cases of croup, it is far better to administer the remedy at once, making a culture at the same time, than to delay the treatment until a diagnosis has been made by bacteriologic examination. The first injection should be large enough to control the disease. One large dose given early is far more efficacious than the same amount in divided doses. Severe cases and those in which the administration of antitoxin has been delayed, or cases which are progressive because of an insufficient first dose, should be given a large intravenous injection whenever feasible. In this way the full value of antitoxin is obtained at once, whereas the absorption from 
the subcutaneous injection is so slow that many hours must elapse before any great amount of antitoxin has found its way into the general circulation. It must be warmed to the body temperature and given very gradually.

Technique. The operator's hands should be cleansed with soap and water and alcohol or 1 to 1000 bichloride.

The skin of the patient should be cleansed with soap and water and alcohol, or a good scrubbing with alcohol may answer, or the skin painted with the tincture of iodine.

Antitoxin is now put up by a number of reliable firms, sent in containers which are themselves the syringes, with sterile needles accompanying. This needle may be dropped into alcohol before using, as an extra precaution. If such conveniences are not at hand, a glass syringe or one that, like it, may be subjected to boiling should be used.

The needle should be boiled and attached by a short piece of sterile rubber tubing to the syringe. This is intercalated to prevent breaking or bending or wounding other structures if the child struggles.

A syringe from which the plunger may be withdrawn is preferable because the serum may more readily be poured into the barrel of the syringe than drawn up through the needle. When this is done the little air must be expelled through the needle before it is used.

Site. An excellent one is the loose tissue of the back at the angle of the scapula, into the loose tissue of the abdominal wall or into the huttock or in the nipple line between the nipple and costal margin. This last is a site upon which the patient rarely lies and if a local reaction occurs, local applications are readily made, and if it is desired to give it into the muscle in an urgent case the buttock serves well. I prefer the side of the buttock.

As has been said, in profoundly toxic cases an intravenous injection is to be preferred, if possible.

(For technique of intravenous injection see Pneumonia, Chap. IX.)

Evidences of Improvement. First, a pause in the spread of the membrane; then, in twelve hours to twenty-four hours the membrane softens, loosens, recedes, disintegrates. With this the swelling of the mucous membranes diminishes and there is an amelioration of the general condition, a betterment of the pulse, and a lowering of the temperature.

In laryngeal cases, the stridor lessens, there is an improvement in the cry or voice and the cough is looser. In nasal cases the breathing is less obstructed and the discharge lessened.

Disagreeable or dangerous results of antitoxin administration. Skin rashes are by far the most common, but far less frequent with 
the use of the refined and concentrated serum than with the old. An erythema may be seen in a few hours, but it soon disappears. After a period of days, seven to fourteen, a rash occurs, either erythematous, that may be mistaken for scarlet fever (the mucous membranes of the mouth and pharynx are not affected, a distinguishing feature from scarlet fever) and which may desquamate; morbiliform, mistaken for measles, or most commonly, urticarial, which may give much annoyance.

This eruption is often accompanied by other manifestations of intoxication; a little temperature, swelling of the glands and at times enough joint pains to suggest rheumatism and perhaps a little albuminuria. The whole process and the period intervening between it and the injection suggest a period of incubation of an infectious disease and is attributed to the effects of certain proteins in the serum and their effects on the tissues to the production of toxic substances. It occurs commonly after the first injection and is not to be confounded with that more sudden and explosive reaction that may follow an injection repeated ten days or longer after the first injection, the result of a sensitization of the tissues by the first serum introduced, constituting the phenomenon known as anaphylaxis.

The most important part of the treatment of this condition is an explanation of its meaning to the parents or patient who may be alarmed by its manifestations.

The pains may be allayed by fomentations applied to the joints or by the use of acetyl salicylic acid (aspirin). The urticaria is best treated by sponges of water containing sodium bicarbonate or bran. Often adrenalin (epinephrin) 1:1000 in doses of five to fifteen minims affords marked relief. It lasts but two or three days.

Sudden Deaths. Very rarely sudden deaths or a condition threatening death occurs. It is possible that some of these sudden deaths may be attributed to status lymphaticus; but more commonly they have occurred in people who are asthmatics and of these a considerable number are of that type of asthmatics whose attacks are excited by the presence of horses. These people seem to be sensitized to some protein of the horse given off in the secretions or discharges of the animal which are carried a considerable distance through the air.

Injection of antitoxin into these people introduces directly the substance to which they are sensitized and an anaphylactic seizure is the result.

I saw one attack in a physician, a victim of horse asthma, precipitated by an immunizing dose of antitoxin given by himself. The attack was characteristic of its kind and copied the reaction in the animal exactly. 
The reaction was immediate, before the needle was withdrawn. He was seized with most urgent dyspnœa, intense cyanosis and prostration. His lungs were filled to the utmost and he was incapable of expelling air from his chest. He was relieved only by having his chest encircled by the arms of his attendant and compressed until air was expelled that might be replaced.

So great was this distension that acute emphysema ensued with rupture of the air into the mediastinum and fascial planes of the neck. Atropine and morphine and adrenalin were liberally administered. Cupping also afforded some relief.

Asthmatics should receive the antitoxin with great precaution.

For the determination of sensitization and the method of disensitization see Pneumonia (Chap. IX).

In sensitized patients Park recommends a concentrated antitoxin, calling attention to the fact that antitoxin can now be procured with the value of 3000 units to 1 c.c. Give of this 0.2 c.c. If no bad results ensue give in an hour another 0.2 c.c. and in another hour 0.4 c.c. and repeat this amount at hourly intervals until the desired dose is attained.

Immunizing Dose. The immunizing dose touched upon should be 500 units in infants and 1000 in older children and adults.

The effect lasts but a short time. Park says that at the end of five days 90 per cent. has been eliminated and at the end of two weeks 99 per cent. Hence, in the presence of continued danger a second dose should be given at the end of a week and certainly at the end of two.

Park also advises the use of human serum, which can be obtained containing 50 units to 1 c.c. for immunizing asthmatics.

Laryngeal Diphtheria. The gravity of this condition and its frequency, for it is said to occur in 40 per cent. of children attacked under three years, make it imperative to appreciate its earliest manifestation.

While it is true that a catarrhal laryngitis may occur in pharyngeal diphtheria, such an assumption should not modify our procedure in the least.

When any laryngeal symptoms occur in the course of a pharyngeal diphtheria, it is to be treated as such and treated promptly. When, however, the laryngeal diphtheria is primary, the differentiation from catarrhal laryngitis and spasmodic croup is not so easy. If the child has been exposed, the assumption should be that the case is diphtheritic and one should not wait upon the return from the culture. When there has been no exposure, however, one has to obtain a culture and study the course of the disease.

A positive culture, of course, settles the matter, but a negative culture 
does not exclude it, as some 40 per cent. of the cases coming to operation and studied by McCollom were negative.

Spasmodic croup is usually at its worst at night and with the morning finds a decided amelioration or cessation of the spasm, but when the spasm continues into the succeeding day or dyspnœa comes on, that is, the lesion shows progression, the condition should be assumed to be diphtheritic, on the symptoms alone.

The symptoms are, in the beginning, hoarseness of the voice and cough, which takes on a metallic character. This is in the catarrhal or earliest stage. Soon true croup begins, characterized by spasm of the larynx with stridor and dyspnœa but with remissions. It is due to the same irritative causes as spasmodic croup. This is the second stage. Following this comes the evidences of continuously increasing obstruction with dyspnœa, cyanosis, restlessness, prostration, feeble pulse, a rising temperature and stupor. No such clear cut stages are to be anticipated, however, in each case.

The stridor is of a peculiar, hissing or sawing character which continues throughout inspiration and expiration.

The important symptoms are hoarseness, metallic or croupy cough, restlessness, prostration, increasing dyspncea taking on a sawing character.

McCollom adds one other symptom upon which he lays great diagnostic stress, namely, a rigidity of the sterno-cleido-mastoid muscles, which he says is always present and even early, both in adult and children.

Antitoxin should be given, as noted above, at the earliest moment and in large doses, 10,000 units and repeated in six hour intervals, if there is spread and in six or twelve hour intervals until decided improvement is seen.

Intravenous administration is always to be preferred.

In young children the condition may prove fatal in thirty-six to fortyeight hours. One sees, then, how urgent the early administration is.

Before antitoxin treatment 90 per cent. of these cases came to operation; since the introduction of the serum, 40 per cent.

Other Treatment. Besides antitoxin nothing has more than a palliative effect and one should not allow any measure undertaken to delay the administration of antitoxin an instant.

Steam. Inhalation of steam seems to relieve the spasm to some extent, though of course not the obstruction.

The best way to administer it is with the croup kettle or two or three croup kettles directing their steam as close to the child's head as is safe. A tent may be made by putting a sheet over the upper third of the bed, so 
that the body will not be bathed in the steam or one may lash an open umbrella to the head of the bed and pass a sheet over this or put a clothes-horse around the head of the bed and drape a sheet over this. This should not be kept up for long periods, as the heat is depressing.

The steam lessens the spasms and is said to facilitate the discharge of secretions.

Hot fomentations to the throat have been advised and some authors say relief is sometimes afforded by the ice-bag applied to the neck.

\section{INTUBATION}

\section{By Miner C. Hill, M. D.}

Procedure. 1. Select intubation tube of suitable size for age of child, according to scale, and be sure that braided silk thread is attached.

2. When tube is on the obturator the broad flange of the head should point away from the instrument. The silk thread is then to the operator's right.

3. Assistant wraps child in a mummy sheet and places some padding under the neck or holds the patient's head over edge of table. Mouth gag is inserted in left side of mouth.

4. Operator takes his position on right side of patient, inserts left index finger along tongue, parallel with median line, until tip of finger locates epiglottis pulling it forward.

5. Handle of intubator is held in right hand close to the patient's chest with one finger through loop of thread and thumb upon the releasing knob.

6. The tube is then advanced along midline of tongue parallel to left index finger until epiglottis is reached.

7. As the tube engages the larynx the handle of intubator is raised and the tube is quickly, but without force, inserted into the larynx. Any undue force is liable to cause a false passage.

8. Tip of left index finger holds tube in place while releasing knob is pushed forward and obturator is withdrawn.

9. When tube is felt securely in place tip of left index finger is again placed upon head of tube while loop of silk is cut and withdrawn.

10. A tracheotomy set should always be in readiness in case intubation is unsuccessful.

A successful intubation is made evident by a characteristic expiratory cough and inspiratory whistling sound and the patient's attitude changes from one of horrible restlessness and fighting for air to one of peaceful quiet and a desire to sleep. 
The patient should be watched intently after intubation.

Occasionally the tube is coughed up and with it a membranous cast of the larynx and reintubation may not again be necessary.

If the tube is coughed up and dyspnœa returns, intubation must be repeated using the same tube and not one of next larger size, for fear of causing pressure necrosis of the cricoid cartilage.

Indications for Intubation. Laryngeal diphtheria is diagnosed by croupy cough, stridor, interference with voice production, increasing dyspnœa and cyanosis. No signs of pharyngeal or nasal involvement need be present. When the dyspnœa has so far progressed that there is retraction of the episternal notch and supra clavicular regions and a pulse that disappears with each inspiration, intubation is indicated. If one wait until there is cyanosis the exhaustion will be so great that the chances of successful intubation are greatly diminished.

Post-intubation Treatment. Our first apprehension after intubation is the danger of expulsion of the tube during a fit of coughing and constant watching is necessary. If the cough is troublesome, the croup kettle and the use of antipyrin or small doses of codeine may give relief.

The next difficulty is the feeding of these cases. At first it is often difficult for the patient to swallow, particularly liquids. Milk toast, thick cereals, custards and ice cream are preferable to liquid food. To facilitate swallowing the patient may be fed while reclining with the head lower than the body (Casselberry Method). When even this method fails, gavage or rectal feeding may be employed.

\section{EXTUBATION}

When the temperature subsides, usually about the fourth day, it is safe to see if the child can do without the tube. The sooner the tube can be removed the less danger there is of pressure injury to the larynx.

Procedure. 1. Have near at hand an introducing instrument with a tube of the same size as the one to be removed.

2. Wrap child in a mummy sheet to prevent struggling, and insert mouth gag.

3. Place tip of left index finger upon head of tube and left thumb against cricoid cartilage externally.

4. Introduce extractor, parallel to median line of tongue, guided by left index finger until one is sure that tip is within lumen of tube.

5. Raise handle until it touches the upper incisor teeth, then the jaw of the extractor will pass well within the lumen of the tube. Now press the lever on handle and, reversing the motions used in intubation, remove the tube. 
The secret of successful intubation or extubation is in keeping the instruments, throughout the whole manipulation, parallel with the median line of the tongue.

Auto-extubation. May occur early or late in the disease. When it occurs early, on third or fourth day, the patient has usually sufficiently recovered to be able to go without the tube. When it occurs later, from tenth day to third or fourth week, it is usually persistent and tube may be coughed out as many as thirty times in twenty-four hours. This persistent coughing up of the tube is due to a perichondritis at the cricoid level and therefore the tube can no longer be held in place by the retention swell for the firm cartilaginous larynx at the cricoid level is converted into a collapsible tube. This condition occurs in from 3 to 5 per cent. of intubation cases. The treatment of these cases is by a bulbous tracheal tube which is longer than the O'Dwyer tube and reaches well down into the trachea. These tubes will practically put a stop to auto-extubation.

Tracheotomy. For this procedure one should have recourse to surgical text-books.

\section{DIFFERENTIAL DIAGNOSIS}

Spasmodic Croup. Sudden onset and sudden subsidence. No aphonia. No progressive dyspnœa but a succession of attacks during the acute stage with marked improvement during the intervals.

Retropharyngeal Abscess. Absence of aphonia but there is a muffled voice with a throaty stridor. Dyspnœa is greater when mouth is open. Head is held thrown back. Digital examination reveals a fluctuating mass on posterior wall of pharynx.

Foreign Bodies. Onset is very sudden, there is a paroxysmal cough and violent dyspnœa. If the foreign body is not coughed out but becomes impacted death may rapidly follow from occlusion of the glottis.

Bronchopneumonia. When the pneumonia is bilaterial in children, there may be marked retreaction of the chest and sinking in of the epigastrium. There is absence of a croupy cough and the different character of the dyspnœa together with the physical signs in the chest clears the diagnosis.

Subglottic Edema. May give rise to the same symptoms as laryngeal diphtheria and an examination of the larynx is necessary to differentiate.

Phlegmon of Glottis. No croupy cough or aphonia. Examination shows marked inflammatory swelling of epiglottis and arytenoid region. 
Nervous Diseases. In certain acute infections as poliomyelitis, superior basilar meningitis, encephalitis and cerebellar abscesses there may be laryngeal symptoms due to irritation of the basal ganglia. Dr. H. L. Lynah has reported two interesting cases, one, a case of cerebellar abscess where the respiration simulated that of laryngeal obstruction. Evacuation of the abscess relieved the respiratory condition. Another, diagnosed as laryngeal diphtheria, proved at autopsy to be an encephalitis with an enormous increase of fluid in the ventricular spaces.

Recurrent Laryngeal Nerve Paralysis due to mediastinal tumors simulates laryngeal diphtheria but is differentiated by laryngoscopic examination. In this condition the obstruction is below the larynx and there is no loss of laryngeal function. The expiratory dyspnca is greater than the inspiratory and percussion, X-ray and course will clear the diagnosis.

\section{TREATMENT OF SYMPTOMS}

Fever. A high temperature is not characteristic of the disease; on the contrary, the temperature is relatively low. Treatment for it, as such, is not indicated and antipyretics are absolutely contraindicated on account of their depressant effect on the circulation. Sponge baths, luke warm or cool so far as they increase the patient's comfort may be given.

Angina. In many cases there may be very little discomfort in the throat, again the throat and mouth may be extensively involved, with much fetor, while with the invasion of other organisms, the inflammatory reaction and discomfort may be much increased.

External Applications. Cold or heat applied to the neck, preferably the former, afford relief. Cold may be so applied in the shape of the ice-bag, the coil or compresses.

Heat. If heat is found the more grateful, it may be afforded by the use of hot fomentations.

Applications to the Throat. It must be remembered that no applications that can be made will kill the organisms concerned and the best we can do is to make such applications as preserve the maximum amount of cleanliness of the part and so lessen the likelihood of spreading, of inviting the invasion of other bacteria or of permitting decomposition and putrefaction.

We must remember, however, that these very efforts, unless judiciously carried out may do damage to the invaded tissues and hasten the very processes they were designed to prevent.

Applications are made by the gargle, spray, irrigation or swab. A gargle is painful and does not reach the parts most affected and serves 
for little better than a mouth wash. The spray is a better method; but by all odds the best is the irrigation.

The best irrigating fluids are those blandest to the inflamed mucous membranes, such as sodium chloride in physiological solution (one dram to the pint) or 2 per cent. boric acid solution or Dobell's solution diluted two to four times. It should be as hot as the patient can comfortably stand it, $100^{\circ} \mathrm{F}$. in a child, $110^{\circ} \mathrm{F}$. to $115^{\circ} \mathrm{F}$. in an adult.

A fountain syringe or irrigator is held above the head at a height sufficient to give free movement to the stream, but not force; a nozzle of hard rubber or metal, or in older children a catheter may be úsed.

The patient, unless the case is of the mildest, should not sit up in bed, lest cardiac failure should occur, but lying on the side at the edge of the bed, with a towel about the neck and a pus-basin or other receptacle under the chin to catch the fluid. In older patients the tongue may be depressed by a wooden or glass depressor or the nozzle may be inserted between the molars and the stream directed in various directions.

One should use from a pint to a quart or more. If the mouth is foul they may be given every two or three hours, in less severe cases three times a day:

If there is a great deal of mucus, the throat may receive a preliminary spraying with a solution of sodium bicarbonate $\mathrm{gr} . \mathrm{xx}$ to the ounce $(1.30 \mathrm{Gm} .-30$ c.c.), the membranes gently touched with half strength solution of hydrogen dioxide and then the irrigations be given. Sprays of the same substances as the irrigations may be given as often as they afford comfort. No measures that cause pain should be persisted in.

Many authors are impressed with the cleanly appearance afforded by applications of Löffler's solution. (For formula see Scarlet Fever, Chap. XVII.) It should be applied with a swab on an applicator and held pressed against the membrane not swabbed around. This may be made two or three times a day. Cracked ice held in the mouth gives much relief at times.

The pain of swallowing when great may be alleviated by the application of 1 per cent. to 2 per cent. cocaine.

In very foul mouths 1 to 2000 permanganate of potash may be used as an irrigation before using the saline.

Nose. Most authorities are in agreement that in nasal diphtheria; irrigations are indicated. McCollom, however, deprecates the procedure, because he is convinced that the number of middle ear cases increase under it.

It would seem, however, that in the great danger attending these cases, with involvement of the rapidly absorbing naso-pharynx that 
some means of cleansing the area involved were necessary and that irrigation was the only efficacious means.

The same solutions may be used as in the throat, namely, warm physiological salt solution, 2 per cent. boric acid solution or Dobell's diluted one to four times.

A fountain syringe, held at such a height as to just force the solution through in a gentle stream and a glass nozzle or a small rubber catheter, with holes cut in the side are used. It should be done as often as every two hours in severe cases with profuse excoriating discharges, less often in the milder cases.

If a fountain syringe is not at hand or difficulty is experienced in its use, a piston syringe may be substituted.

The patient should keep the mouth open during the procedure and the irrigation should be directed first to the side most obstructed, so that a damming up behind the obstruction will not force the fluid back into the Eustachian tube.

If irrigation is objected to, applications gently made with a swab and sprays must take its place. Calomel may be insufflated.

Where there is much hemorrhage from the nose, adrenalin in dilution of 1 to 2000 to 3000 may be used; in some cases dry packing of the nares is necessary or if that fails the gauze used for packing may be saturated with adrenalin, 1. to 3000 .

Thromboplastin and Kephalin contain thromboplastin from brain tissue which is a coagulant. They may be applied locally to the bleeding point on cotton or gauze and left in situ for 5 to 10 minutes. Coagulen is a preparation from blood platelets put up as dry powder. A 10 per cent. solution of this is applied to the bleeding vessel on cotton or gauze. Care must be used in removing the application lest the blood clot formed be loosened. This may be eliminated by not allowing the cotton to dry and leaving some of the solution in situ.

Hemostatic serum (hemoplastin) may be applied on gauze or cotton packing. If it does not stop the bleeding after 5 to 10 minutes, $1 / 2$ to 2 c.c. of the clear solution may be injected locally at the site of the hemorrhage.

Cardio-Vascular Apparatus. The prophylactic treatment of circulatory failure lies in the promptness and thoroughness of the serum treatment.

No complication in diphtheria, excepting laryngeal involvement and its sequelæ, is so grave as that which includes the circulatory apparatus.

One may distinguish an early and a late form of circulatory disturbance. The early form manifests the same evidences of circulatory 
inefficiency as one sees in any other acute infection and is in the main due to the same cause, namely, a vaso-motor failure, referable particularly to the vaso-motor center. However, it must be said that such experimental evidence as we have points to a greater implication of the cardiac muscle in diphtheria than in the case of the other acute infections; but still, it is the vaso-motor center that has to be chiefly considered in this disease too.

What has been said about the cardio-vascular apparatus in discussing scarlet fever (see Chap. XVII), is applicable in all details both with reference to its etiology, its symptomatology and its treatment here, and the reader may consider that discussion as referred to this subject.

These circulatory disturbances may come on at any time in the first two weeks, depending on the severity of the infection, but at the end of the second week up to the fourth week, seldom later there occurs a

Late circulatory failure, which has been attributed to degenerative changes in the heart, especially its nervous mechanism and has been spoken of as pneumogastric paralysis.

It comes in convalescence, at a time when other nerve degenerations are made manifest.

It is a most serious condition, often abrupt, usually first appreciated by an attack of vomiting. This becomes repeated, is accompanied by precordial distress, epigastric pain and tenderness, dyspnœa, great restlessness, sometimes a clear mind, sumetimes marked cerebral disturbance, syncopal attacks, pallor, sweating, very feeble pulse, strikingly slow, though sometimes rapid, and death.

Sometimes the first premonition is given by a sudden change of the pulse rate to a considerably higher or slower rate.

Occasionally one encounters such functional disturbances as auricular fibrillation and heart block. The latter may be attributable to degenerative changes in the bundle of His though this is not always demonstrated at autopsy.

One of the most ominous facts about the heart of diphtheria is the tendency to sudden syncope and death and coming out of a clear sky, without a particle of warning in many cases, even when assiduously watched for. Nearly every writer of note frankly admits his inability to give a reasonable explanation for this appalling accident. There may be lesser manifestations with very slow and irregular heart as the major evidence of disturbance and recovery take place, but one is never sure of the result and faces the possibility of a turn for the worse or a sudden issue.

The early and characteristic vomiting, the cerebral disturbance, the vaso-motor implication, the association with other paralyses and its 
time of onset has suggested the sudden death as a manifestation of cerebral degeneration rather than essentially cardiac. But that there is a toxic effect wrought on the myocordium not evidenced by histological findings or by the character of the histological changes found is shown by the sudden failure of the organ when neither sign nor symptoms are evidenced and the child has begun to resume normal activities.

Diphtheria is another disease in which toxic arteriosclerosis occurs as is shown by slight increase in blood pressure and increase in second aortic sound and thickened palpable arteries. This is likely to disappear after a few weeks. Furthermore aortitis occurring after diphtheria has been often noted. It is said to be a necrosis confined to the media.

The treatment begins with efficient and prompt serum administration at the earliest moment of diagnosis; it continues by great care to avoid even slight physical exertion during and well into convalescence in all cases, even the mild, but especially those that have been very toxic and when there have followed nerve paralyses, or there is evidence of kidney involvement. Therefore daily examinations should lay stress on the deviation from the normal heart rate, high or low, muffling or splitting of heart sounds, irregularity of the pulse and least of all murmurs; upon subjective sensations such as dypsnœa, precordial distress, gastric nausea or vomiting, syncope or even pallor. It must be further emphasized that the later the minor cardiac disturbances such as changes in rate, rhythm or sound occur, the more serious is the cardiac complication. It should be further emphasized that the subjective disturbances noted above are even more ominous than the objective.

The condition is most dire and sudden death threatens with the least exertion, such, for example, as is induced by vomiting or sitting up in bed, or mental excitement.

For this reason absolute rest is imperative and nothing will do so much to secure and relieve the distress and pain and anxiety as morphine sulphate, hypodermically, every four hours if needed, in such doses as to effect the purpose; e. g., gr. $1 / 48(0.0015 \mathrm{Gm}$.) in the child of two years, gr. 1/24 (0.002 Gm.) to gr. 1/16 (0.004 Gm.) at six and gr. 1/12 (0.005 $\mathrm{Gm}$.$) or gr. 1 / 8(0.008 \mathrm{Gm}$.) at twelve years.

With late cardiac manifestations food must be cut down in quantity especially at any one feeding; less food given at more frequent intervals. This applies when no gastric symptoms have occurred. On the appearance of gastric disturbance, food by the mouth must be stopped as the vomiting makes its retention impossible and is perhaps aggravated by it. Such nourishment as is given must be by the rectum. Procedures of all kinds, about the mouth, nose, etc., must be decreased to a minimum. 
As nutrient enemata one may use the following:

\section{Nutrient Enemata.}

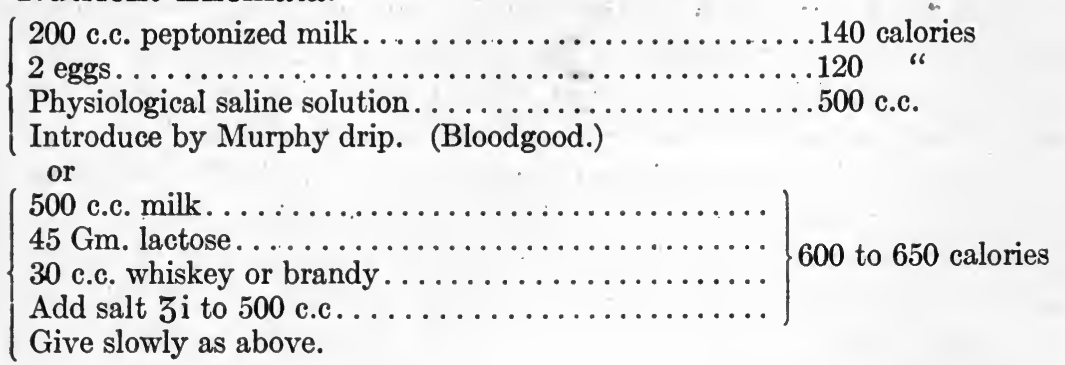

Heat over the precordium may allay pain and distress to some degree. There is no unanimity of opinion about drug administration in this condition.

I believe, however, that those drugs that we use in circulatory failure earlier in the disease and in other acute infectious diseases should be used here; caffeine, camphor, strychnine, which affect the medullary centers, adrenalin (epinephrin) and digitalis. If we accept the theory of pneumogastric degeneration in this condition and fear the effect of digitalis on this structure, we may administer atropine with the digitalis, to eliminate the vagus influence, giving gr. $1 / 100(0.0006 \mathrm{Gm}$.) of the sulphate with each dose of the digitalis.

Of the vaso-motor stimulants I have said my favorites are caffeine and camphor. The rationale of caffeine is discussed under scarlet fever. (See Chap. XVII.) It may be used hypodermically as the double salt of sodium salicylate or sodium benzoate and caffeine. For a child of 5 years the dose is $1 / 2$ to 1 grain $(0.030-0.060 \mathrm{Gm}$.); at 11 years $1-2$ grains $(0.060-0.120 \mathrm{Gm}$.); adult 5 grains $(0.33 \mathrm{Gm}$.).

Camphor, while it has little obvious effect on either a normal heart or vaso-motor center, has been demonstrated experimentally to produce a stimulating effect on both when they have been depressed by poisons. Upon the heart it seems to have a marked effect on the function of irritability. Its mode of administration in circulatory failure and its results are found under scarlet fever. (See Chap. XVII.) It is best given in olive oil or sesame oil hypodermically. The dose at 5 years is $1 / 2$ to 1 grain $(0.030-0.060 \mathrm{Gm}$.); at 11 years $1-2$ grains (0.060-0.120 Gm.); adults 3 grains $(0.2 \mathrm{Gm}$.).

Strychnine: The dose of the sulphate is at 5 years gr. $1 / 200-1 / 100$ (0.0003-0.0006 Gm.); at 11 years gr. $1 / 100-1 / 80(0.0006-0.00075 \mathrm{Gm}$.$) ;$ adults gr. $1 / 60-1 / 30$ (0.001-0.002 Gm.).

An enteroclysis of hot salt solution $3 \mathrm{i}$ to $0 \mathrm{i}(4 \mathrm{Gm}$. to 500 c.c.) may be 
of great value in collapse or on the occasion of syncopal attacks. It should be given with a rectal tube, to which is attached a $\mathrm{Y}$ tube, one branch of the $\mathrm{Y}$ being attached to a rubber tube to carry off the water, while the other is attached to the syringe. By pinching the discharge tube the amount in the bowel and its discharge can be regulated. This apparatus can be utilized with little disturbance to the patient, a matter of the greatest importance during the attack.

The hot saline raises the body heat which is subnormal as a rule and by direct contact with the mucous membrane over a considerable area affords a powerful stimulant to the peripheral vaso-motor supply of the splanchnics; thus raising blood pressure effectually. A cup of hot coffee may well be added to the saline. The temperature of the fluid should be $110^{\circ} \mathrm{F}$. to $115^{\circ} \mathrm{F}$.

A saline injected into the tissues, at a temperature of $100^{\circ} \mathrm{F}$., that is, a hypodermoclysis, is another excellent stimulant.

Cold Air. The splendid effect of cold air upon the circulatory system obtains in this disease as well as in other acute infections. (See Open Air Treatment of Pneumonia, Chap. IX.)

It must be mentioned that most of the authors advise the use of alcohol as a stimulant, 5 ss. to $5 \mathrm{i}$ a day in divided doses in children and more in adults as a cardiac stimulant and as having some beneficial effect on the toxemia.

I am opposed to this teaching, both because it seems irrational to add day after day to the nerve tissues undergoing the degenerative effects of the toxin a substance which is itself capable of inducing a similar process and because I do not believe it is a true stimulant.

That it has some food value cannot be denied, but that it has advantages over the other class of foodstuffs in this condition is more than questionable.

Paralyses. Upon the nerve structures the poison of this disease has a selective action. Nearly all the nerves are susceptible to its action, but some more than others.

The results are rather partial disability, paresis, than total paralysis and the continuance of this disability is brief. The importance of the lesion lies in the vital significance of the nerve structure affected, such as the medullary centers or the vagus nerve.

The preventive treatment depends on the promptness with which antitoxin is administered, for once the toxin is in combination with the nerve tissue there is no known way of ousting it and antitoxin is believed to be ineffectual in achieving such a desideratum.

It depends, too, on the sufficiency of the early doses, for it seems as if the neurotoxic substance was that portion of the toxin designated 
the toxon, which has a less avidity for the antitoxin than the rest of the toxin particle and hence is not neutralized until the rest is satisfied.

Another preventive measure, prevontive rather of the disaster than of actual nerve involvement, is to assume that the nerve may be affected and insist on absolute rest and recumbency in all but the very mildest cases and even in these to realize that risk is not eliminated.

The effects of the toxin on the nerve structures are not evident until it has time to produce a certain degree of degeneration, so that the clinical evidences of paralysis are not as a rule manifest until the end of two weeks.

The likelihood of its occurrence is increased in proportion to the severity of the disease. The earliest evidences are seen in the palate and the heart; hence, the insistency on rest and recumbency when the patient is seemingly entered upon convalescence. The plain facts of the dangers and risks in a case at this time must be told the parents or the patient if an adult or older child.

It is more common in young children, but in pure laryngeal cases is relatively rare.

The incidence of the complications is set at figures that range from 11 per cent. to 40 per cent.

Heart involvement has just been discussed.

Soft Palate. This is the most frequent of the paralyses. It is important, too, because of the difficulty of feeding in decided cases, as the food will regurgitate through the nose.

This entails feeding by gavage, through the stomach tube, which may be more readily accomplished in the adult or through the nose in a child. This manner of feeding will not be continued long, because of the brief period of paresis.

Pharynx. When the pharyngeal nerves are affected, swallowing is rendered difficult and choking may ensue. Here again, the feeding by the tube is indicated.

Respiratory Muscles. Either set, intercostals or diaphragm or both, may be involved. Everything to lessen respiratory effort, such as weight of clothes or doubled up position should be avoided. Not infrequently, affection of the pharyngeal muscles and larynx occur simultaneously. Secretions collect in the bronchi or food particles gain entrance and quantities of secretions gather in the pharynx. The cough is ineffectual to expel these secretions and foreign body pneumonia threatens. The foot of the bed should be raised a trifle, but not enough for the pressure of the abdominal viscera on the diaphragm to further embarrass respiration. The secretions from the pharynx may be as- 
pirated off with a small rubber tube and a syringe and the swab of cotton on a dressing forceps or other form of applicator may be used.

Atropine sulphate gr. 1/100 (0.0006 Gm.) three or four times a day may lessen the secretions. Occasional compression of the chest may help to expel the bronchial contents. The other forms of paralysis, such as the oculo-motor, the facial and that of the extremities (the upper are very rare) need no especial treatment, considering the natural progress of events.

The only drug that it seems rational to administer is strychnine, in doses of gr. $1 / 400(0.00015 \mathrm{Gm}$.$) to gr. 1 / 150(0.00045 \mathrm{Gm}$.$) at two$ years, and gr. 1/100 (0.0006 Gm.) to gr. 1/40 (0.0015 Gm.) at twelve years three or four times a day.

Nephritis. There seems to be a considerable difference of opinion about the frequency of nephritis in diphtheria and its clinical importance.

There is, as in every acute infection, a trace of albumin that is referable to the acute degeneration of the epithelium of the tubules and tufts; but there may also be an acute diffuse nephritis, with no constancy of lesion, that is the glomerular changes may predominate in one and the interstitial in another. There seems, however, to be a good deal of tubular involvement as a rule with an abundant albuminuria. Dropsy is rare and uræmia not common. In severe cases anuria may prove fatal. Serious kidney complications come on earlier in diphtheria than in scarlet fever, are less serious both during the disease and later. The urine should be examined daily.

The treatment again begins with early and effective serum therapy. When developed, however, it is to be treated like the nephritis of scarlet fever (see Scarlet Fever, Chap. XVII), but hot packs are less well borne as, indeed, are any measures that entail much manipulation with consequent strain upon the heart.

\section{OTHER COMPLICATIONS}

Bronchopneumonia. This complication is to be particularlydreaded in the laryngeal cases which come to operation. It is to be treated like any bronchopneumonia. (See Pneumonia and Streptococcus Pneumonia, Chaps. IX and X.)

Adenitis may occur in severe angina and in mixed infections. It is treated with the application of ice and incision as soon as fluctuation is detected.

Otitis is said to occur in 4 per cent. of the cases. It may be followed by mastoiditis, sinus involvement or cerebral invasion. (For treatment, see Otitis in Scarlet Fever, Chap. XVII.) 
Convalescence. In no other disease must the convalescence be handled with more care and discretion.

The dangers of cardiac failure, even when there have been no clinical evidences of the involvement of that organ and even in mild cases make it imperative to explain the situation fully to the patient or in the case of children to the parents.

If the case has been mild the patient may be propped up in bed at the end of the third week and sit up in bed at the end of the fourth and a little later be put in a chair and then allowed to walk in a few days. All this time, the effect on the circulation must be watched.

Some authors make it a rule in mild cases to allow the patient out of bed a week after the throat has cleared up, especially applying the rule to adults or those in whom the tonsil alone has been mildly affected.

All, however, are in agreement that in severe cases the patient should remain in bed six or eight weeks and in no case be allowed up as long as there are signs of heart involvement.

When the patient is up good food and good air are of prime importance. For the anemia that in some degree is sure to have followed iron may be used. Blaud's pill, made fresh, or the Vallet's mass in doses of gr. iii to gr. v (0.20-0.30 Gm.) three times a.day.

Strychnine Sulphate in doses of gr. $1 / 200(0.0003 \mathrm{Gm}$.) to a child of five years and gr. $1 / 40$ to gr. $1 / 30(0.0015-0.002 \mathrm{Gm}$.) to an adult three times a day.

Quinine in doses of gr. $1(0.060 \mathrm{Gm}$.) three times a day have been credited with tonic properties. It can be combined with the strychnine.

In children Cod Liver Oil, in doses of $1 / 2$ to 1 dram (2-4 c.c.) three times a day is given for the same purpose.

Release from Quarantine. A patient should not be released from quarantine until two successive cultures taken from the sites of the lesion are negative. Three are safer at three-day intervals and an effort should be made to get material from the crypts of the tonsils by gentle expression of their contents with a spatula. Cultures should also be taken from the naso-pharynx and nose. In the larger number this occurs in a week after the throat is cleared from membrane, but it may be three, four, five or more weeks before the cultures are returned negative.

When the bacteria are persistent an effort may be made to rid the throat or nose of them by a spray, irrigation or application of an antiseptic. Holt advises bichloride 1 to 10,000 mixed with glycerin one part in eight. This is rarely successful. See Carriers.

If the organisms are not to be gotten rid of by such means, the cultures should be tested for their virulency. 
On release from quarantine the patient should be given a bath with warm water and soap and a shampoo with the same. This should be followed by a bath of 1 to 5000 bichloride of mercury.

Sterilization and Fumigation. For discussion of terminal fumigation, see Scarlet Fever, Chap. XVII. The disinfection is to be carried out as in a case after scarlet fever: the washable clothing and linen should be boiled for an hour after soaking over night in carbolic 1 to 50 to 1 to 20 . Mattresses, blankets, pillows and unwashable clothes may be subjected to steam under pressure. If not obtainable, submitted to formaldehyde gas.

Articles of no value and all toys should be burned or destroyed.

Articles made of metal or china ware may be immersed in 1 to 20 carbolic acid.

All the woodwork, the floor, the ceiling and walls should be rubbed down with cloths wet in 1 to 2000 bichloride of mercury.

The room should then be fumigated with formaldehyde gas. The room must be sealed with strips of paper pasted over cracks and holes and the gas generated from one of the numerous devices on the market, such as paraldehyde candles and left closed twelve to twenty-four hours.

McCollom recommends the method of the Maine State Board of Health. This consists in putting potassium permanganate (commercial) in a pan or other receptacle and pouring over it formalin (40 per cent.). It is used in the proportions of $61 / 2$ ounces of the permanganate to 1 . pint of formalin. This quantity will fumigate 500 cubic feet of space.

The room should then be repainted, repapered and recalcimined.

In case of death, the funeral should be private, the body wrapped in strong antiseptics and the coffin sealed.

Carriers. The detection, disposal and treatment of carriers constitute the most serious problem in combating the disease. It is the carriers that perpetuate the disease and they constitute such a considerable number of the community, often entirely unsuspected either by themselves or others, that their elimination can be achieved only by stamping out the disease through rendering a community immune.

The examination of the upper air passages of large numbers of the civil population lead us to the conclusion that about 1 per cent. are carriers; so far as it can be determined one-half of these have had no contact with cases of diphtheria.

Happily, not all these carriers harbor virulent organisms. The percentage of virulent organisms depends on previous contact with the disease.

Wadsworth found that carriers who had had the disease yielded 90 per cent. of virulent organisms from the day of onset up to one year; 
that healthy contacts, that is, individuals who had been in touch with the disease during an epidemic but had not themselves had it, showed 80 per cent. of virulent bacilli, while among healthy non-contact carriers only 10 per cent. of the organisms were virulent.

Studies of doctors and nurses in contact with diphtheria demonstrate a considerable number of carriers.

A few important points to remember about carriers are as follows; A single negative culture has been shown over and over again to be fallacious. The surface of the tonsils and naso-pharynx may be clear when the deep crypts of the tonsils are still infected.

Nasal cultures should be taken in each instance as well as throat cultures. In one large series of carriers over 25 per cent. of nasal cultures were positive.

Chronic carriers are such by virtue of some pathologic condition of the upper air passage or accessory sinuses. In the great majority of cases the bacilli are to be found in the tonsils, often in both tonsils and nose; occasionally in nose alone; next is the naso-pharynx.

Cultures from chronic carriers should be tested for virulency. This is done by injecting a guinea-pig intracutaneously with cultures of the organism. Virulent bacilli at the end of 24 to 48 hours give rise to an area of redness and induration followed later by necrosis.

Cats and dogs may be carriers and for that reason, if for no other, should be excluded from the sick-room.

Treatment. Operative and non-operative.

Non-operative treatment has universally failed. Sprays of all kinds, argyrol, Dobell's solution, Dichloramin T, Dakin's solution, crystal violet, antitoxin in water have all proved equally ineffectual.

Operative-Correcting the condition that affords a pathologic basis, is the only measure that promises success. All these cases should be submitted to examination by a competent nose and throat man. Diseased tonsils should be enucleated and adenoids removed, septum deformities, erosions or other abnormalities receive attention, and accessory sinus disease be corrected.

Patients with atrophic rhinitis and other chronic inflammation of the nasal passages prove the most persistent carriers.

It is interesting that all chronic carriers seem to give a negative Schick.

Diphtheria cases should show 3 negative cultures at 3 -day periods before discharged.

Prophylaxis. The subject of prophylaxis has been covered on the sections on Schick reaction, active immunization and carriers. 


\section{SUMMARY}

In the case of children examine the throat as a routine in all conditions.

\section{Taking of cultures.}

In all inflammations of the throat in a child, whatsoever be the clinical diagnosis and howsoever mild, take a culture for KlebsLöffler bacilli.

Persistent and especially bloody and excoriating nasal discharges in children demand a culture.

Laryngitis, especially of a croupy character and more particularly croup lasting into the day makes a culture necessary.

For technique of taking culture, see text.

\section{Disposition of the family.}

Other children in family kept from school.

Adults in family kept from contact with children.

Health authorities should be notified.

Authorities should inspect the school or suspected groups.

Children should be isolated and cultures taken from throats and nose.

Adults should submit to the same procedure, as they may become carriers.

Schick reaction should be done on all members of the family.

All the children and adults reacting positively to the Schick should be immunized at once.

Immunizing dose. (Read text.)

Infants 500 units.

Children 1,000 units.

Adults 1,000 units.

Children whose cultures are negative should be removed from the home if the patient remains there.

If these children cannot be removed and react positively to the Schick test, they should have the immunizing dose repeated every three weeks; every two weeks is safer.

(For particulars see below under immunizing dose.)

Children having a positive culture should be isolated, but never with the patient.

If case cannot be isolated at home he should be removed to the hospital.

Schick test. (See text.)

Should be done on all members of the family.

Active immunization, see text.

Should be done on all those reacting positively to Schick.

\section{Room.}

(See Room under summary in Scarlet Fever, Chap. XVII.) 


\section{Nurse.}

Schick test.

Active immunization, if Schick is positive; both passive and active.

(See Nurse under summary in Scarlet Fever, Chap. XVII.)

Special precautions to avoid infection by cough or sneeze in treating throat or nose.

Secretions should be burned at once.

Nurse should use mild sprays of boric acid 2 per cent. to 4 per cent., or half strength Dobell's solution; but no astringents.

Should receive an immunizing dose of 1000 units every three weeks and better every two weeks.

Should avoid coming in contact with children when off duty.

Should avoid close contact with others, realizing danger of her coughing, sneezing and kissing.

\section{Physician.}

Should submit to Schick test and active immunization if Schick is positive.

(See Physician under summary of Scarlet Fever, Chap. XVII.)

Should use mild sprays but no astringents.

If especially exposed, as by a cough into his face, should receive an immunizing dose.

Should realize that he may be a carrier.

Precautions in the sick-room.

(See same in summary of Scarlet Fever, Chap. XVII.)

Temperature of room, same as in Scarlet Fever. (See Chap. XVII.)

Bed.

(See Chap. IX.)

\section{Patient.}

Must go to bed even in mildest cases on account of danger to the kidneys, and cardio-vascular apparatus.

Bath.

Soap and tepid water each day. Sponge between blankets, or in warm room.

Nightgown.

Open down side to facilitate examinations.

\section{Diet.}

For general principles see Chap. II.

For details of diet see summary of Scarlet Fever, Chap. XVII.

Difficulties of feeding accruing from angina or paralysis are met by Feeding by gavage.

500 c.c. (1 pint) of milk. $50 \mathrm{Gm}$. (2 ounces) sugar.

1 egg.

Give three times a day.

Can add cream to above mixture. 
Technique of gavage.

Adults, use stomach tube.

Children over three years, nasal tube.

Infants, stomach tube.

Rectal feeding.

Less satisfactory, but may be necessary.

Nursing infant.

Take from mother's breast.

Feed with mother's milk obtained by pump or massage to assist with wet nurse's milk obtained in the same way.

Water should be given freely and offered every hour or two.

Fruit juice may be given made into lemonade, orangeade, etc.

An insufficient water intake because of painful swallowing is to be met by

Water by the rectum or Murphy drip.

Care of mouth, nose and genitals.

(See same in summary under Scarlet Fever, Chap. XVII.)

\section{Bowels.}

(See same in summary of Scarlet Fever, Chap. XVII.)

\section{Dose.}

Determine by

1. The severity of the disease.

2. The day of the disease when first seen.

3. Somewhat by age (Wright):

In mild cases (simple congested throat with positive culture or one patch on one tonsil)

Give $3000-5000$ units.

Repeat if there is no improvement in twelve hours.

In more severe cases (both tonsils involved)

Give 5000-6000 units.

Repeat in six hours if there is no improvement, and repeat at six to twelve hour intervals.

In still more severe cases (spreading from tonsils onto pillars or pharynx)

Give 8000 units, intravenously.

Repeat at six to twelve hour intervals.

Toxic cases.

1. If a pharyngeal case is toxic.

2. If there are laryngeal manifestations, hoarse cry, stridor or laryngeal cough.

3. If in addition to pharynx nose is involved,

Give 10,000 units, intravenously.

Repeat dose in six or four hours.

Watch continuously.

In malignant cases (rapid spread, marked toxemia).

Give 15,000-20,000 units intravenously.

Repeat at twelve, six or four hour intervals. 
In severe or laryngeal cases under two years

Give larger doses; three days ill, three times the ordinary dose (Holt).

Park as the result of a large clinical experience and from" his experimental data believes a single dose only is necessary, and that the initial dose should be large.

His dosage is as follows.

Units in case.

Mild. Moderate. Severe. Very Severe.

Infants under 1 year......... 2,000 $3,000 \quad 10,000 \quad 10,000$

Children 1 to 5 years......... $3,000 \quad 5,000 \quad 10,000 \quad 10,000$

$\begin{array}{llll}\text { Children } 5 \text { to } 9 \text { years......... } 4,000 \quad 5,000 & 10,000 & 15,000\end{array}$

$\begin{array}{llll}\text { Persons over } 10 \text { years......... } 5,000 & 10,000 & 10,000 & 20,000\end{array}$

(For New York Board of Health dosage, see text.)

Mode of administration.

Subcutaneously.

Blood contents of antitoxin reaches maximum between third and fourth days.

Intramuscularly.

Somewhat earlier.

Intravenously.

In all severe cases.

Blood content of antitoxin reaches its maximum immediately.

Technique of administration.

Operator's hands, cleansed with soap and water, then alcohol or 1-1000 bichloride.

Skin of patient cleansed with soap and water, then alcohol or paint with Tincture of Iodine.

Boil needle and a small length of rubber tube to attach needle to syringe and prevent breaking of needle, if child struggles.

Syringe, boil. Use one from which plunger can be withdrawn and allow serum to be passed into the barrel.

Expel air before using.

Antitoxin usually sent in a syringe as a container, sterile and ready for use.

Site of injection.

Loose tissue of back at angle of scapula.

Loose tissue of abdominal wall.

Loose tissue of anterior chest in nipple line between nipple and costal margin.

Buttock, especially for intramuscular.

Basilic vein for intravenous. Compress vein lightly above.

Draw a drop of blood to prove entrance of needle into vein, before injecting.

Evidences of improvement. (See text.)

Disagreeable and dangerous results (anaphylaxis) of antitoxin administration. (See text.) 


\section{Precautions.}

If one wishes to take great precautions he may inject one or two drops at first, and if no reaction occurs in an hour give the rest.

Precautions in asthmatics should always be taken.

Use concentrated and purified antitoxin.

Give 0.2 c.c. for first dose.

Repeat in an hour.

Give 0.4 c.c. third dose; repeat same hourly till full dose is attained.

Give atropine sulphate, gr. 1/100 (0.0006 Gm.) at the start.

Treatment of anaphylactic shock.

$$
\begin{array}{ll}
\text { Atropine sulphate............. } 1 / 50 & (0.001 \mathrm{Gm} .) \\
\text { Morphine sulphate... } \cdots \ldots \ldots \text { gr. } 1 / 4 & (0.015 \mathrm{Gm} .) \\
\text { Adrenalin (epinephrin) } 1: 1000 \ldots \text { m. xv } & (1 \text { c.c.). }
\end{array}
$$

Expelling air from chest by brute force.

\section{Immunizing dose.}

Infants, 500 units.

Children and adults, 1000 units.

Repeat at intervals of ten days to two weeks.

Precautions.

Especially asthmatics. (See above under summary.)

\section{Laryngeal diphtheria.}

These should include any exposed child with croupy symptoms.

Take culture.

Even with a negative culture suspect and watch closely.

If croup persists by day treat as true diphtheria.

Give 10,000 to 20,000 units and repeat in four, six, or twelve hours.

Give into vein always, if it is possible.

\section{Other treatment. Symptomatic.}

Inhalations.

Steam, using croup kettle or kindred device. (See treatment of Angina in summary of Scarlet Fever.)

Too long steaming may be depressing.

Hot fomentations.

Ice-bag to neck.

Intubation.
Tracheotomy. (See text.)

\section{Fever.}

Rarely demands treatment.

Sponge baths of luke warm or cool water.

\section{Angina.}

Cold.

Ice-bags and bladders.

Ice-coil.

Cold compresses. 
Heat.

Fomentations.

Applications to throat.

Irrigations.

Salt solution $3 \mathrm{i}$ to $0 \mathrm{i}$ ( $4 \mathrm{Gm}$. to 500 c.c.).

Two per cent. boric acid solution.

Half to quarter Dobell's solution.

Technique.

Read text carefully.

Sprays.

Use same solutions as in irrigation.

Gargles.

Painful and of but very little use.

Avoid astringents.

Give cracked ice to suck.

Pain of swallowing.

Paint with 1 per cent. to 2 per cent. cocaine solution.

Foul mouth.

1-2,000 potassium permanganate solution before the bland irrigation.

\section{Nasal diphtheria.}

Irrigations. Exercise great care lest ears become involved.

Solutions same as those used on throat. (See Angina, above.)

Use glass nozzle or catheter with holes cut in sides and an irrigator or fountain syringe.

Hold just high enough to cause a gentle flow.

Frequency; every two hours in severe cases; less often in less severe.

Piston syringe may be used, lacking a fountain syringe.

Patient should keep mouth open during the procedure.

Begin first on the side most obstructed.

Swabs and sprays if irrigation cannot be done.

Use same solution.

\section{Hemorrhage from nose.}

Adrenalin (epinephrin) 1-2000 or 1-3000 by swab on applicator.

Dry packing.

Packing with gauze soaked in adrenalin (epinephrin) 1-3000 up to $1-1000$.

Thromboplastin and coagulen. (See text.)

\section{Circulatory failure.}

Early, due to toxemia as in other infectious diseases. Late.

Treat as in circulatory failure in Scarlet Fever. (See Chap. XVII.)

Prophylactic.

Early and efficient serum treatment.

Absolute rest. Sitting up in bed may kill.

Morphine hypodermically, every four hours if needed to keep patient quiet. 
Dose.

Child of two years, gr. $1 / 48(0.0015 \mathrm{Gm}$.$) .$

Child of six years, gr. 1/24-1/16 (0.0030-0.004 Gm.). Child of twelve years, gr. 1/12-1/8 (0.005-0.008 Gm.). Adult, gr. 1/8-1/4 (0.008-0.015 Gm.).

Stop food by mouth.

Feed by rectum.

Reduce all procedures about the patient to the minimum compatible with needs.

For precordial pain and distress.

Heat over the precordium.

Drugs. (See text.)

Caffeine.

Camphor.

Digitalis.

Strophanthin.

See their use under CirStrychnine. culatory Failure in Scarlet Adrenalin. Fever, Summary (Chap. XVII). (See text.)

For those who fear the effect of digitalis on the vagus in inducing heart block, the use is advised of atropine sulphate, gr. 1/100 $(0.0006 \mathrm{Gm}$.) with each dose of digitalis or strophanthin.

For collapse or syncopal attack.

Hot saline rectal irrigations at $110^{\circ} \mathrm{F},-115^{\circ} \mathrm{F}$.

For technique, see text.

Hypodermoclysis of salt solution $3 \mathrm{i}$ in $0 \mathrm{i}\left(4 \mathrm{Gm}\right.$. in 500 c.c.) at $100^{\circ} \mathrm{F}$. 亏 viii-xvi (240-500 c.c.).

Cold air.

(For the technique of open-air treatment; see Pneumonia, Chap. IX.)

\section{Paralyses.}

Preventive measures.

Early and efficient serum treatment.

Absolute rest during danger period up to fourth week.

(Rolleston says cardiac and palatal paralyses occur in first two weeks.)

Inform the patient of the risk; and the need of absolute rest.

Heart.

(See summary under circulatory failure.)

Palatal.

Most frequent and short duration, also early.

Feeding by gavage.

Stomach tube in infants and adults.

Nasal tube in children.

Pharyngeal.

Feeding by gavage.

Respiratory.

Lessen weight of bedclothes.

Avoid positions embarrassing respiration such as doubling up.

Raise foot of bed a trifle to facilitate discharge of secretions from bronchi, but not to embarrass respiration. 
Aspirate food particles from pharynx with rubber catheter and syringe.

Swab secretions from pharynx with cotton on dressing forceps or on some other applicator.

Occasional compression of chest to help expel bronchial secretions.

Atropine sulphate, gr. 1/100-gr. 1/200 (0.0006-0.0003 Gm.) according to age to lessen secretions.

Give three or four times a day.

Face and extremities need no special treatment.

Nerve tonic and stimulant may be used.

Strychnine sulphate.

Dose for child of two years, gr. 1/400-gr. 1/150 (0.00015-0.00045 Gm.).

At twelve years, gr. 1/100-gr.1/40 (0.0006-0.0015 Gm.) three or Kidneys. four times a day.

The urine should be examined each day.

\section{Nephritis.}

Preventive.

Early and efficient serum therapy.

Treat like nephritis in Scarlet Fever. (See Summary of Scarlet Fever, Chap. XVII.)

Hot packs and other manipulations causing much handling are not well borne.

\section{Bronchopneumonia.}

Treated like any other bronchopneumonia. (See Chap. IX.)

\section{Adenitis.}

(See treatment of adenitis in Scarlet Fever Summary, Chap. XVII.)

\section{Otitis.}

(See treatment of otitis in Scarlet Fever Summary, Chap. XVII.)

\section{Convalescence.}

Explain to the parents the danger to the heart if the patient is allowed up too soon.

Mild cases.

Flat in bed until end of third week.

Increase number of pillows two, three, four and five.

Sit up in bed at end of fourth week.

In a few days in chair, then

In a few days allow to walk.

Severe cases.

Six or eight weeks in bed.

In no case allow up while there is any sign of heart involvement. Food.

Fresh air. 
Anæmia.

Iron.

Blaud's pill (Pil. ferri carbonatis), gr. ii-v (0.15-0.30 Gm.) three times a day.

Vallet's mass (Massa ferri carbonatis), gr. ii-gr. v (0.015-0.30 Gm.) three times a day.

or

Vinum ferri amarum $3 \mathrm{i}-\mathrm{ii}$ three times a day for children.

\section{Tonics.}

Strychnine sulphate.

Dose for child of five years, gr. 1/200 (0.0003 Gm.) three times a day.

Dose for adult, gr. 1/40-gr. 1/30 (0.0015-0.002 Gm.) three times a day.

Quinine.

Doses of gr. i $(0.06 \mathrm{Gm}$.) three times a day.

May combine with strychnine, cod liver oil (oleum morrhuæ) for children 3 ss. $-\mathrm{i}(2-4$ c.c.) three times a day.

\section{Release from quarantine.}

Three negative cultures at three-day periods should be obtained before discharge.

If bacilli persist

Spray with bichloride of mercury 1-10,000 adding glycerin one part in eight (Holt).

(See carriers, below.)

Bath and shampoo with warm water and soap.

Follow with bath of bichloride 1-5000.

\section{Sterilization and fumigation.}

(For details see Scarlet Fever Summary, Chap. XVII.)

For method of fumigation of Maine State Board of Health see text. Funerals should be private.

Body wrapped in strong antiseptic and coffin sealed.

\section{Carriers.}

Cats and dogs, as possible carriers, must be excluded from the sickroom.

Convalescents released without culture or only one negative culture are possibly carriers.

Individuals in contact with the patient are possibly carriers (50 per cent. to 100 per cent. of the members of the immediate family were found to be carriers; 87 per cent. of an infected school were found to be carriers).

Bacilli in carriers should be treated for virulency to determine the danger entailed by an individual to his environment.

Deep crypts of tonsils as well as the surface should be examined. 
Cultures should be taken from nose and naso-pharynx.

Nose and accessory sinuses inspected for abnormalities and infections that favor the carrier condition.

\section{Treatment.}

Non-operative:

Sprays of all kinds proved inefficient.

Spraying the throat every two hours with a virulent culture staphylococcus and an occasional swabbing with the same. Use of antitoxin does not affect the organisms.

Operative:

Correction of abnormalities.

Diseased tonsils, adenoids, removed.

Accessory sinus disease treated. 


\section{CHAPTER XIX}

\section{MEASLES}

Measles is a disease affecting individuals of all ages, but particularly those in the first five years of life.

All children are highly susceptible. The only exception to this is in the first five or six months of life, during which the chances of escape in an epidemic are relatively good.

Measles is a serious disease, a fact not sufficiently appreciated by the laity and often overlooked by the profession.

In adults the occasional case of measles impressed us but little as a serious disease, though we were familiar with the history of its ravages among savage people when first introduced, but the epidemic of measles that invaded our camps during the late war gave it a new and alarming significance.

What the etiological agent in this disease is, we do not know, but Hektoen declares it to be present in the nasal secretions, in the blood, and in scrapings from the skin in the early eruptive period.

The mortality among infants and delicate children is very high, 15 per cent. to 35 per cent. in institutions, 4 per cent. to 6 per cent. at home under better environment (Holt), and while the mortality is low among older children and adults, complications such as pneumonia and ear involvement may be highly dangerous or fatal, while the susceptibility to tuberculous invasion is greatly enhanced.

The mortality in camps complicated by streptococcic infection exaggerated naturally its menace in civil life but emphasizes the warning not to consider measles a trivial disease.

No more pernicious teaching can be imagined than that as a child will probably have measles some time, the sooner it is exposed and has it the better. The older the child is, other things being equal, the better will it withstand the disease and its complications.

It is not the measles, per se, that affords the danger, but the fact that it reduces the resistance of the mucous membranes of the respiratory tract to the onslaughts of the pyogenic organisms, the staphylococci, the streptococci and the pneumococci, and, as has been said, renders the soil fertile for the tubercle bacillus.

The incubation lasts from 10 to 14 days; it is highly infectious from the appearance of the first symptom; hence, precedes the diagnostic 
rash by several days and facilitates the spread of the infection. It is the secretions of the nose and eyes that are especially contagious and the infectivity disappears with the catarrhal discharges and so is short lived. It is not air borne, but coughing and sneezing can convey it over considerable distances. A third person coming directly from a case can convey the disease, but the virus is readily killed and is rarely conveyed by the third person, the physician or other, as is the case with scarlet fever.

Distribution of the Family. Every child directly exposed, except very young infants, are almost certain to become infected. The chances of escaping are not comparable to those exposed to scarlet fever. The young infant, being less susceptible, is still capable of acquiring the disease and as the mortality is high in this group of patients, every effort to avoid the infection should be taken. The child should be removed to another house until the period of incubation of measles has passed. The chances of isolation in the same house are almost nil in contrast to the partial success of such attempts in scarlet fever. Absolute isolation of all in contact with the patient is necessary for success.

It cannot be too emphatically insisted that no person having a cold, sore throat or infection of the upper air passages should come in contact with the patient lest he be a carrier of streptococcus and hence a real danger to the patient.

Whether another house shall be exposed to the almost certain infection by removal to it of the other older children of the family is a question, but as the infection does not cling to the house and room, as in scarlet fever, I feel that inconvenience should not be set against a chance in behalf of any child.

Quarantine for such children may be broken at the end of two weeks, if there are no catarrhal signs, no Koplik's spots and no temperature. Three weeks would meet the demands of great precaution. Of course, all children in the infected household should be excluded from school until the patient is convalescent and the period of incubation for those who have not had the disease is passed.

Room. Fear for the eyes and for the lungs has condemned the patient traditionally to darkness and to a room deprived of air. This tradition even the profession has had great difficulty in disregarding and in no illness is the room more devoid of cheer and comfort than in measles.

Light is a most potent ally in combating infection and air is not only necessary to life, but is a most valuable therapeutic agent.

If an attempt at isolation is to be made, the choice and manage- 
ment of the room must be identical with that demanded in a scarlet fever case. (See Scarlet Fever, Chap. XVII.)

It is true that the eyes have to be considered in the treatment of measles and a glaring light direct in the eyes is to be avoided; this may be done by placing the bed or moving the bed from time to time to avoid it, by letting the eyes rest on a darker surface, such as a hanging of green material, by a screen or by the use of colored glasses. At the most the room should be slightly darkened. The degree of comfort or discomfort of the patient is really the best regulator of the quantity of light.

Of air there can never be too much. Draughts are, of course, to be avoided. The temperature is to be kept at $65^{\circ} \mathrm{F}$. or $70^{\circ} \mathrm{F}$, but in a toxic condition a colder air is highly beneficial, provided the body is well protected. If the cough is severe a degree of moisture, obtained by the use of croup kettles or other generators of steam may alleviate it, but the air is not to be rendered heavy with moisture. Clear cold air will often prove more efficacious; patients react differently to these measures.

When conditions permit of it, two adjoining rooms may be devoted to the patient, thus giving the opportunity for a thorough ventilation and exposure to bright sunlight to the room for the time unoccupied.

Two children sick of measles should never be treated in the same room, lest complicating diseases like pneumonia be transmitted.

In hospitals and institutions, if separate rooms cannot be secured the patients should lie in separate cubicles. These I saw in operation years ago in Grancher's clinic at L'hospital des Enfants Malades in Paris. They reminded me of horse stalls, the partitions being of glass, 3 to 4 feet high raised from the floor to allow the air to circulate. The glass afforded the nurse in charge an uninterrupted view down the ward. In our camps gauze screens were used and are readily adaptible to an emergency.

The Nurse. When isolation is carried out with a view of protecting other children of the family, the nurse should be as carefully restricted to the sick-room and her own room as in scarlet fever. When there are no other children in the family, such restrictions are hardly necessary. She should, however, have no direct contact with adult members who have never had the disease.

Again, in her outings, she should keep away from children, but need observe no such precautions as if on a scarlet fever or diphtheria case.

If the nurse has recently been in contact with an acute infectious case, it would be wise to determine whether or no she was a carrier of streptococcus hemolyticus. If she has more than one child in charge in a family, she should wear a gauze mask to prevent her acting as a carrier, having a mask for each room. She should also wear a gown. 
The Physician. The rarity with which this disease is transmitted through the third person makes the extraordinary precautions exercised by the physician when on a scarlet fever case unnecessary, particularly if he is for a time in the open air after a visit and does not go directly from a measles case to another child.

He should, however, wear a gown in the sick-room and cleanse his hands with soap and water and alcohol or other antiseptic before leaving.

Wearing a mask in the sick-room will help to prevent his acting as a carrier.

Precautions in the Sick-Room. While the virus is far less persistent than that of scarlet fever and diphtheria and so the danger of transmission less, one ought to observe the same precautions with reference to the infectious material contaminating the articles in use by the patient as in these other conditions. This will be found in detail under Scarlet Fever.

When there are no children in the house and isolation is not carefully observed, the soaking of the clothes in disinfectants before going to the wash, if that is done at home, need not be carried out.

Bed. For the proper kind of bed and the preparation see Scarlet Fever, Chap. IX. The covering should be light unless cold air is admitted to the room, just enough to afford the patient comfort.

\section{PATIENT}

Nightgown. Should be of linen or cotton, unless cold air is admitted to the room, or draughts cannot be avoided; then the flannel nightgown is to be preferred. It should be open down the front so that the chest can be readily exposed for examination.

Bath. Great fear seems to be entertained, by many physicians, of the bath. I cannot see any danger, but much good, in a cleansing bath of soap and water with the sponge. If the patient is exposed, the room should be previously warmed to a little over $70^{\circ} \mathrm{F}$. If concern is excited by this hygienic procedure, the patient may be put between blankets and one part after the other exposed and bathed or the patient may be sponged under the blanket. Not only does it keep the skin clean and assist the inflamed structure in its normal functions, but it is decidedly refreshing to the patient. The bath water should be lukewarm or cool.

Diet. The energy demands of the body continue during an infection as in health; for a man at rest, indeed, the demands during fever are greater, because of certain additional demands made by the pyrexia, per se, and by the destruction worked by the toxins of the disease.

The derangement of the functions of the alimentary canal during 
a prolonged and severe infection is remarkably little (see Diet in Acute Infectious Disease, Chap. II), but such as there is is more marked at the incipiency of the infection. In short acute infections, then, there is no necessity for urging food to meet theoretical demands, both because of the temporary derangement of alimentation and because of the brief period of the infection. At such a time anorexia should be respected as of conservative significance. When, however, the infection is prolonged in its regular course, or prolonged because of added, that is, mixed infection, the necessity for supplying enough food becomes of great moment.

Measles runs a short febrile course and the amount of food can be left to the patient's inclination; but if complicated by a bronchopneumonia, continuing for some time, the ealoric and protein needs must be reckoned.

In young infants on the bottle, the food should be diluted with water or thin cereal water one-half or one-quarter.

In older infants the milk had better,be diluted.

In older children and adults, milk and gruels made of arrowroot, barley, wheat flour, cornstarch, farina or imperial granum or small quantities of the cereals themselves with milk or cream and sugar, jellies of barley, tapioca and sago, boiled rice, milk toast; or some of the other modifications of milk such as buttermilk, koumys, matzoon, afford variety. If the fever continues, add bread and butter, eggs, custard, scraped beef, vegetable soups. As the fever becomes normal, add small quantities of scraped beef, raw oysters, chicken or squab, finely minced. The additions are made in convalescence of the shorter attacks.

For the caloric values of articles here mentioned, see Scarlet Fever, Chap, XVII.

Water is to be administered freely. It is to be given whenever asked for, but it is also to be offered during each waking hour. Plain or alkaline waters, lemonade, orangeade, imperial drink, may be used. The latter may be sweetened freely, every ounce of sugar adding 120 calories of food.

The administration of water is too often neglected, while it is infinitely more important than many drugs, the intervals of whose administration is jealously observed by physician and nurse.

Skin. A warm cleansing bath is given each day and a second may be given if it adds to the patient's comfort.

Often there is a good deal of itching and burning. This may be relieved by sponging with a solution of sodium bicarbonate, $3 \mathrm{i}$ to 3 pints ( $4 \mathrm{Gm}$. to 1,500 c.c.) of water or dabbing on bran water, made by putting a handful of bran in a muslin or cheesecloth bag and moving this about in a gallon (4 liters) of water until it becomes milky. For more severe 
itching 1 per cent. to 2 per cent. carbolic acid (phenol) in oil or vaseline may be used.

When desquamation begins the body may be anointed with vaseline after the daily bath. ${ }^{1}$

R

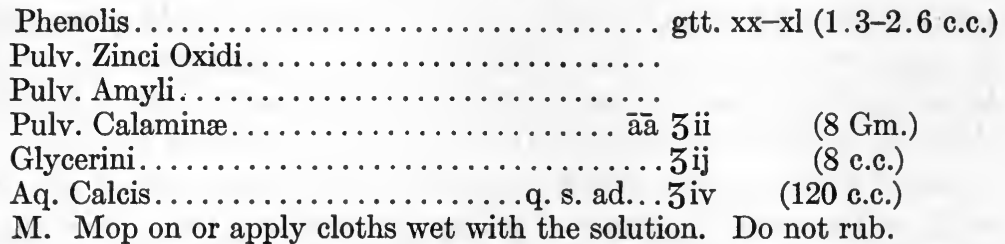

Mouth. When one considers that the most common and serious complications of measles is due to the presence in the mouth of pyogenic organisms, the staphylococci, streptococci and pneumococci, which have invaded the air passages to the production of bronchitis and bronchopneumonia, one appreciates the importance of measures aimed at keeping the mouth and nose clean.

After each meal the mouth should be rinsed with normal salt solution ( $3 \mathrm{i}$ to the pint) ( 4 c.c. -500 c.c.) or 2 per cent. to 4 per cent. boric acid solution of $1 / 2$ or $1 / 4$ strength Dobell's solution. Swabs on wooden tooth-picks, saturated with the same solutions, should be used to cleanse the teeth and the spaces between the teeth and cheeks or lips. For sordes or coated tongue, half strength solution of hydrogen dioxide, peroxide of hydrogen (official) may be applied first, the tongue gently scraped with the edge of a whale-bone and the solutions mentioned then used.

If the mouth is dry equal parts of 2 per cent. boric acid and liquid petrolatum with a little lemon juice affords a pleasing application.

Sprays of the same solutions should be used for the throat. Gargles are not efficacious for this purpose.

Nose. Dried secretions should be moistened with sweet oil and then cleansed with the same solutions as the mouth. The swab on the tooth-pick may be used for these purposes. Sprays of the same materials should follow.

Eyes. The eyes should be cleansed twice a day, or oftener, if needed, with saturated boric acid solution. Vaseline may be smeared on the edges of the lids to prevent gluing. One drop of 20 per cent. solution of argyrol in each eye every two hours from the beginning of conjunctivitis until it clears up is an excellent procedure. (Bingham.)

${ }^{1}$ Hubbard recommends for itching, if much surface is involved, the prescription cited. 
The genitals should be attended to and kept free from irritating secretions, using the saline or boric acid solutions.

Bowels. When first seen a free catharsis should be given. To the infant or very young child, castor oil, $3 \mathrm{i}$ to 3 ii ( $4-8$ c.c.) or calomel, gr. $1 / 10$ to gr. $1 / 4(0.006-0.015 \mathrm{Gm}$.) at 10 to 15 minute intervals until 1 grain $(0.060 \mathrm{Gm}$.) is taken. In older children and adults, a salt, Epsom, Rochelle, Glauber's, or Sodium phosphate in doses of 3 ii to iv (8-15 Gm.) in $1 / 2$ glass of water, alone or preceded by calomel gr. $i$ to gr. iss. $(0.060$ $0.10 \mathrm{Gm}$.) in divided doses.

To children Liquor Magnesii Citratis $亏$ vi to viii (180-240 c.c.) is more grateful or milk of magnesia 5 ss. ( 15 c.c.) following the calomel.

The bowels should be moved by an enema or Liq. Mag. Cit. or Hunyadi water at least every other day.

Fever. A moderate degree of fever, that is $104^{\circ} \mathrm{F}$. or below is to be let alone or even $105.5^{\circ} \mathrm{F}$. if only for a few hours. As a rule measles is not accompanied by greater degrees of fever than these mentioned and the course is short. When, however, a sudden impulse of temperature to $106^{\circ} \mathrm{F}$. or above occurs or a temperature of $104^{\circ} \mathrm{F}$. to $105^{\circ} \mathrm{F}$. persists, an effort should be made to reduce it. Craster has shown that the liability to a fatal issue increases directly as the temperature.

Drugs should not be used for this purpose. Nothing equals cold water as an antipyretic.

For young infants sponging is the best, for children the pack or sponge and for older children and adults the pack or bath.

The younger the child, the warmer the water used. Baths for children should be graduated. The water used in these procedures should begin at $95^{\circ} \mathrm{F}$. to $98^{\circ} \mathrm{F}$. and be gradually reduced to $85^{\circ} \mathrm{F}$. or lower, depending on the reaction of the patient and the fall in temperature. One should be satisfied with a fall to $102^{\circ} \mathrm{F}$. and at this point intermit the procedure. Ice to the head adds to the efficacy of the other measures.

If there is any sign of collapse during the bath, one should take the patient out, wrap him in warm blankets, put heat to the extremities and give warm drinks.

If with a high temperature the extremities are cold, the patient's color poor and pulse small, the hot mustard bath should be given with ice applied to the head.

The mustard bath is made by using mustard in the proportion of a tablespoonful to the gallon. Put the mustard into a small part of the water at a tepid temperature to cause the formation of the oil from the mustard, then add the rest of the water, in a few minutes bringing it up to the temperature of $100^{\circ} \mathrm{F}$. After the child is immersed to the neck, warmer water to bring the whole to $105^{\circ} \mathrm{F}$. can be added if desired. The 
child may be left in five to ten minutes, then dabbed dry, rolled in a warm blanket, with heat at the feet and ice at the head.

Cardio-Vascular. Except in the rare instances of malignant measles and in bronchopneumonia as a complication, the heart does not demand serious attention. When, however, the circulation fails in this condition, the same causes may be deemed operative as in other acute infections, and the same measures should be undertaken to combat it. These are dealt with at length under scarlet fever. In no way is the treatment modified because the infection is measles instead of scarlet fever.

As most of the cases are infants or young children, the doses should be suitable for them.

Digitalis and strophanthin are the only reliable drugs. Eggleston's rule of 2 minims of tincture of digitalis per pound of patient to accomplish digitalization is applicable; e. g., a child of 30 pounds would call for 60 minims of tincture or 6 grains of digitalis. This should be administered in 36 hours. One would give 10 minims every 6 hours or in more urgent cases 20 minims; for the first dose strophanthin might be used, $1 / 10-1 / 5$ of a milligram.

The digitalis must be known to be fresh and of guaranteed assay and it must be remembered that a minim of tincture is not a drop. Always measure the digitalis in a minim glass.

The above dosage is a guide but the dose must be modified to attain results. (See Pneumonia, Chap. IX.)

Caffeine gr. 1/8, 1/4, or 1/2 (0.008-0.015-0.030 Gm.) in the form of one of the soluble double salts of salicylate or benzoate every two or three hours, camphor, gr. $1 / 5$ to gr. $1 / 2(0.012-0.030 \mathrm{Gm}$.) in oil 10 per cent., adrenalin (epinephrin) 1 to 1000 for sudden collapse, $\mathrm{m}$. ii to $\mathrm{m}$. $\mathrm{v}$ (0.150-0.33 c.c.) into a muscle, may be tried with less anticipation of results than from the digitalis bodies or may be used if digitalis fails.

For respiratory failure these same drugs and atropine sulphate gr. $1 / 400$ (0.00015 Gm.)

Nervous Symptoms. In the severe cases the cerebral manifestations may be pronounced; restlessness to delirium and even convulsions or there may be stupor.

These expressions of toxemia in the cortex of the brain are to be combated like those impinging on the vaso-motor and respiratory centers at the base, by the use of hydrotherapy.

The warm sponges and baths for sedative effects and the colder ones for stimulating. These baths, sponges and packs are to be used as described under the section on Fever.

An ice cap to the head has a quieting effect. 
If these measures are not efficacious or not attainable and drugs must be used, in very young children antipyrin in doses of 1 grain $(0.060$ $\mathrm{Gm}$.) and sodium bromide in doses of gr. iii to gr. iv $(0.20-0.25 \mathrm{Gm}$.) three or four times a day may be tried. In older children of five or six, bromide in doses of gr. $\mathrm{v}(0.30 \mathrm{Gm}$.) three or four times a day, in adult gr. $\mathrm{xv}$ to gr. $\mathrm{xx}(1-1.30 \mathrm{Gm}$.) at the same intervals. Acetphenetidin (phenacetin) in doses of gr. i to gr. ii $(0.060-0.125 \mathrm{Gm}$.) to young children may prove sedative, repeated as with the others.

For sleeplessness in children, the baths and the small doses of bromides and antipyretics mentioned are the best.

In adults trional, sulphonethylmethane in gr. $\mathrm{x}(0.60 \mathrm{Gm}$.) doses and chloralamid in doses of $\mathrm{gr} . \mathrm{xx}(1.30 \mathrm{Gm}$.) may be used and repeated in two or three hours if needed.

For wild delirium, sacrificing sleep and rest, morphine will be necessary, hypodermically, gr. $1 / 4(0.015 \mathrm{Gm}$.) in the adult, gr. 1/16 (0.004 $\mathrm{Gm}$.) in the older children, gr. $1 / 24$, to gr. $1 / 48(0.003-0.0015 \mathrm{Gm}$. $)$ in the younger.

\section{COMPLICATIONS}

Bronchopneumonia. Of the serious complications of measles bronchopneumonia is far and away the most common and is responsible for the great majority of the fatal cases.

In private practice the incidence of pneumonia is given at about 10 per cent., but is much more common in institutions. The mortality is very much higher, too, in hospitals than in private practice. In the hospital a child with pneumonia should be promptly removed from the environment of the simple cases, as the condition will spread readily among the cases. An abundance of air, close attention to the toilet of the mouth and avoidance of exposure of the body to draughts and chilling are prophylactic measures.

Most of the cases occur in the first five years of life and the mortality is many times higher in infants under two than in the older children of the first lustrum.

The appearance of bronchopneumonia in a case of measles changes the whole aspect of the case. The treatment henceforth is that of pneumonia, modified not at all by the fact that it complicates measles.

During the late war measles in camps was so complicated by streptococcus hemolyticus that it modified the picture of pneumonia cases to no inconsiderable extent and was accompanied by a high per cent. of empyema and other streptococcic lesions. For discussion of these cases see streptococcic pneumonia (Chap. X). 
The patienf must be kept in bed, unless a young infant, when it may be taken up from time to time. The older patients in bed must be turned from time to time to avoid hypostasis and encourage the discharge of secretions. The temperature of the room should be kept from $65^{\circ} \mathrm{F}$. to $70^{\circ} \mathrm{F}$. unless the cold air treatment is instituted when especial provision in making the bed should be made. As the temperature is more sustained and as an additional toxemia must be combated, more frequent sponging adds to the comfort and refreshes the patient. The care of the mouth must be carried out more rigidly than ever and if the breath is foul or there is stomatitis a phenol solution such as the following should be applied:

R
Phenol (Watery solution 1-20)

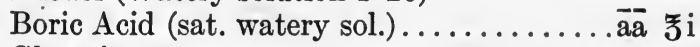
Glycerin....................
M.
(30 c.c.)
(240 c.c.)

The diet should be more liberal than at the beginning, to meet the caloric needs, adding at least 25 per cent. to the requirements in health. (See Scarlet Fever, Chap. XVII, for the caloric requirements.)

The importance of the ingestion of a sufficiency of water must be emphasized, offering it every hour.

Open Air Treatment. A considerable experience with this mode of treatment has made me an enthusiastic advocate of it. The results are so striking and so satisfactory that it is tragic that the physician who is convinced is so handicapped by the traditional fear of the cold entertained so largely by the laity. The most enthusiastic party concerned is the patient. It must be emphasized, however, that the technique must be correct and carefully carried out. Precise instructions about the making of the bed, the protection of the patient, the watchfulness of the nurse, must be given. Detail is set forth in the article on Pneumonia, Chap. IX.

Improvement is seen in the lessening of the nervous symptoms, better sleep, improved appetite, better pulse and respiration.

Objections are made to the cold air treatment in the case of very young and delicate children and in cases of capillary bronchitis. Certainly, watchfulness must be more vigilant in these cases. As for the capillary bronchitis, some react exceedingly well to the open air treatment, while others do not do so well. The response of the individual case must be studied.

Next in value to the cold air in the combat with toxemia is cold water. It may be applied in the shape of cold chest compresses, cold 
sponging, the graduate bath; beginning at $95^{\circ} \mathrm{F}$. and reducing to $85^{\circ} \mathrm{F}$. or the evaporation bath.

In the early stages mustard paste, one part of mustard to five or six of flour in infants and one in four in older children, depending more or less on the reaction of the skin, may be applied. These may be applied every 4, 6 or 8 hours. (See Pneumonia, Chap. IX.) Poultices and jackets add weight and are of little value.

Fever. Continuous high temperature is exhausting. Its effects are to be met by frequent sponging with tepid water, or, if the degree of toxemia is marked with the colder applications, sponges, packs or baths.

Hyperpyrexia calls for extraction of heat, as explained in the preceding section on fever.

Cough. This is likely to be severe in the cases with much bronchitis. In some the effects of the open air is highly satisfactory, in others the applications of the chest compress, but many show an increase of the cough or a "tightening" of the breathing, that finds relief in a warmer, moisture laden air. The effects of steam from a croup kettle in a tent made with a sheet over the upper part of the crib, or over a clothes horse or screen often has a happy effect. If the results of the plain steam are not satisfactory, compound tincture of benzoin, or creosote may be added, a teaspoonful upon the water of the kettle. A degree of moisture may be obtained by water heated in shallow dishes elsewhere in the room. It is not desirable to load the atmosphere of the room with steam. It and the heat generated are depressing, nor should the child be kept too long under the tent. The room should be thoroughly aired two or three times a day, the child being removed to an adjoining room during the ventilation.

A harassing cough sacrifices rest and sleep: It must be met by small doses of codeine phosphate, gr. $1 / 24(0.003 \mathrm{Gm}$.) to gr. $1 / 12(0.005 \mathrm{Gm}$.) in children according to age, in older children and in adults gr. 1/8 $(0.008 \mathrm{Gm}$.) to gr. $1 / 4(0.015 \mathrm{Gm}$.) every two hours. Holt advises Dover's powders in doses of gr. $1 / 10(0.006 \mathrm{Gm}$.) every two hours to a child of one year.

I have no great faith in expectorants and fear the disturbance to the stomach they may set up.

When the secretions are abundant or the child is weak, efforts at expulsion may be ineffectual. Hot and cold water alternately applied, or hot mustard baths may excite to increased respiration and save from a threatened suffocation. Oxygen may be used diluted with air and the chest cupped.

Delirium and sleeplessness are ameliorated by the effects of cold 
air. A warm bath sometimes invites to sleep. An ice bag to the head is often helpful. For headache and extreme restlessness, antipyrin gr. $\mathrm{i}$ (0.06 Gm.) or phenacetin gr. i $(0.06 \mathrm{Gm}$.) or gr. ii $(0.15 \mathrm{Gm}$.) or Dover's powders gr. $1 / 10(0.006 \mathrm{Gm}$. $)$ to infants and larger doses to older children every three or four hours.

Care of the diet and bowels and sufficiency of water are of prime importance when respiration is embarrassed, as the formation of gas produces tympanites; the gas presses up on the diaphragm and encroaches on the breathing space and disturbs the heart action. To relieve this mild salines as suggested, enemata, or enteroclyses of warm salt solution, fomentations (see Typhoid Fever, Chap. V), the introduction of a rectal tube or a Murphy drip may be of value.

Cardio-vascular failure has been dealt with under that heading above.

For edema of the lungs consult Pneumonia, Chap. IX and summary.

Bronchitis. Much more common and far less alarming as a complication is bronchitis in measles. In fact, a certain degree of bronchitis may be anticipated in a well-developed case. The treatment has been outlined under pneumonia, for all gradations of bronchitis are to be met with merging insensibly into pneumonia and all severe cases of bronchitis, involving the bronchi of small size are to be considered as and, indeed, are pneumonia.

For the milder grades, one may try as described above, mustard pastes, the chest compress, inhalations of steam or steam medicated with benzoin, creosote or eucalyptol, the latter especially when the secretions are thick and viscid.

For the cough, if severe and exhausting, Dover's powder or codeine in doses described.

Laryngitis. A laryngitis may occur early or late. When occurring early it probably is not diphtheritic, but a culture of the throat should be taken. If occurring late it probably is diphtheritic and should be treated as such, not awaiting upon the return of the culture which is to be made, but giving antitoxin at once, 10,000 units.

Occurring early it is to be treated like laryngitis under other conditions.

Local Applications. Heat or cold in the shape of compresses, though to apply cold the ice bag or coil may be used, but are not so easy of application as the compress.

Cold Compress. Old linen cut into a strip folded three or four times lengthwise, long enough to go about the neck and pin. Wring out of cold water at $60^{\circ} \mathrm{F}$. Apply to the throat and outside of this a dry flannel. Renew hourly. 
Hot Compresses. A thick piece of old flannel. Lay it in a crash towel, pour over it boiling water, wring it in the towel by twisting the ends in opposite directions, flirt in the air to expel the excess of hot steam, apply, and outside of it a dry flannel, renew every ten or fifteen minutes for three or four times and apply dry flannel. Repeat at three or four hour intervals.

Inhalations. Steam from a croup kettle under the tent as above described. Compound tincture of benzoin, turpentine or eucalyptus oil may be added, $3 \mathrm{i}$ (4 c.c.) upon the water of the kettle.

Warm drinks and warm milk. A glass of hot milk in the morning will often relax the parts and aid to expel the secretions. If the secretions are thick and the patient old enough to permit it, sprays of sodium bicarbonate $\mathrm{gr}$. $\mathrm{xx}$ to the ounce $(1.30 \mathrm{Gm}$. to 30 c.c.), telling the patient to inhale as the spray is injected, will "cut" the secretions and help to discharge them. Sprays in an oily medium, as liquid petrolatum, of menthol, eucalyptus or camphor 1 per cent. or a combination of each 1 per cent. may be found grateful.

If the cough is harassing, small doses of codeine as for bronchitis may be administered.

Otitis. Measles is second to scarlet fever only in the frequency of ear involvement. It is said to be affected in 10 per cent. of the cases, but the degree of impairment and the seriousness of the sequelæ is much less. Nevertheless the ear should be regularly inspected. The treatment is in the application of dry heat, 5 per cent. carbolic (phenol) in glycerin to relieve the pain and incision when bulging is obvious. For details of the treatment, see Scarlet Fever, Chap. XVII.

Adenitis. Marked involvement of the cervical glands occur in some 2 per cent. of the cases. For treatment, see Scarlet Fever, Chap. XVII.

Ulcerative Stomatitis. Noma. Both these conditions may complicate measles. The latter, though fortunately rare, is more commonly a complication of measles than of any other disease. Not only is it highly fatal, 70 per cent. to 100 per cent. in different series, but if it spares, it leaves a lamentable deformity and scarring of the part affected, most often the face. Its relation to ulcerative stomatitis is believed to be close, and it is believed that the noma represents a more virulent process in a less resistant individual than does the ulcerative stomatitis. Both are probably due to the fusiform bacillus of Vincent.

It is for this reason that such great care should be exercised on the oral toilet in measles.

When ulcerative stomatitis occurs, in addition to measures advised in the care of the mouth, one should administer chlorate of potash, 
gr. ii $(0.130 \mathrm{Gm}$.) every one or two hours and use a chlorate of potash solution as a mouth wash; the strength should be $1 / 4$ per cent. to $1 / 2$ per cent. or if this is painful more dilute. If the process is not bettered by these efforts one may touch the spots with 10 to 50 per cent. nitrate of silver, or chromic acid 5 per cent. ur tincture of iodine.

If the process is succeeded by noma or begins as a noma one uses as an irrigation potassium permanganate, making a claret colored solution (1:5000), and has recourse to more heroic measures in addition to those named.

The edges of the gangrenous process is touched with fuming nitric acid or with pure carbolic acid (phenol) followed by pure alcohol. The gangrenous tissue should be previously clipped away or the edges curetted.

The best opinion, however, favors free excision, going out into the healthy tissue, and applying the Paquelin cautery to the edges. This should be done before the toxemia has robbed the patient of what little resistance may be left.

MacGuire recommends the application of a thick paste of subnitrate of bismuth and water at frequent intervals. I have seen most intense ulcerative stomatitis clear up under this treatment.

In a hospital the child affected should be removed from the ward and isolated, as the disease seems contagious.

Noma may attack the vulva and is to be similarly treated.

Gastro-Intestinal. As the rash fades or in convalescence an ileocolitis may occur and is often of serious import. It should be treated as under other circumstances. During the early hours no food should be given, but water freely, in small quantities frequently; then the diet should be barley water, arrow-root water, rice-water, broths, chicken, mutton, veal or beef broth. The broths may be thickened with farinaceous foods, barley, arrow-root, wheat flour. When the acuteness of the symptoms have passed and decided improvement in the stools have occurred milk in the shape of boiled skim milk may be begun, cautiously at first.

The bowels should be moved with castor oil, $3 \mathrm{i}$ ( 4 c.c.) to a child of a year and more in accordance with age. The colon should be irrigated daily with saline solution, $3 \mathrm{i}$ to a pint ( $4 \mathrm{Gm} .-500$ c.c.), using 2 to 3 quarts (2-3 liters), at $100^{\circ} \mathrm{F}$. This will often suffice, but if the stools persist, bismuth subnitrate in doses of gr. $\mathrm{xx}$ to $\mathrm{gr}$. $\mathrm{xxx}(1.30-2 \mathrm{Gm}$.) every two hours, should be given, the dose decreased with improvement in the stools. It can be given, shaken in a little water, through which it quickly diffuses in suspension.

The drug is insoluble, hence, the large doses can be given to the child 
as well as the adult. The object is to give enough for it to exert its protective effect on the considerable extent of the gut involved. The castor oil should be repeated every two or three days. If oil cannot be retained Rochelle salt, $3 \mathrm{i}$ to 3 iv $(4-15 \mathrm{Gm}$.) can be substituted.

Only when the gut is certainly clean and when the diarrhea resists other measures should opium be used, in children in the shape of paregoric, $\mathrm{m} . \mathrm{v}(0.30$ c.c.) equal to gr. $1 / 48$ opium $(0.0015 \mathrm{Gm}$.) or its equivalent in powder, tincture or Dover's powder, every two or three hours. Lengthen the interval promptly with improvement. The temptation to use opium early and to continue it instead of seeking more legitimate measures of relief must be strenuously combated. In severe pain or copious exhausting diarrhea, morphine sulphate hypodermically, gr. 1/48 to gr. $1 / 24(0.0015-0.0003 \mathrm{Gm}$.) at a year to two years may be used. For lesser pains of a colicky nature fomentations should be applied to the intestine.

Diet rather than drugs should afford relief.

Eyes. More or less conjunctivitis is common enough and is to be treated with frequent applications of boric acid solution, 2 per cent. to 4 per cent. If severe, cold cloths, squares of linen or cheesecloth may be applied frequently and over a considerable period of time. These cloths are put on bits of ice in saturated boric acid solution and constantly renewed, as they grow warm upon the eyes. The lids are kept separated with vaseline smeared along the edges. In purulent conjunctivitis silver salts may be used, such as argyrol.

In only the weakly and ill nourished patients may one anticipate the serious complications, an involvement of the cornea with ulceration. These cases need expert advice.

In blepharitis apply yellow oxide of mercury ointment, gr. i $(0.060$ $\mathrm{Gm}$.) of oxide to 3 ii to iv of vaseline (8-15 Gm.)

If there is much photophobia a drop of atropine sulphate in $1 / 2$ per cent. solution can be applied to the eye to dilate the pupil. Use cautiously.

The eyes must be shaded or the room somewhat darkened.

Heart complications, such as endocarditis or pericarditis are very rare and are to be treated as under other circumstances.

Kidney involvement, other than a transient albuminuria, is an uncommon occurrence. If nephritis does occur it is to be met as in scarlet fever. (See Scarlet Fever, Chap. XVII.)

Diphtheria. As has been said, any membrane on the tonsils, or pharynx should demand immediate culture to determine whether it is diphtheritic or not, to be treated accordingly. If laryngitis intervenes in the course of measles, antitoxin should be given, 10,000 units, 
without awaiting the report on the culture. In the hospital all cases should receive an immunizing dose of antitoxin.

When many cases of measles are thrown together as was the case in our camps during the late war and when these men have been in intimate contact with many cases of infection of the upper air passages, before isolation can be effected, and with many more who are carriers of the streptococcus hemolyticus, secondary infection with this organism is inevitable and it was this secondary infection that made Measles the horrible plague it proved to be. Complications became, under these circumstances, more numerous, more varied and severe. They ranged through an extensive gamut, from tonsillitis adenitis, sinusitis, bronchitis, otitis, erysipelas, to bronchopneumonia of the streptococcic type, empyema, peritonitis, meningitis, and septicemia and have to be treated as streptococcic infections. See Streptococcus Pneumonia, and Empyema, Septicemia, Erysipelas, Cerebrospinal Meningitis (Chaps. X, XLV, XLVI, and XXV).

Tuberculosis. Measles renders the patient peculiarly susceptible to tuberculosis, making readily possible an infection or lighting up of an old process or causing the breaking down of and extension from tuberculous bronchial glands. Cough and fever persisting should lead to repeated careful examinations of lungs and sputum. Other tuberculous complications, such as meningitis and acute military tuberculosis may follow. Such possibilities again heighten the importance of sedulously avoiding infection.

Convalescence. The most important matter in consideration of the period of convalescence is the susceptibility to tuberculosis. Avoidance of exposure to a tubercular environment or prompt removal from one, in case any member of the family has or is suspected of having tuberculosis; avoidance of colds, plenty of fresh air and sunlight, an abundance of good food and tonics, the last in fact the least, constitute our efforts. One may administer iron, Blaud's pill or Vallet's mass in doses of gr. ii to gr. v (0.130-0.30 Gm.) three times a day according to age, or the bitter wine of iron, which contains 5 per cent. of the citrate of iron and quinine, in doses of $3 \mathrm{i}$ to ii (4-8 c.c.) three times a day. Strychnine or Tr. nucis vomicae, in doses according to age and cod-liver oil. This latter is best administered to children without effort to conceal the taste, in doses of 3 ss. to i ( $2-4$ c.c.) in older infants, and $3 i$ to ii (4-8 c.c.) in children.

The child should be kept in bed until the rash has quite disappeared and then in the absence of fever or complications may be allowed up and in another week or ten days be allowed out.

Release from Quarantine. The patient should be given a warm 
bath with soap and water and a bath of bichloride 1 to 5,000. The hair should be shampooed.

Disinfection. For discussion of terminal disinfection, see Scarlet Fever, Chap. XVII. The virus of measles does not cling to rooms or articles long. If the room occupied is thoroughly cleansed and aired for some days and children are not allowed to occupy it for two or three weeks, the rigorous disinfection given to a room after scarlet fever is not necessary, but in an institution or when children must occupy the room, such rules as laid down for disinfection in scarlet fever should be carried out. Such precautions will always appeal to the careful parent.

Clothes and utensils may be disinfected as in scarlet fever. (See Chap. XVII.)

\section{SUMMARY}

\section{Distribution of the family.}

Exposed children are almost sure to catch the disease; still they ought to be isolated until the incubation period is passed, i. e., two weeks, then kept away until the patient is well.

Young infants have a greater chance to escape, and their isolation is the more imperative.

Exposed children should be kept from school until the patient has convalesced and the incubation period is passed.

\section{Room.}

In hospitals, separate cubicles.

Choice and management.

(See summary under Scarlet Fever, Chap. XVII.)

\section{Eyes.}

May be protected by the position of the bed.

By the use of screens.

By the use of colored glasses.

By slightly darkening the room.

Patient's comfort the best regulator of light.

\section{Fresh air.}

Temperature of room $65^{\circ} \mathrm{F} .-70^{\circ} \mathrm{F}$.

In toxic condition, cold air, with body properly protected. Two rooms adjoining - one to be exposed to fresh air and sunlight, when not occupied, is excellent.

Never treat two patients in the same room on account of the contagiousness of the complications.

\section{Nurse.}

Nurse for each patient, or if impossible wear a mask for each patient.

Must have no contact with other children or susceptible adults either when she is on duty or during her hours off duty.

Wear gown. 


\section{Physician.}

Should not go directly to another child.

Wear gown and gloves in sick room.

Wash hands on leaving with soap and water.

Follow with alcohol or 1-1000 bichloride.

Wear mask to avoid becoming a carrier.

\section{Precautions in sick-room.}

(See summary under Scarlet Fever, Chap. XVII.)

If there are no other children in the family, soaking clothes in disinfectant before going to the family wash is not necessary.

\section{Bed.}

Choice and preparation.

(See summary under Scarlet Fever, Chap. XVII.).

\section{Patient.}

Nightgown, cotton or linen, or if cold air is admitted, flannel.

Open all the way down the front or sides to facilitate examinations.

Bath.

Soap and tepid or cool water.

Between blankets, if desired.

Room temperature $70^{\circ} \mathrm{F}$. or over during the bath.

\section{Diet.}

Do not urge food during early days.

During short course of a normal measles leave quantity to the patient's inclination.

When prolonged by complications the protein and caloric needs must be considered.

(See Chap. II and Chap. XIV.)

Young infants.

Dilute the milk mixture one-quarter to one-half with water or thin cereal water.

Older infants.

Milk had better be somewhat diluted.

Older children and adults.

Milk gruels of arrowroot, barley, wheat-flour, cornstarch, farina or imperial granum or small quantities of cereals with milk, cream and sugar, jellies of barley, tapioca and sago, boiled rice, milk toast, milk modifications, eggs, ice cream. As the fever declines, bread and butter, custard, raw oysters, chicken and mutton broths, thickened with rice, arrowroot, etc. With normal temperature scraped beef, squab, chicken, lamb chop minced.

Water freely.

Alkaline waters, lemonade, orangeade, imperial drink.

Fruit juices may be sweetened.

(For caloric values see Typhoid Fever, Chap. XIV.) 
Skin.

Cleansing bath each day.

Itching and burning.

Bicarbonate of soda $3 \mathrm{i}$ to $0 \mathrm{iii}$ water $(4 \mathrm{Gm}$. to 1,500 c.c.).

Bran water dabbed on skin.

A handful of common bran in a cheesecloth or muslin bag swished about in a gallon of water until milky.

When more severe.

One per cent. to 2 per cent. of phenol in vaseline or olive oil.

When desquamation begins.

Anoint with vaseline after daily bath.

Mouth.

(For details see summary under Scarlet Fever, Chap. XVII.)

Nose.

Moisten dried secretions with sweet oil.

Use swabs on wooden toothpicks as applicators or sprays of same solutions as in mouth.

Physiological salt solution $3 \mathrm{i}$ to $0 \mathrm{i}(4 \mathrm{Gm} .-500$ c.c.).

Two per cent. boric acid solution.

Quarter to half strength Dobell's solution.

\section{Eyes.}

Cleansed twice a day or oftener.

Use saturated (4 per cent.) boric acid solution.

Anoint the lids with vaseline to prevent sticking.

\section{Genitals.}

Keep clean with 2 per cent. to 4 per cent. boric acid solutions.

Bowels.

When first seen.

Infant or very young child.

Castor oil 3 i to 3 ii (4-8 c.c.).

or

Calomel, gr. $1 / 10$ to gr. $1 / 4(0.006-0.015 \mathrm{Gm}$.$) every ten to fifteen$ minutes until gr. $\mathrm{i}(0.060 \mathrm{Gm}$. $)$ is taken.

Older children and adults.

Salts. Epsom, Rochelle, Glauber's or Sodium phosphate 3 ii to 3 iv $(8-15 \mathrm{Gm}$.) in half glass of water. Give alone or preceded by calomel, gr. i to gr. iss. $(0.060-0.10 \mathrm{Gm}$.) in divided doses.

Children.

Liquor magnesii citratis $§$ vi to $₹$ viij $(180-240$ c.c.) or milk of Later. magnesia 5 ss. (15 c.c.).

Move bowels at least every other day by enema, liquor magnesii citratis or Hunyadi water. 
Fever.

When moderate, below $104^{\circ} \mathrm{F}$. let alone.

Hyperexia.

Sustained above $104 \mathrm{~F}$., or sudden above $105^{\circ} \mathrm{F}$., use cold water.

Infants, sponge.

Children, pack or sponge.

Older children and adults; bath or pack.

Baths for children, if given, should be graduated.

The younger the child the warmer the bath.

Begin at $98^{\circ} \mathrm{F}$. to $95^{\circ} \mathrm{F}$. and cool down to $85^{\circ} \mathrm{F}$.

Be satisfied when patient's temperature falls to $102^{\circ} \mathrm{F}$.

Keep ice-bag, cold cloths, to head or sponge head with cold water.

Collapse during bath.

Wrap in warm blankets.

Heat to extremities.

Hot drinks.

High temperature, cold extremities, bad color and small pulse.

Mustard bath.

₹ss. (15 Gm.) mustard to 1 gallon (4 liters) water.

Add mustard to a small amount of luke warm water to develop the oil, then add hot water to $100^{\circ} \mathrm{F}$.

Put child in.

Bring temperature up to $105^{\circ} \mathrm{F}$.

Leave in five to ten minutes.

Dab dry and roll in warm blanket.

Put heat to feet and ice bag to head.

\section{Circulatory failure.}

(See Scarlet Fever, Chap. XVII, and see text.)

\section{Nervous symptoms.}

Delirium.

Warm baths or sponges.

Sponges, packs, baths. (See above under Fever.)

Ice bag to head.

Drugs.

Only when other measures are not efficacious or attainable.

Young children.

Antipyrin, gr. i $(0.060 \mathrm{Gm}$.) three times a day.

Phenacetin, gr. i to ii $(0.060-0.130 \mathrm{Gm}$.).

Sodium bromide, gr. iii to iv $(0.20-0.25 \mathrm{Gm}$.) three times a day.

Older children.

Sodium bromide, gr. v $(0.30 \mathrm{Gm}$. $)$ three or four times a day.

Adults.

Sodium, potassium or ammonium bromide or any combination of these, $\mathrm{gr} . \mathrm{xv}$ to $\mathrm{gr}$. $\mathrm{xxx}(1-2 \mathrm{Gm}$.) three or four times a day. 
Sleeplessness.

Children.

Warm baths.

Bromides.

Antipyrin.

As above under Delirium.

Phenacetin.

Adults.

Trional, gr. $\mathrm{x}$ to $\mathrm{xv}(0.60-1 \mathrm{Gm}$.) as a powder or in warm water, whisky, brandy or wine.

Repeat in two hours if needed.

Chloralamid, gr. $\mathrm{xx}$ to gr. $\mathrm{xxx}(1.30-2 \mathrm{Gm}$.) in powder or cold water (heat decomposes) or wine, whisky or brandy, and repeat in two hours if needed.

Wild delirium.

Morphine sulphate hypodermically, gr. 1/48-1/4 (0.0015-0.015 Gm.) according to age.

\section{Complications.}

Bronchopneumonia.

Remove from the ward or other cases to prevent its spread to others.

Treat as a pneumonia.

(See Pneumonia, Chap. IX.)

(See text.)

It includes

Cold air.

Watch cases of capillary bronchitis.

They often do better in a warmer air.

Early stages.

Mustard paste to whole chest every three or four hours.

Fever.

(For technique see Pneumonia, Chap. IX.).

Sponges, packs and baths of cold water.

Cough.

Open air.

Chest compresses.

Steam inhalations under tent.

Benzoin.

Creosote.

Water left in shallow dishes in room for moisture.

Don't get air too moist.

Remove child once in a while to air the room.

Codeine phosphate, gr. 1/24-1/4 (0.0025-0.015 Gm.).

Dover's powder, gr. $1 / 10$ (0.006 Gm.).

Every two hours at one year (Holt).

Expectorants not advised.

Feeble expulsion of secretions.

Applications alternately of hot and cold water.

Hot mustard baths. (See above.) 
Oxygen inhalations diluted with air.

Cupping chest.

Changing position from time to time.

Give water freely.

Treatment of cardiac failure and edema of lungs. (See Pneumonia, Chap. IX.)

Tympanites.

Mild salines.

Enteroclysis.

Murphy drip.

Rectal tube.

Fomentations.

(For technique of above measure see Pneumonia, Chap. IX.)

\section{Bronchitis.}

Treat like a mild pneumonia.

\section{Laryngitis.}

Early; probably not diphtheritic.

Culture should be taken, however.

Late; probably diphtheritic. Early.

Give antitoxin at once, 10,000 units, preferably into vein.

Heat.

Compresses, Technique. (See text.)

Cold.

Compresses, Technique. (See text.)

Ice coil.

Ice bag.

Inhalations.

Steam.

Steam medicated with compound tincture of benzoin, oil of eucalyptus or turpentine, $3 \mathrm{i}$ (4 c.c.) to the pint (500 c.c.) water.

(For technique, see Scarlet Fever, Chap. XVII.)

Warm drinks, especially in the morning.

Sprays.

Bicarbonate of soda, gr. $\mathrm{xx}$ to $5 \mathrm{i}(1.30 \mathrm{Gm} .-30$ c.c.). Sprays of oils.

Menthol.

Eucalyptus.

Camphor.

Individually or in combination in 1 per cent. strength.

For cough.

Codeine phosphate or sulphate, gr. $1 / 24$ to $1 / 4$ (0.0025-0.015 Gm.) according to age.

Otitis.

Daily examination of the ears.

For earache.

Dry heat.

Five per cent. phenol in glycerin. Drop in ear. 
Bulging.

Incise.

(For details and technique, see Otitis in summary of Scarlet Fever, Chap. XVII.)

\section{Adenitis.}

(See Adenitis in summary of Scarlet Fever, Chap. XVII.)

\section{Ulcerative Stomatitis.}

Usual solutions for cleanliness.

Chlorate of potash, gr. ii $(0.13 \mathrm{Gm}$.) in water internally every two hours. (Doubtful importance.)

Chlorate of potash $1 / 2$ per cent. to $1 / 4$ per cent. solution locally mouth wash.

Silver nitrate 10 per cent. to 50 per cent solution-touch ulcers.

Tr. Iodine, or 5 per cent. chromic acid solution. Paint on ulcers.

MacGuire's method; see below under Noma.

\section{Noma.}

Irrigate with potassium permanganate (make deep claret red solution).

Clip away gangrenous tissue or curette the edge.

Touch the edge after curetting with fuming nitric acid, or liquified phenol, followed by absolute alcohol.

Better yet free excision, cut into healthy tissue and touch edge with Paquelin cautery.

MacGuire's method; cover affected area every two or three hours with a thick paste of water and subnitrate of bismuth. Remove child from other cases.

\section{Noma of vulva.}

Treat in same way as noma in mouth.

\section{Gastro-Intestinal}

\section{Ileo-Colitis.}

Stop food during early hours.

\section{Then}

Give water in small quantities very frequently.

Barley-water, arrowroot or rice water, mutton, veal or beef broth, then thicken with farinaceous foods, barley, arrowroot, rice, wheat flour, then as stools improve add boiled skim milk and gradually get back on diet.

Castor oil, 3 i to ii (4-8 c.c.) at one to two years.

Colon irrigation to be given daily.

Use salt solution $3 \mathrm{i}$ to $0 \mathrm{i}\left(4-500\right.$ c.c.) 2 to 3 quarts at $100^{\circ} \mathrm{F}$.

If loose stools still persist give bismuth subnitrate, $\mathrm{gr} . \mathrm{xx}$ to $\mathrm{gr} . \mathrm{xxx}$ (1.30-2 Gm.) every two hours, the interval being lengthened as stools decrease. Give it shaken in a little water.

Castor oil should be repeated every second or third day. 
If oil is not retained give,

Rochelle salt $3 \mathrm{i}$ to iv (4-15 Gm.).

If above measures fail, and only when the gut has been thoroughly - emptied by the cathartic, give

Opium, gr. $1 / 48(0.0015 \mathrm{Gm}$.) or its equivalent m. v (0.30 c.c.) paregoric or equivalent amounts in tincture or Dover's powder. Lengthen intervals with improvement.

Copious and exhausting movements.

Morphine sulphate hypodermically, gr. 1/48 to gr. 1/24 (0.0015$0.0025 \mathrm{Gm}$.) at one to two years.

Colicky pains.

Fomentations; technique, see Typhoid Fever summary, Chap. XIV, or Dysentery, Chap. XVI.

Very severe pain.

Morphine sulphate hypodermically, gr. $1 / 48$ (0.0015 Gm.) at one year to gr. 1/24 $(0.0025 \mathrm{Gm}$.) at two years.

Eyes.

Conjunctivitis.

Careful cleansing with saturated boric acid solutions.

If severe.

Cold cloths, i. e., squares of linen or cheesecloth, wet in boric acid solution and kept cold on ice until used.

Apply vaseline to margins of lids to prevent sticking.

Purulent conjunctivitis.

Irrigation with boric acid solution.

Instillation of silver salts, e. g., argyrol.

Ulcers of cornea.

Should seek expert advice.

Blepharitis.

Yellow oxide of mercury, gr. $\mathrm{i}$ to 3 ii or 3 iv vaseline $(0.30$ to 8 or $15 \mathrm{Gm}$.).

Smear on lids.

Photophobia.

Shade eyes or darken room.

Atropine sulphate to dilate the pupil, $1 / 2$ per cent. solution. Use cautiously.

\section{Heart complications rare.}

Treat as under other circumstances.

\section{Kidney complications rare.}

Nephritis.

Treat as in Scarlet Fever. (See summary, Chap. XVII.)

\section{Diphtheria.}

If there is a membrane on the tonsils, take a culture.

\section{Early laryngitis.}

Take a culture. 
Late laryngitis.

Give antitoxin 10,000 units into vein. Don't wait for return on culture.

Hospitals give all cases an immunizing dose.

(See Diphtheria, Chap. XVIII, for treatment.)

\section{Tuberculosis.}

If cough and fever continue, suspect tuberculosis.

Examine lungs repeatedly.

Examine sputum repeatedly.

\section{Convalescence.}

Avoid exposure to a tuberculous environment.

Avoid taking cold.

Fresh air.

Good food.

Drugs.

Iron.

Blaud's pill, better Vallet's mass in capsule gr. ii to $\mathrm{v}$ (0.15$0.30 \mathrm{Gm}$.) three times a day.

Vinum ferri amarum (bitter wine of iron) $3 \mathrm{i}$ to ii (4-8 c.c.) three times a day.

Strychnine sulphate gr. 1/200 to gr. 1/60 (0.0005-0.001 Gm.).

Tincture of nux vomica $\mathrm{m}$. $\mathrm{i}-\mathrm{x}(0.06-0.60$ c.c.), according to age, three times a day.

Cod liver oil.

Allow up.

3i-ii (4-8 c.c.) three times a day.

When rash and fever are gone.

Allow out.

A week or ten days later.

Release from quarantine.

Give bath of soap and water.

Follow with bath of bichloride 1-5,000.

Give shampoo.

\section{Disinfection.}

Room cleaned and aired for a few days.

Put no children in this room for two or three weeks.

The cautious parent and institutions will prefer disinfection as in Scarlet Fever. (See summary, Chap. XVII.)

Clothes and utensils may be disinfected as in Scarlet Fever. (See Chap. XVII.) 


\title{
CHAPTER XX
}

\author{
RUBELLA
}

\section{(GERMAN MEASLES)}

Rubella may be considered the mildest of the exanthems. Such importance as it has rests on the fact that mild cases of scarlet fever or measles may be mistaken for it and entail great risk to others.

The rash is likely to be confluent, which simulates scarlet fever and is formed especially on the abdomen and the inner aspect of the thigh.

It is hardly safe to make a diagnosis of German measles in an isolated case. In an epidemic the diagnosis is relatively easy.

In addition to the rash the most striking feature of the disease is the enlargement of the superficial glands, especially the posterior cervical, posterior auricular and suboccipital glands.

In isolated cases or in the early cases of an epidemic it is much safer to isolate the case and treat it as a scarlet fever suspect until the appearance of further cases settles the doubt.

This disease occurs much more frequently in adults than any of the exanthems, though it is rare after middle life.

The incubation period is from two to three weeks.

Isolation. Many physicians think it hardly necessary, so mild is the disease and so very rare the complications, but if the physician, like the author, believes no infection is so trivial as to be neglected, other children will be sent away, if that can be done without exposing other children, and will be kept from school until the period of incubation expires, that is three weeks. If this is not done these "contacts" should be watched, and with any evidence of catarrh, trivial at the most, or with frank enlargement of the glands of the neck, they should be isolated.

The fever is, with the rarest exception, of little moment and complications so unusual as to be suspected of being coincidences. However, in an epidemic in and about Little Rock, Ark., observed by Geiger there occurred as complications acute arthritis in a considerable per cent. of the cases, two cases of acute nephritis and one of endocarditis. Such complications are to be treated as indicated under Acute Rheumatic Fever, for Arthritis and Endocarditis (Chap. III), Nephritis under Scarlet Fever (Chap. XVII).

It is well to have the patient keep the bed during the few days of 
temperature; allow out of bed a couple of days after and out of the house in a couple of days more.

Sponge baths once or twice a day for cleanliness and comfort, moving the bowels at the beginning with calomel or salts or both and avoiding constipation after, making the diet fairly liberal, milk and milk products, broths, gruels, bread and butter, toast, cereals, eggs, rice, custards, ice cream during the febrile period and meat and vegetables after the febrile period, giving water, or lemonade freely, taking care of the mouth, by the use of boric acid or Dobell's solution constitute the treatment.

Fresh air andsunlight and good nursing are the sum total of treatment.

There is scarcely the necessity for the rigid fumigation and disinfection one carries out in scarlet fever and measles.

If the room is thoroughly cleaned and thoroughly aired for a few days, it meets all the requirements. If disinfection is done, the rules may be found under Scarlet Fever, Chap. XVII.

\section{SUMMARY}

\section{Isolation.}

Contacts should be kept from other children until the incubation period of three weeks are passed.

Because the disease is so trivial protest is made against keeping contacts from school through incubation period.

At least, the slightest sign of catarrh or enlargement of cervical glands should demand isolation.

In sporadic cases isolation is imperative, because cases so diagnosed are repeatedly mistaken diagnoses of mild Scarlet Fever.

Bed.

If there is fever, keep patient in bed until gone.

\section{Baths.}

Sponge of soap and water for cleanliness.

\section{Bowels.}

Move at the beginning with castor oil $3 \mathrm{i}$ to iv (4-15 c.c.). Calomel in divided doses gr. $1 / 4(0.015 \mathrm{Gm}$.) every quarter hour for four doses.

Follow by salts $3 \mathrm{i}$ to iv (4-15 Gm.) or salts, Epsom, Rochelle or Glauber's alone in same doses or liquor magnesii citratis $\xi$ vi to viij (180-240 c.c.) or milk of magnesia 5 ss. (15 c.c.).

Diet.

Fairly liberal.

During fever milk or milk products, broths, gruels, bread and butter, toast, cereals, eggs, rice, custard, ice cream.

Water, lemonade, orangeade, imperial drink, freely. 
Care of mouth:

Use boric acid solution, 2 per cent. to 4 per cent., or Dobell's solution, half to quarter strength.

Complications.

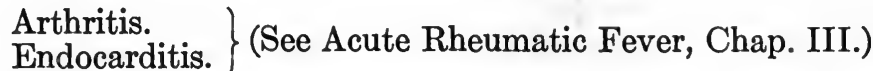

Nephritis. (See Scarlet Fever, Chap. XVII.)

Fresh air.

Allow out of bed two days after fever subsides.

Allow out in two days more.

Cleanse room and air it thoroughly. 


\section{CHAPTER XXI}

\section{VARICELLA}

\section{(CHICKEN POX)}

VARICELla is essentially a disease of childhood and is so because it is so contagious that few children avoid it; but adults who have so far succeeded in doing so are equally susceptible when exposed. The contagion is usually direct, but it may be conveyed by a third person, particularly if the conveyance is immediate.

Distribution of the Family. It is so trivial in its effects that much disturbance of the family to effect isolation seems hardly warrantable and, moreover, other children are pretty sure to have been infected before the disease is recognized; for, as a rule, the first suspicion of its presence is aroused by the eruption. There are, however, exceptions to the statement just made and that in the case of delicate children. Mild though it be in the vast majority of instances, it is possible for it to run a severe course and unexpected and rare complications may ensue. We would not willfully, therefore, expose children and the children of the family should be kept from other children to prevent the spread of disease and that, of course, means that children should be kept from school. Some writers say it is warrantable to let children attend school for the first ten days after exposure, but that ought to mean only when the first exposure is certainly known and when there is no contact of the school child with the patient.

Contacts, if removed from the patient, should be kept from other children until the period of incubation has passed, which should be considered in such cases as three weeks.

Room. A light and well aired room should be chosen for this patient and isolation may not be so strict as in the more severe exanthems, unless any individuals are coming into contact with other children.

The physician can convey the disease, though with a thorough airing and avoidance of visiting a child immediately, it is not likely to happen.

Patient. If there is no fever nor malaise, as is frequently the case, confinement to the bed is unnecessary, but if either of the above mentioned conditions obtain, the patient should remain in bed.

When the eruption is abundant a regular cleansing bath cannot be given without the risk of breaking and infecting the vesicles, an accident which it is desirable to prevent. 
The diet in ${ }^{\circ}$ the light case need in a properly fed child be scarcely modified. If fever of a light grade is present, all but solid foods may be given; if, as rarely happens, the fever is high, food should not be forced, for the fever will subside in a day or two. In the meantime, milk and milk preparations and farinaceous gruels may be given.

Water should be allowed freely.

Skin. The one object of real consideration is the skin; for the nature of the eruption leads to scarring, which on the face, especially of a girl, is to be rigorously avoided.

The eruption itches, the young child scratches and even older children, who endeavor to avoid doing so are sure to lacerate some of the vesicles, inadvertently or in their sleep and infect the lesions, causing an increased damage to the skin and in some instances setting up erysipelas.

The eruption should be kept as dry as possible and dusting powders of sterile talcum powder or equal parts of starch, zinc oxide and boric acid may be used for this purpose.

In young children the scratching can be avoided by putting the arms in splints, by putting on stiff cardboard cuffs, reaching well above and below the elbows, thus preventing the bending of the arms and scratching of the face. Less efficacious, but less trying for the little one, are wrapping the hands in gauze and cutting close the nails.

When pustules form, especially on the face, they should be evacuated by incising the edge of the pustule with a small spear-pointed lancet or a Hagedorn needle, squeezing out the pus and in very bad ones irrigating with a fine pointed dropper with rubber bulb attached, using boric acid or diluted peroxide of hydrogen, half to quarter strength. The vesicle should not be denuded.

Some men advocate painting the vesicles with equal parts of tincture of iodine and alcohol as a protective.

To control the itching the skin may be dabbed with a solution of bicarbonate of soda 1 dram to the pint $(4 \mathrm{Gm}$. to 500 c.c.) or stronger, or 1 to 5 per cent. phenol in sweet oil or vaseline be applied. In intense itching even 10 per cent. is used, but only over confined areas.

The following prescription has been advised by Bethea:

R

Phenolis............................ v

or

Camphoræ................................. xv

M. et adde.

Hydrg. Ammon. ..........................

Ung. Sulphuris.........................ad $\xi^{\mathrm{i}} \mathrm{i}$

Sig: Apply as directed. 
In severe cases there may be some stomatitis. The mouth should be cleansed after each feeding and a mouth wash of salt solution .6 per cent., Dobell's solution half strength, or 2 to 4 per cent. boric acid solution used as a preventative.

Bowels. When first seen the child should be given a mild saline, milk of magnesia 3ii-iv (8-15 c.c.), or liquor magnesii citratis $\zeta$ iv to viii $(180-240$ c.c.).

Constipation should be avoided by a repetition of the dose or an enema.

Fever. The fever is so slight as to require no treatment as a rule. In the rarer cases cold air or dabbing the skin carefully with cool water or an evaporation bath might be tried.

Nervous symptoms are largely due, when they occur, to the irritation of the skin. The applications to the skin advised allay them in a measure, but bromides in doses of gr. $v$ to gr. $x(0.30-0.60 \mathrm{Gm}$.) according to the age, two years to ten years, are indicated. This may be repeated every four to six hours.

Complications. Any complication is unusual, but now and then the following occur; stomatitis (for treatment see Measles, Chap. XIX), conjunctivitis (for treatment see Measles, Chap. XIX).

Corneal ulcer, which may be treated with 1 per cent. atropine, powdered dionin and 5 per cent. yellow oxide of mercury (Wyler).

Nephritis (for treatment see Scarlet Fever, Chap. XVII).

Herpes Zoster, which is to be treated by careful protection, keeping the part dry by applying sterile dusting powders or painting with collodion, the object being to prevent infection, to which the trophic changes . in the lesion render it peculiarly susceptible and erysipelas.

Convalescence is rapidly established and if it has been a severe attack iron may be indicated as a tonic, but in my estimation fresh air and food are far better for such a purpose.

Release from Quarantine. The patient may be considered as no longer a source of infection when the last scab has dropped off. By this time he is ready to go out and is allowed to do so after a thorough cleansing bath and shampoo.

Disinfection. The room needs only good cleaning and airing for a few days.

\section{SUMMARY}

Distribution of the family.

Rare for any member of the family to escape it.

Usually is so light that isolation of other children is hardly advisable.

Exception should be made in the case of sickly children, who should be removed from the environment of the patient. 


\section{School.}

Some physicians let exposed children go to school for ten days but only when date of first contact is surely known.

Contacts should be kept from other children for three weeks, the incubation period.

\section{Room.}

Light and well aired.

\section{Physician.}

Wash hands on leaving case.

Avoid seeing another child at once.

\section{Patient.}

Should go to bed, if there is fever or malaise; otherwise, not necessary.

\section{Bath.}

Cannot be given when the eruption is abundant as the vesicles may break and become infected.

\section{Diet.}

There need be little change from the usual unless there is fever; then exclude solids.

Water should be given freely.

\section{Skin.}

Danger of scarring on the face.

Keep eruption dry.

Use sterile talcum powder or

R

$$
\left.\begin{array}{c}
\text { starch } \\
\text { zinc oxide } \\
\text { boric acid }
\end{array}\right\} \text { equal parts. }
$$

To keep young children from scratching and infecting, put cardboard splints around the arms at the elbows, or wrap the hands in gauze, or cut the nails short.

\section{Treatment of pustules.}

Evacuate by incising edge of pustules with small lancet or Hagedorn needle. Gently syringe out pus.

Irrigate the bad ones with a fine pointed dropper, using boric acid solution, or

Peroxide of hydrogen, quarter to half strength.

\section{Itching.}

Bicarbonate of soda solution.

Phenol, 1 per cent. to 5 per cent. in olive oil or vaseline.

If severe, 10 per cent. over a limited area.

Bethea recommends: 
R

Phenolis gr. v

or

Camphorae. gr. $\mathrm{xv}$

M. et. adde.

Hydrg. Ammon.............................. gr. $x$

Ung. Sulphuris q. s. ad........................ ₹ $\mathrm{z}$

\section{Stomatitis.}

Mouth cleansed after each feeding with

Physiological salt solution $3 \mathrm{i}$ to $0 \mathrm{i}$ ( $4 \mathrm{Gm}$. to 500 c.c.) or

Dobell's solution, half strength, or boric acid solution, 2 per cent. to 4 per cent.

(See Measles, Chap. XIX.)

\section{Bowels.}

When first seen a mild saline of milk of magnesia 3 ii to iv (8-15 c.c.) or liquor magnesii citratis ${ }^{3}$ iv to viii (120-240 c.c.).

\section{Fever.}

Cold water dabbed on skin.

Cold air.

\section{Nervous symptoms.}

Usually due to itching.

Apply sedatives to the skin (see above).

Bromides gr. $\mathrm{v}$ to $\mathrm{x}(0.30-0.60 \mathrm{Gm}$.) according to age, every four to six hours.

\section{Complications.}

Stomatitis. (See Measles, Chap. XIX.)

Conjunctivitis.

(See Measles, Chap. XIX.)

\section{Corneal ulcer.}

1 per cent. atropine sulphate.

Powdered dionin.

5 per cent. yellow oxide of mercury.

Nephritis.

(See Scarlet Fever, Chap. XVII.)

Herpes Zoster. Protection against infection. (See text.)

\section{Convalescence.}

Fresh air.

Abundant food.

Iron, i. e., bitter wine of iron (vinum ferri amarum) $3 \mathrm{i}-\mathrm{ii}$ (4-8 c.c.) three times a day after meals.

\section{Release from quarantine.}

When the last scab has come off.

Give a cleansing bath and a shampoo.

\section{Disinfection.}

Clean and air the room. 


\section{CHAPTER XXII}

\section{PERTUSSIS}

\section{(WHOOPING COUGH)}

$\mathrm{By}$ the layman whooping cough is looked upon as a disease of lesser significance, an annoying inconvenience rather than a danger; by the physician in general, in spite of the statistical evidence of its high mortality, its gravity is not duly appreciated. Measles and whooping cough are exceedingly dangerous diseases when attacking young children under two years, but the low mortality among the older children seems to determine the attitude of the public to the disease in general. Adults are immune only by virtue of a previous attack.

Whooping cough is highly contagious; few children exposed to it escape the infection and the incidence of the disease among the very young, six months and under, a period which seems to enjoy a relative immunity to many of the other infections of childhood together with the frequency of bronchopneumonia of severe grade and the difficulty of feeding when vomiting is frequent, makes it a disease to be dreaded and by every possible means averted.

The mortality under one year is said to be about 25 per cent.

Unfortunately, the very period during which it is most contagious is that in which it displays nothing characteristic to afford a warning of its real nature and permit of avoidance, for in the early or catarrhal stage, it is looked upon as a coryza and a tracheitis or bronchitis, until the persistency of the cough, its periodicity, its paroxysmal character or the vomiting accompanying the paroxysm give the hint of the real condition, while the one or two weeks of this stage has offered abundant opportunity to infect all susceptible individuals who are in contact with the patient. When the paroxysmal or whooping stage has arrived the infectivity has materially diminished, some good authorities think has passed, though such an opinion seems to me at the present time not sufficiently substantiated to act upon. Probably three weeks correspond fairly well with the infectious period; but six weeks for a quarantine are safer. The etiological agent is a bacillus, morphologically, but not culturally identical with the influenza bacillus of Pfeiffer. It was described in 1906 by Bordet and Gengou. The incubation period is 
variously given from a few days to a little more than two weeks. Three weeks is a safe figure for practical purposes.

Symptomatology. The course may be divided into three stages: first the catarrhal with symptoms of a "cold," tracheitis, bronchitis, cough and a little fever; the cough arouses suspicion by its persistency, its relative severity at night, and by a curious coughing down the scale so to speak, until the last breath is exhausted and the face is suffused or blue. This lasts about ten days. The second period is the spasmodic, culminating in the typical whoop. It must be remembered, however, that a whoop may be absent throughout; another significant result of the paroxysm of coughing is the vomiting, which has diagnostic significance even in the absence of the whoop. The duration of this period cannot be definitely fixed and may last for weeks; though commonly the whole course of the disease is about six weeks. The third period is that of decline of cough and other symptoms. It not infrequently happens that paroxysmal coughing and even a whoop will recur for months on the occasion of a cold or irritation of the trachea or larynx that will be mistaken for a recurrence.

The blood may show a mild leucocytosis, up to 15,000 to 25,000 , and the differential count a relative increase in lymphocytes, even up to 80 per cent. at times.

The most serious features of the disease are bronchopneumonia as a complication, the malnutrition incident on the vomiting, and tuberculosis as a sequel. Others will be discussed under treatment.

Excellent results with the complement fixation test reported by the workers of the New York City Board of Health make it urgent to utilize it, in suspected cases, when other children are endangered, provided the technique of the reaction can be properly carried out. This test seems to be more reliable than the agglutinin test. The latter has little or no value after the first week and it is necessary that it must be positive in a dilution of not less than $1: 200$. It is only fair to add that all investigations do not agree about the value of these tests.

Distribution of the Family. As has been said the long delay of the diagnostic symptoms during the most infectious stage makes infection of other children almost certain when whooping cough has not been expected, but in the presence of an epidemic, the first signs of coryza or the first cough should make the individual a suspect and measures for protection of the other children be taken. So serious is this condition to infants and to weakly children that the attempt should be made under any circumstances to prevent infection. The most efficacious means is by removing the other children to another house, unoccupied by children or if that cannot be done, to endeavor to preserve 
a quarantine of the affected case in the house. This last is especially difficult, because of the long course of the disease and because of the unwisdom of cooping up in the house the infected child, who has no complications. This quarantine should last six weeks.

The children who are removed from an infected house become suspects and must themselves be isolated until the period of incubation of the disease has elapsed. The incubation is usually set as one to two weeks, but to avoid the exceptional case these "contacts" should be isolated for three weeks.

The mode of infection is by direct contact, the organism being borne in the fine spray of a cough or sneeze or even in all probability in the act of laughing and talking. It is to be remembered, however, that it is possible for a third person to carry the disease, when the conveyance is rapid; this is important to remember, when the mother or other person in contact with the patient may be tempted to go from one to the other.

Another important fact to be kept in mind is that the infection can undoubtedly be conveyed in the open air and realizing the carelessness of nurses and mothers, too, in taking the affected children about, when allowed out, in the presence of an epidemic young infants should be kept from gatherings of children and decidedly so if any of them are coughing.

The prophylactic use of vaccines in children who are exposed is now generally appreciated. See Prophylaxis.

Room. A large room with the possibilities of light and air should be chosen or a room opening off a balcony, verandah or even fire-escape. It is well when the opportunity affords to select two rooms opening into each other; this to permit of frequent ventilation, by changing the patient from one to the other. It is possible that the rooms become so infected that the patients reinfect themselves, so that disinfection of the room with a formaldehyde candle, lamp or other contrivance, every few days is advisable.

Too much fresh air one cannot have, but draughts, high winds and dust are to be avoided.

Open air is most desirable, and the patient can be kept on the balcony a good part of the time, but in the colder weather, the frequent attacks of coughing, that must displace the coverings, as the child sits up, makes it less feasible than in other infections of the bronchial tree. Where such exposures are frequent it is well to keep the temperature about $70^{\circ} \mathrm{F}$., not allowing it to go much below $65^{\circ} \mathrm{F}$. In mild cases children may be dressed and taken out in the open air in suitable weather, but only when it can be guaranteed that no contact with healthy children may take place. A change of climate if a child can travel and entail no 
risk to others is often of great help; especially from the North in Winter to a milder clime.

Clothing should be flannel next the skin whether in bed or up and about to avoid the chill that comes from wet clothing drenched with the sweats after a paroxysm and from the frequent exposures.

Overclothing, which keeps the skin perpetually moist and enhances the possibilities of bronchial involvement and burdens the chest, is to be avoided.

Those children who are very young, very weak or have considerable bronchitis have to be watched with more care in the open air and many good men prefer to keep them to the room at an even temperature, with frequent ventilation.

Nurse. Considering how highly contagious the disease is and that adults as well as children are susceptible to it, it is hardly just to ask a nurse to take a case of pertussis unless she has already had the disease, for while the danger to an adult is minimal, the length of time that she would be debarred from exercising her profession would work an injustice. A nurse who is willing might be given prophylactic vaccines.

The nurse should realize that while rare, still direct conveyance of the disease through the third person is possible and should avoid contact with other children while off duty.

While on duty the nurse should not leave her patient, especially if it is a young child or infant, for suffocation may occur in a paroxysm or a convulsion may ensue.

Physician. The physician should avoid the small risk of conveying the disease by wearing a gown and washing his face and hands on leaving the sick-room. He should not make his next visit on a child unless some little time in the open air intervenes.

If he himself has not had the disease, he should be careful not to stand directly in front of the patient during a paroxysm, unless there is some special need. Perhaps a mask, like a chloroform mask over the nose and mouth, the gauze or cover of which has been wet with 1 to 20 carbolic acid may help in avoiding infection. Vaccine might be used prophylactically, though the risk to him is not as great as to the nurse.

Precautions in the Sick-Room. Strict isolation to the quarters assigned the patient, as long as he is confined to his room, and when the patient is permitted to go out of doors careful avoidance of contact with other children is the rule to be observed.

All secretions are to be received on cloths and burned or into receptacles and sterilized.

Bed linen, night clothing, towels and so forth should not be sent 
to the family laundry until they have been boiled an hour separately or allowed to soak overnight in 1 to 20 carbolic acid.

Cats and dogs are believed to earry the infection and are to be excluded from the sick-room. Two children should not be treated in the same room.

Bed. For treatment in the open air the bed is made in a special manner, see Pneumonia, Chap. IX.

All patients with whooping cough are not to be confined to bed. The indications for remaining in bed are the presence of a febrile reaction or any serious complication.

In this disease the nerves are particularly affected and trivial excitation can precipitate the paroxysm; among these the slight chill from being put into a cold bed.

Baths. A cleansing bath should be given every day with soap and tepid water. This may be done on a blanket or between the folds of a blanket, exposing only one part at a time.

Diet. For older patients with the milder form of attack, who are not confined to the house, no material change in the diet need be instituted, provided, of course, that the diet is suitable for a child of a given age.

It is the association of vomiting with the paroxysms and the gastrointestinal complications that may occur that make feeding in whooping cough a difficult problem.

When there are no serious complications, gastro-intestinal or pulmonary and the vomiting entails merely a loss of food ingested, the deficit can be made up by'a little tact and perseverance.

After the explosion of the paroxysm of coughing with which the vomiting is so closely associated, there is a period of quiescence for both the organs of respiration and digestion.

One should take advantage of this to offer food, so that as large a portion of the meal as possible may be digested and passed along into the intestinal canal before the next paroxysm is due.

On the other hand, as the time for the succeeding paroxysm draws near, the ingestion of food excites the coughing reflex and precipitates the paroxysm.

When the paroxysms become very frequent, every hour or every half hour, no considerable period is left between paroxysms for gastric digestion; then the problem is to introduce such food as requires a brief stay in the stomach and carries with it a maximum amount of fuel.

In early childhood and infancy the staple article of diet is milk, plain or modified, and when one remembers the physiology of milk digestion, the precipitation of the curd, the digestion at the periphery and the 
three hours required for its complete removal from the stomach, one sees the benefit accruing from small quantities of milk taken frequently, thus affording the maximum surface for digestion and the use of means or methods that lessen the bulk of the curds. Among these measures are dilution with water, half and half affording the optimum for rapidity of digestion, or the use of lime water, 1 in 20 or the use of a cereal water barley or arrowroot as a diluent instead of plain water, or the use of milk gruels, using barley, wheat flour, rice, arrowroot, or some of the malted or farinaceous foods used for infant feeding, or whey, buttermilk, koumys, or predigested milk or animal broths may be given or their food value added to by thickening with farinaceous foods, custards baked or soft, jellies or gelatin carrying sugar, junket, soft egg or albumin water, but always in giving these liquid foods, some estimate must be made of the amount of fuel value they represent in the twenty-four hours, for too often is it forgotten that considerable bulks of liquid food may be ingested that have almost no energy value, for example, animal soup and broths.

Wet toast, milk toast or softened rusks, boiled rice and cereals with milk and cream or sugar may be added. No dry food should be given, lest the articles irritating the pharynx precipitate the attack of coughing.

In infants on milk mixtures, the dilutions should be increased, and the feedings made in smaller amounts at more frequent intervals.

In the worst cases with gastro-intestinal indigestion, every effort is set at naught and one has recourse to rectal feeding, but with small hope that it will be borne sufficiently long or be attended with sufficient success to more than meet short-lived emergencies.

Care of the Patient. In the lighter cases, going out of doors, only the daily bath in the morning, flannel next the skin, taking care not to overclothe, ordinary oral hygiene and cleansing the nose with sprays of boric acid solution 2 per cent. or Dobell's solution quarter strength or with the same applied on a swab of cotton, with destruction of the secretions during the catarrhal stage, are all the measures indicated. When serious complications of the respiratory tract or of the alimentary tract ensue, the care of the mouth is of great importance.

Bowels. Unless there are complications the bowels are to be kept open with mild cathartics, such as cascara in the older children and milk of magnesia in the younger.

Fever. The fever in uncomplicated cases is so trivial as to require no treatment; it is, however, an indication for keeping the patient confined to the bed.

Cough. The paroxysmal cough is the characteristic symptom 
of pertussis. The frequency and severity of these paroxysms determine in no small measure the prognosis in the disease.

Not only is the cough in itself exhausting, but in the severe cases it may be responsible for suffocation, convulsions or cerebral hemorrhages. More than this it is accompanied by vomiting, so frequent at times that it is absolutely incompatible with adequate nutrition and in the very young and weakly is the lethal factor.

Efforts in the treatment of whooping cough are largely directed to the diminution of the number of paroxysms, to afford rest and make possible a sufficient feeding.

If vaccines are of value their early use is obviously indicated to ameliorate this symptom.

There is no reason to believe that any of the empirical measures used heretofore shorten the disease; they merely ameliorate the condition of the patient; hence, if the disease is mild and the paroxysms relatively infrequent, no treatment should be given other than that determined by the hygienic and dietetic measures already laid down.

If we can avoid drugs we should do so, for it must always be remembered that a drug does many things to the organism besides that which we particularly desire and some of these drug effects are deleterious, as for example, the disturbance of digestion in a disease in which so much depends on the stomach. In cases of moderate severity we may, then, with advantage, see what local measures effect before having recourse to medication.

\section{LOCAL PROCEDURES}

Mechanical Support. Few simple devices have been rewarded with more success than the one recommended by Kilmer, the application of an elastic belt to the abdomen, chest or both.

The support given by this contrivance lessens the vomiting in no small measure and modifies the frequency of the paroxysm. It finds its greatest application in infants and weakly children with poor abdominal tone, such as obtains in rickets. Kilmer's instructions are as follows: "A stockinette band is placed upon a baby . . . in the same manner as is done by orthopedists before applying the plaster-of-Paris jacket. This band extends from the axilla to the pubes and fits the baby snugly. Two shoulder straps are used to prevent the band from slipping down. Upon this stockinette band a single width of elastic bandage is sewn, extending entirely around the body and covering the abdomen. The bandage is sewn on when very slightly on the stretch." If the vomiting is not controlled the belt may require a little tightening. The effect is particularly noticeable on the vomiting. A similar elastic bandage may be sewed on 
the stockinette to cover the chest and diminishes the number of paroxysms. These may be worn separately or together. ${ }^{1}$

All forms of excitement and highly emotional states should be avoided; gentle persuasion in nervous children exerted, which while it cannot abort a true paroxysm, may lessen the cough often suggested to the child which precipitates a paroxysm. Fresh air, avoidance of dust, prevention of chilling by exposure to draughts, cold beds, etc., constitute no mean part of the treatment.

Numerous sprays, local applications to the air passages and insufflations have been advised. They are of more than doubtful efficacy, while they often excite and alarm the child and aggravate the condition.

Inhalations. Inhalations of medicated steam will sometimes lessen the number and severity of the paroxysms and lessen the cough of bronchitis, occurring between the paroxysms or render more liquid and easy of discharge the mucus of a bronchitis.

It must be remembered that fresh air is of first importance in whooping cough and that it should not be sacrificed to long inhalations, which themselves from the heat and dampness may become depressing.

Again it must be remembered that one case may find more relief from one agent than from another, or that better results are afforded by occasional change of the medicament employed.

One may try the compound tincture of benzoin first, three or four times a day, more especially at night, when the paroxysms are likely to be worse and sacrifice sleep, or the frequency and length of time may be determined by the degree of relief afforded.

A dram or two of compound tincture of benzoin is placed upon the surface of hot water; the drug is carried with the steam and inhaled.

Simple devices may be used, such as a pitcher, a carafe, or a simple kettle to contain the hot water, a cone of paper arranged over the mouths of the vessels or the spout of the kettle. If the latter is used, it may be kept steaming and a rubber tube attached to the spout may be attached at the other end of a funnel, to facilitate the inhalation.

Another simple device that is especially applicable to children is as follows:- the child lies on the side of the bed, a pitcher filled with boiling water, to which the medication is added, is placed on the floor on a level lower than the face, a stiff piece of cardboard, previously warmed to lessen condensation of the steam, is bent into the shape of a half cylinder and, as an inverted trough, conducts the steam from the pitcher to the face.

Croup kettles of various designs may be used, but one must always 1"Whooping Cough-A New Method of Treatment." Theron W. Kilmer. New York Medical Journal, June 20, 1903. 
be on the sharp lookout for fire when the alcohollamp is used. Electric heaters are of course to be preferred when accessible. Of the different designs of croup kettle my choice is for one made by Lewis and Conger of New York on designs of Holt. It adds safety to convenience.

The inhalations are altogether more efficacious if given under a tent. There are numerous ways of contriving this; ropes or bandages about the four posts of the bed, or where posts are lacking improvised posts at the corners of laths or canes, over which a sheet may be thrown. A sheet may be thrown over an open umbrella. It is better that the whole body should not be included in the tent, only the head. This can be done by arranging four improvised posts, two on each side, a little way apart, connected by rope or bandage with a sheet or rubber sheet over them.

Next to benzoin try creosote. This may be given in the same way; a dram on the surface of the hot water used in any of the receptacles mentioned, in the way mentioned. In some of the croup kettles, such as Holt's, a sponge carried in the spout receives the creosote and the steam passing through the sponge conveys the vapor of the drug.

A mixture of creosote (3ii) (8 c.c) and compound tincture of benzoin (亏 ii) (60 c.c.) affords a good combination.

Chloroform. When the paroxysms are frequent or severe, threatening convulsions, or asphyxia, a few drops of chloroform on a handkerchief, sponge, towel or the hand should be given to inhale.

Other Measures Advised. Nägele has advocated pulling the jaw down and forwards, after the manner of the anæsthetists, to interrupt the laryngeal spasm.

Smith, quoted by Ker, says that prolonged spasm may be broken by plunging the infant's hands into cold water.

In the most severe form of spasm, intubation may be necessary and does afford relief. For technique see Diphtheria, Chap. XVIII.

For inhalation one may try oil of eucalpytus, in the same proportions as the benzoin, or menthol $\mathrm{gr} . \mathrm{xv}$ in $3 \mathrm{i}$ to ii ( $1 \mathrm{Gm}$. in 4-8 c.c.) of compound tincture of benzoin as recommended by Holt, or cresolin, two or three times in the twenty-four hours. There is on the market a special form of vaporizer for cresolin.

Drugs. No end of drugs have been recommended to relieve the paroxysms of pertussis, which in itself constitutes the proof of their very limited value.

The condition to be attacked is a hyperexcitability of the neuromuscular apparatus of the larynx, as well as a general nerve excitation. It would seem rational, then, to select drugs that have a sedative effect on the nervous system and those are the drugs that have been shown empirically to be the most efficacious. It must be remembered, however, 
that these drugs all have undesirable effects as well, the most of them being depressant to the circulation and so should not be used indefinitely or recklessly. Again it is to be remembered that individuals react differently to the different drugs and if results are not obtained after full dosage, it is better to try another rather than keep on with the first in hopes that the action is merely delayed.

My own preference is for the drug especially advocated by Holt and now used very extensively, antipyrin.

Antipyrin. This drug is easily soluble in water and has a slightly bitter taste, but hardly enough to make it desirable to disguise the taste. It should be given in liberal quantities.

Holt advises for a child six months old gr. i $(0.060 \mathrm{Gm}$.) every three hours and, if there are no untoward symptoms increase the dosage up to every two hours. At a year one may begin with a grain and a half $(0.10 \mathrm{Gm}$.) and at two years gr. ii $(0.125 \mathrm{Gm}$.) every four to six hours, the dosage being increased up to every two hours.

As the paroxysms are as a rule worse at night, one can with advantage combine with the antipyrin sodium bromide, in doses of $\mathrm{gr}$. ii $(0.125 \mathrm{Gm}$.) at six months, gr. iii $(0.20 \mathrm{Gm}$.) at one year and gr. iv $(0.25 \mathrm{Gm}$.) at two years to be administered in the latter part of the day and night.

By some it is deemed advisable to stop the drug after a week, having recourse to some other drug, as bromide for a few days, alternating by periods with antipyrin.

The drug is contraindicated in weakly children with impaired circulation or with pneumonia.

Bad effects rarely come from such dosage as is advised unless the patient has an idiosyncrasy for the drug.

The most common idiosyncrasy is marked by an erythematous eruption or localized edema. More rarely one sees evidences of collapse, pallor, weak pulse, low temperature and cyanosis, but this is less common and less marked than after acetanilid.

The continuance of the drug must depend on results and the reaction of the child to the drug.

Belladonna. No drug has enjoyed a reputation for efficiency in pertussis comparable with belladonna. Belladonna is ranked as an antispasmodic and its pharmacology is sufficiently worked out to justify this classification. Its effects on motor nerve endings supplying smooth muscle structures explains its good effects in many forms of spasm, but will not explain the results obtained in the spasm of the striped muscles of the larynx. Pharmacologists have not adequately explained its clinical results in this disease.

Cushny has suggested that perhaps it owes its efficacy to its content 
of hyoscine operating to depress the irritability of the respiratory center. If this is true, one would not substitute atropine for the Galenical preparations of the drug. It is possible that a depression of sensory.nerve endings in the mucous membrane of the trachea affected may play a rôle.

Two facts must be kept in mind in using the drug to control the paroxysms. (1) That it has to be used to the physiological limit, that is, to the point of the earliest toxic manifestation to get results; and (2) that there are many individuals that have an idiosyncrasy for this drug; hence, the beginning dose must be small, a tentative dose.

The most consistent advocate of this treatment was Jacobi, whose definite instructions in the usage of this drug in his editor's note to the article on Pertussis in Modern Clinical Medicine cannot be improved upon and which I quote here.

Taking the case of a child of two years, he says, "Give 6 drops of Tr. Belladonna three times a day; unless the drug cause a 'feverish' flush on the cheeks within half an hour, which must last half an hour or more, it has no effect. If 6 drops have no such effect, give 7, 8, 9 or more every time. The effect must be attained every time, three times daily. Give as many drops as are required to accomplish that end. After a few days more drops will be required. After about a week the full dose will probably have to be doubled."

I would advise before starting with so large a dose as advised by Jacobi that a small dose of $\mathrm{m}$. i ( 0.060 c.c.) of the Tincture be used, for in cases of idiosyncrasy the susceptibility is demonstrated to small doses. If there are no untoward effects one may rapidly mount to the dose advised.

If there be no idiosyncrasy one feels free to give large doses, for this is one of the drugs that children bear proportionately better than adults, taking as J. Walter Carr says at four or five years as large doses as adults.

One may give doses at more frequent intervals, every four hours, if preferred, but when the full dose is attained, to the production of flushing with its attendant disagreeable manifestations, I think the fewer doses are to be preferred.

If we are treating an infant of six months, one begins with a dose of $\mathrm{m}$. ss. to $\mathrm{i}(0.006$ c.c.) three times a day or the fluid extract m. 1/10 ( 0.006 c.c.), at one year double the dose, at two years begin with $\mathrm{m}$. iii ( 0.20 c.c.) of tincture and increase a minim ( 0.060 c.c.) a dose every day to "flushing."

Beside the flushing one may anticipate dryness of the mouth, some hoarseness and dilated pupils.

Idiosyncrasies for the drug are shown both by the symptoms just specified occurring with a minimum dose or by a general erythema, 
suggesting scarlet fever, a talkative delirium and more rarely by vomiting and prostration.

If the delirium is wild it may be controlled by morphine, while prostration or collapse may be combated by caffeine, camphor and strychnine. The bladder should be emptied by catheterization, if retention occurs, and in severe cases saline infusions help to encourage diuresis and elimination.

Bromides. These drugs are hardly potent enough to be of much avail unless pushed to a point of general depression, but combined with antipyrin may enhance the sedative effect of the latter. They may be used with the antipyrin in the latter part of the day and more especially when there is much insomnia. The dose for a child of two should be gr. iii $(0.20 \mathrm{Gm}$.) with each dose of the antipyrin used.

Some authors recommend, however, that they be used alternately a week at a time.

Opium and Its Derivatives. The efficiency of opium in lessening cough, and in inducing sleep sorely tempts the physician and the parent to an unwise usage. It must be remembered that the disease is to be long drawn out, that a certain degree of tolerance will be established and that children are highly susceptible to its toxic effects. For all these reasons it should be used sparingly and only when imperative, that is, when the other sedatives specified are without effect and danger threatens from the exhaustion induced by the paroxysms.

Of opium itself, the best preparations are Dover's powder and paregoric (Tr. opii camphorata). Of its derivatives, codeine and heroine.

Reserve their usage for the severe cases or confine the dosage to night administration.

Try first the effect of the least harmful of these preparations, codeine. It may be given in solution as the phosphate or in tablet form to the older children as sulphate, the dose being gr. 1/100 (0.0006 Gm.) at six months, gr. 1/60 (0.001 Gm.) at one year, gr. 1/40 (0.0015 Gm.) at two years up to gr. $1 / 8(0.008 \mathrm{Gm}$.) or gr. $1 / 4(0.015 \mathrm{Gm}$.) in the older children.

Of the paregoric $\mathrm{m}$. iii-iv $(0.20-0.25$ c.c. $)$ at six months, $\mathrm{m} . \mathrm{v}-\mathrm{x}(0.30$ 0.60 c.c.) at one year, $\mathrm{m} . \mathrm{x}-\mathrm{xx}(0.60-1.30$ c.c. $)$ at two years up to 3 ss. ( 2 c.c.) at five years.

Of the Dover's powder gr. $1 / 8(0.008 \mathrm{Gm}$.$) at six months,$ gr. ss. $(0.030 \mathrm{Gm}$.) at one year, gr. i at two years and gr. ii $(0.125 \mathrm{Gm}$.) at five years.

Talbot with due warning of possible danger entailed, urges the necessity of adequate doses of opium in cases threatened with exhaustion, even to the point of producing prolonged sleep. 
These drugs are best administered at bedtime and in severe cases may be administered again during the night.

\section{MEASURES RECOMMENDED BY OTHER AUTHHORITY}

Quinine. This drug has long been in use in pertussis. The general instructions are to administer it late in the disease and then in large doses of gr. i to gr. iss. $(0.060-0.10 \mathrm{Gm}$.) for every year of the child's life, two to four times a day. It is hard to see what benefits can accrue so late in the disease to offset the discomforts or possible toxic effects of so large dosage.

Heroine. A derivative of morphine, is preferred by some men to codeine. The dose of the hydrochloride is gr. 1/100 (0.0006 Gm.) at one year, to gr. 1/24 $(0.003 \mathrm{Gm}$.) four to six times a day or to be used at night as codeine or opium.

Many other drugs have been recommended, the only excuse for using which lies in the fact that when the more tried measures fail, one feels justified in using any means that holds out any promise whatsoever.

Bromoform has had considerable vogue, but it is the consensus of opinion that little is to be expected from it, while toxic manifestations are readily elicited. To children old enough it may be given dropped on a lump of sugar. If not so, it is best given in emulsion, but it must be remembered that it easily settles out of the emulsion; hence, thorough shaking must precede its usage each time, and it is well to have a fresh emulsion made before the bottle is exhausted, to avoid the large dose that is almost sure to result in the bottom of the bottle from this fact. The dose is a $1 / 2$ minim to 5 minims $(0.030-0.30$ c.c.) three times a day. It must be used with caution. Personally, I prefer not to use it.

Fluoform, in 2 per cent. solution in water in doses of $3 \mathrm{i}$ (4 c.c.) of the solution every two hours has been recommended.

Another fluorine compound is a difluordiphenyl; it is used locally on the chest in the shape of an ointment. This is known as antitussin. I have had no experience with these preparations.

Benzyl benzoate is highly recommended by A. W. Bingham of New York in doses of 20 drops of the 20 per cent. solution given in milk at three-hour intervals. This dose is suitable for children as young as two years. Corresponding doses are given in infants.

Specific Treatment. Specific treatment by the use of sera and vaccines awaits upon the certain identification of the etiological agent. This certain identification has not come, but the claim of Bordet and Gengou that they have isolated the bacillus responsible for the disease seems so good, that this organism, isolated from the bronchial mucus 
raised by the paroxysm in the earlier stages of the disease, has been utilized for the production of a vaccine.

Although vaccines have been used extensively in the past five years since the first edition of this book there is not yet an agreement as to their value; but I think that many excellent pediatricians look with favor upon their use and as no bad results, genreal or local, have attended the treatment so far, I should be inclined to use the treatment.

Talbot gives an injection every other day for three or four doses; first dose $1,000,000,000$; second dose $1,500,000,000$; third dose 2,000,000,000; fourth dose $2,000,000,000$. This dose is for children two years or over. For children under two years half that dose. The Department of Health of the city of New York recommends for children under one year an initial dose of 250,000,000 followed by 500,000,000, 1,000,000,000 and $2,000,000,000$ and $2,000,000,000$ at intervals of two to three days. An autogenous vaccine may be used, if the organism is recovered, but in most instances one has recourse to commercial products. Huenekens emphasizes that the vaccine should be very fresh, not more than a week old and no preservative be used.

Various sera have been tried without encouraging results.

Insomnia. Sleeplessness is so much the result of the frequency of the paroxysms that measures that relieve the latter improve the former. The doses of bromides advised towards the latter part of the day either in combination with the antipyrin or alone gr. ii $(0.20 \mathrm{Gm}$.) are helpful.

Chloral is well borne by the child and it may be administered at night in doses of gr. ii $(0.12 \mathrm{Gm}$. ) at six months, gr. iii or gr. iv (0.20$0.25 \mathrm{Gm}$.) at one year and if not efficacious the dose may be repeated in two hours. It is somewhat irritating to the stomach; hence, it may be given by the rectum in a couple of ounces of warm milk.

Trional has also been used for the purpose, gr. ii to gr. iv (0.12-0.25 Gm.) at two years to be repeated if needed.

Threatened exhaustion from loss of sleep may make the use of opium imperative in cases specified above.

Complications. Vomiting regularly accompanies the disease, but may become so persistent and severe as to constitute a complication.

The first consideration is that of the diet which has already been discussed under that heading.

The application of the abdominal support, such as the Kilmer belt, in many instances lessens the severity and frequency of the vomiting in no small measure. (See mechanical support described above.)

When the vomiting occurs with the paroxysms, those measures which lessen the attacks of coughing will diminish the vomiting, but at times 
the vomiting may occur apart from the paroxysms, as an expression of gastric irritation. In these cases, small doses of bicarbonate of soda, gr. v $(0.30 \mathrm{Gm}$.) with the food may be helpful, while in the more severe cases cocaine hydrochloride, in doses of gr. $1 / 6(0.010 \mathrm{Gm}$.) twice a day has been recommended. (Eustace Smith quoted by Ker.)

Diarrhea is to be treated by a consideration of the diet, an occasional dose of calomel in divided doses, gr. 1/10 (0.006 Gm.) every ten minutes for 10 doses or gr. $1 / 4(0.015 \mathrm{Gm}$.) every $1 / 4$ hour for 4 doses, followed by bismuth subnitrate, gr. $\mathrm{xx}$ to $\mathrm{gr}$. $\mathrm{xxx}(1.30-2 \mathrm{Gm}$.) every three or four hours and by occasional irrigations. The small doses of calomel act, too, as an antiemetic.

Ulcer of the Frenum. An ulcer of the frenum of the tongue occurs now and then in whooping cough. It is usually superficial but may be deep. It is due to the projection of the under surface of the tongue upon the cutting edge of the incisors.

Efforts at healing are made by boric acid mouth washes and occasionally touching the ulcer with silver nitrate stick.

If it resists we may call dental aid to protect the tongue against the teeth or if the process is serious sacrifice the teeth, which, of course, are first teeth.

Hemorrhages. These occur in various parts of the body, but require no treatment (or expectant or symptomatic treatment in case of cerebral hemorrhage), except nasal hemorrhages. These at times may be sufficiently severe to require the application of adrenalin, in a spray of 1 to 10,000 or if the bleeding point can be seen, on a pledget of cotton, 1 to 1,000 .

Very rarely the nares may require plugging.

Coakley advises as a hemostatic tannic acid, mixed with water to the consistency of a syrup and applied on a pledget of gauze.

Bronchopneumonia. This complication is what makes whooping cough the serious disease it is. It is responsible for the vast majority of the fatal cases, estimated by some authorities at 90 per cent. It is prone to occur after the paroxysmal stage is well established, in the second or third week.

The treatment is that of bronchopneumonia under other circumstances and will be found described under Pneumonia, Chap. IX, and under Measles, Chap. XIX, and Streptococcus Pneumonia, Chap. X.

It is necessary to emphasize the importance of the open-air treatment of these cases, the adoption of which has materially lowered the mortality of this dread complication.

The only contraindication is a laryngitis of severe grade.

Such treatment must be carried out with rigid regard for proper 
technique, which will be found described in the chapter on Pneumonia, Chap. IX.

Convulsions. Next to bronchopneumonia this is the most fatal of the complications of whooping cough. The nervous system is in such an irritable condition that slight sources of irritation may precipitate a convulsion and, indeed, convulsions may occur in relatively mild cases; they occur in all degrees of severity, from trivial short-lived spasms to prolonged and fatal attacks.

The physician is rarely at hand at the beginning of the convulsion and until he arrives great alarm is experienced by the family and for their sakes, as well as for that of the child, some instructions should be given to meet the condition. Perhaps the best measure to be pursued by the parent is the giving of a hot mustard bath or pack and because of the lesser disturbance and handling of the patient demanded by the latter procedure, the pack is to be preferred.

Into a quart of luke-warm water a tablespoonful or handful of mustard is thrown, a towel dipped in it and wrung out and wrapped about the baby. The child is then wrapped about with a blanket, an ice-cap put at the head or lacking the ice-bag, cracked ice in a towel, a hot water bottle at the feet, a small fold of a towel tucked in the corner of the mouth to protect the tongue from the teeth, but with care not to obstruct the breathing and then the child kept as quiet as possible. The pack is to be continued for ten or fifteen minutes, then the child is wrapped in a dry blanket, heat kept about the body and extremities and cold at the head.

The bath is prepared by using mustard, a tablespoonful to the gallon; use a small part of the water at a tepid temperature to prepare the mustard and then add the rest of the water, raising the temperature of the bath to $100^{\circ} \mathrm{F}$. (It is to be remembered that hot water kills the ferment in the mustard and no oil will be generated to effect an irritation.) The bath can later be raised three or four degrees. It is continued some ten minutes and then the child is dabbed dry, wrapped in a blanket and treated as after the pack.

The physician's first effort is to promptly interrupt the convulsion and his next to prevent its recurrence.

To interrupt the convulsion nothing is better than a few whiffs of chloroform, given with care, not crowded down upon the face. As soon as the desired result is attained, the prevention is sought in the administration of chloral, a drug toward which a child has considerable tolerance and particularly when convulsed. It is better administered by the rectum to avoid its irritating effects on the stomach. The irritation to the rectum is prevented by the use of an ounce or two of warm milk 
as a solvent. The dose at six months is 3 or 4 grains $(0.20-0.25 \mathrm{Gm}$.), at one year 5 or 6 grains $(0.30-0.35 \mathrm{Gm}$.), and by some given in doses of gr. $\mathrm{x}(0.60 \mathrm{Gm}$.). At two years 8 or 10 grains $(0.50-0.60 \mathrm{Gm}$.). The dosage will depend somewhat upon the severity of the attack. If there is twitching at the end of an hour the dose may be repeated.

The drug may be expected to take effect within a half hour.

Some practitioners add bromide to the chloral in doses of approximately twice as much bromide as chloral. If, however, after the use of the chloroform and chloral the convulsions recur, morphine should be used.

The drug should be used hypodermically and in sufficient doses. At six months the dose of the sulphate usually recommended is gr. 1/48 $(0.0015 \mathrm{Gm}$.), at one year gr. $1 / 24(0.003 \mathrm{Gm}$.$) , and at two years \mathrm{gr}$. $1 / 16(0.004 \mathrm{Gm}$.$) , and this dose, if not effectual may be repeated in a$ half hour to an hour.

Those who are imbued with the traditional fear of morphine in infancy may begin the initial dose with a slightly smaller amount, but satisfactory results will hardly follow in severe cases unless the larger doses are used.

In obstinate cases I would recommend a lumbar puncture. I have seen very satisfactory results in a few cases of continued convulsions, in other conditions follow this procedure.

Other Measures Recommended. Holt recommends oxygen inhalations as of value in some cases which have resisted other efforts.

Still recommends urethane to prevent recurrence of convulsions in doses of gr. $13 / 4(0.10 \mathrm{Gm}$.) three times a day for an infant of nine months and in doses of gr. iii to gr. viii $(0.2-0.50 \mathrm{Gm}$.) three times a day for children six to ten years old.

Release from Quarantine. There is a great disagreement about the length of time a case may be a source of danger, ranging from six weeks to six months. It seems certain that the most contagious period is the catarrhal and early paroxysmal and a whoop may continue long beyond the infective period. As the infectious stage lasts only about three weeks as a rule, six weeks would seem a liberal period for quarantine.

Convalescence. Another reason for dread of this disease is it prolonged course. The average duration of the paroxysmal stage is six weeks, but many run a much longer period and especially in the winter, when pulmonary complications always threaten. The paroxysms often last for months, in many cases long after the infection has surely passed. How much this continued cough and whoop is due to a curious irritability of the laryngeal muscles in the presence 
of what would otherwise be a trivial catarrh of the mucous membranes of the laryngo-tracheal tract or to a mere habit it is difficult in many instances to determine. The continued administration of sedative drugs to subdue this occasional cough is pernicious.

In the young children and infants the treatment during convalescence resolves itself into a dietetic one, for the degree of malnutrition is in many cases grievous and the powers of repair much reduced.

Often a change of air is of benefit and these children seem to do particularly well at the shore, in a warm climate and even a sea voyage may be of great benefit.

Of tonics, iron in the shape of the bitter wine of iron, vinum ferri amarum, in doses of $3 \mathrm{i}$ ( 4 c.c.) in water three times a day or Vallet's mass or Blaud's pill gr. ii to gr. $\mathrm{v}(0.20-0.30 \mathrm{Gm}$.) according to age is indicated and in infants cod-liver oil, best given clear in doses of $\mathrm{m}$. $\mathrm{xv}$ to $3 \mathrm{i}(1-4$ c.c.) three times a day. No drugs should be used if they induce gastric disorder or if there is anorexia and a coated tongue. The bowels should be attended to by the use of the milk of magnesia in doses of $3 \mathrm{i}$ to iv (4-15 c.c.) or cascara, best in the form of some elixir or aromatic preparation.

Fresh air by day, the sleeping porch by night are of vital importance in clearing up the residuum of bronchial catarrh and avoiding colds; baths with cool sponges following and progressively becoming colder, exercise in the open to the point of comfortable fatigue, avoidance of wet feet or exposure are the paths to health and these measures are the more important in view of the fact that next to measles no disease is so commonly followed by tuberculosis as pertussis.

Removal of large tonsils and adenoids may hasten the disappearance of the paroxysms and the completion of convalescence.

Prophylaxis. It is agreed that vaccine used prophylactically is of real value. It should be as fresh as possible. Three subcutaneous injections are usually given, one every third day. Amount used-Children, 500 millian, 1000 million and 2000 million; adults 1000 million, 2000 million and 3000 million. Department of Health, The City of New York.

\section{SUMMARY}

\section{Distribution of the family.}

Separate the suspects from other children. Remove infants from the same house, if possible.

Contacts should be in isolation for three weeks.

Contacts may receive a prophylactic treatment with vaccine. (See text.) 
Remember modes of conveyance by spraying of coughing, sneezing, laughing and talking, for this can occur in the open air; hence, avoid environment of a case even in the open.

During an epidemic keep children from gatherings of children, parties, etc.

\section{Room.}

Light and air.

Approach to verandah or balcony, or two rooms, for change from one to the other, permitting of thorough ventilation.

Occasional formaldehyde disinfection of room.

\section{Open air.}

Milder and uncomplicated cases may be kept up and out of doors but must avoid contact with other children.

Change of climate in selected cases.

More severe and complicated, open air, if patient can be kept covered.

Avoid draughts, high winds and dust. If feasible, temperature of room should be from $65^{\circ} \mathrm{F}$. to $70^{\circ} \mathrm{F}$.

Two children should not be treated in the same room.

\section{Clothing.}

Flannel next the skin. Avoid over-loading.

\section{Nurse.}

Should not be urged to take the case unless she has had pertussis. Avoid contact with other children.

Should never leave her patient when on duty if a young child or infant, lest it suffocate in a paroxysm or have a convulsion.

Prophylactic vaccination.

\section{Physician.}

Should wear gown.

Should wash face and hands on leaving case.

Should not go at once to another child.

If he has not had pertussis he may wear a small mask, like a chloroform mask, wet with 1-20 phenol.

Should avoid standing in front of a patient during a paroxysm.

Prophylactic vaccination.

\section{Precautions.}

Strict isolation to quarters assigned, if in the house.

Avoidance of other children if allowed out of doors.

Secretions should be received on gauze and burned.

Bed-linen, night clothes, handkerchiefs, towels, etc., boil an hour or soak overnight in 1-20 phenol, then launder.

Domestic pets may carry the disease and should be excluded. 
Bed.

For preparation of bed for open air, see Pneumonia, Chap. IX.

Indications for remaining in bed.

When there is fever.

When there are complications.

When there is great nervous irritability.

Bath.

Cleansing bath of soap and warm water daily, on a blanket or between blankets.

Diet.

Older patients and mild uncomplicated cases, no change.

When vomiting is present and gastro-intestinal symptoms are present.

Feed right after a paroxysm as there is then a period of quiescence and avoid feeding near an anticipated paroxysm, as it precipitates it.

When paroxysms occur hourly or oftener.

Give small quantities of high calorie food frequently.

Milk plain or modified.

Dilute with water half and half.

Use lime water 1-20 of milk.

Dilute half and half with barley or arrowroot water.

Milk gruels of barley, wheat flour, rice, arrowroot, malted or farinaceous infant foods.

Whey, buttermilk, koumys, animal broths thickened with cereals and farinaceous foods, custards, baked or soft, jellies with sugar, junket, soft egg, albumin water, wet toast, milk toast, softened rusks, boiled rice and cereal.

Avoid dry foods, they irritate and provoke paroxysms.

Dilute infants' milk mixtures.

Rectal feeding.

Only in most severe cases. Cannot be utilized for any considerable period.

\section{Hygiene.}

Daily baths.

Ordinary oral hygiene.

Sprays for nose of 2 per cent. to 4 per cent. boric acid solution or quarter to half strength Dobell's solution.

\section{Bowels.}

Use cascara in older children and milk of magnesia in the younger or other simple and mild cathartics.

\section{Fever.}

In uncomplicated cases needs no treatment.

Dictates remaining in bed. 


\section{Cough.}

May cause suffocation, convulsions, cerebral hemorrhage, exhaustion, frequent vomiting and malnutrition.

Avoid excitement or emotional state.

Avoid dust, chilling, cold beds, draughts; quiet child's alarm by assurance. Fresh air.

Mild cases require no treatment. Drugs only upset.

\section{More severe cases.}

Local procedures.

Mechanical support, including Kilmer belt. (See text.)

Sprays and local applications to the nose of doubtful value.

Inhalations.

Medicated steam.

Compound tincture of benzoin or creosote, $3 \mathrm{i}$ or ii (4-8 c.c.) on hot water, or a combination of creosote $3 \mathrm{ii}$ (8 c.c.) and compound tincture of benzoin 5 ii (60 c.c.).

May use pitcher, a carafe, simple kettle, with paper cones or regular croup kettle.

Best under tent. (For technique, see text.)

May use in place of benzoin, creosote or oil of eucalyptus in same manner.

Chloroform.

When severe and threatening convulsions or asphyxia.

Few drops on handkerchief, sponge or towel.

Other measures to interrupt prolonged and dangerous spasm.

Pull jaw down and forward as in giving an anesthetic (Nägele).

Plunging hands in cold water (Smith quoted by Ker).

Most severe form of spasm.

Intubation. (See Diphtheria, Chap. XVIII.)

\section{Drugs.}

Antipyrin.

At 6 months gr. i $(0.06 \mathrm{Gm}$.) every three or every two hours.

At 1 year gr. iss. $(0.1 \mathrm{Gm}$.).

At 2 years gr. ii $(0.15 \mathrm{Gm}$.) every six to four hours (Holt).

Towards night combine with bromide of soda.

At 6 months gr. ii $(0.15 \mathrm{Gm}$.).

At 1 year gr. iii $(0.20 \mathrm{Gm}$.).

At 2 years gr. iv $(0.30 \mathrm{Gm}$.).

Don't use it in weakly children and in pneumonia.

Idiosyncrasies for antipyrin. (See text.)

Belladonna.

Has to be used to the physiological limit.

One should begin with a small dose as idiosyncrasies are not uncommon.

Jacobi's dose.

Six drops of the tincture of belladonna three times a day. This should cause a feverish flush on the cheeks in half an hour 
and last half an hour; if it does not the dose must be increased up to $7,8,9$, or more drops.

One must get the flush each time.

It is better to begin with smaller doses than the above, e. g.,

At 6 months begin with $\mathrm{m}$. ss.-i (0.030-0.060 c.c.).

At 1 year, m. i-ii (0.060-0.150 c.c.).

At 2 years, $m$. iii $(0.20$ c.c.).

Increase a minim a dose a day up to the point of inducing flushing.

For other signs of full dose and for idiosyncrasies, see text.

Bromides.

Best combined with antipyrin as above.

Opium and its derivatives.

Use only when exhaustion threatens and other measures fail.

Best used at night.

Codeine best.

Use phosphate or sulphate.

At 6 months, gr. $1 / 100$

At 1 year, gr. $1 / 60$

At 2 years, gr. $1 / 40$

Older children, gr. $1 / 8$ to gr. $1 / 4$

(0.0006 Gm.).

$(0.001 \mathrm{Gm}$.$) .$

(0.0015 Gm.).

(0.01-0.015 Gm.).

Paregoric (Tr. opii camphorata).
At 6 months m. iii-iv
$(0.20-0.30$ c.c. $)$.
At 1 year $m . v-x$
(0.30-0.60 c.c.).
At 2 years $m$. $x-x v$
At 3 years 3 ss.
(0.60-1 c.c.).
$\left(\begin{array}{ll}2 & \text { c.c. }) \text {. }\end{array}\right.$

Dover's powders Pulv. ipecac et opii.).

At 6 months gr. $1 / 8 \quad(0.008 \mathrm{Gm}$.).

At 1 year gr. ss.

At 2 years $\mathrm{gr}$. $\mathrm{i}$

At 5 years $\mathrm{gr}$. ii

$(0.030 \mathrm{Gm}$.$) .$

(0.060 Gm.).

(0.15 Gm.).

Measures recommended by other authority.

Quinine. (See text.)

Heroine. (See text.)

Bromoform. (See text.)

Fluroform. (See text.)

Antitussin. (See text.)

Benzyl benzoate, 20 drops of 20 per cent. solution given in milk every 3 hours (dose for two years of age).

Specific treatment.

Vaccines. (See text.)

Insomnia.

Relieved by measures relieving paroxysms, especially bromides and antipyrin.

Chloral at night.

At 6 months gr. ii $(0.15 \mathrm{Gm}$.).

At 1 year gr. iii to iv $(0.20-0.30 \mathrm{Gm}$.). 
May be repeated in two hours.

Give by rectum in 2 ounces of warm milk.

Trional.

At 2 years gr. ii to gr. iv $(0.15-0.30 \mathrm{Gm}$.).

With threatened exhaustion give opium in doses as given above.

\section{Complications.}

Vomiting of severe type.

Diet. (See above in summary.)

Belt. (See above in summary.)

When accompanying cough.

Measures used to control cough.

(See above in summary.)

Unaccompanied by cough.

Measures to relieve gastric irritation.

Sodium bicarbonate gr. v $(0.30 \mathrm{Gm}$.) with the food.

Cocaine hydrochloride, gr. 1/6 (0.010 Gm.) twice a day (Eustace Smith by Ker).

\section{Diarrhea.}

Regulation of diet.

Calomel occasionally, gr. 1/10 (0.006 Gm.) every ten minutes for ten doses, or gr. 1/4 (0.015 Gm.) every quarter hour for four doses, followed by

Bismuth subnitrate, gr. $\mathrm{xx}$ to gr. $\mathrm{xxx}(1.30-2 \mathrm{Gm}$.) every three or four hours.

Occasional irrigations.

Ulcer of the frenum.

Use boric acid (2 per cent. to 4 per cent. solution) as a mouth wash.

Silver nitrate, touch occasionally with the stick.

Dental assistance to protect tongue.

If severe may extract the incisors responsible, if they are of the first set.

Nose bleed (epistaxis).

Spray with adrenalin $(1-10,000)$ or apply adrenalin $(1-1,000)$ on cotton to bleeding point. If severe, plug nares.

Tannic acid mixed to the consistency of a syrup applied on gauze (Coakley).

\section{Bronchopneumonia.}

(See Pneumonia, Chap. IX.)

Open air.

(For contraindications, see Pneumonia, Chap. IX); also severe laryngitis.

\section{Convulsions.}

Parent or nurse may initiate treatment with hot mustard bath or pack.

(For technique, see text.) 
To interrupt convulsion,

Chloroform, give a few whiffs.

When the convulsion is interrupted,

Prevent recurrence.

Chloral.

At 6 months gr. iii to gr. iv $(0.20-0.30 \mathrm{Gm}$.).

At 1 year gr. $\mathrm{v}$ to gr. vi or even gr. $\mathrm{x}(0.30-0.40-0.60 \mathrm{Gm}$.). At 2 years gr. viii to gr. $\mathrm{x}(0.50-0.60 \mathrm{Gm}$.).

Give by the rectum in 1 or 2 ounces $(30-60$ c.c.) of warm milk. If there is twitching at the end of an hour repeat the dose.

Bromides may be added to the chloral in doses of gr. ii $(0.15$ $\mathrm{Gm}$.) of the bromides to gr. i $(0.060 \mathrm{Gm}$.) of the chloral.

If the convulsions recur in spite of this, use

Morphine sulphate
At 6 months gr. $1 / 48$
At 1 year gr. $1 / 24$
At 2 years gr. $1 / 16$
$(0.00125 \mathrm{Gm}$.$) .$
(0.0025 Gm.).
(0.004 Gm.).

Give hypodermically.

If convulsions or twitching persist, repeat dose in one-half to one hour.

In obstinate cases.

Try lumbar puncture.

(For technique, see Cerebro-spinal Meningitis, Chap. XXV.)

Other measures recommended.

Oxygen inhalations (Holt).

Urethane (Still). (See text.)

\section{Release from quarantine.}

When paroxysms cease unless unduly prolonged. (See text.)

Usually about six weeks.

\section{Convalescence.}

Diet, especially, in malnutrition of infants and young children important.

\section{Change of climate.}

Especially to sea-shore.

Warm climate.

\section{Tonics.}

Iron.

Bitter wine of iron (Vinum ferri amarum) 3i-ii (4-8 c.c.) three times a day.

Vallet's mass (Massa ferri carbonatis) gr. i-iii (0.060-0.20 Gm.) three times a day, or

Blaud's pill (Pil. ferri carb.) same dose, gr. iii $(0.20 \mathrm{Gm}$.).

Cod liver oil $\mathrm{m} . \mathrm{xv}-3 \mathrm{i}(1-4$ c.c.) three times a day.

Keep bowels in order. 
Hygiene.

Fresh air by day.

Sleeping porch at night.

Exercise in the open air.

Avoidance of wet feet.

Watch constantly for any signs of tuberculosis.

\section{Prophylaxis.}

Vaccines.

Fresh as possible.

Dosage. (See text.)

Intervals. (See text.) 


\title{
CHAPTER XXIII
}

\author{
MUMPS
}

(PAROTITIS)

THIs disease of uncertain etiology affects characteristically, but not exclusively, the parotid glands. It is rare in infancy, finds its maximum incidence in the second and third lustra, affects many young adults, but is again rare in later life.

It is very rarely fatal, but entails much discomfort and in its complications sometimes serious results.

There is good reason to believe that the organism isolated and described by Laveran and Catrin, a diplococcus, is the causative agent of the disease.

Symptoms. Usually the enlarging gland is the first symptom. A few, however, will have signs of a general infection with pains variously distributed over the body. The jaws feel stiff and there may be a temporary loss of taste. Sour food may provoke a pain in the jaw, but this is not constant. It has often been thought an early and significant symptom. The parotid swells, giving rise to a characteristic appearance of the face. Sometimes the sublingual or submaxillary glands may share in the affection or may be exclusively attacked. With the parotitis there may be a sense of fullness in the ears and some degree of deafness. The white blood count ranges from 5000-8000 and there is an actual and relative increase of lymphocytes.

Distribution of the Family. I am a firm believer in the prevention in every individual of any kind of infection where possible; so, while many look upon mumps as of slight importance, if not as an amusing incident, such serious consequences can, to be sure rarely, ensue that I advise isolation of the patient or removal of the other children and young adults to other premises.

The period of incubation is from twelve to twenty-six days; hence, these contacts should not expose susceptible individuals until the expiration of twenty-six days.

I am aware that only rarely will such a recommendation be followed by the wage earner and that much protest will be voiced in cases of the school children, but, at least, in the case of the latter, they should not be allowed to go to school longer than ten days after exposure, which period 
trespasses close on the appearance of prodromal symptoms if they are infected and during this period of invasion they are highly contagious.

These contacts should not return to the environment of the patient until the quarantine has been raised.

Room. A well lighted and well ventilated room as free from furnishings as possible should be chosen and a bed, preferably of the hospital type.

A patient who has any fever or other evidence of constitutional reaction to his infection should be confined to bed. Mild cases in children in whom neither the one nor the other obtains may be allowed up, but confined to the room. To male adults, the high incidence (about 30 per cent.) of orchitis as a complication, its distressing symptoms and the possibility of atrophy should be explained with the statement that remaining in bed until the parotid swelling has subsided plus a margin of safety, in all some ten days, is believed to lessen very materially the probability of this complication.

The infection comes from the buccal and nasal secretions and these should be destroyed.

Infection through the third person is very rare, because the infecting organism is very short lived, but the physician should wash his hands thoroughly on leaving the patient and avoid an immediate call on another susceptible individual. The nurse should take similar precautions in her absence from the sick-room.

Baths. A cleansing bath with soap and water should be given every day.

Diet. The difficulty of eating makes a fluid diet or a semi-solid diet a necessity. Milk and milk preparations, cereals, milk broths, meat soups, plain or thickened with farinaceous foods, or with cracker soaked in them, custard, soft eggs, soft toast and similar substances may be used, but the quantity should be such as to satisfy the patient's appetite. If there be no fever and the patient's condition will permit the use of his jaws, a more solid food, meat, fish and vegetables may be used.

Water or drinks made of fruit juices may be allowed and in febrile cases urged.

Acid food or drinks often cause a sharp pain in the jaws and has been looked on by many as characteristic, but this does not always occur and the giving or withholding of such articles depends purely on the absence or presence of pain when taking them.

Care of the Patient. Since the infectious material is resident in the secretions of the nose and mouth and since a mixed infection of the parotid gland leading on to suppuration may occur and presum- 
ably by way of Steno's duct, the hygiene of the mouth becomes of importance.

One may use boric acid solution 2 per cent. to 4 per cent. as a mouth wash or a Dobell's solution, diluted $1 / 2$ to $3 / 4$. These same solutions may be used in the nose in a spray or applied with a swab on an applicator or wooden tooth-pick.

Bowels. At the beginning of the treatment the bowels should be effectually moved by calomel or a salt, or both, as gr. 1/4 (0.015 Gm.) calomel every fifteen minutes until gr. iss. $(0.10 \mathrm{Gm}$.) are taken, followed in two hours by $5 \mathrm{ss}$. (15 Gm.) of Epsom, Rochelle or Glauber's salt.

During the illness the bowels may be moved by a mild saline, like a Seidlitz powder, citrate of magnesia or Hunyadi water or its equivalent in one of the many aperients on the market.

Fever. Only rarely is the fever of any moment. Its discomforts are to be met by cool sponging. In the early stages, if accompanied by pains or aches acetanilid, gr. iss. $(0.10 \mathrm{Gm}$.) or phenacetin gr. iii $(0.20$ $\mathrm{Gm}$.) can be given at hourly or two hour intervals.

Treatment of the Parotitis. In the majority of cases no treatment is required and no discomfort is experienced beyond a stiffness in the muscles of the jaw. It is altogether better not to interfere at all in such cases, as is often done merely because there is a swelling and either the doctor or the patient feels that something ought to be done.

The swelling lasts a week to ten days, but the second parotid may be affected so late as to drag the period out.

When there is pain and marked discomfort in the glands efforts at relief may be made first by topical application, of heat, cold or drugs.

\section{LOCAL TREATMENT}

Heat. In milder cases heat is afforded by the patient himself by the application of a dry dressing, as of absorbent cotton or of nonabsorbent cotton, covering the affected part liberally and held in place by a bandage.

Dry heat can also be applied by the use of the hot water bottle or a bag, the size of which is adapted to the swelling, filled with fine scouring sand or salt and heated in the oven.

Hot fomentations are often efficacious. Two or three layers of thick flannel are laid in a towel and boiling water poured upon them. The water is wrung out of them by twisting them in the towel by turning the ends of the towel in opposite directions.

The degree of heat should first be tested, a flirt in the air made to throw off an excess of steam or hot vapor, the edges turned under to 
prevent any drip of hot water and a dry flannel placed over them. They should be replaced every few minutes before they get cool and this kept up for fifteen to twenty minutes at a time. When taken off the glands should be covered with dry cotton. This may be repeated as often as it affords comfort.

Cold. Some patients find more relief from cold than from heat. Cold may be applied in an ice-bag. The circular ice-bag is the best. The pieces of ice should be about the size of the end of one's fingers, only enough water put in to cover the ice and then the air pressed out and the cover screwed on.

This makes the bag apply itself closely to the part, which it will not do if it contains air. Under the bag should be a layer of flannel, between it and the face. Instead of the ice-bag an ice poultice may be used.

Ice Poultice. "Cut two pieces of oiled muslin the required shape and size, place them together and turn over the edges about an eighth of an inch all round. Bind with adhesive plaster, leaving unbound a small section at the top till the ice has been put in. Fasten the corners securely, strengthening them with extra pieces of adhesive plaster. Mix the ice after breaking it into pieces the size of a walnut, with one-third as much flaxseed or bran, which will absorb the water as the ice melts, and with a small amount of salt, which will intensify the cold.

"Cover the poultice with gauze before applying, and hold it in place either with a binder or a four-tailed bandage, as the position requires." Quoted from Practical Nursing by Maxwell and Pope. Salt should be used cautiously.

Drugs. It is questionable whether any of the ointments and liniments applied do actual good. Among those which have been recommended, I will mention camphorated oil (linimentum camphoræ), Belladonna ointment of the pharmacopeia, Guiacol, 1 in 20 of vaseline and lanolin equal parts, and ichthyol ointment 10 per cent. to 25 per cent.

Now and then a gland goes on to suppuration. In this case it should be incised.

While the seat of the infection is peculiarly the parotid glands, other glands may at times be affected, such as the submaxillary, the sublingual, the testicle, the ovary, the mammary glands, the pancreas and still more rarely the lachrymal and thyroid glands. Of course, the appreciation of their involvement by this specific infection comes only when the parotid swelling tells the tale or in the presence of an epidemic, when, even in the absence of a parotid swelling the significance of the glandular swellings is understood. 
The treatment of the submaxillary and sublingual glands is the same as that of the parotid. When the testicle is involved one may speak of

Complications. Orchitis. It may be prefaced that testicular pains during mumps are not uncommon even when the organ is not apparently involved. This complication is very rare in boys before puberty, but after that period its frequency is given by some authorities as high as 33 per cent. With the involvement of the testis there is usually an exacerbation or recrudescence of the constitutional symptoms. While in some cases the discomfort is trivial, in many the pain is severe. This may be the earliest and only organ involved. The diagnosis in this case would deperd on the presence of an epidemic or exposure.

Ker calls attention to the fact that accompanying the orchitis there may be a slight urethral discharge. Even in the presence of the parotitis this discharge may be misunderstood as gonorrheal or on the other hand an actual gonorrheal discharge might be assumed to be a part of the orchitic involvement. A smear for the detection of the gonococcus of Neisser should be taken.

Remaining in bed during even a trivial attack of mumps lessens the likelihood of an orchitis very materially. If the patient is up and about, he must, of course, be made to go to bed.

A proper suspensory, to support the drag of the inflamed organ must be applied as the first and most essential measure and, indeed, in the milder cases little else is required.

A ready contrivance for affording support is a pillow stuffed between the thighs well up against the perineum; or the thighs may be brought together and a sheet of adhesive plaster 3 or 4 inches wide spread across the thighs on which the scrotum may rest. The disadvantage of these methods is that they interfere with a freedom of motion in the bed. A " $T$ " bandage, one arm going about the waist and the other between the thighs, to support the scrotum, split just beyond the point of support, each half to be attached above to the part around the waist on either side or, if accessible, one of the many forms of support on the market.

A dry poultice may be used, which consists of an abundance of cotton about the scrotum, which retains the heat of the body.

A time-honored application is: Lotio Plumbi et Opii; Lead acetate, 128 grains $(8.5 \mathrm{Gm}$.); Tincture of opium, 4 drams ( 15 c. c.); and water to make 1 pint ( 475 c.c.). (Shake before using.)

The bowels must be kept freely open with salines during the run of orchitis.

The condition is likely to last from three to seven days and resolve without further trouble. The danger lies in the fact that in rare cases atrophy of the testis occurs. In the females either the ovaries or the 
mammary glands may be affected. There is tenderness over the site of the ovaries but the organs are rarely enlarged. Hot applications over the abdomen or over the mammary glands, using the "measures advised for the parotid glands, may be used.

Vulvitis, too, is an unusual manifestation in the female. It is to be treated by local applications of boric acid solution, Sitz baths and hot compresses. Dr. Joseph Sailer reports 14 cases in 4000 or 3.5 per cent. He found it to occur most commonly on the 5 th to 8 th day, but it sometimes comes on late in convalescence.

Pancreatitis. The vomiting, sometimes severe, the pain and tenderness in the epigastrium and left hypochondrium, might be readily attributed to a gastric disturbance, if one did not have in mind the possible involvement of the pancreas. Jaundice may occur. My friend, Dr. Geo. Blumer, tells me he has seen a case go to operation, disclosing an extensive fat necrosis.

The treatment consists in the application of heat or cold over the site of pain and tenderness and a limitation of diet and especially on fat, both to afford the gland a relief from excitation of food taken and the dyspeptic disturbances that might ensue from insufficiency of the pancreatic ferments. The trouble is short-lived and the diminution in food will have no significance.

Thymus gland. Dr. Sailer reports the rare involvement of this gland, 0.1 per cent. of his cases.

There was localized edema over the manubrium, pitting on pressure. This may extend from the cricoid cartilage to the middle of the gladiolus and on either side of the mid-clavicular line, with a circular or oval outline. There is no tenderness to pressure nor nausea. The X-ray detects the enlarged outline of the gland.

Meningo-encephalitis. The large number of cases of mumps occurring in our camps during the late war bore in upon us the frequency and importance of this complication. Probably it is with rare exception, the sole cause of death. Haden reported 9 cases in a series of 476 of mumps, nearly 2 per cent. There is headache, nausea and vomiting which may be severe, slight rigidity of the neck, perhaps Kernig's signs, but not well marked, drowsiness and high fever. The spinal cord fluid is clear, under an increase of pressure, shows a pleocytosis, lymphocytosis usually predominating, and Fehling reducing bodies. in 60 per cent. of the cases.

It may be the earliest manifestation of the infection and simulate very closely a tubercular meningitis. In one such case I made the diagnosis on the basis of its occurring during an epidemic of mumps and confirmed by a tender testicle, developing later an orchitis. 
It is assumed that one is dealing with a meningo-encephalitis. I am not conversant with pathological reports on such cases. One wonders if the pituitary or pineal glands might not be involved to account for the symptom complex.

Lumbar Puncture is a valuable therapeutic measure, as it affords much relief to the headache.

An ice-bag applied to the head may be efficacious to relieve headache. In severe cases morphine may be indicated in doses depending on age. (See Cerebro-spinal Meningitis, Chap. XXV.)

Nephritis, arthritis, have both been mentioned in the literature as having complicated this disease, but they are of extreme rarity and are to be treated, when they occur as under other circumstances.

No other treatment of the condition is needed, except bromides for nervousness, trional for sleeplessness, small doses of phenacetin for pain and in the very severe cases small doses of morphine.

Release from Quarantine. Isolation should continue about ten days after the swelling has ceased, but in prolonged cases it may be cut down to a week after the disappearance of the local trouble.

Convalescence. Is usually rapid and needs little else than fresh air and good food. If a tonic is desirable, one may use iron, the bitter wine in children in doses of $3 \mathrm{i}$ ( 4 c.c.) three times a day and the Blaud's pill in the older cases in doses of $\mathrm{gr}$. iii $(0.20 \mathrm{Gm}$.) to gr. v (0.30 Gm.) three times a day.

Disinfection. The infective material is easily destroyed on exposure to light and air and many men think it unnecessary to do more than clean and ventilate the sick-room.

If, however, one wishes to take extreme precautions it may be disinfected and the clothes treated as described under Scarlet Fever. (See Chap. XVII.)

\section{SUMMARY}

Distribution of the family.

Contacts should not expose susceptible individuals until incubation period of three to four weeks has passed.

\section{Patient.}

Should be confined to room.

If there is fever or complications, to the bed.

Remaining in bed lessens the incidence of orchitis.

Room.

Good light and ventilation. 


\section{Physician.}

Should wash his hands carefully on leaving patient and not go directly to a susceptible person.

\section{Nurse.}

Should take similar precautions.

\section{Baths.}

Daily cleansing bath of soap and water.

\section{Diet.}

Fluid or semi-solid on account of difficulty of eating.

(For suitable articles, see text.)

Acid foods or drinks often cause sharp pain in jaws.

\section{Care of mouth.}

Remember the danger of mixed infection of the parotid and its suppuration.

Boric acid solution 2 per cent, to 4 per cent.

Dobell's solution half to quarter strength.

\section{Nose.}

Use sprays or cotton swabs on wooden toothpicks wet with same solutions.

\section{Bowels.}

At the beginning use calomel or salt or both.

Calomel gr. 1/4 $(0.015 \mathrm{Gm}$.) every fifteen minutes until six doses are taken and follow in two hours by Rochelle or Epsom salt 5 ss. (15 Gm.).

During illness use milder salines, such as citrate of magnesia, Seidlitz powder, Hunyadi or similar water.

\section{Fever.}

Rarely marked.

Cool sponges.

If accompanied by pains and aches,

Acetanilid gr. iss. $(0.10 \mathrm{Gm}$.) or phenacetin gr. iii $(0.20 \mathrm{Gm}$.) every three hours or until relieved.

\section{Treatment of the Parotitis.}

Often no treatment indicated.

Pain and discomfort.

Topical treatment.

Heat.

Hot-water bag.

Bags of hot salt or scouring sand of suitable shape.

Dry poultice.

Thick applications of absorbent or non-absorbent cotton, held in place by a bandage. 
Hot fomentations.

Cold.

(For technique, see text.)

Ice bag.

(For technique of application, see text.)

Ice poultice.

Drugs.

(For technique, see text.)

Ointments and liniments of doubtful value.

Camphorated oil (Linimentum camphoræ).

Belladonna ointment official, or

$\mathrm{R}$

Guaiacol, 1 part.

Vaseline, 10 parts.

Lanolin, 10 parts.

M.

Ichthyol ointment (in vaseline) 10 per cent. to 25 per cent.

Suppurating glands.

Should be incised.

\section{Complications.}

\section{Orchitis.}

Suspension.

Pillow stuffed between thighs.

Sheet of adhesive plaster across the thighs, on which scrotum may rest.

A " $T$ " bandage.

Dry poultice.

Abundance of non-absorbent cotton.

Hot lead and opium lotion on gauze or absorbent cotton.

Lead acetate, gr. cxx $(8 \mathrm{Gm}$.).

Tincture of opium, 3 ii (8 c.c.).

Water to make 1 pint (500 c.c.).

Keep bowels open.

Ovaries.

Hot applications to abdomen.

Mammary glands.

Much such measures as in orchitis.

Vulvitis.

Boric acid solutions 2 per cent. to 4 per cent.

Hot compresses.

Hot sitz baths.

\section{Pancreatitis.}

Limit fat in diet.

Apply heat or cold over site of pain. 
Nephritis. (See Scarlet Fever, Chap. XVII.)

Arthritis. (See Scarlet Fever, Chap. XVII.)

Nervous system.

Bromides gr. $\mathrm{x}$ to $\mathrm{gr} . \mathrm{xv}(0.60-1 \mathrm{Gm}$.) in water three times a day, gr. $\mathrm{xv}$ to $\mathrm{gr} . \mathrm{xxx}(1-2 \mathrm{Gm}$.) at night.

Meningo-encephalitis.

Lumbar Puncture. (For technique, see Cerebro-spinal meningitis, Chap. XXV.) Ice-bag.

Severe headache or delirium.

Morphine according to age.

(See Cerebro-spinal Meningitis, Chap. XXV.)

\section{Sleeplessness.}

Bromides gr. $x v$ to gr. $x x x(1-2 \mathrm{Gm}$.) in water at night.

Trional gr. $\mathrm{x}$ to gr. $\mathrm{xv}(0.60-1 \mathrm{Gm}$ :).

Pain.

Phenacetin gr. iii-v (0.20-0.30 Gm.).

Morphine gr. $1 / 10$ to gr. $1 / 8(0.006-0.008 \mathrm{Gm}$.).

Release from quarantine.

Ten days after disappearance of local trouble.

Convalescence.

Fresh air.

Good food.

Iron if tonic is needed.

Disinfection.

Clean and ventilate room. 


\section{CHAPTER XXIV}

\section{GLANDULAR FEVER}

THIs is essentially a disease of childhood, occurring only occasionally in adult life, and is characterized by glandular swelling especially of the neck.

It begins as a rule on the left side.

Axillary and inguinal glands may be involved and possibly some of the deeper sets.

Liver and spleen have been found enlarged in some cases.

The adenitis is a result of an acute infection but the nature of the infecting agent is not known.

Isolation. The disease is undoubtedly contagious, hence, the child affected should be kept from contact with the other children.

Suspects and children who have been exposed should be kept from susceptible individuals for a week or ten days, for the incubation period while still undetermined is believed to be about a week.

Room and Bed. If the patient be confined to the bed, and that is only when fever or complications are present, they should be selected as in other contagious diseases.

Diet. If there is anorexia or vomiting the food is not pressed until these symptoms have subsided, then the ordinary diet for febrile cases is allowed. See Chap. II.

Water is given freely.

Bowels. Calomel, a salt or castor oil should be given at first. If there is much nausea or vomiting calomel in divided doses may be used, e. g., gr. 1/10 (0.006 Gm.) every ten minutes for ten doses, followed in two hours by milk of magnesia 5 ss. to i (15-30 c.c.) or liq. magnesii citratis 5 iv to viii (120-240 c.c.) or if there is no nausea the salt alone or castor oil in doses of 3 ii to 5 ss. (8-15 Gm. or c.c.) according to age may be given.

Throughout this disease the bowels should have attention, an enema or mild saline like liq. magnesii citratis being given every other day.

Care of Mouth and Nose. Mild alkaline sprays and mouthwashes such as quarter strength Dobell's solution or 2 per cent. boric acid solution, or warm physiological salt solution may be used. 
There is likely to be a mild grade inflammation of the tonsils or phar$y n x$, but bearing no relation to the adenitis.

That the glands sometimes suppurate and that otitis, parotitis, nephritis has been known to occur, accentuates the necessity for care of the mouth, the possible source of such complications.

Toxemia. At the onset the usual malaise, headaches, pains and fever of an infection may occur. If these are marked small doses of acetphenetidin (phenacetin) gr. i to gr. ii (0.060-0.12 Gm.) every two to three hours or aspirin gr. ii to gr. v $(0.12-0.30 \mathrm{Gm}$.) or salicin gr. iii to gr. $x(0.20-0.65 \mathrm{Gm}$.) at the same intervals may be used to relieve discomfort.

Fever. The temperature is not high as a rule but may reach $104^{\circ} \mathrm{F}$. or $105^{\circ} \mathrm{F}$. Sponging with cool water may make the patient more comfortable.

Adenitis. The large tender glands are the "hall-marks" of the disease. They become obvious after twenty-four to forty-eight hours and increase to the size of pecan nuts, remaining discreet. As a rule they begin high up under the sterno-cleido mastoid of the left side spreading later to the right side. They cause the patient to hold the neck stiff and at times to experience some pain on swallowing; moreover, these glands are very sensitive to the touch. All the glands of the neck are likely to become involved, even "a fine network of glands about 1 c.c. in diameter, like the spots placed at the intersection of the strands of a large mesh veil, covers the entire lateral and posterior aspects of the neck," as Haas picturesquely puts it. Beside the superficial glands, cervical, axillary, inguinal, epitrochlear, the mesenteric causing pain in the region of umbilicus, bronchial and mediastinal perhaps causing the croupy cough, may be involved.

Relief of pain and discomfort may be sought in the measures applicable to adenitis from other causes, namely, heat, in the shape of hot fomentations (see Scarlet Fever, Chap. XVII) or poultices, or hot-water bag or cold in the shape of the ice-bag or cold compresses. (See Scarlet Fever, Chap. XVII.)

Ichthyol is a favorite application in 25 per cent. to 50 per cent. in vaseline.

Very rarely a gland may suppurate, when it is to be treated surgically by incision and evacuation of pus.

For glands which are slow in receding Haas recommends Fowler's solution (liq. potassii arsenitis) $\mathrm{m}$. ii to $\mathrm{x}(0.12-0.65 \mathrm{Gm}$.) in water three times a day.

Complications. Beside the occurrence of suppuration in an affected gland, otitis media and nephritis are the only serious compli- 
cations, fortunately rare. They are treated as under circumstances. (For nephritis, see Scarlet Fever, Chap. XVII; for otitis, see Scarlet Fever, Chap. XVII.)

The course is usually mild and although the glands do not resolve for two to four weeks the malaise and fever disappear after a few days, though slight recrudescences may occur with involvements of other glands or recrudescences of the process in the same glands or set of glands with febrile manifestation may be repeated several times. The case impresses one at times as a streptococcus invasion and certain bacterial findings in the throat and suppurating glands strengthen the impression. Again the condition is at times difficult to differentiate from mild scarlet fever for erythematous rashes in glandular fever are occasional happenings. The late glandular relapses and nephritis increase the perplexity of the diagnostician.

Convalescence. Severe secondary anemia may occur and in severe cases may be quite striking. Iron may be administered for this.

In the younger children the bitter wine of iron (Vinum ferri amarum) $3 \mathrm{i}$ to 3 ii (4-8 c.c.) three times a day, or in older children Vallet's mass (Massa ferri carb.) gr. ii to gr. $\mathrm{v}(0.12-0.30 \mathrm{Gm}$.) three times a day, or Blaud's pill (Pil. ferri carb.) in same doses.

As a general tonic small doses of the tincture of nux vomica, $\mathrm{m}$. ii to $\mathrm{m} . \mathrm{x}(0.12-0.65$ c.c. $)$ or strychnine sulphate $\mathrm{gr}$. 1/150 to gr. 1/60 (0.00045-0.001 Gm.) three times a day according to age.

Fresh air and good food are the best tonics.

Prognosis is good. The only cases to be dreaded are those complicated by Nephritis.

Prophylaxis. We know so little about the mode of conveyance of this disease and indeed of its true nature that we are unable to effect efficient prophylactic measures beyond isolation of the sick child in the family.

\section{Treatment.}

\section{SUMMARY}

Isolation.

The child should be kept from contact with other children.

Suspects and children who have been exposed should be kept from susceptible individuals, a week or ten days.

\section{Room.}

Light and airy.

Diet.

Do not force feeding during period of anorexia.

Later follow rules in Chap. II.

Give water freely. 
Bowels.

Calomel gr. 1/10 (0.006 Gm.) every ten minutes for ten doses, followed by

Milk of magnesia $₹$ ss.-i (15-30 c.c.) or

Liquor magnesii citratis $5 \mathrm{iv}$-viii (120-240 c.c.) or if there is no nausea

A salt alone, or

Castor oil 3̈̈i-iv (8-15 c.c.).

Enema or mild saline every other day.

\section{Mouth and throat.}

Washes and sprays of

Two per cent. to 4 per cent. boric acid solution.

Dobell's solution, quarter strength.

Warm physiological salt solution (3i of common salt to 1 pint, $4 \mathrm{Gm}$. to 500 c.c.).

\section{Toxemia.}

Pains and aches of sthenic period.

Phenacetin gr. i-gr. ii (0.060-0.12 Gm.) every two or three hours or

Aspirin gr. ii to gr. v (0.12-0.30 Gm.) at same intervals or

Salicin gr. iii to gr. $x(0.20-0.65 \mathrm{Gm}$.).

\section{Fever.}

Sponging with cold water.

\section{Adenitis.}

\section{Heat.}

Fomentations.

Poultices.

Hot-water bottle (See Scarlet Fever, Chap.XVII.) Cold.

Ice-bag.

Cold compress.

Ichthyol 25 per cent. to 50 per cent. ointment over the affected glands.

\section{Surgery.}

Incision of suppurating glands.

Delayed resolution.

Fowler's solution (Liq. potassi arsenitis) $\mathrm{m}$. ii to $\mathrm{m} . \mathrm{x}(0.12-0.65$ Gm.) three times a day.

\section{Complications.}

Rare.

$\left.\begin{array}{l}\text { Otitis. } \\ \text { Nephritis. }\end{array}\right\}$ (See Scarlet Fever, Chap. XVII.) 
Convalescence.

Anemia.

Iron.

Vinum ferri amarum (Bitter wine of iron) $3 \mathrm{i}$ to $3 \ddot{\mathrm{ii}}$ (4-8 c.c.) three times a day for young children.

Vallet's mass (Massa ferri carbonatis) gr. ii to gr. v (0.12-0.30 Gm.) or

Pil. ferri carbonatis (Blaud's) same doses.

Tr. nucis vomicæ $m$. ii to $m$. $x(0.12-0.65$ c.c. $)$ three times a day or

Strychnine sulphate gr. 1/150 to gr. 1/60 (0.00045-0.001 Gm.) three times a day.

Fresh air.

Good food.

Prophylaxis.

Isolation of sick child. 


\section{CHAPTER XXV}

\section{CEREBRO-SPINAL MENINGITIS}

A GENERAL infection, due to a definite organism, the diplococcus of Weichselbaum (diplococcus intracellularis meningitidis), the characteristic symptomatology of which is referred to the site of its greatest local activity, the meninges of the brain and spinal cord.

It is one of the few diseases for which a specific treatment has been achieved and for this reason an etiological diagnosis is important, that is, the recognition of the causal organism, which may be recovered from the blood before it appears in the spinal cord or in some instances before there are cerebro-spinal symptoms. Indeed, in the presence of an epidemic, cases of meningococcic septicemia have been determined which never developed cerebro-spinal symptoms even though no intraspinal therapy had been used.

It is now recognized that there are three groups of meningococci, the normal or regular, the para or irregular and the intermediary group which cross agglutinates with the other two. The importance of this lies in the appreciation of the fact that unsatisfactory serum therapy may be due to an insufficient representation of the immune bodies produced by a certain group in the polyvalent serum which is used.

Distribution of the Family. One has to consider the possibility of infection of other members of the family and the danger which other members of the family may be, in the capacity of carriers, to those outside.

So rarely did fresh cases break out in the wards of hospitals admitting these cases and so rarely did physician or nurse acquire the disease from the patient, that for a long time it was assumed that the disease was not contagious and even to-day such facts as these and that in large epidemics, in the vast majority of instances, only one member of a family is affected is hard to understand, but more careful study and experimentation has demonstrated the contagiousness of the disease and the important rôle of the carrier. It is because of the high mortality of the disease and the dread possibilities even in case of survival, that Public Health authorities have included it among the contagious and reportable diseases and it becomes incumbent upon us to pursue every means to prevent our patient from being the source of yet another case.

The insistence on that isolation that has become habitual in the case 
of scarlet fever has not yet been voiced by writers on the subject, but it seems to me such isolation is as imperative in the case of the one as the other, for the high mortality and the wretched sequences of the one balance the high incidence of the other.

The patient, then, should be insolated with nurse or attendant.

Other children should be removed, if possible, and to quarters where they shall not come in contact with children, for though they may not develop the disease they may still be carriers of the infection capable of infecting others.

How long these "contacts" should be isolated is a difficult matter to decide. The incubation period has not been very well determined, but the average time seems to be 7-14 days.

However, some of the observations suggest that in some cases, at least, it may last a month, though usually it is a much shorter period. If laboratory facilities are at hand suspects should have their nasal secretions examined to determine whether or no they harbor the diplococcus in the nasal passages. If the children cannot be removed, communication direct or indirect with the patient must be carefully avoided. Adults, though still susceptible, are far less so than children, but, with the exception of those members of the family whose presence in the sick-room is necessary to the patients, no contact with the patient should be allowed.

Adults in the family must remember that infection of fresh cases has occurred curiously frequently through a third person; hence, they should avoid contact with children. If the adults come in contact with children in the course of their daily duty, they should remove from the environment of the patient and observe a period of isolation, as in the case of the children cited above.

In all cases where possible these "contacts" should have their nasal secretions examined to determine whether they are "carriers" or no, and, if so, should avoid children at least as long as the epidemic lasts. Indeed with knowledge that the carrier is a menace to whomsoever he comes in contact with, it would seem reasonable to insist on an isolation of such carriers until they are free from the infection. This was the rule insisted on in camps during the recent war.

The number of people who in contact with the sick become "carriers" is surprisingly large, Elser and Huntoon, during an epidemic, determined that at least 70 per cent. were in this class.

Apart from the epidemic the "carriers" were rare, but occasionally a person becomes a permanent "carrier" and can convey the disease.

That so few of the persons harboring the organisms in this manner become themselves the victim of the disease would bespeak the prob- 
ability of some "bther factor in the determination of the infection and with our present views of infection, we seek it in the lowering of the individual's resistance. For this reason, it becomes doubly important to avoid during an epidemic minor infections, such as rhinitis, tonsillitis, etc., at other times trivial, lest it afford the opportunity for the diploccus of Weichselbaum to gain entrance into the blood.

"Contacts" should use a mild spray for the throat and nose, such as 2 per cent. boric acid or Dobell's solution, quarter strength, or one of the mild equivalents on the market. Under no circumstances should strong astringents or strong bactericidal solutions be used, lest the irritation set up in the mucous membrane lessen the resistance of the structures to the invasion of the infecting organism.

Nurse. The nurse from her intimate contact with the patient is very likely to become a carrier. For this reason she should avoid coming into contact with children during her hours off duty and remember that coughing, sneezing and kissing are means by which the organism may readily be conveyed to another.

She should clean her hands and face with soap and water and alcohol or bichloride 1 to 1,000 and spray her nose and throat before going out. As a precautionary measure she should use one of the mild sprays advised above from time to time while on duty.

Physician. The physician has always to think about the possibility of carrying the disease; but, fortunately, it is rarely that he can be shown to have done so. The organism is readily killed by drying and exposure to sunlight and the physician's contact with the patient is brief. He should, however, carefully.cleanse his face and hands with soap and water and alcohol or 1 to 1,000 bichloride and spray his throat and nose before leaving the case for another call and should make his stay with the patient as brief as is compatible with the performance of his duties. He should, moreover, spend some period in the open air before another visit is made and endeavor not to make his next call upon a child.

Room. A large well ventilated room, free of furnishings, with bare floors or floors covered with some cheap material which may be destroyed after the patient has been removed, such as carpet lining covered with unbleached muslin, should be chosen. A verandah leading from the room and a bath room immediately contiguous are advantages. Light and air are potent allies in destroying the infection.

If the light hurts the eyes, suitable screens may be arranged to protect the patient.

Avoidance of noise, jars, irritations of all kinds are to be observed on account of the marked hypersensitiveness of the patient. 
Precautions in the Sick-Room. Since the infective material is known to reside in the nasal and oral secretions, all these should be received on rags or muslin that may be destroyed or into vessels in which they may be disinfected with carbolic 1 in 20 or bichloride of mercury 1 in 500 .

The thermometer should be left in the sick-room and kept in carbolic 2 per cent. to 5 per cent. or in formalin.

Eating utensils should be boiled, but if sent out of the sick-room for that purpose should be previously soaked in 1 to 20 carbolic acid for twenty minutes to a half hour. Urinals, bed-pans, etc., should be disinfected with 1 to 20 carbolic (phenol) or 1 to 500 bichloride, while clothes should be soaked overnight in 1 to 50 to 1 to 20 phenol and then boiled a half hour to an hour before being put in the family wash.

Bed. The bed is to be prepared in the usual way. It is doubly imperative to avoid wrinkles, crumbs, dampness of the sheets from secretions, because of the tendency to form bed-sores in this condition. If the emaciation is marked, the nutrition of the skin badly impaired or any hint of a bed-sore observed, it is well to have a water-bed or air mattress.

The extreme sensitiveness of the patient to cold makes a demand on more clothing than in other febrile conditions.

The Patient. Much depends on the skill, fidelity and patience of the nurse in attendance. The demands are often continuous and insistent, the pains and discomforts sacrifice rest and sleep and so strength. For this reason and because of the long course many of the cases take, all should be done to economize the strength and health of the nurse, in the choice of a room, the nearness of the bath room, the quiet and seclusion of her own rest hours. Two nurses should be on the case, one for night and the other for day.

The patient should wear a nightgown of light flannel or if this is irritating, cotton. It should be open all the way down in front, to make the necessary examinations possible with the least disturbance to the patient.

Diet. During the early hours or days of the infection when the fever is high and the evidences of intoxication acute, the feeding is not to be urged against the anorexia, but as the disease is peculiarly associated with emaciation and is likely to be long drawn out, the feeding after the first few days, even in the presence of fever or stupor is to be increased, given at regular two and three hour intervals.

Often at a later stage the appetite is keen, if not ravenous, and it should be indulged abundantly.

At first the food should be liquid, milk or one of the milk prepara- 
tions, such as buttermilk, koumys or if there is any gastric disturbance, skim milk, whey or peptonized milk.

Cereal gruels or well cooked cereals and farinaceous foods, such as arrowroot, barley, rice, cornstarch, farina, imperial granum, cornmeal, oatmeal, if it occasions no gas formation, jellies of barley flour, tapioca, sago, milk toast, veal, mutton, chicken or beef broths, may be used. These may be thickened with barley or other flour. Later, ice cream, eggs, custard, potatoes, oysters, scraped meat, puréed peas, small string beans or spinach and asparagus tips.

In infancy the modification of the milk is to be changed to a somewhat greater dilution.

In stupor or in case the patient cannot swallow, the feeding must be by gavage or nasal feeding; it is well to change occasionally from one to the other. As a rule in the older patients the oral route is preferable, while in children the nasal is the better.

Drinks. Water, fruit juices in water, carrying sugar, not merely to sweeten but to add materially to the food ingested, are administered.

It cannot be too much emphasized that the drinks should be pushed. The demands of the tissues are great and the apathy of the patient defeats these demands unless the nurse offers fluids frequently and urges the patient to drink.

The feeding should be at intervals of two to three hours and as the case becomes subacute, some definite idea of the actual amounts of heat units ingested and energy needs of the case must be kept in mind. (See Chap. II.)

Skin. A cleansing bath of warm water and soap should be given each day. Bed-sores are prone to occur. The emaciation accentuating the bony prominences makes it imperative to relieve the pressure upon these points by frequent change of position, mechanical devices such as rings and cushions, by keeping these parts scrupulously clean and dry and by frequent rubbing to keep the circulation active in the skin and by the use of alcohol rubs and talcum powder, by avoiding wrinkles in the sheets, crumbs in the bed and by most gentle handling. If sores are imminent the air mattress or water-bed should be used and increasing care given the threatened parts.

Mouth and Nose. In the care of the mouth and nose in this disease two things are to be kept in mind: first, the comfort and welfare of the patient; second, the danger his secretions entail to others.

The mouth should be cleansed by having the patient rinse his mouth with plain water, followed by 2 per cent. to 4 per cent. boric acid solution or Dobell's solution, half to quarter strength, used as a mouth wash or spray. After eating, the nurse should see that all dead spaces in 
the mouth and interstices of the teeth are freed from food particles by the use of small cotton swabs on wooden tooth picks or other simple applicator, wet with the same solution.

If there is sordes on lips and teeth or the tongue is heavily coated, half strength official hydrogen peroxide may be applied to soften the deposit and in case of the tongue the edge of a whalebone or equivalent instrument may be used as a scraper, then followed by the solutions mentioned. For a dry mouth equal parts of 2 per cent. boric acid solution and albolene with a little lemon juice is excellent.

The nose may be sprayed by the same solutions.

All secretions should be destroyed, preferably by burning, and danger in handling these secretions should be kept in mind by the nurse, who should carefully wash her hands and have recourse to an antiseptic, such as alcohol or 1 to 1,000 bichloride after the ministrations.

Eyes. There is likely to be a conjunctivitis of mild or moderate grade. It is believed that these secretions, too, are infectious and for that reason they should be destroyed with the same care as in the case of nasal secretions. For the eyes nothing is better than mild boric acid washes of 2 per cent. strength.

Bowels. The bowels should be freely opened at the beginning of the illness and attention given them throughout the course.

For an initial catharsis, calomel in doses of gr. $1 / 4(0.015 \mathrm{Gm}$.) every quarter hour for four or five doses, followed in two or three hours by milk of magnesia in dose of 5 ss. ( 15 c.c.) is suitable and is especially good in cases of vomiting, in which the divided doses of calomel is credited with an antiemetic action and the milk of magnesia acts as a gastric sedative. If there is no vomiting, castor oil 3 ii to iv (8-15 c.c.) or Rochelle salt, 3 ii to iv (8-15 Gm.) may be used.

Later, milk of magnesia, 3 ss. (15 c.c.), Liquor Magnesii Citratis, $\zeta$ iv to viii (180-240 c.c.), Hunyadi water, 3 iii to vi (90-180 c.c.), or cascara preparations may be given, or an enema may effect the purpose. If there is much vomiting during the course, the enema will be the better choice of methods. If hyperæsthesia is very marked, the oral administration may be less annoying.

Treatment of Nausea. Almost invariably vomiting occurs with the onset of the disease. Sometimes it continues for days. It is also a feature of the long-standing cases.

Not only is the vomiting exhausting in itself, but it makes feeding difficult. The vomiting is probably central and may occur without nausea. Measures directed at the stomach itself would seem then to promise little unless some gastric irritability in addition existed. We may, however, try such expedients as cracked ice, a mustard paste, one 
of mustard to four of flour, to the pit of the, stomach, sodium bicarbonate gr. $\mathrm{x}-\mathrm{xv}(0.60-1 \mathrm{Gm}$.$) in a little water, bismuth subnitrate gr.$ $\mathrm{x}-\mathrm{xv}(0.60-1 \mathrm{Gm}$.) stirred in a little water, or cerium oxalate gr. iii to $\mathrm{v}$ $(0.20-0.30 \mathrm{Gm}$.) in the same way, or all in combination, bromides in 5-15 grain (0.33-1 Gm.) doses.

Feeding is sometimes possible by the stomach tube, when otherwise rejected.

\section{Treatment of symptoms.}

As one might anticipate from the nature of the lesion, symptoms referable to cerebral irritation are dominant.

Restlessness, delirium and sleeplessness demand attention.

The application of the ice-cap or ice-coil is a helpful measure and the sedative effects of warm sponge baths are to be kept in mind.

Of the drugs in the milder cases one has recourse to bromides, for the more severe, codeine and morphine or even hyoscine hydrobromide in doses determined by age and severity, e. g., bromides gr. iii-v $(0.20$ $0.30 \mathrm{Gm}$.) three times a day at one year; gr. $\mathrm{v}-\mathrm{x}(0.30-0.60 \mathrm{Gm}$.) at five years; for adults gr. 15-20 (grams 1-1.3).

Codeine sulphate gr. 1/60-1/30 (0.001-0.002 Gm.) at one year and gr. $1 / 10-1 / 5(0.006-0.012 \mathrm{Gm}$.) at five years; for adults gr. 1/8-1 (0.0080.060 gram $)$ or in severe cases morphine gr. 1/200-1/100 (0.0003-0.0006 Gm.) at one year to gr. $1 / 30-1 / 20(0.002-0.003 \mathrm{Gm}$.) at five years. For adults gr. 1/8-1/4 (0.008-0.004 grams).

Hyoscine hydrobromide to a child of five years may be given if above drugs fail, in doses of gr. 1/800-gr. 1/400 (0.000075-0.00015 Gm.); gr. $1 / 200-1 / 100$ (0.0003-0.0006 gram) to an adult.

Convulsions. Slight twitchings may be controlled by the use of chloral, best given by the rectum in small amounts of warm milk reinforced by bromides by mouth or rectum.

Severe convulsions require morphine.

The circulation, attacked by the toxins of the disease and feeling the effects of their action and that of intracranial pressure on the medullary centres, needs support and yet its impairment is not, as a rule, as great as one might anticipate.

For continuous support we rely on digitalis or strophanthin or for immediate demands on caffeine.

Do not use strychnine on account of its exciting effects on motor centres.

Dose. In adults digitalis is given in the form of the infusion 3 ss. (15 c.c.) or $\mathrm{m} . \mathrm{xxx}$ of the tincture $(2$ c.c.) three times a day for three or four days, or in emergency strophanthin gr. 1/120-1/90 (0.0005-0.00075 $\mathrm{Gm}$.) into vein or muscle, followed by digitalis as mentioned. Caffeine 
in the form of a soluble salt gr. v (0.30 Gm.) every four to two hours. Children are given smaller doses according to age or weight.

It is believed by certain investigators that brain volume may be lessened by the use of hypertonic glucose solution. If this be true those symptoms referable to increased intracranial pressure, such as restlessness, delirium, sleeplessness and to a certain extent circulatory disturbances should be relieved by the measure. They advise giving intravenously a 25 per cent. glucose in the amount of 250 c.c. taking one hour for the administration. The solution should be sterilized in an autoclave. This injection is to be repeated at 12-hour intervals until evidence of reduction of intracranial pressure is observed.

Bladder. Distension is likely to occur in the stuporous and incontinence is likely to mean an overflow.

Evidence of such a state of affairs is to be sought for by palpation and percussion and efforts at relief afforded by the application of hot stupes over the hypogastrium or if this fails by the use of the catheter.

Drug Treatment. There is no drug that can be called specific; though urotropin has been greatly recommended with the hope that its presence in the cerebro-spinal fluid (which is demonstrable) might prove bactericidal. Our knowledge of the action of urotropin, however, points to its efficiency as a bactericide only in the presence of an acid medium. It probably has no action whatsoever in the alkaline cerebro-spinal fluid. It is still recommended but with diminishing fervor.

Specific Treatment. Upon the determination of the diplococcus intracellularis meningitidis as the causative agent of the disease, there followed efforts to find a specific substance to combat it and its effects. The fact that the toxic substance of the organism was found to belong to that class of poisons known as endotoxins, i. e., toxins bound up in the body of the organism itself and not excreted by it as is the case with the diphtheria bacillus, and the fact that antitoxins to this kind of toxin have not been determined made the outlook for success in this direction no better than in the case of other diseases due to endotoxin-bearing bacteria, such as pneumonia, streptococcæmia, etc.

The effort was made, however, to obtain an active serum by the immunization of animals with the meningococcus and as the first efforts at treatment followed the usual lines of subcutaneous or intravenous injections, disappointing results ensued, until the suggestion to inject the serum into the spinal canal reaped a rich reward.

In spite of theoretical considerations the serum does seem to have some antitoxic power, but in no sense owes the total of its efficacy to this. What it does seem to do is to inhibit the growth, induce the death 
and initiate the disintegration of the diplococcus, while at the same time it has opsonic qualities, encouraging phagocytic activity.

Mode of Preparation. The serum is obtained by injecting into animals (the horse is the animal now preferred) at weekly intervals, increasing quantities of the meningococcus. At first dead bodies are used and it is found that better results ensue if the autolysate (products of self-digestion) of the organism is used alternately with the diplococci themselves. The whole course takes from four to six months. Later the live diplococci take the place of dead ones.

Blood is then withdrawn from a vein, the cells separated from the serum and the latter preserved for use.

Dosage. The dosage is limited only by the capacity of the spinal canal. The dose may be taken at 30 c.c. with these modifications: (1) That if more than this amount of cerebro-spinal fluid can be withdrawn from the spinal canal, an equal amount of the serum should replace it. (2) That if less than 30 c.c. is withidrawn from the canal, the dose should approximate 30 c.c. as near as possible without inducing pressure symptoms or rather without forcing against rapidly increasing resistance. Doses less than 30 c.c. are much less efficacious.

Sophian as a result of his studies lays great stress on the blood pressure. He takes a fall of $20 \mathrm{~mm}$. as an indication for halting the injection. Then he waits a few minutes and tries again; if further fall occurs he stops, as he has noted that after a fall of $20 \mathrm{~mm}$. a further fall is apt to be precipitate and dangerous. He gives the average dose as controlled by blood pressure to be

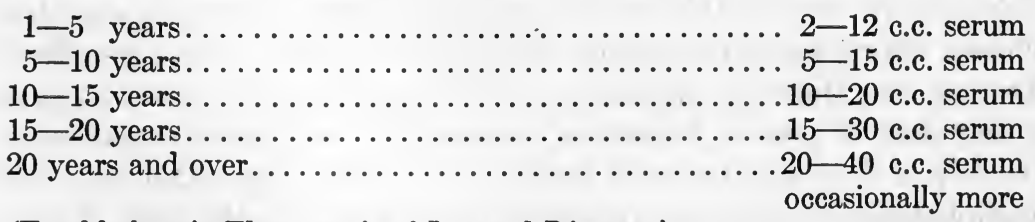

(Forchheimer's Therapeusis of Internal Diseases.)

This precaution of Sophian's is rarely observed in practice and experience does not emphasize the necessity.

Frequency. The dose should be repeated each day for three or four days or longer; longer if the diplococci are found on the stained spread of the cerebro-spinal fluid withdrawn to be still persistent or if active symptoms of the disease continue, even if the fluid seems free from diplococci. It is only in the exceptional case, whose symptoms yield promptly and in which the diplococci have disappeared that fewer doses are permissible.

Normal cerebro-spinal fluid reduces Fehling's solution. In cerebro- 
spinal meningitis this reduction does not occur. With an amelioration of the condition this reduction gradually returns and excellent observers have come to look upon its return as one of the indications for discontinuing the administrations of the serum. It may be added that this loss of reduction of Fehling's solution occurs in some of the other acute inflammations of the spinal cord.

Results. The results are seen both in the amelioration of symptoms and in the change wrought in the cerebro-spinal fluid.

In the latter the diplococci free in the fluid disappear, then the intracellular organisms undergo change, that retard or prevent their growth on ordinary media, interfere with their staining properties, then disintegration and autolysis take place with diasppearance of the bacteria. The cerebro-spinal fluid, itself, becomes clearer; the pus cells disappear, Fehling's solution is again reduced, while in the blood the leucocytosis makes way for a normal count.

The general symptoms show improvement in the lessening of the nervous manifestations, such as delirium, in a lowering of temperature and better circulation and respiration. The improvement is more often gradual, the temperature going down by lysis, but a large per cent. of the cases show a true crisis in the fever and a precipitate improvement in the other symptoms. The disease is shortened by the use of the serum, complications are less frequent and serious sequelæ are lessened.

Bad Results. A disagreeable rather than a dangerous result is serum sickness which occurs much as after diphtheria antitoxin is administered. (See Diphtheria, Chap. XVIII.)

The more dangerous accidents enumerated above, including collapse, have been attributed to anaphylaxis, rapid lysis of the cocci, the effect of the preservative trikresol and increased intracranial pressure. Worth Hale's studies attributed the dangerous results to the trikresol used to preserve the serum as well as to intracranial pressure, but Flexner believes the accidents in the use of serum cannot be attributed to anaphylaxis nor to the preservatives and would attribute them all to the increased intracranial pressure.

Lumbar Puncture. This procedure is absolutely essential to the diagnosis and to the serum therapy and in addition affords no small measure of relief from certain of the symptoms.

It is an exceedingly simple procedure and practically free from danger, but is often approached by him who undertakes it for the first time with trepidation and seems to the parent who witnesses it an heroic undertaking, both of which facts make for delay in this urgent measure.

Site. The site of puncture is determined by a line drawn across the spine at a level with the highest points of the crests of the ilia; a tape 
or a string stretched between these points gives the point on the spine. The spine of the vertebra nearest this point is the fourth. The point of insertion of the needle is between it and the vertebra above, that is, between the third and fourth or between it and the vertebra below, that is, between the fourth and fifth.

In children choose the exact mid-line; in adults choose a point $3 / 8$ to $1 / 2$ inch to the right or left of the mid-line, directing the point of the needle inward, i. e., towards the mid-line, going in between the laminæ. This rule is often violated, the mid-line being chosen, but the likelihood of breaking off the needle and danger of not getting into the canal is enhanced by so doing.

Position of the Patient. The patient should lie on his side at the edge of the bed or table with his back towards the operator. An assistant then bends the child forward, by gently pressing the head down and the knees up so that the spine curves boldly outwards, thus separating the spines of the vertebræ from each other and making the puncture easier. This position is preferable to the sitting posture chosen by some operators.

Preparation of the Patient. The skin all about the site of puncture should be cleansed with soap and water and then with alcohol, or one may paint the site of puncture with tincture of iodine after a preliminary cleaning with alcohol.

Preparation of the Operator. The hands should be cleansed with the same precautions as for a more considerable operation; thoroughly with soap and water, followed by alcohol or one may use sterile gloves.

Preparation of the Apparatus: All the apparatus used should be rendered sterile by boiling. Appropriate needles or trochars and canulas, a syringe and test-tubes constitute the paraphernalia.

Anæsthetic. It is better to do without an anæsthetic. The pain is but slight, as patients have testified, and the use of an anæsthetic complicates the procedure. If an older child or adult is fearful of pain or the parents insist, a local anæsthetic in the shape of ethyl chloride to freeze the site of puncture, or cocainization with 1 per cent. to 2 per cent. cocaine is to be preferred to general anæsthesia; if this may not be employed, a few whiffs of chloroform may be given. Deep anæsthesia is entirely unnecessary and adds discomforts and dangers.

Needle. One may use a needle or a trochar and canula. The needle should be stiff of a fairly good calibre. The exploratory needles used for paracentesis of the thorax or other cavity or antitoxin needles, the bore and length depending on the age of the patient, may be used, or a trochar and canula or a special lumbar puncture needle, after the pattern of Quincke. 
Needles are now supplied that bend but do not break, which avoids an embarrassing accident.

Needles of large calibre leave a wound from which the fluid continues to leak and increases the danger of infection. I have found the exploratory needles more satisfactory than trochar and canula.

The Tap. When the point of entrance has been determined, it is well to use the thumb of the left hand, with the edge of the nail on the upper border of the lower vertebra bounding the space as a guide and push the needle along the back of the nail firmly and slowly until a lessened resistance tells of entrance into the space, of which the flow of fluid is corroborative, when the open end of the needle is released or the trochar is withdrawn. Care should be taken not to rudely push the needle across the cerebro-spinal cavity against the body of the vertebra as bleeding ensues and interferes with an examination of the fluid.

The depth to which the needle is carried is in children about an inch. In adults it is considerably more and the needle should be at least 4 inches long.

The flow may be drop by drop, but is usually continuous or may spurt in a stream, depending on the pressure and the bore of the needle.

The amount varies from a few c.c. to 50 or 60 c.c. or more. Twenty to 30 c.c. are common figures. (Normal infants 10 to 20; older children 15 to 25 . Pfaundler.)

Collecting the Fluid. One should have test-tubes 15 to 20 c.c. at hand, sterile or rendered sterile by boiling with the needles and syringe. The open end of the tube is put under the flow and filled threequarters full, leaving room to introduce a sterile cotton plug without touching the fluid. If the fluid runs a little bloody at first this tube as soon as the fluid runs clear, makes way for another tube to catch the clearer fluid. Let all run that will, eatching in several tubes.

Sophian takes the blood pressure during the tapping and if it falls $10 \mathrm{~mm} . \mathrm{Hg}$. he discontinues the flow.

After plugging the tubes they should be set upright and left undisturbed until examined.

Appearance of the Fluid. The fluid should be purulent or turbid, but the rule is not invariable. When the case is one of meningitis clinically, a fluid which is purulent or turbid indicates serum at once without awaiting the examination of the fluid. Again if the fluid is bloody and obscures the appearance of the fluid the serum should be given.

Only in case the fluid is watery clear should one await for the results 
of an examination, for in this case it is in all probability a case of tubercular meningitis, serous meningitis or poliomyelitis.

In other words always give the patient the benefit of every doubt in giving him the serum, for it is in itself harmless.

Injection. The injection should be by the gravity method. It should be made very slowly. The container is filled with serum, which should be warmed to about the temperature of the blood, the air expelled, and then connected with the needle; the patient watched carefully for evidences of collapse, poor pulse, cyanosis, embarrassed breathing, upon the appearance of which some of the serum should be allowed to flow back into the apparatus until improvement occurs. It is a wise precaution to wait a few minutes after the injection of the serum before the needle is withdrawn so as to make unnecessary another puncture if signs should develop. After the needle is withdrawn, remove the iodine from the skin with alcohol, apply sterile gauze to the puncture, affixing it by adhesive strips.

Some clinicians deem they facilitate the diffusion of the serum in the cord by raising the foot of the bed.

Special gravity apparatus is on the market or one may use the barrel of a 15-25 c.c. syringe as a funnel, to which is attached a 12-14 inch long rubber tube about $1 / 4$ inch in diameter with metal end piece to fit end of needle (Sophian).

This method has replaced the older syringe method, as it is difficult to produce a trauma of the cord by it as the inflow is more even and slower and if bad symptoms arise, the fluid can be drawn off by simply lowering the container.

If breathing is bad, artificial respiration may be instituted.

Atropin sulphate in doses gr. 1/80 to gr. 1/50 (0.00075-0.0012 Gm.) and cocaine hydrochloride gr. $1 / 8$ to gr. $1 / 4(0.008-0.015 \mathrm{Gm}$.) is advised (Sophian).

Reaction. Headache or pain in the back and limbs may ensue or on the other hand the patient may be rendered more quiet, but as a rule no bad reaction follows.

Headache may be relieved sometimes by raising the pillow. If it is very severe morphine may be used.

Dry Tap. Dry taps are usually due to failure in technique. For this reason all preparations for puncture should be carried out with precision; the patient held firmly in the position desired, the landmarks carefully determined and the thrust made firmly with constant regard to the direction. With repeated punctures the patient becomes more alarmed and the whole procedure made more difficult. It means in the great majority of cases that the canal was not entered. If one 
feels sure that he has entered the space and no fluid is obtained, he may introduce a sterile wire through the needle to clear it from a plug of tissue that may be occluding it and if not successful in this manœuvre, withdraw the needle and go in a space above or below.

The dry tap may be due to inspissation of the fluid. Raising the patient to a sitting position may initiate the flow; gentle suction of the syringe may be tried or a little salt solution or a little serum may be gently forced in or the use of two needles in adjacent spaces with saline irrigation between them has been advised.

In true dry tap one may force much smaller amounts in at more frequent intervals. No great pressure should be used.

In this city the serum is now prepared by the Board of Health (New York).

The Possible Bad Results. The statement made that the procedure practically entails no danger it is thought by some should not be taken too literally. Wegeforth and Latham contend that a puncture should not be lightly undertaken as they say' "The two cases just recorded indicate the possibility of the removal of cerebro-spinal fluid acting as a factor facilitating the infection of the meninges from the blood stream." They contend that in cases of septicemia suspected of being meningococcic, an examination of the blood should be made to determine the nature of the infecting organism and if the invading organism be found to be a meningococcus that an effort should first be made to sterilize the blood stream by the use of the antimeningococcic serum before the cerebro-spinal system is involved. This has been done on several occasions and by different workers. They would delay lumbar puncture, then, until the clinical symptoms of invasion of cerebro-spinal system is sufficiently definite before going into the cord. If the blood stream shows no infection at all the risks of puncture are diminished. Even then they advise that minimal quantities of fluid be withdrawn for laboratory tests with small bore needles so that the pressure relations within the cerebro-spinal cavity be disturbed as little as possible.

Another unhappy result that follows, especially on too frequent tappings, comes from traumatism done by the needle and serum to the cord and meninges, setting up a myelitis and involvement of the nerve roots, which affords an explanation of the pain and stiffness of the back and legs, so often felt, atrophy of certain muscles and vasomotor disturbance.

Early Usage. There is no one fact more emphasized by statistics than that every hour of delay in the usage of the serum adds an appreciable percentage to the mortality. Flexner and Jobling, analyzing over 350 cases, show that when the serum was administered 
on the first to the third day, in 123 cases the mortality was 16.5 per cent., on the fourth to the seventh day in 126 cases the mortality was 23.8 per cent., and later than the seventh day of 112 cases the mortality was 35 per cent.

Intravenous Treatment. The intensive study of cases in camps during the late war has made it more and more probable that the disease instead of localizing itself in the brain and cord primarily is a septicemia in the beginning, localizing itself secondarily in the cerebro-spinal system. Herrick was able to get positive blood cultures in about $1 / 3$ of his cases and claims that when the best laboratory technique is available these figures rise to 50 per cent. and even to 80 per cent.

In 45 per cent. of his cases he was able to recognize the infection before the meningitis occurred and this stage of premeningitic generalized infection he found to vary between a few hours and several days, the average 48 hours.

The diagnosis of these early cases is, of course, materially aided by the presence of an epidemic. The patient looks and feels ill, not infrequently there is an early tonsillitis or laryngitis of varying degrees of severity; there is a moderate fever and the patient complains of a severe headache; this, however, is not constant. More significant is the apathy and emotionless expression of the patient. The conjunctivæ are reddened, often strikingly inflamed, and the organism may be recovered from the secretions. Of diagnostic significance is the petechial rash, coming out rapidly in crops. This is seen on the trunk and less constantly on the extremities, face, conjunctivæ and in the mucous membrane of the mouth. These may be few and discrete and give the appearance of an extensive purpura.

There may be at this early stage of the infection none of the signs of brain and cord involvement other than may be attributed to any severe infection and some inequality and irregularity of reflexes and the spinal cord fluid shows no more than one finds in other acute infections. Blood cultures yield the specific organisms in the proportions cited. Herrick's plea for the prompt and efficient use of the serum by the intravenous route is logical and the following is largely abstracted from his article in the Journal of the A. M. A., Vol. 71, No 8, Aug. 24th, 1918, p. 612.

The patient is first given a desensitizing dose of 1 c.c. of the serum subcutaneously; an hour later an intravenous injection of antimeningococcus serum is given. As a further precaution to avoid anaphylactic reactions the first 15 c.c. are given very slowly at the rate of 1 c.c. a minute, the rest more rapidly. The dose is 80 to 150 c.c. serum, depending on the degree of toxicity, but the larger doses are always the safer.

If during the injection any untoward symptoms appear the injection 
is immediately intermitted. These symptoms are pallor or cyanosis, weak, rapid and irregular pulse or dyspnœa or vomiting.

After two or three hours it is perfectly safe to make another effort and success rarely fails. Even patients sensitized by previous serum may be so handled. Herrick advises atropine and morphine before the administration or at any rate if symptoms cited occur. Epinephrin, too, is of value. (See serum treatment of Pneumonia, Chap. IX.)

Frequency. In severe cases the serum should be repeated every 8 to 12 hours throughout the acute stage of 2 to 4 days, in milder cases every 24 hours.

In Herrick's experience the average number of injections given were between 4 and 5 and the total serum 400 c.c. He gave one patient 12 doses, totalling 1050 c.c. Success may in a measure be checked up by blood cultures, due allowance being made for imperfections of technique. If on the evidences of septicemia a puncture of the spinal cord is made, which is the rule, it seems to me that a small needle should be used and only such amounts withdrawn as will establish diagnosis.

Wegeforth and Latham would have us puricture the cord only when signs of meningitis have developed and after intravenous injections of serum.

Herrick puncturing early and finding clear fluid lets the cord alone; when signs of meningitis occur he would withdraw only enough fluid from the canal to lessen its pressure to normal and that only about $a$ half hour after intravenous injection (italics mine) and then introduce not more than 30 c.c. into the cord. After this he would drain the cord freely on the occasion of further intraspinal therapy, utilizing the effects of such drainage on the choroid plexus to increase the permeability to the antibodies in the blood stream.

His average intraspinal injections numbered four. The efficacy of the intravenous treatment undoubtedly lessens the number of spinal injections needed. If 8 or 10 intraspinal injections are not efficacious, it is wise to interrupt the treatment, draining only so often as to relieve pressure symptoms. Some men (Olitsky) believe drainage quite as effectual as serum. The lack of success in any given case may be due to the serum. It is well, then, when meningococci are recovered by culture to determine by them the agglutization power of the serum used. Even when this cannot be done, it is well to try a serum from another source as possibly derived from similar strains.

Fulminating Cases. The overwhelming rapidity of these cases does not permit of timely intervention on the part of the physician. The patients are profoundly affected when seen. These are cases in which intravenous injections as advised are urgent. 
With meningeal involvement more frequent, dosage by the spinal route, as Dunn urges, seems rational. He advises a dose at once and another as soon as the beneficial effects begin to wane, which"may be in a few hours, or to give the dose at twelve-hour intervals.

The best result I have ever obtained was after intraspinal injections of 6 to 8 hour intervals, gradually lengthened as improvement followed. This was before intravenous treatment was urged. The frequent drainage of pus may have contributed materially to the fortunate issue.

Chronic Cases. In the prolonged cases, as long as there are diplococci in the fluid or fever or pain or symptoms of activity of the process in any terms, except a rigidity of the neck and Kernig's sign, which are apt to persist for some time after complete disappearance of other symptoms, the treatment should be continued. Dunn has advised as a routine that after four doses on consecutive days, a wait of a few days follow and then again a course of four treatments be given and again a wait and so on until all signs disappear.

It would seem advisable to change the serum used in the cases or to try the effects of drainage alone for a time.

Sophian has used in the chronic cases meningococcic vaccine as an adjuvant to the serum treatment. The doses are $50,000,000$, up to $1,000,000,000$ or $1,500,000,000$ every three to five days.

Hydrocephalus in the chronic cases demands taps at daily intervals or once in two, three, or four days, depending on symptoms.

Relapses. Relapses occur in a small per cent. of the cases, though Ker found that 15 to 20 per cent. of his cases relapsed.

A relapse is to be treated like a fresh case, in all the details specified.

Exacerbations in the course of a case are to be treated in the same vigorous manner.

When the spinal fluid is too thick to run through the needle, it has been advised to use a sterile physiological salt solution to irrigate the cord and then inject the serum.

When for other reasons the tap is dry and particularly in infants a puncture into the ventricles has been made, the cerebro-spinal fluid withdrawn and the serum given into the ventricles.

Puncture of the Ventricles. In posterior basal meningitis, the use of serum by the intradural method is hazardous and useless. The hydrocephalus demands puncture of the ventricles daily or less often according to the severity of symptoms.

If the fluid is infected, serum is injected into the ventricles as into the cord.

This procedure is not difficult in infants. The anterior-fontanelle 
is used. One enters at the lateral border, pushes the needle down, back and in to the depth of 2 to $4 \mathrm{~cm}$.

Use the same needle as in lumbar puncture.

In older individuals the puncture must follow a trephining.

Results. The mortality in different epidemics in different localities showed about 75 per cent. The analysis of the first 400 cases after the introduction and use of the antitoxin reversed the figures; 75 per cent. recovered and 25 per cent. died.

Of infants under one year, before the introduction of antitoxin the mortality was approximately 100 per cent.; a recovery a rarity. After the introduction of antitoxin, of the first twenty-two treated 50 per cent. recovered.

In 1914 Flexner reported on 1,294 cases treated with his serum. The mortality was 31 per cent.

For those treated on the first to the third day the mortality was 18 per cent., on the fourth to the seventh day 27 per cent. Later than the seventh day 36.5 per cent.

The highest mortality was under one year, 49.6 per cent.; the lowest from five to ten years, 15.1 per cent.

Thirty per cent. of the cases reported by Flexner ended by crises.

In a few cases, he says, the meningococci seem to be serum fast; and in a number of relapses the cocci seem to have acquired serum fastness and defeat the effort of the serum.

Complications. Changes in mentality, varying from irritability and changes in disposition to profound damage together with different paralyses may ensue.

Relief of intracranial pressure must be afforded by frequent tappings, by the use of the vaccine and serum.

Eye complications are varied in type, in part due to effects on centres from pressure or meningo-encephalitis and to be treated by tapping and the use of serum and vaccines; and in part local, such as severe conjunctivitis or even suppurative processes, panopthalmitis with blindness.

These are to be treated as under other circumstances, but the use of the serum locally may help materially. The ocular muscles may be involved.

Ear. Otitis is very common, occurring in over 10 per cent. of the cases and is to be treated by paracentesis.

For treatment of Otitis Media see Scarlet Fever, Chap. XVII.

Deafness is another unfortunate issue.

Joints. An involvement of the joints takes place in 15 per cent. of the cases and may be a polyarthritis, lasting weeks or months. 
Vaccines (see text) are indicated.

Fluid may be removed from a joint and serum injected (e. g., 1015 c.c. into knee) with great benefit.

Pains may be relieved by the use of salicylates or local measures. (See Rheumatism, Chap. III.)

During the acute stages pneumonia is fairly common, pleurisy, peritonitis, pericarditis, endocarditis less so. With intravenous treatment the complications are less frequent. For measures directed at relief of symptoms and support of circulation, see Pneumonia and Rheumatism, Chaps. IX and III.)

Pyelitis is fairly common.

Meningococci may be found in the urine even before they are determined in the cerebro-spinal fluid.

Urotropin should be used. (See Typhoid Fever, Chap. XIV.)

In severe cases, subcutaneous or intravenous use of serum and the use of vaccines would be indicated.

Convalescence. This period varies greatly. It may be fairly prompt, long delayed from weakness and poor nutrition or complicated with permanent damage to some centres in brain or cord or with some of the conditions mentioned.

Air, sunlight, an abundance of good food and good nursing are the main indications.

It has been advised (Herrick) to use vaccine during convalescence to prevent recurrences, e. g., 3 to 5 injections at 6-day intervals of 50 to 200 millions of killed autogenous meningococci; for some prolonged convalescences or recurrences would seem to be due to organisms protected by exudates undergoing mucoid transformations or perhaps derived from nose, accessory sinuses, eye, ear or pericardium. This would seem to be a wise measure.

Orchitis and epididymitis are other rare complications. For local treatment see Mumps (Chap. XXIII).

Prophylaxis. Protection of the individual.

Serum may be used subcutaneously, just as diphtheria antitoxin, to afford passive immunity and it has been claimed that it affords such immunity over a period of two to three weeks.

It would be indicated for those who find themselves in immediate contact with a patient; doctors, nurses, and members of the patient's family.

What is believed to afford a more lasting and effectual protection is afforded by vaccines.

These vaccines have been given in the same doses and at the same intervals (weekly) as the antityphoid vaccine; namely, 500,000,000- 
$1,000,000,000$; but Sophian, because of the rather striking pain in the neck, which alarmingly suggests the real disease, after the initial injection, recommends as a first dose, $100,000,000$, followed in a week by $500,000,000$ and in another week by $1,000,000,000$.

Carriers. Fifty per cent. and in some epidemics even a much higher per cent. of contacts become carriers, and infection through the third person is common; 95 per cent. of these carriers do not suffer themselves.

All contacts should be examined and those found to be "carriers" should be quarantined until their nasal discharges are negative for the meningococci on two occasions, and Sophian advises that those contacts be quarantined a week even when the discharges are negative.

As the incubation period is nearly two weeks it would seem wise to quarantine for this period.

As Major Russell has remarked, it is the chronic carrier with large number of meningococci always present that constitutes the real danger rather than a man with a few organisms. He must be kept under observation.

In quarantine they should use the mild antiseptic sprays, such as 2 per cent. boric acid solution or quarter strength Dobell's solution.

Sophian lays great stress on the use of saline douches three times a day, followed by a spray of peroxide of hydrogen $1 / 2-1$ per cent.

Others recommend the blander silver preparations, but astringents that irritate the mucous membrane and so lower its resistance should never be used.

\section{SUMMARY}

\section{Distribution of the family.}

The disease is contagious and isolation should be obligatory.

The children of the family and other "contacts" should be kept from other children, as possible carriers.

The children of the family should be isolated over a period that probably covers the illy defined incubation period, i. e., three to four weeks.

The nasal passages of the suspects should be examined for diplococci.

Adults, too, should be kept from the sick-room unless their duty keeps them there.

Adults of the family should not come in contact with other children and if their duty brings them in contact with children, they should remove from the environment of the patient and submit to the isolation period before seeing children again.

Contacts should have their nasal secretions examined. If they are positive, they should keep from children during the epidemic and if possible, isolate themselves until the cultures are negative. 
Avoid infections of nasal passages, especially during epidemics of colds and sore throats.

"Contacts" should use a mil'd spray for the throat and nose, 2 per cent. boric acid solution or quarter strength Dobell's solution

Never use strong astringents.

\section{Nurse.}

Some cases a night and day nurse.

Very likely to become a "carrier."

Avoid contact with children.

Remember the ease of conveyance by kissing, coughing and sneezing.

Before going out clean hands and face with soap and water followed by al cohol or 1-1,000 bichloride and

Spray throat and nose with the mild solutions mentioned.

Should spray throat and nose from time to time while on duty.

\section{Physician.}

Should make his visits as short as is compatible with his full duty.

On leaving, wash face and hands with soap and water followed by alcohol or bichloride $1-1,000$.

$\mathrm{He}$ should not see another child at once.

\section{Room.}

Choose with reference to light.

Air.

Verandah or porch approach.

Bath room near by.

Should be stripped of furnishings.

Carpet lining or unbleached muslin on the floor.

Screens for the eyes, if sensitive to the light.

Avoid jars, noise and other sources of irritation.

\section{Precautions in the sick-room.}

Nasal and oral secretions should be received on rags and burned or disinfected in 1-20 phenol or 1-500 bichloride.

Thermometer.

Should be left in sick-room and kept in 2 per cent. phenol or in formalin.

Eating utensils.

Boil in sick-room, or if sent out of sick-room soak in phenol 1-20 for twenty minutes to half-hour, then send out to be boiled. Clothes.

Soak overnight in 1-20 or 1-50 phenol, then boil half-hour before sending to the family wash or laundry.

Urinals, bed-pans, etc.

1-20 carbolic or $1-500$ bichloride.

Bed.

(For technique of bed making, see Pneumonia, Chap. IX.)

Especial care should be taken on account of bed-sores. 
With any threat of bed-sores

Air mattresses or

Water-bed.

Clothing somewhat more abundant than usual on account of extreme sensitiveness to cold.

\section{Patient.}

Isolation.

Nightgown should be of light flannel or, if irritating, cotton.

Should be open all the way down the front to facilitate examinations.

Diet.

Early days do not force.

Later consider the body needs.

Articles of diet. (See text.)

In infancy modified milk is to be further diluted.

In stupor or dysphagia.

Nasal or oral gavage.

Nasal better in children; oral in infancy.

Drinks should be forced.

Water, alkaline waters, fruit juice, as lemonade, etc.

Care of body.

Skin.

Cleansing bath of soap and water daily.

Bed-sores.

Prevention. Change of position.

Scrupulous dryness; use of rings and cushions.

Rubbing of skin with hands.

Use of alcohol and talcum powder.

Care of bed in avoidance of wrinkles and crumbs of food.

When sores threaten, use air mattresses or water-beds.

Sores should be handled on surgical principles.

Mouth and nose.

Remember that the secretions are infectious.

Rinse mouth with plain water, then with

Boric acid solution 2 per cent. to 4 per cent. or with

Dobell's solution quarter to half strength.

Teeth.

Brushed with soft brush or cotton swabs, wet with above solutions, care being taken to free interstices from particles of food. Remove particles between gums and cheeks.

Sordes and coated tongue.

Soften with half strength official peroxide of hydrogen, then scrape: tongue with edge of whalebone.

Follow with the boric acid or Dobell's solution.

Dry mouth and tongue.

Use 2 per cent. boric acid solution.

Albolene āā.

Flavor with lemon-juice. 
Nose.

Soften hard secretions with olive oil.

Spray with boric acid or Dobell's solutions.

Burn all secretions as infectious.

Nurse should carefully wash hands and use alcohol or 1-1,000

Eyes.

bichloride as an antiseptic after these ministrations.

Mild conjunctivitis is common.

Secretions are probably infectious and should be burned.

Cleanse eyes with 2 per cent. boric acid solution:

Care of bowels.

Open freely at the beginning.

Calomel, gr. 1/4 (0.015 Gm.) every quarter hour for four or five doses, or gr. 1/10 (0.006 Gm.) every ten minutes for ten doses. Follow in two to three hours by milk of magnesia $5 \mathrm{ss}$. $(15 \mathrm{Gm}$.) if there is vomiting,

Castor oil $3 \mathrm{ii}-\mathrm{iv}(8-15 \mathrm{Gm}$.) if there is no vomiting; or

Sal Rochelle 3ii-5 ss. (8-15 Gm.) or

Epsom salt 3 ii- 5 Ss. (8-15 Gm.) in half to three-quarter glass of water.

Throughout the illness use,

Milk of magnesii $3 \mathrm{ii}-5$ ss. $(8-15 \mathrm{Gm}$.) or

Liquor magnesiæ citratis 5 iv-viii (120-240 Gm.), Hunyadi or similar water.

Enemata, especially if there is vomiting, though hyperesthesia may make it a too distressing procedure.

\section{Nausea.}

A feature of the onset is vomiting, and it may continue for some time.

One may try

Cracked ice.

Mustard paste, 1 in 3, 4, 5, or 6 of flour to pit of stomach.

(For technique, see Pneumonia, Chap. IX), or

Sodium Bicarbonate......gr. x-gr. xv (0.60-1 Gm.) or

Bismuth Subnitrate......gr. x-gr. xv $(0.60-1 \mathrm{Gm}$.) or

Cerium Oxalate.......... gr. iii to gr. v $(0.20-0.30 \mathrm{Gm}$.) or

Lavage.

a combination of the three.

Treatment of symptoms.

Restlessness, delirium, sleeplessness.

Ice-cap or ice-coil.

Warm sponge baths.

Bromides, in doses gr. $\mathrm{x}$ to $\mathrm{gr}$. $\mathrm{xxx}(0.60-2 \mathrm{Gm}$.) according to age. More severe.

Codeine phosphate in doses of gr. 1/48-gr. ss. (0.0015-0.030 Gm.) according to age. 
Very severe. Morphine sulphate in doses of gr. 1/48-gr. 1/4 (0.0015$0.015 \mathrm{Gm}$.).

Hyoscine hydrobromide in doses of gr. 1/300-gr. 1/100 (0.0002$0.0006 \mathrm{Gm}$.) in adults.

\section{Convulsions.}

Slight twitchings.

Chloral best given by the rectum in warm milk.

Dose, gr. $\mathrm{v}$ to gr. $\mathrm{xxx}(0.30-2 \mathrm{Gm}$.) according to age.

Bromides may be added to the chloral in double the dose of the latter, and may be given by mouth or rectum.

Severe convulsions.

Morphine sulphate, doses of gr. $1 / 24$ to gr. 1/3 (0.003-0.020 Gm.). For details of treatment of convulsions in children, see index.

\section{Circulation.}

So far as influenced by the intracranial pressure, do a lumbar puncture.

For continuous support use digitalis in doses of gr. ss. to gr. iii $(0.030-0.20 \mathrm{Gm}$.) three times a day (3ss. to 5 ss. of infusion $(2-8$ c.c.). or $\mathrm{m} . \mathrm{v}$ to $\mathrm{m}$. $\mathrm{xxx}(0.30-2$ c.c. $)$ of the tincture.

Strophanthin in emergency in doses of gr. 1/120 to gr. 1/60 (0.0005-0.001 Gm.).

For immediate demands. Caffeine in

doses of gr. ss. to gr. v $(0.030-0.30 \mathrm{Gm}$.) every two hours as a soluble salt of caffeine sodium salicylate or sodium benzoate.

Intravenous injection of glucose. (See text.)

\section{Bladder.}

Watch for distension.

Apply hot stupes over the epigastrium.

Catheterize.

Urotropin.

Doubtful value.

Dose, gr. v (0.30) two to four times a day well diluted.

\section{Specific treatment.}

For preparation of serum and the theory of its action, see text.

\section{Administration of serum.}

Intravenous treatment. As the infection is probably in the beginning a septicemia intravenous treatment is indicated.

(For discussion, see text.)

Diagnosis may be made before cerebro-spinal symptoms develop. (See text.)

Determine sensitization by intradermal method. (See Pneumonia, Chap. IX.) 
If this cannot be done, in cases of known sensitization or best in any case, give desensitizing dose of 1 c.c. subcutaneously, one hour later give full dose; first 15 c.c. at rate of 1 c.c. per minute. Give rest more rapidly.

\section{Dose 150 c.c.}

If untoward symptoms appear:-cyanosis, pallor, dyspnœa, vomiting-cease injection for one hour and then try again. Atropine sulphate gr. 1/150-1/100 (0.0044-0.0066 Gm.) or morphine sulphate gr. 1/4-1/8 (0.015-0.008 Gm.). Epinephrin (adrenalin) $1: 1000 \mathrm{~m}$. xv intravenously.

\section{Frequency.}

Eight to twelve hours in severer cases; 24 hours in less severe during the acute stage. Usually takes 4-5 doses.

Mode of administration.

(See Pneumonia, Chap. IX.)

\section{Intraspinal treatment.}

Unless cerebro-spinal symptoms occur it is best not to puncture the cord, or to use small needle and withdraw small amounts.

On the appearance of cerebro-spinal symptoms puncture the cord preferably about a half hour after an intravenous injection, removing enough to relieve pressure and introducing not more than 30 c.c. of serum into the canal; if further spinal therapy is necessary, drain cord and follow procedure below.

\section{Dosage.}

Only limited by the capacity of the spinal canal. Rule, 30 c.c. or if more than this amount of cerebro-spinal fluid is withdrawn from the canal, replace an equal amount of serum.

If less than 30 c.c. of cerebro-spinal fluid is withdrawn, approximate the dosage of serum to 30 c.c. but without forcing against resistance.

Sophian is guided by blood pressure readings. (See text.)

For Sophian's table of dosage at different ages, see text.

\section{Frequency of dosage.}

Once a day for three or four days or longer if meningococci persist in the cerebro-spinal fluid or the symptoms are active.

Favorable results are shown by amelioration of symptoms and change in cerebro-spinal fluid.

(For details, see text.)

\section{Lumbar puncture.}

Site. In a line with the iliac crests. Crosses spine nearest to fourth lumbar.

Go in between fourth and third lumbar or between fourth and fifth lumbar.

In children enter needle in exact mid-line of vertebral column. 
In adults enter $3 / 8^{\prime \prime}-1 / 2^{\prime \prime}$ to right or left of mid-line and direct needle inward toward the middle line. The mid-line, however, is preferred by many operators.

Position of the patient.

On his side with back toward the edge of the bed or table, head and knees approximated.

Preparation of patient.

Wash site with soap and water and alcohol or

Paint the site with tincture of iodine.

Preparation of the operator.

Prepare as for a surgical operation.

Soap and water and alcohol or sterile gloves.

Preparation of the apparatus.

All should be sterilized by boiling; needles, trochars and canulas, syringe and test tubes.

Anæsthetic.

Better none; pain is trivial.

If fear makes it advisable freeze site with ethyl chloride or use 1 per cent. to 2 per cent. cocaine solution or give a whiff of chloroform.

Needle.

Should be stiff and of fairly good calibre, such as is used for thoracentesis or diphtheria antitoxin administration.

Trochar and canula.

Quincke needle.

The Tap.

Guide needle along upper thumb-nail pressed against upper border of lower vertebra bounding the space to be entered.

Lessened resistance tells of entrance of needle into canal.

Flow through needle corroborates entrance.

Don't push needle across space into body of vertebra, lest bleeding ensue and spoil fluid for examination.

Depth of entrance.

In children about $1^{\prime \prime}$, in adults more.

Needle should be $4^{\prime \prime}$ long at least.

The flow.

May be drop by drop; but is usually continuous or in a spurt.

The amount.

Normal infants, 10 to 20 c.c.

Older children, 15 to 25 c.c.

Adults, 20 to 30 c.c.

May vary in meningitis from a few c.c. to $50-60-100$ c.c.

Collecting fluid.

. Sterile test-tubes 15 to 20 c.c.

Fill not more than three-quarters, so that plug of cotton will not touch.

If fluid runs bloody, catch this in one tube and the clearer in the other.

Let all flow that will, or 
-. If blood pressure falls $10 \mathrm{~mm}$. during the tapping it is well to discontinue. (Sophian).

Plug and set upright.

Appearance of fluid.

Usually turbid or purulent.

Don't wait for examination, but give the serum.

If blood obscures it, don't wait, give serum.

If watery clear, wait and examine. May be tuberculous or serous meningitis or poliomyelitis.

Give patient benefit of any doubt by administering the serum.

The injection.

Fill the container with serum warmed to blood heat; expel all air.

Inject slowly by gravity method.

Some advise raising foot of the bed to facilitate diffusion.

Watch for poor pulse, cyanosis, embarrassed breathing, collapse and let serum flow back until improvement occurs.

Keep the needle in situ for a few minutes to avoid necessity for another puncture, if symptoms develop (Dunn).

Remove excess iodine with alcohol. Seal puncture, with sterile gauze pad.

If bad symptoms develop as above,

Do artificial respiration.

Cocaine hydrochloride, gr. 1/8-1/4 (0.008-0.015 Gm.) hypodermically (Sophian).

Atropine sulphate, gr. 1/80-gr. 1/50 (0.00075-0.001 Gm.). Adult dose.

Children, dose gr. 1/800 to gr. 1/100 (0.00008-0.0006 Gm.).

Reaction.

As a rule none of consequence. May be headache, pain in back and limbs.

Raise the pillow.

Morphine sulphate, dose, smallest that will relieve pain; gr. 1/48 to gr. 1/4 (0.0015-0.015 Gm.).

Dry tap.

Usually due to failure in technique.

Canal is not entered.

Needle is plugged with tissue.

Try a space above or below.

Fluid may be inspissated.

Sitting patient up may initiate the flow; gentle aspiration with syringe may be effectual.

Saline irrigation or serum under slight pressure, two needles in adjacent spaces with saline irrigation.

True dry tap.

Use small amount of serum under gentle pressure at shorter intervals. Early usage of first importance.

(For statistics, see text.)

Possible bad results.

(See text.) 


\section{Intravenous treatment.}

Simultaneous intraspinal therapy if signs of meningitis occur.

Begin as soon as diagnosis can be made by symptoms and positive blood culture.

Method.

Desensitize patient by subcutaneous injection of 1 c.c. of horse serum.

After one hour an intravenous injection of 80-150 c.c. of antimeningococcic serum. (See text for details.)

\section{Frequency.}

Every 8-12 hours until desired reaction occurs. Average 4-5 injections.

\section{Fulminating cases.}

More frequent doses, twelve hours or less (Dunn).

Also intravenously in doses of 75-100 c.c. are urgent early.

Chronic cases.

Continue treatment as long as diplococci persist, or there are active symptoms, such as fever and pain. Change the serum used as possibly elaborated from different strains.

Stiff neck and Kernig's sign may outlast the infection.

Give a dose four days in succession, then wait a few days, another series and so on, until signs disappear (Dunn). Try effect of drainage alone for a few days.

\section{Sophian uses}

Meningococci vaccine in addition to the serum.

Begin with $50,000,000$ and increase up to $1,000,000,000$ or $1,500,000,000$.

Give at three to five day intervals.

Hydrocephalus.

Tap every day or once in two, three or four days according to symptoms.

\section{Posterior basic meningitis.}

Tap ventricles daily or less often according to symptoms.

Intradural injection of serum dangerous.

If ventricular fluid is infected inject serum into ventricles as into cord.

Tapping ventricles.

Infants.

Enter by anterior fontanelle at lateral border, push needle down, back and in 2 to 4 c.m.

Use same needle as in lumbar puncture.

Older individuals.

Trephine.

Complications.

Mental disturbances.

Frequent tapping. 
Serum.

Vaccines.

Eye.

Special treatment.

Serum applied locally.

Panopthalmitis with blindness.

Oculo-motors may be involved.

Ear.

Otitis.

(For treatment, see Scarlet Fever, Chap. XVII.)

Joints.

Vaccines.

Remove fluid and inject serum.

Arthritis (Salicylates, see Chap. III, Rheumatic Fever).

\section{Pneumonia.}

(See Pneumonia, Chap. IX.)

Serum under skin or into vein.

Vaccines.

\section{Endocarditis.}

As under other circumstances.

Serum into vein or under skin.

Vaccines.

\section{Pyelitis.}

Urotropin.

Vaccines.

When severe.

Serum under skin or into vein.

Orchitis.
Epididymitis. (See Mumps, Chap. XXIII.)

\section{Relapses.}

Treat as a fresh case.

\section{Exacerbations.}

Treat vigorously as a fresh case.

\section{Prophylaxis.}

Individual.

Passive immunity.

Serum subcutaneously.

Lasts two to three weeks.

More lasting immunity.

Vaccines.

One hundred million, a week later 
Five hundred million, and then a week later One billion.

\section{Carriers.}

Quarantine until discharges are twice negative.

\section{Convalescence.}

Period varies greatly depending on damage done.

Fresh air, sunlight, good food, careful nursing.

Herrick advises vaccines to prevent recurrences, giving 50 to 200 millions of killed meningococci, 3-5 injections at 6-day intervals. 


\title{
CHAPTER XXVI
}

\author{
POLIOMYELITIS
}

\section{(INFANTILE PARALYSIS)}

THis disease, so dreaded because of its frightfully crippling effects, is undoubtedly of microbic origin.

A minute globular organism, so minute as to pass through the pores of a Berkfeld filter, has been detected in the central nervous system, cultivated and made to reproduce the disease in monkeys by Flexner.

The virus is conveyed by individuals infected or commonly by "carriers"; that is, those who having been in contact with the sick, though not themselves infected, can carry the virus to a susceptible person and infect him.

The rôle played by insects in transmitting the disease is questionable.

The portal of entry seems to be the upper air passages, especially the nose, and the virus passes from here to the olfactory lobes, spinal cord fluid and thence to the nervous tissue for which it has a special affinity.

Protection of the Community. The focus from which all epidemics radiate is the patient sick with the disease in question. Protection begins then with sufficient isolation of this individual, so that there shall be no immediate contact between him and a susceptible individual and this can be readily accomplished when understanding and willingness on the part of those concerned coöperate.

But another source of infection beside the patient frankly sick is the abortive and ambulatory case and only a sufficiency of skill in diagnosis of this group of cases can be of avail in eliminating him as a source of infection.

Another source of infection is the "carrier." The carriers may be divided into two groups; first, those who have been in contact with the sick and though uninfected themselves carry a virulent organism about with them and those who have had the disease, have recovered and yet carry about virulent organisms, like the diphtheria and typhoid carriers. Flexner has called attention to these four groups. Flexner and Amos have concluded that healthy and chronic carriers are rare.

Isolation. Those in contact with the patient should be kept isolated, so far as possible for three weeks and if this is not possible 
should at least avoid contact with children; children should not be allowed to go to school or to public gatherings.

It is believed that the nasal mucous membrane has a distinct protective action against infecting organisms. If this be so any antiseptics used in the shape of sprays or local applications which in any measure injure the innate protective mechanism may lessen this protection. For this reason the use of sprays is of a doubtful value.

The abortive cases are to be especially looked for in epidemics.

A diagnosis of an abortive case of poliomyelitis is at best conjectural and when occurring as a sporadic case scarcely possible, but in an epidemic symptoms of an acute infection, in which the febrile reaction is accompanied by excessive irritability and hyperæsthesia, should arouse suspicion; sweating, stiffness of the neck or gastro-intestinal symptoms may be accompanying features.

When such a diagnosis is suspected the spinal cord fluid should be examined. This is clear and shows a ground glass or opalescent appearance due to an increase of the number of leucocytes. If shaken up a foam forms that lasts from one-half to one hour due to an increase of the globulin content. These tests are of value only if positive and in the absence of blood.

The cytology varies with the stage of the disease. There is always a pleocytosis. As a rule in the first week there is a lymphocytosis, though rarely in the very early days there may be a polynucleosis.

The globulin is scant during the first week, but increases steadily and is in excess even 8 to 10 weeks after the onset.

Later in the second and third weeks, the cells decrease, the proportion of polymorphonuclears decrease and the proportion of lymphocytes and mononuclears increase. The globulin reaction becomes more striking. Rarely do the cells reach a thousand; more commonly a few hundred or only 10 to 100 . The reduction of Fehling's solution unlike that of cerebro-spinal meningitis persists.

The fluid may appear normal.

This picture may be given by tuberculous.meningitis and syphilitic myelitis, so there is absolutely no diagnostic feature in the symptomatology or findings, but such a spinal fluid added to the general symptoms given above, occurring during an epidemic makes a diagnosis of poliomyelitis fairly certain and from the practical standpoint imperative. The blood shows a leucocytosis; sometimes as high as 30,000 , and an increase of 10 per cent. to 15 per cent. in the polymorphonuclears.

These cases are to be isolated in the same way as the frank cases and those exposed to such a case treated as those exposed to a frank case. 
More difficult is the problem of chronic carriers. We know that the infection resides in the secretions of the nose and in the saliva and has been found in the tonsils and pharynx.

The organism has been found, described and cultivated by Flexner and his workers and the infectivity of secretions determined by inoculation into monkeys, but a ready method of determining the infectivity of the secretions of suspected "contacts" and of patients recovered from the disease awaits elaboration. In the meantime, such contacts and convalescents should be kept especially from children.

Isolation of the Patient. I feel that sufficient emphasis has not been laid on complete isolation of these cases. In the last New York epidemic I saw and treated a great many cases in the open wards and did not see a spread of the disease within the ward, but I believe that the rarity of cases among contacts gives us a false sense of security. Hill's studies in Minnesota (quoted by Lovett and Richardson) on the relative transmissibility of this disease with other well known contagious diseases showed that 22 per cent. of those exposed to Scarlet Fever contracted the disease, to Diphtheria 17 per cent., to Infantile Paralysis 6 per cent. Studying exposures in single families, 40 per cent. contracted Scarlet Fever, 30 per cent. Typhoid Fever, 29 per cent. Diphtheria and 17 per cent. Infantile Paralysis; moreover, in some epidemics as high as 40 per cent. of houses or families affected showed more than one case.

These figures as well as the knowledge of the infectivity of the dust from infected rooms (Thro), the conveyance of the disease by healthy individuals, all seem to me to demand as strict an isolation for these cases as for Scarlet Fever and I recommend the rules laid down in that chapter as applicable here. See Scarlet Fever, Chap. XVII.

Rules for Disinfection in the Patient's Room. All secretions and excretions should be destroyed, those from the nose and mouth best received on rags or gauze and burned. For the mode of disinfection of secretions, articles in use about the patient, clothes and bedclothes, see Typhoid Fever, Chap. XIV.

Doctors and Nurses. It should be remembered that cases have appeared in or through individuals only once in contact with a patient and that a short time.

The physician and nurse should take the same precautions, then, as when treating a scarlet fever case and the precautions to be taken in the sick-room and on leaving the sick-room should be the same. See Scarlet Fever, Chap. XVII.

Room. A light, well-ventilated room should be chosen, stripped of furniture, carpets, rugs, hangings, etc., leaving bare floors or linoleum, so that the floors and wall may be wiped with damp cloths occasionally 
and these destroyed; for it has been shown that the dust of a room harboring a case is infective and capable of transmitting the disease.

Such a room should be screened, for it is possible that insects may convey the disease, as they probably do typhoid fever by becoming contaminated with secretions; for virus passes through the gastrointestinal canal of man and remains active; but that the disease is regularly conveyed by an insect, e. g., the fly, as was once suspected, is not proven.

A convenient bath room and a separate exit from the house are to be considered in the choice.

Bed. The bed should be of the hospital type, a half or at the most three-quarter bed, of iron with woven wire springs and a firm mattress. The bed should be made with care (see index), and the sheets drawn smooth and free from wrinkles.

One must remember the great hyperæesthesia and paralysis and how much a well made bed makes for comfort. Moreover, numerous mechanical contrivances will be used if paralysis and contractures develop and this type of bed facilitates such efforts.

Care of the Body. Cleansing baths of warm soap and water should be given each day and oftener if the sweating is severe. These are to be followed by sterile talcum powders in abundance.

The mouth is cared for as in any of the acute infections and the same measures applied to the care of the nose and genitals. See Pneumonia, Chap. IX.

It must be remembered, however, that the virus is present in the secretions of the mouth and nose and that all secretions should be burned or otherwise destroyed.

The nurse remembering the danger to herself in handling these secretions should wear gloves and sterilize her hands.

Diet. During the acute stage the diet should consist of milk or milk preparations, cereals, bread, broths, plain or fortified with cereal or farinaceous flours, eggs, custard, rice, ice-cream.

Water or lemonade, orangeade, or other drinks flavored with fruit juices and fortified with sugar may be used.

Later a liberal diet may be allowed and the appetite satisfied.

Treatment of Symptoms. When the diagnosis is made or suspected, the patient should be put to bed for the most important part of his treatment, rest.

When one recalls that the essence of the disease is an inflammatory and degenerative process in the motor centres of the cord and bulb it should go without saying that they should rest; and that means that the muscles they subserve must rest; hence, the whole body. A clue to the 
continuance of this activity of the process is given by the persistence of the hyperæsthesia. When this disappears, it may be assumed that repair has begun in the centres.

Until this time the patient should not be persuaded to try the paralyzed or paretic limbs; but rather forbidden to do so. Even when there are no constitutional symptoms or hyperæsthesia accompanying the paralysis it is better to keep the patient in bed for a couple of weeks.

Bowels. When first seen a cathartic should be given. Castor oil 3ii- i (8-30 c.c.) a salt, Rochelle or Epsom 3j-5i (4-30 Gm.) according to age or calomel gr. i to gr. ii $(0.060-0.120 \mathrm{Gm}$.) in divided doses, followed by a salt.

Later cascara, compound licorice powder or aloin in doses suited to the age may be given or milder salines such as liquor magnesii citratis, Hunyadi water, milk of magnesia or occasionally stronger salts as above or castor oil.

Enemata are usually necessary even when cathartics are given, for constipation is a rather striking feature and more marked when the abdominal muscles are paretic.

Urine. In the early days retention may occur. It is necessary then to be on the lookout, as catheterization may be necessary. One should try, first, however, the simple device of running water, water sprinkled on the hypogastrium or heat or cold applied to the hypogastrium.

\section{PREPARALYTIC PERIOD}

Specific Treatment. Efforts to accomplish a cure by specific therapy has been made along two different routes. First by the use of immune sera derived from a horse by the injection of the organism responsible for the disease and second by the use of serum from a patient convalescent from the disease. It has not yet been definitely settled what is the etiological agent concerned in this disease. Claims are made by Flexner and his co-workers at the Rockefeller Institute for globoid organisms which pass a Berfeld filter and by Rosenow of Chicago for a pleomorphic streptococcus. Both these workers, using the organisms they have isolated, have produced an immine serum which each has used in the treatment of the disease with, what has seemed, satisfactory results. The serum is given intraspinally, intravenously and intramuscularly. Nuzum and Willy using the serum obtained with the Rosenow organism have reduced the mortality to 11.9 per cent. of 159 cases as contrasted with 38 per cent. in 100 cases untreated during the same period.

Their method is to use the serum as early as possible, giving $15-30$ 
c.c. intravenously very slowly, repeating it every 12 hours if necessary; at the same time they give 5-15 c.c. intraspinally after removing about the same volume of spinal fluid. The injection should be made slowly and without pressure as the lumen of the blood vessels is diminished by the perivascular infiltration.

They claim that in 12 to 24 hours there is a critical fall of temperature, a slowing of the pulse and a general improvement in the patient. If given early paralysis may be prevented. They have seen paralysis clear up under late serum treatment.

Sera from patients recovered from or convalescent from acute poliomyelitis was used in the epidemic of 1916. Opinions differed as to the efficacy of this measure, but the majority agree that results were similar to those described above after the administration of immune horse sera. It seems to me to offer greater promise than the horse serum and I should always use it in preference to the immune horse serum when it is available.

The method consists of drawing blood under aseptic conditions from donors convalescent as recently as possible, and who are shown to be free from syphilitic taint or other infection. After the blood is allowed to clot at room temperature it is placed in the ice box to await separation of the serum. The serum is then decanted and, if possible, centrifuged to obtain a product free from corpuscles and hemoglobin. It may then be given fresh or inactivated by the addition of sufficient tricresol to produce a final product, containing 0.2 per cent. of tricresol, i. e., 2 c.c. of tricresol to 998 c.e. of serum, or it ean be inactivated by heating in a water bath to $56^{\circ} \mathrm{C}$. for half an hour.

The method of administration is the same as outlined above for immune horse serum. The size of the intraspinal dose should not be more than 15 c.c. and introduced only after the removal of an equal or greater quantity of fluid. The size of the dose, too, is modified by the knowledge that sera obtained from patients recovered years before rather than months is apt to be less potent. The serum should be given intravenously at the same time. The dose is limited only by the difficulty of obtaining the serum. One would give 40-60 e.c. or even more if the quantity at hand is abundant. The repetition of the dose depends on the severity of the toxemia and the reaction of the patient to the serum and upon the degree of pleocytosis in the spinal fluid.

The serum must be administered early in the disease to prevent paralysis. It is still a question what effects the serum may have upon paralysis already established. Draper says that "generally speaking patients who show counts below 100 in the first twelve to eighteen hours are less apt to develop paralysis then those who show 500 or more." 
Fever occurs in this period and disappears soon after the onset of paralysis. It is not a feature of the disease and requires no treatment.

Hyperæsthesia, pain and sensitiveness of muscles or limbs to pressure or handling are characteristic if not constant.

Hyperæsthesia and fear of pain on handling lead to striking irritability to which undoubtedly meningeal involvement adds.

The pain may be spontaneous or elicited only on handling. These discomforts are usually more marked in paralyzed limbs, but may occur in limbs which are not paralyzed and, indeed, during the paralytic period.

The discomfort induced by movements often causes the child to hold the part so quiet that it simulates paralysis.

Even the weight of the bed-clothes may cause distress and in such cases cradles over the limb to support the clothes are helpful. At times the application of a light splint to immobilize a limb is grateful to the patient.

Heat is especially soothing and may be applied as fomentations or with the hot-water bag; wrapping the part affected in a thick layer of cotton batten subserves the same purpose.

Drugs. When irritability is the preponderating feature, bromides, either the potassium salt or triple bromides may be given with benefit.

When pain is dominant a member of the coal-tar group, phenacetin, antipyrin or acetanilid is indicated. (See Summary for doses.)

Salicylates, such as the sodium salts or acetyl salicylic acid (aspirin) are less depressing than the aniline derivatives and sometimes as effectual.

For more severe pain codeine or even morphine may be necessary. (See Summary.)

Sometimes drowsiness and apathy are present instead of irritability, but even then wakefulness may be a feature.

Insomnia which makes such inroads on strength is met by bromides or a mild hypnotic such as trional sulphonethylmethane or by codeine. Stiffness and pain in the muscles of the neck, especially on bending the head forward is common and demands care in handling and intelligent arrangement of the pillows.

Applications of heat should be useful.

Convulsions are very rare and should indicate warm packs and morphine to control the convulsion and bromides to prevent recurrence.

Gastro-intestinal symptoms are so common as to attract attention and during an epidemic a febrile attack accompanied by vomiting should elicit suspicion and concern. 
The vomiting and anorexia demand rest for the stomach.

The food is stopped entirely or cut down to small amounts. Sodium bicarbonate or bismuth may be used but it is not commonly persistent nor requires much interference.

Diarrhea may occur, but constipation is the rule. Severe diarrhea indicates regulation of food; castor oil and bismuth subnitrate.

Tonsillitis and pharyngitis are sometimes present and the virus has been recovered from the tonsils.

Hot saline irrigations may be used. (See Scarlet Fever, Chap. XVII.) Sprays of mild antiseptics and argyrol 15 per cent. to 25 per cent. may be applied.

Use of Urotropin (Hexamethylenamine). Because, when administered by the mouth, this drug was found in the spinal cord fluid and because when given to monkeys it was thought that the subsequent infection was modified in severity, it came to be quite extensively used; but careful observations of clinical results following its use holds out no hope from its use and, moreover, the efficacy of urotropin (hexamethylenamine) depends upon its breaking up to set free formaldehyde. This occurs only in acid and not in alkaline media such as the cerebro-spinal fluid.

Pathology. The disease has been called a meningo-encephalopoliomyelitis, and this term will explain the types of the disease we observe. But it is even more than this for it affects both the white and gray matter of the brain, the cord and the intervertebral ganglia and the abdominal ganglia. Flexner's studies lead him to this conclusion: that the route of infection is in the vast majority of instances, practically always, by the nasal mucous membrane to the lymphatic channels of the olfactory lobes, to the cerebro-spinal fluid, by this to be distributed to the nerve tissues of the cerebro-spinal axis, for which the virus has a special affinity.

Conveyance by the blood must be exceedingly rare in human beings and curiously enough the avidity of nerve tissue for the virus is not sufficient to derive it from the blood vessels unless damage has previously been done to them or to the choroid plexus.

Types of the Disease. Wickman, in his monograph on the disease, which to avoid the common name connoting the pathology, he calls in honor of the two great students of the condition the HeineMedin's Disease, divides the clinical pictures into eight types,

1. Spinal (the most common) poliomyelitis.

2. Landry's paralysis (ascending or descending paralysis).

3. Bulbar type.

4. Encephalic or cerebral type. 
5. Ataxic type.

6. Polyneuritic type.

7. Meningitic type.

8. Abortive type.

These types are not clear cut and merge into one another.

For this reason fewer and more inclusive groupings have been advocated, such as

1. Spinal type.

2. Bulbar type.

3. Cerebral type.

4. Abortive type. (Müller.)

or

1. Upper motor neurone type.

2. Lower motor neurone type.

3. Abortive type. (Peabody, Draper and Dochez.)

The abortive type, whose symptoms are those of a preparalytic period has been discussed.

Wickman has divided this Type into four groups,

1. A group showing symptoms of any general infection.

2. A group showing much meningeal irritation (meningism).

3. A group in which pain is a feature (influenza-like).

4. A group with predominant gastro-intestinal disturbance.

These serve to fix the attention on poliomyelitis during an epidemic.

The ascending and descending spinal types, Landry's paralyses, are rare and highly dangerous types.

The polyneuritic and ataxic types are among the rarer manifestations of the disease.

Meningo-encephalitic Type. As has been said sharp differentiation between the types of Wickman does not obtain; hence, it is better to consider the meningeal and encephalitic cases together.

The symptoms are such as one might expect from involvement of the brain and its covering; headache, irritability, restlessness and sometimes convulsions. In some, apathy or stupor predominate; vomiting is likely to occur. There are pain and stiffness of the neck, disturbances in the rhythm of pulse and respiration; tache cérébrale and there may be Kernig's sign.

As the hydrocephalus develops Macewen's sign may be noted and in infants the fontanelles bulge. The involvement of the centres causes palsies, facial, monoplegias or hemiplegias, opthalmoplegia, increased reflexes and, later, spasticity. 
These symptoms are to be met as described under the section on the preparalytic period and under cerebro-spinal meningitis, Chap. XXV.

Bulbo Spinal Type. Again a sharp differentiation between the bulbar and spinal types is useless, if possible, and they will be considered together.

This is the common well-known type of the disease that gave to us the term anterior poliomyelitis.

Most authorities recognize distinct stages that have decided significance from the standpoint of therapeutics.

1. The acute stage; from the onset to and through the spread of paralysis.

2. The stage of recovery in those muscles capable of recovery.

3 . The stage of residual and permanent paralysis.

The Acute Stage. All that pertains to the general symptoms of this stage, aside from the paralysis has been dealt with above under the pre-paralytic period.

The essence of the therapy is rest of function of the affected parts and hence, the inflamed cord centres supplying those parts. Rest means not only a comfortable bed, careful nursing, devices to find comfortable positions for the painful members, but also freedom from excitement, entertainment and visitors and, particularly, avoidance of pernicious measures of mechanico- or electro-therapeutics, often insisted on by the parents in an eagerness to see something done for the condition.

Beside affording rest we ameliorate the discomforts.

Lovett calls attention to the importance of recognizing the tendency to contracture in these first two or three weeks and taking measures to prevent it. This is especially likely to occur in the Achilles tendon. Lovett advises gentle stretching of the muscles and, as a prevention, a box covered with a blanket placed at the bottom of the bed against which the soles of the feet may rest and preserve for the feet a right angle to the legs. The weight of the bed-clothes, which, pressing on a paralyzed part, exaggerates the deformity and encourages contracture, must be taken off by wire cages, stretching clothes across the crib or similar devices. Light splints may be applied to correct the position; pillows and folded blankets may be used for the same purpose and the position of a joint should be changed from time to time.

The best guide to the length of the period of the active cord involvement is the continuance of tenderness in the muscles and joints. It may last two or three months and during this time no active measures should be applied to the affected muscles. When the bulb is affected one may see ocular palsies, facial palsy and involvement of the tongue, the pharyn $x$ and muscles of deglutition. These latter may compel feeding 
by nasal or stomach tube and entail constant care to keep the mouth and pharynx free from secretions and food particles that may induce a foreign body pneumonia.

Involvement of Muscles of Trunk and Diaphragm. Paralysis of the respiratory muscles is the usual cause of death. The intercostal muscles are almost always affected before the diaphragm.

Death due to this cause may be direct or due to pulmonary complications sequential to the paralysis.

Either set of muscles are capable of carrying on the respiration. It is the involvement of both that is fatal.

In what seems extensive involvement of these muscles hope lies in the fact that improvement may be rapid and substantial because the depression of centres often overflows the site of actual damage. When the dyspnœa becomes accentuated oxygen may be administered and respiratory stimulants such as atropine, caffeine and strychnine may be given hypodermically and, these failing, artificial respiration tried, but when one bears in mind the pathological basis of this failure in function he realizes how futile these measures must necessarily be.

The paralysis of the abdominal muscles greatly aggravate constipation and makes the patient more dependent on cathartics and enemata.

Retention of urine must be borne in mind and the measures described above undertaken.

Paralysis of the Extremities. The distribution of the paralysis is weird, often affording combinations topographically and functionally remote from each other.

The legs are involved more commonly than the arms and one leg more commonly than both. The onset of the paralysis is lawless; it being the first manifest symptom in some cases or occurring any day after the onset, though the majority of cases show paralysis within three days. However, the onset of the paralysis may be delayed a week or two.

Again it is difficult to forecast the extent of the ultimate paralysis by the extent in the early days, for the early paralysis may be due to other causes than actual cell destruction such as edema and pressure of exudate on centres, depressing effects of toxins on centres, or only partial and not necessarily an eventual crippling involvement of a group of cells, in the cord, so that in a very extensive early paralysis complete recovery or slight damage only may follow. Unhappily there is the obverse of the shield and prognosis waits on time.

What is to be done in the acute stage and even more important what is not to be done has been detailed above.

When the acute process has subsided (see above) one should 
begin the treatment with massage. Massage must be begun gently and for short periods of time at first; gradually increasing up to fifteen to twenty minutes two or three times a day.

Heat in the shape of hot applications or baking is a useful adjuvant to the massage. Electric light bulb suspended from a cradle makes a simple hot air bath. Care must be taken lest excessive heat damage the tissues.

In this way the nutrition and tone of the muscle is subserved.

Electricity probably has a very limited value. Its use has undoubtedly been greatly abused and led to the neglect of more valuable measures, less striking in their exposition and requiring more skill and perseverance.

Galvanism is applied to the nerve trunks and faradism to the muscles, if they will respond to faradism. Galvanism is supposed to affect the nutrition of the muscles the more, but the contraction under the faradic current constitutes a more distinct exercise.

Passive Movements. Passive movements play an important part in the prevention of contractions, as has been noted above. They also, like massage, have a favorable effect upon the contraction in the affected part.

Active Movements. There is no doubt that the best exercise is that developed along normal lines; that is voluntary movements.

It must be remembered that the extensive distribution of motor centres governing any group of muscles is in a longitudinal direction and that some centres are very likely to escape and that these centres may be educated to take on the function of the destroyed centres in no small degree. Persistency is all important. In intelligent adults an understanding of the problem is of great assistance, but the vast majority of the patients are children and very many of them little children, so that coöperation on their part has to be elicited by indirect methods.

The warm bath, affording both heat and buoyancy, has been advised, in which the child is persuaded to effort by a desire to play with floating toys, or similar devices by which strong desire is made to elicit voluntary effort may be resorted to.

In older children efforts may be systematized.

At first, assistance must be given by the operator to effect the proposed movements, but as the patient's ability is increased less and less help is given, then resistance exercises are begun in which the operator makes gentle and then increasing opposition to the patient's movements.

How long these exercises shall be persisted in before recourse to mechanical expedients is had is a nice question, but it should be months. Good authority puts it at a year and a half to two years, and even 
then one is not sure that improvement may not go on, even when it has been discouragingly slow.

If the patient has achieved all he can with the impaired muscles he still may so educate other groups of muscles that they may take over the function, in some measure, of those rendered helpless by the lesion.

In the case of the legs, the effort to walk should be made, but in a severe case this is obviously impossible without mechanical appliances to afford help.

Apparatus. The apparatus may take the form of crutches, corsets, jackets, braces or splints and is distinctly an orthopedic problem and competent authority should be sought.

At last the problem may become a distinctly surgical one and,

Operative Treatment Is Indicated. Lovett in a masterly article outlines the indications as follows:

1. To correct fixed deformity.

2. To improve muscular function.

3. To secure stability of useless joints.

Such operations are instanced by transplantation of a tendon of an active muscle to the insertion of a paralyzed muscle to take over the function of the latter. This should not be an early operation but undertaken only when failure to improve in the paralyzed muscle is without question.

Efforts to stabilize flail joints by arthrodesis, or anchylosing of joints by removing their articular cartilages and getting bony union between the members of the joints.

This is especially applicable in the ankle, but such operations should not be undertaken without first carefully acquainting oneself with the indications and contraindications in individual joints.

Other devices may take the place of arthrodesis, such as the use of silk ligaments mentioned by Lovett.

Contractures may require cutting and stretching; especially about the ankle and knee.

Prognosis. The mortality runs about 15 per cent., but varies in different epidemics and at different ages.

The percentage of recoveries investigated in one series of 234 cases (quoted by Lovett) showed 25 per cent. to be complete and in another series of fifty-seven cases 28 per cent. completely recovered and 31 per cent. more recovered function, but showed some atrophy. The mortality varies in different epidemics and in one considerable series showed less than 10 per cent.

It seems to be higher in infancy and after ten years than between these periods. 
Prognosis of extent of damage. This is difficult to determine in the acute stage, for as has been said, what seems like an extensive damage involving trunk muscles may clear up entirely or the apparent damage may persist and spread and in rare cases the lesion may light up again after subsidence of acute manifestations.

Muscles which contract at all after the acute symptoms subside hold out hope of recovery in part or whole and efforts to improve the power of such muscles should be persisted in for many, many months.

A single muscle in a group functionating in common is more likely to recover than when associated in its paralysis with more members of the group or the group as a whole, in its paralysis.

\section{SUMMARY}

\section{Isolation.}

Of "contacts" for three weeks.

Use of mild antiseptic sprays for the nose and throat by "contacts," e. g., 1 per cent. official peroxide of hydrogen, of doubtful value.

Look for abortive and ambulatory cases. (See text.)

Of patients.

(See summary of Scarlet Fever, Chap. XVII.)

Disinfection of patient's room.

(See summary of Typhoid Fever, Chap. XIV.)

Doctors and nurses.

(See summary, Scarlet Fever, Chap. XVII.)

Room.

High and well ventilated.

Near bath room.

Strip of furniture and furnishings.

Screen.

Bed.

Half or three-quarters. Hospital type preferred.

Woven wire springs; firm mattress.

Care of body.

Cleansing baths of soap and warm water.

Sterile talcum powders.

Mouth.

(See Pneumonia summary, Chap. IX.)

Burn secretions. They contain the virus. Nurse should wear gloves in handling them. 
Diet.

Acute stage.

Milk, milk preparations, cereals, bread, broths thickened with cereal or farinaceous flours, eggs, custard, rice, ice-cream.

Water, fruit juice drinks, such as iemonade, orangeade.

May fortify with sugar.

After acute stage.

Liberal diet. Satisfy appetite.

Treatment of symptoms.

Rest, of body and especially the limbs and muscles impaired; the latter until all hyperæsthesia has disappeared.

Do not urge patient to move muscles.

Do not massage.

Do not use electricity.

During the acute stage.

\section{Bowels.}

Castor oil 3 ii- $5 \mathrm{i}$ (8-30 c.c.).

Salts (Epsom, Rochelle) 3i- $\mathrm{z}$ i (4-30 Gm.).

Calomel, gr. i to gr. ii (0.060-0.120 Gm.),

followed by a salt.

All these in dose according to age.

Later.

Cascara, compound licorice powder, aloin, liquor magnesii citratis, milk of magnesia, Hunyadi water; dose according to age.

Occasionally a stronger salt or castor oil.

Enemata. Required especially when abdominal muscles are paretic.

\section{Urine.}

Watch for retention.

Try running water.

Sprinkle water on hypogastrium.

Heat to bladder.

Cold over bladder.

Catheterize.

\section{Preparalytic period.}

\section{Convalescent serum.}

More valuable than immune horse serum. (For method of obtaining, see text.)

Administered both intravenously and intraspinally.

Method of administration. (See Cerebro-spinal Fever, Chap. XXV.)

Dose by vein $20,40,60$ c.c., depending on quantity of serum available.

Intraspinally not more than 15 c.c. after the removal of an equal or greater amount of spinal fluid. Frequency depends on toxemia, reaction and pleocytosis of the spinal fluid.

Must be administered early. 
Immune horse serum.

Time of administration early as possible.

Dosage 15-30 c.c. intravenously very slowly.

Repetition every 12 hours until favorable reaction obtained.

Simultaneously give 5-15 c.c. intraspinally after removing an equal volume of spinal fluid.

Fever.

Rarely requires attention.

Luke-warm water sponges.

Hyperæsthesia, pain, sensitiveness of muscles.

Remove weight of bedclothes, by cradles and similar devices.

Light splints to painful limbs.

Heat.

Fomentations.

Hot-water bag.

Wrap part in thick layer of cotton batten.

Drugs.

Bromides, especially when there is much irritability, gr. $\mathrm{v}$ to gr. $\mathrm{xv}$ $(0.35-1.0 \mathrm{Gm}$. $)$ three or four times a day, according to age.

Phenacetin, gr. iss. to $\mathrm{gr}$. $\mathrm{xv}(0.1-1.0 \mathrm{Gm}$.), according to age.

Antipyrin, gr. i to gr. $x(0.060-0.65 \mathrm{Gm}$.), according to age.

Acetanilid, gr. ss. to gr. $\mathrm{v}(0.030-0.35 \mathrm{Gm}$.), according to age.

Lesser doses, say one-third, may be repeated at two, three or five hour intervals.

Salicylates.

Sodium salicylate or aspirin (acetyl salicylic acid).

gr. i to gr. $\mathrm{xx}(0.060-1.35 \mathrm{Gm}$.), according to age.

These doses may be repeated at two, three or four hour intervals if pain continues.

Codeine phosphate or sulphate.

For more severe pain.

gr. 1/48 to gr. 1/4 (0.0015-0.015 Gm.), according to age.

Morphine in most severe cases.

Insomnia.

gr. $1 / 120$ to gr. $1 / 4(0.0005-0.015 \mathrm{Gm}$.$) , according to age.$

Bromides, gr. $\mathrm{v}$ to gr. $\mathrm{xxx}(0.35-2 \mathrm{Gm}$.), according to age.

Trional, gr. $\mathrm{i}$ to gr. $\mathrm{x}(0.060-0.65 \mathrm{Gm}$.), according to age.

Codeine sulphate or phosphate, gr. $1 / 48$ to $\mathrm{gr} .1 / 4(0.0015-0.015$ Gm.), according to age.

Stiffness and pain in muscles of the neck.

Heat.

Fomentations.

Convulsions.

Rare.

Warm packs.

Morphine sulphate to control, gr. $1 / 48$ to gr. $1 / 4(0.0015-0.015$ $\mathrm{Gm}$.$) , according to age.$

Bromides to prevent occurrence, gr. $\mathrm{v}$ to gr. $\mathrm{xv}(0.35-1.0 \mathrm{Gm}$. every three to four hours. 
Gastro-intestinal.

Vomiting.

Stop food.

Sodium bicarbonate, gr. v to gr. $\mathrm{xv}(0.35-1.0)$ in water."

Bismuth subnitrate or subcarbonate, gr. v. to gr. $\mathrm{xv}(0.35-$ $1.0 \mathrm{Gm}$.) suspended in water.

Diarrhea.

Regulate food, cut down cream and sugar in milk.

Give castor oil 3 ii- 5 ss. (8-15 c.c.).

Follow with

Bismuth subnitrate, gr. $\mathrm{xv}$ to $\mathrm{gr} . \mathrm{xxx}$ (1-2 Gm.) every two hours.

Tonsillitis and pharyngitis.

Hot saline irrigations, argyrol 15 per cent. to 20 per cent.

(See summary in Scarlet Fever, Chap. XVII.)

Urotropin. (Hexamethylenamine.)

(See text.)

\section{Paralytic period.}

Rest to paralyzed limbs.

Avoiding contractions.

Gentle stretching of muscles.

Rest at bottom of bed for soles of feet in drop of foot.

A box covered with a blanket sufficient.

Take weight of clothes from paralyzed parts with cradles and similar devices.

Light splints to correct position.

Pillows, folded blankets, for same purpose.

Change position of joint from time to time.

Bulbar type.

Muscles of deglutition involved.

Keep mouth free from secretions and food particles.

May have to feed with stomach or nasal tube.

Involvement of respiratory muscles.

Dyspnœa.

Oxygen inhalations.

Atropine sulphate hypodermically, gr. 1/1000 to gr. 1/60 (0.00006$0.001 \mathrm{Gm}$.), according to age.

Caffeine sodio-salicylate or benzoate, gr. $1 / 4$ to gr. v (0.015-0.35 Gm.) or

Strychnine sulphate, gr. 1/500 to gr. 1/30 (0.00012-0.002 Gm.) hypodermically, according to age.

Artificial respiration.

\section{Paralysis of extremities.}

After acute stage subsides.

Massage.

Gentle at first for very short time. Increase up to 15-20 minutes two or three times a day. 
Heat.

Fomentations.

Baking.

Electricity.

Galvanism to nerve trunks.

Faradism to muscles that respond.

Passive movements.

To prevent contractions.

To improve circulation in affected structures.

Active movements.

Best method to develop impaired centres and muscles.

Keynote of success is persistency.

Warm bath a valuable adjuvant. (See text.)

Resistance exercises.

Apparatus.

Crutches, corsets, jackets, braces, splints.

Operative treatment.

Transplantation of tendons.

Arthrodesis.

Silk ligaments.

Operation for contractures.

Prophylaxis.

Avoidance of contacts.

Isolation of patient.

Destruction of secretions.

Members of affected families to avoid contact with children and crowded places.

Children to avoid gatherings of children during an epidemic.

Schools are common centres of the spread of the disease.

Avoid taking children to affected localities, especially in summer. 


\title{
CHAPTER XXVII
}

\author{
SMALL POX
}

\section{(VARIOLA)}

Small pox still jealously guards the secret of its origin. A parasite, the Cytorrhyctes variolæ, first described by Councilman, bids fair, however, to elucidate the mystery. And yet, many of the symptoms of the disease must be attributed to the concomitant action of pyogenic organisms and in all probability it is to these that most of the fatal issues are due.

Jenner's observation that individuals who had suffered from cow pox became by virtue of that fact protected from small pox and his application of that knowledge in terms of vaccination constitutes one of the most dramatic episodes in medical history and is too familiar to want reiteration here. Jenner furnished the means of eliminating the disease forever and only ignorance and criminal carelessness or wilful neglect has prevented the passing of what once was a scourge to the human race.

There seems to be among physicians a wide-spread belief that the infectious organism or virus may be conveyed through the air as well as by contact, which makes isolation more imperative and more difficult; but so good an authority as Rosenau doubts any other mode of conveyance than a direct one, through discharges, secretions or objects, including insects, in contact with such.

In any case efficient treatment begins with isolation as soon as the diagnosis is made or even suspected.

Hospital treatment of the patient is preferable both from his standpoint and that of the community, but if the treatment must be carried out in the home, the physician must realize that a greater obligation rests upon him to establish and maintain isolation and teach the family their part in carrying it out. Skillful nurses can hardly be dispensed with.

Room. The room should be as remote from the rest of the household as possible with a separate approach from the outside to avoid unnecessary contact of nurses and attendants with other parts of the house or members of the family. It should be light, well ventilated, kept at $65^{\circ} \mathrm{F}$. to $70^{\circ} \mathrm{F}$. and screened against insects, whose access 
to the discharges and secretions affords a highly probable mode of conveyance.

The room should be stripped of all unnecessary furnishings and its treatment and separation from the rest of the house carried out as in Scarlet Fever, see Chap. XVII.

Ready approach to a bath room, itself under isolation, facilitates the work about the patient. An open hearth, making possible destruction of discharges on the spot by fire, is a desideratum. The discharges should always be burned. Rules for disinfection of bedding, towels, bed clothes, utensils, etc., may be found under Typhoid Fever, Chap. $\mathrm{XIV}$, and be considered as applicable here.

Nurses. If the nurse has not had the disease, she or he should be vaccinated afresh. The nurse should consider herself as isolated, so far as compatible with her duties. A separate kitchen should be afforded her and, if this is not possible, all dishes and utensils should be treated like those used by the patient. If she goes out at all she should take every precaution not to convey the disease and follow the rules laid down for a nurse attending a Scarlet Fever case. See Chap. XVII.

Treatment of Other Members of the Family or Exposed Individuals. These should all be vaccinated with virus from more than one source to insure a "take." It is certainly desirable to quarantine those exposed, but not always possible. Those who show a successful vaccination may be released when this is obvious; others kept in quarantine for six to eighteen days; but if this is not possible they should be kept under the closest surveillance for the incubation period of sixteen to eighteen days, and isolated on the slightest suspicion of infection.

The apartments vacated by a small pox case must be thoroughly disinfected (see Summary) together with their clothes, toilet articles, utensils, tools or articles with which they may have come in contact since their infection.

Physicians should look upon themselves as potential sources of danger to the community. Except in emergencies they should make their visit to the patient the last one in the day and in any case spend some time in the open air before calling on another patient. They should take every precaution on the occasion of a visit to avoid contamination, should make their visit no longer than is necessary to do their full duty to the patient and then disinfect themselves as thoroughly as possible on leaving. For full details of precautions to be observed by the physician on his visit, see Scarlet Fever, Chap. XVII.

Visitors should not be allowed unless circumstances arise that make it imperative and then should observe all those precautions specified for the physician. 
Bed. The best bed is of the hospital type, an iron half or threequarters bed with woven wire spring and a firm mattress. For the details of bed-making, see Chap. IX.

In very severe cases, especially with skin complications such as boils, abscesses, bed-sores, an air bed or water bed may add greatly to the patient's comfort.

Diet. With the onset there may be decided anorexia and during this period no effort should be made to force the feeding. The diet should be preferably milk or milk preparations or if patients cannot take milk, and the vast majority of those who say they cannot are found to bear it well on trial, one may use cereal waters, cereal "prepared foods" used extensively in infant feeding, then broths fortified with cereals or farinaceous flours and egg albumin water. Later, however, consideration must be had for the theoretical demands of the patient and a fairly high caloric diet may be aimed at. The rules laid down in Typhoid Fever, Chap. XIV, are applicable here. The condition of the mouth in Small Pox makes the ingestion of food more difficult than in Typhoid and the patient must not be nagged and fatigued to ingest definite quantities fixed upon by these theoretical considerations.

Water should be given in abundance. Definite orders should be given the nurse not only to supply the patient's demands but considering the sicker patients too sick to appreciate their needs or to ask for water to offer it to them every hour when awake.

Fruit drinks, lemonade, orangeade, etc., may be used and afford an excellent vehicle to carry sugar, the food value of which contributes materially to the patient's needs.

Alkaline waters, such as Vichy or carbonated waters, if grateful, may be used.

Care of the Body. One has but to visualize the lesion to realize the great discomfort the patient must suffer and the amount of nursing skill and fidelity required to contribute to his comfort.

Mouth. During the pre-eruptive period the mouth should receive the attention usually given it in acute infections.

It should be rinsed after each feeding with water and then with 2 per cent. boric acid solution or half to quarter strength Dobell's solution or the equivalent in one of the many milder antiseptics on the market, the use of which may be more grateful to the patient. The teeth may be cleansed with a very soft brush and a good tooth paste or with a cotton swab on a toothpick saturated with one of the above solutions, particular care being taken to free from food the interstices of the teeth and the dead spaces between the gums and cheek. Dry coating on the tongue may be softened by half strength official peroxide of hydrogen and 
scraped with edge of a whalebone. When, however, the eruption appears, the painful vesicles in the mouth and throat add much to the difficulty of affording proper care. No harsh instrument such as the toothbrush or whalebone can be used. The same solution may be used but irrigations will afford comfort; indeed, much the same measures may be used as in Scarlet Fever. (See Chap. XVII.)

When the mouth is foul, permanganate of potash, a claret colored solution, 1 to 4,000 , may be used as an irrigation or this may be preceded by half strength peroxide of hydrogen. Chlorate of potash gargles may be used, 1 per cent., or less if painful. Ulcers may be touched with silver nitrate solution, 5 per cent. to 10 per cent. When the throat is painful, ice applied to the neck as in tonsillitis may afford relief.

Nose. The nose should be kept free from secretions, by the gentle use of such solutions as have been mentioned in the care of the mouth, as sprays and by cotton swabs, on wooden toothpicks as applicators, dipped in the same solutions. Dried secretions may be moistened first by the application of sweet oil or vaseline.

Eyes. The need of careful attention to the eyes cannot be too much emphasized. At the height of the eruption, it and the attendant edema make proper care of the eyes no easy task.

Boric acid solution, 2 per cent. to 4 per cent., should be applied on wipes to keep the lids clean and be dropped in the eyes. When the eyes close from the edema a warm boric acid solution must be used as an irrigation, the point of a glass irrigating nozzle being gently forced between the lids. To prevent the lids from sticking together an application may be made to their edges of vaseline or $\mathrm{gr}$. i $(0.06 \mathrm{Gm}$.) of yellow oxide of mercury to $3 \mathrm{i}(4 \mathrm{Gm}$.) vaseline.

Frequent and prolonged applications of cold compresses dipped in a 2 per cent. to 4 per cent. boric acid solution or physiological salt solution tend to lessen the edema and discomfort.

When the conjunctivitis is very severe, 5 to 20 per cent. argyrol solutions are useful applications.

When corneal ulcerations occur a 1 per cent solution of atropine sulphate should be used.

Skin. One can imagine the task imposed on the skill of the nurse and the ingenuity of the physician to keep the skin, the site of a multitude of pustules, hot, painful and itching, in even relative comfort. In the pre-eruptive period, sponge baths of soap and warm water are proper and desirable, but during the height of the eruption sponge bathing is no longer feasible. Some authors urge, however, the use of prolonged warm baths, keeping the patients immersed for hours at a time. It is claimed for these warm baths, that they reduce the fever, 
lessen the nervous excitation, are a sedative to the skin and macerate the pustules, thus effecting an earlier discharge of their contents. Certainly the procedure seems a rational one and the criterion of its sucoess is the response of the patient. The temperature of the water should be about $95^{\circ} \mathrm{F}$. It is best carried out in a long bath-tub at the bed side with a comfortable hammock arrangement attached to the sides of the tub to suspend the patient and facilitate moving him.

Burning and Itching. This may be delayed by prolonged warm baths at $95^{\circ} \mathrm{F}$. or the efficacy of the baths may be enhanced by the addition of carbonate of soda, 2 to 10 ounces of soda to a bath-tub of water (30 gallons).

As the itching and burning is most intense in the face and hands, cold compresses may be applied to them with comfort. Hot compresses, especially to the extremities, are sometimes found more grateful.

Rührah has mentioned the efficacy of alum solutions 1 per cent. to 2 per cent., or baths in a 1 to 1,000 solution ( 1 pound of the alum in a bath-tub of 500 liters).

When watery solutions are not applied oily substances find favor, for they both alleviate the discomforts and keep the pustules soft and facilitate their discharge. Opening these pustules to relieve the patient of the effects of absorption from them appeals to me. Of course, all applications must be frequently changed, especially in the pustular stage, as the dressings soon become drenched with discharges.

Ointments are especially applicable when the crusts begin to form.

Simple vaseline or sweet oil may be applied to the skin or to dressings, but the itching is more quickly ameliorated if 3 per cent. to 5 per cent. carbolic (phenol) is added to the one or the other.

Some clinicians advocate glycerin one part to two of water as an excellent application.

The itching is sometimes intolerable; yielding to it lacerates the skin and invites such secondary infections as abscesses and erysipelas. In children mechanical restraint may be necessary to prevent the scratching, such as splints at the elbows and thick "boxing-glove" like bandages to the hands. Innumerable applications have been advocated, their very number declaring the difficulty of the problem. Hubbard uses on the face white precipitate ointment (ointment of ammoniated mercury) linen mask. Appreciating the possibility of absorption he changes occasionally to ordinary zinc oxide ointment. He also uses cold watery solutions of glycerin $3 \mathrm{i}$ to $亏 \mathrm{i}$ (4-30 c.c.) applied on absorbent cotton.

Rūhrah advocates spraying with alcohol to relieve the itching and sometimes adds $1 / 2$ per cent. to 1 per cent. menthol to this. He further 
speaks of the value of spraying with carbolic acid (phenol) in water solution 1 to 40 .

Schamberg advocates warmly the use of undiluted tincture of iodine. He begins this early and applies it to the face once or twice a day, if the skin is not too sensitive. In some cases a dilution of one-half may be used. He claims not only increased comfort under the application but a modification in the severity of the eruption, a decrease in the secondary infections and a relief from offensive odors. (Practical Treatment, Musser and Kelly.)

If the face becomes dry and uncomfortable under the treatment, they apply such ointments as cold cream. Powders, too, have been used to allay the itching. Hubbard (Hare's Modern Treatment) speaks highly of one made of equal parts of boric acid, lycopodium, corn-starch and subgallate of bismuth. He applies this freely.

The offensive odor demands the physician's attention.

The application of iodine lessens this.

Schamberg uses iodoform 2 parts, boric acid 10 parts, and talcum 28 parts, dusting it on freely after a bath.

Aristol (thymol jodide) may be used instead of iodoform.

To Separate the Scabs. Warm sponge or tub baths facilitate the separation as do ointments or plain vaseline.

Care of the Skin. When the scabs fall off the skin is often very tender and the application of bland and sterile toilet powders are grateful.

Complications such as furuncles, abscesses and erysipelas are to be treated on surgical principles.

If the head is shaved at the beginning of the disease local treatment of the eruption on the scalp will be greatly facilitated.

\section{SYMPTOMATIC TREATMENT}

Bowels. When first seen the patient should be given an initial catharsis of a salt, Rochelle, Epsom, or Glauber's, ₹ss.-i (15-30 Gm.) in three-fourths glass of water or this may be preceded by calomel in divided doses gr. $1 / 4(0.015 \mathrm{Gm}$.) every quarter hour for six doses and two or three hours later followed by the salt.

Later the bowels should be moved daily or every other day by enemata or milder salines, such as Hunyadi water, milk of magnesia or one of the milder salines on the market.

The initial stage, which marks the period between the onset and the eruption, usually lasts three days. It is rife with discomforts. The onset is sudden and characterized by the severity of pains in the 
head and back, the nervous manifestations of the toxemia and the fever.

A chill usually precedes the febrile impulse, but requires no especial consideration.

The fever as a rule is maintained during the initial stage at about $104^{\circ} \mathrm{F}$. Such a degree of temperature requires no interference. Cool sponges, however, afford the patient comfort.

At the end of the initial stage the temperature goes down to normal or to but a little above and stays down until the stage of suppuration, when it goes up again.

Excessive high temperatures are best controlled by the cold pack or prolonged tepid baths at $95^{\circ} \mathrm{F}$.

Headache is severe, often intense. It is best treated by the application of the ice-bag or ice-coil.

In the early stages, the sthenic period of the fever, small doses of phenacetin, gr. iii to gr. $\mathrm{v}(0.2-0.3 \mathrm{Gm}$.) at half-hour intervals for three or four doses, or acetanilid in doses of gr. iss. to gr. ii $(0.10-0.120 \mathrm{Gm}$.) at the same intervals for three or four doses may be safely administered if the patient has not cardiac trouble. Later when the circulatory apparatus has felt the effects of the toxins of the disease all members of the coal-tar series should be avoided, because they are themselves depressing.

If the headaches are agonizing, as they sometimes are, morphine may be imperative.

The same drugs may be used to relieve the backaches.

Delirium, may occur in the initial stage, but is more common and violent in the suppurative stage. Fresh air and the ice-bag applied to the head may give some measure of relief. Prolonged tepid baths are probably more efficacious.

The patients must be very carefully guarded lest they leave the bed and do themselves harm. Sometimes mechanical restraint is necessary. Sheets drawn fairly snugly and firmly secured with the hands held under them is the most humane method.

If delirium becomes so decided as to require drugs, morphine is the best to use, in doses of gr. $1 / 8$ to gr. $1 / 4(0.008-0.015 \mathrm{Gm}$.) of the sulphate hypodermically and this may have to be given three times in the day.

Insomnia, when continued night after night becomes a serious consideration, as it takes a mighty toll of the patient's strength. Wellventilated rooms, good nursing, enough water to drink, all conduce to good sleep. The ice-bag is a help, the prolonged warm baths contribute; but in spite of these measures drug assistance may be needed. The mild hypnotics may be tried at first, such as trional gr. $\mathrm{x}$ to gr. $\mathrm{xx}(0.65-1.30$ $\mathrm{Gm}$.) in a little whiskey or in powder form, washed down with water, or 
in a little warm fluid like barley water or milk; or chloralamid may be used, gr. $\mathrm{xv}$ to gr. $\mathrm{xxx}(1-2 \mathrm{Gm}$.) in whiskey or as a powder, but not in warm menstrua, which decompose it.

Either of these drugs may be repeated in the same dose in two or three hours if necessary.

Bromides in doses of gr. $\mathrm{xxx}(2 \mathrm{Gm}$.) may be given in the early evening or this dose may be given in the late afternoon and repeated in the evening. One may use the potassium salt or the mixed or "triple" bromides, potassium, sodium and ammonium gr. $\mathrm{x}(0.65 \mathrm{Gm}$.) each. Administer in water. Chloral in gr. $\mathrm{x}$ doses $(0.65 \mathrm{Gm}$.) may be tried in the initial stage. It is given in water or dilute whiskey, brandy or wine. It should not be given later when the centers are depressed.

Too many attempts with these milder hypnotics should not be made to the sacrifice of the patient's strength, but morphine should be used in doses of gr. $1 / 8$ to gr. $1 / 4(0.008-0.015 \mathrm{Gm}$.) of the sulphate hypodermically.

Gastro-intestinal symptoms are not striking, but there may be nausea and vomiting at the onset. This rarely requires treatment, but if retching continues copious draughts of warm water may afford relief or small doses of sodium bicarbonate gr. $\mathrm{x}$ to $\mathrm{gr}$. $\mathrm{xx}(0.65-1.20 \mathrm{Gm}$.) or bismuth subnitrate $\mathrm{gr} . \mathrm{x}$ to $\mathrm{gr} . \mathrm{xv}(0.65-1 \mathrm{Gm}$.).

Respiratory Symptoms. There may be some laryngitis and this is best treated by inhalations of steam or medicated steam, using compound tincture of benzoin, oil of eucalyptus, menthol or oil of pine; but the effort to inhale and the discomfort of the heat attending the administration may outweigh the benefit accruing to their use.

Some bronchial involvement is common, if not constant. In some cases bronchopneumonia may occur. For the treatment of these conditions, so far as the eruption will permit, see Pneumonia, Chap. IX.

Edema of the glottis is a much severer complication and should this intervene demands intubation (see Diphtheria, Chap. XVIII) or tracheotomy.

Circulation. When evidences of a failing circulation occur, all those measures applicable to the same condition in other acute infections are indicated. For detail, see Pneumonia, Chap. IX.

Release from Quarantine. This should be permitted only when the desquamation is completed, and this occurs last in the thick skin of the hands and feet. Warm baths and the use of soap facilitates the desquamation. Sweet oil or vaseline softens the dry skin and hastens its separation.

Finally the patient should have an antiseptic bath of 1 to 10,000 bichloride after a thorough soap and water bath and a shampoo. 
He should then be removed to a clean room and given clean clothes or his old clothes only after a thorough disinfection.

Disinfection. All those articles that have come in contact with the patient and which can readily be spared should be burned when possible.

Such articles as clothing, bedding, mattresses, carpets, should be disinfected by superheated steam. If this is not possible, mattresses and pillows had better be burned, washable bedding boiled thoroughly and carpets be submitted to the disinfecting vapors used to disinfect the room.

Formaldehyde vapor gas is the best as it is not injurious to any fabric or color. Sulphur dioxide is also efficacious but its injurious effects on metals and fabrics must be remembered.

For details of disinfection, see Scarlet Fever, Chap. XVII.

The dead should be cremated or the body wrapped in sheets saturated in strong antiseptics and buried deep.

\section{VACCINATION}

That after more than one hundred years of the beneficent results of vaccination there should still be found bitter opponents to the procedure, forces us to accept them as psychological aberrants, in no way amenable to facts or reason.

Were vaccination universally and properly carried out there would be no small pox to treat, for the sporadic case and the poorly protected community that furnish the factors necessary for an epidemic would cease to exist and a disease whose mortality is 45 per cent. to 50 per cent. and in individual epidemics even higher would become of historic interest only.

Time for Vaccination. The first vaccination should be in early infancy. Before the fifth month the reaction is less than later. As an accoucheur it was my custom to vaccinate the infant before the lying-in period was completed. The results were most satisfactory both with reference to the character of the "takes" and the absence of constitutional reactions. Revaccination should be done between the ages of ten and fourteen and again and as often as the individual is directly exposed or an epidemic prevails in his community, unless a previous vaccination dates back to only one or two years.

Vaccination or revaccination is to be insisted on in every member of the patient's family exposed and revaccination should be practiced by the physician and nurse on the occasion of every small pox patient seen, unless the interval is less than a year. A failure to "take" on 
revaccination may be due to many other causes than lack of susceptibility, so that in case of marked exposure, a surety of technique and freshness and activity of virus must be had and a repetition of the inoculation is the better part of wisdom.

The average period of protection is usually accepted as seven years, but this period must not be accepted as an excuse for not revaccinating after exposure.

All persons who have been exposed should be revaccinated, nor should the length of time after exposure enter into the question of whether the exposed individual should be revaccinated or not, for even if the protection afforded be not complete it may be relatively so. Early in the incubation period a vaccination will prevent the disease. In these cases the typical vaccination pustule has time to develop before the febrile period is due. In the middle of the incubation period vaccination will serve to modify the severity of the disease; late it will have no effect.

Previous vaccinations enhance the efficiency of the last done during the incubation period. This period is ten to twelve days.

Technique. The virus must be active and secured from recognized sources.

Virus furnished in capillary tubes, each a single inoculation, is preferable to the dried virus on points. If kept, it must be kept cold, as high temperature destroys it or lessens its strength.

The site of inoculation is best rendered clean by soap and water, best by green soap and boiled water, and this followed by alcohol. When dried it is ready for the inoculation. The site preferred is the arm, usually the left, at the insertion of the deltoid. The resulting scar, which in a woman is deemed undesirable at this site, may lead one to choose the leg. The place commonly chosen is on the outer aspect of the leg (not thigh) just below the head of the fibula.

The inoculation. A slight wound is made at the chosen site. Some prefer scraping with the edge of a scalpel, others a scarification by cross-hatching, and others incisions. Incisions are insisted on in Germany and recommended in England. They are made with the edge of a scalpel or point of a needle in Germany, $1 \mathrm{~cm}$. long and $2 \mathrm{~cm}$. apart, 4 in number.

I prefer a small cross-hatch of about $1 / 8$ inch square made with a needle sterilized in a flame. If there has been exposure three or four of these $3 / 4$ inch apart.

Blood should not be drawn by any method to a greater extent than to produce bloody serum. The virus is then applied to the abrasion and rubbed gently in with a sterile needle, using the eye-end or a sterile wooden toothpick, using the blunt end. The serum and virus is then 
allowed to dry in the air. The only protection necessary is several layers of clean gauze sewed into the sleeve of the undervest, drawers-leg or stocking over the site of the inoculation. Once thoroughly dry; a folded clean handkerchief may be used. If the child cannot be trusted to avoid scratching, this gauze should be secured to the part by adhesive strapping, but should be loose over the inoculation.

Shields, pads and other protective contrivances do very much more harm than good.

After the first day bathing, including the site of the inoculation, need not be avoided.

If the vesicle ruptures it is to be cleansed with saturated boric acid solution and 10 per cent. boric acid ointment (made with vaseline) may be applied.

If it becomes infected it is to be treated exactly like any infected wound, on surgical principles.

If the vaccination "takes," on the third or fourth day a papule appears, and on the fourth or fifth day a vesicle with umbilication, showing an infiltration.

The vesicle becomes mature on the seventh, shows the pustule on the eighth with a second umbilication. An areola has formed about the developing vesicle and on the ninth day this begins to fade, on the eleventh or twelfth day the scab forms and slowly separates in the third or fourth week, leaving a pit which heals with a scar showing the characteristic pits or foveations which are the sign of a successful vaccination.

General Symptoms. With the appearance of the vesicle and during its development there may be, but not necessarily, a febrile reaction with attendant discomforts such as headache, backache, etc., simulating a mild attack of grip. The part inoculated may itch or burn or a considerable inflammatory reaction with a resulting sore arm may ensue.

The regional lymph glands are usually swollen and often tender.

Immunity is established about the time of the pustule formation, on the eighth day.

Complications of Vaccinations. These are percentually small, but in one or other form must be met by every active practitioner. The most common result from infection of the wound by pyogenic organisms.

All too frequently one sees deep and broad ulcerations; less commonly, abscesses, cellulitis, lymphangitis, phlebitis, suppurating glands, gangrene, erysipelas and pyæmia. As a less serious complication we see occasionally an impetigo contagiosa. When one considers the careless and often filthy habits of many who are compulsorily vaccinated, the numbers of serious infections seem incredibly small. 
These complications are nearly all surgical problems and to be treated on surgical principles.

Another serious but fortunately rare complication, which from its dramatic course always attracts public attention, is Tetanus.

It is true that this unfortunate infection, which has been traced more than once to the virus (and then several such cases, directly traceable to the same supply of virus), does occur; but I am convinced that in the vast majority of instances and in all sporadic cases it is due to infection of the wound by the patient.

A case seen recently by me in consultation was such an one, for no other case from this virus had been reported to the Board of Health and the technique of the practitioner was irreproachable. The child was saved by prompt intraspinal administration of tetanus antitoxin. (See Tetanus, Chap. XL.)

Generalized vaccinia occasionally occurs as a result of vaccination.

It appears usually during the development of the vesicle to a pustule, comes out in crops and may last some time, in rare instances three to four weeks.

The same thing may occur as the result of auto-inoculation. This is due to conveyance of the virus from the ruptured pustule to other slight abrasions on the body, most commonly by scratching.

Contraindications. During illness or if recently exposed to the exanthemata; if there is any suppurative process in the body or of the skin; in illy nourished infants or in adults suffering from severe constitutional or organic disease. It is contraindicated in bleeders for obvious reasons.

It is better not to vaccinate during menstruation, unless the need is urgent.

Revaccination. The course is not always the same as in the first vaccination.

It may be in those cases in whom the immunity has disappeared; but those in whom a partial immunity persists, an anaphylactic state or "allergie", show what the students of anaphylaxis entitle "an accelerated reaction"; that is, a shortened incubation period and pustule formation (the sixth day); or "an immediate reaction" with an incubation period of less than twenty-four hours and the formation of a papule or erythematous halo about the site of the inoculation, the equivalent of the now familiar Von Pirquet's tubercular reaction.

Conviction, vision, courage, persistency in advocacy, were all needed to establish this amazing contribution to human welfare one hundred years before the birth of immunilogical studies and all these were found in Jenner. 


\section{SUMMARY}

Isolation.

Hospital treatment preferred.

Home treatment.

\section{Room.}

(For choice anditreatment of room, see Pneumonia and Scarlet Fever, Chaps. IX, XVII.)

Disinfection during illness of discharges, clothes, objects. (See Typhoid Fever, Chap. XIV.)

\section{Nurses.}

Should be revaccinated.

Isolated from rest of household.

(See Scarlet Fever, Chap. XVII.)

Treatment of other members of the family or exposed individuals.

Vaccinate with a virus from more than one source.

Quarantine if possible.

Release when vaccination is successful.

If vaccination does not "take", quarantine or keep under closest observation sixteen to eighteen days.

\section{Physicians.}

Revaccinate.

Make case last call of day if possible.

Stay only long enough to fulfil duties.

Do not call on a susceptible person at once, but keep in open air for a time.

(For rules of visit and disinfection on leaving see Scarlet Fever, Chap. XVII.)

Bed.

(For choice and technique of making bed, see Chap. IX.)

Diet.

Don't force during early stages when there is much anorexia; milk, milk preparations, cereal waters, cereal foods (infant foods), broths fortified with cereals or farinaceous flours, egg albumin.

Later, try to approximate food needs. (See Typhoid Fever, Chap. XIV.) Condition of mouth makes it difficult.

Water, offer freely, every hour.

Fruit drinks, lemonade, orangeade, etc., fortified with sugar. Alkaline waters.

Care of the body.

Mouth.

Pre-eruptive period.

Rinse after each feeding with water, then with 
Boric acid solution 2 per cent. to 4 per cent. or

Dobell's solution half to quarter strength.

Teeth cleansed with soft toothbrush and good paste or with cotton swabs on wooden toothpicks, wet with above solution.

Free interstices of teeth and dead spaces between cheeks and gums from food.

Soften coating on tongue with half strength official peroxide of hydrogen. Scrape with edge of whalebone.

Eruptive period.

Cannot use toothbrush or whalebone.

Irrigations of above solutions.

When mouth is foul.

Permanganate of potash solution, claret colored $(1-4,000)$. Chlorate of potash gargles 1 per cent. or less.

Ulcers.

Touch with silver nitrate solution 5 per cent. to 10 per cent.

Nose.

Dried secretions softened with sweet oil or vaseline.

Cleansed with above solutions applied with swabs on toothpicks or as

Sprays.

Eyes.

Cleansed with 2 per cent. to 4 per cent. boric acid solution.

When closed with edema

Gentle irrigation of conjunctival sacks with warm boric acid solutions 2 per cent. to 4 per cent.

Cold compresses of boric acid solution, frequent and prolonged.

Prevent lids sticking by applying vaseline to edges or 1 part of yellow oxide of mercury to 60 of vaseline.

Severe conjunctivitis.

Five per cent. to 20 per cent. argyrol solution once or twice a day.

Corneal ulcerations.

Skin.

Apply atropine sulphate solution 1 per cent.

Pre-eruptive period.

Sponge baths of soap and warm water.

Eruptive period.

Prolonged warm baths, at $95^{\circ} \mathrm{F}$. in tub.

Burning and itching.

Warm baths at $95^{\circ} \mathrm{F}$.

Bicarbonate of soda may be added to the bath.

Cold compresses.

Alum solutions 1 per cent. to 2 per cent.
Alum baths 1 to 1,000 solution.

Simple vaseline.

Sweet oil.

Three per cent. to 5 per cent. phenol in oil or vaseline.

Glycerin 33 per cent. solution in water. 
To prevent scratching in children.

Mechanical appliances.

Splints to elbows.

Bandages to hands.

(For other appliances, see text.)

Cold cream.

Powders.

Sterile toilet powders.

$\mathrm{R}$
Boric Acid, Lycopodium,
Corn Starch.
Hubbard.
Subgallate of Bismuth, equal parts.

Offensive odors.

$R_{*}$

Iodoform, 2 parts,

Boric Acid, 10 parts, Schamberg.

Talcum, 28 parts.

Aristol (Thymol Iodide).

To separate scabs.

Warm baths.

Vaseline.

Apply sterile toilet powders to tender skin after scabs fall off.

Furuncles,

Abscesses, $\}$ Apply surgical principles.

Erysipelas.

Symptomatic treatment.

Bowels.

Calomel, gr. 1/4 (0.015 Gm.) every quarter hour for four doses, then in two hours,

A salt, Epsom, Rochelle or Glauber's $₹$ ss. to i (15-30 Gm.) or a salt alone.

Later.

Milder salines.

Milk of magnesia.

Citrate of magnesia.

Hunyadi water or

Enemata.

Fever.

Cool sponges.

Prolonged warm baths at $95^{\circ} \mathrm{F}$.

Excessive fever.

Cold packs.

Headache.

Ice-bag or ice-coil.

Early stages.

Phenacetin gr. iii to gr. v $(0.20-0.30 \mathrm{Gm}$.) every half hour for three to four doses. 
Acetanilid, gr. iss. to gr. ii $(0.10-0.12 \mathrm{Gm}$.) at same intervals.

Do not use these when circulation is depressed.

When very severe.

Morphine sulphate, gr. 1/12 to gr. 1/4 (0.005-0.015 Gm.).

Backache.

Same drugs as for headache.

Delirium.

Fresh air.

Ice-bag.

Prolonged warm baths at $95^{\circ} \mathrm{F}$.

Mechanical restraint, best with tightly drawn sheets.

Morphine sulphate hypodermically, gr. $1 / 8$ to gr. $1 / 4(0.008-0.015$ Gm.).

Insomnia.

Air.

Ice-bag to head.

Prolonged warm baths at $95^{\circ} \mathrm{F}$.

Trional gr. $\mathrm{x}$ to $\mathrm{gr} . \mathrm{xx}(0.65-1.30 \mathrm{Gm}$.) in whiskey or in powder. May repeat in two hours if needed.

Chloralamid, gr. $\mathrm{xv}$ to gr. $\mathrm{xxx}(1-2 \mathrm{Gm}$.) in whiskey or in powder. (Do not give in warm drink.) May repeat in two hours.

Bromides, gr. $\mathrm{xxx}$ in evening or late afternoon and repeat in evening, either potassium bromide or the mixed, "triple," bromides of potassium, sodium and ammonium.

Chloral, gr. $\mathrm{x}(0.65 \mathrm{Gm}$.) in water, whiskey or wine. Do not use except in sthenic period.

Morphine sulphate hypodermically, gr. $1 / 8$ to gr. $1 / 4(0.008-0.015$ Gm.).

Gastro-intestinal symptoms. Not marked. (See text.)

\section{Respiratory symptoms.}

Laryngitis.

Inhalations of steam, plain or medicated with

Compound tincture of benzoin.

Oil of eucalyptus.

Oil of pine, a teaspoonful or two in the hot water, or

Menthol, a few drops of saturated alcoholic solution in the hot water of the inhaler.

Cold compresses to neck.

\section{Bronchopneumonia.}

(See Pneumonia, Chap. IX.)

\section{Edema of glottis.}

Intubation. (See Diphtheria, Chap. XVIII.)

Tracheotomy.

\section{Circulation.}

(See Pneumonia, Chap. IX.) 


\section{Release from quarantine.}

When desquamation is complete.

Soap and water bath and shampoo, then

Give antiseptic bath of 1-10,000 bichloride of mercury.

Remove to clean room and put on clean clothes.

Disinfection.

(See Scarlet Fever, Chap. XVII.) 


\section{CHAPTER XXVIII}

\section{TYPHUS FEVER}

THE infecting agent in typhus fever has not been definitely determined, but the isolation by Plotz of New York of a Gram positive pleomorphic bacillus, termed bacillus typhi exanthematici from the blood of typhus patients, which fixes complement in the serum of convalescents from the disease, makes a fair claim to the solution of the problem. The mode of conveyance has been discovered, thanks to the brilliant work of Nicolle and Conseil, Anderson and Goldberger, Ricketts and Wilder, and Gavino and Girard. To this work Ricketts yielded his life, a martyr.

Again, as in Malaria, an insect plays the rôle of the intermediary; this time the louse, Pediculus vestimenti, conveying the organism by its bite from the infected patient to a susceptible individual. This constitutes the most important contribution to our knowledge of the disease ever made and explains many observations on its epidemicity; its association with crowding, wretchedness, its "short striking distance," its prevalence in cold climates and in cold season, etc. It also renders precise efforts to prevent its spread or its occurrence.

In these latter years typhus fever had been considered a rarity in the Northern States. Recently a symptom complex which had many features in common with mild typhus fever was reported by Brill of New York. A considerable number of these cases was observed in New York, Chicago and other Northern cities, and, for a time, was considered as possibly a new disease. It has been shown, however, experimentally, that this disease is identical with Mexican (Tabardillo) and European Typhus and that it is conveyed by the same means. The incubation period of typhus lasts as a rule from eight to twelve days; exceptionally as short as four or as long as fourteen. The invasion is abrupt and the duration lasts from twelve days in a child to twentyone to twenty-four in an adult. In the monkey the period has been observed to be twelve days.

The onset of the disease is rapid, characterized by the sharp rise of temperature which reaches its maximum on the second or third day and is accompanied by very severe headache.

The temperature is sustained throughout the disease and falls by crisis or rapid lysis on the 12 th to 14 th day. The characteristic erup- 
tion appears on the third to the fifth day, first on the abdomen, then on the chest, the shoulders, the back and the extremities. It takes two to three days to reach its full development and there are no second crops. The rash suggests measles, but later becomes petechial.

Nervous manifestations, delirium or stupor and progressive and severe toxemia are the striking features of the disease.

Cardiac weakness may appear early, but is much more pronounced with the appearance of the rash. The blood count shows a slight leucocytosis of about 10,000 and a polymorphonucleosis of 80 per cent. to 85 per cent. The mortality varies in different epidemics from 15 per cent. to 60 per cent. The sporadic cases as seen in New York and termed Brill's Disease are relatively benign, the mortality not reaching more than 1 per cent. to 2 per cent.

\section{TREATMENT}

Isolation. The knowledge of the mode of conveyance makes our efforts at prevention of spread more successful than in the days when it was supposed to be conveyed by fomites or more mysterious agents. The keynote to prevention of the spread lies in the destruction of the body louse. The patient should have a room chosen with reference to an abundance of light and air and convenience for nursing.

In epidemics, treatment in tents is preferable to the more poorly ventilated wards.

The choice of the bed and the manner of its preparation should be exactly the same as in Typhoid. (See Typhoid Fever, Chap. XIV.)

As soon as the diagnosis of typhus fever is made, the patient should be undressed and the clothes disinfected. The hair of the head, pubes, axilla and chest should be shaved and the hair burned.

The patient is then scrubbed with soap and water and bathed with dilute ( 1 per cent.) carbolic acid or 50 per cent. alcohol. After the skin is dry the entire body is sprayed with crude petroleum to destroy lice and ova. The patient is put to bed in an environment free from vermin.

The hair on the pubes and in the axilla or in hairy individuals that on the chest, back or elsewhere should have mercurial ointment applied to these parts.

If the head is not shaven but the hair merely clipped, the eggs may be loosened from the hair by soaking the head in vinegar overnight and wiping off the eggs with a towel wet with bichloride.

It must be remembered that it is the body louse found in the clothing that is the transmitting agent. 
The clothes worn by the patient should either be destroyed by burning or the lice killed by immersion in boiling water. Boiling five minutes is said to kill both lice and eggs; a good measure of time increases the assurance. Live steam is an excellent measure applicable to extensive disinfection as practiced in delousing plants during the war.

The bed where the patient has been sleeping should be scrubbed with soap and water and treated with 5 per cent. solution of carbolic acid; the mattress and the blankets subjected to steam, the sheets and spreads boiled; the room is disinfected with sulphur dioxide (use 5-8 pounds per 1,000 cubic feet and leave the room sealed 12-24 hours), or the walls and floors may be sponged thoroughly with crude petroleum and after 12 hours scrubbed with soap and water.

Nurses and attendants should wear a one-piece garment which can be tucked into high boots, rubber gloves and close-fitting head gear to prevent pediculi gaining access to the skin.

Dr. A. C. Burnham of the American Red Cross writes us of the following simple device for disinfecting clothes:

"In an ash can or boiler place several bricks, add sufficient water to half cover them. Loosely pack clothes in a wire cage (of chicken or other similar wire) and suspend it by hooks to the rim of the can so that it rests on the bricks, but swings free of the water.

Close the can tightly and place ten to fifteen pounds of weight on the cover so that the steam will be under pressure. Boil the clothes for one hour; this will kill all the lice and most of the nits."

Burnham says he found in clothing not badly infested the nits could be removed by carefully pressing with a very hot iron. This has the advantage of sparing woolen garments from no inconsiderable shrinkage. Of course the garments should be turned inside out and carefully pressed along the seams.

Furs and shoes, if dry, may be disinfected by exposure to hot air $\left(60^{\circ}\right.$ C. $)$ for 30 minutes.

In military service where delousing measures are carried out on a large scale, treatises on military practice should be consulted.

Care of the Patient. A cleansing bath of soap and warm water should be given daily. Late in the disease particular care should be given to prevent the formation of bed sores by turning the patient from one side to the other frequently, by rubbing the points of pressure, keeping the parts dry, and, when the parts show signs of pressure by persistent redness or bluish discoloration, using rings or cushions to lift the part off the bed. When bed sores form, if superficial, they are to be treated by scrupulous cleanliness and the utilization of some drying powder such as stearate of zinc, or zinc oxide, talcum and starch mixtures. If the sores 
are more extensive they should be treated on surgical principles and the utilization of a water bed or air bed may be necessary.

The mouth and nose should be treated as in other. infections by keeping them clean with such mild antiseptics as boric acid solution 2 per cent. to 4 per cent. or some mild alkaline solution of which there are many on the market. This care should be exercised after every feeding and particular attention should be given to the removal of food particles from between the teeth and from the dead spaces in the mouth between the gums and teeth.

In the severe cases the mouth becomes very dry, the tongue heavily coated and sordes appears upon the teeth and lips.

The ease with which otitis and parotitis ensue upon a foul condition of the mouth emphasize the importance of these measures.

The treatment will be found detailed in the article on Typhoid Fever (Chap. XIV).

Diet. Of prime importance is the administration of an abundance of water; a sufficiency of water diminishes dryness of the mouth, torpidity of the bowel and heightens the efficiency of the emunctories.

The mental condition of the patient is such that one should offer him water at frequent intervals, at least hourly, and not wait for expression of thirst on his part. All the water should be given that the patient will willingly take, and this may amount to three, four or more quarts a day. The administration of food is determined by the same physiological necessity as in any continued infection (see Diet in Acute Infectious Diseases, Chap. II), and the rules laid down for diet in Typhoid Fever hold for Typhus, except that it is to be remembered that the urgency for a high caloric diet is not so great because the infection runs a shorter course, and further, that the degree of toxemia as a rule makes the feeding more difficult than in Typhoid Fever.

One may have to depend almost entirely upon liquids or semi-liquid foods, such as are set forth in the Typhoid dietaries.

Fever. The fever is usually high and is sustained often at $104^{\circ} \mathrm{F}$. to $105^{\circ} \mathrm{F}$. throughout. Except in rare cases of hyperpyrexia the treatment directed at the fever is really directed at the toxemia and its effects upon the circulation and respiration; hence, simple antipyretics, such as the coal tars, are contraindicated; first, because the fever, per se, is not a menace to the patient, but, except in hyperpyrexia, is rather a purposeful reaction of the body harboring toxic material; secondly, because these drugs are direct and potent depressants of the circulation.

The antipyretic measures, however, which are legitimate are those 
which are at the same time stimulating to the vital functions and are used because of this latter effect.

They are, first, the use of cold water, and one may have recourse to the same hydrotherapeutic measures as in Typhoid; the baths, the slushes, the packs, the sponges, with the same indications and contraindications as in Typhoid (for which see Typhoid Fever, Chap. XIV); second, open air. The preparation of the patient and his bed for open air treatment is identical with that of Pneumonia (see Pneumonia, Chap. IX) and, as in Pneumonia, the delirium of the patient necessitates the constant presence of nurse or attendant to prevent the patient from leaving his bed and doing himself some injury.

Circulation. Except in fulminating cases the circulation does not show the depressing effects of the toxin until the second week; then the rapid heart, the low blood pressure and the change in the quality of the heart sounds bespeak its depressing effects. One may use digitalis and other circulatory stimulants in the same manner as described for failing circulation in pneumonia. (See Pneumonia.)

It must be remembered, however, that more potent than these drugs are the effects of fresh air and cold water as described under Pneumonia.

Bowels. Early in the disease the intestine should be thoroughly evacuated by the use of Epsom or Rochelle salt in doses of $1 / 2$ ounce to 1 ounce $(15-30 \mathrm{Gm}$.) or by castor oil in the same dose or by calomel, grains $11 / 2$ to 2 grains $(0.10-0.12 \mathrm{Gm}$.) followed in four hours by a salt as above.

If there be nausea or vomiting, calomel is especially indicated but in divided doses, $1 / 4$ grain $(0.015 \mathrm{Gm}$.) every quarter hour until six or eight doses are taken. Later the bowel is to be moved by an enema. In this disease constipation and meteorism occur not infrequently and when the circulation is embarrassed or the lung involved the latter constitutes a true danger. It is to be combated in the same manner as in Typhoid. (See Typhoid, Chap. XIV.)

Nervous Manifestations. These are particularly shown in the severe headaches, the delirium or the stupor. The headache is often intense and is best met by the use of the ice-cap; in the more violent headache morphine may be necessary.

Delirium and Headache. During the first half of the first week the mind is usually clear; then delirium or stupor intervenes; the delirium is violent and hallucinations of a terrifying character occur. The patient is peculiarly alert and requires continual watchfulness. The delirium increases during the development of the eruption. It is to be treated by the application of the ice-bag to the head; in milder forms by bromides, 
15 to 30 grains $(1-2 \mathrm{Gm}$.) three or four times a day; but in the severe types necessitates the use of morphine. Lumbar puncture may afford relief. Instead of delirium the patient may go into stupor.and coma; all the nervous symptoms are likely to be less severe or are ameliorated by use of the cold bath or by treatment in the open air, but in the latter case some restraint and constant guarding are necessary.

The temperature as a rule subsides by crisis or rapid lysis occupying a couple of days. If the defervescence occupies a much longer period one should think of the possibilities of complications.

Convalescence. Convalescence is usually rapid; the diet is increased and the patient is allowed to sit up in bed, then in the chair, and then to walk around. These efforts are determined by the patient's returning strength and by evidences that the circulation has regained its strength as shown by no great increase in pulse rate when these efforts are made.

Complications. Laryngitis is fairly common and may be treated by inhalations of steam, or steam medicated with compound tincture of benzoin, oil of pine, or the oil of eucalyptus, a teaspoonful on the hot water in the croup kettle or a substitute for it in the shape of a pitcher or pail.

Edema of the larynx sometimes occurs and may require intubation, tracheotomy or scarification. Bronchitis and Pneumonia as well as Pleurisy and Empyema can take place, and are to be treated as under other circumstances. Frequently turning the patient lessens the congestion that favors the onset of these complications.

Diarrhea. While constipation is the rule, diarrhea sometimes is seen. Profoundly toxic patients may suffer from incontinence. Proper care of the bowels from the start renders the onset less probable; it is to be treated by Bismuth subnitrate in 30 grain $(2 \mathrm{Gm}$.) doses every two hours, or by small doses of castor oil, 10 minims ( 0.60 c.c.) every two hours, to which 1 minim $(0.060 \mathrm{Gm}$.) of the deodorized tincture of opium may be added with benefit. In either case it is well to administer a large dose of castor oil, $1 / 2$ ounce to 1 ounce (15-30 c.c.), every second day.

Parotitis. This complication may result from neglect of the mouth. If mild, it requires no treatment or the application of an ice-bag, but if suppuration follows it must be treated on surgical principles.

Otitis may result from infection from the mouth. In all stuporous patients and children the ears should be daily inspected. The condition is to be treated as under other circumstances.

In some epidemics or in some individual cases, the disease may be so mild as to require little treatment other than good nursing; again 
it may be fulminating in character defying all effort, and resulting in death in three or four days. Relapses are exceedingly rare.

Disinfection. This consists in the destruction of the parasite, the louse, either by burning the clothing worn by the patient or by disinfecting it as described above. An assurance must be had that the patient's body is freed from the pediculi and the room must be disinfected by burning sulphur, either the sticks or the flowers, 5 pounds per 1000 cubic feet, leaving the room closed twelve to twenty-four hours. Formaldehyde is of doubtful value in the destruction of the parasite.

Prophylaxis is determined by a knowledge of the mode of conveyance of the disease. The doctor and nurse are both in great danger in handling fresh patients and their louse-burdened clothes. Protection by gloves and gowns and caps are obvious; prompt disinfection of clothes and patients must follow.

Delousing plants in infected communities should be of great assistance. Instruction of the public by every possible means should be used.

It has been suggested that crude naphthalene finely powdered be dusted on the underclothing, nightdress and sheets in infested areas.

\section{SUMMARY}

\section{Isolation.}

The disease is conveyed by the body louse.

Clothes worn by patient should be burned or

Boiled or pressed. (See text.)

Hair shaven from head, pubes, axillæ.

Burn hair.

Patient scrubbed with soap and water, sponged with 75 per cent. alcohol.

Spray entire body with crude petroleum.

Apply mercurial ointment to the axilla, pubes.

Put to bed in a vermin-free room and bed.

Disinfect former bed, mattress, bedclothes and room. (See text.)

\section{Room.}

Choose with reference to light, air and convenience for nursing.

Bed.

(See Typhoid Fever, Chap. XIV.)

\section{Care of the body.}

Daily cleansing bath.

Try to prevent bed-sores by turning patient.

Rubbing the point of pressure.

Keeping the parts dry.

Using rings or cushions. 
For superficial sores.

Keep sores scrupulously clean.

Use drying powders such as

Stearate of zinc,

Zinc oxide,

Talcum and starch mixtures,

Aristol.

Deep sores.

Treat on surgical principles.

Use water bed or air bed.

Mouth and nose.

Use mild antiseptics, such as 2 per cent. to 4 per cent. boric acid solution, especially after feeding.

When mouth is in bad condition, treat as in typhoid fever. (See Chap. XIV.)

Bowels.

As in other infectious diseases. (See Typhoid Fever, Chap. XIV.)

Diet.

Rules for feeding and details for feeding may be borrowed from Typhoid Fever. (See Chap. XIV.)

Water should be given freely, and, if patient is stuporous, offered every hour.

\section{Symptomatic treatment.}

Fever.

Hydrotherapy, baths, slushes; packs and sponges as in typhoid fever. (See Chap. XIV.)

\section{Open air.}

Just as in Pneumonia. (Chap. IX.)

\section{Circulation.}

Just as in Pneumonia. (See Chap. IX.)

\section{Nervous manifestations.}

Headache.

Ice-cap or coil.

Morphine in intense headache.

\section{Delirium.}

Great watchfulness.

Ice-bag.

Bromides, gr. $\mathrm{xv}$ to $\mathrm{gr}$. $\mathrm{xxx}(1-2 \mathrm{Gm}$.) in water three or four times a day.

Morphine sulphate, gr. 1/12 to gr. 1/4 (0.005-0.015 Gm.), hypodermically.

\section{Stupor and coma.}

Cold baths.
Open air treatment. (See Pneumonia, Chap. IX.) 


\section{Convalescence.}

Usually rapid.

Increase diet.

(See text.)

\section{Complications}

\section{Laryngitis}

Medicated steam, using

Compound tincture of benzoin, or

Oil of pine, or

Oil of eucalyptus,

$3 \mathrm{i}$ (4 c.c.) in a croup kettle, or pitcher or pail with paper cone to conduct it.

Edema of the larynx.

Intubation.

Tracheotomy.

Scarification.

\section{Bronchitis,}

Pneumonia,

Pleurisy,

As under other circumstances.

Empyema.

\section{Diarrhea.}

Prevention-proper care of bowel from beginning.

Castor oil in small doses or

Bismuth subnitrate (See text.)

(See summary under Bacillary Dysentery, Chap. XVI.)

\section{Parotitis.}

Precautions, proper care of the mouth.

When mild no treatment, or see Parotitis or Mumps (Chap. XXIII). Suppurative - treat on surgical principles.

\section{Disinfection.}

Destruction of body louse.

See above for treatment of clothes and patient's body.

Room.

Burn sulphur, 5-8 pounds per 1,000 cubic feet.

\section{Prophylaxis.}

Doctors, nurses and attendants should take precautions to prevent infection.

(See text.)

Delousing. (See text.)

Use of crude naphthalene powder. (See text.) 


\section{CHAPTER XXIX}

\section{PLAGUE}

Plague is a disease due to an infection by the Bacillus pestis bubonica isolated by Yersin in 1894 and often accredited to Kitasato. This organism affects peculiarly the lymphatic glands, the swelling of which gives the characteristic appearance to the infected and the designation "bubonic plague."

The especial carriers of the organisms are rats and the fleas infesting them afford the communication between the rat and man.

Rats, however, are not the only carriers; for example, in this country the California ground squirrels have been shown to be infected and conveyance may be made through other insects than fleas, and through the patient by pus, vomitus, sputum, sweat and urine.

While the bubonic form of the plague is the common one, the predominance of other clinical symptoms may warrant other designations, as when the lungs or intestine are the organs especially affected; hence, Pneumonic or Intestinal Plague; or, when the toxemia dominates the picture and is termed Septicemic or Fulminating Plague or the opposite obtains and the toxemia and local manifestations are slight, Pestis minor or Abortive or Ambulatory Plague.

So rapid and wide-spread is the plague, so frightful its consequences that the consideration of the community overshadows that of the individual and renders the chapter on prophylaxis by far the most important in the story of Plague. This will be considered presently.

Therapy. An article of this kind can deal only with the general principles applicable to the individual. A stricken community demands the accumulated fruits of experience in precautions, details and technique that can only be afforded by more exhaustive treatises or infinitely better yet by the presence and control of men of actual experience in plague epidemics.

Isolation. The knowledge that each individual is a centre of infection, through his secretions, makes his isolation imperative and that this infection may be conveyed by insects, such as flies and ants contaminated by these secretions or through the bites of infected insects, as the flea, demands screening against all insects.

Room. The room should be well aired and accessible to sunlight, as darkness and dampness favors the persistency of the organism; an 
ideal is a temporary shack or shelter rather than in an old and ratinfested house.

The bed should be of the hospital type; of iron, hence, readily kept clean. The room should be stripped of everything except the absolutely necessary.

Physician and nurse should wear gowns, caps and especially rubber gloves and in pneumonic cases protect the face with gauze masks such as are used by surgeons, which may be kept moist with some antiseptic solution, and glasses to protect against infection coughed into their faces. Doctors and nurses should take advantage of such protection as vaccine by Haffkine's or Shiga's method will afford.

Sputa should be received on gauze or rags and these should be burned. Vomitus, feces and urine should be disinfected and clothes, bed linen, utensils and instruments sterilized as in Typhoid Fever. (See Typhoid Fever, Chap. XIV.)

Cleansing baths, the care of mouth, nose, eyes, skin and genitals all demand the same consideration as in Typhoid Fever. (See Chap. XIV.)

Diet. Without forcing the food against the patient's distaste, an effort should be made to approximate food needs, however remotely. Milk and milk preparations, cereals, broths thickened with cereals or farinaceous foods, moistened bread or toast, eggs, may all be used. Water should be given abundantly.

Bowels. An initial cathartic with divided doses of calomel, gr. $1 / 4(0.015 \mathrm{Gm}$.) every quarter hour for six or eight doses, followed in two hours by a salt, Rochelle, Epsom, or Glauber's ₹ss.-3i (15-30 Gm.) may be given. Later, at two-day intervals milder salines or enemata may be used.

Bladder. It must not be forgotten that in the very ill urine may be retained and, if lesser measures of heat or cold over the hypogastrium fail, catheterization, with all precautions, must be undertaken.

Fever. The temperature is rarely high enough to demand especial consideration. It lasts, as a rule, from a week to ten days and is very irregular. Sometimes, however, the fever lasts for several weeks and then the increased caloric needs and protein destruction, effects of the toxemia, demand particular consideration of the dietary.

Rarely excessive fever requires antipyretic treatment which is best afforded by cold water in the shape of sponges and packs or if the buboes are not discharging or too painful as tubs. Antipyretic drugs are depressing to the circulation and should not be used.

Circulation. Good nursing, fresh air, food and cool or cold sponging are the best guarantees of a good circulation. 
If drugs are to be used the indications are the same as in the other acute infections. (See Pneumonia, Chap. IX.)

Nervous Symptoms. Headache-is best.controlled by the ice-bag applied to the head.

Delirium may demand restraint.

Sleeplessness is to be met by the milder hypnotics or morphine may be needed to control these symptoms.

Buboes. As the swelling increases rapidly the pain may be very great. Perhaps the best means to relieve this is the application of the ice-bag. At times the pain may be so severe as to demand morphine. Hot fomentations are also used to hasten suppuration.

When fluctuation occurs incision is indicated and the wound treated on surgical principles.

Diarrhea. When this occurs the best drug to use is bismuth subnitrate. Some writers consider opium contraindicated, as defeating the effort at elimination of toxins.

\section{SPECIFIC TREATMENT}

Serum. An anti-plague serum comparable to antidiphtheria serum has never been elaborated, but, especially, the Yersin-Roux has been tried with results that have elicited different interpretations.

I quote from Wherry's article in Forchheimer's Therapeusis of Internal Disease.

"The discussion concerning the value of the Yersin-Roux antiplague serum has apparently been satisfactorily disposed of by Chosky. His series includes 1,081 cases treated in India during 1905-1907. In this series only every alternate case was treated with the serum, the others acting as controls. Very mild cases and severely septicemic and moribund cases were excluded.

"The general mortality was reduced to 49.6 per cent. In cases treated during the first day of the disease the mortality was 30 per cent.; this increased to 52 per cent., among those treated on the second day, and to over 60 per cent., among those treated on the third day."

Of course the value of such statistics increases with their number.

As the general mortality of the Plague in India at this time was 89.9 per cent., the reduction under the serum treatment was remarkable; but in addition the disease was shortened, and the complications lessened.

Early administration is all important and a sufficiency of serum 100-200-400 c.c. should be given and early repeated, in six and eight 
hours and again at weekly intervals as the condition of the patient seems to demand.

Intravenous administration is always to be preferred, but intramuscular and subcutaneous are often combined with it.

The best serum at present seems to be the so-called Yersin serum, obtained by inoculating horses with dead organisms, and later with increasing quantities of living virulent organisms.

Sequelæ. Aside from the rarer sequelæ prolonged suppuration of buboes and cardiac weakness in convalescence are matters of moment.

Convalescence. Fresh food and fresh air and prolonged rest with watchfulness over the state of the circulation are the essential measures.

Disinfection. This should be thorough and effective and much the same measures should be pursued as in Scarlet Fever. (See Scarlet Fever, Chap. XVII.)

Prophylaxis. Since the Indian Commission has so clearly demonstrated the rôle of the rat and his fleas and with our knowledge of the contagiousness of the secretions of infected men, prophylactic measures can be directed with definite ends in view.

It must not be forgotten, however, that while rats are the usual carriers, other animals may harbor and convey the infection and that in our own country the California ground squirrel especially plays this rôle.

Jackson, in Hare's Modern Treatment, sums up protective measures for the community in this way:

"(1) Active warfare against rats and other plague affected rodents, and their fleas;

(2) quarantine applied to persons, goods and animals;

(3) disinfection of cargoes shipped from infected ports;

(4) isolation of the sick and proper disposal of the dead;

(5) international notification between governments of the occurrence of plague within their respective territories;

(6) lastly, but perhaps first in importance, the early recognition of the presence of the plague and rapid diagnosis in individual cases, both dependent upon laboratory workers."

Warfare against the rat entails the building of rat-proof structures in ports; the prevention of ingress and egress of rats when ships are in port, by protection of hawsers and raising of planks at nightfall; the proper disposal of garbage on which they feed, stopping up of their holes and the use of poisons and traps. In some communities cats have been employed for the purpose, though not entirely a safe procedure as cats have been shown to develop a chronic form. The presence of plague 
in any community is so dire a threat to its welfare, the possibility of spread in the community so great that it immediately becomes a public health problem. It is beyond the scope of a handbook of this type to elaborate upon the details that should be carried out by public health officers.

Immunization. Prophylactic measures may be applied to the individual as well. Perhaps the most efficacious of these methods is the use of Haffkine's prophylactic inoculation. It consists of a broth culture of bacillus pestis killed by heating and preserved in 0.5 per cent. carbolic acid and injected subcutaneously in amounts of 0.5 to 4 c.c. repeated in larger amounts after 10 days. The reaction is often quite severe.

That it has protective power, that it has modified both the incidence and mortality statistics seem now to have been proved beyond a doubt; but the protection is not absolute even though immunity seems to be marked in the second year and remains in a slight measure in some cases for five years.

A modification of this method has been devised by Shiga that has the advantage of quicker preparation.

If given within a few hours after first appearance of symptoms it seems to have some curative value.

\section{SUMMARY}

Isolation.

Room.

Well aired and light and screened from flying insects.

Bed.

Hospital type preferred.

(For technique of bed-making, see Typhoid Fever, Chap. XIV.)

\section{Physicians and nurses.}

Wear gowns and gloves and in pneumonic cases wear gauze masks to protect face against the patient's cough.

Prophylactic vaccination is a wise precaution.

\section{Care of patient.}

Care of body and mouth, nose, eyes and genitals as in any acute infectious disease. (See Typhoid Fever, Chap. XIV.)

Sputa should be received on gauze or rags and burned.

Vomitus, feces, urine disinfected and clothes, bed-linen, utensils, and instruments sterilized as in Typhoid Fever. (See Chap. XIV.) 
Diet.

Do not force, but keep in mind food requirements. (See Chap. II.) Milk, milk preparations, cereals, broths thickened with cereals, or farinaceous foods, moistened bread and toast, eggs.

Water should be given freely.

\section{Bowels.}

Initial cathartic.

Calomel in divided doses, gr. 1/4 $(0.015 \mathrm{Gm}$.) every quarter hour for six or eight doses, followed in two hours by

Epsom salt, Rochelle salt or Glauber's salt, 5 ss. to 5 i (15-30 Gm.) Later. in three-quarters glass of water.

Milder salines, Hunyadi, citrate of magnesia,

Milk of magnesia or enemata every day.

\section{Retention of urine.}

Apply heat (hot stupes) or cold to epigastrium. If these fail catheterize.

\section{Fever.}

Cold sponges.

Cold packs.

Cold tubs.

\section{Circulation.}

(See Pneumonia, Chap. IX.)

\section{Nervous symptoms.}

Headache.

Ice-bag or coil.

Delirium.

\section{Restraint.}

Bromides, gr. $\mathrm{xxx}(2 \mathrm{Gm}$.) one to three times a day.

Morphine hypodermically, gr. 1/8-1/4 (0.008-0.015 Gm.).

Hyoscine hydrobromide, gr. 1/150 (0.0004 Gm.).

Sleeplessness.

Bromides, gr. $\mathrm{xxx}(2 \mathrm{Gm}$.) in water.

Trional, gr. xv $(1 \mathrm{Gm}$.) in a little hot water or whiskey or as powder. Chloralamid, gr. $\mathrm{xxx}(2 \mathrm{Gm}$.) in cold water or whiskey or powder. Morphine sulphate, gr. 1/8-gr. 1/4 (0.008-0.015 Gm.).

\section{Buboes.}

Ice-bag.

Morphine sulphate for pain, gr. 1/8-gr. 1/4 (0.008-0.015 Gm.).

Hot fomentations to hasten suppuration.

Incise when fluctuation is detected.

Treat on surgical principles. 
640 TREATMENT OF ACUTE INFECTIOUS DISEASES

Diarrhea.

Bismuth subnitrate, gr. xxx ( $2 \mathrm{Gm}$.) every two hours.

Specific treatment. (See text.)

Convalescence.

Fresh food.

Fresh air.

Prompt rest.

Disinfection.

(See Scarlet Fever, Chap. XVII.)

Prophylaxis.

(See text.) 


\section{CHAPTER XXX}

\section{DENGUE}

\section{DANDY FEVER. BREAK BONE FEVER}

Dengue, essentially a tropical disease, is confined in the United States to the Southern tier of States. Although epidemics have been reported as far north as Philadelphia.

It is characterized by a suddenness of onset, and a rise of temperature with severe headache and a striking soreness of the ocular muscles, giving rise to exquisite pain with each movement of the eyes and pains all over the body that closely simulate Influenza but with especial localization about the joints. These manifestations of intoxication last two to five days and are followed by a drop in temperature often critical and accompanied by nose bleed, diarrhea or a sweat and by a remission or intermission of symptoms of twelve hours to three days, only to be in turn followed by a second febrile period of twenty-four to thirty-six hours. An erythema may accompany the first period, but the characteristic rash, a measles like eruption, occurs with the second access of fever involving hands first, spreading to arms and later involving lower extremities and trunk.

The blood picture is a leucopenia and relative lymphocytosis.

Profound depression of spirits accompanies the attack and the convalescence is slow. The mortality is, however, very low.

This disease, like Malaria and Yellow Fever, is in all probability transmitted by a mosquito (Culex fatigans) as suggested by Graham of Beirut, though as yet not definitely proved. Some workers have suspected the stegomyia to be the host.

Ashburn and Craig, by transmitting the disease through the filtered blood of an infected person, have made it more than probable than the infecting agent belongs to the group of ultramicroscopic organisms.

Therapy. The room chosen for the patient should be as cool and airy as possible or better yet a shaded portico. This should be screened against mosquitoes, not merely as a protection to the patient, but as a protection to others against the patient.

The bed should be selected with a view to comfort as the bodily pains are so great as to make any position one of discomfort. (See Rheumatic Fever, Chap. III.) 
Diet. No effort should be made to force food during the period of anorexia, but after this has passed milk and milk preparations, cereals, bread-stuffs and eggs, cereal broths or mutton and chicken broths thickened with rice, sago or barley may be given as freely as the patient will accept them, while during the long and tedious convalescence a greater variety of foods temptingly prepared and with a consideration of the patient's caloric needs (see Chap. II) should be offered.

Water should be given freely throughout the febrile periods or fruit juices such as lemonade or orangeade or juice of grape fruit.

Bowels. At the beginning of the disease the bowels should be freely moved by small doses of calomel and salts, e. g., calomel gr. 1/4 (0.015 Gm.) every quarter hour for six to eight doses, followed by 5 ss. (15 Gm.) of Epsom or Rochelle salt, and every second day a mild saline such as a Seidlitz powder or liquor magnesii citratis $弓$ viii-xii (240-360 c.c.) or Hunyadi water may be administered.

There should be no purging as the condition does not demand it and the patient's pains and discomforts are greatly aggravated thereby.

Pains. The milder anodynes such as salicylates in gr. $x \quad(0.65$ $\mathrm{Gm}$.) doses every two hours or phenacetin (acetphenetidin) gr. iii to $\mathrm{v}$ $(0.20-0.30 \mathrm{Gm}$.) at like intervals should be tried first, but the pains are commonly too severe to yield to such measures and one should have recourse to codeine sulphate or phosphate gr. $1 / 8$ to gr. $1 / 4(0.008-0.015$ Gm.) every two hours.

At times, however, the headache and pain is so acute that one must use morphine. It is always well to administer this drug hypodermically rather than by mouth and in the smallest doses that will effect the desired results, e. g., a beginning dose of gr. 1/12 (0.005 Gm.).

Local Treatment of Pains. An ice-bag to the head may mitigate the severity of the headache while hot cloths, stupes or poultices may give relief to aching back and joints.

Fever. No effort is to be made to combat the fever as such, as hyperpyrexia is very rare, but measures to assuage the discomforts incident upon the temperature are legitimate, such as cool sponges, cooling drinks, ice-bag to head.

Tub baths add too much to the patient's pains in the handling to warrant the procedure unless hyperpyrexia should obtain.

Circulation. The pulse is characteristically slow about the third day, but the circulation is rarely threatened. Should one be dealing with a poor circulation as the result of an antecedent condition, the toxemia, or both, the indications are the same as in a case of Pneumonia. (See Pneumonia, Chap. IX.) 
Nervous Symptoms. To a patient suffering from Dengue nothing is more gratifying than to be let alone. Quiet and good nursing and the exclusion of visitors are of first importance to him.

Insomnia may be overcome by excluding causes of nervous irritation, by the artifices of efficient nursing and lastly by drugs, e. g., sulphonethylmethane (trional) gr. $\mathrm{x}(0.65 \mathrm{Gm}$.) in the early evening and a repetition of the dose two hours later if needed or chloralamid gr. $\mathrm{xxx}$ $(2 \mathrm{Gm}$.$) at the same intervals.$

If pain is the cause of the insomnia codeine phosphate gr. 1/4 (0.015 Gm.) to gr. ss. $(0.030 \mathrm{Gm}$.), may be used to assist the operation of the hypnotics, but in cases of severe pain only morphine, as already specified, will be of use. With this the other hypnotics are unnecessary.

Complications. Occasionally hemorrhages may occur from nose, stomach, intestine or uterus. Milder hemorrhages need no consideration, but if abundant or in weakly patients, the same measures are to be pursued as in hemorrhages from other causes. (See Typhoid Fever, Chap. XIV.)

Convalescence. It is curiously protracted for an infection so little fatal and is to be hastened by a sufficiency of food, fresh air, quiet and milder tonics such as nux vomica and iron.

Prophylaxis. Accepting the theory that the mosquito is the transmitting agent of the disease efforts should be made to rid the community of the pests by draining swamps, obliterating or treating with oil all stagnant water, ponds, pools, cisterns, and removal of smaller containers, such as buckets, cans, etc., together with screening of dwellings and protection of the exposed parts of the body at nightfall. Especially, as has been said, the infected patient should be screened.

Diagnosis. Features of diagnostic significance are the two febrile periods separated by the remission, the severity of the pains, the slow pulse, the rash of the second period, the low white cell and polymorphonuclear count (e. g., 3,200 whites and 51 per cent. polymorphonuclears) and the protracted convalescence.

\section{SUMMARY}

\section{Room.}

Cool and well ventilated.

Shaded and screened porch or veranda desirable.

Bed.

Preferably of hospital type, with woven wire springs, firm mattress and smoothly laid sheets. 
Diet.

Do not force during period of anorexia.

Milk, milk preparations, cereal broths, mutton or chicken broth thickened with rice, barley or other cereal or farinaceous flour.

\section{Water.}

Give freely.

Lemonade, orangeade and other diluted fruit juices. May add sugar to increase food value.

\section{Bowels.}

Calomel and salts or a salt alone.

Calomel, gr. 1/4 (0.015 Gm.) every quarter hour for six to eight doses. Epsom salt 5 ss. (15 Gm.).

Rochelle salt 5 ss.-i (15-30 Gm.).

Seidlitz powder.

Liquor magnesii citratis 5 viii to xii (240-360 Gm.).

Hunyadi water.

\section{Treatment of symptoms.}

Pain.

Local.

Hot fomentations. (See Pneumonia, Chap. IX.)

Poultice. (See Pneumonia, Chap. IX.)

Sodium salicylate or acetylsalicylic acid (Aspirin), gr. x (0.60 Gm.) every two hours.

Phenacetin (acetphenetidin), gr. iii to gr. v (0.20-0.30 Gm.) every two hours.

Codeine sulphate or phosphate gr. $1 / 8$ to gr. $1 / 4(0.008-0.015$ Gm.) every two hours.

Morphine sulphate hypodermically, gr. 1/12 to gr. 1/8 (0.005$0.008 \mathrm{Gm}$.).

Fever.

Cool sponges.

Cool drinks.

Ice-bag to head.

\section{Circulation.}

Rarely threatened.

(If stimulation is indicated, see Pneumonia, Chap. IX.)

\section{Nervous symptoms.}

Marked irritability allayed by quiet and good nursing.

\section{Insomnia.}

Sulphonethylmethane (Trional), gr. x $(0.60 \mathrm{Gm}$.). (May repeat in two hours.)

Chloralamid, gr. $\mathrm{xx}$ (1.30 Gm.). (May repeat in two hours.) 
Codeine phosphate, gr. $1 / 8$ to gr. $1 / 4(0.008-0.015 \mathrm{Gm}$.). (If due to pain.)

Morphine sulphate, gr. $1 / 12$ to gr. $1 / 8(0.005-0.008 \mathrm{Gm}$.). (If due to pain.)

Complications.

Hemorrhages. (See text.)

(If severe same as Typhoid Fever, Chap. XIV.)

Convalescence.

Protracted.

Increase food intake.

Fresh air.

Strychnine sulphate, gr. 1/60 to gr. 1/20 (0.001-0.003 Gm.) or

Tincture of nux vomica, $\mathrm{m} . \mathrm{x}$ to $\mathrm{m} . \mathrm{xv}(0.60-1 \mathrm{c.c}$.$) three times a day.$ Iron as carbonate, gr. $\mathrm{v}(0.30 \mathrm{Gm}$. $)$ three times a day, as either Blaud's pills, or Vallet's mass.

\section{Prophylaxis.}

War on the mosquito.

(See Malaria, Chap. XV.) 


\section{CHAPTER XXXI}

\section{ASIATIC CHOLERA}

Asiatic Cholera is due to a definite organism, the so-called "comma bacillus" discovered by Koch in 1883 or more technically, the vibrio choleræ asiaticæ.

It is conveyed by the dejecta of the infected directly or through the medium of water and always gains entrance through the mouth.

Its toxin and endotoxin acts specifically on the endothelium of the intestinal canal to induce those alterations in function that constitute the symptoms of the disease, namely, excessive diarrhea with collapse.

The mortality is high, approximately 50 per cent. and is especially fatal to the two extremes of life.

Isolation. During a cholera epidemic the slightest diarrhea should . be looked upon as suspicious and the patient should be put to bed and everything be done to conserve his strength for the coming struggle.

The problem of protection against infection is a simple one, for we know the source of the infection through the feces, its mode of conveyance through hands, articles, insects, food and drink contaminated by the dejecta and the portal of entry through the mouth.

Isolation prevents the ignorant and careless who come into contact with the patient from becoming infected and spreading the disease and prevents added infection to the patient.

Doctor and nurse by an appreciation of the facts just stated should, by use of gowns and gloves, careful cleansing of hands, sterilizations of contaminated objects, and destruction by fire and strong antiseptics of excreta, protect themselves and those with whom they may come in contact.

Room. During an epidemic, of course, the vast majority of patients must be treated in hospitals, camps, etc., but for the favored few, who may be treated under more fortuitous environments, a room, as cool and as well ventilated as possible should be chosen and this stripped of all furnishings and hangings. In fact the source and mode of conveyance of this disease is so like typhoid fever that the details of room, bed, sterilization of articles used, and care of the body are identical problems. (See Typhoid Fever, Chap. XIV.)

Diet. At first it is well to eliminate all food-stuffs, except barleywater, which is best given hot. Later other farinaceous gruels, such 
as arrowroot water, rice water, whey, and then cautiously animal broths thickened with farinaceous foods, and then milk.

These are dietetic measures similar to those we use in the gastroenteritis of infancy.

Water is given abundantly in small quantities freely and not too cold.

Early Treatment. The sooner the patient is put to bed after the looseness of the bowels appears the better. He should be covered warmly, given hot drinks, hot weak tea or lemonade and hot stupes or poultices should be applied to the abdomen.

At this early stage opium may be given, but later in the disease it is a dangerous drug. Indeed, Stitt advises against its use in any stage.

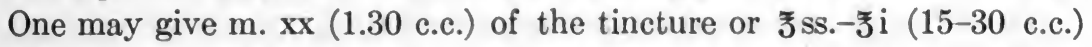
of paregoric or in smaller and frequent doses, as m. ii ( 0.13 c.c.) of the tincture or gr. ii $(0.13 \mathrm{Gm}$.) of Dover's powders or $3 \mathrm{i}$ (4 c.c.) of paregoric every hour.

Cathartics. Rogers, whose masterly article in Hare's Modern Treatment, should be read with attention by all who deal with this disease, decries the use of cathartics in cholera at any stage.

Stage of Collapse. Rogers' treatment while not specific, is deduced so logically from what we know of physiological requirements, that I shall summarize it for the purposes of this article with a frank confession of the shortcomings of such a summary.

Rogers recognizing the obvious fact that the loss of fluids from the tissues constitutes the danger of the disease and that its replacement is the procedure that the logic of the situation demands, finds his success in the appreciation of the significance of certain minor details.

He recognized that not only is there a loss of fluid by the tissues, but also a marked loss of salts, both of which should be replaced, and argued that if salts were introduced in hypertonic solution osmotic currents could be determined toward the blood from the bowel instead of away from it as induced by the toxic agents. Some of the tropical workers report that equally good results have been obtained from the use of normal saline as from the hypertonic solution.

The condition of the blood Rogers estimated by determining its specific gravity. This he does by blowing a drop of blood into mixtures of glycerin and water of known specific gravities from 1040-1076 corresponding to the normal specific gravity of the blood and noticing whether it sank, rose or remained for a time suspended. A small urinometer suffices to determine the specific gravities of the glycerin and water mixture.

The state of the circulation is gauged by the sphygmomanometer. If the pressure is $70 \mathrm{~mm}$. or over, saline may be absorbed from the 
bowel and unless indications are urgent treatment may be begun in this manner.

He uses a solution of sodium chloride gr. 90 to one pint $(6 \mathrm{Gm}$. to 500 c.c.) at blood heat $1 / 2$ to 1 pint (250-500 c.c.) introduced very slowly two hours or by the Murphy drip method.

This is kept up until the kidneys act freely.

If, however, the blood pressure is below $70 \mathrm{~mm}$. Hg., or the patient has severe cramps, is restless or cyanotic a saline should be given intravenously as the subcutaneous method is often ineffectual due to failure of absorption. Owing to the collapse of the vein it is usually necessary to cut down upon the vein to insert the needle.

The efficacy of this method Rogers attributes largely to the concentration of the solution. The formula he advises is as follows:

$$
\begin{aligned}
& \text {.Sodium Chloride.................... } 120 \text { grains. } \\
& \text { Calcium Chloride.................... } 4 \text { grains. } \\
& \text { Potassium Chloride..................6 grains. } \\
& \text { To each pint of water (500 c.c.). } \\
& (0.250 \mathrm{Gm} .) \\
& (0.370 \mathrm{Gm} .)
\end{aligned}
$$

This is injected at body temperature.

Three to four pints are usually injected, aiming to bring the blood pressure up to $110 \mathrm{~mm}$. Hg. The injection is carried on at the rate of about 1 pint in five minutes.

A salvarsan apparatus with a large container may be used for this purpose. (See Pneumonia, Chap. IX.)

Indications for interruptions of the injection or lessening the rate of inflow are headache, dyspnœea or sense of oppression in chest.

Rogers' second point of attack is on the toxins of this disease before absorption.

For this purpose he advocates highly the use of calcium or potassium permanganate, preferably calcium as an oxidizing agent.

He gives it in the drinking water, 4 or more grains to the pint $(0.25$ $\mathrm{Gm}$. to 100 c.c.) (much more than 4 is not readily swallowed) in such quantities as the patient can be made to drink, or in gr. ii $(0.125$ Gm.) pills coated with salol (phenyl salicylate) or keratin every quarter hour for four doses, then every half hour until gr. xx are taken, in four hours, then omit four hours (or take gr. v $(0.30 \mathrm{Gm}$.) bismuth salicylate every hour for these four hours), then one pill every half hour for four hours, then stop four hours and so on over again until stools change color and become less (usually in twenty-four hours).

At the beginning of the second day 16 grains more are administered.

Lately Rogers has been using atropine sulphate gr. 1/100 (0.006 Gm.) morning and night with satisfactory results.

Sellards uses 0.5 per cent. saline solution and 0.5 per cent. sodium 
bicarbonate solution, injecting in the adult as much as two litres of saline at an interval of 15-20 minutes. The improvement is often very great, but purging continues and collapse is almost certain to return. He repeats the infusion at 6 to 8 hour intervals. He notes that patients who recover begin to show inprovement on the second day.

Sellards did not find that the hypertonic solution used by Rogers and others afforded the results that theory seemed to promise.

Other substances, such as colloidal solutions, added to the saline failed to convince Sellards of an additional value.

Vomiting. As lesser measures one may try cracked ice or a mustard paste to the epigastrium or subcarbonate of bismuth in $\mathrm{gr} . \mathrm{xv}(1 \mathrm{Gm}$.) doses or if the vomiting is very severe lavage may be tried. In the early stages a hypodermic of morphine sulphate gr. $1 / 4(0.015 \mathrm{Gm}$.) is warrantable and perhaps more likely to be efficacious than any other measure, but during the stage of collapse Rogers maintains its use constitutes a real danger.

During collapse warmth to the surface of the body is indicated by the applications of hot blankets and hot-water bottles. Hot drinks are also given, friction to the skin and extremities and stimulants administered (see below). Heat, however, should not be applied during intravenous injection as the febrile reaction accompanying it may pass over into hyperpyrexia.

Stage of Reaction. With a cessation of vomiting, a diminution in the stools, improvement in their consistency and color (bile retained), a slowing of the pulse, and warming of the body surface, the stage of reaction sets in and brings hope with it and yet this period is fraught with no inconsiderable dangers that has warned the physician to abate no jot or tittle in his vigilance.

The possibilities for disaster in this stage rest in hyperpyrexia, suppression of urine and uræmia.

Hyperpyrexia. Large bodies of statistics attribute 10 per cent. of deaths to hyperpyrexia. Rogers believes it due not so much to pyrexia as the rapid absorption of toxins from the gut as the circulation picks up under the febrile reaction.

He believes his permanganate treatment has lessened the mortality from this cause. He also warns against the use of heat applied to surface of the body during saline infusion.

Anuria is to be met by the supply of fluid to the tissues by frequent administrations by mouth and rectum and if specific gravity of blood is not too low (Rogers) by vein as saline solutions.

Sellards recommends intravenous injections of 2 per cent. sodium bicarbonate in place of normal or hypertonic saline as being more effica- 
cious in this rondition, while be believes it is equally potent in relieving collapse.

The kidneys may be cupped, cardiac stimulants such as strophanthin gr. 1/120-gr. 1/60 (0.0005-0.001 Gm.) or rapid vaso-motor stimulants such as adrenalin $\mathrm{m}$. $\mathrm{x}-\mathrm{xv}(0.60-1$ c.c.) into muscle or $\mathrm{m}$. $\mathrm{ii}-\mathrm{v}$ $(0.125-0.30$ c.c.) into vein to be used.

Uremia is particularly likely to occur even in the milder cases, in the state of reaction, and Sellards' studies show it is connected intimately with an acidosis. Because of this tendency toward acidosis Sellards early in the stage of reaction increases the strength of the solution used in collapse to 1.5 per cent. of sodium bicarbonate and omits the sodium chloride.

If the urine does not become alkaline to litmus or but little urine is passed he increases the strength to 2 per cent. sodium bicarbonate.

Proper precaution in the sterilization of the sodium bicarbonate solution must be taken to prevent its conversion into sodium carbonate. This may be done by passing carbon dioxide gas through the sterilized solution until the carbonate is reconverted into the bicarbonate. The gas is passed through sterile tubes and filtered through sterile cotton before it enters the solution.

Even 60 to 90 grams of sodium bicarbonate was given intravenously within 24 to 48 hours with most satisfactory results.

The method of intravenous infusion is described in detail under the administration of serum in pneumonia, Chap. IX. The rate is much more rapid than in serum injection. Two litres may be given within 15 to 30 minutes. The intervals are 6 to 8 hours or on the reappearance of collapse. Bicarbonate is discontinued when the urine becomes alkaline.

Specific Treatment. Serum has been used but unfortunately as yet with little result. Prophylactic inoculations of the cholera vibrio by Haffkine have been adopted. Such treatment after evidences of infection occur is of no value and there are theoretical reasons for the belief that it is then distinctly harmful.

Stimulants. A sufficiency of water in the circulation is the best stimulant and that the intravenous injection seeks to assure. However, if this fails, we must use whatever other means we have at hand that gives us any promise of assistance and look for such among the drugs.

Probably no drug is comparable to digitalis for sustained effect upon the circulation and should be administered in doses of $\mathrm{m} . \mathrm{xx}$ (1.30 c.c.) of the tincture every six hours. It requires some thirty-six hours or more, however, for it to exert its effect and it seems to me that for more immediate effect strophanthin in doses of mg. 1/2-1 (gr. 1/120-1/60) in- 
travenously would be indicated here as in other acute infections accompanied by circulatory failure.

For vaso-motor stimulants one may have recourse to camphor gr. $\mathrm{v}(0.30 \mathrm{Gm}$.) in oil or caffeine sodio-salicylate or caffeine sodio-benzoate gr. v $(0.30 \mathrm{Gm}$.) in watery solution every four hours or alternate at twohour intervals or strychnine sulphate gr. 1/40 $(0.0015 \mathrm{Gm}$.) every three to four hours.

All should be given deep into a muscle or if collapse is severe into a vein.

Sellards would allow coffee in small amounts as being both agreeable and possibly beneficial to both heart and kidneys.

Prophylaxis. Wherry has divided this subject into

(1) Communal and (2) Individual Prophylaxis.

Communal Prophylaxis. This means proper quarantine, sanitary supervision and control of water and food supply, bacterial examination of the excreta of all suspects, which includes all persons coming from foci of infection, many of whom may be true carriers, themselves free from any symptoms of the disease.

Isolation of Suspects. This should entail a detention of at least five days.

Individual Prophylaxis. This entails the realization that the infection enters by the mouth; that it comes by way of drinking water; hence, all water should be boiled; that it comes by way of food; hence, only recently cooked foods, carefully protected from flies, and no raw foods should be eaten; that it comes from fingers or utensils soiled by excreta; hence, careful sterilization of utensils, protection of hands by gloves, when in contact with patients, and by cleansing in sterile water and antiseptic solutions; that it comes from flies or other insects bearing excreta on their legs to food and drink; hence, screening of patient and of food; that anything which reduces the resistance of the epithelial lining of the intestine enhances the likelihood of an attack; hence, especial attention should be given to diet during a cholera epidemic. This means eating in moderation, avoidances of all questionable messes and highly seasoned and garnished dishes; raw fruits and vegetables, large quantities of very cold water and alcoholic excesses.

Vaccines have been used as a prophylactic measure with some degree of success.

These vaccines have been variously prepared and administered and it seems probable that their use will become more extensive.

Complications. These are numerous but pneumonia, prolonged dysentery, profound weakness determining a long convalescence are the most common. 
Convalescence is to be met by a careful return to a sufficient diet, good air and rest.

I cannot do better than quote bodily the summary of treatment from Rogers to whose excellent article I owe so much.

Summary of Rogers' Treatment. "In the first place, the bloodpressure and specific gravity of the blood should be estimated and the axillary and rectal temperature taken. If the blood-pressure is 70 millimeters of $\mathrm{Hg}$. or over, the patient quiet, and the general condition good, rectal saline injections may be tried, a careful watch being kept on the pulse, so that any further fall may at once be detected and more active measures adopted. If the specific gravity is very high, such as 1070 , transfusion should not be delayed. If the blood-pressure on admission or later falls below 70, and especially if the patient is restless, cyanosed, and suffering from severe cramps, no time should be lost in giving an intravenous injection, the hypertonic Ringer solution being used. Sufficient should be injected to obtain not only a fair, but a strong, full pressure-pulse, so as to at once restore the urinary secretion. Four pints are commonly necessary in a severe case in an adult male, and proportionately less in women and children. It should be given at body heat unless the rectal temperature is below $90^{\circ} \mathrm{F}$., when the temperature of the solution should be a few degrees higher until the surface temperature is restored. A careful watch should be kept on the temperature reaction which always follows intravenous salines, and the necessary measures taken to prevent an excessive rise occurring.

"In the acute stage nothing should be given by the mouth except the permanganate, drinks and pills, and some antiseptic, such as bismuth salicylate. If the acute stage is prolonged, barley-water may also be necessary. If the collapse recurs, the hypertonic injection must be repeated, being given at a slower rate if the specific gravity of the blood is not much raised, and at a subnormal temperature if there is fever present.

"If a second hypertonic injection is required, the prognosis becomes much graver, although a majority of even such cases may be saved.

"Once the stage of copious evacuations and collapse is past, the allimportant indication is to dilute the blood to at least the normal point, and to raise the blood-pressure high enough to restore the secretory activity of the kidneys, and so insure the excretion of the toxins and the prevention of the uræmic complications. If the specific gravity of the blood remains above normal, and is not rapidly reduced by continuing the salines by the bowel and the water by the mouth, a further smaller subcutaneous or slow intravenous injection of normal saline solution is 
indicated. In addition, cardiac tonics and vaso-constricting drugs are of great service.

"Great caution is necessary in adding to the diet during early convalescence, animal albumins being especially liable to induce a relapse, while starvation for two or three days is less injurious in otherwise healthy subjects whose age is not at either extreme of the scale." (Hare's Modern Treatment.)

For Sellards' treatment, see text.

\section{SUMMARY}

\section{Isolation.}

Of all real cases and all suspects (those who during an epidemic have diarrhea).

Destruction of all dejecta and sterilization of objects coming in contact with the patient or his excretions.

(For details, see Typhoid Fever, Chap. XIV.)

\section{Doctor and nurse.}

Should use gowns and gloves in performance of duties about patient.

Cleanse hands with soap and water, and follow with alcohol or 1 to 1,000 bichloride, even if gloves were worn.

\section{Room.}

Chosen with reference to ventilation.

Stripped of all hangings and ornaments.

Take especial care to keep flies from the room and dejecta.

(For details of care of room, care of the bed, and sterilization of objects, see Typhoid Fever, Chap. XIV.)

\section{Diet.}

At first only barley-water, best given hot.

Little later other farinaceous gruels.

Arrowroot water.

Rice water.

Animal broths thickened with farinaceous foods (cautiously).

Whey, then

Milk.

Water freely in small quantities frequently, not too cold.

\section{Rest.}

Put to bed at earliest sign of diarrhea.

Give hot drinks, weak tea or lemonade.

Cover warmly.

Hot stupes or poultices to abdomen.

(For technique of stupes, see Chap. XIV.)

(For technique of poultices, see Chap. IX.) 
Opium.

May be given early, not late.

Tr. opii $\mathrm{m}$. $\mathrm{xx}$ (1.30 c.c.).

Paregoric 5 ss. to i (15-30 c.c.).

May divide above doses and give at short intervals

Dover's powder gr. $1 / 5(0.010 \mathrm{Gm}$.) every hour.

\section{Cathartics.}

Should not be used at all.

Rogers' treatment of hypertonic salt solutions and potassium permanganate. (See text.)

\section{Vomiting.}

Cracked ice to suck.

Mustard paste to epigastrium. (For technique, see index.)

Bismuth subnitrate in doses of $\mathrm{gr} . \mathrm{xv}(1 \mathrm{Gm}$.).

If severe, lavage.

Morphine, gr. 1/4 (0.015 Gm.).

\section{Collapse.}

Warmth to body.

Blankets.

Hot-water bottles.

Friction to the extremities.

Stimulants, see below.

Conditions arising during the

Stage of reaction.

Hyperpyrexia.

Cold sponges.

\section{Anuria.}

Fluid to tissues. (See Rogers' treatment, text.)

Cup kidneys. (For technique of cupping see index.)

Cardiac and vaso-motor stimulants, see below.

Specific treatment.

(See text.)

Stimulants.

Sufficiency of fluids to the tissues.

Digitalis, m. $\mathrm{xx}(1.30 \mathrm{Gm}$.) every six hours.

Strophanthin in urgent cases gr. 1/120 to gr. 1/60 (0.0005-0.001 $\mathrm{Gm}$.) to initiate the digitalis.

Camphor gr. v $(0.30 \mathrm{Gm}$.) in oil 5 per cent. or

Caffeine sodium benzoate, gr. v (0.30 Gm.) either every four hours or alternate one with the other every two hours, or

Strychnine sulphate, gr. 1/40 (0.0015 Gm.); all given into muscle or vein.

For immediate effect

Adrenalin $\mathrm{m}$. $\mathrm{xv}$ ( 1 c.c.) into muscle or $\mathrm{m}$. iii $(0.20$ c.c.) into vein. 


\section{Prophylaxis.}

1. Communal.
Quarantine.
Supervision and control of water and food supply.
Bacterial examination of excreta of all suspects.
Isolation of suspects for five days.

2. Individual.

Boil water.

Don't drink large amounts of cold water. Avoid alcohol.

Eat no raw foods.

Eat only recently cooked foods.

Protect food from flies.

Eat in moderation.

Sterilization of utensils.

Protection of hands with gloves when in contact with patients.

\section{Convalescence.}

Careful return to sufficient diet.

Fresh air. 


\title{
CHAPTER XXXII
}

\author{
MALTA FEVER
}

Malta Fever in the great ocean of diseases, has like its habitat Malta seemed until recently, an island and a small island at that, of interest only to the physician practicing there or treating the English soldier invalided home, but an article by Ferenbaugh in August, 1911, suddenly made us aware that we harbored the disease in this country in the great State of Texas.

This disease, which affected so widely the British troops stationed in Malta, Gibraltar and a few other Mediterranean localities was found by Bruce in 1887 to be due to a definite organism, the bacterium melitensis. The mode of its distribution was elucidated by the Mediterranean Fever Commission in their work of 1905-7. They showed that it came largely through the use of the milk of goats so plentiful in the island; that 50 per cent. of the goats were infected, acting as carriers, and that 10 per cent. of them secreted the bacteria in their milk.

In Edwards County, Texas, and in adjacent territory an unclassified slow fever had been recognized under various local designations and had even been associated with the goat and by some called "goat fever." The investigators mentioned found all their cases to be in the goat raising country, among those drinking uncooked goats' milk or handling goats (for the Mediterranean Fever Commission found these animals excreted the bacteria in their urine and feces as well as in their milk), and though the organisms were not found in the milk of the Texas goat, 34 per cent. gave a positive reaction to the agglutination test.

The complement fixation test has since been used in the investigation with positive results.

Gentry and Ferenbaugh give the warning that some of the so-called typhoid fever cases in these sections might prove to be Malta Fever.

The incubation period seems to be about two weeks.

The disease is characterized by the peculiar course of the fever, the curve of which, rising and falling like the waves of the sea, have given it the name of undulating fever.

It is very drawn out, often lasting months (averages give 90-120 days-some last a year or more) and is rife with discomforts, such as headache, backache, neuralgic pains, swollen painful joints, perspiration, gastric disturbance, and not uncommonly an orchitis. 
Relapses are the rule and are repeated.

Treatment. Here, as in most diseases, our best treatment lies in husbanding the patient's strength for his own battle with the infection.

This is achieved by rest, consideration of the diet and good nursing.

Bed. Certainly during the febrile exacerbations and during the period of pain the patient should be kept in bed and will undoubtedly be willing, but during the remissions an insistency on the bed may not be feasible, but a maximum amount of quiet should be enjoined.

Each febrile period is considerably prolonged and the bed should be chosen with a view to comfort and convenience as in Typhoid Fever. (See Chap. XIV.)

In a state of so great discomfort good nursing counts for much; quiet should be secured, every annoyance avoided and the bed-pan be insisted upon.

The room should be cool and shady and screened against insects.

The urine and feces which contain the organisms should be destroyed, and clothes, bedclothes and utensils sterilized as in Typhoid Fever cases.

Nurses and physicians appreciating the mode of infection should guard against it by wearing gloves when handling contaminated objects and carefully clean their hands. The rules given under Typhoid Fever serve here. (See Chap. XIV.)

Care of the Body. Sponge baths with soap and water, talcum powders, alcohol rubs, frequent changing of position keep the skin in condition and avoid bed-sores. The mouth and nose should be kept free from secretions, the tongue and teeth especially looked after.

It has been advised, on account of the neuralgias and joint pains, that just as in rheumatism the clothes next the skin should be of flannel or contain wool, and this the more especially because the perspiration is profuse and, when cotton garments become wet through, chilling of the skin may ensue. Of course frequent change of clothes for the same reason is demanded. For details (see Typhoid Fever, Chap. XIV.)

Diet. The general rule for feeding in fever should be followed (Diet in Acute Infectious Diseases, Chap. II) with a view to caloric needs and that, especially, because the fever is long drawn out. As, however, the gastro-intestinal canal is in an irritable condition in the early days, food should not be forced and should at first consist of diluted milk, with soups, cereal waters and jellies, and meat broths (if desired) which may. be thickened with cereals or farinaceous meals to enhance their food values.

Goat's milk should not be used, but if deemed imperative it should be boiled or at the least be pasteurized to kill any bacteria melitenses that may be present. 
Drinks. Water, saline waters or drinks containing fruit juices should be given freely.

Bowels. The bowels should be freely opened at the beginning with calomel and salts or salines alone, Calomel gr. iii to v (0.2-0.35 Gm.) followed by Epsom or Rochelle salt 3 ss. to i (15-30 Gm.). The calomel may be given in divided doses of gr. $1 / 4(0.015 \mathrm{Gm}$.) every ten to fifteen minutes for eight or ten doses.

Constipation is the rule throughout the disease and the use of enemata or drugs cannot be dispensed with. Calomel has been much used because in addition to its cathartic action it has been assumed to exert a good effect upon the disease itself and jalap for the same reason.

Of course this usage rests on individual opinion and not on a rational basis.

Better for this purpose it seems to me are the milder salines, citrate of magnesia, Hunyadi water and the like, cascara, compound licorice powder, aloin and occasionally more drastic salines like Epsom, Rochelle or Glauber's salt or castor oil.

Retention of urine sometimes occurs and should be looked for. Hot fomentations to the hypogastrium or the sound of running water may relieve this; if not the catheter must be used with all due caution to prevent infection.

Specific treatment has as yet been illy developed; vaccines have been used in the more chronic forms of the disease and good results have been reported. It would seem a legitimate field of endeavor, but their use should not be undertaken in the acute manifestations of the disorder except by men well versed in the theory and application of vaccine therapy, as real harm may be done.

In the suitable cases the dosage has been 5,000,000 bacteria and the number of doses one to ten (Basset-Smith). Some use as high as $50,000,000$.

Symptomatic Treatment. The discomforts of the disease are so great that a considerable demand is made on medical procedure.

Pain. Neuralgic pains, joint pains, backache and headache are constant and characteristic phenomena.

When it is possible the measures of physical therapy should be given preference, such as the ice-bag to the head, hot cloths to the back and joints or mustard plasters or poultices or massage or light rubbing with or without liniments and counter-irritants such as chloroform liniment or a 10-15 per cent. methyl-salicylate or menthol or the two combined.

The joints should be treated as in rheumatism, swathed in cotton batten or flannel and if it affords more comfort, mobilized. (See Rheumatic Fever, Chap. III.) 
If drugs are used, one may try acetyl salicylic acid (aspirin) or other form of salicylate in doses of gr. $\mathrm{x}$-gr. $\mathrm{xv}(0.65-1 \mathrm{Gm}$.) every two hours or coal tars, acetphenetidin (phenacetin) gr. iv to gr. viii $(0.25-0.5 \mathrm{Gm}$.) at the same intervals or acetanilid gr. ii to gr. iv $(0.15-0.25 \mathrm{Gm}$.)

These drugs, however, have been very disappointing and in the severest cases morphine has to be used.

Fever. When within proper limits, $104^{\circ} \mathrm{F}$. or less, this requires no other treatment than that directed at comfort such as follows a cool sponge bath; but in hyperpyrexia $105^{\circ} \mathrm{F}$. or above or even $104^{\circ} \mathrm{F}$. or less, when very sustained, measures aimed at it should be pursued and none are comparable to cold water.

The procedures and their details when there is sustained fever will be found under Typhoid Fever (see Chap. XIV and may be adapted in regard to temperature, length and frequency of bath and, indeed, in all particulars. In cases of sudden high temperature the rules given under "Rheumatic Fever" (Chap. III) should be followed. Hyperpyrexia is one of the real dangers of the disease and should be watched for and provision made for prompt treatment. When cold water cannot be obtained, antipyretics may be used but they are illy efficient and dangerously depressant. In the lower temperatures they should not be used.

Vomiting. During vomiting food should be stopped or if the vomiting is persistent give small quantities of egg albumin, beef-juice, or milk peptonized or diluted in water or cereal water. Champagne is sometimes well borne, lessens vomiting and furnishes a little fuel in its alcohol content.

Cracked ice may be used, mustard paste 1 part of mustard to 3 or 4 of flour, or mustard leaf may be applied to the pit of the stomach, bismuth subnitrate gr. $\mathrm{xv}$ to gr. $\mathrm{xxx}(1-2 \mathrm{Gm}$.), bicarbonate of soda gr. $\mathrm{x}$ to gr. $\mathrm{xv}$ $(0.65-1 \mathrm{Gm}$.$) , cerium oxalate \mathrm{gr} . \mathrm{v}$ to $\mathrm{gr} . \mathrm{x}(0.35-0.65 \mathrm{Gm}$.) may be given singly or in combination.

In very severe cases cocaine hydrochloride gr. $1 / 5(0.012 \mathrm{Gm}$.) may be of value, or calomel in gr. 1/10 (0.006 Gm.) doses, or if exhaustion threatens morphine sulphate gr. $1 / 8$ to gr. $1 / 4(0.008-0.015 \mathrm{Gm}$.) hypodermically.

Sleeplessness may require bromides in early evening in gr. $\mathrm{xxx}(2$ $\mathrm{Gm}$.) doses or trional gr. $\mathrm{x}$ to $\mathrm{gr} . \mathrm{xx}(0.65-1.35 \mathrm{Gm}$.) or chloralamid gr. $\mathrm{xv}$ to $\mathrm{gr}$. $\mathrm{xxx}(1-2 \mathrm{Gm}$.) or in prolonged or persistent sacrifice of sleep chloral gr. $\mathrm{x}$ to gr. $\mathrm{xv}(0.65-1 \mathrm{Gm}$.) or even morphine gr. 1/8 (0.008 Gm.) hypodermically.

Toxemia. If this is marked the hydrotherapeutic measures mentioned under hyperpyrexia will be found most efficient.

If the circulation is threatened digitalis should be given in doses 
of the tincture $\mathrm{m}$. $\mathrm{xxx}$ ( 2 c.c.) three or four times a day for three or four days or the equivalent dose of the infusion 3 ss. ( 15 c.c.) or powdered leaf gr. iii $(0.2 \mathrm{Gm}$.).

For more immediate effect the vaso-motor stimulants caffeine in soluble form, double salt of sodium salicylate or benzoate gr. v (0.35 $\mathrm{Gm}$.) every four or every two hours intramuscularly or camphor gr. $\mathrm{v}(0.35 \mathrm{Gm}$.) in oil, 10 per cent. by the same method at the same intervals.

Orchitis. Is not an uncommon complication. It is to be treated like orchitis from other causes. The testicle is supported, hot fomentations applied or "dry poultices," i. e., a heavy non-absorbent cotton dressing and counter-irritants used, of which light application of the actual cautery is the best. (See Parotitis, Chap. XXIII.)

Convalescence. Fresh air and plenty of good food are the best tonics during convalescence, though iron as carbonate gr. ii to gr. $\mathrm{v}$ (0.15-0.35 Gm.) arsenic gr. 1/40 to gr. 1/30 (0.0015-0.002 Gm.) and strychnine gr. $1 / 40$ to gr. $1 / 30(0.0015-0.002 \mathrm{Gm}$.) may be used singly or in combination.

Prognosis is good, the mortality is only about 2 per cent. to 3 per cent. and immunity against a second attack is probably conferred.

Prophylaxis. The etiology makes the problem clear.

The infected goat should be eliminated from the herd, and tests made of herds in infected districts from time to time. When this is not possible the goat's milk in infected districts should not be drunk or at least should be boiled or pasteurized before using.

Knowledge should be disseminated in infected districts of the possibility of infection from handling the goat, whose urine, feces and milk excrete the organism.

Care should be taken lest the water supply or food be contaminated by the excreta.

Filth harbors the infection while sunlight readily kills the bacterium; hence, the importance of cleanliness and light.

The patient's excreta, too, containing the bacteria are sources of infection and should be properly disposed of as mentioned above.

It is well to screen the windows and doors against insects that may bear infected excreta on their bodies to food; for by food the disease is nearly always conveyed.

As in typhoid fever some of the patients become carriers after convalescence and have been shown to excrete the bacteria for two years. 


\section{SUMMARY}

\section{Rest.}

In bed; certainly, during exacerbations.

Bed.

(For technique of bed-making, see Typhoid Fever, Chap. XIV, or Dysentery, Chap. XVI.)

\section{Room.}

Cool and shady. Screen against flies.

Urine, stools, bedclothes, utensils, etc. (See Typhoid Fever, Chap. XIV.)

\section{Physicians and Nurses.}

Should wear gloves in handling secretions.

(See Typhoid Fever, Chap. XIV.)

\section{Care of the body.}

Sponge baths, daily, with soap and water.

Talcum powder.

Alcohol rubs.

Frequent change of position.

Mouth, teeth, etc.

(See Pneumonia, Chap. IX.)

Woolen next to the skin on account of neuralgias, joint pains and abundant perspiration.

\section{Diet.}

Early days.

Diluted cow's milk (not goat's), soups, cereals, jellies, broths thickened with cereals or farinaceous flours.

\section{Drinks.}

Water, mineral waters, lemonade, orangeade, grape-juice, Imperial drinks, freely.

\section{Bowels.}

In the beginning.

Calomel gr. iii to gr. $\mathrm{v}(0.20-0.30 \mathrm{Gm}$. $)$ or in divided doses, followed by a salt, Epsom or Rochelle, 5 ss. -5 i $(15-30 \mathrm{Gm}$.) or a salt may be given alone.

Throughout the disease constipation is the rule.

Calomel.

Citrate of magnesia.

Hunyadi water.

Cascara, e. g., fluid extract m. xv to m. xlv (1-3 c.c.) in water. Compound licorice powder, $3 \mathrm{ss}$. to $3 \mathrm{ii}(2-8 \mathrm{Gm}$.) given in water. Aloin, gr. ss. to gr. i $(0.030-0.060 \mathrm{Gm}$.). 


\section{Retention of urine.}

Hot fomentations to epigastrium.

(For technique, see Typhoid Fever, Chap. XIV.)

Sound of running water.

Catheterize.

\section{Specific treatment.}

(See text.)

\section{Treatment of symptoms.}

Pain and neuralgias.

Ice-bag to head for headache.

Fomentations to back and joints or mustard plasters.

Poultices.

Chloroform liniment.

Methyl salicylate and menthol 15 per cent. in vaseline.

Acetylsalicylic acid (Aspirin), gr. $\mathrm{x}$ to gr. $\mathrm{xv}$ (0.60-1 Gm.) every two hours.

Acetphenetidin (Phenacetin), gr. iv to gr. viii (0.25-0.50 Gm.) every two hours.

Acetanilid, gr. ii to gr. iv (0.120-0.25 Gm.) every two hours.

Very severe.

Morphine sulphate, gr. 1/8 to gr. $1 / 4(0.008-0.015 \mathrm{Gm}$.). Fever.

Cold water, sponges, packs, baths.

(For technique of bath, see Typhoid Fever, Chap. XIV.)

Vomiting.

Cracked ice to suck.

Mustard paste to epigastrium.

(For technique, see index.)

Bismuth subnitrate, gr. $\mathrm{xv}$ to $\mathrm{gr} . \mathrm{xxx}(1-2 \mathrm{Gm}$.).

Sodium bicarbonate gr. $\mathrm{x}$ to gr. $\mathrm{xv}(0.60-1 \mathrm{Gm}$.).

Cerium oxalate gr. v to gr. $\mathrm{x}(0.30-0.60 \mathrm{Gm}$.) or combination of above drugs.

Very severe.

Cocaine hydrochloride gr. 1/5 (0.012 Gm.).

If exhaustion threatens.

Morphine sulphate, gr. $1 / 8$ to gr. $1 / 4(0.008-0.015 \mathrm{Gm}$.) hypodermically.

Sleeplessness.

Bromides, $\mathrm{gr} . \mathrm{xxx}(2 \mathrm{Gm}$.) in water in early evening.

Trional, gr. $\mathrm{x}$ to gr. $\mathrm{xx}$ in warm water, in whiskey, in powder.

Chloralamid, gr. $\mathrm{xv}$ to gr. $\mathrm{xxx}(1-2 \mathrm{Gm}$.) in cold water, in whiskey or in powder.

If more resistant.

Chloral, gr. $x$ to gr. $\mathrm{xv}(0.65-1 \mathrm{Gm}$.$) in water.$

Morphine sulphate, gr. 1/8 (0.008 Gm.) hypodermically.

Toxemia.

Circulation.

(See Pneumonia, Chap. IX.) 


\section{Complication.}

Orchitis.

(See Parotitis, Chap. XXIII.)

Convalescence.

Fresh air.

Good food.

Iron.

Arsenic.

\section{Prophylaxis.}

Elimination of infected goats.

Herds tested.

Milk should not be drunk.

Instruction in regard to the infectivity of milk, urine and feces of infected goats.

Care of the water and food supply.

Disinfection of patient's excretions.

Screen room of patient against insects.

Determination of "carriers." 


\section{CHAPTER XXXIII}

\section{ROCKY MOUNTAIN SPOTTED FEVER}

\section{(TICK FEVER OF THE ROCKY MOUNTAINS)}

THIs disease as the name would indicate pervades the Rocky Mountain Districts, including the States of Washington, Oregon, California, Nevada, Idaho, Utah, Montana, Virginia and Colorado. It has been characterized as an "acute, endemic, non-contagious, but probably infectious, febrile disease, characterized clinically by a continuous moderately high fever, severe arthritic and muscular pains and a profuse petechial or purpural eruption in the skin, appearing first on the ankles, wrists and forehead, but rapidly spreading to all parts of the body" (Maxey). It is transmitted by a tick, Dermacentor venustus.(Dermacentor andersoni). The virus with which the tick becomes infected is entertained by certain rodents on which they feed, chipmunks, ground squirrels, mountain rats.

The virus will not pass through a Berkfeld filter and for a time was believed to be bacterial, but Wolback has isolated a micro-organism which he believes to be a parasite of a different nature. He can identify it neither as a bacterium nor protozoon. He proposes for this causative agent of Rocky Mountain Spotted Fever the term Dermacentroxenus rickettsi in honor of Rickett's pioneer work in this disease. This virus was shown to be transmitted hereditarily in ticks.

It occurs almost wholly in the Spring and is at its height in May or June. This corresponds exactly with the period of activity in the life history of the tick.

The incubation period is usually 4 to 6 days, the extreme limit being 3 to 12 days. It requires on an average 10 hours of feeding time for the tick to infect.

Symptomatology. The onset is abrupt with a chill though the chill may be preceded by a few days of malaise and it has been noted that when such is the case as in most instances in Idaho, the disease is less fatal.

The discomfort suggests a grip attack with severe pain in bones, joints and muscles, small of back and head; a little dry cough and photophobia are common and epistaxis occasionally. The temperature rises fairly quickly, reaching $102^{\circ}$ to $104^{\circ} \mathrm{F}$. on the second day and continues 
to $104^{\circ}$ to $105^{\circ} \mathrm{F}$. and in exceptional instances $106^{\circ} \mathrm{F}$. and $107^{\circ} \mathrm{F}$. in the second week and it begins to fall by lysis toward the end of the second week and strikes normal at the end of the third.

The pulse at first is not very rapid, but later increases out of proportion to the temperature, i. e., a pulse of 120 to $102^{\circ} \mathrm{F}$. of fever is common. In the more severe cases the pulse increases and signifies a bad outlook. The spleen is enlarged and palpable early in the disease. The rash usually appears on the third day, but varies between the second and seventh. It is noted first on the wrist, ankles, back, forehead, arms, legs, chest and lastly abdomen. It is well out in 24 to 36 hours after appearance; but later it may appear on palms and soles and the mucous membrane of the propharynx. The rash at first is a rose-colored macule disappearing on pressure, but gradually it darkens to a purple and at the end of a week fails to disappear on pressure. It then tends to become petechial and considerable subcutaneous hemorrhages may be seen. Often the rash is confluent. It begins to disappear as the fever begins to go down, but often remains for some time as pigmented spots. When remaining discrete this pigmentation has been likened to the markings on a turkey egg.

In severe cases, owing to occlusion of the vessels of the skin, necrosis of the skin of the fingers, toes, prepuce, scrotum, lobes of ears, or of the soft palate may occur. A general desquamation follows the subsidence of the rash. Restlessness, sleeplessness, stupor are common and, in severe cases, delirium and coma before death. Even convulsions have been reported.

Constipation is the rule, vomiting in severe cases and slight jaundice in the second week.

The blood shows a slight leucocytosis and a noticeable increase in the large mononuclears.

One attack appears to confer permanent immunity. The mortality varies in different localities. In Montana, in the Bitter Root Valley, it has run above 70 per cent., in Idaho about 5 per cent. Outside of the Bitter Root Valley it runs between 7 per cent. and 13 per cent.

This disease has many points of resemblance to Typhus Fever.

Treatment. These cases occur in localities where all the niceties of nursing are difficult of attainment and in environments where the most must be made of what is at hand. The disease runs a rather long course and is rife with discomforts and in some localities almost universally accompanied by a high and dangerous degree of toxicity.

The coolest, best ventilated room should be chosen or the case may be treated in the open air, if shade and protection can be afforded. So far as possible, the choice of the bed, its care, the care of the body, baths, 
care of mouth, nose, eyes, skin and genitals should be carried out as directed under Typhoid Fever (Chapter XIV); the only danger of infection being through the bite of the tick, the disposition of excretions and the precautions in handling the patient insisted on in Typhoid Fever need not be observed beyond the usual rules of sanitation and cleanliness.

Diet. As there is not much gastro-intestinal disturbance after the onset and as the course is prolonged, an abundant dietary would seem indicated and the diet advised for Typhoid Fever would seem logical. Water, alkaline drinks and fruit juices should be liberally administered.

The Bowels. An initial cathartic may be given of castor oil 5 ss. $-5 \mathrm{i}$ (15 to 30 c.c.) or of a salt, Epsom, Rochelle or Glauber's 5 ss. to 5 i (15 to 20 grams) which may or may not be preceded by calomel gr. iss. to ii $(0.066-0.120 \mathrm{Gm}$.$) , better in divided doses, especially if there is nausea$ and vomiting. One gives gr. $1 / 4(0.015 \mathrm{Gm}$.) every $1 / 4$ of an hour until the above dose is completed. For the discomforts, aches and pain aspirin in 10 grain doses $(0.66 \mathrm{Gm}$.) at 2 or 3 hours intervals may be used or very small doses of acetanilid gr. iss. combined with bicarbonate of soda gr. i $(0.06 \mathrm{Gm}$.) and citrated caffeine gr. ss. $(0.03 \mathrm{Gm}$.) at half hour intervals until 6 doses are taken, then every two hours, but this should be only in the early stages and never when the circulation is impaired. If pains are intense it is better to use codeine phosphate gr. $1 / 8$ to gr. $1 / 2$ ( 0.008 to $0.03 \mathrm{Gm}$.) at 2 to 4 hour intervals. The drug is more potent administered hypodermically. In the worst cases, and especially if sleep is lost, morphine sulphate should be used gr. $1 / 8$ to gr. $1 / 4$ ( 0.008 to 0.015 Gm.) hypodermically. Headache is relieved by the above measures and an ice-bag (see Pneumonia, Chap. IX) may be applied to the head.

Fever. Unless very high or long sustained had better be left alone. Cool sponges afford relief and are a tonic to the general nervous system. Antipyretics should not be used, for they are for the most part depressants to the circulation. Excessive temperatures may be controlled by cold sponges or cold baths, such as the slush baths and the cold pack. (See Typhoid Fever, Chap. XIV and Scarlet Fever, Chap. XVII.)

Insomnia. The exhaustion that ensues upon loss of sleep cannot be overemphasized. If sleeplessness is due to pains and discomforts or exhaustion is threatening there is no use of temporizing with milder hypnotics. Morphine sulphate should be used hypodermically in doses of $\operatorname{gr}: 1 / 8(0.008 \mathrm{Gm}$.), to be repeated if necessary. If there is delirium begin with morphine sulphate gr. 1/4 $(0.015 \mathrm{Gm}$.). For milder cases one may use bromides in doses of $\mathrm{gr} . \mathrm{xv}$-gr. $\mathrm{xxx}$ (1-2 Gm.) either potassium bromide or the mixed, sodium, ammonium and potassium salts. Give in a glass of water in the early evening or trional in doses of 
gr. $\mathrm{v}$ to $\mathrm{xv}(0.33-1 \mathrm{Gm}$.) or chloralamid $\mathrm{gr}$. $\mathrm{xx}-\mathrm{xxx}$ ((1.33 to $2 \mathrm{Gm}$.). Delirium indicates the use of morphine as specified above and hyoscine hydrobromide gr. 1/200 to gr. 1/150 (0.0003-0.00045 Gm.) may be used with caution, for it is a depressant. An ice-bag to the head and cool sponges are helpful. The circulation should receive serious attention. It is well to digitalize the heart early to anticipate a depression that may steal on us unawares. For the procedure consult the chapters on pneumonia or influenza. In urgent cases the digitalization should be as rapid as in those diseases, but in more moderate cases more time may be taken to attain digitalization and then the dose may be dropped to gr. iii to gr. iss. (0.20 to $0.10 \mathrm{Gm}$.) a day. (See Pneumonia and Epidemic Influenza, Chaps. IX and XII).

Emergency cases must be treated with intramuscular or intravenous administration of strophanthin. (See Pneumonia, Chap. IX.)

There is no specific treatment.

Immune serum has been tried without result. A great variety of drugs have been advocated but without any perceptible beneficial effects. Midrie and Parsons as the result of experimental work on guinea pigs have recommended the use of 5 per cent. sodium citrate solution intravenously in doses of 60 c.c. ( 5 ii) twice a day.

Complications. Pneumonia is an infrequent complication and is to be treated as under other circumstances.

Prophylaxis. The knowledge that the disease is always conveyed by the tick and that it takes the tick an hour or more to become attached to and feed upon the host, makes a systematic search for ticks on the person after exposure a very important prophylactic measure.

\section{SUMMARY}

\section{Treatment.}

Coolest and best ventilated room or

Treat in open air.

For care of the body, mouth, nose, skin. (See Typhoid Fever, Chap. XIV.)

\section{Diet.}

Abundant, if disease is protracted. (See Typhoid Fever, Chap. XIV.)

\section{Fluids.}

To be given in abundance. (See Typhoid Fever, Chap. XIV.)

\section{Bowels.}

Initial cathartic of castor oil, 5 ss. to $5 \mathrm{i}(15-30$ c.c.) or A salt, Epsom, Glauber's or Rochelle (15-20 Gm.). 
May or may not be preceded by calomel, gr. iss. to ii $(0.060-0.120$ Gm.). Better given in divided doses especially if there is nausea.

Aches and pains.

Acetyl salicylic acid, gr. $10(0.66 \mathrm{Gm}$.) at 2 to 3 hour intervals or Acetanilid, gr. iss. (0.10 Gm.) combined with sodium bicarbonate gr. i $(0.06 \mathrm{Gm}$.) and citrated caffeine, gr. ss. $(0.03 \mathrm{Gm}$.) at halfhour intervals until 6 doses are taken and then every two hours. Take only in early stages and never if circulation is impaired or

Codeine phosphate gr. $1 / 8$ to gr. $1 / 2(0.008$ to $0.03 \mathrm{Gm}$.) at two to four hour intervals or

Morphine sulphate, gr. 1/4-1/8 (0.008 to $0.015 \mathrm{Gm}$.).

\section{Headache.}

May be relieved by above measures or ice-bag. (For technique, see Pneumonia, Chap. IX.)

\section{Fever.}

Give no drugs.

Let alone unless high or long. sustained.

Cool sponges for relief.

Excessive temperatures use cold sponges, cold baths-such as slush baths and cold pack. (For technique, see Typhoid Fever, and Scarlet Fever, Chaps. XIV and XVII.)

\section{Insomnia.}

If due to pain or exhaustion, morphine sulphate hypodermically, gr. 1/8-1/4 (0.008-0.015 Gm.).

Repeat if necessary.

Insomnia with delirium.

Morphine sulphate, gr. 1/4 (0.0015 Gm.) hypodermically.

Less urgent insomnia.

Bromides, gr. $\mathrm{xv}-\mathrm{xxx}(1-2 \mathrm{Gm}$.).

Trional, gr. v-xv (0.33-1 Gm.).

Chloralamid, gr. $\mathrm{xx}-\mathrm{xxx}$ (1.33-2. Gm.).

\section{Delirium.}

Morphine as above.

Hyoscine hydrobromide, gr. 1/200-1/150 (0.0003-0.0045 Gm.).

Ice-bag to head.

Cool sponges.

\section{Circulation.}

Well to digitalize the heart early. (See Pneumonia, Chap. IX.)

In urgent cases rapid digitalization. (See Pneumonia, Chap. IX.)

Emergency cases.

Strophanthin intramuscularly or intravenously. (See Pneumonia, Chap. IX.) 


\section{Specific Treatment.}

Immune serum has been tried without results.

\section{Complications.}

Pneumonia infrequent. (See Pneumonia, Chap. IX.)

\section{Prophylaxis.}

Search for the tick after exposure in tick-infected localities. 


\section{CHAPTER XXXIV}

\section{LEPROSY}

To no other unfortunate has the term "unclean" clung throughout the ages so insistently as to the leper.

Its importance to us is that Leprosy has invaded North America and while its distribution is not so uniform as is the case with most of the infectious diseases, still its distribution is no respecter of climate and in our own country it is found in Minnesota in the North and Louisiana in the South, just as abroad it finds residence in the cold of Norway and the heart of India. Fortunately in this country the numbers are not large nor the spread rapid, still its mutilations carry no less horror to-day than in biblical times, even though more knowledge protects the community from unreasoning fear and the unfortunate from ill-usage. At least we have fixed the cause in a definite organism, the bacillus lepræ and learned something of its habits ${ }^{1}$ and the present time is witnessing no inconsiderable impulse to investigation in this field among our own workers.

Three facts of practical importance about bacillus lepræ are, first, that in all possibility its portal of entry into the human organism is the naso-pharynx, second, that it is discharged by way of all the secretions including discharges from sores, and thirdly, that it is peculiarly tenacious of life even in an adverse environment.

The organisms infect all the organs and tissues but have a peculiar predilection for the skin and peripheral nerves and the clinical picture depends on which of these structures is predominatingly affected.

Isolation. The isolation of the leper has been throughout the ages the fruit of experience and recent pleas to free the leper in the community meet with prompt rebuttal in facts educed by a study of his distribution of the infecting germs.

Colonization is the modern method of isolation; a colonization directed by every humane effort to relieve this life-long divorcement from the world.

Precautions of Attendants. It is essential for all who come in contact with the patients to know that the bacilli of leprosy have been found in all the secretions, urine, feces, sweat, milk, sputum, in the secretions from the nose, the vagina, the urethra, as well as in the secre-

${ }^{1}$ Strong in Forchheimer's Therapeusis of Internal Diseases would imply that identification of the organism of Leprosy is by no means certain. 
tions and pus of sores and that all these secretions should be destroyed by burning or, when contaminating useful articles, by boiling or by powerful antiseptics. It should be particularly remembered that the lesions are apt to be early and severe in the upper air passages and that this may be conveyed by the spray of a sneeze or a cough or even in talking. Again it is to be remembered that the bacilli are peculiarly insistent and will remain virulent for months in dried secretions and in soiled linen.

It must be borne in mind, too, that insects may be the means of conveying infection and that non-infected animals may become carriers. That the disease is not highly infectious is shown by the fact that long contact with infected persons seems necessary for its conveyance.

Cleanliness of the skin, the mouth and nose, sufficiency of good, well-cooked food and, in short, careful consideration of the rules of health are doubly important to those exposed to the disease.

Leprosy is a curable disease and that always means that it is the body's own forces that must be called upon. This knowledge accentuates the importance of diet and hygiene, measures that contribute to the body's efficiency.

The fight is a long one and the improvement slow, in this respect like tuberculosis; and so, as in the latter disease, all efforts in the patient's behalf must be persistent.

Diet. After all it is from the food that all the energy used by the body must come, for the production of immune bodies as well as for other purposes; consequently, it should be abundant, well selected as to quality and well prepared.

Fresh Air. The life of the leper like that of the tubercular should practically be in the open, the same provision being made for sleeping out of doors.

Baths. Cleanliness is most important and baths not only keep the pores of the skin open and subserve the functions of that structure, but they have a tonic effect on the nervous system and, when the lesions of the skin are pronounced, serve as a vehicle for suitable medication.

Hot baths are found especially grateful.

Such supportive treatment is of infinitely greater value than any empirics or specifics that we have at our command.

Empirical Treatment. An empiricism means merely a groping; the drugs that find favor are legion and none very satisfactory. Of all the drugs yet employed there is greater agreement on the value of Chaulmoogra oil than on any other.

Chaulmoogra Oil (Oleum gynocardiæ). This oil is expressed from the seeds of the gynocardia odorata.

Mode of Administration. It has been given by the mouth, by 
the rectum, hypodermically and applied to the skin. By far the most common method is by the mouth.

Dosage. The amount given must be considerable to be effective; but, as it is illy borne at first by the stomach, the initial dose should be small and gradually increased.

It is well to begin with 3 or 4 drops at a dose three times a day and increase a drop or two to the dose every three or four days until the limit of tolerance is reached or the dose totals 100 to 130 drops three times a day. The limit of tolerance is determined by the stomach's irritability.

It is perhaps better given before meals, using as vehicles hot coffee, hot milk, cordials or milk of magnesia or it may be given in capsules or in pill-form, although the administration by these last two methods would be feasible only at the beginning.

It has always seemed to me undesirable to give a disagreeable or disagreeing medicine in food, lest a distaste for a useful food be acquired by association.

By Rectum. It has been so administered in milk as a vehicle, but this would scarcely be the preferred route.

Hypodermically. Some irritation may ensue from the oil used in this manner, but when sterilized by heat this is said to be, for the most part or quite, avoided.

The oil so treated as to lessen its irritating qualities has been brought forward under the name of "anti-lepral."

It is said to be less irritating to the stomach. As much as 5 c.c. a day for months has been tolerated when so given. These are also supplied in ampoules of from $1 / 2$ to $1 / 5 \mathrm{mg}$.

Externally. Chaulmoogra oil diluted to 5 per cent. to 10 per cent. with some bland oil has been used as an inunction.

Two rubbings a day are given. Latham and English's system of treatment gives the following formula for its local use; Chaulmoogra, 4 ; soft paraffin, 6 ; hard paraffin, 1 .

There can be no doubt that the rubbing itself would be beneficial to the infiltrated or anesthetic skin, but as compared with its dose by the mouth this and other methods are of dubious value; little is known of its modus operandi in cases which improve, beyond the fact that as fat it has some food value.

Local Treatment. Two and 1/2 per cent. solution of benzoylchloride as a nasal spray and an application to leprous ulcers is said to be followed by improvement in these lesions, but one may have to have recourse to surgical treatment.

The disadvantages in its use rest upon its irritating action on the 
gastro-intestinal canal which induce vomiting and diarrhea. This seems not difficult to avoid if care be taken in its administration.

Opinions differ about its value. Dyer, whose experience in Louisiana, where it is endemic, gives him authority, speaks highly of it in Osler's Modern Medicine, and reports twelve cures in his own experience since 1894. Wooley on the other hand grants no gain to its use and believes improvement due to a better appetite and better functioning of the skin following its use, such as follow certain systems of baths.

It must be said, however, that the results of this treatment have made a considerable impression on most men who have employed it and is perhaps the best weapon at hand at the present moment.

Other Drugs. Among other drugs of less repute are Nastin, a bacterial fat which is diluted in benzoylchloride to lessen its irritative effects to the strength of .05 per cent., called Nastin B, and to .2 per cent. Nastin B. ${ }^{2}$

Bercovitz reporting from China on the method advocated by Heisser, uses hypodermically a mixture of this formula

Camphorated oil......................60 c.c.

Jii

Chaulmoogra oil.......................60 c.c.

茫

Resorcin.............................. grams.

gr. lx

This after sterilization is injected under the skin of the arms and legs in doses of 1 c.c. of the mixture, weekly for three doses, then gradually increase to 3 c.c. weekly. There was always a slight reaction after the first dose, causing a mild headache with malaise and nausea.

With this treatment he combined a bath of 2 per cent. soda bicarbonate taken immediately after the injection three times a week and followed by complete rest for a half hour and by a saline cathartic the next morning, and a compound of iron and arsenic. Inside of four weeks he found a distinct amelioration in all patients so treated. This was the more noticeable in the tubercular type.

One c.c. subcutaneously once a week for five or six weeks is used of the weaker dilution, then is followed by weekly injections of the stronger. This has its advocates but elicits no such commendation as the Chaulmoogra oil.

Calmette's Serum. Dyer attracted by the belief among certain West Indians that the bite of certain reptiles cured leprosy, used Calmette's Antivenomous Serum in doses of 5 c.c. to 20 c.c. under the skin, sometimes as often as every day and found excellent results, reporting three cures.

Specific Treatment. Very naturally in this day of serum and vaccine therapy efforts have been made to utilize the products of bac- 
terial action to enhance the production of immune bodies, and as a matter of course there have been many disappointments. Among the later efforts and one giving some promise is a toxin derived from the bacillus lepræ by Rost and called "leprolin."

Its use, of course, attempts an active immunization. Its dose is 10 c.c. given intravenously at two to three day intervals and further dosage determined as in the use of tuberculin or vaccines, by the reactions induced.

It is said to be contraindicated in the presence of pulmonary or kidney complications.

It is hardly fair as yet to draw conclusions, but judicial clinicians seem to be optimistically inclined toward it.

Tonic Treatment. More or less emphasis is laid on the value of tonics; and strychnine or nux vomica, phosphates and iron are used as in other conditions which are believed to indicate their use.

At the moment of the revision of this work an elaborate study of the chemotherapeutics of Chaulmoogra oil by Walter and Sweeney appeared in which they concluded that the therapeutic action of the drug was due to its "direct antiseptic and bactericidal action on B. lepræ." They suggest that the oil or its acid esters like other fats, may well be stored in the tissue until the concentration becomes bactericidal.

Surgical Treatment. It is important to evacuate any pus. Sores and mutilations demand the removal of diseased tissue of skin or bone. Amputations, excision of nodules, stretching of nerves for pain, etc., may be necessary.

$\mathrm{X}$-Ray treatment is said to reduce the size of the tubercles.

Prognosis. So evil a reputation has leprosy borne throughout all history that the impression one receives of the prognosis is of the worst; and yet a study of the disease shows that it is self-limited at any stage and that a mode of life aimed at improving the general health quickens the process of cure more than anything else can do.

That death or mutilation is the final issue in most of the cases, however, is all too true.

Prophylaxis to the community consists in isolation; to the individual in avoidance of contact with the secretions and discharges. It has long been noted that long contact seems necessary for infection. Extremely interesting results bearing on this fact have followed upon the work of Duval and Gurd on animals. I quote from their article: "Two factors are of great importance in effecting infection. In the first place, a sufficiently large number of organisms must be employed, and, what is still more important, second and subsequent inoculations are more liable to produce leprous lesions than are the primary injections. 
"Such preliminary doses, whether they consist of living or dead organisms, produce a condition of hypersensitiveness or allergy which renders it possible by a second injection of viable bacilli to induce the development of a reactionary lesion. Lesions arising as the result of a second inoculation develop more rapidly, increase in size more quickly, and persist for a longer period than those taking place as the result of a single inoculation, even though very large doses are used. Moreover, the bacilli in these lesions are more liable to lead to metastasis and to a generalized infection. We regard the results of these experiments as having considerable bearing upon the development of the disease in human cases, since we find that it is chiefly among those living in prolonged intimate contact with leprous patients that leprosy develops." (Journal of Experimental Medicine, Aug. 1, 1911.)

I have already spoken of the danger to those in contact with patients that arise from secretions, discharges and spraying of coughing, sneezing, etc., and how these secretions should be disposed of. That the nasopharynx is probably the portal of entry accentuates the precautions that should be taken not to carry the infection by the hands to this locality. The possibility of infection by way of the skin by minute traumata or bites of insects must not be forgotten.

It would seem almost unnecessary to mention the dangers attendant on marriage, and the precautions that should be taken in the employment of nurses, wet nurses or others coming into close contact with members of a household who live in an area where leprosy is endemic.

Another fact of importance is the remarkable viability of the bacilli lepræ, which will survive for months or years in a locality once inhabited by a leper. This makes disinfection of houses and articles subjected to contamination doubly imperative and demands thoroughness. It also emphasizes the importance of colonization.

The dead should be cremated; for the leper bacillus has been found viable months after burial.

\section{SUMMARY}

\section{Isolation.}

By colonization the common method.

All secretions and excretions, urine, feces, sweat, milk, sputum, those from the nose, vagina, urethra, all contain the bacillus and should be destroyed by fire or antiseptics. (See Typhoid Fever, Chap. V.)

Attendants must remember that the spray of sneezing, coughing and talking can convey the organism.

Insects can convey infection.

Attendants must give especial attention to their personal hygiene 
Diet.

Must be sufficient.

Fresh air.

Life in the open; sleep in open air.

Skin.

Must be kept clean by daily baths.

Empirical treatment.

Chaulmoogra oil.

Dose.

Three or four drops three times a day. Increase a drop or two every three to four days until 100 to 130 drops three times a day are given or until irritability of the stomach prevents. May be given in hot coffee, cordials, milk of magnesia, hot milk, capsules; best before meals.

Hypodermically.

External use.

Under sterile precautions give hypodermic injections of:

R

Camphorated oil..............60 c.c.

Chaulmoogra oil................60 c.c.

Resorcin...................... 4 Gm.

M. et S. 1 c.c. each week for three doses. Increase gradually to 3 c.c. once a week.

Follow each dose immediately by a bath of 2 per cent. sodium bicarbonate.

Rest for one-half hour after bath.

Follow each dose by a saline cathartic the next morning.

Duration of treatment-four to six weeks. (Bercovitz.)

Other drugs and measures.

Nastin.

Calmette's Serum. $\}$ (See text.)

Specific treatment.

Leprolin, 10 c.c. intravenously every two to three days.

Tonic treatment.

Strychnine or Nux Vomica.

Iron.

Arsenic.

Phosphates.

Local treatment.

Two and one-half per cent. solution of benzoylchloride as nasal spray and as application to leprous ulcers. 
$\left.\begin{array}{l}\text { Surgical treatment. } \\ \text { X-Ray treatment. }\end{array}\right\}$ (See text.)

Prophylaxis.

Isolation,

Avoid contact with secretions and discharges.

Thorough disinfection of all objects in contact with a case of leprosy. Dead should be cremated. 


\section{CHAPTER XXXV}

\section{ANTHRAX}

Anthrax is a disease that affects peculiarly domestic animals, the horse, cattle, sheep and goats.

Many infected hides are now coming into the country from epidemic centres in China, India, Africa, South America and more cases of anthrax are seen than before the recent war. An investigation of the shaving brush industry traced the source of infected hair not only to points outside the country, but to Chicago as well.

The causative agent is the bacillus anthracis, a large spore-bearing organism, and the first shown to have causative relationship to an infectious disease.

The disease is conveyed to man through contact with animals or their secretions.

It may enter and infect the skin through minute wounds or abrasions, so is most likely to occur on exposed surfaces and in individuals handling the sick animals, their carcasses or hides, wool or hair such as drovers, farmers, veterinarians, butchers, porters of hides, wool sorters, tanners, etc.

The skin, too, may be inoculated by the bite of the stable-fly, and by the use of the shaving brush.

The lesion is called "malignant pustule." At first a papule, then vesicles and pustules, soon with deep and extensive involvement of the subjacent and adjacent tissues, appear. Or it may take a malignant edematous type in which pustules may be absent or an erysipelatous type, difficult to differentiate from ordinary facial erysipelas without bacterial findings.

The external form begins with a minute red, hard pimple at the site of inoculation. This papule becomes a vesicle and is soon surrounded by an area of edema; the vesicle becomes a pustule, turns black and in 36 hours forms a black eschar that gave it the French name charbon. The lymphatics become swollen and painful in 3-4 days.

Of the cutaneous lesions those occurring in the head and neck region are especially dangerous and are associated with extensive edema. The mortality in these cases is given at $40-45$ per cent. against 12.5 per cent. in upper extremities and 1.2 per cent. in trunk and lower extremities.

Characteristic is the freedom from pain, in spite of the extensive 
infiltration. Sooner or later, depending on the virulency, the symptoms of general infection follow. It may be rapidly fatal; if not it runs a course of 9 or 10 days. Fortunately the tendency of the disease in man is to remain localized.

On the other hand it may enter the body through the air passages or by way of the mouth. Infection of the lungs occurs so commonly among one class of workers that their occupation has given a name to the disease, the "wool-sorter's disease," due, of course, to inhalation of spores borne in the dust raised by their work in the wool.

The course is rapid; two, three or four days. The symptoms point to an involvement of the lungs but neither subjective nor objective signs are characteristic. Rapid asthenia sets in, followed by death.

When the infection is by way of the mouth the intestine is affected.

There is a selective action on the lymphatics resembling that in typhoid fever. The inflammation is intense with edema and hemorrhagic extravasations and there may be perforation of all the coats with seropurulent peritonitis.

This usually occurs through eating of infected and improperly cooked meat or drinking infected milk, or it may come from food contaminated in the handling or from the patient's hands. It may, however, localize in the intestine by the blood route. This form is rare in man. It is manifested by stormy gastro-intestinal symptoms that resemble a poisoning or may simulate intestinal obstruction. It is rapidly fatal. One speaks of it as "Intestinal Anthrax." Death follows in two to six days.

Treatment. The treatment aims at supporting the patient's strength, as in any other infection, the use of specific remedies, the treatment of the local lesion and the relief of symptoms.

As it is only the external form of infection that we can hope to attack with success and that only when seen early, we will begin with a consideration of the local treatment.

Local Treatment. This is distinctly a surgical problem. It would seem to me that a lesion rife with such fatal possibilities as malignant pustule should have the most radical treatment and that complete excision going well wide of the lesion out into the sound tissues should be practiced. ${ }^{1}$ It is universally advised that, in addition, the open wound should be thoroughly cauterized with phenol (liquified carbolic acid) or with actual cautery. In addition, some authors advise injections of carbolic acid into the tissues at several points around the area of excision; as the bacilli on their way to the lymphatics may be harbored there. It

${ }^{1}$ Carey reports a cure by this method since the above was written. A.J.M.S., May, 1920, Vol. CLIX, No. 5, p. 742. 
may be used in 3 per cent. watery solution. Others prefer liquified carbolic acid as less likely to be absorbed from the site of injection.

There seems to be of late a growing preference for an expectant treatment over excision, using bichloride of mercury dressings, boric acid dressings, or alcohol compresses (70 per cent.). Gray ointment has found some favor. Rest to the part affected is of cardinal importance.

To give specific direction for excision I cannot do better than borrow from Dudley's article in the Journal of the A. M. A., Jan. 5th, '18.

He cleanses the lesion thoroughly with soap and sterile water, rinses with sterile water, paints with 8 per cent. (or stronger) phenol "( 1 part of ordinary carbolic acid to 12 parts of water $)$ " and rinsed with alcohol.

He then paints the lesion with collodion to prevent contamination of the line of incision.

Following this preparation of the pustule, 8 per cent. phenol is injected into the tissues all about the lesion to wall off the infection. This requires usually some 6 c.c. One-quarter inch outside the phenolized zone he injects 5 to 6 syringefuls of 25 per cent. alcohol (6-10 c.c.) These, he says, are about $11 / 2$ inches outside the centre of the lesion. The line of incision is painted with 8 per cent. phenol solution and an area $21 / 2$ $31 / 2$ inches in diameter excised. After the excision the base and edges are painted with pure 95 per cent. phenol and neutralized at once with absolute alcohol. The skin is cleansed with alcohol and a wet dressing then is applied of boric acid solution 2 to 4 per cent., 20 per cent. alcohol or hypertonic salt solution.

If in spite of this, slight edema appears at the angle of the wound, further steps are taken. He injects 3 to 4 syringefuls (6-10 c.c.) of 8 per cent. phenol into the edematous tissue and if this fails to cure incises freely into the line of the edema and puts in gauze drains and injects more 8 per cent. phenol. Finally he applies an ice-bag to the area.

Dr. D. F. Dudley in a personal communication says: "I have come to believe that excision of pustules near the eye or nose is always a failure as the region cannot be well prepared with the usual strong antiseptics and since a large amount of tissue cannot be excised in these locations. Lesions about the eye and nose should be walled off with the phenol solution and treated by serum; incisions in the edema but not into the pustule may be made later if necessary and phenol solution again injected. I am now preparing a paper on 'The Choice of Treatment'this is necessary because of difficulties in certain locations and because I have found that the age, health, presence of some other trouble such as heart, lungs or high blood pressure are factors to be considered before taking a chance either on serum or excision. I can't emphasize too 
much the importance of choosing the cases which shall be excised instead of using serum and vice versa."

At Camp Hancock, Ludy \& Rice have dissected out the lesion with a nose cautery after infiltrating the surrounding tissue with $30-50$ c.c. of anti-anthrax serum. The line of incision should go at least $1 / 2$ inch from the border. They dress the wound over in 24 hours with a solution of phenol 3 parts; camphor 7 parts; glycerin 40 parts; and alcohol 180 parts.

At Guy's Hospital, following excision, ipecac has been sprinkled on the wound and has been administered at the same time internally.

Carbolic Acid. Carbolic acid has been used, too, around the site of the infection and as originally recommended, as often as every hour or even more frequently, 10 to 15 minims of the 3 per cent. watery solution or even the liquified phenol.

Hot poultices are applied to the infected area.

Bloodgood and McGlannan favor this treatment when excision will result in much mutilation.

In one case successfully treated with antitoxin, the local treatment consisted only of soaking in hot bichloride solution and the application of a Bier bandage.

As another caustic, caustic potash has been recommended.

The wound is dressed with a wet dressing.

Specific Treatment. Serum from immunized sheep or asses has been used in the treatment of animals and more lately of man. In the cases reported 30 to 80 c.c. of the serum was used daily both intravenously and subcutaneously.

One should favor liberal doses.

Ludy and Rice give 75 c.c. of anti-anthrax serum with 50 c.c. of physiological sodium chloride solution intravenously and 75 c.c. of the serum intramuscularly. The serum is repeated every 8 hours, if needed. Dudley, using the serum furnished by the U. S. Bureau of Animal Industry gives 35 c.c. intravenously for the first dose, followed in 8 to 16 hours by a second intramuscularly or intravenously; this is repeated if necessary.

Rajan continued local treatment with intramuscular-some 50 c.c. at first, somewhat smaller doses on such succeeding days as serum is indicated.

At Bellevue in some of the cases 40 c.c. of serum was given into the vein every four hours and into the tissues about the pustule about 10 c.c. in multiple punctures of $1 / 2$ to 1 c.c. each, thus thoroughly infiltrating them, at 4-hour intervals. This seemed fairly effectual.

The serum may be obtained from the Department of Agriculture. 
The specific serum should be injected about the pustule or if excised in the adjacent area. One uses an antitoxin needle and syringe. The needle is inserted just outside the margin of the lesion and directed toward the subcutaneous tissue at the base of the eschar, using 10 to 15 c.c. of the serum. This is done, of course, with intravenous and intramuscular administration of the serum.

The general mortality in a considerable series not receiving serum was given as 27 per cent., but may be very much higher in individual series.

The results reported certainly make the use of the serum imperative. The mortality has been lowered to 6 per cent. or less by the use of serum.

There has been much controversy as to what constitutes the specificity of the serum. By some it is maintained that the results are due merely to the reaction provoked by any foreign protein, provocation of leucocytosis, possibly a mobilization of ferments, the phenomena of the so-called shock therapy; for this reason, other non-specific sera have been advocated, especially normal beef serum, which seems to be less likely to provoke disagreeable reactions than horse serum. The methods have been by hypodermic injection or in severe cases or septicemic cases intravenously. The dose is $10-30$ c.c. or more.

I should incline to more liberal dosage, as the amount administered does not determine toxicity, and to frequent administrations, daily or oftener in severe cases.

The reaction will be a sharp rise in temperature, leucocytosis, then a fairly prompt fall of temperature below the level determined before the injection, with an improvement in symptoms general and local. (Hyman \& Levy.)

Graham and Detweiler in one successful case of septicemia, with recovery of the organism from the blood, made use of chloramin-T (Dakin) intravenously with the serum. They used 100 c.c. of the chloramin-T and 80 c.c. of the serum. They attributed much of the good results to the use of the chloramin-T. I have had no personal experience with this method.

See Jour. Am. Med. Asso., Mar. 9, 1918, p. 671, Vol. 70, No. 10.

When constitutional symptoms are manifest, as they are from the beginning in internal anthrax and as they are after a greater or less period in malignant pustule, supportive treatment should be vigorously begun.

This, of course, means rest in bed in a room chosen with a view to convenience and ventilation and one where isolation may be maintained. 
If this room is accessible to porch, verandah, or lawn, where open-air treatment may be maintained, it is a distinct advantage.

The body should have proper attention, such as baths for cleanliness, care of mouth, nose, genitals, attention to pressure points, in other words all that pertains to good nursing. (See Typhoid Fever or Pneumonia.)

Especial attention should be given to the destruction of discharges from the pustules and the secretions and the excretions of the body, which contain the anthrax bacilli.

The diet should be sufficient and based on the same considerations as determine the dietary in other acute infections. (See Diet in Acute Febrile Conditions, Chap. II.)

The severity and stormy course of the infection in internal anthrax and the violence of gastro-intestinal symptoms in the intestinal form of anthrax, will, of course, modify these rules considerably.

Symptomatic Treatment. The temperature is rarely high and requires no consideration, per se.

Sooner or later the circulation begins to wane and stimulation becomes necessary. To the failure of vaso-motor centers such drugs as caffeine or camphor are directed, in doses of $\mathrm{gr.} v(0.33 \mathrm{Gm}$.) at two to four hour intervals, the former as a soluble salt of sodium salicylate or benzoate and the latter in oil, 10 per cent. Both are used under the skin or better still into the muscle.

Strychnine may also be used in doses of gr. 1/40 to gr. 1/30 of the sulphate (0.0015-0.002 Gm.) at three to four hour intervals.

To support the heart, digitalis in sufficient doses, gr. ix to gr. xii $(0.6-0.8 \mathrm{Gm}$.) a day of the leaf or its equivalent of the tincture 3 iss. to ii $(6-8$ c.c.) or of the infusion fresh 5 iss. - ii $(45-60$ c.c.) until results are obtained or 30 or 40 grains have been given. From this point proceed cautiously with 3 to 6 grains a day.

If the need is urgent strophanthin into muscle or vein gr. $1 / 120-\mathrm{gr}$. $1 / 60$ (mg. $1 / 2$ to 1 ), followed by digitalis.

It is my belief that in the circulatory failure of any acute infectious disease digitalis or strophanthin is far more valuable than any of the stimulants named.

Headache may be relieved by the ice-bag; restlessness and sleeplessness by codeine phosphate gr. $1 / 4$ to gr. ss. $(0.015-0.030 \mathrm{Gm}$.) or if severe, by morphine sulphate gr. $1 / 8$ to gr. $1 / 4(0.008-0.015 \mathrm{Gm}$.). Insomnia may be relieved by chloral gr. $x$ to gr. $\mathrm{xv}(0.66-1 \mathrm{Gm}$.), if the circulation is not impaired.

Vomiting will require cessation of food or small quantities of liquid food, cracked ice, sodium bicarbonate or bismuth subnitrate in $\mathrm{gr} . \mathrm{x}$ to 
gr. $\mathrm{xv}(0.66-1 \mathrm{Gm}$.$) doses or oxalate of cerium gr. \mathrm{v}$ to $\mathrm{gr} . \mathrm{x}(0.33-0.66$ Gm.) or a combination.

Diarrhea will need the administration of large doses of bismuth gr. $\mathrm{xxx}$ to gr. $\mathrm{lx}(2-4 \mathrm{Gm}$.) every two hours, or opium gr. 1/4 to gr. ss. $(0.015-0.03 \mathrm{Gm}$.) at the same interval or starch enema with opium as tincture $\mathrm{m} . \mathrm{x}$ to $\mathrm{m} . \mathrm{xv}(0.66-1$ c.c.).

Delirium will demand restraint, the use of morphine or chloral in doses given or hyoscine hydrobromide gr. 1/200 to gr. 1/150 (0.00030$0.00045 \mathrm{Gm}$.).

Convulsions demand the use of inhalations of chloroform during the attack and morphine and chloral between.

Prophylaxis. Successful stamping out of the disease in man can only be accomplished by stamping it out in animals.

Up to 1917 the U. S. Department of Agriculture had not succeeded in finding an efficient and practical disinfectant for hides. (Brown \& Simpson.)

Certainly more care should be given to disinfection of tools, utensils and vats in tanneries. Instructions should be given to the handlers of hides.

Infected animals should be killed and remembering that the spores are peculiarly resistant and that they are aërobic it is important to disturb the carcasses as little as possible and to bury them deep.

This same resistance of the spores and the fact that they are excreted in urine and feces make it difficult to stamp the disease out of the fields in which the infected animals have been pastured.

It must be remembered, too, that these fields are a menace to healthy animals.

\section{SUMMARY}

\section{Treatment of malignant pustule.}

Complete excision, well out into sound tissue.

Cauterize this open wound with liquified phenol (liquified carbolic acid) or with

Actual cautery.

Carbolic acid either liquified or $\mathrm{m} . \mathrm{x}$ to $\mathrm{m} . \mathrm{xv}(0.60-1$ c.c.) of 3 per cent. watery solution has been injected all about the excised area.

Another method-Injections of phenol as above about site of infection. Poultice to the infected area (Bloodgood and McGlannan). Wound is dressed with wet dressing.

Dudley's method. (See text:) .

\section{Specific treatment.}

Serum 30 to 80 c.c. daily under the skin or into vein. 
Bellevue Method:

40 c.c. intravenously every 4 hours

10 c.c. every 4 hours into tissue about pustules given in multiple punctures of one to one-half c.c.

Serum from Department of Agriculture.

When constitutional symptoms are manifest.

Rest in bed.

\section{Room.}

Cool, light, well ventilated.

\section{Bed.}

Hospital type.

Care of body.

Daily bath.

Mouth.

Nose.

Genitals.

(See Typhoid Fever, Chap. XIV or

Bed-sores. Pneumonia, Chap. IX.)

Discharges from pustules, secretions and excretions must be destroyed by fire or disinfected. (See Typhoid Fever, Chap. XIV.)

Diet.

(See Chap. II.)

Circulation.

Digitalis.

Strophanthin.

Caffeine.

Camphor.

Strychnine.

(See text, or Pneumonia, Chap. IX.)

\section{Headache.}

Ice-bag.

\section{Restlessness and sleeplessness.}

Codeine phosphate, gr. 1/8-gr. $1 / 4$ (0.008-0.015 Gm.).

Morphine sulphate, gr. 1/8-gr. 1/4 (0.008-0.015 Gm.).

Chloral, gr. $\mathrm{x}$-gr. xv (0.60-1 Gm.).

\section{Vomiting.}

Stop food.

Cracked ice.

Mustard paste to epigastrium.

Bismuth subnitrate, gr. xv $(1 \mathrm{Gm}$.).

Sodii bicarbonate, gr. $\mathrm{x}(0.60 \mathrm{Gm}$.).

Cerium oxalate, gr. v (0.30 Gm.). 


\section{Diarrhea.}

Bismuth subnitrate, gr. $x x x-3 i(2-4 \mathrm{Gm}$.) every two hours. Opium, gr. 1/4 (0.015 Gm.) every two hours.

Starch enema with tincture of opium $m . x-m . x v(0.60-1$ c.c.).

Delirium.

Restraint.

Morphine sulphate, gr. 1/8-gr. 1/4 (0.008-0.015 Gm.).

Chloral, gr. xv (1 Gm.).

Hyoscine hydrobromide, gr. 1/200-1/150 (0.0003-0.00045 Gm.).

\section{Prophylaxis.}

Kill infected animals and bury their bodies deep.

Avoid use of infected fields. 


\section{CHAPTER XXXVI}

\section{GLANDERS OR FARCY}

This disease is peculiarly common among horses and the horse kind, and is communicated by them to man; so that it is in hostlers, drivers, farmers and others in intimate contact with the horse, the mule or the ass that the disease is found.

The causative agent is the bacillus mallei, which is found in the purulent discharges and in the secretions from the infected mucous membranes and is inoculated into the air-passages or skin abrasions in man. There is a difference of opinion as to whether it may enter through the unbroken skin.

When the infection is received through the air-passages and the lesion is internal it is called Glanders; when through the skin and the lesion is external it is called Farcy.

The local effect of the bacillus is the formation of nodules of an inflammatory character, which break down and form pustules and ulcers, whose discharges carry the infection.

The disease may run either an acute or chronic course; so that we have Acute or Chronic Glanders and Acute or Chronic Farcy.

Treatment. The disease is a toxemia with local manifestations and the treatment that which is applicable to all infections, i. e., measures aiming at the support of the body in its contention with the disease, specific treatment, if there be any, attention to the local lesion and relief of distressing or dangerous symptoms.

For the first, rest in bed, with consideration for all that constitutes rest, good nursing, good room, quiet and mental rest, a sufficiency of diet and fresh air, preferably in the open, meet the demands. (See Treatment of Acute Febrile Conditions and Diet in Fever, Chaps. I and II.)

The mortality of the disease is high, but, as would be expected, least in the chronic form and when confined to the surface of the body, chronic farcy.

Chronic Farcy. The picture is that of a pyemia, in which the local lesion may precede or follow the toxemia, with, sooner or later, multiple abscesses, ulcers and fistulæ slow in progress and perhaps with improvement followed by relapse, and death from emaciation, 
asthenia and overwhelming by poisons; or at any time acute glanders intervenes and hastens the fatal issue.

Besides the supportive treatment specified above one turns to the use of those substances that in a sense may be termed specific, as contributing to Nature's own efforts and in the same terms.

Unfortunately, the results are but little encouraging, and yet any assistance in so dread an infection is welcome. Of such agents one may mention mallein.

Mallein is a toxin obtained from the bacillus mallei much as tuberculin from the tubercle bacillus and has many parallelisms with the latter.

Its particular usage is as a test for the presence of the infection, used like tuberculin for a kindred purpose and provoking like the latter in a true case a febrile response and a local reaction.

Like tuberculin, too, it has been suggested as a curative agent, provoking in graduated doses increasing response in the tissues in terms of immune bodies. Definite rules of dosage cannot be laid down, but advice should be sought from State or Municipal Laboratories preparing the same and acquainted with the reaction obtained in animals. Few have used it, and their praise is qualified.

Vaccines. More hopefully one turns to the use of vaccines and though the reports of successful cases are scanty, nevertheless some have been noted by competent observers.

Park (Forchheimer's Therapeutics of Internal Diseases) advises $20,000,000$ as a beginning dose and gradual increase up to $200,000,000$ or more at four or five day intervals. Dosage is governed by reaction as in use of other vaccines; i. e., by fever, local redness about site of injection or lighting up of local lesions.

A moral obligation to familiarize himself with the theory and practice of vaccine therapy rests on the man who uses an agent, which used carelessly is potent to convert a local process into a hopeless general one. (For words of warning, see Theobald Smith, Jour. Am. Med. Association, May 24, 1913.)

Serum reactions, used as diagnostic tests, such as the agglutination test and the complement fixation test, bespeak the presence of immune bodies; which together with the results in establishing immunity in animals by vaccines encourage the use of serum of immunized animals as a curative agent but unhappily the hope elicited has not been sustained.

Treatment of the Local Lesions. The word of warning not to convert a local lesion into a generalized infection has been well given, for too vigorous curettage or rough handling may break down or thin 
the walls of abscess or ulcer, nature's barriers, to the defeat of her intent to localize the infection by these means.

Abscesses as in other conditions should be incised and evacuated, thoroughly irrigated and a strong antiseptic applied, but not roughly rubbed about.

Liquified phenol (carbolic) is a suitable substance and this may be followed by alcohol to remove the excess. Wet dressings of bichloride may be used or iodoform gauze or tincture of iodine and a light packing of gauze to keep the wound open.

Ravenel in Osler's Modern Medicine advises as a caustic a 1 to 10 solution of chloride of lime.

If the lesion is small and well localized excision is advisable.

Chronic Glanders. Less hopeful than chronic farcy, indeed, almost hopeless is this condition in which the upper air-passages and the lungs are involved, coming on in the majority of cases as a result of the chronic farcy.

The treatment is, of course, the same, except that not as much can be expected from the vaccines and still less from the use of mallein.

The nose with its ulcerations, necrosis of bones and sinus involvement calls for local treatment, irrigations of saline solutions, boric acid or in the case of much necrotic tissue irrigations with permanganate of potash solution, of a deep claret color or applications of peroxide of hydrogen. Insufflations of iodoform have been suggested.

The annoying cough may be alleviated by inhalations of steam or medicated with compound tincture of benzoin, oil of pine, creosote, or eucalyptus. A teaspoonful or two may be added to the inhaler or a perforated zinc inhaler (Robinson's) may be worn over nose and mouth with some mixture as creosote, chloroform and alcohol equal parts, 10 to 15 drops on the sponge of the inhaler. Later codeine sulphate or phosphate in doses of gr. $1 / 8$ to gr. $1 / 2(0.008-0.030 \mathrm{Gm}$.) at two or three hour intervals may be demanded and still later and especially with the distressing dyspnœa morphine sulphate gr. 1/12 to gr. $1 / 8(0.005-0.008 \mathrm{Gm}$.) at four-hour intervals.

Acute Farcy. Here both local and general symptoms are so severe that life is despaired of. The picture is one of acute septic poisoning, and intense local infection, erysipelatous or forming ulcers, like gangrene and widespread pustular eruption.

Acute glanders presents the same picture as acute farcy with the additional distress attendant upon involvement of the air-passages.

Beside the supportive treatment and relief of symptoms, nothing can be added; for vaccines here can do no good.

Circulatory and respiratory needs are met by the use of caffeine, 
camphor, atropine, digitalis or strophanthin as in other infections, but are used rather with a feeling that an effort to do something is better than laissez aller; though from the patient's standpoint that is doubtful.

Doctor and nurse should remember that the discharges of the patient are infectious and take precautions accordingly, by burning all discharges and thoroughly sterilizing by heat or strong solutions of carbolic acid clothes and linen contaminated with them; by sterilizing the dishes and implements used by the patient. (See Typhoid Fever, Chap. XIV.) Gloves should be used in doing dressings and handling discharges.

Isolation. Acute cases should be carefully isolated and every warning given of the danger of too close contact even in the chronic cases.

Prophylaxis. As the disease is always conveyed by the horse, the appreciation of the disease in this animal is of first importance and a skillful veterinarian is often necessary to detect the latent cases.

Barnett Cohen ${ }^{1}$ advises as an efficient disinfectant of horse troughs the use of hypochlorite of Calcium (i. e., Chloride of Lime).

He maintains that two parts of available chlorine in' a million in the trough over night will disinfect even in the presence of much organic matter.

Infected animals should be killed and the stables carefully disinfected and the other horses tested from time to time to detect further infection.

Individuals handling horses known to be infected should use gloves and if inoculation is known to have occurred excision of the site is advised.

\section{SUMMARY}

\section{Isolation.}

Must be carefully obșerved.

\section{Doctors and nurses.}

Should wear gloves.

Avoid spray of cough.

Destroy all secretions by burning or disinfecting.

Disinfect all articles used about the patient.

(See Typhoid Fever, Chap. XIV.)

${ }_{1}$ Dissolve $0.3 \mathrm{Gm}$. of Chloride of Lime in some distilled water. Then filter and titrate this stock solution before using each time with standard sodium thiosulphate $\mathrm{N} / 10$ to determine the available chlorine: 


\section{Chronic farcy.}

Specific treatment.

Mallein. (See text.)

Vaccines.

Begin with $20,000,000$ and increase up to $150,000,000$ or $200,000,000$.

Give every four or five days.

Dosage and frequency governed by reaction.

Treatment of local lesions.

(Read text.)

Incise and evacuate.

Abscess.

Irrigate.

Apply liquified phenol (carbolic acid).

Apply alcohol to remove excess of phenol.

Pack wound with wet dressing of bichloride, or iodoform gauze.

Excise, small, well localized lesions.

\section{Chronic glanders.}

Specific treatment, as above.

\section{Care of nose.}

Irrigation with saline, 0.6 per cent. (3i to $0 \mathrm{i}, 4 \mathrm{Gm}$. to 500 c.c.) or 2 per cent. or 4 per cent. boric acid solution.

If much necrosis

Irrigate with permanganate of potash; make solution of rich claret color $(1: 5,000)$.

Apply peroxide of hydrogen.

Iodoform, insufflated has been recommended.

\section{Cough.}

Inhalations of steam, medicated with one of the following

Compound tincture of benzoin.

Oil of pine.

Creosote.

Oil of Eucalyptus.

A good formula is

$3 \mathrm{i}$ or ii (4-8 c.c.) on the water of an inhaler.

R

$\left.\begin{array}{l}\text { Alcohol, } \\ \text { Chloroform, } \\ \text { Creosote, }\end{array}\right\}$ equal parts.

S. gtt. $v-x$ on sponge of a Robinson's inhaler (perforated zinc inhaler for nose and mouth).

Codeine sulphate or phosphate, gr. 1/8 to gr. 1/2 (0.008-0.030 Gm.) every two or three hours.

Morphine sulphate, gr. 1/12 to gr. 1/8 (0.005-0.008 Gm.) every four hours. 
Acute glanders and

Acute farcy

Relief of symptoms, as above.

Circulatory stimulants.

Digitalis.

Strophanthin.

Caffeine.

Camphor.

(See Pneumonia, Chap. IX.)

Respiratory stimulants.

Caffeine.

Atropine.

Strychnine.

Prophylaxis.

Killing of infected horses.

Disinfecting stables.

Testing all animals in contact with sick horses.

Wearing gloves in handling sick horses.

Excise site of any inoculation.

Disinfection of horse troughs. (See text.) 


\section{CHAPTER XXXVII}

\section{FOOT AND MOUTH DISEASE}

\section{(APHTHAE EPIZOOTICAE)}

THIS is essentially a disease of animals, but may include man among its victims. It is extremely infectious.

Among animals cattle are the most susceptible; hogs, sheep and goats next; horses, cats and dogs occasionally; certain fowl rarely. Commonly severe among animals, it is relatively mild in man, though there are unfortunately exceptions to this rule.

The cause is as yet unknown, no organism proven definitely responsible for the disease having been isolated.

The virus has been shown to pass through a Berkfeld filter; hence, is to be classed as ultramicroscopic.

It occurs more frequently in Summer than in Winter.

The incubation period is put in Osler's Modern Medicine from two to ten days. It begins in man with a fever and other signs of toxemia. There may also be a gastro-intestinal catarrh, especially in children. The mucous membrane of the mouth is swollen, reddened and glazed. The palpebral conjunctivæ and mucous membrane of the genitals are also involved and in two or three days there appears the characteristic vesiculation in the mouth and between the fingers and toes and frequently on the dorsal surface of the hands and feet that gives the name to the disease. These vesicles contain clear serum, which later becomes cloudy and is extremely infectious. They heal without a scar. Salivation accompanies the vesiculation in the mouth. Immunity is said to be very short lived. Recurrences have been noted in 10 to 12 days.

Clough, whose article on this condition in the Johns Hopkins Bulletin of October, 1915, I especially recommend, epitomizes the disease as follows:- "A mild febrile infectious disease, characterized by the appearance of an erythema and a superficial vesicular eruption over the mucous membrane of the mouth and on the skin of the hands and feet; by salivation, by swelling, burning and paraesthesias of the affected parts, with subsequent desquamation; and by healing of the ulcers without scar formation."

With the appearance of the eruption the temperature declines.

Treatment. There is no specific remedy; so the treatment re- 
solves itself into efforts at relieving the effects of the toxemia and the ulcerations in the mouth and on the skin.

The same principles are applicable in this as in other. infectious diseases.

Rest in bed with the choice of a comfortable narrow bed and firm mattress and a room capable of thorough ventilation and accessible to the sun.

Isolation. The disease is contagious, so only those who minister to the needs of the patient should be admitted.

Physicians and nurses should use gloves in handling the patient, treating the ulcers and handling the secretions. These latter should be burned.

Articles coming in contact with the patient such as thermometers, dishes, bed-pans, urinals, bed clothing, should be handled as in other infectious diseases. (See Typhoid Fever, Chap. XIV.)

Diet. Feeding is difficult on account of the condition of the mouth; the ulcerations affecting it in particular and extending into the pharynx, œsophagus, the larynx and trachea. This, of course, makes chewing and swallowing very difficult.

The food should be liquid or of nearly fluid consistency and concentrated; milk, and milk fortified with milk sugar and cream, say an ounce of each to each glass of milk and soups made of milk and farinaceous flours or cereals, cereal jellies and eggs, may be taken as the basis of such a dietary.

It may be necessary to use the stomach tube or nasal tube or have recourse to rectal feeding, especially in children or illy nourished individuals. Water should be given freely. It must be determined that the milk does not come from infected sources and if there is an element of doubt it should be boiled.

Fever is rarely high or long continued. If any antipyretic measure is used at all, hydrotherapy in the shape of cold sponges offers the only legitimate one.

Daily sponge baths for cleanliness should always be given.

Circslation. Evidences of faltering circulation should be met by the same measures used in other infections. (See Pneumonia, Chap. IX.)

Care of Mouth. This is of the most vital importance for here a fatal sepsis may begin or pain and discomfort sacrifice nutrition to the danger point.

The best means of keeping the mouth in condition is by abundant irrigations of warm saline solution (see Scarlet Fever, Chap. XVII), or one may use boric acid 2 per cent. or quarter strength Dobell's 
solution. This should be done after each feeding and it were well to give a small irrigation before a feeding on account of the abundant secretions from the salivary glands gathering in the mouth and on account of the detritus from the ulcers. This is especially important in the morning.

Food must be removed from the dead spaces between the gums and teeth by swabs on wooden toothpicks or other applicators; one of the above solutions is used to moisten the sponges.

The best treatment for the ulcers is to touch each with silver nitrate stick or with small swabs wetted with 25 per cent. to 50 per cent. silver nitrate.

If the mouth is very foul one may irrigate with permanganate of potash solution made to a deep claret color (1:5000).

Chlorate of potash solutions may be used as mouth wash or gargle, 1 per cent. to 2 per cent., or if painful, of lesser strength. The nose, too, must receive similar attention.

In severe cases the palpebral conjunctivæ may be involved.

Eye washes of boric acid solutions 2 per cent. to 4 per cent. should be used and if there is much swelling and pain, cold compresses, using the same solutions, may be applied.

Vesicles and Ulcers of Skin. These are usually confined to fingers and toes and region of mouth and nose, but in some cases may be more generally distributed.

The ulcers are usually shallow. Drying powders, like sterile talcum powder, is best used on vesicles and ulcers or such a powder as aristol on the latter.

If there is annoying itching those measures recommended for the same condition in Varicella, Measles and Small Pox may be applied. (See Chaps. XXI, XIX, XXVII.)

It must be remembered that the contents of these blebs are contagious.

Gastro-enteric Symptoms. These at times may be severe with much colicky pain and diarrhea.

A thorough cleaning out with castor oil followed by small doses of the oil $\mathrm{m}$. $\mathrm{x}$ ( 0.65 c.c.) every two hours or by bismuth subnitrate 3 ss. $(2 \mathrm{Gm}$.) every two hours with local applications of heat to the abdomen may relieve the distress, or small doses of opium may be necessary, m. i ( 0.06 c.c.) of deodorized tincture, every two hours.

The duration is about two weeks, though it may last somewhat longer.

Death is not a common issue except among sickly children.

Prophylaxis. The disease is transmissible through the contents 
of the vesicles of diseased animals and through the saliva, also through the milk or the products of milk, e. g., butter, cheese and cream of diseased animals; hence, workers about animals and children drinking the milk are peculiarly prone to contract the disease, and, as might be expected, the mouth is first affected in those drinking the milk. Cattle affected should be killed and a thorough disinfection of the stable, etc., follow.

Suspected milk should not be drunk or should be sterilized and all suspected meat destroyed.

\section{SUMMARY}

\section{Treatment.}

Local, of ulcerations in mouth and skin.

General, to relieve effects of toxemia.

\section{Rest.}

Bed, hospital type preferred.

Room, well ventilated and lighted.

Quiet.

Isolation.

Physicians and nurses wear gloves in handling patient and secretions.

Treatment of objects in contact with patient and of secretions. (See Typhoid Fever, Chap. XIV.)

\section{Diet.}

Liquid or of fluid consistency.

Milk, which may be fortified with sugar or cream.

Soups of milk, cereals, farinaceous flours.

Cereal jellies.

Eggs.

Avoid milk from infected sources, or if in doubt boil it.

May have to use stomach or nasal tube or have recourse to rectal feeding.

Water, give freely.

\section{Bowels.}

Initial dose of castor oil $\overline{5}$ ss. to $\mathrm{i}(15-30$ c.c.) preferred if there is diarrhea, or a salt $§ \mathrm{ss} .-\mathrm{i}(15-30 \mathrm{Gm}$.).

\section{Fever.}

Rarely requires interference, or at the most cool sponges.

\section{Circulation.}

(See measures used in Pneumonia, Chap. XIV.) 
Care of mouth.

Abundant irrigations of warm saline (see Scarlet Fever, Chap. XVII) or of 2 per cent. boric acid or quarter strength Dobell's solution.

Well to precede feeding by a lesser irrigation; especially in the morning.

Swabs or toothpicks wetted with solutions mentioned to free dead spaces between cheeks and teeth and interstices of teeth from food.

Ulcers of mouth.

Touch with silver nitrate stick or 25 per cent. to 50 per cent. silver nitrate solution.

Chlorate of potash solution, 1 per cent. to 2 per cent., as a mouth wash.

If mouth is foul,

Permanganate solution of a rich claret color 1:5000.

Nose.

Cleansed with saline, boric acid or Dobell's solution.

Eyes.

Boric acid solutions 2 per cent. to 4 per cent. as eye-washes.

Cold compresses of boric acid solutions, 2 per cent. to 4 per cent.

Vesicles and ulcers of the skin.

Drying powders or aristol.

(For itching see Small Pox and Varicella, Measles, Chaps. XXVII, XXI, XIX.)

\section{Gastro-intestinal symptoms.}

Colicky pains.

Hot fomentations.

\section{Diarrhea.}

Preliminary dose of

Castor oil 5 ss. $-\mathrm{i}(15-30$ c.c.).

Follow by castor oil in small doses, m. $\mathrm{x}$ ( 0.65 c.c.), every two hours.

Bismuth subnitrate,

If much pain.

Opium, deodorized tincture, m. i (0.06 c.c.) every two hours.

\section{Prophylaxis.}

(See text.) 


\section{CHAPTER XXXVIII}

\section{PSITTACOSIS}

THIs is a disease incurred from parrots, resembling an atypical, pneumonia; atypical in its physical signs and in its course, and Osler says characterized by an onset like typhoid fever.

An organism belonging to the hog-cholera group was isolated by Nocard and shown by him to precede the disease in other parrots and other animals.

The incubation has been set as short as a week and as long as three weeks or more.

The diagnosis is made when a case and especially several cases occur in a family where there are sick parrots.

It is to be treated like any pneumonia, especially bronchopneumonia, and the same considerations of choice of room, bed, diet, air and attention to the circulation with relief of annoying symptoms obtain in the one as in the other. (See Pneumonia, Chap. IX.)

The disease in cases that get well runs from two to three weeks. The disease is a severe one as the mortality of 35 per cent. to 40 per cent. indicates.

Prophylaxis. Caution in handling sick parrots and disinfection of cages and utensils in contact with the bird.

Isolation of patient, destruction of secretions, especially bronchial secretions.

\section{SUMMARY}

Treat like a Pneumonia. (See Chap. IX.) 


\title{
CHAPTER XXXIX
}

\author{
RABIES
}

\section{(HYDROPHOBIA)}

THIs disease, relatively rare in the experience of the individual practitioner, when once encountered, leaves on his mind a more indelible stamp of horror than any other he will meet.

It is conveyed to man in the vast majority of instances through the bite of the dog. It is true that all mammals are susceptible to it and that here and there on the earth's surface other animals than the dog may play a leading rôle in its transmission, as the wolf in Russia. In this country as high as 4 per cent. has been attributed to the cat, while skunks and horses are to be remembered as sources of rabies.

The virus is transmitted by the saliva of the rabid animal and, like tetanus toxin, has an especial affinity for the nervous system, entering the nerve endings at the site of the wound, travelling by the nerve trunks to the spinal cord and by the cord to the medulla and brain, unless the wound be on the face, whence the route to the brain structures is more direct. In respect to its route and in respect to the variability of its incubation period which depends on the length of the route travelled to the central nervous system, it finds in tetanus toxin a close analogue.

The source of the virus is probably but not certainly known. Its incubation period and its multiplication in the animal body demands a living organism as its origin. The so-called Negri bodies, characteristically present in the nerve centres, and now recognized as the pathological criterion of the disease, are probably the infecting organisms. Competent authorities are inclined to place these bodies among the protozoa and, indeed, to fix them more definitely in suborders and families of this kingdom.

In the nervous system they are especially readily found in the cerebral and cerebellar cortex, in the hippocampus major (in the horn of Ammon) and in the corpus striatum (in 98 per cent. or more).

Less than 10 per cent. of men bitten by rabid dogs develop hydrophobia and yet every man developing the disease is doomed to die. What determines on the one hand this relative immunity and on the other the fatal virulency is one of several puzzling problems this disease 
has offered to the student of infections. The pathological studies of Paltauf have given reason to believe that the immunity of the 90 per cent. is not due to failure of infection, but to the operation of an unknown mechanism of defense in the central nervous system which is efficient before symptoms arise or fails utterly. (For résumé of these studies, see Editorial on Pathology of Rabies in the Journal of the American Medical Association, May 14, 1910.)

Incubation. As in tetanus the length of this period depends on the time taken to pass by the nerve from periphery to centre; hence, the length of the nerve trunk, or in other words, the site of the wound. For this reason the incubation period after bites on the face or head is much shorter than bites on the extremities. Moreover some other factor operates to delay the incubation in some cases, lengthening out to months and even a year or more.

It may be as short as fourteen days.

The average is set by some authors as forty days, but eight to twelve weeks is common enough.

When a man is bitten by a dog suspected of rabies our procedures are:

1. Immediate treatment of the wound.

2. Confirmation of the suspicion.

3. Preventive, antirabic or Pasteur treatment.

Treatment of the Wound. When there is the least suspicion of the dog the wound should be treated drastically by cauterization.

Lacerated bites are worse than others; bites on exposed surfaces more dangerous than those through clothes; for in these much saliva may be rubbed off the teeth; bites on the face or head are more to be feared than those on the extremity, especially, because of the shortness of the incubation period, and the virulency varies in different animals; wolves are the worst (infection as high as 60 per cent.), and cat's bites are said to be more virulent than those of dogs. No considerations, however, of "more" or "less" dangerous should modify the thoroughness of the local treatment.

The wound should be thoroughly laid open, especially punctured wounds, bleeding encouraged, or when seen immediately after the bite, may be sucked (a procedure not absolutely free from danger, but still a very slight risk).

The wound should be thoroughly washed and liberally bathed with an antiseptic like bichloride 1 to 1,000 , then wiped dry and cauterized.

There is a consensus of opinion that fuming nitric acid is the best caustic for this purpose. It may be applied on a glass rod or other suitable applicator and should search all parts of the wound thor- 
oughly. When the pain of the procedure, as in children, might lead one to sacrifice thoroughness, it is well to use a general anæsthetic or a local, as cocaine. After the application of the acid the wound may be washed with a physiological salt solution or a saturated solution of bicarbonate of soda and with alcohol. Park says that wounds so treated by the acid, contrary to general opinion, heal well and with little scarring. There is experimental evidence to show that this caustic lessens the incidence of rabies after infection.

Other caustics, inferior, but still of value, when nitric acid cannot be had are phenol (carbolic acid), and tincture of iodine.

Phenol should be used full strength (95 per cent.) and as thoroughly as the nitric acid.

After the cauterization, the excess should be removed by swabbing with absolute alcohol. Bloodgood and McGlannan (Musser and Kelly, Practical Treatment) use first the carbolic, follow it by the fuming nitric acid and then wash the wound with a saturated solution of bicarbonate of soda.

Of the two other caustics mentioned my preference would be for the tincture of iodine.

Cumming (Journal American Medical Association, May 18, 1912) prefers 5 per cent. formaldehyde applied to the wound twelve hours to either phenol or nitric acid. The actual cautery has also been used. Nitrate of silver is useless.

After the cauterization a dry dressing is applied. If the wound suppurates it is to be treated as from any other cause, kept open and dressed.

The sooner the wound is treated the more efficient will be the procedure; this does not mean, however, that the wound should not be so treated, if seen later. In fact, it should be the routine, scabs on older bites should be removed, the wound cleansed and cauterized as above. Rosenau, calling attention to the length of time the virus may remain latent in the wound, suggests, with reason, the excision of the scab and treatment of the wound as an open one.

In fresh, wounds on the extremity a tourniquet may be advisable until the wound can be cauterized.

If the wound is sucked, the mouth should be carefully and thoroughly rinsed with antiseptic solutions.

Confirmation of the Diagnosis. If the animal manifest the symptoms of rabies, the head should be sent to the laboratory for pathological examination and the preventive treatment be begun at once, because a failure of pathological diagnosis should not controvert a clinical diagnosis where the price of an error would be so terrible. 
When the animal has disappeared and cannot be found, an unwarranted attack by the beast should indicate the treatment, while, of course, a provocation of an animal apparently of a normal behavior weighs against the advisability of the measure.

Where any doubt exists, however, the patient should receive treatment.

When the animal is secured and shows no evidences of rabies he should be confined for at least three weeks. But if rabies has occurred recently in the locality, the treatment should be given in the meantime.

If no symptoms develop in this time the danger may be considered past and the Pasteur Treatment unnecessary (Rosenau, Preventive Medicine and Hygiene).

Of course, if symptoms of rabies develop at any time during this period of observation treatment should be begun at once.

When the wound is on the extremity in an adult the prolonged period of incubation furnishes enough time to establish immunity by the treatment, but when the wound is in a child and more especially on the face or upper extremity the shortened period of incubation makes every day's loss during this term of observation a source of increasing anxiety, but if symptoms in the dog do appear, it will be too late to establish immunity.

Animals in the early stages of rabies are prone to lick their master's hand or face and while the probability of infection by these means are not comparable to that following a bite, the mere fact that Park (Forchheimer, Therapeusis of Internal Diseases) can cite at least eight fatal cases to his knowledge following this mode of infection makes his advice to take the treatment almost an imperative command.

Shooting of a dog before symptoms are obvious defeats the diagnosis, pathological as well as clinical.

Symptoms of Rabies in the Dog. The earliest evidences of the disease are perhaps manifested in a change of disposition of the dog; he is unlike himself, secretive, hiding himself, irritable, uneasy and restless, abstracted, destructive of objects, apt to run away from home and later bites without provocation, a striking symptom in a gentle dog. A change in the timbre of the voice is very significant. In two to four days the furious delirium in which the dog attacks man and animals and objects, followed by paralysis, occurs, or the paralytic stage ensues without that of furious delirium (dumb rabies).

Unlike the human being the rabid dog has no fear of water. The dog dies in a week or less from the first appearance of symptoms.

Two popular beliefs are to be combated; first, that a rabid animal 
always froths at the mouth, and second, that rabies is confined to the hot "dog days" of summer.

Preventive Treatment. Had Pasteur contributed nothing more to human welfare, his work on rabies alone would have raised for him a sign to all time ten-thousand fold better than a "monumentum aere perennius."

The essence of his discovery was that the virus of rabies, localizing in the nervous system could, under certain conditions and environments, be so modified in its virulency as to be used in other animals to provoke the mechanism of defense to the active production of immune bodies; in other words, to establish immunity; and that, too, with an expedition that forestalled the multiplication of the virus already introduced to a degree dangerous to the patient.

Improvements in technique have been engrafted on his treatment, but it fundamentally remains the same to-day as when he devised it.

Pasteur first used the virus attached to the nerve tissue of rabid dogs brought in from the street and to this kind of virus has been given the designation of "street" virus. He soon found that the virulency of this "street" virus was variable and that rabbits injected with it showed an incubation period of fourteen to twenty-one days. In his effort to overcome this variability he found that running it through rabbits, that is, dog to rabbit and then rabbit to rabbit, it became progressively more virulent as measured by the shortening incubation period up to a fixed period beyond which he could not go, of six days.

This virus he called "fixed" virus and found constant in its potency and in its effects for his purpose.

One other mysterious change was wrought in this virus in the process of fixing. It was found to lose virulency in the dog becoming even avirulent in man while it was increasing in virulency in the rabbit. Adequate explanations for these facts are not yet forthcoming and, yet, on it depends its efficacy in treatment.

His next step was to obtain this virus in varying but definite degrees of virulency. This was accomplished by taking the spinal cord (as more easily manipulated than the brain) of a rabbit dead of the effects of the "fixed" virus and drying it over potassium hydroxide in a bottle at $70^{\circ} \mathrm{F}$. kept in the dark. Day by day as the cord desiccates it loses its virulency until the fourteenth day when it is no longer capable of inducing infection. For a brief résumé of the technique of preparation of the virus used by the New York Board of Health, see Park in Forchheimer's Therapeusis of Internal Diseases.

Portions of the cords dried longest are ground into an emulsion 
in physiological salt solution and injected under the skin of the abdominal wall. Each day this injection is repeated, using portions of cords dried a lesser period, i. e., of increasing virulency (for.the rabbit) until the stronger cords are used.

Slight modifications of the original Pasteur scheme of inoculations are adopted by different institutes and laboratories.

The suitable scheme for the virus used is furnished the practitioner by the institution supplying the virus, when it is necessary for him to administer treatment.

The duration of the treatment is from fifteen to twenty-six days, which fortunately is short of the usual incubation period, in most cases, although full immunity does not obtain until four or five weeks are past. Bites about the head and especially in children may, however, be followed by so short an incubation period or the patient's treatment may be begun so late after the bite that immunization cannot be achieved by the original Pasteur scheme. Shorter incubation periods, too, follow the bites of wolves and cats. It becomes a great desideratum, then, to hasten the process of immunization.

It is the custom, therefore, in case of bites about the head to treat the patient morming and evening for the first two days, or to make the treatment more intensive by using the stronger cords earlier.

More recent work, however, has given promise of a quicker method of immunization by the use, from the first of unchanged, fresh virus. Proescher of Pittsburg has been a pioneer in this work in this country. (For details, see Archives of Internal Medicine, September 15, 1911.) He uses the "fixed" virus in the brains of rabbits. Three c.c. of an emulsion in physiological salt solution containing 0.01 gram of "virus fixe" is injected subcutaneously in the abdominal wall and 0.05 grams used in the course of the whole treatment of one injection each day for five days.

If immunity is really established in this time as from his results Proescher believes it to be, certainly a great stride has been made towards the perfection of the procedure of immunization.

When a State or Municipal Laboratory or Institute is at hand, patients should be treated there preferably; but as these are accessible to the relatively few the

Treatment at a distance from the laboratory becomes of great importance.

This is effected by sending pieces of desiccated cord with instructions for the emulsification or the emulsion is sent in a preservative of carbolic or glycerin or is supplied by manufacturers in syringes ready for use, as diphtheria antitoxin has long been put up. 
The U. S. Public Health and Marine Hospital Service have been accustomed to furnish virus to State Boards of Health to be used under their direction and have found the results perfectly satisfactory. More recently commercial houses have facilitated the use of rabies vaccine by despatching appropriate doses with specific directions to the family physician.

The treatment requires no special confinement, but the patient should avoid fatigue or excesses of any kind. Very little or any disturbance occurs as a result of the treatment in the vast majority other than those that may naturally be attributed to the concern of the patient as to the outcome of his infection, mild rashes or slight anaphylactic manifestations.

In rare instances more serious results ensue in an affection of the nervous system, causing neuralgia, paræsthesia and even paralysis, paraplegias, ascending paralysis and death. The fatal issue is extremely rare. Whether this is due to the virus itself or is an anaphylactic phenomenon is not settled.

They occur late in or following upon the treatment. They last a few days to several weeks. Treatment is symptomatic.

There are no contraindications to the treatment.

It is remarkable, as has been commented, that in the 100,000 cases in which this living virus from the rabbit has been used, it has induced rabies in no instance.

Just how long the immunity so established lasts is not determined but probably for some years; however, a patient bitten again by a mad dog should certainly repeat the process of immunization unless the second bite follows close upon the first.

Results of Preventive Treatment. It is hard to confine oneself to the bare statement of facts, when so much that is dramatic invites to an ebulition of enthusiasm. Granted that 10 per cent. of the bites from rabid dogs resulted in rabies. All these cases meant death, without exception.

What the preventive treatment has done is best shown by statistics; 30,000 cases treated by the Pasteur Institute in Paris gave a mortality of 0.5 per cent. to 1 per cent.

Park's statistics of 2405 cases treated by the New York Board of Health virus showed a mortality of 4 per cent. among those bitten on the head and 3 per cent. among those bitten elsewhere; but his statistics further show that if time for the full development of immunity passes, that is two weeks beyond the full course of treatment, the mortality sinks to 0.3 per cent. in the first and 0.2 per cent. in the latter class of cases. 
Symptoms of Hydrophobia. They have been divided into three stages:

1. Premonitory stage, characterized by malaise, irritability, depression, anxiety, change in disposition, difficulty in taking water, hoarseness, some nervous twitchings or rigors, lasts a day or two, at times longer.

2. Stage of excitement. Great thirst and spasms of muscles of deglutition, the real hydrophobia, intense hyperæsthesia that dreads even the breath of bystanders on the skin, convulsions and delusions.

3. Paralytic stage. Paralysis, coma and death.

The paralytic stage may come early and dominate the picture.

Treatment of the Developed Disease. I know of no more distressing duty that falls to the lot of a practitioner of medicine than this nor any that has left the indelible impression on my own mind that this sad experience has impressed.

To my mind it has only one object, to lessen the patient's sufferings and smooth the way to the end.

Another peculiarity that marks the disease as different from others is that there is no partial immunity established; it is all or none. Its onset spells death and the dubious rare report of a recovery (undoubtedly most often a lyssophobia or nervous symptom complex precipitated by fear) gives no warrant to exhaust those measures designed to prolong life to the provocation of renewed agonies and to add hours of suffering without result.

The room should be darkened and quiet, and all noise, talking, unnecessary handling rigorously excluded.

I have heard the patient plead to avoid the draught from an open door, stuff the chinks about the window, stand further away and turn the head while speaking to avoid the air.

Only those who can be of help should be present. What little food can be taken should be concentrated and suggest fluid as little as possible, such as ice cream, custard, or junket. The bed should be of the hospital type to facilitate handling.

- Enemtaa seem to be the only humane way to relieve the intense thirst.

In the early stages of nervous irritability and hyperæsthesia large doses of chloral and bromides by the rectum may cause some sedative effect. The doses should be liberal gr. xxx $(2 \mathrm{Gm}$.) of chloral and $3 \mathrm{i}$ $(4 \mathrm{Gm}$.) of bromide or even larger doses of both in the adult.

When the convulsive paroxysms come on, only chloroform by inhalation is of use, while morphine hypodermatically may lessen their frequency and the patient's suffering and horror in some measure. 
The amounts of these drugs that shall be given cannot be accurately stated; they must be given in large doses, large enough to accomplish the end desired. Without these two agents to apply, the sufferings of those who are there to minister would be second only to those of the patient.

Other drugs that have been recommended are hyoscine hydrobromide gr. 1/100 and cocaine applied to the larynx.

Attendants, while in no great danger, still should remember that the patient may be a source of infection through his saliva and should take precaution to protect themselves by the wearing of gloves, cauterization of superficial wounds contaminated and restraint of patient during his maniacal periods. One can conceive the necessity of immunization of an attendant accidentally wounded by a patient in delirium.

It would seen logical to use the serum of an immunized animal during the attack, but its use has proven in every instance useless.

Prophylaxis. That such a disease, when preventable, should exist at all within the borders of a State would seem to be a distinct crime against its members. That it does exist in civilized communities is largely due to the difficulty of grasping the just relationship between communal and individual rights.

As the disease is nearly always conveyed to man by the dog, stamping the disease out among dogs practically excludes it in a community. The means to accomplish it are so simple, the inconvenience so slight compared with the results, that it seems amazing that enough opposition should be found to defeat legislative efforts to that end, and yet such is the fact.

Among the measures advised to accomplish this end are

Quarantine. In Australia, where rabies is unknown, the fact is attributed to a law making a quarantine for six months for all dogs brought into the country imperative.

Muzzling. Great Britain has succeeded in eliminating rabies from its confines by an efficient muzzling law.

This can be effected by muzzling all dogs at large for at least six months after the last case of rabies in the community or its immediate environments.

Less drastic and less useful is the use of the leash.

Detention. Valuable but less thorough, is the detention, under observation for three or six months of dogs thought to have been in contact with rabid dogs.

Licensing. A law to license all dogs with destruetion of the unlicensed, lessening the number of dogs by high tax and primitive meas- 
ures, such as holding the owner responsible for damages done by the rabid animal. These latter measures but provoke resentment.

What is needed is a campaign of education in the problem to lay the path to legislation for quarantine or muzzling with compulsory reporting of cases and killing of all animals bitten by rabid dogs.

Rabies in This Country. That the laws against rabies is inefficient is shown by the increase in numbers and distribution of cases. Of course, the States as independent legislative units, have met the problem differently and more or less satisfactorily.

In 1908 Kerr and Stimson, under the U. S. Public Health and Marine Hospital Service, collected 1500 cases who applied for Pasteur treatment that year from thirty States, and 534 infected localities.

There were 111 deaths.

All were from the Eastern three-fourths of the country, none in the Rocky Mountains and Pacific Slope.

Three years later, 1911, Albert reported 4625 persons receiving treatment from 1381 infected localities.

Moreover his report showed the invasion of the Western quarter of the country, where in some localities it was believed the spread was due to skunks.

Finally the incidence of cases by States was determined by the more or less efficient measures adopted for the regulation or stamping out of the disease.

\section{SUMMARY}

When a man is bitten by an animal suspected of rabies.

1. Treat the wound.

2. Confirm the suspicion.

3. Institute antirabic or Pasteur treatment.

Treatment of the wound.

Lay wound open thoroughly.

Encourage bleeding.

Wash thoroughly.

Bathe in antiseptic, e. g., 1-1000 bichloride.

Wipe dry.

Cauterize.

Best caustic is fuming nitric acid; apply on a glass rod or other suitable applicator, searching every part of the wound.

If pain prevents thoroughness, give an anesthetic, general or local.

Then wash the wound with physiological salt solution, $3 \mathrm{i}-0 \mathrm{i}$ (4 Gm.500 c.c.), or with a saturated solution of bicarbonate of soda and with alcohol.

Phenol, 95 per cent., or tincture of iodine may be used as caustic in same manner as nitric acid, but is not so good. 
When phenol is used remove the excess of carbolic acid by swabbing with absolute alcohol.

Method of Bloodgood and McGlannan.

Use first phenol, 95 per cent., then fuming nitric acid, then wash wound with saturated solution of bicarbonate of soda.

Five per cent. formaldehyde applied twelve hours (Cumming).

Actual cautery.

Apply dry dressing.

Do not use silver nitrate for cauterizing.

Suppurating wounds dressed like those from other causes.

Keep open and drain.

\section{Old bites.}

Remove scabs.

Clean wound.

Cauterize as above.

Excise area of scab.

Treat as open wound.

In wound of extremity, if fresh, a tourniquet may be applied until the wound can be cauterized.

Confirm suspicion. (See text.)

Prevention. Antirabic or Pasteur Treatment. (See text.)

Treatment of developed case.

Fatal issue is certain; hence, treatment is palliative to lessen suffering and smooth the way to the end.

\section{Room.}

Must be quiet, avoiding even talking and all unnecessary handling.

Avoid all draughts of air; may be necessary to stuff chinks in windows.

Diet.

Concentrated and suggest fluid as little as possible, e. g., ice-cream, custard, junket.

Thirst is intense.

Can only be relieved by enemata or Murphy drip method.

To lessen nerve irritability.

Early stages.

Chloral and bromides, large doses.

Chloral, gr. $\mathrm{xxx}$ to gr. $\mathrm{lx}(2-4 \mathrm{Gm}$.) by rectum (adult).

Bromides.

Combine with chloral in double the dose of the chloral. Give by rectum in warm milk 5 ii-iii (60-90 c.c.).

\section{Convulsions.}

Chloroform inhalations.

Morphine hypodermically. 
Amounts must be sufficient to accomplish desired results. Cocaine to larynx.

Hyoscine hydrobromide, gr. 1/100.

Maniacal periods.

Restraint.

Attendants must remember that patient's saliva is infectious.

Should wear glasses.

Should cauterize superficial wounds contaminated.

Should undergo preventive treatment if wounded by patient.

Prophylaxis.

Enlightened laws and Health Board regulations.

Quarantine.

Six months for all dogs brought into country (Australia).

Muzzling.

All dogs muzzled for six months after a case of rabies (Great Britain). Less drastic and less effectual.

Use of leash.

Detention for three to six months of dogs in contact with rabid dogs.

Destruction of unlicensed dogs.

Public education. 


\section{CHAPTER XL}

\section{TETANUS}

Tetanus is a disease due to the action of an organism first described by Nicolaier in 1884 and named Bacillus tetani. The symptoms of the disease are effected by the operation of a toxin elaborated by the organism, carried to a distance from the site of infection, entering into and acting upon nerve tissue for which it has a striking affinity. In this the Bacillus tetani is like the Bacillus diphtheriæ.

The toxin affects the motor end plates of the nerves, travels by way of the axis cylinders to the spinal cord and motor nuclei at the base of the brain and arriving here, and only when arriving here, induces by its irritating effects on these centres the characteristic symptoms of the disease.

If the infecting wound brings the toxin at once into intimate contact with motor end plates as a wound of a muscle of the leg, the absorption of the nerve supplying that muscle will be facilitated and evidences of the disease will be manifested in this extremity first; hence, is called "local tetanus" and as the other nerves more remote from the site of the wound are affected the symptoms seem to advance up the body; hence, it is called tetanus ascendens, but if the wound is in the subcutaneous tissue, as it usually is in man, as Ashurst and John explain, the toxins, diffused by way of the lymph channels, act on all motor end plates practically simultaneously and those centres sending out the shorter nerves would actually be reached earliest; hence symptoms would appear in the territory of the short facial nerves with early trismus and in the longer nerves later, hence, Tetanus descendens.

The toxin in all probability also spreads along the cord when carried there by way of the nerve trunks or by way of the general circulation.

The sensory as well as the motor side of the cord is affected and the irritability of the reflexes heightened.

Incubation. After the infection some little time must elapse between the invasion of the tissue by the bacillus and the arrival of the toxins at the centres. This will depend on the activity of the germ in the wound and the facility of transportation for the toxins; for example, the length of the nerve trunk travelled.

The average time is seven to nine days. Under nine days cases are called acute, over nine days chronic. Spores may lie for some time in 
the tissues before they mature and thus the incubation period be lengthened to weeks or even months.

It is believed that the invasion by pyogenic organisms of the tissues in which the spores lie hastens the maturation of the spores.

Prophylactic injections of antitoxin, if it does not entirely prevent the development of the disease, materially lengthens the incubation period.

The symptoms of this disease, then, are attributable to the heightened and uncontrolled operation of the motor centres under the whip of the toxins, and show first and most marked in the territory of those nerve centres first and most affected.

The prodomata occur a day or two after infection and are characterized by restlessness, sleeplessness, sometimes headache, giddiness and excessive yawning and there may be difficulty in urination due to spasm of the sphincter muscle of the bladder.

Then follows trismus (lockjaw) and spasms of the facial muscles giving rise to the so-called risus sardonicus. Stiffness of muscles of neck with retraction; of the muscles of the back with opisthotonus; of the abdomen; curiously enough the muscles of the arms often escape.

There may be dyspnœa and cyanosis due to the spasm of the diaphragm and respiratory muscles.

These contractions are tonic and continuous, interrupted by clonic convulsions, often severe and painful, which are induced by slight irritations.

Moderate fever obtains, sometimes becoming hyperpyrexia, especially, near the end.

Sometimes the disease is restricted to that part of the cord supplying the part wounded. It may show itself as trismus, rigidity or spasm of a limb and not infrequently pain about the wound or there may be spasm of the throat muscle. Such localized tetanus may be seen in those partially protected by prophylactic injections.

Treatment of the Developed Disease. It is to be regretted that the use of "specifics" has too often invited the thoughtless to an undue reliance on them and to a failure of consideration for equally important measures.

Certain measures aiming at the conservation of the patient's energies in his struggle to subdue the infection such as bodily and mental rest, the meeting of energy demands through food intake, and careful nursing are such important measures.

Room. A well ventilated room as remote as possible from the noises of the street or the rest of the household should be chosen; this should be somewhat darkened. The exquisite sensitiveness of the 
sensory-motor apparatus has to be kept in mind and the ease with which slight stimulation of the same precipitates the distressing clonic spasms; so, sudden noises, such as loud talking or exclamations, jarring of doors, windows, or heavy tread, moving of furniture, are to be carefully avoided. Cotton may be put in the ears.

The bed is best of the hospital type, that is one about which the nurse can perform her duties to the patient with the least disturbance to him.

A water bed may prove a source of comfort. Skilful nursing is required to subserve the needs of the body without exciting spasms. Baths should be given by sponges and the water be lukewarm as least likely to provoke a shock and a resultant convulsion.

Diet. Feeding is a difficult task on account of the locking of the jaws and the spasms of muscles the effort excites.

It is obvious that all food must be liquid and as concentrated as possible, as the repetition of the act of feeding is distressing and the danger of aspiration and ensuing pneumonia is always present.

Milk, cereal-waters, eggs raw, all fortified with sugar to increase the caloric value are suitable.

So difficult is oral feeding, that rectal feeding and nasal feeding are sooner or later necessary as adjuvants to or substitutes for oral feeding, and even efforts at subcutaneous feeding have been made. As a nutrient enema Bloodgood advises 200 c.c. of peptonized milk, two eggs and enough salt solution, physiological (roughly, $3 \mathrm{i}$ to $0 \mathrm{i}$ ) to make up to 500 c.c. and administer by the drop method of Murphy. (See Pneumonia, Chap. IX.)

One may give the milk without peptonization and fortify with sugar, adding salt $3 \mathrm{i}$ to the pint $(4 \mathrm{Gm}$. to 500 c.c.) and brandy or whiskey. For example 500 c.c. milk, sugar 45 grams and whiskey or brandy 30 c.c. would furnish $600-650$ calories. If slowly introduced the bowel may be made to retain this amount and in this the spasm of the sphincter may assist. Nasal feeding is done by passing a small flexible rubber tube through the nostrils into the stomach.

To lessen or overcome the spasm that blocks the passage of the tube morphine in doses of the sulphate gr. $1 / 8$ to $\mathrm{gr} .1 / 4(0.008-0.015 \mathrm{Gm}$.) hypodermically may be given beforehand or chloroform may be used during the procedure.

While little reliance can be put on subcutaneous feeding, olive oil has been used and solutions of glucose, 5 per cent. to 10 per cent.

Park mentions the use of normal horse serum, saying that 500-1,000 c.c. can be given. The tissues are in much need of water, which can be supplied best perhaps as a saline solution by the drop method. A pint 
(500 c.c.) may be given four times a day: Fluid can also be given subcutaneously, but with the extreme irritability of the nerve structures it is not a good method in this disease.

Bowels. When seen early a saline can and should be given; later one has recourse to enemata.

Obstinate constipation usually obtains after the disease has once begun.

Urine. Watchfulness to detect the retention of urine that may occur is necessary and its retention entails the use of the catheter.

The second consideration is the elimination of the focus of infection so far as possible. This is accomplished by rigorous local treatment. The wound should be treated on surgical principles, laid wide open, sloughing material removed, packed and allowed to heal by granulation. Ashurst and John advise swabbing the wound with 3 per cent. alcoholic solution of iodine, followed by hydrogen peroxide and packing with gauze saturated with the iodine solution. They say that if amputation becomes necessary the wound of the stump should be left open and treated in the same way. It has been advised to excise the glands related to the lymphatic system draining the affected area if they seem in any way involved. Cauterization is decried as favoring the growth of the anaërobic tetanus bacilli.

Some observers advocate the local administration of dry tetanus antitoxin to the wound after it has been surgically treated and this has been especially advocated in tetanus neonatorum in which the infection comes through the umbilicus.

The antitoxin which will be given at once as a prophylactic measure should be introduced into the tissues around the wound so as to saturate these parts with the antitoxin.

Specific Treatment. The third measure relates to a neutralization of the toxins of the disease by antitoxin. Unfortunately the combination of toxin with tissue has been so completely effected before treatment is begun-for the symptoms of the disease do not appear until this combination takes place-and furthermore the combination is so stable that results comparable to the use of the analogous antitoxin in diphtheria are not obtained. It is the fact, however, that some taxin is still circulating free in the tissues to add its further poisonous action to the cells that makes the use of the antitoxin imperative, for there is evidence that time is required after the toxin has reached the nerve tissue for the union to become stable, and during this period antitoxin if given in sufficient quantity may attract the toxin out of the nerve substance into a union with itself. 
The Antitoxin. The antitoxin is elaborated in exactly the same way as diphtheria antitoxin in the horse, whose serum becomes the vehicle of its administration.

The unit adopted in this country is about ten times the size of the diphtheria antitoxin unit. (See Diphtheria, Chap. XVIII.)

Use of Antitoxin. Our efforts are aimed at getting the antitoxin in contact with the toxins both while circulating free and when in loose combination with nerve tissue. These efforts are effected by giving the antitoxin into the subcutaneous tissue and into the vein to secure the first result and into the cord, into the nerve trunk, into the brain and into the muscle about the end plates to secure the second result. Of these routes the intravenous, the intra-spinal and intra-neural are the important.

It cannot be too emphatically insisted that if results are to be obtained from the use of antitoxin it should be administered as soon as possible after symptoms are evident.

Subcutaneously. So readily is this procedure carried out in comparison with the others that I feel it incumbent on me to emphasize the teaching of the able workers in this field relative to the efficiency of the specific when so administered. Park in his article in Forchheimer's Therapeusis in Internal Diseases says: "A subcutaneous injection is not wholly absorbed for three days. The water holding the antitoxin in solution is quickly absorbed, but the antitoxin is held back. At the end of six hours only 10 per cent., twenty-four hours 35 per cent., forty-eight hours 65 per cent., and at seventy-two hours and ninety-six hours 100 per cent."

In a study on man injected subcutaneously as when given under the skin with 10,000 units Park found the highest antitoxin content of the blood at the end of forty-eight and seventy-two hours amounting to 1 unit per c.c.

He goes on to say that when given into a muscle the absorption is three times as fast, being accomplished in twenty-four hours but even here the absorption is uncertain and not comparable with the intravenous method.

Dosage. It can readily be seen that because of the slowness of absorption and its great dilution in the blood, lymph and tissue juices, little can be expected of it unless administered in massive doses. These doses are limited only by the expense entailed in their purchase. Over 100,000 units in six hours have been advised, 15,000 and more every three hours and in one case actually 224,000 units were given in three days, happily with recovery.

It can only be said that this route should never be preferred to the 
vein if it is possible to get into the latter; if not, the muscle should be preferred to the subcutaneous tissue.

Intra-muscular. As has been said, this route is better than the subcutaneous, but, never should be chosen over the vein. Its use in the muscle about the site of the wound to attack the toxin at the end plates has another purpose.

Intravenous Injections. This method assures rapid contact of antitoxin with such toxins as are still circulating free in the fluids of the body, while the antitoxin content of the blood is more than twice that obtainable by subcutaneous injection.

Dosage. Even by this method the dose should be large and it must be remembered that the efficacy of a given dose is multiplied many fold by being administered early. At the first suspicion of the condition the serum should be administered.

The dose recommended is 10,000 to 15,000 units once or twice in twenty-four hours, and this large dose because, as Park says, though it is many times more than enough to neutralize the toxins present in the blood, the antitoxin experiences difficulties in passing from the blood to the cellular fluids, "the tissue fluids only contain about 3 per cent. of the antitoxic content of the blood." (Park.)

It has been advised to give one large intravenous dose to start with and smaller subcutaneous doses every day or two until symptoms cease or to give lesser doses by the vein every day. I should feel that in so dire a condition it were wise to lean towards the larger doses and shorter intervals, when the expense does not forbid, always preferring the venous route.

Small children may be given one-half the dose and infants one-quarter. Park recommends as "a good working rule," 2,000 units for every ten pounds of patient.

In very young children and infants where the intravenous route is impossible, the intramuscular route is the necessary method.

Perhaps if only one method of administration could be used the intravenous would prove the best, and in practice it should be given preference as a means of getting antitoxin into the body fluids, while as important adjuvants to its operation methods of bringing the antitoxin into direct contact with nerve tissue should be sought.

These methods are the intra-spinal, the intra-neural, the intracerebral and the intra-muscular at the seat of the wound.

Intra-spinal. This is called the best method by Park and with this I heartily concur. It is conceived that the serum so given will come into more immediate contact with spinal centres and that the more likely if the cord or cauda is wounded by the needle. It 
has been thought, too, to diffuse readily into the blood stream from the cerebro-spinal fluids.

The dose should be 3,000 to 5,000 to 10,000 units ( 6 to 20 c.c.) each day until improvement sets in. For table of amounts to be injected, consult table of Sophian. (See Cerebro-spinal Meningitis, Chap. XXV.)

The technique is that of the administration of cerebro-spinal meningitis serum. (See Cerebro-spinal Meningitis, Chap. XXV.) One should combine the intravenous and intra-muscular method with it.

Intra-neural. Of late an especial plea has been made for intraneural injections. It is claimed on the basis of experimentation that antitoxin injected into the nerve travels like toxin by way of the nerve to its centres to operate on the toxins there. The large trunks leading from the wounded area are selected and injected at a site as near the cord as is feasible to expose them. The sciatic, the anterior crural, the obturator and cauda equina have been injected in the lower half of the body, while the strands of the cervical plexus, the hypoglossal and spinal accessory are legitimate points of attack in the upper half.

Ashurst and Jones have put as much as 1,500 units into the sciatic nerve and 750 into the anterior crural.

If injected slowly much is absorbed into the nerve trunk.

The disadvantage is the nicety of surgical procedure demanded. It must be remembered that this is only an adjuvant method and that the other nerves have to be reached by other methods.

This method was not adopted in the work in the late war.

Intra-cerebral. This method was early tried, but the results seem to be so little encouraging and the dangers and sequelæ of the procedure so considerable that I am inclined to advise against it.

Intra-muscular. Injections of antitoxin may be made about the wound, into the muscles to bring the serum in contact with the motor end plates to be first affected.

The instructions of the Board of Health of New York City are to give 3,000 to 5,000 units of the antitoxin intraspinally by the gravity method. This may be diluted with sterile saline making the total volume to at least 5 c.c. An intravenous injection of 10,000 units should be given at the same time to render the blood antitoxic for four or five days. At the end of 24 and 48 hours, repeat the intraspinal injection. At the end of 72 hours (4th day) give a subcutaneous dose of 5000 units.

They furthermore advise that if the intraspinal dose cannot for any reason be given increase the dose to 15,000 to 20,000 intravenously. If neither of these methods is possible give at once an intramuscular dose of 20,000 units or more.

The experience in the late war has afforded an unparalleled opportu- 
nity for the study of this disease and of the efficacy of antitoxin in its treatment. The following table, taken from Fitzgerald's article in Nelson's Loose Leaf Living Medicine, is an outline of the treatment suggested by the Tetanus Committee of the War Office:

\begin{tabular}{c|c|c|c}
\hline Day & Subcutaneous & $\begin{array}{c}\text { Intramus- } \\
\text { cular }\end{array}$ & $\begin{array}{c}\text { Intraspinal } \\
\text { (intrathecal) }\end{array}$ \\
\hline 1st day & $\ldots \ldots \ldots \ldots$ & 8,000 & 16,000 \\
2d day & $\ldots \ldots \ldots \ldots$ & 8,000 & 16,000 \\
3d day & $\ldots \ldots \ldots \ldots$ & 4,000 & $\ldots \ldots$ \\
4th day & $2,000-4,000$ & 4,000 & $\ldots \ldots$ \\
5th day & $2,000-4,000$ & $\ldots \ldots$ & $\ldots \ldots$ \\
7th day & $2,000-4,000$ & $\ldots \ldots$ & $\ldots \ldots$ \\
\hline
\end{tabular}

To get the best results this should be supplemented by intravenous injections in large doses and the rule proposed by Park is an excellent one to follow; i. e., 2,000 units per ten pounds of patient. This will guarantee a high antitoxic content in the blood for four or five days.

Local Tetanus. In local tetanus, antitoxin is used by the intramuscular and subcutaneous methods and need not be given intraspinally.

Drug Treatment of the Disease. All such treatment is empirical, having the weakest kind of basis.

Such, for example, is the use of Phenol (Carbolic Acid).

This method was introduced by the Italian, Bacelli, and its usage has followed his recommendations with slight modifications as to dosage and concentration. He gave 0.30-0.50 grams (gr. v-viiss.) a day. It may be given in 1/2,1,2 or 3 per cent. watery solution and has been used in oil 5 per cent.

It is given hypodermically and at frequent intervals hourly or every two hours. An injection of $\mathrm{m}$. $\mathrm{xx}$ of a 2 per cent. solution at two-hour intervals would give about 5 grains a day; to increase the dose one may make the intervals shorter or the solution stronger, as 3 per cent., which in same amounts and intervals gives about 7 grains.

One should remember that if antitoxin is given at the same time it contains as a preservative 5 per cent. trikresol probably operative in the same direction. Evidences of poisoning by inspection of the urine (smoky urine) should be sought for, but the body seems to be peculiarly tolerant in tetanus.

The statistics seem too good to be true and especially in the absence of experimental corroboration.

When, however, antitoxin is not at hand or in insufficient quantity, phenol should be used. 


\section{DRUGS IN THE TREATMENT OF THE SYMPTOMS}

The most urgent symptom is the convulsion, because of the great exhaustion it entails and because of the fatal spasms that may occur. There are two classes of drugs used for this purpose, one to lessen the irritability of the centres, lessen the severity of the tonic contractions and frequency of the clonic, a milder group; and the other more imperative, quelling the convulsion. Among the first belong bromides and chloral, the most important and mentioned together because they should be used together to get the best effects.

Large doses should be used; for the pharmacological depressing effect on the spinal centres is sought and the condition for which they are used a thousand times more menacing than their toxic effects on circulation or respiration.

Chloral should be given in doses of gr. $\mathrm{xv}(1 \mathrm{Gm}$.) every three hours or gr. $\mathrm{xxx}(2 \mathrm{Gm}$.) every six hours increased to gr. xlv $(3 \mathrm{Gm}$.) and gr. $\mathrm{lx}(4 \mathrm{Gm}$.) every six hours, if the spasm is severe, and heart and respiration are watched. Bromides may be given at double the chloral dose, as potassium bromide or mixed potassium, sodium and ammonium bromide, at the same time or in the intervals.

These are adult doses and should be correspondingly decreased for children. They may be given by mouth through nasal or stomach tube if necessary or by rectum, milk furnishing in the latter method a good menstruum.

Among other drugs belonging to this group but less reliable are atropine, hyoscine, physostigma and chloretone.

Standing between groups one and two is a very valuable drug.

Morphine. This drug not only lessens the spasm, but it relieves pain, induces sleep, and given before any disturbing procedure such as dressing the wound, giving enemas or rectal feeding, passing nasal tube or catheter makes the procedure less trying and less provocative of spasm. The doses should be large, gr. $1 / 4$ to gr. $1 / 3(0.015-0.02 \mathrm{Gm}$.), up to gr. iss. or gr. ii $(0.1$ or $0.125 \mathrm{Gm}$.) of the sulphate a day. Atropine sulphate is well combined with it.

The severity of the clonic spasms and the suffering they sometimes entail demand more dominant measures such as are furnished by the second group of drugs; namely

Chloroform. This is given by inhalation during the exacerbations of the spasms. It has been used continuously over periods of hours and days, but it is the conviction of some observers that such. usage constitutes a danger in itself.

Magnesium Sulphate. Intra-spinal. A method suggested by 
Meltzer of utilizing the depressant effects. of magnesium sulphate when brought into direct contact with the cord was eagerly seized upon, but was found to possess dangers of its own; however, the severity of the spasms, pain and exhaustion may be such as to make the measure in spite of its attendant dangers advisable, but it certainly is not to be undertaken lightly.

A 25 per cent. watery solution of magnesium sulphate is used. The dose 1 c.c. to every 25 pounds of the patient's weight, discounting somewhat for the very obese and large of frame.

The spinal puncture is made as for an exploration (see Cerebrospinal Meningitis, Chap. XXV), except that the shoulders should be somewhat raised to prevent rapid diffusion of the solution upwards towards vital centres.

The needle should withdraw an amount of cerebro-spinal fluid equal to that amount of solution to be used, the syringe then attached to the needle and the salt introduced slowly.

The spasms disappear and do not recur for hours or sometimes days. A recurrence of severe type would indicate a repetition of the procedure. The danger lies in respiratory collapse.

The patient should be under competent observation during the few hours after the injection and artificial respiration and stimulation undertaken if necessary, and it would seem advisable to withdraw what magnesium sulphate may be left in the canal.

\section{DRUGS OF MORE DOUBTFUL VALUE}

Chloretone. This drug in whiskey by mouth or olive oil by rectum has been used in doses of gr. $\mathrm{xxx}(2 \mathrm{Gm}$.) every two hours until the patient sleeps. Even doses as high as 66 and 75 grains $(5 \mathrm{Gm}$.) have been advised.

Atropine. This drug is used in combination with morphine or alone to lessen the spasm and as Bloodgood says finds its principal value in the prevention and relief of the distress due to an oversecretion of mucus in the air passages. The dose of the sulphate should be gr. 1/100 to gr. $1 / 25$ (0.0006-0.0025 Gm.) into the contracted muscles.

It can be repeated at four-hour intervals up to the well-known physiological limit-dilated pupils-excessive dryness of mucous membranes or rash.

Hyoscine. This drug, like atropine derived from the solanaceæ acts like atropine to lessen spasm but is more depressing to the cortical centres and in so far is a desideratum, but it is also depressing to the respiratory and cardiac centres and so has to be watched with care. 
It may be given as the hydrobromide in doses of gr. 1/100 (0.0006 Gm.) twice a day.

Physostigma or calabar bean, best in the shape of its active principle, physostigmine sulphate, has also been administered hypodermically in doses of gr. 1/6 $(0.01 \mathrm{Gm}$.) every three or four hours. Diarrhea or muscular twitching, if it can be made out during the tonic contractions, indicates the physiological limit and interruption of its administration.

Circulatory stimulants may be indicated in the exhaustion following clonic spasms.

Causes of Death. 1. Spasm of the larynx with ensuing asphyxia.

2. Spasm of the respiratory muscles, intercostals and diaphragm with asphyxia.

3. Cardiac failure.

4. Starvation.

5. Hyperpyrexia.

If the patient survives there may be some muscular stiffness for a long time after the subsidence of the attack.

Prognosis. The longer the incubation period the better is the prognosis and the longer the symptoms have continued the better is the eventual outlook; that is, fatal cases are likely to be shortly fatal, or in other words the prognosis depends on the severity of the infection and the resistance of the patient. Extensively distributed spasm is of bad omen and hyperpyrexia very fatal.

Prognosis depends largely on the promptness and efficiency of the treatment and especially the use of antitoxin. While here and there a man of experience expresses himself in this way: "The mortality has not been reduced by treatment save as to prevention" (Hill in Archives of Internal Medicine, December 15, 1911), others, and the majority; take a more cheerful view of the value of therapeusis. Park says, "If every case were given an intravenous injection of antitoxin at the time of diagnosis, and treated well in other respects, probably 50 per cent. would recover" (Forchheimer's Therapeusis of Internal Diseases, Vol. 2 ), while lists of statistics may be found attributing a fall in mortality from 80 per cent. to 90 per cent. down to 45 to 30 and less per cent. under antitoxin. A series of over 200 treated cases studied by Irons shows a mortality of about 61 per cent., a drop of 20 per cent. from the average mortality. Far better results are following the intra-spinal method.

Prophylaxis. Whatever disagreement there may be about the efficacy of treatment by antitoxin there is no dispute about its value as a prevention, though some surgeons believe that tetanus is a rare complication in a wound promptly and properly treated. 
Prophylactic treatment, then, resolves itself into two lines of endeavor.

1. Treatment of the wound.

2. Antitoxin administration.

Treatment of the Wound. The first essential is a knowledge of what kind of wounds under what kind of environment are likely to result in tetanus.

While it is possible that an infection under certain conditions may originate in the intestinal canal, for their presence in the intestine is not a rare finding and in hostlers a common one, or that the bacilli may be resident in the catgut used in surgical procedures, still the vast majority come from punctured and lacerated wounds contaminated by dirt from barns, stables, bites or other wounds from horses, punctured wounds from nails, fish bones in garden soil or barnyard refuse; lacerations, by farm-yard implements, machinery, gun-shot wounds, toy-pistols and giant crackers. The Fourth of July harvest has each year taken the heaviest toll of any cause.

After cleansing the surrounding parts and the wound with green soap, alcohol and ether or painting with tr. of iodine, every suspected wound then should be laid wide open to the very bottom by a free incision, bits of dirt, cloth, wood or powder removed, lacerated shreds separated with scissors or the wound area excised and the wound treated with

Antiseptics. For this purpose a 3 per cent. alcoholic solution of iodine may be used or liquified phenol, followed by alcohol. The wound is then loosely packed with the iodine solution and dressed daily after irrigation with peroxide of hydrogen with the gauze soaked in iodine solution or with iodoform gauze. No caustic should be used as the germs are anaërobic and will thrive best under the eschar produced by such agents. The wounds should be kept open and allowed to granulate from the bottom.

It has been advised to use powdered dry antitoxin in the wound or the liquid antitoxin itself, applying a loose dressing over it. In other words, the wound is to be treated by sound surgical methods.

Use of Antitoxin. This should be given at once. Its efficacy is best demonstrated by the fact that the high death rate from tetanus after Fourth of July wounds has been almost if not quite wiped out in those hospitals where it has been used at the time the wound was dressed. Its use during the late war has placed its value beyond dispute.

Dose. The usual dose is 1,500 units given subcutaneously, or better, into the tissues and muscles about the site of the wound, to protect the 
motor end plates at the site of toxin formation first and the remoter nerves next.

Repetition of the Dose. It has been shown that in eight or ten days all the antitoxin has been eliminated and for this reason a second dose of the same amount should be given. This second dose will almost surely protect. Some cautious men, however, give a third dose and some continued doses at these intervals until the wound is healing in a healthy manner by granulation.

If for any reason a wound has to be opened up a second time a prophylactic dose of 1,500 units should be given again as the tetanus bacilli locked up in the healed wound may become active as a result of the procedure.

Some men have relied on the local use of antitoxin in the wound as described; but, if antitoxin seems indicated at all it should be given as above described except in cases of local tetanus, whether it be used locally or not.

The dosage in a child may be a little less, 1,000 units, but there is no objection and some value in the larger dose.

Serum Disease. Rashes and joint pains as after diphtheria antitoxin may appear, but are of little importance.

\section{SUMMARY}

\section{Incubation time.}

Average seven to nine days. May be weeks or months. (See text.)

\section{Symptoms.}

Due to irritation of motor nerves inducing tonic contraction of muscles interrupted by clonic contractions, which are easily excited by slight stimuli.

\section{Treatment.}

Rest.

Elimination of every stimulus to the highly irritable motor centres.

\section{Room.}

As far away from the noise of the street and the rest of the household as possible.

Avoid sudden noises, such as jarring of doors and windows, heavy tread of feet, moving furniture, loud talking.

Cotton may be put in the ears.

Room should be a little darkened.

Should be well ventilated. 
Bed.

Half-bed of the hospital type, with woven wire springs, firm mattress, smooth sheets.

Water bed may be more comfortable.

\section{Care of the body.}

Baths should be given of sponges of warm water in a warm room, as least likely to provoke a convulsion.

Mouth-best rinsed with clear warm water or saline solution, or 2 per cent. boric acid from time to time unless it incites the clonic contractions.

\section{Diet.}

Feeding very difficult.

Liquid foods; concentrated, administered as infrequently as compatible with body needs.

Milk, cereal waters, eggs; all fortified with sugar.

Sooner or later rectal or nasal feeding must be used in part or wholly.

Rectal feeding.

Two hundred c.c. peptonized milk.

Two eggs.

Salt solution ( $3 \mathrm{i}$ to $0 \mathrm{i}$ ) ( $4 \mathrm{Gm} .-500$ c.c.) up to 500 c.c. or 1 pint and administer by the drop method of Murphy (Bloodgood).

Or 500 c.c. raw milk.

Forty-five grams milk sugar. $600-650$ cals.

Thirty c.c. whiskey or brandy.

Introduce slowly into bowel.

Two or three rectal feedings a day is about all the bowel will take for any length of time.

Nasal feeding.

Use small flexible tube.

If spasm blocks the tube give

Morphine sulphate, gr. 1/8-gr. 1/4 (0.008-0.015 Gm.). (Adult dose.)

Subcutaneous feeding.

Doubtful value.

Olive oil.

Glucose, 5 per cent. to 10 per cent. (real value).

Normal horse serum, 500-1,000 c.c. (Park).

Fluids.

On account of the difficulty of getting enough water by the mouth.

Give physiological salt solution (3i to $0 \mathrm{i}$ or $4 \mathrm{Gm}$. to 500 c.c.) by Murphy drip method by bowel.

Give 5 per cent. glucose solution in same quantities by drop method.

\section{Bowels.}

When seen early.

Saline. Epsom, Rochelle or Glauber's salt, ₹ss. $-53 / 4(15-20 \mathrm{Gm}$.$) ,$ in half to three-quarter glass of water. 
Later.

Enemata-carefully given to avoid exciting spasm.

\section{Bladder.}

Watch for retention; if it occurs use catheter. Precede by morphine.

\section{Eliminate the focus of infection.}

Wound laid wide open.

Remove sloughs.

Pack with gauze, let heal by granulations.

Swab wound with 3 per cent. of alcoholic solution of iodine, follow with peroxide of hydrogen and pack with gauze saturated with the iodine solution. (Ashurst and John.)

Cauterization should not be done.

\section{Specific treatment.}

Use of antitoxin. (For discussion of its limitations and the mode of administration, see text.)

Subcutaneous.

Absorption slow, hence, greatly diluted in blood; requires large doses, limited only by expense, e. g., 100,000 units in twentyfour hours, or

Fifteen thousand every three hours.

Intramuscular.

Better than subcutaneous, but not as good as intravenous.

Dose, 10,000 to 15,000 units once or twice in twenty-four hours.

Intravenous. Better than above but not comparable to intra-spinal.

Can give one large intravenous dose to begin with and subcutaneous or intramuscular doses every day or two following or daily intravenous doses of smaller amounts.

Park's rule. Use 2,000 units for each ten pounds body weight.

In addition antitoxin should be brought into more direct contact with the nerve tissue.

Intraspinal always to be used if possible.

Dose, 3,000 to 5,000 to 10,000 units (6 to 20 c.c.) daily.

(For technique, see serum administration in Cerebro-spinal Meningitis, Chap. XXV.)

For amounts consult Sophian's tables. (See Cerebro-spinal Meningitis.)

Intraneural.

Into large nerve trunks leading from wounded area, as near the cord as feasible to expose them. (As much as 1,500 units have been put into the sciatic nerve.) Not advised.

Intracerebral.

An early method. The results have not been encouraging.

Intramuscular (at site of wound).

To bring serum into contact with motor end plates of wounded area. 
For New York Board of Health method of combined intra-spinal, intravenous and intramuscular dosage, see text.

For the method of the Tetanus Committee of the United States War Department, see text.

These are the best methods.

Drugs.

Purely empirical usage.

Phenol (carbolic acid).

Dose, gr. v to gr. viiss. (0.30-0.50 Gm.) daily.

Given in 2 per cent. solution in oil.

$\mathrm{M}$ : $\mathrm{xx}(1.30$ c.c.) every two hours would amount to gr. v (0.30 Gm.) a day.

Same dose of 3 per cent. would equal gr. viiss. (0.50 Gm.).

Watch urine for smoky urine as evidence of phenol poisoning.

Treatment of symptoms.

Convulsions.

To lessen irritability of centres, and diminish frequency and violence of clonic convulsions.

Chloral.

Dose, gr. xv (1 Gm.) every three hours, or

gr. $\mathrm{xxx}(2 \mathrm{Gm}$.) every 6 hours,

increasing to gr. xlv $(3 \mathrm{Gm}$.) or

gr. lx (4 Gm.) every six hours if necessary. (Adult dose.)

Bromides. In double dose of chloral; either potassium bromide alone or the triple bromides of sodium, potassium and ammonium. Not likely to be effectual alone, but well to combine with chloral. Give in solution in water through stomach or nasal tube, or by rectum in a couple of ounces $(60 \mathrm{Gm}$.) warm milk.

Morphine.

Lessens spasms, relieves pain, induces sleep.

Indicated before any procedure likely to induce convulsions, such as enemas, rectal or nasal feeding, catheterizing.

Indicated during a convulsion.

Doses, sulphate, gr. $1 / 4$ to gr. $1 / 3(0.015-0.020 \mathrm{Gm}$.) up to gr. i or gr. iss. to gr. ii $(0.060-0.10-0.120 \mathrm{Gm}$.) a day.

Atropine.

May be combined with morphine.

Chloroform.

Indicated in severe and painful convulsions.

Inhalation.

Intermittently at periods of clonic convulsion.

Continuous for hours (not free from danger).

Magnesium sulphate.

(For intradural use, see text.)

Other drugs that have been recommended.

Chloretone, gr. $\mathrm{xxx}(2 \mathrm{Gm}$.), every two hours until sleep occurs.

Give in whiskey by mouth or olive oil by rectum. 
Atropine.

Indicated as above with morphine or alone to lessen spasms, also to diminish over-secretion of mucus in air passages.

Dose, of the sulphate, gr. 1/100 to gr. 1/25 (0.0006-0.0025 Gm.) into contracted muscles.

Repeat at four-hour intervals.

Watch for toxic symptoms, widely dilated pupils, great dryness of mucous membranes, rash, delirium.

Hyoscine hydrobromide.

Has same effect as atropine, but more depressant to cardiac and respiratory centres.

Dose, gr. 1/100 (0.0006 Gm.) twice a day.

Physostigma.

Use physostigmine sulphate, gr. 1/6 (0.010 Gm.), hypodermically every three to four hours.

\section{Circulation.}

Exhaustion from convulsions may indicate use of circulatory stimulants.

Use digitalis series for sustained effect and caffeine or camphor for immediate effect. (See Chap. IX.)

\section{Prophylaxis.}

Treatment of the wound.

Cleanse wound with soap, water, and alcohol, or paint with tincture of iodine.

Lay wound open to bottom. Remove foreign particles.

Cut away lacerated flesh or

Excise wound area; then use

Antiseptics.

Three per cent. alcoholic solution of iodine or phenol liquified, followed by alcohol.

Pack loosely with gauze saturated with the iodine solution.

Dress daily, irrigating with peroxide of hydrogen.

Let wound granulate from the bottom.

Powdered or liquid antitoxin has been used in the wound.

\section{Antitoxin administration.}

Should be given at once.

Dose, 1,500 units.

Best into muscles about the site of the wound.

Second dose of same amount should be given in eight or ten days.

Third dose or repeated doses at these intervals until wound is healed are given by most cautious.

In a child 1,000 units may answer, but 1,500 are better. Serum disease, as after diphtheria antitoxin, may follow. It is of small consequence. 


\section{CHAPTER XLI}

\section{INFECTIOUS JAUNDICE (WEIL'S DISEASE)}

THERE is some question as to the propriety of using the terms Infectious Jaundice and Weil's Disease as synonymous. Of late Infectious Jaundice has been taken to mean a definite infection attributable to a spirochete, the spirocheta ictero-hemorrhagiæ, the clinical picture of which is fairly clear cut. This same term had been applied long ago to a group of cases later described by Weil and to which his name is generally attached in the literature. Weil's disease and Infectious Jaundice as now understood have in common the distinguishing features of jaundice and hemorrhages, accompanying a toxemia, but in some minor details vary. It means probably that Weil's Disease covers a heterogeneous group of conditions with which the major manifestations mentioned are associated. We shall discuss here the spirochetal infection; but the measures mentioned, with the exception of specific therapy, are applicable to one and the other.

According to the Japanese workers, to whom we owe so much in the investigation of this disease, the portal of entry is by the skin, broken or intact, or by the gastro-intestinal tract. Excretion occurs in the feces and urine and the organism is readily recovered from the latter, from early in the disease up to 5 to 6 weeks, but usually by the end of the second week. In the body the spirochetes are distributed especially to the kidneys, the liver, the lymph nodes and spleen. They are found in the heart muscle, too.

The most obvious lesion is curiously confined to the duodenum, the pyloric end of the stomach and the upper portion of the jejunum. Here the congestion is intense and the papilla of Vater involved. The obstruction seems to be at the papilla, for the gall-ducts, gall-bladder and liver are but little affected; considering the intensity of the jaundice. It is to be noted that the toxemia seems to be due to the spirochetosis rather than the jaundice and indeed, jaundice may be absent.

The incubation period when infection occurs by the skin is 5 to 7 days. It is seldom later than two weeks.

Symptoms. The onset may be sudden or gradual with the usual symptoms of acute infection, malaise, fever, headaches, generalized pains, cramps in muscles, prostration, anorexia, often vomiting and pain in the upper abdomen, striking constipation and marked congestion 
of the conjunctivæ. Herpes is common. The blood count shows a moderate leucocytosis and polynucleosis, exceptionally both are quite high. The pulse is relatively slow, the blood pressure is normal. In the early days spirochetes may be recovered from the blood and the blood shown to be infective to inoculated animals, but by the fifth day this infectivity is markedly diminished, due probably to the formation of antibodies. By the fifth day spirochetes are usually excreted by the urine. This initial or febrile period lasts six to seven days. The temperature in the beginning may be $102^{\circ} \mathrm{F}$. to $103^{\circ} \mathrm{F}$. and comes down by lysis nearly to normal. During this period hemorrhages are common, occurring from the lungs, nose, stomach, intestine and kidneys, together with, in some cases, purpura, and Dawson and Hume note a "curious purplish discoloration in the abdomen, loins and lower part of the chest in those cases which were most deeply jaundiced."

The second or icteric stage begins at about the end of the first week. Most commonly the jaundice appears on the fifth day, though it may appear as early as the second day or be delayed until near the end of the second week. The degree of jaundice varies. Bile appears in the urine and the stools are clay colored. During this period, too, occur hemorrhages and marked general weakness. Convulsions have occurred in some fatal cases and the heart may show some inefficiency, though this is not characteristic. It is in this second period that fatal issues commonly occur.

A secondary rise of temperature, after an afebrile period of two to ten days takes place at the end of the second week in a certain number of the cases, 30 per cent. to 40 per cent. This rise varies in its duration, but may last several days, be quite irregular, be trivial or be quite as marked as the original fever or even higher.

The third and last period is that of convalescence. It begins with the third week unless delayed by the relapse or after fever, the secondary rise.

The icterus gradually fades and the anæmia and emaciation become more pronounced. Spirochetes are no longer found in the blood, but may still be recovered from the urine, especially in the relapsing cases. Indeed, though diminishing after the twenty-fifth day, they may be found in the urine as late as the 40th day in the average case and even later in the exceptional.

Treatment. That of all acute infections. (See Chap. I.)

The patient is put to bed in a well ventilated room, to which the sun has access, in a comfortable bed. Daily sponge baths are given, the mouth properly attended to and quiet and good nursing afforded.

Diet. Gastro-intestinal symptoms are fairly constant and some- 
times severe. It is useless to force the diet while these disturbances obtain.

It is better to respect the meaning of the anorexia and refrain from giving food until nausea and vomiting cease and then begin tentatively with milk (better skimmed) or broths, such as mutton and chicken (freed from fat), white of egg, thoroughly cooked cereals, toast; enlarging on the diet as the patient demonstrates his ability to handle larger quantities.

Water should be given frequently in small quantities if there is nausea, in larger amounts if the stomach retains it.

Specific Treatment. The Japanese workers Inada, Ido, Hoki, Ito and Wani recommend the use of a specific serum.

As in the use of all specific sera, early administration constitutes a most important factor in success.

Dose. Sixty c.c. should be given in 24 hours, in divided doses at 5 to 6 hours' intervals.

The intravenous route is the best; next, the intramuscular.

It was the experience of the investigators quoted that the serum rarely failed to cause a complete disappearance of spirochetæ from the circulating blood.

The mortality was reduced from 30.6 per cent. to 17.3 per cent in their series.

Bowels. When first seen a saline may be given of Epsom salt or Rochelle salt or if there is nausea and vomiting, divided doses of calomel, gr. 1/4 (0.015 Gm.) every half-hour for five or six doses, followed by the salt; or a salt in divided doses of $3 \mathrm{i}(4 \mathrm{Gm}$.) every quarter hour for five or six doses. Neither the calomel nor the salt should be used in drastic doses. Later enemata or mild salines every day or every second day may be used.

Nausea and vomiting may be alleviated by cracked ice, the application of mustard paste to the epigastrium, stomach washing or the use of such drugs as bismuth subnitrate $\mathrm{gr}$. $\mathrm{xv}$ to $\mathrm{gr}$. $\mathrm{xxx}$ (1-2 Gm.), bicarbonate of soda gr. $\mathrm{x}$ to $\mathrm{gr} . \mathrm{xx}(0.6-1.3 \mathrm{Gm}$.) or oxalate of cerium gr. ii to gr. v (0.15-0.30 Gm.) or a combination; codeine sulphate gr. $1 / 8$ to gr. $1 / 4(0.008-0.015 \mathrm{Gm}$.) or cocaine hydrochloride gr. $1 / 5(0.01$ $\mathrm{Gm}$.) are indicated in more severe cases and even morphine in the worst.

Diarrhea. Attention to the bowels as set forth above, restriction of fat in food and sufficiency of water to lessen the incidence and severity of the diarrhea.

Bismuth subnitrate in doses of $\mathrm{gr} . \mathrm{xxx}(2 \mathrm{Gm}$.) every two hours is the best drug to use; opium being reserved for the most severe forms.

Colicky pains are relieved by hot fomentations. 
Fever. Temperature of $102^{\circ} \mathrm{F}$. to $103^{\circ} \mathrm{F}$. are reached by the second or third day and gradually decreases throughout the first week.

It but rarely requires treatment other than that which contributes to the patient's comfort, afforded by sponge baths.

Nervous Symptoms. Headache, delirium or coma may occur. For the headache the ice-coil is to be recommended, delirium may require small doses of morphine.

Pains in the legs are at times very hardly borne and the applications of hot stupes and mild analgesics, such as the salicylates are required.

Jaundice appears on about the fifth day. This jaundice lasts about two weeks. There is no treatment that probably affects the degree or intensity of the jaundice, but the salicylates as being thought to stimulate biliary output have been much used, either as salol, sodium salicylate or aspirin in doses of gr. $\mathrm{v}$ to gr. $\mathrm{x}(0.3-0.60 \mathrm{Gm}$.) every three or four hours. They have the advantage, too, of acting as antipyretics and analgesics.

Nephritis is a complication that is not uncommon and demands daily examination of the urine.

It is to be treated like acute nephritis under other circumstances. (See Scarlet Fever, Chap. XVII.)

Rarer Complications. Hemorrhages from nose, lungs, stomach, bowel into skin or serous sacks.

Hemorrhages should be treated as under other conditions. (See Typhoid Fever, Chap. XIV.)

The intoxication in infectious jaundice is severe; often the patient sustains much loss of flesh, and strength is but slowly regained.

Fresh air out of doors, a sufficiency of good food and, when feasible, a change of climate does more to shorten the period of convalescence than drugs. If these are used, such simple tonics as strychnine sulphate gr. 1/30 $(0.002 \mathrm{Gm}$.) three times a day or tincture of nux vomica $\mathrm{m}$. $\mathrm{x}$ to $\mathrm{xx}(0.60-1.30 \mathrm{Gm}$.) three times a day are given. Iron may be used.

\section{Prophylaxis.}

Active Immunization. Ido, Hoki, Ito and Wani brought this about by three subcutaneous injections of vaccine at five days' intervals. They used such a suspension as contained 60 to 150 spirochetes in a field, $1 / 12$ oil, immersion, ocular 3 (Leitz) under dark illumination, in 0.5 per cent. carbolic acid. The first injection consisted of 0.5 c.c. of this suspension, the second 1 c.c., the third, 2 c.c. Active immunization should prove a potent prophylactic measure.

In Japan this infection seems to be common among miners; hence, draining of the mines and disinfection of the ground with lime is advised.

The infection through the gastro-intestinal canal can only be avoided 
by such precautions of cleanliness in handling food and boiling water and disposing of infected secretions as are detailed, for example, under Typhoid Fever. (See Chap. XIV.) The urine and stools should be disinfected for at least 40 days and as much longer as the presence of spirochetes in these excretions indicate. Bloody sputum during the attack is a source of infection and must be destroyed.

In that group of cases that cannot be attributed to spirochetal infection and may still be classed under Weil's Disease, the conclusions of Barker and Sladen, resulting from a series of cases may be quoted.

"The sequence of events in our cases, it seems to us probable, was as follows:

"1. Ingestion of tainted meat, containing living paratyphoid bacilli; (2) development of gastro-enteritis due to this microörganism, and (3) the appearance of a catarrhal jaundice due to extension of the gastro-enteritis to the "biliary passages."

\section{SUMMARY}

(For general principles, see Chap. I.)

\section{Diet.}

Refrain from urging food until gastro-intestinal symptoms subside or ameliorate; then

Milk, broths, cereals, white of egg, toast, all tentatively.

Enlarge on diet gradually.

\section{Water.}

Give freely when well borne.

Specific treatment.

(See text.)

\section{Precautions.}

Destroy all secretions.

(See Typhoid Fever, Chap. XIV.)

\section{Bowels.}

Salts, Epsom or Rochelle §ss. to $5 \mathrm{i}$ (15-30 Gm.) in three-quarters glass of water.

If there is nausea or vomiting precede the salt with calomel in divided doses, gr. $1 / 4(0.015 \mathrm{Gm}$.), every hour for four to five doses. Later.

Mild salines, milk of magnesia, citrate of magnesia or Hunyadi water or enemata. 


\section{Nausea and vomiting.}

Cracked ice.

Mustard paste (1 to 4 or 5 of flour) to epigastrium.

Lavage.

Bismuth subnitrate, gr. $\mathrm{xv}$ to gr. $\mathrm{xxx}(1-2 \mathrm{Gm}$.).

Sodium bicarbonate, $\mathrm{gr} . \mathrm{x}$ to gr. $\mathrm{xx}(0.60-1.30 \mathrm{Gm}$.).

Cerium oxalate, gr. ii to gr. v $(0.15-0.30 \mathrm{Gm}$.).

Combine the above. Give every two or three hours in water or in the milk.

Codeine sulphate, gr. 1/8 to gr. $1 / 4(0.008-0.015 \mathrm{Gm}$.).

Cocaine hydrochloride, gr. $1 / 5(0.01 \mathrm{Gm}$.).

Severe cases.

Morphine sulphate, gr. 1/16 to gr. 1/8 (0.004-0.008 Gm.).

\section{Diarrhea.}

Give water freely.

Restrict fat in food (skim the milk).

Boil milk.

Bismuth subnitrate, gr. $\mathrm{xxx}$ ( $2 \mathrm{Gm}$. every two hours).

Most severe cases.

Powdered opium, gr. ss. to gr. i (0.03-0.06 Gm.) every four hours.

\section{Colic.}

Hot fomentations to abdomen.

Headache.

Delirium, coma.

Ice-coil or ice-bag to head.

Severe cases.

Morphine sulphate (hypodermically), gr. 1/12 to gr. 1/4 (0.005$0.015 \mathrm{Gm}$.).

Pains in legs.

Hot stupes.

Salicylate of soda, gr. $\mathrm{v}$ to gr. $\mathrm{x}(0.30-0.60 \mathrm{Gm}$.), every two hours or Aspirin same dose and interval.

\section{Complications.}

Nephritis.

(See Scarlet Fever, Chap. XVII.)

Hemorrhages.

(See Typhoid Fever, Chap. XIV.)

\section{Convalescence.}

Often slow.

Open air.

More food.

Change of climate, when feasible. 
Tonics.

Strychnine sulphate, gr. $1 / 30(0.002 \mathrm{Gm}$.$) , three times a day or$ Tr. nucis vomicæ, $\mathrm{m} . \mathrm{x}-\mathrm{xx}(0.60-1.30$ c.c.), three times a day. Iron.

Blaud's pill or
Vallet's mass
three times a day.

\section{Prophylaxis.}

Active Immunization.

Avoid possibility of ingesting tainted meat and polluted water. (See text.)

In infected districts boil water.

Precautions in handling food.

Disinfect urine and stools for 40 days or as long as spirochetes are present.

Destroy bloody sputum. 


\section{CHAPTER XLII}

\section{YELLOW FEVER}

This disease, in the elucidation of whose mysteries American physicians have played the leading rôle, like Malaria, is transmitted by the mosquito; more definitely by the female of Stegomyia calopus (Sedes calopus). She becomes infected during and only during the first three days of man's illness with yellow fever and herself passes through an incubation period of from ten to twelve days before she can convey the disease to another man. After this she may remain infective for a considerable time; for fifty-seven days in one instance. Although the details of the mode of infection had been marked out elaborately the organisms concerned had defied detection until the careful investigations of Noguchi seemed to be rewarded in 1919. He found in the blood of persons sick with yellow fever an organism that morphologically closely resembles the leptospira ictero-homorrhagiæ of infectious jaundice, but showing different immunological characteristics. This organism he was able to detect in fresh blood by the dark field illumination; he obtained it in pure culture; by the injection of blood and tissue of yellow fever patients, he produced in animals a disease characteristic of yellow fever; he recovered it again from the blood and organs of these animals; and it gave a Pfeiffer phenomenon. Furthermore he was able to transmit the disease from animal to animal.

Symptoms. The onset is sudden with chilly sensations, rise of temperature, headache and backache. After some irregularities the temperature abates about the third or fourth day at which time conjunctival icterus may be seen. After a brief intermission or remission of the temperature and diminution of evidences of intoxication, a renewed fever and toxemia with deepening jaundice, gastric distress, vomiting, even a bloody black vomit, delirium and coma ensues.

Hemorrhages and kidney incompetency are often accompaniments. The pulse is characteristically slow (Faget's sign).

Stitt states that the blood pressure during the sthenic period is very high, sometimes 200 , but begins to fall on second day progressively until in the asthenic period it is very low, sometimes down to 70 .

Isolation. We now know definitely that there are three links in the chain of transmission, any one of which, abolished, will prevent in- 
fection: (1) Stegomyia calopus; (2) its access to a yellow fever patient; (3) its access later to a well person.

The prevention of (2), then, by isolation becomes imperative; moreover, during an epidemic all suspects, suffering from what may be deemed early symptoms of the disease, should be isolated until the diagnosis can be determined. White's instructions as given in his article in the American Journal of Medical Sciences, March, 1913, are so succinct that I will quote them here.

"In dealing with the houses in which known or suspected cases of yellow fever have been discovered, it was my custom first to see whether the house had been properly screened with eighteen-mesh to the inch wire cloth, and to have screening done promptly, using mosquito netting as a substitute for wire cloth, and having the sick-room itself, as a double precaution, screened from the remainder of the house. The sick-room can be easily screened by tacking two ample widths of the netting to the top and sides of the door, and attaching a wooden rod to the bottom of each width, so that the ends projecting in to the door are higher than those at the side resulting in the rod dropping into place and pulling the folds together immediately behind any one passing through.

"With Guiteras I believe in careful screening and in covering all cracks around the sick-rooms; also in the quietness with which the work is done, to prevent disturbing either patient or mosquito. The sickroom window should be screened from a ladder on the outside of the window. In order to prevent escape or entrance through the chimney a cap should be placed on the top, or failing in this, some loose paper should be burned in the fireplace, with a little petroleum to make a rapid flame, and the front of the fireplace covered with a screen of wire netting.

"After the patient is in condition to be removed to another room or house, three days having elapsed since he became ill, he is removed and every crack pasted over into which an insect may hide, and all drawers opened, so that the fumigant used may reach all places in the room, care being taken that no mosquitoes are shut between the outside screen and the window in closing it. The room and its contents are subjected to either sulphur dioxide or cyanide, burning pyrethrum powder or any other fumigant. The whole house is simultaneously treated in the same fashion if the patient can be removed to another house; otherwise we must treat part at a time with more than usual care."

It is seen, then, that after three or four days the patient is no longer a source of infection, but that he is still to be protected against further infection by infected mosquitoes.

His room should be one to which air has the freest access and these 
patients do very well under a tent in the open air. The room should be darkened.

The room, of course, is to be screened until such time as the patient may be removed from the room in which he was taken ill; that is, at the end of the first three days of his illness, during which his blood contains the virus.

If his condition warrants, he is then removed, and the room is fumigated to destroy the mosquitoes. Doctor and nurses should protect parts of the body liable to be bitten and more especially toward nightfall.

The choice of the bed and the care of the bed observe the same rules as in other acute infectious fevers. (See Typhoid Fever, Chap. XIV.)

The certain knowledge of the only mode of conveyance of the disease, i. e., by the mosquito, makes the elaborate use of antiseptics, as, for instance, in typhoid unnecessary.

Rest. There are abundant theoretical reasons and such as appeal to common sense, as set forth in the chapter on Treatment of Acute Infectious Diseases (see Chap. I), that should urge getting a patient to bed on the first suspicion of infection, beside the conviction of those experienced in the treatment of this disease that cases put to bed early are less severe and less likely to be fatal.

Isolation makes rest more easily attained than when friends and visitors have free access to the patients. Bed pans are to be used and in feeding, the glass tube or feeding cup.

Diet. Gastric irritation is a characteristic of the disease and makes the matter of feeding a difficult one. All writers advise against administering food during the first period of three or four days, while the gastric disturbance continues or the fever remains up; even in the second period if above $102^{\circ} \mathrm{F}$.

After four or five days' abstention from food the weakness of the patient may urge upon us the necessity of affording nourishment in some form and recourse may be had to rectal feeding. In severe cases this is almost impossible and in any case great care must be taken not to aggravate the irritability of the colon and rectum.

Agramonte advises feeding in this manner on alternate days, and suggests as a food formula:

Milk....................... 3 ounces (better peptonized)

Whiskey ................... $1 / 2$ ounce

Normal salt solution............... 3 ounces

When conditions as above enumerated would seem to permit of it food may be begun with caution by the mouth, commencing with much diluted milk, with barley water, ice-cream or gelatine jellies. 
Drinks. It is very desirable to administer fluids, if the stomach will entertain them. Alkaline waters seem the best borne.

Carroll advises Vichy or other mineral water, to which is added sodium bicarbonate grains $\mathrm{xxx}(2 \mathrm{Gm}$.) to the pint (500 c.c.), while Anderson is enthusiastic over his effervescent mixture of potassium bicarbonate grains $\mathrm{xxx}(2 \mathrm{Gm}$.) to a dessert-spoonful of freshly expressed and strained lime juice given effervescing in Vichy every two hours. Mild lemonade may also be administered.

All food and drinks should be ice-cold.

Bowels. A saline 3iv-vi (15-24 Gm.) of Epsom or Glauber's salt, best in $3 \mathrm{i}(4 \mathrm{Gm}$.) doses at frequent intervals, should be given. This may be preceded by calomel grains iii to gr. v $(0.20-0.35 \mathrm{Gm}$.) or this may be divided. If not effectual a plain enema should follow. Cathartics are only indicated at the beginning of the disease.

Care of the Body and Mouth. The same rules are to be followed as after any severe acute infection. (See Typhoid Fever, Chap. XIV.)

Specific Treatment. At the time of writing, the experimental work of Noguchi holds out some promise of our ultimately acquiring an immune serum of value.

Treatment of Symptoms. The onset, as has been stated, is sudden and severe and accompanied by headache and backache, often intense.

Headache. It is altogether better to avoid drugs, since the depressant effects of the coal tars and the other pharmacological actions of the morphine series are undesirable, and to rely upon cold applied to the head in the shape of cold compresses or the ice-bag or the cold coil.

Experienced clinicians advocate the application of heat to the trunk and extremities at the same time, as a hot pack, a hot mustard footbath or heat otherwise applied to the feet (see Pneumonia, Chap. IV), and hot drinks such as lemonade if the stomach will stand it. They believe that not only is the headache bettered, but the nausea relieved and the congestion of the kidneys lessened.

Backache. The hot pack, hot stupes, mustard pastes or plasters and rubbing afford relief.

Gastric Distress. Pain, nausea and vomiting are early manifestations often persistent and later in many instances giving rise to the characteristic and ominous black vomit.

No food should be given while the stomach is so irritable; a counterirritant, mustard paste or mustard leaf, may be applied to the epigastrium or the application of the ice-bag to the same site may be tried.

Cracked ice is sometimes efficacious or ice-cold drinks in small quantities frequently, such as the alkalinized Vichy water described above 
and effervescing drinks, as champagne or ginger ale in tea-and tablespoonful doses.

If the violent vomiting still persists one must have recourse to morphine in doses of grains $1 / 4(0.015 \mathrm{Gm}$.) hypodermically.

If tenderness is severe or hiccoughing occurs, Carter highly recommends cocaine hydrochloride, which he gives in doses of one-half a grain to a grain in a capsule to be taken with a minimum amount of water as a preventative of the black vomit. He finds morphine sulphate gr. 1/16-1/12 (0.004-0.005 Gm.) an useful adjuvant if the kidneys do not contraindicate the use of it.

Black Vomit. Among the measures recommended to relieve the serious condition are the hemostatics, such as adrenalin by mouth for its constricting effect on the small arteries. Tincture of ferric chloride in doses of minims 5 ( 0.35 c.c.) every two hours for the styptic effect on the bleeding orifices may be given in limejuice and glycerin; calcium chloride (Guiteras) for its effect on the coagulation of the blood and oil of turpentine in doses of minims $x$ ( 0.65 c.c.) for the same purpose.

If there is much pain in the stomach, Carter recommends cocaine as given above with or without morphine.

Fever. The period of fever is short-lived and rarely excessive and demands no measures except those designed for the comfort of the patient, as sponging with luke-warm or cool water.

If the pyrexia, however, attains high figures or for any time persists above $103^{\circ} \mathrm{F}$., the sponges should be given with colder water from $70^{\circ}$ $\mathrm{F}$., down to $60^{\circ} \mathrm{F}$., or $50^{\circ} \mathrm{F}$., depending on the reaction, at four-hour intervals. Such cold sponging should have a favorable effect on the circulation and on other structures burdened by the toxins.

Urine. Great danger attends the not uncommon involvement of the kidneys and the suppression resulting. Examinations of the urine should be made daily and estimates of intake and output of water noted. The capacity of the kidney for secretion of water is more important than the amount of albumin in the urine. Administration of a sufficiency of water is important, but on account of the gastric irritability difficult of accomplishment. For this reason saline rectal injections are of value given in amounts of 10 to 16 ounces at four-hour intervals, or the Murphy drip may be tried, but care must be exercised not to aggravate the irritability of the intestine from which hemorrhages are so likely to occur. At the same time hot fomentations should be applied to the lumbar region or the area cupped in an effort to relieve the congestion.

Circulation. The condition of the heart and of the principal vessels 
should be determined by frequent examinations and blood-pressure readings.

Depression of the circulation should be met in the same way as in other acute infections. (See Treatment of Acute Infectious Diseases, Chap. I, and Pneumonia, Chap. IX.)

Convalescence. The period depends on the degree of intoxication throughout the illness. In the usual case it is relatively short, so that after the fever has subsided for several days the patient may be allowed to sit up. The patient gets back onto solid food gradually, the condition of the stomach and kidneys affording the criteria for enlarging the diet. The kidneys are rapidly restored to normal condition.

Prophylaxis. Three lines of endeavor to prevent infection are to be pursued.

(1) Destruction of mosquitoes and elimination of their breeding places.

(2) Isolation of the infected individual.

(3) Precautions against bite of stegomyia by non-immunes.

To effect (1), the destruction of the mosquitoes and the elimination of their breeding places, the following measures are undertaken: First, as described under the section on isolation, by carefully screening the room from the first and by removal of the patient after three days of illness with care not to disturb the mosquitoes, followed by fumigation of the tightly sealed room.

White calls the stegomyia calopus a domestic mosquito; that is, that it clings closely to the habitat of man and breeds in water collected in artificial receptacles, cisterns, barrels, tubs, cans, broken crockery, pools, vases, fountains, drains, roof gutters, in fact in any stagnant water commonly to be found about a dwelling.

Of course, all useless litter should be destroyed or disposed of ; cisterns, drains, etc., properly screened with wire netting or constantly covered with a film of petroleum, while small fish devour the breeding mosquitoes in fish ponds and fountains. Swamps, ponds or stagnant waters about the locality infected must be drained, filled in or treated with petroleum.

(2) The isolation of the infected individual to prevent his being a source of infection to others through mosquitoes first biting him has been dealt with.

(3) Precautions against bites of stegomyia by non-immunes are first a knowledge of the habits of stegomyia calopus and second, personal protection.

Carroll says the stegomyia is a twilight mosquito, feeding in early morning and from mid-afternoon until late evening (3 P. м. -10 P. м.), and that in well-lighted places between 9 A. M. and 3 P. M. there is little danger to non-immunes even in infected localities. 
When in an infected locality non-immunes should take care to protect themselves against the bite of stegomyia and that especially at dusk and after dark by wearing mosquito head-nets, heavy gloves and leggings, choosing for a sleeping room as high a room in the house as possible, being assured on retiring that the room is free from mosquitoes, best by fumigating it, sleeping in a screened room and in a screened bed.

They should not expose themselves to the environment of an infected individual unless duty calls and then should take such precautions as are observed by doctors or nurses in attendance.

\section{SUMMARY}

Isolation.

Of both patients and suspects.

(For the technique of isolation, see text.)

\section{Doctor and nurse.}

Must protect themselves against the bite of mosquitoes and more especially toward nightfall.

Bed.

(See Typhoid Fever, Chap. XIV.)

Diet.

Gastric irritation makes the problem a special one. (See text.)

Drinks.

Alkaline waters.

Vichy or other mineral water with sodium bicarbonate gr. $\mathbf{x x x}$ $(2 \mathrm{Gm}$.) to the pint (500 c.c.) (Carroll).

Potassium bicarbonate gr. $\mathrm{xxx}(2 \mathrm{Gm}$.) in a dessert spoonful (3ii or

8 c.c.) freshly expressed and strained limejuice given effervescing in Vichy, every two hours (Anderson).

Mild lemonade.

All drinks ice-cold.

\section{Bowels.}

Epsom, Rochelle or Glauber's salt, 3iv-vi (15-25 Gm.), best in doses of $3 \mathrm{i}(4 \mathrm{Gm}$.) at frequent intervals.

May precede by calomel gr. iii to gr. v $(0.20-0.35 \mathrm{Gm}$.) in one or divided doses.

Care of body and mouth.

(See Typhoid Fever, Chap. XIV.)

Treatment of symptoms.

Headache.

Cold to head.

Ice-bag. 
Heat to extremities.

Hot pack.

Hot mustard foot-bath. (For technique, see Pneumonia, Chap. IX.)

Hot drinks; e. g., lemonade.

Backache.

Hot pack.

Hot stupes.

Mustard paste or plasters.

Gastric distress.

No food by stomach.

Mustard paste or plaster to epigastrium.

Ice-bag to epigastrium.

Cracked ice to suck.

Ice-cold drinks in small quantities.

Alkaline Vichy. (See above.)

Effervescing drinks.

Champagne, $\}$ In teaspoonful or tablespoonful doses.

If pain in stomach severe and black vomit threatens give cocaine hydrochloride, gr. $\overline{\mathrm{sS}}-1(0.03-0.06 \mathrm{Gm}$.) in capsule.

Morphine sulphate, gr. 1/16-1/12 (0.004-0.005 Gm.) may be used as an adjuvant to cocaine.

If vomiting is severe morphine sulphate hypodermically, gr. $1 / 4(0.015 \mathrm{Gm}$.).

Black vomit.

Adrenalin (epinephrin), 1:1,000 in doses of $\mathrm{m} . \mathrm{i}$ to $\mathrm{m} . \mathrm{xv}$ (0.060-1 c.c.). Tincture of the chloride of iron $\mathrm{m} . \mathrm{v}(0.30$ c.c.) in limejuice and glycerin.

Calcium chloride (Guiteras) gr. $\mathrm{x}$ to $\mathrm{gr} . \mathrm{xv}(0.60-1 \mathrm{Gm}$.).

Oil of turpentine $\mathrm{m} . \mathrm{x}(0.60$ c.c.).

If black vomit threatens give cocaine hydrochloride, gr. $\overline{\mathrm{ss}}-1(0.03-$ $0.06 \mathrm{Gm}$.) in capsule.

Morphine sulphate, gr. 1/16-1/12 (0.004-0.005 Gm.) may be used as an adjuvant to cocaine.

\section{Fever.}

Needs consideration only when high or very sustained.

Cold sponges.

Kidneys.

Congestion.

Hot fomentations over lumbar region.

\section{Circulation.}

As in other acute infectious diseases.

(See summary under Pneumonia, Chap. IX.) 
Convalescence.

Usually short.

Up after temperature is normal a few days.

Increase diet as condition of stomach and kidneys warrant.

\section{Prophylaxis.}

1. Destruction of mosquitoes.

(See Malaria, Chap. XV.)

2. Isolation of the infected individual.

(See text under isolation.)

3. Protection of non-immunes against bite of stegomyia.

Noting the feeding time, in morning until about 9 o'clock, and evening after 3 o'clock.

Wear mosquito head nets, heavy gloves and leggings.

Choose as sleeping room one high up.

Sleep in screened room and screened bed.

It is well to fumigate the room before retiring.

Avoid exposure to environment of infected individuals. 


\section{CHAPTER XLIII}

\section{RAT-BITE FEVER}

THIs disease, so recently called to our attention in this country, I am convinced from my own experience with it, is not so rare that any practitioner can afford to disregard it.

It is caused as the name denotes, by the bite of a rat (or rarely of a cat, weasel or other animal), by which the sufferer is inoculated with a spirochete, spirocheta morsus muris.

In due time a symptom complex appears, offering two characteristic features, a relapsing type of fever and an eruption.

Repeated febrile attacks of 4 to 5 days, separated by afebrile intervals of a few days, more especially when these attacks are accompanied by an eruption should always lead us to enquire for a rat-bite or seek local evidences of it. This disease has long been appreciated in Japan, but has only recently elicited our attention in this country.

Not all rats are infected nor are all bites of infected animals necessarily the source of infection, for bites through clothing may be robbed of their virus or abundant bleeding free the wound; bites on exposed parts then are more likely to be infected. There is a curious similarity to rabies in these particulars. There has been no unanimity among investigators as to the causative agent; it having been ascribed to diplococci, spirilla, spirochetæ and streptothrix. Many competent men name the organism a streptothrix muris ratti, but perhaps the strongest argument is put up for the spirocheta morsus muris.

The spirochete is to be found in the blood, in the exanthem and the lymph glands, especially at the height of the fever during the first few recurrences.

Incubation. Arkin gives the average as 12 days and in some instances as short as five days. Other writers fix it at a much longer period, 6 to 8 weeks or even months; thus affording much the same discrepancies as is the case in rabies.

Symptomatology. The rat-bite may or may not have healed during the incubation period, but with the onset of active manifestations the patient experiences a burning and pain at the site of the lesion and a redness with a bluish tint and some induration develops here, surrounded by some edema. Arkin likens its appearance to extra-genital primary 
lesion in syphilis, sometimes the lesion vesiculates. From this inflamed lesion the lymphatics become involved and show streaks of red; this spreads to the regional glands, which become large and tender. Some malaise is experienced, then the patient suffers a chill, followed by an abrupt rise of temperature to $102^{\circ} \mathrm{F}$. to $105^{\circ} \mathrm{F}$., accompanied by headache and pains and aches, nausea and vomiting and in severe cases with mental disturbances, delirium and coma. The febrile period lasts three to six days and then falls by crisis with profuse sweating. During the attack the eruption appears, though in some instances it is delayed to the second or third or later relapses. It is a reddish-blue macular eruption, later becoming a little elevated, varying greatly in size from $1 / 3$ inch to huge blotches and paler in the centre than at the periphery. I mistook this eruption in one case for an erythema multiforme. It may be confined to the area about the bite or to a limited area, but as a rule is more widely distributed, appearing on face, limbs and extremities, even on the palms and soles and in rare instances in the mouth. The nervous system is often much disturbed. After the febrile attack comes an afebrile period of 2 to 6 days when the fever recurs with a fresh outburst of the exanthem, and so these febrile and afebrile periods continue, the chart taking on a characteristic appearance that should suggest the infection at once. These grow less in severity and usually last about two months but have been known to continue for years with long afebrile periods even for months intervening. In severe cases each attack is worse until a fatal issue may occur.

The blood picture is not constant. Usually there is a moderate leucocytosis from 13,000 to 20,000 , dropping during the afebrile period. Sometimes there is a marked increase in mononuclears, sometimes in polymorphonuclears and in some cases a moderate increase in eosinophiles. The diagnosis is clinched by finding the organism in the blood, at the site of the rat-bite, in the exanthem and in the lymphatic glands. The organism stains readily with Giemsa's stain, Loeffler's methylene blue and gentian violet and by other methods such as the India ink and Levaditi's. It shows the spirochetal curves of varying numbers, is short and thick in the blood and longer in the tissue and is flagellated, having a rapid movement in the blood. By inoculation of mouse or guinea-pig they may be demonstrated in five to fourteen days.

Treatment. The course of the disease is long continued, the fever often deviates from the type as given above, in some cases being continuous. The nervous symptoms may be the essential feature in the disease and the exhaustion be absent. These cases may be very acute with continued or remittent fever or the fever may be absent. In mild cases during the afebrile periods the patients are relatively comfortable 
and out of bed. In the more severe form ${ }_{3}$ much careful nursing and medical consideration is needed.

The selection of the room, its ventilation, the choice and preparation of the bed, the care of the body, the mouth and the nose are such as should be exercised in any acute infection and are to be found under Typhoid Fever, Chap. XIV.

Diet. On account of the long infection the diet should be abundant, affording 3,000 calories, or more if the patient desires it; the choice of the food depending on the presence or absence of fever or its degree. One will find suggestions in the dietary given under typhoid fever. Except at the onset of the paroxysms there is but little gastro-intestinal disturbance. Water should be administered freely. Often there is considerable thirst during the febrile periods. Fruit-juices, lemonade, orangeade and alkaline waters may be and should be offered freely.

Bowels. At the beginning of the attack a cathartic may be given of castor oil §ss. $-\mathrm{i}$ (15-30 c.c.) or salts; Epsom, Rochelle or Glauber's salt, 5 ss.-i (15-30 Gm.) which may or may not be preceded by calomel gr. i ss.-ii (0.10-0.120 Gm.) which is often better borne and even more effectual when given in divided doses of gr. 1/4 (0.015 Gm.) at quarterhour intervals. Such divided doses are better borne in nausea, and indeed, are credited with antiemetic properties. Enemata or mild cathartics may be relied on during the later stages.

Pains and Aches which are quite striking in the febrile periods and particularly in the lower extremities as well as headache, hemicrania, neuralgic pains and pain in the bitten part may be relieved by acetylsalicylic acid (aspirin) in doses of gr. x (0.66 Gm.) at 2 or 3 hour intervals or by the use of the coal tars. Of these latter acetphenetidin (phenacetin) and acetanilid are in most common use. The dose of phenacetin for these purposes is gr. iii to $\mathrm{gr}$. $\mathrm{v}(0.20$ to $0.30 \mathrm{Gm}$.) at 2 or 3 hour intervals; acetanilid gr. ii to gr. iii $(0.012$ to $0.20 \mathrm{Gm}$.) at the same intervals. I think excellent results are to be obtained by small doses, for example, gr. iss. of acetanilid at intervals of $1 / 2$ to 1 hour for six doses, then at 2 hour intervals. No coal tar should be used over a considerable period of time and only in sthenic cases, never when the eirculation is imperilled. It is well to combine them with equal or double the amount of bicarbonate of soda which lessens the irritating and toxic effects and with caffeine, citrated, in small doses to increase the anodyne effect. This should be omitted if there is insomnia. If results are not obtained in a half a dozen doses of the above, one should have recourse to more potent drugs, such as codeine phosphate in doses of gr. $1 / 8$ to gr. $1 / 2(0.008$ to $0.030 \mathrm{Gm}$.) by mouth or with more certain effect by the subcutaneous route. In very severe cases morphine may 
be used hypodermatically, but sparingly and in the least doses that are effectual, beginning with gr. $1 / 12(0.005 \mathrm{Gm}$.) of the sulphate.

Sleeplessness. As the disease is long continued and the demand for hypnotics may be considerable it is well to begin with the milder bromides gr. $\mathrm{xv}$-gr. $\mathrm{xxx}$ ( 1 to $2 \mathrm{Gm}$.), trional $\mathrm{gr}$. $\mathrm{v}$ to $\mathrm{xv}(0.33$ to $1 \mathrm{Gm}$.), chloralamid gr. $\mathrm{xx}-\mathrm{xxx}(1.33$ to $2 \mathrm{Gm}$.), adalin gr. v $(0.33 \mathrm{Gm}$.), barbital (veronal) or sodium barbital (medinal) gr. v-viiss. (0.33-0.50 Gm.). changing from time to time, remembering that often the effects carry over to the second night. Give early in the morning. If insomnia is due to pain, codeine and morphine may by used in doses given above.

For Delirium. Use morphine gr. 1/4 (0.015 Gm.) or hyoscine hydrobromide gr. 1/200 (0.0003 Gm.).

Extreme nervousness, paraesthesia and hyperesthesia call for small doses of bromides gr. $\mathrm{x}$ to $\mathrm{gr}$. $\mathrm{xv}(0.66-1 \mathrm{Gm}$.) or trional in gr. ii $(0.120$ $\mathrm{Gm}$.) doses every two hours during the day or luminal in $1 / 2$ grain doses $(0.030 \mathrm{Gm}$.), three times a day.

Dizziness-ringing in the ears, blurring of vision are occasionally symptoms for which, if the source of anxiety and restlessness, bromides may be administered.

Fever. Antipyretics should not be used, but cool sponging afford comfort and in the higher degrees of fever slush baths (see Typhoid Fever) or cold packs (see Chap. XVII) may be tried.

Skin. This requires only cleanliness and the application of drying powders.

Circulation. Failure of the circulation is to be met by vigorous digitalis medication. (For details and the treatment of collapse, see Pneumonia, Chap. IX.)

The inflamed and swollen glands may be painful. Local applications of heat, as fomentations, or cold as an ice-bag or 25 per cent. ointment of icthyol may be utilized.

The glands do not suppurate.

Specific Treatment. There is none that is worthy of the name, but as the causative agent is a spirochete, arsenic combinations are indicated. Arsphenamine (salvarsan) or the neo-arsphenamine has been used with very excellent results in many cases. I think it should be used in every case. The mode of administration is as in syphilis, intravenously, and in the same dosage, $0.40 \mathrm{Gm}$. in females and $0.60 \mathrm{Gm}$. in males, of the arsphenamine; $0.10 \mathrm{Gm}$. to each thirty pound body weight and $0.75 \mathrm{Gm}$. in females and $1 \mathrm{Gm}$. to males.

The effects seem to be the same whether given at the height of the fever or during the periods of apyrexia. Some cases are cured after a 
single dose; others require more. When the drug is effectual the relief of symptoms is immediate.

While the reported results are very gratifying, I have seen a fatal issue after repeated salvarsan injection in adequate dosage.

Mercury has also been recommended. If used one should follow the usual routine in syphilis, especially the hypodermic route.

Complications. Nephritis occasionally occurs and then is to be treated as under other circumstances. (See Scarlet Fever.) Ulcerative myocarditis has been reported and attributed to complicating streptothrix infection.

The disease is often long and exhausting. Throughout a long course the condition of the blood should be studied and iron given for anæmia. My preference is Vallet's mass (Massa ferri carbonatis) in gr. x (0.66 $\mathrm{Gm}$.) doses, three times a day or arsenic may be added, as arsenious acid gr. 1/40-gr. 1/20 (0.0015 to $0.003 \mathrm{Gm}$.) or one may use sodium cacodylate hypodermically in gr. ss. to gr. i $(0.030$ to $0.060 \mathrm{Gm}$.) doses.

Green citrate of iron hypodermically may also be tried in doses of gr. $3 / 4(0.05 \mathrm{Gm}$.).

Convalescence. Fresh air, sunlight, an abundance of food, change of climate, if it can be brought about, and treatment of the anæmia are the indications.

Prophylaxis. Cauterization with fuming nitric acid, thoroughly, even if incision has to be made for it, is advisable.

\section{SUMMARY}

\section{Treatment.}

Room, bed, care of the body, mouth and nose.

(See Typhoid Fever, Chap. XIV.)

\section{Diet.}

3,000 calories or more if desired by patient.

(For details, see Typhoid Fever, Chap. XIV.)

\section{Fluids.}

Offer freely fruit juices and alkaline waters.

\section{Bowels.}

Initial cathartic of castor oil, 5 ss.-i (15-30 c.c.) or

Salts; Epsom, Rochelle or Glauber's, 5 ss.-i (15-30 Gm.).

Calomel gr. iss.-ii $(0.10-0.120 \mathrm{Gm}$.). May be given in divided doses of gr. $1 / 4(0.015 \mathrm{Gm}$.) at quarter-hour intervals.

Enemata or mild cathartics in later stages. 
Pains and aches.

Acetyl salicylic acid (aspirin) gr. $\mathrm{x}(0.66 \mathrm{Gm}$.).

Acetanilid, gr. ii to iii $(0.120-0.20 \mathrm{Gm}$.).

Any one of these is given at two to three hour intervals.

Excellent results are obtained by using small doses at frequent intervals; e. g., acetanilid gr. iss. at $1 / 2$ hour intervals for six doses, then every two hours for six doses.

Use no coal tars except in the sthenic period and when the circulation is good.

May be combined with sodium bicarbonate and citrated caffeine in small doses.

If results are not obtained, use codeine phosphate, gr. 1/8 to gr. 1/2 (0.008 to $0.030 \mathrm{Gm}$.); or if necessary use morphine sulphate, gr. 1/12 $(0.005 \mathrm{Gm}$.) hypodermically. Increase the dose sparingly.

\section{Sleeplessness.}

Begin with milder hypnotics. Bromides, gr. $\mathbf{x v}-\mathrm{gr}$. $\mathrm{xxx}$.

Acetphenetidin (phenacetin) gr. iii to $\mathrm{v}(0.20-0.30 \mathrm{Gm}$.).

\section{Delirium.}

Morphine sulphate..............gr. 1/4

Hyoscine hydrobromide........... gr. 1/200 (0.0003 Gm.).

\section{Nervousness.}

Bromides small doses............ gr. $\mathrm{x}$ to $\mathrm{xv} \quad(0.66-1 \mathrm{Gm}$.). Trional gr. ii $(0.120 \mathrm{Gm}$.) every two hours during the day.

Luminal gr. 1/2 (0.030 Gm.) three times a day.

\section{Dizziness.}

Bromides as above.

\section{Fever.}

Cold sponges or packs.

(See Scarlet Fever, Chap. XVII.)

Skin.

Cleanliness.

Drying powders.

Circulation.

Digitalis. (See Pneumonia, Chap. IX.)

\section{Specific Treatment.}

Salvarsan administered as in syphilis, intravenously, $0.40 \mathrm{Gm}$. to females and $0.60 \mathrm{Gm}$. to males.

Mercury. 
Anemia.

Iron as Vallet's mass gr. $x$ three times a dây.

May add arsenious acid, gr. 1/40-1/20 (0.0015-0.003 Gm.).

Sodium cacodylate, gr. ss.-i (0.0020-0.060 Gm.).

Green citrate of iron gr. 3/4 (0.05 Gm.) three times a day.

Convalescence.

Fresh air sunlight, good food.

Treatment of anemia.

Prophylaxis.

Cauterization with fuming nitric acid thoroughly. 


\section{CHAPTER XLIV}

\section{TRENCH FEVER}

A DISEASE which, contracted in the trenches of the great war, gets its name from the fact. It is essentially a military problem. It will be appreciated from the brief description that follows that its mode of conveyance and spread demand certain conditions peculiarly favored by the exigencies of a military campaign; but, no doubt, now that the war has stamped its picture on our minds it may be appreciated as the cause of certain obscure fevers of civil life. The disease is conveyed by the body louse. The virus is present in the feces of this parasite and is very resistant, withstanding drying of the feces. At first it was believed that the only method of inoculation was through excoriations caused by scratching, but it has been shown that it also may be conveyed by the bite of the louse. It is a filterable virus (Swift). It requires an incubation period in the louse of 7 to 8 days. The louse remains infective up to three weeks after its infection and possibly longer. The incubation period in man is 5 to 20 days. There are very few at any age who are immune to the disease and the immunity after an attack is very short lived, in some instances barely outlasting the attack.

Symptomatology. This varies greatly in individual cases. It is characterized by the general discomforts attendant upon most acute infectious processes, so striking in some cases as to be likened to influenza with its headaches, backaches and pains in the extremities, and flares in temperature or to Dengue with its severe pains in the extremities and post orbital pains; an illusion heightened by the saddle-back temperature of some of the cases, relatively slow pulse and its rash. This sudden onset occurs in about half the cases. Shin pains seem quite characteristic, even if not constant, and pain in muscles and joints may be mistaken for rheumatism. The conjunctivæ are injected. Some authors speak of lateral nystagmus as characteristic; others as being no more common than in other acute diseases. Giddiness may be a feature of the onset. Sweating and polyuria may occur.

The pulse is relatively slow in comparison with temperature, though some observers find its acceleration corresponds with the temperature; the spleen is enlarged and palpable; a characteristic, but evanescent rash occurs in the early febrile periods. It consists of small macules 
like rose spots, disappearing on pressure and distributed especially on the trunk, coming in crops in each relapse.

The blood usually shows a leucocytosis, 14,000-16,000, large mononuclears and lymphocytes are relatively increased, though in some cases decided leucopenia is observed. Leucocytosis is the rule with the fever, and mononuclear increase in the afebrile periods. The temperature may be continuous, of short duration, or lasting two or three weeks and resembling a typhoid or paratyphoid fever curve; on the other hand it may be interrupted, a relapsing type of temperature; there are three or four days of fever, 12 to 24 hours of normal temperature, another period of from 3-4 days and recovery, or with shorter febrile periods of 24 to 30 hours occurring at intervals of $5-6$ days.

Prognosis. The prognosis is good. The disease is not fatal; about 90 per cent. get well promptly on symptomatic treatment; 10 per cent. run a long course.

Treatment. Even if delousing has been accomplished, isolation is necessary, as the infection is conveyed not only by the louse, but by the virus excreted with the urine and saliva of the patient.

If a case were seen in civil practice, one would urge the necessity of rest in bed, even in the lighter cases, as probably having a beneficial effect on the course. The choice of room, bed, care of patient, and preliminary catharsis with enemas and light cathartics later are determined by the same considerations and exercised in the same manner as in Typhoid or Pneumonia or Influenza. (See Chaps. XIV or IX and XII.)

The urine and sputum, both of which are infectious, should be destroyed by heat or the use of such antiseptics as cresol or lysol. (See Typhoid Fever, Chap. XIV.)

The diet should be sufficient to meet the caloric needs of the patient (see Chap. II) and chosen much as Typhoid Fever. (See Chap. XIV.) Water should be freely administered, or fruit drinks, lemonade, orangeade or alkaline drinks.

Aches and pains are to be met by the use of aspirin, the coal tars, acetphenetidin (phenacetin), acetanilid, antipyrin or in more severe cases by codeine or even morphine, for the detailed administration of which see Grippe (Chap. XI).

Insomnia. 'For use of hypnotics, consult Chapters on Grippe (XI), or Pneumonia (IX) or their summaries.

Nervousness. May be lessened by warm sponge baths and the administration of bromides.

In long standing cases anemia ensues. This should be met with a sufficiency of good food, air, sunlight and the use of iron, as Vallet's 
mass or Blaud's pills gr. $\mathrm{x}(0.66 \mathrm{Gm}$.) three times a day with arsenic, as arsenious acid gr. $1 / 40(0.0015 \mathrm{Gm}$.) at the same intervals, combined with iron or as cacodylate of soda hypodermically, gr. ss. $-\mathrm{i}(0.03-0006$ Gm.) a day.

Considering that spirochetes have been suspected as the infecting organism it seemed reasonable to try arsphenamine (salvarsan), but its use was not followed by gratifying results.

Serum from convalescent cases was administered without success.

Richter reported that the intravenous injection of 10 c.c. of 1 per cent. collargol every two or three days during the acute stages was followed by a rapid cure. (Quoted from Swift.)

Complications and sequelæ. A large percentage of cases of D. A. $\mathrm{H}$. (disordered action of the heart) or cardio-vascular asthenia or effort syndrome, in the British Army had trench fever, which played its rôle in all probability in the acquisition of that condition. Small doses of thyroid have been recommended as likely to lessen the incidence of this condition. Leg pains and lumbar pains were sometimes exceedingly persistent.

The patient should not be discharged until the dangers of relapses have probably passed, as he will constitute a pool of virus for the infection of lice and so of other individuals in contact with him.

He should be kept in bed at least a week after the last relapse. $\mathrm{He}$ should then be gotten up gradually and put through graduated exercises. A change of environment, fresh air, sunlight, good food are all helpful. The convalescence is often slow and some five per cent. manifest the neurasthenic symptoms mentioned as a sequel and which probably represent continued infection. These cases require much attention, tact and ingenuity in the handling.

Prophylaxis. Trench fever is transmitted by the louse, Pediculus Humanus, and hence it is very important that any patient suffering from trench fever be carefully deloused. The hair of his head, axillæ, pubis and chest should be shaved and burned. The patient should then be bathed with warm water and soap followed by an alcohol (50 per cent.) sponge. He should then be put to bed in another room in another bed known to be free from vermin. The patient should be daily inspected for nits and lice.

The room previously occupied by the patient and all articles of furniture, clothing, bedding and mattress should be disinfected even though there is no trace of lice, for the virus of trench fever is excreted in the urine and saliva of patients and in the feces of the louse and may still be viable. Those who carry out the disinfection should wear rubber gloves and avoid rubbing the contaminated material on the skin. 
Disinfection may be effected by moist heat at a temperature not lower than $120^{\circ} \mathrm{F}$. for one-half hour.

All persons who have been in contact with patients should be examined for lice and nits and if infected should be deloused and watched for 30 days, for a person may develop the disease after he is freed from the lice.

Bedding and mattresses as well and clothing should be disinfected by heat. (See Typhus Fever, Chap. XXVIII.)

If heat is not used, soaking in a 2 per cent. solution of liquor saponatus cresoli fortis or 2 per cent. solution of crude phenol and soft soap, equal parts at any temperature above $32^{\circ} \mathrm{C}$. for 20 minutes or 1 per cent. solution at $60^{\circ} \mathrm{C}$. to $63^{\circ} \mathrm{C}$. for 20 minutes.

The patient once infected is a source of virus and as the case is often chronic and the relapses very far apart, carriers of this virus are pretty sure to carry the disease into civil life.

\section{SUMMARY}

\section{Treatment.}

Isolate the patient until delousing is completed; when this is accomplished isolation is no longer necessary.

Rest in bed.

Choice of room.

Preparation of bed

Care of patient

Catharsis.

(See Typhoid Fever, Chap. XIV, Pneumonia, Chap. IX, or Influenza, Chap. XII.)

Diet.

Should contain 3,000 or more calories if well taken. (For items of dietary, see Typhoid Fever, Chap. XIV.)

\section{Drinks.}

Water freely, lemonade, orangeade, alkaline drinks, weak tea, cocoa, buttermilk.

\section{Aches and pains.}

Acetyl salicylic acid (Aspirin), gr. v-x (0.33-0.66 Gm.), every 2 or 3 hours;

or:

Acetphenetidin (Phenacetin), gr. iii-v (0.2-0.33 Gm.);

or:

Antipyrin, gr. ii-iv (0.125-0.250.Gm.);

or:

Acetanilid, gr. iss.-gr. iii (0.10-0.2 Gm.) at the same intervals. (See also, Grip, Chap. XI.) 
In severe cases.

Codeine phosphate or sulphate, gr. 1/8-1/2 (0.008-0.030 Gm.) by mouth or hypodermically;

or:

Morphine sulphate, gr. 1/8-gr. $1 / 4$ (0.008-0.015 Gm.), hypodermically;

\section{Insomnia.}

(For choice and use of hypnotics, see Grippe, Chap. XI, or Pneumonia, Chap. IX, or their summaries.)

\section{Nervousness.}

Warm sponge baths.

Bromides in doses of $\mathrm{gr} . \mathrm{xv}-\mathrm{xxx}(1-2 \mathrm{Gm}$.) in water.

\section{Anemia.}

Good food and sufficient.

Fresh air and sunlight.

Iron as Blaud's pills or Vallet's mass (Massa ferri carbonatis) gr. $\mathrm{x}$ $(0.66 \mathrm{Gm}$.).

Arsenic-well to combine it with the iron.

Arsenious acid (Arseni trioxidum), gr. $1 / 40$ (0.0015 Gm.)

Sodium cacodylate, gr. ss. $-\mathrm{i}(0.030-0.060 \mathrm{Gm}$.) given hypodermiccally.

Collargol.

Intravenous injection of 10 c.c. of a 1 per cent solution, every 2 or 3 days during the acute stages. (Richter.)

Sequelæ.

Disordered action of the heart (D. A. H.), the effort syndrome or cardio-vascular neurasthenia. Probably represents low-grade infection.

Good food, fresh air, graduated exercises, tactful handling.

Small doses of thyroid.

Leg pains and lumbar pains.

Local measures.

Aspirin in small doses.

\section{Convalescence.}

Keep in bed at least a week after last relapse.

Get up gradually.

Graduated exercises.

Good food.

Fresh air.

Change of environment, if possible.

\section{Prophylaxis.}

Delousing. (See Typhus Fever, Chap. XXVIII.)

Clothes submitted to steam under pressure. (See Typhus Fever, Chap. XXVIII.) 
Shave pubic and axillary hair. Cut hair on head close.

Bedding and mattresses submitted to heat;-steam under pressure.

Bed clothing if not disinfected by heat may be soaked in 2 per cent. solution of liquor saponatus cresoli fortis.

or:

2 per cent. solution of crude phenol and soft soap, equal parts at any temperature above $32^{\circ} \mathrm{C}$. for 20 minutes;

or:

1 per cent. solution at $60^{\circ}$ C. -63 C. for 20 minutes. 


\section{CHAPTER XLV}

\section{SEPTICAMIA AND PY EMIA}

Although this condition affords a fairly definite picture, it is by no means easy of definition.

Septicæmia is a state of infection in which the infecting organism is not only encountered in the blood, but is multiplying there, and yet evidences of infection with recovery of the infecting organism from the blood does not necessarily constitute a septicæmia but may exemplify a far less serious infection called bacteriæmia and the same organism may be concerned in one or the other condition. For example, the pneumococcus may be, often or usually is, isolated from the blood of a lobar pneumonia and its presence in the blood in no way modifies the picture, course or issue of the pneumonia or on the other hand it may be causal of a true septicæmia.

What relationship of infecting organism and host determines the relatively innocuous bacteriæmia and the profoundly serious septicæmia is not yet clear.

If suppurative foci appear in a septicæmia the process is spoken of as pyæmia.

Toxæmia is a term used to express the effects on the body of the toxins or products of bacterial activity irrespective of whether that activity is local in some tissue or general throughout the body in the blood.

As the essence of septicæmia and pyæmia is the same (presence and multiplication of virulent organisms in the blood), and their difference determined only by the presence or absence of multiple suppurative foci they will be considered together.

The organisms commonly responsible for septicæmia are streptococci; and, indeed, it is a streptococcus septicæmia that the unqualified term "septicæmia" usually connotes to the ear of the general practitioner, but the invasion of the blood by many other forms of bacteria may constitute a septicæmia; e. g., the staphylococci; and a staphylococcus septicæmia is usually characterized by the multiplicity of abscesses accompanying it (pyæmia), the pneumococci, the gonococci, the typhoid bacilli, the colon bacilli, the influenza bacilli, pyocyaneus, proteus, Friedlander's bacillus, micrococcus tetragenus, anthrax, bacillus aërogenes capsulatus, meningococcus. 
The general symptoms of septicæmia and pyæmia (septico-pyæmia) are due to the toxæmia; and the focal and local lesions to the thrombophlebitis and emboli producing infarcts, and abscesses. The valves of the heart and adjacent structures, like the veins, may be the seat of bacterial activity and furnish infective thrombi (bacterial or ulcerative or malignant endocarditis); and the effects they have upon the organs concerned constitute the pathology of the disease.

The early symptoms are due to the toxæmia and the toxæmia is intense; and, in the absence of a local lesion, suggest one of several conditions such as typhoid fever, acute miliary tuberculosis, pneumonia with tardy consolidation, æstivo-autumnal malarial fever.

In children B. coli pyelitis and, more rarely, grippe and otitis media simulate a septicæmia.

A more accurate diagnosis in the early stage awaits the result of blood examination; its cytology, serum reactions and most of all its culture returns.

Later, heart murmurs, hemorrhagic spots (petechiæ), splenic, pulmonary and renal infarcts, cerebral embolism, suppurative foci, retinal hemorrhages, assure a septicæmia; while the other suspected conditions would take on gradually their classic characterization.

The early symptoms are fever, often initiated by chills (and the chills may be repeated or long continued, and, indeed, are characteristic of pyæmia); loss of appetite, delirium or stupor or the typhoid state.

\section{TREATMENT}

Rest. Insistency on rest in its broadest significance should be considered imperative. This means a quiet room, comfortable bed, competent nursing, freedom from worries and anxieties, exclusion of visitors.

Room. Whether in the house or in the hospital, it should be chosen with reference to light, ventilation, conveniences of bath-room, access to the open air by veranda or porch, and remoteness from the noises of the street and of the household. Light is an important therapeutic agent, the operation of which is too little known in its details. Individuals respond differently to light both in its quality and quantity. One has but to consult his own personal experiences in health to realize what an extraordinary effect upon our moods and emotions and so upon our bodily functions light and shade, sunshine and shadow have, aside from the direct effects of the rays of light upon the body cells themselves. In disease these effects of light should be taken into consideration and 
the grateful effects of morning or evening sun and the irritating action of the noon-day glare be provided for or avoided.

The destructive effect of sunlight on germ life is too well known to dilate upon here.

Fresh Air. Maximum ventilation is desirable and a corner room with a number of windows affords this. All that has been said of fresh air in pneumonia obtains here both as regards the rationale of its therapeutic action and the technique of exposing the patient to the open air. (See Pneumonia, Chap. IX.)

The room should be stripped of all unnecessary furnishings and be devoted entirely to the patient.

The use of the same room to sleep or rest in by the nurse is to be deprecated because of the bad results to both in ways readily imagined.

If it is not possible to have more than one nurse, she should be relieved at suitable periods by members of the family, to secure her rest in a quiet room remote from the scene of her duties.

Bed. For details, see Pneumonia, Chap. IX.

Diet. While the most virulent cases of septicæmia run a rapid course and are accompanied by anorexia that makes feeding very difficult, the vast majority run a fairly long course that compels knowledge of the theoretical needs of the patient and the arrangement of a dietary to meet them. The theoretical needs have been detailed in Chap. II, while arrangements of dietaries to fulfil them may be seen under Typhoid Fever, Chap. XIV, Pneumonia, Chap. IX, and elsewhere. (Consult Summaries.)

To epitomize, we endeavor to achieve 3,000 calories daily in the diet and as much more as the patient handles well. The proteid content should be about 80 grams, but need not be rigidly fixed; milk, eggs, bread, cereals, broths (especially those fortified with farinaceous flours), purées, form the basis of this diet and the use of sugar in the milk, in jellies, on cereals, in fruit drinks, and the use of fats, such as cream in the milk, on cereals and butter on bread add to the caloric content of the diet.

Variety is secured by using various milk preparations; butter-milk, koumys, matzoon, zoolak, junket, ice-cream; of eggs by preparing them in different forms; coddled, custard, egg-nog, egg-white, poached; of cereals by using different kinds, incorporating them in broths, or as some of the invalid or infant foods, so numerous on the market; the bread as bread and butter, toast, dry or wet, milk toast, bread and milk, crackers, biscuit.

Frequency of feeding should be every two or three hours as the patients bear it best. 
Fluids should be given in abundance; all that the patients want and to those delirious, stuporous or too sick to ask for it, it should be offered at least every hour. It may be given as water, plain, or if grateful, aërated; as lemonade, orangeade or other diluted fruit juices or Imperial drink. (See summary for formula.)

When first seen the bowels should be freely opened either by castor oil or a salt, Epsom, Rochelle, sodium phosphate, in doses of one or the other of 3 ss. -1 (15-30 c.c. or Gm.) or by calomel gr. ii $(0.12 \mathrm{Gm}$.), best in divided doses, followed by the above or by Hunyadi or equivalent water, or milk of magnesia $\xi$ i to ii (30-60 c.c.) or liquor magnesii citratis 5 viii-xii (240-360 c.c.). Later the bowels should be kept open by the use of the milder salines mentioned above or by enemata.

Care of the Body. For all the details of care of skin, mouth, nose, eyes, genitals, consult Pneumonia, Chap. IX, or Typhoid Fever, Chap. XIV, or the Summaries.

Local suppurative processes in pyæmia, of course, indicate surgical procedures.

\section{SYMPTOMATIC TREATMENT}

Fever. The nature of the infecting organism, the virulency of the toxins, the presence or absence of suppurative foci, all determine differences in the fever curve. The temperature may run continuously high with slight excursions, or show wide excursions, accompanied by rigors and sweating, especially in pyæmia, or may be relatively low in the more chronic cases.

Hyperpyrexia but rarely occurs; but a sustained high temperature of $104^{\circ} \mathrm{F}$. to $105^{\circ} \mathrm{F}$. is more common and has the same deleterious effect upon the vital centres. These cases indicate the local application of cold in the shape of cold packs or cold sponges repeated at three to four hour intervals if necessary, and prolonged until some effect is made upon the temperature. The most beneficial effects of these baths is upon the circulatory and nervous system. When the rise of temperature is initiated by a chill, hot drinks may be administered and heat applied to the feet and body surface until the rigor has passed.

The sweats that follow the febrile exacerbations call for luke warm sponges, change of linen and application of sterile dusting powders.

Sometimes the excursions are so tremendous that collapse may ensue and demand the application of heat, hot drinks, diffusible stimulants, such as the stronger water of ammonia held near the nose on a towel, hypodermics of adrenalin, camphor or caffeine or strophanthin.

At the onset there may be some vomiting, but as a rule this is not 
prolonged or violent enough to demand interference. Cracked ice may be sucked and counter-irritation applied to the epigastrium in the shape of mustard paste.

Should it become more persistent, as it may, one has to modify the diet, giving the food in smaller quantities more frequently, cutting down the cream in the milk or lessening the intake of sugar, trying various modifications of milk. If the stomach tube is well borne, lavage with warm water may be helpful and the food administered by the tube.

In persistent cases rectal feeding may be required.

Nervous Symptoms. Restlessness, insomnia, stupor, delirium, headaches are all common.

Delirium may be low, muttering, a typhoid state or noisy and wild.

The items of importance to be considered in this state are fresh air, cold sponges or packs, a sufficiency of food and particularly an abundance of water. When it is difficult to give the patient enough by the mouth ( 3 to 5 quarts) one should have recourse to colonic irrigations and especially the drip or drop method of Murphy.

Noisy delirium is also to be combated by the application of the ice-bag to the head.

The patient should never be left alone, lest he do himself harm and in the wild cases restraint becomes necessary. The most humane method is by so attaching the bed covers to the side of the bed that his hands and arms cannot escape or the patient sit up, but allowing his body some freedom of movement. At times it is imperative to tie the extremities to the bed; knots must be so made that they will not slip and tighten upon the wrists and ankles to their injury.

Drugs. At times are necessary. If the condition is one rather of restlessness and excitability bromides in doses of 15-30 grains (1-2 Gm.); either the potassium salt or the mixed salts of potassium, sodium and ammonium in equal parts may be administered late in the afternoon and repeated in the evening, as the condition is likely to be worse at this time.

In more severe delirium codeine is indicated, best as the soluble phosphate in doses of gr. 1/4 $(0.015 \mathrm{Gm}$.) hypodermically. In still more severe cases morphine is indicated, given hypodermically as the sulphate gr. $1 / 8$ to gr. $1 / 4(0.008-0.015 \mathrm{Gm}$.) or Majendie's solution $\mathrm{m}$. iv to $\mathrm{m}$. viii (gr. 2/15-4/15;0.008-0.015 Gm. morphine).

If morphine is not well borne or excites as it does in the rare case, one may have recourse to hyoscine hydrobromide in doses of gr. 1/200 to gr. $1 / 100(0.0003-0.0006 \mathrm{Gm}$.) hypodermically.

Insomnia is met by much the same measures, ice to the head, hot 
drinks, heat to the feet, if they are cold, and this is especially so in young subjects; by bromides, trional gr. $\mathrm{xv}(1 \mathrm{Gm}$.), chloralamid gr. $\mathrm{xxx}(2 \mathrm{Gm}$.), any one of which may be repeated in two or three hours, or by codeine and morphine.

Headaches may be due to the irritating effects of the toxins or to a localization of bacterial activity in the brain or its coverings. It may be relieved by the application of an ice-bag or ice-coil or by the use of drugs. The use of coal tar derivatives is to be deprecated as depressing to the circulation. A very severe headache is better controlled by morphine.

Convulsions may occur, but are due in most cases to thrombophlebitis or embolism and not likely to be repeated or prolonged. Morphine sulphate hypodermically in doses of gr. $1 / 4$ to gr. 1/3 (0.015-0.02 $\mathrm{Gm}$.) is the best emergency drug. If convulsions are repeated the same procedures are indicated as in convulsions from other causes. (See Scarlet Fever, Chap. XVII.)

Circulation. Sooner or later the circulatory apparatus is imperilled either by the effects of the toxins on the centres or on the myocardium or by a localization of the process on the endocardium, a malignant endocarditis.

For a detailed use of circulatory stimulants, see Pneumonia, Chap. IX. It is my growing belief that the value of no circulatory stimulant in acute infectious disease is comparable to digitalis and strophanthin. The latter I use in emergency gr. 1/90 to gr. 1/60 (0.00075-0.001 Gm.) intramuscularly or intravenously and repeat in six to twelve hours if needed. Follow with digitalis $\mathrm{m}$. $\mathrm{xxx}(2$ c.c. $)$ of the tincture [ $=$ gr. iii $(0.2 \mathrm{Gm}$.$) of the leaf] or its equivalent in dosage of some other official$ preparation three or four times a day until its pharmacological or toxic action is obvious.

I do not believe in the use of alcohol.

Further treatment is directed to the accidents of the disease. These are determined by localization of the process by thrombophlebitis, by embolism and in pyæmia by suppurative foci.

The process may localize on the endocardium or may originate at this site and the treatment becomes that of malignant endocarditis, its attendant embolisms and circulatory impairment.

It may localize upon the brain, giving rise to a meningitis, and is to be treated like a cerebro-spinal meningitis (see Chap. XXV) symptomatically.

Thrombophlebitis may occur in the veins of the extremities and demand rest, slight elevation of extremities, applications of heat, as fomentations, and protection with cotton batten. 
Thrombophlebitis may occur in deeper organs, giving rise to secondary results. It is this occlusion of the vein by inflammatory products that furnishes a nidus for the organism and with the heart valves furnishes the infective emboli, which are carried to the brain and cause hemiplegia, monoplegia, aphasia or other disturbances of cerebral function.

The treatment is only tentative. Emboli may plug the central artery of the eye and cause blindness, plug the pulmonary arteries and cause infarcts or abscesses and empyema; may plug the coronary arteries, giving rise to sudden and alarming symptoms of dyspnœa and precordial distress or even death. Treatment of conditions following pulmonary infarcts is that of Pneumonia (see Chap. IX) with surgical intervention when suppuration intervenes.

Splenic infarets are common; often of diagnostic import. The pain accompanying them is relieved by local applications of heat or in the worst cases by morphine.

The veins of the liver may become involved and a pylephlebitis, giving the worst prognosis, ensue.

The abdominal viscera may become involved and abscesses result. Visceral abscesses, pylephlebitis, meningitis and endocarditis, all, are of the most ominous significance.

The bones may be affected and osteomyelitis demand surgical interference.

The joints may be the site of a mild arthritis and should be treated as such (see Rheumatic Fever, Chap. III) or may suppurate and need evacuation. The muscles and skin, especially in staphylococcus infection, may be the site of multiple abscesses and require incision.

The kidneys may become involved. Infarcts with hematuria occur, needing only expectant treatment, or an acute nephritis, to be treated as such (see Scarlet Fever, Chap. XVII), or the kidneys become the site of suppuration and, if localized, are amenable to surgery.

Hemorrhages into the skin, petechiæ, are highly diagnostic, especially those in the conjunctivæ. These latter occur peculiarly when the heart is involved.

Erythemata also occur and icterus, but none of these require treatment.

In pyæmia the original site of infection and abscesses secondary to the infection indicate, of course, surgical measures.

Certain differences depend on the nature of the infecting organism.

Of the common forms of infection streptococcus septicæmia is supposed to be the worst, though there is little difference in prognosis 
between it and staphylococcus septicæmia, if, indeed, the latter is not the worse.

Streptococci affecting the heart valves, or the meninges or causing pylephlebitis or visceral abscess lead to an almost certainly fatal issue; although endocarditis of the more chronic type, "endocarditis lenta," "bacterial endocarditis," due to the streptococcus viridans of Schotmüller seems occasionally to get well and even one apparently true case of streptococcus meningitis has been reported cured.

Particularly ominous, too, are the streptococcus septicæmias occurring in small pox, scarlet fever, diphtheria and secondary to surgical procedure.

Of better prognosis is the streptococcus septicæmias of the puerperium.

Staphylococcus Septicæmia is almost certainly fatal, its course being characterized by multiple abscesses.

Pneumococcus septicæmia gives little chance of cure.

Among other forms of septicæmia are those due to diphtheria bacilli (though streptococcus, staphylococcus and pneumococcus are commonly recovered from the blood with it), to typhoid bacilli, colon bacilli, bacillus pyocyaneus, meningococci, bacilli dysenteriæ and even bacilli fusiformis.

Specific Treatment. For only two of the organisms mentioned have we specific sera, bacillus diphtheriæ and pneumococcus type I; for some of the others, sera containing certain dimly visualized antibodies have been elaborated.

Scarlet Fever. Streptococci may be recovered from many cases that run a relatively mild course, a bacteriæmia, and it is difficult to establish a criterion for true septicæmia in the sense of this discussion and, hence, to judge of the efficacy of streptococcic sera.

Nicoll, discussing this subject, accepts a certain clinical picture commencing forty-eight hours or longer after the onset, as constituting sepsis; "no fall of temperature or a renewed rise; abnormal swelling of the mucous membrane of the throat; with or without superficial sloughing; profuse nasal discharge; marked cervical adenitis; rapid, often irregular pulse; mental apathy; active or low delirium."

$\mathrm{He}$, working in the Scarlet Fever Hospital of the Department of Health of New York City, used a serum prepared under the direction of Dr. Park. Eleven strains of streptococci recovered from the blood, throat and other sources from scarlet fever patients were used in the animals to elaborate this serum; hence, a polyvalent serum.

His dose of this particular serum was 200 c.c.; in young children, 50-100 c.c. injected into loose tissue, repeated 2 to 3 times at 6 to 8 hour intervals depending on the results obtained. 
His results warrant his advice to use the serum in all very severe cases of scarlet fever.

Similar efforts with polyvalent sera have been made for some years past, especially in European clinics. One of those best known in Europe is the Moser serum so persistently advocated by the late Professor Escherich of Vienna.

My own impression is that polyvalent streptococcus sera are well worth using in this condition, with the realization that the strains used may or may not be biologically identical with the organisms responsible for the individual case; hence, may be more or less efficacious, depending in a measure on chance.

The dose of the commercial serum is that used by Nicoll. More recently serum from patients convalescent from scarlet fever has been used with some show of success. The dose is just the same as that for the commercial sera.

Vaccine Therapy. Our knowledge of the possibilities and limitations of vaccine therapy is too limited to discuss didactically the application of this measure to septicæmia.

Reasoning that the body is already overwhelmed with virulent toxins, and that the addition of more in the shape of vaccines (endotoxins) is illogical we pause before using them; on the other hand the belief that the response in terms of antibodies may be local at the site of injection urges us to take a chance. Indeed, its use in the severer cases is that of appeal to the last resort and is so far justifiable, but then only with a clear knowledge of the theory and uses of vaccine therapy.

In the more chronic cases the use of vaccines is more encouraging and some cures of malignant endocarditis by the use of vaccines have been reported from reliable sources.

Autogenous vaccines should be used when possible. If the organism cannot be recovered from the blood, local lesion or other reliable source, one may use a polyvalent vaccine with a lesser expectation of success.

Puerperal Sepsis. As I have said the outlook in this form of sepsis is better than in those already mentioned.

Here again the polyvalent streptococcic sera may be used in the streptococcus cases and the vaccines, too, with a greater hope of success, though here, too, it is in the subacute cases that the best results follow.

G. T. Western, studying 96 cases of puerperal sepsis, 39 of whom gave positive cultures (36 were streptococci), treated with vaccine 56 with a mortality of 32 per cent., and treated without vaccines 44 with a mortality of 55 per cent. Even better results have been reported by others. Others report less enthusiastically. I imagine that in this 
particular form of sepsis the management of the case in other respects plays a major part in the results. What the management of a puerperal sepsis case should be I am not competent to judge in the midst of arguments pro and con, but, influenced by environment, quote Ward from the Sloan Maternity Hospital, New York: "A. Obtaining adequate uterine drainage, first, by simple vaginal and intrauterine saline douches. This proving insufficient to control the process, explore the uterus once, and once only to make sure it is empty of foreign products, using the finger for the exploration, and with a minimum of trauma to the uterine tissues.

"B. Treat expectantly secondary foci as they arise, and C. Support the general condition of the patient." (J.A.M. A., April 12, 1913.)

Staphylococcus Septicæmia may be treated on the same principles with sera and vaccines with even less anticipation of results.

Pneumococcus Septicæmia. Hope from sera depends on the determining strain.

Grouping pneumococci according to the classification followed at the Rockefeller Institute, types I and II against which sera have been elaborated may be combated by the use of the sera as in Pneumonia, but practically it has been found that type I serum alone is efficacious.

Types III (mucosus) and IV (a heterogenous group) yield no active sera.

As a vaccine, group I (Neufeldt) may be used as in pneumonia and some believe that the antibodies provoked are in some measure operative against the other organisms, or a polyvalent vaccine may be used.

Again it must be insisted that these measures are adopted by the desire to leave no stone unturned rather than by anticipation of favorable results.

Gonococcus, B. coli, streptococcus mucosus and other forms of septicæmia may be attacked in the same manner, but with a full realization of the paucity of published results and with the sense of responsibility towards the patient in handling these powerful agents.

Transfusion of Blood. At the present time I am using transfusion in septicæmia and in severe infections that may not be truly classed as such, on account of a remarkable experience of my own, the first case in which to my knowledge the method was used for this purpose.

A case of sepsis in a child of twelve, seemingly moribund, recovering after five transfusions from the father's blood.

The case is reported with others by Dr. Lindeman in the Journal of the American Medical Association, October 31, 1914.

Reports of the experience of others since and my own observations 
of improvement in other cases in which the transfusion has been done make me feel that the measure has a place in the therapy of septicæmia.

It is more than probable that the blood of healthy individuals contain in a large per cent. of cases immune bodies to most of the common infections.

The studies of the antitoxin content of normal blood by the Schick reaction for Diphtheria reveals such astonishing positive results, that in the absence of a practical test for other immune substances the use of healthy blood would seem entirely reasonable.

In addition the introduction of fresh blood must be welcome to the patient in an anemic state so decided as that of septicæmia, while other theoretical considerations could be cited for argument's sake.

My experience with transfusion leads me to believe that its value lies in its application to those diseases whose natural history is to recover under favorable conditions, e. g., it would be of value in puerperal sepsis, while it holds out no hope in cases of acute malignant endocarditis.

Methods of transfusion much more facile than the old direct method have been elaborated of late for which special treatises must.be consulted. See Lindeman, Am. Jour. Diseases of Children, Vol. VI, No. 1, Lindeman, Jour. A. M. A., June 7, 1919, Vol. 72, pp. 1661-1665; Satterlee and Hooker, Archives of Internal Medicine, January, 1914, and Unger, Jour. A. M. A., 1915, LXIV, 582; Sanford, Med. Clinics of North America, Nov., 1919, p. 801; Losee, A.J.M. Sc., Nov., 1919, No. 5, Vol. CLVIII, p. 711 .

The donors must be healthy, show a negative Wassermann and prove to have a blood congenial to that of the patient (non-hemolytic).

For the technique of these tests one should consult hand-books of laboratory method. (See Hiss and Zinsser's Bacteriology.)

Theoretically, a parent's blood should be better than a stranger's as more likely to represent the biological characteristics, but this is by no means without exception.

Again, theoretically, a brother's or sister's blood should be better than the parents', but at times it is absolutely uncongenial.

The amounts depend on age, weight, the patient's reaction, and the donor's condition. In my first case 200 c.c. to 300 c.c. were given at weekly intervals for five weeks. In older cases 800 c.c. to 1,000 c.c. may be given. If a good response is obtained it is better to use the same donor than to change, as every now and then in spite of satisfactory laboratory tests an hemolysis in the veins of the recipient occurs with dangerous or disastrous results.

Ottenberg and Libman, however, place implicit reliance in the results of the agglutination and hemolytic test and say, 
"In no case in our series in which hemolysiss or agglutination did not occur in the test-tube were any untoward symptoms observed which could be attributed to these phenomena."

Their article on Blood Transfusion in the Am. Jour. Medical Sciences, July, 1915, is most illuminating.

If the infecting organism is known it is possible to inoculate a donor with vaccines made from it and enhance the protective power of blood before he donates.

Human serum has been advocated especially by Welch.

See New York State Medical Journal, November, 1913.

As the serum contains most of the immune bodies an argument might be made for its selection in preference to whole blood and it certainly is less likely to result in unfortunate accidents.

On the other hand I feel that the whole blood is more likely to give the maximum benefit than serum alone.

Prophylaxis. This begins with proper surgical consideration of foci of infection, but the internist must remember that these foci may be latent in tooth, tonsil, sinus and other anatomical nooks and corners and include all these in his routine examination of patients.

\section{SUMMARY}

\section{Treatment}

Rest.

Quiet room, comfortable bed, competent nursing, freedom from worries and anxieties, exclusion of visitors.

\section{Room.}

Provide for light, air, bath-room facilities, approach to porch or verandah if possible.

\section{Bed.}

Hospital type, half or three-quarters width, woven wire springs, firm mattress.

\section{Diet.}

At onset, don't push.

Later, provide for 3,000 calories, or as much as is well taken, and for 80 grams of proteid.

Milk, buttermilk, koumys, matzoon, zoolak, junket, ice-cream.

Eggs, coddled, poached, custard, egg-nog, egg white.

Cereals, different varieties thoroughly cooked, used in broths, various invalid and infant foods.

Bread, bread and butter, toast, milk toast, bread and milk, crackers, biscuit. 
Cream, butter, cocoa, sugar in milk, in cereals, in jellies, in drinks. Give food every two or three hours.

Fluids.

Water, plain or aërated, lemonade, orangeade, diluted fruit juices.

Imperial drink ( 1 tablespoonful of cream of tartar to 3 pints of boiling water, add sugar and lemon peel to flavor).

\section{Bowels.}

When first seen.

Calomel in divided doses, followed by salts or salts alone or castor oil.

Calomel, gr. 1/4 (0.015 Gm.), every quarter hour for six doses; follow in two or three hours by

Epsom, Rochelle, or Glauber's salt or sodium phosphate; of either 5 ss. to i (15-30 Gm.), or milder salines.

Such as Hunyadi water.

Milk of magnesia, $3 \mathrm{i}-\mathrm{ii}$ ( $30-60$ c.c.),

Liquor magnesii citratis, 3 viii-xii (240-360 c.c.),

Castor oil, 5 ss. $-\mathrm{i}$ (15-30 c.c.).

or

A salt or castor oil without calomel in same or double doses.

Later.

Milder salines mentioned above.

Enemata.

Care of the body.

(See Pneumonia, Chap. IX, or Typhoid Fever, Chap. XIV.)

Local suppurative processes.

Surgical procedure.

Symptomatic treatment.

Fever.

Cool or lukewarm sponges.

Hyperæmia.

Cold sponges.

Cold packs.

Cold baths.

Chills.

Heat to feet and body surface.

Hot drinks.

Collapse.

Heat to body and feet.

Hot drinks.

Diffusible stimulants.

Stronger water of ammonia on a towel held to nose.

Adrenalin $(1: 1000) \mathrm{m}$. $\mathrm{x}$ to $\mathrm{m}$. $\mathrm{xv}(0.65-1 \mathrm{Gm}$.) intramuscularly.

Camphor gr. v (0.30 Gm.) in oil, intramuscularly. 
Caffeine sodium benzoate or sodium salicylate gr. v $(0.30 \mathrm{Gm}$.) in water intramuscularly.

Strophanthin, gr. $1 / 120$ to gr. $1 / 90(1 / 2-3 / 4$ mg.) intravenously or intramuscularly.

Vomiting.

Cracked ice.

Mustard paste 1 in 2, 3 or 4 to epigastrium.

Cut down on diet, and fats and sugars in diet.

Lavage and gavage.

Rectal feeding.

Nervous symptoms.

Delirium.

Fresh air.

Cold sponges or packs.

Abundance of water.

Ice-bag to the head.

Never leave patient alone.

Restraint in mild delirium.

Bromides, gr. $\mathrm{xv}$ to gr. $\mathrm{xxx}(1-2 \mathrm{Gm}$.).

Codeine phosphate, gr. $1 / 4(0.015 \mathrm{Gm}$.) hypodermically.

Morphine sulphate, gr. $1 / 8$ to gr. $1 / 4(0.008-0.015 \mathrm{Gm}$.) hypodermically:

Hyoscine hydrobromide gr. 1/200 to gr. 1/100 (0.0003-0.0006 Gm.) hypodermically.

Insomnia.

Bromides, gr. $\mathrm{xv}$ to $\mathrm{gr} . \mathrm{xxx}$ (1-2 Gm.).

Trional, gr. xv (1 Gm.). Repeat in two or three hours if necessary.

Chloralamid, gr. $\mathrm{xxx}(2 \mathrm{Gm}$.). Repeat in two or three hours if necessary.

Codeine phosphate, gr. $1 / 8$ to gr. $1 / 4(0.008-0.015 \mathrm{Gm}$.) hypodermically.

Morphine sulphate in persistent cases, gr. $1 / 8$ to gr. $1 / 4$ (0.008-0.015 Gm.) hypodermically.

Headaches.

Ice-bag or ice-coil.

Morphine gr. $1 / 8$ to gr. $1 / 4(0.008-0.015 \mathrm{Gm}$.) in severe cases.

Convulsions.

Morphine sulphate, gr. $1 / 4(0.015 \mathrm{Gm}$.) hypodermically.

(See Scarlet Fever, Chap. XVII.)

Circulation.

Digitalis $\mathrm{m}$. $\mathrm{xxx}$ of the tincture $(2$ c.c.) or $₹ \mathrm{ss}$. (15 c.c.) of the infusion or gr. iii $(0.2 \mathrm{Gm}$.) of the leaf three or four times a day.

Strophanthin in emergency, gr. 1/90 to gr. 1/60 (0.00075-0.001 $\mathrm{Gm}$.) intravenously or intramuscularly and repeat in six to twelve hours if needed.

(See Pneumonia, Chap. IX.)

Accidents of the disease.

Endocarditis.

Meningitis. 
(See Cerebro-spinal Meningitis, Chap. XXV.)

Thrombophlebitis.

Rest.

Elevation of extremity affected.

Application of heat, fomentations.

Protection with cotton batten.

Embolism-infarcts.

Of the lung.

(See Pneumonia, Chap. IX.)

Of the spleen.

For pain.

Local application of heat.

Morphine sulphate, gr. $1 / 8$ to gr. $1 / 4$ (0.008-0.015 Gm.)

Arthritis. hypodermically.

(See Rheumatic Fever, Chap. III.)

If joints suppurate, evacuate.

Muscles, abscesses, incise.

Kidneys.

Infarcts-expectant treatment.

Nephritis.

(See Scarlet Fever, Chap. XVII.)

Specific treatment.

Bacillus diphtheriæ septicæmia.

Diphtheritic antitoxin.

Large doses intravenously.

Scarlet fever.

(See severest cases, Diphtheria, Chap. XVIII.)

Polyvalent streptococcic sera.

(See text.)

Vaccine therapy.

(See text.)

Puerperal sepsis.

(See text.)

Staphylococcus septicæmia.

Sera.

Vaccines. $\}$ (See text.)

Pneumococcus septicæmia.

(See text.)

Transfusion of blood.

(See text.)

Use of human serum.

(See text.)

Prophylaxis. 


\section{CHAPTER XLVI}

\section{ERYSIPELAS}

Erysipelas is a disease, deriving its name from its chief clinical characteristic, a redness of the skin due to an inflammation of this structure, resulting from an invasion by the streptococcus pyogenes, sometimes called streptococcus erysipelatis of Fehleisen.

Morphologically and culturally there is no difference between streptococcus pyogenes and streptococcus erysipelatis, but it is probable that the affinity of the organism for the skin is determined by some biological differences and these biological differences constituting strains, and, by mutations, varieties and species, affords one of the most interesting studies of the present moment.

Not only is the skin involved but in rarer instances the mucous membranes. The manner of skin involvement is a very definite one and entirely different from a lymphangitis or cellulitis although the three conditions are but different expressions of the invasion of the skin by the streptococcus pyogenes. The intensity of the inflammation varies, too, from the usual brawny indurated area of erythema, to vesiculation, pustulation, suppuration and necrosis. Any trauma, however trivial, and sometimes too slight to be detected, affords the portal of entry. Again, that something so difficult of definition, which we call susceptibility and affinity determine certain sites of election in the skin. By far the most common site of the infection is the skin of the face (facial erysipelas) constituting 88 per cent. of the whole; the next most common site is the leg. In infants it usually begins about the umbilicus. As a rule it is confined to the part first affected, but may pass from site to site over the whole body and is spoken of as migrating erysipelas.

Infections after trauma, after operation and in parturient women constitute another class.

The symptoms are those attributable to streptococcus toxins, in any case of acute infection by these organisms; after an incubation of three to ten days there is a sudden invasion with chilly sensations or a distinct chill, a rapid rise of temperature, malaise, headache, nausea or vomiting, anorexia, etc. The temperature may run from $102^{\circ} \mathrm{F}$. to $104^{\circ} \mathrm{F}$. and be very irregular, it may remain high, $104^{\circ} \mathrm{F}$. to $106^{\circ} \mathrm{F}$. a few days and then become irregular and fall by lysis, or it may remain high throughout 
and fall by crisis like a pneumonia. The duration of the attack is one to two weeks.

The local symptoms are those of a slowly advancing deep scarlet, brawny indurated area of inflammation with a clear-cut border as a rule. It may advance up or down a limb and has no respect for direction of lymphatic flow. The skin is hot, tense, burning.

In facial erysipelas the usual site of entry is the mucous membrane of the nose and the skin over the nose is first affected. Out on either side, shaped like a butterfly, spreads the expanding area of inflammation, until the whole face, eyes, ears and scalp are involved. As it recedes the swelling diminishes and the skin looks dry and burned.

\section{TREATMENT}

Isolation. Opinions differ about the necessity for isolation. The spread in a ward, in a family and among attendants is relatively rare, so rare as to make the strict rules of quarantine observed in such diseases as small pox, scarlet fever and diphtheria unnecessary if not unjustifiable; but willing to be charged with an ultra conservatism, I prefer to isolate the patient in a separate room in a hospital or in a house, forbidding visitors and having a special nurse or member of the family, who shall be instructed to burn dressings, properly sterilize her hands after contact with the patient and change her clothes before mingling with the family or others. The physician has but to remember that his hands and instruments are the means of conveyance of infection and he should therefore wear rubber gloves in dressing the lesions. Double precaution must be taken by a surgeon and by the obstetrician.

Room. The room chosen should be light and well ventilated and as remote from the rest of the household as possible while fulfilling these requirements.

The facilities of an adjacent bathroom are desirable and access to a verandah; porch or balcony a valuable adjuvant to the patient's comfort and welfare.

An open hearth affords the best means of disposing of infected dressings.

Bed. The hospital type is preferred, a half or three-quarter iron bedstead with woven wire springs and a firm mattress. For technique of bed-making, see Pneumonia, Chap. IV.

Diet. During the early hours anorexia forbids forcing the food. The swollen condition of the face adds to the difficulty of eating and, of course, when, in the rare case, the pharynx is involved, the difficulty is greatly enhanced. When food can be entertained an effort must be 
made to meet the theoretical requirements (see Chap. II). This is the more imperative in the long continued cases; more particularly the migrating forms.

Water and drinks made from fruit juices should be administered freely.

Care of the Body. A sponge bath with soap and warm water should be given daily. The mouth should receive special attention. (For details, see Chap. IV.)

The eyes should be carefully cleansed with boric acid solution 2 per cent. to 4 per cent., and as in the facial form the eyelids may be greatly swollen, especial care must be given to this part of the toilet; cold cloths wet with boric acid solution may be applied to the swollen lids with comfort to the patient.

A cathartic should be given when the patient is first seen. Epsom salt (magnesium sulphate), Rochelle salt (sodium potassium tartrate) or Glauber's salt (sodium sulphate) may be given in doses of $\varlimsup_{\text {ss. }}-\mathrm{i}$ (15$30 \mathrm{Gm}$.) in three-quarters of a glass of water.

One may precede this by fractional doses of calomel gr. 1/10 to gr. $1 / 4(0.006-0.015 \mathrm{Gm}$.) every ten to fifteen minutes until gr. i to gr. iss. (0.060-0.10 Gm.) are taken; the salt following in two to three hours. The smaller doses are especially indicated if there is nausea, as they are credited with being antiemetic. Later one may rely on milder salines, such as liquor magnesii citratis 5 viii (240 c.c.), milk of magnesia 亏ii (60 c.c.), a Seidlitz powder or Hunyadi water to keep the bowels open; or enemata may be given in preference.

Treatment of Symptoms. Prodromal symptoms of malaise, loss of appetite, headache and a little temperature require no consideration. At the onset the discomforts of a high temperature may be relieved by cool sponges and, in very high fever or long sustained, cold baths may be used, much as in Typhoid Fever.

Headachemay be relieved by theice-bag, by small doses of phenacetin gr. iii $(0.20 \mathrm{Gm}$.) every hour for three to four doses or acetanilid gr. iss. $(0.10 \mathrm{Gm}$.) at the same intervals. Later on coal-tar derivatives should not be used.

Nervousness and sleeplessness may be met by bromides in doses of gr. $\mathrm{xv}$ to gr. $\mathrm{xxx}(1-2 \mathrm{Gm}$.) or chloralamid gr. $\mathrm{xx}$ to $\mathrm{gr}$. $\mathrm{xxx}(1.30-2$ $\mathrm{Gm}$.) given in the early evening and repeated if needed late in the evening.

If there is delirium, morphine sulphate hypodermically in doses of gr. $1 / 8$ to gr. $1 / 4(0.008-0.015 \mathrm{Gm}$.) is indicated or in the sthenic period hyoscine hydrobromide gr. $1 / 200$ to gr. $1 / 100(0.0003-0.0006$ Gm.) hypodermically. 
If there are evidences of circulatory failure support must be offered in the same way as in Pneumonia. (See Chap. IX.)

\section{LOCAL TREATMENT}

Facial Erysipelas. Many measures and applications have been advocated. To enumerate them would be only to confuse and in no way to edify or help. I will mention only those that seem to me to have value. My personal preference has heretofore been for ichthyol which I have used in 25 per cent. to 50 per cent. ointment or painted on pure, applied after carefully washing the face with soap and warm water, but the face is more likely to be dry and uncomfortable under the pure ichthyol. Over this one applies gauze, and a roughly made mask with tapes, to keep it in place, may be applied over all. The advance of the lesion has been thought to have been modified by compression of the healthy skin adjacent. The most common way of effecting this is by the application of collodion. This I have done with most of my cases in combination with the ichthyol treatment, sometimes feeling that I had effected something, again seeing no progress whatever. The experience of Inata and Woodyatt at Camp Cody during the war would seem to accentuate the value of this effort to limit the spread by the use of collodion (U. S. P.) applied to the skin within half an inch to an inch in advance of the line of induration. The application must be thorough, leaving no break by which the lesion may spread, repainting if such occurs. They combine this treatment with the application to the area affected of a cloth wet with a cold saturated solution of magnesium sulphate.

My own belief is that it is not easy to convince oneself of the usefulness of any local measure in a self-limited disease, running so short a course and having so low a mortality as facial erysipelas.

Erdman gave the average duration of 500 facial cases at Bellevue Hospital as between six and seven days and the mortality as 5.38 per cent.

Objections to the ichthyol are its odor, its appearance and the besmearing of everything about the patient. The substance is easily removed, however, from the skin or from fabrics by soap and water. Vaseline alone affords a certain degree of comfort.

Erdman with his large experience is convinced that nothing local is better than simple wet dressings. He uses boric acid solutions, keeping the solutions cold with ice and applies frequently to the face gauze dressings dipped in them.

Powders. Bland powders such as zinc oxide, starch, boric acid or a 
combination of them, equal parts, dusted on the skin liberally and covered with a light dressing of non-absorbent cotton relieves some of the disagreeable sensations.

Erysipelas of the extremities runs a little longer course than the facial cases. Erdman's thirty-three leg cases averaged 10.88 days with a decidedly higher mortality (27.37), than the facial cases (5.38 per cent.).

Ichthyol is to be used in the same manner as in facial cases.

A very excellent treatment of these cases is the wet dressing, especially that of aluminum acetate. ${ }^{1}$ Boric acid solutions are also suitable for these cases, but I fail to see any advantage in the use of the stronger antiseptics, such as bichloride of mercury.

Migratory Erysipelas. This is a very severe form of the disease, both because of the extent of the body involved and because it includes so many of the infant cases.

The mortality in Erdman's fifty-six cases was 50 per cent. The duration averaged 14.44 days.

Ichthyol and the wet dressings, may be used in these cases, so far as the extent of the lesion makes these measures practical.

Erysipelas in Infants. Some 8 per cent. of the cases of erysipelas occur in infants under two years of age. The affection is far more serious than in adult life. New-borns are especially liable to the disease. The portal of entry is the umbilical cord. The type is migrating; death usually occurs at the end of six to ten days. Complications are likely to occur.

Treatment begins with prophylaxis and the proper care of cord, buttocks and vulva.

The treatment consists in the use of ichthyol, wet dressings, vaseline and powders as in adult cases detailed above. The mortality of infants under two years averages about 40 per cent.

From two years to sixteen years children are singularly free from the infection (about 1 per cent.).

Specific Treatment. Drug specifics in disease have become in these days of searching criticism a woefully depleted company. Among those to deserve banishment from the category are iron and quinine in erysipelas. I can see no good, whatsoever, from their use, but some disadvantages, such as gastric irritation.

Vaccines. One is loath to abandon the hope held out by the use of vaccines in erysipelas and yet the most extensive series of cases

${ }^{1}$ Formula Aluminis............................ 3 i ss. $\quad(6.00)$ Plumbi acetatis.................... Aq. q. s. ad........................ (1000.00) 
(ninety-five) of which I am cognizant conducted by clinicians and bacteriologists with whom I am personally acquainted and for whose work I have the highest regard leads to these conclusions.

"From our experience with vaccines in erysipelas I must state that the duration of the disease was not at all lessened, the mortality remained at the same level, and there was no immunity guaranteed against recurrence, against spreading of the lesion, nor were complications, such as cellulitis and abscesses prevented; from the statements furnished by the patient, moreover, I could not gather that there was any amelioration of the subjective symptoms." (Erdman, J.A.M.A., December 6, 1913.)

Transfusion of Blood. In severe cases I should consider the problem as one of septicæmia and hold the same attitude towards the measure as in that condition. (See Septicæmia, Chap. XXXIV.)

Complications. Many of these are surgical and to be treated on surgical principles; phlegmon, gangrene, abscesses, otitis media, edema of the larynx, periostitis, purulent arthritis, suppurative adenitis, sinusitis.

Others are medical such as Pneumonia and Pleurisy (see Chaps. IX and VIII), Acute Nephritis (see Scarlet Fever, Chap. XVII), Bronchitis, Tonsillitis, Arthritis (see Chaps. III, V and VII), Endocarditis, Septicæmia and Pyæmia (see Chap. XLV). Most important among these as a cause of death are Septicæmia and Pneumonia.

Immunity seems difficult to establish and the same individual is prone to suffer from the disease again and again. Moreover, relapses are of common occurrence.

Prophylaxis. Proper attention should be given to lesser traumata that furnish the portal of entry, and particularly in those individuals who have already suffered from the disease.

Such slight traumatisms may be looked for in the nasal mucosa and in the skin about the nose, ear and elsewhere about the face.

A vaccination wound may be the site of infection, or an old ulcer of the leg. In infants it is commonly the navel, excoriated buttocks or vulva. Again operative wounds may be infected and every care should be exercised by the obstetrician to avoid contact with erysipelas.

It is a wise precaution to disinfect a patient's clothes before his discharge from the sick-room. 


\section{SUMMARY}

Isolation.

Safer to isolate.

Room.

Light and well ventilated.

Remote from noise of household.

Near bathroom.

Accessible, if possible, to veranda or porch.

Open hearth desirable.

Bed.

Hospital type; one-half or three-quarters iron bedstead.

Woven wire springs; firm mattress.

(For technique of bed-making, see Pneumonia, Chap. IX.)

Diet.

Early hours; don't force.

Prolonged cases. (See Chap. II or Chap. IX.)

Water, lemonade, orangeade, alkaline and aërated waters freely.

Care of the body.

(For details, see Pneumonia, Chap. IX.)

Eyes, 2 per cent. to 4 per cent. boric acid solutions.

Cold compresses to swollen lids.

\section{Bowels.}

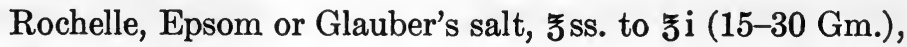

or

Calomel, gr. $1 / 10$ to gr. $1 / 4(0.006-0.15 \mathrm{Gm}$.), every ten to fifteen minutes until gr. $\mathrm{i}$ to gr. iss. are taken, followed in two or three hours by salts in doses named above.

Later.

Milder salines.

Liquor magnesii citratis, 5 viii (240 c.c.).

Milk of magnesia, 5 ii ( 60 c.c.).

Seidlitz powder.

Hunyadi water.

Enemata.

\section{Treatment of symptoms.}

Fever.

If high, cool sponges.

Headache.

Ice-bag to head.

Phenacetin, gr. iii $(0.20 \mathrm{Gm}$.) every hour for three to four doses, in early stages.

Acetanilid, gr. iss. $(0.10 \mathrm{Gm}$.) at the same intervals, in early stages. Nervousness. 
Bromides, gr. $\mathrm{xv}$ to gr. $\mathrm{xxx}(1-2 \mathrm{Gm}$.), either potassium or mixed bromides.

Insomnia.

Chloralamid, gr. $\mathrm{xx}$ to gr. $\mathrm{xxx}(1.30-2 \mathrm{Gm}$.),

or

Trional, gr. $\mathrm{x}$ to gr. $\mathrm{xv}(0.60-1 \mathrm{Gm}$.$) , in early evening and repeat$ if needed.

Delirium.

Morphine sulphate, gr. $1 / 3$ to gr. $1 / 4(0.008-0.015 \mathrm{Gm}$.$) , hypoder-$ mically.

Hyoscine hydrobromide, gr. $1 / 200$ to gr. $1 / 150(0.0003-0.00045$ Gm.), hypodermically, only in sthenic stage.

Circulation.

(See Pneumonia, Chap. IX.)

Local treatment: use of rubber gloves.

Facial erysipelas.

Paint margin of advancing lesion with collodion. (See text.)

Apply to area affected 25 to 50 per cent. ichthyol or cold saturated solution of magnesium sulphate; vaseline.

Wet dressings of boric acid 2 per cent. to 4 per cent. Solutions kept cold with ice.

Powders. Zinc oxide, boric acid and starch equal parts.

Erysipelas of the extremities.

Ichthyol: may be combined with the application of collodion. (See text.)

Twenty-five per cent. to 50 per cent. or pure ichthyol.

Wet dressings.

Aluminum acetate.

Boric acid solutions.

Migratory erysipelas.

Ichthyol.

Wet dressings.

Erysipelas in infants.

Prophylaxis.

Care of cord or excoriated buttocks or vulva.

Ichthyol.

Wet dressings.

Powders.

Vaseline.

Specific treatment.

(See text.)

Vaccines, very little value.

Transfusion of blood.

(See Septicæmia, Chap. XLV.)

\section{Complications.}

Phlegmon, gangrene, abscesses, otitis media, edema of the larynx, periostitis, purulent arthritis, suppurative adenitis, sinusitis. Treat on surgical principles. 
Pneumonia and Pleurisy. ( (See Chaps. IX and VIII.)

Acute nephrîtis. (See under Scarlet Fever, Chap. XVII.)

Bronchitis, tonsillitis, arthritis. (See Chaps. III, V, VII.)

Endocarditis, septicæmia and pyæmia. (See Chap. XLV.)

Prophylaxis.

Attention to slight traumata about nose, ears, face; to old ulcers; to cord, buttocks and vulva in infants.

Disinfection.

Patient's clothes should be disinfected. 


\section{INDEX}

Abdomen, protection of, in bacillary dysentery, 358

Abdominal muscles, paralysis of, in poliomyelitis, 600

Abdominal support in pertussis, 526, 533

Abortive poliomyelitis, 591

Abscess, liver, in amebic dysentery, 377, 378, 379

lung, in epidemic influenza, 254

retropharyngeal differentiated from diphtheria, 464

Abt, on diarrhea in infants, 360

Acetanilid, 221

chemistry of, 221

in bronchitis, 103, 109

in epidemic influenza, 244

in erysipelas, 774

in grip, 218, 231

in Malta fever, 659

in mumps, 547

in pleurisy, 118, 126

in poliomyelitis, 596, 605

in rheumatism, 45

in rhinitis, 72,76

in small pox, 614

in tonsillitis, 83,89

in trench fever, 752, 754

toxic effects of, 223

Acetphenetidin, 45, 66, and gee Phenacetin

Acetylsalicylio acid, 42, and see Aspirin

Acidosis, 46

from salicytates, 46

in epidemic influenza, 251, 267

in rheumatism, 46

Aconite in febrile conditions, 6

in tonsillitis, 83,89

Adenitis in diphtheria, 473

in glandular fever, 556,558

in measles, 499,509

in scarlet fever, 412,437

in tonsillitis, 86

Adenoids, 80

attention to in bronchitis of children, 108, 111 removal of in prophylaxis of pneumonia, 193

in rheumatism, 62

in whooping cough, 537

Adrenalin, in asthmatic attacks in bronchitis of children, 108, 111

in bacillary dysentery, 365,373

in coryzs, 73

in diphtheria, 467,482

in epidemic influenza, 243,245, 248, 249, 267

in pertussis, 534,542

in pneumonia, 144, 145, 177, 178, 204, 248

in rhinitis, $73,74,77,78$

in scarlet fever, 401, 406, 435

in sinus involvement of grip, 228, 233

Age, calorie requirements and, 12

in relation to rheumatism, 30

Agglutination method, in pneumonia, 130

Agramonte, food formula in yellow fever, 737

Air cushions for patient in bacillary dysentery, 357

Albuminuria complicating measles, 501

from salicylates, 40

in diphtheria, 473

in malaria, 336

in scarlet fever, 414

Alcohol, in diphtheria, 471

in malaria, 343

in scarlet fever, 401

in typhoid fever, 299

Alcohol sprays, for itching, in small pox, 612

Algid malaria, 336, 349
Alimentary canal, care of, see under the several diseases

Alkaline salts, as diuretics in scarlatinal nephritis, 420

Alkaline treatment for rheumatism, 46

Alkaline waters, in yellow fever, 738

Allergie following revaccination for small pox, 619

Amberg and Rowntree, on creatinin, 23

Amebic dysentery, $\mathbf{3 7 4}$

appendicostomy in, 383,386

bed in, $355,357,370,375$

care of body in, $356,370,375$

of bowels in, $359,371,376,38$

carriers of, 382,386

complications of, 382

diet in, 358, 368, 371, 374

emetine treatment of, 376,354

empirical treatment of, 375

entamceba coli in, 374

histolytica in, 374

hepatitis in, $377,378,379,385$

ipecac in, 376, 38

pathology of, 375

prophylaxis of, 383,386

quinine treatment of, 381,385

relapses in, 380,385

rest in, $354,370,375$

room in, $356,370,375$

sequelæ of, 382

specific organism of, 374,384

summary of treatment of, 383

surgery in, 383,386

symptomatic treatment of, 386

symptomatology of, 375

Amido-bodies, 24, 34

Ammonia, for prevention of collapse in malaria, 325

for prevention of collapse in pneumonia, 177

for prevention of collapse in septicemia, 760

Ammonium salts, in pneumonia, 166

Anaphylactic reaction in pneumonia, 134, 145, 196

Anderson on yellow fever, 738

Anemia following diphtheria, 474, 485

following typhoid fever, 319

in malaria, 337,351

in rheumatism, 57,68

in scarlet fever, 423

Anesthetic for lumbar puncture in cerebrospinal meningitis, 570

Angina, in diphtheria, 465

in scarlet fever, 398,432

Vincent's, 91, and see Vincent's angina

Anthrax, 678

aim of treatment, 679

bed in, 682,685

care of body in, 683,685

cauterization of pustule, 679

circulation in, 683,685

conveyance of, 678

convulsions in, 684

delirium in, 684,686

diarrhes in, 684, 686

diet in, 683

disposal of discharges, secretions, etc., in, 683

excision of pustule in, 679,680

expectant treatment in, 680

headache in, 683,685

intestinal, 679

isolation in, 682

lesion of, 678

local treatment of, 679,684

organism of, 678 


\section{INDEX}

Anthrax, prophylaxis of, 684,686 rest in, 682

restlessness in, 683,685

room in, 682,685

sleeplessness in, 683,685

specific treatment of, $681,682,684$

summary of treatment of, 684

supportive treatment of, 682

symptomatic treatment of, 683

vomiting in, 683,685

Anti-anthrax serum, 681

Antifebrin. See Acetanilid.

Anti-lepral, 672

Anti-plague serum, 636

Anti-pneumonic serum, 132, 133

Antirabic treatment, 703

Antipyretics,

chemistry of, 221

in febrile conditions, $6,7,9$

toxic effects of, 223

Antipyrin, in bronchitis, 109

in coryza, 72, 73

in fibrinous pleurisy, 118, 126

in influenza, $218,231,245$

in laryngitis, 98,100

in measles, 498

in pertussis, 529,540

in rheumatism, 45

in rhinitis, $72,73,74,76,77,78$

in tonsillitis, 83,89

in trench fever, 752, 754

Antiseptics, in treatment of wounds, 722

Antitoxemic treatment of fever, 1

Antitoxin, administration of, 458, 480

in cerebrospinal meningitis, 567,573

diphtheria, 454

death following use of, 459

disagreeable results of, 458

dosage of, $454,457,479$

early administration of, 457

evidences of improvement due to, 458

immunizing dose of, 460,481

precautions in use of, 481

preparation of, 454

unit of, 454

for hemorrhage of typhoid fever, 296

for laryngeal diphtheria, 461, 481

for laryngitis complicating measles, 498, 508

in pneumonia, 130

paralysis prevented by, 471

tetanus, $715,722,725,727$, and see Tetanus antitoxin

Antitoxins, 453

Antitussin, in pertussis, 532

Antrum, involvement of, in coryza, 75

in influenza, 228, 233

in rhinitis, 75,79 , and see Sinusitis

Apathy in encephalitis lethargica, 271

Aphthæ epizooticæ, 693, and see Foot and mouth disease

Apparatus for paralysis in poliomyelitis, 602

Appendicostomy in amebic dysentery, 383 , 386

Appetite, 11

Appetit-saft, 34

Apple, baked, food value of, 17

Argyrol, in coryza, 73

in rhinitis, 73,77

Arsenic, for anemia in malaria, 339, 340

for convalescence from Malta fever, 660 in chorea, 60

in malarial cachexia, 339

substitute for quinine, 340

Arthritis in erysipelas, 340

in mumps, 551

in pneumonia, 191

in rheumatism, 51

in scarlet fever, 413,417

in septicemia, 763,771

Ascites, in scarlatinal nephritis, 415, 421

Ashburn and Craig, on dengue, 641 Ashurst and John, on tetanus, 711, 714,
717

Asiatic cholera, 646, and see Cholera, Asiatic
Aspiration, in pleurisy with effusion, 120, 127 in pleurisy and empyema in epidemic influenza, 253,268

technique of, 120

Aspirin, constitution of, 60

in bronchitis, 104, 109

in chorea, 60

in coryza, 72

in encephalitis lethargica, 274

in epidemic influenza, 245

in fibrinous pleurisy, 117, 126

in glandular fever, 556

in Malta fever, 659

in pneumonia, 145

in rheumatism, 42

in rhinitis, 72,76

in scarlatinal arthritis, 413

in tonsillitis, 83,89

in trench fever, 752,754

toxic dose of, 43

Assimilation in acute infectious diseases, 18 efficiency of processes of, 3

Asthma, after administration of serum, 145

Astringents in diarrhea of bacillary dysentery, 364

Atropine, in Asiatic cholera, 648

in asthma, after administration of serum in pneumonia, 145

in bacillary dysentery, 365,373

in diphtheria, 473

in lumbar puncture, 572

in pertussis, 530

in photophobia of measles, 494, 501

in pneumonia, 144, 145, 175, 178

in poliomyelitis, 600

in pulmonary edema of epidemic influenza, 249,267

in rheumatism, 57

in sinus involvement of influenza, 228

in tetanus, 720

Auricular fibrillation in epidemic influenza, 257, 269

Auto-extubation, 464

Auto-serotherapy in chorea, 61

in pleurisy with effusion, 124,127

Bacillary dysentery, 353

bed in, 355, 357, 370

care of body in, 356,370

of bowels in, 359,371

carriers, 369

change of environment in, 368

chronic form of, 366,373

collapse in, 373

diagnosis of, 354

diarrhea in, 364,372

diet in, $358,368,371,374$

duration of, 366

empirical treatment of, 361,371

heart in, 366

nausea in, 360

pain in, 362

pathology of, 354

precautions of nurse in, 356,370

of physician in, 356,370

prophylaxis of, 369,374

rest in, 354

room in, 356,370

saline treatment in, $359,362,367,372$

serum in, 360,371

specific treatment of, 360,371

subacute form of, 366,373

summary of treatment of, 370

symptomatic treatment of, 362,372

tenesmus in, 363,372

therapy of, 354

topical applications in, 362, 372

ulcers in, 367,373

vaccine therapy in, $361,366,371,373$

vomiting in, 360

water in, 359

Bacilluria in typhoid fever, 300

Backache in smallpox, 614

in yellow fever, 738,742

Bacon, food value of, 17 
Bacteremia, 757

Baking for paralysis of extremities in poliomyelitis, 601

Barker and Sladen on infectious jaundice, 732

Baruch on hydrotherapy, $5,84,97,157,159$

Bass on blackwater fever, 343

on malaria, 328,329

Bastedo on alkaline salts in rheumatic fever, 43

Baths. See under the several diseases.

as antipyretic measure, 19

Brand, 288

in pneumonia, 161

in typhoid fever, $5,288,291$

mustard, 546

Ziemssen's, 291

Bath-water, disinfection of, 281, 310

Beans, string, food value of, 17

Bed, see under the several diseases

Gatch, 147

importance of in rest, 2

technique of making, 279

Bed linen, disinfection of, 281

Bed pan, disinfection of, 281

in dysentery, 357

in epidemic influenza, 243

Bedsores, in cerebrospinal meningitis, 581

in typhoid fever, 280,310

Belladonna, idiosyncrasy for, 530

in mumps, 548,553

in pertussis, 529,540

in sinus involvement in influenza, 228

in tenesmus in bacillary dysentery, 363

Belt, abdominal, in pertussis, 526, 533

Benzoin, in bronchitis, 105, 109

in glanders, 689

in laryngitis, 96

in membraneous angina in scarlet fever, 401

in pertussis, 527

Benzyl benzoate, in amebic dysentery, 381, 385 chloride, in leprosy, 672 in pertussis, 532

Bercovitz, on treatment of leprosy, 673

Besredka's method of desensitization in pneumonia, 136

Bethea's method in amebic dysentery, 378

of giving quinine in malaria, 329

Bilious remittent fever, 336

Bismuth, for diarrhea, in bacillary dysentery, 364,372

in epidemic influenza, 246

in infectious jaundice, 730

in plague, 636

in typhoid fever, 294

for nausea, in cerebrospinal meningitis, 565

in scarlet fever, 422

in typhoid fever, 295

for noma, in measles, 499

for retching, in smallpox, 615

for vomiting, in cholera, 648

in Malta fever, 659

in scarlet fever, 397

in typhoid fever, 295

in amebic dysentery, 382, 386

in foot and mouth disease, 695

in infectious jaundice, 730

Bitter bush, in treatment of amebic dysentery, 382

Bites, treatment of old. 709

Black vomit of yellow fever, 739, 742

Blackwater fever, 343,352

prophylaxis of, 345,352

summary of treatment, 352

symptoms of, 344

treatment of, 344,352

Bladder, care of. See under the several diseases

Blake, on typing of sputum, 132

Blaud's pill, for anemia, in diphtheria, 474, 484 in scarlatinal nephritis, 423, 441

in trench fever, 755

for convalescence from measles, 502,511

from mumps, 551

from pertussis, 537

in glandular fever, 557

in malaria, 339,35
Blindness, due to quinine, 333

Blisters, application of, 50

in fibrinous pleurisy, 116, 126

in rheumatism, 50

Blood, in amebic dysentery, 375

in encephalitis lethargica, 272

normal human, in scarlet fever, 409

typing of, in pneumonia, 130

Blood pressure, effects of cold water on, 5 of fresh air on, 4

Blood transfusion in erysipelas, 777

in septicemia, 766

method of, 766

Bloodgood, on anthrax, 681

on rabies, 701

on tetanus, 713,720

Body, care of. See under the several diseases

Body louse, in typhus fever, 624, 626

Body mechanism for conversion of energy, 27

Body surface and calorie measurement, 13

Bordet on pertussis, 520

Bowels, care of. See under the several diseases

Bradycardia, in epidemic influenza, 257, 269

Bran bath in scarlet fever, 395

Brand bath, contraindications to, 291

in febrile conditions, 5

in pneumonia, 161

in typhoid fever, 288

method of, $28 \mathrm{~s}$

Bread, food value of, 17

Break-bone fever, 641, and see Dengue

Brill's disease, 625

Bromides, for convulsions, in pertussis, $\mathbf{5 3 6}$ in tetanus, 719

for delirium, in typhus fever, 629, 632

for headache, in pneumonia, 170, 202

for insomnia, in cerebrospinal meningitis, 566

in encephalitis lethargica, 275

in epidemic influenza, 245

in Malta fever, 659

in pertussis, 531,533

in pneumonia, 169

in rheumatic fever, 37

in Rocky mountain spotted fever, 666

in scarlet fever, 407

in septicemia, 761

in smallpox, 415,423

in tonsillitis, 84,89

in typhoid fever, 298, 316

for nervous symptoms, in erysipelas, 774

in measles, 495,506

in septicemia, 761

for restlessness, in cerebrospinal meningitis, 566

in encephalitis lethargica, 275

in scarlet fever, 407

in poliomyelitis, 596,605

per rectum, in rabies, 706, 709

Bromoform, in pertussis, 532

Bronchiectasis, in epidemic influenza, 255

Bronchitis, acute, 101

aches and pains in, 103, 109

care of bowels in, 103, 109

children, management of, 107, 110

cough in, 102,106, 110

counterirritation in, 104, 109

cupping in, 104, 109

diet in, 103, 108

early measures in, 103, 109

etiology of, 101

expectorants in, 106, 107, 110

fever in, 102

fomentations in, 105,109

headaches in, 103, 109

inhalations in, 105, 107, 109, 110

isolation in, 103, 108

local treatment of, 104, 109

pathology of, 101

physical signs of, 102

precautions in, 103, 108

prophylaxis of, 108, 11

room in, 102, 108

summary of treatment of, 108

symptoms of, 102

vaccines in, 108, 11 
Bronchitis, capillary, 101

in grip, 227, 233

in measles, 498,508

in pneumonia, 164

Bronchopneumonia, 101, 128, 179, and see Streptococcus pneumonia

differentiated from laryngeal diphtheria, 464 in diphtheria, 473

in measles, 495,507

in pertussis, 534,542

in scarlet fever, 423

physiology of diet in, 3

specific treatment of, 179

Brook, on quinine treatment of amebic dysentery, 381,385

Brown, on amebic dysentery, 376

Brown, Wade, on blackwater fever, 343

on malaria, 324,337

Brown mixture, in bronchitis, 106, 110

Browne, on scarlet fever, 400

Bubonic plague, 634, and see Plague

Burning and itching, in measles, 491, 505

in small pox, 612, 621

Butter, food value of, 17

Buttermilk, food value of, 17

Cachexia, malarial, 338, 350

Caffeine, double salts of, 6,9

for prostration from belladonna, 531

in anthrax, 683

in bronchitis, 104, 10 9

in cerebrospinal meningitis, 566

in cholera, 651

in diphtheria, 470

in epidemic influenza, 244

in febrile conditions, 6,9

in glanders and farcy, 689

in grip, 219

in malaria, 335,349

in Malta fever, 660

in measles, 495

in pleurisy with effusion, 123

in pneumonia, 176, 178, 204, 205

in poliomyelitis, 600,606

in scarlatinal nephritis, 420

in scarlet fever, 405, 431

in tonsillitis, 83

in typhoid fever, 299

in typhus fever, 629

Calabar bean, for convulsions of tetanus, 721

Calcium chloride in black vomit of yellow fever, 739

lactate for hemorrhage in typhoid fever, 296

permanganate, in cholera, 648

Calmette's antivenomous serum for leprosy, 673

Calomel, anti-emetic effect of, in bacillary dysentery, 360

in bacillary dysentery, 359,360

in cerebrospinal meningitis, 565,582

in dengue, 642

in diphtheria, 452

in erysipelas, 774

in fibrinous pleurisy, 114, 125

in glandular fever, 555

in infectious jaundice, 730

in malaria, 324,346

in Malta fever, 658

in measles, 493,505

in mumps, 547

in paratyphoid fever, 324

in pertussis, 534

in plague, 635

in pneumonia, 151

in poliomyelitis, 594, 604

in rat bite fever, 746

in rubella, 513

in scarlet fever, 397

in septicemia, 760

in small pox, 613,622

in tonsillitis, 82,88

in typhoid fever, 314

in typhus fever, 629

in yellow fever, 738

Caloric balance of human body, 11
Caloric needs, determined by work, 11

in different callings, 12

of resting patient, 13, 27

Caloric output, determination of, 11

Caloric requirements, and age, 12

and body surface, 13

and weighti 13

of febrile patients, 20

of man at rest, 12,27

Caloric value of foodstuffs, 13

Caloric values, Rubner's, 13

Calorie, 11

definition of, 3,11

large, 11, 27, 34

small, 11, 27, 34

Calories of food required by patient at rest, 13

furnished by protein, in adult, 15 in infant, 15

measure of energy at rest, 3

required in sickness, 3

units of, in diet, 17

Camphor, for keeping mosquitoes away, 342

in anthrax, 683

in cholera, 651

in diphtberia, 470

in febrile conditions, 6,9

in glanders and farcy, 689

in grip, 224

in malaria, 335

in Malta fever, 660

in measles, 495

in pneumonia, 176, 204

in scarlet fever, 406,434

in septicemia, 760

in typhoid fever, 299

in typhus fever, 629

solution of in olive oil, 6

Cancrum oris, in Vincent's angina, 93

Cantharides blister, in rheumatism, 50

Capillary bronchitis, 101 complicating measles, 498, 508

Carbohydrates, 16, 26

in typhoid fever diet, 284

Carbolic acid. See Phenol

Cardiac complications in rheumatism, 53, 67 in children, 55,68

Cardiac dilatation complicating pneumonia, 192

Cardiac disturbances from salicylates, 41

Cardiac stimulants, 171, 203

Carpets, disinfection of, in smallpox, 616

Carr, J. W., on belladonna in children, 530

Carriers. See under the several diseases

Carroll, on stegomyia calopus, 740 on yellow fever, 738

Carter on typhoid fever, 298

on yellow fever, 739

Castor oil, in bacillary dysentery, $359,360,361$, 371

in epidemic influenza, 243

in fibrinous pleurisy, 114, 125

in glandular fever, 555

in Malta fever, 658

in measles, 493,505

in poliomyelitis, 597

in rubella, 513

in scarlet fever, 397

in septicemia, 760

in typhoid fever, 292

in typhus fever, 629

methods of administering, 360

Catabolism, protein, influenced by pyrexia, 20

Catharsis, in bacillary dysentery, 359

in diphtheria, 452

in epidemic influenza, 243

in erysipelas, 774

in Malta fever, 658,661

in measles, 493,505

in poliomyelitis, 597

in rat bite fever, 746

in rheumatism, 35

in Rocky mountain spotted fever, 666

in scarlet fever, 397

in smallpox, 613,622

in septicemia and pyemia, 760,769 
Catharsis. in typhoid fever, 292

in typhus fever, 629

Cathartics in febrile conditions, 4, 9

Catheter, use of, for tympanites in typhoid fever, 293

Cattle, foot and mouth disease of, 693

Cauterization, contraindicated in tetanus, 714

in fibrinous pleurisy, 116, 126, 187

in noma of measles, 500,509

in rabies, 700,708

Cecil, on foreign protein therspy in rheumstism, 59

on pneumococcus vaccines, 194

Cell destruction, expression of, 26

Cell, energy transformer, 10 vitality of, 10

Ceratum cantharidis, 50, 67, and see Fly blis. ter

Cerebral disturbances in typhoid fever, 298

Cerebral symptoms from salicylates, 41

Cerebrospinal fluid, in cerebrospinal meningitis, 568,571

Cerebrospinal meningitis, 560

bed in, 563,580

care of bladder in, 567,583

of bowels in, 565,582

of eyes in, 565, 582, 588

of mouth in, 564, 581

of nose in, 564,582

of patient in, $561,563,581$

of skin in, 56.

carriers of, $561,579,589$

causative agent of, 560

cerebrospinal fluid in, 568,571

changes in mentality in, 577

chronic cases of, 576,587

complications in, 577,587

convalescence in, 578, 589

convulsions in, 566,583

delirium in, 566, 582

diet in, 563, 581

disinfection of secretions in, 565,580 of utensils in, 580

disposal of secretions in, 565

distension of bladder in, 567, 583

distribution of family in, 560, 579

drinks in, 564

drug treatment in, 56 ?

ear complications in, 577, 588

early use of serum treatment in, 573

exacerbations of, 576,588

eye complications in, 577,588

fulminating cases of, 575,587

hydrocephalus in, 576,587

incubation period of, 561

intravenous treatment of, 574, 583, 587

intraspinal treatment of, 568,584

isolation of contacts, 561

joint complications in 577,588

lumbar puncture in, 569,584

meningococcic endocarditis in, 578

meningococcic serum and mortality in, 573

mortality from, $\mathbf{5 7 7}$

otitis in, 577

paralysis in, 577

precautions in sick-room in, 563,580

of nurse in, 562, 580

of physician in, 562,580

prophylaxis in, 578,588

puncture of ventricles in, 576,587

pyelitis in, 578, 588

quarantine in, $560,579,589$

relapses in, 576,588

restlessness in, 566,582

room in, 562, 580

septic pneumonia in, 578

serum treatment of, $567,573,578,583$

sleeplessness in, 566,582

specific treatment of, 567,583

summary of treatment of, 579

support of circulation in, 566,583

symptomatic treatment of, 566, 582

treatment of nausea in, 565,582

vaccines in, 576,578
Cerium oxalate, in anthrax, 683

in cerebrospinal meningitis, 566

in infectious jaundice, 730

in Malta fever, 659

in scarlet fever, 397

in typhoid fever, 295, 315

Chaparro amargosa, in amebic dysentery, 382

Charbon, 678

Charta sinapis. See Mustard

Chaulmoogra oil, in leprosy, 671, 672, 674, 676

Cheese, food value of, 18

Chenopodium, oil of, in amebic dysentery, 383

Chest compresses, in bronchitis of measles, 498

in bronchopneumonia of measles, 496

in pneumonia, 157

Chicken, food value of, 17

Chicken pox, 515, and see Varicella

Childhood, laryngitis in, 98, 100

rheumatism in, 30,55

Chloral, in anthrax, 683, 684, 685, 686

in cerebrospinal meningitis, 566

in encephalitis lethargica, 275

in Malta fever, 659

in pertussis, 535,543

in pneumonia, 202

in rabies, 706

in scarlatinal nephritis, 421

in small pox, 615

in tetanus, 719,726

Chloralamid, in dengue, 643

in epidemic influenzs. 246

in erysipelas, 774,779

in Malta fever, 659

in measles, 495,507

in pneumonia, 169

in rheumatism, 37

in scarlet fever, 407

in septicemia, 762,770

in small pox, 615

in tonsillitis, 84,89

in typhoid fever, 298, 317

Chloretone, for tetanus, 720, 726

Chloride of lime, in glanders and farcy, 690

Chloroform, in anthrax, 684

in pertussis, $528,535,543$

in rabies, 706

in scarlatinal nephritis, 422

in tetanus, 719,726

Cholecystitis, in typhoid fever, 298

Cholera, Asiatic, 646

anuria in, 649,654

bed in, 646

cathartics in early treatment of, 647,654

cause of, 646

circulation in, 647

complications of, 651

convalescence from, 652,654

diet in, 646, 653

early treatment of, 647

hyperpyrexia in, 649

isolation in, $646,651,653$

mortality of, 646

precautions in sick-room in, 646, 653

of nurse in, 646,653

of physician in, 646,653

prophylactic inoculation in, 651

prophylaxis of, 651,655

quarantine in, $646,651,653$

rest in, 653

specific treatment of, 650

stage of collapse in, $647,649,654$

of reaction in, 649

stimulants in, 650

summary of treatment in, 653

uremia in, 650

vaccines in, 651

vomiting in, 649,654

water in, 647

Chorea, 59

autoserotherapy in, 61

in rheumatism, 59, 69

local measures in, 60

treatment of, 59,69

vaccines in treatment of, 60 
Chromic acid, in Vincent's angina, 92, 93

Cinchonism, 333

Circulation. See under the several diseases

Circulatory failure in febrile conditions, 6

Citronella for keeping mosquitoes away, 342

Climate, change of, in convalescence from per-

in convalescence from scarlatinal nephritis, 423

Clough, on foot and mouth disease, 693

Coagulen, in nose bleed of diphtheria, 467

Coakley, on nasal hemorrhage, 534

on scarification in edema of the larynx, 99

Coal-tar group, antipyretics of, 7

contraindicated in typhus fever, 628

in encephalitis lethargica, 274

in epidemic influenza, 244, 265

in febrile conditions, 6

in fibrinous pleurisy, 118, 126

in grip, 218,231

in pneumonia, 170

in poliomyelitis, 596,605

in small pox, 614,622

in tonsillitis, 83,89

Cocaine, for cough in bronchitis, 106, 110

for earache in scarlet fever, 411

for lumbar puncture, 570

for vomiting in typhoid fever, 295, 315

in coryza, 73

in diphtheria, 466

in epidemic influenza, 251

in infectious jaundice, 730

in Malta fever, 659

in pertussis, 534

in rabies, 707

in rhinitis, 73

in yellow fever, 739

Codeine, in anthrax, 683

in bronchitis, 106, 110, 498

in bronchopneumonia, 497

in cerebrospinal meningitis, 566

in chorea, 60

in chronic glanders, 497

in dengue 642

in encephalitis lethargica, 274,275

in epidemic influenza, 245, 246, 250

in fibrinous pleurisy, 118,126

in grip, 225,233

in infectious jaundice, 730, 733

in laryngitis, 96,99

in pertussis, 531,541

in pneumonia, 166,168, 170

in poliomyelitis, 596

in rheumatism, 37

in rhinitis, 76

in septicemia and pyemia, 761

in tonsillitis, 84,89

in trench fever, 752,755

Cohnheim, on food, 12

Cold in the head, 71 , and see Coryza

Cold, in febrile conditions, 7,9

in hyperpyrexia, 6

in scarlet fever, 406,432

in septicemia, 760,761

in rheumatism, 49

Cold air, for diphtheria, 471

for measles, 489, 503

Cold applications, for adenitis in glandular fever, 556 bronchitis, 105, 109

for bronchitis, 105,109

for hyperpyrexia, 6,7 in scarlet fever, 401

for membranous angina in scarlet fever, 401

Cold bath in pneumonia, 159

Cold compresses, in laryngitis, 97

in laryngitis complicating measles, 498

in pericarditis in pneumonia, 185

in pneumonia, 157

in rheumatism, 49

Cold pack, for delirium in septicemia, 761

for fever in small pox, 614

in pneumonia, 161

in rheumatism, 53

Cold sponges, for delirium in septicemia, 761
Cold sponges, in pneumonia, 161

Cold water, for bronchopneumonia complicating measles, 496

in pneumonia, 161

in scarlet fever, 407

in typhoid fever, 299, and see Brand bath

treatment, see Hydrotherapy

Cole, on pneumonia, 129

Cole's method of desensitization in pneumonia 136

Coleman, on typhoid fever, 22, 23, 25, 26, 283, $284,287,313$

Collapse, due to antipyretics, 223

during bath in measles, 493

in bacillary dysentery, 373

in diphtheria, 468,483

in malaria, 325

in measles, 494

in pernicious malaria, 335

in pneumonia, 177, 204

in septicemia, 760,769

threatened, in typhoid fever, 299, 318

Collargol, in trench fever, 753,755

Collodion, in erysipelas, 775,776

Colonic irrigation, for ileocolitis complicating measles, 500, 509

in fibrinous pleurisy, 114,126

Conner, on intravenous administration of salicylates, 47 on thrombophlebitis complicating typhoid

Convalescence. See under the several diseases

Convulsions. See under the several diseases

Coryza, 71, and see Rhinitis, acute

occurring in epidemic influenza, 251

Cough, in bronchitis, 102, 106, 110

complicating measles, 498

in bronchopneumonia complicating measles, 497

in coryza, 75

in epidemic influenza, 237, 250

in fibrinous pleurisy, 113,118, 126

in glanders, 689, 69

in laryngitis, 96,99

in pertussis, 525,540

in pneumonia, 161, 200

in rhinitis, 78

source of exhaustion, 1

Counterirritation, in bronchitis, 104, 109

in fibrinous pleurisy, 116, 126

in pericarditis complicating pneumonia, 187

in rheumatism, 50,67

in scarlatinal nephritis, 420

Cowling's rule for dosage, 37

Craig, on dengue, 641

Cream, food value of, 17

Creatin and metabolism, in acute infectious diseases, 24

Creatinin and metabolism, in acute infectious diseases, 22

Creatinin coefficient, 23

Creosote, in bronchitis, 105, 109 complicating measles, 498

in bronchopneumonia, complicating measles, 497

in cough, of glanders, 689

in pertussis, 528

Crockery, disinfection of, 281

Croup, spasmodic, differentiated from laryngeal diphtheria, 464

Croup kettle, in laryngitis, 96, 99

in pertussis, 527

in scarlet fever, 401

Cumming, on rabies, 701

Cupping, in bronchitis, 104, 109

in bronchopneumonis complicating measles, 497

in fibrinous pleurisy, 117

in pneumonis, 165

in pulmonary edema, in epidemic influenza, 249

in pneumonia, 179

in scarlatinal nephritis, 419

technique of, 164 
Cushny on belladonna, 529

Cyanosis, due to antipyretics, 223

in epidemic influenza, 238, 249

Dakin's solution, in anthrax, 682 in streptococcus empyema, 212

Dandy fever, 641, and see Dengue

Deafness, from quinine, 333

from salicylates, 40

in cerebrospinal meningitis, 577

De-amidation, defective, 24

Delafield, method in pleurisy with effusion, 119 , 126

on bronchopneumonia, 208

prescription for dysentery, 361 for pneumonia, 166

Delirium, from belladonna, control of, 531

from salicylates, 40

in anthrax, 684

in bronchopneumonia complicating measles, 497

in cerebrospinal meningitis, 566, 582

in epidemic influenza, 246, 259, 266

in erysipelas, 774,779

in hyperpyrexia, 53

in infectious jaundice, 731

in measles, 494

in plague, 636

in pneumonia, 168

in rheumatism, $40,53,61,70$

in scarlet fever, 407 .

in septicemia, 761, 770

in small pox, 614,623

in typhoid fever, 317

in typhus fever, 629

Delousing, in trench fever, 753, 755 in typhus fever, 627,633

Dengue, 641

bed in, 641,643

care of bowels in, 642,644

circulation in, 642,644

complications in, 643,645

convalescence from, 643,645

diagnosis of, 643

diet in, 642, 644

fever in, 642, 644

headache in, 642

hemorrhages in, 643

infecting agent in, 64

insomnia in, 642,644

local treatment of pains in, 642

mortality in, 641

mosquito carrier of, 641,643

nervous symptoms in, 643,644

pains in, 642,644

prophylaxis of, 643, 645

room in, 641, 643

summary of treatment of, 643

transmission of, 641

water in, 642,644

Dermacentor, in Rocky mountain spotted fever 664

Dermacentroxenus, in Rocky mountain spotted fever, 664

Desensitization, in pneumonia, 135, 136

Diaphoresis, in scarlatinal nephritis, 417, 421 in scarlet fever, 417,438

Diarrhea, in anthrax, 684,686

in bacillary dysentery, 364,372

in epidemic influenza, 246

in infectious jaundice, 730,733

in pertussis, 534,542

in plague, 636,640

in poliomyelitis, 597, 606

in typhoid fever, 294, 315

in typhus fever, 633

Diet. See under the several diseases measure of in calories, 17

physiology of, in febrile conditions; 3 summary of, 27

Dietary, proportion of foodstuffs in, 16

Dietetics, science and art of, 27

Digitalis, in acute febrile conditions, 6 in anaphylactic shock in pneumonia, 145
Digitalis, in anthrax, 683

in bacillary dysentery, 365,373

in cerebrospinal meningitis, 566

in cholera, 650

in diphtheria, 470

in glanders and farcy, 690

in Malta fever, 659

in pleurisy with effusion, 124

in pneumonia, 145,171

in pneumonia complicating epidemic influenza, 247

in rheumatic fever, 54

in scarlatinal nephritis, 420

in scarlet fever, 404, 434

in septicemia and pyemia, 762, 770

in typhoid fever, 299

in typhus fever, 629

Digitalization, 171, 203, 667

Diphtheria, 443

active immunization in, 447

adenitis complicating, 473

anemia in, 485

angina in, 465

antitoxin, 454,479

bath in, 450

bed in, 450

bronchopneumonia complicating, 473

cardio-vascular apparatus in, 467, 469, 482

care of howels in, 452

of genitals in, 452

of mouth in, 451

of nose in, 451, 452, 466

of patient in, 450,478

of teeth in, 451

of throat in, 451,465

carriers of, 475,485

complicating measles, 501

convalescence from, 474,484

culture taking in, 444, 477

death in, 468,483

diet in, 450,478

differential diagnosis of, 464

distribution of family in, 444, 477

extubation in, 463

feeding in, 450, 478

ferer in, 465

hemorrhage from nose in, 467,482

hot fomentations in, 462, 465

immunity to, 448

inhalations of steam in, 461

intubation in, 462

laryngeal, 460, 481, and see Laryngeal diphtheria

antitoxin treatment of, 455,481

differential diagnosis of, 464

dosage of antitoxin in, 455

extubation in, 463

intubation in, 462

symptomatic treatment of, 465,481

late circulatory failure in, 468

malaise in, 443

malignant, dosage of antitoxin in, 455

mortality in, 456

nasal, 451,482

antitoxin treatment of, 455

dosage of antitoxin in, 455

nephritis and, 473,484

open-air treatment of, 471

otitis complicating, 473

paralyses in, 471,483

pharyngeal, dosage of antitoxin in, 455

post-intubation, treatment, 463

precautions of nurse in, 449, 478

of physician in, 449, 478

prophylaxis of, 476

quarantine for, 474,485

rest in, 450,478

room in, 448, 449, 450, 477, 478

Schick reaction for, $444,445,449,450$

serum therapy in, 453

sterilization and fumigation following, 475 , 485

summary of treatment of, 477

symptoms of, 443 


\section{INDEX}

Diphtheria, syncope in, 468, 483 taking cultures.in, 444

treatment of symptoms of, 465

Diplosal, in rheumatism, 44, 65, and see Salicylates

in tonsillitis, 83,89

Disinfection. See under the several diseases

Distribution of family. See under the several diseases

Diuresis in scarlet fever, 439

Diuretics, in pleurisy with effusion, 123, 127

in scarlatinal nephritis, 420

Diuretin, in scarlatinal nephritis, 420

in pleurisy with effusion, 123

Dobell's solution, in bacillary dysentery, 357

in cerebrospinal meningitis, 564, 579

in diphtheria, $452,466,467,476$

in epidemic influenza, 242

in foot and mouth disease, 694

in glandular fever, $\mathbf{5 5 5}$

in measles, 492

in mumps, 525

in pertussis, 525

in scarlet fever, 396,400

in varicella, 517

Dog, symptoms of rabies in, 702

Dogs and prevention of rabies, 707

Dosage, rules for, in children, 37

Dover's powder, in bronchitis, 104, 109

in bronchitis complicating measles, 498

in bronchopneumonia complicating measles, 497

in cholera, 647

in pertussis, 531,541

in rhinitis, 72

Drainage, in streptococcus empyema, 213

Draper, on poliomyelitis, 595

Drinks. See under the several diseases.

Dry tap, in lumbar puncture, 572

Dudley, on anthrax, 680

Dumb rabies, 702

Dunn, on cardiac complications in rheumatic fever, 55

on serum treatment of cerebrospinal meningitis, 576

Duval and Gurd, on leprosy, 674

Dyer, on leprosy, 673

Dysentery, amebic, 374, and see Amebic dysentery

bacillary, 353, and see Bacillary dysentery

Dyspnea, due to impurities in salicylates, 40

in epidemic influenza, 238, 249

in fibrinous pleurisy, 118,126

in pneumonia, 179

in poliomyelitis, 600,606

Earache, in measles, 499, 508

in scarlet fever, 411

Ears, and see Otitis

buzzing in, from salicylates, 40,41

care of, in pneumonia, 149

in scarlet fever, 392, 411

changes in, due to quinine, 333

complications of, in cerebrospinal meningitis, 577

examination of in infections, in children, 443

Edema, angioneurotic, due to antipyretics, 223

in scarlatinal nephritis, 420

in scarlet fever, $421,438,439$

of larynx, 98, 100

intubation in, 99, 100

scarification in, 98, 100

tracheotomy in, 99,100

pulmonary, in pneumonia, 178

subglottic, differentiated from laryngeal diphtheria, 464

Effusions, in rheumatism, 67

Eggleston's rule for digitalization, 171, 203

Eggs, food value of, 17.

Einhorn's tube, use of in typhoid carriers, 305

Elastic belt in pertussis, 526, 533

Electric pad, in fibrinous pleurisy, 115, 126

Electricity for paralysis of extremities in poliomyelitis, 601,607
Elser and Huntoon on cerebrospinal meningitis, 561

Embolism, in pneumonia, 190, 206

in septicemia and pyemia, 763,771

Emetine, in amebic dysentery, 376, 384 toxic dose of, 380

Emphysema, in epidemic influenza, 256

Empyema, 112, 125, 127

in epidemic influenza, 253

in pneumococcus pneumonia, 181, 187

in streptococcus pneumonia, 209, 211

in typhus fever, 630

Empyema, pneumococcus, 112, and see Empyema

Empyema (streptococcus and influenza types), 207,211

aspiration in, 211

Dakin's solution in, 212

drainage in, 213

summary of treatment of, 215

treatment of, 211

Encephalitis lethargica, 271

bed in, 274, 275

care of body in, 274,276

of bowels in, 274,276

convalescence in, 275,276

course of, 273

diet in, 274, 276

drinks in, 274, 276

fever in, 275,276

hyperesthesia in, 275,276

in epidemic influenza, 258

insomnia in, 275,276

irritability and restlessness in, 275, 276

lumbar puncture in, 275, 276

mortality in, 273

pains and headaches in, 274, 276

paresthesia in, 275, 276

pathology of, 271

prognosis of, 273

room in, 274, 275

sequelæ of, 273

summary of treatment of, 275

symptomatology of, 271

Endocarditis, in erysipelas, 777

in measles, 501

in pneumonia, 190

in rheumatism, 53

in scarlatinal nephritis, 422

in scarlet fever, 423

in septicemia, 762,763

in septicopyemia, 758

meningococcic, 578

Endogenous metabolism, 23

Endotoxins, 453

Enemata, in bronchopneumonia complicating measles, 498

in diphtheria, 470

in epidemic influenz8, 243, 251

in erysipelas, 774

in Malta fever, 658

in plague, 635

in pneumonia, 152

in poliomyelitis, 594

in rheumatism, 36

in septicemia, 760

in tympanites, 251, 294

in typhoid fever, 292

milk and molasses, 294

nutrient, 470

peppermint, 294

soapsuds, 36

turpentine, 293

Entamceba coli, 374, 376

histolytica, $353,374,376$

Enteroclysis, in bronchopneumonia complicating measles, 498

in circulatory failure in diphtheria, 470

in scarlatinal nephritis, 422

in scarlet fever, 440

Environment, change of, in bacillary dysentery, 368

Epidemic influenza, 235

abdominal symptoms in, 260

abscess of lung in, 254, 268 
Epidemic influenza, abscess of muscles in, 260, 270

aches and pains in, 244, 265

acidosis in, 251,267

bed in, 240, 263

blood in, 238

bronchiectasis in, 255

care of bowels in, 243, 264

of eyes in, 243, 264

of mouth in, 242, 263

of nose in, 243,264

of patient in, 242,263

of teeth in, 242, 264

carriers of, 261

circulation in, 246, 247, 266

convalescence in, 260, 269

cough in, 237, 250, 267

cyanosis in, 238, 249

delayed resolution, 252,267

delirium in, 246,266

diarrhea in, 246,266

diet in, 244, 265

dyspnea in, 249

emphysema in, 256

empyema in, 253, 268

encephalitis lethargica in, 258

epistaxis in, 238

erythema in, 238

etiology of, 235

fluids in, 244, 265

headaches in, 245, 264, 265

heart and vessels involved in, 256, 268

incubation period of, 237

insomnia in, 245

isolation in, 239, 262

kidneys involved in, 257, 258, 269

meningitis in, 258

mental disturbance in, 259, 268

nervous system involved in, 258

nervousness in, 265

neuritis in, 259

onset of, 237

otitis media in, 260

parotitis in, 260

pathology of, 235

phlebitis in, 257, 269

pleurisy in, 253, 268

pneumonia in, 236, 239, 246, 266

precautions for nurse in, 241, 263

for physician in, 241, 263

prophylaxis of, 261, 270

prostration in, 238

pulmonary edema in, 248, 267

pulse in, 238

respiration in, 238

room in, 240,262

summary of treatment of, 262

sy mptomatology of, 237

temperature in, 238

treatment of, 239,262

tuberculosis in, 255

tympanites in, 251, 267

upper air passages involved in, 256

urine, retention of in, 238

vaccines in, 262

vomiting in, 246, 266

warnings to family in, 242, 263

Epinephrin. See Adrenalin

Epistaxis, in epidemic influenza, 238, 256 in pertussis, 534

Epsom salts, in bronchitis, 103, 109

in dengue, 642

in diphtheria, 452

in epidemic influenza, 243

in erysipelas, 774

in fibrinous pleurisy, 114, 125

in grip, 217, 231

in laryngitis, 95,99

in Malta fever, 658

in mumps, 547

in plague, 635

in pneumonia, 151

in rheumatism, 35

in scarlatinal nephritis, 417
Epsom salts, in scarlet fever, 397

in septicemia and pyemia, 760

in tonsillitis, 82,88

in typhoid fever, 292, 314

in yellow fever, 738

Erdman, on erysipelas, 775,777

Eruptions. See Skin eruptions

Erysipelas, 772

bed in, 773, 778

care of body in, 774,778

of bowels in, 774, 778

of eyes in, 774

of mouth in, 774

circulation in, 774

complications of, 777,779

creatinin in, 24

delirium in, 774,779

diet in, 773,778

disinfection in, 777,780

facial, 772,773

local treatment of, 775,779

fever in, 774,778

headache in, 774,778

immunity in, 777

in infants, 776, 779

incubation period of, 772

insomnia in, 774, 779

isolation in, 773, 778

local treatment of, 775,779

migratory, 776,779

nervousness in, 774,778

of extremities, 776, 779

organism of, 772

prophylaxis of, 777, 780

room in, 773, 778

specific treatment of, 776,779

summary of treatment of, 778

symptomatic treatment of, 774,778

symptoms of, 772

transfusion of blood in, 777

vaccine treatment of, 776

Erythema, from antipyretics, 223

from diphtheria antitoxin, 458

from quinine, 333

from salicylates, 40

in epidemic influenza, 238

in scarlet fever, 387

in septicemia, 763

in varicella, 516 .

Eserine. See Physostigmine

Euquinine, in malaria, 334

Ewing, on typhoid fever metabolism, 25, 286

Exogenous metabolism, 23

Expansion of lung in pleurisy with effusion, technique of, 124

Expectorants, in bronchitis, 106, 107, 110

in pneumonia, 166, 201

Exploratory puncture, in pleurisy with effusion, 120,127

Exsanguination, in typhoid fever, treatment of, 316

Extubation, 463

Eyes, See under the several diseases.

Faget's sign, in yellow fever, 735

Family, distribution of. See under the several diseases

Farcy, 687, and see Glanders

acute, 689,692

chronic, 687,691

serum therapy in, 688

specific treatment of, 688

treatment of local lesions in, 688,689

vaccine treatment of, 688,691

general treatment of, 687

isolation in, 690

mortality in, 687

precautions of nurse in, 690

of physician in, 690

prophylaxis of, 690,692

summary of treatment in, 690

Fat, in acute infectious diseases, 16, 26

in typhoid fever dietary, 284 
Febrile conditions, 1

aconite in, 6

antipyretics in, 7,9

caffeine in, 6, 9

camphor in, 6,9

cathartics in, 5, 9

diet in, 3,8

digitalis in, 6,9

drugs in, 5, 6, 9

fresh air in, 4,8

rest in, 2,8

specific treatment of. See under the several diseases

strophanthin in, 6

strychnine in, 6

summary of, 7

water in, $3,4,5,9$

Febrile patient, calories required by, 20

Febris carnis, 286

Ferenbaugh, on Malta fever, 656

Fever, 1, and see under the several diseases meaning of, 19

open air treatment of, 4

in relation to disease, 19

starvation in, 29

water needs in, 29

Fitzgerald, on tetanus antitoxin, 718

Fixed virus, of rabies, 702

Fleas, and plague, 634

Flexner, on cerebrospinal meningitis, 569, 573 serum, for bacillary dysentery, 361 serum, mortality with in cerebrospinal menin-

Fluoform, in pertussis, 532

Fly blister, in local treatment of rheumatism, 50

Focal symptoms, in encephalitis lethargica, 272

Folin, anslysis of urines, 22, 25

Foodstuffs, standard portions of, 16

Fomentations, application of, 163

in bacillary dysentery, 362

in bronchitis, 105, 109

in bronchopneumonia complicting measles, 498

for buboes, in plague, 636

in diphtheria, 462

in fibrinous pleurisy, 115,126

in glandular fever, 556

in laryngitis, 97

in mumps, 547

in pericarditis of pneumonia, 187

in pneumonia, 163

for retention of urine, in Malta fever, 658

in rheumatism, 49

in scarlatinal nephritis, 420

Food, harm of withholding in fevers, 20

Foodstuffs, proportion of in average dietary, 16

Rubner's figures for caloric value of, 13, 14

Food values, in units of 100 calories, 17

Foot and mouth disease, 693

bed in, 694, 696

care of bowels in, 695,696

of eyes in, 695, 697

of mouth in, 694, 697

of nose in, 697

of teeth in, 694

circulation in, 694

diarrhea in, 697

diet in, 694,696

fever in, 694, 696

gastro-intestinal symptoms in, 695, 697

isolation in, 694

precautions of nurse in, 694,696

of physician in, 694, 696

prognosis of, 695

prophylaxis of, 695

rest in, 694,696

room in, 694,696

summary of treatment of, 696

vesicles and ulcers of skin in, 695, 697

Foreign protein therapy, in rheumatism, 58

Forks, disinfection of, 281

Fowler's solution, in malaria, 339, 351

Fresh air, in convalescence from pertussis, 537 in febrile conditions, 4,8

in leprosy, 671,676
Fresh air, in pneumonia, 155

in septicemia, 759

Friction, in connection with hydrotherapy, 5

Frontal sinus involvement in influenza, 228, 23s

Fuller's alkaline treatment for rheumatism, 46

Fumigation. See Disinfection

Gatch bed, 147

Grstric distress, in typhoid fever, 315

Gastro-intestinal function, disturbance of, in infection, $8,18,28$

Gaultheria, 43, and see Wintergreen

Genitals, care of. See under the several diseases

Genito-urinary infection, in epidemic influenza, 258

Gentry and Ferenbaugh, on Malta fever, 656

German measles, 512, and see Rubella

Glanders, 687, and see Farcy

acute, 689,692

chronic, 689,691

care of nose in, 689, 691

cough in, 689, 691

general treatment of, 687

isolation in, 690

mortality in, 687

precautions of nurse in, 690

of physician in, 690

prophylaxis of, 690,692

summary of treatment of, 690

Glandular fever, 555

adenitis in, 556, 558

bed in, 555

care of bowels in, 555,558

of mouth in, 555, 558

of nose in, 555

of throat in, 556,558

complications of, 556,558

convalescence from, 557, 559

diet in, 555,557

fever in, 556, 558

glands involved in, 555

incubation period of, 555

inflammation of pharynx in, 556

isolation in, 555,557

nephritis in, 556, and see Scarlet fever, nephritis in

otitis in, 556

prophylaxis in, 557, 559

room in, 555,557

summary of treatment of, 557

tonsils in, 556

toxemia in, 556, 558

Glauber's salts, in bronchitis, 103, 109

in erysipelas, 774

in fibrinous pleurisy, 114, 125

in laryngitis, 95,99

in Malta fever, 658

in mumps, 547

in plague, 635

in rheumatism, 35

in scarlet fever, 397

in yellow fever, 738

Glottis, edema of, complicating small pox, 615 , 623

phlegmon of, differentiated from laryngeal diphtheria, 464

Goat fever, 656, and see Malta fever

Goats, carriers of Malta fever organism, 656

Goats' milk, in Malta fever, 657

Goodman, on autoserotherapy in chorea, 61

Gottlieb, on caffeine, in scarlet fever, 405

Graham, on dengue, 641

Gravity method, in lumbar puncture, 572

Grip, 216

antipyretics in, $218,223,231$

antral involvement in, 228, 233

bed in, 217

bronchitis complicating, 227

care of bowels in, 217, 231

carriers of, 230

change of air in, 229

complications of, 225,233

convalescence in, 225, 233

cough in, 225, 233 
Grip, diet in, 217, 225, 231, 233

drugs in, 218, 231

fever in, 220,229, 231, 233

frontal sinus involvement in, 228, 233

mastoiditis complicating, 226, 233

mental depression in, 229, 234

neuritis in, 234

otitis medis complicating, 226, 233

pleurisy in, 227

pneumonia in, 226, 233

prophylaxis of, 230, 234

rest in, 217

rhinitis in, 227

room in, 231

sinus thrombosis complicating, 226, 233

sthenic period of toxemia, 218

summary of treatment of, 231

symptomatic treatment of, 218, 231

tonics in, 225, 233

tonsillitis in, 227

tracheitis in, 224, 232

Growing pains and rheumatism, in childhood, 61

Guiscol, in abscess of lung in epidemic influenza 255

in mumps, 548,553

Guiteras, on yellow fever, 736, 739

Gurd and Duval, on leprosy, 674

Gynocardia. See Chaulmoogra oil.

Haas, on adenitis in glandular fever, 556

Haff kine's vaccine for plague, 635,638

Hale, Worth, on toxicity of acetanilid, 219

on trikresol and meningococcio serum, 569

Halibut steak, food value of, 17

Hanzlik, on rheumatism, 39,44

Headache. See under the several diseases.

Hearing, effect of antipyretics on, 223 effect of quinine on, 333

Heart involvement, in bacillary dysentery, 366 in epidemic influenza, 256, 268

in malaria, 337,350

in measles, 501

in rheumatism, in children, 30

in scarlet fever, 423

Heat, for chill in malaria, 324

for collapse in pneumonia, 177

for earache in scarlet fever, 411

for laryngitis in measles, 498

for otitis in measles, 499

for pain in bacillary dysentery, 552

for parotitis in mumps, 552

in fibrinous bronchitis, 115,126

in malaria, 336

in pericarditis, 186

in pneumonia, 162

in poliomyelitis, 596,597

in rheumatism, 49

Heine-Medin disease, 271 and see Poliomyelitis

Hematuria, from salicylates, 40

Hemoglobinuria, in blackwater fever, 343

Hemoplastin, in nose bleed of diphtheria, 467

Hemorrhage. See under the several diseases

Hepatic abscess, in amebic dysentery, 377, 379

Heroine, for cough, in bronchitis, 106, 110

in epidemic influenza, 250

in grip, 225,233

in laryngitis, 96, 99

in pertussis, 532

Herrick, on serum treatment of cerebrospinal meningitis, 574

Heyn, on rectal administration of salicylates, 47

Hides, and anthrax, 678,684

Hill, Miner C., on intubation in diphtheria, 462

Hill on prognosis of tetanus, 721

on transmissibility of poliomyelitis, 592

Hilprit, on synthetic sodium salicylate, 42

Holt, croup kettle, 96, 527

on diphtheria, 474

on dosage, of diphtheria antitoxin, 456

of quinine, 330

on mortality, in measles, 487

on oxygen inhalations in pertussis, 536

on pertussis, 528

Hominy, food value of, 17
Hooker, on blood transfusions, 767

Horse serum, for hemorrhage in typhoid fever, 296 in poliomyelitis, 605

in tetanus, 713

Horses, glanders and farcy and, 687

Hot air bath, in scarlatinal nephritis, 417

Hot bath, in laryngitis, 95,99

in rhinitis, 72

Hot compresses, in laryngitis of measles, 499

in scarlatinal nephritis, 417,418

Hot fomentations, for angina in laryngeal diphtheria, 465

in diphtheria, 462

in poliomyelitis, 596

Hot milk, in laryngitis complicating measles, 499

Hot pack, in chorea, 60

in scarlatinal nephritis, 417, 418

Hot water bag, in fibrinous pleurisy, 115, 126

Hot water bottle, in rhinitis, 72

Hubbard, ointments used by, in small pox, 612

formula for itching, in measles, 492

Huntoon and Elser, on cerebrospinal meningitis, 561

Hydrocephalus, in cerebrospinal meningitis, 576

Hydrochloric acid, in acute infectious diseases, 18

Hydrogen dioxide, in local treatment of tetanus, 714

in treatment of wounds, 722

Hydrophobia, 699, and see Rabies

Hydrotherapy. See Baths, and also under the several diseases

in febrile conditions, $3,4,7,9$

Hydrothorax, in scarlatinal nephritis, 421

Hyoscine, for chorea, 61

for convulsions of tetanus, 720

for delirium, in anthrax, 684

in epidemic influenza, 246

in erysipelas, 774

in cerebrospinal meningitis, 566

in pneumonia, 169

in Rocky mountain spotted fever, 667

in septicemia in pyemia, 761

Hyperpyrexia. See under the several diseases

Hypertonic glucose solution in cerebrospinal meningitis, 567

Hypertonic solution, in cholera, 647, 652

Hypnotics, in pneumonia, 169, 202

in rheumatism, 37

in small pox, 615

Hypodermoclysis, in diarrhea of bacillary dysentery, 365,373

in diphtheria, 471

with quinine, 331

Ice, for gastric distress in yellow fever, 738

for nausea, in scarlet fever, 422,431

in vomiting of typhoid fever, 295, 315

Ice-bsg, spplication of, 163

for angina, in laryngeal diphtheria, 465

for buboes, in plague, 636

for cerebral disturbances, in typhoid fever, 298

for delirium, in septicemia, 761

in pneumonia, 168

for gastric distress, in yellow fever, 738

for headache, in anthrax, 684

in bronchitis, 104, 109

in scarlatinal nephritis, 422

in septicemia, 762

in small pox, 614

for hemorrhage in typhoid fever, 296

for pains, in dengue, 642

in fibrinous pleurisy, 115, 126

in laryngitis complicating measles, 498

in pericarditis complicating pneumonia, 185

in pneumonia, 163

in rheumatism, 49

in Rocky mountain spotted fever, 667

in typhoid fever, 294

Ice-cap, for headache in pneumonia, 168

in cerebrospinal meningitis, 566

in measles, 494

in scarlet fever, 407,413 
Ice-coil. See also Ice-bag

in cerebrospinal meningitis, 566

in rheumatism, 49

in tympanites of typhoid fever, 294

Ice-collar, in laryngitis, 97

Ice-poultice, in mumps, 548, 553

Ichthyol, in erysipelas, 775,776

in glandular fever, 556

in mumps, 548,553

in scarlet fever, 413

Immunity, active, 454 passive, 454

Imperial drink, in rhinitis, 72

Infantile paralysis, 590, and see Poliomyelitis

Infectious diseases, acute, diet in, 10 to 29

Infectious jaundice, 728

active immunization in, 731

bed in, 729

care of bowels in, 730, 732

colic in, 733

convalescence from, 733

diarrhea in, 730, 733

diet in, 729,732

fever in, 729,731

headache in, 733

hemorrhages in, 731

icteric stage of, 729

incubation period of, 728

jaundice in, 729,731

lesions of, 728

nausea in, 730, 733

nephritis in, 731

nervous symptoms in, 731

organism of, 728

pain in legs in, 731, 733

prophylaxis of, 731, 734

room in, 729

specific treatment of, 730

summary of treatment of, 732

symptoms of, 728

vomiting in. 730,733

water in, 732

Influenza empyema, 211, and see Empyema, influenza type

epidemic, 235, and see Epidemic influenza sporadic, 216, and see Grip

Inhalations, in abscess of lung in epidemic influenza, 255

in bronchitis, 105, 109, 110

in bronchitis in measles, 498

in coryza, 74

in grip, 225

in laryngitis, 96, 98, 99

in laryngitis in measles, 499

in membranous angina in scarlet fever, 400,401

in pertussis, 527

in pneumonia, 165,201

in rhinitis, 74, 77

in tracheitis of grip, 225, 232

oxygen, for dyspnea in pneumonia, 179

in pertussis, 536

steam, in bronchopneumonia, 180

in bronchopneumonia complicating measles, 497

in diphtheria, 461

in glanders, 689

in laryngitis in small pox, 615

in laryngitis in typhus fever, 630

in pneumonia, 165

Inhalers, 96, 99

Insomnia, in anthrax, 683

in dengue, 642,644

in encephalitis lethargica, 272,275

in epidemic influenza, 245

in pertussis, 533,541

in poliomyelitis, 596,605

in rheumatism, 36,68

in septicemia, 761,770

in small pox, 614,623

in tonsillitis, 84,89

Intestinal anthrax, 679

Intestinal infection, and rheumatism, 62

Intracerebral administration of antitoxin, 717 725
Intramuscular administration of antitoxin, 717 , 725

Intraneural administration of antitoxin, 717,725

Intraspinal administration of antitoxin, 716, 725 of magnesium sulphate, in tetanus, 719

treatment of cerebrospinal meningitis, 568,584

Intravenous injection, of adrenalin in pneumonia, 177

of diphtheria antitoxin, 457, 480

of foreign protein in rheumatism, 58

of quinine, 332

of salicylates, 47,66

of saline solution in cholera, 647, 652

of tetanus antitoxin, 716, 725

Intubation, in diphtheria, 462

indications for, 463

in edema of larynx, 99

in pertussis, 528

post-intubation treatment, 463 procedure, 462

Iodine, for itching and burning of small pox, 613 for lesions of farcy, 689

for local treatment of tetanus, 722, 725

for odor of small pox, 613

for membranous angina in scarlet fever, 401

in fibrinous pleurisy, 116, 126

in rabies, 701

in treatment of wounds, 722,725

in Vincent's angina, 92, 93

Iodoform, for odor of small pox, 613

in glanders, 689

in treatment of wounds, 722

Ipecac, in amebic dysentery, 376

in anthrax, 681

in laryngitis, 96, 98, 99, 100

Iron, for anemia, in malaria, 339,351

in scarlatinal nephritis, 423

for convalescence. See under the several diseases

Irrigation, colonic, for ileocolitis complicating measles, 500,509

in otitis of scarlet fever, 410

nasal, in diphtheria, 452,466

in glanders, 689

of throat, in scarlet fever, 400

oral, in foot and mouth disease, 69

rectal, in bacillary dysentery, 362,363

Isolation. See under the several diseases

Itching and burning, in measles, 491, 505

in small pox, 612,621

in varicella, 516

Jackson, on plague, 637

Jacobi, on belladonna, in pertussis, 530,540

Jalap, in Malta fever, 658

Jalap powder, compound, in scarlatinal nephritis, 417

James, on dosage of quinine, 330

on relapses in malaria, 338

Jaundice, complicating pneumonia, 191

Jaundice, infectious, 728, and see Infectious jaundice

Jenner, on small pox, 608

John and Ashurst, on tetanus, 711, 714

Johns, on blackwater fever, 343 on malaria, 324,327

Joints, care of in Malta fever, 658

in cerebrospinal meningitis, 577

in rheumatism, $31,48,50,66$

in scarlatinal arthritis, 413,437

in septicemia, 763,771

Kemp, on rheumatism, 54

$\mathrm{Ker}$, on orchitis in mumps, 549 on pertussis, 528,534

Kerr and Stimson, on rabies, 708

Kidneys, complication of, in diphtheria, 473

in malaria, 336,350

congestion of, in yellow fever, 739

functions of, 393

hemorrhage from, due to quinine, 334

involvement of, in epidemic influenza, 257

in measles, 501

in scarlet fever, 414 
Kidnevs, in septicemia, 763

Kilmer's belt, in pertussis, 526, 533

Klemperer, on gastro-intestinal function, 18

Klercher, on typhoid fever, 24

Knee-joint, paracentesis of, 51

Knives, disinfection of, 281

Koch, on blackwater fever, 344

method of giving quinine in malaria, 329

Koumyss, food value of, 17

Krehl, on toxemia, 21

Krumwiede-Valentine, method of typing sputum. 131

Lamb, food value of, 17

Lambert, on pneumonia, 22, 25

Langmead, on rheumatism in children, 31

Laryngeal diphtheria, 460, and see Diphtheria laryngeal

Laryngeal spasm, in pertussis, 528

Laryngitis, acute, 94

abortive treatment of, 95, 99

care of bowels in, 95, 99

in children, 98,100

cold compresses in, 97, 100

complications in, 98, 100

cough in, 96, 99

diagnosis of, 94

edema of larynx in, 98, 100

etiology of, 94

fomentations in, 97,100

ice collar in, 97, 100

in epidemic influenza, 256

in measles, $498,508,511$

in small pox, 615,623

in typhus fever, 630

inhalations in, 96, 98, 99

local treatment of, $97,99,100$

rest in, 95, 99

smoking prohibited in, 95, 99

sprays in, 97,100 .

summary of treatment of, 99

symptomatology of, 94

Laryngoscope, use of, 94

Latham, on chaulmoogra oil in leprosy, 672 on lumbar puncture, 573

Lawson, Mary R., on malaria, 328, 337

Leeching. See Cupping

Lees, on rheumatic fever, 39, 54

Leprolin, for leprosy, 674

Leprosy, 670

baths in, 671

Calmette's antivenomous serum in, 673

diet in, 671,676

disposal of excreta and secretions in, 670

distribution of, 670

empirical treatment of, 671,676

fresh air in, 671, 676

isolation in, 670,675

local treatment of, 672, 676

organism of, 670

precautions for attendants in, 670,675

prognosis of, 674

prophylaxis of, 674, 677

specific treatment of, 673,676

summary of treatment of, 675

surgical treatment of, 674

tonic treatment of, 674,676

$\mathrm{X}$-ray treatment of, $\mathbf{6 7 4}$

Leyden, von, on gastro-intestinal function, 18

Light, importance of in treatment of septicemia, 758

Lillienthal's method of aspiration, 254, 268

Lindeman, on blood transfusion in sepsis, 767

Lipovaccines, in typhoid fever, 309

Liver abscess, in amebic dysentery, 378

Lockjaw, 712

Loeffler's solution, in diphtheria, 466

for membranous angina in scarlet fever, 401

Loewe, on blood typing in pneumonia, 13i

Longcope, on serum treatment in pneumonia, 130

Lord, on fluid in chest in pneumonia, 180

on lung expansion in pleurisy with effusion, 124

Losee, on blood transfusion, 767
Louse, and trench fever, 751, 753

and typhus fever, 624, 626

Lovelace, on treatment of blackwater fever, 344

Lovett, on operative treatment in poliomyelitis, 602

on prevention of contracture in poliomyelitis, 599

Ludke, on pyrexia, 19

Ludy, on anthrax, 681

Lumbar puncture, for cerebral disturbances in typhoid fever, 298

for convulsions in pertussis, $\mathbf{5 3 6}$

for headache in scarlatinal nephritis, 422

in cerebrospinal meningitis, 569, 584

amount of fluid withdrawn, 571

anesthetic for, 570,585

appearance of fluid in, 571

bad results of, 573

collecting fluid in, 571

dry tap in, 572

flow in, 571

needle for, 570

position of patient in, 570

preparations for, 570

reaction in, 572

site of, 569

the tap in, 57

in encephalitis lethargica, 275

in meningitis following grip, 229

in meningo-encephalitis of mumps, 551

Luminal, for restlessness in chorea, 60

Lung, abscess of, in epidemic influenza, 254 complications of in malaria, 337,350

expansion in pleurisy with effusion, technique of, 124,127

Lusk, on infectious fevers, 20

on functions of protein, 15

Lyssophobia, 706

MacCallum, on pyrexia, 19

on streptococcus pneumonia, 208, 209

Maclagan, on salicin, 37

McCollom, method of fumigation recommended by, 475

on dosage of diphtheria antitoxin, 455

on irrigation, in nasal diphtheria, 466 on laryngeal diphtheria, 461

on scarlet fever, 388,402

McCrae, on prophylaxis of typhoid fever, 303

McGlannan, on rabies, 701

McGuire, on noma, 424, 429

Macaroni, food value of, 17

Mackerel, food value of 17

Magnesium sulphate. See Epsom salts

Magnus-Levy, on metabolism, 12

Malaria, 322

algid form of, 336,349

anemis in $337,350,351$

bed in, 323

cachexia in, 338, 350

care of bowels in, 324,346

carriers, 343

chill in, 324, 346

choleraic form of, 336,350

classes of, 322

collapse in, 325,335

complications of, 336,350

convalescence from, 340

diet in, 323, 346

fever in, 325, 346, 349

headache in, 325,347

hyperpyrexia in, 325,349

isolation in, 342

latent, 338, 350

masked, 338, 350

multiple infectious, 336

nervous manifestations in, 339,351

pernicious, 330

cerebrospinal type of, 335,349

collapse in, 335

comatose form of, 335,349

hyperpyrexia in, 335

irritative form of, 335,349

paralytic form of, 335,349 
Malaria, pernicious, quinine treatment of, 330 348

sequelæ of, 337,350

symptomatic treatment of, 335,349

prevention of collapse in, $325,346,347$

prophylaxis from, $340,351,342$

protection of individual in, 341,352

relapses in, 337,350

rest in, 323,345

room in, 323, 345

sequelæ of, 337,350

specific treatment of, 326,346

summary of treatment of, 345

sweating in, 326,347

symptomatic treatment of, during paroxysm, 324,346

vomiting in, 325,346

Malignant pustule, 678, 679, 684, and see Anthrax

Mallein, use of in farcy, 688

Malta fever, 656

bed in, 657,661

care of body in, 657,661

of bowels in, 658,661

of joints in, 658

carriers of, 660

constipation in, 658

convalescence from, 660,663

diet in, 657, 661

disinfection in, 660

disposal of excreta in, 660

distribution of, 660

drinks in, 658

fever in, 659,662

goats carriers of, 656

hyperpyrexia in, 659

incubation period of, 656

orchitis in, 660

pain in, 658, 662

precautions of nurse in, 657,661

of physician in, 657, 661

prognosis in, 660

prophylaxis of, 660,663

relapses in, 657

rest in, 657,661

retention of urine in, 658,662

room in, 657,661

sleeplessness in, 659,662

specific treatment of, 658

summary of treatment of, 661

symptomatic treatment of, 658,662

symptoms of, 656

toxemia in, 659, 662

vomiting in, 659,662

Mammary gland involvement in mumps, 550

Mania, from salicylates, 40

Manson, theory on malaria, 329

Mask, for physician and nurses in influenza, 241

Mattresses, disinfection of, 282

Maxwell and Pope, on ice poultice in mumps, 549 on mustard bath, 95

on hot air bath, 418

Measles, 487

adenitis complicating, 499

bath in, 490, 504

bed in, 490, 504

bronchitis complicating, 498, 508

bronchopneumonia complicating, 495, 507

care of bowels in, 493, 498, 505

of cardiovascular system in, 494, 498

of eyes in, $492,501,503,505,510$

of genitals in, 493,505

of mouth in, 492

of nose in, 492,505

of patient in, 490, 504

of skin in, 491, 505

complications of, 495,507

convalescence from, 502,511

cough in, 497

diet in, 490, 496, 504

diphtheria complicating, 501, 510

disinfection in, 503, 511

distribution of family in, 488, 503

fresh air in, 489, 503 gastro-intestinal complications of, 499, 509

German, 512, and see Rubella

heart complications of, 501,510

ileocolitis compheating, 500, 509

infectivity of, 487

kidney involvement in, 501, 510

laryngitis complicating, 498, 508, 511 .

mortality in, 487

noma complicating, 499, 509

open air treatment of, 496

otitis complicating, 499, 508

photophobia complicating, 501, 510

precautions in sick room in, 490, 504

of nurse in, 489,503

of physician in, 490, 504

quarantine in, $488,502,511$

room in, 488, 503

sleeplessness in, 495, 507

summary of treatment of, 503

treatment of fever in, 493, 497, 506

of nervous symptoms in, 494, 506

of respiratory failure in, 494

tuberculosis complicating, 502, 511

ulcerative stomatitis complicating, 499, 509

water in, 491, 504

Medinal, in pneumonia, 169

Meltzer, on magnesium sulphate as a test for typhoid carriers, 305

on magnesium sulphate in tetanus, 720

Meningismus, in epidemic influenza, 269

in typhoid fever, 302

Meningitis, cerebrospinal, 560, and see Cerebrospinal meningitis

Meningococcic serum, in cerebrospinal meningitis, 567

dosage of, 568

early use of, 573

intradural administration of, 569

intravenous administration of, $\mathbf{5 7 4}$

mode of preparation of, 568

results of, 573

Meningo-encephalitis in mumps, 550

Mental rest, importance of in febrile conditions, 2

Menthol, in bronchitis, 105, 109

in epidemic influenza, 243

in pertussis, 528

in Malta fever, 658

in small pox, 612

Methyl salicylate. See Salicylates

Methylene blue, as a substitute for quinine, 339 , 351

Meyer and Gottlieb, on caffeine in vasomotor weakness, 405

Milk, analysis of, 16,28

in acute infectious diseases, 16 , and see under the several diseases (diet)

caloric value of, 16,35

food value of, 17

Milk supply, typhoid fever and,'304

Miller, on acidosis, 46

Morphine, for choleraic form of malaria, 336

for convulsions in anthrax, 684

in pertussis, 536

in rabies, 706

in scarlatinal nephritis, 422

in septicemia, 761

for cough, in bronchitis, 106, 110

in glanders, 689

in laryngitis, 96, 99

in pneumonia, 166

for delirium, in anthrax, .684

in epidemic influenza, 246

in erysipelas, 774

in infectious jaundice, 733

in measles, 495

in pneumonia, 168

in scarlet fever, 407

in septicemia, 761

in small pox, 614

in typhus fever, 629

for gastric distress, in yellow fever, 739

for headache, in dengue, 642

in malaria, 325

in pneumonia, 170 
Morphine, for hemorrhage, in typhoid fever, 296 for ileocolitis complicating measles, 501

for insomnis, in anthrax, 684

in epidemic influenza, 246

in plague, 636

in pneumonia, 169

in Rocky mountain spotted fever, 666

in small pox, 615

in tonsillitis, 84,89

in typhoid fever, 298

for late circulatory failure, in diphtheria, 469

for pain, in dengue, 642

in epidemic influenza, 245

in mumps, 551

for restlessness, in chorea, 61

for vomiting, in cholera, 648

in infectious jaundice, 730

in malaria, 325

in Malta fever, 659

in cerebrospinal meningitis, 566

in encephalitis lethargica, 275

in fibrinous pleurisy, 118, 126

in poliomyelitis, 596

in rheumatism, $46,53,61$

in trench fever, 752,755

to facilitate nasal feeding, in tetanus, 719

Morphine suppository, for tenesmus in bacillary dysentery, 364, 372

Moser serum, in scarlet fever, 409

in septicemia, 765

Mosquito, and dengue, 641

and malaria, 322

and yellow fever, 735

destruction of, in prevention of malaria, 341

in prevention of yellow fever, 740

Mouth, care of. See under the several diseases.

Mumps, 545

arthritis in, 551

baths in, 546,552

care of bowels in, 547,552

of mouth in, 546,552

of nose in, 546,552

of patient in, 546,551

of secretions in, 546

complications of, 549,553

convalescence from, 551,554

disinfection after, 551,554

diet in, 546,552

distribution of family in, 545,551

drugs in, 548

fever in, 547,552

gland involvement in, 549,552

in adults, 546

incubation period of, 545

infection through third person, 546

local treatment in, 547

meningo-encephalitis in, 550,554

nephritis in, 551

nervousness in, 551, 554

orchitis complicating, 549,553

pain in, 551, 554

pancreatitis in, 550,553

precautions of nurse in, 546, 552

of physician in, 546,552

release from quarantine in, 551,554

room in, 546, 551

sleeplessness in, 551, 554

summary of treatment of, 551

symptoms of, 545

thymus gland in, 550

treatment of parotitis in. ${ }^{547,552}$

vulvitis complicating, 550,553

Murphy drip, 154

apparatus, 153,154

in bronchopneumonia complicating measles, 498

in cholera, 648

in diphtheria, 451

in pneumonia, 154

in septicemia, 761

in yellow fever, 739

Mustard bath, for convulsions of pertussis, 535

in laryngitis, 95, 99

in measles, 493
Mustard bath, technique, 535

footbath, hot, for headache in yellow fever, 738 in laryngitis, 95, 99

in rhinitis, 72

pack, for convulsions in pertussis, 535

pastes, plasters, and poultices, for backache in yellow fever, 738

for bronchitis, 104, 109

for bronchitis complicating measles, 498

for bronchopneumonia complicating measles, 493,497

for cough in pleurisy, 162

for epidemic influenza, 246, 25

for fibrinous pleurisy, 116, 126

for gastric distress in yellow fever, 738

for nausea in cerebrospinal meningitis, 565

for nausea in infectious jaundice, 730

for nausea in scarlet fever, 422

for vomiting, in malaria, 325

in Malta fever, 659

in septicemis, 761

in typhoid fever, 295,315

in fibrinous pleurisy, 116, 126

in pneumonia, 162, 187

Mustard plaster, application of, 163

Muzzling law, for prevention of rabies, 707

Myositis, in scarlet fever, 414

Nagele method of breaking spasm in pertussis, 528

Nasal diphtheria, 451, and see Diphtheria, nasal

Nasal feeding, in tetanus, 713,724

Nasal hemorrhages in pertussis, 534

Nasal passages. care of in prophylaxis of pneumonia, 192

Nasopharynx, care of in pneumonia, 149

portal of entry of bacillus lepre, 670

Nastin, in leprosy, 673

Nausea. See under the several diseases

Negri bodies, in rabies, 699

Nephritis, in diphtheria, 473,484

in epidemic influenza, 258

in glandular fever, 556

in infectious jaundice, 731

in measles, 501

in mumps, 551

in scarlet fever, 388,414

in tonsillitis, 86

Nervousness, in epidemic influenza, 265

Neuritis, following influenza, 259

Nightgown, special, in rheumatism, 32 in scarlet fever, 392

Nitrate of silver, in amebic dysentery, 383

in bacillary dysentery, 367

for ulcerative somatitis complicating measles, 500

for wound in rabies, 701

Nitrio acid, for noma complicating scarlet fever. 424

for noma complicating measles, 500

for wound in rabies, 700,708

Nitrogen, elimination of, 21

partition, 21

rest, 24

rôle of, in acute infectious diseases, 21

total, 22

Nitroglycerin, in convulsions in scarlatinal nephritis, 422

in pneumonia, 177

Noguchi, on yellow fever, 735

Noma, complicating measles, 499, 509

in scarlet fever, 424

Norris, on fluid in chest in pneumonia, 180

on pericarditis in pneumonia, 184

Normal salt solution. See Saline solution

Northrup's directions for release of scarlet fever patient, 442

Nose, care of. See under the several diseasee (nose, or body, or patient)

Nose-bleed, in dengue, 643

in diphtheria, 467, 482

in pertussis, 534

Nurse. See under the several diseases

Nux vomica. See Strychnine 
Oatmeal, food value of, 17

Odor of small pox, 613,622

Oil, food value of, 18

Oil of chenopodium, for amebic dysentery, 383

Oil of gaultheria, 43

Oil of wintergreen, 43

Oleum betulæ, 43

Oleum gynocardiæ, in leprosy, 671

Open air treatment, of bronchopneumonia complicating measles, 496

of bronchopneumonia complicating pertussis 534

of diphtheria, 471

of fever, 4

of measles, 496

of pneumonia, 155, 168, 199

of typhoid fever, 279

of typhus fever, 630,632

Ophthalmoscope, use of, 94

Opium

in anthrax, 684

in bacillary dysentery, $363,365,372,373$

in cholera, 647, 654

in foot and mouth diseases, 695

in ileocolitis complicating measles, 501

in infectious jaundice, 730

in pertussis, $531,536,543,641$,

in typhoid fever, 294

Orchitis, in Malta fever, 660

in mumps, 549,553

Otitis. See under the several diseases

Otoscope, use of, 94

Ottenberg and Libman, on blood transfusion, 767

Ouabain, in pneumonia, 174

Ovaries, involvement of in mumps, 550

Oxygen inhalations, in bronchopneumonia of measles, 497

in convulsions of pertussis, 536

in dyspnea of pneumonia, 179

in poliomyelitis, 600

in pulmonary edema of epidemic influenza, 249

Packs, cold, in bronchopneumonia complicating measles, 497

in measles, 493

in pneumonia, 161

in typhoid fever, 291

hot, for backache in yellow fever, 738 in chorea, 60

mustard, for convulsions in pertussis, 535

Pain, See under the several diseases

Pancarditis, in rheumatism, 54

Pancreas, involvement of in mumps, 550

Paquelin cautery, in noma complicating measles, 499

Paracentesis, for hydrothorax, in scarlatinal nephritis, 421

for pericarditis of pneumonia, 184

in mastoiditis complicating grip, 226

in otitis complicating scarlet fever, 410

of knee joint, 51

Paraldehyde, for sleeplessness in pneumonia, 170

Paralysis. See under the several diseases infantile, 590, and see Poliomyelitis

Paratyphoid fever, 320

preventive inoculation, 320

treatment of, 321

Paregoric, in cholera, 647

in pertussis, 531,541

Park, on diptheria antitoxin, 454, 456, 460

on rabies, 702, 703, 705

on tetanus antitoxin, $715,716,717$

on vaccine therapy in farcy, 688

Parotitis, 545, and see Mumps

complicating pneumonia, 191

complicating typhus fever, 630,633

in epidemic influenza, 260

in glandular fever, 556

treatment of in mumps, 547,552

Parrots, psittacosis and, 698

Pasteur treatment of rabies, 703

Patient, care of, see under the several diseases

Payne, on specific organism of rheumatism, 30

Peas, food value of, 18
Pediculus vestimenti, typhus fever transmitted by, 624

Pembrey, on respiratory exchange, 4

Peptonized milk, enema in tetanus, 713

Perforation in typhoid fever, 297

Pericarditis, in measles, 501

in pneumonia, 184

in rheumatism, 53

in scarlet fever, 423

Perinephritic abscess, in epidemic influenza, 258

Peritonsillar abscess, 86

Pernicious malaria, 335, and see Malaria, pernicious

Pertussis, 520

abdominal support in, 526,533

avoidance of emotional states in, 527 .

bacillus of, 520,532

baths in, 524, 539

bed in, 524, 539

bromides in, $531,536,541$

bromoform in, 532

bronchopneumonia in, 534

care of bowels in, 525, 539

patient in, 525, 539

secretions in, 523

carriers of infection of, 522

catarrhal stage of, 520

change of climate in convalescence from, 543

chloroform for paroxysms of, 535

clothing in, 523, 538

complement fixation test in, 521

complications of, 533,542

contagiousness of, 520

convalescence from, 536, 543

convulsions in, 535, 542

cough in, 525, 540

diarrhea in, 534, 542

diet in, 524, 539

disinfection of room in, 522

distribution of family in, 521, 537

drugs in, 528, 540

elastic belt in, 526

fever in, 525, 539

fixation test in, 521

fresh air in, 537, 538

hemorrhages in, 534, 542

hygiene in, $525,539,544$

hyperexcitability of larynx in, $\mathbf{5 2 8}$

incubation period of, 520

inhalations in, 527

insomnia in, 533, 541

intubation in 528

isolation in, 521,537

laryngeal spasm in, $\mathbf{5 2 8}$

local procedures in, 526

mechanical support in, 526

mode of infection in, 522

mortality in, 520

nose bleed in, 534, 542

open air treatment of, 522,538

precautions in sick room in, 523, 538

of nurse in, 523, 538

of physician in, $\mathbf{5 2 3}, 538$

prophylactic use of vaccine in, 523, 538, 544

prophylaxis of, 537

quarantine in, 536

release from quarantine in, 536,543

removal of adenoids in, 537

removal of tonsils in, 537

room in, 522, 538

sera in, 532

specific treatment of, 532

summary of treatment of, 537

symptomatology of, 521

tonics in convalescence from 537,543

treatment of severe cases, 526, 540

ulcer of frenum in, 534,542

vaccines in, 532

vomiting in, $520,521,533$

Pestis minor, 634

Pharyngeal diphtheria, antitoxin in, 455

Pharyngeal paralysis in diphtheria, 451, 472

Pharyngitis, in epidemic influenza, 256

in poliomyelitis, 597 
Phenacetin, chemistry of, 222

in bronchitis, 104, 109

in coryza, 72

in epidemic influenza, 245

in erysipelas, $\mathbf{7 7 4}$

in fibrinous pleurisy, 118, 126

in glandular fever, 556

in grip, 218,231

in Malta fever, 659

in measles, 495,498

in mumps, 547

in pneumonia, 161

in poliomyelitis, 596

in rheumatism, 45

in rhinitis, 72,76

in scarlatinal nephritis, 422

in scarlet fever, 407

in small pox, 614

in tonsillitis, 83,89

in trench fever, 752,754

toxic effects of, 223

Phenol, for itching in scarlet fever, 395

for itching in small pox, 613

for itching in varicella, 516

for noms complicating measles, 500

for otitis complicating measles, 499

for wound in rabies, 701, 708

in anthrax, $679,680,681$

in earache of scarlet fever, 411

in farcy, 689

in measles, 492, 505

in tetanus, 718,722

Phenylsalicylate. See Salol

Phlebitis, in epidemic influenza, 257 in typhoid fever, 302

Photophobia complicating measles, 501, 510

Phthalein test of renal function, 415

Physician, precautions of. See under the several diseases

Physiological salt solution. See Saline solution in diphtheria, 466

in rabies, 701

Physostigma, for convulsions of tetanus, 721

Physostigmine, for tympanites in typhoid fever. 294

Pilocarpine, in scarlatinal nephritis, 419

Pituitrin, in pneumonia, 152

in pneumonia of epidemic influenza, 248

in tympanites of epidemic influenza, 251

in tympanites of typhoid fever, 294

Plague, 634

abortive, 634

ambulatory, 634

bed in, 635, 638

buboes in, 636, 63 ?

bubonic, 634

care of bladder in, 635,639

of body in, 635

of bowels in, 635,639

of patient in, 635,638

carriers, 634

circulation in, 635

convalescence from, 637, 640

delirium in, 636, 639

diarrbea in, 636, 640

diet in, 635,639

disinfection in, 637

disposal of excreta in, 635

fever in, 635, 639

fulminating, 634

headache in, 636

immunization in, 638

intestinal, 634

isolation in, 634

nervous symptoms of, 636,639

organism of, 634

pneumonic, 634

precautions for nurse in, 635, 638

for physician in, 635, 638

prophylactic inoculation against, 638

prophylaxis of, 637,638

room in, 634, 638

rôle of rats and fleas in, 637

septicemic, 634
Plague, sequelæ of, 637

sleeplessness in, 636,639

serum treatment of, 636

specific treatment of, 636

summary of treatment of, 638

Pleurisy, 112

dry, 112, 125

types of, 112

Pleurisy, fibrinous, 112

blisters in, 116, 126

care of bowels in, 114, 125

cautery in, 116, 126

coal-tar preparations in, 118, 126

cough in, 113, 118, 126

counterirritants in, 116, 126

cupping in, 117

diet in, 113,125

drinks in, 114,125

drugs in, 117, 126

dyspnea in 118,126

electric pad in, 115,126

fomentations in, 115, 126

heat in, 115, 126

hot water bag, 115, 126

ice bag in, 115, 126

iodine in, 116, 126

local measures in, 114, 126

mustard in, 116, 126

pain in, 113

poultice in, 116, 126

room in, 113,125

strapping the chest in, 114, 126

summary of treatment of, 125

Pleurisy, purulent. See Empyema

Pleurisy with effusion, 118

after-treatment of, 125, 127

aspiration in, 120, 127

autoserotherapy in, 124

convalescence in, 124, 127

Delafield's method in, 119, 126

diuretics in, 123, 127

expanding lung in, 124

exploratory puncture in, 120,127

general care of, 119,126

summary of treatment of, 126

thoracentesis in, 119

treatment of, 119

Pleuritis, 112, and see Pleurisy

Pneumococcus, carriers, 193

serum, 130, and see Serum treatment in pneumonia

types of, 129

vaccines, prophylactic use of, 193

Pneumonia, lobar, 128, and see Peumonia (Pneumococcus)

Pneumonia (Pneumococcus), 128

abdominal pain in, 191

agglutination test in, 130

anaphylaxis in. 134, 145

arthritis in, 191

bath in, 147

bed in, 146, 155, 195

bronchitis in, 164

bronchopneumonia, in, 179

care of body in, 147, 197

of bowels in, 151, 198

of circulation in, 170,203

of ears in, 149

of eyes in, 149

of fissures in, 149

of genitals in, 149

of mouth in, 148

of nose in, 149

of teeth in, 148

of tongue in, 148

carriers of, 193

collapse in, 177, 204

complications of, 180, 205

convalescence from, 192,200

cough in, 161, 200

desensitization in, 135, 136

diet in, 149, 197

drinks in, 151, 198

drugs in, 161, 166, 168, 200 
Pneumonia, dyspnea in, 179, 205

embolism complicating, 190

empyema complicating, 181, 187

enemata in, 152

endocarditis in, 190

etiology of, 128

fever in, 161, 200

following influenza, 226, 233, 236, 239, 246, 266

fluid in chest in, 180

herpetic eruption in, 149

hydrotherapy in, 157

in cerebrospinal meningitis, 588

in grip, 226, 233

in rheumatism, 68

inhalations in, 165, 201

jaundice in, 191

local treatment of, 167

meningitis complicating, 191

Murphy drip in, 154

open air treatment of, 155, 168, 199

pain in, 166

parotitis complicating, 191

pericarditis complicating, 184, 205

peritonitis complicating, 191

pleurisy in, 161

prophylaxis of, 192

relapses in, 191

rest in, 146, 194

room in, 147,195

sensitization in, 134,135

serum treatment of, 130 , and see Serum treatment of Pneumonia

specific treatment of, 129, 195

summary of treatment of, 194

symptomatic treatment of, 155, 160, 200

toxemia in, 167,201

treatment of collapse in, 177, 204

of cough in, 161

of delirium in, 168, 201

of dyspnea in, 179, 205

of fever in, 161,200

of headache in, 170,202

of pulmonary edema in, 178, 205

of sleeplessness and restlessness in 169, 202

of toxemia in, 201

tympänites in, 152, 199

types of, 128, 129

use of rectal tubes in, 152

vaccines, 193

Pneumonia (Streptococcus), 208

empyema in, 209

pathology of, 208, 210

summary of treatment of, 215

symptomatology of, 210

treatment of, $21 \mathrm{C}$

Poliomyelitis, 590

abortive cases of, 591

active movements in, 601

bed in, 593, 603

bulbospinal type of, 599,606

care of bladder in, 594, 604

of bowels in, 594, 604

of patient in, $592,593,603$

carriers of, 590

cerebrospinal fluid in, 591

contractures in, 599, 602

convulsions in, 596, 605

diarrhea in, 597, 606

diet in, 593, 604

disinfection in, 592

gastrointestinal symptoms in, 596, 606

hyperesthesia in, 605

insomnia in, 596, 605

involvement of muscles of trunk and diaphragm in, 600

isolation in, 592,603

meningo-encephalitic type of, 598

operative treatment of, 602

paralysis of extremities in, 600,606

paralytic period of, 600,606

passive movements in, 601

pathology of, 597

pharyngitis in, 606

precautions of nurses in, 592
Poliomyelitis, precautions of physicians in, 592 preparalytic period in, 594, 604 prognosis of, 602

prophylaxis of, 607

protection of community in, 590

room in, 592, 603

serum treatment of, 594, 595, 604, 605

specific treatment of, 594

summary of treatment of, 603

symptomatic treatment of, 593, 604

tonsillitis in, 606

transmission of, 590,592

types of, 597

urine in, 594, 604

Pope and Maxwell, on hot air bath, 418

on ice poultice in mumps, 549

on mustard bath, 95

Potassium chlorate, in foot and mouth disease, 695

for gargling in small pox, 611

for ulcerative stomatitis complicating measles, 499

Potassium iodide, in rheumatism, 46

Potassium permanganate, for membranous angina in scarlet fever, 401

for noma complicating measles, 500

for sterilization, 475

in cholera, 648

in diphtheria, 466

in foot and mouth disease, 695

in glanders, 689

in small pox, 611

Potassium salts, for nervous symptoms of septicemia, 761

in nephritis of scarlet fever, 420

Potato, food value of, 17

Poultices, in adenitis of scarlet fever, 413

dry, for orchitis in mumps, 549

hot, in anthrax, 681

in cholera, 647

in dengue, 642

in fibrinous pleurisy, 116, 126

in pericarditis of pneumonia, 186

in pneumonia, 163

in scarlatinal nephritis, 420

ice, in mumps, 548

in bacillary dysentery, 362

in bronchitis, condemned, 105

Poynton and Payne, on specific organism of rheumatism, 51

Precipitation method, in pneumonia, 130

Pregnancy, use of quinine during, 334

Premature systole, in epidemic influenza, 257, 268

Pressure, in local treatment of rheumatism, 51

Proescher, on rabies, 704

Prophylaxis. See under the several diseases

Protargol, in amebic dysentery, 382, 386

Protein, amount necessary to replace wear and tear, 15

amount needed in fever, 3

amount needed in health, 3

amount needed in infectious diseases, 28

amount needed in typhoid fever, 282

assimilation of, in acute infectious diseases, 18

destruction of by fever, 20

functions of, 14,28

percentage of, in milk, 17

needs, 14, 28

source of hest, 15

toxic destruction of, 20

Protein therapy, foreign, in rheumatism, 58

Psittacosis, 698

prognosis of, 698

prophylaxis of, 698

summary of treatment of, 698

Psychasthenia, in epidemic influenza, 259

Psychoses, in epidemic influenza, 266

Puerperal sepsis, 765

Pulmonary complications, in rheumatism, 56, 58

Pulmonary edema, in epidemic influenza, 248

in pneumonia, 178 Pulmonary embolism, complicating pneumonia, 
Pulmonary exercises, following pneumonia, 192

Pulse, in typhoid fever, effect of baths on, 290 Purgation, in scarlatinal nephritis, 417

Purpuras, in rheumatism, 57

Pustule, in varicella, 516 malignant of anthrax, 678

Pyelitis, in cerebrospinal meningitis, 578

Pyclonephritis, in epidemic influenza, 258, 269

Pyemia, 757, and see Septicemia

abscesses in, 763

in erysipelas, 777

surgical treatment of abscesses, 760

Pylephlebitis, in septicemia, 763

Pyrexia, 19, and see Fever, and under the several diseases

destructive action on protein, 20

factor in feeding in infectious diseases, 18

factor in fever, 19

increased caloric demand in, 28

significance of, 3

and toxemia, parallelism of, 1

Quarantine. See under the several diseases

Quigley, on typing of urine, in pneumonia, 132

Quinine, action of, 326

for children, 329,348

contraindications to, 334

desensitization, 334

dosage, 328

fastness, 329

hypodermic use of, 331,348

hypodermoclysis with, 331

idiosyncrasy to, 334

intramuscular use of, 331

intravenous administration of, 332,348

immunity, 329

preparations of, 328

prophylactic use of, 327,342

substitutes for, 340,351

time for administration of, 327

toxic effects of, 333

Quinine, in amebic dysentery, 381

in blackwater fever, 344, 352

in bronchitis, 104

in diphtheria, 474, 485

in epidemic influensa, 244

in grip, 224, 232

in malaria, 326 to 335 and 347 to 349

in pertussis, 532

Rabies, 699

confirmation of diagnosis of, 701

convulsive paroxysms of, 706, 709

detention of dogs for prevention of, 707

diet in, 709

immunity in, 699

in America, 708

incubation period of, 699,700

licensing of dogs for prevention of, 707

maniacal periods in, 707, 710

muzzling law for prevention of, 707

Pasteur treatment of, 703

preventive treatment of, 702

procedures in, 700

prophylaxis of, 707,710

quarantine in, 707,710

results of preventive treatment in, 705

room in, 706, 709

summary of treatment of, 708

stages of, 706

symptoms of, 706

in $\operatorname{dog}, 702$

transmission of, 699

treatment of, at a distance, 704

of developed disease, 706, 709

of old bites, 709

of wound in, 700, 708

virus of, 699

Rash, due to diphtheria antitoxin, 458

Rat-bite fever, 744

anemia in, 748,750

bed in, 746

blood picture in, 745

care of body in, 746
Rat-bite fever, care of bowels in, 746, 748

of mouth in, 746

of skin in, 747,749

cause of, 744

circulation in, 747,749

complications of, 748

convalescence in, 748,750

delirium in, 747,749

diet in, 746,748

dizziness in, 747,749

fever in, $744,747,749$

fluids in, 746,748

incubation period of, 744

nephritis in, 748

nervousness in, 747,749

pains and aches in, 746,749

prophylaxis in, 748,750

room in, 746

sleeplessness in, 747, 749

specific treatment of, 747,749

summary of treatment of, 748

symptomatology of, 744

Rats and plague, 634,637

Ravenel, on farcy, 689

Rectal administration of coffee, in pneumonia, 176 of digitalis, 172

of salicylates, 47,66

Rectal feeding, in diphtheria, 451

in foot and mouth disease, 694

in late circulatory failure in diphtheria, 469

in persistent vomiting in septicemia, 761

in tetanus, 713

Rectal irrigations, for pain in bacillary dysentery, 363

Rectal tubes, for tympanites in epidemic influenza, 251

for tympanites in bronchopneumonia complicating measles, 498

for tympanites in pneumonia, 152

for tympanites in typhoid fever, 293

disinfection of, 281

Renal complications, from salicylates, 40

Renal function, in nephritis of scarlet fever, 415

Repair of wear and tear, function of protein in, 14,28

Resection of rib, for empyema, 188

Resistance exercises, in poliomyelitis, 601

Respiration, effects of cold water on, 5

effects of baths on, in typhoid fever, 290

Respiratory disturbances, from salicylates, 40

Respiratory failure, in diphtheria, 472

in measles, 494

in poliomyelitis, 600

Respiratory symptoms, in small pox, 615

Respiratory system, care of, in glanders and farcy, 689

Rest. See under the several diseases

calories of energy in, 3

caloric requirements of man in, 12

in febrile conditions, 2,8

physiological significance of, 2

Rest nitrogen, 24

Restlessness. See under the several diseases

Retinal hemorrhages from salicylates, 40

in septicemia, 763

Retropharyngeal abscess, differentiated from diphtheria, 464

Revaccination, against smallpox, 619

against typhoid fever, 308

Rheumatic children, 63

joints of infancy, 30

Rheumatic fever, acute, 30

acetanilid in, 45

acidosis in, 46

adenoids in, 62

age in, 30

alkaline salts in, 43

alkaline treatment of, 46

antipyrin in, 45

arrhythmia in, 41

arthritis in, 48,51

aspirin in, 42

autoserotherapy in, 61

bed in, 32,63 
Rheumatic fever, bowels in, 35,64 bradycardia in, 41 cardiac complications in, $53,55,67,68$ catharsis in, 35 chorea and, 59

complications of, 52,67

convalescence from, 61,70 counterirritation in, 50, 67 delirium in, 40,53

diagnosis of, in infancy, 30 diet in, 33, 63

drugs to relieve pain, 66

dyspnea in, 40

effusions in, 67

etiology of, 30

fluids in, 35

foreign protein therapy in, 58

hyperpyrexia in, 52

in children, 30

in infancy, 30

insomnia in, 68

intestinal infection and, 62

intravenous injection of foreign protein in, 58

joints in, $31,48,50,51$

local applications in, 50, 67

morphine in, 46

paracentesis in, 51

phenacetin in, 45

pressure in, 51

prophylaxis in, 61,70

pulmonary complications in, 56,58

rest in, $31,48,63$

room in, 33,63

salicylates in, 37,64

shock therapy in, 58

sleep in, 36,68

soapsuds enema in, 36

specific treatment of, 37,64

summary of treatment of, 63

symptomatic treatment of, 48,66

symptomatology of, 31

teeth in, 62

theories of, 30

therapy of, 31

tonsils in, 62

vaccine therapy in, 57,68

water in, 35

Rheumatism, 30, and see Rheumatic fever, acute scarlatinal, 413

Rhinitis, acute, 71

abortive treatment of, 72

aches and pains in, 72,76

antral involvement in, 75, 79

complications of, 75,78

cough in, 75,77

direct application in, 73

early treatment of, 72

etiology of, 71

in grip 227,233

in scarlet fever, 402

inhalations in, 74,77

later treatment of, 74,78

local treatment of, 73,77

ointments in, 73

otitis complicating, 75,79

personal hygiene in, 75,79

prophylaxis of, 75,79

removal of obstructions in, 75,79

sinusitis compticating, 75,79

sore throat in, 75

sprays in, $73,74,77,78$

summary of treatment of, 76

symptomatology of, 71

Rice, on anthrax, 681

Rice, food value of, 17

Richter, on trench fever, 753

Ringer's solution, 652

Ringing in the ears, due to quinine, 333

Roast beef, food value of, 17

Rochelle salts, in bronchitis, 103, 109

in cerebrospinal meningitis, 565, 582

in dengue, 642

in diphtheria, 452

in epidemic influenza, 243
Rochelle salts, in erysipelas, 774

in fibrinous pleurisy, 114,125

in grip, 217,231

in ileocolitis complicating measles, 501

in laryngitis, 95, 99

in Malta fever, 658

in mumps, 547

in plague, 635

in pneumonia, 151

in rheumatism, 35

in scarlatinal nephritis, 417

in scarlet fever, 397

in septicemia, and pyemia, 760

in tonsillitis, 82,88

in typhoid fever, 292, 314

Rocky mountain spotted fever, 664

aches and pains in, 664, 668

baths in, 665

bed in, 665

care of body in, 665

of bowels in, 666,667

of eyes in, 666

of genitals in, 666

of mouth in, 666

of nose in, 666

of skin in, 666

cause of, 664

circulation in, 667,668

complications of, 667

delirium in, 666, 668

desquamation in, 665

diet in, 666, 667

fever in, 664, 666, 668

fluids in, 667

headache in, 668

immune serum in, 667,669

incubation period of, 664

insomnia in, 666, 668

prophylaxis in, 667,669

pulse in, 665

rash in, 665

room in, 665

summary of treatment of, 667

symptomatology of, 664

ticks and, 664

Rogers, on cholera, 647,652

on dosage of quinine, 330

on emetine treatment in amebic dysentery, 377

Room. See under the several diseases

Rosenau, on conveyance of smallpox, 608

on rabies, 701

on serum treatment in pneumonia, 130

Rosenow, on rheumatism, 63

theory of etiology of rheumatism, 30

Rost, on leprosy, 674

Rowntree and Amberg, on creatininin in infants, 23

Rubber sheets, disinfection of, 281

Rubella, 512

care of bowels in 513

complications of, 514

diet in, 513

isolation in, 512

summary of treatment of, 513

Rubner's caloric values, 13

Rudolph, on hemorrhage in typhoid fever, 295

Rührah, on small pox, 612

Russell, technique of antityphoid vaccination, 307

Salicin, 45, and see Salicylates

in glandular fever, 556

in grip, 224

in rheumatism, 37,45

in scarlatinal arthritis, 413

in tonsillitis, 83,89

Salicylates, 37

in anthrax, 683

in bronchitis, 104, 109

in cholera, 647

in chorea, 60

in dengue, 642

in epidemic influenza, 244, 245, 265

in fibrinous pleurisy, 117, 126 
Salicylates, in grip, 232

in infectious jaundice, 731

in Malta fever, 658

in poliomyelitis, 596

in rheumatism, $37,39,43,46,60,64$

in scarlatinal nephritis, 413,420

in tonsillitis, 82,89

Salicylates, acidosis from, 46 dyspnea from, 40

intravenous administration of, 47,66

rectal administration of, 47,66

synthetic, 42

toxic symptoms of, 39

Salicylic acid, 37, and see Salicylates

Saline infusions, for hemorrhage in typhoid fever, 297,316

in uremia in scarlatinal nephritis, 421

Saline irrigation, for collapse in pneumonia, 178

for diarrhea in typhoid fever, 294

in poliomyelitis, 597

Saline rectal injections, for renal congestion in yellow fever, 739

Saline solution, enteroclysis of, in diphtheria, 470 intravenous injection of, in cholera, 648 irrigation of nose with in glanders, 689

oral irrigation with in foot and mouth disease. 694

in tetanus, 714

Sa line treatment of bacillary dysentery, 359,362 , 372

Salivary secretion, in acute infectious diseases, 18

Salmon, food value of, 17

Salol, in rheumatism, 45

Salt-bag, hot, for earache, 411

Salt solution, as mouth wash in measles, 492

physiological, for membranous angina in scarlet fever 400

for nose in scarlet fever, 402

Salts. See Epsom salts, and Rochelle salts caloric value of, 13

excreted by intestines, 22

Sanford, on blood transfusion, 767

Salvarsan, in malaria, 340

in rat bite fever, 747,749

in trench fever, 753

in Vincent's angina, 92, 93

Sanitation, in prevention of typhoid fever, 304

Satterlee, on blood transfusion, 767

Scarlatinal nephritis, 414

Scarlatinal rheumatism, 413

Scarlet fever, 387

adenitis in, 412, 437

anemia in, 423,441

anging in, 398,432

arthritis in, 413,437

bed in, 392, and see Pneumonia, bed in

bronchopneumonia complicating, 423

cardiovascular apparatus in, 403

care of bowels in, 397,431

of discharges in, 392

of eyes in, 431

of genitals in, 397, 431

of mouth in, 396, 430

of nose in, 396, 402, 431

of skin in, 395, 430

of teeth in, 396

of throat in, 396, 397, 431

catharsis in, 397

causative agent in, 387

circulatory failure in, 434

contacts, 389

convalescence from nephritis in, 423,441

convulsions in, 422,440

delirium in, 407

desquamation in, 388,430

diaphoresis in, 421, 438

diet in, 392, 416, 429,438

discharge of patient after, 424, 441

disinfection of utensils in, 392, 428

distribution of family in, 388,427

diuresis in, 420,439

drinks in, 395, 416, 430

earache in, 411

edema in, 421, 438, 439
Scarlet fever, eosinophilia in, 388 erythematous eruption in, 387

exfoliation in, 395

fever in, 397, 432

fumigation after, 425,442

headache in, 422,440

heart in, 423, 403

hypertension in, 439, 440

immune human serum in, 408

incubation period of, 387, 427

isolation in, 390

leucocytosis in, 388

muscular twitching in, 421, 439

myocarditis in, 423

myositis in, 414

nausea and vomiting in, 422, 431, 440

nephritis in, $388,414,437$

nervous symptoms in, 407,435

noma complicating, 424,441

normal human blood in, 409

oliguria in, 438

onset and diagnosis of, 387, 427

otitis in, 409, 436

patient in, 392, 428

pericarditis in, 423

polyvalent serum from, 409

precsutions in sick room in, 391, 428

of nurse in, 390, 428

of physician in, 391, 428

prophylaxis in, 426

protection of family in, 388

quarantine in, 424,425,426, and see Distribution of family, and Isolation

recurrence of, 424

relapses in, 424,441

release of patient after, 424

rest lessness in, 407,435

rhinitis in, 402,433

room in, 389,427

septicemia in, 764

serum therapy in, 407,436

sleeplessness in, 407,435

sore throat in, 387

specific treatment of, 407,436

sterilization after, 425,442

summary of treatment of, 427

suppression of urine in, 438

symptomatic treatment of, 397

temperature in, 387

tongue in, 388

uremis in, 421, 439

use of cold in 432

vaccine therapy in, $402,407,411,436$

vomiting in, 397

water in, 395

Schamberg, on iodine in small pox, 613

Schick reaction, in diphtheria, 444, 445, 449, 450 . 477

combined reaction, 447

control, 447

negative reaction, 446

pseudo-reaction, 446

technique of, 445

Seiler's tablets in coryza, 73

in rhinitis, 73

Sellard's dosage of emetine in amebic dysentery, 377

method of determining acidosis, 25

treatment of Asiatic cholera, 648, 649, 651

Sensitization, in pneumonia, 134

determination of, 135

Septic conditions, physiology of diet in, 3

Septic sore throat, in tonsillitis, 85,90

Septicemia and Pyemia, 757

accidents after, 770

arthritis in, 763,771

bed in, 759,768

blood transfusion in, 766

care of body in, 760

of bowels in, 760,769

cerebrospinal meningitis in, 762

chills in 760,769

circulation in, 762,770

collapse in, 760,769 
Septicemia and Pyemia, convulsions in, 762, 770

delirium in, 761, 770

diagnosis of in early stage, 758

diet in, 759,768

drugs for nervous symptoms of, 761

early symptoms of, 758

emboli in, 763,771

erythema in, 763

fever in, 760,769

fluids in, 760,769

form of in puerperal sepsis, 765

in scarlet fever, 764

fresh air in, 759

headache in, 762,770

hemorrhages in, 763

hyperpyrexia, in, 760

icterus in, 763

in erysipelas, 777

in nephritis, 86

in scarlet fever, 764

infarcts in, 763,771

insomnia in, 761, 770

involvement of kidneys in, 763, 771

light in, 758

malignant endocarditis in, 762

nervous symptoms in, 761, 770

organisms of, 757

osteomyelitis in, 763

pneumococcus, 766

polyvalent serum in, 765

prophylaxis of, 768

rest in, 758,768

restraint of patient in, 761

room in, 758, 768

specific treatment of, 764,771

staphylococcus, $757,764,766$

streptococcus, 757,763

summary of treatment of, 768

symptomatic treatment of, 760,769

sweats in, 760

thrombophlebitis in, 762,771

vaccine therapy in, 765

visceral abscesses in, 764

vomiting in, 760,770

Septicopyemia, symptoms of, 758

Serum, administration of, 133,195

anaphylaxis after, 134,145

apparatus, 137

dosage, 146, 196

injection of, 142

preparation of patient and operator, 141

precautions, 136

reaction after, 143,196

shock after, 145

sterilizing apparatus, 140

symptoms after, 144

Serum sickness, pneumococcal, 196

Serum therapy. See under the various diseases

Shad, food value of, 17

Shaffer, on typhoid diet, 22, 23, 25, 283

Shattuck, typhoid diet, 285

Shaving brushes and anthrax, 678

Sheet bath, technique of, 159

Shock, anaphylactic, in pneumonia, 145

Shock therapy, in rheumatic fever, 58

Sick room, care of. See under the several diseases

Silver nitrate, in amebic dysentery, 382

in bacillary dysentery, 368

for oral ulcers in smallpox, 611

for ulcerative stomatitis complicating measles, 500

for ulcer of frenum in pertussis, 534

for ulcers of mouth in foot and mouth disease, 695

in tonsillitis, $84,89,90$

in Vincent's angina, 92, 93

Silver preparations for carriers of cerebrospinal meningitis, 579

Sino-auricular block, in rheumatism, 41

Sinus arythmia, in epidemic influenza, 257, 268

Sinus thrombosis in grip, 226, 233

Sinuses, involvement of, in rheumatic fever, 62 in scarlet fever, 402
Sinusitis, complicating coryza, 75

epidemic influenza, 256

rhinitis, $75, \cdot 79$

Skatol, in undetermined nitrogen, 25

Skin, care of. See under the several diseases

Skin eruptions, complicating rheumatism, 57

due to antipyretics, 223

due to quinine, 333

due to salicylates, 40

Sladen, on infectious jaundice, 732

Sleeplessness. See under the several diseases

Sleeping sickness, 271, and see Encephalitis lethargica

Sliding in bed, to prevent, 147

Slush, in typhoid fever, 291

Small pox, 608

baths in, 611, 621

bed in, 610

care of bowels in, 613,622

of eyes in, 611, 621

of mouth in, 610,620

of nose in, 611, 621

of skin in, 611, 621

delirium in, 614, 623

diet in, 610,620

disinfection in, 616

fever in, 614, 622

headache in, 614,622

initial stage of, 613

insomnia in, 614,623

isolation in, 608, 620

precautions for nurse in, 609,620

for physicians in, 609,620

quarantine in, 615,624

respiratory symptoms in, 615,623

room in, 608

summary of treatment of, 620

symptomatic treatment of, 613,622

treatment of other members of family or exposed persons, 609,612

vaccination against, 616

water in, 610

Smith, Theobald, on vaccine therapy in farcy, 688

Smoking prohibited, in bronchitis, 102

in laryngitis, 95,99

Soapsuds enema, in rheumatic fever, 36

technique of, 36

Sodium benzoate and caffeine in febrile conditions, 6

in Malta fever, 660

in scarlet fever, 405

Sodium bicarbonate, for nausea, in cerebrospina meningitis, 566

in scarlet fever, 422

for laryngitis complicating measles, 499

for retching, in small pox, 615

for vomiting, in anthrax, 683

in poliomyelitis, 596

in epidemic influenza, 245

in infectious jaundice, 730

in measles, 491

in scarlatinal arthritis, 413

in scarlatinal nephritis, 420

in typhoid fever, 295

spray, for membranous angina in scarlet fever, 400

Sodium bromide, in laryngitis, 98, 100 in pertussis, 529

Sodium chloride solution in cholera, 648

for irrigating throat in diphtheria, 466

Sodium phosphate, in bronchitis, 103

in scarlatinal nephritis, 417

in septicemia, 760

in tonsillitis, 82

Sodium potassium tartrate. See Rochelle salts

Sodium salicylate, in Malta fever, 660

in scarlatinal arthritis, 413

in scarlatinal nephritis, 420

in scarlet fever, 405

solutions of, doses, 41

synthetic, 42

toxic dose of, 39

Sodium sulphate. See Glauber's salts

Soft palate, paralysis of in diphtheria, 472 
Sollman on caffeine in vasomotor weakness, 405 Sophian on cerebrospinal meningitis, 568, 571, 576,579

Sordes, in cerebrospinal meningitis, 565

in diphtheria, 452

in measles, 492

in scarlet fever, 396

in typhoid fever, 280

Sore throat, 80 , and see Tonsillitis

diphtheritic, 81

in coryza, 75

in rheumatism, 57,68

in rhinitis, 75

in scarlet fever, 387

Southey tubes, 421

Sparteine, in pneumonia, 175

Spasmodic croup, differentiated from laryngeal diphtheria, 464

treatment of, 98

Specific treatment. See under the several diseases

Spinach, food value of, 17

Spinal fluid, in cerebrospinal meningitis, 568

in encephalitis lethargica, 272

in poliomyelitis, 591

Sponge bath, in cerebrospinal meningitis, 566 in grip, 217

Sponges, cold, in pneumonia, 16 in yellow fever, 739

cool, in chorea, 60

in dengue, 642

in measles, 494

in pertussis, 537

in typhoid fever, 29

Sponging, cold, for bronchopneumonia complicating measles, 496

for fever in measles, 494

Spoons, disinfection of, 281

Sporadic influenza, 216, and see Grip

Spriggs, on excretion of creatinin, 23

Sputum, disinfection of in typhoid fever, 281

typing of, in pneumonia, 131

Standard portions, Fisher's tables of, 17

Starvation, 18, 20, 29

in bacillary dysentery, 368

Steam inhalations. See Inhalations, steam

Steak, food value of, 17

Sterilization after scarlet fever, 425,442

Stitt, method of giving quinine in malsris, 329

on treatment of hepatic abscess in amebic dysentery, 379

on yellow fever, 735

Still, on urethane for convulsions in pertussis, $\mathbf{5 3 6}$

Stimson, on rabies, 708

Stomach, hemorrhages from in dengue, 643

motility of in acute infectious diseases, 18

Stomach tube, in diphtheris, 451

Stomach washing, for vomiting in typhoid fever, 694

in infectious jaundice, 730

Stomatitis, in pneumonia, 148

in varicella, 517, 519

Stools, in acute infectious diseases, 21

disinfection of, 281

Strapping the chest, in fibrinous pleurisy, 114, 126 in pneumonia, 161

Streptococcus hemolyticus, complicating messles, 489, 495, 502

Streptococcus empyema, 211, and see Empyema. streptococcus type

Streptococcus pneumonia, 208, and see Pneumonia, streptococcus

Strong, on leprosy, 670

Strophanthin, in acute glanders and farcy, 692 in anaphylactic shock in pneumonia, 145

in anthrax, 683

in bacillary dysentery, 365,373

in cerebrospinal meningitis, 566

in cholera, 650

in febrile conditions, 6,9

in pneumonis, $145,173,178,203$

in pneumonia of epidemic influenzs, 247

in pulmonary edema of epidemic influenza, 248,267
Strophant'hin, in septicemia, 762, 770

in scarlet fever, 404,434

in typhoid fever, 299

Strychnine, in anthrax, 683

for dyspnea in pneumonia, 179

for nervous symptoms of malaria, 339

in cerebrospinal meningitis, 566

in cholera, 650

in control of prostration from belladonna, 531

in convalescence from diphtheria, $\mathbf{4 7 4}$

from dengue, 643

from epidemic influenza, 261

from infectious jaundice, 733

from Malta fever, 660

from measles, 502

from typhoid fever, 303

in diphtheria, 470

in febrile conditions, 6

in glandular fever, 557

in grip, 225, 233

in pneumonia, 152

in poliomyelitis, 600

in scarlet fever, 406,435

in typhoid fever, 299

in tympanites of epidemic influenza, 252

Stupes, for backache in yellow fever, 738

for pain in bacillary dysentery, 362

for pain in dengue, 642

for tympanites in epidemio influenza, 251

for tympanites in typhoid fever, 293

in cholera, 647

in pneumonia, 152

turpentine, preparation of, 363

Stupor, in measles, 494

in septicemia, 758,761

in typhoid fever, 317

in typhus fever, 629

Subglottio edema differentiated from laryngeal diphtheria, 464

Sugar, food value of, 17

percentage of in milk, 17

Sulphates, ethereal, in endogenous metabolism, 23

Sulphur in metabolism, 24, 26

Sulphur dioxide, as disinfectant, 616,737

Suspensory for orchitis complicating mumps, 549

Symptomatic treatment. See under the several diseases

Symmers, on pneumonia in epidemic influenzs, 236

Tachycardia, in epidemic influenza, 257, 269 in scarlet fever, 403

Tartar emetic, in laryngitis, 98, 99

Teeth, care of. See under the several diseases

Tender toes complicating typhoid fever, 302

Tenesmus, in bacillary dysentery, 363,372

Terpin hydrate, in bronchitis of children, 108

Testicle, involvement of in mumps, 549

Tetanus, 711

antiseptics in, 722

bed in, 713, 724

care of bladder in, 714,725

of body in, 724

of bowels in, 714, 724

of circulation in, 721,727

of mouth in, 724

causes of death in, 721

complicating vaccination, 618

diet in, 713, 724

drug treatment of, $718,719,720$

fluids in, 724

incubation period of, 711,723

local, 718

local treatment of, $714,718,722,725$

mortality in, 721

prodromata of 712

prognosis of, 721

prophylaxis of, 712,727

rest in, 712

retention of urine in, 714,725

room in, 713,723

specific treatment of, 714,725

summary of treatment of, 723

symptoms of, 712,723 
Tetanus, treatment of convulsions in, 719, 726 treatment of developed form of, 712

Tetanus antitoxin, 715, 722, 725, 727 combined intraspinal, intravenous, and intramuscular administration of, 726 dosage, $715,716,717,722,723$

intracerebral, administration of, 717,725

intramuscular administration of, $716,717,725$ 726

intraneural administration of, 717,725 intraspinal administration of, 716,725 intravenous administration of, 716,725 prophylactic use of, 721,727

subcutaneous administration of, 715,725 unit of, 715

use of, 715,722

Tetanus ascendens, 711

descendens, 711

Theobromine, as diuretic in scarlatinal nephritis, 420

Theocine, in pleurisy with effusion, 123

Thermokinetic energy, 11

Thoracentesis, in empyema in pneumonia, 181 in pleurisy with effusion, 119,127 technique of, 181

Throat, care of. See under the several diseases compress, in tonsillitis, 84

examination of in infections in childhood, 443

Thrombophlebitis, complicating typhoid fever 302

in septicemia, 762

in septicopyemia, 758

Thromboplastin, in hemorrhage of typhoid fever. 296

in nosebleed of diphtheria, 467

Thrombosis of pneumonia, 190, 206

Thymus gland in mumps, 550

Ticks, and Rocky mountain spotted fever, 664

Tick fever, 664 and see Rocky mountain spotted fever

Tissue destruction, in acute infections, 450

Tissue metabolism, creatinin as indicator of, 23

Tongue, care of, in pneumonia, 148

in scarlet fever, 396

in small pox, 610

in typhoid fever, 280

coated, in acute infectious diseases, 18

Tonics, in convalescence from diphtheria, 474

from grip, 225, 233

from epidemic influenza, 261

from infectious jaundice, 733

from pertussis, 536

Tonsillitis, 80

adenitis in, 86

care of body in, 83,89

care of bowels in, 82,88

care of heart in, 85

chronic, 87,91

circulation in, 85

complications in, 86

convalescence from, 87,90

cultures in. 81

diet in, 82,88

differentiated from diphtheria, 80

drinks in, 82,88

drugs in, 82,89

etiology of, 80

fever in, 83,89

gargle in. 84,90

in epidemic influenza, 256

in grip, 227,233

in poliomyelitis, 597

insomnia in, 84, 89

local treatment in, 84,89

nephritis in, 86

peritonsillar abscess in, 86

precautionary measures in, 81

and rheumatism in childhood, 30

septic sore throat in, 85

septicemia in, 86

sequelæ of, 86

serum therapy in, 86

summary of treatment of, 89

symptomatology of, 81
Tonsillitis, throat compress in, 84

toxemia in, 85,90

treatment of, 81

urine, examination of, 85

Tonsils, 80

attention to in bronchitis of children, 108, 111

in glandular fever, $\mathbf{5 5 6}$

in rheumatism, 62

removal of, indications for, 88

in pertussis, 537

in pneumonia, 193

Toxemia, in acute infectious diseases, 20

caloric intake and, 28

factor in feeding in infectious diseases, 18

factor in fever, 19

hydrotherapy aimed at, 5

in glandular fever, 556

in Malta fever, 659

in pneumonia, 167

in tonsillitis, 85

meaning of, 757

parallelism of with pyrexia, 1

tissue destruction due to, 450

Toxins, 453

effect on nerve centers in febrile conditions, 5 factor in nitrogen loss in infectious diseases, 20

Tracheitis, 101, and see Bronchitis, acute in grip, 224, 232

Tracheotomy, in diphtheria, 464

in edema of larynx, 99

Transfusion of blood, in delayed resolution in influenza, 252

in erysipelas, 777

in hemorrhage of typhoid fever, 297

in pneumonia, in epidemic influenza, 252

in septicemia, 766

methods of, 767

Trench fever, 751

aches and pains in, 752,754

anemia in, 752,755

bed in, 752

cardio-vascular disturbances in, 753,754

care of patient in, 752

carriers of, 754

catharsis in, 752

complications of, 753

convalescence in, 753,755

delousing in, 753

diet in, 752, 754

disinfection after, 753

disposition of excretions in, 752

drinks in, 752, 754

fever in. 752

incubation period of, 751

insomnia in, 752,755

isolation in, 752, 754

louse and, 751,753

mode of conveyance of, 751,753

nervousness in, 752,755

prognosis of, 752

prophylaxis of, 753,755

rest in, 752

sequelæe of, 753,755

summary of treatment of, 754

symptomatology of, 751

Trional, in dengue, 643

in epidemic influenza, 246

in measles, 495

in mumps, 551

in pertussis, 533

in pneumonia, 169

in poliomyelitis, 596

in rheumatism, 37,68

in scarlet fever, 407

in septicemia, 762

in small pox, 614

in tonsillitis, 84,89

in typhoid fever, 298

Trismus, 712

Tuberculosis, complicating epidemic influenza, 255

complicating measles, 502

Turkey, food value of, 17 
Turpentine, in bronchitis, 105, 109

in black vomit of yellow fever, 739

in laryngitis complicating measles, 499

in pneumonis. 152

in tracheitis of grip, 224

in tympanites of typhoid fever, 293

Turpentine stupes, for pain in bacillary dysentery, 363

in pneumonia, 152

preparation of, 363

Tympanites. in bronchopneumonia complicating measles, 498

in epidemic influenza, 251, 267

in pneumonia, 152, 199

in typhoid fever, 292, 294, 314

Typhoid fever, 277

bed in, 279

bedsores in, 280, 310

Brand bath in, 288

care of alimentary tract in, 292, 314

of body in, 280,309

of bowels in, 292, 314

of circulation in, 298, 317

of teeth in, 280

of tongue in, 280

of urinary tract in, 300,318

carriers of, 304, 319

complications of, 302,318

convalescence from, 303,318

diet in, 282, 311

disinfection in, 281, 310

drinks in, 314

etiology of, 277

gastric distress in, 295, 315

hemorrhage in, 295, 316

bydrotherapy in, 287, 298, 314

isolation in, 304

lipovaccines in, 309

meningismus in, 302

mortality in, 277

influence of hydrotherapy on, 287

open air treatment of, 279

packs in, 291

pathology of, 277

perforation in, 297

phlebitis in, 302

physiology of diet in, 3

precautions for nurse in, 311

for physician in, 311

preventive inoculation for, 306, 319

prophylaxis from, 303, 319

pulse in, 290

rest in, 278

revaccination in, 308

room in, 279,309

slush in, 291

summary of trestment in, 309

symptomatology of, 277

therapy of, 278

treatment of cerebral disturbances in, 298, 316

of convalescence in, 303, 319

of diarrhea in, 294, 315

of hemorrhage in, 296,316

of tympanites in, 292, 314

of vomiting in, 295, 315

use of alcohol in, 299

vaccine therapy in, 301, 318

vomiting in, 295,315

walking, 278

water in, 287

Ziemsen's bath in, 291

Typhoid state, 300

Typhus fever, 625

bed in, 626

care of body in, $626,627,631$

of bowels in, 629,632

of circulation in, 629

of hair in, 626

of mouth and nose in, 628, 632

of patient in, 627

of teeth in, 628

cardiac weakness in, 626

complications of, 630

convalescence from, 630,633
Typhus fever, diet in, 628, 632

disinfection in, $626,627,631,633$

incubation period of, 625

isolation in, 626,631

louse and, 625,626

nervous manifestations in, 629

onset of, 625

open air treatment of, 630,632

otitis complicating, 630

parotitis complicating, 630,633

prophylaxis in, 631,633

room in, 626,631

summary of treatment of, 631

treatment of constipation and meteorism in, 629

of delirium in, 629,632

of diarrhea in, 630,633

of edema of larynx in, 630, 633

of fever in, 628

of headache in, 629

of laryngitis in, 630

of nausea and vomiting in, 629

of stupor in, 632

Ulcerative stomatitis, complicating measles, 499 , 509

in varicella, 517

Ulceration, in foot and mouth disease, 695

Ulcers, in bacillary dysentery, 367, 373

in chronic farcy, 688

in foot and mouth disease, 695

in measles, 501

in pertussis, 554

in small pox, 611,612

Undulating fever, 656, and see Malta fever

Unger, on blood transfusion, 767

Uremia, in cholera, 650

in diphtheria, 473

in scarlet fever, 415

Urea-forming function, 25

Urethane, for convulsions in pertussis, 536,543

Uric acid, in endogenous metabolism, 23

excreted by kidneys in febrile conditions, 22, 24

Urinals, disinfection of, 281

Urinary tract, care of in typhoid fever, 300, 318

Urine, bacteria in, in typhoid fever, 300

creatin in, 24

disinfection of, 281

examination of, in tonsillitis, 85

Folin's analysis of, 22

nitrogen in, 22

retention of, in epidemic influenza, 238

in Malta fever, 658

in plague, 635

in poliomyelitis, 594

in tetanus, 714

in typhoid fever, 300

suppression of, in yellow fever, 739

typing of, in pneumonia, 132

Urticaria, complicating rheumatism, 57

due to antipyretics, 223

due to diphtheris antitoxin, 459

due to quinine, 333

due to salicylates, 40

due to sensitization in pneumonia, 134, 135

due to serum in pneumonia, 144

Urotropin, in bacilluria in typhoid fever, 300

in cerebrospinal meningitis, 567

in poliomyelitis, 597

Vaccination, in small pox, 616

complications of, 618

contraindicstions to, 619

general symptoms of, 618

technique of, 617

Vaccination, preventive against typhoid fever, 306,308

Vaccine therapy. See under the several diseases

Vaccinia generalized, following vaccination, 619

Vallet's mass, for anemia following diphtheria, 474

in scarlatinal nephritis, 423

of trench fever, 755

in convalescence from measles, 502 
Vallet's mass, from glandular fever, 557 from pertussis, 537

Van Noorden, on diet for adults, 416 on gastrointestinal function, 18,26

Varicella, 515,

care of bowels, 517, 519

of patient in, 515,518

of skin in, 516,518

complications of, 517,519

convalescence from, 517-519

corneal ulcer in, 519

diet in, 516, 518

disinfection in, 517, 519

distribution of family in, 515, 517

nervous symptoms in, 517,519

precautions for physician in, 518

quarantine in, 517,519

room in, 515

stomatitis in, 517, 519

summary of treatment of, 517

treatment of fever in, 517, 519

Variola, 608, and see Smallpox

Vedder, method of giving quinine in malaria, 329 on emetine in amebic dysentery, 377,379

Venesection, for pulmonary edema in pneumonia, 178

in uremia in scarlatinal nephritis, 420

Venous thrombosis complicating rheumatism, 57

Ventilation, ill, evils of, 4

Ventricles, dilatation of, in rheumatism, 54 puncture of, in cerebrospinal meningitis, 576

Veronal, for sleeplessness in pneumonia, 169 for sleeplessness in rheumatism, 37

Vincent's angina, 91

care of teeth in, 92,93

diagnosis of, 91

etiology of, 91

malnutrition in, 92, 93

removal of membrane in, 92

severe cases of, 92,93

summary of treatment of, 92

symptoms of, 91

Virus fixe, in rabies, 703

Vitamines, 27, 28

fat soluble A, 27

water soluble B, 27

Voit's figures concerning protein needs, 14

Vomiting. See under the several diseases and cardiac involvement, 46 from antipyretics, 223

from salicylates, 46

Vomitus, disinfection of, 281

Vulva, noma of complicating measles, 500

Vulvitis, complicating mumps, 550, 553

Walking typhoid, 278

Ward, on puerperal sepsis, 766

Wasting, in typhoid fever, 283

Water. See Baths and under the several diseases cold, effects of in febrile conditions, $3,4,5,9$ insufficiency of, in infectious diseases, 18

intake of, in infections, 3,4

locally applied in febrile conditions, 2 needed in fever, 29

Waldeyer's ring, 80

Wallach, on blood typing in pneumonia, 131

Warburg's tincture, in malaria, 334

Weaver, on scarlet fever, 424 on dosage of diphtheria antitoxin, 455
Weaver, on vaccines in scarlet fever, 407

Wegeforth, on lumbar puncture, 573

Weight, caloric requirements and, 12

Weight rule, for dosage, 37

Weil's disease, 728, and see Infectious jaundice

Welch, on use of human serum, 768

Wellman, on dosage of quinine in malaria, 329

Western, on puerperal sepsis, 765

Weston, on vaccines in scarlatinal otitis media, 411

Wet compress, technique, 97

Wherry, on prophylaxis in cholera, 651

Whey, food value of, 17

White's instructions on yellow fever, 736

Whoop, in whooping cough, 521

Whooping cough, 520, and see Pertusgis

Wickman, on types of poliomyelitis, 597

Wintergreen, oil of, 43 , and see Salicylates

Wolf and Lambert, 25

on pneumonia,

Woolen underclothing, in convalescence from scarlatinal nephritis, 423

Wooley, on leprosy; 673

Wool sorter's disease, 679, and see Anthrax

Wound, treatment of in rabies, 700,708

in tetanus, 722

Wright, Sir A. E., preventive inoculation in typhoid fever, 306

$\mathrm{X}$-ray treatment of leprosy, $\mathbf{6 7 4}$

Yellow fever, 735

backache in, 738,742

bed in, 737

black vomit in, 739,742

blood pressure in, 735

care of body in, 738

of bowels in, 738, 741

cause of, 735

circulation in, 739, 742

congestion of kidneys in, 739, 742

convalescence in, 740, 743

diet in, 737, 741

drinks in, 738,741

fever in, 739, 742

fumigation in, 737

gastric distress in, 738, 742

headache in, 738,741

isolation in, 735,741

precautions for non-immunes in, 740

for nurses in, 737, 741

for physicians in, 737, 741

prophylaxis in, 740,743

relief of black vomit in, 739

rest in, 737

room in, 736

specific treatment of, 738

summary of treatment of, 741

suppression of urine in, 739

symptoms of, 735

transmission of, 735

treatment of symptoms in, 738, 741

Yeo, on quinine in grip, 224

Young's rule for dosage, 37

Zeiler's method of using ipecac in amebic dysentery, 376

Ziemssen's bath, in typhoid fever, 291

Zingher, on human serum in scarlet fever, 408 



THIS BOOK IS DUE ON THE LAST DATE STAMPED BELOWे

AN INITIAL FINE OF 25 CENTS WILL BE ASSESSED FOR FAILURE TO RETURN THIS BOOK ON THE DATE DUE. THE PENALTY WILL INCREASE TO 50 CENTS ON THE FOURTH DAY AND TO \$1.00 ON THE SEVENTH DAY OVERDUE.

\section{FEB 241933}

MAR 41942 


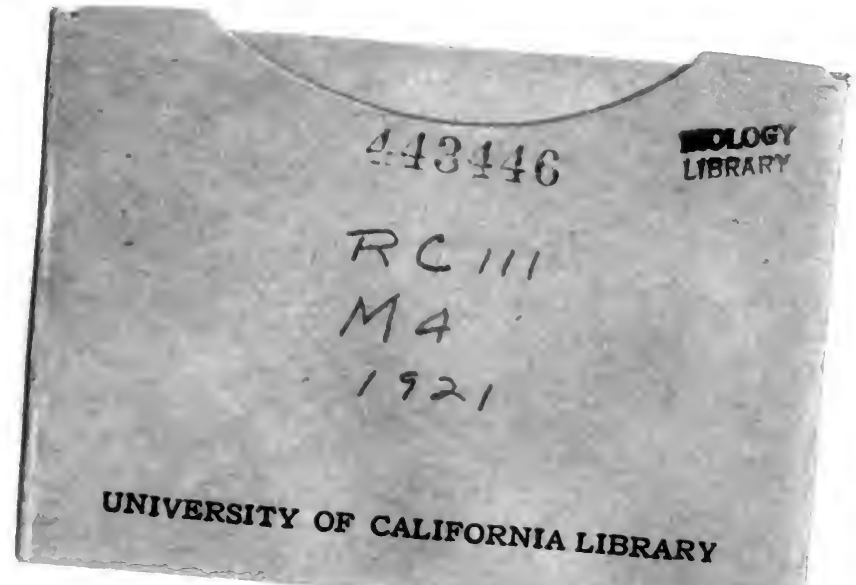


